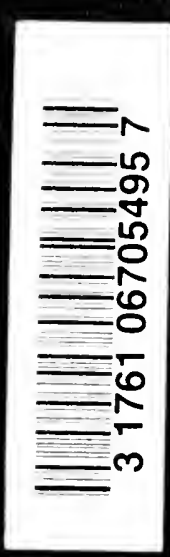




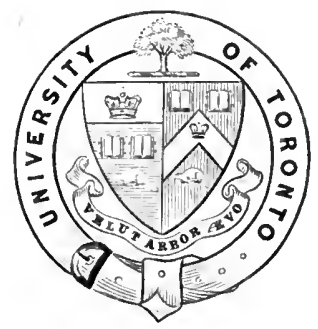

Prescutio to

The Tibrary

of tbc

University of Toronto

bu

D. A.A. Powell. 


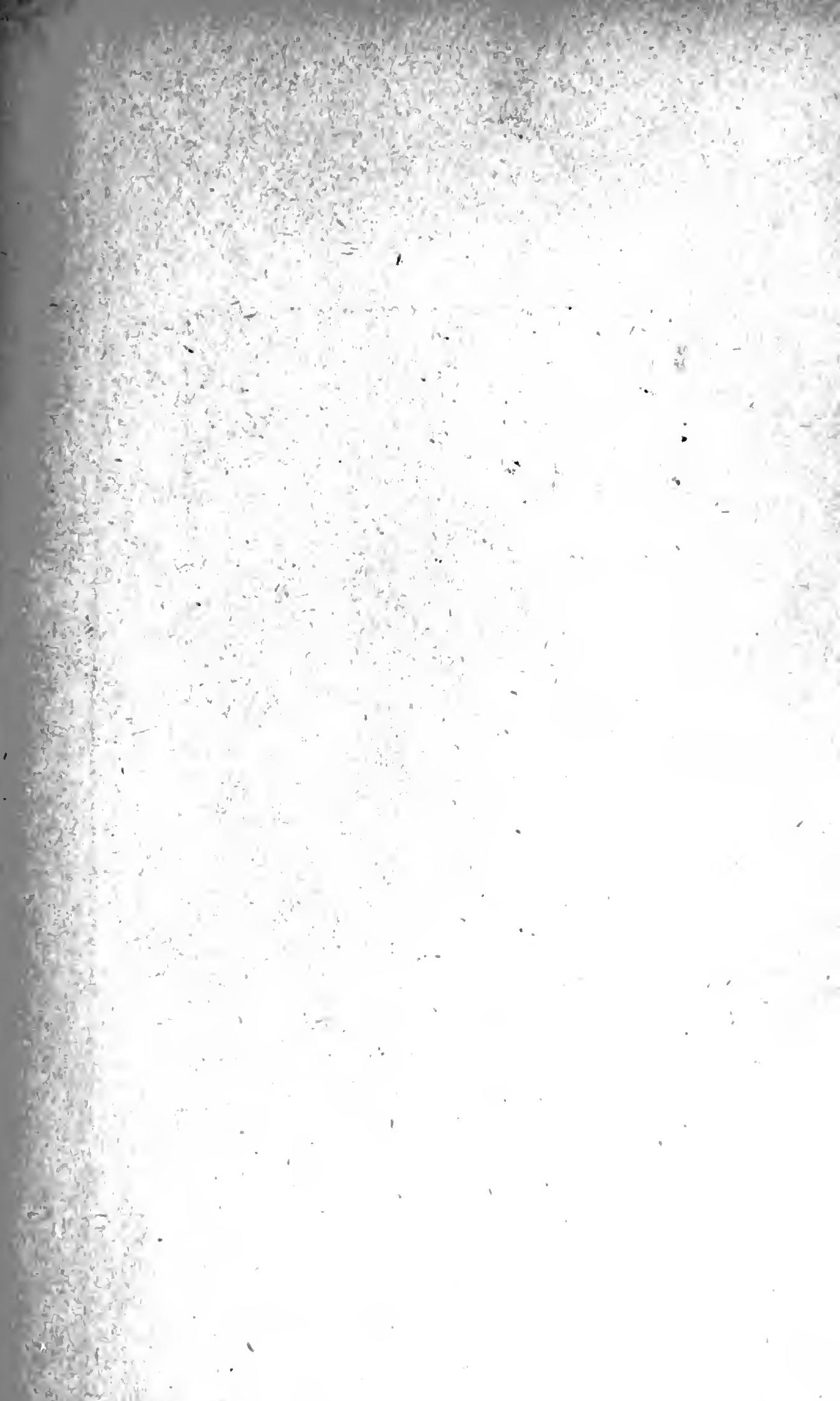


s. 4 1.

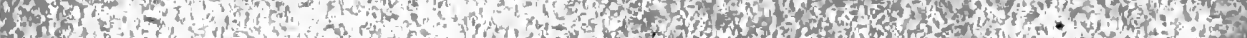

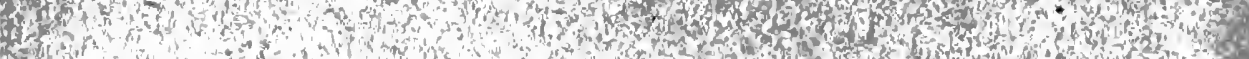
1616

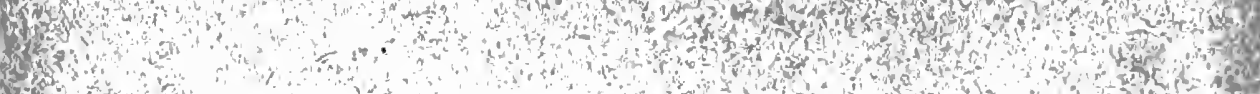

(1)

A.

19.

$4 x \rightarrow x^{2}$

sis:

tot हैं

14

sitas?

afth

Gite.

totitis

and

1.
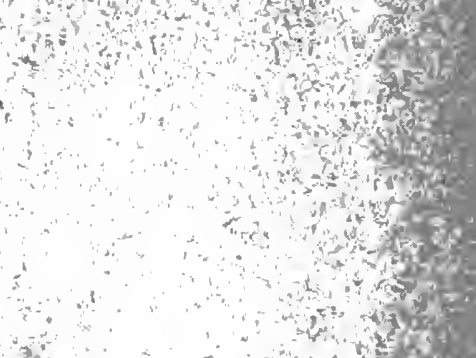

is

wex

:
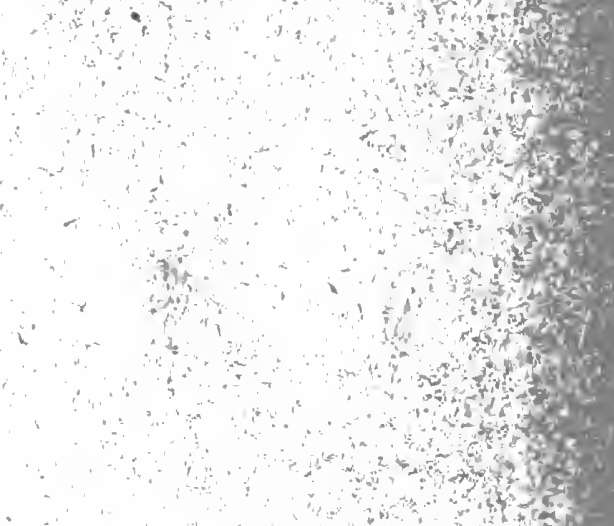

Silo.

int 20.

w.

2y

a de

$\therefore$, $x^{2}$

(ot
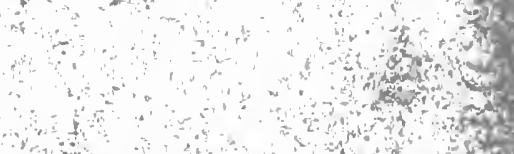

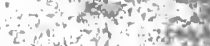

1.

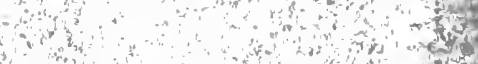

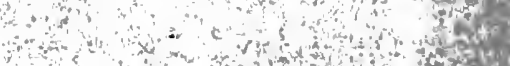

an

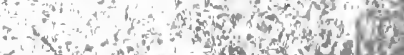

"gi

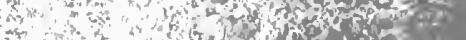


$$
1
$$

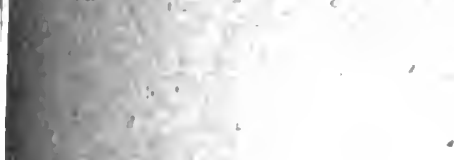

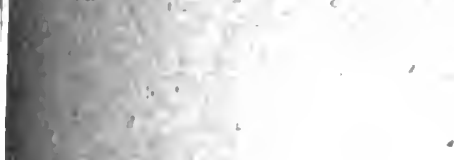
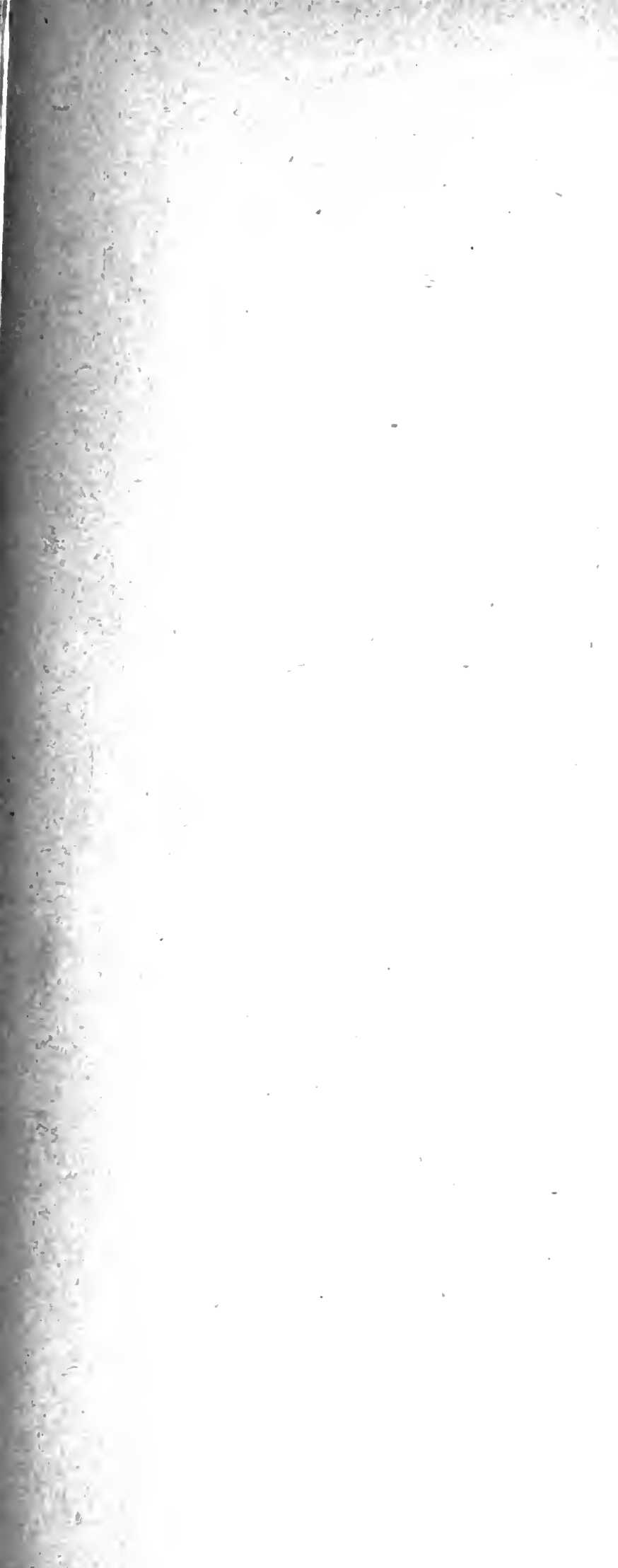



\section{Digitized by the Internet Archive in 2007 with funding from Microsoft Corporation}




\section{CONTRIBUTORS TO THE WORK}

Alexander C. Abbott, M.D. M. Howard Fussell, M.D.

Isaac A. Abt, M.D.

Sir Clifford Allbutt, M.D.

James M. Anders, M.D.

John F. Anderson, M.D.

Bailey K. Ashford, M.D.

J. Harold Austin, M.D.

Lewellys F. Barker, M.D.

M. T. Barrett, D.D.S.

Joseph Colt Bloodgood, M.D.

George Blumer, M.D.

Sir Lauder Brunton, M.D.

Charles W. Burr, M.D.

Richard C. Cabot, M.D.

James Carroll, M.D.

Henry T. Chickering, M.D.

John G. Clark, M.D.

Rufus Cole, M.D.

Warren Coleman, M.D.

Matthew H. Cryer, M.D.

John B. Deaver, M.D.

Clinton T. Dent, M.C.

F. X. Dercum, M.D.

G. E. deSchweinitz, M.D.

Walter J. Dilling, M.B.

Samuel G. Dixon, M.D.

A. R. Dochez, M.D.

George Dock, M.D.

Isadore Dyer, M.D.

David L. Edsall, M.D.

Wm. A. Edwards, M.D.

Charles A. Elsberg, M.D.

Arthur W. Elting, M.D.

John M. T. Finney, M.D.

Charles H. Frazier, M.D.
Thomas B. Futcher, M.B.

John H. Gibbon, M.D.

Joel E. Goldthwait, M.D.

J. L. Goodale, M.D.

Edward H. Goodman, M.D.

William C. Gorgas, M.D.

Maurice C. Hall, Ph.D.

Samuel McC. Hamill, M.D.

H. A. Hare, M.D.

Charles H. Harrington, M.D.

Ludvig Hektoen, M.D.

Albion W. Hewlett, M.D.

Guy Hinsdale, M.D.

John Homans, M.D.

John Howland, M.D.

Guy L. Hunner, M.D.

Chevalier Jackson, M.D.

Henry Jackson, M.D.

Theodore C. Janeway, M.D.

John H. Jopson, M.D.

Thomas C. Kelly, M.D.

E. B. Krumbhaar, M.D.

Charles Krumwiede, Jr., M.D.

Maynard Ladd, M.D.

Egbert LeFevre, M.D.

James Hendrie Lloyd, M.D.

G. Hudson-Makuen, M.D.

W. McKim Marriott, M.D.

Charles F. Martin, M.D.

Edward Martin, M.D.

Charles H. Mayo, M.D.

William J. Mayo, M.D.

Alexius McGlannan, M.D.

R. Tait McKenzie, M.D.

Joseph L. Miller, M.D.
H. C. Moffitt, M.D.

Jesse M. Mosher, M.D.

Sir B. G. A. Moynihan, M.S.

George P. Müller, M.D.

John H. Musser, Jr., M.D.

Edward Osgood Otis, M.D.

Henry K. Pancoast, M.D.

Roswell Park, M.D.

William H. Park, M.D.

R. M. Pearce, M.D.

George Morris Piersol, M.D.

Charles S. Potts, M.D.

Charles W. Richardson, M.D.

David Riesman, M.D.

Samuel Robinson, M.D.

Milton J. Rosenau, M.D.

Leonard G. Rowntree, M.D.

Joseph Sailer, M.D.

Jay Frank Schamberg, M.D.

Henry Sewall, M.D.

Bertram W. Sippy, M.D.

Allen J. Smith, M.D.

William G. Spiller, M.D.

J. Dutton Steele, M.D.

Alfred Stengel, M.D.

Charles G. Stockton, M.D.

Homer F. Swift, M.D.

James E. Talley, M.D.

E. W. Taylor, M.D.

W. Gilman Thompson, M.D.

James Tyson, M.D.

George H. Weaver, M.D.

J. William White, M.D.

Alfred C. Wood, M.D.

H. C. Wood, Jr., M.D. 


\title{
A HANDBOOK OF PRACTICAL
}

\section{TREATMENT}

\author{
BY MANY WRITERS
}

)

JOHN H. MUSSER, Jr., B.S., M.D.

ASSOCIATE IN MEDICINE IN THE UNIVERSITY OF PENNSYlVANIA, PHILADELPHIA

$$
\text { AND }
$$

S

THOMAS CI KELLY, A. M., M.D.

INSTRUCTOR IN MEDICINE IN THE UNIVERSITY OP PENNSYLVANIA, PUILADELPHLA

\section{VOLUME IV}

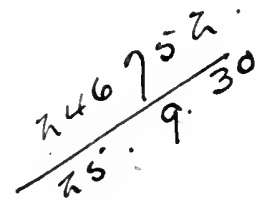

PHILADELPHIA AND LONDON

W. B. SAUNDERS COMPANY 
Copyright, I9I7, by W. B. Saunders Company

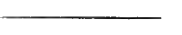

PRINTED IN AMERICA

PRESS OF

W. B. SAUNDERS COMPANY

PHILADELPHIA 


\section{PREFACE TO VOLUME IV}

THE fourth volume of A Handbook of Practical Treatment has been brought out for the purpose of giving the various original contributors opportunity of making in their articles such change or modifications as have occurred in the therapeusis of those diseases the treatment of which they have already detailed. Thus we believe it possible to supplement their contributions and to make them complete, both as to detailed treatment and as to the newer and mormodern procedures. In the treatment of some of the disorders, practically no fundamental or minor change has occurred in the principles as outlined originally. Such diseases have not been considered in this supplementary volume.

It has not been possible in every case to secure as a contributor the author of the original monograph. Death has carricd off not only the two editors of the first volumes, but also many of the authors of individual sections. Two, Dr. J. William White and Sir Lauder Brunton, have died in the past year since rewriting their articles. Several of the original contributors have been unable for various reasons to undertake a revision of their articles. These authors have been replaced, when occasion demanded it, by others, who in order that they might be given a free hand have been asked to rewrite the article completely so that they might have the opportunity of expressing their own views upon the subject and not be compelled to subscribe to ideas and methods with which they might not be fully in accord. As a result of this plan Dr. Leonard G. Rowntree has completely revised the article upon Nephritis; Dr. Maurice C. Hall has rewritten the article on Diseases due to Animal Parasites; Dr. William C. Gorgas has contributed an article on the General Aspects of Yellow Fever; Dr. Bailey K. Ashford has revised fully the section on Tropical Diseases; Dr. Charles S. Potts has considered fully Electrotherapy; Dr. John B. Deaver has reviewed Surgery of the Liver and Gall-Bladder; Dr. Joseph L. Miller has rewritten Diseases of the Blood; and Dr. Joseph Sailer has supplemented the article on Diseases of the Intestines. The editors have made such changes as seem indicated in the articles on Pertussis, Mumps and Asthma. Dr. Henry T. Chickering has collaborated with Dr. Rufus Cole in the preparation of the article on Typhoid Fever, and Dr. Walter J. Dilling with Sir Lauder Brunton in the latter's section on 
the General Principles of Drug Treatment. Drs. George Morris Piersol, Edward H. Goodman and Alexius McGlannan have revised those sections that they originally prepared in association with other authors.

In order to incorporate some of the more specialized new forms of treatment not included in the previous volumes, an article has been contributed by Dr. Homer F. Swift on the Treatment of Cerebrospinal Syphilis; Dr. J. L. Goodale on the Treatment of Hay Fever and Hay Asthma; Dr. John Howland and Dr. W. McKim Marriott on Acidosis in Children; Dr. W. Gilman Thompson on Occupational Diseases; Dr. Samuel G. Dixon on the Governmental Prophylaxis of Disease; Dr. William H. Park and Dr. Charles Krumwiede, Jr. on Specific Therapy; Dr. Allen J. Smith and Dr. M. T. Barrett on Peridental Suppuration; Dr. E. B. Krumbhaar and Dr. R. M. Pearce on Splenectomy as a Therapeutic Procedure in the Anemias; Dr. A. R. Dochez on the Specific Therapy of Pneumococcic Infections; Dr. Joel E. Goldthwait on the Postural Treatment of Abdominal and Thoracic Visceral Disturbances; Dr. Charles A. Elsberg on Surgical Treatment of Diseases of the Spinal Cord; and an article on Erysipelas, the consideration of which was omitted in the previous volumes, has been prepared by Dr. J. Harold Austin. Dr. Alfred Stengel has written an introductory article on the Modern Treatment of Disease. We believe that there have been incorporated in this supplementary volume all the newer methods of treatment that have been shown to be efficacious in the handling of disease, and that the new contributions will present these subjects in a convenient form to the profession, while such changes that have occurred, since the publication of the last volumes, in the treatment of the conditions previously taken up, has been fully covered by the original authors or those who have replaced them.

The editors wish to thank the contributors for their hearty co-operation, which has materially assisted them through the prompt preparation of their manuscripts, as well as the publishers, who have lent every possible aid to the rapid completion of the work: JoHN H. Musser, JR. Thomas C. Kelly.

April, I9I 7. 


\title{
CONTRIBUTORS TO VOLUME IV
}

\author{
ISAAC ARTHUR ABT, M.D.
}

Professor of Diseases of Children, Northwestern University Medical School; Attending Physician, Sarah Morris Children's Hospital of Michael Reese Hospital, Chicago

SiR Thos. ClifFord Allbutt, K.C.B., M.D., HoN. D.SC., F.R.S., F.R.C.P., LOND. (HON.)

Colonel R. A. M. C., Regius Professor of Medicine in the University of Cambridge; Fellow of Gonville and Caius College, Cambridge; Consulting Physician to the Military Hospital for Functional Diseases of the Heart, Hampstead, London

\section{JAMES M. ANDERS, M.D., PH.D., LL.D.}

Professor of Medicine and Clinical Medicine at the Medico-Chirurgical Hospital, Philadelphia; Consulting Physician to the Jewish Hospital and to the Widener Home for Crippled Children, Philadelphia; Officier de l'Instruction Publique

\section{JOHN F. ANDERSON, M.D.}

Formerly Surgeon and Director of the Hygienic Laboratory, U. S. Public Health Service; Consulting Pathologist to the Middlesex County General Hospital and St. Peter's General Hospital, New Brunswick, N. J.

\section{BAILEY K. ASHFORD, M.D., SC.D.}

Lieutenant-Colonel, Medical Corps, U. S. Army; President of a Board for the Study of Tropical Diseases as they Exist in Porto Rico; Collaborator with the

Institute of Tropical Medicine and Hygiene of Porto Rico; Member of a Medical Commission to Brazil, International Health Board; Fellow of the Society of Tropical Medicine of London

\section{J. HAROLD Austin, M.D.}

Associate in Medicine, University of Pennsylvania; Assistant Physician, University of Pennsylvania

LEWELLYS F. BARKER, M.D. Professor of Clinical Medicine, Johns Hopkins University

M. T. BARRETT, D.D.S., M.S.

JOSEPH COLT BLOODGOOD, B.S., M.D.

Associate Professor of Clinical Surgery, Johns Hopkins University; Senior Surgeon to St. Agnes Hospital, Baltimore, Md. 
GEORGE BLUMER, M.D.

Dean and John Slade Ely Professor of the Theory and Practice of Medicine in the Yale Medical School, Attending Physician to the New Haven Hospital

SiR T. LAUder BRUNTON, BART., M.D., D.SC., LL.D. (EDIN. AND ABERD.), M.D. HON. (DUBLIN), F.R.C.P., F.R.S.

Late Consulting Physician and Lecturer on Pharmacology and Therapeutics to St.

Bartholomew's Hospital; Late Correspondant Etranger de la Societe de Therapeutique, Paris

HENRY THORNDYKE CHICKERING, A.B., M.D.

Resident Physician, Hospital of the Rockefeller Institute for Medical Research, New York City

\section{JOHN G. CLARK, M.D.}

Professor of Gynecology, University of Pennsylvania; Gynecologist-in-Chief to the Hospital of the University of Pennsylvania

RUFus COLE, M.D.

Director of the Hospital of the Rockefeller Institute for Medical Research, New York City

JOHN B. DEAVER, M.D., SC.D., LL.D.

Professor of the Practice of Surgery, University of Pennsylvania; Surgeon-in-Chief, German Hospital, Philadelphia, Pa.

FranCIS X. DERCUM, M.D., PH.D.

Professor of Nervous and Mental Diseases, Jefferson Medical College, Philadelphia;

Foreign Corresponding Member of the Neurological Society of Paris; Cor-

responding Member of the Neurologic and Psychiatric Society of

Vienna; Member of the Royal Medical Society of Budapest;

Consulting Neurologist to the Philadelphia General

Hospital

\section{G. E. DESCHWEINITZ, M.D., LL.D.}

Professor of Ophthalmology in the University of Pennsylvania; Ophthalmic Surgeon to the University Hospital, Philadelphia

Walter James Dilling, M.B., CH.B. (ABERD.)

Dr. Robert Pollok Lecturer in Materia Medica and Pharmacology, Glasgow University; Examiner in Materia Medica, Pharmacology, Pharmacy and Therapeutics, Liverpool University; Temporary Captain Royal Army Medical Corps

SAMUEL G. DIXON, M.D., LL.D., SC.D.

Commissioner of Health, State of Pennsylvania

ALPHONSE RAYMOND DOCHEZ, A.B., M.D.

Associate Member of the Rockefeller Institute for Medical Research; former Resident Physician to the Hospital of the Rockefeller Institute for Medical Research, New York City 
GEORGE DOCK, M.D., SC.D.

Professor of Medicine, Washington University Medical School; Physician-in-Chief, Barnes Hospital, St. Louis

ISADORE DYER, PH.B., M.D.

Professor of Diseases of the Skin, Tulane University of Louisiana

Charles A. ElsBerG, M.D., F.A.C.S.

Clinical Professor of Surgery, New York University and Bellevue Hospital Medical College; Attending Surgeon to Mount Sinai Hospital and the New York

Neurological Institute

THOMAS B. FUTCHER, M.B., M.D.

Associate Professor of Clinical Medicine, Johns Hopkins University

Charles HarRison Frazier, A.B., M.D., SC.D.

Professor of Clinical Surgery, University of Pennsylvania; Surgeon to University Hospital, Philadelphia

\section{JOHN H. GIBBON, M.D.}

Professor of Surgery, Jefferson Medical College and Surgeon to Pennsylvania Hospital, Philadelphia

JOEL E. GOLDTHWAIT, B.S., M.D., F.A.C.S.

President of the Corporation of the Robert B. Brigham Hospital; Professor of Hygiene and Physical Education of Smith College; Ex-President of the American Orthopedic Association; Formerly Chief of the Orthopedic Service at the Massachusetts General Hospital

J. L. GOODALE, M.D.

Instructor in Laryngology, Harvard Medical School

EDWARD HARRIS GOODMAN, M.D.

Associate in Medicine, University of Pennsylvania; Assistant Physician to the Presbyterian and University Hospitals, Philadelphia; Pathologist to Children's Seashore House, Atlantic City

WILLIAM CRAWFORD GORGAS, M.D., LL.D., SC.D. Surgeon-General U. S. Army

MaURice C. Hall, PH.D., D.V.M.

Parasitologist, Research Laboratory, Parke, Davis and Company, Detroit, Mich.;

formerly Assistant Zoologist, U. S. Bureau of Animal Industry, and

Assistant Zoologist, U. S. Insecticide and Fungicide Board

HOBART AMORY HARE, M.D., B.SC.

Professor of Therapeutics and Diagnosis in the Jefferson Medical College; Physician to the Jefferson Hospital; former Clinical Professor of Diseases of Children in the University of Pennsylvania 
GUY HinsDale, A.M., M.D.

Associate Professor of Climatology, University of Pennsylvania: Secretary of the American Climatological and Clinical Association; Fellow of the Royal Society of Medicine

JOHN HOWLAND, B.A., M.A., M.D.

Professor of Pediatrics, the Johns Hopkins University, Department of Medicine

GUY L. HUNNER, S.B., M.D.

Associate in Clinical Gynecology, Johns Hopkins University

CHEVALIER JACKSON, M.D.

Professor of Laryngology, Jefferson Medical College; Professor of Laryngology, University of Pittsburgh; Professor of Bronchoscopy and Esophagoscopy,

New York Post-Graduate Medical School and Hospital

THEODORE C. JANEWAY, M.D.

Professor of Medicine, the Johns Hopkins University: Physician-in-Chief, the Johns Hopkins Hospital, Baltimore

JOHN H. JOPSON, M.D.

Professor of Surgery at the Philadelphia Polyclinic: Associate in Surgery in the University of Pennsylvania: Surgeon to the Presbyterian and Children's

Hospitals of Philadelphia; Surgeon to Bryn Mawr Hospital

\section{THOMAS C. KELLY, A.M., M.D.}

Instructor in Medicine in the University of Pennsylvania and Physician to the Medical Dispensary of the University Hospital, Philadelphia; Pediatrist to St. Mary's Hospital; Physician to the Prophylactic Dispensary of the Babies' Hospital, Philadelphia

EDWARD BEll KRUMBhaAR, A.B., M.D., PH.D.

Assistant Professor of Research Medicine, and Associate in Medicine, University of Pennsylvania

Charles KRUMWIEdE, JR., M.D.

Assistant Director, Bureau of Laboratories, Department of Health, New York City; and Assistant Professor of Bacteriology and Hygiene, University and Bellevue Medical School, New York University

MAYNARD LADD, A.B., M.D.

Instructor in Pediatrics, Harvard Medical School: Physician-in-Chief to the Children's Hospital Department of the Boston Dispensary; Consulting Physician to the Children's Hospital, the Floating Hospital, and Nursery for Blind Babies

\section{JAMES HENDRIE LLOYD, A.M., M.D.}

Neurologist to the Philadelphia General Hospital and to the Methodist Episcopal Hospital; Consulting Neurologist to the Elwyn Institute for Feebleminded Children, Elwyn, $\mathrm{Pa}$., and to the State Hospital for the Chronic Insane, Wernersville, $\mathrm{Pa}$. 
G. HUDSON-MAKUEN, M.D.

Late Professor of Defects of Speech in the Philadelphia Polyclinic; Late Consultant

Laryngologist and Otologist to the Roosevelt and Frederick Douglass

Hospitals, Philadelphia

WILLIAMS MCKIM MARRIOTT, B.S., M.D.

Associate in Pediatrics, the Johns Hopkins University

CHARLES F. MARTIN, M.D.

Professor of Medicine, McGill University; Attending Physician to the Royal Victoria Hospital, Montreal

CHARLES H. MAYO, M.D.

WILliam J. MAYO, A.M., M.D., F.R.C.S., LL.D., D.SC.

Alexius MCGLanNan, A.M., M.D.

Professor of Clinical Surgery, University of Maryland, Medical Department, and College of Physicians and Surgeons; Surgeon to the Mercy and St. Agnes Hospitals, Baltimore

JOSEPH L. MILLER, B.S., M.D.

Associate Professor of Medicine, Rush Medical College; Attending Physician, St. Luke's and Cook County Hospitals, Chicago

HERBERT C. MOFFITT, M.D.

Professor of the Principles and Practice of Medicine in the University of California

GEORGE P. MÜLLER, M.D., B.S.

Associate in Surgery in the University of Pennsylvania; Professor of Surgery in the Philadelphia Polyclinic; Surgeon to the St. Agnes Hospital; Consulting Surgeon to the Chester County Hospital, West Chester, Pennsylvania

JOHN H. MUSSER, JR., B.S., M.D.

Associate in Medicine, University of Pennsylvania; Physician to Philadelphia General Hospital; Assistant Physician and Chief of Dispensary, Presbyterian Hospital

EDWARD OSGOOD OTIS, A.B., M.D.

Professor of Pulmonary Diseases and Climatology. Tufts College Medical School,

Boston; Member of the American Climatological and Clinical Association and of the International Tuberculosis Association

HENRY K. PANCOAST, M.D.

Professor of Roentgenology, University of Pennsylvania; Roentgenologist to University Hospital

William Hallock PaRK, A.B., M.D., LL.D.

Professor of Bacteriology and Hygiene, University and Bellevue Hospital Medical College; Director of the Bureau of Laboratories, Department of Health. 
RichaRD MiLls PEARCE, M.D., SC.D.

Professor of Research Medicine, University of Pennsylvania

GEORGE MORRIS PIERSOL, B.S., M.D.

Associate in Medicine in the University of Pennsylvana; Assistant Physician to the Hospital of the University of Pennsylvania and to the Philadelphia General Hospital

\section{CHARLES S. POTTS, M.D.}

Neurologist to the Philadelphia General Hospital; formerly Instructor in Electrotherapeutics and Associate in Neurology in the University Hospital, and

Professor of Neurology in the Medico-Chirurgical College, Philadelphia

CHARLES W. RICHARDSON, M.D.

Ppofessor of Laryngology and Otology in the George Washington University; Consulting Laryngologist and Otologist to Providence Hospital; Attending

Laryngologist and Otologist, George Washington University

Hospital; Episcopal Eye, Ear, and Throat Hospital, and the Foundling Asylum, Washington, D. C.

LEONARD G. ROWNTREE, M.D., SC.D.

Professor of Medicine, University of Minnesota; Physician-in-Chief, University Hospital

\section{JOSEPH SAILER, M.D.}

Professor of Clinical Medicine, University of Pennsylvania; Physician to the Philadelphia General, University, and Presbyterian Hospitals; formerly Professor of Diseases of the Stomach and Intestines in the Philadelphia

Polyclinic

JAY FRANK SCHAMBERG, A.B., M.D.

Professor of Dermatology and Infectious Eruptive Diseases, Philadelphia Polyclinic and College for Graduates in Medicine; Late Consulting Physician to the Municipal Hospital for Contagious Diseases

HENRY SEWALl, PH.D., SC.D., M.D.

Professor of Medicine in the University of Colorado

BERTRAM W. SIPPY, M.D.

Professor of Medicine in the Rush Medical College, in affiliation with the University of Chicago; Attending Physician to the Presbyterian and Cook County Hospitals, Chicago

AllEN J. SMITH, A.M., M.D., SC.D. (HON.), LL.D. (HON.)

Professor of Pathology and Comparative Pathology in the Medical School of the University of Pennsylvania

ALFRED STENGEL, M.D., SC.D.

Professor of Medicine, University of Pennsylvania; Physician to the University Hospital and to the Pennsylvania Hospital 
CHARLES G. STOCKTON, M.D.

Protessor of the Principles and Practice of Medicine and Clinical Medicine, Medical Department of the University of Buffalo; Attending

Physician, Buffalo General Hospital

HOMER F. SWIFT, M.D.

Associate Professor of Medicine, Columbia University; Visiting Physician, Presbyterian Hospital, New York City

JAMES E. TALLEY, M.D.

Visiting Physician to the Presbyterian and Methodist Episcopal Hospitals, Philadelphia

EDWARD WYLLYS TAYLOR, A.M., M.D.

Assistant Professor of Neurology, Harvard Medical School; Chief of Service, Neurological Department, Massachusetts General Hospital

W. Gilman THOMPSON, M.D.

Late Professor of Medicine in Cornell University Medical College in New York City; Consulting Physician to Bellevue Hospital and the Woman's Hospital;

Late Visiting Physician to the Presbyterian and New York Hospitals

GEORGE H. WEAVER, M.D.

Attending Physician, Durand Hospital of the Memorial Institute for Infectious Diseases, Chicago

J. WILliam WHITE, PH.D., M.D., LL.D. (ABERD.)

Late John Rhea Barton Emeritus Professor of Surgery, University of Pennsylvania, etc.

\section{ALFRED C. WOOD, M.D.}

Assistant Professor of Surgery, University of Pennsylvania; Surgeon to the Hospital of the University of Pennsylvania, the Philadelphia General, Howard,

St. Timothy's, and Rush Hospitals

HORATIO C. WOOD, JR., M.D.

Professor of Pharmacology and Therapeutics in the University of Pennsylvania 



\section{CONTENTS OF VOLUME IV}

The Modern Treatment of Disease .........................

By Alfred Stenger, M.D.

The Governmental Prophylaxis of Disease............ 17

By Samuel G. Dixon, M.D., LL.D., Sc.D.

The General Principles of Drug Treatment............. 25

By Sir lauder Brunton and Walter J. Dilling, M.B., Ch.B.

Specific Therapy: Bacterital Vaccines and Sera.......... 33

By William H. Park, M.D., and Charles Krumwiede, JR., M.D.

Hydrology in Military Practice................... 83

By Guy Hinsdale, M.D.

Climate and Climatotherapy.

By Henry Sewall, M.D.

ELECTROTHERAPY

By Cularles S. Potts, M.D.

Roentgen Therapy....................... I 22

By HeNRY K. PANCOAST, M.D.

Miscellaneous Therapeutic Procedures.............. 142

By George P. MÚller, M.D.

Food Intoxication and Poisoning by Reptiles and Insects..... I 57 By George Blumer, M.D.

Drug Poisoning and Drug Habits.................... 163

BY H. C. WOOD, JR., M.D.

Prophylaxis and General Treatment of the Occupational DisEASES.

By W. Gilman Thompson, M.D.

\section{INFECTIOUS DISEASES}

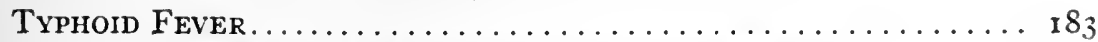

By Rufus Cole, M.D., ANd Hexry T. Chickering, M.D.

Lobar PNeumonia

BY H. A. HARE, M.D.

Specific Therapy of Pneumococcus Infection

By A. R. Dochez, M.D.

DiPHTHERIA

By George H. Weaver, M.D.

SCarlet Fever. Scarlatina 
TUBERCuLOSIS.

$$
\text { By Edward OsGood Otrs, A. B., M.D. }
$$

Septic Toxemia, Septicemia And Pyemia............... 264

$$
\text { By J. HAROLD Austin, M.D. }
$$

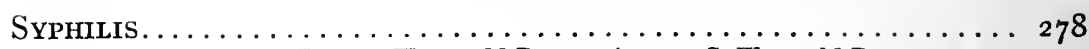
By J. William White, M.D., ANd Alfred C. Wood, M.D.

VACCinia............................... 294 By Jay Frank Schamberg, M.D.

Cerebrospinal Ffver, Cholera Asiatica, Plague.......... 296

Yellow Fever.............................. 299 By William C. Gorgas, M.D.

Rabies, Foot-and-Mouth Disease, and Anthrax.......... 324 By Alexius McGlannan, M.D.

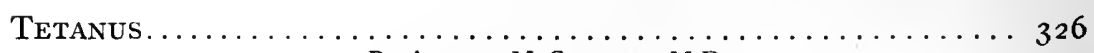
By Alexius McGlannan, M.D.

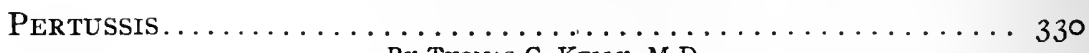
By Thomas C. Kelly, M.D.

Infectious Parotitis-Mumps. $\ldots \ldots \ldots \ldots \ldots \ldots \ldots \ldots \ldots \ldots \ldots \ldots$ By Thomas C. KeLly, M.D.

Amebic Dysentery......................... 337 By Charles F. Martin, M.D.

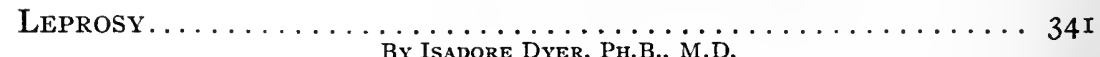

Typhus Fever. . . . . . . . . . . . . . . . . . . 343 BY JOHN F. ANDERSON, M.D.

Rat-Bite Fever...................... 344

By George Blumer, M.D.

BY BAILEY K. ASHFORD, M.D.

The Ocular Complications of the Infectious Diseases...... $38 \mathrm{I}$ By G. E. DeSchweinitz, M.D.

\section{PARASITIC DISEASES}

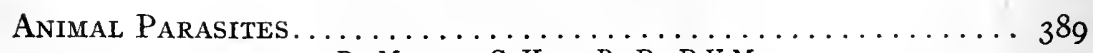
By Maurice C. Hall, Ph.D., D.V.M.

\section{DISEASES OF THE CIRCULATORY SYSTEM}

DisEASES OF THE BLOOD............................. 420 By JoSEPH L. MILLER, M.D.

Splenectomy as a Therapeutic Procedure in the Anemias. . . . 453 By E. B. KrumbhaAr, M.D., and R. M. Pearce, M.D. 
Hodgkin's Diskase..................... 46 I By George Dock, M.D.

Diseases of the Endocrine Glands............... 463 BY George Dock, M.D.

Surgical Treatment of Diseases of the Thyroid Gland....... 470 By Josepal Colt Bloodgood, M.D.

Diseases of the Cardiovascular System............. 476 By Sir Clifford Allbutt, K.C.B., M.D.

\section{CONSTITUTIONAL DISEASES}

Diabetes

By Theodore C. Janeway, M.D.

Treatment of Acidosis in Children.............. 50 I

BY JoHN HOWLAND, M.D., AND W. MCKIM MarRIotT, M.D.

Diabetes Insipidus. . . . . . . . . . . . . . . . 508

BY THOMAS B. FUTCHER, M.B.

ARthritis Deformans.......................... 5 I0 BY Thomas B. Futcher, M.B.

GouT By Thomas B. Futcher, M.B.

Lithemia, Uric Acid Diathesis............... 516 By Thomas B. Futcher, M.B.

\section{DISEASES OF THE RESPIRATORY SYSTEM}

Tonsillitis.

By Charles W. Richardson, M.D.

Defects of Speech. By G. Hudson-Makuen, M.D.

Hay Fever and Hay Asthma.................... 528 By J. L. Goodale, M.D.

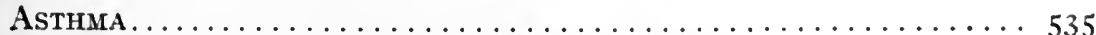

By John H. MUSSER, JR., M.D.

Hemoptysis........................ 54 I

By James E. Talley, M.D.

Pulmonary Edema.......................... 548

By James E. TAlley, M.D.

Pulmonary Colmapse............................ 549

By James E. Talley, M.D.

Embolism, Thrombosis and Infarction of the Lung ....... $55^{\text {I }}$

By James E. Taliey, M.D. 
Treatment of Tracheal and Bronchial Obstruction.........
By Chevalier Jackson, M.D.

Pleuritis............................ 564

By Edward H. Goodman, M.D.

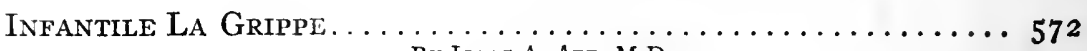
By IsAac A. ABt, M.D.

\section{DISEASES OF THE DIGESTIVE SYSTEM}

General Hygiene of the Mouth.

By George Morris Piersol, M.D.

Chronic Peridental Suppurations, with Especial Reference to Chronic Suppurative Pericementitis or Pyorrhea Alveo-

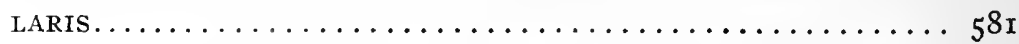
By Allex J. Smith, M.D., and M. T. Barret, D.D.S., M.Sc.

Diseases of the Mouth, Tongue and Salivary Glands . . . . . . 6or By George Morris Piersol, M.D.

Esophagostrostomy aNd Intrathoracic REsection of the Esophagus .........................667 By Charles H. Mayo, A.M., M.D., LL.D., F A.C.S.

Gastric and Duodenal Ulcer....................6 6 By Bertram W. Sippy, M.D.

Surgery of the Stomach and Duodenum............... 629

By William J. Mayo, A.M., M.D., Sc.D., F.R.C.S., F.A.C.S., LL.D.

Diseases of the Intestines. . . . . . . . . . . . . . . 639 By Joseph SaIler, A.B., M.D.

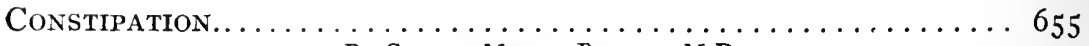
By George Morris Piersol, M.D.

The Surgical Treatment of Intestinal Stasis........... 665 BY JoHN G. ClaRK, M.D.

Digestive Disorders of Infants.................667 By Maynard LAdD, M.D.

Diseases of Liver and Gall-Bladder.............. 685 By Charles G. Stockton, M.D.

Surgery of the Liver and Gall-Bladder... . . . . . . . . . . 693 By John B. Deaver, M.D., LL.D., Sc.D.

Diseases of the Pancreas.................... 706 By James M. Anders, M.D., LL.D.

Visceroptosis......................... 7 I0 By Joseph SAILER, M.D.

The Postural Treatment of the Abdominal and Thoracic Visceral Disturbances................... 7 I 6 By Joel E. Goldthwait, M.D., F.A.C.S. 
Surgical Treatment of Visceroptosis.................. By JoHN H. Grвzos, M.D.

Peritonitis.

\section{THE URINARY SYSTEM}

The Treatment of Nepirritis

By Leonard G. Rowntree, M.D.

Renal Function in Health and Disease. . . . . . . . . . 786 By Leonard G. Rowntree, M.D.

The Cause, Pathological Physiology, and Treatment of the More Important Clinical Manifestatrons of Nephritis... . 800 By Leonard G. Rowitree, M.D.

Surgical Indications in Urinary Tract Diseases.......... 856 By GuY L. HunNer, M.D.

\section{THE NERVOUS SYSTEM}

Surgical Aspects of Certain lesions of tie Central Nervous System. By Charles II. Frazier, M.D.

Diseases of the Spinal Cord. 874 BY E. W. TAYLOR, M.D.

Treatment of Cerebrospinal Syphilis. $88 \mathrm{r}$ By Homer F. Swift, M.D.

The Surgical Treatment of Diseases of the Spinal Cord..... 899 By Charles A. Elsberg, M.D.

The So-Called Functional Nervous Diseases............ go6 By F. X. Dercum, M.D.

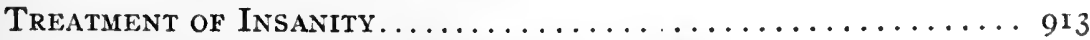
By James Hendrie Lloyd, M.D.

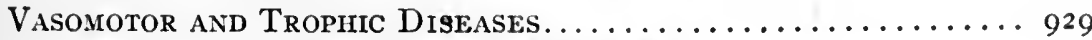
By H. C. MOFFItT, M.D.

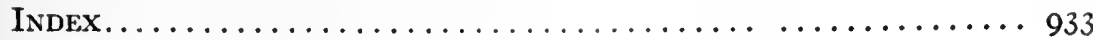





\title{
PRACTICAL TREATMENT
}

\section{THE MODERN TREATMENT OF DISEASE}

\author{
By Alfred Stengel, M. D.
}

That there are currents and counter-currents in the sciences and in such near-sciences or applied arts as medicine scarcely needs discussion, but it is interesting to observe how frequently advancing knowledge leads men to traverse with the new leadership of more modern methods historic ground explored before. Nowhere is this more apparent than in works upon the treatment of disease, and in connection with these it is to be observed that a wide gulf lies between a treatise on pharmacology and one on medical treatment. In the former emphasis should properly be laid upon the data of exact scientific investigation; in the latter the results of clinical experience as well as the application of experimental investigation may suitably be discussed.

Somewhat over forty years ago, Horatio C. Wood, in presenting the first edition of his "Treatise on Therapeutics, comprising Materia Medica and Toxicology, with Especial Reference to the Application of the Physiological Action of Drugs to Clinical Medicine," the first serious attempt made in this country or perhaps in the world to collect in a single volume the data of accurate investigation regarding the action of drugs, says in his preface: "The old and tried method in therapeutics is that of empiricism or, if the word seems harsh, of clinical experience. . . . It is evident that this is not a new path but a highway already worn with the eager but weary feet of the profession for two thousand years.

"Therapeutics developed in this manner cannot, however, rest upon a secure foundation. What to-day is believed is to-morrow to be cast aside, certainly has been the law of advancement, and seemingly must continue to be so.

"Since the profession has toiled so long and found so little, if further progress is to be made, we must question the old methods and search out new ones, which haply may lead to more fruitful fields. - . A primary knowledge of the end to be accomplished, and a secondary acquaintance with the instruments, are a necessity for successful human effort; and until the sway of this law is acknowledged by physicians, medicine can never rise from the position of an empirical art to the dignity of applied science. Until within a comparatively recent period, it has been impossible to comply with this law. But 
through the advances made by the pathologists and by the students of the natural history of disease, we are fast learning the methods in which nature brings the body back to health. When this is donewhen disease is thoroughly understood, we shall have wrought out the first element of the problem, shall have complied with the first requirements of the law.

"The work of the therapeutist is chiefly concerned with the second portion of the law. Evidently it is his especial province to find out what are the means at command, what the individual drugs do when put into the human system."

With these and further thoughts, the then youthful Wood dedicated himself to his life's work of developing a scientific therapeutics based largely upon the physiologic action of drugs as tested in the human or animal body. For him then as for certain distinguished European pharmacologists forty years later, "Experimental pharmacology in its widest significance deals with the reaction of living organisms to various chemical agents or, otherwise expressed, with their behavior under chemically altered conditions of life. Consequently pharmacology is to be looked upon simply as one portion of biology." "How well this work was fulfilled was testified in the dedication of the first edition of these volumes and has similarly been testified by generations of students whose good fortune placed them under the magic spell of Wood's genius. Great and constantly increasing advances in knowledge have been made during the four decades since his book first appeared; but invariably the law which was laid down that a knowledge of the mechanism of disease must precede a scientific therapeutics has held good. If we must contrast the pharmacology of to-day with that of 1874 , we must also contrast the knowledge of disease of to-day with that of the time when bacteriology was in its incipiency, when physiological chemistry was just beginning to yield results, when serology and immunology were unknown, when the physiology of growth and nutrition were problems of the future and pathology was merely a study of damaged structure.

It is manifest that the gain from a cultivation of the study of the physiological action of drugs would be mainly in the direction of greater certainty regarding the influence on certain organic functions - and could not advance beyond the prevailing knowledge of physiology. To use a modern term, the "organotropic" power of drugs was the main purpose of this newer pharmacology. It introduced no means for investigation of the effects of drugs upon the essence of disease itself, nor could it be laid under any proper charge of inefficiency so long as the etiology of disease remained so hidden in obscurity. Ehrlich in his Harben Lecture in 1907, when presenting his chemotherapeutic studies of trypanosomiasis remarks that "Pharmacology has become essentially Toxicology and has become somewhat estranged from the actual curative purposes of medicine. It is, therefore, readily appreciated that the researches upon drugs are 
carried out on healthy animals, and are especially concerned with substances, like alkaloids, which produce toxicologic phenomena that are open to scientific analysis; but it is unnecessary to indicate to my hearers that it is also essential to study the effect of curative agents upon diseased animals. What does it profit to know the manner in which sublimate proves toxic or that in poisoning with potassium iodid, cardiac failure occurs! These facts cannot in any sense form the basis of therapeutic application, nor do they teach us that these substances are capable of curing syphilitic or other processes." Though the reproach of the older pharmacology contained in the earlier part of this quotation is scarcely merited and (perhaps unintentionally) seems to ignore the enormous contributions of the physiologic methods toward an understanding of the effect of drugs on organic functions, Ehrlich's words summarize the problem of the newest pharmacology - the study of the action of therapeutic agents upon the causes of diseases themselves. It is in this etiotrophic or parasitotrophic effect of healing agents that the most striking progress has been made in recent years.

One readily appreciates how those whose studies had led them in to the fields of more accurate medical sciences like etiology and experimental pathology gradually lost interest in the older pharmacology and therapeutics and even became outspoken therapeutic nihilists; but an impartial survey of the successive steps by which progress in the actual treatment of disease has been attained should impose a more appreciative attitude. That quinin, mercury, iron, opium, belladonna, digitalis, and salines have been given to practical therapeutics by the crude methods of other days and in large part still lack their final explanation is proof enough that the past was not all darkness and that the present is far from an age of perfection. It is essential to real progress that we view with tolerance and balanced judgment the beliefs and methods of the older therapeutists and observe a becoming modesty with regard to the advances of these modern days. More than once some remedy lauded by the clinical observers after wide experience has been looked upon with disfavor by more scientific investigators only to be later on proposed anew, as if just discovered, when more recent methods of study had shown its worth. An example in point is quinin, whose value in inflammatory conditions and pneumonia had been asserted by the old clinicians and denied or doubted by the therapeutic sceptics. Recent experiences in ophthalmic practice and even in pneumonia seem to indicate that certain quinin-containing compounds have a demonstrable influence in destroying pneumococci or combating their efiects. Other examples might easily be cited and the persistence with which practical physicians have held to their belief in some mysterious but actual potency of arsenic and mercury in chronic diseases (probably infectious) may here find a hint of explanation.

Let us turn from general to specific considerations and review very briefly some of the methods of treatment introduced into medical 
practice as a result of newer knowledge of physiology, etiology and immunology.

One of the earliest departures in treatment was that in which various glands or extracts of glands from the lower animals have been put to use in the treatment of definite human diseases or in influencing functional activities in man for the purpose of correcting abnormalities resulting from disease. After Ord's studies of the relation of the thyroid gland to myxedema and after the experimental demonstration (Schiff, von Eiselberg, and Horsley), of the corrective effect of implantations of thyroid tissue in thyroidectomized animals, the way was opened to the medical use of thyroid gland in the treatment of myxedema, cretinism and obesity. The first publications of Murray were so conclusive that this treatment was at once established, and now rests upon a secure foundation. Naturally this innovation in therapeutics was speedily followed by investigation of the value of other glands in diseases presumably caused by deficiency in the function of these glands. Thus adrenal, pancreatic, ovarian and hypophyseal extracts have been used in Addison's disease, diabetes, climacteric disorders, and certain forms of diseases of the hypophysis - with variable results. Though usually uncertain and disappointing, the results of these attempts have occasionally opened vistas through which greater success may be seen beyond; but the quickening of interest in the whole subject of the physiology of the endocrine glands and of their relation to nutrition and growth is perhaps the most immediate contribution to knowledge growing out of the original investigations of the functions of the thyroid. Beyond these immediate applications of gland treatment to definite diseases resulting from insufficiency of the glands of internal secretion one must recall the wide range of usefulness of adrenalin, pituitrin and the iodin-containing compounds of the thyroid to form an adequate estimate of the advances that have been made in this department of therapeutics, and it may not be inappropriate to note how greatly these advances have depended upon the study of the physiologic action of the substances in question upon normal animals.

Another chapter in the history of modern therapeutics is that which tells the story of the introduction and cultivation of serum and vaccine treatments. Strict accuracy requires that we go back to Jenner's researches in vaccinia and Pasteur's brilliant discovery of the treatment of rabies, but the uplift of modern immunological treatment dates from von Behring's introduction of diphtheria antitoxin, based as it was upon a definite knowledge of the etiology of the disease (diphtheria) and upon abundant experimental investigations. If animal experimentation had yielded to mankind no other blessing, diphtheria antitoxin would more than repay all the loss of animal life entailed in such investigations from the beginning of scientific medicine. After diphtheria, tetanus, anthrax, glanders, streptococcus infections, pneumonia, epidemic meningitis and many other diseases have been attacked by somewhat similar methods, and though the 
results in most of these cases have been less gratifying than in diphtheria something has been accomplished in all, and directly or indirectly the researches after a serum treatment in each has rendered the disease less dangerous to man. In all of this work it is well to remember that the fundamental principles regarding immunity established by Ehrlich, Bordet and other scientific workers have been the guide to new advances and that no attempt at treatment is justified until it has been tested out in the laboratory of experimental investigation.

Vaccine treatment, the method of combating disease by a process of stimulating active immunity by introducing dead or devitalized cultures of bacteria was suggested by Pasteur's treatment of hydrophobia and first used in a disease of definite etiology by Haffkine in the care of cholera. Its later extensive employment-one is tempted to say its vogue-followed Pfeiffer and Kolle's, and Sir A. E. Wright's investigations in the prophylactic inoculation against typhoid fever, regarding which there is now no more question of doubt than regarding the value of diphtheria antitoxin. The applicability of vaccine treatment in the course of infectious diseases, however, involves considerations other than those that govern protective inoculation. The authorities best qualified to guide in this matter insist that while vaccines are undoubtedly useful and their employment based upon sound principles in cases of local infections their introduction in acute and generalized infections is not founded upon a safe foundation. That vaccines (or sera) injected subcutaneously or intravenously may be used in serious diseases in the belief that they are at all events incapable of harm may be doubted. The use of vaccines in furunculosis, acne, local infectious diseases and the like when autogenous or known to represent the actual etiologic agent is undoubtedly proper and likely to prove beneficial; but the use of "stock" vaccines in conditions, the etiology of which is uncertain or guessed at, cannot well be defended. An impartial investigation of vaccine treatment would seem to show that in the matter of prophylaxis its value has been demonstrated beyond all question, while in the treatment of disease excepting certain strictly localized infections a wholesome scepticism is very much in place.

Chemotherapy represents a new departure in scientific medical treatment and one that has engaged the researches of some of the most brilliant experimenters of modern times. Briefly stated this new method involves the discovery of chemical (as opposed to biological) substances that have the effect of destroying the causes of disease. Very largely this methorl is based upon the specific affinity of certain chemical substances for the agents of disease and the process of study is founded upon the discovery of these affinities. The earliest investigations dealt with various dye-stuffs, but Ehrlich's epoch-making researches led into new fields and the introduction of various arsenical compounds-especially salvarsan. It is true as Ehrlich has claimed in the quotation cited that a study of diseased 
animals is more or less essential to these studies, but it must be remembered that chemotherapeutic researches (in this sense) can only be applied to diseases of known etiology and during their active stages.

The contributions of the synthetic chemist to medical treatment constitute a large factor in modern medicine. We need but recall phenacetin, antipyrin, aspirin, salol, synthetic adrenalin and many others whose uses are beyond question. Chemical industry is so bewildering that sceptics doubt the uses of all new drugs of this kind, but the few that have been firmly established justify the labor that has been expended. After all it may be remembered that the trial of drugs, whose harmlessness is ascertained by experiment, is quite justifiable. An equally free use of sera, vaccines or other agents used hypodermatically might be far less defensible.

In one important direction the treatment of disease has been enriched by the introduction of methods derived from sources outside the domain of the medical sciences. To the physicist primarily is due the credit for the medical applications of the Roentgen-ray, radium and even sunlight itself. 'The time has not yet arrived for a proper appreciation of the ultimate uses of these agencies. Much over-enthusiastic discussion of the value of radiant energy in the cure of malignant diseases has found its way into medical and secular publications but that we shall eventually find these forces beneficial in various local diseases is clearly evident.

A review of modern treatment of disease would be incomplete without reference to non-medicinal methods. If one should seek for the fundamental differences in the treatment of disease at the present day from that of a half century ago one would find it in the essentially changed attitude regarding the possible effects of remedial agents. We seek now for remedies that may destroy the causes of disease or neutralize their effects, or for agents that may correct deranged organic action, rather than for substances that will remove the material results of disease. In chronic organic diseases like diabetes, nephritis, cardiac diseases and the like we are more concerned with the diet, the daily regimen and the exercise of the patient and with baths and other non-medicinal treatments than with drugs.

Finally it must be noted that in the treatment of disease, especially chronic disease, the environment of the individual-his habits, labor, diet, and all external influences-may be more important than more direct causes and may more urgently need correction. 


\title{
THE GOVERNMENTAL PROPHYLAXIS OF DISEASE
}

\author{
By Samuel G. Dixon, M. D., LL. D., Sc. D.
}

After studying the broad and very able treatment of the principles of prophylaxis written by Doctor Harrington and Doctor Abbott, it appears to me that in attempting to supplement their valuable treatise, it would be consistent that I should confine myself to the subject of governmental prophylaxis as applied in the Commonwealth of Pennsylvania.

The earliest effort toward preventive treatment by organized authority in Pennsylvania was local in its nature, applying first to the city of Philadelphia, and developing with the growth of other large cities in the State.

The Acts of 1889 and 1893 relating to cities of the third class and boroughs, provide for the establishing of boards of health in all of the municipalities of the State, conferring upon these boards wide powers, and imposing upon them important duties. The results, however, accomplished by this legislation alone were not satisfactory, at least in so far as State-wide results were concerned. They left the villages and rural communities, now representing over two millions of our people, without any protection or supervision, and there was a lack of central authority to assist and encourage local boards, to direct them in such a way as to secure uniform practices, or to afford them protection from each other or from outlying unincorporated districts.

For twenty years prior to r905, noble efforts had been made by the State Board of Health to supervise epidemics and to organize and encourage local boards of health, but they met with little success only because it was not equipped with the necessary powers or given the necessary means to accomplish its purpose.

In 1905, the State Department of Health was created and upon it was placed the duties:

r. Of directly executing preventive measures in the villages and townships of the State; and in those municipalities of the State in which there were no active boards of health.

2. Of protecting municipalities from each other by supervising their water supplies and systems of sewage disposal.

3. Of encouraging the organization of boards of health in municiVoz. IV,-2 
palities and instructing them in uniform methods of enforcing public health laws.

4. Of securing State-wide registration of births, deaths, and marriages.

5. Of instructing the public and conducting educational campaigns in preventive medicine.

In order to accomplish as nearly as possible the purposes for which the Department of Health was created a number of divisions were organized.

The Medical Division.-Probably the most common cause of the dissemination of communicable disease is the mingling of those who are sick with those who are well. In this day when the people of all classes mingle so closely with each other the public has no way to protect itself from possible contagion except by the careful administration of quarantine laws. Pennsylvania's laws require all physicians to report to the health authorities cases of communicable diseases treated by them, and-upon the basis of these reports quarantine restrictions are to be enforced by the health authorities.

Physicians, of course, must have free access to their patients and are not subject to the ordinary quarantine restrictions applicable to other persons forbidden ingress or egress. It is, however, very important that great care should be taken by practitioners that they do not themselves become the carriers of contagion.

The department insists that physicians when treating cases of contagious diseases should carry with them in a bag intended only for this purpose a gown which extends nearly to the floor, fitting closely at the neck and wrists and entirely covering all clothing. The bag should also contain a hood or protective covering for the hair and a pair of high close-fitting rubbers.

Upon leaving the premises they should thoroughly wash their hands, face, and beard with soap and water, and the mouth and nose should be sprayed with the liquor antisepticus diluted with water.

A positive regulation of the Advisory Board, adopted January 23, I906, requires these precautions in the treatment of smallpox, and recommends the same for other acute infectious diseases.

The medical division of the Department of Health has direct charge of quarantine in townships of the second class and in other districts not supplied with local boards of health. The health officers appointed by the Commissioner of Health receive reports of communicable disease from attending physicians, placard the premises, establish quarantine, and disinfect at the expiration of the quarantine period.

In Pennsylvania we now disinfect for anthrax, anterior poliomyelitis, bubonic plague, cerebrospinal meningitis, chicken pox, Asiatic cholera, diphtheria, German measles, glanders, leprosy, malarial fever, measles, mumps, relapsing fever, scarlet fever, smallpox, typhoid fever, paratyphoid fever, typhus fever, whooping cough, and yellow fever. 
In a number of these we understand the etiology and we have proven that we can kill the organisms that produce them; therefore, there is no question about the advantage of our present system of disinfecting.

In chicken pox, German measles, mumps, poliomyelitis, scarlet fever, smallpox, typhus fever, and yellow fever, we are not acquainted with the etiology or the causative organism has not been absolutely determined. We have, however, reason to believe that they are produced by organisms that reproduce themselves, and we have a right to believe that in general these organisms will yield to our disinfectants as do the communicable diseases the etiology of which we understand. Therefore, as we are dealing with the lives of our people it is better to err on the safe side by continuing our disinfecting after these diseases in which there is doubt as to the organisms that produce them.

There is another reason why we should take this course and that is because there is so much tuberculosis in our homes in the United States. We know that the houses become infected and often transmit that infection to family after family who occupy such premises.

Again in spite of our endeavor to be thorough in our work, there are communicable diseases the etiology of which we do understand that escape us owing to the secretiveness of the families and the disregard of the law by physicians, and, therefore, there is a great unknown quantity that we well may cover by disinfecting for measles and the other diseases, the etiology of which is yet unknown.

A county medical inspector, an experienced physician, supervises the work of the health officers in each county. When a health officer learns of a case which has not been reported or for which no physician is in attendance, he visits the premises, makes an investigation and refers the case to the county medical inspector for diagnosis. If a householder admits the presence of a quarantinable disease, the premises are tentatively placarded until the diagnosis is confirmed.

Separate pamphlets relating to each quarantinable disease and instructing the householder as to the precautions which should be taken are left on the premises when quarantine is established.

The medical division has charge of the medical and sanitary inspection of schools in fourth-class districts. Local physicians are appointed to make these inspections. Defects of the eyes, ears, nose, throat, teeth, and skin are specially noted, and parents and guardians are advised by special letters from the department of the existence of defects, deformities, or diseases detected in their children and are advised to secure medical attention for them. The inspectors also examine the school rooms as to light, ventilation, floor space, methods of cleaning and heating, and the conditions of grounds and toilets. School directors are notified as to the unsanitary conditions existing in their schools and requested to remedy them. If a succeeding inspection discloses the same conditions such districts are certified to the State Superintendent 
of Public Instruction in order that the State appropriations to such districts may be withheld until the unsanitary conditions have been remedied.

The medical division coöperates with local health authorities in the suppression of epidemics-frequently at the request of the local authorities-assuming temporary charge until the epidemic has subsided.

The Division of Sanitary Engineering.-The growth of our municipalities and our commercial interests have brought to our people serious problems as to adequate water supplies and methods of sewage disposal. Large numbers of persons receiving their supply of drinking water from a common source are subject to a common danger from the germs of water-borne disease.

In 1905, typhoid fever had become prevalent to an alarming extent throughout the State. In order that there might be a central control of water supplies and sewage disposal, the Act of Assembly of I905 known as the "Purity of Waters Act" was adopted and this work has been largely carried on by the division of sanitary engineering. Plans are filed in this division of all of the municipal water and sewer systems. Since the passage of the act no additional source of wa ter supply can be obtained without a written permit from the Commissioner of Health. The application must be accompanied by a certified copy of plans and surveys. These are studied in the engineering division and checked by field investigations of the engineers, and are carefully considered by the Commissioner of Health before permits are issued.

Under the Purity of Waters Act the discharge of sewage into the waters of the State is forbidden except that it does not apply to municipal sewage that was being discharged into streams at the time of its passage. It does, however, forbid the discharge of any additional sewage from such municipalities. The growth of the cities and boroughs and the necessity for the extension of their sewers has, however, practically brought all municipal systems within the provisions of the law. Whenever any new system is contemplated, or any addition to any system, application must be made to the Commissioner of Health and such system or addition may only be constructed under a permit or decree issued by joint authority of the Governor, Attorney-General, and Commissioner of Health. All plans and specifications for sewers, sewer extensions, and disposal plants are also carefully studied by the division of sanitary engineering and checked against field observations.

In order that private pollutions of the streams may be discovered and abated the watersheds are patrolled by stream inspectors of the engineering division. Orders of abatement are issued, and if upon re-inspection these orders have not been obeyed, the offenders are indicted for violation of the law.

The division of sanitary engineering assists the medical division in the supervision and suppression of epidemics of water-borne 
disease; secures field data from which the cause of the epidemic may be studied and the source of the infection discovered; and installs and operates temporary treatment plants for the purification of water and sewage.

Tuberculosis Sanatoria and Dispensaries.-For a number of years tuberculosis had been the greatest single cause of death in the State. While universally recognized as highly communicable, this disease can not be managed by ordinary quarantine restrictions applied to other contagious and infectious diseases. The well-todo might have the advantage of favorable climates or of private sanatoria, but for the indigent there was no escape.

In 1907 , the Legislature authorized the Commissioner of Health to construct sanatoria, by and with the consent of the Governor, and to establish dispensaries for the free treatment of indigent persons suffering with tuberculosis. Since 1907 three sanatoria, with a combined capacity of over two thousand (2000) beds, have been constructed and are in operation, and one hundred and fifteen (II5) dispensaries have been established - at least one in every county. Indigent patients are treated at the dispensaries, furnished with medical attendance and medicine, and in many instances are supplied with milk. Each dispensary has one or more trained nurses who visit the homes of the patients and instruct them in preventive measures, and at the same time are constantly looking for new cases which should have dispensary treatment. In the dispensaries applications for sanatorium treatment are filled out and forwarded to the central office of the division at Harrisburg. Waiting lists are maintained for each of the sanatoria and patients are admitted as their turn is reached. By means of the dispensaries and sanatoria many thousands of poor tuberculous patients have been treated and cared for and their families and friends have been better protected from infection.

Tuberculosis is no longer the greatest single cause of death in Pennsylvania.

Vital Statistics.-All civilized governments to-day are recognizing the value of comprehensive systems of vital statistics, and for a number of years our National Government has been urging the several States to adopt such systems. Pennsylvania's registration law was adopted the same year that the Department of Health was created and the bureau was made a part of the Health Department. Within a very few years Pennsylvania, classified prior to 1905 as a non-registration State, was designated by the United States Bureau of Census as the "keystone" in the arch of national registration.

The division has for its head a State Registrar and local registrars are appointed and conveniently located for all parts of the State. Certificates of births and deaths are received by the local registrar and forwarded to the central bureau. Permits for burials, without which no body can be lawfully buried within the State, and transit 
permits, without which bodies cannot be carried by common carrier from one place to another, are issued by the local registrars. Reports of marriages are also received from county officials who issue marriage licenses; and morbidity reports are forwarded to the bureau by the health officers of the Department of Health and the secretaries of local health authorities. All of these reports are valuable for statistical purposes and afford a measure by which the State can estimate the results of public health work. The birth and death records are also of great value to our individual citizens-certified copies may be used in evidence in the courts and in the future these records will be decisive in matters which otherwise could only be determined by tedious litigation.

From the morbidity reports the medical division secures notice of an unusual prevalence of any disease in any locality and promptly orders an investigation to be made by the county medical inspectors or by physicians from the central office in order to ascertain the cause and to prevent the occurrence of an epidemic.

Distribution of Biological Products. - No act of assembly directly imposes upon the Commissioner of Health the duty of distributing biological products to the poor, but under his general powers he is directed to determine and employ the most efficient means for the prevention of disease and the protection of the lives of the people, and the several appropriation Acts authorize the use of money for the free distribution of biological products to the poor.

The discovery of vaccine virus, diphtheria antitoxin, and tetanus antitoxin has removed much of the terror of smallpox, diphtheria, and tetanus, but while these remedies are open to those who have money, their cost in many instances puts them beyond the reach of the indigent. In order that all may have the benefit of modern treatment, both for immunizing and curative purposes, the Department of Health has placed diphtheria and tetanus antitoxins in sufficient quantities in local distributing stations all over the State where practising physicians may secure them upon certifying that their patients are financially unable to purchase them. Vaccine virus is furnished to county medical inspectors and other medical representatives of the department to be used for vaccinating smallpox contacts and the children of the poor. In some instances typhoid vaccine has been furnished to communities where the disease was prevalent. Tubercle bacilli extracts are distributed to the dispensaries and sanatoria for the free treatment of indigent tuberculous patients.

Laboratories.-Questionable cases which are difficult to diagnose are constantly confronting practitioners and can only be definitely determined by laboratory tests which the ordinary physician is not prepared to make. The laboratory division of the Department of Health makes Widal tests, Wassermann tests, examines specimens of blood and sputum, makes bacteriological analyses of milk and water and sewage effluent, and is open to any physician 
in the State who will send specimens for analysis or examination. The laboratory division also prepares tuberculin for use of the sanatoria and dispensaries and conducts laboratory research work.

Division of Housing.-The extraordinary number of foreign laborers coming into Pennsylvania has largely occasioned congestion and conditions of insanitary living in mining and manufacturing towns. Bad housing conditions are not only a menace to the health of those living under such conditions but are a menace to the entire community in which they are situated.

Houses originally intended for but one family were converted into tenements or lodging houses-different rooms and different floors occupied by separate families, and the sleeping apartments frequently used for relays of sleepers. In order to remedy and check such conditions the Act of I9I3 providing for the establishing of the division of housing in the Department of Health was enacted. It is the purpose of this division to make inspections of tenements and lodging houses, to relieve congestion, and to prevent the use of insanitary rooms and apartments for living purposes. The division of housing is authorized to notify property owners about changes and improvements that will be required, giving a stated time in which the requirements must be met, and to file a copy of such notice in the Court of Common Pleas of the county wherein the property is located. In case the improvements are not made within the time specified and no appeal has been taken into the said Court, information may be made against the property owner for violation of the law.

Division of Public Service.-The growth of the business interests of our State and Nation greatly increased the numbers of our citizens who travel from place to place and are dependent upon hotels and public eating places for entertainment. This army of travelers, realizing the dangers to which they were being subjected and from which they could not protect themselves, were insistent upon their demands for more legislation relating to sanitary conditions in the hotels and restaurants of the State.

In order to meet this demand the Act of I9I5 was adopted, providing that no person suffering from a contagious disease should be employed in any public eating place and covering particularly certain communicable diseases which are not included in the general quarantine law, and also providing for the sanitary handling of eating, drinking, and cooking utensils.

It is the purpose of the division of public service to make inspections of all public eating places, to establish a system of medical inspection of employees and generally to enforce the sanitary provisions of the Act of I9r5.

All of these divisions operate directly for the prevention and suppression of disease, and in connection with the work of each division the department seeks to educate the people. Bulletins are published and distributed monthly, short talks on live subjects 
appear weekly in the newspapers in the State, and the correspondence going from the department to individuals or local authorities is intended to be instructive rather than reprimanding or mandatory. As a distinct educational feature, a general exhibit and lecture course reaches in its itinerancies the various parts of the State and is open free to the public.

In order that the prophylactic activities of the department may be successfully and economically conducted, it is also necessary to have a business side to the department's work. The fact that the legislatures have made liberal appropriations makes this part of the work all the more important. The expenditures must be regulated so that the people may get the greatest possible returns and at the same time the appropriations not be exceeded. Emergency work is hard to forecast. For these purposes the department has divisions for auditing, accounting, and purchasing which carefully audit all vouchers, keep careful itemized book accounts, and make detailed reports to the State government of all moneys expended.

A division of supplies sends out stationery and the various materials used by field officers and keeps an accurate account of all shipments.

While each of the various divisions has its own peculiar duties to perform, all work together with each other for the common purpose of suppressing disease, and protecting public health. 


\section{THE GENERAL PRINCIPLES OF DRUG TREATMENT}

By Sir Lauder Brunton and Walter J. Dilling, M. B., Ch. B.

The principles of treatment which were enunciated by the senior author in his monograph have retained almost uniformly the complete confidence of therapeutists. In the interval that has elapsed since I9I I, a number of new remedies and methods have been evolved, some of which have proved of primary and lasting importance, while others have been merely a passing vogue and have been discarded as inferior to established methods of treatment. In a general review of the intervening period the most prominent additions to our knowledge of drug treatment have taken place in the therapy of heart diseases and of syphilitic conditions, while the treatment of disease by vaccine therapy has been simplified and its value endorsed by most competent observers.

\section{RELATION OF DRUGS TO THE CEILS OF THE ORGANISM AND TO DISEASE GERMS}

The interpretation of the influence of drugs upon cells and microörganisms is by no means a simple matter and general conclusions as to the reason for the selective action of certain drugs on special tissues cannot be reviewed with too great scepticism in view of the paucity of our knowledge regarding the internal economy of the cell. The actions of acids and alkalis as caustics, of heavy metals and tannin containing drugs as astringents, and of salines as purgatives can be explained on physico-chemical grounds and actions resembling those they exert on living cells may be elicited also on dead tissues. When we come to the consideration of the specific influence which alkaloids and other complex drugs possess upon individual cells or groups of cells in positions remote from the place at which the drugs were administered, we are faced with a problem, the solution of which is difficult. It is also highly probable that if the solution of this problem were attained in the case of one drug, this key would do no more than unlock a few doors and leave the remainder of the chemical laboratory of the cell unexplored. We know that drugs display a selective affinity for certain tissues or that certain tissues or cells are specially susceptible to the action of certain drugs. From this it is evident that chemotaxis or some form of mutual attraction exists between certain cells and drugs, but this does not necessarily imply that the drug is present in greater concentration in the selected tissue than in other parts of the organism although in some cases this has been shown to occur. While we recognize such facts, the theories 
which have been evolved to elucidate the problem are all incapable of affording a satisfactory explanation of this composite question.

The theory that cellular activity is dependent on a series of ferments which may be influenced individually or collectively by drugs has been fully discussed in Vol. I.

Upon the relationship of chemical constitution to pharmacological action is based an assumption, elaborated by Loew and others that drugs enter into definite chemical combination with the cellular elements or protoplasm and that specific chemical groups possess characteristic powers of modifying the protoplasm of the cell or disturbing its internal economy by interfering with or enhancing its oxidative or other powers. There is a certain amount of evidence that compounds of similar chemical constitution and certain organic chemical groups possess pharmacological actions which resemble each other and which might be attributed to intracellular chemical combination. It is, however, impossible to define with any accuracy the pharmacological action of a drug from its chemical constitution, and although we can modify the action of some organic drugs by the introduction or substitution of side-chains into the molecule, we cannot be certain that the chemical modification we have made will produce the desired change in the pharmacological activity until this is subjected to experimental tests. While the interactions which occur between chemical compounds and living protoplasm may aid in the interpretation of the action of general protoplasmic poisons, they do not serve to explain the selective actions of drugs nor do they help to elucidate the fact that alkaloids of widely different chemical constitution like pilocarpin and muscarin possess almost identical actions.

Evidence has been brought forward to indicate that the physical properties of a substance have some power in determining its action upon living tissues. Drugs which possess a low solubility and diffusibility in the body fluids are as a rule relatively more inert and slower in action than those which are readily soluble and diffusible. At the same time just as ingested fat is accumulated in certain parts of the body, so special tissues appear to possess a peculiar affinity for certain drugs and readily extract them from the circulating fluids of the body. Thus, according to the Meyer-Overton theory, the narcosis induced by various hypnotics is dependent on their relative solubility in the brain lipoids and in water; and that, in virtue of their higher solubility in fats, these hypnotics tend to accumulate in the nervous tissues where they enter into a loose and temporary combination with the lipoids of the nerve cells and thus lower the functional activity of the neurons. It has also been suggested that alterations in the surface tension of cells and of the fluids which surround them may be productive of changes in their functions, while Straub, in the case of the alkaloids, muscarin, and atropin has attempted to show that their antagonistic action on the heart is brought about by a physical change in the cellular membrane whereby mus- 
carin produces its effects during its passage through the membrane into the interior of the cell, while atropin annuls the action of muscarin by altering the cellular membrane in such a way as to retard the rate of its absorption. These hypotheses, although they may serve to explain individual instances of drug actions, are incapable of general application and as the physical properties of a chemical compound or drug are dependent on the chemical structure of the substance, it is futile to anticipate that physical properties alone will serve to elucidate the remarkable variations in the actions of drugs.

The investigations of Langley on alkaloids led Ehrlich to formulate a theory of the action of drugs on cells and microörganisms similar to that which he evolved to illustrate the effects of toxins. He postulated in cells and in microörganisms the presence of different specific chemoreceptors, each possessing the affinity for and attaching to itself a certain chemical grouping. On this theory a cell or microörganism which possessed a large number of these chemoreceptors would be more susceptible to the influence of chemotherapy since receptors were provided for chemical groups of various kinds. The specific remedy, however, must also possess a definite grouping which has a chemical affinity for one of the chemoreceptors of the cell. This grouping (haptophore) serves to fix the therapeutic agent to the cell, but in order to produce its action the drug must be further provided with a destroying or poisonous group (toxophore) which can then bring about the injurious action on the cell. In complex synthetic compounds the haptophoric and toxophoric groups may be connected not directly with one another but may both consist of side-chains attached to a central chemical nucleus. Thus in the case of salvarsan Ehrlich believed that the orthoamido-phenol group constituted the haptophore or fixing body, the trivalent arsenic group, the toxophore or poisonous body and the benzol group constituted the nucleus or link binding the two.

\section{ANTISEPTIC TREATMIENT}

For the sterilization of the cutaneous tissues prior to operation increasing use is being made of alcohol, ether, acetone, etc., as surgical detergents. Solutions of antiseptics such as iodine in alcohol are used for superficial disinfection of the skin on recent wounds. Ether, by virtue of its solvent action on fats, forms a suitable medium where deeper penetration of the antiseptic is desirable, and etherial solutions of soap are efficacious for the removal of the sebaceous secretion of the skin and for softening scabs in skin diseases. Acetone is both a solvent for fats and a powerful antiseptic; it has the advantage also of being a hardening medium and a deodorant. A mixture of equal parts of acetone and alcohol is specially useful for skin disinfection. Valuable results have recently been obtained by the use of a 0.5 solution of hypochlorous acid (eusol) and a mixture of equal weights of bleaching powder and boric acid (eupad) as antiseptics for infected 
wounds and particularly for gas gangrene. Almroth Wright has brought forward evidence to show that antiseptics are by no means essential to the treatment of infected wounds and pleads that physiological methods of treatment-free drainage and the use of lymphagogues, e.g., hypertonic saline solutions-are more satisfactory. The rationale of this may be summed up by stating that with free drainage the bacterial concentration in the wound is lessened, the phagocytes and other defensive measures become predominant and are further enhanced by the augmentation of the flow of lymph.

\section{TREATMENT OF THE RESPIRATORY SYSTEM}

In the various catarrhal diseases caused by microörganisms in the upper air passages vaccine therapy has been used with great advantage in many cases. As far as possible treatment should be carried out by autogenous and not by stock vaccines. They are of more definite curative value in cases of recurring infections with intermissions of health than in chronic conditions where permanent morbid changes have taken place in the mucous membrane. In acute septic infections large doses of vaccines should be given early and repeated as soon as possible if a favorable result is to be attained. Pneumococcus vaccine in the treatment of pneumonia has not proved uniformly successful; it does not hasten the crisis and in the opinion of most observers has no marked effects on the progress of the disease.

The treatment of chronic infective pulmonary disorders, including tuberculosis, by means of inhalations of various volatile oils in the form of fine vapor has been largely recommended. While no conclusive evidence has been brought forward indicating that the treatment has any marked retarding or curative powers on the course of such diseases, there is this to be said in its favor that the volatile oils probably disinfect the sputum to some extent and cover its offensive odor so that the life of the patient is rendered more pleasant to himself and to his associates.

\section{TREATMENT OF THE DIGESTIVE SYSTEM}

The Mouth.-In the treatment of pyorrhea alveolaris therapeutic measures, in addition to surgical interference, have been employed with some success. Ionic medication using a current of 2-4 m.a. with zinc sulphate in 2 per cent. solution applied at the positive pole aids in the treatment of pyorrhea and the employment of an autogenous vaccine in these cases serves to eliminate the symptoms of debility and gastric disturbance resulting from the disease.

The Stomach.-When indigestible food or poisonous materials have been ingested recourse must be made to the stomach pump or to emetics. In corrosive poisoning the use of the former is inadvisable and the selection of an emetic becomes of importance. Emetics may act either on the vomiting center, like apomorphin, or may produce their effect on the stomach by a local irritant action which 
results in reflex contraction of the stomach and diaphragm. It is in this latter way that ipecacuanha, tartar emetic, ammonium carbonate, zinc sulphate, copper sulphate, etc., act. The most certain and rapid means of evacuating the contents of the stomach is apomorphin hydrochlorate administered hypodermically in doses of $0.05^{-0.1}$ grain $\left(0.003^{-0} 0.006 \mathrm{gm}\right.$.) or 5-10 minims (0.3-0.6 c.c.) of a I per cent. solution. Besides their use as such, the emetic class are serviceable from their nauseating powers for liquefying the bronchial secretion where this is tough and difficult to remove; more especially in young children where the coughing reflex is weak, repeated small doses of vinum ipecacuanha until emesis is produced frequently results in the simultaneous clearing of the secretion from the bronchial tubes.

The use of alkalis administered before meals as digestive adjuvants has frequently been defended on the ground that they excite the secretion of the gastric juice. The researches of Pawlow and others have shown that the reverse is the case and that alkalis actually inhibit gastric secretion. Their undoubted value in gastric therapeutics is rather to be attributed to their power of liquefying mucus and thus of permitting more intimate contact between the food and the gastric juice. Cannon has shown that the reaction of the stomach contents is an important factor in determining the opening and closing of the pylorus. A slight acidity of the stomach contents relaxes the tone of the pyloric ring, while acidity on the duodenal side leads to closure of the pylorus. The former condition is to be combated by the use of alkalis and their excessive administration may be avoided by directing that they should be given in solution and slowly sipped. In the treatment of hyperacidity by alkalis discretion in the selection of these is advisable, thus where flatulence is associated with hyperacidity, the use of carbonates and bicarbonates tends to augment the flatulence, and oxides such as those of magnesia or hydrates are preferable; at best such treatment is symptomatic and attention should be directed to the origin of the hyperacidity.

The beneficial effect of bitter infusions and of carbonic acid in dyspepsia has been shown to be due partly to their power of augmenting the peristalsis of the stomach.

The Intestine.- The value of drugs as intestinal antiseptics cannot be said to be in such high estimation as it was in former years. Of most efficacy are probably the preparations of mercury, bismuth salicylate, $\beta$-naphthol and turpentine. Carbolic acid and the sulphocarbolates have been shown to have small and negligible antiseptic powers toward the putrefactive organisms of the intestine. Instead of employing direct antiseptic measures Metchnikoff suggested the use of lactic acid producing organisms which are antagonistic to the growth of putrefactive bacteria. A proper estimation of the value of this treatment is rendered difficult from the fact that tableis and powders purporting to contain the Bacillus caucasicum, but really inert, were placed on the market. In general, however, the results attained by lactic acid therapy even when fluid cultures 
or sour milk was given have been relatively disappointing. In some cases of autointoxication vaccine treatment has yielded good results. The employment of sea-water and artificial sea-water injections in the treatment of mucous colitis and infantile diarrhea has fallen largely into desuetude.

A high degree of protection from typhoid fever by means of inoculations of antityphoid vaccine has been attained in the European war. When inoculated cases develop typhoid, the disease is mitigated to a considerable extent and the mortality lessened. Antityphoid inoculation does not, however, afford protection against paratyphoid fever A or B and many cases invalided home as typhoid prove on bacteriological examination to be paratyphoid. Experiments with a mixed vaccine-antityphoid and antiparatyphoid - have already given hopeful results.

In the treatment of acute and chronic amebic dysentery and hepatitis, hypodermic injections of emetin hydrochlorate $1 / 3$ to I grain $(0.02-0.065 \mathrm{gm}$.) have proved remarkably effectual; temperature is reduced and the diarrhea checked. Bacillary dysentery does not yield to emetin treatment.

Among other remedies directed toward sterilization of the intestine anthelmintics fail to be considered. These may be grouped into two classes-vermicides, which kill the worms, and vermifuges, which drive them out of the intestine. The principal vermicides in employment are for tapeworms, male fern, pelletierine the active principle of pomegranate bark, cusso, cucurbitae semina præparata, embelia and turpentine; and for round worms santonin and buteæ semina. In the treatment of threadworms, enemas containing turpentine, infusion of quassia, or salt and water are more efficacious, while Ankylostoma duodenalis is more readily eliminated by thymol or eucalyptus oil given by the mouth.

In the employment of purgatives increasing use is being made of those which possess a purely mechanical effect on the intestine, such as agar-agar, liquid paraffin, and bland oils. These augment and lubricate the contents of the intestine and reflexly induce mild peristalsis. The evanescent popularity of physostigmin as an intestinal stimulant and hypodermic purgative in intestinal atony brought out its disadvantages in the vomiting and griping which were concomitants of its action and it is now but seldom employed. Extracts of the infundibular lobe of the pituitary gland, on the other hand, have been found of considerable value in paralytic distention of the intestine following operation.

\section{TREATMENT OF CONDITIONS OF THE BLOOD}

The treatment of syphilis and other diseases dependent on the circulation of parasites in the blood stream has been rendered more efficient by the introduction of salvarsan therapy. It was formerly well known that arsenical preparations exerted a beneficial influence 
on the course of many parasitical diseases but their high toxicity toward man rendered their use impracticable. Keeping this fact in view, Ehrlich and his co-workers set themselves to obtain an arsenical preparation which, while powerfully toxic to the parasiteparasitotrop, was less toxic toward the host-organotrop. Their researches culminated in the discovery of the compound dioxydiamino-arseno-benzol or salvarsan. Originally Ehrlich belicved that a single injection of this compound would effectually sterilize the body so far as the syphilitic spirochete was concerned and believed that he had obtained a therapia sterilisans magna. Subsequent investigations have proved that this is not attained by a single injection of salvarsan and modern opinion holds that several intravenous injections of salvarsan-three to seven at intervals of eight days or longer-are required, or repeated injections of salvarsan combined with injections of mercurial cream, thus, three intravenous injections of salvarsan at intervals of six weeks with intermediate weekly injections of mercurial cream containing I grain ( 0.065 gm.) of mercury. Neosalvarsan has the advantage over salvarsan of being readily soluble with neutral reaction; it is also slightly less toxic and may be given by intramuscular injection. Salvarsan therapy produces a rapid disappearance of the syphilitic symptoms in the primary, secondary and tertiary stages of the disease, but even after repeated injections of salvarsan have been made a permanent cure cannot be presumed unless the Wassermann reaction remains negative for a year after active treatment has ceased. Upon parasyphilitic conditions intravenous injections of salvarsan have very little effect but the intrathecal administration of neosalvarsan has been found beneficial in cases of tabes and general paralysis. Salvarsan and neosalvarsan have also proved efficacious in the treatment of yaws but their employment in malaria and pernicious anemia is hardly justified by the results.

Endeavors to treat sleeping sickness on similar lines have been made but without much success. Antimonial preparations appear to have more activity against the trypanosomes than arsenical; injections of antimonial compounds, however, merely banish the parasites from the blood but do not influence those in the spinal cord. The fatal result is merely delayed somewhat in the majority of cases and seldom averted.

Of the internal secretions which are poured into the blood stream by the various ductless glands, the secretion formed by the pituitary gland has recently come into prominence as a therapeutic agent. Extracts prepared from the infundibular lobe of the ox, when injected, raise the blood-pressure and increase the strength of the cardiac contraction chiefly by increasing the general arterial tone. They are, therefore, valuable in counteracting shock and collapse from serious operations and injuries. Pituitary extract also increases intestinal tone and promotes contraction of the atonic uterus while it has distinct diuretic and galactogogue properties. 


\section{TREATMENT OF THE CIRCULATORY SYSTEM}

Recent investigations on the physiology and mechanism of the cardiac beat have elucidated to a certain extent the origin of cardiac irregularities and afforded new indications for the exhibition of cardiac remedies. We now recognize that the stimulus for the myogenic rhythm of the heart is supplied by the inorganic salts of the blood which are distributed to the heart muscle by the coronaries. The sodium ions maintain the osmotic conditions which promote excitability and contractility, alone they lead to diminution in the tonus of the muscle; on the other hand, the.ions of calcium maintain the muscular tone and promote contraction; while potassium ions induce relaxation of the cardiac muscle in diastole. The probable point at which these ions exert their individual actions is the sinoauricular node, a neuromuscular structure at the junction of the great veins and right auricle. The ions excite this structure to rhythmic activity and the contraction wave is propagated through the auricles by the auriculoventricular bundle of $\mathrm{His}$ to the ventricles. The wave travels first to the musculi papillares and thereafter to the rest of the ventricle and returns by way of the spiral fibers to the base in the region of the pulmonary artery. A separate rhythmical center also exists in the auriculoventricular node. Interference with the rhythmic centers or with the conducting mechanism of the cardiac muscle will be productive of cardiac irregularities of various kinds. Thus, auricular fibrillation, where incoördinate contractions of individual muscle fibers of the auricle are followed by irregular ventricular systoles, sinus irregularity, extrasystoles, paroxysmal tachycardia, auricular flutter, the pulsus alternans, and heart-block are recognized. In the medicinal treatment of cardiac irregularities discrimination requires to be exercised. Digitalis is of particular value in auricular fibrillation when failure of compensation sets in; after its administration the pulse falls in frequency while the beats become stronger and more regular in time. The fibrillation of the auricle, however, continues even after digitalis has slowed the pulse. Digitalis produces these effects by lowering the conductivity of the bundle of His, producing a partial heart-block and thus slowing the ventricle. Where digitalis fails to improve the condition of auricular fibrillation recourse may be made to strophanthus or squill and, if the case be urgent, intravenous injections of strophanthin ( $0.5 \mathrm{mg}$.) may be employed. Tachycardia, acute and paroxysmal, is often benefited by digitalis while Adams-Stokes syndrome (heartblock) is aggravated by its use. 


\section{SPECIFIC THERAPY: BACTERIAL VACCINES AND SERA}

By William H. Park, M. D., and Charles Krumwiede, Jr., M. D.

\section{VACCINES}

General Considerations.-The prophylactic use of vaccines in certain diseases has found a rapidly widening and fairly successful application. The therapeutic use in carefully controlled series of cases has, in some instances, been successful and added considerable new data. Partly because of these good results the profession at large has resorted to the almost indiscriminate use of vaccines for therapeutic purposes. This has added little to our knowledge and probably less to the comfort and recovery of the cases treated. The reasons for the widespread use of vaccines are several. The general availability of stock vaccines has probably been the main one. Each report of good results is followed by new stock vaccines and the physician accepts them in good faith and thus keeps up the demand. He may little realize how slight a relationship the bacteria in the vaccine have to the infecting organism in his case, although each may have the same name. The attempt to treat mixed or secondary infections with stock vaccines has led to the use of mixed vaccines in infections of undetermined etiology. This reversion to medieval polypharmacy has become more popular than one would be inclined to believe. Although such methods may have some nonspecific beneficial effect, they are used and advised on the erroneous belief that specific treatment is being used.

The rational use of vaccines as a specific prophylactic or therapeutic agen ${ }^{1}$ presupposes a bacteriological diagnosis or at least the history and clinical signs should warrant the probable diagnosis of a specific disease caused by a specific organism. Furthermore, one must know whether the organism found in all cases of this disease are practically identical. In late complications, too much reliance should not be placed on the history. Thus a chronic joint involvement in a case giving a history of gonorrhea is not necessarily gonorrheal, and the complement reaction should be resorted to if possible before the inoculation of vaccines. In the case of definite clinical types of disease known to be caused by different species of bacteria or by dissimilar varieties of the same species, the clinical history must be supplemented by bacteriological examination. No less important is the method of obtaining material for examination and the method employed in the examination. This should be done under the direction of a man trained in this type of work.

\footnotetext{
${ }^{2}$ See under Non-specific Response to Vaccines p. 38. 
Depending on the bacteriological diagnosis will depend the selection of the vaccine and will also tell whether a stock vaccine is applicable. The following brief summary will be helpful in this regard:

Staphylococcus, at least two pathogenic groups, aureus and albus, the former more virulent. Although little is known about the interrelationship of members of this group experience has shown that mixed stock vaccines are frequently efficacious.

Streptococcus, two large, more or less distinct groups, hemolytic, including the Streptococcus pyogenes and the streptococcus of erysipelas, the usual agent in acute local and general infections; non-hemolytic, including the so-called "Streptococcus viridans," less invasive, the usual agent in chronic endocarditis and focal infections such as chronic joint infections, apical abscess, etc. The hemolytic types tend more to be similar, the non-hemolytic group contains widely different individuals. Based on our own work we are inclined to consider the latter as an almost completely heterogenous group. Stock vaccines, therefore, have a very limited application, especially in the non-hemolytic types.

Pneumococcus, a species subdivided into groups I, II, III and IV on immunity reactions. All of group I are alike in their serum reactions; group II, most members identical, subgroups encountered, antibodies against the main members of group II are active against members of the subgroups, but the antibodies against the subgroups are only active against that subgroup; group III Pneumococcus (Streptococcus) mucosus, probably like group II, most of the members being the same but some differing; group IV completely heterogenous, each individual strain having no immunity relationship with other strains (see section on pneumonia). Stock vaccines of the fixed type can, therefore, be used, if the same fixed type is found to be the infective agent.

In the case of lobar pneumonia about 75 per cent. of the cases are due to members of groups I, II or III and a mixed vaccine of the three types could be used with some justification in case the infecting type could not be determined. In the majority of the milder types of inflammations especially about the teeth and of the mucous membranes of the nose and throat, group IV is encountered.

Gonococcus.-Although closely related, differences are noted. It is possible to group the members according to their immunity reactions and select representative strains for a polyvalent vaccine. Because of this and the difficulties of obtaining autogenous strains, stock vaccines are almost exclusively used. These should, however, contain representative strains.

Meningococcus.-Meningococci tend to fall into groups according to their immunity reactions. Although most members show relationship one to the other or according to groups, this is variable and may be slight or absent. A prophylactic vaccine should, therefore, be preferably made from strains isolated from the cases at the 
beginning of an epidemic. Autogenous therapeutic vaccines for chronic or relapsing cases would likewise be preferable.

Typhoid Bacillus.-All individuals are apparently identical. Stock vaccines as a rule made from a strain known to produce antibodies and cause a minimum reaction are preferable as a rule.

Paratyphoid Bacilli $A$ and B, and Bacillus Enteritidis (Gärtner).These three groups can be easily differentiated and identified by immunity reactions and the appropriate type of vaccine used. Bacteria similar to these but differing in their immunity reactions, so-called "intermediate," or paracolon, bacilli are occasionally encountered, especially in genitourinary infections, in which case autogenous vaccines only are applicable.

Colon Bacillus.-This is a name used loosely to cover very many types similar to those found in the feces. It is evident, therefore, that autogenous vaccines should be employed.

Bacillus Mucosus Capsulatus and Related Types.-This group also called B. aerogenes is closely related to the colon bacilli. They occur in genitourinary infections. B. pneumonix Friedlander causing pneumonia. B. ozæna supposed to have an etiological relationship to atrophic rhinitis and $B$. rhinoscleroma, found in rhinoscleroma, belong to this group. Little is known of the relationship of the various types and autogenous vaccine should be employed.

B. Pyocyaneous and B. Proteus.-Nothing is known as to the immunity relationship of members of these two groups.

Bacillus of Tuberculosis, and Other Acid-fast Types.-Although tuberculin therapy is a subject by itself, it is well to state here that there is every reason to believe, from experimental work, that vaccines of non-virulent acid-fast types or strains of B. tuberculosis claimed to have been rendered non-virulent by passage through non-mammalian animals, are valueless, both for the immunization and for treatment.

Glanders Bacillus.-A vaccine from stock vaccines would be satisfactory.

Hemoglobinophilic Group : B. Influenza.-During widespread epidemics, probably one type is responsible. As encountered in sporadic infections or where found as the predominating organism in inflammations of the respiratory tract or accessory sinuses, dissimilar types are encountered, autogenous vaccines, therefore, should be employed. $B$. pertussis. As far as known this is a distinct type and shock vaccines are applicable. The method of preparation and selection of strains from the standpoint of date of isolation may be a factor in the preparation of a potent vaccine. This is under investigation and should be considered.

Diphtheroid Bacilli.- This term is loosely used to cover miscellaneous types of bacilli resembling the diphtheria bacillus only in that they stain irregularly. Aerobic, microerophilic and anaerobic types are encountered. They have been found in many conditions such as indurated acne, leprosy, in apparently normal and in enlarged 
lymph nodes, they are common in the mouth especially if inflammatory conditions exist and associated with or overgrowing other bacteria in inflammations of the middle ear, pleurisy, etc.

The type encountered in the lymph nodes of Hodgkin's disease is supposed to have etiological importance. In typhus fever, Plotz claims that the type he has isolated in many cases is the etiological agent in this disease. Mallory more recently assumes another type to have etiological importance in scarlet fever.

It can at least be said that autogenous vaccines are preferable in the treatment of the miscellaneous forms of local inflammations.

Polyvalent Vaccines.-It is evident from the above outline that few of the so-called polyvalent vaccines are polyvalent in the sense that they contain representatives of all the types in the group. In many instances, they are really mixed vaccines and their probable specific value in an individual case should be considered and can be determined from the general facts given.

Vaccines of Mixtures of Bacteria, so-called Mixed Vaccines.Two kinds of mixed vaccines are used. The one is a mixture advocated for general use where a bacteriological diagnosis cannot be made. They are gunshot mixtures and have little, if any, justification except when it is probable that the infecting organism is one of those in the mixture. The other type is a mixture of the types of bacteria found associated in certain types of inflammation, in which their use is advocated. This would at first glance seem a satisfactory procedure, but unfortunately the conditions in which they are advocated are in most instances conditions in which are encountered the heterogenous or unrelated types of the different bacterial groups. Mixed vaccines of the autogenous flora, therefore, should be used as a rule.

Types of Vaccines.-Killed Bacteria.-This is the type of vaccine most commonly used and in spite of suggested substitutes is on the whole the most satisfactory type for general use. The usual method of killing the bacteria is by the use of heat. As heat, if excessive, may lower or destroy the immunizing properties of bacteria, the temperature to which they are heated should be the minimum that will insure death of the bacteria. In place of heat, some advise the use of disinfectants such as phenol, trikresol, etc. There is, however, insufficient evidence to definitely prove that this is an improvement over the application of minimum heat.

Bacterial Extracts, Autolysates, Digested Bacteria.-One of the objects in these preparations is to give a preparation that is more easily and quickly absorbed, so that the immunity response will also be more rapid. Another object has been to bring into solution the toxic elements hoping to get a more antitoxic type of response to their injection. As a matter of fact these toxic extracts do not cause the production of antitoxin and, furthermore, they are as a rule, less potent than the whole bacterium in stimulating the production of other antibodies. Unless very carefully used, such products 
may give rest to severe or even serious reactions. In some instances, the method of preparation is a secret one and the product widely advocated.

Atoxic Vaccines. - An attempt has been made to produce vaccines of bacteria freed more or less of their toxic portions, having the protoplasms which is considered the essential factor in the production of immunity. At present, this is in the experimental stage.

Live Bacteria.-The use of live bacteria is not likely to become general, although it is apparently a safe procedure under certain circumstances. It is based on the experience that an unnatural mode of entry of a virus usually leads at most to a local reaction. The advantage would be that the unchanged bacteria should give a higher degree of immunity.

Sensitized Bacteria: Living or Killed.-Besredka and others advocate the use of such vaccines on the ground that the treatment of the bacteria with a specific immune serum lessens or avoids the local and general reaction. This should, therefore, lead to a more rapid development of immunity as larger and more frequent dosage can be employed. Furthermore, the sensitized bacilli are probably more quickly disintegrated and their products more quickly absorbed. The objection to the living vaccines is that their infectiousness is not destroyed by the serum and should they, by accident, reach the normal portal of entry they might cause infection. Dead sensitized vaccines have been used both for prophylactic and curative purposes to a great extent.

The evidence of their superiority is not conclusive. A similar procedure, namely, simultaneous injections of bacteria and immune sera, has been used in certain prophylactic methods. This method is advantageous where the bacterium used is very toxic and in doses adequate for the production of immunity, gives severe reactions.

Deterioration of Vaccines.-As a general rule vaccines should not be used longer than four months after preparation and should be kept in the refrigerator.

Theoretical Considerations, Type of Case, Etc.-The use of specific vaccines is primarily based on the idea that the existing infection is not stimulating the production of sufficient antibodies to cause recovery. Therefore, if $w \epsilon$ inject vaccines in healthy tissues or the blood current, we cause the body to respond with a further production of antibodies, which carried by the blood to the infected focus or foci aids in recovery. Undoubtedly the vaccines stimulate healthy tissues remote from the infected diseased area which, because of slight absorption from this area, might otherwise respond slightly or not at all. On this basis, localized lesions of a subacute or chronic character should be the most promising cases for vaccination. Although this is true in general, there can be no doubt that some of the more general and acute infections are favorably influenced by vaccine therapy. It would seem that when the blood stream was invaded by the bacteria that all the tissues of the body would be 
stimulated sufficiently without the addition of vaccines. Here, however, we must distinguish three classes of infections; first, those diseases in which there is an initial invasion of the blood stream, with or without marked multiplication in the blood, with subsequent localization and practical disappearance of the infective agent from the blood; second, acute local infections, the bacteria being carried into the blood stream in small or moderate numbers, but no multiplication occurring in the blood (bacteremia); and finally, a septicemia with invasion of the blood stream and multiplication.

The first two types, one because of the tendency to localization, the other because of the inhibited multiplication in the blood stream, evidence the response of the protective force of the body but not necessarily the maximum response possible. In such diseases or infections, it may be possible to demonstrate the further production of antibodies due to vaccines, an example being typhoid fever. Furthermore, such injections may lead to a cellular response, viz., polynuclear leukocytosis or an increase if it already exists. It is easy to see, however, how easily injudicious vaccinations might exhaust this ability to respond with serious consequence to the patient. In the last group of cases, multiplication of bacteria in the blood stream with no tendency to localization usually means a breaking down of the barriers of protection and little is to be expected from further stimulation by vaccine. In practice this is generally the case.

From the standpoint of specific response the self-limiting types of infection of short duration are not likely to be benefited byvaccine therapy, primarily because true antibody response to vaccines is not immediate but takes several days to become marked and longer to reach its maximum.

The site of the infection is important. If the antibodies do not reach the focus in sufficient concentration to be effective, vaccine are not likely to be of much help. This probably explains the more or less unsatisfactory results in the treatment of infections of the mucous membranes and bone cavities.

There is a growing tendency to rely on vaccines to make up for insufficient surgical treatment, or delay the latter in the hope that vaccines will be effective. The relief of tension by incision, even if pus has not formed, is followed by a curative response on the part of the local tissues due partly to better circulation. Drainage, likewise, allows the exudation of fresh serum and cells, both factors being of the utmost importance.

Non-Specific Response to the Injection of Specific and NonSpecific Vaccines.-The theoretical considerations already given are based on the fundamental asssumption that the therapeutic value of vaccines are due to specific antibody response. This, however, does not explain certain prompt curative reactions observed after the injection of vaccines as well as other non-specific substances, especially when given intravenously. In some instances, such improve- 
ment may be illusory as the substance injected had a depressor action masking the clinical signs. The increasing number of reports of the curative value of non-specific substances is difficult of explanation and at first glance would seem to overturn our conception of the specific action of therapeutic vaccines. The treatment of typhoid fever by intravenous inoculation is a good example. An injection of the appropriate amount of typhoid vaccine is followed by a chill with rapid rise of temperature and a subsequent progressive fall, sweating, and marked subjective and general improvement. With these phenomena are associated a primary decrease with a sharp subsequent leukocytosis. This reaction in some cases develops into a recovery by crisis, in others the disease resumes its course and a second injection giving similar reaction may or may not cause a critical recovery. A similar crisis can be produced by other substances such as paratyphoid vaccine, colon vaccine, or even albumose.

Similar reactions have with curative effect been obtained in other diseases ${ }^{1}$ using specific and non-specific substances.

An explanation of these results is difficult. One factor is undoubtedly the sharp leukocytic response. The striking improvement cannot be directly attributed to an increase production of specific antibodies, even where a specific vaccine is used. Vaccination during the period of incubation of typhoid fever has little effect on the disease, which should be the case if mere increased production of antibodies was an important factor. The best results have been obtained after the tenth day of the disease when the reaction to the infecting agent has fully developed. Experimentally, it has been shown that the sera of immunized rabbits show a rapid increase in the antibody content when intravenous injections of typhoid bacilli or even non-specific proteins are given.

It would seem that reaction-producing protein acted as a nonspecific stimulus to the release and dispersion of antibodies already produced in response to infection. If the hemopoietic organs are the main source of antibodies, as is probably the case, this stimulus is similar in kind to that causing the cellular release or leukocytosis. Probably the specific vaccine would also stimulate the production of new antibodies to maintain the heightened content in the circulating blood. ${ }^{2}$

A further factor as shown by Jobling and Peterson is probably the mobilization of non-specific ferments as a result of the injection. The serum protease, a tryptic ferment, would act upon the toxic products of the bacilli reducing them to lower and non-toxic forms and explain the sharp improvement. The importance of the mobil-

1 See under Gonococcus infections.

2 Jobling and Peterson (Jour. Am. Med. Assn., I916, lxvi, 1753), whose theoretical considerations are contained in the above outline, in discussing this possibility say "the hematopoietic system has been attuned to respond to a non-specific stimulus with the production of specific substances. Dunklin, working in this laboratory, finds a marked increase in antibodies follows the intravenous injection of proteoses in immunized rabbits." They do not mean actual production but that more antibodies were found in the sera of blood drawn for test. 
ized lipase is obscure. The antiferment content of the serum is also influenced by the injection which may be a further factor. Desensitization or refractory condition of the body cells in that they do not respond to the typhoid bacillus and its products has been suggested in explanation.

The chill and temperature rise are important manifestations, as failing a reaction the curative response is not marked. How far the rise in temperature is due to the injected protein or due to dissolution to toxic fractions of the infecting bacilli is not settled. A similar reaction can be induced in the uninfected individual but a larger dose may be needed; furthermore the reaction is milder in low degrees of infection. The explanation of the beneficial effect of febrile reactions both after vaccines or as an intercurrect phenomenon in various conditions is still wanting.

Undoubtedly changes in the focal area, that is the site of localization of the bacteria, enter into the phenomena as well although an evident reaction may be absent. Whether this is in the form of either local tissue response or local desensitization or both remains to be determined. Using specific vaccines as in gonorrheal joint inflamations, the focal reaction is undoubtedly associated with beneficial results.

In considering the types of infections suitable for vaccine therapy we spoke of the ability of the body to respond in the various degrees of general infection. The ability to respond to non-specific substances parallels the response to specific vaccine.

Although we must agree that non-specific vaccines especially when given intravenously have a curative effect hitherto not sufficiently appreciated, we must not because of this cast aside the previous ideas of the importance of specificity. Non-specific proteins undoubtedly raise the resistance of experimental animals yet no one would consider using them in prophylaxis against specific disease. As a therapeutic measure much has still to be learned about the relative curative value of specific and non-specific proteins even in conditions where the latter are undoubtedly of therapeutic value. Nothing is known, as yet, as to the relative importance of the specific and non-specific response in many other types of disease, both chronic and acute. Theoretically, the added specific response to a specific vaccine should be of value. For the present at least, vaccine therapy should be considered from the standpoint of its historical development, viz., the importance of specificity, and the newer knowledge of the value of non-specific substance considered as a new phase of its development.

For this reason, we consider the discussion of the subject from the specific standpoint and the information given to insure specificity of vaccines as previously outlined, of basic importanice. The moderate knowledge we have of the value of non-specific vaccines is no excuse to ignore specificity in general vaccine therapy. Nor can it be used as a justification of wholesale use of mixed and pseudo- 
specific stock vaccines as they are used on the assumption that they contain bacteria specifically identical with those causing the infection.'

Reaction to Vaccines. - The reaction to vaccines may be local, focal or systemic or general. With proper therapeutic dosage, the local reaction is usually absent, or moderate; focal reaction inappreciable or moderate and the systemic reaction slight or at most moderate. Prophylactic injections may be followed by moderate local and general reactions, or exceptionally both are severe. Some prophylactic procedures give more reaction than others.

Dosage.-For prophylaxis, the appropriate amount and number of injections is the result of experience and should be closely adhered to, unless exceptionally severe reactions are encountered. Children as a rule, stand vaccines very well and full correction of the dose according to weight is unnecessary. For therapeutic use, it is better to start with small or moderate doses and increase the amount till the maximum dose is reached or a reaction encountered. The reacting dose is usually the maximum for the individual. The interval between doses may be 48 to 72 hours while the dose is small and the period lengthened as the dose is increased. For long standing chronic infections, longer intervals may be used from the start. As a rule, reactions of any extent should be avoided. Continued injections increasing in amount frequently lead to a result, after the treatment was about to be abandoned as a failure.

So-called Negative Phase.-Based on opsonic determinations Wright thought that there was an initial fall in opsonins after the administration of vaccines, especially if the dose was large. Unless the dosage be excessive, or vaccines be recklessly administered to a patient already overloaded with bacteria and their products, the possibility of a negative phase need not be considered.

Mechanism of Antibody and Cellular Response.-This has been fully covered by Dr. Hektoen in Vol. I.

Mode of Injection.-The subcutaneous injection of vaccines is most generally employed. Although the intravenous route has lately been advocated especially in typhoid fever, its possible dangers should not be overlooked. The intravascular agglutination of bacteria thus introduced with subsequent cerebral capillary emboli, or serious symptoms of shock because of the rapid dissolution of the bacteria and the formation of toxic substances, are possibilities that cannot be overlooked. In typhoid fever hemorrhage has followed injection. In fact, several deaths can be directly attributed to this mode of injection. Its advantage is that the antibody and cellular response is more rapid.

\section{VACCINES IN SPECIFIC DISEASES}

The following is a brief summary of the status of the prophylactic and therapeutic use of vaccines, in specific diseases and in the various types of infection with the dosage and mode of administration.

1 See also Hektoen, Jour. Am. Med. Assn., I916, Ixvi, I591. 
Staphylococcus Infections.-Staphylococcus vaccines are of great value. The best results have been obtained in chronic or recurrent infections such as pustular acne, furunculosis and sycosis vulgaris. Stock vaccines are of value, but should failure be encountered an antogenous vaccine should at least be tried.

Acne.-Undoubted benefit is obtained in many pustular cases by the administration of staphylococcus vaccine alone. In some cases, however, and especially in the deep indurated types of disease, staphylococci may be of minor importance. Several other types of bacteria have been demonstrated in these conditions, especially the acne bacillus. This bacillus may also have some importance in the development of comedones. Possibly the staphylococcus is essentially only a secondary invader increasing the pustular aspect of the disease. Injections of $\mathrm{B}$. acne vaccine 3 to 5 millions increasing to Io to 20 millions may be tried in these cases either alone or with the staphylococcus vaccine with a hope of better results. B. acne is somewhat difficult to isolate and cultivate and an autogenous vaccine may be difficult to obtain. With our present knowledge, however, it is to be preferred wherever obtainable. Persistence in treatment is often rewarded by improvement and ultimate cure.

Furunculosis.-The influence on existing furuncles is relatively slight in our opinion. On the whole, however, the likelihood of further lesions or of recurrence is materially lessened. The injections should be continued for some time after the disappearance of the lesions and resumed promptly, if there is any indication later of a relapse.

Sycosis Vulgaris. - Vaccines may aid materially in the treatment of this disease.

Other Skin Lesions.-Staphylococcus pustular lesions may be associated with eczema, rosacea, etc. Vaccines may be of help in controlling the inflammation.

Miscellaneous Infections.-In acute local infections vaccines are of doubtful value and surgical procedures usually suffice. Various chronic inflammations may be encountered in which the staphylococcus is either the primary or secondary infecting agent. Vaccines may be of benefit if the location of the infection is favorable.

Other Methods of Treatment.-These should be carried out as thoroughly as though vaccines were not being used, especially surgical treatment. In skin infection we cannot too strongly advise the protection of the surrounding healthy skin from contamination by discharges from existing foci.

Administration.-As a general rule, the more extensive the lesions, the smaller should be the initial dose, which may range from $5^{\circ}$ to Ioo millions, this may then be increased to rooo millions or more. In the more subacute types of infection, moderate focal reaction may not be without benefit, but the dosage as a rule, should be controlled that no sharp aggravation of the local conditions is induced.

Streptococcus Infections.-Local Infections.-Acute local infections due to the streptococcus pyogenes types are surgical conditions. 
The local application, when possible, of streptococcus serum is probably far more valuable than vaccines. When the infection has become subacute, some help may be obtained from vaccines. Various types of streptococci are found in many acute and chronic conditions with or without systemic involvement. Infections such as common colds, bronchitis, sinus involvements, and mouth infections per se are discussed later. Focal streptococcus infection in relation to systemic disease is discussed under this heading. The evidence that puerperal infections are influenced favorably by vaccines, is far from strong. This condition is variable at best and treated cases are often not sufficiently controlled especially by blood cultures to determine the degree of general dissemination, which is a large factor in the mortality.

Erysipelas.-The opinions as to the value of vaccines are as variable as the manifestations of the disease. Erdman ${ }^{1}$ in an analysis of 800 cases of which ninety-five were treated by vaccines concludes, "that the duration of the disease was not at all lessened, the mortality remained at the same level and there was no immunity guaranteed against spreading of the lesion, nor were complications such as cellulitis and abscesses, prevented." Although we encounter more favorable opinions, they are in isolated or small series of cases and their value for that reason slight. Nearly all vaccines used have been stock vaccines and although this may be a factor in the results autogenous vaccines are not feasible in the great majority of cases.

General Infections.-The value of vaccine in septicemia either cryptogenetic or as a complication of a local infection is doubtful. Individual cases vary widely and the available data is meager. There is a slight indication that several large doses of sera followed by autogenous vaccines may be of help. The negative results imply that autogenous vaccines are probably of no value in infective endocarditis due to the Streptococcus viridans, although isolated recoveries have been reported.

Dosage.-In severe or general infections the initial dose should be small, not over 5 to ro millions. In the more subacute cases, the dosage may be larger. The maximum dosage is usually 500 millions.

Pneumococcus Infections.-Pneumonia.-See article by Dochez. etc.

Other Infections.-See under common colds, bronchitis, otitis,

Dosage.- - Io to 500 millions.

Gonococcus Infections.-Urethritis.-There is no definite evidence that vaccines influence this condition, nor that they limit to any appreciable degree, the extension of the infection to adjacent or remote organs or tissues. The reports available show a slight reduction in the number and incidence of complications but not sufficient to warrant the general use of vaccines.

${ }^{1}$ Jour. Am. Med. Assn., 1913, 1xi, 2048. 
Vulvovaginitis.-The reports of those who have used vaccines are not convincing. Although some have seen some benefit, the degree of inprovement has not been marked. Our own experience in the infectious disease hospitals has been, on the whole, disappointing. Pearce ${ }^{1}$ has pointed out, however, that the strains of gonococci from vaginitis in children differ immunologically to a relative degree from those found in adult infections. This may be a factor as most of the vaccines used have probably been of strains isolated from adult sources.

Conditions due to Extension of Infection.-Epididymitis.-Several observers have credited vaccines with a hastened cure. Bruck agrees with this opinion.

Prostatitis.-Although isolated cases have apparently been benefited vaccine therapy, on the whole, has been employed without results.

Pelvic Infections. - There is some indication that vaccines are of value in recent infections, if carefully used. Naturally vaccines should not be considered in place of surgical treatment, if the latter is indicated.

Conditions due to Dissemination.-Gonococcemia.-This condition frequently arises as is evidenced by the occurrence of remote foci. As a rule, the numbers of gonococci are small and the main clinical significance is the development of foci of localization. In some cases, the number may be large and the condition shades into a true septicemia, either mild or severe, and with or without localization. Endocarditis may develop. Theoretically, at least, these conditions should be treated by gonococcus sera, rather than vaccines. The latter might be employed when localizations develop with subsidence of the blood invasion. The experience in this direction is too limited to warrant a statement as to the value of such treatment.

Para-arthritis and Similar Conditions.-Of all types of gonorrheal infection, this has been most successfully influenced by vaccine therapy. In the subacute cases which have not gone on to fibroid changes, the majority have been benefited and in some the results have been startlingly good. A moderate number of chronic cases, generally showing multiple joint involvement with persistent urethral and prostatic infection respond not at all to vaccine therapy.

Gonorrheal Iritis.-Vaccine therapy supervised by a competent ophthalmologist may be of benefit. It should be tried in the recurrent types.

Dosage and Administration.-With stock vaccine killed by heat, the dosage ranges, as a. rule, from 25 to 500 millions. Up to 1000 millions has been used by some. With freshly isolated strains, the initial dose had better be much smaller. The interval may vary from two to seven days, the shorter interval being preferred while the doses are small. The dosage should be increased rather rapidly till some evidence of reaction is elicited and the intervals and dosage then determined by the degree of reaction. Although a reaction of 
any extent should be avoided, a mild focal ${ }^{1}$ or febrile reaction seems not only of value as indicating the limits of dosage, but also because of the better clinical results. Criticisms of the vaccine in some instances have seemed to us to be due to inadequate dosage as the patients improved with larger doses.

Sensitized vaccines have been used by some observers, but their superiority over ordinary killed vaccines has not been demonstrated.

Intravenous Administration.-Bruck and Sommer ${ }^{2}$ have recently advocated the intravenous use of vaccines for diagnostic purposes and also claim a greater therapeutic effect when vaccines are thus administered. A quantitatively greater temperature reaction, showing the existence of a relatively specific response, was elicited in those having gonorrheal infections. Unusually good results are claimed in the treatment of epididymitis, arthritis, and even prostatitis and some cases of urethritis were favorably influenced. This procedure cannot be advocated for general use at present. For this reason we refer those interested from the experimental standpoint to the various articles which have appeared. ${ }^{3}$ We have had no experience with intravenous injections in man except with typhoid bacilli. A preparation known as Arthigon was used by Bruck and others. One c.c. of this contains, according to Schindler, 80 million gonococci. Naturally, the activity of other preparations might vary in action due to methods of preparation or strains employed, even though the dosage be the same. Muller and Weiss ${ }^{4}$ claim that the intramuscular injection of non-specific substances, such as sodium nucleante or milk are as efficacious as the intravenous injection of gonococcus vaccines. Miller and Lusk ${ }^{5}$ also report good results in cases of arthritis following the intravenous injection of typhoid vaccine. These results indicate that the non-specific response to vaccines is of importance in gonorrheal infections. Furthermore the results explain the growing conviction that a reaction following gonococcus vaccine is of therapeutic value.

M. melitensis.-Prophylactic inoculation in doses of 4 millions repeated several times has been employed by Eyre. While the number of cases immunized was small the results indicated a short immunity. Larger doses might give an immunity of longer duration.

Therapeuticly good results have been reported in the treatment of the disease. Eyre believes that even in the acute cases, vaccines lessen the severity and shorten the disease. He used 5 to 10 millions; others have used doses up to 50 millions.

Meningitis (Epidemic Cerebrospinal).-Prophylactic.-Probably the meningococcus primarily invades the blood stream and then

${ }^{1}$ A positive focal reaction is of diagnostic importance, the absence of reaction is not.

2 Munch. med. Wchnschr., I913, Ix, 1185; Menzer, Med. Klin., I913, ix, 1332; Fruhwald, Med. Klin., I913, ix, I799; Kyle and Mucha, Wien. klin. Wchnschr., 1913, xxvi, I 755; Bordack, Munch. med. Wchnschr., I913, lx, 2622; Kreibich, Wien. klin. Wchnschr., I913, xxvi, 2024.

Loc. cit.

Wien. klin. Wchnschr., r916, xxix, 249.

s Jour. Am. Med. Assn., I916, lxvi, 1756. 
localizes in the meninges. If the individual were immunized by vaccine injections the immunity so produced should prevent such invasion or promptly dispose of any meningococcus finding their way to the blood stream. Because of the fact that antibodies are easily produced by vaccine injection, Sophian suggested the general use of vaccines for immunization. Three injections, at week intervals of doses of 100 million, 500 million and I000 million would seem to be satisfactory, based on the antibody production response in a series of persons vaccinated with varied doses. A local reaction is usually produced which may be somewhat severe. A constitutional reaction may be absent or there may be some malaise, or occasionally, a rise in temperature with severe headache and nausea and vomiting. Actual epidemiological data as to the value of prophylactic vaccination is very limited. Of about roo persons in Dallas, few of whom had complete injections, two nurses developed the disease some weeks later but recovered. On 280 families in which the disease had occurred, no cases developed subsequent to vaccinations.

Therapeutic.-In subacute or chronic course where lumbar puncture and administration of serum, although repeated five to six times or more, has little or no effect especially on the presence of meningococci, vaccine therapy may be of value. An autogenous vaccine, if procurable, should be injected at intervals of two to three days. The initial dose is roo to 250 millions to be increased to rooo million or more depending on the reaction. A limited series of cases has been treated in this way by Du Bois and Neal.

Micrococcus Catarrhalis.-See under Common Cold, etc. Dose. -Ten to 500 millions.

Typhus Fever.-Prophylactic.-Plotz and his associates have not succeeded in producing immunity in experimental animals by the injection of bacilli from typhus fever cases. Until successful in this direction, there is little likelihood of the vaccine being efficacious in man. Recently, two workers from the Mount Sinai Hospital developed typhus fever although vaccinated previously.

Therapeutic. - No data are available.

Typhoid Fever.-Prophylactic.-The use of typhoid vaccine for prophylactic purposes had its origin in I896, when Pfeiffer and Kolle injected two men with killed typhoid bacilli and showed that the same protective substances developed in their blood as in that of a person recovering from typhoid fever. During the same year, Wright inoculated two men and published the results of these injections. To him more than anyone is due the credit of placing typhoid vaccination on a practical basis. In $\mathrm{I} 898$, he inoculated 4000 men in India. In I900, with Leishmann, he supervised the inoculation of the troops engaged in the Boer war. In I909, vaccination was started in the United States army under the direction of Russell. The good results obtained in armies has been duplicated in civil life. The early results with vaccines were good, but much better results have been obtained with improved methods of preparing the vaccine 
and with a better knowledge of the optimum number of injections and dosage.

Selection of Vaccine.-Although various types of vaccines have been tried, and although some may have certain advantages, we feel that for the present at least, the vaccine as made for the United States Army is the most reliable for general use. This is a vaccine made from a strain of known antibody-producing properties, the bacilli being killed by the application of a minimum of heat. It would be well to be sure of the vaccine, before using it and that it is not over four months old and that it had been kept in the refrigerator. Unfortunately, some of the vaccines available are relatively inactive due possibly to the methods, preparation, preservation or too long a labelled period of potency, or a combination of these factors.

Of the various modified vaccines, sensitized vaccines have been used most widely. Gay has been its main advocate in this country. He employs sensitized bacilli killed by alcohol. The sensitized live vaccine of Besredka has the drawback of all live vaccines and its general use is not probable. For a fuller analysis of these modified vaccines and bibliography, the reader is referred to the articles noted. ${ }^{1}$

Administration.-With the usual killed vaccine, a strong degree of immunity is conferred only after three injections, given at intervals of seven to ten days. The doses are, first 500 millions, second and third 1000 millions. A child of 50 pounds would require about one-half of that of an adult. For those engaged in business, successive Saturdays are convenient times for injection. If the injections are given about 4 P. M. the reaction, if any occurs will take place while abed. Abstinence from alcohol should be enforced, if possible. The injection should be subcutaneous, if injected more deeply, possibly into the muscle, absorption may be more rapid and the reaction enhanced. The injection should be on the outer surface of the arm (left) about the insertion of the deltoid.

Reaction.-The injection may cause some burning, but this quickly subsides. After the lapse of several hours, a local reaction may develop consisting of a red, tender, swollen area several inches in diameter. Sometimes the reaction is more extensive and severe. The lymph nodes of the axilla may become enlarged and tender and constitutional symptoms may also develop. The latter consist, in most instances, of headache, malaise and some rise in temperature. A severer reaction is present in less than $x$ per cent. of the case injected. In any event, neither the local or general symptoms need occasion any alarm, being of no importance except for the discomfort. The general reaction rarely lasts more than twentyfour hours. There is no relation between the severity of the reaction and degree of immunity which follows. The average frequency and severity of reaction is shown by the following table by Russell:

${ }^{1}$ Gay and Claypole, Arch. Int. Med., I9I4, xiv, 671; and Sawyer, Jour. Am. Med. Assn., I915, lxv, I413; also, Nichols, Jour. Exper. Med., I915, xxii, 780. 


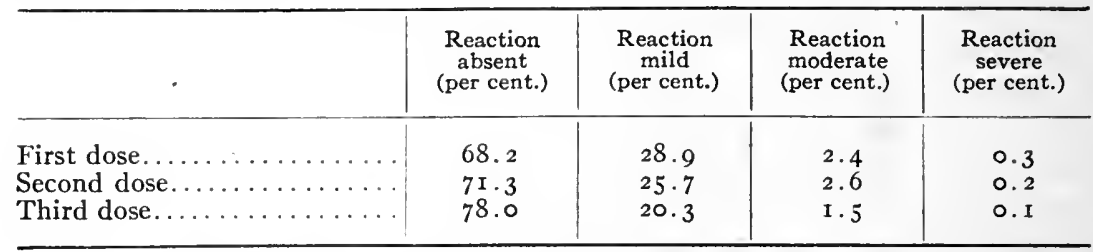

So-called Negative Phase.-The fear that vaccination, during a period of exposure will lower a person's resistance and render him more susceptible to infection is without foundation. Should a person be already infected, the injection also does no harm. It apparently influences the course of the disease very little, except that in several instances we have found that the injection apparently made the onset more acute in character.

Results of Immunization.-The immunity following adequate vaccination is almost complete, and the general results are only exceeded by those following the Jennerian method of vaccination against smallpox. Immunization, however, is no excuse for laxity in the enforcement of other preventive measures. The few cases of typhoid developing after vaccination which we have investigated were among physicians or nurses caring for typhoid cases and in one other case a woman was being nursed by her mother who was found to be a typhoid carrier. Excessive exposure or dose of infectious material evidently will override the immunity conferred by vaccines. Any extensive failure would raise a strong suspicion as to the value of vaccine used. The general observation has been, that should typhoid develop after vaccination, the disease is less severe and the mortality reduced 50 per cent. In the few cases we have had, the mortality was high, but in each instance due to hemorrhage.

Duration of Immunity.-Firth, from a wide experience in the Indian army, concludes that the immunity begins to diminish after two and one-half years. Even after four to five years, however, he finds that the rate among the vaccinated is still only one-fourth of that among the unprotected troops. Revaccination, as practised in our army, that is after expiration of the enlistment period, would seem to be generally satisfactory. The experiences in the present war, however, indicate that under conditions of constant exposure to infection associated with reduced resistance due to strain and privation, immunization should be repeated every year.

Application.-The nearly general compulsory immunization in armies, military and naval organizations, should be extended to institutions, especially general hospitals.

The immediate vaccination of all members of a family where a suspected case of typhoid is found should be enforced wherever possible. Those employed in construction camps, vacationists or those who travel a great deal should be vaccinated. In spite of all sanitary precautions, the typhoid carrier is a constant menace, 
and every person vaccinated if protected when exposed, is not only a case less of typhiod fever, but for every thirty persons so saved from infection, one typhoid carrier is avoided.

Therapeutic Use.-The vaccine has been employed both subcutaneously and intravenously.

Subcutaneous Injection.-Watters ${ }^{1}$ has collected II 20 cases thus treated with seventy-one deaths, that is, a mortality of 6.3 per cent. Probably seventeen cases with fifteen deaths could be omitted from this series on the ground that they were moribund when treated or for other reasons. This would give a corrected mortality of 5 per cent. In the cases where the number of relapses are stated, the incidence was 6 per cent.

A surprising lack of correlation is noted between dosage and mortality and relapse percentages, thus:

\begin{tabular}{|c|c|c|c|c|}
\hline Author & Cases & Relapses & Deaths & Dosage \\
\hline 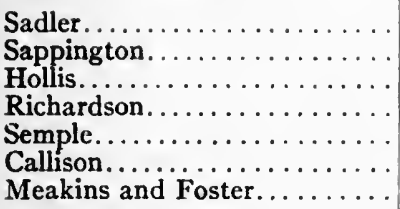 & $\begin{array}{l}92 \\
22 \\
51 \\
28 \\
60 \\
38 \\
41\end{array}$ & $\begin{array}{r}0.0 \\
0.0 \\
15.6 \\
3 \cdot 5 \\
3 \cdot 3 \\
2.6 \\
2.4\end{array}$ & $\begin{array}{r}15.2 \\
13.6 \\
3.9 \\
0.0 \\
3.3 \\
13.1 \\
2.4\end{array}$ & $\begin{array}{c}12-2 \mathrm{~m} \\
5-50 \mathrm{~m} \\
10-250 \mathrm{~m} \\
10-100 \mathrm{~m} \\
50-200 \mathrm{~m} \\
500 \mathrm{~m} \\
1000-2000 \mathrm{~m}\end{array}$ \\
\hline
\end{tabular}

So many factors enter into the death rate that one cannot ascribe the lowered death rate directly to the vaccines. In the different series treated, the rate varies very widely.

In general, those who have used the vaccines, agree that the patients so treated seem brighter, that the temperature averages lower, that the febrile period is appreciably shortened, and that the number of relapses are fewer. The more moderate dosage 250 to 500 millions would seem advisable.

Intravenous Use.-The therapeutic method was introduced by Ichikawa in I9I2. He published a series of cases in 1914, and since that time several hundred cases have been treated by various observers. ${ }^{2}$ In many of the cases so treated, a critical drop of temperature, with very marked improvement, has followed the reaction due to the vaccine, within twenty-four to forty-eight hours. Some failed to respond, others only temporarily, but about 50 per cent. became convalescent. In spite of the curative results obtained, there is insufficient data to warrant an idea as to the influence of this method of treatment on death rate. The method is not without danger. A munber of cases have developed fatal hemorrhage from the bowel or elsewhere after inoculation. Several have died shortly after injection, in two cases of which there was

1 Med. Record, 1913, lxxxiv, 518.

= See Summary of Bibliography, McWilliams, Med. Record, Oct. 10, 1915, and Jour. Immunol., 1916, i, 759; Gay and Chickering, Arch. Int. Med., 1916, xvii, 303.

Vor. 1V. -4 
an associated pneumonia. Any evidence of hemorrhage is apparently a strong contraindication, likewise any involvement of the heart or lungs. The dosage and kind of vaccine employed has differed widely. From 50 to 250 millions typhoid bacilli would seem to be the average dosage. No one kind of vaccine seems better then another, although Gay strongly advocates sensitized vaccines made according to his modification. A distinct reaction with an initial rise in temperature and an associated polynuclear leukocytosis seems essential to success.

Paratyphoid Fever and other Types of Infection due to the Paratyphoid-enteritidis Group.-The paratyphoid bacillus A type produces paratyphoid fever only and is infrequently encountered. The paratyphoid bacillus B type and the enteritidis bacillus are the causative agents in most meat or food poisoning outbreaks of the choleraic or gastrointestinal type. Both produce paratyphoid fever, the former being the mose commonly encountered bacillus in this disease. Infections by these types are relatively uncommon in this country. They are, however, very frequent abroad.

Prophylactic Vaccination.-Because of the similarity clinically, of typhoid and parratyphoid fever and because of the close relationship of the bacilli involved, vaccination with mixed vaccines has been employed in order to protect against the several types of infection. Experimental evidence shows that the antibody response is satisfactory to the individual strains in the mixed vaccine. Where paratyphoid fever is fairly common, this procedure would seem to be of value. No statistics are available as yet. It would seem that vaccines would give little protection against food poisoning where the dose of bacilli or pre-formed toxins was large, although systemic invasion might be averted in some instances due to the immunity conferred by the vaccines.

The combined vaccine is given in three doses, a week to ten days apart, the first dose containing 500 million typhoid bacilli and $25^{\circ}$ million of the paratyphoid A type and of the paratyphoid B type, the second and third doses being twice these amounts.

Bacillary Dysentery.-Because of the high toxicity of dysentery vaccines, Shiga has used the simultaneous injection of vaccine and serum. The results have not been wholly satisfactory, although the death rate among the vaccinated was lowered. The prevalence of infections by different types of dysentery bacilli adds to the diffculty of the problem. Therapeutic inoculation is not practised. It has been claimed that vaccines are of value in the carrier state.

Plague.-As the immunity following prophylactic vaccination is relatively short, the best results are obtained during the epidemics, in which case sufficient protection is given to last over the period of greatest danger. Although the protection against the bubonic plague is only relative, the mortality among those vaccinated is also lowered. No protection is conferred against the pneumonic type. Haffkine advises 3 to 3.5 c.c. of his specially grown broth cultures 
and advocates a second dose after eight to ten days. Kolle grows his culture on agar and advises a dose of $2 \mathrm{mg}$. In either mode of preparation, the bacilli are killed by heat. The reactions following vaccination are relatively severe. Therapeutic vaccination is not used.

Cholera.-Prophylactic inoculation confers a relative immunity against infection, but the mortality among the vaccinated is only slightly reduced. Kolle advises two doses of killed vaccine about a week apart, the dose being 2 and $4 \mathrm{mg}$. of cholera vibrios. Haffkine uses live vaccines. The reactions following vaccination are frequently severe.

Combined Prophylactic Vaccines.-Where two or more preceding diseases, typhoid fever, paratyphoid fever, cholera, plague, etc., are endemic, combined vaccines for immunization against the endemic types would be a convenience; furthermore, under urgent conditions, would save time. Castellani, especially, advises their use based on experimental inoculations in animals and in man. The reactions were not severe except where cholera and plague with or without other types was inoculated. The results with dysentery have been the least satisfactory. The dosage for the various combinations have probably not been sufficiently standardized, nor are field data available on their value in practice. ${ }^{1}$

B. Pyocyaneus and B. Proteus.-These bacilli are mostly encountered as secondary invader in various infections. Under these conditions, according to several observers, some benefit may be obtained by vaccines. The dosage is from 25 to rooo millions.

Glanders.--Vaccines may be tried in the subacute or chronic cases. Only a small number of cases have been treated, but the reports have been favorable. The dosage must be carefully controlled as the vaccine acts like mallein, that is, the infected individual is hypersensitive to the injection of glanders bacilli and their products. The temperature reaction following injection is the best guide to dosage. This rise should be only moderate at most. From Io to 200 millions is the general range of dosage. Injections may be given every two to four days depending on the degree of reaction.

B. Coli and Related Bacilli.-Genitourinary Infections.-In cystitis with or without infection of the upper urinary tract vaccines seem to be of benefit. In pyelitis after the acute symptoms have begun to subside, vaccines may be of aid. The reported results, however, have not been concordant, some observers having obtained benefit, others none at all. Other therapeutic measures should also be employed if any surgical condition interfering with free drainage is present, vaccines alone under these circumstances are of little help.

Other types of infections such as of the drainage openings following appendicitis or cholecystitis as well as pelvic abscesses after drainage have been treated with vaccines. A diminution of fever

${ }^{1}$ See Castellani, Centralbl. f. Bakteriol., 1915, lxxvii, 63, or Jour. Trop. Med., I914, xvii, 326 . 
and of the discharge with a shortening of the period of convalescence seemed to result from their use.

Types of Bacilli Associated and Dosage.-Colon types are most commonly found including the mucoid aerogenes varieties. Intermediates or paratyphoid-like bacilli are also not infrequently encountered. The dosage will vary with the individual strains as some are more toxic than others. An initial dose of 25 millions should be used and the amount of increase based on the reaction. The maximum dose possibly may be 200 to 500 millions or more.

Atrophic Rhinitis.-The Bacillus ozænæ closely resembling the bacillus of Friedlander is constantly found in this condition and vaccines of this organism alone or with the associated cocci have been used. Although some effect has been noted the results have not been satisfactory. Recently Perez has isolated an organism, "Coccobacillus fotidus ozæna" which he believes to be the etiological agent. Vaccines of this organism have given more suggestive results. In some instances it would seem advisable to use vaccines of the ozænæ bacillus as well. The subject, however, needs much further investigation, especially as to the relationship of the various "Perez types" found by different investigators and whether fetid rhinitis varies etiologically. ${ }^{1}$

Rhinoscleroma.-Vaccines of the rhinoscleroma bacillus found in this condition have in some instances seemed to be beneficial. They are at least worthy of trial.

Other Infections due to B. Friedländer and Related Types.These bacilli may be found in pneumonia, and its complications and in inflammations elsewhere in the respiratory tract and accessory sinuses including the middle ear and mastoid. In the acute condition vaccines are probably of no value. The data available are meager, but vaccines may have some value in the subacute infections or possibly as an accessory in some conditions after surgical treatment.

Dosage.-As for similar types (see above), the Perez bacillus vaccine is used in an initial dose of 50 millions, which is increased till a focal reaction in form of coryza, free discharge from the nose, and sensations of heat and fullness are induced.

Pertussis.-Prophylaxis.-Although some protection is conferred by this procedure, it is difficult at the present time to judge of the degree. One difficulty is the lack of reliable statistical data on sufficiently large series of cases with details as to the vaccine used and the dosage employed. One such series, an outbreak in an institution, is reported by Hess. ${ }^{2}$ Of 244 children immunized, twenty developed the disease. Of the unvaccinated children, eighty could be considered equally exposed, of these fifty-nine developed pertussis. Although these figures cannot be taken wholly at their face value, Hess concludes, considering all the associated factors, that the vaccine did materially reduce the number of cases. These results as

1 Horn, Jour. Am. Med. Assn., I9r 5, lxv, 788.

2 Jour. Am. Med. Assn., I914, lxiii, roo7. 
well as those of Sill, Luttinger and others warrant its general trial, especially as the procedure is innocuous.

Therapeutic.-In the above-mentioned outbreak, Hess could see no effect on the disease even in the cases that received both prophylactic and therapeutic inoculations. In fact, some of the severest cases were encountered in the latter category. It is difficult to correlate these results with those of Sill, Luttinger, Hoag and others, who believe that vaccines lessen both the symptoms and the duration of the disease. Recently we have received reports from individual physicians treating small series of cases, which without further analysis seem encouraging. The prompt amelioration in many cases thus reported cannot be considered as due to the production of immune bodies. On the whole, the results suggest that the vaccine is of value and that with more knowledge as to the most satisfactory type of vaccine and dosage better results would be obtained.

Dosage.-For children over one year old, we advise an initial dose of 500 million; two days later I billion is given and two days after the second, a third injection of 2 billion. An interval of several days is allowed to elapse after the third injection. If the improvement is not marked, one may give further injections. Larger doses may be given with some hope of result. No vaccine should be administered on the day the patient feels worse than before, or has a temperature of $100.5^{\circ}$ not attributable to intestinal trouble.

For children less than one year the initial dose is $25^{\circ}$ million while adults of normal weight should receive I billion.

Prophylactic injections are given every third day and consist of three injections of 500 million, 2 billion and 3 billion respectively.

There may be some local reaction (redness, induration) which is insignificant and a slight general reaction, consisting in a little fever. In active cases, there may be a temporary aggravation of the symptoms.

Influenza Bacillus Infections.-No data is available as to the effect of vaccines as a preventive or therapeutic measure in true epidemic influenza. Bacilli of this group are encountered in various acute and chronic inflammations of the mucous membranes of the respiratory tract as well as the accessory sinuses, middle ear, and conjunctiva. Influenza meningitis and pneumonia also occur. Their presence in some of these conditions is not necessarily evidence of their etiological importance. This group of organisms has been included in mixed vaccines for common colds, etc. The value of autogenous vaccines in subacute or chronic conditions where the influenzal types, as based on careful bacteriological examination are apparently of direct etiological importance, is problematical. The data is too meager for any opinion. In a few instances we have had suggestive results.

Dosage.-A small initial dose, to to 20 millions would seem to be indicated and the increase made dependent on the reaction. A maximum dose cannot be given as this will probably depend on the strain 
used as well as on the patient. Two or 500 millions may probably be given.

Tuberculosis.-Tuberculin therapy is usually considered a special subject as the mechanism of its action is so essentially different. The various attempts to use vaccines especially of non-pathogenic acid-fast types have no rational basis. Prophylactic vaccination has been done in a few cases by Webb. As live bacilli only give any appreciable immunity, this method is not likely to be adopted. The danger is not so much in the lesions immediately developed, which give an immunity to infection from without, but in the possibility of later dissemination from these lesions with active tuberculosis should latency not be maintained by the resistance of the subject. Should a complete healing occur, immunity would disappear.

When secondary infection is present, vaccines of the associated bacteria may, according to a few observers, be of benefit. The improvement due to vaccines has not been sufficiently marked to lead to their more general adoption.

Focal Infection in Relation to Systemic Disease.-A discussion of this condition as well as of the means to be employed in the search for the focal infection and the methods applied in making bacteriological diagnosis cannot be given here. Although vaccines are used in some cases, the subject is really more one of symptomatology and diagnosis and on these depends the question of the selection as well as the advisability of a vaccine. The essential factor in treatment is the successful search for, and the treatment or eradication of the focal infection which in itself is commonly followed by improvement or recovery. Vaccines should be considered as an accessory. For instance, the extraction of teeth having apical abscesses is frequently all that is necessary. In pyorrhea with associated joint symptoms, the mechanical treatment is likewise commonly efficacious. If, in pyorrhea, the period of a single treatment be too prolonged and too vigorous, reactions in the joints may be observed. In other words, autoinoculation is apparently caused by the treatment and is a factor in recovery. Whether autogenous vaccines in addition are of value is a question. If we had some way of determining which of the various streptococci in the pocket was of importance, we would have a more rational basis for the application of vaccines. The use of stock vaccines cannot be considered more than a make-shift, in place of careful clinical and bacteriological investigation. Each case is a separate problem for their application. The most frequent bacteria encountered in these conditions are the streptococcus and the gonococcus. The latter is discussed under that heading.

Miscellaneous Conditions.-Common Colds.-Although both prophylactic and therapeutic inoculations have been found beneficial by some, there is some scepticism of their value. Little is known as to the primary etiological agent or agents. Various bacteria, pneumococci, streptoccoci, Micrococcus catarrhalis, influenza bacilli, Bacillus segmentosus, etc., have been found as the predominating organ- 
isms in various outbreaks or in different individuals during the same outbreak. These types may be simply secondary invaders. Kruse and Foster give strong evidence that some outbreaks at least are due to a filtrable virus.

Bronchitis and Other Chronic Respiratory Conditions.-Some have obtained a moderate amount of benefit from autogenous vaccines of the predominating bacteria. In most of the cases we have seen, the benefit was slight. Anatomical changes may be a factor in keeping up the inflammatory process.

Infections of Sinuses and Middle Ear.-In subacute or chronic inflammations, correction of anatomic anomalies and proper drainage are only slightly aided by vaccines, if at all. The general unsatisfactory results in the treatment of these conditions is to a great extent due to anatomic conditions, viz., relatively poor circulation and commensurate inaccessibility to antibodies. A common difficulty in preparing a vaccine is the secondary invasion by one or more types of bacteria. On the whole a moderate degree of improvement is the most to be expected from vaccines in these conditions.

Mouth Infections.-The vaccine therapy of Riggs disease or pyorrhea by vaccines of the predominating easily cultivable bacteria is strongly advocated by several observers. The multiplicity of bacteria, including spirochetes and fusiform bacilli, found in this condition, not to mention the ameba recently ascribed the etiological agent, make one question the curative value of vaccines made from the small fraction of the total flora.

In many cases the vaccines do not even contain all the varieties of one type present in the pockets. Those advocating vaccines employ the usual local surgical treatment, which in the hands of most operators, when used alone, gives equally good results. The final argument of those advocating vaccines that they influence the systemic symptoms should they exist, is another subject (see focal infections).

Selection of Vaccine Dosage, Etc., in Above Conditions.-Because of the varied types encountered not only in different cases but even in the same case, a careful bacteriological examination is evidently a necessary preliminary to obtain the appropriate strains for an autogenous vaccine. Stock vaccines are widely advertised for the various conditions, but as the types of organisms in these conditions are usually members of heterogeneous groups, their value is doubtful to say the least. As Irons remarks, vaccines of mixed cultures usually means unknown cultures. The dosage of the individual types has been given. Where several types are used in the vaccine, the amounts should be so adjusted that prorata fraction of the dose for each can be administered. The degree of reduction of the dose of each type should be nearly proportionate to number included.

Hodgkins Disease. - The etiological importance of the diphtheroid bacilli is not settled. How far the benefit obtained where vaccines were used may have been due in great part to the associated thera- 
peutic measures, is open to question. The dosage ranges from 5 to IO0 millions.

Bacillus Carriers, Chronic.-The importance of the chronic carrier in maintaining typhoid fever in hygienically civilized communities is only equalled by the general failure of the therapeutic measures which have been used. Vaccine therapy continued over long periods has apparently been successful in a moderate number of cases as reported by Meader and Stone. Although the intermittency of excretion and the occasional spontaneous recovery of cases makes one question the results, the method should be more generally tried out. Meader believes that the rise in the bactericidal titre of the blood is essential to success. More recently the method has been advocated for dysentery carriers.

\section{GENERAL OBSERVATION ON THE THERAPEUTIC AND IMMUNIZING USE OF SERA}

The fundamental principles of serum therapy as given by $\mathrm{Dr}$. Hektoen in Vol. I, require no additions. A few practical points, however, are grouped together here before taking up the individual diseases in which there have been additions to our knowledge since the subjects were considered in Vol. II.

The antitoxic sera are alike in that, when present, they absolutely prevent infection and if in sufficient amount they neutralize any quantity of toxin. The antitoxins neutralize the toxins of the whole groups of bacilli producing them; thus diphtheria antitoxin produced in an animal through the stimulation of the extracellular toxin produced by any diphtheria bacillus will neutralize not only the toxin produced by it.but also the toxin produced by every strain of bacilli. The same is true of the tetanus antitoxin. These two microörganisms are also peculiar in that the extracellular toxins produced by these two bacilli are so much more powerful than their endotoxins that we do not need to concern ourselves with the effect of the latter. In immunization the antitoxins and other sera are alike. A subcutaneous injection gives immunity for ten to twenty days. A second injection adds a somewhat shorter period of immunity. The method of administering each serum and antitoxin varies according to the routes by which antitoxin and other antibodies gain access to the toxins and bacteria. In diphtheria the effect on the local tissues attacked is at first of chief importance and then later the widespread action of the poison. In tetanus the involvement of the local tissue is of no importance. The central nervous system is alone serious. Experimentation had shown that given subcutaneously antibodies are gradually absorbed from the local area during a period of two to four days (see Figs. I and 2). Injected in the muscles it more quickly reaches the blood, but still requires two to four hours for an appreciable amount to be absorbed. Given intravenously the entire protective substance is available im- 
mediately to begin to pass from the blood to the tissues. As antitoxin remains for several days in the blood, it is not necessary to repeat a subcutaneous or intravenous dose unless a very prolonged action is required. The antitoxins as well as the other antibodies must be repeatedly injected into the spinal canal as they pass out to the lymph vessels and only return to the extent of I per cent. of that in the blood.

In developed tetanus experience with animals has shown that where the subcutaneous injection always failed, and the intravenous usually did, that the intraspinal generally succeeded. The serum in intraspinal injections should be thin so as to readily pass up the canal and into the ventricles. The use of sera is to combat the bacteria rather than their toxins.

When we use the sera to influence infections due to the organisms causing disease chiefly by their endotoxins we meet many difficulties. 'The strains differ among themselves and we have no such perfect antidote as we have for the extracellular toxin. Indeed, we have to satisfy ourselves chiefly with destroying the bacteria rather than neutralizing their poisons and with many this is difficult or impossible. Again, there are great difficulties in standardizing the sera and in accumulating a large amount of protective substance in a cubic centimeter. The methods of administration differ as in the case of antitoxins, according to the region of the body that the protective bodies are needed.

In cerebrospinal meningitis for instance the disease is chiefly located on the surfaces of the cord and brain and their membranes. To reach these we must inject intraspinally. The strains of meningococci vary somewhat and for this reason by injecting animals with a mixture of strains we obtain a single serum suitable for all. The pneumococci and streptococci have been found to fall into still more distinct groups, and not only did the serum stimulated by the injection of the strains of one group fail to have any influence on the infection produced by the strains of other groups, but many of the strains seemed to be untouched by sera from animals who had been treated by them. The thin sera are used in infections of a septicemic type or of organs which cannot be directly reached. They are given subcutaneously or intravenously in large doses of from 50 to 200 c.c. and like the other bacterial sera repeatęd once or twice daily.

\section{THE ADMINISTRATION OF DIPHTHERIA ANTITOXIN FOR CURATIVE PURPOSES}

Although more than twenty years have elapsed since the introduction of diphtheria antitoxin, good observers still differ in the amount which they believe should be injected and in the method of its administration. Before giving our own conclusions on the proper dosage, it is well to consider several important points upon which this dosage is founded. 
The amount of toxin in any case of diphtheria is comparatively small. One hundred units of antitoxin which would neutralize fifty times the amount of toxin sufficient to kill a six year old child, would surely make harmless all the toxin present in the most malignant case if it could gain access to it in time. If we gave antitoxin, therefore, as many suppose simply in sufficient amount to neutralize the poison in the body of an infected person, comparatively small amounts would be injected, but we have to give very much more than this because of the time it requires for much of the antitoxin to reach the toxin. This can be brought into direct contact with the toxin only by being absorbed into the blood and then passing through the capillary walls to the tissue fluids and cells. The larger the amount of antitoxin that is in the blood, the greater will be the amount that will pass to the tissues. The combined endeavor of the clinical observer and the laboratory worker is to find the suitable amount which will give a sufficient concentration in the blood to neutralize, as quickly as necessary, the toxin in the tissues. In the laboratory, we can test the amount of antitoxin which is absorbed into the blood from any given dose and the amount which passes out to the lymph, while the clinical observer can note the changes which take place as he watches the case after antitoxin treatment.

It is naturally a matter of great importance as to how the antitoxin is administered. When given subcutaneously, the swelling caused by its injection, rapidly disappears from the absorption of the water, but the globulins and antitoxin remains behind in the tissues because of the slow absorption of proteins. By testing many patients, it has been found that it takes twenty-four hours for the major part of the antitoxin to be absorbed by the blood from the subcutaneous tissues and some twelve hours from the muscles (see Figs. I, 2 and 3). For its total absorption it requires two or three days. Through the use of the Schick test, it has been determined that an injection of antitoxin given intravenously passes out to the tissue fluids about ten times as rapidly as when the dose is given subcutaneously and four times as when given intramuscularly. A unit gives most effect when given intravensouly and least when given subcutaneously. If it were not for the fact that it is more difficult to give it intravenously and also that sharper serum reactions occur, the intravenous method would be the only, one used. Another matter which is of importance, is the size of the individual treated. It is self-evident that, if a child weighing 20 pounds is injected with ro, 000 units, it would, on the average, have in its blood five times as much antitoxin per cubic centimeter as a person receiving the same amount who weighs Ioo pounds. The influence of weight on the dose is, however, largely neutralized by the fact that diphtheria in the child is generally more dangerous than in the adult. Every minute of delay in the neutralization of the toxin in a severe case is of importance, but in a mild case, where dangerous poisoning is still remote, slight delay makes little difference. Infants and children are especially liable to 
laryngeal diphtheria so that every case in a child presents a certain gravity which the adult does not present.

The last point to be considered is as to whether a single or a multiple dose should be given. It must be realized that antitoxin has no effect whatever on injury which has taken place. It is as useless here as water on the ashes of a burned-out building. If the
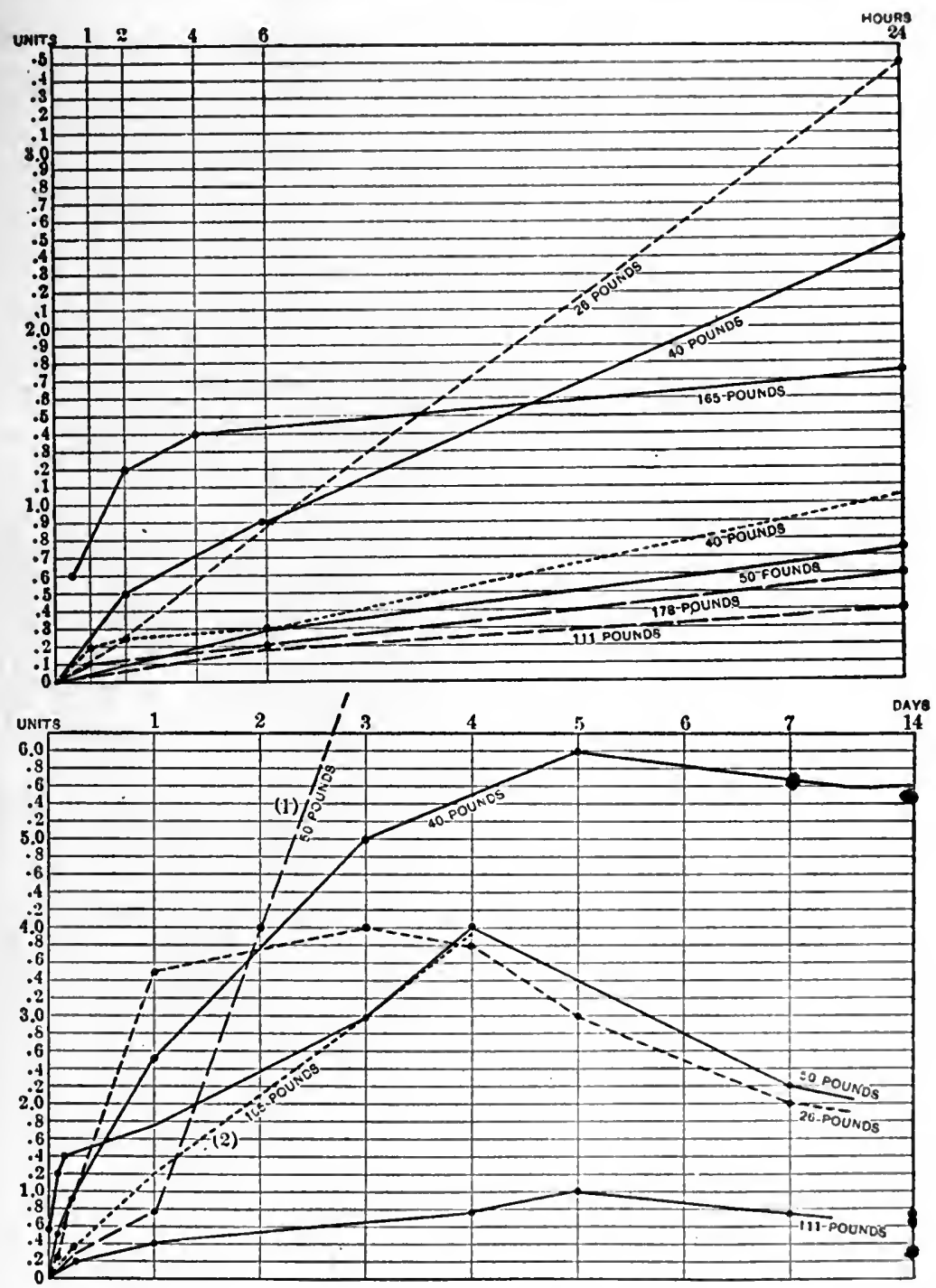

FIGs. I and 2.-Showing the extent and rapidity of absorption of 10,000 units of antitoxin given subcutaneously. Each line represents the antitoxin content of I c.c. of blood of a case at different intervals of time. The weight of each case is given in pounds. The heavy dots show the time of the test bleedings. 
toxin is firmly united with the cell substance, antitoxin is no longer of any service. It is the early and sufficient dose which is important. When we give a divided dose, we simply get the effect of the first half portion during the interval before the giving of the second dose. If the second dose had been given with the first, we would have had its affect added, and so an insufficient dose made adequate. When

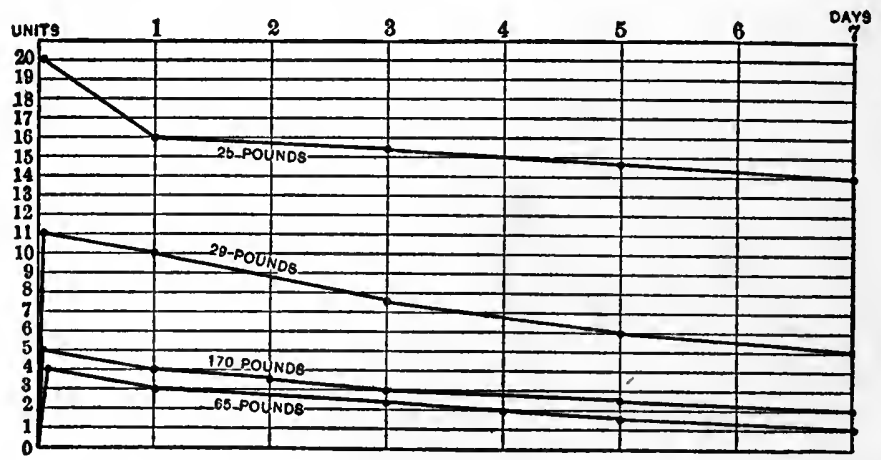

FIG. 3. - The antitoxic power of human blood after an intravenous injection of $10, \infty 0$ antitoxic units.

the first dose has been of a sufficient size, the second and third injection, though harmless, are absolutely useless. The holding back of a part of the first dose so as to give it later, simply delays its action to a time when it cannot have much, if any, effect. In the article on diphtheria by Weaver, the importance of giving a single adequate dose is not appreciated. It must be remembered that the antitoxin given in the first dose is still in the body when the second injection is made.

For the last three years, we have used in the hospitals for contagious diseases only a single dose of antitoxin, which in mild cases, has been given subcutaneously; in moderate cases subcutaneously or intramuscularly; and in very severe cases, intravenously or intravenously and intramuscularly. After twenty years of experience and consultation with physicians in New York and elsewhere, the following dosage, which is that adopted by the Health Department, is advised.

The doses suggested in the table are in our opinion large, and we

Dosage of Antitoxin in Diphtheria. Single Dose Only

\begin{tabular}{|c|c|c|c|}
\hline \multicolumn{4}{|c|}{ Infant, ro to 30 pounds (under two years) } \\
\hline Mild & Moderate & Severe & Malignant \\
\hline 2,000 & 3,000 & $5, \infty 00$ & \\
\hline \multicolumn{4}{|c|}{$\begin{array}{c}5,000 \quad 10, \infty 00 \\
\text { Child, } 30 \text { to } 90 \text { pounds (under fifteen years) }\end{array}$} \\
\hline $3, \infty 00$ & 4,000 & $10, \infty 00$ & $10, \infty 00$ \\
\hline $4, \oplus 00$ & $\begin{array}{l}\text { I0,000 } \\
\text { Adult, }\end{array}$ & $\begin{array}{l}\text { I } 5, \infty 00 \\
\text { d over }\end{array}$ & 20,000 \\
\hline $3, \infty 00$ & 5,000 & 10,000 & I5,, 00 \\
\hline 5,000 & 10,000 & 20,000 & 40,000 \\
\hline
\end{tabular}


Method of ADMinistration

\begin{tabular}{|c|c|c|c|}
\hline & & $\begin{array}{c}\text { 恼 Intramuscular } \\
\text { or }\end{array}$ & \\
\hline $\begin{array}{c}\text { Subcutaneous } \\
\text { or }\end{array}$ & $\underset{\text { or }}{\text { Intramuscular }}$ & $\begin{array}{c}\text { Intravenous } \\
\text { and }\end{array}$ & $\begin{array}{l}1 / 2 \text { Intravenous } \\
\text { and }\end{array}$ \\
\hline 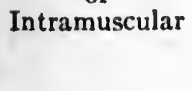 & Subcutaneous & $\begin{array}{c}1 / 2 \text { Intramuscular } \\
\text { or } \\
\text { Subcutaneous }\end{array}$ & $\begin{array}{c}1 / 2 \text { Intramuscular } \\
\text { or } \\
\text { Subcutancous }\end{array}$ \\
\hline
\end{tabular}

think no appreciable advantage would be obtained by giving any larger amounts. Very much smaller doses are still able to do great good as the general blood current soon becomes antitoxic and blocks any further passage of toxin from the diseased tissues to other portions of the body. The feebly antitoxic plasma gradually permeates the body. There will be, however, more delay in the improvement of the local process for the neutralization of the concentrated toxin in the diseased tissues will be slower. The exudate or pseudomembrane will continue to increase for some hours after the complete neutralization of the toxin because the injury to the cells takes time to manifest itself.

\section{THE ADVANTAGES AND DISADVANTAGE OF THE SUBCUTANEOUS, INTRAMUSCULAR AND INTRAVENOUS METHODS OF INJECTING ANTITOXIN IN DIPHTHERIA}

As stated earlier in this article, if it were not for the disadvantages inherent in the intravenous methods there would be no question that it would be the way chosen. It is necessary, therefore, in each case to weight the indication for and against the method.

The disadvantages are, first, the difficulty in giving the serum intravenously in small children and infants. In these, it is necessary to inject it directly into the jugular vein or do a cutting operation to render a vein accessible. The injection, here, requires experience and dexterity and sometimes meets with objections from the side of the family or friends.

The second disadvantage is, that the injection of the serum, even though it is warmed and given slowly, is apt to be followed in from fifteen minutes to one hour by a rise of temperature and a chill. In about 40 per cent. of the cases given the advised doses of antitoxin, a moderate or severe chill will take place which lasts for from five minutes to one hour. With this the temperature may rise from 2 to $5^{\circ} \mathrm{F}$. Within six hours the temperature falls to its previous level or below and no bad effects are noticeable. The reaction may be rather alarming at the time and impresses unfavorably, not only the family, but also the physician.

The necessity of getting the more rapid effect of the intravenous injection must be weighed in each case.

There is no doubt that in cases which have already been sick for from twenty-four to seventy-two hours and which show a great extent of disease as shown locally by the amount of pseudomembrane and of swelling and constitutionally by symptoms due to 
toxic absorption, that every minute's delay in getting the antitoxin to all parts of the body is serious and that in these, an injection of 3000 to 10,000 units of antitoxin intravenously is imperative.

On the other hand, the mild and moderate cases of diphtheria are not, as a rule, generally toxic when admitted and here a subcutaneous or better an intramuscular injection is sufficient. It is true that an intravenous injection would cause a quicker local effect, but the difficulty of administration and the possible chill more than counterbalance this.

The decision in the laryngeal cases is difficult. Some years ago, in coöperation with Dr. Raymond B. Mixsell, we treated 158 cases of laryngeal diphtheria in a manner that every other case got an intravenous injection and every other one a subcutaneous injection. The results were rather surprising in that, while those injected intravenously showed a quicker response, the death rate was apparently unaltered. The serum reactions were also not marked.

The results can be summarized as follows:

If we calculate the total death rate of the series, irrespective of the occurrence of bronchopneumonia, we have the following figures:

\begin{tabular}{|c|c|c|c|}
\hline & Cases & Deaths & $\begin{array}{l}\text { Per cent. of } \\
\text { mortality }\end{array}$ \\
\hline $\begin{array}{l}\text { Intravenous. .............. } \\
\text { Subcutaneous.......... }\end{array}$ & $\begin{array}{l}79 \\
79\end{array}$ & $\begin{array}{l}36 \\
42\end{array}$ & $\begin{array}{l}45 \cdot 5 \\
53 \cdot x\end{array}$ \\
\hline Total. & $\times 58$ & 78 & $49 \cdot 3$ \\
\hline
\end{tabular}

The above percentages show only a slight advantage in favor of the intravenous method.

Upon examining the records of 158 cases, the most startling thing is the prevalence and almost absolute mortality of bronchopneumonia. Forty-two and one-half per cent. of the intravenous series, and 5I.9 per cent. of the subcutaneous series developed bronchopneumonia either before, or a day or two after admission. Out of a total of seventy-seven cases of bronchopneumonia only five recovered, making a death rate of 93.5 per cent. for the bronchopneumonia cases in both series. Of the subcutaneous cases developing bronchopneumonia 93.5 per cent. died. Of the intravenous cases developing bronchopneumonia 88.8 per cent. died.

If we exclude the pneumonia cases, the mortality goes down at once. In the intravenous series four cases died out of forty-three, a death rate of 9.07 per cent. In the subcutaneous series two died out of thirty-eight, a death rate of 5.26 per cent., or a total rate of 7.4 per cent. for eighty-one cases not developing bronchopneumonia. From these results we judge that most cases of laryngeal diphtheria are not suffering from a marked general toxemia at the time of admission and that a large intramuscular or even subcutaneous injection will accomplish most of what an intravenous injection would. 
DIPHTHERIA IMIMUNITY-NATURAL, ACTIVE AND PASSIVE. ITS DETERMINATION BY THE SCHICK TEST

The definite association between the presence of antitoxin in the blood and immunity to diphtheria has been firmly established by the efficiency of passive immunizing doses of antitoxin in protecting individuals against diphtheria, and also by finding a complete absence of antitoxin in those who develop clinical diphtheria.

The presence of natural immunity to diphtheria in a large number of people has become a well-established clinical fact-this immunity is generally associated with the presence of antitoxin, as shown by finding it in measurable quantities in the blood of the majority of such individuals. A small percentage of individuals seem to be protected by bactericidal rather than antitoxic substances. The determination of the presence of antitoxin by the older methods requires much time and the use of guinea pigs. Schick's merit is that he devised a simple clinical test by which we can easily determine the presence of antitoxic immunity.

The reaction depends on the local irritant action of minute quantities of diphtheria toxin when injected intracutaneously. If antitoxin is absent in the cutaneous lymph, or present only in very small amounts, insufficient for neutralization, a positive reaction will appear in twenty-four to forty-eight hours. This is characterized by a circumscribed area of redness and slight infiltration which measures from I to $2 \mathrm{~cm}$. in diameter. It persists for seven to ten days, and on fading shows superficial scaling and a persistent brownish pigmentation. The amount of toxin injected as advised by Schick is $1 / 50$ MLD. for the guinea pig in o.I c.c. of normal saline. We prefer $1 / 50$ MLD. in 0.2 c.c., on account of a fairly severe local reaction seen occasionally with the greater concentration of the toxin in individuals in whom not even a trace of antitoxin is present.

It is important to distinguish the true reaction from a pseudoreaction which is found extremely rarely in young children, in a small percentage of older children, and more frequently in adults, who have antitoxin in either a small or large amount. These reactions are probably local sensitization phenomena of a protein character (local anaphylaxis) since similar reactions can be obtained almost uniformly in these persons with dilutions of an autolysate of the Klebs-Loeffler bacillus (endotoxin) which is free of toxin, or with heated toxin $\left(75^{\circ} \mathrm{C}\right.$. for five minutes) in which the soluble toxin has been destroyed. In a few cases, the beef broth, less diluted however, will give the same result. The pseudoreaction can be distinguished clinically in most cases from the true reaction. It appears earlier (six to eighteen hours), is more infiltrated in some of the cases, less sharply circumscribed, and disappears usually in forty-eight to seventy-two hours. At its height the pseudoreaction is characterized by a small central area of redness surrounded by a secondary areola which gradually shades off into the surrounding skin. It leaves on fading only a faintly pigmented area, which, in our experience, very seldom shows 
superficial scaling. Occasionally a combined true and pseudoreaction is seen. In these cases the true reaction remains, after the pseudoreaction has disappeared, and finally shows the typical scaling and pigmentation. In the rare cases in which the nature of the reaction remains in doubt, is it best to consider the reaction as positive.

It is best to control the pseudo and combined reactions with toxin heated to $75^{\circ} \mathrm{C}$. for five minutes. If the control gives a reaction similar to the original test, and both fade in about three days, the test may be considered as a pseudoreaction and the individual as being immune. If the control test is less marked than the original test, and the evidence of a positive reaction remains after the secondary areola fades in the original test (a distinct area of brownish pigmentation with superficial scaling), it may be considered as a combined reaction and the individual as not being immune to diphtheria.

For the carrying out of the test, it is essential to have an accurate syringe with a sharp, but short-pointed fine needle. The usual I c.c. "Record" tuberculin syringe with a fine platinum-iridium needle answers the purpose well. A standard ripened diphtheria toxin is used, of which the MLD. for the guinea pig has been carefully estimated. One hundred times that amount is diluted with sufficient sterile salt solution to give ro c.c. of a primary dilution. For example, if the MLD. $=0.007$ c.c., then 0.7 c.c. of toxin is added to 9.3 c.c. of saline. The primary dilution keeps in the ice-box for two weeks. For daily use, or whenever required, a fresh final dilution is made by adding $\mathrm{I}$ c.c. of the primary to 99 c.c. of salt solution; 0.2 c.c. of the final dilution represents $I / 5 \circ \mathrm{MLD}$., the amount used in the test. The final dilution should not be used after twenty-four hours. For the greater convenience of the general practitioner and hospital worker, an outfit has been devised, which consists of a capillary tube that contains a little over one MLD. of undiluted diphtheria toxin, a small rubber bulb, similar to the ones used in vaccine outfits to expel the toxin, and a bottle containing ro c.c. of sterile physiological salt solution. By diluting the toxin with the saline, a solution is quickly prepared which is ready for the tests. The outfits, if kept cold, will remain good for six months; the diluted solution should not be used, however, after twelve to twenty-four hours.

The toxin is injected intracutaneously on the flexor surface of the forearm or arm. If properly carried out, a distinct wheal-like elevation with prominent openings of the hair follicles appears at the site of injection. A good guide in the insertion of the needle is to be able to see the oval opening of the needle through the superficial layers of the epidermis. We have found that the toxin diluted so that $1 / 50$ MLD. is represented by 0.2 c.c. instead of o.I c.c. as recommended by Schick, is better for the reason that the more diluted toxin is less apt to cause, in unprotected individuals, severe reactions, with occasional superficial necrosis; another reason is that the larger volume of fluid is more easily handled. 
Though the intensity of the reaction varies in different individuals, a well-marked redness indicates an almost complete absence of antitoxin. Faint reactions point to the presence of very small amounts of antitoxin, which are not sufficient, however, to certainly protect the individual against diphtheria, but do prevent the constitutional poisoning. To prevent the appearance of the reaction, according to Schick, at least $1 / 30$ unit of antitoxin per cubic centimeter of blood is required. This amount he considers sufficient to protect against diphtheria. According to v. Behring, even as little as $1 / 100$ unit of antitoxin will protect against the disease in uncomplicated cases.

The Schick reaction was carried out during the past eighteen months on all patients entering the scarlet fever pavilion of the Willard Parker Hospital. In all I 200 patients were thus tested, and of these, 656 , or 54.7 per cent., gave negative reactions. Only cases giving positive reactions were immunized; those giving a negative reaction received no immunization, but were carefully observed. Although about 20 per cent. of the negatively reacting patients became bacillus carriers during their stay in the wards, no cases of clinical diphtheria developed among them.

The remaining 544 patients who gave positive reactions received in practically all cases some form of active immunization. Among those who reacted insufficiently to active immunization with toxinantitoxin mixtures or vaccines made from the Klebs-Loeffler bacillus, seventy-two developed clinical diphtheria. These received antitoxin. The majority of the cases were of a mild tonsillar or anterior nasal type and all recovered. follows:

According to age the number of reactions obtained were as

Table 1.-Schick Reactions among Scarlet Fever Patients (PARK AND ZiNGHER)

\begin{tabular}{|c|c|c|c|c|}
\hline Age & Total & -Schick & +Schick & $\begin{array}{l}\text { Per cent. } \\
+ \text { Schick }\end{array}$ \\
\hline 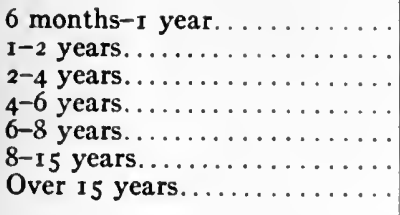 & $\begin{array}{r}10 \\
68 \\
223 \\
249 \\
196 \\
237 \\
167\end{array}$ & $\begin{array}{r}5 \\
22 \\
93 \\
116 \\
123 \\
171 \\
126\end{array}$ & $\begin{array}{r}5 \\
46 \\
180 \\
\mathrm{r} 33 \\
73 \\
66 \\
4 \mathrm{I}\end{array}$ & $\begin{array}{l}50.0 \\
67.6 \\
65.9 \\
53.4 \\
37.2 \\
27.8 \\
24.5\end{array}$ \\
\hline - & I 200 & 656 & 544 & $45 \cdot 3$ \\
\hline
\end{tabular}

A curious result of these tests noted by Dr. A. Zingher, who carried them out, was the fact that children having scarlet fever showed a higher percentage of Schick reactions than those who were healthy.

Several large groups of healthy persons tested gave the following results: 
Table 2.-Summary of Schick Tests in 2700 Normal Children (Zingher)

\begin{tabular}{|c|c|c|c|c|}
\hline Age & Total & -Schick & +Schick & $\begin{array}{l}\text { Per cent } \\
\text { + Schick }\end{array}$ \\
\hline 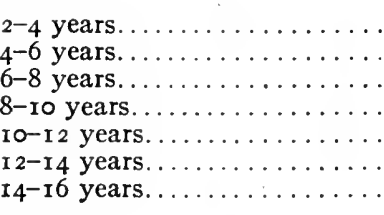 & $\begin{array}{r}62 \\
318 \\
444 \\
597 \\
584 \\
506 \\
189\end{array}$ & $\begin{array}{r}42 \\
236 \\
347 \\
462 \\
459 \\
416 \\
158\end{array}$ & $\begin{array}{r}20 \\
82 \\
97 \\
135 \\
125 \\
90 \\
31\end{array}$ & $\begin{array}{l}32.2 \\
25.7 \\
21.8 \\
22.6 \\
21.4 \\
17.7 \\
16.4\end{array}$ \\
\hline & 2700 & 2120 & $5^{80}$ & $2 \mathrm{I} .4$ \\
\hline
\end{tabular}

From these and other results, we may assume that the percentage of individuals susceptible to diphtheria is greater between the ages of one to four years. It is less during the second six months of life and less in older children and in adults. Our results in scarlet fever cases closely approach those obtained by Schick, whose table shows positive reactions in 7 per cent. of the newborn, in 43 per cent. during the first year of life, in 63 per cent. between two and five years and 50 per cent. between five and fifteen years. In adults the positive reactions were not more than ro per cent.

In the group of normal individuals it is important to note that only from $16-33$ per cent. of the children between four and sixteen years of age gave positive Schick reactions. This fact is of significance in the active immunization with mixtures of diphtheria toxinantitoxin, in that it becomes quite practical to attempt to immunize such a relatively small part of the child population.

In an interesting study of the relationship of the antitoxin content of the blood of the mother and newborn, v. Gröer and Kassowitz found that 84 per cent. of mothers and their newborn show a considerable amount of natural antitoxin. The antitoxin of the infant is evidently derived from the mother through the placenta, and because of its frequency it is regarded as a physiological phenomenon. The mothers also showed a pseudoreaction in 47.5 per cent. of cases. This is an unusually high percentage and the toxic culture fluid must have been exceptionally rich in extract of the bacillus substance. Similar reactions could be obtained in these mothers with the same culture fluid after it was neutralized with diphtheria antitoxin.

With this test Schick attempted to place on a more rational experimental basis some interesting clinical problems in connection with the prophylactic and therapeutic dosage and mode of administration of antitoxin. By making the test at regular intervals previous to the injection of antitoxin, he found that neutralization of toxin in contact with tissue cells was still possible up to a certain number of hours. This was shown by the partial or complete suppression of the reaction. The efficiency of the injected antitoxin depended on the mode of administration and the size of the dose. 
Table 3.-Relative Efficiency of Diphtheria Antitoxin Administered IN Different Ways (PARK AND ZiNgher)

\begin{tabular}{|c|c|c|c|c|c|c|c|c|c|}
\hline Name & Age & Units & Admin. & $\begin{array}{c}20 \mathrm{hr} . \\
\text { Prev. }\end{array}$ & $\begin{array}{l}6 \mathrm{hr} . \\
\text { Prev. }\end{array}$ & $\begin{array}{l}4_{\text {Prev. }} \mathrm{hr} \\
\text { Pre }\end{array}$ & $\begin{array}{l}2 \mathrm{hr} . \\
\text { Prev. }\end{array}$ & Simul. & $\begin{array}{l}2 \mathrm{hr} . \\
\text { Subseq. }\end{array}$ \\
\hline $\begin{array}{l}\text { A. N... } \\
\text { J. R... } \\
\text { I. W.. } \\
\text { W. S.. } \\
\text { A. F.. } \\
\text { G. S... } \\
\text { F. M.. }\end{array}$ & $\begin{array}{l}6^{1} \\
4^{9} \\
5^{6} \\
5 \\
6^{8} \\
6 \\
6^{2}\end{array}$ & $\begin{array}{r}1,000 \\
1,000 \\
1,000 \\
20,000 \\
10,000 \\
10,000 \\
10,000\end{array}$ & $\begin{array}{l}\text { S. C. } \\
\text { I. M. } \\
\text { I. V. } \\
\text { S. C. } \\
\text { I. V. } \\
\text { I. M. } \\
\text { S. C. }\end{array}$ & $\begin{array}{c}+t \\
+++ \\
++t\end{array}$ & $\begin{array}{c}++ \\
++ \\
+ \\
\pm\end{array}$ & $\begin{array}{c}++ \\
++ \\
\pm \\
\pm\end{array}$ & $\begin{array}{c}++ \\
+ \\
-\end{array}$ & $\begin{array}{l} \pm \\
- \\
-\end{array}$ & $\begin{array}{l}- \\
-\end{array}$ \\
\hline
\end{tabular}

S. C. = subcutaneous.

I. M. = intramuscular. $+=$ reaction. $\quad-=$ no reaction.

I. V. = intravenous.

We carried out some parallel experiments (see Table 3) attempting at the same time to see approximately how much more effective the same dose of antitoxin was when given intravenously than when given subcutaneously or intramuscularly. The Schick test was made six hours, four hours and two hours before and at the time of the injection of the antitoxin. With rooo units given intravenously the reaction from the toxin injected six hours previously was considerably weakened, though not entirely suppressed. The reaction from the toxin given four hours before appeared very faint, while the test given two hours before was negative. One thousand units given subcutaneously had no effect on the toxin given four and six hours previously, and only weakened the reaction made by the toxin given two hours before, while that from the simultaneous injections was just visible. The same amount (1000 units) given intramuscularly had a somewhat stronger effect than the subcutaneous injection, and prevented the appearance of a reaction from the simultaneous injection.

To obtain the same effect with the subcutaneous as was obtained with the intravenous injection of antitoxin, from ten to twenty times the amount was needed; 20,000 units given subcutaneously were able to affect the reaction due to the toxin given six hours previously to the same extent as I000 units given intravenously.

\section{IMIMUNITY RESULTS OBTAINED FROM THE USE OF DIPHTHERIA TOXIN- ANTITOXIN MIXTURES}

Behring has recently brought forward the practical application of toxin-antitoxin mixtures in which the toxin had been so neutralized as to be no longer poisonous, but still retaining sume toxin in loose combination. The use of this immunizing agent is the result of a long series of investigations as to the possibility of producing antitoxin through injection of these mixtures. In 1895 Babes carried out successful experiments in guinea pigs and since I897, the horses in the Health Department of New York City, which are used to produce diphtheria antitoxin, have been immunized at first with these neutral mixtures and in 1903 , one of us published the records of a num- 
ber of horses showing that three injections might cause production of several hundred units of antitoxin in each cubic centimeter of serum.

Theobald Smith later made a careful study of the subject in guinea pigs and suggested the toxin-antitoxin injections in children for practical immunization but never tried it.

Behring deserves, therefore, the credit of actually applying the toxin-antitoxin mixtures for the immunizing of persons against diphtheria.

We have personally watched the result in a series of over rooo cases, that had been actively immunized by Zingher with diphtheria toxin-antitoxin. These susceptible individuals were selected by means of the Schick test out of a total of about 10,000 children and adults in ro different institutions.

The mixtures of toxin-antitoxin that were used for immunization were either neutral (66-70 per cent. L+to each unit of antitoxin) or slightly toxic (80-90 per cent. L+to each unit of antitoxin) to the guinea pig. The dose was varied from 0.5 c.c.-I.o c.c., and the number of injections from one to three. The injections were made subcutaneously at intervals of seven days. The local reactions at the site of injection were generally mild; in the older children and adults, the redness and swelling were more marked. General symptoms, like malaise, and temperature of $100-102^{\circ} \mathrm{F}$. were noted in Io to 20 per cent. of the cases; in a few the temperature reached ${ }^{10} 4^{\circ} \mathrm{F}$. The symptoms lasted twenty-four to forty-eight hours, and then rapidly subsided. Both local and general symptoms were especially evident in those who showed a susceptibility to the protein by giving a combined pseudo and true Schick reaction. No harmful after-effects were noted in several thousand injections.

The re-tests with the Schick reaction showed that only 30-40 per cent. became immune three weeks after the first injection; about 50 per cent. at four weeks, $70-80$ per cent. at six weeks, and 90-95 per cent. at eight to twelve weeks. The best results were obtained with the full immunization, consisting of three injections of r.o c.c each, given at weekly intervals. The duration of the active immunity was studied in a group of children that were followed up for over one and one-half years; these cases showed that the active immunity persisted for at least that length of time. It is possible, that the immunity induced by the injections of toxin-antitoxin starts a continued cellular production of antitoxin, which would have otherwise appeared much later in life.

From their results Park and Zingher concluded that it is advisable to immunize children soon after the first year of life so as to afford them a protection against diphtheria, at a time when the disease is most dangerous. In addition such young children, by not having any hypersensitiveness to the bacillus protein, show very mild local and constitutional symptoms after the injections. An immune child population could thus be developed with the result that fresh clinical cases would be prevented and the bacillus car- 
rier would probably soon disappear as a hygienic factor in our communities.

Interesting and parallel results were noted in guinea pigs and horses. Guinea pigs are fairly resistant to active immunization with diphtheria toxin-antitoxin, and in that respect they show an almost complete parallelism to the positive Schick cases among human beings. After injections of toxin-antitoxin, an antitoxic immunity develops slowly from the sixth to eighth week. Horses, on the other hand, as a rule, correspond in their behavior toward small doses of toxinantitoxin to human beings, who are naturally immune. They both give a ready response, even after a single injection of toxin-antitoxin, and show a distinct increase in the antitoxin content toward the end of the first week. Occasionally, a horse is found that has no antitoxin in the control bleeding; such animals respond slowly to small doses of toxin-antitoxin. It is probable that the tissue cells of the naturally immune human beings and the majority of horses have acquired the property of giving a quick and easy response to the stimulation of diphtheria toxin.

In reviewing then the results of our investigations on immunity and some of the more important applications of the Schick reaction we have to emphasize:

I. The great reliability of the Schick test, when the toxin is of proper strength and the method of employing it is correct, in showing the presence or absence of antitoxic immunity to diphtheria. A negative reaction obtained after the age of two or three years indicates that the individual is protected, probably indefinitely, against the disease. The great majority of positive reactions in children are true reactions and indicate an absence of antitoxin, and therefore, unless other antibodies are present, a susceptibility to diphtheria. Among adults the psuedoreaction is seen in a certain proportion of individuals, but this reaction can usually be distinguished clinically from the true reaction. The two reactions may be found combined in the same individual: in such cases the psuedoreaction will disappear in three to four days leaving a distinct circumscribed area of scaling pigmentation due to the positive reaction. The later injection of some of the same lot of toxin either heated to $75^{\circ} \mathrm{C}$. for five minutes or after neutralization with antitoxin as a control will help to indicate the pseudoreactions.

2. The great value of the test in determining clinically the efficiency of the immunization of susceptible individuals, who have been injected with mixtures of diphtheria toxin and antitoxin. For this purpose only positive Schick cases should be chosen, and after the injections, they should be tested one, three, six, and twelve months later to determine whether a sufficient amount of antitoxin had developed early or late to inhibit the Schick reaction, showing thereby the production of an active immunity to diphtheria.

3. The Schick test is of a great help in clearing up the diagnosis of clinically doubtful cases of diphtheria. With a purulent or sanious 
nasal discharge showing the Klebs-Loeffler bacillus it is difficult to decide whether the case is a carrier or a beginning diphtheria. A negative reaction excludes diphtheria, while a positive Schick reaction leaves the diagnosis of diphtheria still a probability. A case of tonsillitis due to streptococcus in a carrier of diphtheria bacilli would, by the use of the culture alone, be thought to have diphtheria and in danger of extension of the disease. A negative Schick reaction would indicate the case to be simply a carrier, and in no danger from the effects of the diphtheria poison.

4. The Schick reaction has added further proof to the clinical and experimental observations that very toxic cases of diphtheria do better when given an early intravenous injection of antitoxin than when it is administered in any other way. It reveals the fact that an intravenous injection of antitoxin is able to partly neutralize toxin six hours after its absorption by the tissues and thus gives us hope in some of the late cases of diphtheria. The results with the Schick test will serve as a reminder that after contact with the tissues for more than a few hours the effect of the toxin can no longer be prevented; that a day, and, in fact, hours of delay in the administration of a therapeutic dose of antitoxin may mean not only the absorption but the final binding of a fatal dose of diphtheria toxin.

5. The results obtained with the Schick test in families seem to indicate that besides infection with virulent diphtheria bacilli, other factors, possibly hereditary in nature, are concerned in the production of natural immunity to diphtheria. The absence of antitoxic immunity in more than 65 per cent. of individuals after an attack of diphtheria and in the majority of positive Schick cases within four weeks after treatment with mixtures of toxin and antitoxin shows that the tissues have never had, and have only slowly acquired the ability to produce antitoxin.

6. The Schick reaction can be applied with advantage in testing the patients, resident staff and nurses of contagious disease hospitals. By its use a considerable saving can be effected in antitoxin during outbreaks of diphtheria in institutions or in homes; it relieves of the fear of contagion and also avoids unnecessarily sensitizing about one-half of the exposed individuals. It should be applied as a routine in both the measles and the scarlet fever pavilions of hospitals. By administering 1000-2000 units of antitoxin in positive Schick cases and reimmunizing the patients if they remain for more than three weeks in the hospital, we should be able to control the development of diphtheria during the convalescence from scarlet fever or measles.

7. The immediate results following the injection of the toxinantitoxin mixture have been somewhat disappointing but the later ones are quite encouraging.

In conclusion we believe that the good late results following the toxin-antitoxin injections and the ability now to recognize clinically those individuals who are susceptible to diphtheria, will 
arouse an interest in the more widespread use of an active immunization with mixtures of diphtheria toxin and antitoxin which will enable us to greatly lessen and, perhaps, finally eradicate the disease. Up to the present time, in spite of the use of antitoxin, the number of cases of diphtheria has but slightly diminished. The brilliant lowering of the death rate has been due as stated by von Behring to the lessened mortality in the treated cases rather than to a decrease in the total number of cases of diphtheria.

\section{ADMINISTRATION OF TETANUS ANTITOXIN FOR PREVENTION AND TREATMENT}

Treatment.-While tetanus antitoxin has proved efficacious in the prevention of tetanus, its employment as a curative agent has been until recently less successful and some who have used it have doubted its value. This failure to produce more uniformly good results is chiefly due to its too late administration, insufficient dosage and the use of the subcutaneous method. While the subcutaneous use of the antitoxin, which at first was the usual method employed, may prove of some value in large doses given in moderate cases within the first few hours after the onset of symptoms, nevertheless it usually fails because of slow absorption and the time required to reach the tissues of the central nervous system. The intravenous method overcomes the fault of the slow absorption, but not that of the difficulty of reaching the toxin in contact with the nerve cells. The intravenous method while far in advance of the subcutaneous is far inferior to the intraspinal. In the light of our present knowledge, even the intravenous method when used alone can be justified only by an inability of the physician, for one reason or another, to give an intraspinal injection. The latter method is being more and more recommended, and when employed before the disease has made too much headway has given excellent results.

From time to time since I900, single cases or small series of cases have been reported in which the antitoxin has been given intraspinally or intraventricularly. Thus Neugebauer ${ }^{1}$ in 1905 reported forty-three cases from Continental and American sources with a mortality of twenty-two, or 5I per cent., and also three patients treated by him of whom two recovered. Abbe and Rogers used it in a number of cases in New York City. Many of these patients received also intravenous or subcutaneous injections or both. The results seemed to some observers to be better than when other methods were used without intraspinal dosage, but on the whole, this method of treatment until recently has not made much headway. In the IgI I edition of this handbook Bloodgood and McGlannan state that because it is necessary to reach the nervous system, intravenous and subcutaneous injections have not given good results and that the serum should be given by lumbar

${ }^{1}$ Neugebauer, Wien. klin. Wchnschr., 1905, xviii, 450. 
puncture or better still by intraventricular injections. Experimental proof of the greater value of the intraspinal method has not until very recently been at all convincing. Permin of Copenhagen, working on rabbits and dogs, showed that local tetanus could be prevented by the simultaneous injection of tetanus toxin intramuscularly and a dose of antitoxin given in the spine, whereas with the same dose of antitoxin given intravenously, local tetanus occurred. Four hours after the giving of a large dose of toxin neither method of preventing the occurrence of local tetanus was efficacious, and when nine hours were allowed to elapse between the giving of toxin and antitoxin, the animals could not be saved.

A series of experiments were undertaken by Nicoll and ourselves, with guinea pigs, with the object of determining to what extent tetanus antitoxin given in the spine has greater curative power, when the disease is actually established, than when given in the circulation. The results are so conclusive in showing that the intraspinal injections save life when all other methods of giving serum fail that one experiment is described in part, in order to bring out more graphically this extremely important fact. The comparative ineffectiveness of subcutaneous injections of tetanus antitoxin in developed cases was incidentally brought out.

March I2, seven pigs were injected deeply into one hind leg with two fatal doses of toxin. The amount was proportioned to body-weight of each. Nineteen hours later they were given antitoxin in the amounts and by the methods designated in Table 4, the inoculated leg being stiff in the degree noted at the time.

Table 4.-Administration and Result of Antitoxin in Experiment

\begin{tabular}{|c|c|c|c|c|c|}
\hline No. & $\begin{array}{c}\text { Weight, } \\
\text { gm. }\end{array}$ & Cond. of leg & Method & $\underset{\text { units }}{\text { Amount }}$ & Result \\
\hline 488 & 250 & Stiff & Control & $\cdots$ & D 2 days. \\
\hline 442 & 295 & Slightly stiff & Control & $\ldots$ & D 3 days. \\
\hline 499 & 350 & Slightly stiff & Control & $\ldots$ & D 3 days. \\
\hline 480 & 275 & Slightly stiff & Heart & 100 & D r3 days. \\
\hline 498 & 275 & Stiff & Heart & 200 & Discharged April ro; drags leg. \\
\hline $45^{\mathrm{I}}$ & 265 & Fairly stiff & Spinal & 10 & $\begin{array}{l}\text { Discharged well April 23; drags } \\
\text { leg. }\end{array}$ \\
\hline 479 & 270 & Stiff & Spinal & 50 & $\begin{array}{l}\text { Discharged well April ro; drags } \\
\text { leg. }\end{array}$ \\
\hline
\end{tabular}

It is to be noted that each pig received exactly two fatal doses of toxin and that the degree of tetanic spasm in the inoculated leg was carefully observed at the time of giving antitoxin. The one surviving intracardial case received twenty times the amount of antitoxin of one intraspinal case and four times the amount of the other.

This experiment was repeated on three series of guinea pigs with similar results. This would seem absolutely conclusive of the superiority of the intraspinal method of giving antitoxin over the intra- 
venous method. An attempt was made to give the antitoxin to four guinea pigs intraneurally. 'The animals lived no longer than those receiving subcutaneous injections.

Permin, ${ }^{1}$ after rendering the blood of rabbits and other animals strongly antitoxic by the intravenous injection of antitoxin, was able, nevertheless, by introducing tetanus toxin by way of the nerves, to produce a local form of the disease, from which he reasons conversely that tetanus toxin, having once reached the spinal centers, cannot be dislodged by antitoxin given by the route of the blood, and therefore, all methods of giving it for curative purposes except that of direct application to the spinal cord can have little or no effect. The statement that an intravenous or subcutaneous injection is useless seems to us of far too sweeping a character, especially since it will be noted that in the foregoing experiment a guinea pig in which the symptoms, when treatment was given, showed every evidence of the toxin having reached the spinal nerve centers, nevertheless recovered after a large dose of antitoxin given in the blood.

The Disappearance of Tetanus Antitoxin from the Spinal Canal.Through passage to the blood the antitoxin injected into the spinal canal rapidly is reduced to about I per cent. of the blood strength. The most accurate information upon this transfer has come from MacConkey and Frazier. The latter considers that there are under normal conditions 60 to 20 c.c. of cerebrospinal fluid, and that it is replaced every three to four hours. This would bring the daily quantity up to 360 to 960 c.c. If, then, we inject 4000 units of antitoxin into the subarachnoid space, we must not expect to find more than about one unit of antitoxin per cubic centimeter of cerebrospinal fluid at the end of twenty-four hours. His actual tests show that the amount of antitoxin present in the cerebrospinal fluid twentyfour hours after an injection of 4000 units varies greatly, the lowest being about $1 / 300$ unit and the highest nearly Io units. In one case, after rooo units had been given daily for four days, the cerebrospinal fluid contained twenty-four hours after the last dose about $1 / 100$ unit of antitoxin. (This would be about the amount returned from the blood.) Then 4000 units were given intraspinally, and four days later, the cerebrospinal fluid contained 1/5 unit. The total amount of antitoxin given did not seem to have any influence on the antitoxin content of the cerebrospinal fluid. Thus, after 20,500 units had been given intraspinally in six days, there was, twentyfour hours after the last dose, only 1/10 unit per cubic centimeter of cerebrospinal fluid. We cannot as yet say what minimum concentration of antitoxin in the cerebrospinal fluid it is necessary to attain in order that our efforts to cure may be successful.

The Frequent Exacerbation of the Symptoms in Spite of the Injection of Ample Antitoxin.-Davies (I I $_{5}$ ) has noticed a tendency to slight improvement followed by exacerbation of the symptoms about the third or fourth day. Dreyfus and Unger (I9I4), who

1 Permin, Carl, Mitt. a. d. Grenzgebiet. d. Med. und Chir., r913, xxvii, 1. 
treated thirty-two cases of tetanus (ten deaths) with large doses of serum-in one case $\mathrm{r}_{52,000} \mathrm{U}$. S. A. units-state that in the cases which recovered they observed the same course of events. The tetanus usually progressed for a day or two, then came to a standstill and eventually proceeded to pass away gradually, with occasionally a recurrence. They also say that after these large doses of serum it was noticed that there was almost always a rise of temperature, of ten to a high degree.

As a result of our series of animal experiments every effort has been made during the past year to get into immediate touch with physicians and hospitals in and about the city of New York having cases of tetanus under their care, and induce them to give antitoxin as soon as possible by the following method:

I. From 3000 to 5000 units into the lumbar region of the spinal canal, preferably under an anesthetic, the volume of fluid injected being brought up to Io or I5 c.c. by the addition of sterile normal saline, the exact amount being regulated according to the age of the patient and the amount of spinal fluid withdrawn.

2. Ten thousand units intravenously at the same time.

3. Repetition of the intraspinal dose in twenty-four hours and forty-eight hours to replace that removed by absorption from the spinal canal and delivered to the blood.

4. A subcutaneous dose of Io,000 units four days later to keep up the antitoxic strength of the blood.

The well-recognized adjuvants to specific treatment - quiet, subdued light, sedatives, including magnesium, etc.-were, of course, understood as a necessary part of the therapeutic measures.

Whenever possible Dr. Nicoll personally attended the case.

The Results of the Intraspinal Injections.-Reports of the first twenty cases have thus been collected. Dr. Nicoll saw about half of the patients personally, in consultation. In others, the physicians have been kept in constant touch by telephone or through a clinical assistant during the progress of the case. In all cases the antitoxin used was furnished by the Department of Health.

A brief abstract of the clinical histories of representative cases follows:

CASE I.-F. D., girl, aged ten years, severe case treated early. Patient fell, striking her forehead on the ground, receiving a lacerated wound $3 / 4$ inch long over one brow. This was properly disinfected and sutured, healing promptly. Seven days later there was a facial paralysis on the side on which the wound was received. Thirty-six hours later, the jaws were firmly locked. Eight hours after this symptom was noted, the patient received 3000 units of antitoxin intraspinally and ro,000 in travenously. Several subcutaneous injections were later given. The tetanic spasms were largely confined to the muscles of the jaw and pharynx, and later, the abdominal muscles; attempts at swallowing and the slightest external irritation caused contractions of the muscles of the throat and larynx, cyanosis, general convulsions and unconsciousness. Such convulsions occurred on fifty or more occasions, together with innumerable minor spasms. Pneumonia developed later, resolution being very long delayed. After a protracted convalescence and extreme emaciation, the patient made a perfect recovery.

CASE 2.-Gustav H., aged twenty-three, carpenter, severe case treated late. He had injured the tips of his fingers in some way, June I 6 . A definite history could not be obtained. Three days before this, the patient had a pustule on his neck, which was 
opened on the 2oth. There was no other history of injury. June 21 (incubation five or eight days), he first noticed that his jaw was stiff; two days later, this was much more marked and had spread to the neck, back, arms and legs. On admission, there was marked opisthotonos, and the jaws were tightly locked. The slightest disturbance caused a convulsion. The patient's body rested on the heels and back of the head. The abdomen was boardlike. Treatment on the first day after admission consisted of 5000 units of antitoxin intraspinally and 5000 intravenously; June 28,5000 subcutaneously. The opisthotonos continued. July I, 5000 units were administered intraspinally, five hours after which the patient became delirious with a rise in temperature to I05. From July 4 to July 8 , the rigidity decreased daily. July 18 , the patient was discharged, cured.

CASE 3.-T. D., woman, aged thirty years. She had given birth to a child two weeks previously in very dirty surroundings. The symptoms of tetanus were very marked, and many convulsions continued for five days. Two days after the onset of symptoms, 10,000 units of antitoxin were given intraspinally and 5000 intravenously; the later dose was repeated three times in three successive days. The patient was discharged cured except for a slight trismus which lasted for three days longer, October II.

CASE 4.- - Severe case with slow development and late use of antitoxin. M. G., woman, aged twenty-two, gave no history of traumatism. Four days before admission to the Lebanon Hospital, there was slight rigidity of the neck and difficulty in opening the mouth. The next day the jaws were completely locked. On the day before admission she had general convulsions. On the day following admission, five days after the onset of symptoms, she was given 5000 units of antitoxin intravenously and 750 units intraspinally. On the second and third days she was given 5000 units intraspinally under an anesthetic. The patient also received injections of magnesium sulphate $(25$ per cent.) subcutaneously and intramuscularly three times a day in doses of 30 c.c. for seventeen days, without apparent relaxing effect. The course of the disease was rather protracted with many convulsions. The patient was discharged cured seven weeks after admission.

CASE 5.- Severe case immediate treatment. L. M., male, aged eighteen years, was admitted to St. Peter's Hospital, March 27, 1915, with a crushed and lacerated wound of the great toe. The wound was immediately disinfected and properly dressed. March 30, the tip of the toe sloughed off. April 6, the patient had a severe chill and complained of sore throat. There was noted a slight stiffness of the jaw. In the afternoon there were marked stiffness of the back, and occasional slight convulsions when the patient was disturbed. Twenty thousand units of tetanus antitoxin were given intraspinally four hours after the occurrence of the chill. On the 7 th, the symptoms increased in severity, convulsions occurring on the slightest irritation. There were marked opisthotonos and rigidity. Ten thousand units of antitoxin were given intraspinally under an anesthetic. On the 8th, the patient's condition was about the same. Ten thousand units of antitoxin were given intravenously. After the tenth day, the convulsions stopped; the rigidity continued some time longer. April 22, the patient was up and about with slight rigidity. He was discharged cured a few days later.

CASE 6.-E. C., boy, aged thirteen years, admitted to Bellevue Hospital, June 30 , 1914, fourteen days previously had fallen and cut his head. Eleven days later he had abdominal pains and vomiting, which were regarded as due to appendicitis. On admission to the hospital he had marked opisthotonos, locked jaws and inability to swallow. A convulsion occurred on the slightest disturbance. He received ro, 000 units of antitoxin intraspinally and 10,000 units intramuscularly. The patient's condition grew worse from the time of his admission and he died within twenty-four hours. The disease during the three days had evidently progressed too far to be influenced by antitoxin.

In judging the effect of antitoxin given intraspinally in the series of cases, it must be remembered that the patients were not selected, but that every case of tetanus reported was given the benefit of the treatment regardless of the clinical condition. The series, therefore, may be said to be fairly representative of this type of the disease occurring in and about the city of New York. A few of these patients would undoubtedly have recovered if the intraspinal injection of antitoxin had not been given or, indeed, without any treatment other than symptomatic. The results obtained, however, in the saving of 
life are so much more favorable than those in previous years, when large doses of antitoxin were recommended to be given by the intravenous and subcutaneous methods, that there can be no reasonable doubt that the low death rate, 20 per cent., here obtained was largely due to intraspinal dosage.

The reports from Europe indicate that in the cases treated the intraspinal method is gradually being accepted by the majority of physicians.

\section{THE SERUM TREATMENT OF DYSENTERY}

The knowledge concerning the serum treatment of dysentery given us by the experience of Shiga has been somewhat extended in recent years. Dysentery has not been prevalent recently in the northern United States. The earlier opinion has been confirmed that bacillary dysentery alone occurs in cold and temperate climates, while in hot climates both bacillary and amebic cases occur about equally. The idea that summer diarrheas were frequently due to the dysentery bacilli has been discarded. The bacilli may be present at times in small numbers in these cases, but they have too little part in the disease process to require specific treatment. The dysentery bacilli may be divided into dysentery bacilli and paradysentery bacilli or into different strains of a group. Like so many other bacteria causing inflammations of mucous membranes the organisms exciting dysentery belong to a variety of strains.

The bacillus isolated by Shiga and proven by him to be the cause of the epidemic occurring in Japan is not only the most widespread but also the most toxic of the strains which later investigations have associated with dysentery. It seems well to call this strain the dysentery bacillus and the others paradysentery bacilli. The most important of these other strains are those isolated by Flexner, Kruse, Hiss, Park and Strong. These are separated by their action on sugars and by complement fixation and agglutination tests. The serum produced by one strain operates better against bacilli belonging to it than against those belonging to the other strains. For sporadic cases and those occurring in the beginning of an outbreak, a polyvalent serum must be used. In later cases in an epidemic where the strain has been identified the monovalent serum is to be used if possible.

The serum in mild cases is given in doses of from to to 30 c.c. twice daily according to the size of the person. In severe cases as high as roo c.c. two or three times in the twenty-four hours can be given and in desperate cases the serum has been given intravenously. If given intravenously, the serum must be warmed and given s'owly. The doses are to be continued from day to day until permanent improvement is established. Injections are usually continued for two or three days. The majority of those who have used the serum confirms Shiga's original belief that the results are good. My own experience in a number of severe cases is on the whole favorable. I 
believe it is unnecessary to use the serum in slight cases. In favorable cases within six to twelve hours the constitutional symptoms frequently improve. The abdominal pains are less, the mental condition is better and the pulse slower and stronger. There is frequently at this time some reduction in the number of stools though they may be more copious and have increased sloughs. The serum on the market is not always labeled as to its type and its strength and some of it is valueless. The necessity of using a polyvalent serum in most cases also lessens its value. In spite of these objections the use of the serum in severe cases is strongly indicated. It is very desirable that records be kept of the cases treated and the results as reported.

Shiga believes the mortality to be reduced by the serum treatment from around 35 per cent. to about 9 per cent. Some report even more striking results, while still others have met with disappointment. Undoubtedly the extent of mixed infections with other organisms, the possibility of some cases having been due to ameba and the use of inactive serum must be considered as being possibly the cause of the failure of the serum in these cases. Ruffer and Willmore showed that the Shiga type of serum had no effect on their cases which were due to $T$ or $Y$ type of paradysentery bacillus. Either a polyvalent serum or a suitable monovalent serum was found effective. The usual serum after-affects may develop.

\section{CEREBROSPINAL MENINGITIS}

The paper by Barker in the second volume of this series needs little change. The intraspinal method of injecting the antimeningitis serum first used and advocated by Jochmann and its value corroborated by Wassermann and Flexner is now the only way of injecting the serum employed. There are a few practical points that should be added.

The Serum.-The methods of producing the serum have not materially changed since Wassermann, Jobling and others developed them in 1905 and later.

Standardization.-Antimeningitis serum has been standardized by the opsonic method, by agglutination and complement fixation.

The opsonic method is the only one definitely known to be connected with the therapeutic actions of the serum, and it is the one that has been most generally accepted in the past. It is open to the objections that it is impossible to make absolutely. uniform preparations which renders the reading difficult and somewhat indefinite and, furthermore, that the serum of some normal horses show a marked phagocytosis. Agglutination is of value in determining the polyvalency of the serum, viz., its probable activity against different strains.

Complement fixation gives sharply defined readings, but no relation has been established between the antibodies involved and 
the therapeutic actions, although from clinical experience the two seem to agree.

As in some instances at least, the antibodies do not run parallel it is advisable that the standardization of the serum should be checked by all three methods.

Effect on Mortality.-It is generally accepted following the reports of Jochmann, Flexner and his coworkers and others that the mortality has been reduced by its use from about 75 per cent. to 25 per cent.

The mortality of sporadic cases is probably not so high as that of epidemics at their height. It is difficult to estimate how many of the cases that we see would die if untreated. Perhaps 60 per cent. is a fair estimate. In r9r4, our mortality in the cases treated by the Department of Health with serum was 26 per cent.-in I9r 5 , 34 per cent. Of course, the diagnosis in sporadic cases is usually made later than in an epidemic. This would tend to lessen the efficacy of the serum.

The duration of the disease is greatly shortened by the use of serum and the sequelæ are probably lessened. A study of treated cases shows that deafness is the most common serious sequela. Mental defects are very rare.

Addition of Preservatives.-Trikresol in dilutions of 0.5 to 0.3 per cent. was used from Igro to October, I9I4, in the serum prepared by the Health Department. For the following year chloroform was added, but in I9I6 we returned to the use of trikresol, which apparently acts as a local anesthetic. . This was used in patients of all ages with no untoward effects referable to it. There were a few cases of shock following the injection of serum, but these became very rare after we began to use smaller doses.

Serum containing trikresol has little irritating action when injected into the spinal canal, while that containing chloroform causes severe pain in practically all cases. This is a strong argument in favor of trikresol to all who are administering serum. Moreover, in dilutions of 0.1 to 0.2 per cent. trikresol has a marked bactericidal action on meningococci in vitro.

Administration.-Method and Size of Dose.-The gravity methoa is far preferable to the syringe. It is very difficult to judge how much pressure is being exerted with a syringe and the danger of even a slight increase in pressure is a most serious one. The ease with which the serum runs in is a valuable index to the amount that can be safely used. While it is generally best to give a smaller amount than that of the fluid withdrawn, sometimes when the amount of fluid is small, a larger amount of serum can be safely injected if it runs in easily. In dry taps, Io, I 5 and even 20 c.c. may sometimes be given. The easy introduction of serum also proves that one is in the spinal canal in cases of dry tap. Again, although a large amount of fluid has been withdrawn, the serum sometimes runs in with considerable difficulty and it is not wise in these cases to administer large amounts. 
It is not usually advisable to give more than 20 c.c. even though much more fluid has been withdrawn. The relief of pressure is, in itself, of therapeutic value. If, however, the case does not seem to respond well and a larger amount of fluid runs in easily, 30-40 c.c. may be given, if the patient shows no change in pulse or respiration and is not rendered very uncomfortable. All these points should be very carefully noted while giving serum. Ordinarily 5 or more c.c. less of serum than the fluid withdrawn should be given. At times, however, as stated above, if the amount of fluid is small and the serurn runs in easily, 5 to ro c.c. more serum may be administered. The case of dry taps has been already referred to. It is safe to withdraw as much fluid as will run out while the patient is in the recumbent position.

Repetition.-The serum should be administered daily until no organisms can be demonstrated by smear or culture. The culture should be incubated forty-eight hours before being pronounced sterile. If, although the fluid is sterile, it is still very purulent, the serum should be administered until the fluid is nearly normal. Injections in this case may be given every other day in some instances. The serum treatment should also be continued until the symptoms have greatly improved. If it is impossible to have the fluid examined bacteriologically, one must rely on the gross appearance of the fluid and the symptoms. The serum should be continued until the fluid is nearly clear, the temperature practically normal and the other symptoms greatly improved. It is safer to give too much than too little serum. If the chemical examination of the fluid can be obtained, a decrease in the albumin and globulin and the good reduction of Fehlings indicates a return to normal.

In cases that are desperately ill injections may be given every twelve hours until improvement begins. It is rarely safe to give less than four injections and fifteen to twenty may be necessary. One or more punctures for the relief of pressure may be indicated after the meningitis has cleared up. It is obvious that no exact rules about the administration of serum can be laid down. The greater the experience, the greater will be the skill in administering it.

Anaphylaxis.-Although, in a fairly large number of cases, the administration of serum has been extended over a period of two to four weeks either continuously or intermittently when there have been relapses, there has never been a severe anaphylactic reaction. This is due to the intervals between the injections being only one to three days. Serum rashes occur occasionally.

\section{ANTISTREPTOCOCCUS SERUM}

The experimental use of a serum obtained from a horse immunized by repeated injections of living streptococci is quite successful when used to protect against a later infection or one in the beginning stage. It is only moderately successful in developed cases. Its use in human beings is complicated in many ways. 
The serum acts chiefly upon the infecting organisms by its opsonins. The streptococci unite with these and are thus altered so as to be taken up by the leukocytes and perhaps by other cells and later destroyed by the cell ferments. Before the virulent streptococci unite with the opsonins they are little or not at all absorbed by the leukocytes. It is more than twenty years since Roger and Mamorek used the serum for therapeutic purposes. Many favorable and unfavorable reports have been made by different observers since that time. In 1902, Moses advocated its use in scarlet fever because streptococci had at least as a mixed infection much to do with the seriousness of the complications and toxemia.

Unfortunately, virulent streptococci belong to many strains which differ among themselves absolutely as to their relation to antibodies. These are two main groups, one the hemolyzing streptococci found especially in infections of the subcutaneous tissues and the other resembling more the pneumococci and being found mostly in respiratory infections and complications growing out of them. These two main groups are divided into subdivisions and these strains often differ among themselves. Like the pneumococci they also differ in the amount of protective substances their infections give use to. There are no standards established to control the strength of the sera so that the honesty and capacity of the firms sending out the sera must be carefully weighed. As the serum ages, it gradually loses in value, so that a serum more than six months old is undesirable. Weaver and Tunnicliff found only three out of seven lots of serum purchased after being in stock two years to have retained any value.

When an antistreptococcus serum is to be used the attempt should be made to determine whether it is for the hemolyzing or non-hemolyzing type (viridans). This can be done by cultures in twentyfour hours. A polyvalent serum for this type is then used. In scarlet fever, erysipelas, cellulitis and abscess, the hemolyzing type of antistreptococcic serum is used. Sometimes it is so difficult to decide the probable type that both varieties of polyvalent sera are mixed together. The infections due to the viridans strains have not shown a very favorable response to the serum injections.

Observations have convinced us that large doses of serum are necessary in treatment. As with all therapeutic sera, the size of the individual to be treated and the severity of the infection influence the size of the dose. The serum is given usually intravenously in amounts of $5 \circ$ to $15 \circ$ c.c. and repeated every twenty-four hours for two or three days, if favorable results are noted and the infection remains active.

The serum should be warmed to the body heat, given very slowly and injected with all necessary precautions.

In some cases the effect appears to be striking. The temperature falls $3^{\circ}-6^{\circ}$, the mental and general conditions improve and the organisms disappear from the blood. In many others no results appear whatever. These failures of the serum may be 
due to the presence of strains not affected by serum or of different strains than those used in the treatment of the horses.

Local Application.- Horse serum as such seems to have a favorable influence upon ulcerated and infected surfaces. In infected wounds and cavities, like the uterus, the frequent applications of antistreptococcus serum seem to do considerable good and certainly do no harm. Studdiford has used serum locally considerably in postpartum uterine infections. Applied to streptococcic throat infections it seems to have had good results.

The use of antistreptococcic sera for prevention of infection is favored by many. The protection lasts about ten to fourteen days. The usual dose is ro or 20 c.c. The serum is protective only against streptococci similar to those with which the horses were treated.

The decision as to the advisability of giving antistreptococcic serum in any given case is often very difficult. It is seldom given except in severe cases. The somewhat deleterious serum affects which may follow must be considered as of some weight. In septicemia the results have usually been disappointing. In toxic scarlet fever, if possible, convalescent human serum should be employed, but if this is not available, the polyvalent hemolyzing streptococcus serum is in my opinion, advisable. In suitable cases the local use of the serum is always to be urged.

Serum in Infections Due to Pneumococci: see article by Dochez.

\section{ANTISTAPHYLOCOCCUS SERUM}

The use of the sera so far produced have not shown any practical value in immunization or treatment.

\section{THE THERAPEUTIC USE OF HUMAN CONVALESCENT BLOOD HAVING SPECIFIC ANTIBODIES}

Convalescent serum or whole convalescent blood has been used in the treatment of early toxic cases of scarlet fever both here and abroad and has given encouraging results in the limited number of cases observed thus far. Reiss and Jungman recommended the intravenous injection of 50 to Ioo c.c. of pooled convalescent serum, while Zingher makes use of the intramuscular injection of whole convalescent blood, citrated or non-citrated, which he injects in quantities of $\mathrm{I} 20$ to 240 c.c. The blood causes no local inflammatory reactions in the muscles, and is rapidly absorbed. The convalescent serum or fresh whole blood is obtained from patients who are two to three weeks convalescent from scarlet fever. These donors should be free from syphilis and tuberculosis.

The effect of convalescent serum or whole blood in the uncomplicated early toxic cases of scarlet fever is seen in a critical drop in temperature, begining about six hours after the injection and ending in from twenty-four to thirty-six hours; an early fading of Vor. $1 \mathrm{~V},-6$ 
the rash; improvement in the circulation and character of the pulse; and especially in the general condition and mental symptoms of the patient. . Zingher reported the results obtained at the Willard Parker Hospital with intramuscular injections of whole convalescent blood in fifteen very toxic cases of scarlet fever, selected out of a total of 900 admissions. A striking effect was noted in five cases, improvement and final recovery in six more, while four patients died from various septic complications.

In the later septic cases, seen from the fifth to tenth day of disease, complicated by an extensive streptococcus exudate over fauces and tonsils, enlarged and tender cervical glands, a poor circulation and showing general septic temperature, fresh normal blood, injected in quantities of $\mathrm{I} 20$ to 240 c.c. and repeated if necessary in three to four days has shown very beneficial effects in some desperately ill cases. Fresh normal blood has no specific action in septic cases of scarlet fever, but it supplies definite nutritive, stimulating, and normal bactericidal substances.

The recent epidemic of poliomyelitis has given an opportunity for the use of intraspinal injections of human serum from recovered cases. At the time of writing this article the results of the injections seem to be somewhat favorable. The serum is injected as in cerebrospinal meningitis. Sometimes only one injection is given, but usually two or three. The amounts given are somewhat smaller because of the scarcity of the serum. The most active serum is obtained from those who have had the disease within the year. After five years the serum may or may not be better than that from persons who have not had the disease. 


\title{
HYDROLOGY IN MILITARY PRACTICE
}

\author{
By Guy Hinsdale, M. D.
}

The European war has brought about a notable change in the uses to which British and Continental watering places are devoted. The presence of a large number of invalid soldiers and sailors for whom spa treatment is indicated has naturally prompted the medical officers at the base hospitals to take the necessary steps to afford this treatment in suitable cases.

The numerous French resorts, such as Vichy, Barèges and Amelie-les-Bains, are so used and the British spas have received large numbers sent over from Continental battlefields. A committee of the Royal Society of Medicine, in response to the request of the War Office, has furnished authoritative information for the use of bath treatments for wounded and invalid soldiers. This includes:

I. A summary of the medical and surgical maladies most commonly met with in the military hospitals, for which after-treatment as a health resort is indicated.

2. A list of British spas, as well as other bathing establishments, with the factors influencing the preference for one or another, in the treatment of such maladies.

Acute Cases. - The use of medical baths for wounded and invalid soliders will naturally begin in many cases in the hospital. Hydrotherapy in acute cases is threefold and comprises:

r. Cooling applications, local or general, in fever, circulatory excitement and inflammatory congestion.

2. Baths at a higher or subthermal temperature $\left(90^{\circ}-98^{\circ} \mathrm{F}\right.$.), which are sedative to the nervous system and have an extensive use in nervous mental and circulatory disorders.

3. Thermal and hyperthermal baths $\left(98^{\circ}-\operatorname{II} 2^{\circ} \mathrm{F}\right.$.), which primarily stimulate the metabolism and circulation.

Only those cases which are passing out of the acute, or have reached the chronic; stage can be suitably sent to health resorts. The wise use of baths and other physical measures tends to regulate metabolism, both local and general, and the promotion of excretion together with a stimulative or sedative action upon the circulation and the nerve centers.

\section{SURGICAL}

Contusions and Bruises (Later Stages).-When congestion has abated, cooling and sedative are replaced by stimulating (hot) baths, or by the Scotch (alternately hot and cold) douche. 
Sprains and Strains of Joints.-In an early stage, alternate hot and cold applications; later, effusions are removed by douches of increasing strength and alternating temperature; to obviate contraction and immobility, deep, or full, thermal baths, such as are given at Bath, Buxton and Woodhall Spa. To relieve pain local hyperthermal baths and electric radiation.

Fractures near Joints with Immobility.-Thermal baths, 105 ${ }^{\circ}-$ IO $4^{\circ} \mathrm{F}$. with movements and rubbing in the tub. In painful cases prolonged baths at lower temperatures $\left(95^{\circ} \mathrm{F}\right)$. reduce edema, relieve pain and restore local circulation. It is well known that in hot baths it is possible to move stiffened and painful joints when otherwise it would be impossible. Long-continued baths at a somewhat lower temperature reduce edema and favor a restoration of the local circulation.

Dr. Fortescue Fox whose advice in regard to medical baths for invalid soliders has been sought by the British War Office says: ${ }^{1}$ "It is a golden rule in hydrology that every kind of thermal bath is contraindicated, not only in the presence of cardiac weakness but also in febrile cases, and in acute or subacute inflammatory conditions of all kinds; such baths are applicable only in chronic disease where the tissue reactions are defective and sluggish or pervented. It may often be observed in the history of a somewhat practical case that there comes a moment when acute conditions have subsided and when a stimulating or thermal bath treatment is indicated, though previously inapplicable. Such stimulating measures would be injurious in the early stages of serious sprains, on periarticular contusion, or in a traumatic arthritis or synovitis in which it is above all necessary to allow the acute reactions to subside. But two or three months after the accident, should the pain or swelling persist, and muscular atrophy set in, salt or sulphur or thermal baths, according to individual conditions, together with massage, movements and douches have their proper and individual place."

Fractures Imperfectly Healed; Unresolved Effusions.-Here, again, it is necessary to carefully judge of the temperature required in the bath. Continued bathing at a moderate or subthermal temperature favors asepsis and cicatrization of wounds. Higher temperatures may be injurious, especially if an unhealed cicatrix leads to an intermuscular sinus. We may order in cases of unresolved effusions douches of alternating temperature, massage douches, thermal baths such as are given at Bath, Buxton and Harrogate.

Fractures with Osteitis and Necrosis. - Thermal baths (Bath, Buxton and Matlock); sulphur baths (Strathpeffer, Harrogate); brine baths (Droitwich, Saltburn-on-Sea, Margate).

Wounds Unhealed; Painful Scars.-Prolonged baths. The English committee notes that an even warmth maintained for some hours operates with the moisture, so as to increase the peripheral

\footnotetext{
${ }^{1}$ Trans. Roy. Soc. Med., Jan. I4, I9I5.
} 
circulation, soothes the nerve endings and promotes nutrition, especially in debilitated subjects. Sulphur and nearly isotonic salt waters have been found to favor the healing of wounds (Llanwyrtyd, Llandrindod, Woodhall Spa).

Cases Where Arterial Circulation is Locally Deficient; Effects of Pressure; Frost-bite.-Thermal treatments by local vapor baths as with the apparatus Bertholler (Bath, Buxton, Droitwich) or dry heat in hot radiation chambers (Tyrnaner apparatus and local baths with incandescent lamps).

Cases after Operation.-The English committee makes the following differentiation as to choice of a spa and climate, indicated by the condition of the circulation and of the nerve centers:

For a sedative action, Bath, Leamington, Sidmouth, Forquay, Rothesay.

For a stimulant sedative action, Strathpeffer, Llandrindod, Southport, Dunblane, Ilfracombe, Ramsgate, Bournemouth, Malvern.

For a stimulant action, Buxton, Harrogate, Droitwich, Woodhall Spa, Nairn, North Berwick, Peterhead, Folkestone, Scarborough, Nargate, Brighton.

\section{MEDICAL}

A. Rheumatic Disorders.-Fatigue Fever and Muscular Rheumatism (Acute Fibrositis), the Result of Fatigue and Exposure.-These disorders form the largest class of cases in armies in war as well as in peace. Long-continued and extreme physical exertion, especially in untrained troops, are the natural and obvious causes. Life in the trenches, the inevitable exposure to cold and dampness largely augment the sick roll. Dr. Fox notes that an excessive muscular metabolism causes, in this condition, an accumulation of waste products within the muscles and a slight general septic intoxication, the symptoms being exhaustion and stiffness and acute pain on movement. If the muscular work is too greatly prolonged, what has been called "organic exhaustion" ensues, , perhaps with cerebral disturbance. In slighter cases immediate relief is obtained by stimulating the circulation and sweeping away the waste products. Even at the front suitable treatment is now afforded by means of hyperthermal baths $\left(105^{\circ}-110^{\circ} \mathrm{F}\right.$.) of thirty seconds to three minutes duration; very brief in cases showing exhaustion. Such baths have been found highly stimulating to the circulation and a powerful aid to the elimination of waste products. Such baths are given at all French and British Spas. Sometimes a little ammonia is added to the water.

Pain, Severe.-Baths of thermal indifference $\left(93^{\circ} \mathrm{F}\right.$.) very prolonged. At later stages vapor, or Russian, baths relieve nervous irritability and muscular spasm. Sedative bath resorts (Bath, Leamington).

In Chronic Cases.-Higher temperatures and douches as at Bath, and the more bracing stations of Buxton, Harrogate, Woodhall Spa, Droitwich and Malvern. 
Sciatuca and Lumbago.-(Droitwich, Woodhall Spa, Buxton, Bath (in winter). Local applications of Fango or peat (Matlock, Strathpeffer,. Harrogate).

Convalescence from Rheumatic Fever.-Internal use of sulphur waters, acting on the bowels (Harrogate, Llandrindod); acting on the kidneys (Strathpeffer, Llanwyrtyd, Lucan).

Chronic Articular Rheumatism, Synovitis and Degenerative Arthritis.-The results of injury or exposure especially in those who have occupied cramped positions in the trenches are often successfully treated by a combination of eliminative waters and thermal baths; the sulphur spas such as Harrogate, by sodium chloride waters, such as Droitwich and Woodhall Spa; with thermal baths and douches with massage, as at Buxton in summer or Bath in winter. Warm, dry, sunny climatic resorts are to be preferred.

B. Circulatory Disorders.-Defective Peripheral Circulation.After fevers and wounds, natural thermal waters (Bath, Buxton), peat baths (Strathpeffer, Harrogate, Buxton) or brief hyperthermal baths and massage at all the spas. With sluggish elimination, if the heart is sound, a course of Turkish baths and massage at hydrotherapeutic institutions.

Cardiac Dilatation, Graves' Disease, Tachycardia.-Cooling treatment is indicated; heat is to be avoided. Sedative (antithermal) baths at $90^{\circ}-95^{\circ} \mathrm{F}$; ; still or effervescing (carbonated). These produce their best effects when the climatic influence reinforces that of the bath employed.

C. Nervous Disorders.-These are greatly benefited by baths. Nervous shock, neurasthenia and psychasthenia from traumatism, mental strain or operation can be treated at most resorts, many circumstances governing the choice. Hot baths and climates are seldom advisable but marine and woodland stations are advised and whether sedative or more or less stimulating treatment is given will depend on the peculiar needs of the individual case.

When a stimulant effect is desired hot air and other thermal baths and douches are to be preferred; but where, as in the case of very many soldiers invalided home, a more profound exhaustion is shown by irritability, depression and insomnia, sedative baths, given at a lower temperature and more prolonged are recommended. The treatment of military cases of nervous disease naturally follows much the same lines as in civil practice. No distinction need be drawn.

It will be, naturally, a great advantage in all nervous cases especially if the advantages of climatic treatment can be combined with hydrotopic treatment. From the base hospitals to the health resort is the logical procedure.

Irritative Conditions, Excitement or Insomnia.-In a gouty or rheumatic case, gentle eliminative treatment at a quiet and restful spa (Strathpeffer, Landrindod, Leamington Moffat, Cheltenham and, in winter, Bath). 
In Depressed and Atonic Subjects and in Neuralgia.-Cold hydrotherapy and invigorating douches at all the spas and medical baths. Bracing stations such as Harrogate, Buxton and Malvern; marine baths at Cromer, Peterhead, North Berwick Scarborough, Eastbourne, Folkestone, Nargate. When elimination is defective, Turkish baths, douches and massage at any bracing resort.

In Melancholia.-Slighter forms, purgative and diuretic waters.

Palsies.-Post Hemiplegic or Following Injury to Nerves with Wasting.-Thermal baths with massage, (Bath, Buxton). Douche baths may be used in appropriate cases.

Peripheral Neuritis.-The underlying condition requires eliminative treatment, diuretic, saline, sulphur or sodium chlorid waters. Bath treatments: in early stages sedative and subthermal; later, more stimulating and resolvent (Peat, Strathpeffer; Fango, Mortlock; brine, Droitwich). Functional, the same as for neurasthenia.

Digestive, Hepatic, Respiratory and Cutaneous Disorders.Catarrhal Dyspepsia in Neurasthenics.-Mild sodium chlorid, sulphur or thermal waters, with tonic regimen.

Dyspepsia with Anemia.-Harrogate, Brighton, Tunbridge Wells; bracing marine resorts, Nairn, Scarborough, Yorkshire, Folkestone, Weston-Super-Mare (Somerset).

Constipation and irritation of the colon, after enteritis and dysentery, purgative waters and tonic regimen, Harrogate, Cheltenham, Leamington, Landvindod; intestinal lavage, Buxton, Harrogate, Woodhall Spa.

Respiratory Catarrhs, Asthma, Incipient Tuberculosis.-In sensitive subjects, Ventnor, Falmouth, Hastings, Rothesay; in atonic subjects, Eastbourne, Brighton, Cromer, Southport, Tunbridge Wells; in gouty subjects, Bridge of Allan, near Stirling, Scotland, Llandrindod, Mid-Wales.

Chronic Eczema.-Sulphur waters and baths, Strathpeffer, (Ross-Shire), Llanwyrtyd (South Wales), Lisdoon-varna (County Clare, Ireland), Ripon (Yorkshire).

\section{PRINCIPAL CHARACTERISTICS OF SPAS}

The principal characteristics of the spas mentioned may be summarized as follows:

Bath (Somerset).-One hundred feet. Thermal waters $1^{\circ} 4^{\circ}$ $120^{\circ} \mathrm{F}$., radioactive, with salts of lime; tonic and diuretic. Wellappointed deep thermal baths, douches and accessory appliances, with massage, vapor baths, etc. Climate mild, equable and sedative, in summer suitable only for persons able to support a warm climate; well adapted for weakly and debilitated subjects in winter, autumn or spring.

Indications.-(I) All forms of chronic rheumatism, fibrositis and arthritis; (2) stiffness and limitation of movement after wounds; (3) neuritis; (4) wasting following nerve injuries; (5) traumatic neurasthenia; (5) frost-bite. 
Bournemouth (Hampshire).-A mild dry climate, with pine woods. Indicated in traumatic neurasthenia, convalescence from pulmonary affections. Turkish and sea baths.

Bridge of Allan (near Stirling).-Waters containing chlorids of calcium and sodium, suitable in dyspepsia, venous engorgement, catarrh, gout and rheumatism.

- Brighton.-A sea front facing south, sheltered from the north and with an invigorating air.

Indications.-Convalescence, neurasthenia, general debility and want of tone, anemia. Douches, sea baths and Turkish baths.

Buxton (Derbyshire).-One thousand feet. Waters, subthermal, $\left(82^{\circ} \mathrm{F}\right.$.), radioactive with calcium and magnesium bicarbonates, diuretic, chalybeate. Thermal establishment well equipped, for bath and auxiliary treatments, swimming and immersion baths, douches and massage; effervescing and peat baths, hot air and hydrotherapy. Climate bracing; elevation, rooo feet.

Indications.-All forms of chronic rheumatism; neuritis; sprains and strains; stiff joints after wounds; gout.

Cheltenham.-One hundred and fifty feet. Aperient waters (sulphates and chlorids).

Indications.-Dyspepsia, hepatic disorders and chronic gout. Baths are given.

Droitwich (Worcestershire).-Two hundred feet. The premier British Salt Spa. Brine, nearly saturated, radioactive. A powerfully stimulating bath; local applications and douches. Preferable in summer.

Eastbourne.-A dry and sunny south coast resort facing southeast. Turkish and sea-water baths.

Folkestone (Kent).-A sunny sea-bathing health resort, sheltered by the Downs; tonic and bracing. Turkish and other medical baths.

Harrogate (Yorkshire).-Five hundred, feet. Sulphur and sodium chlorid waters and Chalybeate Springs; aperient and diuretic. Sulphur,douche, peat, efflervescing and other baths, besides intestinal lavage. Elevation 400-600 feet. A bracing spa, in cold winter.

Indications.-Muscular and articular rheumatism and gout; stiffness of joints; unhealed wounds; dyspepsia and constipation; disorders of the liver and colon; anemia.

Leamington (Warwickshire).-Two hundred feet. Waters containing sodium chlorid and sulphates, being diuretic, aperient and tonic. There are Turkish, effervescing, brine, douche and electric baths.

Indications.-Dyspepsia, hepatic disorders, glycosuria and gout; also chronic rheumatism, stiff joints, sciatica and lumbago.

Llandrindod (Mid-Wales).--Seven hundred feet. Waters saline, sulphurous and strongly diuretic.

Indications.-Digestive disorders, nervous fatigue, gouty and rheumatic affections and injuries.

Margate.-A bracing seaside resort in Thanet, Kent. 
Mattock (Derbyshire).-A natural subthermal water $\left(70^{\circ} \mathrm{F}\right.$.) principally used for baths. The climate is mild and suitable for winter visitors especially as Mattock Bath when the water has its source.

Indications.-Rheumatism, neuritis, injuries of nerves, muscles and joints and neurasthenia.

North Berwick.-A sunny and bracing sea-bathing resort.

Scarborough (Yorkshire). - A bracing east coast resort. Aperient sulphated water. Bathing facilities.

Strathpeffer Spa (Ross-Shire).-Two hundred feet. Strong sulphur and chalybeate waters; diuretic and tonic. Sulphur, peat and effervescing baths, douches, etc. The most northerly spa in Great Britain.

Indications.-Rheumatism and gout; nervous breakdown, concussion, shock; digestive and cutaneous affections.

Torquay (Devonshire).-An attractive seaside resort facing south and west.

Indications.-Nervous and muscular fatigue, insomnia; chronic cardiac and renal affections.

Tunbridge Wells (Kent).-Invigorating air; elevation $400-500$ feet. Hydrotherapeutic treatment.

Ventnor.-South side of Isle of Wight. Warm, equable, marine resort; marine and radiant heat baths.

Wood Hall Spa (Lincolnshire).-Thirty-seven feet. Hypertonic salt waters.

Indications.-Rheumatism, arthritis; nervous shock; sciatica. Baths, intestinal lavage, etc.

\section{FRANCE}

There are three principal mineral water stations in France operating in connection with the military establishment. These are Vichy with 2 I 2 beds, Barèges with 225 beds and Amélie-les-Bains with 4 I 8 beds. No doubt at the present time the capacities of these "hôpiteaux d'eaux minéralis" have been greatly increased and include other hospitals at Plombières, Bourbon-l'Archambault, Bourbonne-lesBains and Aix-les-Bain. We have made some inquiry as to the extent of the service in these spas but so far very little information has been elicited. We know, however, that they are all busy looking after the sick and wounded and data will no doubt be forthcoming later. In all campaigns from the time of the Romans, in the wars of the middle ages and the Napoleonic wars these spas have served a most useful purpose. Dr. Fox reminds us that in France cold water dressings were strongly advocated in surgery by the great Ambroise Paré as long ago as 1553; and that they were much used by the surgeons of Napoleon.

\section{AMERICAN SPAS}

The question has never been raised, but it is a natural one; can American spas be in like manner differential and classified on a basis 
of military use? We trust the need for this may never arise but it is possible. The first requisite is an inventory of such resources, their situation, ownership, accommodations and special adaptability for medical or surgical affections. The United States Government owns only one spa, the Arkansas Hot Springs, where it maintains the Army and Navy hospital. The State of New York owns the principal Saratoga Springs. There are probably not over a score of other spas, worthy the name, under private ownership, some of them more or less identified with certain affections for the cure of which a reputation has been acquired. But the habit of selection of spas for special indications has not been developed in America in anything like the same degree as in Europe.

Beginning with the seashore resorts suitable for ocean bathing they would obviously be unpopular for civilians and probably unavailable in war time, especially in the upper Atlantic Coast and near the larger seaports. But it may be said that Deanville, Fronville and Berck Plage on the French coast and Brighton, Eastbourne and Isle of Wight resorts, together with Blackpool on the Irish Sea have their counterpart in Newport, Southampton, Coney Island, Long Branch and Atlantic City, Cape May and Old Point Comfort. The American seashore watering places, as a rule, have better sands and safer waters than the European resorts mentioned.

Balneologic and hydrotherapeutic facilities are more extensively provided, and during much longer seasons, at the interior spas only the more prominent of which can be here enumerated. It would be difficult to impartially characterize each one. In the case of hostilities involving any considerable number of troops the selection of convalescence camps or the use of mineral water resorts would be influenced almost entirely by military circumstances. Choice would probably be made near the coasts so that patients might be the more readily transported.

Arranged geographically we have:

In Maine, Poland Spring; a valuable depurative indifferent water for internal use and a new bathing establishment.

In New York, Saratoga Springs, I 75 miles from New York City; laxative saline waters, carbonated; bathing facilities as yet inadequate. Richfield Springs, Sharon Springs and Clifton Springs, well provided with bathing facilities. Glen Springs (Watkins), making a special feature of "Nauheim" baths for cardiac cases.

In Pennsylvania, Bedford Springs.

In Virginia, the Hot Springs and the Warm Springs. These resorts are provided with natural thermal waters used for baths at their natural temperature. The Hot Springs are not excelled in America in equipment and clientele. Gout, rheumatism and nervous diseases are chiefly treated. Cases of syphilis are not received. Elevation, 2400 feet. There is a good hydrotherapeutic establishment at "The Chamberlain," Fortress Monroe, on the Chesapeake 
Bay. In time of war this would be taken over by the Government as an adjunct to the military post close by.

In West Virginia, the White Sulphur Springs with a modern equipment as to bathing and accommodations. Like the Hot Springs it is open all the year.

In Michigan, Mount Clemens; a saline sulphurous water used only for baths. Numerous establishments.

In Indiana, French Lick Springs with a good reputation for baths and the internal use of the waters for purgative purposes. The waters are cold.

In Arkansas, the Hot Springs. These are thermal springs of of light mineralization, used chiefly for bathing. Visited by thousands annually for the relief of rheumatism, gout, syphilis and affections of the nervous system and skin.

In Colorado, Glenwood Springs; a lightly mineralized thermal water. An excellent bathing establishment.

In California, a very large number of spas, chief of which are Paso Robles, Arrowhead Hot Springs, Harbin Hot Springs, Nkaiah Springs, Klamath Hot Springs.

In Arizona, Castle Creek Hot Springs, very limited and not easily reached.

There are many other springs in the United States, with more or less local reputation but, as a rule, with primitive or inadequate equipment, often without much medical supervision, taking all cases that present themselves. As Dr. Fox says of English resorts, and it is probably true in America, there is a tendency to introduce all the methods of treatment belonging to other places. In the endeavor to create a universal provider there is, perhaps, a danger lest the original virtue of the place, perhaps the waters by which its reputation was first established, should be in the new development neglected and forgotten.

\section{BATHING IN TYPHOID FEVER}

The European war has proved to be no exception to other wars in the occurrence of typhoid fever, and its congeners paratyphoid $\mathrm{A}$ and B. The law on compulsory vaccination in the French Army came into effect October I, I9I4, and in troops so immunized the incidence of typhoid has been smaller than in those unvaccinated but nevertheless the disease has proved a serious feature of the campaign. About 1400 cases of typhoid occurred among the British troops in France and Belgium in the first eighteen months of the war. In the earlier months grave forms predominated and it has been shown that legitimate typhoid fever with Eberth's bacillus may, though rarely, be met in the vaccinated in which case it runs a mild course. Cardiac complications, due probably to the inevitable prostration and hardships of the trench warfare are common. Phlebitis is reported to be less frequent. Tuberculosis is reported 
to be a not uncommon sequel. Complications, therefore, may influence hydrologic treatment.

D. H. Grenet ${ }^{1}$ makes the unqualified statement that "cold baths constitute far and away the best treatment of typhoid fever" and this statement is interesting in view of the tendency now noted in American hospital practice to resort to sponges, sprinkling and other means in place of the Brand method generally followed ten or fifteen years ago. It is reported that Dr. Glenard in France maintains the superiority of the cold bath. However, in military practice this is not always possible and substitutes are used. Among these are cold packs. The bed is covered with oil cloth, the patient is wrapped in a sheet wet in cold water and over that is placed a blanket. The wet cold sheet is repeated every three or four hours, on each occasion for a period of five or ten minutes. We are not informed at what bodily temperature these measures are instituted. The lotion douche is also used. The head of the bed is elevated, or the patient is placed on a long table elevated at one end. Four thicknesses of blanket are placed on the table and over that an oil cloth leading to a receptacle on the floor. After placing the patient on the table thus prepared he is covered with a sheet, in which he is wrapped, the lower end of which hangs over the free edge of the table so as to drain into the receptacle. Cool water is then poured over him through the nose of an ordinary garden watering pot using 7 to ro gallons as each bath. Ice bags are sometimes applied to the abdomen, especially on first admission to the hospital. Sometimes both measures are applied, i.e., permanent application of ice to the abdomen and three tepid baths progressively cooled on each following day. Murphy's drop method is also employed in France with excellent results. They are using a solution of $50 \mathrm{gm}$. of glucose to the liter. Ordinary canesugar may be used if glucose is not available. The solution is placed in a douche can with suitable attachments of tubing and catheter and at an elevation sufficient to allow about 60 drops a minute so that the whole quantity is used in about three hours. Under the influence of this treatment the temperature falls, the tongue clears, the urine is increased and there is a rapid improvement in the general state.

The necessity for baths for troops in the field has produced the invention of the bath train now employed in the European war. One of these trains has two cars with 30 bath tubs each; two tank cars to supply the water; two locomotives to draw the train and supply the hot water; one car for undressing; two cars for dressing; four freight cars with clean linen, etc.; a sleeping car (wagon-lit) for the personnel of the train and two or three cars for the disinfection of the clothing. The arrangement permit I 200 men in the course of ten hours to take a shower bath and have all their clothing thoroughly sterilized.

${ }^{1}$ Le monde médical, December, x 9 I 5. 


\title{
CLIMATE AND CLIMATOTHERAPY
}

\author{
By Henry Sewall, M. D.
}

The medical concept of climate is made up of three groups of variables which may be specified as the physiographic, the social and the psychic.

The first group has to do solely with the physical factors of meteorology and their vital influence, and comprises the range of medical climatology usually so-called. Practical experience, however, has shown that climatic therapy must take account not only of disease but of the social and moral attributes of the individual patient. Therefore, the would-be philanthropy that transfers an indigent consumptive, for example, from a "bad" to a "good" climate, from the familiar scenes and tender care of home to a strange environment among people who are indifferent or even hostile and where the very necessities of life are lacking but for charity, such philanthropy is abortive of good and prolific of harm. Again, even when a patient is in affluent circumstances, nostalgia may overshadow the brightest foreign environment, one of whose chief therapeutic features must always depend upon the stimulation of a happy outlook and renewed interest in passing events. Without this social and psychologic standardization of the individual seeking advice, the most refined knowledge of the physiological relations of climate must be futile for successful therapy.

The analytic tendency of scientific medicine has put the presentday climatologist somewhat in the position of an apologist for his wares. An interesting paradox has developed within the scant generation during which time the open-air treatment of tuberculosis has been established on a rational basis. While realizing the salutary effect of removing certain invalids from a stuffy room to the fire escape, the roof or the backyard of a house, the medical mind has largely lost appreciation of the fact that a still wider change in environment must be expected, under favorable conditions, to operate even more powerfully in the desired direction. If the medical practitioner will but consent to consider the climatic problem as here defined he will then be in position to benefit in a very rich and fallow field of investigation, namely, the influence of actual climates on the human organism.

Medical climatology can only emerge from its epoch of empiricism into something like a science through an understanding of the relations existing between the vital functions and the various physical attributes of climate. Productive research in this area 
has been exceedingly meager. In only two directions has there been within the last decade a notéworthy advance in our knowledge of the physiologic influence of environment, namely, through studies pursued at low barometric pressures and in researches into the rationale of ventilation.

The important work of Zuntz $z^{1}$ and his colleagues, carried out in the Alps at an elevation of 14,960 feet has been amplified and extended by Haldane, Douglas, Henderson and Schneider on Pike's Peak, elevation I4, roo feet, in Colorado. ${ }^{2}$ The latter authors concluded that all modifications of vital activity in such situations are due solely to diminished oxygen pressure in the air. They hold that, while under ordinary conditions gas exchanges in the lungs are carried out under the physical law of diffusion, when the alveolar oxygen tension falls to a certain critical level the alveolar epithelium assumes the function of active oxygen secretion into the blood.

Nothing would seem easier than to settle the now ancient controversy as to the effect of high altitudes on hemopoiesis and the red blood count. Zuntz and his colleagues seemed to have proved that there is an active stimulation of the red bone marrow in animals kept at high elevation, with an increase in their hemoglobin of 20 to 30 per cent. Haldane and his associates came to essentially the same conclusions as to the effect of the lowered barometric pressure upon the blood. But Bürker ${ }^{3}$ and his colleagues, working at a moderate level, maintain that, although there is a true polycythemia under such conditions, the increase in the red blood count is much less than has usually been attributed to elevations up to say, to,000 feet. Finally, the work of Loevenhart and his associates seems to have effectually disposed of the objections of those who, like Abderhalden and others, have explained the apparent altitudepolycythemia as due to concentration of the blood resulting from excessive evaporation, or to abnormal vascular distribution of the corpuscles caused by altered barometric pressure. ${ }^{4}$

Loevenhart confined animals (rabbits) in ventilated chambers in which, while the total barometric pressure was kept constant, the partial pressure of oxygen was lowered at will to correspond to that which it assumes at various elevations above sea level. The blood changes under these conditions were found to be identical with those associated with low pressure at high elevations.

Recent studies on acidosis have emphasized the importance of the tension of carbon dioxid in the alveoli of the lungs as an index of the acid-alkali balance of the body. ${ }^{5}$

When the balance swings toward an acid reaction the respiratory center is stimulated to excessive ventilation through which volatile

${ }^{1}$ Zuntz, Loewy, Müller and Caspari, Höhenklima u. Bergwanderungen, Igo6.

2 Phil. Trans., Roy. Soc., I913, cciii, 185.

${ }^{3}$ Bürker, et al., Ztschr. f. Biol., I913, lxi, 379 .

${ }^{4}$ Loevenhart, Arch. Int. Med., I9I5, xv, I059; also Dallwig, Kolls and Loevenhart, Am. Jour. Physiol., I915, xxxix, 77.

${ }^{5} \mathrm{Cf}$. Peabody, Am. Jour. Med. Sci., igr6, cli, I84. 
$\mathrm{CO}_{2}$ is removed and the total acidity is kept within bounds. Conversely, the finding of a low alveolar $\mathrm{CO}_{2}$ tension signifies acidosis.

Many years ago Mosso, ${ }^{1}$ in his admirable studies on the high Alps, was led to explain "mountain sickness," and other disturbances associated with low barometric pressure, as a result of deficient tension of $\mathrm{CO}_{2}$ in the jucies of the body. For this condition of subnormal $\mathrm{CO}_{2}$ tension he coined the word "acapnia."

In a series of physiological researches Yandell Henderson ${ }^{2}$ has sought to establish the importance of acapnia in the production of pathologic phenomena, specifically surgical shock.

It would be imprudent at this date to draw a definite analogy between the conditions of alveolar $\mathrm{CO}_{2}$ tension as found in altitude acapnia and in clinical acidosis. The suggestion may not be farfetched, that in the estimation of the acid-alkali balance of the body we may possibly possess' a most useful objective and early criterion of the vital influence of climatic change.

Belief in the therapeutic and prophylactic virtues of the open air was founded, like that in vaccination for smallpox, upon empiricism. Trudeau's demonstration of the ruling influence of environment on the course of induced tuberculosis in rabbits served to establish the broad relations between vital resistance against disease and surrounding conditions. Aside from conditions of food and drink, we are brought by no agent so intimately in contact with our environment as by the air in which we live and breathe. The vital relations of the air may all be considered under that topic of practical hygiene known as ventilation.

In spite of all the research that has been devoted to the subject, it cannot be claimed that the rationale of ventilation has been completely disclosed. The old "chemical" view that the deleterious agent in rebreathed air was $\mathrm{CO}_{2}$ gave way to the conception that exhalations from the lungs contained poisonous organic effluvia which were accountable for the symptoms witnessed in people and animals occupying closed spaces. Then it was proved that the morbid sensations, and possibly the disturbances of metabolism as well, caused by breathing "close" air could be accounted for by the very simple explanation that the physical conditions under which air becomes "close" impair the normal radiation of heat from the body. These conditions are high temperature combined with excessive humidity in the surrounding air. When means are adopted to keep the rebreathed air cool and dry a person can remain in an unventilated space in comfort when the proportion of poliuting effluvia is very high. But it may well be urged against the conclusiveness of experiments which show that the feeling of well-being is strictly under control of the combined temperature and humidity of the air, that the environmental changes induced in them were excessive and too rapid to permit of physiological adjustment. That this

1 A. Mosso, Life of Man on the High Alps (Trans.), I 898 .

Yandell Henderson, Acapnia and Shock, Am. Jour. Physiol. 
criticism is well taken is clear from the results of the prolonged observations of the New York State Commission on Ventilation. ${ }^{1}$ This body. found that groups of people could carry on various sedentary occupations for three to six hours daily for periods of several weeks with no sense of discomfort or loss of physical or mental power under conditions of ventilation, temperature and humidity which, if rapidly induced, would have been intolerable. The only physiological signs of the untoward environment were a diminution of vital energy and of appetite for food. In a special research two members of the Commission were forced to conclude that, even with the correction of temperature and humidity, there is something about close air that militates against the physiological well-being. The present writer ${ }^{2}$ has pointed out that though it is clear that the sense of well-being is determined more or less completely by the physical factors, temperature, humidity and motion of the air, there has been no demonstration whatever that these are the essential agents in ventilation. The facts of anaphylaxis make it obvious that effluvia from the lungs or bodies of certain animals can be absorbed by and excite marked disturbances in other creatures which are appropriately sensitized. We are entitled to suspect, though we are not yet in a position to maintain, that the fundamental necessity for ventilation lies in a widespread tendency for rebreathed air to set up harmful vital reactions such as the immunologist is wont to demonstrate.

Certain people, a class especially if not altogether composed of those whose pulmonary apparatus has become "sensitized" to one or another volatile noxa, are liable to suffer symptoms of bronchial congestion when remaining in a crowded room. When we realize that practically everybody is probably more or less sensitized toward a wide range of antigens which, if they occur in the air breathed, must provoke more or less biologic response, it is obvious that no mere demonstration in chemistry or physics is adequate to solve the problem of ventilation; to show what constitutes physiologically pure air or how to get it.

1 Am. Jour. Pub. Health, rg 5 , v.

2 Interstate Med. Jour., I9I6, xxiii, 23. 


\section{ELECTROTHERAPY}

\section{By Charles S. Potts, M. D.}

Continual advances are being made in the study of electricity as used in medicine, both in the indications for its use and the methods of application. At the present time the following forms of current are employed. Constant or galvanic, induced or faradic, sinusoidal, static and high frequency. Advances in the use of the latter three have been especially marked. The passage of the electric current through the body causes changes in functions and tissues and to use it intelligently some knowledge of these varied physiological actions ${ }^{1}$ is essential. All of the forms of current above mentioned do not produce these changes in the same degree. Some are not caused at all by certain currents, while others may be excited in greater degree by some than others. In the space at command it will not be possible to do more than give an outline of these various phenomena, neither will it be possible to give in detail the many indications for and methods of application of the electrical current. ${ }^{2}$ A knowledge of the physiological actions will enable the reader to find for himself many indications.

\section{ELECTROPHYSIOLOGY}

The Constant Current.-The constant or galvanic current possesses all of these properties to a greater degree than any of the others and if only one form of current was available it should be the one to select. The methods of obtaining it are given in Vol. I, page 600 et seq. It may be here stated, however, that the obtaining of the current from the main (electric light or other circuit) is preferable whenever possible, and that many excellent types of apparatus are now made for this purpose.

The activity of this current is manifested principally at the poles (polar effects) but there are also changes taking place between them (interpolar effects).

The former consist of the production of electrolysis, phoresis, and electrotonus. By its electrolytic action the tissues of the body, composed principally of salines in watery solution, in the polar regions are decomposed into their ultimate elements or ions. Those which are electropositive or repelled by the positive pole or anode are known

1 This term as used in this article comprises not only pure physiological effects as electrotonus but also its electrolytic, phoretic, heating and mechanical properties.

2 Only medical indications are considered in this article. Surgical indications are also many and increasing.

Vol. IV. -7 
as cations and appear at the negative pole or cathode. Those which are electronegative or repelled by the negative pole are known as anions and appear at the positive pole. Of the former the principal ones are hydrogen, alkalies and the bases, of the latter they are oxygen, acids, and substances taking the place of acids as iodine, chlorine, etc. Movement of the ions from the anode to the cathode is known as cataphoresis, from the cathode to the anode as anaphoresis. This will take place through porous substances and membranes and is known as electric osmosis. The general term for the phenomena is phoresis. If the current is strong enough caustic and destructive effects are manifested at each pole. At the anode, the acids thus generated sear the tissues and may be utilized to stop hemorrhage, a firm clot being produced. At the cathode not so much irritation is caused by the alkalies but considerable destruction of tissue is caused. The blood being driven from the anode to the cathode, there will be more or less depletion at the former and hyperemia at the latter. Currents of ordinary strength do not cause these changes to a visible degree but they are constantly taking place both at the poles and in the region between them. There is also a constant flow back and forth between the poles. Of much importance are the electrotonic effects. For practical purposes it may be stated that these consist of a lessening of the excitability and conductivity of a nerve at the anode and an increase of these functions at the cathode. The former is called anelectrotomus and the latter catelectrotonus. In other words the anodal action is sedative and the cathodal stimulating.

The interpolar effects are similar but not so strong; consequently as the current passes through the body there is a constant electrolytic decomposition of the tissues, the cations going toward the negative pole and the anions toward the positive. This interchange causes metabolic and vascular changes which facilitate the absorption of morbid exudates and adhesions which may exist in the interpolar region. The polar actions may be summarized as follows:

Cathode or negative:

I. Physiological: stimulating (catelectrotonus).

2. Physiochemical: accumulation of the positively charged ions of hydrogen, sodium, potassium, calcium and ammonium (cations). Formation of an alkaline caustic, i.e., the hydrates of sodium, potassium, calcium and ammonium. Production of a soft, retractile eschar.

Cataphoric influence causing the accumulation of fluid and basic ions at the pole. With mild currents nutrition promoted; with strong currents denutritive or destructive effect.

Anaphoric influence by which the electronegative ions as sulphur, chlorine, iodine, etc., may be made to pass through the skin and be diffused through the body (osmosis). First effect of current, ischemia; second, hyperemia; ultimate effect, equalization of circulatory conditions. 
Anode or positive:

I. Physiological: sedative (anelectrotonus).

2. Physiochemical: accumulation at the pole of negatively charged ions of oxygen and chlorine. Formation of an acid caustic, i.e., sulphuric, hydrochloric, nitric and phosphoric acids.

Cataphoric influence causing loss of hydrogen and basic ions; loss of fluid; coagulation (of blood). Starvation of tissues. Production of a dry, hard, and non-retractile eschar. Diffusion through the tissues of ions of oxidizable metal electrodes or of ionized solutions. All salts in solution may be diffused from the anode. Antiseptic: first effect of current ischemia; second, hyperemia, but much less marked than at the negative pole. ${ }^{1}$

The interpolar effects are:

r. Electrolysis.

2. Transference of fluids from the positive to the negative pole (cataphoresis). It must be remembered that the physiochemical actions are only marked when strong currents are employed.

A valuable function of the constant current is its power to produce muscular contractions. These are produced only when there is a sudden increase or decrease in the density of the current, as when the circuit is either suddenly broken or closed. In other words a constant current flowing through a muscle or nerve without interruption will cause muscular contraction but if it is suddenly broken a contraction results. In this connection it is important to remember that the weakest current which will cause a contraction in a normal muscle is when the cathode is used as the exciting electrode, if the current is increased in strength, contraction will be obtained when the circuit is either opened or closed with the anode as the exciting electrode, i.e., over the supplying nerve or motor point of the muscle being treated or tested. The relative strength of current required to cause contraction in a normal muscle may be indicated as follows, the amount required increasing from left to right:

$$
\mathrm{CaClc}, \mathrm{AnClc}, \mathrm{AnOc}, \mathrm{CaO} c \text {, }
$$

$\mathrm{Ca}=$ cathode $\mathrm{An}=$ anode; $\mathrm{Cl}=$ closure; $O=$ opening; $C=$ contraction (see Vol I, page 6ro et seq.).

\section{FARADIC OR INDUCED CURRENT}

The faradic current being one in which there are rapid alternations and a momentary duration of current, polar and interpolar effects as caused by the constant current are absent (see Vol. I, page 604). There is, however, an extremely sudden increase and decrease in current density (page 104) and hence it causes muscular contractions, which if the current is interrupted very rapidly are tetanic in character. It does not cause contractions in muscles that are paralyzed from a lesion in the peripheral motor neuron (motor

${ }^{1}$ Modified from Cleaves, Jour. Advanced Therap., Dec., r908, p. 645. 
cranial nerve nuclei, anterior horns of gray matter of the spinal cord, peripheral nerves), if the lesion is severe enough to cause reactions of degeneration (Vol. I, page 6 $\mathrm{I}_{3}$ ). When rapidly interrupted and an electrode with five points, as a wire brush, is used, it stimulates nerves of common sensibility (touch, pain). This, if long continued or a larger electrode is employed, will eventually act as a sedative and may relieve pain and muscle spasm.

\section{THE SINUSOIDAL CURRENT}

The sinusoidal current is an alternating current derived from an alternating-current dynamo, an apparatus which is now made by most makers of electrotherapeutic appliances. The sinusoidal current differs from the faradic in that in the former the increase in the current from zero to maximum and back to zero again, first in one direction and then in the other, is gradual. A tracing of such a current forms a sine curve as shown in Fig. 4. From $a$ to $m$ represents

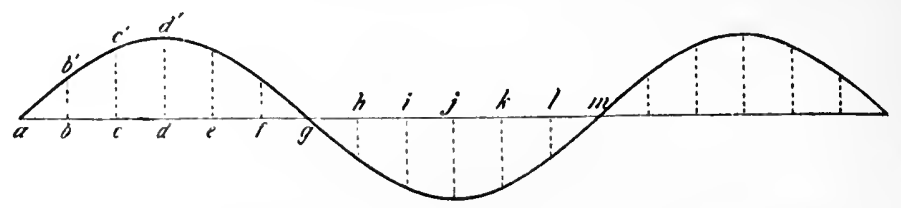

Fig. 4.-Cycle of a sinusoidal current or e.m.f. (Potts" "Electricity.")

one complete alternation or cycle. In the faradic current the increase from zero to maximum is very sudden and the return to zero almos equally so. The actual duration of the e.m.f. (Vol. I, page 602) is but momentary at each make and break of the circuit in the primary coil. A tracing of this current would appear as Fig. 5. Owing to the alternating character of the sinusoidal current, its polar effects are slight. It is, however, an efficient contractor of muscles

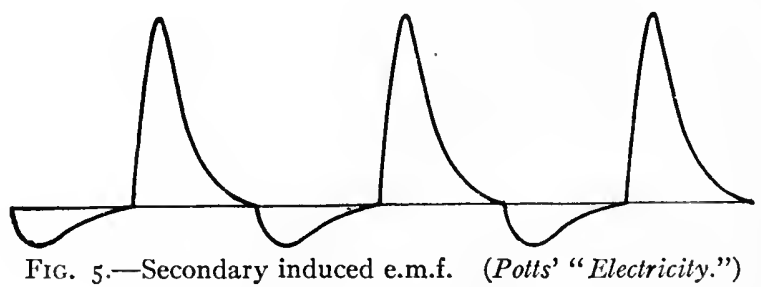

and this is especially so of involuntary muscle as in the stomach and intestines. It is practically painless and will cause muscles to contract which are paralyzed from a lesion of the peripheral motor neuron (page ro3). To cause clonic muscular contractions the alternations must be slow (20-60 per minute, 28 being the preferable number). If rapid alternations as 2000 or more per minute are used, clonic contractions cease and a tetanic one occurs. Such action 
in the case of muscles in a state of pathological spasm will eventually act as a sedative. It acts similarly in sensory nerves and relieves pain.

\section{STATIC CURRENT}

The static current (see Vol. I, page 605) is one of great e.m.f. or voltage and of small amperage (about 5 milliamperes from a powerful machine). It is unidirectional and hence has polar action, not however to anything like the same extent as the constant current. Electrolytic and phoretic action is practically absent, but the negative pole stimulates and the positive is sedative. The action of the current differs according to the method of using it (see page 106). Sparks cause muscular and tissue contraction, by which when local stasis or congestion is present, the fluid contents of the tissues are forced through the blood-vessels and lymphatics. Pain is thus relieved and exudation removed. ${ }^{1}$ The spark from the positive pole is less painful than that from the negative. Brush discharges cause dilatation of the capillaries and a local leukocytosis. The spray or breeze, especially if positive, the insulation being negative, is soothing and relieves pain. The static bath has some tonic effect and it is claimed acts more or less upon metabolism; and the excretion of urea, the pulse rate and, respiratory combustion being increased by its use. The static wave current has marked contractile power, thus relieving local congestion and pain. It also increases metabolism.

\section{CURRENTS OF HIGH FREQUENCY}

Currents of high frequency are produced by discharging one or more condensers through a coil of wire (solenoid). The condensers are charged by either a static machine, induction coil or stepup transformer. The frequency of alternations or oscillations is extremely great. Those of the induction coil may amount to a few hundred per second, these may amount to millions. They are known as the D'Arsonval, resonator and Tesla currents according to the method by which they are obtained. Dia-

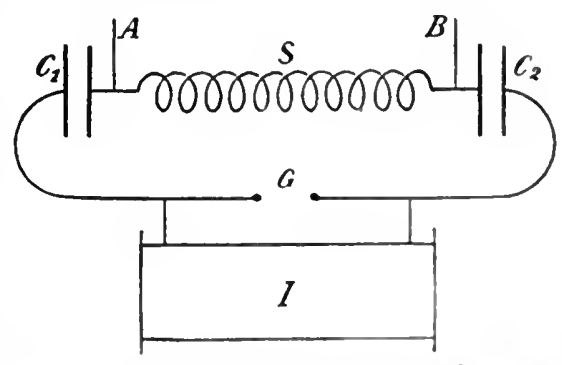

FIG. 6.-D'Arsonval oscillator with two condensers. (Polls" "Electricily.") grammatically the D'Arsonval apparatus may be represented as in Fig. 6, where $I$ represents an induction coil, $C^{\prime}$ and $C^{2}$ the condensers, $S$ the solenoid consisting of a few turns of heavy wire, $G$, the spark gap and $A$ and $B$ the points where the electrodes are attached. When the condensers which may be either Leyden jars or other form

1 Visible muscular contractions are not caused as may be with the constant, faradic or sinusoidal current, unless either a very powerful spark or the static induced current is used. 
of condenser are charged, they discharge through the solenoid and across the spark gap, producing oscillations of great frequency.

The resonator depends upon the principle of resonance. A coil of wire may be tuned to act in unison with the oscillations produced in another coil and thus increase the effect, just as a tuning fork vibrating over a column of air which is of such a length that its free vibrations are of the same frequency as those of the fork, will set into vibration the column of air and thus increase the sound.

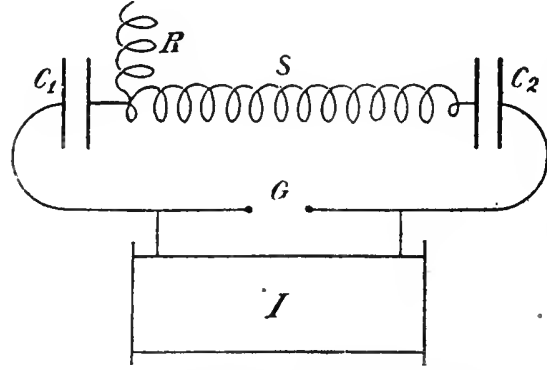

FIG. 7.-D'Arsonval ošcillator with resonator. (Potts" "Electricity.")

Diagrammatically the apparatus is represented in Fig. 7, where $R$ represents a coil of wire attached to the solenoid of the apparatus shown in Fig. 6. When properly tuned an electrode which ends in a joint attached to the end of $R$ will give off a powerful effluve or discharge manifested as an arc of purplish light, and fine sparks radiating from the end of the electrode for a distance of from 2 to I 2 inches. If brought too close to a conductor (a patient) a strong spark will pass.

The Tesla current is derived from a secondary coil shown as $S^{\prime}$ in Fig. 8 which is placed inside the primary solenoid $S$ and consists of five or six times as many turns as the primary, and is composed of finer wire. The electrodes are connected with the secondary coil. The effects are similar to those obtained from the resonator. The most valuable effects of these currents are the production of hyperemia and heat. In these respects they excell all other forms of the electrical current and are probably the most valuable agents we have for these purposes. The heat differs from that obtained by other artificial methods, in that it occurs from within and is very slowly dissipated. All the tissues between

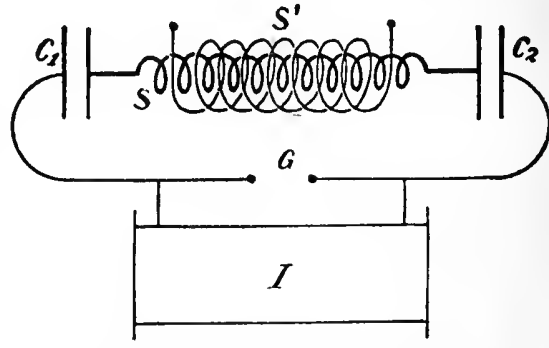

FIG. 8.-Connections of. Tesla transformer. (Potts' "Electricity.")

the two poles are heated; therefore deep structures can be influenced and the effect remains for some time. This property has been termed diathermia or thermopenetration. In addition the secretion of sweat, and the quantity of solids in the urine are increased, the blood-pressure temporarily decreased, hyperemia is produced at the location of the electrodes which causes a relief of deep venous congestions. After the action of the current has subsided freshly oxygenated arterial blood enters into the previously anemic and 
(before the treatment) venous congested areas. It will readily be seen that such action increases phagocytosis and promotes metabolism.

Sparks can also be obtained which exert a similar influence to the static spark but are not so efficient. The eflluve and vacuum electrode applications are sedative and may be used to relieve pain.

In summing up it may be said the constant current is most useful for exercising paralyzed muscles, if the paralysis is due to disease of the peripheral motor neuron, for relieving the pain of a functional neuralgia if the affected nerve is near the surface, and for its electrolytic effects as when it is desired to clot the blood in an aneurysm, or to remove exudation as in an ankylosed joint. For the latter purposes it is not so efficacious as the high frequency and static currents.

The faradic current is useful to exercise paralyzed muscles, due either to lesion of the central motor neuron or of the peripheral motor neuron if they will respond to it. It may also be employed to relieve pain and sensory paralysis of the skin. The faradic current is rather painful and if obtainable the sinusoidal current is preferable. This current also is most advantageous when it is desired to produce contractions of involuntary muscle.

The static current is most valuable when a vibratory effect is desired to relieve local congestion and exudation. This is best caused by the static induced current. High-frequency currents are efficacious in relieving local inflammation, pain, and exudation and are most useful to increase metabolism.

In addition, it must not be forgotten that electricity has a valuable psychic influence which is most useful in neuroses such as hysteria; for this purpose either the static or high-frequency currents should be the first choice. If these are not at hand the faradic may be of service.

\section{METHODDS OF APPLICATION}

Constant, Faradic and Sinusoidal Currents.-In Vol. I, pages 606-608, are mentioned the essential appliances needed to administer the constant and faradic currents. It may be here mentioned that for office work a current derived from an electric light circuit is preferable whenever possible. If this current is alternating in type, a converter must be used to convert it into a direct current. A number of appliances are now on the market to supply the sinusoidal current. When using either the constant, faradic or sinusoidal currents certain general rules should be observed (see also Vol. I, pages 607,608$)$. The electrodes must be covered and moistened except when it is desired to treat the skin. The best covering is absorbent cotton which should be changed for each patient. Before beginning the treatment see that all switches are turned to zero, then increase the current strength very gradually 
until the required strength is reached, after the treatment is finished turn all switches to zero. In the case of children and nervous people it may be well for the first few treatments to use a weaker current

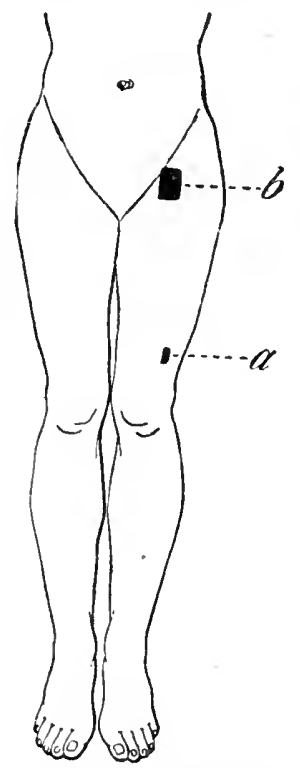

FIG. 9.-Diagram showing position of electrodes for causing contraction of a muscle. The active electrode should be negative. $a$, Active small electrode over motor point of vastus externus muscle; $b$, indifferent large electrode over anterior crural nerve. (Potts' "Electricity.") than is required until their confidence is secured.

Do not place the electrodes over bones that are thinly covered by soft tissues, unless necessary, as it is painful. Do not use very strong currents, if interrupted, on the head or face.

As the current passes the resistance of the skin becomes less, owing to the producing of hyperemia at the electrodes, hence the current strength will become greater and may have to be reduced.

When muscular contractions are being caused, it must be remembered that too long and too strong applications may do harm. This is especially so in muscles that are much atrophied, when such treatment instead of stimulating the muscle will exhaust it. The current should only be of sufficient strength to cause contraction and about a dozen contractions for each muscle will be sufficient. If very strong currents are required to cause contractions it may be well to use the constant current without interruption, placing the cathode over the affected muscles. As a rule three treatments a week are sufficient.

Treatment is contraindicated as a rule in those with fever, those greatly debilitated from some exhausting disease, very old people and menstruating and pregnant women.

\section{When using} the constant current, if stimulation is desired the negative pole or cathode should be the active one; if a sedative effect the positive pole or anode must be used. The question of polarity is not important when employing either the sinusoidal or faradic currents; the rapidity, however, of the alternation is. To cause muscular contractions they should be slow, to

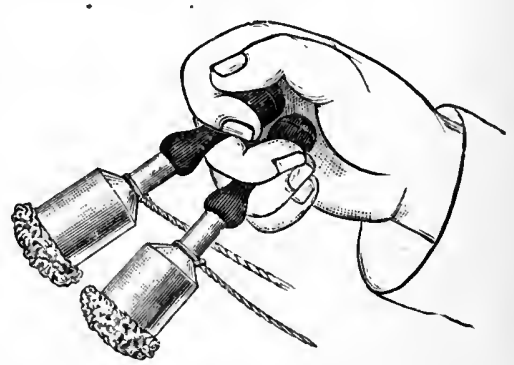

FIG. I0.-Method of holding electrodes in one hand. (Mills.) produce sedative effects rapidity is desired. When treating paralyzed muscles, the indifferent electrode, a fairly large one, may be placed either at some indifferent point, or when possible over the nerve supplying the affected muscle and the active electrode, a small one, over the 
motor point of the muscle, Fig. 9. If using either the faradic or sinusoidal currents both electrodes may be held in one hand as shown in Fig. ro and placed over the muscle being treated. When it is

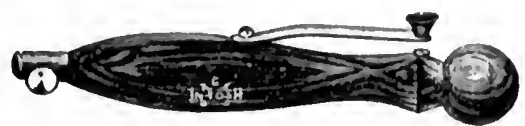

FIG. II.-Universal handle with interrupter.

desired to interrupt the constant current, it must be done by the operator opening and closing the circuit by either reversing the commutator (Vol. I, page 607), using an electrode with a switch in the

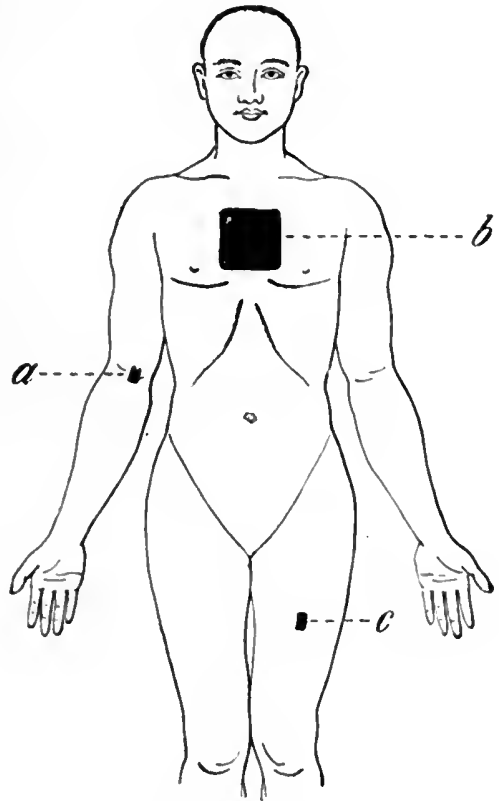

FIG. I2.

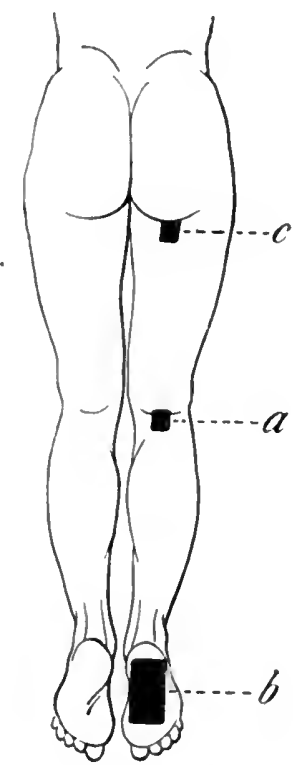

FIG. I3.

Fig. 12.-Proper position of the electrodes to obtain polar action in muscle or nerve. If stimulation is desired, the active electrode should be the kathode; if sedation, the anode; $a$, small active electrode over the biceps muscle; $b$, large indifferent electrode over the sternum; $c$, small active electrode over the median nerve. (Polts" "Elcctricily.")

FIG. 1 3.- Showing the position of the electrodes in passing a current down a nerve, in this case the sciatic. The anode is placed above or below according as it is desired to have a descending or ascending current. The upper electrode should be placed in the lumbosacral region. a, Electrode in popliteal space, or, if it is desired to influence the branches, it may be placed on the sole of the foot; $b$, large plate electrode on sole of foot; $c$, electrode over sciatic nerve. (Polls" "Electricity.")

handle, Fig. I I, or by alternately placing it on and removing it from the muscle. This may be done with a brushing motion and is useful when a group of muscles is to be treated.

The faradic and sinusoidal currents are interrupted automatic- 
ally. If there is no means of doing this slowly it may be done by placing the electrode on and taking it off the muscleas described above when using the constant current. When it is desired to obtain the polar action of the constant current, as in utilizing the sedative action of the anode in treating neuralgia, the indifferent electrode must be some distance from the active one. The relative positions and size of the electrodes are shown in Fig. I2. If it is desired to pass the current up or down the course of a nerve the electrodes are placed as in Fig. I3. If it is desired to influence organs that are deeply situated as the stomach or spinal cord, large electrodes and a strong current are required. The electrodes should be so placed that the organ is between them. It may be stated as a general rule that whenever very strong currents are being used, large thickly covered electrodes must be placed over the skin. When it is desired to concentrate the current upon a small superficial area, the active electrode must be small.

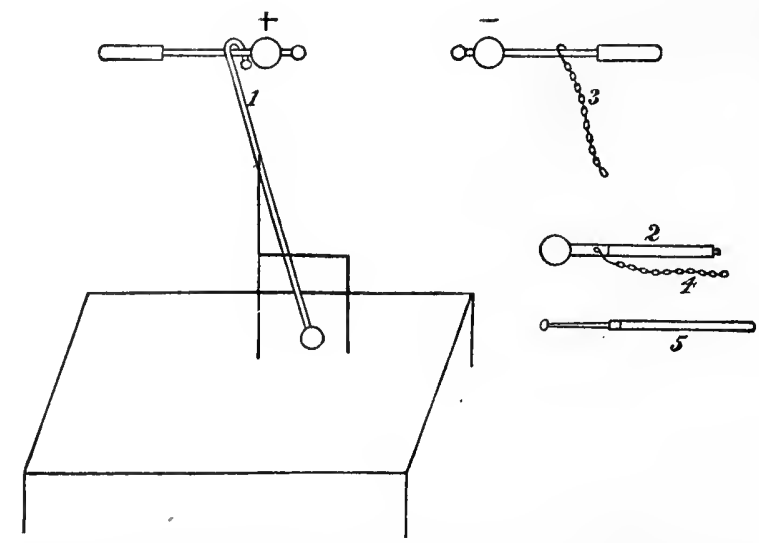

FIG. 14.-1. Shepherd's crook; 2, ball electrode; 3, ground chain to water pipe; 4, ground chain to gas pipe; 5 , spark director held in operator's hand. (Snow.)

Static Current.-Moisture or dampness interferes with the action of the Static machine. It is, therefore, important to keep it in a dry, sunny room and to keep an agent that will absorb moisture, as quicklime, within the case.

The precautions mentioned on page Io4 regarding using weak applications for the first few treatments are equally applicable when using this current. When employing the breeze, metal pins and buttons must be removed or sparks may result. When it is applied to the head, celluloid combs and pins also must be removed or they may catch fire. If the patient feels weary and sleepy after the treatment it has been either too strong or too prolonged.

Sparks applied to bony prominences are very painful. Strong sparks should not be applied to the head, or face, and none should be applied to the breast, nails or genitalia. When constitutional effects 
are desired the treatment should last from fifteen to thirty minutes.

By the aid of the following condensed directions and diagrams of Dr. Arnold Snow ${ }^{1}$ the technique is made plain:

To give sparks:

I. Seat or stand the patient on the insulated platform so that he is opposite the prime conductor of the positive pole $(+$ in diagram). Connect either the shepherd's crook or a chain with the positive pole of the machine and place it on the platform. 'The nearer it is to the patient's feet the stronger the effect. 'The ground chain (3) should connect the negative pole with earth by a metallic conductor (gas or water pipe). A ground chain (4) should also connect the ball electrode with the earth in a similar way, and must be held by the operator with a chain holder alongside the electrode so that it will not touch the patient, Fig. I4. Ordinarily

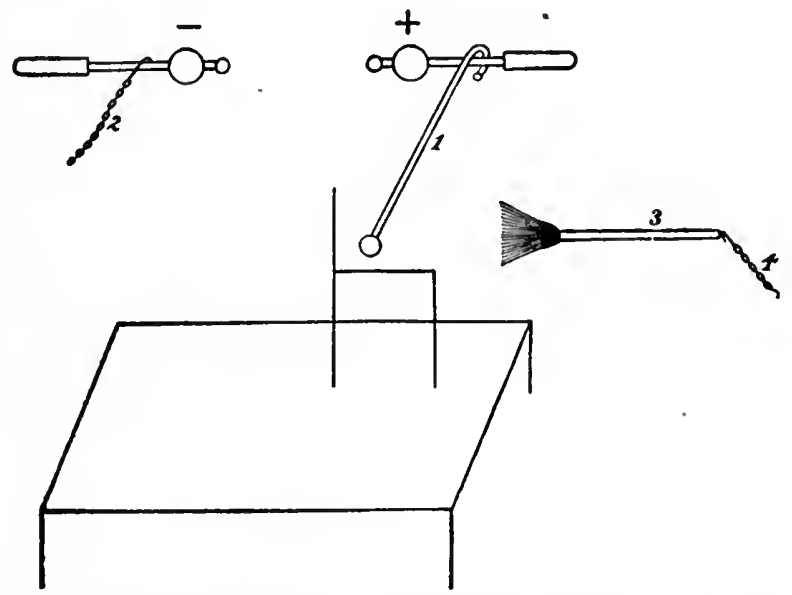

Fig. 15.-1, Shepherd's crook held by patient; 2, ground chain to water pipe; $\dot{3}$, wire brush electrode; 4, ground chain to gas pipe. (Snow.)

the discharging rods are kept apart, if it is desired to weaken the discharge, they may be brought together so that a spark passes between them or the operator may place his foot on the insulated table. The ball electrode is brought toward the area being treated until a spark passes, then quickly withdrawn, this being repeated five or six times.

To administer the spray:

I. The poles of the machine are widely separated.

2. The patient on the insulated stool holds the shepherd's crook on a chain connecting him with either side of the machine, preferably the positive. The other side is grounded.

3. The operator then administers the breeze by a to-and-fro motion of either a brush or point electrode. It must not be brought too close to the patient or a spark may be discharged. (Fig. 15.)

1 Jour. Advanced Therap., Aug., 1906, p. 380. 
To administer the breeze:

I. The patient is seated on the insulated stool and the poles of the machine widely separated.

2. The shepherd's crook on a chain extends from the positive pole either directly to the patient or to the insulated platform. The other pole is grounded.

3. The stand is placed with a point electrode near the part to be treated, or a crown electrode is placed over the head.

4. A stronger effect may be obtained by connecting the electrode directly with the negative pole, Fig. I6.

To obtain the brush discharge:

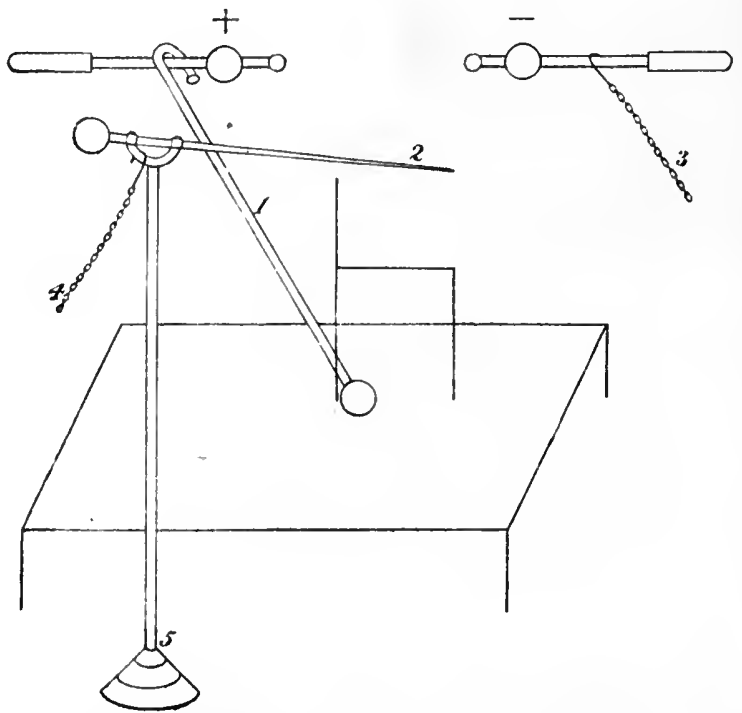

FIG. 16.-I, Shepherd's crook; 2, point above patient's head; 3, ground chain to water pipe; 4, ground chain to gas pipe; 5 , stand for holding point or crown electrode. (Snow.)

The patient is thoroughly insulated and the platform connected with the negative side of the machine if a sedative effect is desired and with the positive, if a stimulating. The opposite side is grounded.

The discharging rods are kept wide apart and the speed of the machine increased until the desired result is obtained. The electrode, made of wood capable of absorbing moisture, is grounded to a separate one from that of the machine. It must be kept about I 5 inches from the patient. The resulting effect is a more or less spluttering discharge of violet color rich in ultraviolet, violet and blue-violet rays and ozone. It is not similar to the brush discharge from the terminal of a resonator or Tesla coil, Fig. I 7. The machine must be gradually stopped before removing the electrode from the patient, and all celluloid and metal pins, combs and buttons must be removed. The discharge from the negative side is usually 
employed and is said to be efficacious in the treatment of sprains, neuritis, neuralgia and neurasthenia.

To obtain the static wave current:

I. Ground one pole of the machine to a gas or water pipe. It makes no difference which.

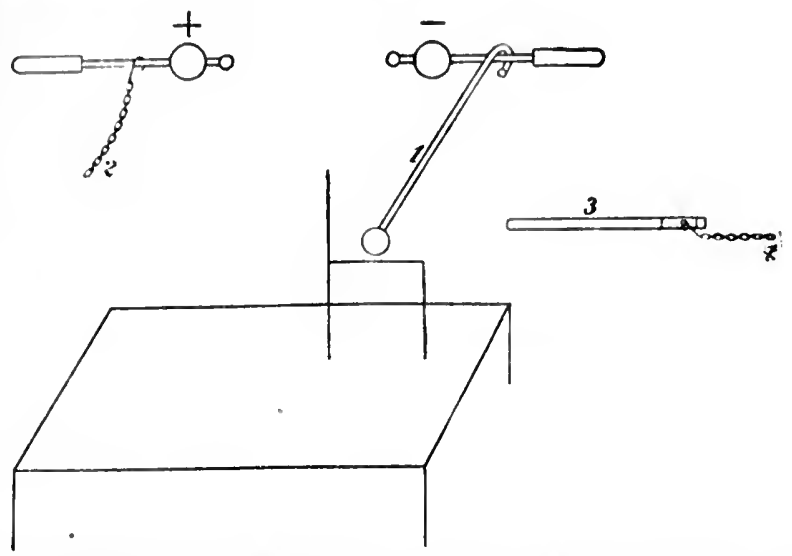

Fig. 17.-1, Shepherd's crook held by patient; 2, ground chain to water pipe; 3 , wooden electrode with metal sleeve, 4 , ground chain to gas pipe. (Snow.)

2. Place the patient on an insulated platform.

3. Place an electrode made of lead or block tin, over the part being treated and connect it with the other side of the machine.

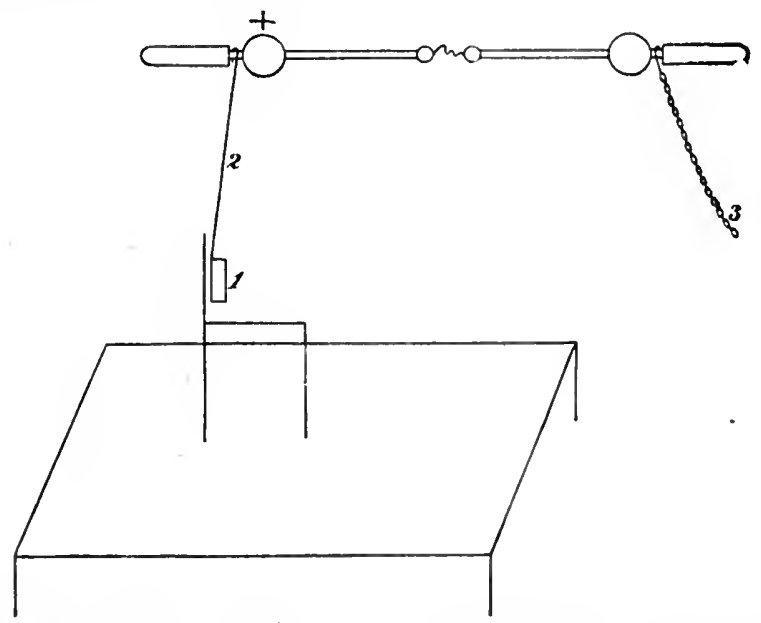

Fig. 18.-1, Metal electrode on patient; 2, connecting wire; 3, ground chain to water pipe. (Snow.)

4. Close the discharging rods and start the machine. Then gradually separate them until discomfort is felt, either as a burning sensation or muscular contraction, Fig. I 8 . 
Nothing must be between the electrode and skin of the patient. Several electrodes placed in different machines if desired can be connected with the same side of the machine. To obtain a general time influence a long narrow electrode is placed along the spine. When local effects are desired the electrode, shaped according to the necessities of the case, is placed over the part. To obtain the static bath or static insulation, sometimes employed for either general tonic or sedative effects:

I. The patient is placed upon the insulated platform which is connected with one side of the machine, negative if sedation, positive if stimulation is desired.

2. Ground the side not connected with the patient, open the discharging rods and start the machine, Fig. I9. The static breeze may be combined with this. The static machine may be used to charge a resonator or Tesla coil (page I02). Current of very high

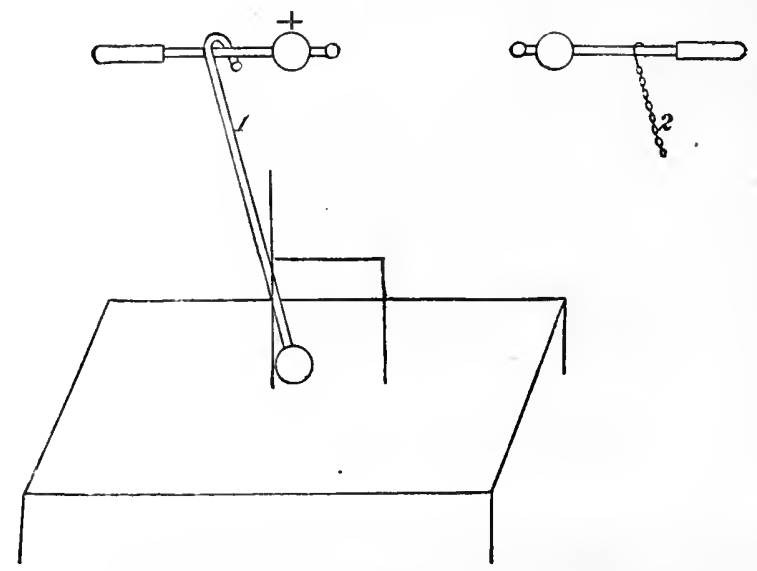

FIG. I9.-r, Shepherd's crook; 2, ground chain to water pipe. (Snow.)

potential may be so obtained which if slowly interrupted cause marked muscular contractions and hyperemia. If very rapidly interrupted muscular contractions will cease. The patient must be on the insulated platform and one side of the machine grounded. A metal, glass vacuum or effluve electrode, Figs. 20, 21, may be used. If the effluve is given it must be taken from the side of the Tesla coil opposite the positive pole of the static machine. If desired, stronger effects may be obtained by connecting the patient to the machine with a metal plate placed over the back, feet or abdomen.

High-frequency Currents.-The general precautions as regards strength of current, etc., given on page ro4, also maintain when these currents are used. The conducting cords must not come into contact with the patient as a painful spark will probably result. Such currents will pass through glass, and the effect is increased if 
made to pass through some woolen material, either the clotning or if a vacuum electrode is being used, a piece of woolen stuff wrapped about it. High-frequency sparks will cause burns. They do not require a complete circuit for their transmission but may be given off from one pole.

The contraindications for their use are low arterial tension, degenerated heart muscle, acute inflammation of any of the viscera,
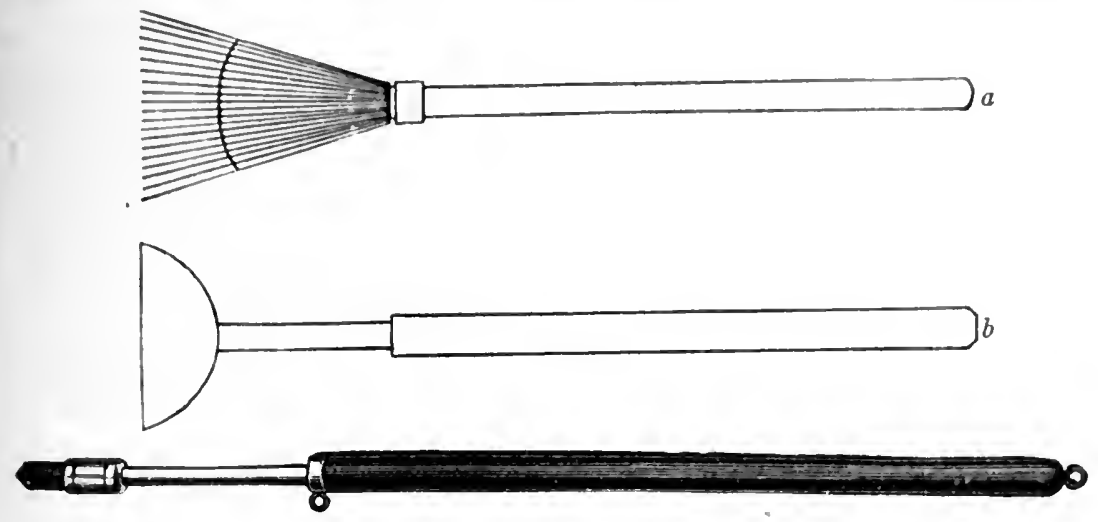

$c$

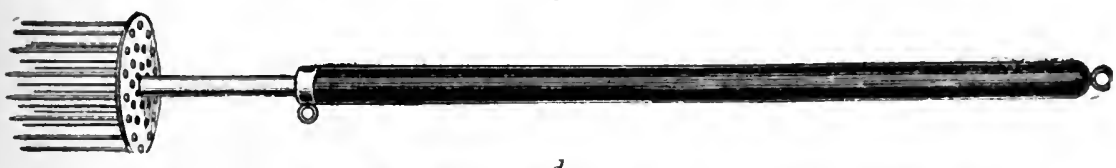

$d$

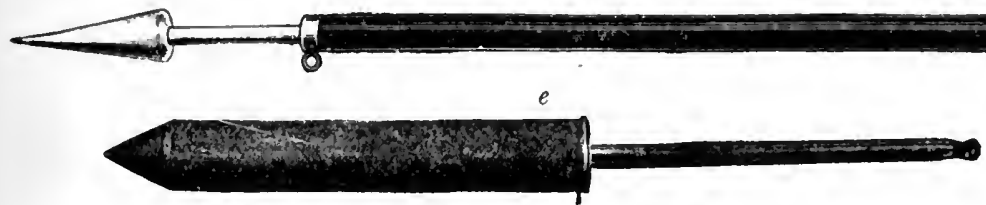

$f$

FIG. 20.-Effluve electrodes. $a$, Wire brush electrode; $b$, bell electrode; $c$, carbonpoint electrode; $d$, multiple-point clectrode; $e$, single brass-point electrode; $f$, resonator electrode. This is a form of electrode designed to be attached to the Tesla coil terminals or to the Oudin resonator by a cord, just as are the regular vacuum electrodes. It gives a fine, long, soft effluve and is used to apply the effluve or the spark. 'The latter is readily obtained by holding the electrode somewhat closer to the patient than when administering the effluve. (Potts' "Elcctricity.")

infectious fevers and advanced tuberculosis (they are used in the treatment of the incipient stage).

To obtain currents of comparatively low voltage and high amperage, such as are employed for autocondensation and diathermy, the D'Arsonval apparatus is preferable. The resonator and secondary coil of the Tesla apparatus give currents of higher voltage and less amperage. They are used to obtain unipolar currents such as the effluve or spray discharge when glass vacuum electrodes are employed. If a static machine is used to charge, the contractile 
effect is very great (page ro2). A twenty-plate Holtz machine should be employed with large (5 gallons) Leyden jars (see Journal of Advanced Therapeutics, April, I9r5, page 179). The higher the frequency the less the discomfort of the patient as the muscular contractions are less or absent. The current strength is regulated by increasing or decreasing the strength of the exciting current, by increasing or decreasing the length of the spark gap and in the case of a resonator, putting it more or less out of tune.

The effluve or spray discharge is obtained by employing an electrode similar to those shown in Fig. 20 , attached by a cord to the terminal of a resonator or one terminal of the secondary of a Tesla coil. The voltage should be high enough to get the effect at least 8 inches from the patient. If moved near enough a spark will result,

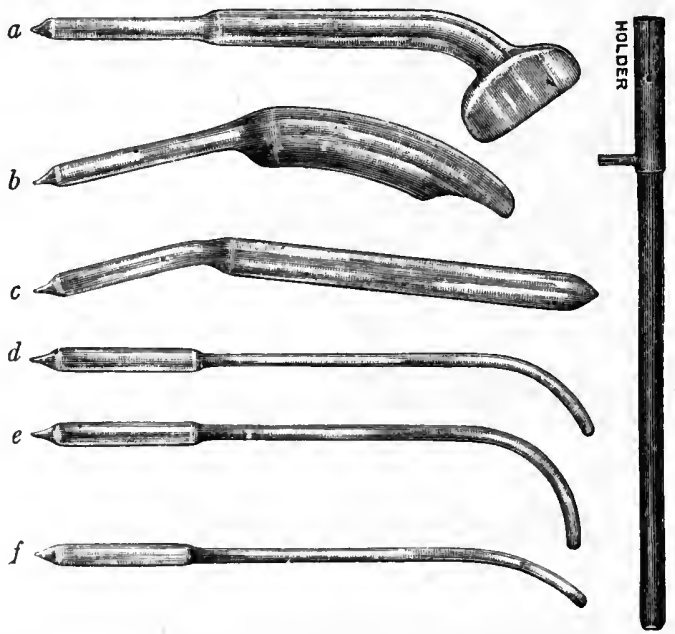

FIG. 21.-Vacuum electrodes. $a$, Surface; $b$, uterine; $c$, rectal; $d$, throat; $e$, urethral; $f$, nasal. (Potts' "Electricity.")

and they can be used for that purpose if desired. Local sedative and analgesic effects may be obtained by using the effluve.

To obtain local hyperemia and heat and consequent sedation glass vacuum electrodes may be employed, Fig. $2 \mathrm{I}$. The current passing through them makes a different color according to the intensity of the vacuum; the lower the vacuum the darker the color. In those usually used it is violet or purplish, which has led many to call the current so employed the "violet rays." If the electrode is held in close contact with the patient only a feeling of heat is experienced. If moved away from the surface a multitude of fine sparks result which are more or less painful. Metal ball or point electrodes may be attached to one terminal of a Tesla coil and sparks obtained.

General metabolic effects and reduction of blood-pressure are obtained by the methods of autocondensation and diathermy. 
Autocondensation is obtained by laying the patient on a couch, on which is a sheet of metal covered by some insulating material as rubber or a compressed fiber. ${ }^{1}$ In the hands are held metal electrodes connected with one end of the coil and the plate is connected with the other. This treatment may also be administered while sitting in a chair and appliances are made for the purpose. From 500-1000 milliamperes or more may be given to a patient by this method, the length of treatment being from ten to twenty minutes.

In employing diathermy electrodes made of block tin are used, which may be cut into any shape or size desired, one being connected with each terminal of the solenoid. The sharp corners and edges must be either turned up or bound with adhesive plaster to prevent sparking, which may cause disagreeable burns. The surface of the skin under the electrode may be dampened with water. The electrodes must be far enough apart to prevent sparking between them, and may be held in place either by a bandage or adhesive strips. They must be in close contact with the skin. A size of 3 by 5 inches for each rooo milliamperes of current employed gives the best results. If the electrodes used are smaller, the current strength may be proportionally less. Too high a degree of heat will damage cells which is not the desire in medical therapeutics although it may be in surgical. To obtain general metabolic effects electrodes may be placed on each arm and each leg,' and the electrodes must be large, and those of each side connected with the same side of the coil. For local effects one should be placed so far as possible on each side of the place that it is desired to treat. This should be done with great caution in the case of the thoracic or abdominal viscera for if ulceration (as gastric ulcer) should exist hemorrhage might be caused. If general heating is being obtained it is important that the patient sweat; otherwise too great a strain may be placed upon the kidneys. It must also be used with caution in advanced arteriosclerosis where there are either marked calcareous deposits in or thinning of the vessel walls as the hyperemia caused may lead to rupture and hemorrhage. ${ }^{2}$

\section{PHORESIS}

The property of phoresis, page 98 , may be used to cause drugs to pass through the skin and influence the structures beneath. In doing this attention must be paid to whether the substance is an anion and diffused from the cathode (anaphoresis). or whether it is a cation and diffused from the anode (cataphoresis). Hence if we wish to introduce the salt of an alkaloid as hydrochlorid of cocain it should be placed on the anode, as the active base (cocain) will go toward the cathode and the acid will remain at the anode. On the

1 Fiber electrodes are made that can be placed in an ordinary chair and a metal electrode held in the hand.

${ }^{2}$ See Jour. Advanced Therap., Sept., r913, p. $35^{6}$ and Am. Jour. Electrotherap. and Radiol., Jan., 1916, p. 5 and Feb., 1916, p. 79.

Vol. IV. -8 
other hand, if we wished to introduce arsenic, in the form of arsenite of potassium, it should be placed on the cathode, the arsenic as arsenious acid going toward the anode, and the potassium will remain at the cathode. The following table shows the more important substances employed. Those marked "electronegative" should be placed on the cathode, those marked "electropositive" on the anode.

$\begin{array}{ll}\text { Electronegative } & \text { Electropositive } \\ \text { Sulphur } & \text { Silver } \\ \text { Iodine } & \text { Mercury } \\ \text { Arsenic } & \text { Copper } \\ \text { Phosphorus } & \text { Lime } \\ \text { Bromine } & \text { Magnesium } \\ & \text { Calcium } \\ & \text { Sodium } \\ & \text { Potassium } \\ & \text { Alkaloids }\end{array}$

The drug should be in watery solution. The absorbent cotton covering the electrode may be saturated with it or one of the electrodes devised for the purpose may be used. Of course a proper dose only should be employed. This can be obtained by dissolving soluble tablets or triturates containing such a dose in water. The active electrode is placed over the part it is desired to influence and the other is placed at some indifferent point. In the case of a joint, one may be placed in each side of it. The current should be from 5 to 20 milliamperes or $I_{5}$ to 30 cells in strength according to the sensitiveness of the parts where the electrodes are placed and should flow until the electrode is dry. Drugs cannot be introduced for any great distance by this method; hence only local effects can be produced in the skin or directly under it. The blood-vessels will, however, take it up and hence it may exercise a general effect. The method is, however, employed only when a local influence is desired and is usually chiefly in diseases of joints, some skin diseases, neuritis and neuralgia if the nerves are near the surface and for the producing of local anesthesia by cocain or its congeners.

\section{SPECIAL ELECTROTHERAPEUTICS}

Under this heading the more important medical diseases in which treatment with some form of the electrical current may be of benefit will be considered.

\section{DISEASES OF THE NERVOUS SYSTEM}

The earliest use of electricity in medicine was for the purpose of exercising paralyzed muscles. For this purpose it is still of great service when properly used (see page 103). In selecting the proper current to use the dictum of $\mathrm{H}$. C. Wood still holds good, i.e., "use the current which gives the best contraction with the least pain." It is of no use to employ a current which will not cause contraction; hence to the employment of the faradic current in many cases of 
peripheral neuron (page 103) lesion is a waste of time. In many of these cases the constant current must be used, using preferably the cathode as the active pole if the muscles will contract, if not use the anode. It cannot be repeated too often not to exhaust the muscles by too strong a current and too many contractions, and if they will not react to a current of moderate strength it is well to merely utilize the stimulating influence of the cathode by rubbing that electrode over them. The sinusoidal current is also useful in such cases. It must also be remembered that the current should not be used to contract muscles when there is an acute inflammation of any part of the nervous system. Therefore, it should not be employed in hemiplegia following apoplexy, and acute inflammations of the brain, cord or peripheral nerves until the acute symptoms have subsided. In acute neuritis it may be used to relieve pain, a constant current being passed up or down the affected nerves, Fig. Io, or the heating properties of the high-frequency current (page ro2) may be employed. When treating paralyzed limbs in which there are contractures, as is often the case in hemiplegia, only the weaker muscles should be treated, that is the extensors. In such cases an attempt may be made to relax the overacting flexor muscles by rubbing over them the anode of the constant current for five or ten minutes. These currents, preferably the sinusoidal, may be used whenever it is desired to passively exercise muscles as in obesity, degeneration of the cardiac muscle, etc.

In sciatica and other forms of neuritis the high-frequency current by the method of diathermy (page 102) is of great value. In the case of the sciatic nerve one block tin electrode ( 3 by 5 or larger) is placed on the buttock over the sciatic notch and the other of similar size may be placed either just above the popliteal space or lower down if desired.

Numbness and other forms of paresthesia may sometimes be relieved by the use of the faradic dry brush. The high-frequency effluve or vacuum electrode application is often of service in acroparesthesia.

In neuralgias of nerves that are near the surface the sedative effect of the constant-current anode may relieve the pain. The preferable procedure is to place the anode over the nerve, the other and larger electrode being placed some distance away. The current must be increased and decreased gradually and the applications last about five or ten minutes. If the trigeminal nerve is affected the supraorbital, infraorbital and mental foramina would be the proper locations for the anode. Cocain may be placed on it if desired.

The headache of neurasthenia may at times be relieved by employing either the static breeze or high-frequency effluve. The general application of these currents may be used for their influence in metabolism.

Any of the symptoms of hysteria may yield to any form of current, that which is likely to make the biggest impression being preferable. 
In exophthalmic goiter the heart's action may be slowed by the constant current, placing the anode over the seventh cervical vertebra and rubbing the cathode up and down the side of the neck over the nerves. A weak current should be used and the application made two or three times daily for ten minutes or more.

In occupation neuroses of the spasmodic type the anode of the constant current may be rubbed over the muscles. In the paralytic type the cathode can be similarly used. High-frequency current, employing a vacuum electrode or the effluve, may also be employed.

\section{DISEASES OF THE MUSCLES AND JOINTS}

Myositis or muscular rheumatism, especially if localized, as in "stiff neck" and lumbago, is successfully treated with electricity. High-frequency currents, applied either by the vacuum electrode or thermopenetration, so as to get the heating effect and followed by sparks are of great service. Static sparks and the static wave current are also of service. The constant current as strong as can be borne, applied by rubbing the anode over the muscles for from five to ten minutes may give good results.

In rheumatoid arthritis either the high-frequency or static currents are often of service in relieving pain and exudation in the affected joints if actual ankylosis has not occurred. High-frequency currents may be applied by using a vacuum electrode, first keeping it in close contact with the joint for ten minutes for the heating effect and then withdrawing it far enough to spark the joints. Diathermy is also of great service. The static current is best applied when possible by means of the static wave current. Sparking the joints is also of service. The constant current may be passed through the joint, placing an electrode in each side. The property of phoresis can also be utilized to introduce iodine or other drugs into it. Autocondensation or other general measures may be used to influence metabolism. Chronic arthritis due to other causes can be similarly treated.

\section{DISEASES OF THE STOMACH}

The value of electricity in the treatment of diseases of the stomach has been disputed. Many authorities, however, employ it with asserted success, and the experience of the writer is in accord with them. It may be used to increase motility and secretion, to diminish secretion and in some cases for its psychic influence. The active electrode in general use is that of Einhorn, which is flexible and can be swallowed. Bassler has devised an improvement on this, which he claims is easier to introduce. His instrument consists of two parts, the electrode proper and an introducer, Fig. 22. His description of how to use it is as follows:

"The patient is first made to drink a tumblerful or more of water. . . . The introducer is lubricated with glycerin, then 
the electrode (which has been immersed in water) is mounted by slipping the spherical end on to the stem of the introducer, after which one turn of the cable is made around the end of the introducer shaft. The entire instrument is inserted into the patient in the same way as is a stomach tube. At the desired point in the esophagus, or better in the stomach, the electrode is freed by a simple direct withdrawal or by first making one or two short in-and-out movements of the introducer, the cable being freed from the hand. The introducer is then withdrawn leaving the electrode within the patient. Another swallow or two of water can then be taken. Care must be taken not to have more than one turn of the cable about the end of the introducer, and it is advisable to free the cable from the hand after the capsule has been delivered below the laryngopharyngeal junction. At the end of the treatment the electrode is withdrawn in the same way as is the simple deglutible instrument; that is the string is drawn upon until the ball end is held at the back of the larynx,

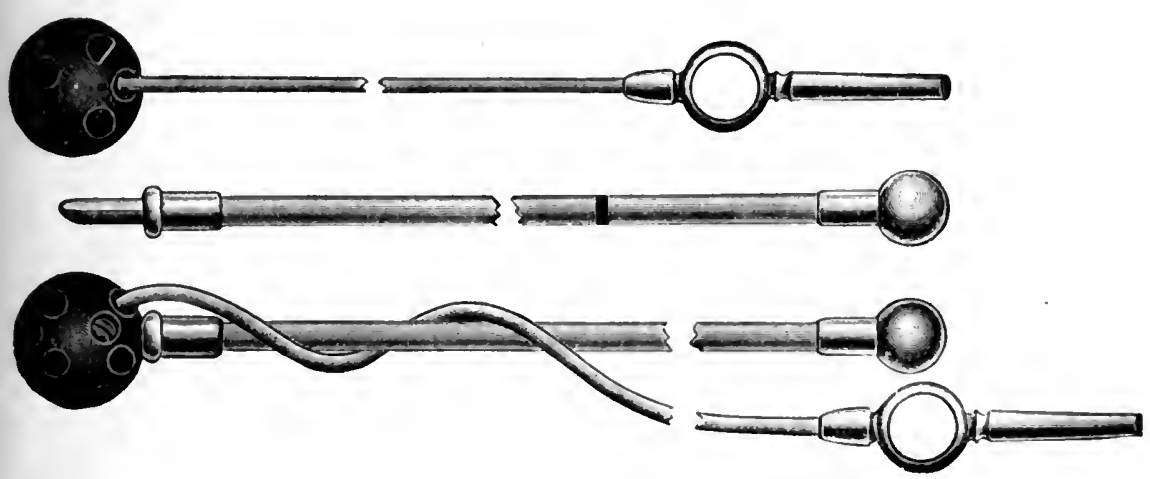

Fig. 22.-Bassler's modification of Einhorn's stomach electrode.

then with an effort at swallowing (when the introitus of the esophagus opens), the end of the instrument is delivered." The treatment must be given when the stomach contains nothing but a tumblerful or more of water. If the stomach is markedly dilated, the patient should lie down; otherwise the sitting position is proper. 'The indifferent electrode should be of fair size (about 3 by 3 ), and may be placed on the back or epigastrium. Weak currents should be used. If the constant current is used, 5 milliamperes are sufficient. The current should be increased and decreased in strength gradually. The treatment should last from five to ten minutes. To improve motility and tone the slowly interrupted faradic or sinusoidal current should be used preferably. The rapidly interrupted faradic current or constant current with the cathode within the stomach may also be employed.

In the irritative sensory and secretory conditions, as gastralgia, hyperchlorhydria and other conditions found in the neuroses, the constant current is the one of choice. In conditions of hyperesthesia 
the anode should be the active electrode, in cases of excessive secretion this electrode may also be used or the poles may be alternated by means of the polarity changer. Some cases of chronic gastritis also do well with this method.

If it is not desired to introduce the electrode within the stomach, one electrode may be placed on the back and the other over the stomach. If this organ contains water and the patient is made to lean forward, some of the current will certainly pass through it. Polar influence, however, cannot be gotten in this way. If the abdominal walls are relaxed they should be treated as paralyzed muscles to increase their tone. In ptosis this may be of service. About three treatments a week, should be given, diminishing them in frequency as improvement occurs.

Spasm of the esophagus especially if of hysterical origin is well treated by introducing an electrode, such as are devised for the purpose (a stomach electrode will do) and using either the constant or faradic current, preferably the latter.

\section{DISEASES OF THE INTESTINES}

Relaxed conditions of the abdominal walls should be treated as stated under diseases of the stomach. Constipation if chronic and due to the weakness and lack of tone in the intestinal muscles (intestinal stasis) often yields to the sinusoidal current. Large electrodes, from 3 by 6 to 6 by ro inches should be employed. One may be placed over the lower part of the back and the other placed on various parts of the abdomen, and pressed in deep. One electrode may be placed on the abdomen, and one (rectal electrode) in the rectum. The faradic and constant currents may also be used. The application must not be too strong or too long continued (about five minutes) or exhaustion of the muscles may be caused.

\section{DISEASES OF THE BLOOD-VESSELS}

The treatment of saccular aneurysm of either the abdominal or thoracic aorta by employing the blood-clotting properties of the anode of the constant current has met with some measure of success. The operation, known as the Moore-Corradi method, consists in introducing into the sac through a hollow needle a fine wire which coils within the sac, and then passing a strong galvanic current through this wire which is attached to the anode of the battery. In thoracic aneurysms the needle can be introduced directly over the highest point of swelling, in those of the abdominal aorta, an abdominal section must be done first to expose it. The wire used must be of either gold, or silver, gold being preferred by D. D. Stewart and Hare. According to the observations of Hare, copper as an alloy is dangerous as such wire becomes brittle and may break. ${ }^{1}$

${ }^{1}$ Jour. Am. Med. Assn., April 18, 19r4, p. I218. 
He prefers yellow-gold wire as distinguished from red gold. The technique advised by the late D. D. Stewart is as follows: The needle is hollow, made of gold and insulated to within $1 / 4$ inch of its tip with porcelain or shellac. This is introduced with aseptic precautions into the sac where it is nearest the surface and away from its mouth; then through it with the same precautions, is introduced a fine coiled wire of either silver or gold, of about 30 gauge and so drawn that it will assume snarled, spiral coils. The amount required depends upon the size of the sac, viz., for one of about 3 inches in diameter, 3 to 5 feet is sufficient; for a diameter of 4 to 5 inches, 8 to ro feet should be used. The end of the wire is then attached to the positive pole of a source of constant or galvanic current, and a large clay pad electrode is placed in the abdomen to which the negative pole is attached. The current strength is then gradually increased until it reaches from 50 to roo milliamperes, ${ }^{1}$ which must be maintained for an hour or more and then gradually reduced to zero. The wire is then detached, the needle carefully withdrawn by rotation and counterpressure, and the released external portion of the wire gently pulled upon and cut close to the skin, the cut end being pushed beneath the surface. This latter procedure may be facilitated by drawing the skin at the site of puncture slightly to one side when introducing the needle. The opening should be sealed with gauze and collodion. The skin may be anesthetized before the puncture with either cocain or ethyl chlorid. Before the operation the source of current must be carefully tested. The following record of a case operated upon by Hare shows how the current should be regulated:

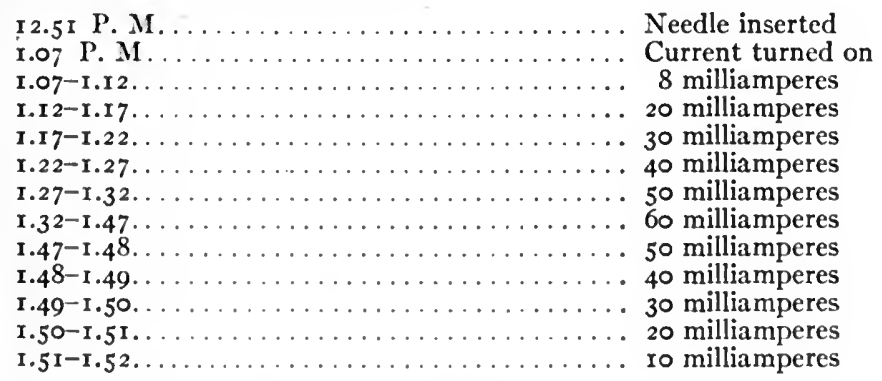

Current turned off at 1.52 P. M. This schedule is, of course, not absolute but may be varied both in current strength and duration according to the exigencies of the case.

The clay pad electrode is made of potter's clay which should be kept in the consistence of soft mud by the admixture of water. It should be made fresh for each application and should be heated. Pans can be secured for the purpose which have a double bottom. In these boiling water can be kept within the space between the two bottoms. Before putting the clay in the pan several layers ${ }^{3} 50$ milliamperes is usually sufficiently high. 
of gauze should be placed in the bottom, and should be large enough to project over the sides of the pan, so that the clay can be lifted out. The clay is spread over this gauze about an inch thick, and it should be large enough to cover the abdomen. After it is placed on the patient, a plate of metal (block tin) containing a binding post to which the coil from the battery can be attached is pressed into the clay. The needle and wire must, of course, be carefully sterilized. Hare (loc. cit.) is of the following opinions as a result of operating on twenty-five cases:

I. The fact that no accident has occurred in any of the twentyfive operations I have performed leads me to believe that this seemingly radical operation is not a dangerous one, the more so as I have no knowledge of any accident having taken place when the operation was properly performed by others.

2. The operation is the only one that offers material hope of prolonging life, this having been prolonged in one case at least five years.

3. It is justified by the prompt relief of pain which nearly always ensues and lasts, unless the growth, extending in another direction, creates a new source of pain.

4. The diagnosis of saccular aneurysm should always be confirmed by roentgen-ray examination as this operation is contraindicated in fusiform aneurysm for obvious reasons.

5. Great care is absolutely necessary that properly prepared wire be employed.

Hunner's results in twenty-three cases, seventeen thoracic and six abdominal are: four were cured; of these three were thoracic and one abdominal. In nine cases there was relief of symptoms and prolongation of life.

The dangers are sepsis; the development and rupture, especially in large multilocular aneurysms, of a secondary sac due to rapid filling of the main sac by coagulum and the shunting of the blood stream against a portion not receiving a special strain before; emboli breaking from the sac wall during or after the insertion of the wire; in abdominal aneurysm the closing of important vessels by these suddenly being filled with clot. ${ }^{1}$

Arteriosclerosis.-The high blood-pressure often present in this condition may be lowered more or less effectively by high-frequency currents. For this purpose the D'Arsonval current is the one of choice, administered either by autocondensation or general diathermy. A current strength of from 200 to 800 milliamperes should be used for about fifteen or twenty minutes daily for a week or two and then every other day for several weeks. In this connection it must not be forgotten that a moderate amount of increased pressure

${ }^{1}$ For further information see Stewart, Am. Jour. Med. Sci., Oct., r892; ibid, Aug., r896; Phila. Med. Jour., Nov. r2, r898; Guy Hunner, Johns Hopkins Med. Bull., Nov., r900; Freeman, Trans. Am. Surg. Assn., rgor, p. 359; Matas, Trans. South. Surg. and Gyn. Assn., I9oo, p. 286; Beardsley, Therap. Gaz., Mar. I5, I9II; H. A. Hare, Jour. Am. Med. Assn., Apr. 13, I912; ibid, Apr. 18, 1914. 
is desirable in certain cases, as for instance when there is degeneration of the cardiac muscle or marked atheroma of the arteries. In such cases the method should not be used. It is of value in early cases where the increased tension is due to arterial spasm due to toxins formed in the intestines and elsewhere. As the treatment also increases the elimination of solids by the kidneys, it should be used with caution when these are not acting well. It is recommended to drink freely of water before and after treatment. The following contra-indications have been given by Carriére: ${ }^{1}$ In albuminuria, if the chlorid elimination is less than $3 \mathrm{gm}$., if nitrogen elimination is below normal, if there is less than $800 \mathrm{gm}$. of urine in twenty-four hours, and if the renal permeability to methylene blue is prolonged or retarded. If the excretion of urine falls below I liter with symptoms of nitrogen or chlorid retention, it should be stopped. Advanced interstitial nephritis is a contraindication; care must be taken in any event not to lower the tension too suddenly or too much.

Raynaud's disease, which is due to venous and arterial spasm may be benefited at times by the use of high-frequency currents (infra). Another method is to place the hands in hot water in which the cathode of the constant current is immersed and the anode placed at an indifferent point. The current should be as strong as can be borne and the seance last for from ten to fifteen minutes, the object being to obtain the dilating influence of the cathode on the vessels. Acroparesthesia may be similarly treated.

In that form of obliteration of the caliber of the blood-vessels of the legs and feet, termed by Buerger thrombo-angiitis obliterans, ${ }^{3}$ the symptoms of which resemble those of Raynaud's disease and intermittent claudication, Johnson obtained excellent results by local diathermy. ${ }^{3}$ He placed a large absorbent cotton pad, wet with a solution of sodium bicarbonate as a cap over the toes, and another similar one below the knee. Over these metal plates were placed which were connected with the coil. A current of 500 milliamperes was employed daily for thirty minutes. It is important to increase the current strength gradually and cautiously to avoid burns. The writer saw this case before and after treatment-in fact had advised amputation in the near future. This was over a year ago and amputation has not yet been done; the patient can walk and is free from pain which was not the case before the treatment was given. In these cases a vacuum electrode may be used if ulceration has not occurred and the constant current used as advised for Raynaud's disease may be tried if the high-frequency current is not available.

Electricity is of service in the treatment of many diseases of the skin, eye, throat and nose, genito-urinary organs and surgical conditions, a consideration of which is not in order in this place.

${ }^{1}$ Paris méd., Jan. 20, I9ı.

2 Am. Jour. Med. Sci., Oct., rgo8.

- Am. Jour. Electrotherap. and Radiology, Feb., I9r6, p. 72. 


\title{
ROENTGEN THERAPY
}

\author{
By Henry K. Pancoast, M. D.
}

It would seem pertinent at the beginning of this supplementary chapter to announce the recent adoption and general acceptance of a definite and scientific nomenclature for use in connection with this branch of medicine. It comprises the addition of the prefix "roentgen" to all words implying the use of roentgen-raysin medicine. For example roentgenology, roentgenologist, roentgen diagnosis and roentgen therapy and similar words and terms are now generally employed in place of older ones, many of which were incorrect, unscientific or ambiguous.

Roentgenology occupies rather an unique position in medicine for the reason that the roentgenologist and the physicist are closely allied in their investigations and work. While each is working along his own line they are mutually assisting each other. The study of the phenomena exhibited by the roentgen-ray tube continues to be one of intense interest to the physicist and he is constantly making new discoveries and developing new forms of apparatus. The roentgenologist is working in unison and making practical applications wherever the results of the investigations and the devices of the physicist can be found to afford improvements in technique or widen the field of usefulness of the roentgen-ray in diagnosis or treatment. With the two branches of science working in unison it can be readily understood why roentgenology has made such rapid strides in a comparatively few years. Unfortunately, however, these rapid advances have imposed a most difficult task upon those who have attempted to compile this subject into book form. The original chapter on roentgen therapy in the first volume of this work may be cited as an example of the necessity for complete revision of a literary contribution that was thoroughly up-to-date a few years ago. Scientific accuracy demands that many contradictory statements must now be made, in addition to the supplementary information regarding new or improved methods or devices that is offered in the pages to follow.

Two very important advances were made in connection with the roentgen-ray in the year of I9I3. The discovery that the rays could be reflected or refracted led to a clearer understanding of their identity, and the designing of the Coolidge tube practically revolutionized rontgen therapy and materially aided in increasing the accuracy and scope of roentgen diagnosis.

Attempts to reflect or refract roentgen-rays date back to the time of their discovery, but it was not until I9I3 that Friedrich and Knipp- 
ing, and later, W. L. Bragg, following the investigations of Laue, were able to demonstrate that the rays could be regularly reflected by the planes of cleavage in crystals. No polished surface from which light waves can be regularly reflected can be made by any known mechanical means so smooth but that countless infinitesimal irregularities remain. While these may be disregarded in the case of the comparatively long wave lengths of light, it is now known that roentgen-rays are of such short wave length that no mechanically polished surface is sufficiently smooth to reflect them. Roentgen-rays may be regarded as atomic in length, ${ }^{1}$ and any reflecting surface would have to be practically atomically smooth. Such surfaces are found in nature along invisible parallel planes of atoms in crystals, and roentgen-rays have been successfully reflected by utilizing the planes of cleavage in certain crystals. With a knowledge of the distances between the atoms it has been possible to calculate the wave length of roentgen-rays, and such calculations have shown that roentgen-rays are closely allied to ultraviolet light waves, and it is at present believed that the rays and light are identical. It is also believed that the penetration of roentgen-rays is dependent upon their wave lengths, the shorter the wave the more penetrating the impulse.

The Coolidge Tube.-As has been previously stated (Vol. I, page 634) the produetion of the roentgen-ray impulse depends upon the bombardment of the target of the tube by a negative corpuscle traveling from the cathode at an enormous velocity. The negative corpuscle is furnished by the atoms of the small amount of gas remaining in the tube after it has been adequately exhausted. The energy of the impulse, or its penetrating quality, depends upon the velocity with which the corpuscle strikes the target, and this in turn depends upon its initial velocity imparted to it by the voltage of the charge delivered at the cathode, less the impediment to its flight offered by the presence of gas atoms in its path. Furthermore, the voltage of the charge will be largely dependent upon the relations between the resistance of the tube or amount of gas to supply corpuscles and the volume of current delivered or to be carried. All of these factors in roentgen-ray energy or penetration are subject to constant changes, and the output of a tube is a heterogeneous volume of rays of varying intensities and degrees of penetration. As long as a small amount of gas has to be left in a tube, it is never possible or feasible to drive all of the gas out of the metal electrodes, and after forced operation for a variable period the metal parts become so heated that they evolve a large amount of occluded gas, and the resistance of the tube is lowered to such an extent that the penetration of the output becomes too low for practical purposes and may do harm. It will be readily understood, therefore, that the one essential factor in elec-

s Visible light waves vary from $7.7 \times 10^{-5}$ to $3.6 \times 10^{-5} \mathrm{~cm}$. in length, while roentgen-rays are practically in the neighborhood of $10^{-8} \mathrm{~cm}$., and gamma rays are still shorter. 
trical conduction and roentgen-ray production is responsible for many of the limitations of the roentgen-ray tube.

In r $^{1} 3$, Prof. W. D. Coolidge devised a tube which bears his name and is constructed and operated along entirely different lines from the tubes that had previously been in use. In connection with the elaboration of our present conception of matter, it has been determined that metals and solids contain free negative corpuscles, which are more or less responsible for heat and electrical conduction. The better the conductor the more free electrons it contains. Furthermore, these free corpuscles can be ejected from the body by heat. In 1905 , Wehnelt constructed a tube in which a strip of platinum contained therein was raised to a bright heat by a low-voltage current independent of the discharge current. On this platinum strip was mounted a piece of lime, and when it in turn became heated it emitted large numbers of negative corpuscles which were utilized in place of the corpuscles derived from gas atoms. This tube was not a success, mainly because of the low voltage at

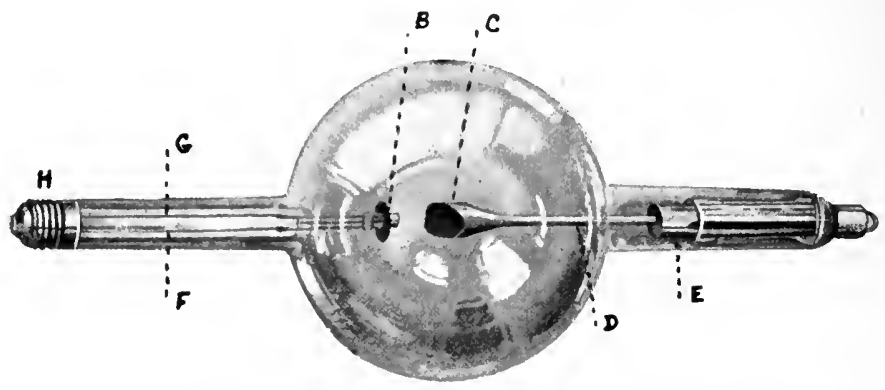

FIG. 23.-Coolidge roentgen-ray tube.

which it had to be operated, and the resulting soft quality of the roentgen-ray output. Other experimenters tried platinum and carbon, but these could not be satisfactorily freed from gases. The experiments of Coolidge with tungsten wire proved successful and led to the construction of the tube that is now in general use in therapeutic work.

The essential principle of this tube is the absence of practically all gas, the conduction of the discharge through it depending upon electrons driven out of the heated cathode by the high-tension current. As a type it is frequently designated as the "electron tube" in contradistinction to the older type of."gas tube." No discharge current can pass through it unless the cathode filament ( $A$, Fig. 24) is heated, and the volume of the current that can be conducted depends primarily upon the temperature of the filament and the number of electrons thus made available. The speed of the corpuscles and the resulting penetration of the roentgen-ray output depend solely upon the voltage at the electrodes, and are not altered and do 
not vary as in the gas tube because of the presence of occluded gas in the metal electrodes.

The cathode (Fig. 24) is composed of a filament $(A)$ consisting of a flat closely wound spiral of tungsten wire, the free ends of which are connected by conductors ( $F$ and.G, Fig. 23) with the cathode terminal $(H)$ which is constructed like an ordinary screw plug. Over the latter may be screwed a socket in which terminate the leads from

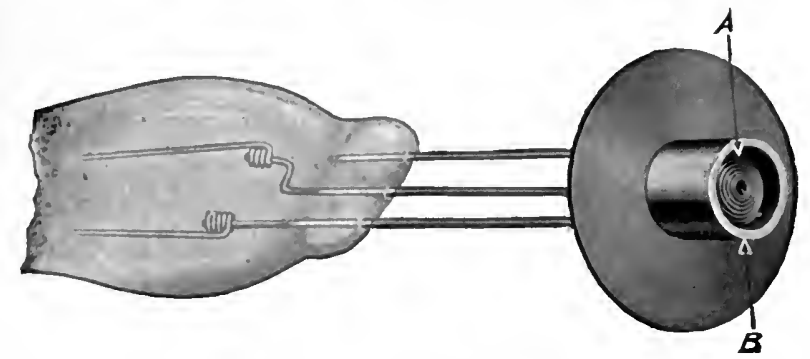

FIG. 24.-Cathode of the Coolidge tube. (General Electric Co.)

a storage battery. So far, the tube and cathode resemble in construction an ordinary incandescent lamp operated on a low-voltage battery circuit. The filament is surrounded by a focusing device ( $B$, Fig. 24) consisting of a molybdenum cylinder which projects just beyond the spiral. Its object is to focus the cathode stream upon the target (C, Fig. 23) and prevent any electron discharge backward.

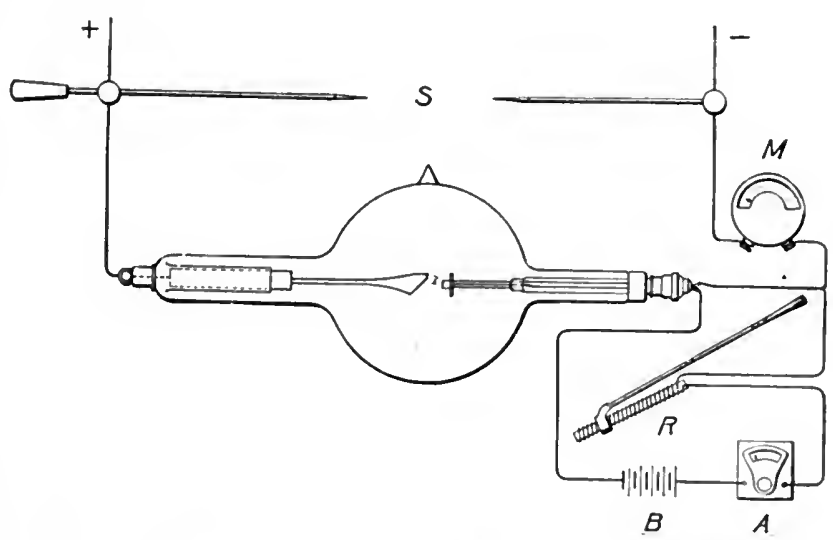

Frg. 25.-Diagram illustrating the operation of the Coöidge tube.

The target or anticathode (C, Fig. 23) consists of a piece of wrought tungsten supported by a molybdenum rod $(D)$ and attached to a split iron tube $(E)$ which connects with the anode terminal.

The method of operating the tube is diagrammatically shown in Fig. 25. The cathode terminal is connected by a socket in the circuit from a I2-volt storage battery $(B)$ and in this circuit is an ammeter 
$(A)$ and a rheostat $(R)$. The lead from the positive terminal of the transformer or coil $(S)$ is connected to the anode terminal of the tube, and the negative lead, with milliammeter $(M)$ in series is connected to some convenient part of the battery circuit. In this way the entire battery circuit during the operation of the tube is brought to the full potential of the secondary discharge through the tube and must, therefore, be as fully insulated as the tube or any other part of the discharge circuit.

Before operating the tube the battery circuit is closed through the tube filament, and the heating current is varied by a long insulated handle on the rheostat somewhere between 3 and 5 amperes, as shown on the ammeter. This will give a temperature in the filament between $1700^{\circ}$ and $2350^{\circ} \mathrm{C}$. After adjusting the rheostat at about the point determined through experience, the primary switch of the transformer or coil is closed. The milliammeter will not register unless the discharge is passing in the proper direction and if it does not, the current must be reversed. The primary rheostat and the battery circuit rheostat must then be adjusted to suit the requirements. Suppose, for example, it is desired that a discharge of 6 milliamperes shall pass through the tube backing up a parallel spark gap of 9 inches, which is an average adjustment for deep therapeutic work. The battery rheostat is adjusted until the ammeter shows the usual reading which is near 5 amperes through the filament circuit. The primary rheostat is then set on the contact button that will give 6 milliamperes and the battery rheostat further adjusted until the tube is found to back up 9 inches of spark gap.

If the filament current and discharge current remain the same the tube will run continuously without any adjustment and deliver a constant discharge, except that after the target becomes intensely heated there will be a slight drop in resistance which will require a little adjustment of the battery rheostat. The tubes as now constructed cannot be operated at a greater spark length than ro inches without danger of puncture. As the entire cathode stream is directed against the target there is no fluorescence in the tube, and there is no unusual heating of the anterior hemisphere as noted in the gas tube, which would seem to indicate the absence of secondary cathode rays and positive ions from the target. In a large measure this eliminates the undesirable indirect radiation from the glass walls. Until the target becomes intensely heated the discharge will pass in one direction only, because the source of electrons is solely in the cathode filament.

The greatest advantage of this tube is in therapeutic work, for the reason that it can be operated at a higher voltage and deliver a higher penetration output and for a longer time than any other tube. It has in this way revolutionized deep therapeutic work. Because of the great penetration and volume of the output, the tube should never be used unless it is adequately enclosed in a perfectly safe box or shield, which must be sufficiently well insulated to guard against 
short-circuiting a ro-inch spark. The complete enclosure of the tube means the retention of a great amount of heat and this necessitates an adequate air cooling device, either by compressed air through the shield or exhaustion of the heated air. Fig. 26 shows one of the most satisfactory treatment tube shields now on the market. Only the terminals of the tube $(A)$ project outside, and a non-conducting opaque material fits around the cathode extremity $(B)$. The box is lined with 1/8-inch lead. A hairdresser's blower fan $(C)$ operating in series with a 32-candlepower lamp forces air into the box, whence it escapes through an opening in the top and also along the anode extremity of the tube. In this way the tube is kept sufficiently cool

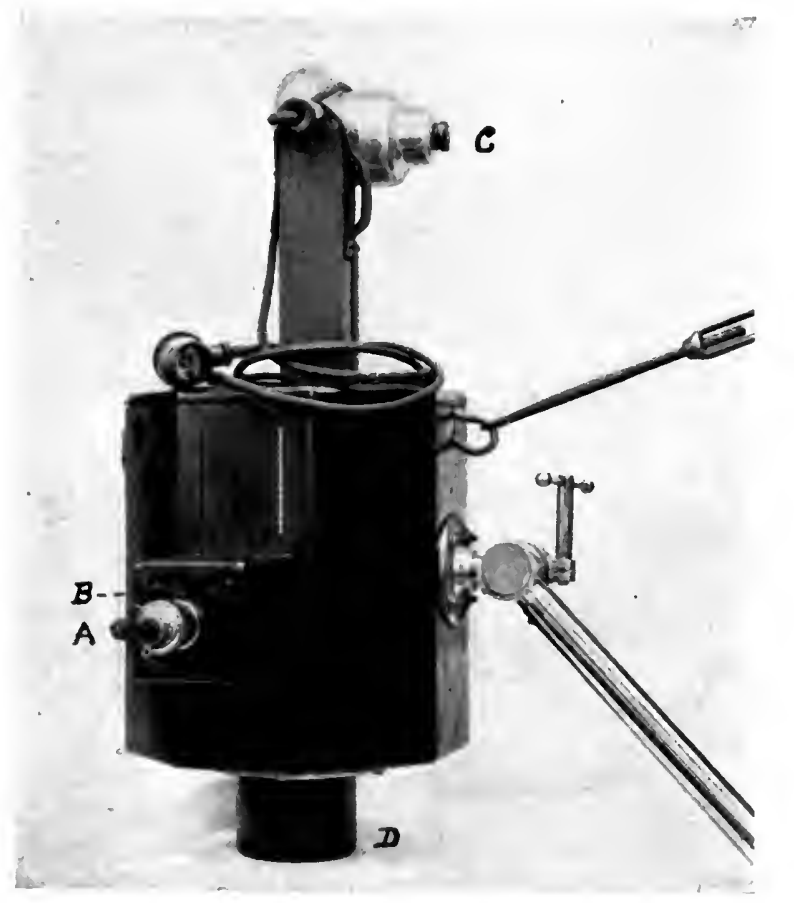

FIG.26 . Protecting tube box for Coolidge tube.

to permit its operation for practically an indefinite period. Metal diaphragms and cones $(D)$ of various shapes and sizes may be easily fitted into the bottom of the box. 'The lid is hinged to permit the tube to be inserted (Fig. 27). The aluminum filters are placed between the cone and the opening in the bottom of the box.

The Coolidge tube has not only simplified deep roentgen therapy but has increased its efficiency as well, by making it possible to deliver a comparatively heavy roentgen-ray output continuously, so that deep areas may be exposed to cross-fire radiation through a large number of ports of entry within a reasonable time. The time factor is still an important one, however, and in order to cut down the 
length of applications and permit more work to be done, Dr. Coolidge is now devising a tube with a water-cooling device in connection with the target, whereby the output can be greatly increased with a corresponding saving in time. Instead of the usual solid target, a copper-backed piece of tungsten is soldered in the end of a copper tube, inside of which is a smaller tube through which the water is forced against the back of the target and then escapes through the space between the two tubes. The enormous heat developed at the target may be readily realized from the statement of Dr. Coolidge

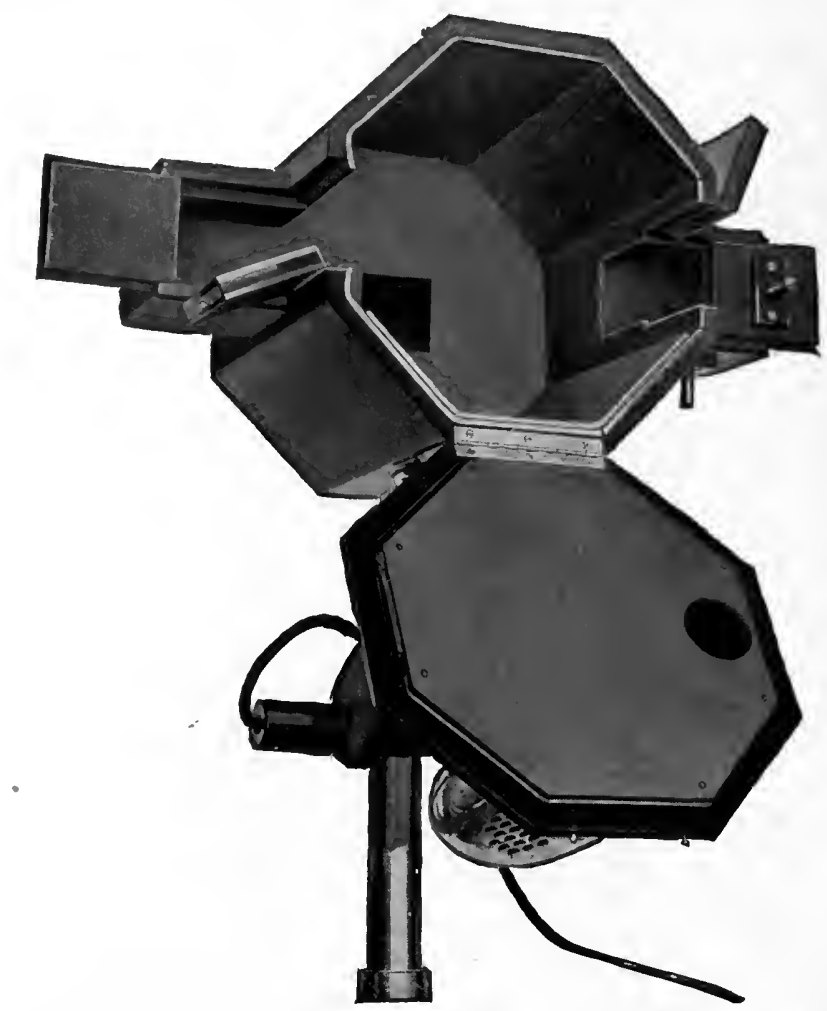

FIG. 27.-Interior of protecting tube box for Coolidge tube. (Waite and Bartlett Mfg.Co.)

that "An energy input of 200 milliamperes at 70,000 volts is sufficient to raise the temperature of a liter of water from $20^{\circ} \mathrm{C}$. to the boiling point in thirty-three seconds," and in order to prevent boiling it would be necessary "to pass through the target at least 2 liters of cold water per minute." The simplest device proposed is one similar to the water-cooling system of an automobile. A rotary pump run by a small motor pumps the water into the tube, thence it passes to a cellular or tubular radiator against which a fan is directed. The tube itself must of course be kept cool by an air current. During recent experiments such a tube was operated continuously for forty- 
two hours on a current of 100 milliamperes at a potential of 70,000 volts. This would mean an enormous saving of time when it is considered that 5 to ro milliamperes is the usual operating current at the present time.

Dosage.-The two most radical departures in the technique of roentgen therapy made in the past few years have been in the general adoption of massive dosage and treatment of deep lesions by crossfire methods. Massive dosage has been made a safe and accurate procedure by the perfection of dependable devices for measuring the intensity of radiation at the surface treated. Deep therapy has been rendered still more safe by the use of filters for absorbing the softer or less penetrating rays that would be absorbed in the skin and superficial tissues, thus permitting a much greater intensity of decply penetrating radiation.

Two instruments are in more general use and are to be recommended for the measurement of dosage - the Kienböck quantimeter and the Hampson radiometer. The quantimeter of Kienböck consists of two essential parts:

I. The normal scale is a long strip of standard photographic paper divided into ten sections of gradually increasing shades from white to black. These sections are numbered from $\circ$ to 10 , and each shade represents the amount of change that will be shown by the standard photographic paper following exposure to definite quantities of roentgen-ray energy, after development under standard conditions of time, temperature and strength of developer. Shade number $\mathrm{I}$ is taken as the unit and is known as $\mathrm{I}-\mathrm{X}$ unit, shade number 5 would represent $5-\mathrm{X}$ units and number ro, ro- $\mathrm{X}$ units. 2. The test strips are small strips of standardized photographic paper enclosed in black envelopes. One of these is placed upon the surface of the area treated, and after the exposure is completed the strip is removed from the envelope in the dark room, and developed in a standard developer at a definite temperature for a definite time. After fixing, it is matched with the color scale, and the number of the section to which its shade corresponds is noted. This determines the number of $\mathrm{X}$ units of the exposure. Aside from the trouble and the care required in development, the one great objection to this method is the fact that the dosage cannot be determined for some time after the exposure. However, after a few trials, dosage with a given Coolidge tube can be standardized by noting the time required to give the desired number of $\mathrm{X}$ units with a definite milliamperage of current, distance and spark-gap resistance. The Kienböck $\mathrm{X}$ unit is the one usually employed in describing dosage. $\mathrm{A}$ single $\mathrm{X}$ unit represents a very small dose, and ro- $\mathrm{X}$ is the average normal dose sufficient to produce a very mild safe erythema of the skin, or epilation in such conditions as sycosis, provided no filter is employed. This dose corresponds to tint B of the Sabouraud-Noire scale, or tint 4 of the Hampson scale, to be described later, or to $5-\mathrm{H}$ units of the Holzknecht radi- 
ometer scale. It has been determined that I $\mathrm{mm}$. of aluminum absorbs about the same amount of radiation as $I \mathrm{~cm}$. of water or muscle tissue. Therefore, by placing over the test strip at the time of exposure the "tiefenmesser," composed of a step form aluminum strip increasing I mm. in thickness with each step, one may determine approximately the roentgen dosage at different centimeter depths in the tissues. ${ }^{1}$ To be more accurate, strips may be placed in cavities at about the depth of lesions treated, as in the vagina or rectum in the treatment of uterine fibroids, and the exact dosage determined at the level of the lesion.

The Sabouraud-Noire pastille method consisted in placing a small pastille composed of a piece of cardboard coated with barium

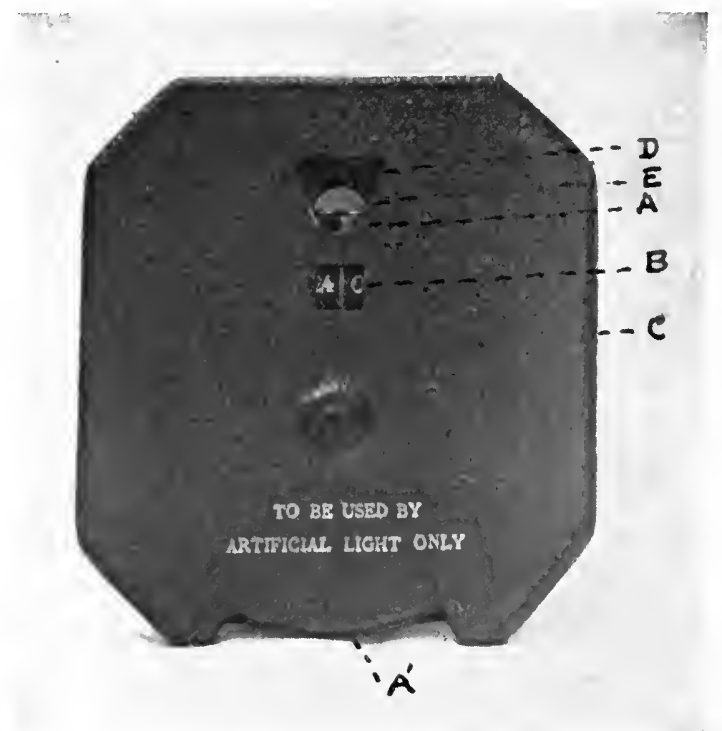

FIG. 28.-The Hampson radiometer.

platino-cyanid crystals at a point halfway between the target of the tube and the skin surface. It was supported by a metal holder. The exposure was continued until the pastille changed color by losing its green tint until it matched a standard " $\mathrm{B}$ "tint, which would represent the amount of exposure sufficient to produce either no visible effect or a very mild safe erythema and epilation. The objections to this method are the difficulty of placing the pastille at the distance required and the inability to judge dosage less or greater than the tint $B$, should an intermediate or more intense dose be required.

The Hampson radiometer was an instrument devised to meet all the objections to the Sabouraud method. It consists essentially of a rotating disk $\left(A-A^{\prime}\right.$, Fig. 28$)$ around the outer edge of which are arranged as sectors 25 carefully graded tints corresponding to

${ }^{1}$ It has recently been proven that this is not dependable. 
the color changes induced in the Sabouraud pastilles of barium platinocyanide under the action of roentgen rays. The zero tint $(B)$ is the same tint as the fresh unexposed pastille. Were the pastille placed in the same position as in the Sabouraud method, tint 16 would represent the color change corresponding to tint $\mathrm{B}$, but as it is placed on the surface treated instead, tint 4 on the scale represents the same dosage as is obtained at tint B. 'Tint 4 also corresponds to the dosage of Io-X units on the Kienböck radiometer scale, and tint 8 , to $20-\mathrm{X}$ units. The rotating disk is enclosed in an outer case $(C)$ which has cut in it a small aperture $(D)$ through which the tints can be viewed one at a time. The pastille (E) is placed in the aperture just above the tint scale and the latter is rotated by the thumb at $\left(A^{\prime}\right)$ until the exact match in tint is obtained. Another small aperture $(B)$ just below shows the number of the tint. It is absolutely necessary that the matching of the pastille be done under artificial light. The pastilles soon regain their original color when exposed to daylight in a moist atmosphere. The advantage of this instrument over the Kienböck quantimeter is the ability to determine the exact dosage at once. The treatment can be immediately resumed should the desired tint not be obtained.

One of the most radical changes in the technique of roentgen therapy in the past few years has been the universal adoption of the massive dose method in the treatment of the majority of diseased conditions in which this agent is employed. By referring to the previous section on roentgen therapy (Vol.r, page 644), it will be noted that the fractional dose method was generally advocated and massive doses were advised against because of the danger, mainly from the lack of means of accurate measurement of roentgen-ray intensity. When the older method was in vogue we were obliged to depend upon the dosage factors of time, distance and intensity, the latter being estimated approximately by the volume of current passing through the tube. Penetration was a very variable and uncertain factor that had to be adjusted as nearly as possible to suit the depth of the lesion treated. In most instances in which maximal effects were desired, it was customary to repeat fractional doses at varied intervals until the necessary reaction was obtained. In the case of deep lesions treated through healthy skin, the applications were repeated to the point of safe skin tolerance. With the use of the Coolidge tube it is now possible to deliver an output of constant intensity and of comparatively homogeneous penetration for practically an indefinite time, and, by the use of the instruments of measurement just described, the exact dosage or volume of output needed to produce a certain reaction, and it is possible also to measure and apply such a dose with accuracy. It is now perfectly safe, therefore, to treat any condition by the massive-dose method, provided such a technique is indicated. It should be borne in mind, however, that there are many lesions in which the fractional dose method is still to be employed, but instead of employing the older crude 
dosage factors of time, distance and intensity, it is advisable and safer to use one or more of the instruments for accurate dosage estimation. This applies particularly to the treatment of many skin lesions such as eczema.

Filtration.-The Coolidge tube delivers an output of far more homogeneous penetration than the older gas tubes, and this remains practically constant as long as the filament current and discharge current remain the same. This being the case, it is permissible to use the parallel spark gap as the means of estimating penetration. The output of the Coolidge tube has not an absolute homogeneous penetration, however, and the bundle of rays it delivers is always made up of a definite proportion of softer rays than are required, and in the treatment of deep lesions where high penetration is essential, the skin receives a considerable proportion of rays of less penetration than can reach the area treated. In addition to being of no value, these rays only tend to hasten a reaction in the skin and lessen the intensity of deep radiation that can be applied to the lesion. In order to eliminate these less penetrating rays as far as possible it is now customary to employ filters that have absorptive properties approaching those of the skin and subcutaneous tissues. Sole leather and aluminum are the filters that have been most generally employed, and at the present time aluminum is almost universally used. It is customary in the treatment of deep lesions, such as uterine fibroids, to employ at least $3 \mathrm{~mm}$. of aluminum as a filter. In their passage through the aluminum, however, the primary rays produce a certain amount of characteristic secondary radiation in themetal, and as this has comparatively feeble penetration it is advisable to eliminate it as a source of danger to the skin by the interposition of a second substance, such as blotting paper or leather. When filters are employed the pastilles or test strips should be placed on the surface treated, under the filter.

The technique of the treatment of superficial lesions is so widely different from that of deep therapy that each must be discussed separately. It is impossible in such a limited survey of the field of roentgen therapy as can be made in these few pages to enter into the details of technique in any diseased conditions. Full particulars as to technique must be acquired by experience and teaching, or from special text books on the subject. Space permits only of reference to pathologic conditions in which roentgen therapy is applicable, statements as to the value of the treatment and a general explanation of the method to be employed when this is necessary for an understanding of the principles involved. In the treatment of superficial lesions there have been two notable changes in technique during the past few years. In the first place, it is now customary to employ the massive-dose method in dealing with many conditions, but this is by no means to be recommended as a routine procedure, especially in connection with eczema and many other skin diseases. It is highly desirable, however, in those conditions in which epilation is the result 
to be obtained, as in sycosis, favus, and tinea tonsurans, to use maximum doses and to accomplish the result at a single sitting. 'The exact dose necessary is well recognized and has already been referred to. Where epilation of the entire scalp is desired, the head is mapped out into a number of areas of such size and shape that each will receive the same dosage, and each is then given a carefully measured epilation dose, the face being carefully protected. The second change in superficial technique has been the employment of rays of deeper penetration than were used in former years. Instead of using a tube with a resistance of as low as $1 / 2$ inch to $1 \frac{1}{2}$ inches (Vol I, page 648) it is now customary to have it back up a parallel spark gap of at least 4 to 5 inches, and many roentgenologists use even higher penetration than this. The amount of absorption by the superficial tissues is so great with tube resistances even as high as 6 inches that there is little or no effect produced in the subcutaneous tissues when an erythema or epilation dose is delivered to the surface. Malignant lesions such as epithelioma, and possibly lupus vulgaris, are to be regarded as exceptions in many instances. Many epitheliomata, especially, require very destructive dosage, and if they are comparatively superficial care must be taken not to have the penetration so high that a destructive dose will be delivered to entirely healthy tissues beneath, although the destructive effect must be carried to the very deepest portion of the growth.

It is very necessary at this time to call attention to certain corrections that must be made in the technique advised in many instances in the original section on roentgenotherapy in Vol. r. Wherever definite dosage was advised and was based upon the time, intensity and distance factors, this should now be disregarded. Modern technique implies the use of exact dosage estimation by one or more of the instruments advised for the purpose. Fractional doses can be measured just as readily in this way as massive doses. For example, instead of the dosage recommended in psoriasis (Vol. I, page 65I), it is entirely unnecessary to repeat fractional doses, measured by the older method, until an erythematous reaction appears, because we know just what dosage, when carefully measured, will produce such a reaction at a single sitting. As a matter of fact, it is wiser to apply one-third to one-half a full dose in psoriasis and then wait for a reasonable time, say two to three weeks, and then repeat the dose if necessary.

Leukoplakia Buccalis.-Roentgen therapy cannot now be regarded as the method of choice in the treatment of this condition. While it may be efficient in the average case in which the area involved is accessible, there are many selected cases which may react far better to desiccation or radium. The former is particularly applicable to circumscribed areas, especially when they are thick and elevated. In flat, and particularly, smooth areas on the inner surface of the cheek and over the tongue, a half or full strength radium applicator may be used to good advantage, with adequate filtration. 
Radium tubes in filter capsules can be readily applied to lesions on the alveolar process where the teeth are missing, or in the sulcus between the cheek and gums.

Senile Keratosis.-Radium or desiccation are equally as applicable as the roentgen-ray in the treatment of this condition.

Acne Vulgaris. - As a slight correction to the technique previously advised in cases of this condition in which roentgen therapy is indicated, it is perhaps best to administer a carefully measured erythematous dose divided into from two to four sittings over a period of about three weeks, and then to wait long enough for any reaction to fully subside and the effect to become manifest before repeating the procedure, should it be found necessary to do so.

Lupus Vulgaris.-The lesions in this condition should receive full massive doses carried beyond the amount necessary for a mild erythema. The dose may be repeated after three or four weeks. If the lesions are deep, it is advisable to employ a filter, and increase the penetration and intensity accordingly. Small areas, especially around the eyelids, may be treated often to better advantage by radium.

Warts.-To the measures previously advised in the treatment of warts should be added the use of radium. An application lasting a few minutes to half an hour, depending on the size and depth, usually suffices.

Synovial Lesions of the Skin.-Occasionally synovial sacs are found under the skin above the nail and springing from tendon sheaths. The appearance is frequently like that of a smooth wart. These lesions are usually difficult to eradicate surgically and in a few reported instances they have been successfully removed by the use of radium.

Keloids.-Roentgen therapy is the best general treatment for these growths. When they can be excised readily it is usually best to resort to surgery first and follow the excision by postoperative roentgen radiation or the application of radium. The latter agent seems to yield better results when it can be employed to advantage.

Nevus.-(a) Flat Superficial Variety.-Radium therapy is undoubtedly the best method of dealing with these lesions in suitable cases. If moderate pressure upon the area will produce more or less blanching, the treatment is likely to be very successful, but if pressure causes little or no appreciable effect, a very favorable result can hardly be expected. The more superficial the lesion the better the cosmetic effect. Caution and patience are always essential and idiosyncrasy is always to be a foremost thought, as no two cases, and frequently no two areas in the same lesion, will respond alike. Each case should be studied by itself and the applications should be made cautiously at first until the proper dosage is determined. Flat applicators are necessary and proper filtration is as essential as the correct length of exposure. In the more superficial lesions, only a mild reaction should be produced, but in deeper ones it may be neces- 
sary to induce a reaction sufficient to bring about encrustation. Too vigorous treatment is likely to be followed by unsightly telangiectases.

(b) Cavernous Variety.-In lesions of this variety radium likewise produces excellent cosmetic results, and especially when "crossfiring" is possible. The exposure must be longer and filtration greater than in the flat lesions. When pulsating vessels are present these should first be ligated.

Pruritus.-It was formerly the custom among many roentgenologists and dermatologists to treat this condition by comparatively soft radiation. The best results undoubtedly follow radiation with high penetration delivered through filters of from 2 to $3 \mathrm{~mm}$. of aluminum. Fractional doses should be administered, but these should be carefully measured in order that their repetition may be kept within perfectly safe limits.

\section{DEEP THERAPY}

This term implies the treatment of lesions at varying depths below the surface of the body or skin by roentgen rays of very high penetration delivered through adequate filters to remove the softer rays, and by the "cross-fire" method when this can be used to advantage. It is in the treatment of deep lesions that roentgen therapy has made the greatest strides in the past few years, owing to the unusual perfection in tubes, apparatus and technique. Careful study and experimental work have shown that what in former years passed as deep therapy was little more than a useless waste of energy when compared to modern methods, and it is surprising that any results were ever obtained by the practices of eight or ten years ago. The very simple experiment of placing a Kienböck test strip in the vagina or rectum and subjecting it to radiation delivered through the abdomen will demonstrate the fact that at that depth the tissues will receive only from one-seventh to one-twelfth the dose, or even less, delivered at the skin surface when using the rays of greatest penetration now possible, and filtered through 3 to $4 \mathrm{~mm}$. of aluminum. The explanation lies in the absorption of the rays by the intervening tissues and the loss in intensity according to the law of inverse squares. It is inferred, therefore, that in order to deliver even the mildest destructive dose to a lesion 2 or 3 inches below the skin surface the rays must be directed to the lesion by cross-firing through at least seven to twelve or more ports of entry, giving the maximum safe dose that the skin will tolerate at each port. As the result of hundreds of thousands of applications of rays from Coolidge tubes backing up a parallel spark gap of 9 to $9 \frac{1}{2}$ inches and filtered through at least $3 \mathrm{~mm}$. of aluminum with leather added, it has been conclusively demonstrated that the skin will tolerate about twice as much radiation as could be given without the use of a filter. In other words, $20-\mathrm{X}$ Kienböck units, or eight units on the Hampson 
radiometer scale can be administered with no more and possibly less reaction than would follow one-half the same dosage without such a filter, with rays of like penetration.

Special Applications for Deep Therapy.-In the exact interpretation of the term, deep therapy implies the treatment of lesions at some distance from the surface, as intrathoracic, intra-abdominal and intrapelvic neoplasms and conditions which depend for symptomatic amelioration or cure upon the induction of a premature menopause by radiation of the ovaries. In all such cases cross-fire radiation is essential and can be carried out to excellent advantage. There are many other conditions, however, in which the lesions or structures to be treated are near the surface and may not always be amenable to cross-firing, but in which the same deep penetration and filtration are to be employed, as, for example, tuberculous adenitis and exophthalmic goitre.

Tuberculous Adenitis. - The same indications are recognized in the treatment of this condition as in former years, but the more modern technique is now employed. The region to be treated is mapped out into suitable areas, and each area is given a full dose of filtered rays, carefully measured. The application can then be repeated in three to four weeks, if necessary. Some roentgenologists prefer to divide the full dose.

Leukemia.-The improved technique in roentgen therapy during the past few years has, unfortunately, not altered the ultimate prognosis in this disease, although it has possibly bettered the general results. The employment of rays of higher penetration and adequate filtration has made it possible to administer more intensive radiation to the bones, but cross-fire radiation directed against the bone marrow does not seem to possess any advantages whatever, and in fact, judging from the amount of absorption that is known to take place in the tissues and the approximate intensity of rays reaching the bone marrow, it seems hardly likely that the comparatively small amount of radiation reaching this structure in the past has been sufficient to exert any direct destructive effect whatever upon leukemic cells. Certainly the attempt to cross-fire and deliver a destructive dose is followed by no better results than the older method of administering much smaller dosage. The greatest improvement in technique has been in connection with splenic applications. It is not now regarded as safe or rational procedure to expose the entire spleen at one time as was done in former years. The entire skin surface over the enlarged spleen should be mapped out into small areas from the size of a silver dollar to about twice that size. A full deep filtered dose should then be directed through one, or not 'more than two, of these areas daily, thus cross-firing through the spleen. Such a procedure is perfectly safe if not followed too early in a case showing toxic manifestations of the disease. The bone marrow should, of course, receive the same careful radiation as was formerly recommended and the bones should receive the prelimi- 
nary treatment until it has been satisfactorily demonstrated that the patient will not react in any unfavorable manner. The above method will be found far more efficacious in reducing the size of enormous spleens such as were formerly found to be so rebellious, and it will be found that the organ can be exposed to far more radiation without risk of injury to the skin.

Aside from the difference in the manner of radiating the spleen there has been little change in the technique of treating leukemia. Arsenic should be used in conjunction only when indicated, and then in small doses only. Benzol may act favorably in many cases but in some it may do harm, and in very exceptional cases only should it be employed in conjunction with roentgen therapy. There have been many reports of cases more or less successfully treated by radium applications over the spleen, but there is no reason why radium should have any advantage whatever over carefully applied deep roentgen therapy with proper cross-firing.

Pseudoleukemia.-Under modern deep therapy and cross-firing the prognosis in this condition is better than with the older methods, mainly for the reasons that more radiation can be made to reach the enlargements, both superficial and deep, without injury to the skin. When local enlargements are found in the neck or axilla, and especially when they exist in both localities, the chest should be very carefully examined, and if any mediastinal involvement is suspected, it should receive careful prolonged cross-fire treatment. In fact, this procedure is advisable in most cases with cervical lesions even though mediastinal glands are not discovered by the stereoscopic roentgenograms. If the enlargements are general or occur in one or both groins, pelvic and abdominal involvement should be suspected and treated. Even in superficial enlargements as in the neck, cross-firing should be practised as far as is practicable. In many instances in which the glandular enlargement in the neck is localized, more adequate cross-firing may be accomplished by the use of radium, provided it can be employed in sufficiently large quantities. In such cases also, it is often advisable to resort to surgery to remove the small remaining glands after they have become much reduced in size, but only in distinctly localized lesions. In all instances a superficial gland should be removed for confirmation of the diagnosis by the microscope. It may also be advisable, when the enlargements are more or less general and inaccessible, to remove one or more glands for the purpose of making a serum or vaccine to assist in the treatment. In cases with very large cervical glands the following precaution should always be observed: Sometimes the lesions may undergo a definite increase in size after an application, and if the entire area of the neck is treated at one time, the increase in size immediately following may be sufficient to exert dangerous pressure upon the trachea. Finally, a case should not be treated until the diagnosis is assured, and then, if the enlargements are extensive it is best to treat only one or 
two small areas the first day in order to determine whether or not there will be any undesirable reaction. The number of areas treated each day may then be increased gradually.

Polycythemia.-Added experience has shown that splenic treatment in these cases is more desirable, but if there is no response, applications to the bone marrow may be tried. Deep-therapy methods with cross-firing are, of course, indicated when the spleen is treated. In recent years some excellent results have been obtained with the use of radium, even when roentgen therapy has failed. If the former agent is employed, cross-firing should, of course, be practised.

Exophthalmic Goiter.-There has been a decided change in the technique employed in the treatment of this condition during the past few years, and this has seemed justified by the better and more uniform results obtained. There seems to be ample proof in literature for the assumption that more or less hyperplasia of the thymus gland accompanies the thyroid condition in a large percentage of cases of Graves' disease. Certainly thymic enlargement is definitely found in many cases and there is reasonable ground for believing that the two conditions are closely associated or that there is an intimate relation existing between them. In a large number of cases of Graves' disease in whom the thymus area was experimentally exposed and the thyroid carefully protected, ${ }^{1}$ many were apparently symptomatically cured by this treatment alone, while several others showed decided improvement. These experiments have been responsible for a decided change in the technique of treatment of exophthalmic goiter. A review of the literature prior to the past few years is of no value in determining the efficacy of roentgen treatment of this condition, as the technique has frequently been so crude, and in some instances, ludicrous, that statistics are entirely unreliable. The technique as now followed is based upon sound principles and far better results should be obtained in the future than in the past, provided sufficient time is allowed for a definite effect. A single series of applications without results means nothing, as the majority of cases require more than one series. In cases with small glands, each lobe should receive a full dose of $20-\mathrm{X}$ units delivered through $3 \mathrm{~mm}$. of aluminum and leather, and if the gland is large, cross-firing may be necessary on each lobe, with an application in the midline as well. In addition to this, the thymic region should receive from one to four applications through as many ports of entry, even though there may be no clinical or roentgenologic evidence of enlargement. A second series should not be given in less than three or four weeks. Following this, one or more series may be necessary, but as soon as there is a decided improvement in symptoms, the intervals should be further apart, in order to guard against the possibility of inducing hypothy-

1 Walter, Roentgenization of the Thymus Gland in Graves' Disease, Jour. Am. Med. Assn., April 24, I915. 
roidism. It is not advisable at the start to give an entire series of applications at one sitting as there is danger of stimulating the secretion of the gland and increasing the severity of the symptoms. It is best at first to treat only one, and never more than two areas at one time, waiting a few days before exposing any more. 'This is very essential in cases exhibiting very severe symptoms of hyperthyroidism. It should be borne in mind that the object of the treatment is to combat hyperthyroidism, and that when this is accomplished the applications should be discontinued. In perhaps the majority of instances there will be little or no change in the size of the goiter or in the exophthalmos.

Needless to state, whatever other forms of treatment that may be necessary and which will not conflict with the roentgen treatment, should be carried out in conjunction with the latter. For example, rest is usually essential, and anemia should receive careful attention.

It is appropriate to call attention at this time to the fact that there are many other lesions occurring in the neck for which heavy roentgen dosage must be administered, and that the thyroid gland may be subjected to sufficient radiation to materially affect its function. This is often unavoidable, but whenever the neck is exposed to heavy dosage the thyroid should be protected as much as possible.

Thymic Enlargement.-Until a few years ago, thymic enlargement in infants sufficient to cause dangerous pressure symptoms frequently ended fatally unless the pressure was relieved by operative procedures. Operation of course relieved the urgent pressure symptoms and no more, and if the case fully recovered it was due to the subsequent relative and actual normal involution of the gland. Partial thymectomy is, of course, a serious operation and attended with a definite mortality. Fortunately in recent years the use of the roentgen-ray in the treatment of thymic enlargements has made this condition one to be little feared and has yielded excellent curative results without endangering the life of the patient in the least, if properly applied. The first authentic instance of a case of thymic enlargement successfully treated by the roentgen-rays was reported in $1907 .{ }^{1}$

A study of the histology of the thymus will show why persistence of the enlargement responds so readily to roentgen exposure. Primarily the gland is developed by an epithelial invasion from the third branchial cleft. Were this the sole important origin of the gland tissues it is not likely that roentgen treatment would have any very desirable effect. Later the gland is invaded by lymphoid cells closely resembling the small lymphocytes of the blood in many respects. After the second year the original thymic structure is gradually replaced normally by fibrous and fatty tissue, although some of the characteristic tissues remain. In infancy the lymphoid structure of the gland is in excess, and it is to this, particularly, that the abnormal enlargements are due. It is probable that the lymphoid tissue of the thymus responds to roentgen exposure very much

${ }^{1}$ Friedlander, Arch. Pediat., July, I907. 
in the same manner as do the lymphoid cells in leukemia, and comparatively little exposure, certainly far less than an ordinary destructive dose, is required to promote a decided shrinking of the gland. Experiments upon animals ${ }^{1}$ have proven that prolonged roentgen exposure not sufficient to harm the skin or cause alopecia will produce a complete disappearance of the typical thymus tissue, leaving nothing but a fibrous structure. Such a complete atrophy is unneccesary and undesirable in the treatment of patients, and only sufficient exposure should be given to relieve pressure symptoms. About half to three-fourths of a full dose is usually all that is required at one time. Recurrences of symptoms are almost to be expected and the treatment should be repeated as soon as there is any evidence of pressure returning. Such patients should be kept under close observation until all danger from recurring enlargement is passed. Roentgen treatment is to be employed in cases in which thymic enlargement is sufficient to cause pressure symptoms and is not applicable to those cases in which symptoms are referable to the status lymphaticus.

Uterine Hemorrhage.-Uterine hemorrhage is a symptom referable to a large number of etiological factors and in certain well-defined causes deep-roentgen therapy has become an established form of treatment in selected cases. No reference will be made to malignant disease. The cases in which roentgen therapy is applicable may be divided into well-defined groups: (I) Cases with marked menorrhagia or metrorrhagia without any evidence of disease of the pelvic organs. This group may be further divided into adult women approaching the menopause, and young girls or young adult women, mainly because of the greater difficulty in treating the latter class and the more undersirable feature of inducing sterility. (2) Cases with hemorrhage due to polypoid endometritis. (3) Some cases of hemorrhage arising in connection with inflammatory conditions of the adnexa. (4) Uterine myomata.

It has been definitely established through experiments on animals and human beings that the testicles and ovaries are among the most susceptible organs of the body to roentgen-ray exposure in so far as inhibitory action upon their functions is concerned. It is probable that the effect of roentgen therapy in ameliorating uterine hemorrhage in the conditions just mentioned is largely if not entirely due to the inhibitory action upon the ovaries, with the exception of uterine myomata in which the effect of reducing the size of the growth is due, in addition, to the direct effect of the rays upon the tumor mass. The depressing effect upon ovarian function lessens the menstrual flow and suppression of function finally obliterates it. The relief of hyperemia or congestion tends to promote atrophy of the myoma and the direct action of the rays continues the atrophy and absorption. In myomata and the worst cases of hemorrhage due to other causes a complete obliteration of function of the ovaries is neccessary. 
The nearer the age of the patient is to her menopause the less the amount of treatment required to induce an artificial menopause, and also, the less the contraindication to sterility. In younger patients, the ovarian function is more active and the resistance to radiation is far greater, and it is, therefore, more difficult to obtain results. Because of this difficulty and for the reason that sterility is more undesirable, roentgen therapy has not so wide a field of application in young adults as in women near or at the menopause.

Before roentgen therapy is instituted in any case it is absolutely essential that the cause of the hemorrhage be determined beyond all possible doubt, and most especially must malignancy be excluded. It must be definitely proven that the case is one in which such treatment is indicated, and is to be preferred to any other procedure. Unless the roentgenologist is a gynecologist as well, he should treat no cases that have not previously been examined and passed upon by a competent gynecologist, who should remain as a consultant on the case throughout the treatment.

The most important indications and contraindications to roentgen therapy may be best considered in connection with fibroids. Fortunately we have in this method a procedure that can be applied safely and successfully to all cases in which there is the least contraindication to operation, and especially in women approaching or past the menopause. In such patients the contraindications to operation become indications for roentgen therapy, and they may be briefly enumerated as follows: anemia, organic heart or kidney disease, diabetes, pulmonary disease and exophthalmic goiter. The special contraindications to roentgen therapy are pedunculated tumors, fibroids undergoing malignant degeneration, sloughing fibroids, those complicated by operative diseases of the adnexa and in cases where life would be endangered by delay during the treatment.

The application of the treatment implies a technique that requires the services of the specialist in roentgenology. In any other hands it is not only ineffectual but extremely dangerous, but when properly applied, the dangers are nil. A description of the technique would be of interest only to the roentgenologist, and would be superfluous in these pages.

In gynecology is to be found one of the most successful fields for radium therapy, which is applicable in practically the same classes of cases as roentgen therapy. Fortunately, radium is an agent which can be easily handled by the gynecologist, and with safety, if he is careful to learn the physical properties of radium and the principles of its therapeutic action. It is essential that he should possess or have access to a quantity sufficient for the purpose for which it is to be used. There are many instances in which radium therapy is superior to roentgen therapy, and as a rule it can be employed with far less chance of inducing sterility than can the roentgen rays, which is a distinct advantage if sterility is not required and is undesirable. 


\title{
MISCELLANEOUS THERAPEUTIC PROCEDURES
}

\author{
By George P. Müller, M. D.
}

\section{BLOOD-LETTING OR VENOUS SECTION}

As mentioned in the previous article, this subject has received but little attention and is but little used by the practicing physician. Laurence $^{1}$ of the Harvard Medical School reports upon a series of cases in which venous section was performed for valvular disease of the heart with decompression, for chronic nephritis with hypertension, cardiac hypertrophy, circulatory failure, and impending uremia, and for cases of hyperpiesis. He believes that the venous section in these cases acted more promptly and more surely than drugs, it lowered blood-pressure, and at the same time produced a more efficient circulation when performed in cases of hypertension, but it does not lower normal arterial tension. The amount of blood to be drawn is to be decided by the point at which relief is obtained, the error generally being on the side of too small an amount. It may be repeated as often as the indication arises, within reason.

\section{ASPIRATION OF THE CHEST}

In addition to the remarks on page 685 , Vol. I, I would emphasize the good features of the method devised some years ago by Samuel Robinson. Instead of freezing the skin with ethyl chlorid, etc., as mentioned previously, Robinson injects novocain into the skin and then into all of the tissues to the pleura. This is done with a small needle and just as though one were making the puncture for the pus. In a few moments the large needle can be introduced painlessly and the specimen obtained. I would also emphasize the necessity of having clean sharp needles for this purpose.

\section{SALINE IRRIGATIONS AND INFUSIONS}

The use of physiologic salt solution for various medical and surgical conditions has become so universal that it may be considered a routine procedure. In the previous article I referred to the fact that Thies had called attention to certain dangers attending the indiscriminate infusion of salt solution. In I9I I attention was called by Evans ${ }^{2}$ to the dangers attending the indiscriminate use of normal salt solution, without previous knowledge of the condition of the blood-pressure, the ability of the heart to handle large amounts of

${ }^{1}$ Boston Med. and Surg. Jour., I9I6, clxxiv, 263.

2 Jour. Am. Med. Assn., rgI I, lvii, 2126. 
fluid successfully, or the functional capacity of the kidneys to excrete successfully the large amount of chlorid thus forced into them. He quotes Brooks' fatal case and reports a nearly fatal one due to the mistake of giving concentrated (stock solution) instead of normal solution. He particularly condemns its use in the toxemia of acute infections, in pneumonia, and in incompetency of the heart and kidneys. Sokolova ${ }^{1}$ showed by some experimental work that the injection of large amounts of fluid may prove directly injurious for an exhausted body. Many other protests have been published from research laboratories against the use of sodium chlorid in cardiorenal cases with chlorid retention, water retention, and many consequent evils. On the other hand, Henkel ${ }^{2}$ after careful observation believes that there is no reason to refrain from saline infusion even when there is edema, or heart defect, or nephritis. He has made much use of salt in eclampsia and has never witnessed harm but rather benefit.

An interesting article has appeared by Trout. $^{3}$ As a result of correspondence with 232 hospitals, he ascertained that the method of preparation of the so-called sterile salt solution varied from careful attention to the most minute details and the use of potassium, calcium, and sodium chlorid in varying proportions, filtered and sterilized, to the simple placing of two teaspoonfuls of table salt in I quart of tap water. Furthermore, following the directions of preparation given by the majority and using the amount of fluid said to be absorbed by the rectum by many, there would be forced into an already weakened patient in the space of twenty-four hours the average salt consumed as a condiment by a normal man in one month. For these reasons and because of the improvement in some patients with nephritis when placed on a salt-free diet, Trout believes that ordinary tap water is just as efficient in the relief of thirst and absolutely without any untoward effects. Several other communications have appeared upon this same subject.

\section{AXILLARY SUP}

The method of introducing fluid into the circulatory system adopted by Arbuthnot Lane is described by Foster." "The fluid used is normal saline solution which is placed in a reservoir to which is connected a "Y" rubber tube with glass connections. The single tube of this "Y" is sufficiently long to permit it to be coiled within a porcelain enamel basin filled with hot water kept at $110^{\circ}$. The saline solution is also poured into the reservoir at this same temperature. Such an arrangement allows of a drop of from $5^{\circ}$ to $10^{\circ}$ before reaching the hypodermoclysis needles. The reservoir is placed at a proper elevation so that sufficient column pressure is exerted to

${ }^{1}$ Russky Vratch, I9r4, xiii, abs., Jour. Am. Med. Assn., I9I4, 1xiii, I6I 5.

2 Münch. med. Wchnschr., I910, lvii.

Surg., Gynec. and Obst., 1913, xvi, 560.

- Surg., Gynec. and Obst., I914, xix, 248. 
easily force the saline into the subcutaneous tissues. The hypodermoclysis needles are always kept very sharp that they may pierce the skin with ease. They are 3 inches long, with the lumen large enough to permit a free stream of the fluid. Before introducing the needle the operator should be sure that no air remains in the tubes and that the saline running from the needles is of the proper temperature. This is a very important part of the technique as there is no reason for the procedure if the fluid is cool. We do not desire to take any heat from the body, but rather to add to the local temperature if it is necessary.

After the patient is fully under the anesthetic the chest is laid bare. The arms are secured at the sides by a retentive strap attached to the wrists and running beneath the buttocks or small of the back as the case may be. Any danger of extreme pressure upon the arms and forearms upon the table edge is avoided by padding with towels.

A section of the skin area just inside of the external border of the pectoralis major muscle over its most prominent part and above the mammary gland is cleansed with antiseptic soap. The suds are then wiped away and the skin perfectly dried with sterile gauze. This skin area is then painted with full-strength tincture of iodine. After this technique has been carried out in the strictest surgically clean sense, we have two circular skin areas, one over each pectoral muscle, about 3 inches in diameter.

While the fluid is running the needles are introduced piercing the pectoralis muscle in a direction outward and slightly tilted downward until the needle point is seen to push the loose skin of the axilla before it. The point is then moved about slightly to make sure it is properly situated and free.

A very important point when introducing the needles is to make sure they enter no part of the mammary gland. Should any portion of this gland intercede, the needle becomes occluded and seepage rendered imperfect, which may discourage the beginner. The technique of introducing the needles is the criterion for successful drinking during the operation. At the completion of the operation the needles are withdrawn and the skin apertures sealed with collodion and sterile cotton. It is very important that these apertures be sealed very tightly and watched a moment to detect leakage. The upper anterior chest and axilla are full and round. In fact, the loose subcutaneous space in the axilla is taken up and the space itself practically obliterated, and, as before stated, the appearance of the chest is that of a well-padded football player."

\section{BISMUTH PASTE}

Ten years have elapsed since the bismuth treatment of suppurating sinuses and abscess cavities was introduced into surgery and in the previous volume we described the method used by Beck. In his most recent article, Beck ${ }^{1}$ summarizes his experiences up to the ${ }^{1}$ Jour. Am. Med. Assn., ×9r6, lxvii, 2I. 
present time. He now uses a mixture composed of subnitrate of bismuth 30 per cent. and petrolatum, 70 per cent. In empyema cases he uses a ro per cent. mixture. He believes there is no advantage in substituting the subcarbonate for the subnitrate of bismuth in order to avoid bismuth poisoning.

The bismuth should be thoroughly incorporated with the petrolatum and entirely free from small lumps of pure bismuth. It also should be free from water, nor should water be allowed to drop into it during sterilization. The mixture should be thoroughly heated until liquefied and should be injected without undue force but the sinus tract should be filled up. The following is given by Beck as the technique to be followed in a case of tuberculous coxitis of long standing with multiple sinuses and it may be taken as an example of the method:

"I. Preliminary to the treatment, a set of stereoroentgenograms of the affected region is taken, to make sure that there are no foreign bodies or sequestrums present. If they were present, they might be overlooked after the bismuth had been injected, because the shadows produced by the bismuth would obliterate the shadow of the foreign body.

2. Bacteriologic examination of the secretion is the next step. Smear preparations, cultures, and, in some instances, inoculation of guinea pigs, are made.

3. The sinus is now ready for injection. No attempt should be made to irrigate the sinuses with antiseptic solutions, nor should any drying-out process be tried. The skin surrounding one of the sinuses is washed with alcohol, and the tip of the glass syringe, which has been filled with the liquefied paste, is placed firmly against it, and then the paste slowly but firmly forced into its channel until it is seen to escape from the nearest opening. Then the finger is quickly placed against this opening to prevent the escape of the paste, and the injection is continued until the patient begins to complain of some pressure. If there are many openings, an assistant must occlude all of them with his fingers during the injection, in order to be certain that all the branches of the sinuses have been filled.

4. After the injection another set of stereoscopic roentgenograms is taken, which will give a clear picture of the entire network of sinus tracts and sometimes be the means of tracing the path to the focus from which the disease originated.

5. A sterile bandage is then applied and the patient put to bed, for a few hours, or a few days, depending on the severity of the case. In subsequent treatments the patients are usually allowed to walk about immediately after the injection.

6. The first dressing is done the following day, if the discharge, which before injection was creamy or profuse, has changed to a serous consistency, it is to be regarded as a favorable sign, and a microscopic examination will usually prove it to have become sterile. If the discharge is sterile, the sinuses need not be reinjected Vor. IV. -10 
unless they later become reinfected. It is not intended that the paste remain in the sinuses. It will gradually exude, and within a week only traces may be found by fluoroscopic examination or by roentgenogram.

7. Should the discharge persist and remain purulent, the injections should be repeated at intervals of from five to six days for a reasonable length of time (from four to six weeks), and if after this period there is no appreciable improvement, one must search for the cause. A sequestrum may be present, or the focus of the suppuration be in such location as to be inaccessible to the paste, as, for instance in the cancellous structure of long bones.

In such cases, either the foreign body must be removed or, as the case may be, the infected cancellous structures of bone curetted, and the bismuth injections resumed."

\section{BLOOD TRANSFUSION}

Blood transfusion was employed with reputed good results long before the advent of modern surgery. It never became a therapeutic procedure of value until Carrel's work on the suture of blood-vessels had been done and Crile had introduced the metal canula which goes by his name and which has been modified many times. In I909, Brewer and Leggett ${ }^{1}$ introduced the use of paraffin-coated glass tubes for the conduction of the blood. Bernheim ${ }^{2}$ next introduced a metal tube in two pieces, one of which was introduced into the donors' vein and the other into that of the recipient, and these were subsequently joined together. In I9I I, Curtis and David ${ }^{3}$ introduced the use of large paraffin-coated bulbs, producing accurate measurement and satisfactory transfer of blood from donor to recipient. In I9I3 Kempton and Brown ${ }^{4}$ devised a cylinder for the receipt of blood from the donor and its subsequent transference to the recipient which, together with certain modifications by Vincent and by Percy, has proven to be the simplest and best means of blood transfusion.

A somewhat different line of investigation has been followed by the Mount Sinai Hospital group. Lindeman ${ }^{5}$ advocates the use of a number of syringes which draw the blood from the donor and introduce it to the recipient through ingenious trocar canulas.

Unger ${ }^{6}$ has recently devised a somewhat more complicated apparatus, the working of which, however, is said to be quite simple. This "modified syringe method" promises much, especially where it is impossible to secure a good assistant.

Finally, Hustin, ${ }^{7}$ Richard Weil ${ }^{8}$ and Lewisohn ${ }^{9}$ have advocated

1 Surg., Gynec. and Obst., r9o9, ix, 293.

2 Surgery of the Vascular System, r9 3.

Jour. Am. Med. Assn., rgr I, lvi, 35 .

- Jour. Am. Med. Assn., r9r3, lxi, i 17.

- Am. Jour. Dis. Child., July, r913, p. 28.

- Jour. Am. Med. Assn., r915, lxiv, p. 582.

7 Ann. et bull. Soc. roy. de Soc. méd. et nat., Bruxelles, 1914, No. 4, ro4.

8 Jour. Am. Med. Assn., I91 5, lxiv, 425.

- Surg., Gynec. and Obst., 1915, xxi, 37. 
the use of a sodium citrate solution added to the blood to prevent coagulation.

At the time the earlier volume of this book was published, transfusion was a formidable procedure, accessible only to those who possess especial skill in blood-vessel surgery and who had practised upon the lower animals the technique of blood-vessel anastomosis. Even with the Crile, Elsberg, Landon, or other canulas, clotting at the point of junction was frequent and the amount of blood transferred was never known. All this has changed with the introduction of the Kempton tubes. It is now possible for any one possessed of average surgical ability to learn to do transfusion, to do it successfully, and to know exactly how much blood is passed over.

In order to transfuse it is neccessary to have a donor who is not only healthy and whose blood gives a negative Wassermann reaction, but whose plasma neither hemolyses nor agglutinates the patient's red cells and whose cells are neither hemolyzed nor agglutinated by the patient's plasma.

A certain knowledge of the normal reactions of human blood is necessary for a proper understanding of this subject, but it is out of place here, and we can only describe the tests in use at the present time to determine whether the donor is satisfactory or not. In large institutions a number of prospective donors are examined and placed into groups, because it has been found that all adult individuals belong to one of four possible groups, so far as their agglutinins and hemolysins are concerned.

Moss ${ }^{1}$ characterizes the groups as follows:

Group I. Serum agglutinates no corpuscles. Corpuscles agglutinated by sera of groups II, III, and IV.

Group II. Serum agglutinates corpuscles of groups I and III. Corpuscles agglutinated by sera of groups III and IV.

Group III. Serum agglutinates corpuscles of groups I and II. Corpuscles agglutinated by sera of groups II and IV.

Groups IV. Serum agglutinates corpuscles of groups I, II, and III. Corpuscles agglutinated by no sera.

It is only necessary to determine to which group the patient belongs and then one of the donors of this group can be summoned and without further test the blood used.

Vincent and Minot $^{2}$ have noted that one may determine to what group a patient belongs (using both his cells and serum) if one has on hand serum and cells of only group II or group III. One may see how this is possible by referring to the characteristics of the different groups. For instance, if an individual's cells agglutinate with group II serum, he belongs to group I or III. If group II cells are agglutinated by the individual's serum, he must belong to group III or IV. Previously we learned that the individual could belong to group

1 Johns Hopkins Hosp. Bull., I910, xxi, 63.

2 Boston Med. and Surg. Jour., 1916, clxxiv, 667. 
I or III only, therefore, he cannot belong to group IV and must belong to III. Similarly it may be worked out for the other groups.

Methods for Testing Donors for Transfusion.-Rous and Turner ${ }^{1}$ have recently described a method for testing iso-agglutination which has been taken up by many of the laboratories. The blood is collected separately from the patient and donor in a mixing pipet such as is used for counting leukocytes. The pipet is rinsed beforehand with ro per cent. sodium citrate in water. 'The citrate solution is drawn up to the mark one; the pipet is then rapidly filled with blood from a puncture of the ear or finger and without pause the mixture is expelled into a small narrow test-tube. There is thus obtained the citrated blood containing slightly less than I per cent. of citrate. The pipets which they have employed hold only 0.25 c.c. of fluid. This much blood is easily obtained from a single puncture. There is no objection to increasing the flow by pressure. Should it cease before the pipet is full, the blood must be at once expelled into the test-tube, in order that it may mix with the citrate and clotting be avoided. The mixture is then taken up again, a new puncture made, and the pipet completely filled. After each blood is obtained, the pipet is rinsed with citrate, then with distilled water, then with fresh citrate, and it is ready for another blood. If several donors are to be tested, two pipetfuls of citrated blood should be obtained from the patient. It is best to take them from different puncture wounds, in order to avoid a possible clotting in the pipet. From these citrated specimens of blood, mixtures are made by means of capillary pipets, as follows:

I. Nine parts of patient's citrated plasma with I of donor's.

2. One part of patient's citrated plasma with I of donor's.

3. One part of patient's with 9 of donor's (if desired).

After the mixtures have stood for about fifteen minutes, a drop is placed on a slide and examined microscopically to see if agglutination has occurred or not. Though not necessary, it is desirable to add a drop of salt solution to the drop from the mixture, before looking for agglutination, so that the red cells appear more separated and one can see the agglutinated masses more easily. If there is no clumping in the mixtures, the two bloods do not agglutinate, and will not hemolyze each other. If clumping occurs in the first mixture, and to a less degree in the second mixture, it is certain that the blood of the patient agglutinates, and may, perhaps, hemolyze the donor's. Clumping in the second mixture, with little or none in the first, indicates that the plasma of the patient agglutinates the cells of the recipient.

Minot has modified Rous and Turner's method and performs the test as follows:

Place 3 to 4 drops of a 1.5 per cent. citrate solution in 0.9 per cent. salt solution in a short (about $2 \mathrm{~cm}$.) test-tube, with a relatively large diameter (about $\mathrm{I} \mathrm{cm}$.); then collect in this tube 9 drops of blood from ${ }^{2}$ Jour. Am. Med. Assn., I915, lxiv, r980. 
the recipient and $\mathrm{I}$ from the donor, and in another tube similarly collect 9 drops from the patient and I from the recipient. He prefers to use only these two dilutions, though one may also use a dilution of I drop from each individual, using 2 drops of citrate solution. 'The drops of blood are collected from a prick in the ear, trying to allow the blood to fall directly into the citrate, but it makes no difference even if the blood runs down the side of the glass. One does not need to have the drops exactly the same size, and the amount of blood does not need to be accurate, but approximate. After the mixtures have been mixed by shaking, and have stood for about fifteen minutes, one then takes out a drop and examines it microscopically for agglutination as described above. There is no reason why the citrated plasma cannot be collected separately by the above method, and the mixtures made from these, in which case one may have as a control, if one desires, the citrated plasma of each case.

Methods of Indirect Transfusion.-Of the various methods advocated and of the devices invented it seems necessary only to describe the method of use of the "Kimpton-Brown" tubes, the Unger "syringe-canula" method, and the citrate method.

Kimpton-Brown Glass Cylinders.-The special instruments consist of the tube and an ordinary cautery bulb. The tubes are of two sizes and are graduated up to Ioo c.c. and to 250 c.c. respectively. The safety and success of the operation depend upon the proper preparation of the tube. It is thoroughly cleaned with water, alcohol and ether, and dried. A small lump of paraffin or of "Vincent's mixture" (stearin, I part; paraffin, 2 parts; vaseline, 2 parts) is placed in the tube and the latter corked. The tube is then wrapped in a towel and sterilized in the autoclave. The tube is removed from the autoclave while still quite hot and then rotated until the entire inner surface is coated with the paraffin mixture, the excess being allowed to run out through the side arm. It is necessary to do this rapidly before solidification can occur. It is important to watch the canula tip and the side arm to see that no paraffin blocks the lumen. If obstruction occurs it can be removed by heating over an alcohol flame and then shaking the paraffin into the tube. The tubes are then wrapped in a sterile towel, next in sterile cotton, and kept until needed.

For the performance of transfusion, knives, scissors, mosquito hemostats, tissue forceps, small artery clamps, and hypodermic syringes for injection of the local anesthetic are needed. A bloodpressure armlet is almost essential to effect compression of the veins. Everything should be prepared and checked up before beginning the operation.

Under strictly aseptic precautions and under local anesthesia, a vein at the bend of the elbow of the donor is exposed and freed for a distance of $\mathrm{I}$ inch, and a vein in the arm or any other accessithe part of the recipient is similarly exposed. Each vein is ligitul, that of the donor distal and that of the recipient proximal to the 


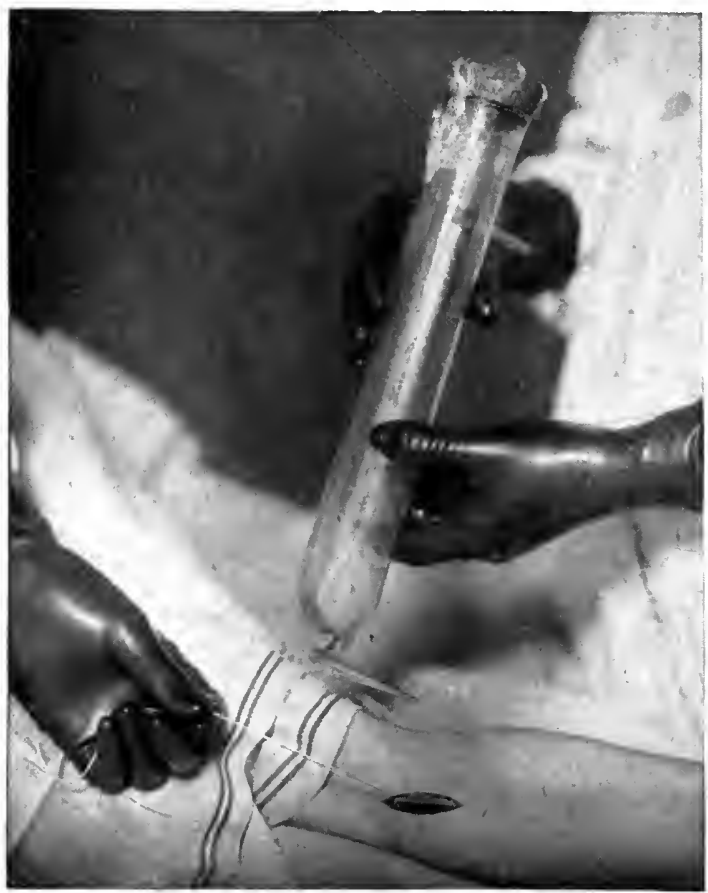

FIG. 29.-Donor's vein exposed, ligated centrally, and tube ready to be introduced into vein, the tip directed peripherally. (Mason, in "Surgery, Gynecology and Obstetrics.")

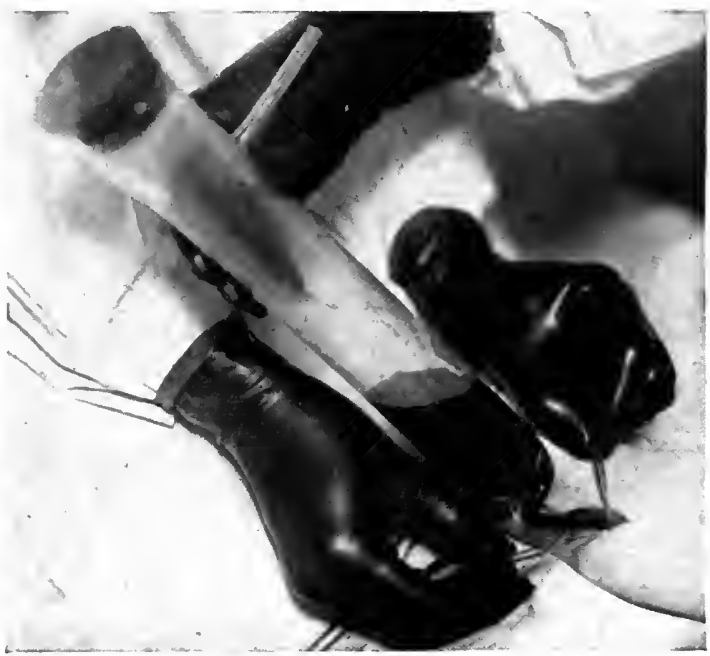

FIG. 30.-Tube in donor's vein, and blood flowing into the tube. (Mason, in "Surgery, Gynecology and Obstetrics.") 
incision in the vein. The wound should then be thoroughly washed in salt solution, rapidly dried, and some liquid paraffin dropped with a dropper over the vein. The adventitia is next stripped off

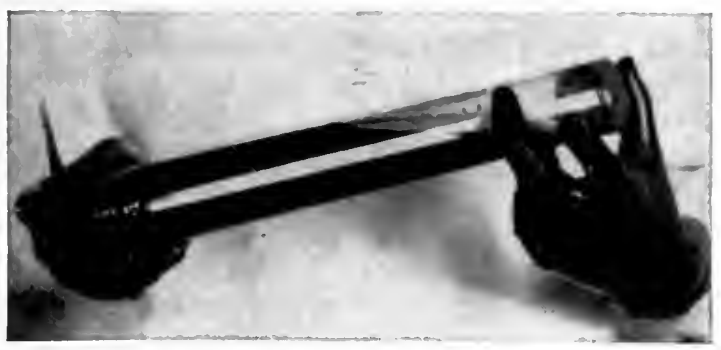

FIG. 31.-Proper method of holding the tube while transporting blood from donor to recipient. (Mason in "Surgery, Gynecology, and Obstetrics").

the anterior aspect of the vein and a Crile artery clamp applied so as to check the flow of blood when the vein is opened. A longi-

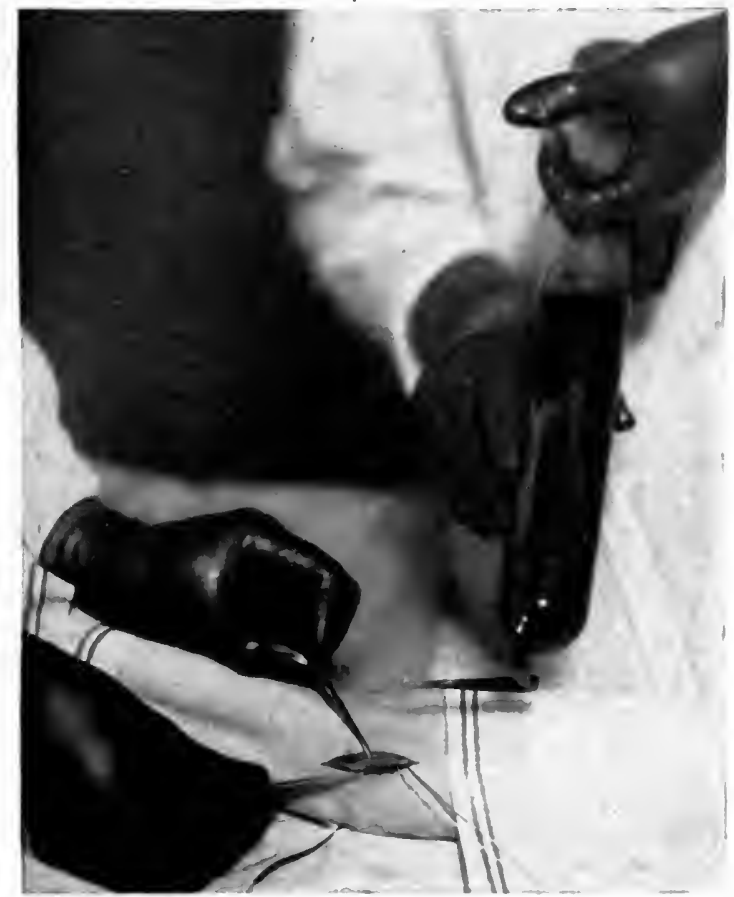

FIG. 32.- Recipient's vein exposed ligated distally, and tube filled with blood ready for introduction into vein, the tip of the tube directed centrally. (Mason, in "Surgery, Gynecology and Obstetrics.")

tudinal incision about $5 \mathrm{~mm}$. long is made with a sharp knife, preferably a cataract knife, and the vein instantly washed out with some salt solution in a dropper and then a few drops of paraffin are dropped 
over the incision. The edges of the incision are grasped with mosquito hemostats which hang by their own weight on the arm.

On the arm of the donor the rubber cuff of the blood-pressure apparatus should be placed before covering with the sterile towels and should now be inflated to about 60 to $80 \mathrm{~mm}$. of mercury. The canula tip of the Kempton tube is then slowly introduced into the vein and, of course, toward the hand, and the Crile clamp released. The blood should instantly flow into the tube and in from five to ten minutes fill the larger sized one. The donor is directed to open and close the hand slowly where the filling is proceeding slowly. When full, the tube is quickly withdrawn and turned on its side in such a manner that the tip of the tube and the end of the side tube are uppermost; at the same time the Crile clamp is replaced in posi-

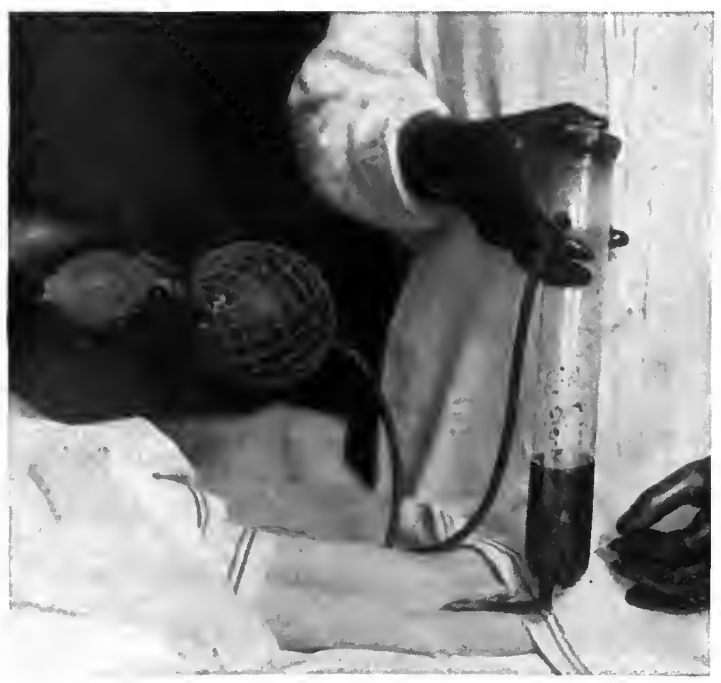

Fig. 33.-Tube in position, the bulb attached, and the blood being slowly forced into the vein of the recipient. (Mason, in "Surgery, Gynecology and Obstetrics.")

tion to check the flow of blood. The tube is then introduced into the vein of the recipient toward the heart and the cautery bulb attached by its tube. Very slight pressure with the bulb is then exerted and the blood flows into the vessels of the recipient at any rate desired. If more than 250 c.c. is needed, a second and a third and even a fourth tube are used to transfer the blood across. I have several times tried the use of the same tube over again but believe that it is attended with the great danger of inducing embolus.

The two most disagreeable complications in the technique which I have encountered are traumatism to the walls of the donor's vein with subsequent thrombosis, and blowing out of the corks while pumping the blood into the recipient.

Both can be avoided, however, with care and if the former occurs 
it may be necessary to open another vein; while, if the cork blows out, the nurse's hand carefully placed over the open end will serve the same purpose. The cork supplied with the tube is too coarse and the rubber tubes are not at all satisfactory. At the conclusion of operation it is necessary to immediately rinse the tubes in very hot water. This will soften the paraffin and wash out any blood clots.

Vincent Tubes.-These are similar to the Kimpton-Brown tubes but the canula tips are ground to fit into large-sized needles which are plunged directly into the veins without making an incision. A canula may be tied into the recipient's vein if it is very small and a sharp one used for the donor. The technique is then the same as above.

Percy Tubes.-These tubes devised by Percy ${ }^{1}$ differ somewhat from the Kimpton-Brown tubes as seen in the illustration. Percy coats these tubes with paraffin and then sucks into the tube 2 ounces of sterile liquid paraffin. This floats over and completely covers

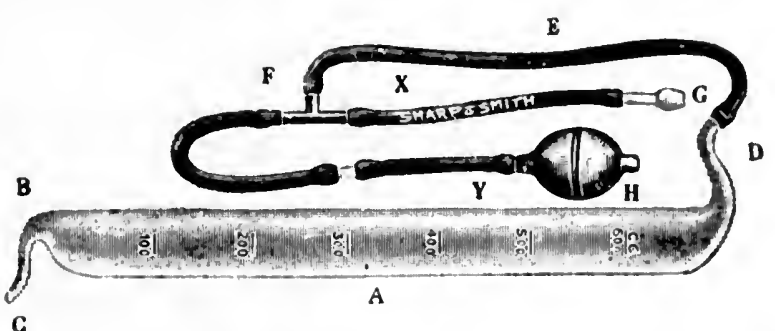

Fig. 34. - Instrument used in indirect method of transfusion of blood. (Percy.)

the blood, thus protecting it from the air. He uses a large tube holding about 600 c.c., and assists the flow from the donor by suction.

Unger "Syringe-canula" Method.-In I9I5 Unger" described an interesting instrument for syringe transfusion. The instrument. is well shown in the illustration. Unger advises that at least four of the 20 c.c. reformed Record syringes be kept on hand in case of clotting although in a later paper he states that by spraying the syringes with an ether spray clotting can be minimized or entirely avoided. By means of the stopcock $(C)$, a valve is manipulated which first allows the blood to be drawn up into the syringe from the donor and then allows it to be injected into the recipient. In the first position, the assistant is slowly injecting saline through the tubes and recipient's vein, thus kecping it clear; and in the second position the tubes and vein of the donor are kept clear. In other words, the operator simply fills and empties the syringe containing blood, while the assistant maintains a slow steady pressure on the saline syringe. As soon as the syringe begins to work with difficulty it is changed for a new one. The canulas and tubing are sterilized

'Surg., Gynec. and Obst., 1915, xxi, 360.

2 Jour. Am. Med. Assn., 1915, Ixiv, 582. 
and then placed for a few seconds in boiling paraffin. The tubes are then shaken in the air in order to distribute the paraffin equally, to get rid of the excess, and to hasten hardening.

Citrate Method.-The names of Hustin, Weil and Lewisohn are associated with blood transfusion by the citrate method. Lewisohn's technique ${ }^{1}$ is as follows: "The donor is put in a table, a tourniquet applied to the arm, and the vein punctured with a canula. The blood is received in a sterile glass jar (500 c.c.) containing 25 c.c. of a 2 per cent. sterile solution of sodium citrate at the bottom. While the blood is running into the glass receptacle, it is well mixed with the citrate solution by means of a glass rod. After 250 c.c. of the blood have been taken another 25 c.c. of citrate solution are added. If less than 500 c.c. of blood are taken (i.e., in infants), the amount of citrate solution added to the blood is reduced accordingly. In cases where we expect to take more than 500 c.c. of blood

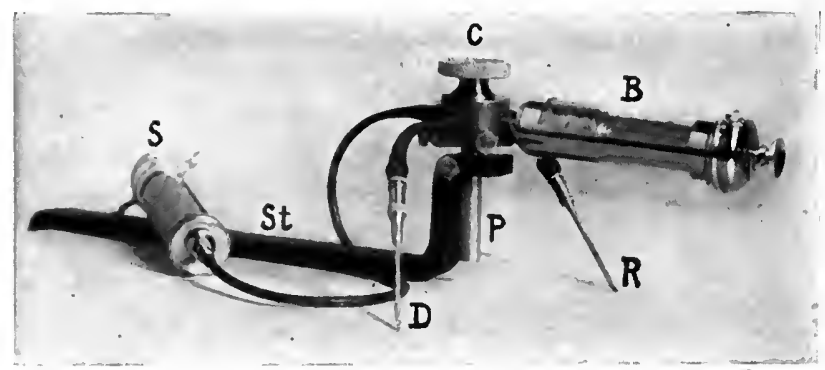

FIG. 35.-Unger's instrument for syringe transfusion: $C$, stop-cock; $B$, blood syringe connected to blood outlet; $S$, saline syringe connected to saline outlet; $D$, donor's canula connected to donor's outlet; $R$, recipient's canula connected to recipient's outlet; Si, stand; $P$, pedestal by which the stop-cock is raised or rotated.

we have another glass container ready to be used in exactly the same manner. The glass jar containing the blood is then put aside and covered with a towel to safeguard against contamination. I have not found it necessary to immerse it in hot water or surround the jar with an asbestos covering. The blood is then taken either into the recipient's room or the recipient is brought into the operating room. I consider it a great advantage that this method does not require donor and recipient to be in the same room; this lessens the psychical shock of the whole procedure for the patient. In fact, the donor's blood may be collected in the laboratory or office and carried to the patient's bedside (Kaliski). Another great advantage of the citrate method is that as there is no connection between the donor and recipient the donor is safeguarded against contagion of any disease or infection which the patient may have."

"The recipient's vein is then punctured or exposed by a small incision; the canula is introduced and attached to a salvarsan flask ${ }^{1}$ Surg., Gynec. and Obst., 1915, xxi, 37. 
or a glass funnel. It is advisable to fill the rubber tubing connection between the flask and canula with some saline solution, so as to prevent air from getting into the circulation. After the connection is made, the blood is poured into the salvarsan apparatus. In order to prevent sudden overloading of the circulation it is advisable (especially in larger transfusions) to stop the flow of blood from time to time by compressing the rubber tubing. After the blood has been injected the canula is removed and the transfusion thus ended. The whole procedure can be performed with the greatest ease and without any hurry, because the citrated blood, as we have seen above, can be kept for two or three days in the glass jar without danger of clotting."

It will thus be seen that Lewisohn uses an 0.2 per cent. solution. of sodium citrate and believes this is the point which prevents clotting and does not produce any deleterious symptoms. In a recent article by Garbat, ${ }^{1}$ the statement is made that sodium citrate, per se, is not harmful even if repeated injections are given over a long period of time. He employs an 0.25 per cent. final solution as it was repeatedly observed that an 0.2 per cent. solution was not sufficient to keep the blood of some donors from clotting.

Several fatalities have been reported in the literature, notably by Simon, ${ }^{2}$ and the method is still on trial. An interesting article upon the use of anticoagulins in transfusions is that of SatterleeHooker. $^{3}$ They have experimented with metaphosphate of sodium on animals and believe it has a more powerful anticoagulative effect than sodium citrate in proportion to its weight. They also warn of the toxic effects of sodium citrate as well as metaphosphate of sodium.

\section{ANEURYSM OF THE AORTA}

"This lesion is essentially a fatal disorder, although exceptionally spontaneous cure takes place. Accordingly, treatment can, as a rule, be only palliative, relieving symptoms and prolonging life. Deligation is inapplicable from anatomical considerations. Rest, ergot, iodids, gelatin, calcium chlorid, adrenalin chlorid and introduction of foreign substances, with or without the passage of a galvanic current, have been employed, Horsehair, catgut, needles, watchspring, and wire of various kinds have been introduced, with varying result, and a galvanic current has been passed through conducting substances. Wiring with electrolysis has been employed in a moderate number of instances, with satisfactory results in the majority. The procedure has proved simple and safe, but it is applicable only to sacculated and not to fusiform aneurysms."

The technique is relatively simple if one has the proper apparatus and the procedure followed by Hare is to be commended because

\footnotetext{
1 Jour. Am. Med. Assn., I9r6, lxvi, $15+3$.

2 Jour. Am. Med. Assn., I91 5, lxv, I 339 .

3 Jour. Am. Med. Assn., t916, lxvi, 618.

Eshner, Am. Jour. Med. Sci., October, rgro.
} 
of his exceptionally large experience. In his most recent article ${ }^{1}$ he states that he has performed this operation twenty-five times and that in no case has any accident occurred. One must have a hollow needle, not too large, insulated with French lacquer, and preferably made of gold. He emphasized that only specially prepared wire can be used and the alloy should be platinum and not copper because a wire made with gold and copper is liable to be eaten out by the current. He believes that silver wire, because it does not coil, is an unsuitable agent.

On the other hand, Hunner ${ }^{2}$ and Finney $^{3}$ use a wire made from a silver and copper alloy in proportion of 75 parts copper to I000 parts silver. Most recent writers arbitrarily use ten feet of wire. It is also necessary to have a large electrode because a small one produces considerable pain. Finally, a meter which will register milliamperes is necessary. If the streak current is used a table should be insulated and the operator wear thick; rubber-soled shoes, and stand on a perfectly dry floor.

The most superficial part of the aneurysm should be selected and after freezing the skin or injecting with weak novocain solution the needle is pushed into the aneurysm, the spurting of blood announcing the success of the procedure. The wire, which has been wound tightly upon a wooden spoke in order to give it a coil, is carefully fed through the needle until the full amount has been introduced. The shoulder of the needle must not come in contact with the skin, otherwise an electrolytic burn may result. The wire is then attached to the positive pole and the negative attached to the pad on the patient's back. It is absolutely necessary to follow this order because disorganization rather than clot formation occurs at the negative pole. The electric current should then be turned on and slowly raised during a period of forty-five or fifty minutes up to about 75 ma. At the end of this time the current should be slowly reduced to the zero point in about the same time and then the needle carefully withdrawn, the wire cut as short as possible, and the point pushed beneath the skin. During the operation the patient should be made as comfortable as possible, otherwise it will be necessary to cut short the operation, and full aseptic precautions should be taken to avoid infection.

For obvious reasons the operation is contraindicated in fusiform aneurysm and the diagnosis of the sacculated variety should always be confirmed by roentgen-ray examination. The operation is done with the intention only of prolonging life and of relieving pain, and these furnish the indications for it.

1 Jour. Am. Med. Assn., I914, lxii, 1217.

2 Johns Hopkins Hospital Bull., November, Igoo.

3Ann. Surg., 1912, p. 661. 


\section{FOOD INTOXICATION AND POISONING BY REPTILES AND INSECTS}

\section{By George Blumer, M. D.}

Since the publication of the first edition of this work there are two phases of the problem of food intoxication concerning which a considerable amount of information has accumulated. One of these is the relation of hypersusceptibility or anaphylaxis to food poisoning, and this was touched upon in the previous article. The other is the relation of certain diseases, such as beri-beri, pellagra, and scurvy to the deficiency of certain principles in the food. These principles have been usually described as vitamins and the diseases dependent upon their lack are, therefore, spoken of as the avitaminoses.

\section{FOOD POISONING FROM FOOD HYPERSUSCEPTIBIIITY OR ANAPHYLAXIS}

During the past ten years the amount of literature which has accumulated concerning the phenomenon of anaphylaxis is enormous. It has become clear that the phenomenon, far from being a laboratory manifestation of only theoretical interest, has a direct bearing on the clinical manifestations of a variety of diseases. In order to make this relationship clear it seems advisable to sketch in brief form the outstanding features of the condition.

The term, anaphylaxis, may be defined in a broad way as hypersensitiveness or hypersusceptibility to an alien protein. This hypersusceptibility may be inherited or may be acquired, and this is true not only of anaphylaxis as produced in the laboratory but also of the human manifestations of the condition. In laboratory experiments it can be shown that animals can be sensitized to any alien protein. If a rabbit or a guinea pig, for example, is injected with a small dose of egg albumin (sensitizing dose) and then receives, after a period of incubation of from seven to fifteen days a second injection of the same substance (reacting dose), the phenomena of hypersensitiveness appear. It is generally assumed at present that the phenomenon is merely a phase of immunity and results from a reaction between the reacting dose of the protein and specific antibodies which formed after the introduction of the sensitizing dose.

From the point of view of human pathology the importance of the phenomenon lies in the fact that under certain circumstances individuals may be hypersensitive to any alien protein, no matter how harmless this may ordinarily be. They may be hypersensitive to the proteins of any of the ordinary foodstufis which are without 
deleterious action upon normal individuals, and this hypersensitiveness may be either inherited or acquired. In many instances the exact time at which and method by which the sensitization took place cannot be determined. It is clear, however, that the sensitizing substances need not necessarily be introduced parenterally but that the alien protein may be absorbed from the mucous membranes of the respiratory tract or those of the digestive tract. It is probable that in both instances the mucous membrane is not normal; indeed, there is good evidence that so far as the digestive tract is concerned absorption of unsplit proteins only occurs when catarrhal conditions are present or where dietary errors, such as a diet containing excessive amounts of sugar, favor such absorption.

It is clear, therefore, from what has been said, that particular individuals may be naturally sensitive or may have been accidentally sensitized to any of the proteins of food. As a matter of practical experience it has been found that sensitiveness to certain kinds of food is relatively common, while sensitiveness to other varieties is rather rare. Hypersusceptibility to milk and eggs is not very infrequent, and most physicians have doubtless met with such cases. There are in the literature occasional instances of hypersusceptibility to a great variety of foods-shell fish, meat, strawberries, tomatoes, buckwheat, and a variety of other foodstuffs come to mind.

The clinical manifestations of food hypersusceptibility vary considerably in intensity and in the localization of their symptoms. In a general way, unusually sensitive individuals suffer when poisoned from the phenomenon of anaphylactic shock. In an infant with hypersensitiveness to cow's milk the administration of this substance results in nausea, vomiting, diarrhea, swelling of the tongue or lips, urticarial or erythematous skin eruptions, dyspnea, and in severe cases, great general prostration, feeble pulse, a clammy skin, and the usual manifestations of severe shock. In the many chronic forms of food anaphylaxis the manifestations may affect predominantly the skin. Of recent years this has been particularly emphasized by the dermatologists and pediatricians who have demonstrated that certain types of eczema and other chronic skin lesions, both in the infant and in the adult, are associated with hypersusceptibility to foodstuffs. It seems likely, too, that the erythema multiforme and its visceral manifestations have the same etiology, and that in some cases of bronchial asthma the irritant is introduced as a food.

The treatment of these various manifestations of anaphylaxis demands of necessity a recognition of the causative food. This can be decided by one of three methods, direct experiment, the employment of skin reactions, and animal inocculation. The method of direct experiment, in which the suspected protein is administered to the patient, is often satisfactory from the point of view of diagnosis but is naturally likely to result unpleasantly to the patient. For this reason the employment of skin reactions is more satisfactory. A von Pirquet test is made with a solution of the suspected protein and, 
in case of hypersusceptibility, a marked skin reaction takes place within an hour or two; indeed, in some instances constitutional manifestations occur. Inasmuch as the hypersusceptibility is transferable through the blood serum, tests may be made by animal inoculation. One or 2 c.c. of the patient's blood are introduced into a rabbit or guinea pig and at the end of twenty-four hours the animal is inoculated with a small dose of the suspected protein. A positive reaction would, of course, indicate a correct diagnosis.

The prevention of anaphylactic food poisoning is suggested by the conditions which favor its occurrence. In individuals with gastrointestinal disturbances alien proteins should be used cautiously as they may be absorbed unsplit at such times and sensitize the individual. In infants who have become sensitized to cow's milk it has been shown, for example, that very often there is a history of an attempt to wean the child resulting in slight gastro-intestinal disturbances. The child was then taken off cow's milk and was nursed again for a period of a week or two and the manifestations of milk poisoning appeared when the child was once more put upon cow's milk. It is not desirable where possibilities for absorption exist to give a single dose of a protein food and then allow an interval long enough for the incubation period. It is better to give repeated doses at intervals of a few days.

The treatment of the developed condition takes the form of the treatment of the actual attacks and the treatment aimed at overcoming the hypersusceptibility. In the attacks of anaphylactic shock, the usual stimulative measures must be applied; warm th to the extremities, hot drinks, cardiac stimulants, etc. Experience has shown that atropin (gr. 1/200-1/100 (0.0003-0.00065 gm.) of the sulphate hypodermically) is the drug which must be relied upon in the severe cases. Adrenalin (8-I 5 minims (0.5-I.o c.c.) of the I:I000 solution intramuscularly or intravenously) and barium chlorid (gr. $1 / 2-2(0.03-0.12 \mathrm{gm}$.) in solution) have also proved of value.

The cure of hypersensitiveness to an alien protein is brought about by the methods of immunization. The particular protein to which the patient is hypersensitive must be administered in gradually increasing doses. It will be necessary in many instances to begin the immunizing process with exceedingly minute doses, otherwise the patient will suffer from a reaction with each dose. The increase of the dose, too, will often have to be so gradual that considerable time will be consumed before a cure is accomplished. Experience has shown that in some instances there is a natural tendency for the hypersusceptibility to disappear. This is true of anaphylaxis to milk. In other instances it has been possible to produce a condition of immunity by the method above described.

\section{THE AVITAMINOSES}

The nutritional studies of the past decade have taught us that we must revise our conceptions of the physiology of food in certain 
respects. It has generally been held until recent years that, provided a sufficient quantity and correct proportion of protein, carbohydrate and fatty food, with their necessary contents of purins, lipoids, salts, and water were consumed, adequate nourishment of the body would result. The researches of Casimir Funk, of Thomas Osborne, Mendel and others, have shown that the presence of a new group of substances in food is essential both to adequate growth and to normal development. These substances were named by Funk "vitamins" and the diseased conditions which result from their lack in food are spoken of as the "avitaminoses." The substances named are associated with a variety of foods, are usually combined with certain protein elements of the food, and are essential to the proper nourishment of the individual. They have been isolated in a state of purity in a few instances and their relation to the experimental forms of certain diseases, such as beri-beri, has been well established. Many of our views concerning them are still hypothetical but so much has been learned regarding the results of their absence from food and so much can be legitimately assumed concerning their relation to certain human diseases that their discussion is profitable in this place. The three diseases which seem most clearly at the present time to come under the head of the avitaminoses are beri-beri, pellagra, and scurvy. Inasmuch as treatment of scurvy is discussed in another part of this volume only beri-beri and pellagra will be considered.

\section{BERI-BERI}

This is a disease that occurs mainly among those nationalities in whom rice is the staple carbohydrate food, namely, the Chinese, Japanese, East Indians and Koreans. The disease, however, is not unknown in temperate climates, and in both Europe and the United States has been occasionally observed among the inmates of institutions for the insane. The most characteristic form of the disease, namely, that associated with the consumption of polished rice, has been more extensively studied than any of the avitaminoses. As a result of this study it has become clear that in rice eaters the disease is due to the use of polished rice, i.e., rice from which the pericarp has been removed. Experience has shown that the consumption of the pericarp with the rice causes the disappearance of the disease in areas where it was formerly endemic. The disease is characterized mainly by the occurrence of an extensive neuritis affecting not only the peripheral but also the central nerves, like the vagi, and associated with atrophy of the extremities (dry form) or with extensive edema (wet form). The symptoms are those of a profound peripheral neuritis, often associated with great cardiac weakness. The prophylaxis of the disease consists, in rice-consuming countries, in the avoidance of polished rice. In these countries the pericarp must be consumed as well as the endocarp. Where the disease occurs in temperate climates among a population in which rice is not a carbo- 
hydrate staple it will be found that those attacked have been fed upon too restricted a diet and a study of the diet is necessary to determine what particular elements are lacking.

After the disease has declared itself also, the main indication is to provide the lacking principle. The resultant neuritis must be treated on the same general principles as neuritis from other causes. The cardiac weakness, which is such an important feature in some cases, must also be treated by cardiac stimulants and the usual measures adopted to counteract this condition.

\section{PEILAGRA}

There is still difference of opinion as to the exact nature of this disease. In the judgment of the writer the evidence which has been presented by Goldberger and his co-workers in the Public Health Service is sufficiently convincing to substantiate the view that this disease is due to a deficiency in vitamins. There is a possibility that the overconsumption of maize may play some rôle in the production of the disease, but inasmuch as complete recovery may occur under a diet containing maize, this seems somewhat doubtful. The evidence adduced suggests that the chief causal factor in pellagra is probably not the lack of any single vitamin but rather a general deficiency in vitamin-containing foods.

The disease is characterized by general debility, digestive disturbances, and skin eruptions which have a more or less seasonal occurrence. Usually in the spring the patient develops an erythematous eruption followed by a roughening and dryness of the affected part and sometimes by the formation of crusts. At the same time dyspepsia, diarrhea, and often salivation occur. The patient becomes weak and loses weight. After a few months these manifestations disappear only to recur again the following spring, and finally after many recurrences the patient develops marked changes in the nervous system, increasing paralysis of the legs, and pronounced mental deterioration, usually in the form of mania or melancholia with suicidal tendencies.

The prevention of pellagra is largely an educational matter. In the United States the disease is fairly well limited to certain of the southern States where the dietary of the inhabitants has been too restricted and has consisted very largely of molasses and corn with an insufficiency of milk, eggs, meat, and certain vegetables, particularly the leguminous vegetables. The conditions favoring the development of pellagra in a community are associated to some extent with poverty and to some extent with agricultural and dietetic ignorance. The inhabitants of these regions must be encouraged to produce their own milk, keep chickens, and particularly to raise those varieties of beans capable of being stored in a dry form so that these may be employed as an important part of the winter diet.

The treatment of pellagra must be along the same dietetic lines.

Vor. IV. -11 
Experience has abundantly shown that treatment of the disease by medication is at best accessory. Treatment by general tonics and drugs, such as alterative drugs like arsenic, while of value, is by itself of little use. The patient should be provided with and urged to take an abundance of fresh milk or buttermilk, fresh eggs, a reasonable amount of fresh meat, and the leguminous vegetables in a fresh or dried but not in a canned form. It will, of course, be necessary to regulate the form of administration of foods in cases of severe gastrointestinal disturbances, but even pronounced diarrhea is not in these cases a contraindication to feeding. The greatest difficulty in many instances is to prevent the patient from going back to his former faulty dietetic habits. 


\title{
DRUG POISONING AND DRUG HABITS
}

\author{
By H. C. Wood, JR., M. D.

\section{GENERAL ANTIDOTES}

So-called chemical antidotes to poisons may act either (I) by forming relatively insoluble compounds or (2) by virtue of physical properties which delay the absorption of the poison. As examples of the first group of antidotes are the use of albumen in corrosive sublimate poisoning, of iodine in the alkaloidal poisonings, and of sodium chlorid against silver nitrate. While the chemical precipitant must depend on the nature of the poison and an exact diagnosis might at first thought seem necessary, there are certain substances which are antidotal to so many of the common poisons that if for any reason diagnosis be difficult or the proper antidote be not at hand they may be used with good result.

Albumen overcomes the caustic action of most of the local irritants such as corrosive sublimate and other metallic salts, the mineral acids, and caustic alkalies. It may. be administered in the form of white of egg, milk, or hashed meat.

Magnesium oxide neutralizes mineral acids, many of the irritant metallic salts as corrosive sublimate, delays the absorption of alkaloids, and is next to the hydroxid of iron the most efficient antidote for arsenic. Two or three teaspoonsful may be stirred up in a tumblerful of water and of this mixture 2 ounces (6o c.c.) given every few minutes, or the milk of magnesia may be used in doses of 2 to 3 ounces ( $60-90$ c.c.), at frequent intervals.

Iodine in the form of the compound solution, commonly known as Lugol's solution, precipitates all the alkaloids and in the author's opinion is greatly superior to the more frequently recommended tannic acid in most alkaloidal poisonings. Of this 5 to ro minims (0.3-0.6 c.c.) in 3 or 4 ounces of water may be administered at a dose.

The second group of antidotes includes those which delay absorption by their physical properties. These of course are useful chiefly against those poisons which have a systemic action. It must be remembered that these absorbents only delay and do not prevent the entrance of poisons into the system and they should therefore always be followed with emetics and cathartics; nevertheless it has been experimentally shown that suprafatal doses of alkaloidal poisonings are borne without other treatment than the administration of absorbents.

Charcoal is antidotal to many alkaloids, most of the metallic salts, phosphorus, etc., and has long been used as a general antidote. The animal charcoal (bone black) is superior to the wood charcoal 
although the latter may be employed. Either one of them may be given in tablespoonful doses.

Fuller's earth is a mixture consisting chiefly of aluminum silicate, the basis of clay. There are many varieties of clay of this character upon the market all of which appear to have more or less absorbing properties, although there are wide variations between them. Their relative value seems to depend on their physical state rather than their chemical composition. The substance known as "Lloyd's reagent," which is prepared by elutriation of Fuller's earth, is probably the most valuable of this group of antidotes. Ordinary Fuller's earth-Terra Silicea Purificata, U. S. P.-is, however, a very valuable antidote especially in alkaloidal poisonings but the form of clay known as kaolin is greatly inferior. According to the experiments of Fantus ${ }^{1}$ it requires from 8 to 200 parts of Fuller's earth to absorb I part of poison, according to the poison and the source of the clay. As practical remedy Fuller's earth may be given in doses of $\mathrm{I}$ to 2 tablespoonsfuls. Its antidotal value is not the same for all alkaloids being relatively slight for strychnin but. very marked for morphin.

\section{CORROSIVE SUBLIMATE POISONING}

The effects of mercuric chlorid on the system after its absorption are of such violent character that any hope of saving the life of the patient depends upon the prevention of absorption, hence the prompt evacuation of the stomach and the neutralization of the poison are of the utmost importance. The most widely used and probably the best chemical antidote for corrosive sublimate is albumen, preferably in the form of white of egg, although milk and hashed meat may also be used. It must not be forgotten, however, that although the free use of egg white will lessen the immediate caustic action of the poison it does not prevent the constitutional results. Therefore, even if the patient has vomited it is well to wash the stomach out with the stomach tube or, if this be not obtainable, to insure its complete emptying by the use of emetics and large amounts of tepid water. Saline purgatives should also be given to prevent absorntion taking place in the intestioes.

After the danger of the immediate caustic effects is past there still remains grave peril to life in the later inflammatory changes which take place, probably during the process of elimination. These late injuries practically always involve the kidney and usually also the alimentary tract. The subsequent treatment of bichlorid of mercury poisoning, therefore, should consist in efforts to dilute the drug in the blood and, especially, in the excretions as much as possible, and to encourage elimination through the skin. For the first purpose large amounts of water are strongly indicated. If the vomiting has been controlled the patient may drink freely by the mouth. If there be not purging, large amounts of water can often

\footnotetext{
${ }^{1}$ Jour. Am. Med. Assn., I9r 5, lxiv, 1838 .
} 
be introduced advantageously by Murphy's enteroclysis, commonly known as the "drip method." Much more certain, however, is the absorption from the subcutaneous tissue and a quart a day of physiological salt solution may be introduced hypodermically. To encourage excretion through the skin the use of hot packs and other sudorific measures are important. Frequently there is violent purgation and the temptation is strong to stop the diarrhea by the use of tannin or opiates but unless it be so violent as to threaten exhaustion the use of active astringents should be avoided because they prevent elimination through the mucous glands. Bismuth subcarbonate, however, is valuable for its protective effect and may be used freely. Demulcent drinks, as acacia and the like, should be used to soothe the irritation of the throat and stomach.

In the advanced stages of bichlorid poisoning the inflammation of the kidney is so violent that there may be almost complete suppression of function. The author has seen a case in which less than 2 ounces of urine was secreted in five days. ${ }^{1}$ Partly for the purpose of maintaining elimination of bodily waste and partly in the hope of aiding the excretion of the metal through the skin, the use of sweat baths is generally advised. Occasionally Edebohls' operation of stripping the kidney has been performed with the idea of relieving the intense congestion and permitting the resumption of excretory function. Despite the severe damage to the kidney these patients, however, do not commonly show uremic symptoms and the author therefore does not feel that the operation is commonly indicated and the clinical results have not been encouraging.

\section{DELIRIUM TREMENS}

At the time the patient is first seen by the physician there is usually. not much likelihood of any of the poison being in the stomach or even intestines, but, unless there is an extraordinary asthenia, an emetic dose of ipecacuanha and a calomel purge at the beginning of the treatment are advantageous, not only to rid the alimentary tract of any unabsorbed alcohol but also to aid in the reëstablishment of gastric and biliary secretions. By some apomorphin is preferred, on the theory that it not only has the beneficial effects of an emetic, but also exercises a sedative influence on the nervous system; it is asserted that, at least in the milder cases of this condition, the hypodermic injection of $1 / 10$ grain $(0.006 \mathrm{gm}$.) of apomorphin hydrochlorid produces an emesis which is immediately followed by a refreshing sleep.

The most important factor in the treatment of mania à potu is the relief of the exhaustion. This is to be met, as in other similar states, by full nutrition and rest. The diet should be an easily assimilable one, highly nutritious and stimulating. Broths and meat extracts, especially the latter, may be stimulating and appetizing but they are certainly not nutritious and while they are useful as adju-

\footnotetext{
${ }^{1}$ Jour. Am. Med. Assn., 1915, lxiv, 507.
} 
vants should not be relied upon. The treatment of the digestion should be precisely along the same lines as after acute alcoholism (see Vol. I, page 793) with the exception that the more nutritious foods such as milk or eggs are to be added to the diet.

Rest of course is essential. The violent muscular exertion often expended in the maniacal excitement means not only a strain upon the enfeebled circulation and an exhaustion of the reserve store of energy but often leads to bodily injuries. The question of restraint in violent patients becomes a matter, therefore, of considerable importance. While manual restraint is preferred by many writers, in most institutions this is impracticable because of the number of attendants required. Under ordinary circumstances a folded sheet across the chest with ends firmly fastened to the bed is sufficient or, in very violent cases, muslin bandages may be applied to the wrists and ankles and the free ends securely fastened to the sides and foot of the bed respectively. Care should be taken to see that the bandages do not cause excoriations. Nerve sedatives, such as bromids or scopolamin, suggest themselves to quiet the excitement but although commonly used they rarely have much effect.

An all-important question is that of procuring sleep. So essential is this that there is a good deal of truth in the old belief that if a patient with delirium tremens sleeps he will recover. The ordinary somnifacient remedies, however, are almost completely inefficient in a fully developed delirium tremens, and it is extremely doubtful whether the too free use of chloral, paraldehyde, morphin, and the like may not do as much harm by their depressant influence upon metabolism as they do good by their tendency to produce sleep. Ranson ${ }^{1}$ has published the statistics of 500 cases of delirium tremens treated in the Cook County Hospital (Chicago). He finds that the mortality in fully developed delirium tremens was actually higher when these hypnotic drugs were used than when they were not; on the other hand in the predelirium stage the use of the bromids or chloral or similar drugs greatly lessened the occurrence of delirium and consequently the mortality.

Nevertheless we believe that the hypnotics properly used are valuable adjuncts in the treatment of delirium tremens. One reason for the bad statistical result quoted above is the reckless size of the doses employed. The desire to quiet the patient is so strong that the physician keeps on piling in somnifacients with complete forgetfulness of the total dose absorbed. It must be remembered that the aliphatic series (which includes chloral, paraldehyde, veronal, trional, etc.) are essentially alike in their effects and to administer two of these drugs is equivalent to a double dose of one of them. On the other hand, morphin and scopolamin (hyoscin) belong to entirely different pharmacological groups and enhance the hypnotic effect without proportional increase of toxicity. A combination of paraldehyde I fluidram (4 c.c.), morphin $1 / 4$ grain (0.015 gm.), and scopolamin

${ }^{1}$ Jour. Am. Med. Assn., I909, lii, I 224. 
$1 / 100$ grain $(0.00065 \mathrm{gm}$.) given simultaneously and not repeated for twenty-four hours will prove, we believe, of material assistance. The bromids are not useful as somnifacients, but may be used to quiet the nervous restlessness in the waking periods. Ten grains $(0.6 \mathrm{gm}$.) of either ammonium or sodium bromid may be given every two or three hours.

The question of the use of alcohol is one about which there is so much difference of opinion and so little real evidence that one cannot afford to be dogmatic on the subject. It is very doubtful whether it really improves the patient's chances of recovery, especially if the aliphatic narcotics-which are closely related to it both chemically and physiologically - have been employed, and it is probably wiser to omit it.

Foremost among the symptomatic measures indicated in mania a potu is the stimulation of the circulation. In the studies of Ranson the only drug which, statistically at least, had any influence on the mortality was ergot. This undoubtedly acts by its effect upon the vasomotor system, increasing the circulation and consequently the nutrition of the brain. The fluidextract of ergot is by far the most efficient preparation and may be given in doses of $1 / 2$ to I fluidram (2-4 c.c.). Full doses of digitalis have been highly lauded by many writers. Strychnin ( $1 / 20$ grain ( $0.003 \mathrm{gm}$.) every two or three hours) and other circulatory stimulants should be used as indicated.

Recently at the Philadelphia General Hospital, lumbar puncture followed by the intraspinal injection of magnesium sulphate has been used with good effects. About 20 c.c. of the spinal fluid are withdrawn and 5 c.c. ( 75 minims) of a 25 per cent. solution of magnesium sulphate injected.

\section{THE OPIUM HABIT}

The treatment of a drug habit naturally divides itself into three stages: withdrawal of the narcotic, the restoration of the diseased nervous system to an approximately normal state, and the prolonged convalescent period during which it is necessary to guard the patient against a relapse. An undue amount of emphasis has been laid upon the first stage. The various so-called "cures" are mostly simply" methods of abbreviating the period of suffering or alleviating its intensity. It must not be forgotten that these patients are physically, mentally, and morally below par and the obliteration of the acute craving for the drug does not restore their nervous system to a normal condition, and until their moral tone is built up they are almost certain to relapse to their former state on the slightest provocation. Time alone can accomplish this. It is absurd to imagine that any medication or system of therapeutics can regenerate a thoroughly diseased organism in a few days.

To be permanently successful the treatment of a drug habit necessitates the removal of the original cause of the addiction. If there be a continuing demand for physical relief, such as constantly recur- 
ring pain or asthmatic paroxysms, it is too much to expect a person whose moral resistance has been destroyed by prolonged use of narcotics to abstain from obtaining relief. It might appear superfluous to add also that one can hardly hope to cure a patient who does not desire to be cured were it not for the fact that it is so often attempted. In many cases the most important step is to implant a real desire for reform.

Withdrawal Stage.-The first requisite in the treatment of a drug addiction is to obtain absolute control of the patient. It is essential that the physician know with complete certainty that the patient is receiving no drug, of any kind, not ordered. It is extremely difficult in the ordinary case to have this absolute control in the patient's home. There are so many opportunities for the clandestine use of the drug and the members of his family are so excited by his suffering that the patient can never be left alone a minute either day or night in his own home. On the other hand, in properly managed hospitals or institutions, if visitors are not admitted, one may be reasonably certain that no unordered narcotic is reaching the patient. The question whether the treatment can be better carried out at a special institution for drug addiction than at a hospital is one about which there is at present considerable difference of opinion. Although this class of patients oftimes cause so much trouble that many general hospitals refuse to admit them, good results may be achieved outside of the special sanitaria.

The first question to be settled is the rapidity of withdrawal. The general trend of medical opinion today seems more and more toward a very rapid or immediate cessation of the narcotic. Excepting patients in very bad physical condition or when the dose has been extremely large, the following withdrawal plan may be used. First, it is necessary to determine the quantity of drug which the patient has been taking; the patient's statements in this matter are often not reliable. After this amount has been determined, the drug is abruptly stopped. On the first day the patient receives no opiate of any kind, on the second. day he receives at night a single dose of about one-third his ordinary daily ration. After this none of the drug is exhibited unless the symptoms become alarming, in which case it may be necessary to administer a small dose of the narcotic on the fifth or sixth day. Under this plan of withdrawal there will be ordinarily intense restlessness or even actual pain but the unpleasant symptoms can be greatly lessened by skillful treatment.

The author does not believe that routine systems of management are any more suitable for narcomania than for any other disease, but there are certain measures which are so frequently of service that they require especial mention. Among these may be named first, purgation. None of the various explanations which have been given of the beneficent effects of purgation in drug addictions are completely satisfactory but its utility seems abundantly 
established by clinical eviderce. The cathartic employed is not a matter of any great importance except that it must be powerful enough to act not merely as a laxative but as a rather strenuous purge. Perhaps as useful as any is 1o grains ( $0.6 \mathrm{gm}$.) of blue mass followed in eight hours by an ounce $(30 \mathrm{gm}$.) of magnesium sulphate or of Rochelle salts, the latter to be repeated every two hours until free purgation is obtained. 'This catharsis should be repeated daily for the first three days of the withdrawal and as frequently as may seem necessary thereafter.

Among the most useful sedatives for the control of the intense restlessness preference should be given to the drugs of the solanaceous series. Some authorities prefer scopolamin (hyoscin), some atropin, and some a mixture as represented in hyoscyamus. Here again the effects on the individual patient should be the determining factor in choice of drug. Whether scopolamin or atropin is used it is generally necessary to administer doses larger than ordinarily employed but it does not seem usually advisable to push them to the point of toxic mania, as is sometimes recommended. Full doses (30 to 40 grains $(2-3 \mathrm{gm}$.) three or four times a day) of the bromids are often of service. Other sedatives, as valerian, occasionally exercise some effect.

A number of writers have recommended ethylmorphin (dionin) or codein for the relief of the abstinence symptoms. While these drugs are less powerful than morphin or heroin in their effects upon the nutritive processes, as far as we know their actions are precisely similar in kind, and the author feels that they should be used very circumspectly if at all. Hydrotherapeutic measures such as cold full bath, cold pack, douche baths, and similar procedures which tend to excite the peripheral circulation, are often of great service. Narcomaniacs, like other neurasthenics, are very susceptible to suggestion. For example in patients who have been using the drug hypodermically, injections of distilled water will often have a remarkable sedative action, and the most violent symptoms of heroin "sniffers" may be at least temporarily assuaged by the sniffing of milk sugar if it is called heroin.

During the withdrawal period there are various troublesome abstinence symptoms which may arise that require symptomatic relief. One of the most common of these abstinence symptoms is disturbance of digestion as shown by loss of appetite, intense nausea, and very frequently vomiting. The nausea is usually much worse in those patients who have not been thoroughly purged. During this period of gastric hypersensitiveness it is ordinarily not advisable to force food upon the patient as it is liable to be immediately rejected and twenty-four or forty-eight hours rest for the stomach is of great assistance in restoring digestive function. If there be extreme emaciation small quantities of milk containing a dram of whiskey may be administered for a short period until the stomach will accept solid food. For the nausea, alkalies- 
as soda or magnesia are of great value and may be advantageously combined with the local gastric stimulants-such as peppermint or ginger. While during the first few days of the withdrawal period there is usually very marked constipation, after the opiate has been completely eliminated from the system there is apt to occur a profuse diarrhea which may prove a serious drain on the patient's strength. For this diarrhea we have found a combination of aromatic sulphuric acid with one of the tannin containing drugs such as gambir or kino, to be usually efficacious. For the insomnia which is an almost universal symptom, the aliphatic hypnotics as chloral, trional, paraldehyde, or veronal, may be used, but in moderate dose usually fail to produce the desired effect and in large quantities are depressant to the nutritive functions. Moreover, the physician must not lose sight of the fact that these drugs may themselves give rise to a habit. The hot bath or pack are often serviceable for their sleep-producing effects. While the wakefulness of course is unpleasant for the patient it is rarely dangerous and one or two nights' loss of sleep should not occasion alarm. If, however, sleep does not come and the moderate doses of the ordinary somnifacient drugs have failed, a single dose of morphin may be given, of course without permitting the patient to know what he is getting.

In general the hospital treatment should last at least two weeks or, in most instances, longer. Whether it is safe for the patient to leave the institution at the end of this time will depend on conditions in his own home. Certainly he is not cured of his habit and requires careful medical attention. Generally they are so debilitated physically and nervously that a more or less protracted form of rest cure is required. The treatment during this period of upbuilding is along the same general lines as in other forms of neurasthenia. Nutritious easily digested food and ample rest are the main necessities; digestive stimulants, hematinics and circulatory tonics should be exhibited as indicated. If there be undue nervous excitability bromids may be used but the stronger narcotics should be sedulously avoided. Proper hydrotherapeutic measures to tone the circulation and to stimulate metabolism, graduated exercises as the strength increases should be employed. Careful attention to the general health and comfort of the patient is of the utmost importance as the most trivial diseases may become determining factors in the success of the cure.

One trouble which at this stage of the treatment will tax severly the ingenuity of the physician is to provide mental distraction. While the acuteness of the craving will have been largely blunted by this time there is, nevertheless, a sense of discomfort and an active recollection of the relief which might be provided by the narcotic. Mental idleness always increases the sharpness of the pain and the fact that the sufferer is perforce forbidden from the customary distractions of his life increases greatly the seductiveness of his temptation. Not only, therefore, should 
all possible effort be directed to diverting his mind from his own distresses but he should be most carefully watched in order that it may be made as difficult as possible for him to obtain an opiate.

The duration of the second stage of treatment may vary anywhere from one to six months. Gradually there is acquired a sufficient degrec of physical and moral strength to permit of the assumption of a part of his normal daily life. But even after the patient has recovered his strength sufficiently to return to ordinary habits the physician must not flatter himself that the cure is permanent. At the least departure from the even tenor of his way there will always be grave danger of the patient flying for relief to the old enemy. He should report frequently to the physician not merely in order to be sure that he is not getting the narcotic but also for an immediate correction of those slight physical ills which his still unstable nervous system so magnifies that they may become the cause of a relapse. A cure cannot be regarded as permanent until the patient shall have gone for some two years without the use of his narcotic.

\section{COCAII}

While the habitual use of cocain is one of the most serious of drug habits in its effects on the general health and while permanent cure is not usually accomplished, the withdrawal of the drug is much easier than the withdrawal of the opiates. The users of cocain take the drug for the sake of the pleasurable sensations produced while the users of morphin continue it only for the prevention of the suffering which follows its deprivation; it has been well said that cocainism is a vice, morphinism a disease. In treating the cocain habit the drug should be immediately withdrawn. Usually no worrisome symptoms occur and all that is needed is to upbuild the patient's strength and general moral tone. 


\title{
PROPHYLAXIS AND GENERAL TREATMENT OF THE OCCU- PATIONAL DISEASES
}

\author{
By W. Gilman Thompson, M. D.
}

The prophylaxis of the occupational diseases concerns two main objects: (I) the humanitarian, which is in common with all efforts to conserve the health of the community; and (2) the promotion of efficiency in labor which invariably suffers through ill health of the workman, and the cares or worries incident thereto. This fundamental economic principle has recently so impressed a mumber of large industrial corporations, employing thousands of workmen, that they are seeking to extend the hygienic care given the laborer to his home and family, realizing that a man works better and more actively when not harassed by domestic anxieties, or unhealthful and therefore depressing home environment.

The prophylaxis of the industrial diseases should be considered under: (I) the hygiene of the workroom or factory; (2) the hygiene of the workman. Otherwise stated, this concerns what can be done to protect the workman, and what the workman may do to protect himself. Generally speaking, special emphasis should be given to the former method, for the average workman, through ignorance or stubbornness, is notoriously slow in practising suggestions for self-protection.

The methods of prophylaxis may otherwise be subdivided into those which are generally applicable in any hazardous industry, and those which are special, being applicable to particularly hazardous industries.

\section{GENERAL HYGIENE OF THE WORKROOM OR FACTORY}

Space.-The factory health laws in those states in which they exist require from 250 to 300 cubic feet of air space per workman as a minimum, but it is most difficult to adjust this matter through legislation, owing to the varied nature of the hazards encountered, and the frequently changing processes of manufacture, often in the same building. Moreover, the cubic air space above the workman should bear definite relationship to floor space, and this is defined also in some of the factory laws. Much depends, too, upon whether or no artificial ventilation, as by exhaust ducts, is employed, and in some industries the casual requirements for ventilation exceed those of routine work. For example, in a brass foundry at the moment of "drawing" the molten metal zinc found may fill a large room almost to suffocation, and in general foundry work should be con- 
ducted only in one-story buildings with lofty roofs and open skylights. A cotton-spinning mill with a minimum air space may be unhealthful, but may be rendered healthful by properly maintained artificial ventilation. A "sweat shop" may be brought within the legal requirements of air space, but still remain unhealthful if leaky gas stoves are employed for heating the pressing irons. 'The writer has seen a paper box factory in which the boxes were piled as fast as they were made, so as to occupy more than half the legalized air space of the workroom.

Ventilation.- The ventilation of factories is more or less under State control through the factory laws, but these laws obtain in only a few States and are very incomplete. At best they cannot control the ventilation problems in many industries where manufacturing processes are subject to frequent change. They comprise twofold problems: first, the ordinary ventilation of any large building in which many employees may be crowded together; and second, the ventilation of buildings in which steam and other moisture, toxic fumes and dusts, excessive temperature, products of incomplete combustion resulting from the use of gas or petroleum for heating apparatus or for lighting, products of evaporation as from tannery tanks, etc., and many other deleterious conditions must be dealt with. There are no more difficult hygiene problems to deal with by legislation than those of ventilation. Then there are the large groups of workmen who are not employed in buildings but must work in foul air, as in scraping paint inside the double bottoms of warships. varnishing the inside of large closed tanks, scraping or sandpapering paint in small ill-ventilated compartments, cleaning out sewers and the precipitation tubes of cement works, etc. In some occupations such as working in cotton-spinning mills, artificial moisture must be supplied and windows may have to be kept closed. Open windows, desirable as they always are, render the use of a closed system of ventilation with blow fans and exhaust ducts impracticable unless great suction power is employed. Where fans and ducts are used their operation is expensive and there is a tendency to economize ventilation at the expense of the health of the employee. Wherever possible swivel or casement windows which admit of regulated opening should be installed with transoms above. Outside dust may be controlled by wire or cheesecloth window screens and steam or water radiators should be placed beneath or near windows. The use of clectric fans thoroughly to change the air of factory rooms is most desirable, and vertical flues placed in the wall and leading to the roof aid ventilation like a fireplace chimney, especially when fitted with a gas jet to favor an upward current of warmed air. Workmen naturally like to be comfortable and dislike drafts from open windows, but it is a decided error to regard personal "comfort" as a criterion for estimating the fitness of an atmosphere to breathe. A person may feel quite "comfortable" in an atmosphere so impregnated with illuminating gas as to prove fatal. 
Flooring.-The principles involved in proper flooring are as follows: (a) To prevent constant dampness of the feet where, as in tanneries, paper mills, steam laundries, etc., water or condensed steam keep the floors constantly wet. In such cases slats or grllls should be employed to keep the feet an inch or two above the floor. To prevent flat-foot, sciatica and in women, dysmenorrhea and undue fatigue by constant standing, almost in one position on too hard a surface, as for example in tending looms or other machinery. Instances may be found where one foot being used to operate a treadle or trip a lever, the extra pressure put upon the other foot leads to the gradual grinding of quite a deep hole in the floor. In all such work mats of some sort should be used to lessen strain. (c) To promote cleanliness, wherever much dust is produced the flooring should be of asphalt, concrete, tiling, cement, or similar impervious material to permit of daily washing. This is also important where animal products are manufactured as in tanneries, slaughterhouses, bone fertilizing plants, etc., so that the flooring may be scoured with antiseptics. (d) To prevent jarring by heavy machinery in motion, such as looms or engines. The writer met with cases of neurasthenia in young girls traceable entirely to the incessant jarring of the floor where they worked by a heavy engine in the room adjoining. When the flooring was properly braced the trouble ceased. Rubber mats lessen vibration. (e) Whereas floors should be smooth, as a rule, to facilitate cleansing, they should not be allowed to become slippery. In a factory where an oil spray was used to lay dust, several serious injuries occurred from slipping on the floor which might have been prevented by a rubber mat or a few bevelled slats. In rubber manufactories where much soapstone is used, the floors become very slippery.

Lighting.--Modern factories, as a rule, are being well lighted, but in old buildings in which electric lighting has been recently introduced, the lights are usually so placed, as to strain the eyes. When possible, swinging or fixed lights should be placed behind or at the side of the workman, rather than in front, and a diffuse well-screened white light is always preferable. In a very dark machine shop into which adequate lighting was finally introduced, one of the workmen made the significant comment that he "wore out fewer shoes, because he did not hit his feet against so many projecting angles." In addition to injuries to the eyes, inadequate or misplaced lights give rise to headache and a degree of nervous disorder which is rarely fully appreciated.

Lockers.-It is most important that all lockers be constructed of openwork galvanized or other metal, non-absorbing, and free to air currents. The locker room should be provided with special heating apparatus to dry the workman's clothing which may be wet from outside storms, and his overalls which may be wet through perspiration, or by the steam or water encountered in many kinds of work. 
Toilets.-Where State factory laws regulate the number of toilets, a minimum is required of one for each 25 employees, with separate rooms for the sexes. Directions for their proper use and care should be posted conspicuously, particularly where ignorant foreigners are employed. The closets should be made verminproof with impervious washable floors, and flushing should always be automatic. A cold, damp, dark, malodorous closet tends to shorten the process of defecation and induce constipation.

Cuspidors should be placed wherever men are employed in indoor manufacturing to receive tobacco juice as well as sputum. They should be cleaned daily.

Drinking water should be made accessible so that too much time is not taken from work to obtain it. An abundant supply is particularly desirable where the nature of the work induces excessive perspiration, as among founders, stokers, kilnmen, et al. The water should not be too cold as such workmen are apt to distend the stomach by drinking large quantities, but if they have good water they may drink less beer at the lunch hour.

Lunch rooms.- Separate lunch rooms are now supplied by many large corporations and do much to promote health and cheer among the workmen. In no case should workmen employed with lead or other toxic materials be permitted to eat lunch on their dust-covered work tables, or in fact anywhere in the workroom, so easily are poisonous dusts conveyed to the mouth with food or by soiled fingers. It is a mistake to permit shortening of the lunch hour. As an experiment in a paper manufactory where several hundred girls are employed, it was lengthened by fifteen minutes with a surprising gain in economic efficiency.

Cleaning and dust removal should be conducted daily in the workroom as soon as the employees have left for the day and in exceptional cases also during the lunch hour. Dry sweeping of dust is always to be condemned. Window ledges, work tables, etc., should be wiped with moist cloths, and floors should be swept or mopped after moistening with moist sawdust, paper fragments or similar material, or after spraying with water, steam or an atomized oil. In some cases vacuum cleaning may economically be employed.

\section{SPECIAL HYGIENE OF THE WORKROOM}

It is impossible within the limitations of this article to more than allude to some of the many important prophylactic measures which come under this heading.

Dust Protection.-Wherever grinding, buffing or polishing is in process, as in emery grinding of cutlery, and brass polishing or where sandpapering of paint or sandblasting of castings is employed, strong suction apparatus should be used, applied through ducts adjusted as near as possible to the work. With sandblasting of large castings an additional current of air should be directed from 
behind or above the workman to direct the sand and metal dust into the exhaust duct. In sorting pipe, horsehair and similar materials it is often desirable to have the workman stand over a perforated grill or have such grill set into the workbench, covering a large exhaust duct. In general, heavy dusts should be removed downward through the floor and lighter dusts from in front of, or above, the workman, as in pottery grinding. The polishing and grinding of small articles such as those made of glazed pottery or china, metal work, etc., should be conducted within a cabinet into which the workman's arms are passed through slits or cuffs let into"a canvascovered side, while he looks in through a celluloid window. An exhaust duct is also applied within the cabinet. Emery wheels should be protected by close-fitting sheaths and adjustable shields to protect the workman's eyes from sparks, leaving only such a small portion of the wheel exposed as comes into contact with the object to be ground. The method of wet grinding should be employed wherever the nature of the work permits, as in glass grinding.

Grinding and mixing of raw materials conducted on a large scale, as in grinding cement, mixing paints, etc., should only be conducted in separate apartments specially ventilated, and when possible in tightly closed receptacles to prevent access of dust to other parts of the building. The same rule applies to barreling or packing dusty products, such as chrome salts.

Protection of Receptacles.-Tanks, kettles, etc., containing acids, lye, dies, boiling oil, etc., or other substances liable to injure the skin or eyes through spattering should never be filled to the top, and should be fitted with covers, connected with exhaust ducts wherever toxic fumes arise.

"First-aid" kits should be kept at hand wherever emergencies are liable suddenly to endanger the employees, as for example where a carboy of acid might explode, toxic fumes suddenly get beyond control, ammonia vapor escape (as in cold-storage plants). Such an outfit should provide aromatic spirits of ammonia, hypodermatic cardiac stimulants, eye lotions containing boric acid, oil (for acid burns), etc., carron oil (for dressing for burns), antiseptic solutions (for application to cuts or abrasions), compress, bandages, clamps and artery forceps, tourniquets, absorbent cotton, swabs of gauze for sponging, etc. Wherever employees are subject to the possible hazard of being suddenly overcome by toxic fumes, gases or vapors, as in gas works, powder plants, etc., emergency apparatus for artificial respiration and oxygen helmets for rescuers should be at hand.

Hours of Work.-In some occupations, as in caisson work, bisulphid of carbon processes, cleaning out large tanks which have held toxic materials, varnishing the interiors of ill-ventilated receptacles such as beer vats, etc., it is neccessary to restrict the workman's period of employment to one or two hours, or sometimes a few minutes a day. In certain occupations involving hazards from chemical substances, it is desirable furthermore to have fre- 
quent change of occupation in the same industry to prevent the accumulative effects of poisons.

\section{OCCUPATIONAL HYGIENE OF THE WORKMAN}

There are several reasons why workmen do not as a rule coöperate actively in protecting themselves and it is an interesting fact that their "unions" concern themselves almost exclusively with wage problems and hours of work to the almost complete neglect of enforcing rules of hygiene. The individual workman of ten naturally errs in this neglect through ignorance of a health hazard, or, if he recognizes it, believes that while others may be affected, he himself is so strong that he will prove immune. Thus the miner, long accustomed to firedamp and other explosives, may finally neglect the most obvious precautions. The writer has seen metal grinders wearing their goggles on top of their forehead, and workers in dusty trades cast aside their respirators. Another reason for such neglect is that the workman dislikes to take extra trouble or appear "fussy," or he fears to lose his job by appearing to complain of unhealthful conditions which surround him. Nevertheless, much can be done through instructing and encouraging him in the the use of simple precautionary measures. Such instructions should be given in printed form as well as orally, and some manufacturers go so far as to offer rewards for the best record in following them.

Bathing.-A full hot bath should be taken at least twice a week (or oftener where the work begets excessive perspiration), soaking and soaping the body well. If the work involves exposure to irritant dust or fumes, the body should be sponged with warm water and well rubbed each night before retiring. The skin may thus be kept free from eczema or dermatitis, and more restful sleep induced. Workers exposed to metal fumes, especially those of lead, should be afforded facilities for shower bathing before returning to their homes. At meal time and before returning home, in all dusty trades the workman should be allowed at least ten minutes for thorough washing of the face and hands, and among lead workers the use of nailbrushes also is to be emphasized particularly. The British factory laws prescribe in great detail the provision of at least one washbasin, or 2 feet of porcelain-lined wash trough, for each workman, and also the type of fluid soap, nailbrushes and individual towels to be used.

Clothing.-The use of overalls or special working gowns or suits should be insisted upon in whatever occupations the clothing is liable to become saturated with moisture, dust or any substances peculiar to the occupation. In lead smelting works and similarly hazardous places, a complete change of clothing for working is most important and the overalls, etc., should frequently be washed. Working clothes should always be loose fitting and the wearing of

VoL. IV. -12 
tight collars, garters, belts and corsets should be discouraged. Wool absorbs more water, perspiration, dirt, odors and germs than linen, and cotton occupies an intermediate position, but wool is superior to either as a protective against cold. Where cold winds and heavy rains are to be endured, as among motormen and sailors, leather and rubber or other waterproof material reinforce the other clothing. Dr. George H. Kober, who has made a special study of the most desirable workman's clothing for the President's Homes' Commission of $\mathrm{r} 898$, states that "texture has nothing to do with protection from the direct solar rays; it depends entirely on color, and white is the best. As a protection against fire (as in foundries) leather clothing is generally worn," but any clothing may be rendered fireproof by a solution of 20 per cent. of sodium tungstate with 3 per cent. of phosphate of soda in starch sizing. Aprons and caps, when not of leather, should be of washable material. Loose sleeves and blouses should not be worn near machinery in which they might become entangled. Moreover, they gather much dust. Where liquid dyestuffs, acid solutions, boiling oil, etc., are used, large aprons of rubber, leather or oilcloth should be worn.

Care of the Feet.-Wherever the workman has to stand for long hours, and particularly where he has to push treadles with the feet it is most important that he have properly fitted shoes. Flatfoot, blisters and bunions are readily acquired through faulty positions in standing and easily may become incapacitating. It is desirable in many industries to have special shoes to be worn only while at work. Irritating dusts may work their way into ill-fitting shoes and cause ulcers, eczema or other lesions. Workers in nitroglycerin and dinitrobenzol should wear thick soled shoes in good repair, for these substances may be absorbed through contact with the feet. Where floors are constantly wet rubber shoes or boots may be worn, but their continued use is bad for the feet, and wooden shoes may be preferable. Stockings with holes or hard seams should not be worn, and all socks or stockings should frequently be washed, for accumulated sweat irritates the feet. Nightly soaking of the feet in warm water does much to lessen fatigue and prevent excessive local sweating. Flat-foot should be treated promptly by means of massage, suitable exercises of the muscles and properly adjusted supports.

Care of the Hands and Nails.-The hands should always be washed after going to the toilet and before meals. The use of individual towels should be insisted upon. Fingernails should be kept short to prevent tearing and accumulation of dirt. This direction, as well as that of thorough scrubbing with soft soap and a nailbrush, is particularly important for all workers in paint and other lead preparations. Workers in packing houses, bone fertilizer plants, tanneries, slaughterhouses, etc., are liable to infection through abrasions of the fingers, especially "hangnails," and where such abrasions exist they should be protected by collodion, adhesive 
plasters, etc. All cuts or abrasions should promptly be cleansed and disinfected with iodin, carbolic or other disinfectant solution obtained from the emergency kit. Canvas, leather and rubber gloves are used to protect the hands of workmen who are obliged to immerse the hands in strong acid or alkaline solutions, irritant dyeing solutions, etc. Where this is not possible, kceping the hands well greased with tallow or other fat or oil affords some degree of protection from irritation.

Care of the Hair.-The hair, when not protected, affords a means of conveying dust into the home. Workmen in dusty trades, and where the fumes of molten lead or zinc, etc., may condense in the hair, should keep the hair short and be close shaven, washing the head daily, and shampooing it at least twice a week with green soap. In this manner seborrhea and eczema may be avoided. Lead smelters and workers in white lead manufacture should wash the head before going home to prevent dissemination of lead dust in their homes. Stiff, close-fitting hat brims impede the circulation and impair the nutrition of the hair. Paper or linen caps should be in much more general use than they are in this country.

Care of the Eyes.-Workmen who have constantly to look at bright moving polished surfaces, as in polishing steel, should protect the eyes by colored glasses. Close-fitting goggles should be used where irritant dusts like those of chrome, cement, etc., are liable to enter the eyes, or where irritant fumes or gases obtain, and the eyes should be bathed on quitting work with a saturated solution of boric acid. Eye shields, spectacles and goggles are made of such materials as mica, wired glass, wire mesh, celluloid, etc., either plain or colored and should be worn by metal grinders, stonecutters and all those liable to have fine sharp particles injure the eyes. When a foreign body is lodged in the cornea, a fellow workman is usually asked to remove it which he often does with a dirty toothpick or straw, thereby setting up an inflammation often serious. Instead of this the workman should endeavor to float it out with boric acid solution or a drop of sterilized oil, and failing this, only an expert should be allowed to remove it. In excessively hazardous work as in sand blasting or dealing with strong irritant fumes, as those of ammonia or nitric acid, protective helmets are used, covering the entire head and shoulders, fitted with mica or celluloid windows, valve respirators, and supplied by compressed air forced through a flexible hose. In this manner not only the entire head is protected, but the workman obtains fresh uncontaminated air.

Care of the Mouth and Nose.-For protection of the mouth and nose against irritant dusts, such as those of lime and chrome salts which are liable to produce ulceration a douche and gargle should frequently be used of warm normal salt or saturated boric acid solution. The nose may be protected further by greasing the interior of the nostrils with lard or vaseline. Acid vapors tend 
rapidly to erode the enamel of the teeth. To counteract its effects the mouth should be rinsed frequently with an alkaline solution, and the teeth should be cleansed twice a day with a simple toothpaste or castile soap. It is most important for workers in mercury and phosphorus to permit no decay of the teeth to gain headway, as caries and abscess of the jaw may quickly follow. Tobacco chewing is particularly bad for lead workers. They carry the "quid" in a dirty pocket, or handle it with soiled fingers and thus convey lead dust or its salts to the mouth, and thus acquire systemic poisoning. In lead and chrome works the employees should brush the teeth before eating. Besides the systemic poisoning resulting from pyorrhea, carious teeth and gingivitis the poor condition of the mouth impairs appetite and digestion and lessens the workman's efficiency.

Position while at Work.-To the writer's clinic come from time to time workmen who have become seriously deformed from malpositions while at work. Such, for example, are young carpenter's helpers, who elevate the shoulder and curve the spine in carrying heavy timbers, hand shoemakers, who curve the spine forward compressing both thorax and abdomen, working girls, who stoop constantly over machines, postmen, who carry heavy mail sacks over the shoulder, etc. Such deformities are preventable to a great extent by corrective exercises and deep breathing.

Exercise.-Exercise is a difficult matter to regulate for many workmen, especially those whose work is sedentary. They may either live so near their work as to require little walking, or so far from it that they always ride to and fro. Again they may overexercise and fatigue certain groups of muscles from the nature of their work, or they are too fatigued by their day's work to take any normal exercise in the open air. In such cases deep breathing exercises conducted at home in front of an open window twice a a day are of great service, or the use of Indian clubs or other aids to exercise which favor chest expansion and development often proves of great value. Such corrective exercises need consume only a few minutes each day but should be persevered in. In other cases, overexercise and strain constitute the hazards which obviously. can only be mitigated by rest or change of occupation. The workman should be instructed as to the value of properly regulated exercise in its relation to prevention of constipation, anemia and lassitude.

Sleep.-Often the workman's home surroundings in a crowded, ill ventilated tenement prevent restful or adequate sleep. He should be instructed as to the value of abundant fresh air in the sleeping room, of bathing the body and especially the feet before retiring, securing comfortable bedding with light but warm covering. Those who perform night work should sleep at once in the day, before doing other things. Excessive noise may be shut out by cotton in the ears and light by a dark bandage over the eyes. In winter, cold feet 
often prevent sleep-a condition easily rectified by filling a few beer bottles with hot water and encasing them in socks, when they are placed at the feet.

Exposure to Cold.-Many occupations present the hazards from cold of frost-bite, freezing and chapped hands. Frozen fingers or ears should be rubbed with snow or ice, then with cold water, and thoroughly dried and bandaged. Frozen parts should never be brought near an open fire or heater. The subsequent hypersensitiveness of the skin should be treated by rubbing vigorously twice a day with salt and cold water. "Chapped" hands should be kept thoroughly dried after washing, without rubbing and at night camphorice (lard, vaseline and camphor) may be applied, or balsam of Peru may be used, and the hands protected by loose gloves, worn during sleep.

Avoidance of "Colds."-The workman should be made to realize that colds are induced more often through fatigue and indigestion with lack of fresh air, than by drafts. Keeping the skin and digestion in good order, avoidance of foul air, abstention from alcoholic beverages, and cleansing the nostrils and gargling the throat with simple antiseptics are among the best prophylactic measures.

Excessive Heat.-During very hot humid weather one should move slowly, drink no alcoholic beverages of any kind, eat but little meat and take a lukewarm bath to remove perspiration before retiring. In the experience of the writer nearly all cases of genuine insolation occur among hard drinkers. Outdoor workers in excessive heat (as in laying asphalt pavements) should protect the head by a cloth wrung out in cold water and worn beneath a light-colored hat. Stokers and foundrymen after excessive perspiration often come out into the cool air and sit in a draft which chills the exterior of the body and causes congestion of internal organs, particularly the kidneys, or a localized myalgia or neuralgia may ensue. Acute enteritis is often induced by sudden chilling of the abdomen after previous exposure to great heat. Enough cool water should be drank in hot weather to replace loss from perspiration, but large draughts of iced water may induce gastric catarrh and dilatation. In a large factory in which the workmen suffered much from diarrhea during the summer it was entirely controlled by substituting moderately cold water for the large quantities of iced water which the workmen were in the habit of drinking.

Food.-In occupations involving hard work and muscular strain it is well for the workman to eat heartily at breakfast. House painters and other lead workers who may not have access to soap and nailbrushes to cleanse the hands thoroughly before eating may use two pieces of clean paper in handling their sandwiches, etc., to protect the food from contact with lead. In some industries such as caisson work, cleaning out flues, "blue beds" of lead carbonate, etc., involving much strain, it is well to supply the workman with simple luncheons on the premises, such as bread and milk, hot soups, coffee, etc. 
Physical examinations of employees before entering upon any laborious work are insisted upon in many industries and may do much for the prophylaxis of occupational diseases, as well as determining the physical fitness of the employee for the particular subdivision of work in which he is to be employed. Such examinations should include particularly detection of possible hernias, cardiac valvular lesions, incipient tuberculosis, general muscular strength and fitness of the eyes. 


\title{
INFECTIOUS DISEASES
}

\author{
TYPHOID FEVER
}

\author{
By Rufus Cole, M. D.
}

AND

\section{Henry T. Chickering, M. D.}

During the past few years considerable progress has been made in the prophylaxis and treatment of typhoid fever. The advances that have taken place have been made possible by a more complete knowledge of the nature of the pathological physiology of the disease and a better understanding of the immunological processes that accompany it. The study of the metabolic activities of the body during typhoid fever has influenced the dietary treatment of the disease, while the discovery of various immune substances in the blood of those immunized actively to the typhoid bacillus, has caused renewed interest in prophylactic vaccination and specific vaccine therapy. ${ }^{1}$ While these discoveries in large part have not dealt directly with the treatment of the individual patient, yet they have had and will in the future have, in increasing degree, a marked effect in lowering the incidence of this malady and will undoubtedly also lead to further decrease in mortality and should be fully understood by those having direct care of such patients.

\section{PATHOGENESIS}

The work of Metchnikoff and Besredka(I) in I9II, who produced the disease in anthropoid apes by feeding materials contaminated with B. typhosus completes the chain of evidence which established this microörganism as the cause of the disease.

It is well known that typhoid fever is a general infection, the localizing lesions being most marked in tissues rich in lymphoid elements. The frequency with which typhoid fever is accompanied by a bacteremia was first noted by Schottmüller(2), Auerbach and Unger(3), and Cole(4), in I900 and I90r. More recently, Schottmüller(5) and Jochmann(6) abroad and Buxton and Cole$\operatorname{man}(7)$ in this country have emphasized the fact that the bacilli are usially found circulating in the blood from the onset of the disease until a few days before defervescence and in rare instances

${ }^{3}$ For a more detailed description of the generally accepted methods of prophylaxis and treatment, the reader is referred to the section on typhoid fever in Vol. II, page 176 . 
in the first few days of the afebrile period. Moreover, the severity of the disease usually depends more on the intensity of the bacteremia than on the extent of the localized lesions, such as splenic tumor, intestinal ulceration, and mesenteric glandular enlargement which are probably secondary to the blood infection. In fact, deaths from typhoid fever without intestinal lesions, while uncommon, are occasionally seen and reported.

The microörganism usually enters the blood by way of the gastrointestinal canal. In a few cases the bacilli have been obtained from the blood within twenty-four hours after the onset of the fever (6). The invasive power of the typhoid bacillus seems to be greater for man than for lower animals. Whether or not infection takes place apparently depends not only on differences in the degree of natural immunity, but also largely on the size of the infecting dose. If heavily contaminated food is ingested by a number of persons, the percentage of those contracting the disease is large. The epidemic reported by Sawyer(8) is a case in point. This epidemic was traced directly to the eating of contaminated spaghetti by a large number of persons. The heat employed in cooking the spaghetti was insufficient to kill the organisms, and actually aided in their multiplication. Of the one hundred and fifty individuals at the dinner, some of whom are known not to have partaken of the dish, ninety-three contracted the disease.

The importance of the size of the infecting dose in relation to the occurrence of the disease in individuals who have been immunized to the typhoid bacillus will be discussed later.

The proliferation of the typhoid bacilli in the body is accompanied by a very characteristic reaction on the part of the tissues of the host. Mallory(9) believes this to consist chiefly in a marked proliferation of the endothelial cells lining the blood-vessels and lymph spaces due to poisons elaborated by the typhoid bacilli in the intestinal canal and in the tissues. The large endothelial cells become markedly phagocytic. Sections from tissues having a rich blood supply, namely, the liver, spleen, bone marrow, and lymphoid tissues of the intestine and of ten the lungs, show the capillaries and blood spaces closely packed with phagocytic endothelial cells, macrophages and leukocytes. The blood and lymph spaces may become so congested with these cells and their products of degeneration that the blood supply may become entirely occluded, causing small areas of focal necrosis and intestinal ulceration. Arima(Io) found that following the intravenous injection of rabbits with toxic products of typhoid bacilli, 70 per cent. of the animals showed characteristic lesions in the intestines and mesenteric lymph nodes. Mallory has described sections of the intestinal lesions obtained intravitam or immediately after death which are extraordinarily free from bacteria, and those bacteria that are seen are not infrequently colon bacilli which have invaded these tissues secondarily after ulceration has taken place. Some cases that apparently 
die of severe toxemia (heavy bacteremia) in the first or second week of the disease may show no intestinal ulcerations whatever, only the general hyperplasia of the lymphoid elements of the intestine and other organs previously mentioned. It is quite probable that differences in toxicity of strains of the typhoid bacillus occur. Stenitzer(II) has stated that only certain strains produce toxic filtrates. While as yet no one has been able to differentiate typhoid bacilli into distinct biological groups characterized by differences in antigenic properties, it is probable that different races show definite differences in their rate of growth, toxicity and adaptation to unfavorable surroundings.

The typhoid bacillus may invade the central nervous system or produce localized lesions in bones or soft parts, though often these lesions are due to secondary invaders. Here the typhoid bacillus may take on certain pyogenic characteristics, the lesions showing well-marked leukocytic as well as endothelial cell infiltration. $\operatorname{Koch}\left(\mathrm{r}_{2}\right)$ and Chiarolanza(r3) have demonstrated that both in man and in experimental animals the gall-bladder usually becomes infected from the blood stream. A chronic infection may exist in the gall-bladder or urinary bladder for years without causing symptoms of disease. Koch has pointed out that such cases as these, the so-called "carriers," form important and dangerous sources for the spread of the disease. Prigge(I4) estimates that about 5 per cent. of individuals having had typhoid fever become chronic disseminators of typhoid bacilli. In a few instances chronic carriers of the typhoid bacillus may have no knowledge of having had the disease. Women are more prone to become carriers than men. Of 5 or temporary or chronic carriers or disseminators col, lected by Prigge, $7 \mathrm{r}$ per cent. were women. Thus far this carrier state, both experimentally in animals and in man, has failed to yield to therapeutic agents.

While infection with paratyphoid bacilli " $A$ " and " $B$ " are relatively rare in this country, still sporadic cases due to these organisms are continually appearing, which often present a syndrome suggestive of dysentery, cholera, or food poisoning. Of sixty-five cases of typhoid fever recently reported by Gay( $\left.\mathrm{I}_{5}\right)$, there were two caused by Bacillus paratyphosus "B." In the European war zone many cases of paratyphoid fever have been reported. Leishman(I6) reports the occurrence of about 1200 cases in northern France during the first fifteen and one-half months of the war, with a mortality of ' $\mathrm{I} .5$ per cent. Paratyphoid " $B$ " infections made up onehalf to two-thirds of the cases. Carriers seem to be the principal source of the infection. Basset-Smith( $\left.\mathrm{I}_{7}\right)$ has reported seventy cases of typhoid fever from the Dardanelles region of which $4 \circ$ per cent. were due to B. paratyphosus "A". Sarrailhé and Clunet(r8) have recently reported a mild typhoid-like epidemic from that same district, which was also characterized clinically by gastrointestinal disturbances and by jaundice. From a large proportion 
of these cases they isolated an organism which was not agglutinated by antiparatyphoid "A" or "B" serum, but which differed culturally only slightly from the well-known paratyphoid strains.

The significance of the bacteremia in typhoid fever has not received the attention it deserves. Frequently cultures of the blood yield information of value in diagnosis and prognosis, and may serve as a criterion of the efficacy of a given therapeutic measure.

From the standpoint of early diagnosis, the blood culture is more valuable than the agglutination reaction, the Gruber-Widal test, inasmuch as a positive culture can usually be obtained early following the onset of the disease, whereas the Widal reaction usually appears later. In making blood cultures it is important to use relatively large quantities of blood, for the moderately severe cases of typhoid may present only a mild bacteremia, frequently no more than one to three colonies of B. typhosus appearing after inoculation of I c.c. of blood.

Though a positive Widal reaction does not usually appear until the second week of the disease, a strong reaction may sometimes be present as early as the fifth to the seventh day. On the other hand, in severe cases in which the Widal test may be negative even as late as the seventh week of the disease, the blood culture may still be positive at this time.

With a positive blood culture the exact etiologic agent can be determined, while from the Widal reaction alone it is not always possible to state whether the infection is due to B. typhosus or paratyphosus "A" or "B." Recently Bernard and Paraf(rg) and Rist (20) have published a carefully studied series of cases in which the agglutinins were stronger for certain of the allied organisms than for the organism causing the disease, the latter being determined by direct cultivation. The knowledge of the particular, organism concerned is especially important from the standpoint of prognosis as the average mortality from typhoid fever is from 9 to 12 per cent., while that from paratyphoid infection is I per cent. or under.

With the increasing frequency of prophylactic vaccination both in military and civil communities, the Widal reaction becomes a less dependable diagnostic aid, since the vaccination is usually followed by the appearance of agglutinins in the sera of the individuals thus immunized. If, however, on repeated examination the Widal reaction becomes progressively stronger, this is believed to be an indication of current infection.

In rural communities the Widal reaction will probably have to be relied upon, but in cities free blood cultures should be one of the functions of the public health laboratories. The early diagnosis of suspected cases of typhoid fever is very important from the public health standpoint, as precautions aimed to prevent the spread of the infection are not enforced with the same stringency in doubtful cases as in those in which the diagnosis is certain.

In addition to the Widal reaction and blood culture, the ophthal- 
mo-reaction as a diagnostic aid in typhoid fever has been advocated by Chantemesse (2 I), Floyd and Barker(22), and Austrian(23). While the reaction is usually positive during the febrile period of the disease if a carefully prepared antigen is used, it is also occasionally positive in conditions other than typhoid, especially in tuberculosis.

Careful bacteriological examination of the blood of typhoid fever patients is also of great aid from the standpoint of intelligent prognosis. Schottmüller and more recently Landsberger(24) have shown that the more serious cases are those that have a marked bacteremia. As the disease advances toward a lethal outcome, there occurs a progressive increase in the number of bacteria in the blood stream. Cases that have two or three colonies per cubic centimeter of blood are almost always severely ill, while those having 25 to 200 colonies per cubic centimeter of blood rarely recover. In practically every case having hemorrhage or perforation, even though late in the disease, B. typhosus can be cultivated from the blood. As long as bacilli are present in ro c.c. of blood, a continued course of fever can be predicted. When cultures are sterile, I 5 or 20 c.c. of blood being used, defervescence is usually close at hand. On the other hand, with the onset of a relapse, the bacteria reappear in the blood.

If, as is believed, the severity of typhoid fever is largely dependent upon the intensity of the bacteremia, one has in the blood culture a guide for estimating the efficacy of a given method of treatment. If the use of any therapeutic agent, which in itself does not have a deleterious effect on the tissues of the patient, is accompanied by sterilization of the blood with any degree of regularity, one may assume that its action is beneficial. This method has been used as an indication of the effectiveness of antipneumococcus serum in the treatment of lobar pneumonia by Cole(25) and in the treatment of typhoid fever by intravenous injection of sensitized vaccines by Gay(15). It is extremely interesting to note that Schottmüller(5) found no appreciable change in the number of typhoid bacilli in the blood of several of his patients following a course of antipyretics, a result which was to be expected. This, so far as is known, was the first attempt to estimate the efficacy of a therapeutic agent by a quantitative determination of the concentration of the bacilli in the blood. Testing a therapeutic measure in this way would seem to be of more value than judging from mortality statistics alone, especially when a relatively small number of cases is investigated, and in a disease like typhoid fever which varies so widely in its severity from year to year and in different localities.

Technique of Blood Culture.- Twenty to 25 c.c of blood is withdrawn from a vein at the elbow with a glass syringe under aseptic precautions. Ten c.c. of the blood so obtained is mixed with 200 c.c. of ro per cent. bile broth and another ro c.c. of blood is added to 50 to I $\infty 0$ c.c. of agar and plates are poured. If growth occurs, 
subcultures are made in glucose, litmus milk and lactose media, and smears and hanging drops are examined for morphology, staining reactions and motility. The organism is finally tested for agglutinability with antityphoid serum (anti-paratyphoid sera being also used if gas is produced in the sugar media).

Technique of Widal Reaction.-The macroscopic method here described has certain advantages over the more commonly used microscopic test. With this method clear serum which has been separated from the clotted blood is used. In a series of test-tubes is placed I.8 c.c. of normal salt solution in the first tube and I c.c. of salt solution in the remaining tubes. To the first tube is added 0.2 c.c. of the serum to be tested and their mixture thoroughly effected. One c.c. of this mixture is then added to the second tube and the procedure repeated with each succeeding tube. In this way a series of dilutions of $I: I 0, I: 20, I: 40, I: 80, I: I 60$, $I: 320$, and on up to $I: I 280$, or $I: 2560$ is quickly and accurately made. To each tube is then added a drop of a stock saline suspension of an easily agglutinable agar-grown strain of $\mathrm{B}$. typhosus, which has been killed by the addition of formalin ( 40 per cent.) in a concentration of 0.1 per cent. Agglutination usuallyoccurs after one-half to two hours' incubation at $38^{\circ} \mathrm{C}$., but final readings should be made after twenty-four hours in the ice-box. A positive test is one in which the bacteria have flocculated at the bottom of the tube leaving a clear supernatant fluid. With this method the reaction is never doubtful. It is either positive or negative. The strength of the reaction is determined by the greatest dilution of the serum in which agglutination occurs.

\section{PROPHYLAXIS}

Typhoid fever is a preventable and unnecessary disease. Its occurrence in a community is dependent to a large extent upon the efficiency of its public health service and its practising physicians. In no disease is the motto of the Department of Health of New York City more applicable. "Public health is purchasable. Within natural limitations a community can determine its own death rate."

Fortunately the typhoid bacillus does not thrive well outside the human body. Infection is generally spread by relatively close contact either directly with individuals who are excreting the bacilli or through the medium of contaminated food, milk, or water. Consequently efforts to prevent the spread of the disease should be directed in three main channels:

I. Protection of the water and food supply from contamination.

2. Detection and elimination of carriers.

3. Prophylactic vaccination.

\section{GENERAL PROPHYLAXIS}

I. Protection of the Water and Food Supply from Contamination.-While the problems of safeguarding the food and water supply 
of a community are more those of public hygiene, still the individual physician may render service in the successful solution of these problems by intelligent coöperation with the proper authorities and by stimulating a public consciousness of their importance. From the standpoint of efficiency and ultimate economy, a State health organization with centralized authority is of greatest importance. A corps of well-trained sanitary inspectors could become immediately cognizant of the outbreak of disease in any particular district. The attention of the whole State organization could then be brought to bear immediately on the problem. In a few of our largest cities an organization of this sort has been developed which investigates most thoroughly the incidence and source of all cases of typhoid fever occurring in the city. An active public health service demonstrates its usefulness not only in the detection of disease, but in its prevention. In relation to typhoid fever, its activities are numerous; such as the introduction and enforcement of measures to prevent the sewage pollution of potable waters, the supervision of local water supplies, making sanitary surveys of watersheds, the regulation of the production of food to be consumed in raw state and the inspection of places where such food is offered for sale, the regulation of the production of the milk supply and its pasteurization, the introduction of measures to ensure the sanitary laundrying of clothing, and other functions too numerous to mention. A most important duty of the health department of a city or State is to see that the isolation of the individual case is adequately carried out. Such isolation will be most effective if the proper measures be instituted early. This can be done only if an early diagnosis is made and this is much facilitated by means of Widal tests and blood culture. The municipal health authorities should, therefore, be prepared to make these examinations free of charge. In all these measures the practising physician can be of the greatest assistance, first by making every effort to arrive at an early diagnosis, second, by reporting all positive or suspected cases; third, by seeing that the proper measures are instituted for preventing the patient from becoming a source of danger to others, and finally by using his influence in every possible way to assist and support the health authorities in their efforts to prevent the spread of the infection.

For a detailed description of measures employed to prevent the spread of the disease from patients actively suffering from typhoid fever, the reader is referred to Vol. ii, pages $18 \mathrm{r}-189$.

The authors have found it useful to provide the family of a typhoid fever patient with a set of printed instructions regarding the more simple measures to be employed as follows:

I. Isolate patient in a single room.

2. One individual should be responsible for the patient's care.

3. Screen windows against flies. Kill those in the room.

4. Provide washstand, basin, pitcher, and slop jar in the patient's room. 
5. Provide a basin to contain 2-3 quarts of 5 per cent. lysol solution. (If expense is not burdensome, 70 per cent. alcohol may be used. Its disinfecting qualities are as great and it is less irritating to the hands).

6. Provide a small wine glass filled with 70 per cent. alcohol for the thermometer.

7. Provide a galvanized-iron wash tub containing 3 per cent. lysol to receive soiled linen. Allow linen to stand in this for one hour before boiling for thirty minutes.

8. Disinfect excreta by adding twice the volume of 5 per cent. lysol to the contents of bed pan, mix thoroughly and wash into slop jar. For each volume of urine add one-half volume of 5 per cent. lysol, and allow to stand at least two hours. Store bed pan and urinal in tub containing 5 per cent. lysol.

9. Pin a small paper bag to the edge of the bed to receive the pieces of linen into which the patient expectorates, and later burn.

Io. Cover door knobs with gauze kept moist with 5 per cent. lysol.

II. The person caring for the patient must not prepare the food for the family.

12. The patient's attendant before eating should wash hands thoroughly with soap and water and rinse with 70 per cent. alcohol, allowing the same to evaporate to dryness: 5 per cent. lysol may be used.

13. Never place fingers in mouth, and handle food to be eaten as little as possible.

I4. Food and dishes to be returned from sickroom should be received in a large dishpan and boiled before handling.

I5. Bathe patient at least once a day with soap and water and 70 per cent. alcohol.

2. The Detection and Elimination of Carriers.-The second great source for the spread of typhoid fever is the chronic carrier of the typhoid bacillus. Most important is the certain detection of the persons who of ten without knowledge of having had the disease, go on excreting live virulent typhoid bacilli year after year. The carrier state is so resistant to medical treatment that continuous isolation would be manifestly an unfair hardship. This menace would be lessened if the danger of this carrier condition to the community were clearly recognized and efforts were made to instruct such carriers in personal hygiene and habits of cleanliness. The excreta of all food handlers should be carefully examined for the presence of typhoid bacilli. If present, such individuals should be strongly advised or prevented by law from seeking positions as milkmen or cooks, or occupations that are intimately associated with the preparation and serving of food. The detection of typhoid carriers by serological tests is very unsatisfactory. They may or may not have positive Widal reactions. Repeated careful bacteriological examinations of the urine and stools offer the best evidence of the carrier state. Stokes and Clarke(26) have reported the results of a systematic 
search for typhoid carriers among 800 convalescents. A definite bacteriological diagnosis was made in 165 cases. As of ten as possible, they examined the excreta on two successive days following a purge the night before, the specimen being cultured as soon as possible after voiding. A thick emulsion of the feces was made and the solids allowed to settle for about twenty minutes. Six large loops of emulsion were then transferred to ro c.c. tubes of 2 per cent. peptone water to which 0.5 c.c. of $\mathrm{r}: \mathrm{r} 0,000$ brilliant green was added. The tubes were allowed to incubate for eight to ten hours and then streak plates on Endo's medium were made. Suspicious colonies were then picked off and their fermentative and agglutination reactions determined. By this method they discovered 65 temporary carriers of which three became chronic.

3. Prophylactic Vaccination against Typhoid Fever.-The third effective weapon to prevent the occurrence of typhoid fever is antityphoid vaccination. The earlier encouraging results of Pfeiffer and Kolle(27) and Wright(28) and Leishman(29) have been fully confirmed by the splendid statistics of Russell giving the results of inoculation in the United States army. During a period of five years, up to June 30 , I9I4, Lyster(30) has reported that I45,688 men in the United States army have been inoculated. During the last three years of this period, when vaccination was made compulsory, only thirty-eight cases developed, and of these, twenty-eight cases had either not been vaccinated at all or only partially vaccinated, having entered the service apparently in the incubation period of the disease. Before vaccination became general, from 200 to 282 cases of typhoid fever occurred in the army each year. This record covered a period during which a large number of the troops were mobilized on the Mexican border, in a region, which from point of view of climate and topography compared with the region where an encampment of troops in the Spanish American war was stationed. In this camp there occurred between 1729 and 2693 cases of typhoid with 248 deaths. On the Mexican border there were twice as many troops and two mild cases developed. This truly phenomenal record has not been duplicated in other countries, especially among the nations of Europe now at war. This may be due to several extraordinary factors incidental to the mobilization of huge numbers of troops. In the first place, vaccination was not made compulsory in the French army until October, I9r4. The Belgian army was only inoculated after a four months' campaign. Vaccination against typhoid in the British army is still voluntary. At the present time, I915, Rist estimates that fully 20 per cent. of the military population in France has not been inoculated against typhoid. The larger the proportion of non-immunized individuals in a community, the greater the chance of an epidemic starting and spreading to those immunized. Moreover, it has developed that in certain localities paratyphoid fever seems to be endemic and infections due to the paratyphoid organisms have made up a large proportion of the incidence of the disease. Of 
Rist s series of 215 cases, 73 per cent. were due to paratyphoid types, while in 325 cases reported by Bernard and Paraf, 76 per cent. were paratyphoid fever. It has further been learned that immunization against the typhoid bacillus does not protect well against paratyphoid organisms; therefore the incorporation of paratyphoid " $\mathrm{A}$ " and " $\mathrm{B}$ " strains in the prophylactic typhoid vaccine seems to be clearly indicated.

Although prophylactic vaccination when administered in the usual way, subcutaneously in two or three doses, does not confer absolute protection against typhoid infection, nevertheless there remains no question of the great efficacy of this prophylactic measure. Such protection must, of course, be relative, dependent upon the magnitude of the exposure and the general condition of the individual at that time.

This relative immunity is clearly illustrated by the fact that a large proportion of those contracting the disease after prophylactic vaccination are intimately associated with the sources of infection, as for instance, the relation of nurse to patient and laboratory worker to culture tube.

Antityphoid Vaccines and Methods of Administration.-Vaccines prepared in various ways have been recommended for prophylactic use by numerous investigators. Only the more commonly used types of vaccines will be discussed here.

A. Killed Cultures of the Typhoid Bacillus.-Wright(3I) prepared his vaccine by growing typhoid bacilli in bouillon and killing them with heat at $60^{\circ} \mathrm{C}$. for one hour. Pfeiffer and Kolle used agar for a culture medium and after washing the growth off in salt solution killed it at $56^{\circ} \mathrm{C}$. The United States army vaccine as prepared under Russell's $\left(3_{2}\right)$ direction, is composed of a single strain of low virulence, the Rawlings strain, which is the same as that used in Wright's vaccine. This strain was originally selected because of the ease with which it was possible to produce a smooth emulsified vaccine. It is grown on agar and after emulsification in salt solution, is killed by heat at $55^{\circ}$ or $56^{\circ}$ for one hour. Trikresol is added in a concentration of 0.3 per cent. as a preservative. Vincent(33) advocated the use of a polyvalent whole vaccine or an autolysate of a culture of B. typhosus killed with ether. Other observers urge the use of a phenol to sterilize the vaccine, believing that it is better to use chemicals than heat for killing the organisms, as by the latter method some of the antigenic qualities are thought to be lost.

B. Extracts of Bacteria.-Extracts of cultures of the typhoid bacillus have been used for purposes of immunization. The extracts have been prepared by various means, freezing with freezing mixtures or liquid air, drying and grinding, autolyzing in distilled water, subjecting cultures to the action of a Buchner press, etc. Vaccines prepared by these methods have not been so generally used, however, as have those previously described. 
C. Living Cultures of Typhoid Bacillus.-Castellani(34) and Besredka (35) have advised the use of vaccines composed of living non-virulent organisms. Most authors agree that experimentally a higher grade of immunity can be induced by the use of live than by dead vaccine, but most observers feel that there is too great an elcment of danger in the injection of live organisms.

D. Sensitized Cultures of the Typhoid Bacillus.-In I902 Besredka(36) introduced a new factor in vaccine therapy - that of sensitization of the vaccine, by subjection to the action of a corresponding immune scrum. Experimentally Metchnikoff and Besredka(37) found that a vaccine so treated was one-fourth to one-fifth as toxic for guinea pigs as a vaccine not thus treated. Larger doses could be borne with less discomfort and immunity be established more quickly. Chimpanzees injected with this vaccine developed a lasting immunity against live virulent typhoid bacilli and yet did not become carriers. Gay and Claypole (38) have published results which seem to show that experimentally a modified sensitized typhoid vaccine is more effective than a non-sensitized vaccine. The GayClaypole sensitized vaccine is prepared from polyvalent agar grown strains of B. typhosus and is sensitized by a potent immune rabbit serum. The sensitized bacteria are killed by precipitation in alcohol. Under aseptic precautions the bactcrial residue is dried to a constant weight, ground, and weighed amounts are then extracted in salt solution. This extracted bacterial residue, after as much as possible of the soluble portion is removed, is then emulsified in normal salt solution with the addition of phenol in a concentration of 0.5 per cent. as a preservative. Their experimental results indicate that the bacterial residue is less toxic than the whole vaccine and that it is also more antigenic.

In connection with this work on antityphoid vaccination, Gay and Claypole(39) noted the phenomenon of hyperleukocytosis, which occurs not only in rabbits but in man following the intravenous injection of vaccine and to a greater degree with a sensitized than a non-sensitized vaccine. This phenomenon is thought to be intimately connected with the mechanism of immunity in typhoid fever.

In spite of the extraordinary success following the use of typhoid vaccines composed of single strains, there is some experimental evidence to indicate that there are antigenic differences in different strains of typhoid bacilli and for that reason a polyvalent vaccine may be indicated.

Following the subcutaneous injection of typhoid vaccine for prophylactic purposes a so-called "reaction" occurs. Its severity depends largely on differences in individual susceptibility and partly on the type of vaccine used. Following the injection of the PfeifferKolle vaccine, which contains from I to $2 \mathrm{mg}$. of bacteria per dose, severe reactions, not infrequently accompanied by chills and fever as high as $4 \mathrm{I}^{\circ} \mathrm{C}$., occur in about 75 per cent. of those inoculated. VoL. IV.-13 
With the other kinds of vaccines there is little cause for apprehension about the reaction.

In the vast majority of cases there is only a local reaction which appears a few hours after injection of the vaccine. There may be slight redness and swelling about the point of injection. In about four to six hours there may be a feeling of stiffness in the muscles about the axilla and slight swelling of the axillary lymph nodes if the vaccine is injected in the usual site near the insertion of the deltoid muscle.

The local reaction may be accompanied by headache, general malaise, and slight rise in temperature which usually disappears within twenty-four hours. When non-sensitized vaccines are employed, usually 500 million organisms are injected in the first dose and rooo million in the two subsequent doses, at weekly intervals. If sensitized vaccine is used, Gay recommends giving three doses of 800 million organisms each, at two- or three-day intervals. In general there is said to be less reaction from the use of sensitized vaccine than unsensitized vaccine even though given at the short intervals stated.

A summary of the intensity of the reaction from the use of a variety of vaccines has been made by $R$. $\operatorname{Kraus}(40)$ from a large series of cases.

\begin{tabular}{|c|c|c|c|}
\hline Vaccine used & $\begin{array}{l}\text { Reaction slight, } \\
\text { no fever, } \\
\text { per cent. }\end{array}$ & $\begin{array}{c}\text { Moderate reaction, } \\
\text { temperature } 37-3^{\circ} \mathrm{C} . \\
\text { per cent. }\end{array}$ & $\begin{array}{l}\text { Severe reaction, } \\
\text { temperature } 38-40^{\circ} \mathrm{C} . \\
\text { per cent. }\end{array}$ \\
\hline 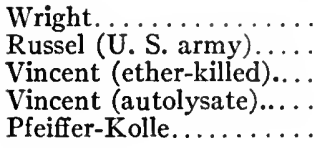 & $\begin{array}{l}82 \cdot 5 \\
94 \cdot 3 \\
81 \cdot 48 \\
94 \cdot 3 \\
0.0\end{array}$ & $\begin{array}{l}13.0 \\
5 \cdot 3 \\
14 \cdot 81 \\
5 \cdot 7 \\
25 \cdot 0\end{array}$ & $\begin{array}{r}4.5 \\
0.4 \\
3.7 \\
0.0 \\
75.0\end{array}$ \\
\hline
\end{tabular}

Harris and Ogan(4r) report that with the Russell, U. S. army vaccine, a mong $128,9 \circ 3$ individual vaccinations the temperature rose to 103 to $104^{\circ}$ with malaise, vomiting, diarrhea, or chills as follows:

$\begin{array}{ccc}\text { First dose } & \text { Number vaccinatea } & \begin{array}{c}\text { Per cent. of severe } \\ \text { reactions }\end{array} \\ \text { First dose................ } & 45,680 & 0.3 \\ \text { Second dose............ } & 44,321 & 0.2 \\ \text { Third dose...... } & 38,902 & 0.1\end{array}$

Kolle and Hetsch(42) and Kutscher(43) believe the strength of the immunity obtained from vaccination depends largely on the severity of the reaction. The very small number of severe reactions accompanying the use of U.S. army vaccine together with the remarkably low morbidity would seem to refute this idea.

If the injections are made in the late afternoon, the reaction, if any, will be present at a time when the individual is quiet in bed, and will have practically disappeared before morning. If the injection is accidentally made directly into a vein, quite alarming reac- 
tions follow, which are characterized by fever, chills, prof use perspiration and prostration. This can easily be avoided if care is taken to see that the needle is not in a vein by making gentle suction with the piston of the syringe after the needle is beneath the skin before injecting the vaccine.

While it is not definitely known whether the injection of the antityphoid vaccine renders an individual temporarily more susceptible to infection, in other words produces a so-called negative phase, the weight of evidence is against it. Recently Bull(44) was unable to demonstrate any decrease, and even noted an increase in the antibody content of rabbits' blood following large doses of killed or living typhoid bacilli. However, it is best to vaccinate when the individual is in good health and free from fever. In the presence of typhoid fever in a family or community, those individuals who present no evidence of infection should be vaccinated. At the same time the strictest precautions against contact with the sources of infection must be insisted upon. It is well known that a certain period of time must elapse before the development of even relative immunity from prophylactic antityphoid vaccination. Occasionally cases of typhoid fever develop in those who have been vaccinated while attending patients ill with the disease, though there is no evidence to believe that the incidence is greater in vaccinated than in unvaccinated persons who are greatly exposed to infection. But the danger of a false sense of security which may be derived from vaccination in the presence of typhoid fever cannot be too strongly emphasized.

The Department of Health of the City of New York(45) has recently published the results obtained from the vaccination of individuals who had been exposed to typhoid fever, they being chiefly members of families in which there were one or more cases of the disease. Of 8ror individuals thus exposed, 534 submitted to two or three doses of the New York City Department of Health's antityphoid vaccine. Of these none developed the disease. Of the 7567 individuals who were not immunized, which includes 127 who received one dose only and were not considered immunized, I6I developed typhoid fever, a little over 2 per cent.

In 1905 Leishman (46) called attention to the presence of various immune substances in the blood of individuals immunized against typhoid fever, and his observations have been repeatedly confirmed. In 90 to 94 per cent. of 750 cases, M. K. John(47) found that agglutinins were present in the blood of persons immunized with either Pfeiffer-Kolle or Johan vaccines.

Dakeyne (48) has reported that in 80 per cent. of 280 vaccinated soldiers, agglutinins were present, and the percentage of positive tests was twice as large in the case of those receiving two doses of vaccine as in those receiving only one dose. Authors differ as to the value of the presence of strong agglutinins as an index of immunity. Both Garbat and Meyer(49) and Gay and Claypole have noted that the agglutinins are much weaker in the serum of those immunized 
with sensitized vaccine than in the serum of those immunized with unsensitized vaccine. On the other hand, they have shown that the bacteriotropic and bacterio-inhibiting powers of the blood are greater when sensitized vaccines have been used for immunization than when non-sensitized vaccines have been used. Karaffa and Korboutt $(50)$ found that the protective properties of immune typhoid horse serum (when tested in guinea pigs) were greater when sensitized vaccines were used for immunization than when unsensitized vaccines were used.

If the immunizing properties of a sensitized typhoid vaccine are as great as those of a non-sensitized vaccine, and if the results of the use of sensitized vaccine are as good as both the experimental studies and the practical tests in civil communities as reported by Sawyer(5I) would indicate, it is the vaccine of choice, for it has been shown that it is less toxic than the non-sensitized vaccine.

The fact that the use of a sensitized vaccine does not produce agglutinins to any appreciable extent in the blood of those immunized is of some clinical importance, as the previous use of non-sensitized vaccine for prophylactic purposes has caused considerable confusion in the diagnostic use of the Widal reaction in cases of possible typhoid infection.

The exact duration of immunity following antityphoid vaccination is unknown. The agglutinins that appear after vaccination usually disappear rapidly after eight or nine months. At best the immunity is relative. At least three injections of vaccine should be given, more would probably confer greater immunity. Vincent $\left(5_{2}\right)$ advises a course of four treatments and revaccination each year. Leishman and Wright believe the immunity lasts about two and onehalf years. In the United States army the men are revaccinated with each reënlistment term of three years. Gay and Claypole have recommended the use of the typhoidin skin reaction as an index of the duration of immunity. They advise revaccination when this test becomes negative.

Since Widal and Sicard(53) first noted the appearance of specific antibodies, agglutinins, in the serum of animals injected with mixtures of different bacteria, an observation which was later confirmed by Castellani(54), both Castellani and Vincent(55), as well as other observers $(56)$, have advocated the use of a mixed typhoid, paratyphoid " $A$ ", and paratyphoid " $B$ " vaccine. Recent events in Europe have demonstrated the importance of immunization against all these forms. Where mixed vaccines have been used, antibodies against each have been demonstrated in the blood of individuals thus immunized. Furthermore, in the Japanese navy, Kabeshima $(57)$ reports that the use of typhoid-paratyphoid vaccine has reduced the incidence of paratyphoid infection to a large extent. tions:

Castellani advises a vaccine made up in the following propor- 


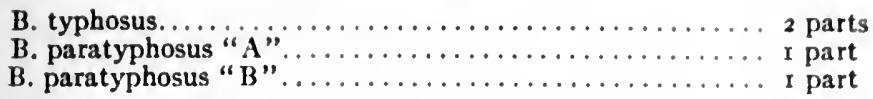

Reactions are no more severe where mixed vaccines are used than when single strains are employed.

In this country where the paratyphoid " $B$ " form of infection is more common, it would be advișable to incorporate a larger proportion of " $B$ " vaccine than " $A$ " vaccine.

The efficacy of antityphoid vaccination is so clearly established that the general adoption of prophylactic vaccination by the.civilian population is indicated. A comparative table drawn from the reports of about 20 different authors showing the relative incidence of the disease in vaccinated and non-vaccinated individuals has been compiled.

\begin{tabular}{|c|c|c|}
\hline & $\begin{array}{l}\text { Morbidity, } \\
\text { per cent. }\end{array}$ & $\begin{array}{l}\text { Mortality, } \\
\text { per cent. }\end{array}$ \\
\hline $\begin{array}{l}\text { accinated }{ }_{3} 34,669 \ldots \ldots \ldots \ldots \ldots \\
\text { on-vaccinated } \\
\text { I } 47,94 \mathrm{r} \ldots \ldots \ldots \ldots\end{array}$ & $\begin{array}{l}6 \text { r } 9 \text { or } 0.46 \\
4056 \text { or } 2.7\end{array}$ & $\begin{array}{r}13 \text { or } 4.2 \\
282 \text { or } 12.6\end{array}$ \\
\hline
\end{tabular}

Russell(59) has drawn attention to the frequency of typhoid fever in children. In 1909 there were 10,722 cases of typhoid in the United States, of which 3366 were under twenty years of age. $\mathrm{He}$ has vaccinated over 359 children from two to sixteen years of age. The reaction is not as severe as in adults, and robust children may safely be given typhoid vaccine at the same time they are vaccinated for small pox.

Goldscheider and Kroner(60) have observed 300 cases of typhoid that have developed in those previously vaccinated. They compare the course of the disease in these persons with that in a similar group of non-inoculated individuals as follows:

\begin{tabular}{|c|c|c|}
\hline Severity of disease & $\begin{array}{l}\text { Non-inoculated, } \\
\text { per cent. }\end{array}$ & $\begin{array}{l}\text { Inoculated, } \\
\text { per cent. }\end{array}$ \\
\hline $\begin{array}{l}\text { Light. ...... } \\
\text { Moderate. . }\end{array}$ & $\begin{array}{l}36.55 \\
24.85\end{array}$ & $\begin{array}{l}50.13 \\
25.88\end{array}$ \\
\hline Severe.... & 25.8 & 17.52 \\
\hline
\end{tabular}

In many cases those previously inoculated had higher fever with the onset but the fastigium was much shorter and the general course of the disease milder.

Hohlweg(6r) reports that the percentage of positive blood cultures is lower in those previously vaccinated than in those not so treated, which supports the clinical impression that the course of the disease is actually milder.

\section{TREATMENT}

During the past few years wide interest has developed in the specific treatment of infectious diseases. Of the acute infections, typhoid fever would seem to offer a more favorable opportunity for the artificial stimulation of immunity than other conditions which pursue a more rapid course. 
Specific treatment may take the form of passive immunization, serum therapy, or active immunization, vaccine therapy.

Passive Immunization.-The theoretical considerations upon which the use of an antiserum in the treatment of typhoid fever are based have been discussed at length in Vol. II, page 203 .

In spite of the remarkable mortality statistics, 4.2 per cent., drawn from a series of rooo cases of typhoid fever treated with the antitoxic typhoid serum of Chantemesse(62), this therapeutic agent has been little used outside of Paris.

It is by no means clear just what type of immune serum is of most value. Sheep, goats and horses, as well as smaller animals, have been used as sources of immune sera. These animals have been immunized in a variety of ways, by the subcutaneous or intravenous injection of dead or live typhoid bacilli, their extracts, or filtrates of the culture media in which the bacteria have been grown.

Rodet $\left(6_{3}\right)$, on the basis of wide experimental and clinical experience, concludes that a serum produced from horses by the intravenous injection of dead bacteria, later followed by living organisms, as well as by toxic bacterial extracts, offers the best vehicle for transference to the patient of protective bodies against typhoid infection. Rodet has reported about ninety cases treated with such an antiserum with definite benefit in about one-third of the cases.

In the light of the recent work of Bull(64), who studied the action in vivo of antityphoid and antipneumococcus sera on rabbits infected by the intravenous injection of $B$. typhosus or pneumococcus, it would seem that a highly agglutinating serum would be the most effective agent for sterilizing the blood in human typhoid. He found that the injection of an immune serum in his experimental animals was accompanied by an immediate agglutination of the live virulent typhoid bacilli in the blood stream. After agglutination or opsonization of the bacilli had occurred they were then phagocyted by the leukocytes of the circulating blood and to a less extent by the fixed phagocytic cells of the body. If the animals were immediately sacrificed, it was found that large accumulations of leukocytes containing phagocyted bacteria have taken place in the liver, spleen and bone marrow.

If single large doses of serum were given, it was noted that many of the larger clumps of agglutinated bacilli failed to be phagocyted, and after a certain time had elapsed, bacilli began to reappear in the blood, the infection continuing to a lethal termination. On the other hand, if small doses of serum were given frequently over a period of several days, especially in the case of pneumococcus infection which is the more virulent, the animal was protected against enormous doses of bacteria. This work is of great interest and furnishes many suggestions as to the most effective use of an immune serum.

It is interesting to note that Andriescu and Cuica(65), using an immune serum prepared by Besredka(66), report that the bacteremia disappears after its use. Fifteen cases were treated with repeated 
doses of serum using up to 300 to 400 c.c. subcutaneously. They noted a marked decrease of toxic symptoms and all the patients but one who were thus treated recovered.

Progress in the serum therapy of typhoid fever has been greatly retarded by the difficulty that has been encountered in determining accurately and easily the potency of the immune serum to be used. As the typhoid bacillus does not produce a soluble toxin as does the Klebs-Loeffler bacillus of diphtheria, it has been impossible to produce an antitoxic serum which would correspond to the antitoxic diphtheria serum. Most antityphoid sera are of the agglutinating bacteriolytic type, the protective action of which can be determined only roughly on the basis of protection tests in mice or guinea pigs.

If serum is to be employed, one that has been prepared for intravenous use is to be recommended. Before instituting treatment, inquiry should be made concerning any previous injections of foreign serum and for symptoms suggestive of asthma. If the above factors are absent, a preliminary subcutaneous injection of 0.5 c.c. of the serum to be used should be given from twelve to twenty-four hours before intravenous treatment is begun. This procedure not only facilitates the detection of individuals who are-hypersensitive to the parenteral injections of a foreign protein, but also in the light of Besredka's work, serves to desensitize the individual, at least temporarily. It is rare to encounter an individual who is sensitive to horse serum, the medium usually used. But where hypersensitiveness to a foreign protein does exist, serious anaphylactic reactions have resulted when this preliminary precaution of desensitization has not been employed. If the subcutaneous treatment is followed by symptoms of serum alergy, local redness and swelling at the point of injection, urticaria and respiratory difficulty, careful desensitization should be tried according to the method of Besredka. 'If there is no evidence of hypersensitiveness, sterile serum can be given intravenously even in amounts as large as roo c.c. with safety, if the serum is given slowly, allowing fifteen or twenty minutes for the procedure. It is advisable to proceed very carefully with the first 5 c.c. of serum injected, allowing five minutes to elapse, and stopping if any signs of respiratory difficulty, flushing of face, restlessness or notable acceleration of the pulse appear. The authors have never experienced any anaphylactic reactions in a limited use of antiserum in typhoid fever and in a wider experience with immune serum in the treatment of lobar pneumonia, the above-noted precautions having been taken.

A rare and usually fatal complication of typhoid fever is meningitis. If the typhoid bacillus can be isolated from the spinal fluid, the use of an antityphoid serum would seem to be definitely indicated. While it has been impossible to find any reports of the serum treatment of typhoid meningitis, the fatal outcome of this condition justifies the cautious intraspinous administration of a potent anti- 
serum, using the same technique that is employed in the serum treatment of epidemic cerebrospinal meningitis.

A limited number of cases of typhoid fever have been reported which were treated with the sera of convalescent typhoid fever cases, with good results. The limited supply of such sera, together with the very obvious disadvantage of the danger of transferring other infections from the donor to the recipient must necessarily limit the scope of this form of treatment.

Recently several observers, notably Koenigsfeld(67), Petrovitch(68), and F. Ramond and G. Gauvert(69), have reported in the aggregate 555 cases of typhoid fever treated by autoserotherapy with apparent benefit in a large number of the cases. The procedure is as follows: the patient is bled, the serum separated from the clot and injected subcutaneously into another part of the patient's body.

This method of therapy has little experimental basis and seems largely empirical. Its ultimate usefulness has not yet been determined.

Active Immunization.-The treatment of typhoid fever by the use of vaccines has interested a large number of physicians since the initial work of Fraenkel(70) in 1893 when he reported the results of this method of therapy in fifty-seven cases. While progress in the vaccine therapy of typhoid fever was hindered for several years by doubt as to the specificity of the treatment by the work of $\operatorname{Rumpf}(7 \mathrm{I})$ and Kraus and Buswell(72), who thought they obtained equally good results by the injection of vaccines composed of $\mathrm{B}$. coli or $\mathrm{B}$. pyocaneus, still an increasing number of cases have been treated with each succeeding year, so that in 1915 Krumbhaar and Richardson (73) were able to collect reports on some I800 cases thus treated. While the statistics drawn from these cases have not shown any striking decrease in mortality, the impressions obtained from study of the clinical course of the disease under this form of treatment have been favorable. The dosage employed has been from 250 to I000 million bacteria injected subcutaneously every three or four days, depending on the type of vaccine used. The difficulty has been to find some practical method of determining the best vaccine to use, when and how to use it, and the proper dosage. It is notorious that conclusions drawn from the results of treatment of few and noncontrolled cases, in a disease that varies as much as typhoid fever, are extremely fallacious. However, attempts have recently been made to measure with some degree of accuracy the changes that take place in the blood of patients treated with vaccines. In a series of cases reported by Gay and Chickering( I $_{5}$ ), though small in number, the effect of vaccine treatment was noted in the blood by estimation of the changes in the number of bacteria in the blood, the production of leukocytosis, and changes in the strength of the agglutinin reaction. Still more work on the bacteriotropic and protective power of the blood of patients thus treated, as well as its complement 
fixing power, is now in progress. Experimentally it had previously been found that the intravenous injection of sensitized vaccine in non-immune rabbits caused a temporary leukocytosis of high grade. It was also found that the vaccine sterilized the blood of a large proportion of rabbits within a few hours after injection, and that it also prevented a large proportion of rabbits becoming chronic carriers. Moreover, the rabbits tolerated large doses of vaccine without harm. It was on the basis of this experimental work that Gay was encouraged to attempt the intravenous treatment of typhoid fever by the use of sensitized vaccine sediment, the method of preparation of which was described on page 193. This form of therapy had also been tried by Thiroloix and $\operatorname{Bardon}(74)$, who used, however, non-sensitized vaccine, and by $\operatorname{Ichikawa}(75)$ in $\mathrm{r} 9 \mathrm{r} 3$, who used a vaccine sensitized with the serum of convalescent patients.

When vaccines are given in typhoid fever by the subcutaneous route in moderate doses there follows practically no reaction. There may be a slightly higher rise in the evening temperature and possibly slight localized tenderness at the point of injection. On the other hand, when vaccines are used intravenously, there follows a very characteristic reaction. A general shaking chill occurs about onehalf hour to one hour after the injection of the vaccine, if the dose has been large enough, which may last from a few minutes to onehalf hour. This is accompanied by a rise in temperature of $1^{\circ}$ to $3^{\circ}$ in a period of as many hours. The temperature then falls as quickly as it has risen and it may reach normal or subnormal within eight to twelve hours. During the chill the patient may feel either hot or cold. If the patient complains of any discomfort, it is from the fatigue due to the spasm of the muscles during the chill. Occasionally there may be slight cyanosis and some acceleration of pulse and respiration. The rise in temperature is frequently accompanied by a leukopenia, the white blood cells falling to 2000 or 3000 . With the fall in temperature there may be a profuse sweating, which may last for several hours. It is during this period and in the few hours following that the hyperleukocytosis occurs. This may rise as high as 20,000 to 40,000 accompanied by a relative increase in the number of the polymorphonuclear cells.

When blood counts are made only at twenty-four-hour intervals, this hyperleukocytosis may be entirely missed, as it is usually transitory, the count returning to the original one in twenty-four to thirty-six hours. In some of Gay's carefully studied cases blood counts were made every two hours for twentyfour to thirty-six hours. With the fall in temperature the patient feels symptomatically well, the headache and delirium disappear, and in many cases the patient is hungry. In several cases the blood which was positive for typhoid bacilli immediately before treatment, became sterile twenty-four hours later, and remained so. At the same time the strength of the agglutinin reaction increased. In favorable cases the temperature may remain normal or rise in the evening for three or four days, and then remain normal. 
Thirty to 35 per cent. of the cases which abortively recover in this way make a very striking impression and while critical recovery from typhoid fever does occur in cases treated symptomatically, it is rare or is by no means as common as has been reported by the numerous authors whose observations have been compiled by Gay. Another third of the cases which do not recover in an abortive fashion seem to be definitely improved. The blood culture may remain positive for three or four days and then become sterile after a second or third treatment. The temperature will usually average a degree lower after each treatment and finally return to normal by slow lysis. While the results in these cases are not as striking as in those of the first group, and it is difficult to say what would have been the result without treatment, still the clinical impressions drawn from such cases are very favorable.

There is still another third which seems to be little influenced by specific intravenous treatment. In this group of cases the fever takes its usual course, the blood culture remains positive in spite of treatment, and the Widal reaction usually is feeble. While the vaccine seems to have no ill effects, the course of the disease is not shortened. It is in these severe cases which do not respond quickly to vaccine therapy that Gay has used a combination of serum and vaccine treatment. While the number thus treated is extremely small, in a few cases the blood was cleared of bacteria by the use of a strongly agglutinating antiserum and the temperature then fell to normal after another intravenous vaccine treatment. As soon as the blood becomes sterile when ro to 20 c.c. of blood are used for culture, improvement is rapid and convalescence usually close at hand. In cases which recover quickly after treatment it is very difficult to keep the patient in bed. He feels well and is anxious to eat. But, as one knows nothing of the effect of this form of treatment on the intestinal lesions, great caution should be exercised in permitting the patient to get up and go about.

The intravenous use of vaccines in the treatment of typhoid fever does not prevent the occurrence of relapses. It would seem that the reaction produced by the injection of vaccine causes a sudden mobilization of at least some of the factors which make up the mechanism of immunity. This sudden acquisition of immunity, if followed by further suitable stimulation, may be sufficient to prevent a relapse. It was found from a limited experience with the use of sensitized vaccine intravenously that a large proportion of the cases which relapsed were those that had recovered abortively after one or two doses of vaccine. Moreover, when the usual subcutaneous prophylactic vaccination was used as soon as intravenous medication seemed no longer necessary, the occurrence of relapses practically ceased. Whether this experience will be confirmed by the treatment of larger numbers of cases remains to be seen.

While vaccine treatment of typhoid fever is believed to be a very valuable therapeutic aid, it is not a method of treatment to be used 
in a routine fashion. There is no doubt but that the indiscriminate thoughtless overdosage of typhoid fever patients with vaccines has produced harm. This is especially the case with the intravenous use of vaccines. Too large doses of vaccine may produce most alarming symptoms and Biedl has reported one case that died three hours after treatment. The danger, however, does not lie so much in the vaccine as in its injudicious use by the inexperienced physician. At present this method of treatment should be reserved to hospital treated cases where the results of such treatment may be accurately and carefully watched.

Following Fraenkel's first publication a discussion arose as to the specificity of the reaction following vaccine treatment. Recent studies have shown that many foreign substances when injected into the blood of normal or ill individuals produce essentially the the same type of reaction as do typhoid vaccines in typhoid fever patients. But even though the general reaction following the injection of the typhoid vaccines is not of a specific character, it is well known that typhoid vaccines injected subcutaneously or intravenously do stimulate the formation of specific immune bodies which are in some way related to immunity.

Besides the sensitized vaccines employed as above described, practically all the varieties of vaccine which have been used for prophylactic purposes have also been employed therapeutically, and they have been injected subcutaneously and also intravenously. They have been given at different intervals from one to seven days and in dosages varying from to to 500 or 1000 million. A resume of the cases reported treated with various vaccines by both the subcutaneous and intravenous route has been made.

\begin{tabular}{|c|c|c|c|}
\hline . & $\begin{array}{l}\text { Number of } \\
\text { authors }\end{array}$ & $\begin{array}{l}\text { Number of } \\
\text { cases }\end{array}$ & $\begin{array}{l}\text { Benefited } \\
\text { per cent. }\end{array}$ \\
\hline 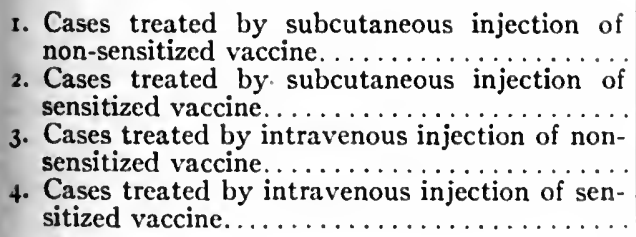 & $\begin{array}{c}13 \\
14 \\
9\end{array}$ & $\begin{array}{l}732 \\
455 \\
259 \\
260\end{array}$ & $\begin{array}{l}58.0 \\
57.0 \\
63.0 \\
78.5\end{array}$ \\
\hline
\end{tabular}

Though the intravenous treatment of typhoid fever by means of vaccines is still in its infancy, it would seem to be a method which may be productive of much good in shortening and ameliorating the disease.

\section{DIET IN TYPHOID FEVER}

The problem of nourishment in typhoid fever, the course of which usually averages thirty days or more, must always be of importance. The considerations which have influenced the dietary management 
of the disease from the time of Hippocrates to the present time have been discussed in considerable detail in Vol. II, page 2 ro, and will not be repeated here. In recent years there has been a steadily increasing tendency to give food of greater caloric value, if not of greater variety, in typhoid fever. In this country the foremost advocate of very liberal feeding in typhoid fever has been Coleman(76) and his collaborators. Up to I II I Coleman had collected considerable evidence of the usefulness and harmlessness of such a regime and the past four years have seemed to confirm his earlier experiences with this method of treatment. Briefly reviewing Coleman and DuBois' (77) investigations concerning the metabolic activity of the body during typhoid fever, it was found that the total metabclism seems to increase and diminish with the body temperature, there being an average increase over that of the normal individual of 40 per cent., but figures over 50 per cent. were occasionally encountered. It was also found that the metabolism of liberally fed patients was scarcely raised above that of fasting typhoid patients. In other words, there is a marked increase in the activity of body functions, but food in itself does not produce this activity. Food, in the typhoid fever patient, therefore, exhibits little (3-5 per cent.) or no specific dynamic action. In typhoid fever, protein, fat and carbohydrates are oxidized to the same or approximately the same products as in health, and in their oxidation give off standard units of heat. There is much evidence of a toxic destruction of protein as is shown by the distinctively negative nitrogen balance to cover the heat production. When typhoid fever patients are storing fat on an abundant diet they may be losing weight and body protein. The observers found it was impossible to maintain nitrogen equilibrium by feeding excess of protein or fat, but on the other hand, the use of carbohydrates in excess spared the body protein. Theoretically these patients need 38.5 calories per kilogram of body-weight, but in order to keep such a patient in nitrogen and weight equilibrium it may be necessary to increase this figure from 50 to IIO per cent.

DuBois has studied the question of absorption af food in typhoid fever in six cases and finds that even during the febrile period, protein, fat and carbohydrate may be absorbed almost as well as in health. In these experiments the patients were studied over a period of five to twenty-one days and were given a diet composed of:

$$
\begin{array}{lll}
\text { I000 c.c. milk } & \text { I00-200 gm. lactose } & \\
300-400 \text { c.c. of } 20 \text { per } & 2-3 \text { eggs } & \text { representing } \\
\text { cent. cream } & 2 \text { slices toast and butter } & 2000-3000 \text { calories. }
\end{array}
$$

When carbohydrates were given in amounts under $300 \mathrm{gm}$. a day reducing bodies were found in the stools only in traces. When amounts over $300 \mathrm{gm}$. were given, 2 to $3 \mathrm{gm}$. of such bodies were found. The nitrogen in the stools averaged I.I $2 \mathrm{gm}$. a day and never exceeded $1.8 \mathrm{gm}$. which is within normal limits, the percentage of loss being 7.1 per cent. The loss of fat through the stools during the first three weeks of the disease was 7.2 per cent., later falling to 
4.5 per cent., with an average loss in all cases of 6.02 per cent., whereas the normal loss is about 3 per cent.

With a diet composed chiefly of milk, cream, butter, lactose and eggs in amounts representing from 3000 to 5000 calories a day, Coleman(78) has shown that it is possible for a patient to gain weight even during the febrile period. In one case food up to 93 calories per kilogram of body-weight a day was given. $\mathrm{He}$ also showed that this increase in weight was not due to water retention. On the basis of calorimetry experiments Coleman believes the average typhoid fever patient requires more than 40 calories per kilogram of body-weight per day, but he emphasizes the necessity of determining the optimum amount of food to be given in each individual patient. Shaffer and Coleman's best results were obtained by giving 62 to $94 \mathrm{gm}$. of protein a day, representing 10 to $\mathrm{I} 5 \mathrm{gm}$. of nitrogen. Fats, while utilized at all stages of the disease, seemed to be better borne in the later stages, in some cases as much as one-half the total energy of the food given was in the form of fat. As noted above, carbohydrates prevent protein losses better than fats or protein itself and form the most important source of the energy requirement.

Coleman furnishes the protein portion of the diet largely in the form of soft-boiled eggs, giving four to six a day. Fats are given in the form of cream, ice cream, butter, and yolk of egg. He has given as much as 200 to $250 \mathrm{gm}$. of fat a day without any digestive disturbance. In some cases fat seems to cause nausea, vomiting, and diarrhea and consequently must be given more sparingly. Milk supplies fat and protein as well as carbohydrate, and is given in as large quantities as the individual patient can digest, usually $1 / 2$ to 2 quarts a day. If tympanitis occurs, or curds appear in the stools, the quantity is reduced. In some cases a special idiosyncrasy seems to exist for milk, and in these cases its place has to be supplied by other articles of food. The largest part of the carbohydrate requirement is supplied by lactose, of which Coleman has given as much as $\mathrm{I} 3 / 4$ pounds a day. He finds that lactose is well absorbed and more easily taken than other sugars. Large quantities of lactose may be taken in lemonades and orangeades.

In a limited number of cases Torrey(79) has investigated the changes that take place in the intestinal bacterial flora of typhoid fever patients on the Coleman high calorie diet. He found that on a diet consisting of a daily average of 50-100 gm. of protein, 75-100 $\mathrm{gm}$. of fat, and 250-300 gm. of carbohydrate, the intestinal flora tended to become simplified in regard to the variety of bacterial types observed. In cases that bore this diet well, fermentative types predominated, represented largely by Bacillus acidophilus. With patients on a purely milk diet this transformation did not take place in such a marked degree as occurred when a more liberal carbohydrate diet was employed. Coleman has utilized these observations of Torrey in a few cases by feeding Bacillus acidophilus in addition 
to the high calory diet and has noted a decrease in such digestive disturbances as distention, when such a condition has been present.

The following combinations are suggested by Coleman(80). They may be given in the earlier stages of the disease, adding four to six eggs to make up the protein requirement. Some of the cream may be given in the form of ice cream. In certain cases, if patients have no difficulty with mastication, custards, mashed potato, cocoa, apple sauce, toast and cereals are given, though these articles are usually more suitable later in the course of the disease. It is difficult to give more than 3000 calories a day in the early part of the disease. When the temperature is falling and in convalescence from 4000 to 6000 calories daily may be given.

Diets:

Calories

For rooo calories a day:

Milk, I quart (rooo c.c.) . . . . . . . . . . . . . 700

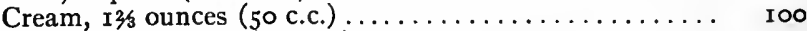

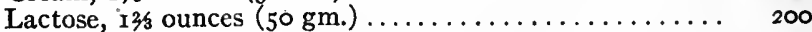

This furnishes eight feedings, each containing

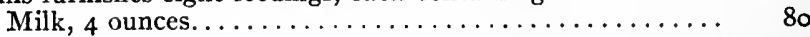

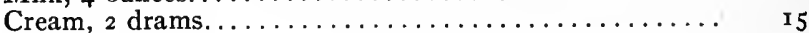

Lactose, 6 drams. . . . . . . . . . . . . . . . . . 24

For I 500 calories a day:

Milk, I 12 quarts $(1500$ c.c.) .................. 1000

Cream, I3/3 ounces .......................... 100

Lactose, 3 13s ounces (roo gm.) ........................ 400

This furnishes six feedings, each containing

Milk, 8 ounces........................

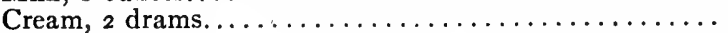

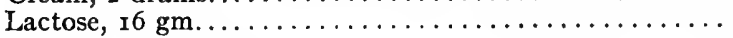

I60

I 5

64

For 2000 calories a day:

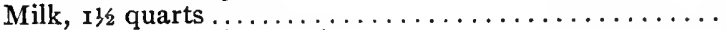

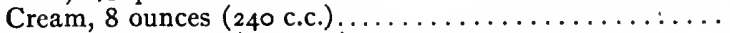

Lactose, 4 ounces (I 25 gm.)................

This furnishes seven feedings, each containing

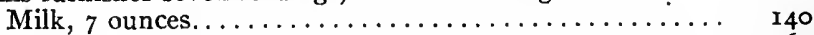

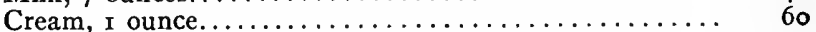

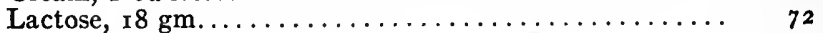

For 2500 calories a day:

Milk, r1 12 quarts ......................... 1000

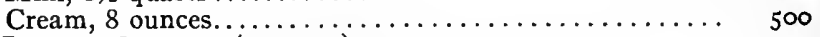

Lactose, 8 ounces $(250$ gm.)................ 1000

This furnishes seven feedings, each containing

Milk, 7 ounces. ..................... 140

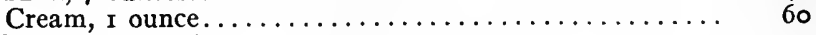

Lactose, $36 \mathrm{gm}^{1} . \ldots \ldots \ldots \ldots \ldots \ldots \ldots \ldots \ldots \ldots \ldots \ldots \ldots$

For 3000 calories a day:

Milk, r1s quarts ....................... 1000

Cream, I pint $(480$ c.c.).................. 1000

Lactose, 8 ounces....................... 1000

This furnishes eight feedings, each containing

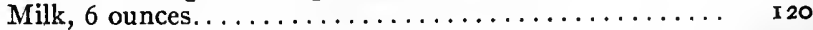

Cream, 2 ounces......................... I 20

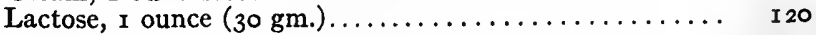

${ }^{1}$ If this and the following combinations are too sweet, a portion of the milk sugar may be given in some other form. 
For 3900 calories a day:

Milk, $1 \$ \xi$ quarts ......................... 1000

Cream, I pint......................... 1000

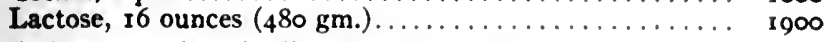

This furnishes eight feedings, each containing

Milk, 6 ounces.......................... $\quad$ I 20

Cream, 2 ounces........................ $\quad 120$

Lactose, 2 ounces....................... 240

The diet for this day furnishes 3910 calories.

$\begin{array}{lrrr}\text { Hours } & \text { Total } & \text { Calories } \\ \text { Milk, } 6 \text { ounces........ } & \text { 9 A.M.; I, 3, 7 P.M. } & \text { 1260 c.c. } & 860 \\ \text { Cream, 2 ounces....... ro P.M.; 1, 4 A.M. } & 420 \text { c.c. } & 840 \\ \text { Lactose, ro gm...... } & 70 \text { gm. } & 280 \\ & & \frac{280}{1980}\end{array}$

\begin{tabular}{|c|c|c|c|}
\hline At II A.M. & Calories & At 5 P.M. & Calories \\
\hline Egg, I....... & 80 & 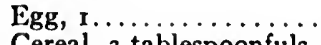 & 80 \\
\hline Mashed potato, $20 \mathrm{gm} .$. & 20 & Cereal, 3 tablespoonfuls. & 150 \\
\hline Custard, 4 ounces. . & 250 & Cream, 2 ounces. & 120 \\
\hline Toast (or bread), i slice. & 80 & Applesauce, I ounce.... & 30 \\
\hline Butter, $20 \mathrm{gm} . \ldots \ldots \ldots$ & I 50 & Tea & \\
\hline Coffee & & Cream, 3 ounces. & 180 \\
\hline Cream, 2 ounces. & I 20 & Lactose, $20 \mathrm{gm}$. . & 80 \\
\hline & & & \\
\hline
\end{tabular}

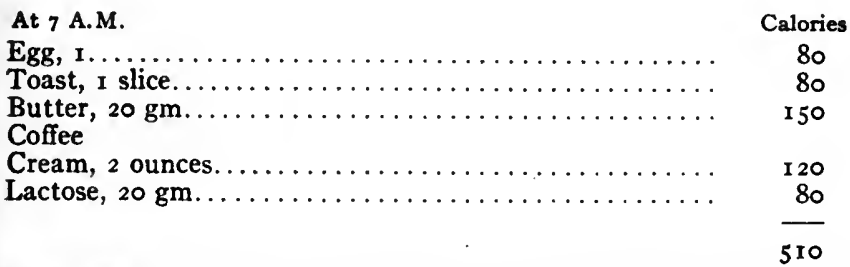

Milk sugar lemonade may be substituted for the milk mixture at three o'clock.

The diet for this day furnishes 5580 calories.

$\begin{array}{lrrr} & \text { Hours } & \text { Total } & \text { Calories } \\ \text { Milk, } 5 \text { ounces...... } & \text { 9 A.M.; II, I, 3, 7 P.M. I } 200 \text { c.c. } & 820 \\ \text { Cream, } 2 \text { ounces... } & \text { Io P.M.; I, 4 A.M. } & 720 \text { c.c. } & 1440 \\ \text { Lactose, I5 gm.... } & & \text { 120 gm. } & 480 \\ & & & \frac{4840}{2740}\end{array}$

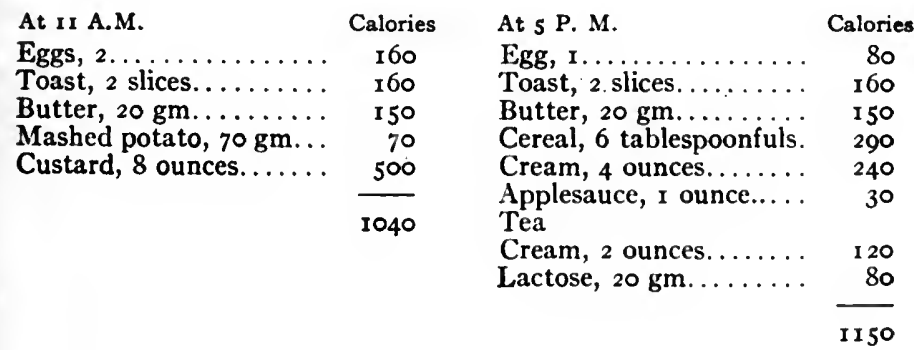




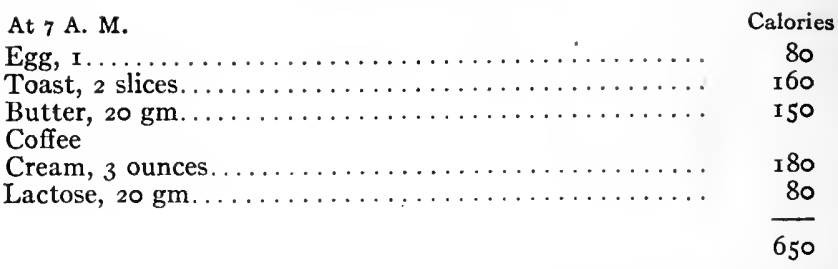

A great deal of the success of this treatment depends on the way in which the diet is administered to the individual patient. The amount of food should be increased cautiously. The abdomen and stools should be carefully watched. When absorption is not taking place the amounts given should be reduced. If the amount of lactose is increased too rapidly, it may cause nausea, vomiting and tympanites. Too much cream may cause diarrhea.

Coleman (80) has reported the results of the use of the high calory diet in I ro cases. Of these eleven died, a mortality of ro per cent., but of the eleven dying, in only three was it possible to give the liberal diet a trial. During the same period in the same and allied hospitals the mortality in 773 cases was 16.5 per cent. Relapses occurred in 20 per cent. of Coleman's cases, which is rather a high figure, but in the control cases 26 per cent. of the cases suffered relapse. Hemorrhage occurred in I 2 per cent. of the series and perforation in only one case. The majority of the patients lost some weight during the course of the disease, but in most cases the loss was under ro pounds. The greatest loss was 20 pounds in one case. Some patients gained I to 2 pounds during the febrile period. Gains in weight during the first two weeks of convalescence varied from 3 to ro pounds.

The high calory diet did not seem to shorten the course of the disease, but the toxicity of the disease seemed to be definitely lessened. Convalescence was materially shortened.

While it may not be possible to utilize the Coleman treatment to its utmost advantage in all cases because of the lack of coöperation on the part of the patient, this work has been of extreme importance in that it has clearly shown that in typhoid fever there is a very marked increase in metabolism and that by the treatment suggested the increased demands to maintain a metabolic equilibrium may be satisfied without danger.

\section{BIBLIOGRAPHY}

\section{TyPHOID FEver}

I. Metchnikoff, E. and Besredka, A., Recherches sur la fièvre typhoide experimentale, Ann. d. l'Inst. Pasteur, rgr r, xxv, I93.

2. SCHотTMÜLLER, H., Über eine das Bild des Typhus bietende Erkrankung hervorgerufen durch Typhus ähnliche Bacillen, Deutsch. med. Wchnschr., 1900, xxvi, 5 II.

3. AuERBACH, M. and Unger, E., Über den Nachweis von Typhusbacillen in Blut Typhuskranken, Deutsch. med. Wchnschr., 1900, xxvi, 796.

4. Cole, R., Frequency of Typhoid Bacilli in the Blood, Johns Hopkins Hosp. Bull, I9Or, xii, 203. 
5. SchotTMÜlleR, H., Die Typhösen Erkrankungen, Handbuch der Inneren Medizin, 1912 , bd. 1, Mohr and Staehelin.

6. Jocrmans, G., Lehrbuch der Infektionkrankheiten, Springer, Berl., 1914, p. 1.

7. Boxton, B. H. and Coleman, W., The Bacteriology of the Blood in Typhoid Fever, Amer. Jour. Med. Jci., 1907, cxxxiii, 896.

Coleman, W. and Buxton, B. H., The Bacteriology of the Blood in Convalescence from Typhoid Fever, Jour. Med. Research, I909, xxi, 83 .

8. SawYer, W. A., Ninety-three Persons Infected by a Typhoid Carrier at a Public Dinner, Jour. Am. Med. Assn., 1914, lxiii, 1537.

9. Mallory, F. B., The Principles of Pathologic Histology, Saunders Co., 1914, p. 162.

ro. Arrma, R., Uber die Typhustoxine und ihre pathogene Wirkung, Centralbl. $f$. Bakteriol., I91 2, 1xiii, 424.

31. Stenitzer, R., Typhusantitoxin, Kraus and Levaditi, Handbuch der Immunitätsforsch., I909, ii, 217.

12. Kock, J., Typhusbazillen und Gallenblase, Zeitschr. f. Hyg., 1909, lxii, 1.

13. Chiarolanza, R., Experimentelle Untersuchungen über die Beziehungen der Typhusbazillen zu der Gallenblase und den Gallenwegen, Zeitschr. f. Hyg., rgog, lxii, I r.

14. Prigge, F., Bazillenträger und Dauer ausscheider, Arb. a. d. Kais. Gesund., I9 2 , xli, 276 .

15. GAY, F. P. and Chickering, H. T., Treatment of Typhoid Fever by Intravenous Injections of Polyvalent Sensitized Typhoid Vaccine Sediment, Arch. Int. Med., I9r6, xvii, 303 .

16. Leishman, W., Discussions on Paratyphoid Fever, Brit. Med. Jour., I915, No. 2865,780 .

17. BAsset-Smith, P. W., Discussions on Paratyphoid Fever, Brit. Med. Jour., I915, No. 2865,780 .

18. Sarrailhe, A. and Clunet, J., "Camp Jaundice" and the Paratyphoid Epidemic at the Dardanelles, Lancet, 1916, $\mathrm{i}, 664$.

19. Bernard, L. and Paraf, J., La Séro Agglutination et le Diagnostic de l'Infection Eberthienne des Infections Paratyphiques, Presse Médicale, 1915, xli, 333.

20. Rist, E., Etudes sur la fièvre Typhoìde, Ann. méd., I916, iii, 54 and 88.

21. Chantemesse, A., L'Ophthalmo-diagnostic de la fièvre Typhöide, Deutsch. med. Wchnschr., r 907, xxxiii, 1572.

22. Floyd, C. and Barker, W. W., General Susceptibility in Typhoid and Colon Infection as shown by the Ophthalmic Test, Jour. Med. Research, r9o9, xv, 95 .

23. Austrian, C. R., The Ophthalmo-Reaction in Typhoid Fever, Johns Hopkins Hosp. Bull., I9 2, xxiii, I.

24. Landsberger, O., Zur Prognosestellung bei Typhus abdominalis, Med. Klin., I9I $5, \mathrm{xi}, 1078$.

25. Cole, R., Treatment of Pneumonia by Means of Specific Serums, Jour. Am. Med. Assn., I9I 3, lxi, 663.

36. Stokes, A. and Clarke, C., A Search for Typhoid Carriers among 800 Convalescents, Lancet, I916, $\mathrm{i}, 566$.

27. Preiffer, R. and Kolle, W., Experimentelle Untersuchungen zur Frage der Schutzimpfung des Menschen gegen Typhus Abdominalis, Deutsch. med. Wchnschr., 1896 , xxii, 735 .

28. Wright, A. E., On the Results Which have been Obtained by Antityphoid Inoculation, Lancet, London, I902, No. 4r $23,65 \mathrm{I}$.

29. Leisharan, W. B., Harben Lecture, Antityphoid Inoculation, Jour. Roy. Inst. Pub. Health, London, I910, July, Aug., Sept., xviii, 385-401, 499, 51 3-535.

30. Lyster, WM., Vaccination against Typhoid Fever in the United States Army, Jour. Am. Med. Assn., I915, lxv, 5 IO.

31. Wrigrt, A. E. and SeMpLE, D., Remarks on Vaccination against Typhoid Fever, Brit. Med. Jour., 1897, I, 256.

32. Russell, F. F., The Prophylaxis of Typhoid Fever by Means oi Vaccines, Forchheimer's Therapeusis of Int. Diseases, $1914, v, 182$.

33. VinCENT, H., Sur l'immunisation active de l'homme contre la fièvre typholde, Compt. rend. Acad. d. sc., I9I $2, \mathrm{clv}, 480$.

34. Castellani, A., Typhoid and Paratyphoid Vaccination with Live Attenuated Vaccines (Mixed Vaccines), Lancet, London, I913, i, 595; Observations on Typhoid Vaccination in Man with Attenuated Live Cultures, Centralbl. f. Bakteriol., I909, lii, 92.

35. Metchnikoff, E. and Besredka, A., Des Vaccinations Antityphiques, Ann. d. l'Inst. Pasteur, I9I I, xxv, 865.

Voc. IV.-14 
36. Besredka, A., De l'immunisation active contre le peste, le cholera et l'infection Typhique, Ann. d. l'Inst. Pasteur, rgo2, xvi, 918; Deux Ans de Vaccination Antityphique avec du Virus Sensibilisé Vivant, Ann. d. l'Inst. Pasteur, I9r3, xxvii, 607 .

37. Metchnikoff, E. and Besredka, A., Des Vaccinations Antityphiques, Ann. d. l'Inst. Pasteur, I913, xxvii, 597.

38. Gay, F. P. and Claypole, E. J., An Experimental Study of Methods of Prophy. lactic Immunization against Typhoid Fever, Arch. Int. Med., I914, xiv, 671.

39. Gay, F. P. and Claypole, E. J., Specific Hyperleucocytosis, Arch. Int. Med., IgI4, xiv, 669 .

Gay, F. P. and Claypole, E. J., Studies in Typhoid Immunization, Arch. Int.

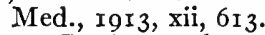

40. KRAUS, R., Bermerkungen über Schutzimpfungen une eine Bakteriotherapie des Typhus abdominalis, Wien klin. Wchnschr., I9I4, xxvii, I443.

4I. HARRIS, L. I. and OGAN, M. L., Experiences of the New York Health Department in Typhoid Immunization, Jour. Am. Med. Assn., I91 5, lxiv, 3.

42. Kolle, W. and Hetsch, H., Typhus abdominalis, bd. 1, p. 266; Die Experimentelle Bakteriologie und die Infektionskrankheiten, mit besonderer Berücksichtigung der Immunitätslehre, Urbain und Schwarzenberg, Berl., I9II.

43. Kutscher, K. H., Abdominaltyphus, Kolle und Wassermann, Handbuch der pathogenen Mikroorganismen, iii, I9I3, 7I7.

44. Bull, C. G., The Influence of Typhoid Bacilli on the Antibodies of Normal and Immune Rabbits, Jour. Exper. Med., I916, xxiii, 419.

45. Weekly Bulletin of the Department of Health, City of New York rgr6, v, 65 .

46. Leishman, W. B., An Investigation upon the Blood Changes Following Antityphoid Inoculation, Jour. Hyg., I905, v, 380 .

47. Johs, M. K., Uber Vergleichende Typhusschutzimpfungen, Deutsch. med. Wchnschr., I915, xli, 827 .

48. Dakeyne, D. I., Agglutination Reactions of the Blood of Soldiers Inoculated against Typhoid Fever, Lancet, London, I915, clxxxix, 540.

49. Garbat, A. L. and Mever, F., Uber Typhus Heilserum, Zeitschr. f. exper. Path., IgIO, viii, I.

50. Karaffa-Korboutr, Contribution a l'Etude du Sérum des Chevaux Immunises avec le Vaccin Antityphique de Besredka, Compt. rend. Soc. d. Biol., 19r4, lxxvi, 279 .

5r. SAWYer, W. A., The Efficiency of Various Antityphoid Vaccines, Jour. Am. Med. Assn., I915, lxv, I413.

52. Vincent, H., Sur la Vaccination antityphique, Jour. State Med., Igr 2, xx, 321.

53. WIDAL, F. et SiCARD, A., Etude sur le serodiagnostic et sur les reaction agglutinante chez les typhiques, Ann. d. l'Inst. Pasteur, I897, xi, 353.

WIDAL, F., Les associations microbiennes dans l'organisme, Chap. vii, Superposition des actions humorales propres á chaque microbe dans les infections combinees Congres de Montpellier, I892.

54. Castellani, A., Die agglutination bei gemischter Infection und die Diagnose der letzteren, Zeitschr. f. Hyg. u. Infektionskrankh., I902, xl, I; Antityphoid Vaccination with Attenuated Live Cultures, Lancet, London, I909, ii, 528; Typhoid and Paratyphoid Vaccination with Live Attenuated Vaccines; Mixed Vaccines, Trans. Soc. Trop. Med. and Hyg., I909, vi, 57, and Lancet, London, I91 $3, \mathrm{i}, 595$.

55. Vincent, H., Sur la vaccination de l'homme contre le fièvre typhoilde, Bull. d. l'Acad. de méd., 3d Series, Igro, lxiii, 615; Sur la vaccination antityphique. Vaccin par autolysat et vaccin bacillaire. Principes fondamentaux de leur preparation, Compt. Rend. Soc. d. Biol., I9I I, ii, 269; La vaccination antitypholdique dans l'armee francais, Rapport du xviie Congrés international de Medicine, Londres, I9I3.

56. Cummins, S. L. and Cumming, C. C., Preliminary Note on Immunization against B. Paratyphosus A, Jour. Roy. Army Med. Corps, I91 2, xix, 389;

Dreyer, G., Walker, Ainley E. W., and Gibson, A. G., Typhoid and Paratyphoid Infection in Relation to Antityphoid Inoculation, Lancet, London, I9 $5, i, 324$.

57. KABESHIMA, T., Uber typhus and Paratyphusschutzimpfung mittels gemischter Typhus und Paratyphusvaccine und die Ergebnisse der Schutzimpfung in der Kaiserlich Japanischen Marine, Centralbl. f. Bakteriol., orig., I914, lxxiv, 294.

58. Castellani, A., Further Researches on Combined Vaccines, Centralbl. f. Bakteriol., orig., I9I 5 , lxxvii, 63 .

59. Russeli, F. F., Antityphoid Vaccination in Children, Jour. Am. Med. Assn., r9r3, $\mathrm{lx}, 344$. 
60. Goldscheider, and Kroner, K., Uber den Einfluss der Typhusschutzimpfungen auf die Typhuserkrankungen bei der Armee im Herbst und Winter, 1914-1915 Berl. klin. Wchnschr., 1915, lii, 933,968 , 1001.

6x. Horrweg, Uber den Einfluss der Typhusschutzimpfung auf den Nachweis der Typhusbacillen im kreisenden Blut., München. med. Wchnschr., 1915, lxii, 538 .

62. Cranteuesse, A., Cinq. Ans de Sérotherapie Antityphoide, La Presse medicale, 1906, No. 16, 121 .

63. Rodet, A., Die Serotherapie beim Typhus, Handbuch der Serum Therapic, A. Wolff-Eisner, 1910,164 .

64. BuLL, C. G., The Mechanism of the Curative Action of Antipneumococcus Serum, Jour. Exper. Med., 1915, xxii, 457; The Fate of Typhoid Bacilli When Injected Intravenously into Normal Rabbits, Jour. Exper. Med., I915, xxii, 475.

65. Andriecsu, C. and Cuica, M., De l'Action du Serum antityphoique des Besredka sur l'Evolution de la fièvre Typhoỉde, Ann. d. l'Inst. Pasteur., I9 I3, xxvii, I 70.

66. BesredkA, A., Des moyens d'empécher les Troubles anaphylactiques, Compt. rend. Soc. Biol., 1909, lxvi, 125.

67. Koenigsfeld, H., Ein neues Prinzip der Serumtherapie bei Infektionskrankheiten mit besonderer Berücksichtigung des Typhus abdominalis, Munch. med. Wchnschr., I9I 5, lxii, 253 .

68. Petrovitch, Traitement de la fièvre typhoïde par l'homosèrothérapie dans l'armée, serbe, Presse Médicale, 1915, No. 35, 288.

69. RAMOND, F. and GouBERT, G., L'autohemothérapie apliquée au traitement de la fièvre Typhoïde, Bull. d. l'Acad. méd., I9 I 5, 3d series, lxxiii, 205.

70. Fraenker, E., Über spezifische Behandlung des abdominal Typhus, Deutsch. med. Wchnschr., I893, xix, 985 .

71. RUMpF, T., Die Behandlung des Typhus abdominalis mit abgetödteten Culturen des bacillus Pyocyaneus, Deutsch. med. Wchnschr., 1893, xix, 987.

72. Kraus, F. and Buswell, H. C., Über die Behandlung des Typhus abdominalis mit abgetödteten Pyocyaneus Culturen, Wien. klin. Wchnschr., 1894, vii, 51 I, 595.

73. Krumbiaar, E. B., and Richardson, R., The Value of Typhoid Vaccines in the Treatment of Typhoid Fever, Am. Jour. Med. Sci., 1915, cxlix, 406.

74. Thtrolorx and Bardon, Vaccin Typhique intraveineux, Centralbl. f. Bakt. Ref., $19 I_{4}, 1 x, 212$ (3d ref.).

75. Ichrkawa, S., Mitteil d. med. Gesellsch., zu. Osaka, April, 191 2, x, No. 5; Abortive Treatment of Typhoid and Paratyphoid, Sei-I-Kwai Med. Jour., Tokyo, 19I4, xxxiii, No. I 2, p. 73 or Zeitschr. f. Immunitatsforsch., I9 I 4, xxiii, 32.

76. Shaffer, P. A., and Coleman, W., Protein Metabolism in Typhoid Fever, Arch. Int. Med., 1909, iv, 538 .

77. Coleman, W., and DuBois, E. F., The Influence of the High Calory Diet on the Respiratory Exchanges in Typhoid Fever, Arch. Int. Med., I9I4, xiv, 168; Calorimetric Observations on the Metabolism of Typhoid Patients With and Without Food, Arch. Int. Med., I915, xv, 887.

Coleman, W. and Gephart, F. C., Notes on the Absorption of Fat and Protein in Typhoid Fever, Arch. Int. Med., 1915, xv, 882.

DuBors, E. F., The Absorption of Food in Typhoid Fever, Arch. Int. Med., I9I $2, x, 177$.

78. Coleman, W., Weight Curves in Typhoid Fever, Am. Jour. Med. Sci., I912, cxliv, 659 .

79. Torrey, J. C., The Fecal Flora of Typhoid Fever and its Reaction to Various Diets, Jour. Inf. Dis., 191 $5, x v i, 72$.

80. Coleman, W., The High Calory Diet in Typhoid Fever: A Study of One Hundred and Eleven Cases, Am. Jour. Med. Sci., 1912, cxliii, 77. 


\title{
LOBAR PNEUMONIA
}

\author{
By H. A. Hare, M. D.
}

Too much emphasis cannot be laid upon the need of fresh air in this disease and Northrup of New York deserves a great deal of credit for his insistence on this point. At first advocated by him for children suffering from pneumonia and proved of extraordinary benefit in these cases, it is now widely employed in adults as well. A sleeping porch, if it can be made, should be provided for the patient as life out-of-doors does better for pneumonia patients than a room or ward with open windows. At the Jefferson Medical College Hospital we have had for some years "pneumonia roof wards" and I would rather part with all drugs than with this method of treatment. I have repeatedly seen a patient with a good color and comfortable breathing become dusky colored and oppressed within five minutes after being brought into the wards for a physical examination and then improve promptly on being put out in the air. All my patients stay out under canvas regardless of temperature, rain or snow, and the danger of the nurse becoming infected by the disease is lessened, notwithstanding the exposure to the elements. She should, of course, dress carefully for her task. So valuable is this method that it is to be considered as the greatest advance in the therapy of this disease yet made, unless it be the recognition that excessive medication does more harm than good.

Care should be taken that the patient in winter weather is provided with a warm cap, which comes over the ears, and is given enough covering, but not too much. A hot water bag may be put at his feet if need be. So, too, either two mattresses should be put under him or a single mattress should be covered with a rubber blanket and two ordinary blankets to keep his under surface warm. Even when the bed pan is used it is not neccessary to take him indoors in most instances, and, unless a thorough physical examination of chest is required, an examination of the heart can be made through a small opening in the bed clothing. When the temperature reaches normal and the weather is bad he is taken indoors.

The amount of heat units the patient requires is not the number needed by a patient at rest in bed but the number needed by a man doing hard work, since the increased respiratory rate is a form of severe physical exercise which any one in health can determine if he will breathe as hard for five minutes as the patient often does for hours. The fever also uses up heat units or energy, but this is to be compensated by food and not by endeavoring to reduce the temperature by drugs. 
When the heart seems to be in very grave danger of failure an intravenous injection of strophanthin as prepared by Bochringer in ampoules ready for use, may be employed. A better preparation, because it can be given hypodermically without irritation as well as intravenously, if need be, is strophanthone, made by Parke, Davis and Company, also put up in ampoules ready for use. I have used this with great satisfaction but both strophanthin and strophanthone are very active preparations and ought not to be used as a rule oftener than once in twenty-four hours. When all stimulants fail, autopsy may show that a chicken fat clot has made the effect of any drug impossible of development.

Recently in cases of delayed resolution the use of the roentgenrays applied to the chest wall seems to have induced good results. I have no experience with this method. Often a supposed delayed resolution is in reality an empyema or or a thickened pleura.

And, finally, the physician should constantly, if possible several times a day, use the sphygmomanometer to determine not only the systolic pressure but more important the diastolic pressure and pulse force. Gibson in Edinburgh and the writer in this country showed conclusively some years' ago that when the systolic pressure, as expressed in millimeters of mercury, is well above the pulse rate per minute the condition of the patient is usually favorable and he does not need stimulation. If the figures are identical he is in danger, and if the pulse rate exceeds in number the figures of systolic pressure he usually dies. So, too, if the difference between the systolic pressure and the diastolic pressure constantly decreases it is indicative of lack of cardiac power and fatigue of the heart muscle, particularly if all the pressures are falling. If the cause is fatigue several hours' sleep induced by morphin with active stimulation may do good. If the failure is due to myocardial degeneration, remedial measures can do little. Very frequently the heart muscle, before the illness develops, is so impaired that it has "no margin of safety" to keep it going under strain and toxemia. 


\title{
SPECIFIC THERAPY OF PNEUMOCOCCUS INFECTION
}

\author{
By A. R. Dochez, M. D.
}

Diplococcus pneumoniæ or the pneumococcus, to use its more frequent designation, is a parasitic microörganism of wide distribution that is responsible for many and often fatal infections in man. Strange though it may seem, this organism which posesses the capacity to cause a variety of dangerous pathological conditions also leads a more or less harmless saprophytic existence in the buccal secretions of from 50 to 75 per cent. of healthy individuals. In fact, the pneumococcus was first isolated by Pasteur(I) not from pathological lesions but from the sputum of a case of rabies and by Sternberg(2) from his own saliva. A. Fraenkel(3) and Weichselbaum(4), working independently, were the first to establish the pneumococcus as a pathogenic agent for man and to determine beyond doubt its etiological relationship to lobar pneumonia.

Pneumococcus can be readily recognized in microscopic preparations made from the lesions which it produces. It stains with the ordinary aniline dyes, and in animal tissues appears as a lanceshaped diplococcus surrounded by a well-defined capsule. Chain formation as a rule does not occur in the animal body, the organism growing usually in single pairs of closely approximated cocci. In the tissues the organisms are generally present in large numbers, and can be cultivated with great regularity, provided that proper media are used. Good growth can usually be obtained in beef or veal infusion broth, the reaction of which should be about +0.6 per cent. acid to phenolphthalein and which should not be heated above $100^{\circ} \mathrm{C}$. in the process of sterilization. The addition of animal sera or of whole blood greatly enhances the growth, as does also the addition of certain sugars such as glucose. The latter, however, causes the organism to die rapidly in fluid cultures. Incubation at $37^{\circ} \mathrm{C}$. is most favorable to growth, and the greatest concentration of organisms is attained at the end of about eighteen hours. Pneumococcus grows well on blood agar plates on which it forms rather small translucent, moist ring-shaped colonies with a central elevation. In the periphery of the colony the hemoglobin is changed to greenish brown methemoglobin. Hemolysis rarely occurs. The organism is easily identifiable on artificial cultivation, the most salient features being its characteristic morphology, usual ability to ferment inulin, and readiness with which it is dissolved in bile or solutions of the bile acids. The latter test is a very serviceable one in distinguishing the organism from streptococcus which at times it may resemble morphologically. 
By far the most serious disease for which the pneumococcus is responsible is acute lobar pneumonia. In addition to this important condition, pneumococcus is the etiological agent in many instances of acute bronchitis and bronchopneumonia. It also plays an important role in affections of the upper respiratory tract such as the common cold and disease of accessory sinuses of the nose. Pneumococcus frequently invades the middle ear where it causes a severe inflammatory process which not infrequently extends to the mastoid antrum and may even penetrate the brain and meninges. Meningitis is an extremely serious manifestation of pneumococcus infection and is fortunately rather rare during the course of lobar pneumonia, although it is somewhat more frequent in children with middle-ear disease of pneumococcus origin. Most of the focal infections with pneumococcus outside the lungs occur as complications of lobar pneumonia and are in order of their frequency empyema, pericarditis, endocarditis and purulent arthritis. Abscess of the lung is not an infrequent sequela of lobar pneumonia, but almost always represents invasion of the lung with secondary organisms, such as streptococcus and staphylococcus. Primary pneumococcus peritonitis is occasionally observed and seems to be most frequent in young children. Inflammatory conditions of the eye, such as conjunctivitis and corneal ulcer are commonly due to pneumococcus infection. The conditions mentioned above are, however, comparatively rare compared with the high incidence of lobar pneumonia, and inasmuch as the object of specific therapy in pneumococcus infections has been to combat this disease, a short consideration of its character is important in order to understand the difficulties and limitations of such a method.

Lobar pneumonia is an endemic disease of widespread character. It attains its greatest incidence in the temperate zones though it may occur in the tropics where, in certain instances, it may assume epidemic proportions. The disease attacks individuals of all ages but is most common during the period of greatest physical activity, affecting males who perform hard outdoor labor more often than females who lead a comparatively sedentary existence. The greatest mortality is observed among the aged. The etiological agent is almost always the pneumococcus, although pneumonia of the lobar type may be produced by streptococcus, staphyloccccus and the Bacillus pneumonia of Friedländer.

The disease itself is one of the severest of the acute infections encountered in temperate climates, equaling and sometimes surpassing even tuberculosis as a cause of death. The onset of the infection is usually sudden and without obvious premonitory signs. However, it may be said that pneumococcus finds conditions most suitable for development and invasion of the lungs when it is implanted on previously inflamed mucous membranes as is demonstrated by the great frequency with which pneumonia is superimposed on the common cold and the increased incidence of the disease that is commonly noted 
during epidemics of influenza. One of the characteristic features of beginning infection is a chill, which may vary from simple chilly sensations to a hard rigor lasting often as long as half an hour. This may be followed by nausea and vomiting, and soon the cough, pain in the chest, and bloody tenacious sputum make their appearance. The patient rapidly passes from what previously may have been a state of good health into one of great urgency. Respiration becomes rapid and of a labored, often shallow, character. The temperature rises to a high point and maintains its elevation with slight variations throughout the course of the disease. The pulse rate is rapid, almost always above roo to the minute, and in the severe cases shows a tendency to increase as the critical period of the disease is approached. The two most important criteria for determining the progress of the disease are the rate of spread of infection in the lung and the degree of invasion of the blood by the pneumococcus. It is undoubtedly an error to maintain that the amount of lung involvement has no relationship to the outcome of the disease. The whole mechanism of resistance in pneumonia is designed to localize the infection and when this is successfully accomplished the result is usually favorable.

The natural tendency, however, of every pneumococcus infection of the lungs is either to spread locally or to cause a serious invasion of the blood, and the condition of the patient responds with the accuracy of a barometer to the degree to which such an aim is achieved. With every extension of the disease process the condition of the patient becomes more precarious, and he is most likely to succumb during one of the periods of active growth of the pneumococcus. The ordinary duration of pneumonia is from seven to ten days, although the period of defervescence occurs not infrequently either before or after this period. At the crisis the individual passes sometimes within a few hours from a state of profound distress into one of comparative comfort. Pneumococcus is therefore a watchful enemy that makes use of each opportunity to extend its activities. It gains momentum with every advance and tests to the utmost the resistance of the infected individual. If success is to be achieved by specific therapy a method must be devised which can match the extraordinary resourcefulness and rapidity with which the organism develops its attack.

\section{SERUM THERAPY}

Ever since the discovery by Pasteur that animals could be successfully immunized against infectious microörganisms, the goal of investigators in medicine has been to make use of this process in the curing of human disease. The study has been one of great fruitfulness and from it there have developed many facts of importance in the interpretation of infectious diseases. Attempts to control bacterial diseases by means of artificially produced immune animal sera have developed along two somewhat different lines. The observation by Roux and Yersin(5) that the diphtheria bacillus 
on artificial cultivation produced in the medium of its environment a toxin which is analogous in its activities to that produced during growth in the animal body, and the demonstration by v. Behring $(6)$ that laboratory animals could not only be rendered immune to the poison of the diphtheria bacillus, but that this immunity could be conveyed passively to normal untreated animals by injection of the blood serum of the immunes, represent the first great advance in the subject of serum therapy. Hope was engendered by these discoveries but it was soon found on further investigation that comparatively few infectious microörganisms give rise to tangible toxic bodies, and that because of this fact the scope of the method is greatly limited.

Numerous attempts have been made to develop a soluble toxin from the pneumococcus, but, accepting diphtheria toxin as a standard for comparison, these attempts must all be adjudged failures. Toxic bodies have been recognized in culture fluids in which pneumococcus has been grown, and a certain degree of immunity to them can be produced, but the toxic content is so small and the immunity so achieved seems to differ in no way from that attained by the use of the bacterial bodies themselves. We are, therefore, forced to conclude that the mechanism of immunity against pneumococcus is quite different from that involved in infection by the diphtheria bacillus. The failure to develop antitoxic sera has, therefore driven investigators to seek another method for the control of pneumococcus infection. The knowledge was acquired many years ago that the serum of an animal immunized actively against a living virulent microörganism would, in some instances, protect other normal animals against many times the lethal dose of this organism. Such sera are designated as anti-infectious or antibacterial, in order todistinguish them from the true antitoxic sera. Their activity is directed not against a soluble toxin produced by the bacterium, but against the living organism itself, and results either in the destruction of the infectious agent or a limitation of its capacity to deve op. A. Fraenkel (7) was the first to make the fundamental observation that rabbits which had withstood an injection of a living pneumococcus culture were subsequently immune to lethal doses of fully virulent organisms. This observation was fully confirmed by numerous other investigators, and it soon became apparent that a high degree of active immunity could be produced in animals either by treating them with killed cultures of pneumococcus or by the utilization of both killed and living organisms.

When this fact had been established beyond doubt, the step was but a short one to the attempt to make use of the blood serum of animals actively immunized to pneumococcus for the protection of non-immune animals against pneumococcus infection. The early efforts met with considerable success, and it was found quite easy to guard susceptible animals against infection if a certain quantity of the immune serum were given either previous to or simultaneously with the dose of the infecting microörganism. On the other hand, 
attempts at curative results were not nearly so successful, and it was necessary to give serum within a very short period of the time of infection in order to observe any therapeutic effect, since pneumococcus infection in small laboratory animals is usually septicemic in character and rapidly fatal. Subsequent observers(8) by developing a slower type of pneumococcus infection have observed favorable therapeutic results when serum has been administered some hours after infection has occurred.

The comparative success of antipneumococcus serum in animal experimentation has seemed to justify an attempt to make use of this method in the treatment of lobar pneumonia in man. Investigations concerning the effect of such immune serum on the course of the disease in human beings were first made by G. and F. Klemperer(9) more than twenty-five years ago. They tested the efficacy of serum of rabbits highly immunized to pneumococcus in eighteen human cases and were of the opinion that a certain amount of benefit was derived from its use. The amount of serum employed was small in each instance and in the light of what is known today striking results were not to be expected. Since that time the subject has been studied more or less continuously, and with the passage of years more and more has been added to our knowledge until at the present time treatment of pneumonia with specific antiserum bids fair to become a permanent and useful method of therapy. During this period numerous sera have been prepared by different investigators and it may be of some interest to trace briefly the history of the development of the method. The antipneumococcus sera that have had the widest application to human cases have been those of Pane, Römer, Neufeld and Händel abroad, and the serum prepared by the Rockefeller Institute in the United States. Commercial houses have marketed antipneumococcus sera and advised its use for a number of years but the statistics following its employment have not been collected and are not available for study.

Pane, working in Italy, has prepared a serum by immunization of the donkey to pneumococcus and his serum has been applied in a considerable number of human cases. Pane(10) himself has treated thirty-two cases with this serum. According to this author the serum brings about an improvement in the subjective feelings of the patient and causes a reduction of the temperature. Of the treated cases three died, a mortality of about 9 per cent. The three fatal cases were treated in an advanced stage of the disease. Before this Washbourn(II) had successfully treated two cases of pneumonia with a serum made by immunization of a pony. Eyre and Washbourn(I 2) made a further study of Pane's serum and tested its potency against five strains of pneumococcus, four from individuals who were the subjects of pneumococcus infection and one from normal saliva. They found that the serum had protective value for four of these strains, but failed to protect rabbits against infection with the fifth. A number of other investigators have employed Pane's 
serum and thought that they observed a certain degree of favorable effect. Banti and Pieraccini(r $\left.\mathrm{r}_{3}\right)$ treated twenty-one cases with this serum without apparent benefit and Spolverini(I4) observed the same effects when normal serum was used as when immune serum was administered. Anders(I5) has reviewed the serumtreated cases of pneumonia in the literature up to r904 and comes to the conclusion that the slight apparent lowering of the mortality is insufficient to justify its general application, but is sufficiently striking to warrant a continuation of the study.

The antipneumococcus serum made according to the method of Römer has received a rather wide application in Germany, both in the treatment of lobar pneumonia and in pneumococcus ulcer of the cornea. Römer(r6) attempted to improve the method of preparation of the serum. The strains of pneumococcus used for immunization were collected from a variety of human sources and, with as little manipulation after isolation as possible, were injected into the animal to be immunized. Animal passage was avoided because it was thought that such a procedure might alter the antigenic qualities of the organism. In addition to this unusual procedure, a variety of animals were selected for immunization on the grounds that human serum might fail to complement the antibodies of one animal serum, whereas it might possess a suitable complement for that of another species. Such complicated methods have subsequently been found not to be necessary and have been discarded. The reports of the use of this serum in lobar pneumonia are not altogether convincing as to its value. Römer(r 7 ) himself reports very beneficial effects on ulcus corneæ serpens. Pässler(I8) treated twenty-four cases of human pneumonia with Römer's serum with twenty recoveries and four deaths. Of those that died, two were practically moribund at the time of treatment, and one had such severe complications as endocarditis and meningitis. The serum was given subcutaneously in repeated doses of from ro to 30 c.c. In favorable cases the course of the disease seemed to be shortened and there seemed to be a beneficial effect subjectively and on the respiration and circulation. He advises the use of the serum in severe cases of pneumonia. Other observers have obtained somewhat less noticeable results and Jürgens(I9) claims to have noted no favorable effects following the use of the serum. A summary of the results of several observers shows that of forty-four cases treated with Römer's serum, 25 per cent. died, which is about the normal mortality in pneumonia of the type of case treated, a result that seems to indicate that the serum in question had no very marked influence on the death rate in pneumonia.

Neufeld and Händel(20) working in Germany, have been largely responsible for the further development that has taken place in the preparation and use of antipneumococcus serum. They emphasized the importance of using the intravenous inoculation of large doses of virulent pneumococcus in the immunization of animals. 
The strains chosen as antigens were obtained from human cases of pneumonia and their serological relationships were carefully studied. Eyre and Washbourn and Römer had previously recognized the biological differences among strains of pneumococcus and had made use of a multiplicity of strains, but Neufeld and Händel tested the antigenic relationship of one strain to another. There developed from this study the fact that certain strains of pneumococcus are closely related to each other immunologically and on further investigation it developed that the majority of pneumococci studied by these authors were of the same variety and that an immune serum prepared from one of these strains would protect animals equally well against infection with all the others. In addition to this type organism, certain other atypical organisms were encountered which were resistant to this serum. These strains however, gave rise to a potent immune serum when injected into animals, but this serum protected only against the organisms used for immunization and against none of the strains included in the major variety. Two of the atypical strains which were examined exhibited crossprotection. From these studies it at once becomes evident that the character of pneumococcus concerned in infection is of the utmost importance from a standpoint of specific therapy, and until it is known that the organism in any particular case is susceptible to the action of the serum employed, judgment cannot be passed on the efficacy of such a serum.

The titration of the potency of antibacterial sera has always offered difficulties and such methods as have been devised are not nearly so accurate as those employed in the standardization of diphtheria antitoxin. Attempts had previously been made to determine the protective power of antipneumococcus serum by measuring the amount necessary to protect mice or rabbits against infection with rather small doses of pneumococcus. Such methods are necessarily rough and Römer for a time maintained that the only useful measure of the potency of this serum was to test it in infected human beings. Neufeld and Händel have developed a method for titrating antipneumococcus serum which seems to be fairly satisfactory, and permits a certain standard of efficiency to be maintained. Mice are injected in duplicate with a given quantity of serum, usually 0.2 c.c., and about two hours afterward with varying doses of a culture of standard virulence. In this way the minimal lethal dose of culture is estimated; and the number of lethal doses against which the measured quantity of serum protects is determined. No definite unit has so far been established by them, other than that 0.2 c.c. of a good serum should protect against approximately 0.I c.c. of culture of which $0.00000 \mathrm{r}$ c.c. is fatal for mice. In the course of this study the fact was observed that 0.2 c.c. of immune serum protects quite constantly against o.I c.c. of a virulent culture, but that if the serum is reduced in amount a level is soon reached at which no protection results, even against 
minimal doses of pneumococcus, although theoretically the serum contains many times the requisite amount of antibodies. From this they deduce that protection is related to the concentration of antibodies in the animal, and not to the actual quantity. The necessary concentration for animals has been determined, and from this they estimate that a curative dose in man should be at least 75 c.c. of a serum equal in potency to the one with which the studies were made. They advise the use of the intravenous method of administration. Antipneumococcus serum previously had been given for the most part subcutaneously, and in very much smaller doses, so that these two factors may explain in part at least the inadequacy of antipneumococcus serum in the past.

The serum of Neufeld and Händel has now passed beyond the experimental stage and is being prepared commercially in Germany for the treatment of human cases of lobar pneumonia. Weitz(2I) has reported the treatment of thirty-eight cases of pneumonia with this serum. The initial dose was from ro-40 c.c. given intravenously, and this was repeated every twelve hours until two and frequently several injections had been given. Of sixteen cases treated on the second day, twelve ran an abortive course, one of which had 90 colonies of pneumococcus per cubic centimeter of blood upon the first treatment. Two cases showed defervescence of the fever on the third day, ten on the fourth and one on the fifth day. In three cases there was no shortening in the period of the disease. One individual, an alcoholic, died, but at the autopsy the blood was sterile, although before treatment ro c.c. of blood contained from 2000-3000. colonies. Of fourteen cases treated on the third and fourth day, nine had a normal temperature within two days of the beginning of treatment, two showed no appreciable alteration of the duration of the pneumonia, and three died. The latter were said to have had mixed infections. Four out of seven cases treated first on the fifth to sixth day died. These results are by far more convincing as to the usefulness of antipneumococcus serum than any previously reported. The report of Weitz is interesting in that it shows clearly the influence of serum on blood infection in pneumonia, and the great importance of its administration early in the disease. In the light of what we now know the results seem extraordinarily good, since apparently no attempt was made to determine whether or not the organism in each particular case was susceptible to the action of the serum.

Geronne(22) has reported the treatment of twelve cases of pneumonia with the serum of Neufeld and Händel. Three of these were in children. In the early cases he found that doses of from ro to 20 c.c. given intravenously were insufficient, and later used from 40 to 80 c.c. in a single dose. He observed a marked improvement in the general condition of the patients, a lowering of the temperature and in certain cases apparent shortening of the disease. The local condition of the lung did not seem to be materially in- 
fluenced. Controls injected with normal sheep serum were uninfluenced by the treatment. Neufeld thinks that failure to alter the lung lesion is relatively unimportant and that the chief benefits to be derived from the use of antipneumococcus serum are the control of the septicemia and the possible prevention of new areas of involvement in the lung.

During the past six years a study of lobar pneumonia has been carried on continuously at the Hospital of the Rockefeller Institute. The principal object of this endeavor has been to develop if possible a means of treating the disease by specific methods, and in order to attain this object our main effort has been directed along the lines of specific serum therapy. The hope that an immune serum may prove efficacious in treatment is based upon the fact that at the period of recovery from pneumonia certain specific substances not previously demonstrable develop in the blood of the infected individual. Previous investigators have shown that the serum of patients after the crisis in pneumonia possesses the power to protect animals against experimental infection with pneumococcus. The truth of these observations had, however, been denied and the starting point of our investigations has been a further study of this question. Observation of a series of individuals from whom the blood was removed at varying intervals of time, both before and after the crisis, has shown the serum to contain no recognizable antibodies in the precritical period. However, rather sharply at the time of the crisis substances make their appearance in the blood which are capable of protecting mice against many times the minimal lethal dose of pneumococcus. These bodies persist for a variable length of time after the crisis and are most active against the strain of pneumococcus from the infected individual, somewhat less so against strains of the homologous group, and not at all against heterologous strains. Chickering (23) has studied the development of agglutinins and has shown that the curve of these bodies follows closely that of the protective substances. These studies indicate that at the time of recovery from lobar pneumonia there develop in the blood of the patient antibodies which are in every way analogous to those found in the serum of artificially immunized animals, and we must assume that they are in part at least concerned in the mechanism of recovery. The attempt to supply these substances in artificially prepared sera is, therefore, a logical one.

The fact has been recognized for some time that distinct biological varieties of pneumococcus exist. Eyre and Washbourn, and. Neufeld and Händel have pointed out that a serum which possessed protective power against one strain of pneumoccocus might fail entirely in such action if another strain of organism were employed. In view of this fact, Dochez and Gillespie(24) have made a study of a large number of strains of pneumococcus freshly isolated from lobar pneumonia, in order to determine the degree of variation that occurs and what particular varieties were dominant, that the latter 
might be chosen for purposes of immunization. The results of these studies have shown that all the pneumococci encountered could be divided into four general groups which have been arbitrarily numbered I, II, III, IV. This grouping depends upon the presence of specific biological relationships which are determined by the reactions of protection and agglutination. The dominant types are groups I and II. If horses be immunized against strains belonging to either of these two types a serum of comparatively high potency is developed, and such a serum is found to protect animals equally against infection with all strains of the homologous group, but has no such protective value for strains belonging to the other groups. Organisms of these two groups closely resemble each other morphologically and culturally. Group III consists of strains of the pneumococcus mucosus so-called because of the large moist colonies and the mucous exudate which they cause in infected animals. They are quite distinctive culturally. Because of the low potency of sera derived from immunization to these strains, it has not seemed likely that such sera would possess any therapeutic value in man. Group IV comprises a heterogeneous series of strains of pneumoccocus that are individual in their immune reactions. A serum prepared from any one of these strains protects only against the organism used for immunization, and not against strains belonging to groups I, II or III. A classification of strains of pneumococci obtained from a large number of cases of pneumonia is given in the following table:

Table I.-Ceassification of Disease-producing Types of Pneumococcts

\begin{tabular}{|c|c|c|}
\hline Type of organism & Number of cases & Percentage \\
\hline $\mathbf{I} \ldots \ldots \ldots \ldots \ldots \ldots$ & 78 & 34.97 \\
\hline $\operatorname{II}, \ldots \ldots \ldots \ldots \ldots \ldots$ & 75 & 33.63 \\
\hline III $\ldots \ldots \ldots \ldots \ldots \ldots$ & 22 & 9.86 \\
\hline IV.. & 48 & $21 \cdot 5^{2}$ \\
\hline Total number of cases. & 223 & \\
\hline
\end{tabular}

From this table it is evident that lobar pneumonia in man is due to a variety of distinct races of pneumococcus. The significance of this fact in the problem of specific serum therapy is obvious. It at once becomes evident that no constant results can be expected from the use of a serum prepared from a strain whose exact relationship to other strains of pneumoccoccus is unknown. Furthermore some means must necessarily be devised for determining whether a serum to be used in any particular case of pneumonia is active against the type of pneumococcus responsible for the disease in the individual under observation. Up to the present time there has been produced at the Rockefeller Institute a serum of high protective value for organisms belonging to group I and a serum of somewhat lower potency against organisms of group II and these sera are suitable for therapeutic application in man. As I have said before, immunization of animals to pneumococcus type III gives a serum of such low value that it does not seem useful at the present time to test its efficacy in man. Group IV consists of a series of independent strains 
not related to one another serologically and because of this fact it is manifestly impossible to prepare a serviceable serumagainst organisms of this group, since such a serum would be limited in its activity to the particular strain used for immunization. Although quite a large number of infections are due to organisms of this group, the need of a specific serum is not so urgent because of the low mortality encountered in such infections. There is some variation in the virulence of the different groups for human beings and the mortality statistics of a certain number of cases not specifically treated is given in the following table:

TABLE II

\begin{tabular}{|c|c|c|c|}
\hline Type of organism & Number of cases & Deaths & Percentage \\
\hline $\begin{array}{l}\text { I. } \\
\text { II } \\
\text { III } \\
\text { IV } \ldots \cdots \cdots \cdots \cdots \cdots\end{array}$ & $\begin{array}{l}28 \\
25 \\
17 \\
33\end{array}$ & $\begin{array}{l}7 \\
9 \\
8 \\
2\end{array}$ & $\begin{array}{r}25 \\
36 \\
47 \\
6\end{array}$ \\
\hline & IO3 & 26 & 25 \\
\hline
\end{tabular}

At the hospital of the Rockefeller Institute specific serum therapy for the reasons mentioned above has been limited to cases infected with organisms belonging to groups I and II. The sera employed are univalent in character and in order to treat any individual it is necessary to determine promptly the type of pneumococcus with which he is infected. The most rapid and suitable method for obtaining the organism concerned consists in inoculation of fresh sputum into the peritoneal cavity of a mouse. By proper supervision it is usually possible to obtain a small portion of sputum coughed up directly from the lung. This is washed through a series of sterile salt solutions, ground up in a sterile mortar with about I c.c. of bouillon and injected directly into the peritoneal cavity of a mouse. In this locality pneumococcus grows with great rapidity whereas contaminating mouth bacteria usually fail to develop. The degree of growth can be easily determined by inserting a capillary glass pipette through the abdominal wall, drawing out a little fluid, staining and examining microscopically. When the organisms have become sufficiently numerous, usually in from four to eight hours, the mouse is killed and the peritoneal cavity washed out with salt solution. Such fluid contains bacteria and leukocytes. The cellular débris is removed by centrifugalization at low speed, leaving the bacteria in the supernatant fluid, which is subsequently pipetted off and the organisms thrown down at high speed. They are then resuspended in salt solution, and their agglutination reaction tested with the various types of sera. Should the pneumococcus in question agglutinate typically in type I or type II serum, treatment is commenced at once with the appropriate serum. If antipneumococcus serum is of any therapeutic value in man, such efficacy as it may 
possess has the best chance to show itself under the conditions in which the serum employed is known to be specifically adapted to the organism in question.

The method of administration of the serum may be described briefly as follows: On admission the patient receives 0.5 c.c. of horse serum subcutaneously in order to test for hypersensitiveness. As soon as the type of organism has been determined, and the danger of anaphylaxis ruled out, he receives intravenously from 50 to 100 c.c. of serum diluted one-half with freshly prepared salt solution. This dose is repeated twice daily until the condition of the patient indicates that no more serum is neccessary. Usually from four to five such treatments are required, though in some instances more serum has been given without harmful effects other than the characteristic serum disease which so often follows inoculation of animal sera.

This method of specific therapy of lobar pneumonia has now been carried on for a sufficient length of time to give clear indications concerning the value of the procedure. Treatment of pneumonia with serum type I has given unexpectedly good results, whereas treatment of cases with whole serum type II has in our hands proved unencouraging. With the latter serum other investigators have had more success. In a series of sixty-five cases treated with serum type I, the mortality has been 7.5 per cent. Reference to Table II shows that the average mortality among treated cases in this type of infection has been 25 per cent. showing a reduction in the mortality of about 18 per cent. These statistics include one individual who was moribund at the time of the first treatment, and another who died of pulmonary embolism after recovery from the pneumonia. Such results indicate that in pneumonia due to type I pneumococcus serum therapy causes a lowering of the mortality of this disease. Inasmuch as this method of treatment has been applied for a number of years with continued evidence of beneficial effects one feels justified, even in the absence of a very large number of treated cases, in the hope that treatment with type I serum will prove of permanent benefit.

The effect of serum on the temperature in general is as follows: In quite a number of cases the intravenous injection of the serum is followed in about half an hour by a sharp reaction accompanied by a chill during which the temperature may rise considerably. Following this the patient may have a sweat and the temperature may fall to normal. A subsequent rise usually occurs and the phenomenon may repeat itself after the second injection. It is unusual to have more than two such reactions. Frequently no chill or rise in temperature occurs and defervescence takes place slowly over a period of several days. Sometimes the temperature curve is uninfluenced and the drop to normal occurs by crisis at the customary time. In general, there is some shortening of the duration of the febrile period, and a striking absence of the Vol. VI. -15 
increased pulse rate and fever that are so often seen in untreated cases during the time when the pneumonic process is spreading. As a rule the tendency to the rising pulse rate commonly observed in severe instances of the disease is absent. The general condition of the patient is almost always benefited by the administration of serum, the toxic symptoms are diminished, and cyanosis less marked. One of the most important clinical criteria for judging the efficacy of the serum is whether or not the lung lesion continues to spread after the initiation of treatment. In a number of instances when treatment was begun early there has been no apparent increase in the amount of lung involvement. The portion of the lung originally involved in some cases goes on to complete consolidation, but only very exceptionally has the process spread to a new lobe during the course of treatment. On the other hand, the consolidated lung shows no tendency to an increase in the rate of resolution and quite a number of cases show delayed resolution. It has been thought that serum may in some way be responsible for this delay, although it is not an uncommon occurrence in cases of pneumonia not treated with serum. In a number of instances empyema has followed recovery from the pneumonia itself, but so far all such cases have terminated favorably following the usual operative procedures.

As has been said before, one of the important features of severe instances of lobar pneumonia is the frequency with which invasion of the blood by the pneumococcus occurs, and the determining influence that such invasion may have on the course of the disease. The effect of the administration of antipneumococcus serum upon such a septicemic condition is, therefore, one of the important criteria in judging the efficacy of the serum. In every case so far studied sterilization of the blood has followed promptly after the treatment. Such a result is usually attained after a single dose and only very exceptionally, thereafter, has it been possible to cultivate the pneumococcus by blood culture. Early destruction of the microörganisms in the blood probably has a significant influence in preventing the development of meningitis and metastatic infection elsewhere in the body.

When lobar pneumonia runs a normal course in individuals not specifically treated immune bodies make their appearance in the patient's blood, first, at the period of critical fall in the temperature when such occurs, or during the time of slower defervescence that is sometimes observed. Previous to this period no such immune bodies are demonstrable. It has been possible to study the appearance of such bodies in the blood of patients treated with antipneumococcus serum, and it has been found that they are present in recognizable quantity immediately following the first injection of serum, and that by subsequent treatment they can be kept throughout the course of the disease at a level quite as high as that normally attained at the time of crisis. This means that small amounts 
of the patient's serum, taken at any time during the disease following the first treatment contain sufficient immune bodies to protect small animals against many times the lethal dose of pneumococcus. The fact that these substances are demonstrable in considerable concentration throughout the course of the disease would seem to indicate a favorable action of the serum. The results here detailed apply in their fullest extent only to cases of pneumonia due to pneumococcus type $I$ infection and treated with the corresponding serum.

As I have said before, we have discontinued the treatment of pneumococcus type II pneumonia with whole immune serum of that type because of the absence of any demonstrable beneficial effect on the outcome of the disease. The work of Gay and Chickering(25) and of Chickering(26) on new methods of concentration and application of this serum has, however, enabled us to continue the specific treatment of this form of the disease with some hope of success. Although an insufficient number of cases have been treated up to the present time to permit of satisfactory judgment of the results, a short description of the method is of some interest. Gay and Chickering found that all the immune bodies can be precipitated from an antipneumococcus serum by adding to it a solution of the bodies of the penumococcus. Such a precipitate has the power to protect mice against experimental infection with pneumococcus. Chickering subsequently showed that extraction of this precipitate with salt solution after certain manipulations causes the immune bodies, and probably only a small part of the antigen, to be redissolved in the salt solution. In this way the greater part of the immune bodies are recovered in a much condensed form and can be used for treatment. In addition to the immune bodies a certain amount of antigen is also present, which, when injected, causes some degree of active immunity. The preparation is non-toxic and can be used either subcutaneously or intravenously, and gives promise of better results than have been obtained by the use of whole serum in this type of infection.

From this review it is seen that there has been more or less continuous progress in the methods of preparation and administration of antipneumococcus serum. In the early days but little attention was paid to the antigenic characters of the organisms used in its preparation, and the methods of immunization were such that only sera of relatively low potency were produced. The work of Neufeld and Händel marked a great step in advance in showing the necessity of taking into account the different varieties of pneumococcus in the preparation of serum, and by developing adequate methods of titration they have ensured a reliable standard of potency. In addition they contributed valuable information concerning the usefulness of large intravenous doses of serum in the treatment of the disease. At the Hospital of the Rockefeller Institute immune sera of high potency have been produced to the 
main varieties of pneumococcus, which include about 70 per cent. of all cases of lobar pneumonia. The methods of administration have been so perfected that it is known in advance whether or not the type of serum employed is effective against the particular organism concerned. The serum has been used in sufficient dosage to give it every opportunity to exert whatever beneficial effect it may possess. By this method it has been possible to treat successfully a great majority of cases of pneumonia due to type I pneumococcus. Treatment with type II serum has been less efficient and the method has been modified in a way that gives greater promise for the future.' A method, therefore, has been developed which makes the intelligent application of antipneumococcus serum possible in about 70 per cent. of all cases of lobar pneumonia, and it is to be hoped that with further study, methods of increasing the efficiency of the serum may be developed so that a constantly increasing number of cases of lobar pneumonia may yield to specific therapy.

\section{VACCINE THERAPY}

Pneumococcus vaccines have been used extensively in the treatment of lobar pneumonia, and different observers vary in their opinions concerning the efficiency of this method from enthusiastic support to more or less unqualified condemnation. The object of such a form of therapy is to stimulate to a point of increased usefulness the forces by means of which the body normally overcomes infection, and to attain this result, if possible, in a shorter period of time. Such an attempt is scientifically valid and worthy of trial. Its success is dependent upon the question whether an individual who already harbors an overwhelming dose of the infectious agent can be stimulated to increased resistance by the addition to this burden of relatively small quantities of the same organism. Offhand this would seem like an illogical method of procedure, but there is some indication that there exist certain unknown factors in the steps that lead toward immunity which may make such a trial advisable.

Protection of animals against infection with pathogenic microörganisms by previously inoculating them with non-lethal doses of the same agent is a common observation in immunological work. Small animals can readily be protected against infection with pneumococcus by this method. Such a type of prophylactic vaccination has been successful in a number of instances, notably in smallpox and typhoid fever. The value of such methods has developed the hope that the same procedure might be used with success during the course of disease. Indeed, in one instance at least, rabies, it has been possible in a large percentage of cases to protect individuals after infection has occurred by vaccination with a modified virus of the disease. The good results in this instance 
are probably due to the long incubation period of the disease, and the method is of no value when acute symptoms have developed. The extensive work of Wright and his associates in treatment of local infections by bacterial vaccines has been a great stimulus to interest in this method of therapy. The good results obtained by a number of observers following Wright's technique and the apparent usefulness of Koch's tuberculin in certain types of tuberculosis has inspired a hope that is still seeking justification. In the main, however, attempts at the therapeutic use of vaccines in the acute infectious fevers have, up to the present time, not successfully demonstrated their value. That further progress will be made seems likely, inasmuch as at the present time the treatment of typhoid fever with typhoid vaccine of various types is being energetically studied and the reports so far published seem definitely favorable.

In reviewing the studies of the treatment of lobar pneumonia by pneumococcus vaccine, one is impressed with the difficulty of appreciating the method at its proper value. Many of the published reports are so favorable as to cause a certain amount of distrust concerning the adequacy of the standards of judgment, and in addition one gets the impression that only the favorable results have found their way into the literature, whereas the failures remain unreported. But little laboratory work on the curative value of pneumococcus vaccine has been undertaken, and much of the evidence in favor of its efficacy in human pneumonia is impressionistic in character, objective tests of value not having been sought for to any extent.

Stoner, $(27)$ in this country, has summarized the treatment of quite a large number of cases of pneumonia with pneumoccocus vaccine. In all, $5_{50}$ cases are included in the review. These include cases treated by the following observers: fourteen by Wolfe, of which eleven recovered, whereas the death rate in the untreated controls was 40 per cent.; thirteen by Boelke, all of whom recovered and exhibited some shortening of the duration of the disease, the average length of which after inoculation was three days; eighty-three cases by Leary, of whom seventy-one recovered, a mortality of 14.4 per cent., which is considerably lower than has been observed in an equal series of untreated cases in ordinary hospital practice; one case by Batten which recovered; seven cases by Harris, four of which were benefited by the treatment showing an early crisis, and three in whom the ordinary course of the disease was uninfluenced; by Allen, one instance of delayed resolution with recovery; twenty-four cases by Wilcox with only one death; six cases by Craig, all of which recovered; and six cases by Fisher with four recoveries. Of the $x_{55}$ cases reported by Stoner, $\mathrm{r} 35$ cases recovered and twenty died, a mortality of somewhat less than I 3 per cent. Comparison of these results with the average hospital statistics shows that the death rate is considerably lower than that observed even in the less severe pneumonia years. 
An analysis of the vaccine treated cases of pneumonia reported by Leary (28) shows that of the eighty-three cases so treated, thirtyfour were alcoholics, a class in which the prognosis is almost invariably bad. Of these thirty-four cases only six died, a mortality of 17.7 per cent. which is extremely low for individuals with a wellmarked alcoholic history. Of the remaining forty-nine cases but two died, a death rate for this series of 4.08 per cent. and a total mortality of 9.7 per cent. for the entire series of eighty-three cases. As far as one can judge from a study of Leary's series, no care was exercised in selecting a strain of pneumococcus adapted antigenically to the case in question, nor was the existence of distinct varieties of pneumococcus even considered. From this it would seem that quite a large percentage of the cases must have recovered independently of any specific influence of the vaccine. In going through the literature numbers of equally favorable results are encountered. Craig,(29) in I9r3, reports marked beneficial effects from the use of vaccines in an unstated number of cases of pneumonia. He observed shortening of the period of the disease, lowering of the temperature, lessening of the toxic symptoms, and especially a greater proportion of recoveries. Williams, in one of the Bulletins of the New York City Board of Health, mentions a series of ninety-one cases of pneumonia treated with vaccines, of whom fifteen died, a mortality of 16.4 per cent. Of these the majority of deaths occurred among the cases with complications. In seventy-two uncomplicated cases there were but two deaths. Such evidence could be further multiplied, but sufficient has been given to show the general tenor of the reports concerning vaccine therapy of pneumonia in the United States.

In Germany but little attention has been given to methods of active immunization during the course of lobar pneumonia, although much time has been spent in the study of active immunization of animals, and in the preparation of antipneumococcus sera. Neufeld thinks that by the subcutaneous inoculation of killed bacteria, there is little hope of influencing the course of an acute infectious disease such as pneumonia in which in a large percentage of cases the infectious agent gains entrance to the blood.

Vaccine therapy has always been accorded a favorable hearing in England due largely to the influence of Wright and his co-workers. A number of English investigators have studied the effect of pneumococcus vaccine on the course of lobar pneumonia. Eyre(30) accredits considerable value to the action of vaccines on chronic pneumococcus infections of the lung in which he finds that they cause a heightened opsonic index of the blood. Although he has had no experience with such treatment in acute lobar pneumonia, he is inclined to view it with favor. His views concerning the value of the opsonic index as a standard of increased immunity were developed some years ago, and in view of the more recent studies of Wright (3I) concerning the doubtfulness of its applicability to pneumococcus infections, Eyre's conclusions are open to question at 
the present time. Allen(32) gives enthusiastic support to the use of pneumococcus vaccine in the treatment of pneumonia. He advises the use of a stock vaccine immediately, and the employment of autogenous vaccine as soon as it can be prepared. Failure of successful results in many instances, he thinks, is due to use of the vaccine in insufficient quantities. Morgan(33) has reported the treatment of forty-three cases of lobar pneumonia with vaccine. His results seem to be extraordinarily good. Of the forty-three cases so treated, only two died, one of these from a complicating nephritis following the subsidence of the pneumonia. He employed repeated doses of 50 million bacteria, but thinks this dosage a little large, and recommends the administration of from $I_{5}$ to 30 millions at each dose. Some of the cases recovered by crisis which seemed to be induced by the administration of the vaccine, and others more gradually by lysis. Morgan thinks that the temperature in some cases is an index of the usefulness of the treatment, but finds that the most noticeable effect is an abatement in the severity of the general symptoms. He did not find the opsonic index a reliable method for measuring the degree of immunity induced, and recognizes the importance of developing some objective method for determining the efficacy of the treatment. Charteris $(34)$ has reported the treatment of nineteen cases by similar methods in which he was unable to determine any difference in the course of the disease in the treated cases from that in the untreated.

In $19{ }_{3} 3$ Rosenow and Hektoen(35), working in the United States, reported the treatment of a large series of cases of lobar pneumonia with a modified form of pneumococcus vaccine. The vaccine is prepared by allowing pneumococci to undergo partial autolysis in salt solution. In this way the bacteria are freed from their toxic portion which is of no service in the process of immunization, and the non-toxic immunizing portions are retained. The dosage of this special vaccine is from to-20 billions, in some instances given only once, and in others at daily intervals. The cases treated by Rosenow and Hektoen are divided into three groups and may be summarized as follows:

The first group consisted of thirty cases treated by physicians in private practice, and of these three died. This group showed the best results because of the class of the patients and the earliness with which the methods could be applied.

The second group is made up of thirty-five cases treated at the Cook County Hospital. The death rate among these was 25.7 per cent. This is about the average hospital mortality, but somewhat low for the type of pneumonia observed in that particular hospital.

In the third series are included 294 cases, I 46 of which received injections of the vaccine, and 148 served as controls. No selection was practised, alternate cases being chosen for treatment and controls. Of the treated cases thirty-four died, a mortality of 23.3 per cent. and of the controls fifty-six died, a death rate of 37.8 per cent., 
showing a diminution of 14.5 per cent. in the mortality of the treated, as compared with the untreated group. Many of the patients gaive an alcoholic history, and numbers were treated for the first time only after the disease had become well established. The earlier the first injection was given, the better the result obtained. In favorable cases injection of the vaccine was followed by a slight rise in temperature and later by a drop, the temperature subsequently remaining at a somewhat lower level. Repetitions of the injection sometimes caused the temperature to drop to normal in from three to five days after the onset of the disease. No harmful effects were observed in any of the treated cases. Those cases in which the results were not so good were treated somewhat late in the disease, the average time of injection being about the fifth day, which is a disadvantage in any form of specific treatment of pneumonia and particularly for such methods as have in view the development of active immunity. The use of the vaccine seemed to have no noticeable effect upon the development of complications or sequelæ. Crisis occurred somewhat earlier in the treated cases than in the untreated, especially when the treatment was begun early. In view of the fact that the mortality was lower each year in the injected cases, and that all were severe types of pneumonia, nearly one-half having an alcoholic history, Rosenow and Hektoen think that this form of treatment is of some service in the management of lobar pneumonia. No further report of the method has been made, and whether it has continued to prove of value cannot be said.

The work reviewed above covers the principal reports concerning the use of vaccine therapy in pneumonia. At the present time some use is being made of the method of sensitized vaccine, but it is too early to discuss the usefulness of this procedure. Although the published reports of vaccine treatment in pneumonia indicate no small measure of success, they fail for certain reasons to carry conviction. There is a striking lack of laboratory experimentation concerning the utility of pneumococcus vaccine, and with the exception of a few seemingly unreliable observations on the opsonic index, no objective measure of therapeutic effect has been developed, such as the diasppearance of the bacteriemia or the demonstration of an increase in the specific immune bodies in the blood of treated individuals. From an experimental standpoint, it is difficult to find support for the use of vaccines in a disease of short duration such as pneumonia, in as much as immune bodies do not appear in any considerable concentration in the blood of inoculated animals much before the tenth day, the usual time of normal termination of pneumonia. In addition, in the severe forms of pneumonia, the infectious agent enters the blood and it is difficult to see how additional small quantities given subcutaneously can materially alter the general result. It is true that vaccines may have an effect that is not objectively recognizable at the present time, but in the absence of any demonstration of such a fact, before their efficacy in the treatment of 
pneumonia can be conceded, at least some of the discrepancies mentioned must be eliminated.

\section{CHEMOTHERAPY}

As in many acute infectious diseases, certain drugs have been advocated from time to time in the treatment of lobar pneumonia. It seems unnecessary to give a detailed list of these therapeutic agents and their effects, inasmuch as the supporting evidence is in most instances unsubstantial. Considerable progress has, however, been made along the lines of chemotherapy in lobar pneumonia in the last few years and there is promise of continued advance. Chief interest has centered in the quinin derivatives prepared by Morgenroth, and, to a certain extent, in the possibilities of camphor.

Camphor has been recommended on clinical grounds alone in the treatment of pneumonia for a number of years. Aside from its possible value as a circulatory stimulant, the suggestion has been made that it exerts a bactericidal action against the pneumococcus. Such a hypothesis is susceptible to experimental investigation, and some work has been done with a view to elucidating this question. Boehncke(36) has studied recently this alleged bactericidal action experimentally, and claims that by the administration of camphor in oil, that he was able to protect animals against a fatal dose of pneumococcus if the camphor were given before infection. Therapeutic use of the drug in rabbits, however, was not so successful since it was impossible to prevent a fatal issue if treatment was begun subsequent to the injection of the pneumococci. By large prophylactic doses, rabbits could be protected against a surely fatal dose even when administered intravenously. Stein(37) has recently confirmed the prophylactic value of camphor as determined by Boehncke. She found that if mice were injected with camphor four hours before they received a fatal dose of pneumococcus, the animals were protected. If the interval were shortened to two hours, or if the drug were given at the time of infection, no effect could be demonstrated. All strains of pneumococcus were not equally susceptible to the action of the drug, six out of nine examined proving refractory to its action. The clinical results so far reported, and even the experimental work, does not as yet give much support to the efficacy of camphor as a chemotherapeutic agent in the treatment of pneumonia.

To Morgenroth(38) and his assistants we owe the first real progress that has been made in the attempt to control pneumococcus infection by means of a chemical compound with specific bactericidal action. Morgenroth and Halberstaedter had observed that quinin and certain synthetic derivatives of this drug could be used to protect mice against experimental infection with Trypanosoma brucei. Because of certain characteristics which trypanosomes have in common with pneumococcus, they decided to test the efficacy of the quinin derivatives in pneumococcus infection in animals. Several 
derivatives, quinin, hydrochinon, hydrochlorisochinon, ethylhydrocuprein, and propylhydrocuprein were employed in the experiments. The most satisfactory results were obtained by Morgenroth and Levy by the use of ethylhydrocuprein. An attempt was first made to protect mice against pneumococcus by administering the drug in watery solution previous to the infecting dose of pneumococcus. Of ninety-three animals treated in this way, twenty-two died during the manipulations. Of the seventy-one animals remaining, eighteen died in the same time as the controls, and fifty-three survived the controls at least twenty-four hours. Of these eighteen animals, 25.5 per cent. withstood completely the infecting dose of pneumococcus. These results prove the drug to be of definite value in protecting mice against experimental infection with pneumococcus since these animals are so susceptible that they succumb with unfailing regularity to very minute doses of pneumococcus. Morgenroth and Kaufman were able to establish for the drug a curative, as well as a protective, value. Of twenty-two mice treated in from five to six hours after intraperitoneal injection with pneumococcus, only two died as soon as the controls, eight lived six days, whereas seventeen control animals were all dead in forty-eight hours, and twelve of the twenty-two or 54.5 per cent. survived. Such results are very striking and show that ethylhydrocuprein undoubtedly effects a sterilization of the blood in mice, inasmuch as in the above experiments the animals must have already developed a septicemia at the end of six hours. Further studies showed that the best experimental results are obtained by administering the drug in oil suspension instead of in watery solution. The toxic dose of the drug for mice is but little below the curative dose, and when the drug is given in solution in water, absorption is too rapid and the action cannot be maintained for a sufficiently long period of time without intoxicating the animal. When given in oil, absorption is slower, and the effect of the drug on the pneumococcus is thus prolonged over a considerable period of time. Of the animals treated prophylactically in this way, from 80 to 100 per cent. survived. The curative results were also much improved especially if the administration of the drug was repeated every twenty-four hours for a few days.

Morgenroth has shown that ethylhydrocuprein is active against more than a single strain of pneumococcus. Moore(39) has investigated this question further and finds that the drug exerts an equal bactericidal action in vitro against the various biological groups of pneumococcus, but has no such specific action against streptococcus. Animals can also be protected effectively with ethylhydrocuprein against infection with the different types of pneumococcus, and such protective action is effective against many multiples of the minimal fatal dose. Moore has shown that the serum of rabbits that have received a single dose of ethylhydrocuprein exerts first a bactericidal and later an inhibitory action on the growth of pneumococcus in vitro. This effect is most marked when the 
base (optochin ${ }^{1}$ base) is given in oil subcutaneously, somewhat less when the hydrochlorid of the drug is given in water subcutaneously, slight when the base is given in oil intramuscularly, and least evident or even absent when the hydrochlorid in water is introduced directly into the stomach. To get these effects by the intravenous route, toxic doses must be given, and even with toxic non-fatal doses the effects do not last long. When the base is given in oil subcutaneously to rabbits, the maximum bactericidal effect is obtained one hour after administration, and this passes over to a simple inhibition of growth in about four hours. Moore has also found that the blood of man becomes bactericidal for pneumococcus after the administration of $0.5 \mathrm{gm}$. of the drug given by mouth or subcutaneously. This investigator has studied the effect of combining optochin therapy with specific antipneumococcus serum in the treatment of pneumococcus infection in animals. He finds that a single small dose of ethylhydrocuprein which of itself has practically no protective effect against infection of mice with pneumococcus, is capable of increasing the threshold value of the type homologous antipneumococcus serum at least fifty times. This effect is proportionately many times greater than a simple summation of the protective effects of these two agents. No such effect was obtained when the antiserum used is one produced against a strain of pneumococcus from a group other than that to which the infecting pneumococcus belongs. These experiments give a solid experimental foundation for the usefulness of ethylhydrocuprein as a valuable agent in combating pneumococcus infection, and warrant a trial of its efficacy in the treatment of lobar pneumonia in man.

Already ethylhydrocuprein or optochin, as it is more commonly called, has had wide application in the therapy of pneumococcus infections in man. Only a few years have elapsed since its initial use, and the results have not as yet been sufficiently correlated to pass final judgment concerning its efficacy. They are, however, of sufficient promise to justify a review of some of the more important publications. Fraenkel(40) was one of the first to use the drug in human beings, and found that of twenty-one cases of pneumonia treated nine or 43 per cent. showed no noticeable change following the exhibition of the drug; in six cases, 28.5 per cent., a doubtful result was obtained; and in the other six cases, 28.5 per cent., a marked beneficial action of the drug was noted. In the cases in which there was beneficial effect of the drug on the pneumonic process, the temperature fell on the fourth or fifth day, in four falling within twelve hours after the first treatment and in two by lysis. The general character of the cases was mild and Fraenkel thinks that more experimental study is necessary before the drug could be said to be suitable for human application. Ethylhydrocuprein has certain toxic effects in man mostly referable to the special senses

1 Trade name for ethylhydrocuprein. 
of sight and hearing, and Fraenkel noted several instances of amblyopia following its administration. The loss of vision, however, was not permanent. Parkinson(4I) has treated nine cases of pneumonia with optochin and found that three had crises somewhat earlier than is usual, two patients died, and in the remaining four no noticeable therapeutic effect was observed. In three cases out of seven the pupils became widely dilated during treatment, but there were no instances of amblyopia. He thinks that ethylhydrocuprein has no therapeutic effect on pneumonia in man and shows that toxic symptoms may appear after the administration of $\mathrm{I} \mathrm{gm}$. by mouth or $0.5 \mathrm{gm}$. subcutaneously.

With increasing experience much improvement has taken place in the dosage and method of administration of the drug so that there have been an increasing number of favorable reports. Baerman(42) has reported the treatment of thirty-one cases of pneumonia. In some of these, serum obtained from individuals convalescing from pneumonia was combined with ethylhydrocuprein in the treatment. Of five cases treated by intramuscular injections of the base in oil, favorable results were obtained in three cases and two died. One of the latter had a pneumococcus bacteremia, and it is possible that the treatment caused a diminution of the number of organisms in the blood. Seven cases were treated with the acid salt by mouth in repeated doses of from $0.25 \mathrm{gm}$. to $0.5 \mathrm{gm}$. No amblyopia was observed. Six of the patients treated in this manner seemed to derive benefit from the treatment, and recovered, whereas one died. Nineteen cases were treated with combinations of serum of recovered patients and ethylhydrocuprein. Two methods of administration of the drug were employed, by mouth, and intramuscularly in oil. Of the nineteen cases so treated, four died and the remainder recovered, seeming to derive some benefit from the treatment. In some instances pneumococci were present in the blood and when this condition existed, treatment either caused a disappearance or diminution of the organisms present. Baermann expresses the opinion that the drug has unmistakable curative value in pneumonia, especially when combined with immune serum. The better results obtained by this author are probably due to his improved technique of administration.

Since these early reports, treatment of pneumonia with optochin has become more general, especially in Germany. Leschke(43) has recently reviewed a large series of treated cases, and the results are best shown in the following tables which are taken from his report.

In these tables cases in which the temperature dropped to normal before the sixth day are regarded as favorable, those in which it became normal on the seventh or eighth day as doubtful, and after the eighth day as uninfluenced. A great difference is observed in the cases treated before the third day as compared with those treated later. In the former instance the mortality was only 5 per cent. in 
Table I.-Early Cases. Treatment on First to Third Day of Disease:

\begin{tabular}{|c|c|c|c|c|c|c|c|c|c|c|c|c|}
\hline Author & 岁 & 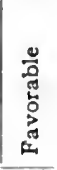 & 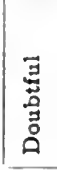 & 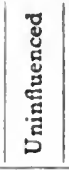 & Died & 息 & 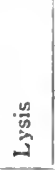 & $\left|\begin{array}{l}\tilde{z} \\
0 \\
\frac{\tilde{d}}{\tilde{J}} \\
\tilde{z}\end{array}\right|$ & 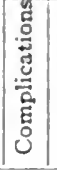 & 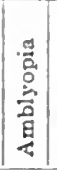 & $\mid$ & Dosage, gm. \\
\hline lermann & 14 & 7 & 4 & 3 & I (sepsis) & so & 2 & 2 & . . & . . & . & $\begin{array}{l}2-4 \\
6-8\end{array}$ \\
\hline $\begin{array}{l}\ldots \\
\ldots \ldots \\
\ldots\end{array}$ & $\begin{array}{r}15 \\
12 \\
3\end{array}$ & $\begin{array}{r}7 \\
12 \\
\ldots\end{array}$ & $\begin{array}{l}4 \\
\therefore\end{array}$ & $\begin{array}{l}3 \\
3 \\
3\end{array}$ & $\begin{array}{l}\mathrm{I}(70 \mathrm{yrs}) \\
\quad \cdots \cdots \cdots\end{array}$ & $\begin{array}{l}1 \\
8 \\
2\end{array}$ & $\begin{array}{l}4 \\
4 \\
.\end{array}$ & i. & $\because$ & $\because$. & $\because$ & \\
\hline Iess-Moritz.... . . & 28 & 19 & 6 & 3 & & . & .. & $\because$ & $\ldots$ & 4 & 2 & $6-8$ \\
\hline 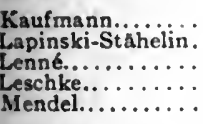 & \begin{tabular}{r|r}
11 & \\
9 & \\
5 & \\
9 & \\
12 &
\end{tabular} & $\begin{array}{r}10 \\
7 \\
2 \\
8 \\
12\end{array}$ & $\begin{array}{l}\because \\
\text { I } \\
2 \\
I \\
.\end{array}$ & $\begin{array}{c}\mathbf{I} \\
\mathbf{I} \\
\mathbf{I} \\
\cdots \\
\cdots\end{array}$ & 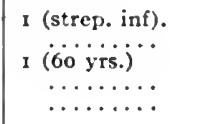 & $\begin{array}{r}5 \\
6 \\
-5 \\
5 \\
4\end{array}$ & \begin{tabular}{r}
5 \\
2 \\
\hdashline \\
4 \\
8
\end{tabular} & $\begin{array}{c}2 \\
4 \\
\because \\
i \\
2\end{array}$ & $\begin{array}{l}\because \\
\because \\
\because \\
\therefore\end{array}$ & $\begin{array}{l}\because \\
i \\
\cdots \\
\cdots\end{array}$ & $\begin{array}{l}1 \\
\because \\
y \\
\cdots\end{array}$ & $\begin{array}{r}3 \\
4-12 ? \\
1-3 \\
10 \\
5\end{array}$ \\
\hline 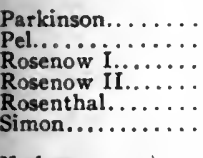 & \begin{tabular}{r|r}
3 \\
3 \\
15 \\
24 \\
6 \\
26
\end{tabular} & $\begin{array}{r}3 \\
3 \\
12 \\
14 \\
6 \\
13\end{array}$ & $\begin{array}{l}\because \\
\ddot{6} \\
\ddot{7}\end{array}$ & $\begin{array}{l}. \% \\
\ddot{3} \\
4 \\
0 \\
6\end{array}$ & $\begin{array}{l}\ldots \ldots \ldots \ldots \\
2 \quad \cdots . . . . . \\
\text { I (empyema) } \\
\text { I (typhoid) } \\
3 \text { (gangrene of } \\
\text { lung.) } \\
\text { lung }\end{array}$ & $\begin{array}{r}\because \\
\because \\
9 \\
12 \\
4 \\
11\end{array}$ & \begin{tabular}{r|}
$\because$ \\
4 \\
4 \\
2 \\
$\mathrm{II}$
\end{tabular} & $\because:$ & $\begin{array}{l}\because \\
\because \\
4 \\
\because \\
\therefore\end{array}$ & $\begin{array}{l}\cdots \\
\because \\
\because \\
\cdots \\
\cdots\end{array}$ & $\begin{array}{l}\because \\
\because \\
\because \\
\therefore\end{array}$ & $\begin{array}{r}\mathrm{I}-3 \times 0.5 \\
3 \times 0.5 \\
6 \times 0.25 \\
6 \times 0.25 \\
6 \times 0.25 \\
6 \times 0.25\end{array}$ \\
\hline Vetlesen............ & 9 & 8 & . & I & ......... & 7 & 2 & 2 & .. & .. & $\cdots$ & $3 \times 0.5$ \\
\hline & 204 & 150 & 27 & \begin{tabular}{r|}
27 \\
$13 \%$
\end{tabular} \mid & 12 & 84 & & 15 & 5 & 5 & 5 & \\
\hline
\end{tabular}

Table II.-Late Cases. Treatment after Third Day of Disease

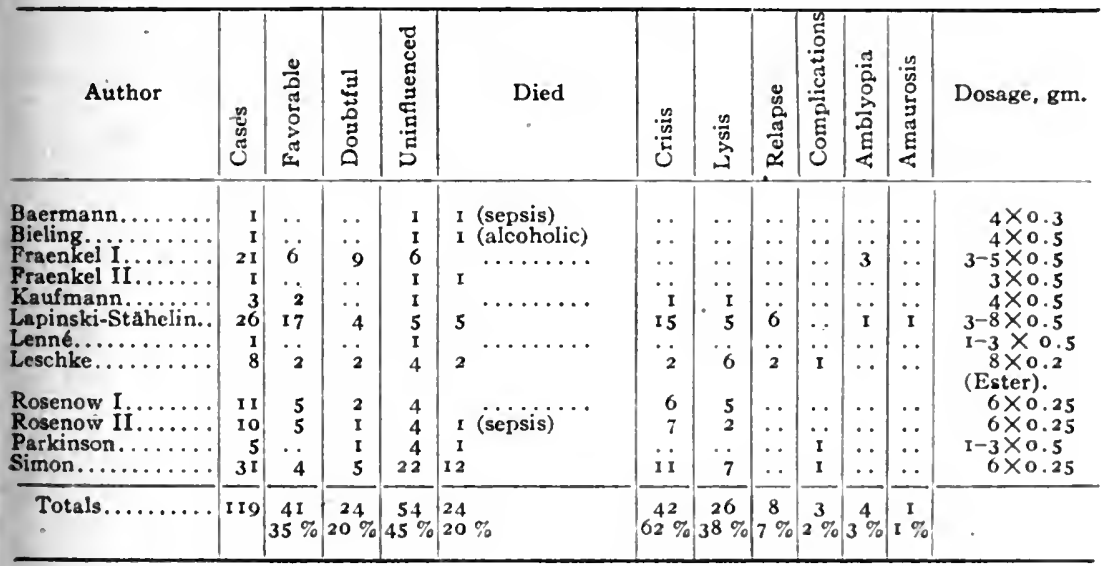

204 cases, and in the latter 20 per cent. in I I 9 cases. The mortality in the total 323 cases II.I per cent. Such a death rate compared with the ordinary mortality statistics in pneumonia observed in the United States is exceptionally low. Damage to vision occurred in quite a number of instances. This was less common when the drug was given in repeated small doses, and a total dosage of $1.5 \mathrm{gm}$. per day was not exceeded. The results tabulated by Leschke seem very good and possibly exaggerate somewhat the efficiency of the drug. A great deal of careful study of the effect of optochin in lobar pneumonia is being carried on at the present time, and it should soon be possible to formulate a reliable opinion concerning its efficacy. 
Determination of the optimum dosage of optochin is an important part of the investigation. Experience has taught that it is unsafe to give individuals of average weight more than I.5 gm. per day. This has been given in two ways, in three divided doses of $0.5 \mathrm{gm}$. each and in a greater number of smaller doses of from $0.2 \mathrm{gm} .-0.25$ $\mathrm{gm}$. each at regular intervals. The smaller dosage seems somewhat better, since too long intervals elapse between the larger doses and in this time the bactericidal power of the blood may disappear and the pneumococci gain an opportunity to grow. Moore and Chesney(44) have made a quantitative study of dosage, and find that the best method of administration is, in a man of average weight, an initial dose of $0.45 \mathrm{gm}$. and $0.15 \mathrm{gm}$. every two and onehalf hours until the required I. $5 \mathrm{gm}$. per twenty-four hours is reached. This dosage is continued until symptoms abate unless the appearance of toxic manifestations of the drug requires the cessation of treatment. In this manner, the blood rapidly becomes bactericidal, and the bactericidal action is maintained at a high point throughout the disease. ${ }^{1}$ The drug shows a slight cumulative action as the treatment progresses. If larger doses are given at intervals the bactericidal titer is irregular and may disappear altogether between the first doses. Toxic symptoms are apparently dependent on the concentration of optochin in the blood rather than on the total amount administered, and if single large doses are given; greater concentrations than are safe may be attained for a short period of time.

Eye symptoms are regarded as an indication to discontinue the use of the drug, but deafness does not necessitate stoppage. The damage to the special senses that has been observed up to the present time has never persisted as a complete loss of either sight or hearing except in one case reported by Oliver. ${ }^{2}$ In the eye, when toxic symptoms appear, there is a widening of the pupils, dimness or complete loss of vision. In the extreme cases, the eye grounds show pallor of the retina with great narrowing of the retinal vessels. Blindness may persist for one week or more, and though central vision later becomes normal, marked contraction of the visual fields and narrowing of the retinal vessels may persist. Such a result in a certain percentage of the cases treated with optochin constitutes a considerable menace, though it is discounted somewhat by the seriousness of the outlook in most cases of lobar pneumonia. Toxicity of the drug seems to depend somewhat on idiosyncrasy which may be similar to the increased sensitiveness of certain individuals to quinin. The condition in the eye resembles that seen in quinin

1 Moore and Chesney have shown that a dosage of from 0.024 to 0.026 grams of optochin per kilogram of body weight is necessary to ensure the development of bactericidal power in the blood. They think that in treatment the total daily dose should be based on body weight rather than that an arbitrarily fixed daily quantity should be given. This work is about to be published and those who are interested should read the original article.

${ }_{2}$ Oliver, Brit. Med. Jour., I9r6, 580, reports an instance of permanent blindness following the administration of optochin ( 5 gr. q. 3 h. Total dosage $120 \mathrm{gr}$.). 
amblyopia. Symptoms may develop after a relatively small total dosage of the drug.

Ethylhydrocuprein has been used in the treatment of pneumococcus infections other than lobar pneumonia. The most interesting of these are pneumococcus meningitis, and pneumococcus ulcer of the cornea. In the former the drug has been given in great dilution intraspinally, but the number of such attempts is so small at the present time as not to warrant discussion. Considerable experience has been had, however, in the treatment of ulcus cornex serpens with optochin and the results have been very favorable. The drug is applied directly to the lesion in from I to 2 per cent. watery solution. In this strength there is no damage to the corneal epithelium and the ulcerated areas are said to heal with unusual rapidity. The treatment is accompanied by some burning sensation which can be prevented by the use of a local anesthetic. The scars left are much more delicate than those following other methods of treatment especially cauterization.

From the results reviewed in this article it is evident that the specific therapy of pneumococcus infections has advanced greatly in the last few years. Two methods, one the use of specific antisera and the other the use of a synthetic drug have been developed, and with each reliable effects already seem to have been attained. With further improvements in the technique of administration, and possibly by combinations of the two, it is to be hoped that a constantly increasing number of favorable results in the treatment of lobar pneumonia and the secondary pneumococcus infections will be observed.

\section{BIBLIOGRAPHY}

I. Pasteur, Bull. d. l'Acad. med., i $88 \mathrm{I}$.

2. Sternberg, Bull. Nat. Board of Health, I88I-82, ii-iii.

3. Fraenkel, A., Zeitschr. f. klin. Med., $1885-86, x, 426$.

4. Weiciselbaum, Wien. Med. Jahrb., i 886, ix, 775.

5. Roux and YerSin, Ann. d. l'Inst. Pasteur, I888, ii, 629; I889, iii, 273.

6. Bemring, V., Deutsch. med. Wchnschr., I 893 ,

7. Fraenkel, A., Zeitschr. f. klin. Med., I885-86, x, $40 \mathrm{r}$.

8. Neupeld and Ungermans, Centralbl. f. Bakteriol., Abt. i, Ref. igr 2, liv, Beih., p. $7 \mathrm{I}$.

9. F. and G. Klemperer, Berl. klin. Wchnschr., i 891 , xxviii, 833 .

10. Pane, Centralbl. f. Bakteriol. Orig., $1897, x \times i, 664$.

II. WashbourN, Brit. Med. Jour., I $897, \mathrm{i}, 5$ Io.

12. EYRE and WAshbourn, Brit. Med. Jour., i 899, ii, I 247.

13. Banti and Pieraccisi, Seltermana Med., Florence, I 899, liii, 285.

14. Spolverini, Dull. d. r. Acad. med., Rome, I900, xxvi, 79.

15. ANDERS, Jour. Am. Med. Assn., I 904 , xliii, i 777.

16. RöMre, Serum therapie der Pneumokokken Infecktion der Menschlichen Cornea, J. F. Bergmann, IViesbaden, I 899 .

17. RÖMER, Loc. cit.

18. PASSLER, Deutsch. klin. Med., I905, Ixxxii, $36 \mathrm{r}$.

19. JURGens, Charite'-Ann., Berlin, I907, xxxi, $7 \mathrm{I}$.

20. Neufeld, Zeitschr. f. Hyg., I902, xl, 54.

Neureld and HaNDEL, Zeitschr. f. Immun., I909, iii, I 59; Berl. klin. Wchnschr., I91 2, 680; Arb. a.d. k. Gsndhtsamte, 1908, xxviii, 572 .

21. WeIrz, Med. Klin., Berlin, I912, viii, 1072. 
22. Geronne, Berl. klin. Wchnschr., rgr 2, ii, r699.

23. Chickering, Jour. Exp. Med., I9I4, Xx, 599.

24. Dochez and Girlessie, Jour. Am. Med. Assn., I913, lxi, 727.

25. GaY and Chickering, Jour. Exper. Med., I9I 5, xxi, 389 .

26. Chickering, Jour. Exper. Med., igr 5 , xxii, 248

27. Stoner, Am. Jour. Med. Sci., IgI I, cxli, I86.

28. LEARY, Boston Med. and Surg. Jour., 1909, clxi, 714.

29. Craig, Med. Record, I9I 2, lxxxii, 465.

30. EXre, Lancet, $1908, \mathrm{i}, 539$.

3I. WrIGHT, Lancet, I9I4, clxxxvi, I.

32. Allen, Lancet, I909, ii, 780 .

33. Morgan, Proc. Roy. Soc. Med., I9ro, iii, Suppl. 65.

34. Chartesrs, Glasgow Med. Jour., I9r 2, Ixxvii, rg.

35. Rosenow and Hektoen, Jour. Am. Med. Assn., 1913, 1xi, 2203.

36. BoEhnCKe, Berl. klin. Wchnschr., r913, 1, 818.

37. Stein, Zeitschr. f. klin. Med., I9I6, lxxxii, 249.

38. Morgenroth and Levy, Berl. klin. Wchnschr., I9II, ii, I560, 1979.

MORGENROTH and HaLBERSTAEDTER, Berl. klin. Wchnschr., rgrI, ii, 1558.

Morgenroth and KaUfManN, Centralbl. f. Bakteriol., I9I2, liv, Beih. 69.

39. Moore, Jour. Exper. Med., I915, xxii, 269; Jour. Exper. Med., 1915, xxii, 389; Jour. Exper. Med., r915, xxii, 55 I.

40. FraEnKel, Berl. klin. Wchnschr., I9I 2, xlix, 664.

41. Parkinson, Zeitschr. f. Chemother., Orig., I9r3, ii, I.

42. BaermanN, Zeitschr. f. exper. Path. u. Therap., I9I4, l, 476.

43. LeschKe, Deutsch. med. Wchnschr., I9I4, I359.

44. Moore and Chesney, Proc. New York Soc. of Serology, April 7, 1916, Jour. of Immunology. 


\section{DIPHTHERIA}

\section{By George H. Weaver, M. D.}

Diphtheria is an infectious disease caused by the diphtheria bacillus. Diphtheria is characterized by a pseudomembranous exudate upon a mucous membrane or the abraded skin, in which exudate Bacillus diphtheria is always present. The mucous membranes most often affected are those of the throat and larynx, but the infection may extend from these locations to the adjacent nasal, aural, ocular, or respiratory mucous membranes. Rarely it extends to the esophagus and even invades the stomach. Especially in children, the vaginal mucous membrane may be involved. Intact skin is not affected, but when abraded, as by a blister, eczema, etc., it may become the seat of infection, and likewise the surfaces of wounds may be involved.

The leading clinical symptoms are sore throat, fever, and some general malaise. The related lymph glands are swollen. With involvement of the larynx there is a croupy cough and hoarse voice, and later signs of obstruction. The principal symptoms of systemic poisoning are progressive heart failure, diminished amount of urine which contains albumen, and vomiting. An individual predisposition is essential for the development of diphtheria. Virulent bacilli are sometimes carried in perfectly healthy throats without harm. Immunity to diphtheria depends upon the presence of diphtheria antitoxin in the blood and susceptibility depends upon its absence. By means of the Schick reaction it has been possible to test large numbers of persons for the presence or absence of diphtheria antitoxin in the blood. From such studies we know that the proportion of persons who are susceptible to diphtheria varies with age, being about 25 per cent. in infants, 50 per cent. from five to fifteen years, and 25 to 50 per cent. in adults. Carriers constantly have considerable amount of antitoxin in the blood. A temporary immunity follows an attack of the disease, but it is often soon lost, and sometimes appears to be followed by increased susceptibility to infection. Relapses and recurrences may occur. Among local predisposing conditions may be mentioned enlarged tonsils, adenoids, catarrhal conditions, either from measles or other causes. These local conditions are very important in establishing and continuing the condition of carriers. The disease is least prevalent in summer, but occurs throughout the year.

Treatment. - Knowing that the pathogenic action of the diphtheria bacillus depends upon toxins which it forms, and having a specific antidote for these poisons, our principal concern is to neutralize the toxins with the antitoxin at the earliest possible moment, and before 
extensive irreparable damage has occurred. In addition to this, mechanical obstruction of the larnyx must often be relieved by operative measures, and the effects of secondary infections must be counteracted as much as possible.

The general measures directed toward preventing the dissemination of the infection from persons suffering with the disease, include thorough quarantining and isolation from others. Certain measures may also be employed to prevent the disease in exposed persons. The use of cleansing and mildly antiseptic gargles and mouthwashes by attendants help to keep the parts in a healthy condition, and thus local conditions are avoided which predispose to the establishment of an infection. Susceptibility to diphtheria may be overcome by measures which introduce preformed antitoxin into the body or which stimulate its production in the body. The former is accomplished by the injection of antidiphtheritic serum from the horse, the latter by injections of diphtheria toxin and antitoxin in the mixture of which there is a small quantity of unneutralized toxin-Behring's vaccine. Injections of 500 to I000 units of diphtheria antitoxin produce quite perfect protection for about three weeks. The immunity produced by Behring's vaccine is more lasting but has the disadvantages that it requires time for the elaboration of the antitoxin and that, according to Park, antitoxin does not usually appear in those persons who previously possessed none at all. Through the use of the Schick test these immunizing measures can now be restricted to those who require them, i.e., to those who possess no antitoxin. Prophylactic injections are to be recommended for susceptible nurses who care for diphtheria patients. The prophylactic use of antidiphtheria serum is most useful in dealing with children under ten or twelve years of age. For such children the immunizing dose is 500 to 1000 units. Protecting injections are to be given to all the children of a family in which a case of diphtheria occurs; if the people are poor and the children cannot be carefully watched, this is more imperative. In the treatment of the individual case of diphtheria we are able to employ a remedy which is absolutely specific, antidiphtheritic serum.

The standard antidiphtheritic serum for the United States is prepared in the Hygienic Laboratory, United States Public Health and Marine Hospital Service. This standard serum is distributed to licensed manufacturers and others working in this line. By this means a standard strength of the antidiphtheritic serum sold in the United States is insured.

The liquid antidiphtheritic serum, which is the one usually employed, is a yellow, clear fluid having at most a very slight precipitate, and having a slight odor of the preservative. It is put up in bottles containing from 500 to $10,000^{\prime}$ units. By using high value serums the injection of large quantities is avoided. The average dose of antidiphtheritic serum for purposes of immunization is 500 units; for therapeutic purposes, 5000 units. 
The modes of introduction of diphtheria antitoxin in the order of their efficiency are intravenous, intramuscular and subcutaneous. Intravenous injections are used in very toxic cases in which the most rapid effects are desired. Except in these cases of great urgency the intramuscular route is to be employed, the relatively slow absorption from the subcutaneous tissues causes this last route to be restricted to cases in which immunization only is sought. The skin at the point of injection is to be sterilized as for a surgical operation, and the punctures subsequent to the injection should be protected by a pledget of sterile cotton held in place by collodion. Intravenous injections are usually made into one of the large superficial veins in front of the elbow. Intramuscular injections are best made into the mass of muscles on the outside of the thigh. Subcutaneous injections should be given where the tissues are loose.

Antitoxin should be given at the earliest possible moment. The value of early administration is well shown by McCollom's experiences. In the South Department of the Boston City Hospital I9o cases of diphtheria among doctors, nurses and employees were treated with antitoxin without a death. Antitoxin was given to all in large doses at the onset. In many cases this could be done while there was only congestion of the mucous membrane. By this means the formation of a membrane was often prevented. In all cases the diagnosis was confirmed bacteriologically.

Extensive study has shown that most cases which would be recognized clinically as diphtheria prove to be so upon bacteriologic examination. All such cases and all suspicious cases should receive the antitoxin as soon as possible after coming under observation, not waiting for the results of examination by cultures. We have no means of accurately measuring the amount of toxin in the individual case and in arriving at the dose of antitoxin to be given the clinician must attempt to estimate this as closely as possible. The factors to be especially considered are the length of time the patient has been sick, the location and extent of surface involved, and the virulence of infection as shown by the toxic symptoms in the patient. An effort should be made to give in the initial dose all the antitoxin required. Better results follow this method of treatment than are secured from the administration of repeated smaller doses. The primary dose required will vary from 2 or 3000 units to 30,000 units. The size of the individual should be considered in estimating the dose. An absence of improvement after eighteen to twenty-four hours should lead to a repetition of the dose. When the toxins have been neutralized, this is evidenced by a decline in the fever, by a marked improvement in the general feeling of the patient, and by a reduction in the local discomfort. Locally, the membrane ceases to extend, its color changes from grayish-yellow or grayish-green to a lighter yellow, and soon becomes sharply outlined upon the red underlying surface, and begins to 
loosen and peel off at the borders. In cases with laryngeal stenotic symptoms relatively large doses are indicated. The initial dose in such a case should never be less than 8000 or 10,000 units, and it should be repeated at short intervals until relief is obtained.

Local treatment of the nose and throat has perhaps been too much neglected since the introduction of antitoxin. It has been such a relief to be rid of the necessity for the active local treatment which was formerly carried out under great protest of patients. It must not be forgotten that the diphtheria antitoxin acts specifically against the products of diphtheria bacilli alone, and that the effects of secondary infection must be met by suitable measures. It is advisable to keep the mucous membranes of the nasal, pharyngeal, and buccal cavities as clean as possible by the use of mild antiseptic washes. Strong antiseptic and irritating solutions are to be avoided. The disappearance of the diphtheria bacilli appears to be more facilitated by washes of physiologic saline solution than by strong antiseptics. 


\title{
SCARLET FEVER. SCARLATINA
}

\author{
By George H. Weaver, M. D.
}

In reviewing the previously published chapter upon scarlatina, I was struck by the necessity of accentuating one of the most important details in the management of a case of scarlet fever and upon which I wish to lay particular stress now, the question of quarantine.

If it appears that some of the measures previously outlined in the prophylaxis of scarlet fever are rather extreme; still they are justified by the conditions. They are warranted by the good results obtained by their conscientious use. Imperfect isolation and disinfection are not only of little value, but they give a false sense of security in the presence of danger.

There is a tendency among sanatoriums to dispense with fumigations and antiseptics and to depend more upon thorough washing with soap and water, and upon the action of air and sunlight in the disinfection of the recovered patients and of the rooms which they have occupied. In suitably constructed hospitals and in some private houses, the mechanical cleansing seems to be efficient and fumigations unneccessary. In many private houses one will hesitate to entirely abandon the use of antiseptic agents. Much good can be done by liberal repapering of walls, and painting.

Efforts have been made to obtain a specific treatment for scarlet fever. Various drugs have been advocated at different times, but have failed to stand the test of use. Acting upon the assumption that the symptoms and organic changes which occur in scarlet fever are due to a toxin which is neutralized by an antitoxin when recovery takes place, several attempts have been made to secure curative results by injecting blood serum from convalescent patients into scarlet fever patients during the acute stage. The convalescent serum was supposed to contain antitoxin for the scarlet fever poison. While Huber and Blumenthal ${ }^{1}$ and v. Leyden ${ }^{2}$ are inclined to ascribe some favorable effects to the convalescent serum, Rompel ${ }^{3}$ and Scholz ${ }^{4}$ have observed none. Later reports on the use of serum from convalescent scarlet fever patients by Reiss and Jungmann, ${ }^{5}$ by R. Koch, ${ }^{6}$ and others seem to indicate that it possesses marked value in suitable cases. The serum is collected from eighteen to twenty-four days

1 Berl. klin. Wchnschr., I897, No. 31 .

'Die Behandlung des Scharlachs mit Reconvalescentenserum, Deutsch. Arch. f. klin. Med., 1902, Lxxiii, 6 r6.

Zur Behandlung des Scharlachs mit Reconvalescentenserum, Münch. med. Wchnschr., 1903,38 .

- Die Serumbehandlung des Scharlachs, Fortschr. d. Med., I903, xxi, 353.

- Deutsch. Arch. f. klin. Med., 19r 2, cvi, 70.

- Münch. med. Wchnschr., 1913, 1x, 2611. 
after the beginning of the scarlet fever. The donor of the serum must be free from syphilis as shown by a negative Wassermann, free from tuberculosis and must not have been the victim of septic complications. The serums from several convalescents are mixed, a more efficient serum being thus secured since the proportion of immune antibodies varies in different patients. The serum must be sterile and is administered in doses of 40 c.c. for children and roo c.c. for adults. The best results follow intravenous injections and this is insisted upon by most of the advocates of this form of therapy. The effects of the serum are most marked in toxic cases and in the early stages of such cases the convalescent serum is said to act in a specific manner. Zingher ${ }^{1}$ urges the use of convalescent blood in the early toxic or malignant cases, and normal blood in the septic cases. He avoids the collection of serum by using freshly drawn citrated whole blood, which is injected into the muscles instead of into a vein, in doses of $2 \frac{1}{2}$ to 8 ounces; 2 c.c. of a ro per cent. solution of sodium citrate is added to roo c.c. of blood to prevent coagulation. The rapid absorption of the serum from the muscles places such injections almost on a par in efficiency with intravenous injections, and the simple technique speaks in its favor. Because of the difficulty in determining with certainty that the person yielding the serum for injection is not infected with tuberculosis or syphilis, this method of treatment must be reserved for the most severe cases. Patients who are likely to recover under expectant treatment should not be subjected to the possibility of infection with these diseases.

Numerous reports of the use of antistreptococcus serum in cases of scarlet fever have appeared. In most instances those who have employed this remedy have done so with the purpose of combating the complicating streptococcic infection which is so frequent, and not with the idea that it would cure or cut short the original disease. While the curative value of antistreptococcus serum in pure streptococcic infections is still a matter of dispute, the most conflicting results following its use, it is hardly advisable to pass final judgment upon its value in cases of scarlet fever with secondary streptococcic infection.

During the last few years the treatment of various infections by injections of the casual bacteria killed in various ways, usually by heat, has found wide use. After observing the effects of injections of streptococci, killed by suspension in 25 per cent. galactose solution, in a considerable number of cases of scarlet fever, $\mathrm{I}^{2}$ have concluded that such injections given relatively early in the course of the disease, exert little if any influence over the later occurrence of complications due to streptococci. However, in some cases of subacute and chronic streptococcic infections which complicate

1 Jour. Am. Med. Assn., 1915, lxv, 875 .

2 Weaver and Boughton, Jour. Inf. Dis., 1908, v, 608; Boughton, Jour. Inf. Dis., r910, vii, 99 . 
scarlet fever and persist during convalescence, injections of galactose killed streptococci, especially homologous strains, appear to exert a very pronounced curative effect. Of such killed streptococci, the suitable dose for a primary injection is about 100 millions, and for subsequent injections the dose may be increased to 500 millions. Too large initial doses have been observed to be followed by harmful results. 


\title{
TUBERCULOSIS
}

\author{
By Edward Osgood Otis, A. B., M. D.
}

There has been no essential change in the general treatment of tuberculosis since the first edition of this work was issued. The "hygienic-dietetic" or "open-air" method still stands supreme as the one successful form of treatment. Now and again some new remedy is exploited as a specific, such as the notorious Freedman's vaccine, only upon investigation and trial to be proved worthless. Tuberculin is the only one of the special forms of treatment which maintains its place to a greater or less degree. There is still a difference of opinion, however, as to its value. Some, after trial have not been convinced as to its benefits and have abandoned its use, while others of large experience with it believe that in properly selected cases and in small doses it is of assistance in effecting the arrest of the disease, and makes the arrest more permanent. While such a divergence of opinion and experience as to its merits exists, one can only say that it may do some good and if cautiously employed does no harm.

The Sanatorium.-The sanatorium, wherever situated, as the best means of carrying out the open-air treatment, is becoming more and more recognized, and such institutions are being multiplied throughout the land, both public and private. For the majority of cases sanatorium treatment offers the best chances of recovery or of putting the curable patient upon the road to recovery, for increased experience has shown that this latter, viz., giving the patient a good start toward an arrest, is about all that can be accomplished during the average residence in the sanatorium.

It will still be a long time, however, if ever, before sufficient accommodations in such institutions will be available for all or the majority of curable cases; hence, many patients from necessity or choice will have to take the treatment at home, but with a strict adherence to the principles of the open-air method success can be attained under the guidance of the family physician. Furthermore, in many "open" climatic resorts, the "cure" is successfully effected, provided the medical supervision is competent and continuous.

Graduated Exercise.-The importance of graduated exercise, carefully planned and supervised, is becoming more fully recognized as an integral part of the treatment at the proper stage. It is not of so much importance what the kind of exercise is, as that it should be capable of being gradually increased and adapted to the individual patient, and readily supervised by the physician or attendant. 
The reader is referred to the section upon exercise under this subject for the details in the application of this measure.

After-treatment.-Since it has been more fully realized that sanatorium treatment does not in so many cases complete the "cure" but only begins and advances it up to a certain point, greater attention is being given to the so-called "after-treatment" and the avoidance of relapse, which in the past has, unfortunately, so frequently occurred.

After the return from the sanatorium, the patient should be kept under observation for an indefinite period, and he should be impressed with the vital importance of continuing the same general plan of life, so far as his circumstances will allow, which he pursued in the sanatorium, or while taking the cure.

\section{ARTIFICIAI PNEUMOTHORAX}

The one striking addition to the therapeutics of pulmonary tuberculosis within the last few years is artificial pneumothorax, the introduction into the intrapleural space of a harmless and slowly absorbable gas for the purpose of collapsing the diseased lung and thus immobilizing it. The operation is simple, and, as now done, it is comparatively devoid of danger and without shock. In cases where it is successful the results are often striking; it has saved many lives otherwise doomed. Its application, however, is limited, and it is often unsuccessful. As Balboni well says, "it is an unusual measure, the creation of a pathological condition in order to cure or relieve another pathological condition." Forlanini in 1892 first induced artificial lung collapse as a therapeutic measure and has been and is one of the principal exponents of this operation. Murphy, in this country, without the knowledge of Forlanini's work, also devised the same operation and published his results in 1898. About this time (1906-I9II) Brauer and his associates published the results of a large number of cases treated by this method, and since then the operation has been widely employed all over the world, and the literature upon the subject has become quite enormous.

Experience with the operation has, therefore, accumulated rapidly and we are in a position to form a fair estimate of its value, although opinion differs as to the kind of cases to which it is applicable. Both Brauer and Saugman confined their cases to those which gave an absolutely unfavorable prognosis. "If you are to judge of the results of the treatment with artificial pneumothorax," says Saugman, "it must at once be understood that this treatment deals with very seriously attacked, third-stage patients, of whom a great part, indeed most of them, without this treatment would have practically no chance of recovery or healing."

The present tendency seems to be, however, to employ the operation in earlier cases, regardless of the stage, which are progres- 
sive, and which, after a fair trial; fail to improve under carefully applied open-air treatment.

The two universally accepted indications have been:

I. Extensive unilateral, progressive or chronic lesions which fail to respond to the ordinary hygienic-dietetic treatment, the opposite lung being comparatively free from disease; and

2. Recurring more or less severe hemoptysis or a single severe hemorrhage which fails to yield to ordinary treatment, provided, of course, one is sure from which lung the hemorrhage comes.

Other conditions in which the operation has been advocated or employed are:

I. Uncomplicated, unilateral, slowly progessive cases; with free pleura, of any degree of lesion (Forlanini).

2. Moderately advanced cases which have not improved under the ordinary hygienic-dietetic treatment.

3. Rapidly progressive cases.

4. Bilateral cases, not acute, with the disease not advanced on both sides (Forlanini).

5. Advanced, progressive tuberculosis, tending directly toward a fatal result (generally contraindicated but sometimes a favorable result is obtained, or at least distressing symptoms are alleviated).

6. Persistent, harrassing cough.

7. Abscess of the lung and bronchiectasis.

8. Early cases which are afforded no opportunity for the usual open-air treatment.

9. Moderately or far advanced cases who themselves desire something more done for them than the usual treatment.

"The present tendency," says Sachs, " "grown out of experience is toward a greater conservatism in the selection of cases, a selection based on thorough preliminary study and full consideration of the possible effect of compression in the individual case."

In order to effect a successful compression there must be no pleural adhesions, or only such light ones as can be readily broken by slight pressure-a condition, unfortunately, not always present. Many cases, however, which from the extensive disease one would suppose would have adherent pleura, are found to be free and the compression is readily accomplished.

The fluoroscopic or roentgen-ray examination is essential in determining the condition of the lung as to probable adhesions, as well as the condition of the opposite lung, and subsequently, both before and after reinflations, the most reliable guide is the roentgen-ray picture. Although the operation is done without this guide, it is to a degree working in the dark, for the most careful physical examination may mislead one.

Each individual who is submitted to this operation should be under careful continuous observation, and hence, in the great majority

1 Trans. National Association for the Study and Prevention of Tuberculosis, 1915, p. 150 . 
of cases it should be done only under hospital or sanatorium supervision. Moreover, the patient should be kept at rest after each inflation, at least for the first months of the treatment, and this can best be done in an institution. When, therefore; artificial pneumothorax is contemplated, a residence for the first few months in a hospital or sanatorium should be insisted upon. The operation should be explained to the patient, and he should be assured of its painlessness and comparative freedom from danger. With the information obtained from the fluoroscopic picture or a good roentgenray plate, and from the physical examination, the site of the puncture is chosen, which will depend upon the location of the disease, and the probable absence of adhesions. Somewhere between the scapular and postaxillary line, or below the angle of the scapula, is perhaps the most common point to find the lung free.

If the.patient is in an excited, nervous condition, one should either wait until this is allayed, or give a subcutaneous injection of 16 grain (0.01 gm.) of morphia a half hour before the operation. Balboni and others do this as a routine procedure to "diminish the discomfort and cough due to the pressure excited by the gas." The patient should be in the recumbent position, preferably in bed. A pillow under the side, and the hand of the side to be operated upon placed on the head or on the opposite shoulder helps to widen the intercostal spaces. The field of operation is carefully disinfected and painted with tincture of iodine. The skin is frozen with ethyl chlorid, and a small puncture made through it with a sharp-pointed instrument, such as a tenotomy or cataract knife, or one that can be released by a spring. Through this minute puncture the local anesthetic is injected with an ordinary hypodermic syringe. Either novocain in combination with adrenalin, as in the following:

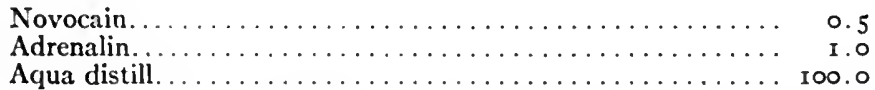

can be employed or eucain also combined with adrenalin, the combination being obtainable in ampules-thirty minims of the former and one or two ampules of the latter will generally be sufficient to produce anesthesia of the parts.

As one slowly advances the needle through the tissues, they are infiltrated in front of the needle point, and when the parietal pleura is reached a little more of the solution is injected in order to anesthetize the pleural site of operation.

After a few minutes the pneumothorax needle is slowly introduced through the path previously anesthetized, at right angles to the chest wall. Of course everything should be done under careful antiseptic precautions, and whatever apparatus is employed one should make sure beforehand that it is in perfect working order.

When the pleural cavity has been reached it will be indicated by the characteristic respiratory oscillations as shown by the up- 
and-down movements of the fluid in the manometer attached to the apparatus. These oscillations are always negative in pressure, and it is absolutely essential to obtain them before attempting to inject the gas. If they do not appear, the needle may be cautiously advanced or withdrawn a little, and if one then fails to obtain them, the needle should be withdrawn and the attempt made at another spot. "The closer the manometer is followed," says Floyd " "the more safely will the operation of pneumothorax be performed. It is never advisable to allow an inflow of nitrogen into the chest unless an excursion of $\mathrm{x} .5$ to 2 c.c. in the meter column is obtained with respiration."

The needle should always be maintained in a perpendicular position to the chest wall and never diverted from this position to one side or the other.

If all goes well and the characteristic oscillations indicate that the needle is between the two pleuræ, the gas is allowed to flow in under a gentle pressure. At the first inflation, not more than 300 to 500 c.c. should be introduced, unless for hemorrhage. If pain or distress occurs, the inflow of gas should be stopped and the needle withdrawn. After removing the needle, a pad of sterile cotton or gauze is firmly applied over the puncture. At first, reinflations should be made every two or three days in amounts varying from too to 500 c.c. until a more or less complete compression of the lung is obtained, indicated by neutral or slightly positive readings of the manometer, and also shown by the roentgen-ray and the physical examination. When this is accomplished, the intervals between the injections are lengthened from a week to ten or twelve days, according to the absorption of the gas, and also, as King and Mills ${ }^{2}$ say, depending upon "the symptoms, especially the amount and character of the sputum, temperature, etc."

As time goes on, the time can be still further lengthened from two to four weeks, the object being to maintain as perfect collapse as possible, and when this exists, the absorption of the gas takes place more slowly, and, hence, reinflations are required only at long intervals.

The length of time the compression should be maintained will, of course, depend upon the results obtained, but as the procedure is generally applied in more or less advanced cases, it is obvious that it must continue a long time, for months if not several years. Saugman considers that the treatment should be continued two years or more, but it must be remembered that he is referring to advanced cases. The main object to be attained is an arrest of the disease and the disappearance of all evidence of active disease, this condition to have existed for several weeks at least, then reëxpansion may be allowed to slowly take place.

In the case of a serious hemorrhage, a larger amount of gas

1 Trans. Am. Climatol. Assn., r9r3, xxix, I8r.

2 Therapeutic Artificial Pneumothorax, Am. Jour. Med. Sci., Sept., 19r3, p. 33 o. 
will have to be introduced at one time, rooo c.c. or more. In the case of adherent pleura, the operation is obviously impossible, and attempts to separate adhesions are dangerous; occasionally, however, slight adhesions are easily separated and a successful result obtained.

Besides the evidence obtained from the roentgen-ray as to the probable existence of adhesions, the manometer reading will show a reduced negative pressure and a lessened range of fluctuations or even a positive pressure as when the gas distends a pleural pocket. Even if a complete collapse is not obtainable, a partial one has often given good results. "The complete collapse of the lung in most cases, " says Floyd, "if of any duration, whether of phthisis or abscess of the lungs, is generally impossible, but in a large proportion sufficient collapse can be obtained to give good results. It is often remarkable to see how small an area of pneumothorax will favorably influence the pulmonary condition."

Nitrogen gas is generally employed to produce the compression on account of its comparatively slow absorption, but sterile atmospheric air can also be used, or oxygen; some operators use the latter for the first inflation, recommended by Prof. Deneke of Hamburg ${ }^{2}$ on account of its rapid absorption, so that if the needle has entered a vessel and the gas gets into the blood stream, there is less likelihood of producing gas embolism.

As a rule, the gradual collapsing of the lung by repeated small injections of gas causes but few symptoms: there is more or less dyspnea at first, and the heart action becomes more frequent, temporarily the cough and expectoration may be increased, and so may the fever if it previously existed, or if not, there may be a rise of temperature. There is sometimes a loss of weight. The function of the opposite lung is increased, and the heart is displaced. The diaphragm is depressed, pushing down the organs below. Adjustment to the changed condition of the organs involved soon, however, takes place; but the patient should be kept at rest during the earlier inflations while the readjustment is taking place and the pneumothorax is becoming established. Later the patient can be about during the intervals of injection.

When a satisfactory collapse has been obtained there follows, in a relatively short time, a marked relief of the symptoms, fever is reduced or ceases, cough may entirely disappear, the sputum is diminished and the bacilli lessen or entirely disappear, night sweats cease, and, in brief, there is a general all-around improvement in both local and general condition. The whole aspect of the case is changed, and from a state of hopelessness there emerges renewed hope, and a reasonable prospect of a permanent arrest of the disease. This, however, cannot always be expected to happen, for there are many failures.

1 Op. cit.

'Dle Kunstliche Pneumothorax, Seine Technik und Seine Erfolge, Prof. Dr. Th. Deneke, Hamburg. 
Complications.-Although, in the great majority of cases, the operation is a simple one and devoid of danger, yet serious and even fatal accidents have occurred, and this should always be borne in mind. Most of them, however, have happened from a faulty technique, and can be avoided by care in the manipulations-there should be rigorous antiseptic precautions; thorough local anesthesia; and the gas should never be allowed to enter until the characteristic oscillations are observed in the manometer tube and the pressure is shown to be negative.

Gas Embolism.-Fatal gas embolism, although rare, has occurred and is a possibility; it happens when the needle, instead of being in the pleural cavity, is in a blood-vessel, either in the lungs or in a pleural vessel connected with the pulmonary circulation, and the gas is allowed to flow in. It has most frequently occurred in refills. If not fatal, hemiplegia may result. This untoward accident can almost always be avoided by never allowing the gas to be injected until the manometer readings are satisfactory.

Pleural Reflex.-Pleural reflex or shock is another accident which is alarming and may be fatal. Balboni ${ }^{1}$ doubts if this can happen if a preliminary subcutaneous injection of morphia is given and thorough anesthesia of the parts is produced. The symptoms resemble those of shock-loss of consciousness, faintness, pallor, cyanosis, transitory blindness, facial paralysis or hemiplegia, small and rapid pulse, dilated pupils and shallow respiration. These symptoms may be only transitory or may continue longer, gradually subsiding or, rarely, may be at once fatal. By some operators pleural shock is considered the most serious danger of the operation. If it occurs, the usual means for the treatment of shock in general should be employed, such as the diffusible stimulants, nitroglycerin, morphia, caffein, alcohol, heat, etc.

Emphysema.-Emphysema, either deep or subcutaneous, may happen from the escape of gas into the tissues along the needle track. Subcutaneous emphysema may be caused, according to Balboni, from (a) large wound (large needle), (b) injection of nitrogen at high pressure, $(c)$ lack of tensity of the tissues, $(d)$ excessive thinness of the subject, $(e)$ paroxysm of coughing following an injection.

If a small needle is used and excessive pressure in the introduction of the gas is avoided, this accident will rarely if ever happen. More or less discomfort may be experienced, but it is not dangerous. Further treatment is prevented until the absorption takes place. Deep, or mediastinal emphysema occurs when the nitrogen perforates the costal pleura and is injected into the lung and enters the mediastinum, producing deep pressure symptoms which develop in a few hours after the introduction of the gas. The symptoms are a sensation of fullness and stiffness about the neck and shoulder, sore

${ }^{1}$ Forlanini's Artificial Pneumothorax, Boston Med. and Surg. Jour., 1914, clexi, Nos. 19 and 26. 
throat and difficult deglutition, with perhaps the taste of nitrogen and a peculiar bubbling sensation on the injected side (Balboni). These symptoms disappear within a few hours or a few days. The roentgen-ray will indicate the true condition of the case, for no evidence of a pneumothorax will be observed.

Serous Pleural Effusion.-This complication occurs in a very considerable number of cases, some authors placing it as high as 50 per cent. It can happen at any time during the treatment, whether the lung is wholly or partially compressed. The effusion is regarded as an exudate, and is, in the majority of cases, tuberculous. The onset may be insidious or may be of the form of a more or less inflammatory process.

The treatment is generally conservative and requires no active interference except when excessive or acute symptoms persist, such as pyrexia, increased cough and expectoration, distress in the chest, etc. The presence of a moderate effusion does not contraindicate the continuance of the treatment, only refills, obviously, become less frequent. Careful manometric observations should be be made to control the pressure.

Infection of the pleura may occur from without through faulty technique, but with scrupulous antiseptic precautions this accident can be avoided.

Displacement of the Heart.-The displacement of the heart and interference with the blood supply by pressure or a twisting of the vessels may cause temporary disturbance, such as syncope, cyanosis, etc. As a rule, however, adjustment to the changed condition soon takes place and no ill effect results.

Various other complications or accidents are possible, such as rupture of the mediastinum, gastric disturbance from downward pressure of the diaphragm, pneumothorax from puncture and rupture of the lung, or hemorrhage from puncture of the opposite lung. These accidents are all rare and it can again be stated that the operation is practically without danger if one observes a careful technique and is guided by the indications given by the manometer. How few fatalities occur in this operation is evidenced from the fact that in 10,000 operations reported by Forlanini upon I34 patients, two fatalities occurred from gas embolism and twelve cases had serious symptoms which, however, were not fatal; and in 5,000 operations by Saugman on 186 patients, two fatalities occurred at a first injection.

Lillingston (quoted by Johnston of Grand Rapiäs, Michigan) in the London Lancet of September $1_{3}, 1_{9} 1_{3}$, gives the following precautions to be observed in attempting the operation:

"I. Don't inject the gas without satisfactory manometric oscillation, or at a pressure exceeding the atmospheric pressure when beginning a first injection.

"2. Don't spare anesthetics (local).

“3. Don't create a high intrapleural pressure when beginning 
a first injection. A pressure of $40 \mathrm{~cm}$. of water may cause no discomfort during injection, but it may be more than doubled by a fit of coughing and a leak may spring in the pneumothorax.

"4. Don't induce a pneumothorax during menstruation when reflex excitability may be raised.

"5. Don't puncture on the first occasion in many different places in search for a free pleural space; patients have collapsed after the sixth or seventh puncture who have tolerated the first punctures well. It is better to continue the search for a free pleura in a day or two.

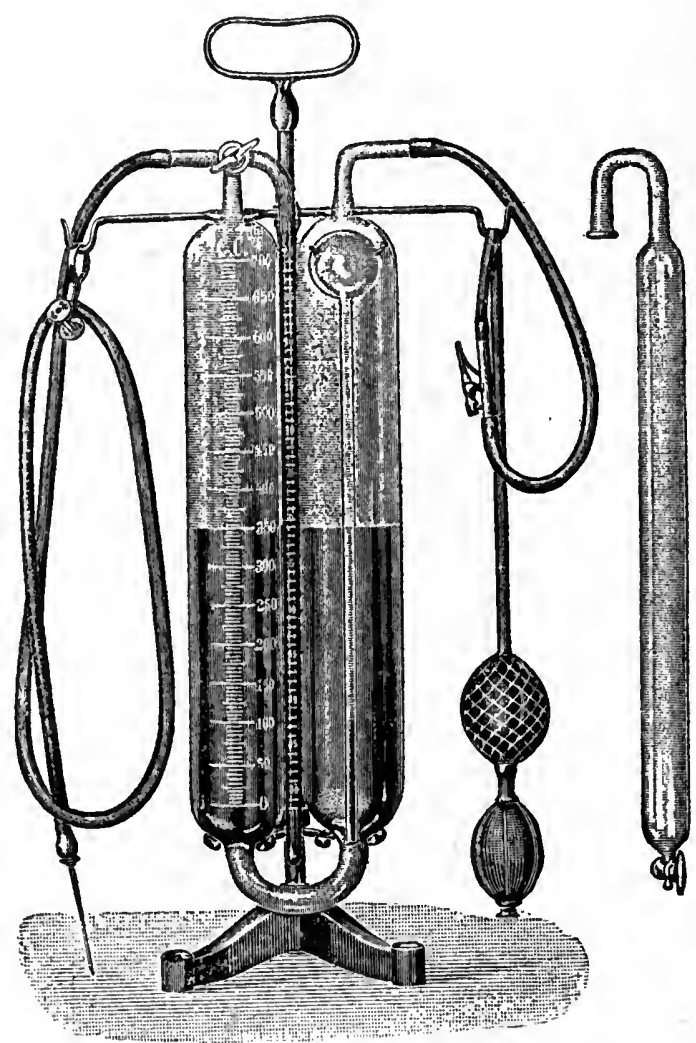

FIG. 36.-Forlanini's latest apparatus for pneumothorax.

"6. Don't inject gas rapidly at a low temperature. Brauer has once seen symptoms of pleural reflex after the use of cold gas.

"7. Don't use large needles or needles with rough surfaces, and don't let the rubber tube drag on the needle during an inflation.

"8. Don't inject until the patient's posture is easy and comfortable.

"g. Don't hesitate to withdraw the needle at the earliest sign of collapse. 
"ı. Don't operate without brandy and a hypodermic syringe of ether handy."

Contraindications to the Operation.-The conditions which preclude the application of the operation are considered by various authorities to be the following:

I. Extensive involvement of both lungs.

2. Cardiorenal disease.

3. Severe intestinal tuberculosis.

4. Extensive pleural adhesions.

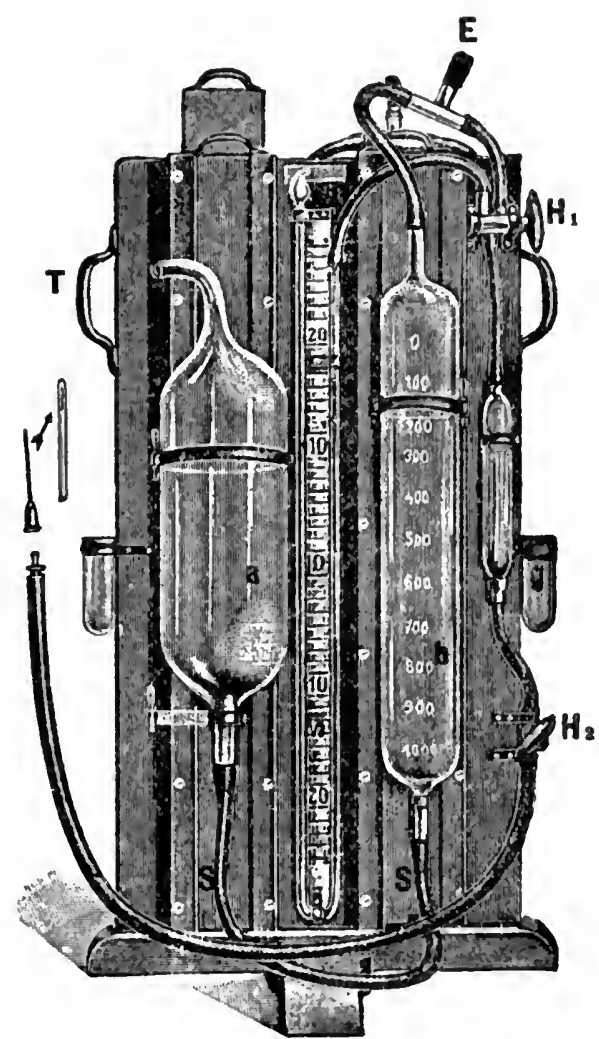

Fig. 37.-Apparatus of Prof. Deneke, Hamburg.

5. Great fear of the operation.

6. Alcoholism (Balboni).

7. Dry pleurisy or pleurisy with effusion.

8. Grave complications.

9. Distention of the abdominal cavity due to gas (Knopf). "This condition must be removed on account of an otherwise serious condition from dyspnea or heart complications."

Contraindications to Reinflation.--The following contraindications to reinflation are given by Kessel and Taschman: ${ }^{1}$

Vor. IV. -17

1 Arch. Int. Med. I914, xiv, 2 Io. 
(a) Marked pain.

(b) Urgent dyspnea.

(c) Sübcutaneous emphysema.

(d) Extension of the disease on the opposite side.

(e) Large pleural effusion.

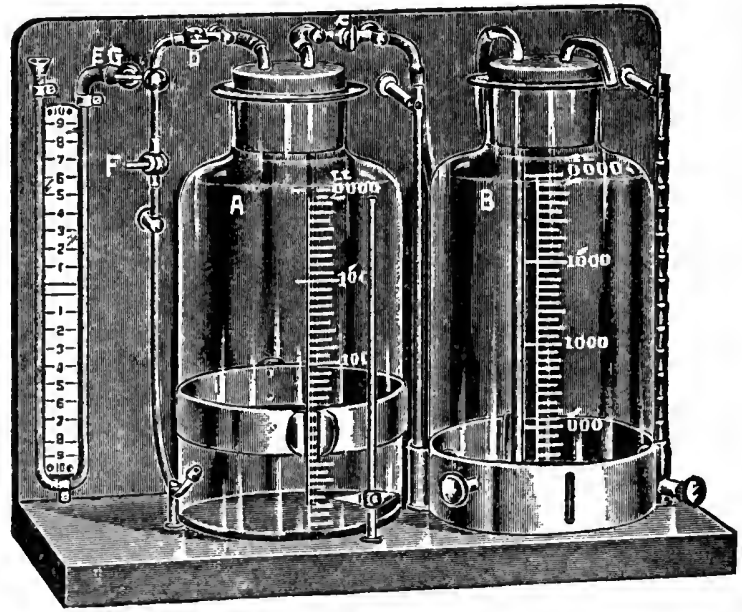

Frg. 38.-Dr. Samuel Robinson's apparatus for artificial pneumothorax.

Two bottles of 2000 c.c. capacity are employed. One is stationary and filled with water containing 2 drams of pyrogallic acid to take up any oxygen which may enter in conjunction with nitrogen. Nitrogen gas is then forced into stationary bottle $A$ displacing the water back to bottle $B$. At completion of this displacement the apparatus is ready for use. On opening certain cocks the water in bottle $B$ replaces the nitrogen in bottle $A$ gradually filling it. The difference in the water levels of the two bottles represents the pressure under which the nitrogen is injected, the rapidity of its injection being regulated by the size of the opening in any one of the cocks. When bottle $B$ is full the maximum pressure is obtained amounting to about $\mathrm{I}_{4}$ c.c. of water. As the water levels approach one another, bottle $B$ may be raised as in Fig 37 , thus maintaining the pressure until most of the nitrogen has been displaced, when the pressure is necessarily reduced. With this hydrostatic mechanism the pressure may be varied at will, never attaining the dangerous limit.

The arrangement of cocks $d, e$, and $f$, corresponds to the substitution of a three-way cock at point $g$. In other words, with cock $d$ closed and $;$ and $f$ open, a direct connection is made between the thoracic cavity and the manometer. With cock $f$ closed and $c, d$, and $e$ open, connection is made between the confined nitrogen and the manometer, thus recording the pressure represented by the difference in water levels of bottles $A$ and $B$. With cock $e$ closed and all others open the nitrogen passes directly from bottle $A$ into the pleural cavity.

This apparatus has the advantages of stability and portability. The fixation of all cocks and tubing to the backboard facilitates their opening and closure, while the use of rubber tubing with clips such as employed in earlier apparatus proved a constant source of annoyance. (Courtesy of Messrs. Codman \& Shurtleff, Boston.)

The Apparatus.-Forlanini's apparatus, or some modification of it, is prehaps the one more commonly employed (Fig. 36). Almost everyone who has done much work in artificial pneumothorax has either modified or added something to this or some other apparatus, or, in some instances, has been obliged to make his own instruments. Whatever the form of apparatus, one seems to be as efficient as 
another in skilful hands. Forlanini originally had no manometer connected with his apparatus, but Saugman introduced it in 1907 , and it is now universally employed, and by its use artificial pneumothorax is bereft of most of its dangers.

The fluid in the manometer tube may be either colored water or mercury. Sometimes two manometer tubes are attached to the same apparatus, one of water and one of mercury.

The main essentials of the apparatus are a glass reservoir for the gas and another, connected with the former, and containing water which either by gravity or by the pressure of a rubber bulb

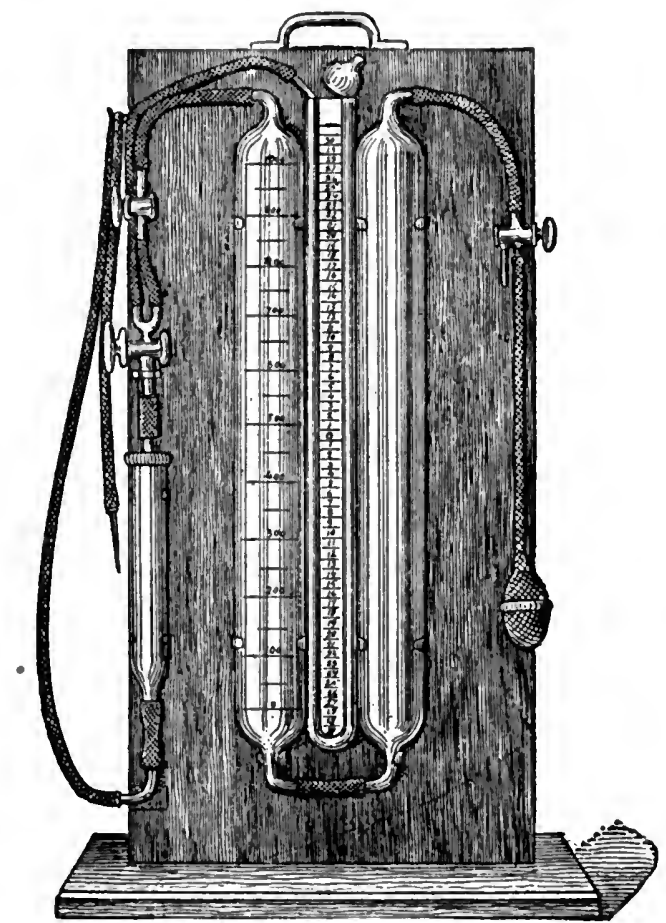

Fig. 39.--Dr. V. Muralt's apparatus.

is forced into the gas reservoir, thus by gentle pressure forcing the gas from the reservoir into the pleural cavity. The various connections are made with rubber tubing and stopcocks. In the tube through which the gas passes from the reservoir into the pleural cavity a filter chamber containing sterilized absorbent cotton or gauze is inserted.

Prof. Deneke of Hamburg has devised an apparatus which permits either oxygen or nitrogen gas to be used (Fig. 37), and thus one can employ at the first injection oxygen gas, so that if by any chance the needle may have entered a vessel, the oxygen gas, on account of its rapid absorbability, will be much less likely to produce gas em- 
bolism. By simply turning a stopcock, nitrogen gas can be substituted for the oxygen gas.

A favorite form of apparatus in this country is that of Robinson and Floyd (Fig. 38). Other forms of apparatus are shown in the illustrations (Figs. 39, 40, 4I).

There are also many kinds of needles recommended and used by operators, some simple and others more complicated, as shown in the

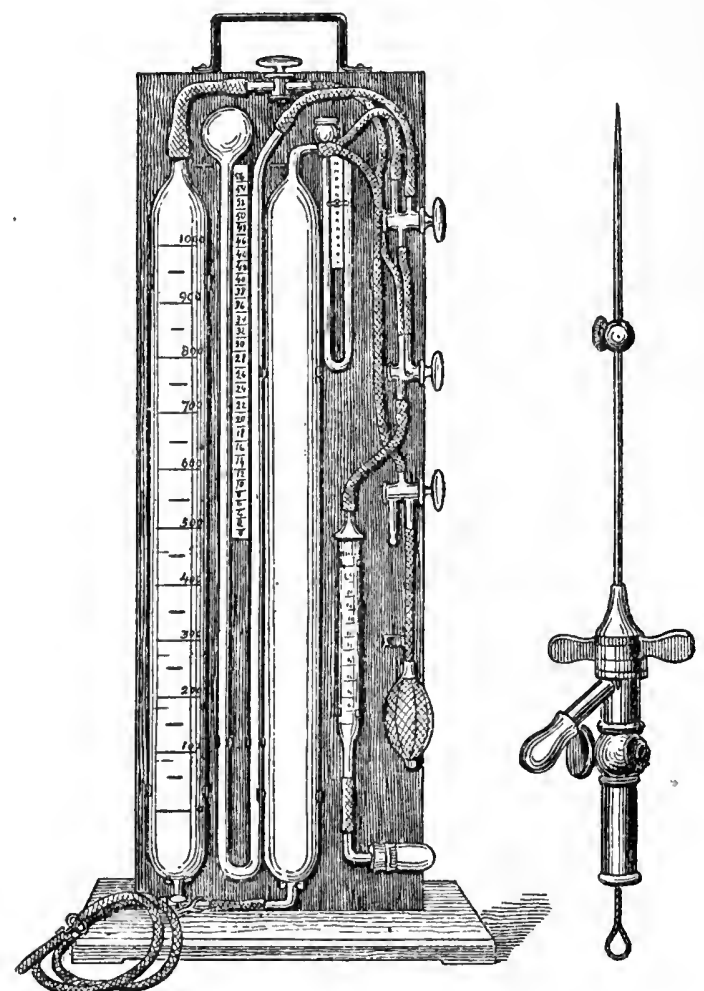

Fig. 40.-Apparatus of Dr. Kornmann, Davos.

illustrations (Figs. $37,42,43,45$ ). The essential thing in the choice of a needle is to have one that is simple, easily manipulated, and which can be readily cleaned and sterilized. The point should not be cut at as sharp an angle as the ordinary aspirating needle. Some needles have the opening at the side near the point, and others at the extremity. A small bore needle is preferable, particularly for reinflations. 


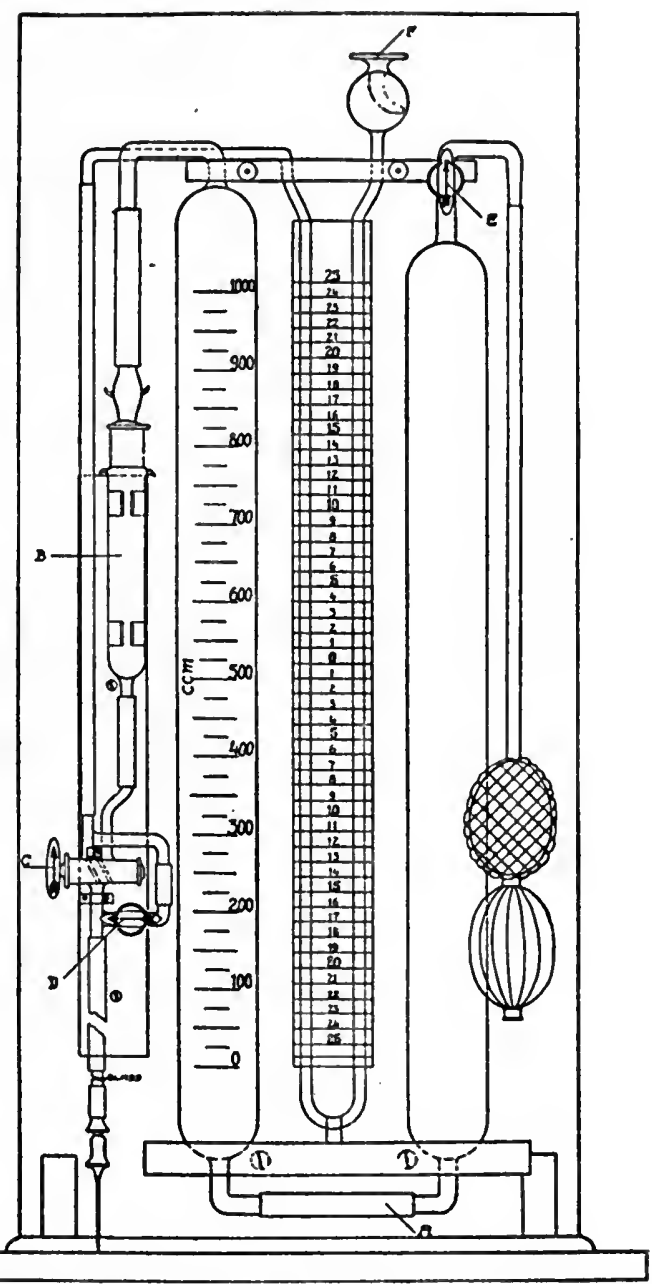

Fig. 41.-Apparatus for artificial pneumothoras. (Forlanini, Saugmann, Knnopf.) 


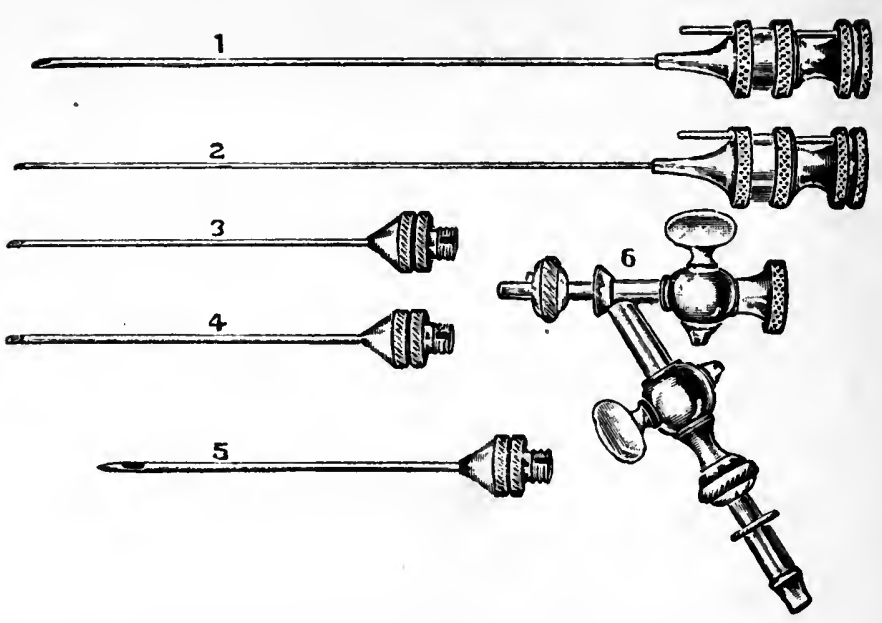

Fig. 42.-Dr. Balboni's needle is so constructed that it may be detached from the stopcock and either of the other sizes attached. This needle may be obtained with only two points, i.e., No. 3 and 4 , each having a separate stilette. Needle point No. 5 has an opening on the side, Nos. 3 and 4 on the end.

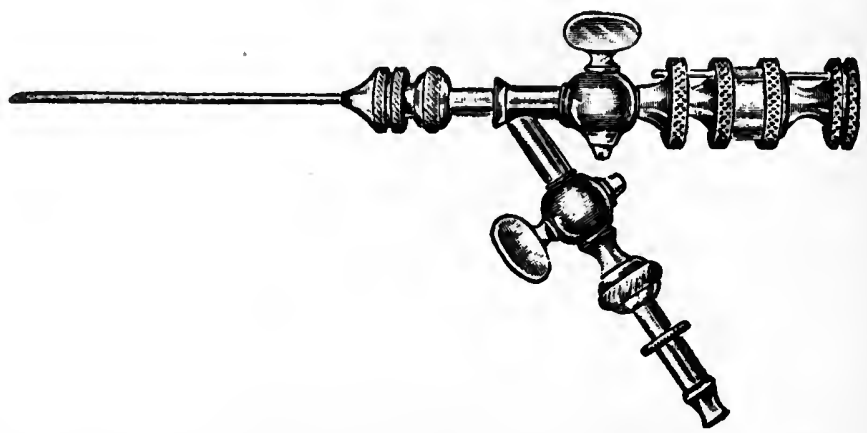

Frg. 43.--Illustrates a needle which Dr. Cleaveland Floyd has designed for use in pneumothorax therapy. It is of the type of Brauer's needle with, however, several changes in construction which experience has demonstrated to be of value.

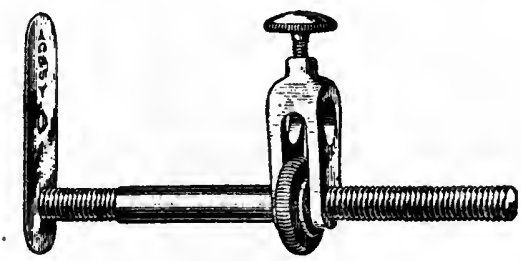

Frg. 44--Dr. Baldwin's adjustable guard for Robinson-Floyd artificial pneumothorax needle, designed by Dr. E. R. Baldwin, Saranac Lake, N. Y. The advantage in using a guard is obvious. In the primary operation of pleural puncture the point of the needle may be held at the exact depth desired when the manometer indicates its entrance into the pleural cavity. This prevents the accidental pushing of the needle too deeply and thus wounding the lung in motion, especially if the patient coughs unexpectedly or moves suddenly. (Courtesy of Messrs. Codman and Shurtleff, Boston.) 


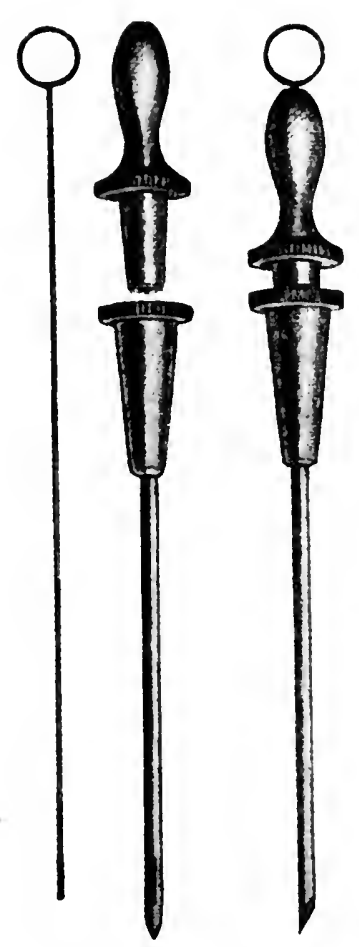

Fig. 45.-Needle for the application of artificial pneumothorax. 


\title{
SEPTIC TOXEMIA, SEPTICEMIA AND PYEMIA
}

\author{
By J. Harold Austin, M. D.
}

A consideration of toxemia, septicemia and pyemia in the broadest sense of these terms could be properly extended into a discussion of infection in general, for in all of the infectious diseases there is toxemia, and in many there is an invasion of the blood stream by the infecting organisms, hence septicemia. In common usage, however, the terms septic toxemia, sapremia, septicemia, septicopyemia and pyemia are employed in connection with a particular group of infections somewhat vague in its limitations, but which in a general way may be said to comprise the infections arising from pyogenic organisms. The bacteria most frequently concerned are undoubtedly the streptococci, but as will be noted later, a very considerable number of different microörganisms may give rise to the clinical pictures which we consider characteristic of the group. In this restricted sense by septic toxemia or sapremia, we understand the presence of bacterial toxins in the circulation derived from a focus or foci of pyogenic infection and giving rise to certain constitutional symptoms. By septicemia we understand the general invasion of the blood and tissues by these pyogenic organisms whether the general infection follows local suppuration, develops secondarily in the course of some other disease or occurs apparently as a primary condition without any obvious portal of entry. The constitutional symptoms of septicemia are naturally those of septic toxemia and are due to the same factor, the circulating poisons set free directly or indirectly by the activities of the infecting bacteria. There is, however, this difference-in septic toxemia arising from a local focus, removal or adequate drainage of the focus is followed by the disappearance of the toxemia; when septicemia is established, removal or treatment of the primary focus will in most instances be of no avail, the organisms continuing to proliferate and produce toxin throughout the body.

When, in the course of a general invasion of the blood and tissues by pyogenic organisms, multiple secondary foci of suppuration appear, the condition is termed pyemia. The distribution of the infecting organisms in pyemia is frequently accomplished by fragments of thrombi which becoming separated from a local infected thrombus perhaps on a cardiac valve, perhaps in a thrombosed vein, pass as emboli to various parts of the body and cause suppuration wherever they lodge. Septicopyemia is a term used by some authors to include both septicemia and pyemia.

The conditions which most frequently render the individual liable to septic infection may be grouped as follows: 
I. Abrasions of the skin or mucous membranes; contusions; lowered vitality or necrosis of any portion of the tissues; collections of extravasated blood or of exudate in the tissues or serous cavities. All of these are factors that predispose to local pyogenic infection. When such infection has occurred the degree of absorption of toxins and the danger of invasion of the general blood stream by the organisms is increased if the fluids in the infected focus be confined under - tension. Among the conditions belonging in this group are the uterus after parturition or abortion, especially if there be retained placenta or blood clots; extensive wounds especially when associated with contusion of the surrounding tissue; gangrenous areas; cold abscesses when exposed to the possibility of secondary infection.

2. Certain diseases in which secondary streptococcic or other pyogenic infection is prone to occur. These include scarlet fever, diphtheria, erysipelas, smallpox and measles, and more rarely, lobar pneumonia, typhoid fever and tuberculosis. Indeed, the most serious features of the first five diseases named are, as a rule, duc to secondary streptococcic infection.

3. Any chronic enfeebling disease in which not rarely the terminal event is a streptococcic or other pyogenic infection, either localized, or more often a septicemia, usually proceeding to a rapid fatal ending.

In addition to these conditions predisposing to septic infection, of importance in the etiology of septicemia and pyemia are focal infections, which although sometimes obviously grave, may sometimes appear to be of the most trifling character or, indeed, may give no symptoms whatever until they become the origin for a more or less severe, possibly fatal, septicemia or pyemia. Among such focal infections are subacute or latent endocarditis, thrombophlebitis in any part of the body, boils, felons, abscessses, infections in the nasal sinuses, tonsils, ears or mastoids, peridental abscesses, osteomyclitis or osteoperiostitis, suppurative foci in the abdomen or thorax such as pylephlebitis, pelvic, hepatic or subdiaphragmatic abscess or suppurating retroperitoneal or mediastinal lymph nodes, conditions that are sometimes almost wholly devoid of localizing signs.

The most frequent infecting organisms are the pathogenic streptococci, the hemolytic streptococci, the Streptococcus viridans, and the Streptococcus mucosus. Other organisms that may give an indistinguishable clinical picture are Staphylococcus pyogenes aureus, Bacillus pyocyaneus, Micrococcus tetragenes, Friedländer's bacillus, pneumococcus and Bacillus aërogenes capsulatus, more rarely Bacillus coli, Micrococcus gonorrhœa, Bacillus typhosus and Bacillus proteus. Only by the blood culture can the exciting organism in a case of septicemia or pyemia be identified, and this examination should be made repeatedly in all septic cases when possible. It must be remembered that in many cases, the bacteria are present in the blood only for short periods and at infrequent intervals, usually during and immediately after a chill or rise in tem- 
perature. The importance of looking for anaërobic organisms must likewise not be forgotten.

The symptomatology of these conditions is one that varies greatly depending upon the virulence of the infecting organism and the strength and reacting powers of the patient. On the one hand, we have certain infections such as those due to the Streptococcus viridans, very insidious in onset, giving rise merely to a low, irregular fever with mild toxic manifestations, to a gradually progressive anemia and to increasing weakness. Such infections often run their course over many months, and may readily be misinterpreted in the early stages, the patient being looked upon as merely "run down." On the other hand, we have the most fulminant forms of septic infection of sudden onset and characterized by high remittent fever, chills, sweats, headache, general muscular pains, anorexia, vomiting, diarrhea, prostration, dry mouth, delirium and stupor.

The fever, while varying with the virulence of the infection, tends to be of the characteristic septic type with marked daily fluctuations in the temperature. These fluctuations are, as a rule, most pronounced in the cases of pyemia. The temperature may, however, resemble that of typhoid fever or may closely simulate that of malaria.

Petechix or ecchymoses are frequently to be seen in the skin; less often erythema scarlatiniforme, multiforme or nodosum, roseolæ, pustules, herpes, or erysipelatous inflammations develop. Hematogenous jaundice may be present. The pulse is usually accelerated; rarely there is bradycardia; extrasystolic arrhythmia is common, and functional systolic cardiac murmurs are frequent. The bloodpressure is usually low. The blood shows a progressive secondary anemia which may become quite severe, and a polymorphonuclear leukocytosis is almost invariably present. The spleen is usually not palpable, but may be enlarged and tender. In addition to the cerebral manifestations of the toxemia, there may develop meningitis, acute exudative nephritis, endocarditis, secondary septic arthritis, fibrinous or purulent pleuritis, pericarditis or peritonitis or bronchopneumonia.

In pyemic cases, in addition to more pronounced fluctuations of temperature, and more frequent chills and sweats, there may be local pain and tenderness at the sites of secondary abscess formation together with local suppuration.

In the presence of an obvious local infection associated with septic symptoms, the problem in diagnosis is to distinguish between simple septic toxemia and the graver conditions of septicemia or pyemia. The distinction cannot be based upon the int nsity of the symptoms alone. Only by the demonstration of the organisms in the circulating blood or by finding a metastatic focus can the existence of a septicemia or pyemia be shown. Of greater difficulty and importance, is the diagnosis of septic infection in the cases without obvious foci of primary infection. Depending upon the 
virulence of the microörganisms and the rate of absorption of the toxins, these infections may resemble, to a most misleading degree, in the mildest forms the constitutional symptoms of early tuberculosis, in the severe forms typhoid fever or malaria. Enlargement of the spleen is of some importance in that it is a much more characteristic feature of typhoid fever or malaria, than of septic infection. The Widal reaction, blood culture, and repeated examinations for malarial plasmodia especially for the crescents, are the most reliable methods of differentiation. It is likewise possible to erroneously consider as septic infection, the severer forms of scarlet fever or smallpox.

The discovery of the primary focus in cases of cryptogenetic septic infection may require a prolonged investigation. When this focus is one that is amenable to surgical treatment and if there be not as yet too severe a septicemia or pyemia, the discovery and treatment of the primary focus frequently determines the prognosis. Hence search for the same becomes an imperative duty. Probable sites have already been listed in the section on etiology. The most painstaking and often repeated physical examination, the roentgen ray, and with proper discretion, the exploratory needle are to be employed for its discovery. The need for frequent repetition of physical examination in these cases deserves especial emphasis, for the signs of the local foci of ten vary greatly from day to day in their conspicuousness. A roentgenogram of the roots of the teeth may occasionally expose an unsuspected abcess, and this is particularly to be considered where there is extensive bridge or crown work.

In surgical and puerperal cases, the toxemia and sometimes a septicemia subside after adequate local treatment and drainage. The same is often but less constantly true after drainage of deepseated abscesses, appendicitis or cholecystitis; the existence of septic thrombophlebitis or of other neighboring or distant secondary foci renders eradication of the infection more difficult. Recovery from the milder septicemias is frequent; the severer forms are fatal in from a few days to weeks while certain types, especially the Streptococcus viridans continue for months, but are, as a rule, eventually fatal. In general the prognosis depends on the kind of infecting organism, the general vigor of the patient and the number of secondary foci developed.

Treatment.- The first indication is the identification of every accessible focus of infection and the institution of suitable treatment. Whether this treatment is to be surgical drainage or removal or not, requires most careful consideration: first, of the nature of the local lesion, whether a localized collection of pus or a diffuse septic process, the latter as a rule being less amenable to surgical treatment; second, concerning the surgical accessibility of the focus; third, concerning the ability of the patient to stand the operative procedures; fourth, in the milder forms of septic intoxication such as those arising from a tonsillitis, the danger of causing a dissemination of the organisms 
by surgical manipulation during the acute stage of the infection; fifth, concerning the danger under certain conditions of exposing by surgical interference, a focus of lowered resistance to infection with an organism more virulent than that already at work. When surgical interference is considered contraindicated, such local measures as cold or heat, ichthyol, evaporating lotions or in the case of open wounds, a continuous drip of normal saline may prove beneficial. Secondary foci should be promptly detected and appropriate treatment instituted as in the case of primary foci.

In the treatment of infected wounds during the present European War the following antiseptic developed by Dr. Dakin has been found to be especially valuable. "Dissolve in a large bottle I40 grams of dry carbonate of soda with ro liters of sterile water. Add to this 200 grams of chloride of lime (bleaching powder) and shake well. After half an hour siphon the clear fluid into another bottle through a cotton plug or filter paper and then add 40 grams of boric acid to the clear filtrate. This solution is neutral to litmus, is non-irritating and is the proper strength for wet dressings and irrigations. A stronger stock solution of 4 per cent. may be made, but the quantity of boric acid to be added must be determined exactly, so that the solution is just acid to phenolphthalein; otherwise the solution decomposes quickly. The solution should be made fresh every three or four days, and the dry stock ingredients should be kept in covered receptacles. Besides its proven efficiency, another point worthy of consideration is that this solution can be made up, even in small amounts, at a cost of only about 5 cents for ro liters.

"This antiseptic is used for wet dressings and as an irrigation. Large infected wounds, particularly those showing a necrotic or gasforming tendency, are treated as follows: After the surgical condition, such as a compound fracture or a lacerated, contused wound, has been treated by incising and clearing the parts of any loose material (foreign), drainage is accomplished in the usual way by the use of the fenestrated drainage tube introduced into the depth of the wound when thought necessary. In addition to this, a small soft-rubber fenestrated tube is introduced into the depth of the wound and allowed to protrude beyond the surface for 4 or 5 inches. The wound is then lightly packed with gauze pads saturated with the antiseptic solution and completely covering the small rubber tube in the wound, except that part which protrudes. Several larger gauze pads are now placed over the wound and secured lightly by a bandage, while the small rubber tube protrudes outside the dressings. This small rubber tube is now connected with a Murphy drip apparatus containing the antiseptic, which latter is allowed to enter by slow drops, usually about 30 to 50 drops a minute, the rapidity depending on the character and gravity of the infection. These dressings are renewed once or twice a day, according to indications, and the drops kept up as long as the infection lasts, the condition of the wound and the constitutional manifestations being the general 
guide in carrying out the treatment. As the infection subsides the tube is removed and a moist pad merely left in the wound at the time of the daily dressing.

"Another method of applying this antiseptic to the wound, which has also given very satisfactory results, is to inject into the wound from 5 c.c. to ro c.c. of the antiseptic every one to two hours through the small protruding rubber tube. The amount to be injected depends on the size of the wound, while the interval between injections is made longer or shorter, depending on the character of the infection. It is considered advisable to keep the dressings more or less saturated for the first forty-eight hours, and after that the general condition of the wound and the patient will indicate the proper course to pursue with reference to further treatment. By this method wounds are usually dressed every morning, and as the infective process subsides a less energetic treatment is instituted. This method is specially applicable to wounds about the face and neck and also in large lacerated or contused wounds of the other parts. A combination of continuous nightly irrigations with daily injections is sometimes used, particularly if it is found that by use of injections at night the patient's rest and sleep is seriously interrupted.

"When properly carried out this treatment will usually practically sterilize a wound in from three to four days, depending on the character of the wound and the extent of the infective process. When the wounds are treated early and the foreign infective material removed they rapidly clear up and have sometimes been actually closed by suture. after several days, with the subsequent uncomplicated healing of the wound. Amputation for badly infected compound fractures has been rarely resorted to when this method is used, and has only been performed when complicated by serious blood-vessel injury which prevented proper nutrition of the part.

"A very valuable feature of this antiseptic is its remarkable power of quickly separating and dissolving necrotic material in a wound. It very frequentiy happens in the wounds of modern warfare that a certain amount of devitalized tissue in the wound becomes necrotic and usually remains for a longer or shorter period as a medium for saprophytic bacteria and consequent prolongation of the infective process. With an antiseptic which rapidly cleans the wound of this necrotic and sloughing material, the surgeon has a most important adjunct in satisfactorily treating this class of wounds. In view of the fact that the gas-forming bacilli (perfringens) undoubtedly thrive best in the presence of devitalized, necrotic, or sloughing tissue, the value of this antiseptic in dealing with this character of infection will at once be appreciated. The use of this antiseptic and its mode of application in the treatment of serious and complicated wounds has been successfully demonstrated many times, not only in Dr. Carrel's hospital, but also in a number of other base hospitals and field-ambulance units."1

${ }^{1}$ Fauntleroy: Medico-Military Aspects of European War.-U. S. Government Printing Office. 
Next to the treatment of the local foci, the most important measures at the present time are those which tend to conserve and build up the strength and resistance of the patient. The indications to this end are first to secure the maximum amount of rest, an abundance of fresh air, as much nourishing food as the digestive powers of the patient can care for, to maintain, if possible, the appetite, and to secure a normal but not an excessive elimination through the bowels and kidneys. Absolute confinement to bed in the open air with a liberal diet of milk, eggs, gruels, beef juice, rice, cornstarch and arrow-root preparations is the best treatment for cases of sepsis. When the digestion permits, potato, macaroni, and such meats as chicken, lamb chops and minced ham should be allowed. Unfortunately only too often loss of appetite or even nausea and vomiting complicate the treatment. The appetite may then be stimulated by such dainty dishes as caviar, or by sherry, champagne, a bitter tonic, or kumyss. Occasionally bismuth, small doses of hydrocyanic acid or a combination of cerium oxalate, cocain and codein may control the vomiting. If vomiting be persistent, the nutrition may be sometimes in part maintained by the use of nutritive fluids per rectum, and of these, the best is probably a 3 to 5 per cent. dextrose solution in water. This is to be given by the drop method. It is superior to the older nutritive enemata.

Water should be given by mouth at frequent intervals, a glassful every hour or two during the day, except when replaced by liquid nourishment. When vomiting is present, water must be supplied by enteroclysis or hypodermoclysis.

Diarrhea is best controlled by modifications of the diet, the use of pasteurized or peptonized milk with lime water or of albumin or barley water. Bismuth and chalk mixture may be given but some caution is to be used in checking the diarrhea with opium unless it be quite excessive.

Cool or tepid sponging or even the full cold bath is of value both for the control of high fever and as a cardiac and vasomotor stimulant and may be used to advantage every three hours for either hyperpyrexia or falling blood-pressure. As a cardiac stimulant, alcohol has long been advocated, being given usually in the form of whiskey or brandy in full doses. Its value is at present, however, seriously debated by many observers. The habits of the patient in this regard are to be considered, and if alcohol appears to improve the appetite and spirits of the patient, it is probably desirable, but if the contrary is the case, it is better omitted. Instead, hot drinks or hot enemata serve a useful purpose as temporary cardiac stimulants. Camphorated oil and caffein hypodermically and adrenalin and normal saline intravenously are also useful as temporary cardiovascular stimulants. Digitalis may be given for more long-continued effects. It must be kept in mind, however, that any medication which destroys the appetite or lessens the patient's ability to handle his food, will inevitably do more harm than good. 
Sometimes digitalis may be given hypodermically in suitable preparations for short periods of time to avoid local irritating action upon the stomach. Pain frequently demands the use of codein, morphin or opium in some form for its relief, or occasionally a salicylate may be effective. In the regulation of the bowels great care must be taken to use either enemata or mild cathartics and to so adjust the dosage of the latter as to secure a laxative action without free purgation, as frec purgation is too depressing in its effects. The same objection is valid against such active eliminative measures as diaphoresis by vapor baths or by pilocarpin. Diuresis is to be secured by the water administered, possibly with the addition of one of the alkaline diuretics. Caffein diuretics are best avoided because of the frequent presence of acute degenerative renal changes. Coal-tar antipyretics are absolutely contraindicated because of their depressing effects. Concerning the value of quinin, there is a difference of opinion. By some authors, it is recommended in very large doses, Io grains every four hours until toxic symptoms appear; by others, in small doses; still others recognize no beneficial effects and in this group the author includes himself.

For sleeplessness, fresh air is the most useful remedy. Veronal, trional, paraldehyde and the bromids may be used, if necessary. They should be given early in the evening when used, and should not be given every night.

For delirium and nervous excitement, tepid sponging and an ice bag to the head frequently suffice. The sedative drugs, bromids, morphin, and hyoscin are variable in their action and the latter especially may increase the mental excitement.

Specific treatment has been directed first, toward increasing the number of circulating leukocytes; second, toward increasing their efficiency by raising the patient's opsonic index and third, toward destroying the microörganisms by antiseptics or by specific antisera. The preparation of an antitoxin such as is employed in the treatment of diphtheria has proved impossible owing to the fact that for the production of such a specific antitoxin, it is necessary that the microörganism shall produce a soluble toxin. Of the pathogenic microörganisms only two, the diphtheria and the tetanus bacilli, produce such toxins; neither of these organisms is concerned in the conditions here under condiseration.

A variety of substances have been observed to increase the number of circulating leukocytes, among which may be mentioned the intravenous injection of normal saline and the subcutaneous injection of albumoses and of nucleins. The latter especially has been used with apparently favorable results by certain observers. There are several preparations of nucleins and nucleic acid standardized according to their phosphorus content which may be used for this purpose. They may be given in a dose of ro c.c. of 5 per cent. solution hypodermically once daily, for three or four days out of 
every eight days; or smaller doses may be given continuously. Their use should be controlled by daily leukocyte counts, as there is some evidence to suggest that while acting at first as stimulants to the bone marrow, they may subsequently depress it.

To increase the opsonic index, autogenous vaccines have been employed. In those cases characterized by a well-marked virulent septicemia, the injection of vaccines seems irrational and contraindicated, nor is there any conclusive evidence of the value of vaccine treatment in such a case. When, however, the condition is one of toxemia from a focal infection or of long-continued mild septicemia from an organism of low virulence, the use of an autogenous vaccine appears more rational and experience supports in some degree, the value of such treatment. It is unfortunately just these cases, however, in which the difficulty of obtaining the offending organism in culture is greatest, and often accordingly the preparation of a vaccine is impossible.

With the evidence now at hand concerning the uselessness of a vaccine prepared from a strain other than that responsible for the infection and with the growing evidence of a great multiplicity in the variety of strains responsible for these infections, the use of a stock vaccine even though prepared from a half dozen strains of organisms is a method of treatment that has no rational basis and deserves condemnation. That such treatment can be beneficial is improbable in the highest degree; that it may be positively detrimental is quite possible.

In the effort to destroy the infecting organisms or inhibit their growth by the introduction of antiseptics, a variety of substances have been used. Among these are quinin, salicylates, hexamethylenamin, sulphocarbolates, various silver salts, including especially collargol, various preparations of iodine and mercury. Failure, as a rule, has followed all of these attempts as yet, although individual observers have become enthusiastic over this or that substance. Quinin is still recommended, as already noted, by some. Collargol has probably received wider support than any of the other substances. While originally advocated on the basis of a possible direct antiseptic action, Dunger has advanced evidence to show that it acts by destruction of the older leukocytes of the circulating blood. This results in a stimulation of the bone marrow probably from products of the disintegrated leukocytes, perhaps the nucleins, and in consequence the blood receives a larger accession of fresh, young leukocytes. This hypothesis is not as yet established. The usual dosage is from 0.05 to $0.2 \mathrm{gm}$. in 2 to 5 per cent. suspension, given intravenously daily for several successive days. Care must be observed to avoid injecting any sediment as in a few cases dyspnea and collapse have occurred, attributed to this error in technique. Typically, the injection is followed in from one-half to four hours by a chill and sharp rise of temperature and within twelve hours by a sharp fall in pulse rate and temperature with improvement in the subjective symptoms. 
Serum Treatment.- Up to the present time, the treatment of septicopyemia by specific sera has proved most disappointing. Possibly in part this may be due to the factor already mentioned in discussing vaccine therapy, namely, the multiplicity of strains of organisms involved. The preparation of antiserum against the particular strain of organism active in a particular case, requires too long, except possibly in the more chronic forms of septicemia, even if facilities and funds for such work be available. In the latter group of cases, we are confronted, however, with the difficulty in obtaining cultures of the offending organism. It is the custom of some to employ a mixed stock antistreptoccoccus serum when streptococcic septicemia has been demonstrated by a positive blood culture or when as in cases of puerperal septicemia, the diagnosis of streptococcic infection may be made with reasonable certainty without awaiting the results of blood culture. To be of value the serum must be used early in the infection and must be given in large doses, 50 to Io0 c.c. per day either subcutaneously or intravenously. The cost of such treatment frequently constitutes a serious obstacle, which is the more to be considered in view of the questionable benefit resulting from the treatment. Further studies may reveal some more effective method of specific therapy against these infections. For the present, however, chief reliance must be placed upon the treatment of the local foci, the maintenance of the strength of the patient and the relief of symptoms as they arise.

In cases that recover, the convalescence must be a prolonged one, and the patient be kept at absolute rest in bed for at least two or three weeks after complete disappearance of fever.

\section{ERYSIPELAS}

Erysipelas is a contagious disease characterized by local inflammation of the skin and rarely of the underlying subcutaneous tissues due to a local streptococcic infection and accompanied by more or less severe constitutional symptoms and toxemia.

Etiology.-Three views regarding the streptococcus responsible for this condition are in vogue, each supported by competent observers: (I) That it is a specific streptococcus-the Streptococcus erysipelatis-closely related to the Streptococcus pyogenes but distinct from it; (2) that it is identical with the Streptococcus pyogenes; (3) that while essentially identical with the latter organism, it acquires while infesting the lymph spaces of the skin certain peculiarities which for the time being render it distinguishable from the common strain of the hemolytic streptococcus pyogenes. The infection is typically localized, not a septicemia, and the organisms are found in the dermal lymph spaces most abundantly at the advancing edge of the lesion. Occasionally, fortunately rarely, a septicemia arises and perhaps leads to secondary localizations of a similar character or to pyemia. The infection may be clearly a wound infection as 
in surgical or puerperal erysipelas, or, in the so-called medical erysipelas, the point of infection may not be obvious. The commonest site for the latter type of the disease is the face, usually beginning about the nose or mouth. Even in these cases, however, the disease is probably essentially a wound infection, for about the nose as the result of fingering or of the excoriating discharges of a coryza minute abrasions are frequently present to serve as points of entrance for the microörganisms. Spread of the disease is probably by direct transmission upon the hands of attendants and upon the utensils, linen and other articles that have come into contact with the patient. Aerial transmission is of little, if any, importance. The inflamed surface and probably the desquamating scales from the healing area are the source of the organisms. It should be possible, therefore, with proper precautions on the part of the patient and attendants to entirely prevent the transmission of the infection. At the same time, prudence justifies the custom of excluding erysipelas cases from wards and rooms occupied by surgical or puerperal cases or by young children. Certain conditions appear to favor the occurrence of the disease. It is more common in middle life than at the extremes of age. Certain individuals exhibit a special susceptibility; those weakened by acute fever, by chronic wasting disease or by improper feeding or overwork, are more liable to the infection. The damp changeable months of Spring and Fall afford a disproportionate number of cases.

The pathology of the lesion is that of a simple inflammation with infestation of the dermal lymph spaces of the afflicted area with the streptococci. The regional lymph nodes show secondary acute lymphadenitis and the organs and tissues generally exhibit the degenerative changes common to acute infections. Suppuration is exceptional; indeed, the occasional development of suppuration in the subcutaneous tissues is to be looked upon as a complication, rather than as a symptom of the disease itself.

Symptoms.-The incubation period after infection is probably from a day to a week. The onset is, as a rule, sudden with little or nothing of prodromal manifestations and occurs frequently with a chill or chills and sudden elevation of temperature. The local lesion may be evident with the onset of constitutional symptoms or not appear for a day or two thereafter. The inflammation may be contiguous to the wound or abrasion, but not rarely develops at some slight distance, perhaps $1 / 2$ inch or more from the probable point of infection. Thus in medical erysipelas a frequent point of beginning inflammation is the bridge of the nose when the infection occurs in the nares. Facial erysipelas commonly appears about the nose, angles of the mouth or border of the scalp. The lesion is a somewhat brawny and edematous, raised, reddish area, tense and tender and hot to the touch and with an irregular but sharply demarcated outline, the edge being especially elevated and reddened. The lesion characteristically spreads from day to day. Close to, 
but beyond, the periphery there may be seen small reddened areas that represent the beginning of more extensive involvement. Facial erysipelas commonly extends over both cheeks onto the forehead and involves the eyelids and ears, frequently permitting the tip of the nose to escape. Certain anatomical lines, such as the hair line, may check the progress of the lesion, but it may extend over the scalp or down onto the neck. The older areas of the lesion eventually exhibit desquamation. The regional lymph nodes may be enlarged and tender. Occasionally metastatic areas develop at a distance on the extremities or trunk, constituting erysipelas migrans. Occasionally, too, the character of the lesion may be unusual, exhibiting vesicles or pustules on the surface or very rarely the deeper subcutaneous tissues may be involved by suppuration. Cases with suppuration exhibit very severe constitutional symptoms. The degree of constitutional disturbance is variable. It may be slight; on the other hand, marked nervous symptoms, headache, even delirium, intense exhaustion, coma, gastrointestinal symptoms, such as vomiting or diarrhea, and in the drunkard a delirium tremens are sometimes seen. The fever is remittent in character and exhibits diurnal fluctuations similar to those of other septic infections. As a rule, with extension of the local process, a rise of temperature is observed, but occasionally the local lesion progresses when the patient has become afebrile.

A comparatively rare, but especially serious form of the disease, is that attacking the mucous membranes of the mouth and pharynx and characterized by great pain, redness and edema of the affected areas. Edema of the glottis is a dangerous complication of this type of the disease. In infants and young children, the navel or vagina are common sites of origin, and the condition at this age is a grave one.

Among the complications are subcutaneous abscesses, gangrene, meningitis, arthritis, phlebitis, pleuritis, pericarditis, nephritis, tonsillitis, pneumonia and pyemia. Relapses have been observed in about ro per cent. of cases, the lesion recurring in the same area usually after an afebrile period of from three to five days. Repeated relapses may occur. As a rule, however, a brief immunity follows an attack-for from five weeks to three or four months.

The diagnosis rests upon the association of a spreading brawny inflammation of the skin exhibiting a raised, reddened, sharply demarcated margin with the constitutional symptoms, sudden onset, chills, moderate high fever and polymorphonuclear leukocytosis.

Certain eczemas, erythemas and urticarias may closely simulate the local lesion but do not give the constitutional symptoms. A cellulitis either primary or overlying a deep suppurative focus may be confusing, but does not have the characteristic sharply demarcated border, usually exhibiting instead the most pronounced inflammatory features at the center of the lesion.

The disease is a self-limited one and the average duration of the 
uncomplicated case is from two to three days to two weeks. The migrating or relapsing form may last many weeks. The prognosis is influenced by age; being unfavorable under five years of age and becoming again more unfavorable with increasing years above forty-five. The general average mortality is from about 4 to 7 per cent. In the chronic alcoholic or in the presence of any of the septic complications, the prognosis is graver and surgical or puerperal erysipelas is more serious than the medical form.

Treatment.-To prevent the spread of infection, all articles coming into contact with the patient should be disinfected and the patient should be kept isolated from puerperal cases, young children, or individuals with open wounds. Nurses and others in contact with erysipelas should protect even trifling abrasions upon the hands or should wear rubber gloves. All linen about the patient should be frequenty changed and sterilized by boiling; dressings should be collected as removed and burned. As desquamation sets in, the part may be protected by vaseline or boric ointment and frequently washed with weak boric acid or permanganate solution. The general treatment of the patient demands rest in bed, fresh air, water freely, a rather full nutritious diet of simple, easily digested foods and the keeping of the bowels open without active purging, essentially the general treatment described in considering septicemia in the previous section. Stimulation is frequently required and and for this purpose strychnin, digitalis, ammonia and camphor may be employed. In this, as in the control of restlessness, insomnia and hyperpyrexia, the symptomatic treatment is identical with that of septicemia. Of great importance in this as in other acute infections is the free administration of water by mouth to promote diuresis. It is the writer's habit to give a glass of water hourly, except when a liquid feeding is due, until the urinary output has reached a liberal figure $-\mathrm{r} 500$ to 2000 c.c. per day-after which the amount may be slightly reduced. : At the onset of the condition, a course of calomel followed by a saline purge is advisable. Thereafter the bowels are to be kept open by laxatives, but active purgation is to be avoided.

Of drugs given internally and supposed to exert a specific effect upon the process, three have in the past been held in especial esteem: the tincture ferri chloridi given in water in doses of 5 to 8 minims every second or third hour; quinin in amounts up to ${ }_{5}$ to 30 grains daily; and camphor in 3-grain doses every two or three hours. The iron and either the camphor or quinin have been used simultaneously. The claim is made that by these drugs the temperature is reduced, the toxemia lessened, and the course of the disease shortened; the statistics on which these recommendations are based are not conclusive, however. The writer is disposed to spare the stomach as far as possible for a rather full diet of milk and eggs, etc., rather than to risk disturbing the digestive tract with large doses of drugs of uncertain value. 
The results with antistreptococcic sera and vaccines are as yet of unsettled value, although favorable reports have been given by occasional observers.

Of local measures, there is a great variety. To relieve the pain and discomfort, the application of some wet dressing such as leadwater and alcohol is probably of greatest value. On the face this is done by the use of a mask with holes cut for the nose and mouth but with the eyes left covered. Saturated solution of magnesium sulphate or a 25 to 50 per cent. solution of chthyol have been very highly recommended, and the latter is probably one of the best of local dressings, although on the face it is objectionable because of its odor. The use of ice bags has been discountenanced on the ground that it lowers the vitality of the skin. The use of ointments receives less support than wet dressings, except in the later stages, during desquamation, when a boric or zinc oxide ointment or a I per cent. carbolic ointment may be used.

To prevent the extension of the lesion, the older methods of painting with iodine or silver nitrate about the lesion are generally admitted to be useless. Painting the skin just beyond the advancing edge of the lesion with collodion or ichthyol collodion ( 50 per cent.) was said to discourage extension by exclusion of air from the underlying skin; but the method is probably useless. Injection of a ring of 2.5 to 5 per cent. carbolic, intradermally $\mathrm{I}$ inch beyond the margin of the lesion or crossed scarification in a ring an inch outside of the lesion followed by spraying with 5 per cent. carbolic, has been recommended. With these methods the author has had no experience, but the danger of phenol poisoning must be kept in mind in any such procedure.

In the treatment of buccal or pharyngeal erysipelas, frequent gargling with an alkaline wash and the constant sucking of ice, aid in keeping down the edema. If edema of the glottis becomes of a threatening grade, scarification of the larynx or tracheotomy may have to be done. Hot foods and steam inhalations have been accredited with increasing the tendency to edema of the glottis in this form of the disease and are to be avoided.

The occasional complications of erysipelas, suppuration and gangrene, require treatment by the usual surgical methods. 


\section{SYPHILIS}

By J. William White, M. D., and Alfred C. Wood, M. D.

The therapy of syphilis has been profoundly influenced by three recent discoveries-the recognition of the Spirochæta pallida (Treponema pallidum) as the specific cause of the disease by Schaudinn and Hoffmann in April, I905; the Wassermann reaction, announced by Wassermann, Neisser and Bruck in May, 1906, and the introduction of salvarsan (therapia sterilizans magna) by Ehrlich in December, I910. The great value of these additions to medicine is universally recognized.

The diagnosis of syphilis is now possible, in the first few days after the appearance of the primary lesion by the presence of the spirochete in the serum obtained from the chancre. A little later, the disease may be detected by the Wassermann test. The proper use of salvarsan or neosalvarsan, in conjunction with the remedies heretofore employed, has added very greatly to the certainty of prompt and permanent cure. Finally as we may now determine the exact measure of the success or failure of our medication by the Wassermann test, the treatment of this disease has been removed from the realm of empiricism and placed on a plane with the highest standards of modern medical science.

In considering the recent additions to the treatment of syphilis, the headings employed in the original article (see Vol. II, page 460) will be followed. Reference will be made only to those topics on which there has been some important addition since the first edition was published.

Diagnosis.- Up to the time the Spirochrta pallida was demonstrated to be the cause of syphilis, and the Wassermann reaction was found to be uniformly present in untreated or unsuccessfully treated cases, the best syphilographers declined to make a diagnosis or to advise treatment until certain "secondary" symptoms were manifest. In the average case this period was about nine weeks from the time of infection.

The diagnosis may now be made, and should be made in the earliest stage of the disease by examining the suspicious lesion (chancre) for the presence of the Spirochæta pallida. This is done by expressing a little serum from the lesion, a drop of which is placed on a slide, a drop of normal salt solution added, a cover-glass placed over the fluid, and the slide examined by means of the "dark field illuminator." If it is found to be impossible to express any serum the surface of the lesion should be scarified with a scalpel sufficiently to permit of the escape of a little serum, without drawing 
blood. Very careful and persistent search must be made for the spirochetes or they may be overlooked. If the lesion is but one or two days old, and no organisms are found, the examination should be repeated again in two days.

While this examination requires special apparatus and some experience, the importance of reaching an early diagnosis is such that every effort should be made to have it done. No difficulty will be experienced in this matter in any city or town of reasonable size, and the means of travel now make it easy for any one to reach a specialist in this line, for this test.

It cannot be stated too emphatically that the diagnosis of syphilis should be made at the very earliest possible moment, and vigorous treatment instituted at once. There is now no occasion to wait for "developments" of any kind after the primary sore (chancre) has appeared. - If the patient should first come under observation at a later period, the Wassermann test will establish the diagnosis.

Treatment.-In the first edition this was discussed under two heads: first, the steps necessary to prevent the patient from transmitting the disease to others; and, second, the elimination of the poison from the patient's system.

The first of these, need not be repeated here, but should be carefully read (see Vol. II, page 463 ).

The second, the elimination of the poison from the patient's system, has been very largely modified on account of the great advance in our knowledge in regard to: (a) the nature of this "poison;" $(b)$ the diagnosis; and $(c)$ important additions to our resources in treatment. The term "elimination of the poison from the patient's system" is used advisedly. Everyone undertaking the treatment of a case of syphilis should fully understand that there is a vast difference between treating the symptoms of the disease and fully clearing the system of the germs, or curing the patient. It is a matter of common observation that the chancre, the various secondary manifestations and many tertiary lesions yield readily to treatment, but are only too prone to recur at a later period. Anyone who contemplates for a moment the great number of persons suffering from the various manifestations of late syphilis, and recalls that most of these persons were "treated" and were supposed to be cured, must feel impelled in every case that comes under his care, to secure a prompt diagnosis and to treat the patient until the last trace of the poison is destroyed. This is now possible, and we have the means in the Wassermann test of checking our results, so that we need no longer remain in doubt when to stop the treatment.

A. Initial Lesion.- Since it is now possible to make a diagnosis in the earliest stages of the chancre much of what appears under this head in the original article has become obsolete.

Excision of the chancre is still to be advised, if situated on the prepuce, or where its removal will not cause any undue mutilation. 
Removal may be contraindicated by anatomical considerations such as the possibility of troublesome hemorrhage, interference with functions, etc.

While the removal of chancre as soon as possible after its appearance will not abort the disease, it is reasonable to suppose that by eliminating this nidus of active multiplication of the treponema the system will not be so freely saturated with the organisms as though it was allowed to remain. It should be understood, however, that removal of the chancre does not in any way modify the usual subsequent treatment.

Constitutional Treatment.-The advice of the best syphilographers in the past to withhold specific treatment until a positive diagnosis could be made has lost none of its force, and should still be rigidly observed. However, the former custom to wait for secondary symptoms is no longer permissible. The "positive diagnosis" is now reached by examining a drop of serum expressed from the chancre. By this means effective treatment may be begun at once.

Formerly we had but a single drug with which to treat syphilis at this stage, namely, mercury, which however, was administered in many forms. In December, I9ז0, Ehrlich placed salvarsan (606) upon the market; subsequently he brought out a modification, neosalvarsan (9I4). Both of these substances have been found highly efficient, and they share with mercury the distinction of being specific remedies for the disease.

Success in thoroughly overcoming the systemic infection depends more upon early, energetic treatment than upon any other factor. Full doses of salvarsan or neosalvarsan and of mercury administered before the patient becomes Wassermann positive, that is before the appearance of secondary symptoms, is more effective than any line of treatment carried out at a later period. After the treponema once become well established, throughout the system, it is difficult to wholly eradicate them.

Primary and Secondary Syphilis.-The weight of opinion at this time is definitely in favor of treating syphilis in both the primary and secondary stages with a combination of salvarsan or neosalvarsan and mercury.

It will be readily understood that no hard and fast rules can be laid down that will be suitable for every individual, but the treatment must be modified according to the age, sex and general condition of the patient, as well as the stage of the disease. It should also be stated that authorities differ widely in regard to the administration of salvarsan. It is the custom with some to administer two or three doses at intervals of from three to five days. We believe, however, these frequently repeated doses are not always well borne and may even lead to serious consequences.

As a basis of a safe and efficient working formula, the following may be suggsted as applying to an adult, otherwise in good health and having sound organs: 
I. Give the usual dose of salvarsan or neosalvarsan considering the age, sex, condition of general health, etc., of the individual, intravenously (see dose table page 285 ).

2. In two days begin a mercurial course (inunctions of mercurial ointment, hypodermatic injections, or mouth administration) and continue until the physiologic effects of the drug are noticed. This may require ten days to three weeks.

3. A second salvarsan or neosalvarsan injection.

4. Repeat the mercurial course as before.

5. A third salvarsan or neosalvarsan injection.

6. A third mercurial course, continued for about six weeks, using any of the forms mentioned, and pushing the dose up to the limit of tolerance while watching carefully for any signs of mercurial intoxication. If these should appear the drug should be discontinued until the evidences have disappeared, and then resumed, using slightly smaller doses.

7. Discontinue all treatments and after four to six weeks have a Wassermann test made. If this is negative no further treatment need be ordered. Two or three months later another Wassermann test should be made, and if still negative this test may be repeated at the end of another six months, and again at the end of a year. If all of the tests, carefully carried out, remain negative, the patient may be considered cured. If, however, the reaction is positive, there should be another neosalvarsan injection followed by active mercurial administration, and the treatment carried out as in the first instance. The number of additional doses of neosalvarsan and the prolongation of the mercurial treatment will be determined by the degree of the reaction. A strongly positive report would indicate not only active, but more prolonged treatment; whereas a very mildly positive report would indicate a shorter treatment. After this second course, an interval of a month or so is allowed to pass, when another Wassermann test should be made. Although the foregoing plan is more prolonged than that recommended by some writers, the necessity of completely eradicating the infection is so imperative that to stop short of absolute sterilization of the system is certainly censurable.

While it is not possible to guarantee permanent immunity from recurrences in every case by any system of therapy, as before stated, the greatest percentage of cures will result if the treatment is begun in the earliest days of the disease. After secondary symptoms are well established and especially after tertiary lesions have developed, it will be found much more difficult to render the patient permanently Wassermann negative.

Tertiary Syphilis.- In the tertiary manifestations there does not seem to be the same need for large doses of salvarsan; moderate doses ( $0.45^{-0} 0.6 \mathrm{gm}$. for women, and 0.6 to $0.75 \mathrm{gm}$. for men) repeated over a longer period will be found most satisfactory. Between the neosalvarsan injections the patient should be given the so-called 
mixed treatment, i.e., mercury with iodine, in some form, preferably potassium or sodium iodid. Or the mercury may be given by inunctions or by hypodermatic injection (Vol. II, pages $475-478$ ), and the iodid by the mouth. As in the case of the primary and secondary stage of the disease, after a vigorous course of treatment has been carried out, for from three to six months, the patient should be given a period of rest, and the Wassermann test made. If this is positive, the treatment should be repeated, and at intervals of a few months the result again checked by the Wassermann test. It is not possible in these late cases to render all patients permanently Wassermann negative, but a consciencious effort should be made to do so.

Hereditary Syphilis.-Salvarsan and neosalvarsan are recommended in the treatment of hereditary syphilis. An indication of the appropriate doses will be found on page 285 . The introduction of the needle into a vein will naturally be more difficult. Unless the operator is very expert, it may be necessary to expose the vein by incision.

The former treatment (Vol. II, pages 488-490) should becarried out as energetically as though the salvarsan had not been used. The results of the treatment should be determined by the Wassermann test, just as in the acquired disease.

\section{SALVARSAN $^{1}{ }^{1}$ (DIOXYDIAMIDO-ARSENO-BENZENE DIHYDROCHLORID, 606)}

Arsenic was occasionally prescribed in the treatment of syphilis in the past, but its use was quite empiric and did not attract any attention until $\mathrm{I}_{90}$, when Uhlenhuth showed that atoxyl exerted a specific influence on fowl spirochetosis, which led to the suggestion that the remedy might be useful in syphilis. Professor Paul Ehrlich became interested in the subject and began a series of experiments in search of a remedy, the administration of which would destroy the Spirochrta pallida in the body without injury to the patient. After 605 distinct synthetic preparations had been tested out and discarded the next combination proved to be a success, hence the designation "606." Subsequently the term salvarsan was adopted. After the most exhaustive laboratory tests, the remedy was placed in the hands of a few representative physicians in the larger medical centers, and after having thoroughly established its value was finally placed on the market. Although salvarsan did not meet the hope entertained by Ehrlich, that a single dose would entirely free the system of spirochetes, it was found to be at least as effective as mercury, more prompt in its action, and for certain manifestations of syphilis superior to the latter.

'Salvarsan and neosalvarsan, being powerful remedies, "any irregularity in the manufacture of which may result in the formation of byproducts of enormous toxicity," and any errors in the administration of which exposes the patient to grave dangers, the writers feel that the rules formulated by Ehrlich should be implicitly followed. An effort has been made to present these faithfully in the following pages. While certain variations might be made without harm resulting, the safe rule is certainly to follow the instructions so carefully and scientifically worked out by Ehrlich, Bertheim and Hata. See also footnote, p. $28 \%$. 
Salvarsan is a yellow powder, which dissolves in water with a strongly acid reaction. It contains about 34 per cent. of arsenic and corresponds with the following formula: $\mathrm{C}_{12} \mathrm{H}_{12} \mathrm{~N}_{2} \mathrm{O}_{2} \mathrm{As}_{2} .2 \mathrm{Hcl}+$ ${ }_{2} \mathrm{H}_{2} \mathrm{O}$. Any irregularity in its manufacture may result in the formation of byproducts of enormous toxicity. Salvarsan is decomposed on exposure to the air, becoming poisonous and unfitted for use. Hence carefully weighed quantities are put in glass ampules which, after being filled with a neutral gas, are sealed. Under these conditions the drug will remain unchanged indefinitely. Before use the ampule should be carefully inspected to see that there are no cracks or flaws in the glass. The contents of any ampule showing these defects should be rejected.

The ampules are referred to by number, from $I$ to 6 , and contain 0.1 gm., 0.2 gm., $0.3 \mathrm{gm}$., $0.4 \mathrm{gm}$., $0.5 \mathrm{gm}$., and $0.6 \mathrm{gm}$. respectively.

Indications.- Salvarsan is employed in the treatment of primary, secondary and tertiary syphilis, including the various manifestations that accompany these different stages.

Contraindications.-Salvarsan should be withheld or employed with great caution in cases showing evidences of serious disturbances of the cardiovascular system, in fetid bronchitis, pronounced cachexias, unless the direct consequence of syphilis, and kidney disease. In affections of the eye, even if the result of syphilis, and in patients who have an idiosyncrasy against arsenic, the dose employed in the beginning should be relatively small.

-When symptoms of lues cerebralis or meningitis are present, especially early meningitis, caution should be exercised in the dosage, and the treatment commenced with small amounts.

As symptoms of meningitic troubles, severe headaches, insomnia, rhachialgia, motor asthenia, changes in psychical condition, speech troubles, giddiness, vomiting, cramp and nervous symptoms such as sparks before the eyes, singing in the ears, etc., are typical.

Dose.-For intravenous administration the doses usually employed for persons showing no contraindications are:

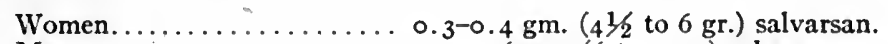

Men.................. $0.4^{-0} 0.6 \mathrm{gm}$. (6 to 9 gr.) salvarsan.

Patients who are feeble, or who have affections of the central nervous system, heart, kidneys or eyes, if at all suitable for this treatment should be given relatively small doses, $0.1-0.3 \mathrm{gm}$. Children may be given 0.2 to $0.3 \mathrm{gm}$. ( 3 to $4 \frac{1}{2}$ gr.), infants 0.02 to $0.1\left(1 / 3\right.$ to $1 \frac{1}{2}$ gr.) according to age. Ehrlich advised repeating the doses mentioned in three or four weeks, if the first was well borne. If the amount administered was unusually small for any reason it may be repeated at shorter intervals than when the maximum quantity, considering the age, sex, and condition of the patient, has been given.

It has become the practice of some syphilographers to repeat the doses at short intervals, even in three to five days. It is the 
opinion of the writers that this is fraught with danger.' Many of the fatalities that have followed the intravenous administration of salvarsan, have been in cases receiving the second or third dose at intervals of a few days. It has been shown that traces of arsenic remain in some of the internal organs (liver) for a considerable time after the administration of salvarsan. Repeating the dose too soon may, therefore, cause toxic symptoms from the cumulative action of the drug.

Administration.-Salvarsan may be introduced into the system, subcutaneously, intramuscularly or intravenously. It has also

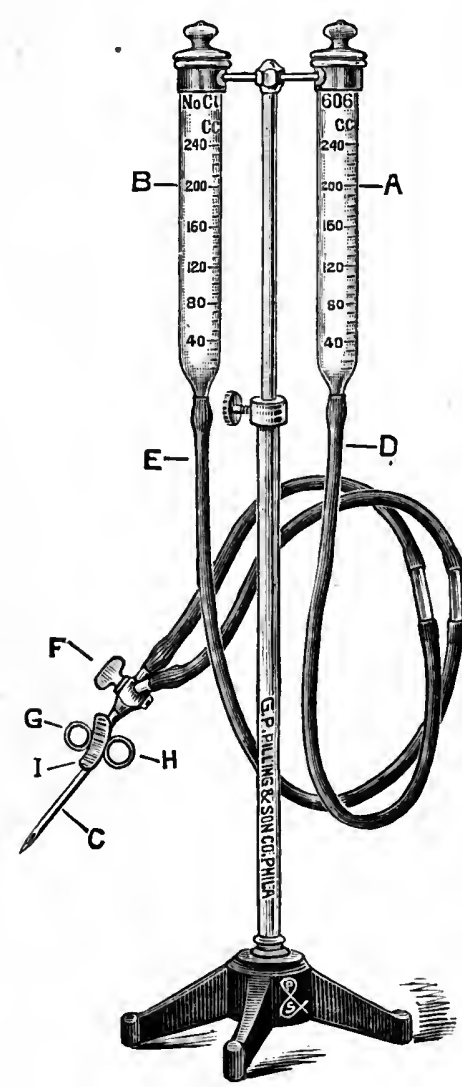

Fig. 46.-Boehm apparatus for been given by the mouth and in suppositories. The subcutaneous and intramuscular administrations have been largely discontinued, on account of the great pain and the extreme reaction which follow the injections. Abscesses and sloughing have been reported in a number of cases. While it is possible that the more serious sequels of this kind may have been due to bacterial contamination, nevertheless the drug is so irritating to the tissues that it does not lend itself well to this form of medication. The internal use and the incorporation in suppositories have not been given sufficient trial to warrant any statement as to the value of either of these methods. It is now almost universally advised to administer the drug intravenously, although intramuscular injections of neutral suspensions in oil have proven very satisfactory in the hands of some who have employed them.

Apparatus Required.-(I) Suitable glass reservoirs connected to a hollow needle by 3 or 4 feet of rubber tubing (Fig. 46). (2) A cylindrical glass-stoppered bottle, graduated to 300 c.c. and containing about 50 small glass beads. (3) Glass funnel for the purpose of straining the solution through wet cotton or gauze. (4) Alcohol lamp. (5) Fifteen per cent. sodium hydroxid solution. (6) Sterile 0.5 per cent. normal salt solution. (7) Sterile freshly distilled water. (8) Alcohol. (9) Tr. iodine. (Io) Sterile cotton or gauze sponges. (II) Collodion. (I2) Piece of bandage or fillet for tourniquet. 
Preparation of the Patient.-The patient should be prepared for the administration of salvarsan in the same manner as for neosalvarsan (see page 289 ).

Intravenous Administration.-(a) Preparing the Solution.Into a sterile, narrow-necked, glass-stoppered bottle, graduated to 300 c.c. and containing about 50 sterile glass beads, 30 to 40 c.c. of sterile, freshly distilled water are placed. The outside of the glass ampule containing the proper dose of salvarsan having been sterilized by alcohol, the neck, at the point where it widens into the body of the ampule, is slightly scratched with a file, sterilized by alcohol, or by passing through a flame. One end of a thin glass rod is then heated to redness in a Bunsen burner or spirit flame, and quickly applied to the scratch on the neck of the ampule. If this is properly done a straight crack running horizontally around permits the neck to be easily broken off, when the contents may be poured into the vessel containing the distilled water. Solution readily takes place with the aid of vigorous shaking. After the solution has become absolutely clear and no undissolved particles remain, it is neutralized by the addition of the proper quantity of a 5 per cent. solution of caustic soda. This solution is made as follows:

\section{SODIUM Hydroxid SOLUTION}

Purified sodium hydroxid...................... 5 gm.

Sterile, freshly distilled water $\ldots \ldots \ldots \ldots \ldots \ldots \ldots \ldots \ldots \ldots \ldots \ldots \ldots \ldots, 8,5$ c.c.

The proper quantity of this solution to be added to the various doses of salvarsan is as follows:
$0.1 \mathrm{gm}$. salvarsan about 0.19 c.c. $=$ about 4 drops.
$0.2 \mathrm{gm}$. salvarsan about 0.38 c.c. $=$ about 8 drops.
$0.3 \mathrm{gm}$. salvarsan about 0.57 c.c. $=$ about 12 drops.
$0.4 \mathrm{gm}$. salvarsan about 0.76 c.c. $=$ about $\mathrm{r} 6$ drops.
$0.5 \mathrm{gm}$. salvarsan about 0.95 c.c. $=$ about 20 drops.
$0.6 \mathrm{gm}$. salvarsan about $\mathrm{I} .14$ c.c. $=$ about 24 drops.

The addition of the caustic soda produces a precipitate which, however, dissolves on shaking the vessel. Sterile 0.5 per cent. saline solution, prepared from chemically pure sodium chlorid and sterile, freshly distilled water, is added until the total volume represents $50 \mathrm{c}$.c. for each $0 . \mathrm{I} \mathrm{gm}$. of salvarsan used. If the solution should not become entirely clear, or if a slight turbidity should occur after standing a short time, a drop of caustic soda solution should be added, and if after two or three minutes the turbidity remains another drop is to be added, and so on until the solution is perfectly clear, but always waiting a short time between each drop in order that the addition of an excess may be avoided. It will be observed that each 50 c.c. of this solution contains $0.1 \mathrm{gm}$. ( $\mathrm{r} / 2 \mathrm{gr}$.) salvarsan.

In the preparation of the alkaline solution for intravenous injection it is imperative that the salvarsan, by shaking with glass beads, be at first completely dissolved in a little distilled water 
(not saline solution) and that the solution does not show, even when examined by transmitted light, any gelatinous, drop-like particles whatever, before the caustic soda solution is added. The caustic soda solution should not be added gradually, in portions, but run into the solution in one lot. The precipitate produced thereby must likewise be completely dissolved again prior to the further dilution of the alkaline concentrated salvarsan solution with 0.5 per cent. saline solution. Chemically pure sodium chlorid is an indispensable requisite. Ordinary table salt or spring water is just as unsuitable as the physiologic saline solution which contains sodium carbonate.

b. Introduction into the Vein.-The technique of the intravenous administration of the salvarsan solution is identical with that for neosalvarsan (see page 290).

After-effects.-During the administration, patients not infrequently state that they can taste the medicine. If the water employed in making the salt solution and the salvarsan solution was freshly distilled and sterile, and if the salt used was chemically pure, and further if the efforts to secure asepsis throughout have been entirely successful, relatively little inconvenience is experienced by the patient. Occasionally, within an hour or two, the temperature may rise, reaching in some cases $104^{\circ}$. With the higher temperatures, there is apt to be a rigor or even an actual chill. Occasionally vomiting may occur and headache may be complained of. The occurrence of higher temperatures is probably due to some bacterial contamination, or the use of water that is not freshly distilled and sterile. Diarrhea is apt to ensue in from six to twelve hours, but passes off without requiring any treatment.

Occasionally after a second or third injection, given at short intervals, the symptoms may be very severe; convulsions and coma with death having been reported.

As a rule the beneficial effect of the treatment may be noticed within the first two or three days, and in favorable cases a remarkable improvement may be observed. It is stated that the spirochetes can rarely be found in the lesion the day after the administration. The Wassermann reaction usually becomes weaker and weaker.

Occasionally the first effect of the salvarsan appears to be an aggravation of the symptoms. This lasts but a very brief period and is followed by signs of improvement. In some cases of latent syphilis a negative Wassermann reaction becomes positive after the administration of salvarsan. This has lead to the custom of giving a "provocative" dose of salvarsan in suspicious, but doubtful, cases that are Wassermann negative. The immediate after-care of the patient is the same as that recommended after neosalvarsan administration (page 289).

Subcutaneous and Intramuscular Injections.-For subcutaneous and intramuscular injections an all-glass syringe, having a needle I1 $1 / 2$ to 2 inches long with a caliber of about 20 , should be used. 
For subcutaneous administration salvarsan has been prepared (a) in a simple trituration in fatty oils (oil of sweet almond, oil of sesami, olive oil) making a ro per cent. mixture; $(b)$ in ro per cent. solution in sterile freshly distilled water; $(c)$ in neutral suspension. The latter is prepared in a sterile porcelain dish as follows: to $0.5 \mathrm{gm}$. of salvarsan is added 8 drops $(0.45 \mathrm{gm} .=0.38$ c.c. $)$ of caustic soda solution. To this is added at first drop by drop with constant trituration the proper quantity ( 5 to Io c.c. sterile freshly distilled water). The suspension produced is tested with litmus paper in order to be sure of a neutral reaction. According to the reaction a drop of caustic soda or of dilute hydrochloric acid may be added.

In the subcutaneous administration the injection is made between the shoulder-blades at the side of the vertebral column and in a downward direction. In all cases the locality of the injection must be previously disinfected with alcohol or iodine tincture. The fluid should, after subcutaneous or intramuscular injection, be distributed by careful massage over as large an area as possible.

With sensitive persons the locality of the injection may be completely anesthetized by a previous injection of 2. c.c. of a 0.5 per cent. novocain solution. Hydropathic measures, as moist compresses, hot baths, etc., or even the application of warmth, may be successfully employed to prevent any after-pains or reactive painful infiltrations. It is advisable that the patients remain in bed for some time after an injection, under supervision of a reliable attendant.

Intramuscular Injection.-For intramuscular administration the appropriate quantity of salvarsan, say $0.5 \mathrm{gm}$. is placed in a sterile mortar with (for this quantity of salvarsan) I9 drops of $I_{5}$ per cent. caustic soda solution, and then from 5 to ro c.c., of freshly distilled water added. The injection is made in the upper external sections of the gluteal muscles, and should be made deeply but very slowly in order to avoid undue traumatism to the tissues. The neighborhood of the sciatic nerve must be scrupulously avoided. The ro per cent. trituration in oil has also been employed intramuscularly.

Salvarsan has also been administered intramuscularly in the so-called fractional doses, injections of $0.1-0.2 \mathrm{gm}$. suspended in oil being given every second day until a total maximum of $1.2 \mathrm{gm}$. (I8 gr.) of salvarsan has been employed.

\section{NEOSALVARSAN ${ }^{1}(9 \times 4)$}

During his investigations, Ehrlich observed that formaldehydesulphoxylates were capable of restraining for a considerable time

1 According to Ehrlich, salvarsan and neosalvarsan are equally efficient, therapeutically; the advantages being somewhat on the side of the latter on account of lesser toxicity and greater simplicity in administration. For the purposes of this discussion the terms are used interchangeably. Practically, every statement applying to one is equally true of the other. The indications and contraindications are broadly identical, and where one is mentioned, the other may be substituted, bearing in mind, however, the difference in dosage, and that the technique of preparing the solutions for administration differs widely in the two cases. This detail must be rigidly observed. 
the autoöxidation of solution of salvarsan. This led to the introduction of neosalvarsan, which is obtained by the action of formaldehyde-sulphoxylate on dioxydiamido-arsenobenzene.' Experiments on animals and clinical test appeared to show that neosalvarsan possesses the following advantages over salvarsan: (I) Neosalvarsan dissolves very easily in water and gives a solution completely neutral in reaction. (2) Neosalvarsan is better tolerated subjectively. (3) The activity of neosalvarsan is at least as great as that of salvarsan. (4) Neosalvarsan is suitable for intramuscular injection. (5) The employment of neosalvarsan is preferable for patients who exhibit hypersensitiveness toward salvarsan (facial edema).

It is claimed that any variation in the exact technique of manufacture may lead to products possessing a high degree of toxicity, therefore, only such neosalvarsan as has been tested biologically, under the conditions laid down by Ehrlich is put upon the market.

Neosalvarsan is a yellowish powder having a peculiar odor and dissolving readily in water with a neutral reaction. It contains as the active constituent dioxydiamido-arsenobenzene-monomethane-sulphinate of sodium $\left(\mathrm{C}_{12} \mathrm{H}_{11} \mathrm{O}_{2} \mathrm{As}_{2} \mathrm{~N}_{2} \cdot \mathrm{CH}_{2} \mathrm{O}\right.$.SONa), together with inorganic salts.

\section{Dose.-}

$0.15 \mathrm{gm}$. neosalvarsan corresponds to dose $I=0 . I \mathrm{gm}$. salvarsan.

$0.3 \mathrm{gm}$. neosalvarsan corresponds to dose II $=0.2 \mathrm{gm}$. salvarsan.

$0.45 \mathrm{gm}$. neosalvarsan corresponds to dose III $=0.3 \mathrm{gm}$. salvarsan.

$0.6 \mathrm{gm}$. neosalvarsan corresponds to dose IV $=0.4 \mathrm{gm}$. salvarsan.

$0.75 \mathrm{gm}$. neosalvarsan corresponds to dose $\mathrm{V}=0.5 \mathrm{gm}$. salvarsan.

$0.9 \mathrm{gm}$. neosalvarsan corresponds to dose $\mathrm{VI}=0.6 \mathrm{gm}$. salvarsan.

The average single dose for men is 0.6 to $0.75 \mathrm{gm}$., for women 0.45 to 0.6 gm., and for children, according to age, from 0.15 to $0.3 \mathrm{gm}$. For suckling children doses of $0.05 \mathrm{gm}$. neosalvarsan may be administered.

As a maximum single dose for men $0.9 \mathrm{gm}$. and for women 0.75 gm. should not be exceeded.

The dose, the required number of repetitions, and the intervals after which each injection is to be made, depend upon the stage of the disease, and the condition and age of the patient. Definite normals cannot, therefore, be fixed. As a rule, however, the quantity of neosalvarsan may be used which corresponds to the dose of salvarsan which would have been injected in the particular case.

A combined treatment with mercury, as experience with salvarsan has already shown, is always to be recommended.

In cases of early syphilis and especially in those exhibiting derangement of the central nervous system (headaches, etc.); it is advisable to subject the patients to a brief preparatory mercurial treatment, followed by cautious treatment with neosalvarsan in small doses of 0.15 , maximum $0.3 \mathrm{gm}$.; the second injection should be made with the same doses. For subsequent injections doses of $0.4,0.6,0.75$ and at the most $0.9 \mathrm{gm}$. may be used. 
In early chancres, of at most a fortnight's existence, which still exhibit a negative Wassermann reaction and no swelling of lymphatic glands a more drastic initial treatment may be followed.

The indications and contraindications are the same as for salvarsan, but as a rule neosalvarsan is better borne.

Administration.-Neosalvarsan is almost universally administered by intravenous injection. It has also been employed occasionally by subcutaneous and intramuscular injection.

Apparatus, Etc., Required.-(I) Suitable glass reservoirs, attached to a hollow needle by 3 or 4 feet of rubber tubing. (2) Graduated glass measure (i 20 c.c. capacity). (3) Glass funnel. (4) Alcohol lamp or Bunsen burner (to sterilize file and heat rod to break ampule). (5) Bandage or other material to make tourniquet. (6) Flask of sterile salt solution (250 c.c.). (7) Alcohol. (8) Tr. iodine. (9) Sterile cotton or gauze sponges. (ro) Collodion.

All of the apparatus may be sterilized by boiling, preferably, or by 95 per cent. alcohol and subsequently rinsed with sterile water.

Preparation of the Patient.-Inasmuch as neosalvarsan temporarily interferes with the digestive function, and may cause nausea and vomiting, or diarrhea; or both, the patient should be instructed to take a laxative the day before the administration, to eat lightly afterward and to take no food for four to six hours prior to the time of the treatment.

After-care of the Patient.-After the administration it is well for the patient to remain recumbent for a few hours, when, if no untoward symptoms have developed, he may go about, avoiding, however, any severe exertion or fatigue. The food should be restricted for the balance of the day, and, perhaps, even on the following day. If the proper precautions have been observed, there is usually very little reaction, and it is only necessary to restrict the patient to moderation in activities and in diet. With scrupulous asepsis and the proper preparation of the patient, the treatment may safely be given in the office. In the absence of the proper facilities, however, the patient should be sent to a well-equipped hospital.

Preparation of the Solution.-The ampoule should be carefully inspected to see that it has not been damaged, as it would be unsafe to use the contents if cracked or broken.

The ampoule having been sterilized by immersing in aıcohol, or by thoroughly rubbing the surface with gauze or cotton wet in alcohol, is opened by scratching the neck with a file that has been sterilized, and applying the end of a small glass rod that has been heated to redness in a flame. This usually results in a straight crack around the neck. Occasionally, on account of a warm temperature or the heat of the hands, the neck may fly off with considerable force.

It was originally advised to dissolve the neosalvarsan in sterile freshly distilled water at room temperature, but sterile 0.4 per cent. saline solution is now preferred. The saline solution should be recently prepared from chemically pure sodium chlorid and freshly VoL. IV. -19 
distilled water. The temperature of the salt solution at the time the neosalvarsan is added should not be above that of the room, i.e., $68^{\circ}$ to $7 \mathrm{I}^{\circ} \mathrm{F}$. Heating of the neosalvarsan solution will result in its decomposition. About 25 c.c. of the salt solution is advised for each $0.15 \mathrm{gm}$. neosalvarsan. The apparatus having been sterilized by boiling, or by 95 per cent. alcohol, should be assembled and a quantity of freshly distilled, sterile water run through, to rinse the tubing of either sediment or alcohol.

The proper quantity (25 c.c. for each 0.15 gm. neosalvarsan) of distilled water or of 0.4 per cent. saline solution, prepared as directed, is placed in a sterile measure, and the neosalvarsan dusted slowly on the surface of the fluid. Added in this way solution occurs immediately. (If poured too rapidly the neosalvarsan falls to the bottom of the vessel in masses which dissolve slowly. It is desirable to avoid stirring or agitating the mixture as this promotes oxidation.) The fluid should at once be gently poured into the appropriate glass reservoir, through a funnel containing a little sterile cotton, which has first had sterile water run through. Particular care must be taken to expel all air bubbles from the entire length of tubing.

(b) Introduction into the Vein.-The intravenous administration of neosalvarsan must be carried out with the most thorough asepsis at every step. Various forms of apparatus have been recommended, but the most practical, in the opinion of the writers, is a simple gravity apparatus, the best form of which is illustrated in Fig. 46. It consists of two graduated cylindrical glass reservoirs (for neosalvarsan the capacity of each should be at least i2 2 c.c.; for salvarsan 300 c.c.). The reservoirs are supported on a suitable stand, and are connected by about 4 feet of rubber tubing with a hollow needle of about 20 gauge, attached to a two-way stop cock. Short sections of glass tubing should be inserted in the rubber tubing near the stop cock, to act as windows. Normal salt solution, made from chemically pure sodium chlorid, and sterile freshly distilled water, is placed in one reservoir, and the solution of neosalvarsan (or salvarsan) in the other. Before beginning the administration, be sure that all air bubbles are expelled from both rubber tubes.

The patient should be in the semirecumbent position with one arm fully exposed. A tourniquet is placed about the arm close to the axilla and made sufficiently tight to obstruct the venous return, but not firm enough to occlude the artery. While the distention of the veins is taking place, the operator should thoroughly cleanse the surface of the forearm and arm with alcohol or tincture of iodine. If the veins do not stand out with sufficient prominence the patient is told to clench and unclench the hand a few times. The most prominent vein in the neighborhood of the bend of the elbow being selected, the needle is gently inserted into its lumen, the tourniquet released, and the stop cock turned to permit the salt solution to flow. If the needle is in the vein it will be observed that the level of the fluid in the reservoir is steadily falling and there will be no swelling about 
the needle; if the needle is not in the vein, the fluid will not flow freely and a localized subcutaneous swelling will occur around the point of the needle, due to the extravasation of the fluid in the cellular tissue. The flow must be at once stopped, and the needle introduced at another point, having first rendered the veins prominent by tightening the tourniquet as before. If any unusual difficulty is experienced, the needle may be disconnected from the tube, when the entrance of the point in the lumen of the vein will be promptly announced by the flow of blood. The needle must then be kept steadily in position while the tubing is attached and the fluid allowed to flow into the vein. As soon as the fluid is found to be flowing freely without any signs of swelling in the neighborhood of the needle, the stop cock is turned to allow the neosalvarsan solution to flow. It is most desirable to ascertain whether the point of the needle has properly entered the vein with salt solution in this manner, as the neosalvarsan is exceedingly irritating to the tissues, causing great pain and an extensive phlegmon that is absorbed very slowly. After all the neosalvarsan solution has passed, the stop cock is again turned to permit a few cubic centimeters of salt solution to flow in. In this way all traces of the drug are removed from the needle and vein, and irritation of the tissues avoided. When the needle is withdrawn pressure should be made over the puncture until bleeding ceases, when the point may be covered by collodion. Salvarsan is even more irritating to the tissues than neosalvarsan.

Very close attention should be given to the administration throughout. It may be observed, for instance, that the solution is not flowing; this may be due to stoppage of the needle by blood clot, by particles of solid matter in the solution, or by the point of the needle impinging against the wall of the vein. The occurrence of a blood clot in the needle may be prevented if the flow of the solution is not allowed to become interrupted. The occlusion of the needle by solid particles may be prevented by straining the solution; and if it is a matter of contact of the point with the wall of the vein, a little readjustment of the needle will start the flow again. It may also be observed that a swelling is occurring around the point of the needle and the patient may complain of a burning pain, this would indicate that the needle point has been dislodged permitting the fluid to escape outside of the vein. The flow must be at once stopped, the needle withdrawn and a new insertion made at another point. To avoid this complication, the needle should be steadied with the greatest care during the entire administration.

The administration may be made, but less satisfactorily, by means of a single glass reservoir attached to 3 or 4 feet of rubber tubing carrying a hollow needle at the other end (Fig. 46). Some form of clip or stop cock must be applied to the tubing to control the flow, and a short section of glass tubing should be inserted within a few inches of the needle to permit constant observation of the fluid at that point. 
The neosalvarsan solution having been prepared, normal salt solution is placed in the reservoir, all air bubbles expelled from the tubing, the needle inserted in the vein as before described (page 290) and the clip released. When the salt solution is found to be flowing into the vein properly, the portion remaining in the reservoir is quickly poured out and the neosalvarsan introduced. This change should be made quickly, dextrously, without interrupting the flow through the needle and without permitting air to enter the tubing.

After-effects.-The reaction to neosalvarsan is usually much less than to salvarsan. Patients, as a rule, state that the taste of the drug is perceptible during its administration. There may be a rise of temperature within an hour or two. Generally this is moderate, but marked pyrexia, and chill may occur. The fever passes off in a few hours. Nausea and vomiting are very rarely observed, if the patient's stomach was empty at the time of the treatment, but diarrhea lasting a few hours is not uncommon.

The chill and fever are probably due to some fault in the technique, and should not occur if $(a)$ absolute asepsis is obtained at eyery step; $(b)$ if the water employed is freshly distilled and sterile; and $(c)$ if the sodium chlorid is chemically pure, and the solution is sterile and made with freshly distilled water.

The preparation of salvarsan for administration is so much more complicated that there is correspondingly greater opportunity for a break in the aseptic chain. This may explain the more marked reaction after salvarsan in many cases.

The beneficial results observed after neosalvarsan correspond with those noted under salvarsan.

Neosalvarsan has been administered in concentrated solutions by Ravaut, Duhot, Emery, Strauss, Stern and others: for example, 0.45 to $0.6 \mathrm{gm}$. of neosalvarsan dissolved in ro c.c. of sterile distilled water; and 0.75 to 0.9 in I5 c.c. of sterile water. It is claimed by thus reducing the quantity of fluid, the so-called water faults, are eliminated. Whether this practice will prevail or not must be decided by future experience. It may be stated, however, that Ehrlich never advised the use of such concentrated solutions.

Subcutaneous and Intramuscular Injections.-For this form of administration about 3 c.c. of freshly distilled water should be used for each 0.I $5 \mathrm{gm}$. of neosalvarsan. On account of the pain, soreness, induration and possible abscess, after these injections, they have not been extensively used. Suspension in a neutral fat has been found in practice to be much better tolerated, and is to be preferred if this form of administration seems desirable.

\section{MERCURY}

Notwithstanding the great enthusiasm, which has followed the introduction of salvarsan and neosalvarsan and the undoubted value of these remedies, it does not seem probable, at this time, that the 
use of mercury can be safely dispensed with. Unquestionably the best average results are obtained by using a combination of one of the above compounds with the mercury the latter also being employed in full doses in the earliest stages of the disease. It will be understood, of course, that the possible toxic effects of mercury will be constantly looked for, and the drug stopped immediately upon the appearance of the first signs of hydrargyrism (Vol. II, page 479)

The application of mercurial ointment by inunction is a very effective means of introducing the drug into the system, and has the merit of sparing the alimentary tract. It is not suitable for general use, however, as many patients object to the attendant inconveniences.

During the past few years the hypodermatic method of administration has grown rapidly in favor and at present there are on the market a number of preparations which are suitable for use in this way. While most of the forms of mercury cause some local pain, if the details of asepsis are carefully observed, abscesses are practically unknown.

The pain may be largely or entirely overcome by first injecting a small quantity of 0.5 per cent. novocain solution. (A number of formulæ will be found in Vol. II, pages $477-478$, but it is more convenient and safer to use the preparations put up in ampoules by manufacturing chemists).

As the hypodermatic use of mercury has increased in favor, the administration by mouth has become less popular. The writers are convinced, however, that the mouth administration is being undeservedly neglected. There are certain patients who decline to submit either to the mercurial inunctions or to the hypodermatic injections, and there are many cases in which for one reason or another the administration by the mouth is desirable. For these reasons, the method should not be given up. It is undoubtedly true, however, that this form of medication requires more careful oversight than either of the other two mentioned. The particular form of the drug and the dose must be carefully suited to the individual. It is also proper to state that a certain number of patients are unable, on account of disturbances to the alimentary tract, to take any form of mercury by the mouth in sufficient dose to be effective; in these cases resort must be had to one of the other methods. 


\section{VACCINIA}

\section{By Jay Frank Schamberg, M. D.}

Bacteria-free Virus.-The method of propagating vaccine virus at the present day is by transmitting it from calf to calf by cutaneous inoculation. Various bacteria from the skin are commonly present in the virus but these are in large part destroyed or attenuated by the germicidal activity of the glycerin which is added to the virus. When the virus is fresh or "green" the number of bacteria is considerable, but after it has been kept at a very low temperature in contact with the glycerin for one to three months, the virus is practically freed of extraneous organisms and is ready for use.

Vaccine establishments, licensed by the U. S. Public Health Service, are obliged to carry out various animal tests before the virus is placed upon the market. Organisms cultivated from the virus are injected, usually intravenously into animals, to determine their non-pathogenicity, before the virus is permitted to be distributed for use.

While glycerin has a much more feeble effect upon the organism which produces vaccinia than upon the extraneous bacteria present, there is reason to believe that in the course of time it tends to attenuate the activity of the virus. The ideal virus would be one in which extraneous organisms had never gained entrance.

Efforts have been made, from time to time, to produce a bacteriafree vaccine. The goal has recently been achieved by Noguchi who accomplished the result by inoculating vaccine lymph into the testes of rabbits and bulls.

Hansel and Convent, Paschen, Tyzzer, and von Prowazek and Miyaji had previously injected vaccine virus into the testes of animals with various objects in view, but it remained for Noguchi to produce a bacteria-free vaccine virus and test out its purity and clinical results on animals and on the human species. By exerting great care in the cutaneous vaccination of a rabbit and by shaking the virus subsequently with ether, Noguchi obtained a virus free of sporebearing bacteria. This he inoculated under aseptic conditions into the testes of rabbits and bulls. Sixty transfers from one rabbit to another were made within one year. During the first transfer the virus is perhaps weaker than the original skin virus, but the potency increases as the transfers are continued. The multiplication of the virus within the testicle reaches its highest point about the fourth or fifth day after inoculation. The quantity of the virus then remains stationary until the eighth day, and then diminishes so that at the end of five weeks no more virus can be detected; the testicle is excised 
at the end of four days and the testicular pulp is mixed with sterile 50 per cent. glycerin.

The vaccinations in man have given rise to typical vesicles, the process reaching its maximum development on the seventh or eighth day. The lesions were unusually free of undue inflammation and there was neither pain nor itching. It has not yet been demonstrated that this method of propagating vaccine material can be used on a large scale for the commercial production of vaccine virus.

If a bacteria-free vaccine can be commercially produced by this or any other method, it will constitute a revolutionary advance in vaccine propagation. 


\title{
CEREBROSPINAL FEVER, CHOLERA ASIATICA, PLAGUE
}

\author{
By Lewellys F. Barker, M. D.
}

Since the appearance of the three articles on the treatment of the above diseases in the last edition of this book, there have been no essential changes in the treatment of any of these disorders. I will, therefore, simply mention briefly such points as I think need particular accentuation in the treatment of the several diseases.

\section{MENINGITIS}

Specific therapy in the form of Flexner's serum, or antimeningitis serum, is of course essential in the proper treatment of this disease. I wish to lay particular stress upon the necessity of making an immediate and rapid diagnosis, for the statistics that have appeared in the last few years definitely show that in order successfully to combat meningitis it is necessary to inject the specific serum at the earliest possible moment. I would, therefore, strongly urge the necessity of a prompt diagnosis, and as soon as a suspicion arises of the existence of meningitis, lumbar puncture should be performed. This operation is one requiring a certain amount of skill, and every physician should possess it. A small quantity of the cerebrospinal fluid is withdrawn and if it is turbid the antimeningitis serum should be injected without waiting to study culturally or morphologically the organisms that are in the fluid. The amount of serum that is withdrawn should be about 20 to 40 c.c. depending upon the age and size of the patient. The amount of injection should be approximately 5 c.c. less than the amount that has been withdrawn. The serum should be injected rapidly according to the results of the studies from the Research Laboratory of the Department of Health in New York. In mild cases serum may be injected daily for four days; in severe cases it may be introduced twice during every twenty-four hours until there is improvement. The subsequent administrations should depend very largely upon the reaction of the patient to the disease, and upon the results of bacteriological examination of the spinal fluid.

Though there has been no essential change in the general management of the disease, nor in the prophylaxis, it is well to keep in mind the fact that, though many people may be truly meningococcus carriers, apparently it is only the occasional carrier that may throw off germs that are capable of causing infections in other people. Under all circumstances, however, if a carrier be found, it is advisable to attempt to free him from the organisms. Dubois and Neal assert that they have had excellent results from 
swabbing out the nose and throat of the carrier with a weak solution of argyrol.

\section{CHOLERA ASIATICA}

Prophylaxis.-In recent years, much more than formerly, attention has been paid to the subject of bacillus carriers. During the epidemic at Manila in 1908 an examination of the stools in apparently healthy persons was done as a routine, and all those who were in contact with cholera cases, or who were in the neighborhood of epidemic regions, were thus studied. It was found that about 7 per cent. of those examined were healthy carriers. The average length of time that vibrios are carried is probably about twenty days, though cases have been reported in which the vibrios have been isolated from the stools as long as six months after their original discovery. Furthermore, cases have been reported in which the vibrios are found only occasionally on bacteriological examination of the dejecta. In such instances it is to be presumed that the vibrios are harbored as are typhoid bacilli, in the bile passages and gall-bladder. Although the bacillus carrier is rather a rarity he nevertheless undoubtedly occurs, and therefore, in order thoroughly to carry out proper quarantine regulations, it is necessary to have a stool examination made before discharging the person who has been sick with cholera from quarantine.

In rgrr at the instigation of Surgeon Allen J. McLaughlin, the Secretary of the Treasury issued an order that the feces of all those coming to U.S. ports from cholera districts should be examined bacteriologically, and that quarantine should be continued until stool examination showed negative results. This order represents a real advance in quarantine methods as applied to cholera, for it not only shortens the quarantine detention period by several days but also furnished an almost absolutely safe method of preventing the entrance of vibrio carriers into this country. In making the stool examination as carried out by the U.S. Public Health Service a rather simple technique is employed. The fecal material is planted in testtubes containing a peptone solution devised by Goldberger in place of the ordinary peptone solution, and is then placed directly on ordinary 3 per cent. neutral agar plates. The peptone-enriching solution of Goldberger is an alkaline egg-peptone and alkaline-meat-infusionpeptone solution. These solutions are employed because in them the vibrios are not overgrown by other bacteria of the intestinal flora. The fecal material is grown in the solution for three, six and twelve hours, at the end of which time agar plates are streaked with this material. For rapid diagnosis, a suspicious vibrio colony is tested on a glass slide with drops of $\mathrm{I}: 200$ immune cholera serum. A positive reaction is subsequently confirmed by agglutination tests to determine to the limits of agglutinability (agglutination titer).

Treatment.-In the previous edition, mention was made of the various methods of introducing fluid into the body to replace that 
lost by the severe vomiting and diarrhea. Since the original article was published, Sir. L. Rogers has advocated a treatment that seems to have had a very definite effect in reducing the mortality of the disease. In order to combat collapse, Rogers recommends the intravenous injection of hypertonic saline solution. The solution that is employed contains I 20 grains (4 gm.) of sodium chlorid, 6 grains $(0.4 \mathrm{gm}$.) of potassium chlorid and 4 grains $(0.25 \mathrm{gm}$.) of calcium chlorid in a pint (500 c.c.) of sterile water. In adults, the initial dose was usually about 3 pints of this solution, or in bad cases of collapse, even 5 or 6 pints were employed.

The original injection is repeated only if collapse occurs or is threatened. The indications of threatened collapse are cyanosis, restlessness, an extremely low-tension pulse, abdominal cramps, suppression of urine and severe vomiting with diarrhea. It has been recommended, in addition to the hypertonic salt solution, to attempt to maintain the effect of a preliminary dose by injection into the rectum of ordinary saline solution every hour in small quantities. The results of Rogers' method of treating cholera have been brilliant, and represent a marked advance in the treatment of this formerly extremely lethal disease. For example, Sympton reports a series of sixty-nine cases that he has treated with a mortality of 21.7 per cent. as compared with a mortality of 76.1 per cent. for the epidemic as a whole. Of the drugs that have proved useful in aiding to combat collapse, pituitrin and epinephrin are probably the best.

\section{PLAGUE}

In the treatment of plague there is little to be added to what has already been stated in the previous article. No specific treatment that has had sufficient trial to show its effect has as yet been devised. Vaccine therapy has been employed with results that are at least hopeful. At some future time, this form of therapy may be made of some avail.

In regard to the prophylaxis of this disease, as I pointed out in my previous article, the prime necessity is to destroy the rodents that are apparently indirectly the transmitters of the disease. The disease is acquired by the rat through the bite of fleas, Xenopsylla cheopsis and Ceratophyllus fasciatus. The logical methods, therefore, of combating the disease are first, the destruction and extermination of the rats; and secondly, the rat-proofing of houses in localities in which the disease is endemic. Rat-catching is an extremely difficult and expensive procedure. It is much more logical to rat-poof buildings properly and prevent the rats from coming into houses and wharves where they may grow unrestrictedly, than to go to the expense of attempting to catch them one at a time after they have had the opportunity of breeding and growing. 


\section{YELLOW FEVER}

\section{By William C. Gorgas, M. D}

Sir Patrick Manson, than whom no medical author is better able to describe in a few terse sentences the essential features of a disease, defines yellow fever as follows: "An acute, specific, very fatal febrile disease occurring epidemically, or as an endemic, within a peculiarly limited geographical area. Though subject to great variations, its typical clinical manifestations may be said to be characterized by a definite course consisting of an initial stage of a sthenic nature, rapidly followed by an adynamic condition in which such evidences of blood destruction as black vomit, albuminuria, and hematogenous jaundice are liable to occur. One attack confers permanent immunity. The germ is transmitted by the domestic mosquito, Stegomyia calopus (S. fasciata)."

Yellow fever was, as we know, the much feared "vomito" of the West Indies; the dreaded "yellow jack" of the English speaking sailor. I believe that clear ideas as to our present knowledge of the prophylaxis and treatment of yellow fever can best be conveyed by a brief preliminary outline of the history and the occurrence of the disease, including its symptoms and diagnosis.

Until quite recently, the West Indies were known as the endemic home of yellow fever. From time to time, it would spread from thence to the contiguous continents, north of the Gulf Coast of the United States, west to Mexico and south to the northern parts of South America, to Rio de Janeiro, and even as far to the south sometimes as Buenos Ayres; also the west across the Isthmus of Panama to Ecuador and Peru. It was endemic also in Africa, but only on the west coast, and especially in Sierre Leone.

Yellow fever was first recognized in the West Indies about the middle of the $I 7$ th century. It probably existed in Mexico when America was discovered. It came to Havana in the year I76I, and never entirely disappeared from that city until I9OIjust 140 years later. The circumstances attending this disappearance we will consider at some length a little later.

It has invaded the territory of the United States, most often entering at New Orleans and affecting the Gulf States to a greater or less extent at different times. The epidemic of 1853 was perhaps the most notable of all. The latter reached Memphis, where there was an appalling mortality from the disease.

There was a large epidemic in the City of Philadelphia in the year 1793. Once it got to Boston, and even as far north as Quebec. It has been mainly a disease of harbors and coasts for reasons which will appear later. A few times it has extended as far as Europe, 
principally to the shores of Spain, or rarely to Italy. Seaports in France and England have sometimes been infested, but never seriously.

On our side of the Atlantic, yellow fever is still present in Brazil and Ecuador. The port of Guayaquil, in Ecuador, has been for some years a menace to our people on the Isthmus of Panama, and has had to be watched with great care on account of the yellow fever and plague always present there. Apparently epidemics require a temperature of about $75^{\circ} \mathrm{F}$. or a mean winter temperature of $65^{\circ} \mathrm{F}$. The mosquito appears not to bite at temperatures below $62^{\circ} \mathrm{F}$.

Yellow fever has in the past caused great losses among the Spanish and other troops in the West Indies, also formerly among the French in Mexico: the French in the Island of Haiti were once practically annihilated. There were over rooo cases among the United States troops during the Civil war. After that time, it did not seriously affect the army except at, a few small border posts. For a period of ten years before the Spanish war, there were no cases at all, except a few in Florida. When we invaded Cuba, however, it was a different story. Among our troops at Santiago there occurred about 600 cases and seventy deaths. Quite a number of cases occurred among Americans in Havana before preventive work there had had time to give results. But Igor saw the last of it, except a little, as we shall see later.

It would be difficult to realize, unless one had lived in the extreme southern United States, some twenty years ago, what a terrible and calamitous thing this yellow fever was to us. Hardly a family there that had not, at one time or another, perhaps often, paid tribute to it in treasure and in lives. Because it had such awful results on a community, both in the ruin of commerce and in the filling of the cemeteries, but more likely, perhaps, because of its mysterious nature and its unexplained mode of spreading, it was wont to cause great panic among the people. Shotgun quarantines, for the most part useless as we now know, were often maintained against one's neighbors. Valuable property was destroyed because we thought that fomites carried the infection. Great and unnecessary discomfort and trouble were caused the traveling public for months at a time.

But now all this is changed. We no longer fear the introduction of the once so dreaded disease, nor, even when introduced, do we longer anticipate its spread-and why not? This greatly to be desired result was attained mainly by the discovery of the method by which yellow fever is spread, and we of the Medical Corps of the army take great pride in the fact-which is also now generally well recognized-that the main part in this brilliant work of discovery was undertaken by our own men, and principally by the immortal Walter Reed. I think I may venture to digress a little here, and give a brief account of how these things came about, endeavoring. 
to cover the main points in the history of the search for the knowledge of the method by which yellow fever is spread.

The first thing of real interest or importance that happened in this connection, the first theory more particular than vague and indefinite statements about fomites, and bad air, and miasms, and so forth, was the announcement made by Dr. Carlos Finlay, in a communication to the International Sanitary Conference at Washington in $188 \mathrm{I}$, that he had formed the opinion, after mature thought and study, that the infection of yellow fever was conveyed by an independent agent from the sick to the well. Later, in the same year, he announced to the Havana Academy of Sciences that this agent was the mosquito. He guessed right, indeed, and most remarkable to say, he even picked out the right mosquito, and stated that the fever was carried from patient to patient by that one kind and by no other species. Finlay's statement was received with great incredulity, polite and otherwise, by physicians and scientists. Another interesting thing about Finlay, he did not allow himself to be coaxed or laughed out of his unorthodox theory; he stuck to it and stoutly maintained his thesis against all opponents up to the very time that the proof of its correctness was forthcoming. He carried out practical experiments for many years in the endeavor to prove to the skeptics that he really was right in his conclusions. That he made some mistakes in these experiments, that he sponsored an incorrect organism, indeed that he was not a trained laboratory investigator, should not be held against him. In fact, I sometimes think we do not give him enough credit; not that we are at all inclined to detract from his work in any way, but we are so much dazzled by the light cast by our own so brilliant luminaries, that we perhaps may not see the true effulgence of the lesser ones. Finlay's work, indeed, strikes one as one of the most remarkable instances of deductive reasoning of which we have any account in medicine.

In the meantime, various scientists and practical men were hard at work on the question and from time to time announced a solution. Besides Finlay's find, Freire of Brazil brought forward a coccus, and so on.

Later, when the bacteriologist Sanarelli introduced his famous bacillus, almost everyone thought, for a time, that the problem had been solved at last. In fact, I believe that Sanarelli was awarded a very valuable prize as the discoverer of the organism which had so long been unsuccessfully sought for. Sternberg, however, our former Surgeon General, and the foremost bacteriologist of his day in America, had long been studying this same question, even having spent much time on the ground in Cuba. He always doubted the correctness of Sanarelli's conclusions and the Reed Board later proved without much difficulty that the Sanarelli bacillus was not causative of yellow fever and was probably a variety of the hog cholera bacillus. The same line of work practically threw out Sternberg's own bacillus 
"X," which he had found in yellow fever cadavers and had thought might possibly stand in a causal relation to the disease.

About this time another Richmond appeared in the field, this time, indeed, a practical physician, Dr. Henry R. Carter of the Public Health Service, who had had a great and varied experience in the diagnosis and treatment of yellow fever, and the handling of yellow fever epidemics all over the South. He made the second great step forward in our knowledge of its etiology. He noticed that, after the introduction of a case of yellow fever into uninfected territory, there always elapsed a period of about fifteen or twenty days before other cases (usually a goodly number) would occur. The idea suggested itself to him that some kind of development must here be taking place, some kind of incubation, as it were, of the causative organism outside of the original patient, before it had the power to reproduce the disease in others. It was by this time generally conceded that there must be some kind of living organism at work, though it had not been found. In furtherance of this idea, he called this stage the period of "extrinsic incubation" of yellow fever and announced the discovery in a paper in the New Orleans Medical Journal of May, I900. This was also a remarkable piece of observation and reasoning, and entitles Dr. Carter to a prominent place in our galaxy of scientific heroes.

The next step, and it was the beginning of the end of this baffling mystery, was the appearance of the Reed Board at Havana to make an extensive study, on that favorable spot, of all the etiological theories advanced, with the strong hope that something more definite might be found and the problem perhaps somewhat advanced on the road to its final solution. This board consisted of Reed, Carroll and Lazear (later Agramonte was added). After about six months, spent in the various preliminary investigations which resulted in the elimination from the problems of Sanarelli's and other bacilli, Reed became rather favorably impressed with the idea of Finlay, so much so, at any rate, that it decided him to look into the theory of insect convection. He then devised the now famous set of experiments which so much redounded to his own fame and that of our corps, and which conferred such an inestimable boon on humanity for all time to come. His procedure in brief was as follows: A camp was established in November, I900, in the open country, a few miles from the city of Havana, and a mile from other habitations of man, for the purpose of the experiments. Reed had previously, however, as the result of the observation of certain cases, especially of one where all other measures of infection could be positively excluded, announced his belief that the mosquito served as the intermediate host for the parasite of yellow fever.

The camp was strictly quarantined and no one permitted to leave it, except the few immunes attached to it. These were volunteer hospital corps men, civilians and newly landed Spanish immigrants. Temperatures of the non-immunes to be experimented upon were 
taken, and recorded, until the full time of incubation for yellow fever had passed, with the design, of course, of eliminating the possible introduction of the yellow fever in a manner foreign to the purpose of the experiment.

At the end of this period, the volunteer non-immunes were allowed to be bitten by Stegomyia mosquitoes which had, at various periods of time, previously bitten typical cases of mild or severe yellow fever, these cases being at various stages in the progress of the disease.

We have not the space, and indeed it is not necessary, to go into minute details of these cases. Suffice it to say that the results were positive and the non-immunes acquired typical cases of the fever in the judgment of an experienced board of diagnosticians, including the celebrated Dr. Juan Guiteras, who had an unusually great experience in the practical handling of yellow fever. Other non-immunes were infected by the injection of the blood taken from cases of yellow fever.

A second series of experiments was undertaken for the purpose of determining whether the disease could be propagated in any other way than by the mosquito, e.g., by fomites or infected baggage, bedding and clothing. For this purpose a securely screened, small frame house was constructed at the camp. Non-immunes were allowed to sleep in this house together with bedding, etc., contaminated by contact with cases of yellow fever and by the discharges from the same, and brought in boxes. In fact, their beds were made up with those articles. The articles were shaken about, packed and unpacked each day by the non-immunes. After a period of about three weeks, during which the conditions of the experiment had been kept up, there had resulted no cases of the fever. Some of these men were subsequently infected by the mosquitoes or blood. In all, there were twenty-two cases of experimental transference, fourteen by bites and six by injection of blood, and two by blood serum (previously passed through a Berkefeld filter).

To make a long story short, it was realized by everyone who had followed the experiments, that the dark places had been made light, that the long-studied problem had been solved at last, and that, in fine, the matter of transference of yellow fever from one patient to another was a mystery no longer.

The definite conclusions finally arrived at by the Board were as follows:

I. The mosquito Stegomyia calopus serves as the intermediate host for yellow fever.

2. Yellow fever is conveyed to the non-immune individual by the bite of a mosquito that has fed at least twelve days previously on the blood of the sick with this disease during the first two or three days of its inception. Biting before that time, it does not convey the disease (Finlay had thought that it did). 
The disease can also be experimentally conveyed by the inoculation of blood taken from a patient during the first two days of the disease.

\section{A previous attack conveys immunity.}

4. The period of incubation is from a little less than two to a little more than five days.

5. Yellow fever is not conveyed by fomites, and hence disinfection of the same is not necessary in this disease. (Note that many thousands of dollars had been formerly spent in such disinfection, aside from the infinite time and trouble expended).

6 . The specific cause had not been discovered.

These results have since been confirmed by other observers.

It should be remembered that during the course of the work at Havana, Dr. Lazear, one of the members of the Reed Board, contracted a fatal case of the fever, thus becoming a true martyr to science.

The next dramatic step in the history was the application of Reed's results to practice, the freeing of Havana and of Cuba from the horrid pest from which they had never once been free for generations past. I had the great honor and pleasure of directing, personally this part of the work, and in this I had the hearty coöperation and support of the Governor General, Leonard Wood, and the Chief Surgeon of the Department, Colonel Valéry Havard, the latter also a member and General Wood a former member of our corps.

There were many discouragements at the start; there always are in these campaigns, because it takes a long time to begin to get evident and spectacular results. However, the theory was right, the application of it scientific and thorough, and the results were bound to come. They did in due time, and the world waked up to the fact that science had achieved another victory and conquered another plague. A big epidemic in New Orleans in 1905 was brilliantly handled along the same lines by White of the Public Health Service. We must not forget that Kean of the medical corps had great success in stamping out a recurrence of yellow fever in Cuba in I908, due to a relapsing of the methods we had instituted there.

Porter, of Florida, for many years its Health Officer and a retired officer of the medical corps, has done great work in that State. Perry of the Public Health Service had kept the South American fever out of Panama by his wonderfully efficient quarantine.

Ross, of the navy, had large experience and great reputation in the early days. Nearly all of these men we are proud to claim as members of our corps, or of the sister medical services.

So when one thinks of yellow fever, or of modern preventive medicine in general, he should not fail to remember Finlay and Guiteras and Agramonte of Cuba, Ross of the navy, Carter, White and Perry of the Public Health Service, Lazear and Carroll and, above all, Walter Reed of the army. These are the big outstanding 
names in yellow fever, and yellow fever the big example of the results of science applied to the investigation and management of infectious diseases. Palmam qui meruit ferat. Their reputation and history should serve, in some future investigation, as inspiration for similiar work elsewhere.

Since the Cuban work, various organisms have been brought forward as the causative one; most have been definitely disproved. The last one is that of Seidelin: extremely minute bodies in the red cells which he thinks consist of chromatin granules with cytoplasm; he calls it Paraplasma flavigenum, a protozoön. Inoculation and mosquito experiments with it are said to have failed, as also complement-deviation tests, so that this organism will probably be thrown out also. However, let us not be too dogmatic, when we think of Finlay and his mosquitoes.

Experimentation since the time of the army board's work in Cuba by Reed and Carroll, as well as by other observers, has given us further information as to the poison, although we do not yet know its nature.

It was found that the virus in the blood is destroyed by a temperature of $55^{\circ} \mathrm{C}$. for ten minutes. It passes through the Berkefeld filter and also through the Chamberland " $F$," but is arrested by the Chamberland "B." The virus does not infect if laid on a raw surface, and the infectivity of the blood lasts two to five days, reaching the latter limit if protected with vaseline. The disease is therefore caused by a filterable virus or an ultramicroscopic organism, or by an organism which, like the spirochete of syphilis, is invisible under ordinary staining methods.

The French Commission, working in Brazil, claimed that a certain degree of immunity was conferred by the injection of infected serum heated to $55^{\circ} \mathrm{C}$. for five minutes, and that the serum of convalescents has certain degree of prophylactic and curative properties. This matter is, however, still sub judice.

Pathology.-There is usually pronounced rigor mortis, which sets in early. The skin is of a decided saffron color, especially in dependent parts.

There may be petechix in the skin and extravasation of blood in the muscles.

The internal tissues and fluids may be tinged yellow. The blood does not coagulate readily. The changes in the stomach and small intestines and in the liver are the most characteristic. The stomach always contains more or less black acid material of a thin or tar-like consistency, and usually the same material in the small intestine. The mucous membrane of these organs shows patches of arborescent congestion and often swelling or softening of the mucous membrane.

The condition of the liver is almost pathognomonic. It is not much enlarged, but of ten friable and of a mottled yellow color that has been described as café au lait, or like boxwood. These Vor. IV. -20 
colors may be uniform or in patches, alternating with others of a brownish color. Petechial hemorrhages in the liver are not uncommon, as also in the kidney and brain.

The kidneys are sometimes congested, sometimes pale, usually showing the condition of parenchymatous degeneration.

The spleen is not characteristically changed, nor are the heart and lungs. The brain and meninges are frequently hyperemic.

Microscopically the blood shows no constant or characteristic changes. There are profound fatty degeneration and cloudy swelling of the liver cells, the other organs of the body being more or less similarly affected, as a a rule, especially the kidney. The renal tubules generally show infarcts. There is also fatty degeneration of the capillaries and smaller blood-vessels, which probably explains the tendency to hemorrhage in this disease.

Symptoms.-In a few cases, some of the usual prodromata, including vertigo, so common in febrile affections, may occur in yellow fever, but as a rule the attack comes on suddenly, "like lightning," as one author expresses it; perhaps most often during the night. There is a decided rigor, lasting a quarter of an hour or so. The temperature rapidly rises to $102^{\circ}$, $103^{\circ}$, or $104{ }^{\circ} \mathrm{F}$., according to the severity of the attack; sometimes even over $105^{\circ}$. The face is flushed and glossy looking, and perhaps also the upper part of the trunk, of ten amounting to erythema; the conjunctiva are injected, the eyes glistening and there is often photophobia. There is extremely severe and intolerable cephalalgia, the pain being in the frontal portion of the head usually, and behind the eyes, also severe backache, and often pain in the extremities There are nausea and generally vomiting, first of food and then of mucous material, colorless or bile tinged. The pulse is rapid, full and bounding. There is usually at this stage considerable tenderness on pressure over the pit of the stomach. The characteristic yellowness has not yet appeared. The tongue is usually furred and red at the tip and edges, and rather small and pointed. The gums are swollen and spongy; later, perhaps, a red line appears at the margin and blood can be made to ooze out by moderate pressure. The urine is somewhat diminished in quantity, acid, and of high color, and shows no albumin at this time. There is generally considerable nervous restlessness, and great thirst is usually present. There is sometimes epistaxis, or, in women, there may be a bloody flow from the uterus.

This stage in the ordinary case, lasts twenty-four to seventytwo hours. At succeeding visits during this time, it will be noticed that while the temperature rises or remains stationary, there is a gradual drop in the pulse rate. It may have been 120 or 130 at the start; it gradually drops to Iro, roo, 90,80 , sometimes finally down to 50 or 40 , while the temperature is still high. I think it well to make a particular note of this fact. It will be found stated in all the descriptions of the disease, but is perhaps, not sufficiently 
emphasized. The phenomenon was noted by an old French practitioner of New Orleans, many years ago and named after him "Faget's law." If one is not careful, when it comes to prognosis, this slow regular pulse in yellow fever may put him off his guard.

There is found in most of the text books, the classical description of three stages in yellow fever: First, the stage of invasion or of congestion or of initial fever which we have just described; second, the period of calm or of remission; and third, the period of reaction or of secondary fever. Now, while one sometimes sees this succession of events rather plainly marked out in a case of yellow fever, I do not believe it is at all constant or good as a clinical description of the disease. Guiteras has shown, I think, that it is only onc of the phases assumed by the temperature curve and symptoms; he calls it the "remitting type" when there is a fall in temperature on the third, fourth, or fifth day, rising again thereafter.

Perhaps the second rise is due to hemorrhage or secondary infection. It is in these cases, with the appearance of a calm stage, that the patient feels well, wants to get up or to eat, and if allowed to do so, probably dies. In addition, Guiteras describes a "descending type," usually mild, in which the fever gradually drops, with slight oscillations, the first or second day; and a "continued type," the temperature keeping up throughout the disease. This last variety is usually fatal. So far as the temperature curve can be classified, I think these types of Guiteras really describe approximately what we see clinically. In reality there is nothing absolutely diagnostic about the temperature curve. I would note here that, in the mild cases, the temperature quite constantly reaches its highest point on the "second day.

Some describe the invasion, the disease proper, and the termination. This is a much better division, I think. Touatre speaks of the period of congestion and the period of infection or toxemia. Again not bad, if we disregard the inference as to etiology; of course, the whole thing is infection, as we know. Sternberg and others describe yellow fever as a disease of one paroxysm, like most of the other acute infections, as scarlet fever, for instance; and that is perhaps the best way to look at it.

Let us go on, however, with our description of the disease proper, to use that division for purposes of simplification. We have considered the symptoms of congestion that occur during the first two or three days of the fever in the ordinary cases. Then, as a rule, the congestion and the pain diminish, though the capillary circulation remains sluggish, and there is either a gradual return to the normal, in the most favorable cases terminating in recovery, or, in the severe cases, there are signs of blood stasis, with hemorrhages from the mucous membrane, or symptoms of malignant icterus. With this comes the most common termination - death, though some of these cases do recover.

The different courses of the temperature curve we have described. 
The redness of the skin and the injection of the eyes disappear about the second day.

Icterus first appears in the conjunctiva, in severe cases, about the beginning of the third day, reaching the face and finally the skin of the entire body, and appears in a great variety of shades in different cases-lemon to even mahogany, but some shade of yellow in nearly all cases. Touatre describes a dirty yellow color in the conjunctiva due to congestion and often seen in the first day or two. This may be confused with the real jaundice which, as we have seen, comes later.

In general, the later the jaundice appears and the lighter the color, the less serious the disease, but no rules like these are entirely reliable in yellow fever. The unexpected often happens.

The urine becomes albuminous on the second or third day of the disease, from a mere trace to $5-\mathrm{I} 5$ per cent. with perhaps hyaline casts. This may diminish in favorable cases, or, in severe cases, may increase by the third or fourth day to $30-50$ per cent., or more, with many coarse granular and epithelial casts stained dark by bile pigment. Red blood cells are present when there is hemorrhage. In bad cases, the urine also becomes very scanty and may finally be totally suppressed.

About the fourth or fifth day, sometimes earlier in very severe cases, small black specks, like pieces of flies' wings, someone puts it, and which are really particles of blood, begin to appear in the vomitus; later the whole vomit becomes dark or black and looks like coffee grounds, the famous "black vomit" of yellow fever. This is owing to the escape of blood from the congested stomach wall. Sometimes, for the same reason, tarry stools are passed. One other characteristic thing may be noted here, and that is that this material is expelled by the stomach apparently without effort; it simply wells up, as it were, and gushes out over the bedclothes and the floor. This gives one a very dramatic picture, I can assure the reader. Black vomit is noticed mainly on the fourth and fifth days, very rarely on the third, and then only in exceedingly malignant cases. If it appears earlier than this, Touatre says that by careful examination one will usually find it coming from epistaxis, or something of that kind. Fatal cases, and they are nearly always fatal where there is extreme jaundice and scant highly albuminous urine and black vomit, go on to anuria and complete suppression, to delirium, perhaps convulsions, to asthenia and coma, to terminate in death. Persistent hiccough may appear and is' a very bad prognostic sign. The tongue may become fissured and bleeding. Hemorrhage may occur from the other mucous membranes or from the uterus. Petechiæ in the skin may appear. At any period, however, the symptoms may begin to improve, gradually subside and recovery take place.

Toward the end, in fatal cases, it is quite usual for the temperature to drop quickly at the same time that the pulse rate rapidly goes up. A sudden fall of temperature accompanied by a rise in 
pulse rate is of very ominous significance. Death usually occurs between the fifth and ninth days of the disease, though it may take place earlier or later.

In some moderate cases, as observed by Carroll, there is often a striking depression without other bad symptoms, when the temperature has not been high and of only a few days' duration.

There is nothing typical about the blood count in yellow fever; of course, there is secondary anemia in severe cases.

Relapses may occur, especially if any dietetic indiscretions are committed, and are very dangerous.

Yellow fever in children does not differ in symptoms especially from that in adults, except in the one very important point that it is less fatal, in fact rarely so. Touatre says the mortality is less than in measles.

Diagnosis.-I believe I may say that, excepting in the very light cases, which we would often miss in any infectious disease except what the presence of an epidemic and connecting cases help us to decide, the diagnosis of yellow fever is quite easy. As Touatre would say, it actually obtrudes itself by the end of the second day. We do not really need the clinical symptoms of black vomit, extreme jaundice, albuminuria and suppression of urine, indeed, when these have come, there isn't much use as far as our particular case is concerned, in making any diagnosis.

The fall in the pulse rate, of which we have spoken, and the divergence of the pulse and temperature curves occur in the majority of cases. To detect this, frequent observations should be made and careful records kept.

The prevailing symptoms of congestion, the peculiar localization and the great severity of pains, the erythematous blush, the appearance of the eyes, the general facies, the tongue and the gums, which things one soon learns to appreciate, the great tenderness on slight pressure over the pit of the stomach, are quite peculiar also to the disease. Many experts in old times made the diagnosis mainly in this way.

The fact of the presence of an epidemic in the vicinity, the history of exposure to infection in the season of the year corresponding to the height of temperature necessary to the invasion of yellow fever, all this sort of information may help us to decide, especially in the light cases.

In the severest cases there may be albumin even at this early stage and the preliminary hemorrhage of congestion, as opposed to that of degeneration later, may, as we have seen, appear.

In the small epidemic at Fort Barrancas, Florida, in 1897 , we were able to pick out the first cases of yellow fever from a number of malarial cases, although there was supposed not to be another case within a hundred miles or so. The blood was examined, of course, but we did not decide from that, nor from the peculiarities of the temperature curve, as we had not then learned to appreciate 
Faget's law, and not, as I remember, until about the end of the third day. The condition of the urine was the most suspicious feature. Considerable albumin developed and a bunch of coarse black granular casts strewn all over the field and looking like a broken bunch of cigars. I did not recall having observed a similar phenomenon before and could find no other explanation for it than yellow fever. The patient, in two or three days more, had developed black vomit and suppression, and so on, and promptly died.

In malarial remittent fever, we have the microscopic examination of the blood to help us, and also the inapplicability of. Faget's law. In blackwater fever, the difference in the urinary symptoms; and if the color should appear to be similar, the fact that in one case there is hemoglobinuria, in the other hematuria; besides, the history of the case and the difference in the general run of the symptoms will help us to decide.

In blackwater fever, we should expect an increase in, and perhaps a pigmentation of the large mononuclears in the blood film. In Malta fever, we have the agglutination of the Micrococcus melitensis; in relapsing fever, the spirochetes in the blood. If there should be a question of typhoid fever, though this is not likely, there are the blood culture and the Widal test to aid us.

The main thing that confuses us, and perhaps, if I said the only thing, I should not be far wrong, is dengue fever. The territorial range of the two diseases is about the same, at least on the American continent, their seasonal distribution and many similar facts in their epidemiology, and, to superficial observation, the general symptomatology of light cases, may appear confusing. We know, of course, that there is no black vomit, nor icterus, nor anuria in dengue fever and most important point of all, no deaths. Touatre says it causes no tears to flow and that such, alas, is not the case even in the mildest epidemic of yellow fever. So there is no excuse for doubt at all about a severe case of yellow fever. The trouble is with the mild cases. Even here, I think, if we are careful, we do not run much chance of confusing them.

Faget's law does not apply in dengue. While the temperature sometimes runs quite as high as in yellow fever, the pulse in dengue is correspondingly elevated. If there is slowing of the pulse, it is toward the termination of the case or in convalescence and not at the start as in yellow fever. One of the main symptoms is dengue and one of the principle points of differentiation is the presence of a polymorphous eruption, sometimes accompanied by edema. We do not have this in yellow fever, as we have seen it in dengue.

In yellow fever, the severe and distressing pains are chiefly and characteristically frontal headaches and rachialgia, the violent pains in dengue are essentially articular and muscular. The peculiar syndrome of the invasion symptoms, which 1 have described, is not seen in dengue. Dengue generally reaches its highest point of temperature at the outset; yellow fever later. However, this 
rule is not invariable. It is not by any one of these things alone, that one makes the diagnosis, but by considering them all together.

Prognosis.-The mortality of yellow fever is, on an average, say, about 25 to 30 per cent.; in permanent residents perhaps ro per cent., or less, although epidemics vary widely in this regard. The type of the epidemic is probably the main thing in determining the fatality. There is no rule about epidemics except that it has seemed that those in New Orleans when they began early, as in May, June or July, were very severe, when they began late, as in August or September, they were mild. We do not know why. Perhaps the passing of the germs through several hosts increases their virulence. Theoretically, the dose of the poison ought to count,.but this idea is of no use to us practically. Lazear, we know, died from one bite of one mosquito. Perhaps the second most powerful determining factor is the resistance of the individual attacked. Children escape mostly without danger; the aged, the alcoholic, and those with organic visceral lesions, especially of the kidney, almost invariably perish. The aged are said to be less frequently attacked, however. Bad hygiene of any kind naturally tends to influence the course of the disease unfavorably.

Susceptibility and mortality have been said to vary directly with the distance from the equator at which the individual had his nativity. While perhaps too dogmatic, the statement has an element of truth. As to the points in any particular case upon which we would base a prognosis, apart from these already mentioned, we must study carefully the individual symptoms. The most important of these, in the early stages of the disease, is the temperature. When the fever continually defervesces, or when there are morning remissions of at least a degree, not exceeding $104^{\circ} \mathrm{F}$., the exacerbation becoming less pronounced each day, then the patient gets well.

Sternberg puts it that there are few fatalities unless the temperature is over $104^{\circ}$, and none if it is not over $103^{\circ}$. When the temperature is between $104^{\circ}$ and $105^{\circ}$, with a tendency to rise instead of falling, the condition is serious, yet perhaps a majority recover. When the fever reaches $105^{\circ}$ or more at the start, or if it once returns to that point after having dropped, the disease in adults is nearly always fatal.

The quantity of urine secreted is a very important point. Considerable urine is a good sign, even though there may be a good deal of albumin. Scanty urine with much albumin means great danger. Complete anuria, or a solid coagulum of albumin, means death in most cases. In this connection, Touatre refers to an old empirical practitioner who had considerable reputation in New Orleans. On being asked how he made such good predictions as to the outcome of his cases, he replied that he asked how the patient was urinating; if this was well he announced a recovery; if it was said that the patient passed very little urine or none at all, he shrugged his shoulders, which meant there was no hope. 
The third important indication we get from the black vomit. I have never seen but one case recover in which occurred the projectile vomiting of the claret-colored material, which the laity know as "black vomit."

The "flyspecks" are another matter. They are liable to appear in severe cases, and the earlier they are seen, the greater the probability of progression to a fatal end. Coincident intestinal or uterine hemorrhage, or bleeding from other mucous membranes indicates a hopeless state.

Early and intense icterus gives a bad prognosis. In spite of these rules, which are pretty reliable, surprising recoveries sometimes occur. The lesion we should learn from this is never to "give a patient up" before the end. This should, however, be the practice of good physicians, as we know, in any disease.

Treatment.-The main principles of present-day methods of treatment of yellow fever may be summed up in two words "non nocere"-do no harm.

This disease, like typhoid fever, affords one of the best examples of the change of professional opinion from the old-time empirical polypharmacy to the more modern rationalism. While formerly the patient was literally drenched with drugs of all kinds, he now gets little or nothing. We have no specific like quinin in malaria or the arsenic preparations in trypanosomiasis. If, as many of us think, however, the causative organism is a minute protozoon of some kind, it is entirely within the range of possibility that some specific treatment will sooner or later be found, perhaps along the line of Ehrlich's synthetic arsenic compounds, perhaps along some other line. The field is large and the laborers many. Let us fervently hope that one of them will meet with success.

As an example of the older methods, an incident I have related elsewhere, which' occurred when I was visiting the wards of the Havana Hospital, will perhaps be instructive and severe to impress this important point on the memory. I was examining a patient in whose case I had been called in consultation, and noted on the history charts that the patient had been given a dose of one or another kind of medicine about every half hour or oftener during the twenty-four, also the remark at the bottom "dosing all the time." I thought the remark correct enough but wondered why the nurse had felt impelled to comment on it. On inquiry, it transpired that the patient had shown a tendency to sleep and the nurse had intended, but for her imperfect English, to say "dozing all the time." In the small epidemic in 1897 , with which I was associated in Florida, the pendulum had begun to swing in regard to the drugging of yellow fever patients, in fact, drugging was already well on the downward grade. We did not administer very much medicine. We gave a preliminary purge of calomel and soda, usually about 5 grains ( $0.3 \mathrm{gm}$.), of the mercurial salt at one dose. If any assistance to the movements 
of the bowels was considered necessary during the further progress of the case, simple enemeta were used.

Touatre gave 3 grains $(0.2 \mathrm{gm}$.) of calomel; to children I grain (0.065 gm.). Carroll recommended sodium sulphate as an initial purge. Generally, also, Southern physicians used to give at the outset what was then known as the "Creole foot bath." The method of giving this was about as follows: A foot tub was about half filled with quite warm water and a pound of freshly ground mustard, which had previously been dissolved in cold water, was added to it. The patient lying on his back, for even then we had begun to appreciate the advantage of absolute rest, the legs were flexed and placed in the water. Patient and tub were then covered with woolen blankets, and, at short intervals, a small pitcher of hot water poured into the tub. The bath is made about as hot as the patient can stand it, taking care, of course, not to burn him. This course of treatment, amounting in effect to a vapor bath, is continued until its effect is shown by bringing the patient into a copious perspiration. Hot water is sometimes given internally at the same time. When the patient is perspiring freely, the bath is removed and he is kept covered with the blankets for fifteen minutes or so longer, then quickly and thoroughly dried. This treatment usually relieves the headache and pains in the back considerably, even if only temporarily.

Present-day therapeutics do not employ the preliminary calomel and the mustard foot bath as a matter of routine, although I am not at all sure that it is not good treatment in cases seen at the very start. The point is, nowadays, that we think that perhaps greater harm may be done to the patient by the more or less active manipulation and movements than good by the purgation and sweating engendered.

At Fort Barrancas, we kept the patient in bed with as little movement as possible, and gave him a very small amount of strictly liquid diet. In another town, not far away, they allowed ice cream (the simplest vanilla flavored kind, of course), and claimed it did no harm, but on the other hand, gave fine results. The epidemic was mild, however, and I do not think therefore that the post hoc, ergo propter hoc argument can be safely applied in this case. The patient would probably have done about as well on any light diet.

As a routine medical treatment, we gave Sternberg's alkaline mixture. This was an outcome of the attempt on his part to find something specific for the disease and he had used it with apparent good results in Cuba. The formula is as follows:

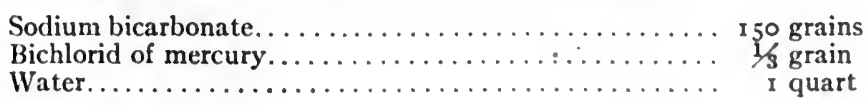

and the method of administration is to give $\mathrm{x} 1 / 2$ ounces every hour.

For the pains, antipyretics are given in moderate doses; for hyperpyrexia, cold water applications or baths. For excessive 
vomiting, sinapisms to the epigastrium, cracked ice and effervescent waters with complete rest to the stomach.

When black specks appeared in the vomitus, tinct. ferri chloridi gtt.v ( 0.3 c.c.) every two or three hours was tried.

For suppression of urine, counterirritation, dry cups to the back over the kidneys, perhaps calomel internally. The patient must be carefully watched all through the case as to the elimination of urine, and the catheter must be freely used. Perhaps what appears to be a suppression of urine may turn out to be only a retention and save us considerable trouble and worry about the case.

Stimulants are given as indicated, especially strychnin and alcohol, the latter being best given in the form of champagne. About the ninth day, a mild solid diet is usually allowed after tentative trial of milk diluted with Vichy water. In convalescence the usual tonic remedies, such as the elixir of iron, quinin and strychnin are indicated.

This is a brief outline of the usual treatment given by most physicians at the time of the Spanish war. Indeed they gave very little medicine even then. Nowadays the expert therapeutist gives still less. All our treatment now is based on the theory that the less the patient is moved the better. Keep him perfectly quiet. Insure absolute rest in bed, with plenty of fresh air, the latter being a very important point. Touatre, in New Orleans, noticed a big difference in the mortality, depending on the good or ill ventilation of the patient's quarters. In removing the patient to the hospital, he should be carried recumbent and not allowed even to lift his hand. At the hospital he is put to bed, and, if necessary, given a cleansing sponge bath with as little disturbance of the patient as possible. The bed pan and urinal are insisted on. The most careful nursing is necessary, and this is one of the great troubles in the big epidemics. There are not enough nurses, they are not well trained or they do not sufficiently appreciate the physician's instructions. A patient apparently doing perfectly well, for instance, gets up out of bed or takes solid food too soon, and dies suddenly from heart failure or has a fatal relapse and so on. We cannot too strongly emphasize the importance of absolute rest in the recumbent position with no exertion at all, extending well into convalescence. This is the first cardinal principle in the treatment of yellow fever; and the second is just as important, this is to give absolutely no food whatever during the first few days, particularly the first three days of the fever. I do not say during the whole active stage of the disease, as some authorities lay down the rule, because it is better to take whatever risks go with the food than to practically starve the patient to death. Of course, this is all a matter of common sense and of individual judgment of the particular case in hand.

Food may be administered by the rectum if the patients cannot take it by the mouth, on account of hemorrhage or irritability. Carroll reports that Spanish physicians in Cuba had good results from the repeated injections of warm olive oil. 
The exceptions need not interfere with our general rule. Patients are encouraged to take all the cracked ice and water that they wish. The alkaline carbonated waters make the best drink you can give, and preferably Vichy. If this or other alkaline effervescent water is not obtainable, you can give bicarbonate of soda, I dram to the quart. Touatre, and his reasoning seems rational, in the attempt to keep up functional activity of the kidneys, uses a sort of forced administration of large quantities of Vichy water (Celestin's), 2 to 3 or 4 quarts in the twenty-four hours. The idea is that perhaps the toxins may be diluted and to some extent eliminated in this way.

The third indication is to reduce temperature and stimulate the nervous system by cold sponging (say every two hours) especially when the fever exceeds about $103^{\circ} \mathrm{F}$. When the fever tends to keep excessively high (say $105^{\circ}$ ), cold baths may be given, at any rate, the cold sponging should be repeated at very frequent intervals, say every half hour in some cases. It has been noted that the nervous excitability, so common in children with this disease, is generally well controlled by means of hydrotherapy. Touatre thought he owed his best success to hydrotherapy, scientifically applied. There are many light cases of the fever, of course, which we do not need to be so strenuous. They will recover without any active therapy of any kind, though the rest to body and stomach are just as important. We have been describing severe and moderately severe cases.

As to the irritability of the stomach, the Vichy water, taken ice cold, tends greatly to relieve it, or perhaps cracked ice. In extreme cases it is best to stop even the intake of water, apply mustard plasters over the stomach and give saline solutions by rectum. No medicine is given whatever.

The cephalalgia and pain in the back may be treated with icebags. In very severe cases, perhaps one or two very moderate doses of phenacetin may be given. It is best, however, to avoid the use of the coal-tar products. Perhaps aspirin would be better.

Opium is dangerous on account of the kidney condition and should not be administered; also cantharides plasters might perhaps do harm. Quinin apparently does no good and is likely to irritate the stomach. Strychnin is a better stimulant than digitalis, as, in spite of the diuretic action of the latter, it is liable to irritate the stomach. Digitalin might be tried, however. Purgatives also have an irritative tendency on the intestinal canal, certainly after the first day. If attention to the bowels is necessary, give light enemata night and morning.

When there is suppression of urinary secretion, or apparent danger of that complication, as shown by the elimination of less than 500 c.c. of urine in twenty-four hours, try copious enemata of normal saline solution, and counterirritation by sinapisms or dry cupping to the back.

For the gastric hemorrhage or black vomit, astringents, such 
as tincture of chlorid of iron, were formerly used, or ergot; and of late, adrenalin has been theoretically advocated. I have about abandoned all medication for this condition, and simply give rest to the stomach. It is often accompanied by a marked condition of collapse and for this, of course, we would carry out a general stimulant line of treatment, such as hot applications, and the administration of strychnin or digitalin or caffein, or hot coffee by rectum, perhaps ether and camphor. Intravenous saline solution should be given a trial in these desperate cases.

Forchheimer, on general principles, recommends the use of Fischer's solution intravenously'

\section{PROPHYLAXIS}

Here the conditions are curiously reversed from what they are in the case of malaria. In that disease, once we have made the diagnosis, and that is not generally difficult, treatment of the individual case is easy and satisfactory. Most of our patients get well; if they do not we feel that the fault does not lie with us or with medical science. When we come to the prophylaxis, however, we meet with perhaps the most difficult problem in preventive medicine, due most largely, as we know, to the ubiquity and elusiveness of the Anopheles mosquito. We do get results, of course, as witness the Panama Canal, but at the cost of infinite labor and trouble, and we cannot entirely eradicate it with the means and money at our command. On the other hand, the yellow fever question is just exactly opposite to the malarial, in a practical way. Treatment is unsatisfactory and uncertain, the best we can do, perhaps, is to let nature take its course and see that the patient is not harmed by improper measures, but, when we come to the prevention, we know now, by several brilliant, practical demonstrations, that the yellow fever in any particular area can be absolutely eliminated within a few months by comparatively easy and inexpensive methods. This is mainly due, as we know, to the peculiar life habits of the Stegomyia mosquito. I would qualify the statement just made by saying that we can get the results, provided we can get and retain the confidence of the laity and especially those in authority in the work that we are doing. This is the most important point of all in this yellow fever prophylaxis. The scientific part of it is easy, though it requires patience and great attention to detail.

The general principles are, of course, just about the same as we use in the case of malaria. Both consist, par excellence, in antimosquito campaigning, as it is easy to appreciate.

What, in brief, are the methods that we pursue?

First in importance, naturally, is the destruction of the breeding places of the Stegomyia mosquito. When the breeding places are once abolished, of course, the race of the mosquito, so far as that place is concerned, comes to an end; and as soon as the already 
existing cases have terminated, also the epidemic. We destroy the existing larva, as we come to them in the work, by turning them out of their containers or by boiling. We usually have an ordinance passed allowing the levying of fines against the householder where Stegomyia larva are found on his premises. This is rather a highhanded sort of procedure, but sometimes they will "stand for it," and the fear of a fine is quite a help in the work; it gives, indeed, an indefinite number of unpaid assistants in the cleaning up and policing of back yards, and things of that sort.

Then we destroy the adult mosquito. This measure, quite important in malaria, assumes an added, indeed an immense, importance in the case of yellow fever, owing to the domestic nature of the Stegomyia.

The second great principle along which we work is to prevent the access of Stegomyia mosquitoes to the sick with yellow fever, and the access of infected mosquitoes to the healthy. If either of these things can be absolutely done, we see again how the epidemic must necessarily end. This occurred, of course, in the successful campaigns in Cuba and Panama. It was also of ten successfully done at Panama with single cases, that is, in the case of patients discovered on ships by the quarantine officers. There has been no recurrence of the disease at Panama since it was originally stamped out.

The third great line of work which, we have seen, was of so much importance in the case of malaria, that of working on the human host by attempts at producing immunity, is still, so far as our present subject is concerned, in the experimental stage, though perhaps something will develop later along this line, judging from analogy in other infectious diseases. Again, however, it is not so important here, as the yellow fever organism does not remain latent in the human host as it does in malaria, this being another thing that makes yellow fever prophylaxis so much the easier.

Captain Craig has written interestingly and rather convincingly to prove that yellow fever, dengue and pappataci fever are similar in a good many ways, as regards their infectiousness and the nature of their viruses and propagation by insects, and that their respective organisms, when found, will turn out to belong to the protozoa, though probably ultramicroscopic in size.

Let us take up briefly, yet as thoroughly as possible, the methods by which we can accomplish the desiderata of which we have just spoken. And first as to the destruction of the breeding places of the Stegomyia. Remember that, in general terms, this is a town fight, as opposed to malaria, where the warfare is suburban and also largely in the adjacent country. We should divide the town into districts, each under charge of a sanitary inspector and who has under him a working force, its size depending on the magnitude of the district. Daily inspections are made, if possible, of all the household premises in the district, in the search for so-called containers of the larva. Theobald calls the Stegomyia "almost exclusive a can and 
pot breeder," and this is true in general terms. However, we find them in many places in the vicinity of dwellings. A favorite place for them is in cisterns and sagging gutters, which were found very important in Havana and Panama, also in imperfect open street drains or even sewers. Sometimes they breed in pools about the houses. Look out for barrels and tubs, and old tin cans and horse troughs and grindstones, for flower pots, and so on. Places in which they are often found are the tins of water in which it is customary to place table legs in the tropics, to keep ants and other insects out of food. Very rarely, they are found in sinks and cesspools, though, as a rule, they prefer clean water. Carter says cesspools are not especially objectionable. I have found the larvæ in privies, however. In a word, look out for any possible collection of water in and about a house. Mr. Le Prince, who was our chief mosquito expert at Panama, was fond of narrating an incident in this connection. In Havana he had reason to believe that the Stegomyia was breeding in a certain house, but the most careful search by experienced inspectors had failed to locate them. Finally he found them in a small collection of water at the bottom of an old barrel of books in the library. So we see that this inspection cannot be too attentive and painstaking and careful of minutiæ. When containers are found, they are emptied out, thus disposing of. what larvæ they contain, and one must be careful to turn the container completely upside down, and empty and drain it or the larvæ will collect in the few drops left in the bottom. Naturally, the sewering and putting in of water pipes into a town will have a big effect in minimizing the Stegomyia, because there will be so many less artificial collections of water left about the town. If one has to leave their water barrels, they must be covered with screened tops. Small ponds may have to be oiled, as also any collection of water that cannot be abolished. This is perhaps the best place to mention a few points in connection with the life history of the Stegomyia which we have not yet considered, and which have some bearing on the means of exterminating it. It may be carried by vessels for indefinite distances and more often in sailing than in steam vessels. Note, in this connection, that, in ports, we have to look for containers also on the boats and about wharves. The mosquito is apparently not often carried on trains, though this is, of course, a possible way.

Apparently once having acquired the yellow fever infection the mosquito retains the power of imparting it to man for the rest of its life: the longest time recorded is fifty-nine days. It is inclined very strongly to remain about the premises where it has fed. This fact, of course, is of great help to us in our work. Probably it is not carried through the air as a rule, more than roo yards. Apparently it does not feed in bright sunlight and it is comparatively safe to go into the infected district between the hours of about 9.00 A. M. and 3.00 P. M. The early morning and evening hour seem especially dangerous in this connection. 
Now as to a very important part of the work, the desiruction of the adull mosquito infected, or thought to be possibly infected. This is usually done in the house from which we take a yellow fever case (or the ship) and usually the contiguous houses on each side. We kill the mosquitoes by sealing up the house and then fumigate it. When a case is reported the screening squad is sent to the house and the room occupied by the patient is securely, screened; the rest of the house is gone over room by room, and also the adjoining houses; the crevices are sealed up by means of paper strips and paste and then the rooms fumigated. Various fumigants have been and can be used, such as sulphur, for instance, or formaldehyde. The one that I prefer is pyrethrum powder, I pound being burnt to each rooo cubic feet of space and the room opened after three hours. This does not actually kill all the mosquitoes, but merely stupefies them, so they should be swept up and destroyed. Great care should be taken in this operation not to set the house afire. We had a disastrous fire caused in this way in the city of Panama, or so it was claimed, and which the Sanitary Department never heard the last of. Sulphur may be used in the fumigation. It has the advantage that it actually destroys the insects. One pound is used for 1000 cubic feet within a period of one and one-half hours. The trouble with sulphur is that it injures fabrics and the method thus becomes unpopular among laymen. Formaldehyde is not reliable as an insecticide. On the completion of the case, the room occupied by the patient is similarly disinfected, care, of course, being taken before that time not to let any mosquitoes out of the screened area, and only immunes being allowed to enter the room. However, as we know, it takes the mosquitoes biting the patient twelve days or more to become infected, and there would be no special danger in allowing non-immunes to enter within these limits of time. This is, of course, presuming the case in question to be the original infecting one. During the course of an epidemic these mosquitoes might be dangerous. It is easy to see what a great protection the twelve-day extrinsic incubation period is to us, and how much it aids us in our campaigns.

Captain Thomason of the army medical corps introduced a useful device in the Cuban work. He devised a sort of big canvas affair like a circus tent which could be placed over several houses so that all could be fumigated at once.

Of course, if our hypothetical case is removed to the hospital, as it ordinarily would be, the whole house is fumigated immediately. The case is transferred in a screened ambulance to a double screened compartment in the fever ward, that is, the ward is screened, and also the compartment. This, of course, is to protect the outside world against the hospital.

I think this will give a pretty good idea of the method of conducting an anti-yellow-fever campaign.

Carter says that in the early stages of the infection of a city we get the best and quickest results from working on the human 
host; later, in an epidemic, the results come from attacking mosquito breeding.

Now in this warfare on the Stegomyia, we do not actually eliminate all of that species of mosquito, though, as I have said, we can approximate much more closely to it than we can with the Anopheles. We simply reduce them to a point below what I have called the "yellow fever point." I will try to explain. We will say, for example, that there are rooo mosquitoes in a certain block, and that the epidemic is still going on. When the number is reduced to Io, the epidemic is stopped. These numbers, all merely imaginary, are used for purposes of illustration only. We have never figured out just how many mosquitoes it takes to make a place infective. Now, from the doctrine of chance, when the number of mosquitoes reaches the lower figure, the opportunity of their biting a patient, carrying out the cycle and biting a nonimmune becomes so small that it practically does not occur. Until the reduction in numbers of mosquitoes does reach that low figure, no matter how much fumigation is practised or how carefully the sick are isolated, there will still be cases, as long as non-immunes are present. This was our experience in Havana, and notably in Panama.

I hope I have made the point plain. It is of great practical and economic importance. It would perhaps cost many times as much to hunt out and kill the last ro mosquitoes, as it did to get the original rooo. We have to stop somewhere. The public would not stand for it otherwise, and as I have said, this matter of dealing with the laity and conciliating one's military or civilian superior officers is so difficult and delicate that probably, in all public health work, certainly in this antimosquito business, it looms up as the biggest problem of all. There is no use in trying to save a man if he simply will not be saved. We must learn how to conciliate, and when to compromise, or we will never have any real success along these lines.

What are the requisites for keeping up the endemicity of yellow fever in a region? These have usually been described by the authorities as two: either $(a)$ a sufficient birth rate, as in a city; or (b) a constant influx of non-immunes from the back country.

These factors have some weight, no doubt, but from a careful study of conditions in the chief endemic centers, notably Havana, Rio de Janeiro and Guayaquil, I am inclined to believe that the one essential element of endemicity in yellow fever is a sufficiently large and well-sustained adult foreign immigration.

We have not yet mentioned the general screening of the houses of the population and the universal use of mosquito bars with the view of keeping out infected mosquitoes. These, of course, are valuable measures and neccessary ones in most places.

As to the expense of the work: It has been figured out that in the case of a large population, five mills per day per individual will suffice to do the work. 
We will now consider for a moment the quarantine regulations against yellow fever. These have been worked out by our Public Health Service, and are devised so as to be effective and at the same time to interfere as little as possible with the necessities of commerce. We have been considering how to stamp out yellow fever when it has once appeared in a place; it is also of great importance to keep it out afterward. This we do by quarantine. In the United States the Public Health Service, which has control of our maritime quarantine, has evolved a set of regulations which are comparatively simple and do not seriously interfere with commerce, and yet which seem to give adequate protection against the importation of yellow fever from Central and. South American ports, where the disease has not yet been eliminated. These regulations are substantially as follows:

The period of detention is six days. At an infected port, the vessel must lie at approved moorings in open harbor and not approach the wharves. The crew are not allowed ashore. The introduction and breeding of mosquitoes are to be guarded against. The vessel is fumigated before stowing cargo or taking on passengers. If a vessel has properly complied with the regulations (this information is had from the bill of health) and arrives at the United States port north of the southern boundary of the State of Maryland without sickness after six days from the date of departure, she may be admitted to pratique after fumigation without detention. If less than six days have elapsed, she is detained to complete that period. If she is over twelve days, she may be held full time, as cases may have occurred and recovered.

South of the said line, she is held for the full period during the closed season (April to October, inclusive) unless the port of departure is known to be free from the fever. If all the crew and passengers are immune, if the vessel is of iron, and sanitary and proper precautions have been taken to prevent the ingress of mosquitoes, she may be admitted even in the South. If cases have occurred aboard, they are removed to hospital in a screened conveyance and placed in a screened ward. Remove and isolate all persons not absolutely required for the care of the vessel, then fumigate simultaneously all parts of the vessel by sulphur dioxid. The vessel may then take on a fresh crew and depart. It is not necessary to disinfect baggage unless it is believed that it may harbor mosquitoes. Adult mosquitoes are not likely to be conveyed in baggage, as they can live only for a few days without water.

Vessels in the fruit trade are not delayed if they have complied with the following regulations:

I. At Foreign Suspected Ports.-No one is allowed to go ashore except the master of the vessel or his representative and then only during the daytime. No one from the shore is allowed on board, except the quarantine and customs officer and the agent of the ship. Laborers taken on must have no intercourse with the shore until discharged. Passengers must have been under observation for three VoL. IV.-21 
days and have health certificates, or come from some elevated and non-infectible point in the interior; the vessel must have a certificate from the consular medical officer as to the sanitary condition of the ship and country, and that the vessel has complied with the regulations. Holds of vessels are to be disinfected with sulphur after discharge of cargo, when necessary.

II. At Foreign Infected Ports.-The rules for suspected ports apply with some additions. No passengers are carried. Between sunset and sunrise, the vessel must be moored at least 200 meters from the wharf. Water containers are guarded against mosquitoes. There is simultaneous fumigation of all parts of the vessel with $\mathrm{SO}_{2}$ (pyrethrum may be used in the engine room) immediately before sailing. Baggage is rigidly inspected to exclude mosquitoes.

III. At Southern Ports of the United States.-If yellow fever is known to exist, the vessel must be six days out, otherwise substitute crew and fumigate the living quarters, and also the whole vessel after unloading. The vessel must carry a physician. If there was yellow fever on board during the trip, a new crew, except the captain, mate and engineer, is substituted. Fumigate the living apartments. Detain the crew until the cargo is discharged, the vessel fumigated and she returns to quarantine.

So much for an outline sketch of the maritime quarantine methods. The old-time land quarantine with its cordons, and shotgun quarantines, and so on, is going out of fashion nowadays. We no longer fear fomites, and sensible regulations can be made as to the intercourse of the people which will reasonably insure safety and interfere as little as possible with the transaction of business.

In Havana, for instance, to prevent the reintroduction of fever from the provinces, non-immunes were kept out, but were simply examined as to the temperature for six days after their entrance.

In the case of troops, we apply the same general principles that we have been considering in regard to fumigation and mosquito destruction. Of course, a good way to avoid this, like any other trouble, is to keep away from it; that is, if this can be done. Do not make expeditions to territory infected with yellow fever during the yellow fever season. If it is intended to permanently occupy a place of that sort, it is a good plan to do the recruiting from the residents of the district who are probably immune. Soldiers should be prohibited from entering infected houses and localities by stringent orders, and infractions of this rule are to be severely punished. Careful attention should be paid to the general hygiene of the troops. Mosquito nets should be used. and suspicious buildings fumigated before occupation.

The old army method in the South, when yellow fever broke out in the vicinity of the post, was depopulation. The command was sent away, usually far enough to the north to get away from the area of country liable to become infected. In some cases this was impracticable, as for instance in 1897 at Fort Barrancas, Florida, 
where I was then stationed. The yellow fever broke out in the post before it was known to have occurred anywhere in the vicinity. In this case, we simply picked up the command and moved it a couple of miles away into a quarantine camp. The maneuver was entirely successful. After the cases, that were incubating when we went out, had been eliminated from the camp, there was not further incidence of the disease. However, it was quite late in the season and the mosquitoes were not very active. 


\title{
RABIES, FOOT-AND-MOUTH DISEASE, AND ANTHRAX
}

\author{
By Alexius McGlannan, M. D.
}

\section{RABIES}

The prophylaxsis of rabies depends on the injection of a vaccine manufactured from the spinal cords of rabbits infected with the disease. The preparation of this vaccine was the crowning achievement of the great work by Pasteur.

A rabbit inoculated subdurally with rabies virus (street virus), develops the disease, and a second rabbit inoculated with an emulsion of the cord from the first follows a similar course. After a certain number of such serial inoculations a virus is obtained which produces a fatal result in a fixed time. This is called the fixed virus and is the starting point for producing the protective vaccine.

The cord of a rabbit dead from inoculation with the fixed virus is removed under aseptic precautions immediately after the death of the animal. This cord is suspended in a drying bottle over a layer of potassium hydroxid and kept at a temperature of $72^{\circ} \mathrm{F}$. for a varying period. As the cord dries the virus becomes weakened with each succeeding day. The virus is extremely susceptible to variations in temperature and, therefore, the cords must be protected from heat and cold. .

Beginning with a weakened virus a portion of the dried cord is emulsified by grinding it with a weak solution of glycerin and this mixture is injected into the patient. Each day a cord which has been drying a shorter time is used for preparing the emulsion, the virus in which, therefore, becomes more and more powerful.

The preparation of the vaccine is so complicated by the maintenance of animals and laboratory facilities that it is impracticable except at Pasteur Institutes or other large plants. Some of the manufacturers of biological products for the treatment of diseases have added rabies vaccine to their list of products.

The emulsion of the dried cord in an ampoule and special syringes containing salt solution are shipped in vacuum containers which protect the virus from variations of temperature. The daily doses are sent in sequence, so that each day the physician receives a supply of material which will be used while awaiting the next shipment. Full directions and suitable record forms are sent by the manufacturer, so that the family physician is now placed in a position to administer this valuable prophylactic treatment and spare his patient the journey to a Pasteur Institute. 


\section{FOOT-AND-MOUTH DISEASE}

With the epidemic of this disease among cattle during the past year there came a number of reported cases in human beings. Apparently the disease is a milk-borne one and many of the human cases occurred in children. The virus has very slight resistance and is easily destroyed by heat and disinfectants. In the presence of a cattle epidemic all milk should be pasteurized as a prophylactic precaution.

Potassium chlorate and potassium permanganate solutions are probably the most efficacious mouthwashes. Erosions should be cauterized with silver nitrate or copper sulphate, or disinfected with tincture of iodine.

\section{ANTHRAX}

Complete excision of the focus by means of the cautery is the best method for treating localized anthrax, provided the operation does not cause great mutilation. Under the latter circumstances pure carbolic acid should be injected into the tissues circumferential to the pustule. Whenever there is evidence of great constitutional disturbance associated with the pustule the antianthrax serum should be given, and, if possible, blood culture made.

The seriousness of an anthrax infection depends entirely on the presence or absence of an anthrax septicemia. Treatment of the pustule, therefore, must be such that a general infection is avoided.

Since the original edition of this work, the value of antianthrax serum in the treatment of anthrax septicemia has been shown in several cases, in which the organisms were obtained from the blood of the patient. Dr. H. M. Silver at the meeting of the Society of the Alumni of Bellevue Hospital, December I, I9I5, presented a summary of these cases. The first case was reported from Grocco's clinic in I899. The patient received I90 c.c. of Sclavo's serum in eight divided doses.

Bandi in 1904 advised intravenous administration of the serum. In his report Dr. Silver records the case of anthrax septicemia successfully treated by Dr. J. E. Mahoney of Enid, Oklahoma, using the Eichhorn serum.

The serum is made by immunizing horses against virulent anthrax bacilli. From 80 to roo c.c. of the serum should be given by intravenous injection as the initial dose. Daily injection of 20 c.c. should follow until the temperature reaches normal and remains normal.

After the first injection there is usually a reaction beginning with a chill, followed by fever reaching $104^{\circ}$ or $105^{\circ} \mathrm{F}$. This fever rapidly subsides and the temperature falls to a level lower than that existing before the injection. 


\section{TETANUS}

\section{By Alexius McGlannan, M. D.}

Reports from the present war prove the great value of the antitoxic serum in the prevention and treatment of tetanus.

In the prophylaxis, Aschoff and Robertson ${ }^{1}$ recommend soaking absorbent cotton in antitoxin and drying it. This impregnated cotton may be applied to the open wound and when it becomes moistened by the secretion, the antitoxin is liberated into the tissues.

Prophylactic Injection of Antitetanic Serum.-The dose of I 500 units is universally accepted as the proper one for protection. Certain of the war bulletins report the use of smaller quantities, but as it is impossible to ascertain whether or not the units correspond with the American standard, these reports cannot be accepted as authoritative. One German unit is equal to about 40 American, while the French unit equals about 25 American. The injection should be given into or around the principal nerve supplying the region of the wound of entrance, between the wound and the spinal column. Exposure of the nerve in an open wound for the purpose of making the injection or for drainage seems unnecessarily radical.

On account of the frequent persistence of the tetanus bacillus in the tissues about the wound of entrance, the protective action of the serum is limited to a period of ten or twelve days. If the wound has not entirely healed in this length of time the injection should be repeated. ${ }^{2}$ For the same reason it is necessary to administer a prophylactic dose before operating on sinuses or the wounds of those patients who have been exposed to the infection.

Infection by contact is possible; therefore, tetanus patients should be separated from other wounded as far as can be, while the wounded that are necessarily companions of the tetanus cases should be given a protective injection. In field surgery contact of the wounded with straw, etc., should be prevented by spreading a tent or blanket under the patient.

In the local treatment of a suspicious wound it is important that the wound surface be kept moist and free from drying scabs. For this purpose some oily dressing, such as balsam of Peru, is valuable. The exposure of the wound to a current of oxygen or air is recommended whenever practicable.

Local antiseptics other than the phenol and iodine already described, seem limited to some form of chlorine application.

${ }^{1}$ Jour. Am. Med. Assn., I915, lxv, 748.

2 Irons, Jour. Am. Med. Assn., 191 5 , lxiv, 1554. 
The English Medical Research Committee ${ }^{1}$ recommends a powder made by mixing finely powdered chlorinated lime with an equal weight of powdered boric acid. The advantage of the powder lies in its easy portability, a factor in military surgery. 'The CarrelDakin antiseptic solution of sodium hypochlorite and boric acid seems a better way to apply chlorine, because impermeable crusts are less likely to be formed.

This antiseptic is made by adding $200 \mathrm{gm}$. of chlorinated lime to a solution of $\mathrm{I} 40 \mathrm{gm}$. of anhydrous sodium carbonate in Io liters of water, and thoroughly shaking the mixture. The precipitated calcium carbonate is allowed to collect in the bottom of the container and the supernatant liquid is filtered through cotton. To this filtrate boric acid is added until the solution becomes faintly acid. Usually about 25 to $40 \mathrm{gm}$. of boric acid will be required. The neutralization of the alkaline solution by boric acid gives this new antiseptic solution its advantage over Javal water and Labarraque's solution, both of which contain available chlorine in the form of alkaline hypochlorite.

The treatment of the wound, however, is not so important as is the prophylactic injection of antitoxin, and if one or the other must be postponed, delay in the wound treatment is the lesser evil. This point is of great importance in military surgery.

After a battle, wound treatment beyond the simplest first aid is rarely possible for many hours or even days after the occurrence of the injury. The injection is a simple procedure, and with a sufficient supply of antitoxin available could be carried out at the advanced dressing station and at the station for the slightly wounded.

Magnesium sulphate by intraspinal injection continues to be a satisfactory method of controlling the convulsions, but the risk of respiratory paralysis must be continually kept in mind and provided for. The pulmotor or some similar mechanical device for artificial respiration should be at hand when the injection is given.

Magnesium Sulphate by Hypodermic Injection.-The administration of the drug by this method is easier than by the intraspinal route, but it is also subject to the same dangers. A 20 or 30 per cent. solution is used and beginning with 5 c.c. the dose is gradually increased until diminished reflex activity is produced. A 5 per cent. solution of calcium chloride or some $1 / 60$ grain (o.00I $\mathrm{gm}$.) eserin tablets should be available for use as an antidote in case of alarming symptoms.

About an hour after the injection the spasms usually relax and the effect of the drug lasts about six or eight hours. The injections are repeated as soon as symptoms return. Tightness of the chest and difficulty in swallowing are usually the earliest signs of returning spasm. Magnesium glycerophosphate has been advised in place of the sulphate in the hope of overcoming some of the bad effects of the latter salt.

${ }^{1}$ Brit. Med. Jour., Aug. r4, rgr 5, p. 26r. 
All manner of desperate means have been advised for control of the spasm of respiration. Section of both phrenic nerves was successfully done by Jehn ${ }^{1}$ for the relief of this spasm. Intertracheal insufflation should be kept in mind, as a means for supplying air without respiratory movement.

For the treatment of tetanus four principles were enumerated in the original article. Of these the third may be subdivided as follows:

I. The neutralization of the toxin in the cerebrospinal fluid and in the blood.

2. Limiting or preventing the development of toxin at the focus of infection.

3. Interruption of the flow of toxin from the wound to the central nervous system.

The first condition is met by the injection of antitoxin into the spinal canal and intravenously. Ashhurst and John ${ }^{2}$ outlined a method of treatment, which has been subsequently modified by the work of Park and Nicoll. ${ }^{3}$ The details and methods of this treatment are considered fully by Park in this volume.

There is a difference of opinion regarding the management of the wound after the disease has developed. On the one hand, excision is advised for the purpose of removing the focus; on the other, it is condemned on the ground that such an operation only opens up new avenues for dissemination of toxin and bacteria.

The best method seems to be non-interference unless the wound clearly requires opening for drainage and disinfection. Occasionally it may be well to make injections of antitoxin into the tissues around the wound. Hydrogen peroxid, oxygen spray, etc., have no specific value in treating the wound.

For the third condition intraneural injections of antitoxin are efficacious. Such procedures as section of all the nerves from a limb and bringing the peripheral ends out of the wound for drainage are unnecessary and unduly radical. The same seems true of the introduction of drainage tubes into the nerve.

It is better judgment to refrain from all avoidable traumatism and to rely on the antitoxin introduced into the cerebrospinal fluid and the blood for combating the toxin.

The nutrition of the patient is of extreme importance. As long as he can swallow without difficulty this is a simple matter. When dysphagia is present, when the spasm of the jaw keeps the mouth tightly closed, the problem becomes difficult. Nutrient enemas help for a short time, but every effort must be directed toward the relaxation of the spasm.

Morphia hypodermically is most useful in early cases when spasm of respiration and deglutition are often the only symptoms.

1 Jour. Am. Med. Assn., r914, 1xiii, I 796.

2 Am. Jour. Med. Sci., June, I913, p. 806.

${ }^{3}$ Jour. Am. Med. Assn., 1915, lxiv, 1982. 
Chloral hydrate in doses sufficiently great to produce a distinct stupor is valuable, but the large doses necessary (20 to $25 \mathrm{gm}$.300 to 375 grains daily) are not without danger.

The results of intraspinous and intravenous administration of antitoxin far surpass those of any other form of treatment and whenever antitoxin in sufficient quantity is available, this should be the method of choice for treating tetanus. With a scarcity of antitoxin, the intraspinous dose should be given rather than the intravenous. Bruce $^{1}$ in a study of 231 cases of tetanus treated, shows the best results to follow repeated intrathecal injections of large doses of antitoxin.

As is true of all infections, the efficacy of the treatment of tetanus varies directly with the period of the disease at which it is instituted. Early diagnosis and prompt treatment give the best hope for success. The early symptoms of tetanus are not marked, but are quite distinct.

Pain and stiffness in the muscles of the region of the wound may be mistaken for a simple myalgia, but is easily differentiated by exaggeration of the local reflexes, which exaggeration becomes progressive. Twitching and stiffness of the muscles when these are tapped is an evidence of increased excitability.

Pain about the mouth, fatigue in chewing, slight difficulty in swallowing without any cause for this condition evident in the throat, is an early and very certain sign in a suspicious case.

Cramps in the chest muscles, generally described as "a stitch in the side" and a sense of tightness in the chest are other early symptoms. Profuse sweating and a rapid pulse precede the onset of distinct lockjaw.

Starting at noises, at lights or in drafts is evidence of undue reflex sensitiveness and occurring in the wounded should give rise to a strong suspicion of tetanus.

In the presence of any of these signs the patient should be placed in the best surroundings possible for quiet and darkness. The intraspinous and intravenous injections of antitoxin given as soon as possible and the general nutrition preserved by frequent feeding of small quantities of easily digested food. Water should be given by rectum as well as by mouth in as large a quantity as can be taken. The spasmodic or paralyzed bladder may require catheterization and enemas may be necessary for emptying the bowels. Whatever manipulations are required must be carried out with the utmost gentleness, and preceded by the administration of opium if they annoy the patient. Every effort must be used to limit the effect of external stimuli on the central nervous system.

${ }^{1}$ Brit. Med. Jour., Oct. 23, 1915, p. 593. 


\section{PERTUSSIS}

\section{By Thomas C. Kelly, M. D.}

During the past five years many advances have been made in our knowledge of pertussis, and there is hope that in the near future, an early diagnosis of this disease will be possible, and that by the use of specific therapy in the form of serums or vaccines, it will be numbered among the easily curable diseases. Observers have endeavored to impress both the profession and the laity with the seriousness of the disorder, so that instead of regarding it as a mere incident of childhood, it is to be thought of as among the serious diseases to be, if possible, avoided, both for the primary danger per se, and also for the numerous serious complications and sequelæ.

The earlier work of Bordet and Gengou, ${ }^{1}$ has been confirmed both by more recent studies by themselves, and by numerous other investigators, Arnheim, C. Fränkel, Seiffert, Wollstein and others, and it is now generally acknowledged that the bacillus first described by Bordet and Gengou is the cause of whooping cough. The organism is found in the mucus from the respiratory tract, more often from below the larynx than above it, and is more abundant at the beginning of the disease than it is later on, apparently disappearing coincident with the paroxysms or initial whoop, whence the lessened danger of contagion from that time on.

The pathological lesions due to the organisms have been described by Mallory, ${ }^{2}$ who found the organisms in large numbers packed between the cilia of the epithelial cells lining the trachea, in the bronchi, and also present in the bronchial secretion and enclosed in the polymorphonuclear leukocytes. The action of the B. pertussis Mallory believed to be purely mechanical, in that it interferes with the normal movements of the cilia, and therefore produces a continual irritation which excites coughing. It also produces a rather mild toxin, as shown by the inflammatory exudate, the lymphocytosis, and the production of the specific antibody. Rhea suggests a possible similarity between the disease as described by Mallory, and canine distemper, common among dogs, who showed the same lesions as were due in man to the B. pertussis, which intimated a possible relationship between the $\mathrm{B}$. pertussis and the $\mathrm{B}$. bronchisepticus. The organism (B. pertussis) as shown by Klimenko ${ }^{3}$ does not ordinarly circulate in the blood, but is probably limited to the mucous membrane of the respiratory tract.

${ }^{1}$ Brit. Med. Jour., Oct. 9, I909, p. I062.

2 Bull. Mass. Med. Soc., Medical Communications, I9r3, xxiv, Ir3.

${ }^{3}$ Vratch, January, I910, and La semaine médicale, June 29, I910, p. 304. 
Diagnosis.-In 1897 Fröhlich called attention to the fact that with whooping cough there was generally an associated leukocytosis. With the view of determining the importance of such knowledge, Kolmer ${ }^{1}$ made a careful study of the blood in whooping cough, and found that in the precatarrhal stage there was a slight leukocytosis with an absolute increased percentage of all forms of white blood corpuscles. This increase is continuous during the catarrhal stage, reaches the climax during the paroxysmal stage, and gradually decreases until it reaches the normal usually at the end of three months.

Recently much work has been done as to the possibility of the early diagnosis of pertussis by means of the complement fixation test. While Bordet and Gengou used the complement fixation test as a control in their earlier work when first describing the B. pertussis, and their observations were concurred in by Delcourt; ${ }^{2}$ Netter and Weil showed that the test did not appear during the catarrhal stage, sometimes during the second week of the paroxysmal stage, and occasionally later during the subsidence of the disease. However, Friedlander and Wagner ${ }^{3}$ claim that by means of this method it is possible to make a diagnosis during the catarrhal or during the early part of the paroxysmal stage. In a series of cases two out of three were positive during the catarrhal stage, nine examined just at the time of the initial whoop were positive, and eighteen during the paroxysmal stage were all positive. They accounted for their good results by the fact that both the antigen and the serum were always perfectly fresh.

More recently Olmstead and Lutinger ${ }^{4}$ have reported their results with a series of over I roo cases or suspected cases of whooping cough. They believe that the best antigen is made by autolyzing an aqueous emulsion of a twenty-four to forty-eight-hour growth of the Bordet-Gengou bacillus for eighteen to twenty-four hours at a temperature of $56^{\circ} \mathrm{C}$. and shaking for several hours. Their conclusions with about 40 per cent. of cases giving a positive reaction augurs well for the future practical value of the reaction; especially applicable will it be for children's hospitals, thereby enabling them to exclude from the general wards cases which would otherwise be treated until too late to avoid possible contagion.

Complications. - In addition to the usual complications described in the previous article by Dr. Hammill, there have recently been reported several of a more uncommon type. Nacht ${ }^{5}$ has reported an unusual case of choked disk with complete blindness which developed during the course of pertussis, and which was cured by trephining, the optic neuritis being due apparently to the increased

${ }^{1}$ Arch. Int. Med., July 15, r909, p. 8r.

2 Bull. Soc. de pédiat., Nov., r 10 .

${ }^{8}$ Jour. Am. Med. Assn., March 28, 19 I 4.

4 Arch. Int. Med., July, 1915, p. 67.

5 Klin. Monatsbl. f. Augenh., May and June, I910. 
amount of cerebrospinal fluid. Conda ${ }^{1}$ reports a case of intussusception occurring in the course of whooping cough, due probably to the severe paroxysms of coughing and an associated diarrhea. Various authors have described several nerve and brain complications which are usually of a convulsive or hemiplegic nature. $\mathrm{Hada}^{2}$ describes a case of pachymeningitis productiva interna, and Henoch believes that all the brain complications of whooping cough are primarily caused by the effect of the toxin, causing various forms of inflammation, such as encephalitis, leptomeningitis, etc., and that frequently the inflammatory process is of a distinctly hemorrhagic character.

Prophylaxis.-Much attention has recently been directed by the profession to the more rigid and intelligent preventive treatment of this disease. The fact that while it is regarded by many with more or less complacency, it is far more serious than is generally supposed, has directed many pediatrists to consider means for its proper subjection. Rucker ${ }^{3}$ has called attention to the fact that Io,000 children die annually from pertussis, and that the death rate per one hundred thousand of population is almost identical with that of scarlet fever and only slightly less than that of measles. The hospital facilities for the treatment of this disease are extremely inadequate, most hospitals refusing to admit such cases to the wards. Pisek ${ }^{4}$ has called attention to the fact that of over 5000 beds for infants and children in New York City alone, there are only 40 set apart for the treatment of whooping cough, and has suggested the use of ferry boats and barges to supply the deficiency.

\section{TREATMENT}

Very little has been added to our knowledge of the general prophylactic and hygienic treatment of this disease since the previous article was published (Vol. II, p. 624). Czerny ${ }^{5}$ considers that the nervous element in whooping cough has received too little attention, and suggests a change in environment, more thorough isolation of the patient and more particular attention to hydrotherapeutic measures. Occhenius has reported a series of $\operatorname{cases}^{6}$ of 107 children, in eighty-four of whom he had obtained beneficial results from the systematic daily application of a 2 per cent. solution of nitrate of silver to the pharynx (in young infants a $\mathrm{I}$ per cent. solution was used). The child was held under firm control, and the pharynx thoroughly painted once daily for a period of two weeks, and while in a few cases the attacks at first seemed more numerous, in the majority of instances there was a decidedly beneficial result as

\footnotetext{
1 Arch. de méd. des enfants, March, I9I4, p. 207.

2 Virchow's Arch., I913, Heft 2, p. 206.

3 Public Health Reports, Oct. 26, I912.

4 Pediat., June, r914, p. 325.

5 Therap. Monatsh., Berlin, vol. xxii, No. I2.

6 Semaine méd., Nov, 26, I913, p. 569.
} 
evidenced by the lessening in the number and severity of the paroxysms. As to the medical treatment, antipyrin, belladonna (in full doses), and quinin, used singly or in various combinations, still seem to be the most efficacious in controlling the paroxysms. Among the more recent drugs suggested for use in this disease is iodine. Cavazzani advocates the use of $4^{-6}$ drops (for a child one year of age), of a solution of $\mathrm{r} \mathrm{gm}$. of iodine, $15 \mathrm{gm}$. of potassium iodid and $\mathrm{I}_{5} \mathrm{gm}$. of aqux destillata, and that by this means the course of the disease is rendered more benign, and the cough less severe. Janiscke ${ }^{1}$ and various Austrian authors have obtained good results by the use of the hydrochlorid of papaverin in $\mathrm{I} / 3$ to $\mathrm{r} / 2$ grain $(0.02-0.03 \mathrm{gm}$.), doses three or four times daily. Fletcher, Lord and others have suggested the cautious use of adrenalin chlorid: Hamburger ${ }^{2}$ reports good results from the application of the faradic current over the larynx and about the neck, during the late "nervous" stage of the disease, and thinks that between 70 and 80 per cent. of the cases were cured after two or three treatments.

While many of the drugs used in the treatment of pertussis undoubtedly do good in certain instances and certain ones in particular have their enthusiastic adherents, there is no one general specific -at the most they render less severe at times the paroxysms of the disease, and at times seem to shorten the duration of the attack. During the declining stage of the infection, one must bear in mind the great benefits from fresh air, sunshine and general tonic and supportive treatment. Simple tonics such as the elixir of iron, quinin and strychnin, or syrup of the iodid of iron, often appear to be the means of enabling the patients to regain their failing strength, and to render less exhausting the attacks of coughing.

Specific Treatment.-Since the discovery by Bordet and Gengou of the cause of pertussis there have been numerous attempts to produce a specific mode of treatment either by serums or vaccines. Vanlain $^{3}$ reports a series of seventy-one cases treated by Duthort of Brussels, in which after a single injection of serum there was a decided abatement of all symptoms, and the course of the disease was considerably shortened. Klimenko ${ }^{4}$ reports a series of thirtyfive cases treated by the subcutaneous injection of serum in which without exception there was a diminution in the number of the attacks of coughing and a bettering of the general condition, and in about one-third of the cases the duration of the disease was shortened.

Numerous investigators have also endeavored to perfect a cure by means of a suitable vaccine. Sill ${ }^{5}$ cites his results in thirtythree cases treated by means of vaccines in which he obtained uniformly good results as to the amelioration of the symptoms, and in the few cases in which he tried the prophylactic use of vaccines,

1 Therap. Monatsh., April, r914.

2 Semaine méd., Nov. 5 , I9r3, p. 540.

Semaine méd., Oct. 29, I9I3, p. 528.

- Arch. d. sc. biol., St. Peterburg, r913; Semaine méd., Oct. 23, 1912.

- Am. Jour. Dis. Child, rgr3, Ivii, 379. 
he thinks his results were quite hopeful. More recently Hess ${ }^{1}$ studied a series of 375 children in the Hebrew Infant Asylum in New York City, and found that while vaccines did very little good in the cure of pertussis, they were decidedly beneficial in preventing the infection.

The whole question of vaccine therapy in pertussis seems to be still in a more or less experimental stage and no absolute conclusions can be drawn as to its final value, but with the increasing number of investigators and their refinement of technique, there is every reason to hope that in the future the disease will be numbered among those in which brilliant and certain results are obtained by this method of treatment.

The method of administration of vaccines in pertussis is discussed in the article by Dr. Wm. H. Park, page $5^{2}$.

${ }^{1}$ Jour. Am. Med. Assn., March 28, 1914, p. 1007. 


\title{
INFECTIOUS PAROTITIS-MUMPS
}

\author{
By Thomas C. Kelly, M. D.
}

During the past five years very little of a positive nature has been added to our knowledge of either the diagnosis or treatment of mumps. While several investigators have called attention to a complication sometimes of an alarming nature, and others have recently endeavored to perfect a protective serum, our conception of the entire disease has advanced but little in recent years.

Merelli $^{1}$ in a series of eight cases, isolated from the blood, and from the testicles in the cases complicated with orchitis, an organism which he called Micrococcus tragenus, and which he believed to be the cause of mumps. This organism differed somewhat from those previously described as the cause of this disease, in that it did not stain by the Gram method or liquefy gelatin. It gave the agglutination test in dilutions as low as $1-500$, but when inoculated into animals gave negative results. Petrelli ${ }^{2}$ and Bonazzi, ${ }^{3}$ working independently, observed that epidemics of mumps were often preceded or accompanied by attacks of pharyngitis, and Bonazzi in one of the epidemics studied by himself, found that there was present in nearly all of the cases, either sore throat, marked hyperemia of the palate, or, as in the majority, numerous small ulcerations on the lips, nose or ears-an observation of moment as it possibly throws some light, upon the mode of transmission of the infection.

Complications.-Among the accompanying features of an attack of mumps there is at times, as pointed out by Tiessier ${ }^{4}$ a well-marked bradycardia- of moderate degree, begining early, occasionally persistent, and most often present in those cases without fever. Besides involvement of the testicle, which is more or less common in adult males, several well-authenticated cases of involvement of the ovary have been reported several years ago by Troitsky ${ }^{5}$ and two cases of a similar nature more recently by Brooks ${ }^{6}$ who found that for some years after the attacks of mumps neither of the patients became pregnant, which fact he regarded as suggestive.

In I8I7 Schmachpfeffer called attention to the involvement of the pancreas during an attack of mumps, and since then this fact has been casually commented upon by numerous observers. In

\footnotetext{
1 Policlinico, Rome, Jan. I2, I913, p. $62 \mathrm{I}$.

2 Semaine méd., r9 3 , p. 453.

3 Bull. d. sc. med. d. Bologna, August, I9r3.

- Semaine méd., Jan. 17, I9I3, p. 32.

Vratch, I903, I, No. I5.

- Jour. Am. Med. Assn., Feb. 1, 1913, p. 359.
} 
r9I3 Allegri ${ }^{1}$ carefully studied four cases in which there were marked symptoms of involvement of the pancreas-all having severe. localized epigastric pain increased on pressure, with nausea, vomiting and marked diarrhea, but without either albumin or sugar in the urine. Edgecombe also reported four cases with acetone and diacetic acid in the urine, and in one of which the Cammidge reaction was present.

Treatment.-Very little that is new has been advocated in recent years for the treatment of this disease. Rest in bed especially during the febrile period is important. The bowels should be kept freely moved, preferably by means of saline cathartics, and during the first few days a simple diuretic fever mixture is sometimes of service. An antiseptic mouthwash, the local application of ice, belladonna or ichthyol ointment, or in some cases hot fomentations to the parotid swelling are all accepted forms of treatment and have changed but little in their value. Should orchitis develop absolute rest in bed is imperative, the testicles should be elevated and lead water and laudanum or some similar preparation applied. Dr. Hugh Cabot, believing that the atrophy of the testicles which occurred in about 60 per cent. of the cases was due to the increased intratesticular pressure caused by the inflammation, advised slitting the tunica albuginia, thereby lessening the tension, restoring the circulation and in many cases preserving the testicle.

With the hope of obtaining a preventive serum for mumps, Hess $^{2}$ recently conducted some studies in immunity in this disease at the Hebrew Infant Asylum in New York City, in which he attempted to immunize children with the blood of convalescent patients, using from 6 to 8 c.c. of the blood drawn from the vein of the donor and injected intramuscularly. None of the children so inoculated contracted mumps, while other children similarly exposed to the contagion did become infected. It may be that further studies along similar lines will result in an easy practical method of at least rendering children immune from this disease by means of protective serum.

1 Policlinico, Rome, Oct. I9, r913, p. I 5 I 8.

2 Pediat., May, I915, p. 230; Am. Jour. Dis. Child., April, 1915. p. 99. 


\section{AMEBIC DYSENTERY}

\section{By Charles F. Martin, M. D.}

The occurrence of dysentery in epidemic, endemic, and sporadic form is, for the most part, confined to tropical countries, although not a few cases are found outside of the tropics.

Though epidemics in temperate climates are comparatively rare, it is a well-recognized fact that in countries where dysentery is common a very large proportion of the population has the infection, though, perhaps, not showing symptoms of the disease. Thus, for example, 30 per cent. of the patients in the Civil Hospitals of these countries are thus affected, and it is said that 5 per cent. of all natives have amebas in their intestines. Europeans are particularly prone to the disease.

The only known rational therapy is by the use of ipecac or its alkaloid emetin.

The Treatment with Ipecac.-Ipecac has been used in the treatment of dysentery for many years, since Piso introduced the root into Europe from Brazil in 1658 . Its great benefits in the treatment of dysentery were demonstrated in Mauritius, and later on by Manson who observed the need of large doses in order to produce effective results.

Leonard Rogers pointed out the clinical value of ipecac in amebic abscess of the liver; and Simon and Brem confirmed his observations. Preliminary preparation of the patients is essential to good results. A dose of castor oil is given the night before and a dose of tinct. opii, 20 minims ( 1.3 c.c.) is recommended half an hour before the first pill is administered. The patient is starved for six hours and is given no water for three hours prior to using the drug. The recumbent posture is imperative once the treatment begins.

The ipecac is prepared in pill form, 5 grains $(0.3 \mathrm{gm}$.) coated with salol or keratin, and given with as little fluid as possible. On the first night eight or ten pills are given, and on each succeeding night the dose is reduced by ro grains ( $0.6 \mathrm{gm}$.) until only two pills are given. Then the Io grains is given every alternate night for two weeks, and a "cure" is effected. Most authorities regard this as almost a specific.

The Treatment with Emetin.-In I89r it was shown by Welch that the alkaloid of ipecac, emetin, had a remarkably curative effect, and that thirty-two out of thirty-four cases thus treated recovered. A more striking evidence of its efficacy was shown experimentally by Vedder in I9I I, who demonstrated in vitro that emetin acted on the amebas especially in the tissues, and that such an effect could be 
produced after absorption in the blood stream. The amebicidal action was proved to be twice as powerful as that of the fluidextract of ipecac. Rogers applied these experiments clinically and it is due to his genius that the hypodermic injection of salts of emetin have been used so successfully. This advance was based on the discovery that contact of the ameba with a normal saline solution of emetin hydrochlorid was fatal in a strength of $\mathrm{I}: 100,000$, and as a result weak solutions were used hyperdermically. One to 2 grains were injected over a period of two days, with remarkable benefit to the patient.

Twenty-four consecutive cases were treated, and in most instances the stools became free from the parasites in two or three days. The average sojourn in hospital was seven days.

Since these observations, good results from this treatment have been reported wherever tried, as witness the publications of Dopter, Chauffard, Lyons, Rouget, Dock and Brem, Simon, et al.

A proper diagnosis is, of course, essential, and a thorough examination is necessary to establish the presence of disease. It has been stated that if in a chronic diarrhea the proctoscope is used, the pathognomonic anatomical picture can usually be found, and for this purpose often the worm stage is essential. Craig is responsible for the dictum that wherever amebas are found in chronic diarrhea, they may be regarded as the cause. Rogers suggests the therapeutic test with emetin.

The Drug.-Emetin is one of the alkaloids of ipecac and exists as an amorphous white powder and occurs in different roots in different proportions, thus rendering some roots more efficacious than others. This, at all events, seems to account for the greater specificity of the Brazilian over the Colombian ipecac. Other alkaloids which exist in ipecac must not be confused with the pure emetin.

As employed today it forms a soluble salt (E. hydrochlorid) put up in tables or in ampoules.

Method of Administration.-Emetin is given by mouth, or subcutaneously, intravenously, and by irrigation of the rectum.

Subcutaneous Method. - The average dose is $1 / 2$ grain ( $0.03 \mathrm{gm}$. in ro-I 5 minims of normal saline solution. This is given daily in one or in divided doses, and is continued according to results as seen in the stools and rectum, i.e., normal stools, absence of amebas, healthier rectum. Children are very tolerant of the drug. At two years of age $1 / 6$ grain (0.0I gm.) may be given every twelve hours for three doses, and for those under one year $1 / 8$ grain ( $0.008 \mathrm{gm}$.) for two to three doses.

Untoward Symptoms.-Excessive doses irritate the bowel, induce malaise, and vertigo, though, when the drug is eliminated, in two or three days these symptoms disappear. Where the injection is given a local induration results.

Per Os.-The drug is coated with keratin and sometimes causes nausea. The tablets are used but are not efficacious in severe cases. 
Intravenous injections may be used in severe cases, but are obviously not required in the milder forms of the disease.

The dose is $1 / 2$ to 2 grains $(0.03-0.15 \mathrm{gm}$.) in the normal saline solution, and in such doses causes but slight disturbance (nausea, flushing of face, dizziness). Larger doses of 5 to 6 grains $\left(0.3^{-0.4} \mathrm{gm}\right.$.) induce dyspnea, vomiting, diarrhea, shock, etc. Four grains is considered a maximum dose for an adult, while 3 is sufficient.

A good practice is to first inject intravenously, or subcutaneously, $21 / 3$ to 3 grains (0.15 to $0.2 \mathrm{gm}$.) of the emetin hydrochlorid in roo c.c. salt solution. Then every second day $1 \frac{1}{2}$ to 2 grains (0.I to o.13 gm.) for four doses. Repeat this procedure every three or four weeks during several months, and a cure is practically assured.

Effects and Results of Treatment.-The drug is practically a specific, acting like quinin in malaria, and causing the parasites to become immobile, rounded, and bulging till they burst or fade. Granular degeneration of the nucleus follows, and fibrillar degeneration of the cytoplasm. The cases treated early result in prompt cure, while in those where the treatment is started after long infection, or an extensive invasion, the results vary. The relief of clinical symptoms is miraculous. In two to three days, the distress goes, as do the griping pains, the painful bloody flux, and aching in the trunk and extremities.

Liver abscesses are actively affected. The presuppurative stage is cured while the true abscess is rapidly healed after combining incision with the emetin treatment. Mild local irritation occurs, but there is no general discomfort from the drug in proper doses.

Duration of Treatment.-The incubation period is long (average sixty-four days), so after a "cure" it is not easy to tell if a relapse is likely, for both amebas and cysts sometimes appear long after treatment.

It is not safe, however, to await symptoms, and it is probably wiser to follow the suggestion of Baermann and Heinemann who repeat cures every few weeks. Some continue later on by giving the drug by mouth, or using ipecac pills, e.g., 20 to 30 grains (I. $3^{-2}$ $\mathrm{gm}$.) on the eighth day at bedtime for three nights, and then less for a while.

Limitations. - In some ro or 20 per cent. the results are not satisfactory, or temporary only. For this reason one must be cautious in mistaking a "clinical cure" for a permanent one, i.e., where the amebas or their cysts may have only temporarily disappeared. No cure is proven till months have passed without recurrence.

Apparently the amebas in the walls and the floor of ulcers are destroyed for the most part, but others that are protected seem to escape the action of the drug. While some authorities think that it is only necessary to destroy the visible parasites, others, e.g., Seguin, say that relapse is sure unless both amebas and cysts disappear permanently from the stool. Some strains of the ameba too, seem emetin-fast, and the question naturally arises do small 
doses give the parasites an immunity, or does a previous treatment with ipecac confer a protection. The latter is evidently not the case, as emetin often succeeds in recurrent cases where a previous "cure" has been undertaken.

Encystment of the ameba takes place, and not uncommonly cysts are found in the stools of healthy carriers, showing that the environment had been unfavorable, and also that the vegetative stage alone is actively productive.

There is no doubt a latent form of the disease, for encysted amebas may, after years, reappear in an acute form, actively vegetating.

The emetin treatment not rarely results in carriers of cysts, in two ways:

I. Convalescents, or

2. Healthy people, as in large numbers of natives of tropical countries.

The disease is brought hither by direct contact, or through flies. 


\section{LEPROSY}

By Isadore Dyer, Ph. B., M. D.

Leprosy is attributed to the Bacillus lepre, discovered by Hansen in 1871 and reported in 1874 ; it was confirmed by Neisser in 1879 . Leprosy probably spreads its organisms through the nose or mouth and possibly by sexual intercourse. It is not proven hereditary.

Experimental inoculation has been successful in monkeys, white mice, and some cold-blooded animals. Both mosquitoes and bedbugs have been found infected with $B$. lepre $\dot{e}$ and a lepra-like bacillus has been found in rats. The diagnosis of leprosy depends on the clinical evidences and the bacteriological findings. Clinical evidences of leprosy are characteristic in the eruptions: Of macules, on the face, neck, shoulders, forearms, back, buttocks, thighs and legs, with a constant tendency to bilateral distribution upon the extensor surfaces. The macules are oval in shape, ringlike in form with the color deeper at the borders and fading to the center, which is sometimes a dirty white. The whole color varies from a bright rose red (in recent lesions) to a reddish brown (Indian red), which is the most characteristic color of the disease. The deeper the color the more analgesic the lesions; bright or new macules are of ten hyperesthetic.

Sometimes the macules are just pigment splotches on the skin. In such cases the body generally is covered and the bronzed hue of the eruption is suggestive.

The tubercle or nodosity of leprosy may develop in the area of the macule, especially on the face. More often the tubercles come in the pendulous portions of the ears, the soft parts of the nose, in the alæ nasi, in the lips, on the chin and on the backs of the hands. Nodosities are more likely on the cheeks, on the forehead, about the genitals and along the course of the ulnar nerve, occurring in the nerve sheath or in the skin above it. Blisters at the finger joints, contracture of hand muscles, discolored and blunted nails and loss of phalanges occur in trophic types. Local analgesia, leathery ears, deformities of the hand, loss of expression in the face (satyriasis or leontiasis) and the peculiar sibilant, nasal, raucous speech are peculiar signs of leprosy.

The bacteriological diagnosis is determined by the examination of scrapings from the lesions of the skin and from the nasal septum, where usually a superficial ulcer is present.

The treatment should be directed at segregating the leper and at improving the resistance of the patient with wholesome surroundings and with such remedies as have been proven efficacious. Many drugs and measures have been used, including sera and vac- 
cines. Strychnin, arsenic, mercury, iodids, salicylate of soda and chaulmoogra oil have seemed the best.

Chaulmoogra oil has survived most of these and remains the only remedy producing consistently good results. It is given best in capsules, before meals, starting with three drops, increasing one drop a day until the maximum dosage for the individual is reached-varying from 40 to I 50 drops at the dose, three times a day.

Heiser, in the Philippines, has given chaulmoogra oil by needle, with good results, using this formula:

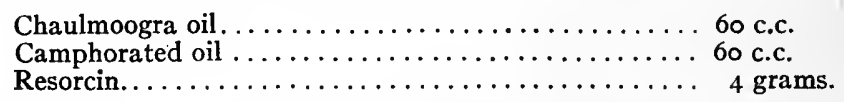

To be mixed and dissolved with heat on a water bath-then filter. Initial dose of one c.c.-increasing to tolerance. Frequent doses at first, gradually remitting as the disease improves.

Strychnin is a valuable adjuvant in $1 / 50$ to $1 / 30$ grain dose three times a day.

Daily baths as hot as can be borne aid the treatment. The diet should be liberal and unrestricted.

The improvement in leprosy is always slow and may not show for months after treatment is begun. After all evidences have gone treatment should be kept up indefinitely. Bacteriological examination should be made from time to time to make sure of cure. Where doubt is entertained, a course of iodid treatment, in increasing doses, may light up lurking leprosy. 


\section{TYPHUS FEVER}

\section{By John F. Anderson, M. D.}

The recognition of the widespread incidence of typhus fever and the severity of the epidemics that have occurred in the eastern section of the great European war, has aroused unusual interest in this disorder.

In IgII it was shown that a disease, cases of which had been studied by Dr. Nathan E. Brill of New York, was identical with typhus fever. Since then it has been definitely determined that the disease is present in practically all of the larger cities of the United States.

In I9r 5 Plotz of Mt. Sinai Hospital reported the cultivation of an organism from cases of typhus fever of both European and American origin. While the work of Plotz seems to be conclusive, it has not yet been confirmed by other workers. Moreover, the work of Plotz himself, as also the work done in the Hygienic Laboratory in Washington, has failed to demonstrate that immunity in animals is conferred by inoculation either with living or dead cultures of the organism described by Plotz. Moreover, Dr. Fricks has recently published a paper on Rocky Mountain spotted fever in which he reports the successful cultivation of an organism similar, morphologically and culturally, to the one described by Plotz, from animals sick with spotted fever.

In spite of the widespread interest in this disease, advances in our knowledge have been concerned entirely with its recognition in a mild form and the apparent demonstration of the causal organism. There has been nothing new brought out in regard to the treatment or prevention of the disease that has not already been fully covered in the article as it appeared in the former edition. Treatment has remained much as it was when this article first appeared.

The widespread prevalence of typhus in the European countries has given an opportunity to apply on a large scale the measures for the control of the disease based upon its transmission by the louse. It has been found that those measures have been effective wherever consistently employed. 


\section{RAT-BITE FEVER}

\section{By George Blumer, M. D.}

Rat-bite fever is an infectious disease which is particularly prevalent in Japan but which has also been observed not infrequently in the United States. It results, as the name indicates, from the bite of a rat, or occasionally from the bite of some animal whose natural habits bring it in contact with the rat '(cat, weasel, ferret). Following the rat bite, which usually heals, there is a period of incubation averaging about two weeks, followed by a characteristic febrile reaction. This usually takes the form of a relapsing fever, the febrile attacks lasting from two to three days and occurring at intervals of from five to ten days. Accompanying the attacks of fever there frequently occur a peculiar erythematous rash and severe muscular pains. Dysphagia is a not infrequent accompaniment of the attacks. As many as 26 paroxysms of fever may occur and the mortality of the disease is about ro per cent. Abortive forms with lack of fever or atypical fever are not uncommon. In these a local reaction in the form of reddening and pain at the sight of the original bite, erythema along the course of the local lymph channels, and the tumefaction of the regional lymph nodes are prominent features. The causal agent is probably the organism first described by Schottmüller as "Streptothrix muris ratti," also observed in this country by Blake and by Tileston. Japanese observers have described a spirochete.

The prevention of the disease naturally involves the extermination of rats and avoidance of contact with these animals. Where an individual has suffered from a rat bite the experience of Miyake has shown that immediate cauterization of the wound either with strong carbolic acid or the actual cautery is a sure preventive.

After the disease has manifested itself there is only one remedy which has shown itself of value and that is salvarsan. At the time of the publication of Tileston's article twelve cases had been treated by salvarsan with cure in ten, or 83 per cent. The drug is used in identically the same manner and the same dosage as in syphilis. 


\title{
TROPICAL DISEASES
}

\author{
By Bailey K. Ashford, M. D.
}

MALARIA

Progress in our treatment of the problem of malaria since IgI I has been greatest in securing clearer ideas of its epidemiology and a better working basis for prophylaxis, but this progress in turn has proceeded from the intense laboratory work done on the subject.

Latent Forms of Malaria, Relapses.-The trend of opinion seems to be toward explaining relapse, even after long periods of freedom from symptoms, by the persistence in the body of a few asexual forms which had escaped the action of quinin, or were perhaps resistant to it. Those who go upon this hypothesis as a rule advocate, therefore, higher doses of quinin than heretofore given and a more persistent and long-continued treatment. The gametes were proven by Thomson to have only a short life in the body, although they are known to be quinin-resistant and their continued presence for long periods in the blood is said by him and others to be due to the fact that they are constantly being formed from the unkilled asexual forms in sluggish cycle. This was quite fully demonstrated by applying Ross' thick film method of staining blood to the search for parasites in cases in which simple smears had failed to demonstrate them.

In a sufficient number of cases the persistence of these asexual forms was demonstrated so that the theory is placed on a fairly firm basis. Placing the life of a gamete at six weeks, a course of quinin in large doses, 20 to 30 grains $(\mathrm{I} .3-2.0 \mathrm{gm}$.) a day, will usually cause their disappearance by destroying the asexual forms from which they proceed. Thomson states that a three weeks' course is sufficient to reduce crescents to one per cubic millimeter of blood, which he considers too few a number to be of importance in propagating the disease. He, therefore, advises that every adult person in the population of an infected locality and children in proportion to their age, be given such a consecutive three weeks' course in each of the four quarters of the year. This is less quinin for the year than if 5 grains a day were consumed, especially if those are exempted who in a quarterly blood census were free from parasites after examination by the thick film method. All persons found harboring crescents should be detained in hospital until non-infective. This is perhaps as yet an irrealizable scientific ideal but great necessity may at any time force the issue in given localities.

Darling states that the limit of infectiousness is $\mathrm{I} 2$ gametes per cubic millimeter or one to every 500 leukocytes. 
Incidentally James' method of staining a thick film is worth citing: A drop of blood is spread in the form of a circle $3 / 4$ inch in diameter and allowed to dry. It is then immersed in 50 c.c. ethyl alcohol containing ro drops of commercial hydrochloric acid until the hemoglobin is dissolved. The specimen is now washed in running tap water from ten to twenty minutes and stained with a Romanowsky stain for two or three minutes, replenishing the stain tissue as it evaporates (personal demonstration by James). It is then well diluted with water, more water being from time to time successively added. The whole process should take ten minutes. It is then washed and examined. The modification of this method which I make in the laboratory of the Institute of Tropical Medicine and Hygiene of Porto Rico is to place the slide after its first bath in tap water in a Petri dish filled with distilled water; then add 1/20 normal sodium hydrate solution with phenolphthalein as an indicator until alkalinity is just produced. After this the Romanowsky stain may be applied.

James states that Deek's method of giving I5 grains ( $1.0 \mathrm{gm}$.) of quinin three times a day by mouth to malarial fever patients for two or three weeks results in fewer relapses than when Io grains ( $0.6 \mathrm{gm}$.) are so given, but he favors intravenous injections in severe cases. A considerable proportion of malaria in Panama is said by him to be due to relapses.

With this doctrine of high dosage of prophylactic quinin we are confronted by Celli's remarkable statistics of success in Italy from small doses. Celli's method was to give 3 grains $(0.2 \mathrm{gm}$.) in the morning and 3 at night daily. This he said practically guarantees a healthy individual against infection in an endemic zone. Both large and small doses given daily are soon well tolerated and the initial ringing in ears and headache disappear, but if several days' interval lie between doses toleration is not so well established. Celli states that 40,000 kilos of quinin are distributed free or bought in Italy annually. In 1887 there were 22,000 deaths from malaria; in I9I 2 only 3000 and the decrease began when quinin first began to be distributed.

Bass, whose recent epidemiologic work has been most prolific in its results, states that the people who are suffering from clinical malarial fever are only a small element in the propagation of the infection. It is the carrier who is not ill and who has even not had malarial fever to whom we must especially direct our attention. He makes of the information furnished by Thayer to the effect that a continued temperature below $65^{\circ} \mathrm{F}$. causes failure of development of sexual forms in anopheles and death of those already developed, a text upon which to apply a wholesome sermon to our Southern States. If mosquitoes cannot act as hosts except during four to six months of the year, then the rest of the year man must be their habitat. As sexual forms live only six weeks he advises for a complete eradication of malaria that a proper amount of quinin be taken by in- 
habitants of infected zones in each of two successive days in each of six successive weeks.

There are other theories concerning relapses advanced by well-known malariologists and each has its adherents:

(1) By parthogenesis of female gametes (Schaudinn); (2) by conjugation of two young asexual forms, which later split into asexual parasites (Craig).

Christophers explains that the proportionate prevalence of the different forms of parasites in any community is dependent upon:

r. The activity of transmission, i.e., the number of infected anopheles.

2. Factors increasing or diminishing the number and continuance of relapses.

Malignant tertian, producing gametes, increases most extensively when transmission is active while quartan, producing few gametes but which has a propensity to relapse even after long periods of quiescence, increases when factors favoring relapse are most in evidence, even though transmission is low. Simple tertian lies between the two.

The most exact evidence of the state of a community with regard to malaria, is furnished by Ross' method of taking the endemic index by a combination of estimating the spleen rate in children and the parasite rate in peripheral blood. The first is more valuable because it is steady, seasonal variation of the disease making the parasite rate uncertain. The percentage of children with large spleen in a malarial district is one of the best bases upon which to depend not only for an estimate of the amount of malarial infection in the population but as a means of checking upon the efficacy of prophylaxis.

One of the most thoughtful studies on epidemiology is presented by $\mathrm{C}$. A. Gill. He states that a community acquires a relative partial immunity by repeated continuous inoculation with fresh parasites on immunity not long sustained in absence of reinfection. Such communities have little fluctuation in their collective immunity, as in tropical endemic zones among an indigenous population where the spleen rate is steady, the pernicious forms are not common, and fulminant epidemics are not to be expected. But in zones where transmission of infection remains in abeyance for long periods, "fulminant epidemics" may occur when that transmission becomes suddenly active. In such zones the spleen rate fluctuates quite violently.

The whole matter depends on the conservation of an equilibrium between the degree of immunity and the amount of infection. When one or the other is temporarily disturbed or lost, epidemics may be looked for.

The cultivation of the malarial parasite in vitro is another conquest in the last few years. This was accomplished by Bass by incubating ro c.c. of defibrinated infective blood at $40^{\circ} \mathrm{C}$. with o.I c.c. of a 50 per cent. solution of glucose. Thomson confirming 
his work emphasizes the fact that while well-grown plasmodium vivax does not tend to clump, plasmodium falciparum, in all save its youngest forms, does, a proof in vitro of the visceral capillary clogging from parasites and consequent gravity of malignant malarial fever. Bass has produced many generations by removing the leukocyte layer by centrifugation.

For local prophylaxis Giemsa recommends as a valuable adjunct to other measures the spraying of rooms and dark corners with the following to rid the house of adult mosquitoes.

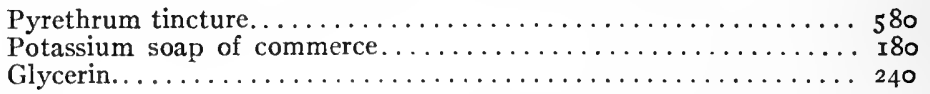

Mix with 20 times its volume of water and spray. Legendre recommends a conical net like a butterfly net to catch adults by hand in houses. It is said to be very efficient. Surgeon General Gorgas. epitomizes the sound elemental points in prophylaxis in his advice to Southern Rhodesia as a result of a study of malaria and blackwater fever there.

I. Selling quinin at cost price.

2. The use of daily prophylaxis given in 5 grain doses.

3. Mosquito proofing of houses.

4. Clearing and draining of all land within 200 yards of dwellings.

5. Use of mosquito nets.

Craig advises the following mosquito repellant for use on the skin :

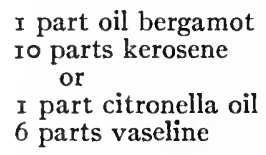

There are two suggestions for diagnosis which have become of recent importance.

I. Thomson has insisted upon the value of a periodic mononucleosis with leukopenia, roughly synchronous with the periodicity of malarial exacerbation, but in which neither parasites can be found in the blood nor symptoms of diagnostic importance noted.

2. The application of Schlesinger's reaction for urobilin present in malarial urine. The test is made as follows: Fill a test-tube onethird full of unfiltered urine. Add an equal quantity of Schlesinger's solution (I part of zinc acetate to ro parts of alcohol) previously well shaken. Then a few drops of a weak tincture of iodin. Filter. If urobilin is present there is fluorescence. The value of this test is only relative as it occurs in the urine of cases of cirrhosis. of the liver and many infective diseases but a negative reaction is quite eloquent as all malarial attacks seem to be marked by urobilinuria.

Quinin Treatment.-Complaint has with justice been made that. most text books give such an array of methods for the dosage and 
manner of administration of quinin that no one method is followed as a normal procedure. It is easily seen that variations in its administration must be required in individual instances but an attempt will be made here to enunciate what seems to be a safe routine practice leaving to the art of the individual physician the task of fitting the variation to the individual's peculiar case. Such cases should not be frequent.

If one achievement in American medicine should arouse in us a healthy pride it is the successful handling of the malaria problem in the Panama Canal by American physicians and it seems unnecessary for us to go vibrating confusedly from one to another person's method when this monumental work can furnish us with a routine practice in the treatment of malaria with every reason to consider the careful decision in method in Panama as final until a manifestly better means be demonstrated of controlling the disease. James states that the routine treatment in Panama is:

r. A preliminary purge of 3 to 5 grains $(0.2-0.3 \mathrm{gm}$.) of calomel followed in twelve hours by 2 ounces $(60$ c.c.) of a 50 per cent. solution of magnesium sulphate.

2 An initial dose of 20 grains ( $\mathrm{r} .3 \mathrm{gm}$.) of a liquid preparation of quinin, best made by adding a drop of hydrochloric acid to every 5 grains $(0.3 \mathrm{gm}$.) of quinin dissolved in 5 c.c. of distilled water.

3. Doses of 15 grains ( $1.0 \mathrm{gm}$.) of quinin in this same form three times a day for a week or until the temperature has been normal for from five to six days.

4. A subsequent reduction of this dose to 30 grains (2.0 gm.) a day for ten days more.

5. When after this treatment there are relapses use I. to $2 \mathrm{gm}$. of quinin in 300 to 500 c.c. of normal salt solution intravenously on two successive days and then continue with 45 grains $(3.0 \mathrm{gm}$. a day by mouth.

He states that when persistent vomiting is an obstacle to quinin by the mouth it may be injected into the tissue well diluted. $\mathrm{He}$ injects deep into the subcutaneous tissue $1.5 \mathrm{gm}$. of the bichlorid dissolved in 20 c.c. of normal salt solution. This seems, however, to give great pain and can be well replaced by intramuscular injections of small amounts.

There seems, however, no objection to giving quinin hydrochlorid in capsules for fastidious patients in the place of liquid preparations, remembering, however, that liquid forms seem more potent. A sweetened effervescent citrate of magnesia may be well substituted for the Epsom salts.

John Pelham Bates, also working in Panama, adds to this general plan a few remarks. He states that the average daily dose can be considered to be 30 grains $(2.0 \mathrm{gm}$.) but that when the case is grave 40 to 45 grains ( $3.0 \mathrm{gm}$.) daily for one to two days is permissible with a subsequent fall to the 30 grain $(2.0 \mathrm{gm}$.) dose.

In pernicious forms 60 to 90 grains $(4.0-6.0 \mathrm{gm}$.) may be given 
but no high dose of this kind should be carried beyond twenty-four hours.

The dose of quinin should be increased:

I. When there are large numbers of parasites in the peripheral circulation, bearing in mind that only a very few may be found in grave forms.

2. When presegmenting and segmenting forms of subtertian and quotidian parasites are seen in the peripheral blood.

3. When the blood exudes from a skin puncture with difficulty, is dark, clots quickly and spreads unevenly. One here is licensed to suspect a plasmodial capillary blocking or a malarial toxemia. Use an intravenous injection in such cases.

To prevent relapses count seven days from the last day of the last febrile attack and make that day the pivotal day of intermittent treatment as follows: 30 grains $(2.0 \mathrm{gm}$.) the day before the pivotal day, 30 grains $(2.0 \mathrm{gm}$.) on the pivotal day and 30 grains $(2.0 \mathrm{gm}$.) the day following. This should be continued eight weeks.

Bass calls attention to the fact that 50 per cent. of quinin is usually eliminated in twelve hours and nearly all in twenty-four. Hence successful treatment of malignant forms, which sporulate not in brood but irregularly, requires adequate doses every few hours. $\mathrm{He}$ is strongly opposed to injection into the tissues and states that when there is urgency to saturate the blood the intravenous method should be utilized. It is never necessary to give over 30 grains $(2.0 \mathrm{gm}$.) thus a day and never more than ro grains $(0.6 \mathrm{gm}$.) at a time as large doses are very dangerous. Even 20 grains (r.3 gm.) produces much shock and 50 grains $(3.3 \mathrm{gm}$.) has killed in several occasions. The best way to avoid these dangers is to give 5 grains ( $0.3 \mathrm{gm}$.) every four hours or to grains ( $0.6 \mathrm{gm}$.) every eight. Bates states that he does not believe in varieties of parasites inherently resistant to quinin, nor in the production of quinin-fast strains, nor that the condition of the patient prevents quinin from acting efficiently, nor that the virulence of the malarial parasite varies in different tropical countries. He further insists that a fever not yielding to quinin in five days is not malaria and cites his experience with 8000 cases. MacGilchrist whose able work on experimental pharmacology is well known makes a strong appeal for the more frequent use of the amorphous forms of quinin both for treatment and prophylaxis. He states that quinin is absorbed not from the stomach but from the intestine. His reasons for advocating amorphous quinin are as follows:

I. Its sparing solubility.

2. Its comparative tastelessness.

3. Its abundantly rapid absorption (as rapid as with a salt).

4. Its reduced bulk and weight.

5. Its absence of hemolytic action.

6. Its cheapness.

He states that $I: I 50$ is the strongest solution of a quinin salt that 
can be intravenously injected without danger of a thrombosis. As a matter of fact 7 grains of quinin bihydrochlorid should be dissolved in from 2 to 3 pints of normal salt. This author cautions against the use of acid salts of quinin as they seem to tend toward the production of hemoglobinuria.

Other Drugs, or Byproducts of Quinin.-Salvarsan and Neosalvarsan.-After a most confusing lot of testimony it may be fairly deduced that both successfully combat the benign forms and may be used when quinin is contraindicative.

Hectine is an arsenic salt of the general nature of arrhenal. Its use in malaria seems empirical and its results doubtful. Intramuscular injections of from to to 20 centigrams are advised. It is mentioned because so vigorously recommended by certain French writers.

The study of the quinin congeners by MacGilchrist has been accomplished in a most scientific and satisfactory manner. We find that the amount per 70 kilos of body weight of each drug necessary to cause the disappearance of a sexual form of benign tertian and malignant parasites from the peripheral blood is as follows:

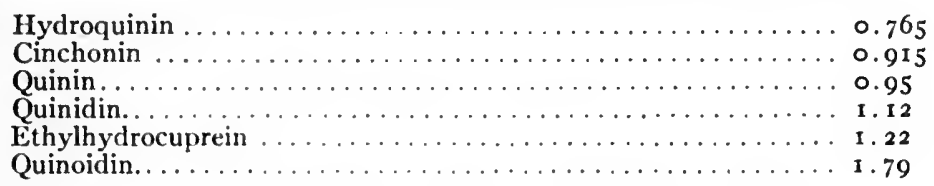

In every way hydroquinin seemed superior to quinin.

$\mathrm{He}$ makes the interesting statement that the antimalarial action of quinin is due to the residual part of the quinin molecule which appears to be a derivation of piperidin, a fission product of piperin, an alkaloid of black pepper. It should be remembered that three centuries ago black pepper was used with evidence of success in treating malarial fever. The results from the use of ethylhydrocuprein or optochin hydrochlorid are rather unlooked for, as clinical reports from a number of sources have been favorable in doses of $0.5 \mathrm{gm}$. t. i. d.

Recently Ross has led a vigorous campaign against the use of quinin in concentrated solution injected intramuscularly, stating that it is absorbed with far more difficulty than when given by the mouth and leaving us to infer that many cases of pernicious malaria for which such injections are used in all faith die from insufficient absorption of quinin. His following is larger and larger every day and numbers among its adherents some of the most noted malariologists. To these arguments can be added the danger of inducing tetanus, and the frequent production of necrosis and abscess.

That intramuscular injections of quinin should not be at present abandoned seems evident from the strong support given this method by good observers but wherever quinin by the mouth or intravenous administration for grave cases can be applied, there is little question 
that these methods should replace an indiscriminate application of intramuscular injection.

Hemoglobinuria is now being treated by intravenous injections of a 5 per cent. glucose or ro per cent. lactose solution. Splenectomy for persistent "ague cake" with cachexia has recently come to the front. It has been found by Rosati that the serum from the splenic vein in such cases is excessively hemolytic for erythrocytes the spleen being a dangerous hemolytic organ. Kopylow synthesizes the indications for splenectomy in malaria as follows:

I. In splenic rupture and torsion of the pedicle apt to occur in floating spleen.

2. In enlarged movable spleen.

3. In large, immovable, painful spleen in which medical treatment has failed.

\section{Principal References}

Bignami, Amico, South. Med. Jour., I9I3, vi, 79.

James, W. M., Jour. Infec. Dis., I9I3, xii, 277.

Bass, C. C., Interstate Med. Jour., I9I3, xx, 92I. South. Med. Jour., 1915, viii, 182. Bass C. C. and Johns, F. M., Jour. Exper. Med., 191 2, xvi, 567 .

Linnell, R. M. C., Trans. Soc. Trop. Med. and Hyg., I9r 5, viii, 239.

Thomson, David, Ann. Trop. Med. and Parasit., I912, vi, 215.

Thomson, J. G., and David, Ann. Trop. Med. and Parasit., I913, vii, I53.

Celli, A., Ztschr. f. Hyg. u. Infektions-krankh., I913, lxxv, I23. Ann. d'Ig. Sper., I914, xxiv, I77.

Christophers, S. R., Scient. Memoirs by Officers of the Med. and Sanitary Depts. Govt. of India, I9I 2, New Series No. 56, Calcutta, Superintendent Govt. printing, India.

Gill, C. A., Proc. 3d. All-India Sanitary Conference, held at Lucknow, Jan. 19-27, 1914, vol. 4, Papers suppl. to Indian Jour. Med. Research, p. I8.

Giemsa, G., Arch. f. Schiffs u. 'Trop. Hyg., 1912, xvi, 565 .

Legendre, J., Bull. Soc. path. exot., 1913, vi, 43.

Gorgas, Wm. C., South. Med. Jour., I9I4, vii, 687.

Bates, John Pelham, Jour. Trop. Med., I913, xvi, I77.

Atkinson, J. M., Lancet, 1913, June 28, p. I802.

Brown, Wade H., Arch. Int. Med., I9I3, xii, 315.

Craig, Chas. F., Office of the Surgeon General, U. S. Army, War Dept., Bull. No. 6, I914.

Wrightson, Wm. D., Am. Jour. Trop. Dis. and Prev. Med., I915, ii, 738.

MacGilchrist, A. C., Indian Jour. Med. Research, I9I3, i, 347.

Parrot, L. M., Rev. de med. et hyg. trop., 1913, x, 89 .

Bass, C. C., Jour. Am. Med. Assn., 1915, 1xv, 577 .

Wright, Thos. E., South. Med. Jour., I9r 5 , viii, 196.

Bates, John Pelham, Jour. 'Trop. Med., I913, xvi, 297.

MacGilchrist, A. C., Proc. of $3 \mathrm{~d}$ meeting of the General Malaria Committee held at Madras, Nov. I8, I9 and 20, I9I2, p. I7 (1913 Simla Govt. Central Branch Press) p. 97.

Bates, John Pelham, Jour. Trop. Med., I913, xvi, I45. Proc. Med. Assn. Isthmian Canal Zone, October, I9I2, to March, I9I3, v, Part 2, p. II 5.

James, W. M., South. Med. Jour., I913, vi, 347.
Rosati, Beniamino, Gazz. internaz. med. chir. igiene, Dec. I4, I912, p. II86, and

Dec. 21 , p. I 201 .

Kopylow, N. W., Arch. f. klin. Chir., I913, ci, 708.

Izar, G., and Nicosia, R., Berl. klin. Wchnschr., I 914 , li p. 385 , Ibid., p. 453 . p. 516.

MacGilchrist, A. C., Indian Jour. Med. Research, I9I4, ii, 3 I5. Ibid., p. 336. Ibid.,

Liefman, Emil, Therap. Monatsh., I9I5, xxix, 260.

MacGilchrist, A. C., Jour. Indian Med. Research, I9I5, iii, I.

Joyeux, Rev. de med. et d'hyg. trop., I9I 2, ix, 227.

Fontoynont and Razafimpanilo, Rev. de med. et hyg. trop., I9I3, x, 77. 
Werner, H., Deutșch. med. Wchnschr., r912, xxxviii, 2068.

Cestan and M. Pajol, Gaz. d. hop., I9r2, lxxxv, I793.

Memmi, G., and Cantieri, C., Riv. crit. clin. med., I913, xiv, p. x. Ibid.. p. 17. Ibid.. p. 33. Ibid., p. 49 .

\section{UNDULANT FEVER}

The widespread nature of what was generally known as "Malta fever" has compelled the use of the above more appropriate term as reports of endemic foci have been received from the most diverse parts of the tropical and subtropical world. The attention of most investigators seems to be chiefly centered in its diagnosis which is by no means as simple as it was considered to be when the agglutination test was first introduced. The symptomatology is suggestive but is not ordinarily susceptible of giving us a definite diagnosis without the assistance of laboratory methods. Indeed, the agglutination test has been the most feasible means of arriving at a conclusion which in view of recent studies seems to be all too frequently fallacious as it has been hitherto practised. A good example of this will be found in the report that Malta fever is to be found in Porto Rico. This error arose from a case in the hospital where I was stationed in I899, an assistant of recognized ability depending upon laboratory methods of diagnosis which today are not acceptable as positive proof. Inasmuch as this diagnosis has not been made from that day to this save in one instance open to the same objection, although agglutination tests have been repeatedly utilized, nor had it ever been made before, Porto Rico cannot be considered an endemic focus. In the first place serum reactions to the $M$. melitensis reveal great difference in strains even to the point that Negré and Raynaud have described M. paramelitensis, and Sergent and Zammit M. pseudomelitensis, neither of which, of course, can be depended upon for the diagnosis of true undulant fever. Not only this but non-specific agglutinins in normal persons and in those with a variety of diseases especially typhoid, typhus and tuberculosis are apt to seriously compromise accurate judgment. To surround the agglutination test with every safeguard the following requisites are to be exacted:

I. An organism not agglutinated by normal serum nor that of diseases other than undulant fever.

2. An organism always agglutinated by the serum from any case of undulant fever.

Negre and Raynaud state that heating the serum to $56^{\circ}$ for onehalf hour will rid it of non-specific agglutinins but this seems to destroy some of the specific agglutinins as well. Many authors look with favor on this method and state that with a specific organism and a high dilution $(I: 400)$ the agglutination may be fairly conclusive. 
P. W. Bassett-Smith states that the relative value of diagnostic tests is as follows:

I. Sero-culture.

2. Agglutination test in high dilution, $I: 400$.

3. Complement fixation.

4. Precipitin tests.

5. Cultures from the urine.

He adds that blood cultures often fail in the advanced cachetic stage.

While infected goat's milk is still shown to be the chief source of spread, mules, asses, oxen, cows, rabbits and fowls have been demonstrated to be carriers. Human carriers are far more important a source of spread than heretofore recognized. The germ is very resistant and laboratory infections have been common. Castellani and Chalmers consider the modes of infection to be grouped as follows:

I. By the alimentary canal.

2. By the respiratory system through inhalation of dust containing organisms from the urine of infected goats (and other animals and human carriers).

3. By the cutaneous system (inoculations) which is rare.

4. Sexual intercourse (possible).

They emphasize the high mononuclear count in differentials and state that while the average duration of the disease is about ninety days it may continue to three hundred.

No important addition to methods of treatment seems to have been discovered. Bassett-Smith recommends autochthonous vaccines and yeast therapy but the value of vaccines seems to be undecided. The dose of vaccine he employs is 100 to 500 millions and of yeast 2 drams.

\section{Principal References}

Castellani and Chalmers, Manual of Tropical Medicine, 2d Edition, New York.

Sergent, Edward, Paris méd., Aug. I, Ig14, p. 224.

Bassett-Smith, P. W., Jour. R. Naval. Serv., 1915, i, r66. Trans. Soc. Trop. Med. and Hyg., I914, vii, I27.

\section{ULCERA PUDENDUM}

In the course of the investigations made by the writer personally in Brazil in I9I6 some cases of the disease were seen, as well as results of treatment and the supposed causative organism. The disease is considered in Brazil to be caused by Calymatobacterium granulomatis. The ulcer generally lasts five to fourteen years untreated and at times spreads over buttocks, thighs and abdomen from its initial point in the genital region. It seems probable that in tartar emetic we have a true specific for this disease. It is administered intravenously in doses beginning with 5 and ending with Io or I 2 c.c. of a $\mathrm{x}$ per cent. solution in physiologic salt solution every other day. Twenty injections are usually required for a cure and often 
many more. Vianna and Aragāo first demonstrated the specificity of the treatment. The results seen in Brazil seem to fully justify their claims. H. C. Souza de Araujo, however, states that we may expect 12 per cent. of recurrences.

In this connection it is well to note that Octavio Torres of Bahia claims far better and safer results in leishmaniasis from freshly made solutions. The dose is frequently given in out-patient clinic in Brazil seemingly without the slightest danger and with the highest degree of efficacy.

\section{Principal References}

De Souza Araujo, H. C., Estudo clinico do granuloma venereo. Trabalho do instituto Oswaldo Cruz., Rio de Janeiro, Brazil.

Torres, Octavio, Reprint from Brazil Medico. "O Valor de Tartaro Emetico na leishmaniose," Communicaçăo a Sociedade Medica dos Hospitaes da Bahia, en Outubro de rgr4, e publicada no Brazil Medico. "Consideraçoes sobre o granuloma ulceroso" Communicaça a Sociedade medica dos hospitaes da Bahia, em ll de outubro de rgr4. Publicaçăo do Brazil Medico.

\section{VERRUGA PERUVIANA}

Heretofore verruga peruviana has been made to include Carrion's disease or Oroya fever by Peruvian physicians, the acute and often fatal fever marking the onset of what in favorable cases is believed by them to eventuate in verruga. Strong and others of the members of the First Expedition to South America of the Harvard School of Tropical Medicine in I9I3 in their report have demonstrated that these are probably two separate and distinct diseases, Oroya fever being caused by Bartonella bacilliformis, a protozoön found in the red blood corpuscle and having an apparent evolutionary stage in the endothelial cells of lymph glands, and spleen. It is not communicable to animals. Verruga, on the other hand, can be easily inoculated into monkeys and is probably a filterable virus, no organism being found. These dualist views are stoutly combated by the Peruvian School lead by Townsend who has also declared that verruga is conferred by the bite of the titira or a species of Phlebotomus.

There is no specific treatment as yet, but it would be interesting, if it has not already been done, to try intravenous injections of tartar emetic as in leishmaniasis and ulcera pudendum in which it has been found to be a specific.

\section{Principal References}

Strong, Richard P., Tyzzer, Ernest E., Sellards, A. W., Brues, Chas. T., Gastraburu, J. C., Report of first Expedition to South America, 1913. Harvard School of Tropical Medicine, Cambridge. Harvard University Press, 19r5, pp. 220. Well illustrated by colored plates and lithographs.

Townsend, C. H T., Cr6́nica med. (Lima), r9r3, xxx, 210. Bull. Entomolog. Research, rgr3, iv, r25. "Jour. Am. Med. Assn., r913, lxi, I717.

\section{LEISHMANIASIS}

Specific infectious diseases caused by protozoa of the genus Leishmania. The constitutional affection has been known by its 
Indian name of kala-azar; the cutaneous disease by the name of oriental sore.

\section{KALA-AZAR}

An endemic disease first studied in India but later shown to exist in various parts of the tropical and subtropical world and caused by Leishmania donovani, a protozoön of 2 to 4 microns by $11 / 2$ to 3 microns, and of elliptical shape. It is characterized by a round, lightly staining trophonucleus and a rod-shaped, deep red kinetonucleus (Romanowsky stains), the protoplasm being non-granular and faintly blue. The parasite is essentially intracellular and is generally found in the cells of endothelial type in the spleen, liver and bone marrow. At irregular times these organisms, by the disintegration of their containing cells are freed and taken up by the mononuclear and polynuclear leukocytes, being usually found only after long search in the peripheral blood.

Kala-azar slowly advances along common lines of communication. The vector, probably some biting arthropod, is as yet unknown, as is the animal reservoir. In 1907 Patton announced the discovery of developmental forms in the bug, Cimex rotundatus, but his vector has not yet been generally accepted. As leishmaniasis of dogs in endemic centers occurs in the Mediterranean form, it had been all but accepted that these animals were the natural reservoirs and that their fleas, in a few of which developmental forms were found, communicated the disease, but this theory has of late been seriously. shaken by recent experimental work. Donovan suspects the Conorrhinus rubrofasciatus.

The disease is characterized by persistently recurring two- to six-week periods of usually high fever, with at first two or three quotidian maxima; great hypertrophy of the spleen and, to an extent, of the liver; moderately severe anemia with an unusually marked leukopenia, relative mononucleosis, and diminished coagulability of the blood; great emaciation; and often a peculiar darkening of the skin. Kala-azar runs its course in from several months to two or three years and is very fatal, although it is probable that even in India some few recover spontaneously. Between the febrile periods are varying intervals of apyrexia with diminution in the size of the spleen and liver, but at last the fever becomes continuous and the patient dies of exhaustion, or, more commonly, of some intercurrent infection to which he is abnormally susceptible. Some of the less constant conditions are ulceration of the intestine with diarrhea, hemorrhages into the intestinal tract and skin, and edema of the lower extremities.

The disease above described is what has been known as Indian kala-azar but in the Mediterranean basin, notably in Sicily, Greece and North Africa a form exists (Mediterranean kala-azar) which has until recently been considered to be confined to very young children, with, however, a very similar clinical picture to that of the Indian 
form. It has been attributed to infection by a distinct species of Leishmania, L. infantum, but the identification of cases among adults of typical kala-azar in these endemic areas, the successful culture in vitro of both organisms which are morphologically indistinguishable, and the reproduction of the same disease by inoculation of experimental animals, is about to simplify the matter by affirming the identity of the two forms, remembering, however, that a possible difference in virulence or strain in the Mediterranean organism may explain certain undeniable epidemiologic and clinical peculiarities. The "ponos" of Hydra and Spezzia (islands of Greece) is stated to be leishmaniasis.

The diagnosis is only positive on finding the specific organism, although by attention to the clinical picture and by exclusion one can arrive at a fairly satisfactory working diagnosis.in endemic centers. The definite diagnosis must be made:

I. By examination of the peripheral blood. The edge of the specimen should be sought in order to get the greatest number of leukocytes. Long search may be successful but a negative result by no means excludes the parasite, at best accidentally in the blood. Intestinal ulceration and a late stage of the disease are said to favor a successful search.

2. By examination of the fluid from a blister raised by some vesicant.

3. By culture in each of a dozen NNN media tubes of drops of peripheral blood.

4. By spleen puncture; dangerous in diffluent organs with reduced coagulability of the blood.

5. By liver puncture, a safer procedure.

6. By etherization of the patient and aspiration of the bone marrow from the head of the tibia.

7. By removal of a small gland (postcervical) under local anesthesia and staining of smears.

Complement fixation and agglutination tests cannot be depended upon.

Treatment.-The patient should be well nourished and, if possible, promptly removed to some healthier region. Existing diseases should be treated and pathogenic intestinal parasites expelled. There is no known specific but very recently Di Cristina and Caronia have adopted Vianna's antimony treatment of dermal leishmaniasis for European kala-azar, with apparently excellent results, confirmed by Rogers' independent experience in ten Indian cases and by Castellani. Quinin in large and continued doses has until now been most generally employed. Castellani's method is the daily administration of $5 \mathrm{gm}$. of quinin, 4 by mouth and $\mathrm{I}$ intramuscularly throughout the afebrile period. It should be kept up in lesser dose in the afebrile period. All arsenical preparations have been individually lauded but none have found general acceptance save as hematinics. This, of course, includes atoxyl, salvarsan and hectine. 
Rogers bases his treatment on the following facts:

I. The patient succumbs not to the fever, but to a secondary infection.

2. The prognosis is bad in direct proportion to the reduction of the polymorphonuclear leukocytes.

3. Spontaneous, complete and permanent recovery occasionally follows noma and pneumonia, provided that there is a vigorous leukocytosis.

He states that sodium nucleate was a disappointment, iodine intravenously a failure, staphylococcus vaccine of only temporary value, and spleen tabloids alone productive of no visible benefit, but that the steady administration of alkalies by mouth, combined with injections of staphylococcus vaccine, and spleen tabloids by mouth is really efficacious.

Prophylaxis.- No one who has read Dodds Price and Rogers' article on segregation measures in Assam will fail to see the importance of place infection. The quartering of fresh coolies in new barracks only a few hundred yards from old infected quarters, where it appears the disease was raging and continued to decimate the occupants, preserved those quartered in the new, uninfected houses in health for sixteen years. Isolation should be insisted upon and disinfection of infected quarters practised. Dogs should be excluded from endemic centers, houses should be treated as if in a plague district as far as fleas are concerned, and great cleanliness should exterminate bugs. Screening with fine wire should complement these measures.

Oriental Sore (Dermal Leishmaniasis).-The large number of confusing names applied to this affection, over 30 being listed by Castellani, is due to its strict endemicity in certain localities and the comparative freedom from infection of others close by (aleppo boil); to its locally popular etiology (pascha-churdj, or fly-bite); or to unproven parasitic causes of times past (mycosis cutanea chronica). Historically it is an "oriental sore" but today it is known to exist in an ever-increasing number of points throughout the tropical world by the demonstration of its true parasitic cause, Leishmania tropica, Wright, 1903 , indistinguishable morphologically from L. donovani. This species is cultivated in the NNN medium and inoculations of animals have been successful. The organism can be demonstrated in the fluid and scrapings of the lesions. While accidental contact infection by inoculation of abraded surfaces and wounds, and auto-inoculation by scratching seems frequent, there is reason to believe that some biting insect conveys the disease. The lesions have been described (pages 7II7 I 2 , Vol. II) but recent reports show that at times it may invade the nose. Its incubation varies from a few days to over six months and some authors note constitutional reaction at its onset.

Brazilian Dermal Leishmaniasis.-This is a clinical variety of oriental sore although the organism is apparently identical and the 
skin lesions the same (Splendore); there is a tendency for it to invade the nose, pharynx and mouth and present 'a frambesial type, la espundia. Brumpt and Pedroso saw sixty-five cases in three weeks in the wooded districts of Sao Paulo and propose the name "American forest leishmaniasis." They state that clinically 90 per cent. of the cases are mild but ro per cent. are malignant. The sores appear on exposed parts of the body and at certain periods of the year. Exposure is most certain in clearing virgin forests and they seem to favor a Tabanid as the vector. That the disease is by no means limited to Brazil is seen by Escomel's report from Peru. Migone describes it in the lumber forests of northern Paraguay as beginning with one or more erythematous papules which itch and are invariably attributed to insect bites. Two or three days later these become pustules and, the latter bursting, leave a little hole from which exudes a mucilaginous liquid which when dried forms a grayish scab. The ulcer attains a diameter of from I to $2 \frac{1}{2}$ $\mathbf{c m}$. producing neighboring edema. Hard, reddish cords, often nodular, map out lymphatic vessels extending therefrom to their glands which are enlarged and tender. These ulcers heal after seven to eight months but the glands remain enlarged. In from five to six months from the original inoculation the nasopharyngeal lesions may present themselves. The septum is often destroyed and ultimately even the nose itself with the formation of large granulomatous masses. This may extend to the cheeks and lips and even the isthmus of the fauces, rendering speech and deglutition difficult. The patient may live for twenty years with this gradually extending infiltration until sepsis, malnutrition and exhaustion cause death. Migone states that of roo laborers in some endemic timber areas, from seventy to eighty have to leave in a couple of months. The appearance of the sores is frequently accompanied by irregular fever and there is mononuclear leukocytosis of from 12,000 to 16,000 . No parasites are found in the blood (Splendore).

The large number of cases seen in the hospitals of Bahia and Rio de Janeiro entitle one to pronounce this form of leishmaniasis one of the most mutilating naso-pharyngeal processes and a very serious menace to country labor in Brazil. The pathogenic action is apparently limited to cartilage and soft tissues and no involvement of bones is seen. No flattening of the nasal bridge was noted in a large number of cured cases, a point of distinction clinically between this and the nasal involvement caused by leprosy and syphilis.

Treatment.- In addition to many topical remedies used in the treatment of all ulcers, Wenyon lauds the ointment of Cardamatis and Melissidis consisting of equal parts of methylene blue, lanolin and vaseline. He was unsuccessful in vaccinating patients with killed cultures of L. tropica. The Brazilian school, first voiced by Vianna and warmly supported by the testimony of Terra, Piraja da Silva, Lapa and others, counsels the use of tartar emetic, I per cent. 
solution intravenously in distilled water or physiologic salt solution, in doses of from 5 to ro c.c. daily or on alternate days. Sterilization is accompanied by filtering through a Berkefeld filter in the cold.

That intravenous injection of tartar emetic is a specific in this disease is evident from personal observation but as many as two hundred injections may be required to effect a cure,

\section{Principal References}

Castellani, A., and Chalmers, A. J., Manual of Tropical Medicine, rgr3, 2d Edition. Leboeuf, I9II, Traite de pathologie exotique, Grall et Clarac, I9II, ii, 309.

Patton, W. S., Sci. Mems. by Officers of the Med. and Sanit. Depts. Govt. of India, New Ser. No. 53 , I912.

Jemma, R., Riforma Medica, I9r2, xxviii, 925.

Di Cristina, G., Pathologica, rg1 2 , iv, 409 .

Di Cristina, G., and Caronia, G., Pathologica, I9I 2, iv, 519. Ibid., p. 534. Ztschr.

f. Kinderheik., I913, ix, I 28.

Splendore, A., Bull. Soc. path. exot., I9I2, v, 4 II.

Wenyon, C. M., Jour. Lond. School Trop. Med., I912, i, 207. Ibid., p. 224. Trans.

Soc. Trop. Med. and Hyg., 1914, vii, 97.

da Silva, Pirajá, Arch. d. Parasit., r912, xv, 401.

Nicolle, Ch., Arch. d. l'Inst. Pasteur, Tunis, I9I 2, No. 4, p. 2 Ig.

Migone, L. E., Bull. Soc. path. exot., I913, vi, I18. Ibid., p. 210. Trans. Soc. Trop. Med. and Hyg., I9I 5, viii, 2 I9.

Basile, Carlos, Atti della Reale Accad d. Lincei, Rendiconti, April 6, 1913.

Donovan, C., Proceedings $3 \mathrm{~d}$ Meeting Gen. Malaria Committee, held at Madras,

in November, I9I 2, p. 211 .

Cochran, S., r913, Jour. Lond. School Trop. Med., r913, ii, I79.

Da Silva, Pereira, Arquivos do Instit. Bact. Camara Pestana, I9I3, iv, I47.

Price, J. Dodds and Rogers, Leonard, Brit. Med. Jour., Feb. 7, I9I4, pp. 285-289. I-I4.

Lemaire, G., Sergent, Ed., and Lhéritier, Rev. méd. d'Alger, January, I914, pp.

Carini, A., Bull. Soc. path. exot., I914, vii, 277.

Terra, F., 4, Bol. da Soc. Brasileira da dermatologia, I913, ii, 58.

Mackie, F. Percival, Ind. Jour. Med. Res., IgI 5, ii, 942.

Rogers, Leonard, Ind. Med. Gazette, I915, 1, I63.

Castellani, A., J. Trop. Med. and Hyg., I915, xviii, 112.

Brumpt, E., and Pedroso, A., Bull. Soc. path. exot., I913, vi, 752.

\section{THE SPIRIILOSES}

The spirilloses to be included under this caption are the relapsing and the tick fevers. While the virus of widely separated regions produces differences in clinical details and especially in virulence, all of these fevers bear a resemblance so close as to permit of their being considered together. Thiroux considers it more logical to divide these spirilloses according to their vectors into:

I. Spirilloses transmitted by the tick.

2. Spirilloses transmitted by some other ecto-parasite (now being generally conceded to be the louse).

Accordingly the first group contains:

(a) The tick fever of West Africa (Koch virus).

(b) The tick fever of East Africa (Dutton virus). Both due to Sp. duttoni.

(c) The tick fever of Columbia.

The second group contains: 
(a) The recurrent or relapsing fever of Europe due to Sp. obermeieri.

(b) The recurrent fever of America due to Sp. Novyi, said by Darling to include the recurrent fever of Panama and Columbia.

(c) The recurrent fever of Bombay due to Sp. Carteri.

(d) The recurrent fever of Algeria due to Sp. berbera.

(e) The recurrent fever of Tonkin (Indo-China).

The differential characteristics, biologically, between these spirilla is today based most safely on agglutination phenomena, as the morphology and the receptivity of laboratory animals to inoculation are often fallacious. Representatives of all these species have been cultivated by Noguchi. Clinically they all present an incubation of a few days to a week, a primary febrile period of several days to a week, an afebrile interval of a week or more, a secondary febrile period of shorter duration and of generally increased violence, and a slow convalescence. Other relapses can occur, but rarely. The fever is usually high, begins abruptly with a chill, and is continuous, with a fall by crisis followed by profuse sudoration. The spleen is enlarged. Typhoid states, icterus, and grave complications may develop. The parasites can usually be demonstrated by direct examination of the blood during the fever but not in the interval. All seem promptly amenable to salvarsan.

Group I (a)-Recurrent Fever of West Africa.-The vector has been shown to be the Ornithodorus moubata. Inoculation of man is not due to the bite itself but to its soiling by the animal and the inoculation of the virus by scratching. Great pain in the spleen and violent vomiting may be observed. The second febrile period lasts from three to four days and there is a return of the parasites to the blood. Successive relapses may occur even to the number of eleven. Severe headache and pains in the limbs and body, great restlessness and frequently delirium are prominent. The patient emerges from his active symptoms much prostrated and emaciated.

(b) Recurrent Fever of East Africa.-No material differences require mention.

Group $2(a)$-Relapsing Fever of Europe.-This need not be considered as it is not a disease of the tropics. Its general characteristics are expressed in the introduction and it is of usually a more benign type, the mortality being about 5 per cent. A second relapse is rare.

(b) Relapsing Fever of America.-This is not unlike the European form. Its mortality is between 2 and 6 per cent.

(c) Recurrent Fever of Bombay.-In this form, instead of the state of well-being experienced in the interval by the sufferer from the European form, there is little improvement in the general condition, and even collapse similar to that seen in cholera may occur at the fall of the temperature.

The varieties of this form are (I) a short, irregular, intermittent fever, '(2) the so-called "bilious remittent," an icteric form. The 
first is a form resembling malaria and only to be distinguished from it by an examination of the blood; the second is a form resembling typhus or something akin to yellow fever with deep jaundice, and a macular eruption. The typhoid state is frequently seen in this form which gives a mortality of about 70 per cent. The prognosis of the Bombay relapsing fever is rather serious, the mortality being about I8 per cent. Of evil omen are a prolonged, irregular fever with descent by lysis, and icterus. While salvarsan is, as in other forms, the drug par excellence, early stimulation should be resorted to and preparations made to meet sudden collapse.

(d) Recurrent Fever of Algiers.-The incubation is about twelve days and the disease is of milder type. A notable feature of this form is its tendency at times to give the picture of a cerebrospinal meningitis without, however, spirilla being demonstrable in the cerebrospinal fluid (Ardain-Delteil, Raynaud, Coudray, and Derrier). It appears proven by Nicolle, and confirmed by Sergent and Foley, that the organism undergoes development in the louse, finally becoming infective in its colomic cavity. He shows that lice are not infective until the eighth day after ingestion of infected blood and that by the nineteenth they lose their power of conferring the disease. ${ }^{1}$ The mere contact of the virus with a healthy mucous membrane suffices to infect man (Edm. Sergent). Conseil states that of 108 untreated cases, seventeen had one febrile period, seventy-nine had two, nine had three, and three had four. The average course consists of two febrile attacks of five or six days with a ten-day interval. Fiftythree of r6o cases had jaundice. The sufferers from the Algerian type, according to the same author, bear their high fever and pulse well and seem to show little debility or prostration.

(e) Recurrent Fever of Tonkin.-This is usually a very severs form. The general mortality is 27 per cent., but in certain epidemice rises to 50 per cent. and over. There is a tendency to icterus which appears on the third to the fifth day and it is of bad import. Vomiting is apt to be severe and is at times uncontrollable. Delirium and typhoid states are common. Death may occur from myocarditis, anemia, hemorrhage, etc. There are three types: (I) the simple in which icterus is observed and the prognosis is good; (2) the typhoid; and (3) the typhobilious, with icterus and often hemorrhages. Onequarter of the cases reported by Gaide, Mathis and Leger were of the typhobilious form and were very fatal.

\section{TREATMENT}

The only drugs worthy of serious mention are salvarsan and neosalvarsan and most authors are unanimous in demonstrating that they act like specifics. Salvarsan must be used in small doses as it

\footnotetext{
${ }^{1}$ The bite of the louse is not infective but inoculation of the infective forms in the cœlomic cavity of the insect is caused by the scratching and crushing of the louse by the victim. The blood is infective for animals in the afebrile period although no organisms can be found by direct examination.
} 
seems to be especially dangerous in these cases, a number of deaths being attributed to it. The minimum efficient dose scems to be from 0.20 to $0.25 \mathrm{gm}$.; it should be administered intravenously. The immediate effect is an aggravation of existing symptoms with a rise in the fever but the disease is usually cut short in twenty-four hours and most authors state that relapses do not occur or are at least rare. Primet, however, while reporting favorably upon salvarsan with which he states he has reduced the mortality from 40 to 3 per cent., gives the idea that relapses are common irrespective of the dose employed. Levaditi proved by animal experimentation that much smaller doses wcre required and a more rapid and favorable effect secured by the administration of salvarsan only in the first "precritical period." This was confirmed clinically by Foley and Vialatte in Algiers, who, however, used 0.05 to $0.1 \mathrm{gm}$. of neosalvarsan per kilo of body-weight. No other arsenical preparation has been found to compare with these two drugs. Galyl and ludyl, however, are considered by Conseil to act more favorably. Antimony seems to be practically of little or no value (Conseil).

Results of Treatment.-Hermant treated fifty-one cases by salvarsan, with four deaths, but the rest were promptly cured. Mouzel reports 373 cases so treated, forty-one by intrarectal and 332 by intravenous injection with only six relapses. Twenty-two died but in the rest from eight to eighteen hours sufficed to dissipate the symptoms and four to five days to put the patient on his feet. It is stated that the mortality was reduced from 4.16 to $I .7$ per cent. and the stay in hospital 66 per cent.

Conseil used salvarsan and neosalvarsan, eleven cases treated by each. With the first all were subjects of a violent reaction, the patients being completely prostrated for from five to twenty hours but ten rapidly recovered from their disease. Neosalvarsan gave a less severe reaction and as good results.

Perthuisot reports from Indo-China in r9i I a mortality of 69 per cent. before salvarsan was employed, in 1912 when it was introduced 7.6 per cent., and in $1913,4.2$ per cent. He never had to give a second injection.

Prophylaxis.-In regions where the spirilla belonging to group 2 cause relapsing fever, general hygienic measures should be instituted and special measures taken to reduce lice. Of self-evident importance are those governmental measures tending to combat the poverty and misery of the masses who usually harbor lice. The tick fevers of group $\mathrm{r}$ are true endemic diseases, and the general and special prophylaxis for these ecto-parasites will here obtain.

\section{Principal References}

Castellani, and Chalmers, Manual of Tropical Medicine, 1913, 2d Edition.

Thiroux, A., Traité, Pratique de pathologie exotique, Grall. et Clarac. I9II, ii, 277.

Gaide, Mathis, C., et Leger, M., Ibid., p. 282.

Nicolle, C., Blaizot, L., et Conseile, Compt. rend. Acad. d. sc., I91 2, cliv, r6 636 . Ibid., clv, 481. Arch., d. l'Inst. Pasteur, Tunis, I9r 2, No. 3, pp. r ro-i I 2. 
Nicolle, Charles and Blaizot, L., Arch. d. l'Inst. Pasteur, Tunis, rgr 2, p. 2ór.

Hermant, Bull. Soc. méd. chir. de l'Indochine, I912, iii, 418.

Mouzels, P., et Nguyên =Xuan-Mai, Ibid., p. 427 .

Legendre, J., Bull. Soc. path. exot., r912, v, 339.

Conseil, E., et Bienassis, E., Bull. Soc. path. exot., I9r2, v, 476.

Conseil, E., Arch. d. l'Inst. Pasteur Tunis, r9r3, p. 31 and p. 67.

Levaditi, C., Bull. Soc. path. exot., rgr 2, v, 524 .

Noguchi, H., Jour. Exper. Med., rgr2, xvi, r99.

Paucot, M., Bull. Soc. path. exot., r9r3, vi, 68.

Sergent, Edm., Compt. rend. Soc. d. biol., I9r3, lxxv, r85.

Perthuisot, Bull. Soc. méd.-Chirug. de l'Indochine, I9r4, v, I8.

Ardin-Delteil, Raynaud, M., Coudray and Derrieu, Bulls. et mems. Soc. méd. des hôp. de Paris, 19r4, xxx, Ir26.

Wm. Lien Teh (G. L. Tuck,) North Manchurian Plague Prevention Service Reports (r9r1-1913), London; Cambridge Univers. Press, r914, p. 98.

Darling, S. T., Trans. xvii Intern. Congress of Med., r9r3, Part 2, p. 279.

Primet, ror 3 , Ibid., p. $28_{3}$.

Foley, H, and Vialate, C., Bull. Soc. path. exot., I914, vii, 569.

Sergent, Edm., and Foley, H, Compt. rend. Acad. d. sc., I914, clviii, r926.

\section{BERIBERI}

"Beriberi is an acute or chronic disease characterized by changes in the nervous system and particularly by a multiple peripheral neuritis with an especial tendency to attack the nerves of the limbs, the pneumogastrics and phrenics $* * *$. It is a disease resulting from faulty metabolism, usually only seen in those persons who eat rice as the staple article of diet and is directly caused by the deficiency of certain vitamines in the food" (Vedder).

There are three principal foci: (I) in Asia (Japan, certain parts of China and India, the Philippines, Dutch East Indies and Malay States), (2) in Africa, and (3) in South America (northern and eastern coast).

The milder forms are characterized by weakness, heaviness, stiffness and numbness of the legs, with cardiac palpitation, and an oppression or pain in the epigastrium; the more severe forms by anesthesia and pain in the legs with motor weakness, generally extending over the body and deepening to paralysis with extreme emaciation and atrophy of the muscles (the dry form), or by weakness and atrophy of muscles with edema (the wet form). In either case cardiac palpitation and dyspnea may bring the patient to the verge of death. The three main conditions are: (I) cardiac insufficiency; (2) peripheral neuritis; and (3) edema. The motor disturbances begin by partial or complete paralysis of those muscles supplied by the anterior tibial and peroneal nerves and in well-developed cases extend all over the body. The sphincters are not involved and the sensory symptoms are varied. A characteristic anesthesia over the tibia is noted. Hypertrophy of the right heart and pericardial effusion of moderate degree are frequent. Aphonia is of bad import as the pneumogastric is thus shown to be seriously affected. The general mortality is 5 per cent.

Etiology. - While the excessive preponderance of polished rice in a dietary is responsible for most outbreaks, a deficiency of the necessary factors, or vitamines, in a variety of foods may lead to the development of the disease. The husked rice grain consists of (I) an 
outer layer, the pericarp; (2) the aleurone layer, thicker and containing much fat; and (3) the starch granule body. In these two outer layers is contained the vitamine, a deficiency of which brings about beriberi. The usual milling of rice terminated in a polishing process which removes all but a negligible part of these first two layers, so that the use of polished or highly milled rice must be substituted by undermilled rice or grains not deprived of these layers in order to prevent the disease. The best indicator of the amount of pericarp in the rice grain is a calculation of the phosphorus pentoxid. Rice having less than 0.4 per cent. of this substance should be rejected for food in beriberi districts. A practical method within reach of most persons is the staining of the grains by Gram's solution; they are then washed and dried. The pericarp will be gray and the denuded parts of the grain will take on a dark blue or black color. The addition of barley, beans and fresh vegetables will also combat beriberi. A vitamine-free food (polished rice diet) will produce beriberi in man in about ninety days. Animals have likewise been made to reproduce the symptoms in a similar manner. Polyneuritis gallinarum has been experimentally produced by feeding polished rice to fowls and is considered by Vedder to be identical with beriberi. The same author so considers infantile beriberi, ship beriberi and epidemic dropsy. He insinuates, however, that dry and wet beriberi are distinct diseases and produced by the lack of different vitamines both contained in rice polishings, or the shed outer layers. Funk, who first demonstrated the vitamines of rice, found a crystalline base which Vedder seems to believe alone cures dry beri-beri, although the. wet form and the cardiac disturbance is relieved in a few hours by the extract of rice polishings which contain both vitamines. So far Funk's base has not been produced in sufficient quantity to make it available for routine use. Vedder states, "Peripheral neuritis per se cannot be the essential lesion in beriberi because degeneration of the nerves occurs before symptoms arise, because advanced degeneration may be present accompanied by no symptoms at all, and because degeneration of the nerves remains long after recovery has occurred." He believes that in dry beriberi the cause of the symptoms is found in degenerations affecting the entire nervous system but particularly the cells of the cord and of the brain. Funk's vitamine "is a building stone which is essential for the normal metabolism of nervous tissue." He thus explains rapid cures in supplying this life-giving factor and explains that as a rule only from Io to I 5 per cent. of the fibrils of affected nerves are degenerated. He seems to believe that infantile beriberi and ship beriberi are diseases differing from dry beriberi in that in the latter there is a deficiency of a vitamine represented by Funk's base. Infantile beriberi shows no paralytic phenomena and there is a pronounced tendency to oliguria. It is the chief cause of mortality in the Philippines and in the vast majority of cases the mother of the suckling child is, or was, a subject of the disease beriberi. Extract of rice 
polishings often produces sensationally rapid cures and gradual benefit is noted by substituting fresh animal milk. Puppies fed on the milk of women whose suckling infants had died of infantile beriberi also died of the same disease.

The case against ship beriberi which Funk classifies with scurvy is a little less clear. Here again polyneuritis is not common, but certain vitamines are evidently deficient, whether rice is or is not a chief article of food, because fresh meat and vegetables prevent and often cure the disease. Some inconsistencies seem to exist in the evidence against epidemic dropsy, so far as it relates to the absolute identity of this disease and ordinary beriberi. Here fever and skin eruptions are superadded to the clinical picture, although the addition of vitamines to the diet appears to control it.

Funk groups together beriberi, polyneuritis of birds, epidemic dropsy, scurvy, ship beriberi and pellagra as deficiency diseases. All present cachexia and marked nervous symptoms and all can be prevented and cured by certain protective substances, the vitamines. The inference to be drawn from the observations of this and other authors is that the vitamines curative of these various conditions differ. Interesting conclusions by these authors may be summarized as follows:

I. The addition of carbohydrates, especially starch and sugar, to a standard dietary hastens the onset of beriberi.

2. Vitamines play a more important rôle in starch metabolism than any other constituent of the dietary.

Treatment.-For the majority of mild and chronic cases a liberal and varied diet with an abundance of fresh meat and vegetables, especially beans and peas, will be sufficient to cure. No canned or overcooked food should be allowed as vitamines are destroyed by very high temperatures. Undermilled rice may be permitted. For the cardiac crises give the extract of I kilo of rice polishings daily (for method of preparing see Vedder's book, "Beriberi") a dose of about 2 ounces of liquid. This will also produce very rapid effect in cases with edema.

Drugs are only needed for definite symptomatic conditions. Constipation in the edematous forms is best relieved by salines. Digitalis is of great value in cardiac crises and even bleeding may be practised in acute dilatation. Muscular cramps are relieved by the bromids. Strychnin is an excellent general tonic.

The patient should remain out of doors and give unaffected muscular groups sufficient mild exercise to prevent the atrophy of disuse. When, however, a patient shows a tendency to cardiac involvement he should be put to bed and reliance should be placed upon electrical stimulation and massage.

In infantile beriberi, the child should be temporarily removed from the breast, given animal milk, and a daily dose of the extract of $100 \mathrm{gm}$. of rice polishings. By treatment of the mother, breast 
feeding may be resumed later but the administration of the extract of rice polishings to the child should be continued. rice.

Prophylaxis.- I. Education of the public to demand undermilled

2. The taxation of highly milled rice by international agreement.

\section{Principal References}

Vedder, Edward B, Captain, U. S. Army, Beriberi, Wm. Wood \& Co., pp. 427, I913. Shiga, K., Centralbl. f. Bakteriol. I Abt., IgI 2, liv, I56.

Funk, Casimir, Jour. Physiol., I912, xlv, 75. Brit. Med. Jour., Sept. 28, I9 2 , p. 787. Jour. Physiol, rgr3, xlvi, 173. Hoppe-Seyler's Zeitschr. f. Physiol. Chemie, 1914, Ixxxix, 373 and 378 .

Fraser, H., and Stanton, A. T., Lancet, 1912, p. I005.

Eijkman, G., I913, Trans. xvii, Internat. Cong. Med., London, Section XXI, Trop. Med. and Hyg., Part I, p. 25.

Wise, K., and Minett, E. P., r913, Report of Trop. Dis. Research in the Government Laboratories of British Guiana for six months, I912-13.

\section{SPRUE .}

A chronic, probably infectious disease of warm climates, characterized by an atrophic inflammation of the mucous membranes of the digestive tract, atrophy of the liver, excessive production of gas in the bowel, and a white or light-colored, abundant, foul-smelling, frothy diarrhea, a marked tendency to the production of a cachectic state, termed by Carnegie Brown, "marasmic toxemia." Bahr's classification of complete and incomplete sprue cannot be too strongly insisted upon. The complete form presents the four cardinal conditions noted in the definition above. Incomplete sprue is divided into (I) intestinal sprue and (2) tongue sprue, whose mere designations are sufficinetly descriptive.

Unlike beriberi which has been demonstrated to be a deficiency disease, sprue is notable in choosing its victims from the highest walks of life where a varied diet is assured, as well as from the ranks of the less fortunate poor. Its ravages are singularly accentuated among the whites of Northern birth but it is also a serious disease among the indigenous population. In Porto Rico, at least, it is practically limited to towns and cities and seems to be very rare in the country where bread is infrequently eaten.

Le Dantec in his description of sprue in 1900 states that it is impossible to disregard the great preponderance of yeasts in the stools when considering the etiology of the disease. Kohlbrugge also calls attention to yeasts in his cases. Bahr studying sprue in Ceylon (I9I 2-I9I4) draws the following conclusions in his report, Researches on Sprue in Ceylon, I9I 2-I9I4, pages I IO-III:

\footnotetext{
"I. Sprue is a specific disease of tropical and subtropical countries, though it is possible that cases occasionally originate in temperate zones.

"2. It is a prevalent disease in Ceylon, especially among the Europeans. Contrary to the opinion hitherto held, it occurs among the natives irrespective of race or mode of life.

"3. This fact, together with the occurrence of the disease in people closely associated
} 
with one another, suggests some specific local influence or some communication of the specific cause from man to man.

"4. Sprue is a variable disease. It may occur in a mild or in a particularly virulent form, and, in common with many other serious diseases, is prone to sudden exacerbations, remissions and periods of latency.

"5. There is evidence that the disease may occur as distinct clinical forms according to the part of the alimentary canal attacked.

" 6 . Researches on the composition of the stools point either to a complete absence or to inadequacy of the intestinal digestive ferments.

"7. Researches on the blood and on the urine are in favor of regarding certain of the more important clinical features of sprue as dependent on an alimentary toxemia.

" 8 . The pathological findings are also in favor of such a conclusion and, if anything, point to an infection with the thrush fungus (Monilia albicans) as being the cause."

Castellani, after a study of yeasts found in sprue patients, states that their great variety negatives the constant presence of any one species and, while recognizing their power to cause some of the symptoms such as excessive gas production, does not believe them responsible for the disease, considering, with Chalmers, that the geographical distribution of sprue would point rather to some unknown protozoön. It should be noted, however, that Castellani's species of Monilia seem to be made on cultural differences in varying sugar media, that author considering as characteristic the fermentation and changes in reaction of the mesdium.

In this connection it is of great importance to record the evident fact that sprue probably does not exist in Brazil. Personal examination of many patients and observation of many others in hospitals in that country failed to reveal even a suspicious case. Moreover not one of the many excellent clinicians interviewed had ever seen a case.

Ashford, working independently, I908-I9r6, upon a suspicion expressed early in I9I 3 in correspondence, and based on epidemiologic observations, that bread might harbor a contaminating specific yeast pathogenic for man and causative of sprue, found in I9I4 an apparently new species of Monilia, not M. albicans, in a typical case of sprue. His conclusions are as follows:

\section{(a) Clinical:}

I. Sprue is usually a mild disease with a veiled picture in which intestinal fermentation is usually present, a tendency to spontaneous cure and a ready submissiveness to a non-carbohydrate diet.

2. Tongue lesions are often clinically (save absence of white membrane) and histopathologically indistinguishable from ordinary thrush, a disease due as a rule to Monilia albicans.

3. Clinically and histopathologically the picture of the tongue is projected on through stomach to intestine.

4. Chronic intoxication supervenes after well-developed sprue, and the liver atrophies without cirrhotic changes, secondary anemia making its appearance.

5. The intestinal lesions produce large, acid, frothy, white stools with excessive gas production, and full of yeasts. The character of these stools does not warrant the belief that serious ulceration takes place.

6. There is a tendency to chronicity and to periods of latency in which decided betterment or apparent cure may take place.

7. Drugs are of little avail save when used symptomatically for definite clinical crises and no specific has yet been found.

(b) Epidemiological:

I. Sprue is a disease of towns and cities where bread is a staple food (the author is referring to sprue as it exists in Porto Rico). 
2. Sprue is apparently rare in the country districts where bread is not at least a daily food and where it is eaten only at long intervals.

3. Family endemics are noticeable.

4. There seems to be a racial predisposition to sprue in persons of northern birth.

(c) Biological:

I. A Monilia, not heretofore described, and of undetermined species is apparently constantly found in cultures from the inflamed tongue or feces of persons suffering from sprue in Porto Rico.

2. This Monilia has been found in the center of a cooked loaf of bread baked in an endemic zone.

3. This Monilia has been found in certain cases of fermentative disturbance of the intestine in which the complete picture of sprue is not developed.

4. The Monilia is found in only 3 per cent. of persons, apparently healthy or, at least, free from any gastrointestinal disturbance.

5. The complement fixation test is positive for all of the reduced number of cascs of complete sprue in which it has thus far been applied and negative for several times that number of persons without sprue.

6. This Monilia is not only productive of mycotic septicemia in small laboratory animals injected with virulent culture but will produce severe and intractable mycotic ulcers in their tissues when they are relatively immunized to the extent of preventing a mycotic septicemia.

Recently Ashford has produced a hypervirulent strain of his Monilia by passage through guinea-pigs, white rats and rabbits which has on feeding healthy animals caused severe and long-continued diarrhea, loss of weight, marked stomatitis, and in some animals, rapid death from a monilia-pneumonia.

Here the matter rests awaiting confirmation.

Treatment.- So far no specific has been found. Santonin and emetin hydrochlorid are alike impotent to influence the disease save in that they may relieve the unconscious sufferer of co-existing ascarides, on the one hand, or Entamebre histolytica on the other.

The dietetic treatment alone has universally commended itself to practitioners in the tropics. The basis of success seems to be the complete removal from the dietary of certain sugars and starches. The Monilia described by Ashford ferments and is stimulated to its most active development by glucose, levulose, maltose, saccharose and galactose. It ferments no other sugars.

The Strict Milk Diet.-After a preliminary purge, preferably castor oil, the patient is instructed to take 8 ounces of milk every two hours on nine occasions each day for four days. During the next four days the dose will be 9 ounces, and thus it increases until after four days at I 3 ounces per dose, the diet can be modified by a reduction of milk and substitution for the amount reduced of a quantity of fruit, fresh vegetables and meats equal to or exceeding the nutritional value of the fraction of milk suppressed.

The milk should be taken cold or very slightly warmed; it should be sipped slowly or taken through a straw; and the time allotted for each ration should not be less than ten minutes. All milk used in Porto Rico has to be boiled but the self-evident value of fresh milk needs no special encomium. Such patients should avoid exertion while on this diet but need not necessarily be kept in bed unless in the more advanced stages of the disease. Experience has taught that the admixture of 3 c.c. of a culture of the Bulgarian strain of Vol. IV. -24 
the lactic acid bacillus to each feeding is of value and in those who can tolerate milk curdled by this organism the results of treatment by a strict milk diet are even better. Castor oil in small dosage should be administered, with lemon juice to disguise the taste, twice a week and strychnin may be required for its general tonic effect.

Thus from the first period of four days when the ration yields 1462 calories daily there is a gradual increase to 2375 on the twentyfifth day. An important addition to this diet may be made after the tenth day in selected cases by permitting weighed quantities of fruit. Strawberries seem to hold first place among English authors but Ashford has secured apparently as felicitous results with the banana. Each banana of the current variety weighs about $2 \frac{1}{2}$ to 3 ounces and a pound gives a nutritional value of 480 . The amount of fruit should be gradually increased as is the milk. Pineapples and alligator pears (aguacates) should not be employed.

The strict milk diet is really the best treatment for sprue and after the first month fresh vegetables not too high in starch, other fruits, salads and animal food may be cautiously added to the dietary until the diet has become quite liberal. Sugars, however, should be withheld for six months or more.

The Strict Meat Diet.-When milk cannot be employed, meat may be used. The latter can rarely be tolerated over a week, but its stimulating effect and its happy effect upon the diarrhea in many cases commends it alone. The classical method is the administration of 2 pounds of chopped and braised meat a day. A filet of beef is chopped fine with a chopping knife (a meat chopping machine giving a grumous mass is not to be employed) salt is added and the total quantity is divided into six equal portions and placed in the ice-box. Every three hours a portion is placed in a hot frying pan with a little butter and lightly browned. It is served hot. Ten ounces of hot water or hot plantain gruel should be previously taken. After a week the beef may be varied at times by vegetable or fruit feeding or by other kinds of flesh. A pound of meat yields I000 calories.

The Strict Fruit, or Fruit and Fresh Vegetable Diet.-The Dutch have cured many cases of sprue in this manner. Fruits have been discussed, so little more need be said. Green vegetables and salads made therefrom are also capable of curing sprue but great care should be taken to furnish an adequate nutritional value. The more succulent vegetables should first be used.

Results of Dietetic Treatment.-The effect of a milk or milk and fruit diet is usually favorable and promptly so. After a few days diarrhea will have disappeared, the tongue improves, and, above all, the mental confusion with restlessness, so striking a symptom in sprue. At first, on a strict milk diet, gaseous distention increases, weakness is at times extreme, and hunger becomes ravenous, but in about a week the unpleasant features of a milk diet are little or not at 
all noted, the color improves, the patient is more cheerful and alert, gaseous distention after alimentation disappears, and diarrhea or constipation will often be replaced by normal movements.

In ninety-eight patients treated by Ashford in Porto Rico:

8.43 per cent. died.

60.2 per cent. were apparently cured.

25.3 per cent. were improved.

6. per cent. were improved.

\section{Principal References}

Bahr. P. H., Report on Researches on Sprue in Ceylon, 1912-r914. Cambridge University Press, London, 1915, p. 155.

Begg, Charles, Sprue, its Diagnosis and Treatment, I9r 2.

Carnegie Brown, Sprue and its Treatment, 1908.

Castellani and Chalmers, Manual Tropical Medicine, 2d Edition, rgr3.

Castellani and Low, G. C., The Rôle Played by Fungi in Sprue, Jour. Trop., I913 xvi, 34 .

Manson, Sir Patrick, Trop. Dis., 5th Edition, 1909.

Kohlbrugge, Die Aetologie de Aphthx Tropicx, Arch. f. Schiffs. u. Trop. Hyg., I90I, vii, 325 .

Ashford, B. K., Notes on Sprue in Porto Rico and the Results of Treatment by Santonin, Am. Jour. Trop. Dis. and Prev. Med., I913, vii, I46.

Is Sprue a Moniliasis of the Digestive Tract? Am. Jour. Trop. Dis. and Prev. Med., I91 5, iii, 32 .

Studies in Moniliasis of the Digestive Tract in Porto Rico, Am. Jour. Med. Sci., $1915, \mathrm{cl}, 680$.

Preliminary Report of the Instit. of Trop. Med. and Hyg. of Porto Rico, San Juan, P. R., rgI4.

The Dietetic Treatment of Sprue, Am. Jour. Trop. Dis. and Prev. Med. 1916, II, 377.

Further Experimentation in Animals with a Monilia commonly found in Sprue, Am. Jour. Med. Sci., 1916, CLI, 520.

Musgrave, Wm. E., Sprue or Psilosis in Manila, Am. Med., March 8 and 15, 1902.

\section{TRYPANOSOMIASIS}

There are two varieties, the African and the American.

\section{AFRICAN TRYPANOSOMIASIS}

This is a chronic infectious disease, characterized by a febrile period of glandular hypertrophy and nervous and mental symptoms, and by a terminal dementia. It is due to infection by protozoa of the genus Trypanosoma, species $\mathrm{T}$. gambiense and $\mathrm{T}$. rhodesiense through the bites of specifically infected glossinx, the first species being usually conferred by G. palpalis, the second by G. morsitans, although all the tsetse flies can act as vectors of trypanosomiasis pathogenic to man (Bruce, Eckmann). The natural reservoir of $T$. rhodesiense is wild game which are themselves unaffected. Kinghorn and Yorke have shown 16 per cent. of infections in wild game in Rhodesia. The trypanosomes pathogenic for man partially develop in the gut of the fly and from thence reach their salivary glands where they alone become infective, a tempera- 
ture of from $75^{\circ}$ to $85^{\circ} \mathrm{F}$. being necessary. Bruce states that $\mathrm{T}$. brucei and $\mathrm{T}$. rhodesiense are identical and that wherever they exist in wild game and G. morsitans is present there, trypanosomiasis will be found in man, in whom, however, some degree of relative resistance is native. While all infected fly areas are exposed to more or less extent, the infection is especially apt to be found in fisherman, porters, and members of expeditionary parties working near the shores of lakes and streams or passing over well-watered forest regions. The trypanosomes are not transmitted by heredity either in flies or animals.

Pathology.-The key to a correct understanding of the pathogenesis lies in the histopathology. There is first an invasion of the peripheral lymphatic system and later of that, especially, of the central nervous system, with an accumulation of lymphocytes around small vessels of the brain, reducing the size of the blood stream by compression, and an invasion of connective tissue and neuroglia: A chronic meningoencephalitis or meningomyelitis is finally produced. At first the trypanosomes are present in the blood and absent from the cerebrospinal fluid which is clear. Later this becomes turbid and mononuclears and trypanosomes are abundant.

Symptomatology.-The period of incubation is from ten days to three weeks. Some authors insist upon the lesion sometimes seen at the site of the fly bite which they refer to as the trypanosome "chancre." Martin and Leboeuf divide the developed disease into three periods:

The period of début extends from the appearance of the parasites in the blood to their invasion of the cerebrospinal fluid, and may be very short or extend to seven years. It is characterized by irregular fever, at times preceded by a chill, high fever and severe constitutional reaction; by painful enlargement of lymphatic glands, particularly of those in the postcervical triangle; by deep hyperesthesia; by a circinate, non-itching, erythematous eruption chiefly on the trunk, at times varied by rubeloid spots and papules, the "trypanides;" and by psychical exaltation, depression and confusion with loss of flesh and energy. The pulse is rapid and there is often insomnia and some anemia. Other symptoms noted by various authors are neuralgic pains, headache, fine tremors of fibrillary character, cramps in the lower extremities, a diminution or abolition of genital functions, ocular disturbances (iridocyclitis), and rapidly appearing and disappearing localized edema.

Period II dates from the appearance of flagellates in the cerebrospinal fluid and is characterized by serious involvement of the nervous system. Most of the symptoms of period I are accentuated but the chief feature is the enfeeblement of the intellect. The changes in the psychic affect the intellectuality, the morals, and the will, and the character of the patient is changed or gradually effaced. There is an expression of hebetude or of satisfaction, with varying periods of expansion, delusions of grandeur, hallucinations or fury, 
in which crimes may be committed, and depression with mental confusion, dream states, or deep melancholy. The sleeping syndrome from which the name of the disease is derived is characterized by Martin and Leboeuf as typical of the disease but in reality it seems to occur in but 5 per cent. of cases noted by most authors. Castellani and Chalmers state that the patient's usual condition is better described as lethargic. The fibrillary tremors of tongue and hands become coarser; there is a shuffling gait and difficulty in maintaining equilibrium. Romberg's sign is present but there is no inequality of the pupils and the Argyl-Robertson pupil is not demonstrable. There may be spasms of muscles, hemiplegia, epileptiform seizures (often of Jacksonian type), and paraplegia. The great profusion of nervous symptoms will depend in each case upon the part of the nervous system most affected at the time. Emaciation and muscular weakness are apt to become extreme. The blood usually shows a moderate decrease in erythrocytes, a still greater diminution in hemoglobin, and a relative mononucleosis but usually no leukocytosis. The duration of this stage is as a rule from three to six months.

In period III mental automatonism reigns, broken only by moments of impulse or fury. There succeeds a condition in which the emaciated dement, dribbling saliva, suffering from bedsores and contractures of his muscles, is incapable of speaking, walking or even standing. The disease may terminate thus with coma or, before this extremity is reached, by an apoplectiform or epileptiform seizure, or by some intercurrent disease. The appetite is usually voracious to the last. The duration of this period may be from two to three months.

Forms and Evolution.-The most characteristic feature of African trypanosomiasis is its remarkable resemblance to syphilis, from the so-called "trypanosome chancre" to the ultimate dementia, corresponding to general paresis. It is "as polymorphic in its course as in its symptoms: it may be acute and rapidly fatal, may go forward by fits and starts, and may spontaneously cure without affecting the meninges after the parasites appear in the blood." It also has a tendency to flare up from some residuum after a long period of apparent good health. Infection by $\mathrm{T}$. rhodesiense is generally more rapid and severe with a duration at times of but four or five months and is said to show a more constant hypertrophy of the epitrochlear than of the postcervical glands, as well as a tendency to interstitial keratitis.

Prognosis.- This should be based on the strain of the infecting trypanosome and the terrain (Martin and Leboeuf). Man is supposed to already possess some natural immunity and Leboeuf states that the poor and underfed negroes of Central Africa have this resistance greatly reduced. There seems to be a great difference in virulence between various strains of trypanosomata in South 
Central Africa. A somber outlook is presaged by relapses, and apoplectiform and epileptiform seizures are of gravest import.

Diagnosis.-(I) By simple direct examination of blood smears, thick or thin. This is more apt to be a failure than not. (2) Nabarro's method of centrifugation of citrated blood, successively removing the supernatant fluid twice and examining the sediments (usually the sediment from the last centrifugation is sufficient). (3) Puncture of an enlarged postcervical gland and aspiration of the gland juice. (4) Centrifugation of the cerebrospinal fluid (usually negative until the advent of period II). (5) Inoculation of animals. These methods constitute the only feasible way of checking upon the results of trypanocides.

Treatment.-There is little to hope for in sera or vaccines unless Novy's demonstration of the injection of living attenuated cultures of $\mathrm{T}$. lewisi in animals can be applied to the human species pathogenic for man. With regard to drugs one must take great precaution to avoid fatal poisoning in an effort to secure chemical sterilization, on the one hand, and especial care, on the other, not to create a drug-resistant strain of trypanosomes by timid dosification. Success depends on early treatment, on the willingness of patient and physician to take risks, and to regularity in treatment.

Arsenic.-Atoxyl is administered hypodermically. The method used in the German expedition to East Africa (Koch), was $0.5 \mathrm{gm}$. on each of ten successive days. Martin and Darre used $0.5 \mathrm{gm}$. for each of five successive days. This drives trypanosomes from the blood, accompanied by a febrile reaction. A method recommended by Martin and Leboeuf is to give $0.75 \mathrm{gm}$. to a person weighing from 35 to $40 \mathrm{kilos}$; $\mathrm{I} \mathrm{gm}$. to patients of 40 to $50 \mathrm{kilos}$; and $0.5 \mathrm{gm}$. to those weighing from 25 to 30 kilos. This may be done daily for eleven days. It is stated that atoxyl should be freshly made up, sterilized at $I 10^{\circ} \mathrm{C}$. in the autoclave and kept in the dark until used. In the routine treatment of dispensary cases Ouzilleau says that he used a Io per cent. solution of atoxyl, boiled and cooled. He administers by hypodermic injection ro c.c. in the flank of adults and from 5 to 8 c.c. in children, who tolerate it well, as a rule. He adds that only the aged, edematous and cachectic take it badly. -

The dangers from atoxyl are nephritis, and its toxic effects on the eye and the intestinal tube, the latter often producing shock. The worst is atrophy of the optic nerve which comes on after a variable time and is permanent.

Many authors consider atoxyl the best single remedy we have in this disease.

Soamin is not so toxic but even this drug has produced blindness.

Arsacetin has also produced blindness and is more toxic (Martin and Leboe).

Arsenophenylglycin is given by Aubert and Heckenroth in two injections of $3.5 \mathrm{gm}$. each and then rest from eight to ten days before 
another dose. They give it in physiological salt solution in dilution of from I : IO to I : 20 , but it should only be used in period I.

Orpiment taken by mouth causes the flagellates to disappear from the peripheral blood and can be used as a prophylactic for prevention of fly infection from human cases. Three grams throughout three days will usually clear the blood but the drug is not safe to deliver promiscuously to ignorant people in quantity. It is well to combine it with opium to prevent diarrhea.

Salvarsan and Neosalvarsan.-Some authors seem to have a good deal of confidence in these drugs while others declare them of littla value. They seem to clear the peripheral blood of parasites for short periods but are probably inferior to atoxyl. Broden, Rodhain and Copin used 2 to $3 \mathrm{cg}$. per kilo of body-weight with success.

"Atoxyl-emetic."-The joint use of atoxyl and "potassium emetic" is better in its results than either of these drugs separately but they should not be used in timid doses. Broden and Rodhain give two doses of $0.10 \mathrm{gm}$. of potassium emetic in eight to ten days with 0.75 to $\mathrm{r}$ gm. of atoxyl in one dose, or in two doses of $0.5 \mathrm{gm}$. each. Martin and Leboeuf give up to $0.10 \mathrm{gm}$. in solution of $\mathrm{I}: \mathrm{I} \infty \mathrm{O}$ every month and precede each dose by isolated injections of 0.75 to $\mathrm{I}$ gm.of atoxyl. Martin and Darré give intravenous quotidian doses of o.ro gm. in a dilution of $\mathrm{I}: 1000$ for fifteen days, resting three weeks between each seance. They give atoxyl in addition in doses of $0.5 \mathrm{gm}$. every five to six days.

Antimony.-Tartar emetic alone makes trypanosomes disappear from the blood with great rapidity (in from five to ten minutes). From 0.05 to $0.10 \mathrm{gm}$. is a dose and it should be administered well diluted and intravenously. It may cause pain in the limb injected, cough, and, exceptionally, syncope. To avoid these accidents the dose of Io cgm. should be given slowly and only to those weighing more than 50 to 55 kilos. Below this weight reduce the dose by I to I. $5 \mathrm{cgm}$. for each 5 kilos less of weight. In a series of I $_{50}$ cases treated by antimony, 0.065 every four days, three died from the effects of the drug. Of thirty-four cases of trypanosomiasis twentyfour were continuing to improve at the time of report (Ranken). This author states that the ordinary symptoms produced by intravenous injection are fever, fall in blood-pressure, diuresis, cough (rarely lasting over ten minutes), pain in the xiphisternal region on the next day and cephalalgia.

The Benzidine Colors.-Tryparosan and trypaflavin are combined with salvarsan in seventeen early cases by Broden, Rodhain and Corin and twelve were considered cured thereby after an observation of from four to seven months.

Results of Treatment.-Of 593 cases treated by Le Fanu:

Atoxyl cured 52.6 per cent. of fifty-two cases.

Arsenophenylglycin cured 77 per cent. of 233 cases.

Salvarsan cured 95 per cent. of twenty cases.

Neosalvarsan cured 82 per cent. of forty-five cases. 
Conclusions from treatment drawn by Mouchet and Dubois:

I. Treatment giving prolonged sterilization of the blood plays a considerable part in prophylaxis.

2. Salvarsan and arsenophenylglycin in moderate doses produced sterilization of three months' duration.

3. Single injections of the above-mentioned drugs sometimes cure.

4. They are best used in the first stage of trypanosomiasis as most of the cured come from this period.

5. The discontinuous treatment is not recommended for the second stage of the disease.

6. Atoxyl-emetic often produces fairly long sterilization.

7. Orpiment has little action in moderate doses and is dangerous in large ones.

Their routine treatment was atoxyl-emetic, atoxyl, 0.5 to I gm. on Monday, and emetic, 0.10 gm. on Thursday. They treated ninety-seven patients thus for from four to six months. Of twentyeight in the first stage, twenty-three were apparently cured and five were worse; they remark that atoxyl cured several in the first stage but that atoxyl-emetic was preferable for the second. Arsacetin was tried in forty cases, three or four injections of I gm. each at weekly intervals, and after this dose was reduced to $0.5 \mathrm{gm}$. The results were as with atoxyl.

Prophylaxis.-The preventive measures to be adopted are obvious from the etiology: destruction of wild game, deforestation, sterilization of the blood of human infected, and the establishment of treatment camps. But campaigns against the disease should secure the highest efficiency with the least destruction, prohibition and expense possible. Even in the face of daily accruing evidence against wild game the English hesitate to advise its destruction. So far a clearing around towns or villages for 300 yards, clearing of shores of streams at fording places, and prevention of the passage of uninspected persons from infected to clean districts have been the favorite measures employed. Depopulation of entire infected areas has been consummated in some parts of Africa. Destruction of flies and their haunts, if it were not so hopeless as a general measure, would be very efficacious and has indeed proved so in Principe Island as seen below.

One thing seems to stand out very clearly in this great problem and that is the necessity for the creation of a special service with police powers to deal with this question alone. With a fixed plan and provided with inspectors, engineers, entomologists, sanitarians and physicians and with a numerous force of laborers, a more or less costly solution might be made.

The individual measures for prophylaxis should embrace the wearing of white clothing and boots, with head nets, when traveling through fly areas, the use of mosquito bars at night, and the immediate disinfection of bites, when perceived, with iodin, a doubtful remedy for an injected virus.

An apparently complete victory has been won over a serious endemic by an organized Portuguese force in the Island of Principe on the west coast of Africa. This island had a population of about 
4000 in 1902 . Trypanosomiasis in 1907 had reduced this population to 3000 despite frequent restocking from the mainland and the death rate in 1903 was 221 per 1000 . As the cacao industry was seriously threatened, a sleeping sickness commission was sent by the Portuguese government. By a system of movable camps, deforestation, drainage, destruction of breeding places, extermination of wild dogs and hogs, isolation of sick, the sterilization of the infected in the early stages, blood and gland examination of the entire population every three months and fly-catching, the disease was completely stamped out in the years I9II-I9I4.

The war upon the fly is so interesting as to call for special remark: pairs of men dressed in white clothing and with a screen of dark cloth covered with bird lime upon their backs were sent to walk all day in the fly areas. Each man picked the flies from his companion's back and at the end of the day these were counted. The total number thus caught was 470,000 in three years. In February of I 9 II, II, 865 flies were caught by I 37 men; in April, May and June of I9r3 only one fly was caught although the fly-catching force had increased to r97. While undoubtedly the flies caught were only a tithe of those starved and driven out by other means, this feature took a part of considerable importance in the campaign and demonstrated the determined nature of the fight put up by all who took part in this remarkable bit of sanitation.

\section{AMERICAN TRYPANOSOMIASIS}

Chagas in 1909 announced the discovery in Brazil of Schizotrypanum cruzi, causing what is now known as the "Molestia de Chagas." Chagas has recently changed the name of this organism to Trypanosoma cruzi. It produces a disease which chiefly affects children but adults are not exempt. The vector usually mentioned and considered responsible for its dissemination is Triatoma megista but later T. sordida, T. vitticeps, Cimex rotundatus, $C$. lectularius, C. infestans and certain ticks have also been incriminated. The organism is frankly dimorphic and invades the blood cells as a merozoite without flagellæ or is found in the blood on escaping from the corpuscle as a trypanosome. An asexual cycle is consummated in the tissues; chiefly the muscles of heart, trunk and extremities, and the neuroglia of the central nervous system. In these tissues the invasion of a cell is followed by multiple binary division until the cell, distended to a pseudocyst, ruptures when the merozoites invade fresh erythrocytes. Animals are susceptible of inoculation (monkeys, rabbits). Before becoming infective bugs require a definite period for the development of the parasite which finally invades its salivary glands. The forms found in the pseudocysts are typically aflagellar. The multiplication of these organisms in heart, muscle, nerves, thyroid, suprarenal glands, etc., determine the protean nature of the symptoms. 
Symptomatology.-The brunt of the disease is apt to fall most heavily on the nervous system where cysts and consequent local reaction on rupture determine serious results, but the thyroid, the suprarenal glands and the heart muscle are profoundly affected in the same manner.

The Acute Form.- The child is suddenly taken with high fever, thyroid hypertrophy, edema of the face, hypertrophy of lymphatic glands especially of the neck, enlargement of the spleen and often of the liver and, in severe attacks, signs of meningitis. The fever disappears after a time but periodically recurs. During the attack trypanosomes can often be found in the blood.

The Chronic Form.-There are marked thyroiditis, loss of hair, hypertrophy of lymphatic glands, a dull expression of countenance with a peculiar bluish bronze pallor, tachycardia, intense nervous disturbances, and, frequently, convulsions.

Chagas divides the disease into five forms:

I. The pseudomyxedematous, characterized by hypertrophy of the lateral lobes of the thyroid, a peculiar light bronze color (said to be due to involvement of the suprarenals), a general enlargement of lymphatic glands, tachycardia, low blood-pressure, convulsions, and, sometimes, fever.

2. The myxedematous, with an atrophied thyroid and the usual signs of myxedema.

3. The cardiac, with arrhythmia, extra systole and sinus irregularities.

4. The nervous, due to various brain and cord lesions, in which diplegia, spastic paraplegia, athetosis, aphasia, pseudobulbar paralysis, etc., are noted.

5. The chronic, with acute or subacute exacerbations, characterized chiefly by a preponderance of fever and causing a high mortality. The sequelæ, termed metaschizotrypanosis by Chagas, include impaired intelligence, infantilism, and idiocy, accompanied by hypothyroidism.

In the course of the disease there seems to be a more or less constant thyroid and suprarenal involvement. The diplegia is explained by bilateral lesions and is one of the most frequent disturbances; it is apt to be accompanied by spasticity. Idiocy, however, may occur without marked motor disturbance. The parasites are not usually demonstrable in the blood in the chronic forms. As a rule there is no globular anemia but a slight hemoglobinemia. Leukocytosis may be observed in acute cases; there is an increase in the large lymphocytes, even in one case to 59 per cent.

Diagnosis.-In the acute form the parasites may be observed in the peripheral blood. For other stages inoculation of animals by blood or cerebrospinal fluid is necessary. The organism is easily cultivated in NNN medium, and takes oval, round, or crithidial forms, trypanosomes not being observed. 'Personal work with Chagas in Minas Geraes revealed 242 cases of this disease in a clinic 
of 1435. The diagnosis was confirmed by him but clinically there was often great similarity to endemic goiter and the differential diagnosis must take into account the demonstrated presence of the Trypanosoma in a sufficient number of cases to make such a diagnosis justifiable for the rest. Such a demonstration in all chronic cases seems to be a physical impossibility for a clinical laboratory when the blood is negative and an immediate positive diagnosis is urgent, although in this instance the diagnoses of the brilliant South American discoverer seem fully warranted. A more or less generalized goiter in a population whose houses are overrun with the Triatoma megistia, the demonstration of the trypanosomes in these bugs and experimental infection of animals by their bite, the occurrence of acute febrile cases in children in whose circulating blood the parasite is demonstrated and the dissemination of crithidial forms in the heart, muscles and other tissues of those chronic cases coming to postmortem would seem to amply justify a clinical diagnosis, in similar cases, of American trypanosomiasis. All of these conditions are fulfilled by Chagas before a locality is considered infected.

The prognosis, as can be deduced from the above description, is really serious but cases are spontaneously cured without apparent lesions.

The treatment is as for African trypanosomiasis.

\section{Principal References}

Martin, Gustave, and Leboeuf, Traité pratique de Pathologie exotique, rgr 2, iii, 302. Castellani, A., and Chalmers, A. J., Manual of Tropical Medicine, I913, 2d Edition. Kinghorn, Allan, and Yorke, W., Tropical Dis. Bull., 1912, i, 126. Ann. Trop. Med. and Parasit., I9 I2, vi, 483.

Broden, A., Rodhain, J., and Corin, G., Arch. f. Schiffs. u. Trop. Hyg., 191 2, xvi, 749. Wolbach, S. B., and Binger, C. A. L., Jour. Med. Research, 191 2, xxvii, 83. Brit. Med., Jour., Nov. 2, 1912, p. I188.

Rodhain, J., Pons, C., Vandenbranden, J., and Bêquaert, J., Bull. Soc. Path. Exot., I912, v, 762 .

Kinghorn, Allan, Yorke, W., and Lloyd, Ll., Ann. Trop. Med. and Parasit., Igr 2, vi, 495. Ibid., vii, p. 183 .

Kleine, F. K., and Fischer, W., Ztschr. f. Hyg. u. Infektionskrankh., I gr 2, Ixxiii, 253. Robertson, Muriel, Phil. Tr. Roy. Soc., London, 1913, Series B, vol. 203, No. B. 298, pp. $16 \mathrm{r}-\mathrm{r} 84$.

Ranken, H. S., Trop. Dis. Bull., 1912, i, 507. Proc. Roy. Soc., 1913, Series B, vol. 86, No. B 586,203 .

Novy, F. G., Perkins, W. A., and Chambers, R. Jour. Inf. Dis., rgr 2, xi, 4 Ir.

Taute, M., Ztschr. f. Hyg. u. Infecktionskrankh., 1913, lxxiii, 556 .

Shircore, J. O., Trans. Soc. Trop. Med. and Hyg., 19r3, vi, I3I.

v. d. Hellen, Arch. f. Schiffs. u. Trop. Hyg., 1913, xvii, 230 .

Macfe, J. W. Scott, Ann. Trop. Med. and Parasit., I913, vii, 339.

Bruce, David, Harvey, David, Hamerton, A. E., and Lady Bruce, 1913, Proc. Roy.

Soc., vol. B 87, No. B 592, pp. 26-35. Ibid., p. 35. Ibid., p. 58.

Eckard, B., Centralb. f. Bakteriol., I Abt. Orig. I 9r3, lxil, 73.

Aubert, P., Monfort, F., Heckenroth, F., and Blanchard, M., Bull. Soc. path. exot., 1913, vi, 632 .

Le Fanu, C. V., Trop. Dis. Bull., 1913, iv, 164.

Kleine, F. K., Ztschr. f. Hyg. u. Infektions krankh, I9 4 , Ixxvii, I84.

Yorke, W., and Blacklock, B., Ann. Trop. Med. and Parasit., I9I4, viii, p. I.

Mouchet, R., and Dubois, A., Beihefte 2. Arch. f. Schiffs. u. Tropenhygiene, roI4, xviii, 5 .

Boletim Oficial do Governo de Provincia de S. Tomé e Principe, 1914, July I5, Suplemento, No. 8, p. 4. 
Da Costa (B. F. Bruto), Sant' Anna (J. Firmino), Dos Santos (A. Correia) and Alvares (M. G. de Araujo), Relatorio Final da Missao da Doença do Sóno da Ilho do Principe (I912-19I4), Arquivos de hig. e patol. exot., 1915, March 30, vol. 5, p. 262.

Ouzilleau, F., Bull. Soc. path. exot., I9I5, viii, I 78 .

\section{AMERican Trypanosomiasis}

Martin, Gustave,'Traite' pratique de Path. exot., Grall et Clarac, r9r 2, iii, 394. Castellani, A., and Chalmers, A. J., Manual of Tropical Medicine, 1913, 2d Edition. Delanaoe, M., and Mme. P., Bull. Soc. path. exot., I9 12, v, 599 .

Dias. Ezequiel, Memorias do Instituto Oswaldo Cruz., rgr 2, iv, 34.

Brumpt, E., Bull. Soc. path. exot., I913, vi, I72. Ibid., I914, vii, I32.

Chagas, Carlos, Rev. med. de S. Paulo, rgr 2, xv, 337.

Neiva, Arturo, Brazil-medico, I913, xxvii, 309. Ibid., I9I4, xxviii, 333. Ibid., 1915, Xxix, I.

Torres, Margarinos, Brazil-medico, r913, xxvii, 321. 


\title{
THE OCULAR COMPLICATIONS OF THE INFECTIOUS DISEASES
}

\author{
By G. E. DE Schweinitz, M. D.
}

TREATMENT OF DISEASES OF THE LID

Blepharitis.-Much of the success in treating severe and chronic forms of blepharitis depends upon the removal of the crusts which form on the margin of the lid in the manner already described on page 745 , of Vol. II. A satisfactory method is the daily gentle scrubbing of the lid margins with the lather of a good neutral soap. For this purpose special soaps (ophthalmic soaps) may be employed. As already mentioned, in the presence of the Morax-Axenfeld bacillus, sulphate of zinc applications, from 0.5 to 5 per cent., are particularly advantageous, and stubborn varieties of blepharitis have been successfully treated by ionic medication with a 0.5 per cent. solution of sulphate of zinc, after the manner of Wirtz. Special electrodes are required.

Herpes Zoster Ophthalmicus.-In the presence of this specific, infectious exanthem, serious involvement in the eye may be anticipated, namely, ulceration of the cornea, iritis and iridocyclitis. These ocular inflammations are most apt to occur when the nasal branch of the first division of the trigeminus is involved, and the vesicles extend to the tip of the nose. It must be the duty of the attending physician to watch carefully for the first signs of conjunctivitis, keratitis, or iridocyclitis, and to employ the remedies elsewhere described. It should also be remembered that as the result of this disease glaucoma, or a glaucoma secondary to cyclitis, may develop, and the tension of the eyeball should be frequently tested, and atropin drops, otherwise often indicated, used with care and constant observation of the tension of the eyeball.

\section{TREATMENT OF DISEASES OF THE CONJUNCTIVA}

Simple Conjunctivitis.-In the treatment of this affection, usually called catarrhal conjunctivitis, in addition to the remedies named on page 747 of Vol. II, attention should be directed to certain etiological factors. Occasionally the affection is scarcely more than an aggravated hyperemia of the conjunctiva, with very little mucopurulent secretion, aggravated necessarily by local irritants, smoke, dust, high wind, especially in automobile riding, and exposure to glare, but these hyperemias may be acute and recurrent, and arise under the influence of metabolic disorders, for example, gout, and are sometimes an ocular manifestation of so-called gastrointestinal 
autointoxication, and if they do not yield readily to the ordinary local remedies, these possible conditions must be investigated.

A form of catarrhal conjunctivitis to which much attention has recently been called is the so-called swimming-bath conjunctivitis, which has been frequently encountered among those who frequent public baths. Usually the secretion is less abundant than that found in catarrhal conjunctivitis of the ordinary type, and sometimes the affection resembles acute trachoma, and rarely "inclusion bodies" have been demonstrated. The treatment does not differ from that already detailed, but this possible exciting cause, especially during the summer months, must not be neglected.

Special Forms of Conjunctivitis.- The prophylaxis and treatment of gonococcic conjunctivitis neonatorum (ophthalmia neonatorum) have been described in detail on pages $748-750$. While the value of Crédé's method, so long firmly established, continues in a sense unchallenged, certainly in institution work and where the birth canal is known to be infected with gonorrhea, or where the suspicion of infection cannot be excluded, nitrate of silver, especially in strong solutions, that is, stronger than 2 per cent. (I per cent. is better), should not be used lest silver catarrh be produced. Recently, in place of nitrate of silver, a 5 per cent. solution of spohol has been highly recommended; indeed, Axenfeld advises its use "instead of the formerly employed Crédé silver drops." With this drug the author has had no experience, but its efficiency should be investigated.

That conjunctivitis neonatorum, which, when the gonococcus is the etiological factor, is the cause of fully 8 per cent. of the blindness in this country, should be listed as a reportable disease is evident, and the distribution by health boards of circulars of advice to midwives and mothers is advisable. Tubes containing the chosen prophylactic, thus far usually a I per cent. solution of nitrate of silver, may be given to trained, educated midwives, as well as to physicians, and with good effect, but compulsory notification represents an effective method at our command. Since all properly managed hospitals at the present time have as part of their equipment a social service, the difficulties of home treatment have been lessened, and the follow-up system has been productive of admirable results. Efforts should be made, however, to compel hospitals to make special provisions, that is, hold in readiness special wards for the treatment of this disease, to which babies can be taken whose home surroundings are not sufficiently satisfactory to carry on the treatment. Such wards would permit not only trained attention, which is essential, but should be so constructed that the mother of the child could come at stated intervals for the purpose of nursing the infant. The author is in hearty accord with George S. Derby's statement that "in the vast majority of severe cases ophthalmia neonatorum should be treated in a hospital."

The treatment of gonococcal varieties of ophthalmia neonatorum has been detailed on page 748 of Vol. II, and the author does not 
find it necessary to alter the recommendations which have been made in these paragraphs. He is well aware that many surgeons deprecate the use of nitrate of silver in this disease. For example, Derby believes the reaction of the silver salt in gonorrheal conjunctivitis is too great to permit its use, and that its bactericidal power cannot assail the deep-seated gonococci. His fear that nitrate of silver may injure the corneal epithelium, or produce too violent a reaction, is well grounded, and certainly its application must be restricted to skilled hands and not repeated more than once in twenty-four hours, argyrol being used in the intervals in the manner already described. That nitrate of silver should be banished from the management of this disease the author is entirely unconvinced. Quite recently E. B. Heckel reports admirable results from the constant irrigation alone with iced physiologic salt solution. Evidently the previous recommendation that ophthalmia neonatorum should be treated in hospitals is confirmed by the very fact that there is not universal agreement in its management, for in hospitals the hourly observations which are possible can readily check a remedy which is acting disadvantageously, or determine the value of one which is acting favorably.

Trachoma.-Although this disease, usually called granular lids, and which depends upon an inflammatory infiltration of the adenoid layer of the conjunctiva, associated with the development of follicles, has not been traced to any general infection, or to any known constitutional disorder, a word should be said in regard to it. As is well known, it is generalized through Egypt, is exceedingly common among the poorer classes in Ireland, Italy, Hungary, Poland and Russia, and Jews of the lower social grades are very apt to be attacked. The Indians of our own country are very frequently affected, and the disease is widely distributed through certain regions in the middle west and the western portions of the United States. The menace of trachoma on account of emigration in our own country is a very serious one. Its exact cause is unknown, just as the exact nature of the so-called Prowazek bodies which are found in the cells and in the secretion of trachoma is unknown. They are so seldom present, however, in other conjunctival diseases, that their detection is highly significant. Their absence, however, does not exclude trachoma.

There is no proof that trachoma is caused by microörganisms of the bacterial group, or by blastomycetes. Transference of the morbid material from a trachomatous conjunctiva to another eye may result in a disease like the one from which it came. In this sense the disease is specifically contagious, although the affection, to quote Axenfeld, is not conspicuously contagious by secretion inoculation. The so-called acute varieties, with much mucopurulent discharge, usually represent an admixture of ordinary granular lids and acute conjunctival catarrh, the latter due, for example, to the Morax-Axenfeld bacillus, the Koch-Weeks bacillus, and even the gonococcus. The so-called acute varieties may be treated 
exactly as purulent conjunctivitis is managed; the chronic varieties require the application of astringents, for example, sulphate of copper in crystal form, or this remedy dissolved in glycerin in 5 per cent. solution. Usually the chronic varieties are best managed by operative interference, expression of the trachoma granules, various types of grattage, and in the chronic varieties by excision of a portion of the tarsus.

Tubercle of the Conjunctiva.-This condition must be distinguished from trachoma, epithelioma and syphilitic ulceration. Bacteriologic and microscopic examination of the affected tissue is often necessary to determine its histologic characteristics. In trachoma the lymph glands are not involved, and the discovery of the Prowazek bodies would tend to establish the diagnosis. Epithelioma is excluded by the age of the subject, as tuberculosis almost invariably occurs in young persons, that is, those under the thirtieth year of life. In addition to the treatment already recommended, Axenfeld ascribes a curative effect to a 50 per cent. solution of lactic acid. Following its instillation the eye must be irrigated with a saline solution.

\section{TREATMENT OF DISEASES OF THE CORNEA}

Phlyctenular Keratitis.-In addition to the remedies and measures mentioned in the treatment of this stubborn disease, the author desires to reiterate, as the result of additional experience, his recommendation that its subjects as they appear in hospital dispensary work shall be treated like other cases of tuberculosis. The follow-up system has proved of the utmost value, and the instruction given to the parents or caretakers as to how the child shall live, what food shall be served, etc., by one of the workers of the social service of the hospital should never be neglected. It is desirable to emphasize that phlyctenular keratitis is not a local disease, but a manifestation of a general condition aggravated by the coexisting nasal difficulties, and often fostered and excited by septic conditions of the alimentary canal, in their turn the result of improper and defective diet.

Ulcers of the Cornea. - Therapeutic recommendations in the management of ulcers of the cornea, whether they are small and single, or large, purulent and infected, have been detailed on pages $754-756$ of Vol. II. It is important to determine if possible the active bacterial element in each corneal ulcer, as this frequently gives the best indication for treatment. Thus, not a few corneal ulcers are now known to contain the diplobacillus of Morax and Axenfeld, and in these circumstances zinc in from I to 5 per cent. solution, applied directly to the ulcer, is almost a specific. Other special remedies determined by like examination are referred to on page 388 . Recently purulent keratitis has been successfully treated by zinc iontophoresis. Whether scarlet-red in the treatment of infected ulcers, a 5 per cent. ointment in an aqueous solution, as recommended by Darier, is an 
advantageous remedy, the author is unprepared to say from personal experience, nor has he used pellidol and azodolen, derivatives from scarlet-red, in 2 per cent. ointment. The application of radium has been advocated by Lawson and McKenzie.

In all types of infected corneal ulcer close attention should be paid to the associated conditions. Of especial importance is the investigation of the condition of the lachrymal passages and the presence of purulent material in the lachrymal sac. The teeth should always be examined, and if faulty the patient turned over to a competent dentist. The frequent relation of carious teeth and pyorrhea alveolaris to corneal ulceration is well established, and there seems little doubt that some serious types of corneal ulceration have been caused by disease of the accessory sinuses, especially the ethmoid sinuses, by purulent rhinitis, and have appeared in association with infected tonsillar crypts, the streptococcus usually being the active bacterium.

Parenchymatous or Interstitial Keratitis.- That variety of nonulcerated corneal inflammation which is most frequent between the ages of five and fifteen, is due in certainly 60 per cent. of the cases to inherited syphilis (rarely to acquired syphilis). Fully ro per cent. of the cases, however, are tuberculous in nature, and the importance, therefore, of submitting the patient to the Wassermann test or to diagnostic injections of tuberculin is evident. Should the lesion prove to be syphilitic, in addition to the treatment previously recommended the author has found the use of salvarsan and neosalvarsan of much value. The injections of neosalvarsan are given at intervals of one or two weeks. Usually small doses are employed. During the intervals the medication should consist of mercurial inunctions, iodid of potassium, or mixed treatment. There is no doubt that this treatment shortens the attack in the sense of causing a more rapid subsidence of the active irritative phenomena than is otherwise the case, although it has not been definitely proved that it hastens the disappearance of the deep-seated corneal infiltrates.

Certain types of deep keratitis, circumscribed parenchymatous forms of the disease, as they appear in adults, are undoubtedly at times an expression of the evil results of overeating and drinking, and occur in those subjects who, in general terms, are liable to gout and who have defective intestinal functions. Other types appear to be an expression of a gastrointestinal autointoxication. The treatment in these cases, in addition to the usual remedies, atropin, dionin, etc., must include a regulation of the constitutional faults which have been referred to.

\section{TREATMENT OF DISEASES OF THE UVEAL TRACT}

Iritis, Iridocyclitis and Uveitis.-In general terms the treatment of these diseases has been described on pages $757-759$, of Vol. II, but especially in the last few years a very great change has taken Vou. IV. -25 
place in our conception of the various types of iritis and iridocyclitis. They should, in a large measure, be regarded, not as independent diseases, but as complications, and the patient must be studied from the general standpoint, because the iritis is one of the manifestations of the toxemia of which he is the subject.

Syphilis continues to hold the first place in the etiologic catalog in relation to this disease, and in addition to the usual treatment, or in place of it, salvarsan and neosalvarsan have proved their great efficiency. If the Wassermann test is positive, other things being equal, a dose of $0.6 \mathrm{gm}$. should be given intravenously (in women 0.4 gm. is usually sufficient). At the expiration of two or three weeks, during which period mercury and iodid of potassium should be administered, if the Wassermann test is still positive, this dose may be repeated. Using neosalvarsan, the injections may be given at comparatively frequent intervals, the dose being regulated according to the circumstances and effects, as often as once in ten days or two weeks. Good results also follow the administration of cacodylate of soda.

While it is the custom, even now, to speak of rheumatic iritis, it may be seriously questioned whether acute rheumatism, that is, rheumatic fever, or articular rheumatism, is ever the cause of a non-purulent inflammation of the iris or ciliary body. It is true that Krückmann describes a special type of iritis which begins with conjunctival hyperemia or non-bacterial conjunctivitis, to which he gives the name rheumatic iritis, but in general terms the relation of rheumatism to the development of iritis is a vague one, and those types of the disease which are seen frequently in the subjects of myalgia, chronic joint lesions (polyarthritis), lumbago, pleurodynia, and other conditions loosely classified as chronic rheumatism, are not examples in the true sense of the term of rheumatic iritis. They have recently been denominated autotoxemic or toxemic iritis, and the term rheumatic is abandoned, or should be. They yield, in general terms, in addition to the local measures of atropin, dionin, etc., to the antirheumatic remedies, the salicylates, which should be exhibited in full doses, 60 to 80 grains per diem, or aspirin, but they do not recover promptly if there is anywhere in the system an area of focal infection.

Almost all of the so-called rheumatic forms of iritis will fall into this category, and therefore, we have come to speak of iritis secondary to mucous membrane infection, and have come to know that the primary source of such infection is most frequently a chronic septic process, especially in the mouth (pyorrhea alveolaris), in the teeth (tooth-root abscesses), in the tonsil, in the nasopharynx, in the accessory nasal sinuses, in the stomach, in the urethra, in the uterine cavity, in the prostate, and in cutaneous boils and furuncles. In this connection the recent researches of Allen J. Smith and M. T. Barret of the University of Pennsylvania, in the etiology of pyorrhea alveolaris and the relation which parasitic amebas 
of the mouth have to this disease are extremely important. It is not sufficient to make an ordinary inspection of the teeth and the pharynx. The roentgen rays must be brought into use, and by their means tooth-root abscesses and similar lesions may be discovered which otherwise would remain undetected.

Much work has been done to connect the development of iritis and uveitis with intestinal sepsis, and although the analyses do not prove that any toxic substance elaborated in the course of a socalled gastrointestinal autointoxication, that is, a toxin formed within the metabolism, can by its toxic properties produce iridocyclitis, they prove the presence of bacterial activity, and indicate a source from which these bacteria or their toxic products may proceed and cause an inflammation of the uveal tract. Therefore, in this sense the gastrointestinal intoxications are important etiologic factors in the development of iridocyclitis.

\section{SPECIAL LINES OF TREATMENT}

Tuberculosis of the Eye.-The general plan of treatment of various types of tuberculosis of the eye has already been recorded but it is desirable that the relation of this infection to chronic inflammations of the uveal tract (the iris, the ciliary body and the choroid) should be emphasized, as well as its relation to certain deep types of keratitis, especially in association with patches of scleritis. While there is some difference of opinion as to the value of tuberculin, as already described in the treatment of these affections, and while its repeated use may do positive harm, it undoubtedly often is singularly efficacious, especially if, as the result of other means, or owing to natural defences, the subsidence of the disease has begun. In these circumstances the tuberculin may give it an additional impetus. The usual methods of administering tuberculin in eye work are described on page $76 \mathrm{I}$ of Vol. II.

Gonococcic Infections of the Eye.- It has been pointed out that many types of so-called rheumatic iritis are really the expression of a systematic toxemia, and there is little doubt that gonorrheal iritis is much more frequent than was formerly believed. Indeed, William Lang goes so far as to say that the gonococcus is the most frequent cause of plastic iritis. Certainly in all types of plastic iritis the blood should be examined for a complement fixation reaction and the posterior urethra for gonococci. There may be a long interval between the acquisition of the gonorrhea and the iritis, frequently several years, and often as long as thirty years. The value of a Neisser bacterin in the treatment of this affection has been described. Often iodid of potassium acts satisfactorily, and relief will follow profuse sweats by means of pilocarpin given hypodermically or with the aid of an ordinary hot chamber or cabinet. The usual local treatment with mydriatics and lymphagogs must be ordered. 
Pneumococcic and Streptococcic Infections of the Eye.-The value of antipneumococcus serum has been described. Recently ethylhydrocuprein (optochin) has been strongly advocated in the treatment of pneumococcic ulcers of the cornea. It may be applied directly to the ulcer in a strength of from $I$ to 2 per cent. by means of a cotton swab and held in place for a minute, the application being repeated as required, or it may be used in a watery solution every hour, or employed in the form of an ointment combined with atropin. If it causes much pain, this may be checked by the instillation of a 2 per cent. solution of holocain. It would seem that this drug, although recommended in pneumococcic ulceration of the cornea, is not without some efficiency in the management of ulcers apparently caused by other bacteria, for example, staphylococci. Rohmer has advocated autoserotherapy in the treatment of hypopyon keratitis. The serum obtained from a blister on the patient's arm is injected beneath the conjunctiva, the dose being I c.c. Apparently there has been little or no advance in the efficiency of antistreptococcus serum or vaccine in sloughing ulcers of the cornea, which cannot at present be regarded as more than a remedy which is supplementary to other forms of treatment. 


\title{
ANIMAL PARASITES
}

\author{
By Maurice C. Hall, Pir. D., D. V. M.
}

Technique of Microscopic Examinations.-The examination of feces for evidences of parasitism should be a routine procedure in tropical and semitropical countries, in such places as the southern United States, or wherever a patient's habits or surroundings are such as to predispose to parasitic infestation. The various methods which have been advocated for examining feces for evidences of parasitism have been summarized and discussed by Hall. These methods range from the simple smear method to the numerous more elaborate and effective methods which aim at a concentration of the parasite eggs by taking advantage of the differences in the physical, chemical and biological properties between such parasitic material and the non-parasitic material. The examination of feces for parasites is not unpleasant when properly carried out; it is an easier matter than the examination of urine or blood and often gives as valuable or more valuable information. A differential blood count which shows eosinophilia, with no other obvious explanation, should arouse a suspicion of somatic or intestinal parasitism, and the latter possibility should be tested by fecal examination. Charcot-Leyden crystals in the feces are at times indicative of helminthiasis.

Concentration of parasite eggs in fecal material is usually based on the difference between the eggs and the fecal matter as regards size, specific gravity, chemical and physical solubility, adhesiveness, staining reaction, and development in cultures. This concentration is attained as follows: By sedimenting or centrifuging in water, in which methods the parasite material, which is heavy, goes to the bottom; by centrifuging in a solution, such as a calcium chlorid solution, having a specific gravity of 1.050 , which brings material lighter than parasite eggs to the top, and then centrifuging the sediment in a solution having a specific gravity of 1.250 , which brings the parasite eggs to the top and leaves heavier matter at the bottom; by sedimenting or centrifuging and then decanting and washing, whereby soluble matter and fine suspended matter are removed; by straining through gauze or sets of graduated sieves of which the finest has a mesh aperture of about 0.120 mm., which will permit the passage of eggs but not of coarse fecal particles; by staining the feces on a slide, as with 5 drops of toluidinblau in 2 per cent. aqueous carbolic acid, which stains the feces blue or purple and the eggs light brown, or by staining with a drop of anilin gentian violet, which stains everything but the parasite eggs, leaving them as clear areas; by placing the feces on a slide, immersing the slide in 
water, and gently removing it, which results in washing off everything but hookworm eggs, which adhere to the glass; by dissolving fatty materials and mineral constituents in such chemical or physical solvents as hydrochloric acid, ether, antiformin or alcohol; or by culturing the feces and examining the cultures for embryo and larval worms; and by combinations of the above methods. Not all of these devices can be used to advantage, but the employment of some of them greatly increases the likelihood of detecting infestation as well as saving time and eye-strain in microscopical examination. of slides. The use of screens and careful washing will also facilitate the detection of gross parasites-entire worms, tapeworm segments, etc.-and will prevent errors due to mistaking citrus pulp vesicles, banana fibers and other ingesta for parasites. When washed clean, the non-parasitic nature of such objects is usually obvious.

The examination of the urine and sputum by the usual routine microscopic method will detect vesical and pulmonary parasitism wherever these cases are capable of detection by microscopic examination.

\section{TAPEWORMS - CESTODES-TENIASIS}

Tapeworm infestation is either intestinal or somatic, as far as parasites of man are concerned. In intestinal teniasis the adult strobilate tapeworm is present in the lumen of the host intestine. This is the most common form of teniasis in man; there are a total of about I $_{5}$ species of tapeworms reported in the strobilate stage from man, of which two or three are of fairly common occurrence in the United States. Diagnosis of intestinal teniasis is by fecal examination.

In somatic teniasis the larval tapeworm, or bladderworm, is present in the tissues of the host. In man the common form of somatic teniasis is hydatid infestation; this and the less common forms of infestation with bladderworms must be diagnosed clinically, by such serological methods as complement fixation, or by the occasional findings of hooks, membranes or small cysts, as may occur in the sputum in pulmonary echinococcosis. In cutaneous or subcutaneous teniasis, the excision and examination of the parasite will determine its identity.

\section{INTESTINAL TENIASIS}

The tapeworms reported from the intestine of man are as follows: Davainea madagascariensis, Davainea asiatica, Dipylidium caninum, Hymenolepis diminula, Hymenolepis nana, Drepanidotania lanceolata, Tania solium, Taniarhynchus saginatus, Taniarhynchus africanus, Taniarhynchus hominis, Teniarhynchus philippinus, Tania confusa, Tania bremneri, Bertiella satyri; Diphyllobothrium latum, Diphyllobothrium cordatum, Diplogonoporus grandis, Diplogonoporus brauni, and Braunia jassyensis. 
Tæniarhynchus saginatus (Tænia saginata).-This tapeworm is rather common in the United States as a result of the American habit of eating rare beef. While over half of the beeves killed in the United States are slaughtered under federal inspection and carefully examined for the larva, Cysticercus bovis, which gives rise to this tapeworm, there is a large amount of meat from animals which are not inspected or which are inspected in municipal inspection in a manner that is frequently unsatisfactory. The cysticerci may safely be regarded as dead after refrigeration at $15^{\circ} \mathrm{F}$. for six days, or at higher temperatures after the lapse of twenty-one days.

Tænia solium.-This tapeworm is so rare in man in the United States that Hemmeter's case is the only valid record known to me. The presence of the parasite in this country is established for the most part from the fact that the larval stage, Cysticercus cellulose, is occasionally found in hogs in abattoir inspection. Its comparative rarity is due to the fact that Americans, in general, prefer their pork well done just as they prefer their beef rare, whereas in some countries, where Tania solium is more common, there is a large consumption of underdone or raw pork, especially in sausage products.

Diphyllobothrium latum (Dibothriocephalus latus).-Most of the records of this parasite in the United States have been from foreigners, and patients from the Baltic provinces, Switzerland and Japan are among those most apt to have it, though it occurs in most, and perhaps all, European countries. Pfender reported a total of forty-two cases known from the United States up to rgro. Cases of American origin are now known to exist, and an endemic focus has been found in the region of the Great Lakes. The larvæ, or plerocercoids, were found by Nickerson in fish caught in these lakes. The Great Lakes supply a large amount of the fish consumed in the United States, either fresh, salted or smoked, and the danger of infestation with this large and injurious parasite in this country is now known to be less remote than was formerly supposed.

Hymenolepis nana.-This parasite, perhaps identical with the rat form, is rather common in children and hundreds of worms may be present.

Hymenolepis diminuta. - This worm, which is normally a parasite of rodents, is rather rare, but it has been reported from man in a number of cases in the United States. The intermediate or larval stage is known to occur in larval or adult meal moths and earwigs, and in adult beetles, and infestation probably arises as the result of the accidental ingestion of these insects or of other suitable intermediate hosts not yet known. The expulsion of the parasite is readily accomplished. Prophylaxis is largely a matter of keeping food products away from insects, though the voluntary ingestion of infested insects is a possibility to be kept in mind.

Dipylidium caninum.-This worm, normally a parasite of the dog and cat, has been reported from man about 80 times, a recent list being that published by Blanchard in 1914. There are three 
American records. The worm has a habit of burrowing into the intestinal mucosa, a habit which not only destroys the integrity of the mucosa and opens a way for pathogenic organisms, but which also results in rendering the expulsion of the worm difficult, since vermifuges affect only the exposed portion of the strobila that lies in the intestinal lumen and leave the imbedded head to renew the infection after the passage of the exposed portion. Prophylaxis is based on a rational treatment and handling of dogs and cats to prevent ingestion of fleas and lice infested with larvæ.

Symptoms of Intestinal Teniasis.-In a general way, it may be said that there is no symptom or symptom-complex constantly associated with intestinal teniasis and in any way pathognomonic. Moreover, the fact that cases are not uncommon where even fairly heavy infestations with worms of various sorts do not seem to give rise to any noticeable symptoms, has led many persons to conclude that intestinal helminthiasis is a matter of no consequence and gives rise to no symptoms. Such cases may within certain limits be compared with the bacterial infections in the case of carriers of $B$. typhosus, etc.: we have the etiologic agent of a disease present and the disease not produced. Intestinal helminthiasis, including teniasis, whether in massive infestations or where few or even single worms are present, may at times give rise to pronounced digestive and nervous disorders, but they are not constant; the cause must be suspected in connection with the history and surroundings of the case and the elimination of other possibilities, and diagnosis is by fecal examination and not on the symptoms presented, as a rule.

Among the digestive disorders are alterations in appetite, which may be decreased or increased, diarrhea or constipation, eructation, nausea, vomiting, unpleasant sinking sensation, pressure and discomfort in abdomen, and even colicky pains, enterospasm, etc. The nervous symptoms are headache, vertigo, fainting, convulsions, delirium, chorea, disturbances of vision and hearing, etc. Anemia, probably due to toxins, is also associated with intestinal teniasis at times, especially with Diphyllobothrium latum, and eosinophilia, regarded by Weinberg and Séguin as a condition especially adapted to the neutralization of certain toxic substances, is also of common occurrence. These conditions should lead one to suspect the probability of intestinal teniasis or other forms of parasitism. Intestinal helminthiasis is an afebrile condition normally.

Treatment of Intestinal Teniasis.- The effectiveness of treatment for intestinal teniasis calls for more attention to detail than is often given. Ineffective treatment, with a failure to expel more than a few segments which might have been expelled equally well by a drastic cathartic, or the failure to remove the head, followed by the recurrence of the strobilate growth, is rather common. It is first of all essential to use an adequate remedy, and some of the standard drugs do not measure up well in experimental tests, sometimes due, no doubt, to the fact that these drugs deteriorate with age. It is 
equally essential that the involved physical conditions of treatment receive attention. A tapeworm head imbedded in the intestinal mucosa and surrounded by a thick coat of mucus is apparently fairly well protected from the toxic effect of vermifuges, and vermifuges commingled with a large bulk of intestinal contents may readily prove to be too much diluted and physically restricted from access to the worms, to exert their action. It would be well, not only to withhold solid food for twenty-four hours and all food for twelve hours, but also to make the administration of the preliminary purgative a routine procedure. In catarrhal conditions, with considerable mucus present, the use of ammonium chlorid to liquefy the mucus has been regarded as a valuable adjunct.

The Anthelmintic.-Oleoresin of male fern appears to be the teniafuge most commonly used at present, so far as evidence is available. It occasionally happens that this drug is reported as ineffective in a given instance where other drugs subsequently used have proved effective, but for the most part it is dependable.

Pelletierin.-Dr. Ch. Wardell Stiles regards Tanret's pelletierin as more efficacious than male fern and also superior in that it is less dangerous.

Pumpkin Seed.-This home remedy is commonly very efficacious, though not entirely palatable and often nauseating. Goldschmidt says that the seeds, without any preparation, may be fed directly to children without difficulty in amounts of 50 to $60 \mathrm{gm}$.

Thymol.-Various writers have reported that thymol is very efficacious against tapeworms. De Vevey gives it in $0.25 \mathrm{gm}$. doses every day for eight days, the worm usually coming away the third or fourth day. The repetition of the dose ensures that the head comes away and he reports no recurrence in twenty-three cases so treated. With thymol one must avoid alcohol in any form and fats and oils, milk, etc. Use salines instead of castor oil.

\section{SOMATIC TENIASIS}

The following worms have been reported as present in cases of somatic teniasis: Tania solium (Cysticercus cellulosa), Multiceps multiceps, Echinococcus granulosus (Echinococcus polymorphus, E. multilocularis, etc.), Plerocercoides prolifer, Sparganum mansoni and Sparganum baxteri.

Tænia solium (Cysticercus cellulosæ).-Cysticercosis in man is due to the ingestion of the eggs of the armed tapeworm, Tania solium, which has already been noted as a rather rare parasite in the United States, though cysticercosis due to this parasite is occasionally found in hogs in this country. It is believed that the disease may occur in man not only as a result of tapeworm eggs ingested in food or water, but also as a result of reverse peristalsis bringing the tapeworm eggs to the stomach in the case of persons infested with the adult tapeworm. It is, therefore, highly desirable that prompt 
treatment be instituted and followed up in cases of intestinal infestation with this worm.

Cysticercosis as a result of infestation with the larva, Cysticercus bovis, of the beef tapeworm, Taniarhynchus saginatus (Tania saginata), is not known to occur. If it were a possibility the common occurrence of the adult worm should result in a fairly large number of cases of cysticercosis due to its larva.

Echinococcus granulosus.-Although there is some likelihood that there are two species of Echinococcus, the disease due to hydatid does not require separate consideration for these two species. At the present time there have been over 250 cases of hydatid disease in man reported from the United States and Canada. This parasite has been found to be alarmingly prevalent in domestic animals in some parts of the United States, notably in certain localities in Virginia, Arkansas and Oklahoma, and the prevalence of hydatid in domestic animals is an index of the danger to which persons are exposed. The prevention of this highly dangerous disease calls for the suppression of the dog nuisance as a measure necessary for the public welfare. Vagrant dogs, dogs without responsible owners who will keep them clean and free from parasites, and who will assume responsibility for their conduct, dogs that carry and spread parasites and various bacterial, fungous and virus diseases, such dogs should be eliminated, and all other dogs should be kept within reasonable bounds and under sanitary conditions.

Sparganum mansoni.-Moore in I9I5 summarized the literature dealing with the twenty-five reported cases of infestation with this parasite. Most of the cases are from Japan; one, Moore's case, is from the United States in a native of Texas. The parasite occurs in the urinary passages, the tissues around the kidneys, the eye or orbital tissues, posterior mediastinum, or subcutaneous. It causes painful swellings of a transitory or intermittent character. The life history is unknown: a similar larva from the muscles of the hog developed to an adult worm in the intestine of a dog, according to von Ratz.

Plerocercoides prolifer (Sparganum prolifer).-Four cases of human infestation with this worm have been reported thus far, one of them from Florida in this country, the others from Japan, by Ijima, Stiles, and Yoshida. The parasite is a bothriocephalid larva occurring in the subcutaneous tissue, viscera and brain, with fatal results in massive infestation. The largest specimen reported was $75 \mathrm{~mm}$. long by $2 \mathrm{~mm}$. wide. The life history of the parasite is unknown. Diagnosis is based on the disclosure of the worms on incising the subcutaneous nodules. No medicinal treatment appears to have been attempted.

Treatment of Somatic Teniasis.- Treatment is surgical. Ransom and Hall have summarized the literature dealing with the action of anthelmintics on parasites located outside of the alimentary canal. Feletti, de Renzi, and Dianoux report cures of hydatid and cysticercosis through the administration of extract of male fern in doses 
of $0.3 \mathrm{gm}$. to $2 \mathrm{gm}$. a day, the total amount in one case amounting to $102 \mathrm{gm}$. Experiments on animals affected with hydatid, cysticercosis and cœnurosis by Moussu, Dévé, and Hall have in all cases failed to indicate any selective action on the parasites and there is reason to question the evidence on which cures were claimed in some cases by the other writers noted. More work is necessary and desirable along this line, as the location of the parasite in somatic teniasis often makes surgical treatment dangerous or impossible.

\section{FLUKES-TREMATODES-DISTOMIASIS}

The flukes which have been reported from man are as follows: Watsonius watsoni, Gastrodiscus hominis, Fasciola hepatica, Fasciola gigantica, Fasciolopsis buski, Fasciolopsis rathousi, Fasciolopsis fiulleborni, Fasciolopsis goddardi, Paragonimus ringeri (Paragonimus westermanii), Agamodistomum ophthalmobium, Amphimerus noverca, Opisthorchis felineus, Opisthorchis viverini, Clonorchis sinensis, Pseudamphistomum truncatum, Yokogawa yokogawa, Heterophyes heterophyes, Echinostoma ilocana, Echinostoma maylayanum (Euparyphium maylayanum (Artyfechinostomum sufrartyfex (?)), Dicrocaelium dendriticum, Schistosoma hamatobium, Schistosoma mansoni and Schistosoma japonicum.

Although over 20 species of flukes have been reported from the intestine, liver, lungs and blood-vessels of man, distomiasis is rarely observed in man in the United States and the cases found here commonly originate elsewhere.

\section{VENAL DISTOMIASIS}

Schistosoma hæmatobium (Bilharzia hæmatobia).-The most important recent addition to our knowledge of this parasite of the portal system and of the veins of the pelvic region, especially of the bladder, is the demonstration of its life history by Leiper. He has shown that this species has a life history similar to that of the other digenetic trematodes, the intermediate host being a snail of some species of the genera Planorbis or Bullinus. The cercariæ which develop in these snails are apparently able to penetrate the unbroken skin of man and of other hosts of the adult worm, though Cawston considers persons with skin abrasions more liable to bilharziasis. The cercariæ may also enter, apparently, by way of the mouth or anus, but probably penetrate the tissues at these places, or in the case of those entering the mouth, may at times penetrate the lining of the cardiac portion of the stomach. Prophylactic measures may therefore be directed toward the prevention of contamination of water supplies by the egg-bearing urine and feces of patients, and the provision of safe drinking water and safe bathing places, keeping out of doubtful or infested waters, wearing a bathing suit as a partial protection, destruction or control of infected dogs, cats or other 
animals, the eradication of snails by suitable measures, and possibly the destruction of fluke eggs and cercariæ. White rats, desert rats, variegated mice, guinea pigs and monkeys were found susceptible to infestation, and consequently in infested regions measures for the control of various wild animals which might serve as collateral hosts would be indicated as prophylactic measures.

This parasite has been reported from the United States in fifteen or more cases.

Schistosoma mansoni.--This species, which has a lateral-spined egg, apparently never gives rise to hematuria, the worms living in the inferior mesenteric vein and the eggs passing out by way of the rectum.

Schistosoma japonicum.-The life history of this parasite also has only recently been discovered. Several years ago, Katsurada and Hashegawa showed that infection occurred by way of the skin and lately Leiper and Atkinson showed that the intermediate host is a snail, Katayama nosophora, and that the cercarix penetrate the skin. Preventive measures are therefore substantially the same as for Sch. hamatobium. This worm is endemic in the Philippines.

This species does not cause hematuria. In the first stage there are abdominal pains, cough and eosinophilia; in the second stage, enlarged liver and spleen, and stools with blood-streaked mucus; in the third stage cirrhotic liver, ascites, emaciation, anemia, weakness, and edematous extremities.

Treatment of Venal Distomiasis.-Bilharziasis or schistosomiasis is commonly treated empirically with male fern and has been treated with a long list of drugs, as arsenic, iron, potassium iodid, mercurials, copper sulphate, quinin, nux vomica, santonin, urotropin, salol, methylene blue, emetin, soamin, salvarsan, thymol, etc., with very variable results clinically and with practically no positive data as to the effect of the treatment on the worms themselves. From the nature of the disease, it is evident that the destruction of the worms would not of itself cure the disease. Looss has pointed out that bilharziasis is a disease characterized by lesions due to the passage through or retention in the tissues of the bladder, rectum, liver, and other organs of vast numbers of eggs, while the parasite itself in the blood-vessels has very little direct pathological significance. By the time the patient goes to a physician for treatment for hematuria, the worms producing the eggs which cause this are perhaps dead or near the end of their existence. Looss looks upon the reported cures, therefore, as resulting from an artificial retention of the eggs in the tissues, causing thereby a suppression of the symptoms but not a cure of the disease. While this seems to be sufficiently obvious, it would also appear to be true that for the many cases which may be recognized clinically in earlier stages, a remedy which could be depended on to kill the worms in the blood would have the great value of thereby stopping egg production and so shut off a further supply of eggs which must be eliminated to the destruction 
and injury of the tissues traversed. It must also be admitted that the worms themselves may give rise to phlebitis, thrombosis and embolism. Aided by prophylactic measures to guard against reinfection, an adequate vermicide by killing the worms and stopping egg production would lighten the burden of inflammatory alteration and destruction and speed the process of resolution. For the present there is no recognized treatment other than palliative- the treatment of a chronic, irremediable cystitis, anodynes in case of pain, etc. Madden advises enterotomy and cleaning of the bowel in rectal bilharziasis when a tumor can be felt, but in general surgical interference is not regarded with favor unless unavoidable.

\section{PULMONARY DISTOMIASIS}

Paragonimus ringeri (Paragonimus westermanii).-Ward and Hirsch find on a comparative study that the species of fluke described from the lungs of a tiger and known for a long time as Paragonimus westermanii is not identical with the lung fluke described from man by Cobbold and now known as Paragonimus ringeri. They also find that the lung flukes reported from the United States from the hog, dog and cat are distinct from the human species, $P$. ringeri, and should be known as Paragonimus kellicotti. The arrangement and form of the cuticular spines are easily studied features of specific importance.

The life history of this worm has just recently been ascertained and published (1916) by Nakagawa and by Yoshida. Their investigations in Formosa and in Japan show that the intermediate hosts are such fresh-water crabs as Potamon (Geothelphusa) obtusipes, P. (Ge.) dehaanii, Sesarma dehaani, and Eriocheir japonicus. The young encysted larvæ were found in the liver of the crab; full grown larvæ were found on the gills. Twenty per cent. of these larvæ from the gills would float on water, indicating the danger of drinking water in which these crabs live. Feeding experiments show that the larvæ penetrate from the intestinal tract to the peritoneal cavity, thence through the diaphragm into the pleural cavity, and so to the lungs.

Treatment of Pulmonary Distomiasis.- There is no known medicinal treatment effective against this parasite. It is, therefore, advisable to see that the sputum and excreta of infested persons are properly disposed of, that questionable water be avoided for drinking or bathing purposes, and that raw or insufficiently cooked crabs be avoided as articles of diet. Where lung fluke occurs in man, dogs may be suspected of being possible carriers. In the course of time, if reinfestation is prevented, the flukes present in the lungs will die and degenerate, or an occasional specimen may be coughed up.

\section{HEPATIC DISTOMIASIS}

Clonorchis sinensis (Clonorchis endemicus).-This fluke is common in China and Japan, and may occur in enormous numbers, 
one case being reported in which over $2 \mathrm{I}, 000$ flukes were recovered from a patient at necropsy, a large number more being lost. The life history has been determined by Kobayashi, who found the intermediate stages subcutaneous and in the muscles of a dozen species of cyprinoid fish, especially in Pseudorasbora parva and Leucogobio güntheri. These larvæ were easily killed by heat, dying when subjected to temperatures around $100^{\circ} \mathrm{C}$. It appears that the larvæ when eaten pass to the biliary ducts of the liver by way of the gallduct, as larvæ were found here and in the gall-bladder fifteen hours after feeding.

No adequate medicinal measure for the removal of this parasite is known. Prophylaxis depends on proper sanitation to prevent the eggs from infested persons getting back to water supplies, and in thorough cooking of fish. The same parasite is carried by dogs, cats and swine, and these animals should be subjected to whatever restraint is necessary in guarding water supplies from possible carriers.

Fasciola hepatica (Distoma hepatica).-Ssinitzin has shown that the encysted cercariæ of this fluke are commonly carried about in water, rather than commonly remaining attached to grass blades and other vegetation as has been usually stated, which fact explains the occurrence of this fluke in man. He has also shown that the idea that the larval fluke ascends the bile-duct to the liver from the intestine is erroneous, and that the fact is that the larval fluke, when ingested, bores through the walls of the digestive tract and attains the body cavity, where it wanders about on the viscera for a time and finally makes its way to the liver and penetrates the capsule, entering the biliary canals near the periphery.

There appear to have been less than thirty cases of the occurrence of this fluke reported from the liver in man, the usual hosts being sheep, cattle and swine. It has been reported once from man in Porto Rico by Stiles. The eating of raw liver by man occasionally gives rise to a peculiar transfer of the adult worm, which apparently crawls up the esophagus from the stomach and attaches to the larynx or glottis, producing asphyxia. This form of infestation is known as "halzoun." The prompt production of vomiting will usually carry out the worm and save the patient. Should this treatment fail, tracheotomy and surgical measures for the removal of the worm would be indicated.

Treatment of Hepatic Distomiasis.--A number of eminent European authorities-Grassi, Calandruccio, Perroncito, Alessandrini, Borini, Railliet, Henry and Moussu-have reported success in removing the common liver fluke, Fasciola hepatica, from the liver of its usual hosts, cattle and sheep, by the use of the ethereal extract of male fern. Subsequently Marek has reported even better results with kamala. While this fluke disease is usually only diagnosed postmortem in man, it would appear that the tentative use of male fern or kamala is indicated in human cases diagnosed antemortem, on the strength of available evidence. It would also appear advisable to 
try out such treatment against Clonorchis sinensis, but if this has been done the writer has found no record of it. The treatment of this disease is regularly symptomatic. As liver flukes usually die and pass out within a year, the patient may be nursed until relieved of the parasites in this way.

\section{INTESTINAL DISTOMIASIS}

Fasciolopsis buski.-This is a large fluke, 2 to 3 inches long. It appears to be rather common in India and occurs in the hog as well as in man.

Heterophyes heterophyes.-This is a very small fluke, less than $1 / 12$ inch long. It occurs in Egypt, Japan and China, and is found in the dog and cat as well as in man.

Watsonius watsoni, Gastrodiscus hominis, Fasciolopsis goddardi, Yokogarea yokogawa, Echinostoma maylayanum and Echinostoma ilocamum, the latter from the Philippine Islands, are other flukes reported from the intestine of man.

Intestinal distomiasis gives rise to very variable symptoms. Such small species as Heterophyes heterophyes appear to be rather innocuous in small numbers, but in heavy infestations this species may give rise to an intense diarrhea, predisposing to cholera or occasioning the death of the patient directly. The diarrheal stools may be streaked with blood. The intestinal flukes are blood suckers, giving rise to lesions of the mucosa with resultant irritation and a destruction of integrity which gives entry to pathogenic bacteria.

Treatment of Intestinal Distomiasis.-The expulsion of intestinal flukes requires the use of adequate anthelmintics. Garrison has used male fern successfully. Guiart recommends the use of thymol in two or three doses of $\mathrm{I} \mathrm{gm}$. each, this treatment to be given in the morning and repeated for a total of three consecutive days; five hours after the last treatment a saline purgative is administered. Dr. Stiles regards it as dangerous to leave this amount of thymol in the intestine over night. Kamala would be worth trying.

Inasmuch as the life histories of these flukes are as yet unsolved, exact prophylactic measures cannot be given, but we may be reasonably sure that the life history calls for an intermediate host which will probably be found to be some such invertebrate as a mollusc, arthropod, etc. It is therefore advisable to follow the prophylactic measures which are serviceable against so many diseases and parasites - the use of safe water supplies for drinking and bathing purposes, the protection of such water supplies against contamination of any sort, the wearing of gloves and boots to avoid contact with polluted soil and water when walking, hunting, etc.

\section{ROUNDWORMS OR THREADWORMS-NEMATODES}

The following nematodes have been reported from man, some of them being true parasites, others worms ingested in food and sub- 
sequently vomited or passed in the feces, others worms introduced into the vagina in douches, etc.: Anguillulina putrefaciens, Rhabditis pellio, Rhabditis niellyi, Strongyloides stercoralis, Gnathostoma siamense, Dracunculus medinensis, Loa loa, Filaria labialis, Filaria bancrofti (Microfilaria nocturna), Filaria perstans, Filaria conjunctiva, Filaria juncea, Filaria hominis oris, Microfilaria romanorum, Microfilaria powelli, Agamofilaria oculi, Agamofilaria georgiana, Agamofilaria palpebralis, Dirofilaria magalhãesi, Gongylonema species, Onchocerca volvulus, Agamomermis restiformis, Trichuris trichura, Trichinella spiralis, Dioctophyme renale, Metastrongylus apri, Hamonchus contortus, Trichostrongylus orientalis, Trichostrongylus probolurus, Trichostrongylus vitrinus, Trichostrongylus instabilis (?Trichostrongylus colubriformis), Mecistocirrus fordi, Oesophagostomum apiostomum, Oesophagostomum stephanostomum thomasi, Oesophagostomumbrumpti, Ternidens deminutus, Ankylostoma duodenale, Ankylostoma ceylanicum, Necator americanus, Syngamus species, Physaloptera caucasica, Physaloptera mordens, Ascaris lumbricoides, Ascaris maritima, Ascaris texana, Belascaris mystax, Toxascaris limbata, Lagochilascaris minor, Oxyuris vermicularis, Gordius aquaticus, Gordius chilensis, Paragordius varius, Paragordius tricuspidatus, Paragordius cinctus, Parachordodes tolosanus, Parachordodes pustulosus, Parachordodes violaceous, Parachordodes alpestris, Gigantorhynchus hirudinaceus (Echinorhynchus gigas), Gigantorhynchus moniliformis, and Echinorhynchus hominis.

The parasitic nematodes make up a very large group of worms, which in turn is exceeded in number by the free-living species. The parasitic forms may occur in practically any organs and tissues, and constitute the largest, most important and most widely distributed group of parasitic worms.

\section{SUPERFAMIII ANGIOSTOMOIDEA}

This group includes the nematodes in which parasitic males are not found, only the small, filariform females being found as parasites. The eggs produced by these females develop, as a rule, into a generation of free-living rhabditiform males and females.

Strongyloides stercoralis (Strongyloides intestinalis).- This parasite usually inhabits the upper intestine, and its presence is diagnosed by the finding of the larvæ in the stools, as the embryos are liberated from eggs deposited in the intestinal crypts, develop there to rhabditiform larvæ, and then make their way down the intestine. This parasite was at one time supposed to be the cause of Cochin-China diarrhea, but as the study of cases of Cochin-China diarrhea showed the absence of this worm in numerous cases, the worm became discredited as a pathogenic organism. There is at present an inclination to regard this worm as of some little pathologic significance. It appears to have little relation to diarrhea, but the burrowing habit of the female and of its larvæ appears to give rise to a cellular proliferation and the larvæ have been found post- 
mortem in the muscles, submucosa and subserosa of the large intestine, surrounded by inflammatory infiltration, in the vicinity of a dysenteric ulcer. These larva have also been found in the lymphatics and as emboli in the liver. Parasites with such invasive force, often present in enormous numbers, must at least be looked upon as capable of doing some little traumatic injury and of opening the way for pathogenic bacteria by their destruction of the integrity of the intestinal mucosa. They have also been associated with fever, enteritis, anemia and eosinophilia.

This parasite occurs in temperate as well as tropical countries. Darling found it in $\mathrm{I} 8$ per cent. of all patients in a hospital examination in the Canal Zone at the Isthmus of Panama.

Treatment of Strongyloidosis.-Owing to the burrowing habits of the adult worm, treatment is very difficult. Male fern and thymol are the two drugs which have been most used and recommended, but repeated treatments are apt to be necessary, and even these may prove ineffective. Dr. Stiles states that sulphur seems to be of some use. Preti recommends glycerine, $50 \mathrm{gm}$. by mouth and $30 \mathrm{gm}$. by the rectum. Prophylactic measures are based on the fact that infestation may occur either by way of the mouth or the skin. Prevention of infection is therefore primarily a matter of sanitary disposal of excreta and a proper regard for cleanliness in the matter of food and water and of care in regard to bathing or other contact with polluted water and soil. Fülleborn finds this parasite readily transmissible to dogs, and finds what is apparently this species very commonly present in dogs in China and Japan. It is therefore necessary to take measures against the dog and other potential carriers which will protect the public from the results of soil, water or food pollution from these sources, and to avoid undue familiarity with dogs.

\section{SUPERFAMIIIY TRICHINELLOIDEA}

This group includes those forms in which at least the anterior portion of the body is very slender and is traversed by an esophageal tube imbedded in a chain of single cells; the eggs are lemon-shaped.

\section{TRICHIIVIASIS}

Trichinella spiralis (Trichina spiralis).-According to Dr. B. H. Ransom, about 1600 cases of trichiniasis, with about 150 deaths, have been reported from the United States. Living trichinæ were found in almost I.5 per cent. of $8,000,000$ hogs slaughtered in this country. Williams found trichinx in 27 of 505 cadavers. It has been estimated that it requires about $1,000,000$ trichina embryos to make a clinical case of trichiniasis, and that in severe cases $50,000,000$ may be present.

Ransom gives the symptoms of trichiniasis as follows: The first stage, due to the parasites in the intestine, is characterized by gastrointestinal symptoms, beginning the day the infested meat is eaten or the following day. These symptoms are usually nausea, vomiting, 
diarrhea, and abdominal pains, though these may be lacking. There follows a general torpor, muscular weakness and twitching, and sensations of pain and tension, especially in the tensors of the limbs. In about a week, edema of the eyelids and face appears. This may disappear and reappear later. The second stage, due to the invasion of the muscles, usually occurs in from ten days to two weeks, and is characterized by muscular pains. Movements of the limbs, or even of the eyes, may be painful; mastication, speech, deglutition and respiration may also be painful. In the fourth and fifth weeks there may be a pronounced dyspnea and sometimes violent attacks of asthma, which may terminate fatally. Profuse sweating commonly occurs in this second stage, but may occur throughout the entire attack. The third stage, due to the encystment of the parasites, begins about six weeks after infestation and is characterized by a diminution of the myositis and a beginning edema, anemia and occasional cutaneous eruptions. Pneumonia is occasionally a complication of this stage.

The fever reaches its maximum, IO $4^{\circ} \mathrm{F}$. or higher, about ten days after the first symptoms appear. Herrick has emphasized the fact that trichiniasis is the only disease due to a metazoan parasite which is constantly accompanied by fever. Prognosis is uncertain. Death occurs usually from the fourth to the sixth week, rarely before the end of the second week or after the seventh. Recovery may require months and weakness may persist for years as a sequel.

Thompson found an eosinophilia of over 20 in more than half of his fifty-two cases, and an eosinophilia of 86 has been recorded.

The symptoms are probably due in part to a microbic invasion of the intestinal mucosa, following the trichinæ. The dyspnea and increased rapidity of respiration are perhaps due in part to the invasion of the diaphragm by the trichinæ.

Treatment of Trichiniasis.-In very recent infestations, treatment may be directed toward the elimination of the worms from the intestines. For this purpose calomel may be given in $0.3-\mathrm{gm}$. doses followed by $15 \mathrm{gm}$. of magnesium sulphate six hours later, and the bowels should be kept open for a week to ensure the elimination of such embryos as may be reached in this way. Thymol in 0.15-gm. doses may be given four or five times in twenty-four hours as an anthelmintic. Obviously anthelmintic or purgative treatment is apt to exert little influence on the females which have burrowed into the mucosa for the purpose of depositing embryos in the lymph spaces, and even less effect on the embryos so deposited. The intestinal irritation due to the local activities of the worms may be allayed by the use of bismuth salts. Bromids and chloral are indicated for the period of muscular inflammation due to the invading and developing worms. The McNerthneys found neosalvarsan intravenously of value in certain stages. They note, however, that Van Cott and Lintz had unfavorable results.

Prophylaxis is a matter of avoiding pork that may contain living 
trichina, and requires primarily that pork and pork products be well cooked. It has been found that meat inspection for trichina is apt to lead to a false sense of security, as such inspection is a tedious, difficult and uncertain process, and also a slow and expensive one. Ransom's investigations have shown that refrigeration may serve as well as heat in the destruction of trichina, providing sufficiently low temperatures are attained. Such pork products as are commonly eaten without cooking-summer sausage, Italian hams, etc.--may be regarded as safe when exposed to temperatures not higher than $5^{\circ} \mathrm{F}$. for not less than twenty days. It is also likely that certain processes, such as salting, may be found to render meat safe under certain conditions, but the necessary experimental data covering this point are not yet available. Other prophylactic measures are rat eradication and preventing hogs from eating other hogs, pork scraps, rats, or slaughterhouse offal.

\section{TRICHURIASIS}

Trichuris trichiura.- It is still generally believed that this parasite usually occasions little or no disturbance to its host, but there is an increasing amount of evidence that in many cases it exerts a marked pathological influence. Gastrointestinal disturbances, enteritis, appendicitis, diarrhea, anemia and a long list of nervous disturbances, neurasthenia, convulsions, hysteria, etc., have been attributed to this worm in cases where these symptoms disappeared on the removal of the worms. Death from whipworm infestation has been reported. The trauma due to the worm's habit of sewing the anterior portion of the body into the mucosa and the microbic invasion following this destruction of the mucosa integrity are obviously injurious features, with the possibility that toxin secretion is responsible for the nervous disturbances noted.

Treatment of Trichuriasis.-The successful treatment of infestation with this worm is commonly regarded as a difficult matter. Experiments indicate that while this is true, the difficulty is not due to any resistance to vermifuge action on the part of the worm, but to the likelihood that vermifuges, after reaching the ileocecal valve, will be carried down the colon without entering the cecum and coming into contact with the whipworms present. The experimental treatment of dogs in a series of experiments carried on in the United States Bureau of Animal Industry shows that a treatment will rarely remove all the whipworms present, that in a larger number of cases treatment will remove part of the whipworms present, while in the great majority of cases it will remove none, any one of several different vermifuges being employed. It therefore appears that any treatment directed against this worm will depend for its efficacy on the factors which determine whether the medicine employed will pass into the cecum, and unfortunately we do not appear to be aware of the nature of these factors or of how to control them. Oleum chenopodii, 
santonin, thymol and chloroform have all been found to remove whipworms at times, and male fern has been reported as efficacious. As matters stand it appears to be less a question of the drug than of the accident of peristalsis which determines the course of the drug beyond the ileocecal valve. It may be found good practice to administer anthelmintics in small doses over a long period of time to ensure the entrance of the drug into the cecum. Wade reports success with 2-grain doses of thymol three times a day over a period of two weeks. The use of enemata, such as strong infusions of quassia or an emulsion of I fluiddram of benzine to a quart of water, have been recommended. Berrio and Mouatt-Biggs have made very strong claims for the latex or sap of the fig tree, Ficus laurifolia and other species, the former commonly known as the "higueron," in tropical America (Costa Rica, Colombia, Venezuela, etc.). Berrio uses doses of 24 to $32 \mathrm{gm}$. followed by castor oil. Mouatt-Biggs gives the latex morning, noon and night, mixed with water, which may be sweetened, or with milk. The dose of the latex is from ro to $40 \mathrm{gm}$., according to the age of the patient. The latex has a syrupy consistency, a milky aspect, and mixes easily with glycerin and water. It has a mild aperient action, does not cause unpleasant symptoms, and is not dangerous. A preliminary cathartic is advised and treatment is followed on the fourth day by the administration of Epsom or Glauber's salts. This treatment is said to be especially effective against whipworms, but it has also been adopted against hookworms by the Venezuela National Board of Health.

The worm has a simple life history, the embryo developing in the egg and the egg being subsequently ingested in contaminated food or water or in some similar fashion. Prophylaxis is therefore a matter of cleanliness.

\section{SUPERFAMIIY ASCAROIDEA}

This group is composed of forms which are usually thick in relation to their lengths, and usually provided with a mouth with three lips. They usually have a simple life history.

\section{ASCARIASIS}

Ascaris lumbricoides.-This is a large active worm of a wandering disposition, capable of inflicting considerable damage. It of ten occurs in rather large numbers, as much as II pounds of worms having been removed from one individual. One child has been reported as passing 5000 worms in the course of three years. In such cases there is a resultant obstruction, irritation, spasm and in some instances death, but even a single worm has been found to cause enterospasm necessitating operation. Its wandering habits lead to its occurrence in the stomach, whence it may be ejected by vomiting, a distinctly unpleasant experience in view of the fact that this worm 
is about the size of a lead pencil. It occasionally travels up the esophagus and enters the larynx, producing asphyxiation, or may go down the trachea to the lungs, with resultant gangrene. It has been known to enter the posterior nares and to traverse the Eustachian tube and make its exit from the ear by way of a perforated tympanum. Specimens, sometimes more than one at a time, have been found in the gall-bladder, the hepatic duct and the common bile duct, as many as six in the last-named location. They are also found in the appendix. There are a number of records of perforation of the intestine by these worms, including some cases where operations on the intestine have been followed by the burrowing of ascarids through the operation area, giving rise to such complications as peritonitis and at times resulting in the death of the patient. Many physicians are inclined to scout the idea of worms in children. It should be borne in mind that this worm is not at all uncommon, the children in some places showing as high as 96 per cent. of infestations. There are no definite symptoms indicative of infestation with this worm, but diagnosis is readily made on the passage of worms or the examination of feces. This latter should be urged as a routine before operation.

Treatment of Ascariasis.-Oleum chenopodii, oil of American wormseed, appears to be extremely effective against ascarids. It may be given in 5- to I5-drop doses in capsule or on lump of granulated sugar three times at one- to two-hour intervals. Two hours after the last dose give 15 to 30 c.c. of castor oil, to which may be added advantageously about 2 c.c. of chloroform. Heiser reports the use of oleum chenopodium by physicians at various places in a total of over 100,000 cases without any bad results. This oil may remove all ascarids present at a single treatment, but it is usually advisable to repeat the treatment. It should be borne in mind that nothing known to us in the way of vermifuges can be depended on to remove all worms present even in repeated doses in all cases, as there are conditions little understood which at times appear to inhibit the action of even reliable vermifuges. Oil of chenopodium appears to be superior to santonin and thymol and has the advantage of being inexpensive and readily obtainable by American physicians. Salant and Nelson find the toxicity of the drug diminished by administering it in oil. It may be given in castor oil or in olive oil followed by castor oil. The wormseed oil itself is distinctly constipating. Some fatalities from chenopodium have been reported.

Prophylaxis against ascarids is a matter of cleanliness, to avoid ingestion of contaminated food and water. It should also be noted that the ascarid of the hog is indistinguishable from that of man and that the two species may prove to be identical. Prophylaxis therefore calls for attention to the hog as a possible source of infestation. There is also an ascarid worm of the dog and one of the cat, which have been reported from time to time as parasites of man. It therefore behooves us to keep these pets at a proper distance, and espe- 
cially to protect children from the bad effects of undue familiarity with them.

\section{OXYURIASIS}

Oxyuris vermicularis. - In addition to the anal pruritus commonly occasioned by the presence of this worm, there are other and more serious consequences which may follow. The worm has been reported in numerous cases as the cause of verminous appendicitis, the inflammation not only arising from the activities of the worms themselves, but also from destruction of epithelium allowing invasion by pathogenic microörganisms. Rheindorf thinks that chronic discomfort in the appendix region, especially sharp pains increasing during exercise, should suggest the possibility of infestation with this very common parasite. Roll has reported a case of Oxyuris as the cause of a fatal ileus.

Treatment of Oxyuriasis.-The parasites are present in the small intestine during the early stages following their entrance into the body, and subsequently migrate to the large intestine. It is therefore necessary to employ vermifuge treatment by mouth as well as by rectum. The use of magnesium sulphate or calomel alone is of value. The frequent injection of copious enemata of water alone is useful and may result in a cure in some cases as the water exerts a destructive osmotic action on the worms. In obstinate cases the employment of corrosive sublimate solution, I: Io, 000 as enemata, has been found satisfactory. In some cases the worms appear to be located in some practically inaccessible portion of the appendix and removal seems to be almost impossible. In such cases Stettiner believes that final success in eradication of the worms may be obtained by excluding carbohydrates from the diet as an adjunct to the anthelmintic treatment.

Prophylaxis is primarily a matter of cleanliness in regard to personal habits. The hands, clothing and bed clothing should be carefully washed and kept clean.

\section{SUPERFAMIIIY STRONGYLOIDEA}

These worms are characterized by the presence in the male of a flattened expansion, called a bursa, at the posterior extremity of the body, this bursa being of service in clasping the female.

\section{ANKYLOSTOMIASIS}

Ankylostoma duodenale and Necator americanus.-These are hookworms, the former being the one most common in Europe and the latter being the one most common in the United States. The latter is evidently of African origin, coming to the United States as a parasite of slaves, and finding unusually favorable conditions in the climate and habits of life among the negroes of the southern 
United States. The treatment and prophylaxis for the two worms are identical, though Ankylostoma duodenale, as a result of its more powerful mouth armature, is regarded as a more difficult worm to remove by vermifuge treatment.

Treatment for Ankylostomiasis.-Until recently the classical treatment for removal of hookworms has consisted in the administration of thymol, or, to a lesser extent, of betanaphthol or eucalyptol. Thymol is commonly given to adults in three doses of $\mathrm{r} .39 \mathrm{gm}$. each at hour intervals and followed by a saline. The precautions noted in regard to its use against tapeworms should be observed. Betanaphthol is commonly given in three doses of $0.6 \mathrm{gm}$. each at hour intervals and followed by a saline. Eucalyptol is given as follows:

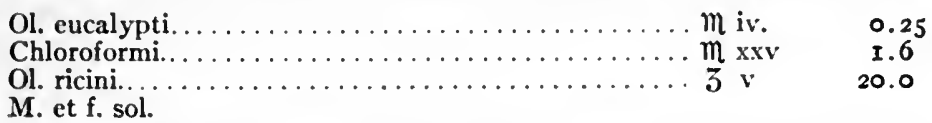

The above dose is to be given and then repeated a half hour later.

As noted elsewhere, oil of chenopodium has lately come into prominence as a remedy for hookworm infestation. Heiser claims that over 100,000 cases of infestation with Ancylostoma and Necator have been successfully treated with this oil. In a series of tests where patients were given two treatments with thymol, betanaphthol, eucalyptol and oil of chenopodium, the following percentage of the entire number of worms removed in both treatments came away on the first dose: eucalyptol, 38 per cent.; betanaphthol, 68 per cent.; thymol, 83 per cent.; oil of chenopodium, 9r per cent. This may be taken as a fair basis on which to figure the percentage of efficacy of these drugs. On the same basis, the results of Bishop and Brosius show chenopodium to be even more efficacious in comparison with thymol. Lane gives the oleum chenopodium in 15 -minim doses on sugar at two-hour intervals, following the last dose with an ounce of castor oil after an interval of two hours. It has already been noted that, contrary to the case for male fern and some other substances, the toxicity of oil of chenopodium is diminished by the simultaneous administration of oil, and the employment of castor oil for purgation is indicated. In experiments carried on in the U.S. Bureau of Animal Industry, testing vermifuges against hookworms in dogs, the worms being very similar to Ankylostoma duodenale, oil of chenopodium has proved more efficacious than thymol, but neither has been as effective as chloroform. Schultz found chloroform very effective. Alessandrini claims that chloroform is a sovereign remedy for internal and external parasites. Combined with castor oil it requires no special preparation of the patient; can be administered at a single dose; does not cause local or general disturbance either immediately or subsequently; is perfectly well tolerated and is not nauseous. He gives it in 3- to 4-gm. doses dissolved in castor or olive oil in a single dose, and claims that it is thoroughly efficacious against hookworms, whipworms, ascarids and pinworms. 
It should be noted in passing that it is possible to give an overdose of this substance by mouth, and that such an overdose will probably result in pneumonia and in irremedial lesions of the parenchymatous viscera with a promptly fatal termination. Dr. Stiles urges some caution as to the use of chloroform against worms. In proper dosage, it has been found in the experiments of the U.S. Bureau of Animal Industry to be very efficacious against hookworms, and it deserves a more extensive test against these worms in man if an efficacious dose proves to be uninjurious. It is useful against whipworms and ascarids, but it appears distinctly less valuable than oil of chenopodium against ascarids. No vermifuge can be depended on to remove Ioo per cent. of all worms of any sort with a single dose, much less to remove all worms of all sorts present, nor is it possible always to remove all of any given kind by repeating the dose once. It is practically always advisable to repeat a treatment at least once after a suitable interval, and if additional worms are passed the treatment should be repeated until the worms are passed after treatment. So far from being a reason for terminating a course of treatment, the removal of worms should be regarded as a reason for repeating it. This is true of chloroform as of other drugs. Chloroform is a powerful and penetrating solvent with a markedly lethal action for all forms of animal life, and there is good reason for supposing that its administration as an anthelmintic should be followed by satisfactory results within certain limits. It will require more experimental and clinical evidence to determine whether it is as efficacious in the large intestine as in the small. It should also be noted that writers have ascribed to oil of chenopodium great efficacy against hookworms in treatment where the chenopodium was followed by castor oil containing chloroform in amounts found sufficient by Alessandrini to remove hookworm. Don't give chloroform twice in less than a week.

\section{SUPERFAMIIY FILARIOIDEA}

The worms of this group have a simple mouth surrounded by papillæ or, with two lips, and are usually notably long, slender worms. So far as known, they all have a complex life history with an intermediate stage in a secondary host, the known intermediate hosts being arthropods of various sorts.

\section{FILARIASIS}

Filariasis is widely distributed and has some manifestations which are of considerable interest. In the United States, Johnson found practically 20 per cent. of 400 persons in Charleston, S. C., to have microfilariæ in their blood.

Filaria bancrofti.-Of the numerous drugs which have been recommended by various authors as of service against this worm, none can be depended on so far as later investigations have shown. Dutcher and Whitmarsh have found a bacillus, B. lymphangiticus, 
which they believe to be responsible for the lesions of what is commonly termed filariasis due to this parasite. Confirmation of their work would necessitate entirely new lines of attack in determining a treatment for this discase.

Dracunculus medinensis.--One case of infestation with this worm, the so-called "Guinea worm," has been reported from a man who had always lived in Philadelphia. Leiper has shown that hydrochloric acid in a strength equal to that in the gastric juice will kill the Cyclops which is the intermediate host of this worm and render the larval worm very active. This indicates that infestation with this worm probably arises from drinking water containing infested Cyclops.

Loa loa (Filaria loa).-Leiper has made the very important discovery that this worm has its intermediate stage in biting flies belonging to the genus Chrysops. These flies bite by day, a fact which is in keeping with the presence of the microfilariæ in the peripheral blood during the day. This highly important discovery at once indicates prophylaxis in the shape of measures directed against the fly and avoidance of its bite.

Schultz has reported success in killing the adult worms in the connective tissue in heavy infestation and the larval worms in the blood by the administration of collargol in dessertspoonful doses of I per cent. solution three times a day, the treatment being continued for over a year. She also finds that when the filarix appear under the conjunctiva, they may be rapidly killed by the instillation of a few drops of this solution.

Filaria perstans.-The adults of this species occur in the connective tissue and deeper fat. The life history is unknown. Other species which are reported from man are $F$. demarquayi, $F$. ozzardi, $F$. volvulus, $F$. powelli, $F$. philippinensis and Dirofilaria magalhãesi, some of which are regarded as the same as other forms reported under other names, and some of which are noted under other genera previously.

A medical treatment that may be depended on to kill adult filarix in the tissues and embryos in the blood remains to be devised. Such work as that of Schultz, for instance, is too recent to have been given adequate test. Robertson believes that pepper and piperine have prophylactic value in food and therapeutic value in the treatment of established cases.

\section{FLIES-DIPTERA-MYIASIS}

Though myiasis is a term meaning infestation with flies, the term is applied only to the condition arising when the larva of various species attack or become parasitic upon man or other animals. It is not properly applicable to the attacks of adult biting flies. Myiasis is rather common, probably much more so than is commonly realized. Banks states, "When we consider that these dipterous larvæ occur 
in decaying fruits and vegetables and on fresh and cooked meats; that the blowfly, for example, will deposit on meats in a pantry; that other maggots occur in cheese, oleomargarine, etc., and that pies and puddings in restaurants are accessible and suitable to them, it can readily be seen that a great number of these maggots must be swallowed by persons each year, and mostly without any serious consequences." Banks quotes Walsh as saying, "We doubt whether, out of 10,000 cases where the larvæ of two-winged flies have existed in considerable numbers in the human intestines, more than one single case has been recorded in print for the edification of the world by competent entomological authority."

We may recognize five varieties of myiasis: External cutaneous myiasis, in which the larvæ are blood suckers attaching to the skin; subcutaneous myiasis, in which the larvæ burrow under the skin; cephalic myiasis, in which the larvæ invade the cavities of the head, including the nose, frontal sinuses, ears, etc.; gastrointestinal myiasis, in which the larva live in the lumen of the stomach and intestines; and vesical myiasis, in which larvæ occur in the urinary bladder, their presence being reported on their passage in the urine.

Cutaneous Myiasis.-While it is quite common to find adult flies attacking man and sucking blood, it is rather extraordinary to find a larval fly, or grub, sucking blood. Nevertheless, the "Congo floor maggot," Auchmeromyia luteola, has this habit, the fly eggs being deposited in the cracks in the mud floors of the native huts in Africa and the resultant maggots coming out at night to suck the blood of the sleeping natives.

Subcutaneous Myiasis.-This condition has been recorded as determined by the larvæ of a large number of species of flies, including Anthomyia pluvialis, Wohlfahrtia magnifica and Dermatobia cyaniventris, and several species of Sarcophaga, Sarcophila, Lucilia, Cordylobia and Hypoderma.

Chrysomyia macellaria (Lucilia macellaria).--Under the name of "screwworm," the larva of this fly is widely and unfavorably known in the United States. It seems to be rather common in Texas, but cases are known from such northern States as Kansas, Iowa and Nebraska. Probably over a hundred cases of screwworm infestation in man are known from the United States, most of them cases of cephalic myiasis. Where subcutaneous infestation with this worm occurs, it is usually as a result of the eggs or living maggots being deposited in wounds.

Cordylobia anthropophaga.-This is a subcutaneous parasite of man in Africa which has become famous as the Ver de Cayor.

Dermatobia cyaniventris.- This is the grub which infests man in Central America and Brazil, and which is known in its earlier stage, when it is club-shaped, as the Ver Macaque, and in its later stage, when oval, as the Torcel. It is reported that the eggs of this fly are carried by large mosquitoes, and infestation with the maggot may occur in connection with mosquito bite. 
Hypoderma lineatum, $H$. bovis, and $H$. diana.-These grubs, normally parastitic in the subcutaneous and other tissues of cattle and deer, and with a life history which calls for their migration to the dorsal region of the infested animal, are known to have been the cause of "creeping eruption" in a number of cases, and are perhaps the agent involved in most cases of the sort.

Sarcophaga carnaria and other species of flesh flies become of great importance in war times owing to the numbers of unburied bodies furnishing habitats similar to those in which they normally breed, and to the fact that under these conditions they frequently infest the wounds of the living and add to the difficulties of nursing and of treatment.

Treatment of Subcutaneous Myiasis.-The essential treatment consists in the extraction of the larvæ. Hypodermic injections of antiseptics have usually failed to kill these. It is sometimes possible to remove them by surgical measures under cocain anesthesia. Cases have been reported in which as high as 87 subcutaneous larvæ have been present, and in such cases surgical measures would have to be used with some caution. In maggot-infested ulcers, the use of chloroform to kill the maggots is perhaps as good treatment as any.

Cephalic Myiasis.-In cephalic myiasis the nostrils, ears and sinuses may be invaded, and such tissues as the soft palate, the turbinates, etc., eaten away, by the maggots of a number of species of flies, such as Sarcophaga carnaria, Sarcophila latifrons, Wohlfahrtia magnifica, Lucilia nobilis, Chrysomyia macellaria, Compsomyia viridula, Calliphora vomitoria, Calliphora limensis, and Oestrus ovis.

Chrysomyia macellaria.-This "screwworm," already noted as causing subcutaneous myiasis, is even more frequently reported as an invader of the nostrils, ears, and sinuses of the head. Either the ova or larvæ may be deposited at the exterior nares, and this is especially apt to occur in the case of persons who are sleeping, intoxicated or sick, or in the case of children. At times hundreds of these maggots are present in the head. The violent inflammation and edema which result are extremely painful and dangerous, the tissues swelling to the point where swallowing and respiration become difficult or impossible. The tissues are attacked and broken down and the accompanying bacterial invasion gives rise to suppuration and necrosis. Death occurs in a large number of cases, several of the published lists of cases showing a mortality of 60 to 70 per cent.

Oestrus ovis.-This grub, normally a parasite in the nostrils and frontal sinuses of sheep, is a not uncommon parasite of man in $\mathrm{Al}$ geria, causing a disease known as "Thim'ni."

Treatment of Cephalic Myiasis.-The maggots in the head cavities are usually accessible to chloroform and may be killed by injection or inhalation of this substance, and extracted by the use of forceps. The injection of a 4 per cent. solution of cocain has been found of value in this procedure. 
Gastrointestinal Myiasis.-The chance of dipterous eggs or larvæ being ingested in food or water is extremely good, as has already been noted. What the subsequent fate of the ingested eggs or larvæ would be for the various species involved is a matter concerning which we have too little information. We know that a number of grubs are normally parasitic in the stomach or intestine of various animals, and that a number of others are normally free-living, but have little data as to how well some of the free-living forms are capable of adapting themselves to conditions in the digestive tract of various animals and becoming facultative parasites. It is, however, fairly well established by a large amount of clinical observation and an extremely small amount of experimental evidence that myiasis of this type may and does occur. The larvæ of the following flies. have been reported from time to time as parasitic in the alimentary tract of man, at times with a resultant gastro-intestinal disturbance of a more or less severe type: Muscina stabulans, Pollenia rudis, Tachina larvarum, Piophila casei, Calobata cibaria, and various species of Eristalis, Aphiochata, Gastrophilus, Musca, Fannia, Sarcophaga, Lucilia, Calliphora and of Drosophila.

Treatment of Gastrointestinal Myiasis.-Apparently these larvæ may be removed at times by the commonly used vermifuges, but little seems to be known about treatment. It is likely that chloroform in 3- to 4-gm. doses in oil would be efficacious. Carbon bisulphid has been found very efficacious against the common Gastrophilus larvæ in the stomach of the horse, and this treatment might. be worthy of a trial in a case determined as being gastrointestinal myiasis.

Vesical Myiasis.-About twenty cases of vesical myiasis have been reported, the flies concerned being as a rule species of Fannia, and usually Fannia canicularis. These larvæ have been reported as passed in the urine of persons of both sexes, and it is assumed that such cases arise as the result of eggs being deposited by the adult fly in the vicinity of the urinary meatus of persons asleep or intoxicated and with the meatus accessible to the fly.

There seems to be no record of treatment for this condition.

\section{ADULT BITING FLIES}

In this group may be included all two-winged biting insects, such as mosquitoes, gnats, horse flies, breeze flies, tse-tse flies, stable flies, horn flies, deer flies, etc. The following genera are involved in the species which are reported as biting man: Phlebotomus, Anopheles, Myzomyia, Pyretophorus, Myzorhynchus, Nyssorhynchus, Cellia, Culex, Aedes, Mansonia, Taniorhynchus, Mycterotypus, Culicoides, Oecacta, Johanseniella, Hamatomyidium, Melusina, Chrysops, Rhinomyza, Tıbanus, Hamatopota, Hippocentrum, Rhagio, Asilus, Glossina, Stomoxys, Stygeromyia, Lyperosia, Hamatocia, Philamatomyia, Hippobosca, Ornithomyia, and some others, more especially some 
mosquito genera not named here. There are scores of species too numerous to list. These diptera are injurious in two ways: They bite, and they carry parasites and diseases.

The damage occasioned when a person is bitten by flies, mosquitoes and other diptera, depends principally on the amount of biting done and the species of insect doing it. Some species attack quietly and have a comparatively painless bite, but they inject a poison into the wound which will later give rise to a considerable itching, inflammation, vesicle formation, etc. Other insects attack viciously and have a large piercing apparatus which causes a sensation like the jab of a needle. Much depends also on the number of bites. Where many bites are inflicted in a short time, the person bitten may be rendered ill or even killed as a result of irritation, shock and loss of blood.

But it is as carriers of disease and of parasites that diptera are of most importance. Diseases and sometimes animal parasites may be conveyed by insects from one person to another, or from other sources to man, in a purely mechanical way, bacteria being carried on the feet of flies from excreta or open sores to wounds, the external mucosa or the food, or being carried on the biting mouth parts of bloodsucking insects from one person to another. Thus the common house fly or typhoid fly is believed to convey the germs of such diseases as typhoid, tuberculosis, cholera and dysentery to man, since it is known that the germs of these and other diseases will adhere to the feet of the fly and that its well-known habits of life, breeding and feeding in filth and then entering dwellings and crawling over exposed food and on the bodies of persons, must lead to the conveyance of those diseases where the germs are readily available to the fly in the sputum and excreta of sick individuals. In a similar mechanical way, such biting flies as the stable fly, Stomoxys calcitrans, or the various horse flies, might carry anthrax from the blood of a diseased animal to man, either by the mere puncture of the germ-soiled biting parts, or by puncture followed by the crushing of the fly on the open wound and the incidental inoculation of the person by the infected blood from the stomach of the fly.

Aside from such mechanical conveyance, diseases are transmitted by insects in cases where the insect serves as the necessary intermediate host of the parasite causing the disease. In this way mosquitoes of the genus Aedes (Stegomyia) transmit yellow fever; those of such genera as Anopheles, Myzomyia, Pyretophorus, Myzorhynchus, Nyssorhynchus and Cellia transmit malaria; those of such genera as Myzomyia, Pyretophorus, Myzorhynchus, Cellia, Culex, Mansonia and Taniorhynchus transmit filariasis; a fly of the genus Chrysops transmits Loa loa; and species of Glossina transmit the trypanosome of sleeping sickness.

The control of diptera as vectors of disease calls for a knowledge of the life history and breeding habits of the fly or mosquito involved. When these facts are known, it is usually possible to devise measures 
for the destruction of the fly eggs or larval stages or of the breeding places. The adult flies may be trapped or access to the human skin or food prevented by the use of screens, gloves, etc.

\section{HEMIPTERA-BUGS}

Bugs of the following genera have been reported as attacking man: Cimex, Eciacus, Anthocoris, Lyctocoris, Reduvius, Rhinocoris, Prionotus, Conorhinus, Triatoma, Rhodnius, Phonergates, Rasahus, Melanolestes, and Reduviolus. Of these, Cimex lectularius and Triatoma megistus are the most important.

Cimex lectularius.- The bedbug molts five times in its development and takes at least one meal of blood before each molt. It follows from this that it is apt to function as a carrier of disease from one person to another. However, while there is at present some reason to suppose that the bedbug may carry kala-azar, relapsing fever, Brazilian trypanosomiasis and other diseases, more work remains to be done regarding these and other possibilities. The bug itself is a pest deserving of attention. To some persons its bite is very unpleasant and results in a severe reaction, and to most persons the presence of these pests will render a night's sleep impossible.

Treatment.-For moderate infestations the use of gasoline and benzine, soaking these into the carpeting and cracks, will usually be found adequate. Due regard must, of course, be paid to the danger from fire where these substances are used. Where the bugs are very numerous it may be necessary to resort to fumigation with hydrocyanic acid gas. This gas demands expert attention and extreme care, as deaths occasionally result from carelessness or ignorance in its use.

Maintaining a temperature of about $\mathrm{I} 20$ to $130^{\circ} \mathrm{F}$. for Io hours by running the heating plant in warm weather has been found effective in killing the bugs and their eggs.

Triatoma megistus.- This is a large and active bug which serves as the transmitter of Trypanosoma cruzi, the cause of Brazilian trypanosomiasis or Chagas disease.

Treatment.-The bites should be disinfected with a I : rooo solution of corrosive sublimate, or some similar disinfectant, to prevent infection. The bugs may be attacked in the same way as the bedbug, and in other ways, owing to their large size, and since they fly at night, doors and windows should be screened against them.

\section{PEDICULOSIS-LICE}

There are three species of lice attacking man: Pediculus vestimenti, the body louse; Pediculus capitis, the head louse; and Phthirius pubis, the so-called "crab louse." Lice are more frequently present on the heads of women, owing to their long hair, than on the heads of men. They are readily transmitted from one person to another by 
contact, common use of combs and brushes, exchange of hats, etc. Under the unhygienic conditions prevalent in times of war, with large bodies of men of all stations of life and of wide variation in personal habits and clegree of cleanliness, with the limited time and facilities for bathing and even for removal of clothing, these insects speedily rise to a pest of primary importance. They are not only a source of irritation which detracts from a soldier's concentration of attention to the dangerous business of war and from his ability to find recuperation in sleep, but they are known to transmit the dreaded typhus fever by their bites, and such diseases as impetigo and favus by mechanical transfer of the etiological agents of these diseases.

Treatment of Pediculosis. - The great European war of I9I4 has brought out a large amount of literature dealing with measures for combating pediculosis. Adequate comparative tests of all the various remedies proposed have not been made as yet, so that while it is possible to cite the various remedies found to be efficacious, it is not possible as yet to select one of these as the best under given circumstances. Zucker has found that engorged lice are more resistant than starved lice, and of course nits or eggs are more resistant than either. He finds that an engorged louse can live a week without food, instead of three days as commonly stated. In actual practice, of course, treatment should provide ample margin over what is needed to kill the most resistant forms.

Lice in clothing, bedding, dwellings, etc., may be killed by heat or fumigation. Carefully ironing the seams of clothing is a useful procedure for individual cases. For extensive outbreaks, as in armies, clothing may be subjected to hot streaming dry air. Zucker finds that engorged lice are killed by exposure to $40^{\circ} \mathrm{C}$. for six hours; to $45^{\circ}$ for two and one-half to three hours; to $50^{\circ}$ for one to one and one-half hours; and to $65^{\circ}$ for fifteen minutes. In practice, Seligmann and Sokolowsky use the following temperatures: $100^{\circ}$ to $110^{\circ}$ for thirty minutes; $120^{\circ}$ to $130^{\circ}$ for twenty minutes; $150^{\circ}$ to $160^{\circ}$ for fifteen minutes. Under the low pressure of a partial vacuum, heat is even more efficacious in killing lice; cars are treated in this way on the German state railroads. Both sulphur and chlorine fumigation, properly used, are effective against lice. Fumigation should be repeated in ten days to two weeks.

The contact insecticides for use against lice on man and clothing are legion. Kerosene oil is highly recommended by Castellani and Jackson as a powerful licecide which practically kills body lice instantly. They used it in wholesale disinfection of troops and prisoners in Serbia, applied to naked men, after bathing, by means of a spray pump or applied directly with cloths or cotton wool, with no bad effects and very little dermatitis. Benzine and gasoline are similarly effective and have a less objectionable odor. The same writers find vaseline alone quite efficacious, and regard the licecidal action of the various ointments as due to the vaseline used as a base. 
Other substances found efficacious are guaiacol, anise preparations, naphthaline, betanaphthol, menthol, turpentine, xylene, dichlorobenzene, cyclohexanol and cyclohexanone. Kinloch finds the most lethal substances to be the chlorine derivatives of methane, ethane, and ethylene, and advises the use of soap solutions of 2 per cent. trichlorethylene or to per cent. tetrachlorethane. Alessandrini advocates the use of 20 to 30 parts by weight of chloroform in any fatty excipient. The British found the N. C. I. powder (naphthalin 96 per cent., creosote 2 per cent., iodoform 2 per cent.) very effective.

\section{SIPHONAPTERA-FLEAS}

Fleas have become of immense interest since it was found that they were of primary importance as transmitters of bubonic plague. It is now known that rats and other rodents serve as a reservoir for bubonic plague and that fleas which have fed on the blood of plagueinfected rats ingest bacilli which multiply in their bodies and transmit the disease to other rats and to man by their bite, the bacilli being regurgitated or entering the wound from the feces of the flea. Prophylaxis must therefore take this insect into consideration and direct measures jointly against fleas and rats. Perhaps the most important transmitters of plague bacilli are the rat fleas, Xenopsylla cheopis and Ceratophyllus fasciatus, but the disease may also be carried by Ceratophyllus anisus, Pygiopsylla ahala, Leptopsylla musculi, Pulex irritans, Ctenocephalus canis, Ctenocephalus felis, Hoplopsyllus anomalus, and Ceratophyllus acutus. The dog flea, Ctenocephalus canis, and the human flea, Pulex irritans, are also suspected of carrying leishmaniasis. The dog flea is also the common intermediate host of the double-pored tapeworm, Dipylidium caninum, which is transmissible to man. Finally the bite of the flea causes a variable amount of distress to different individuals.

Treatment and Prophylaxis.-In a general way it is not difficult to keep persons free from the attack of fleas provided dogs, cats and other animals are kept out of the house or kept free from fleas. It is therefore desirable that houses be rat-proof to keep out the unwelcome rat and the dangerous flea, and that dogs and cats be kept free from fleas by the use of I to 2 per cent. creosol washes or applications of pyrethrum powder or powdered naphthalin. Fleas breed in dust and trash, and their eradication calls for cleanliness and disinfection around the sleeping places or kennels of dogs. Here one may use gasoline or kerosene, with due regard for fire, or pyrethrum powder or flake naphthalin sprinkled liberally about. Fleas without food starve inside of a week as a rule, but as the development from the egg may take almost three months, starvation is not apt to be a useful measure. The vacuum cleaner is of value in keeping down fleas. Oil of pennyroyal is a good repellent in a neighborhood where fleas abound. To relieve itching from the bite, use menthol, camphor, carbolated vaseline, or hydrogen dioxid. 


\section{ARACHNIDA-SPIDERS}

The Acarına, or mites, a group which includes the ticks, and the Linguatulidx, or tongueworms, constitute the two important parasitic groups of the class Arachnida, or spiders, from the standpoint of human parasitism.

\section{ACARIASIS-MITES}

A large number of ticks and of the smaller mites have been found to attack man, often inflicting severe bites or giving rise to a marked dermatitis and pruritus. A list of these includes the following ticks: Argas persicus, A. reflexus, $A$. brumpti, Ornithodoros moubata, $O$. coriaceus, $O$. turicata, O. talaje, $O$. pavimentosus, O. tholozani, Otobius megnini, Ixodes ricinus, I. r. scapularis, I. hexagonus, I. pilosus, I. bicornis, I. putus, I. rasus, Hyalomma agyptium, Amblyomma americanum, A. cajennense, A. dissimile, A. hebraum, Hamaphysalis punctata, Dermacentor reticulatus, D. andersoni or D. venustus, D. variabilis, Boöphilus decoloratus, Rhipicephalus sanguineus, $R$. simus, and Rhipicentor bicornis. Of the above, especial interest attaches to Ornithodoros moubata, the tick which transmits to man the blood parasite, Spirocheta duttoni, the cause of relapsing fever or tick fever. Otobius mengini occurs at times in the ears of man, and as it has been known to cause the death of cattle, on which host it is commonly found parasitic in the same location, it must be looked upon as a dangerous parasite. Dermacentor andersoni or D. venustus is the most important carrier of Rocky Mountain spotted fever, a highly fatal disease rather widely distributed in the western United States. This same tick causes in the United States and Canada what is known as tick paralysis in man and other animals by attaching along the spinal column and at the base of the skull. This paralysis may terminate fatally.

The following mites, other than ticks, have been reported from man: Dermanyssus galline, D. hirundinis, Liponyssus lobatus, $L$. sylviarum, Leiognathus bacoti, L. morsitans, Holothyrus coccinella, Laelaps stabularis, Trombidium striaticeps, Metatrombium poriceps, Microtrombidium meridionale, $M$. pusillum, $M$. wichmanni, $M$. vandersandei, Trombidium akamushi, T. tlalsahuate, T. americanum, $T$. irritans, Cheyletus eruditus, Acaropsis mericourti, Tydeus molestus, Pediculoides ventricosus, Tarsonemus intectus, T. hominis, Neprophages sanguinarius, Notadres cati, Sarcoptes scabiei, S. s. crustosa, S. s. canis, S. s. capra, S. s. dromedarii, S. s. equi, S.s. ovis, S. s. suis, S. s. leonis, S. s. vulpis, S. s. auchenia, S. s. wombati, Tyroglyphus siro, T. longior, T. farina, Histiogaster entomophagus, Rhizoglyphus parasiticus, Carpoglyphus alienus and Demodex folliculorum. Not all of the above records can be regarded as cases of true parasitism, and physicians finding mites on the bodies of patients should secure reasonably good assurance before regarding these as parasitic. Quite a number of the mites named above are parasitic as a rule on mammals other than man, and their occurrence on such animals as 
the rat carries with it the possibility of the transfer to man of such serious diseases as plague. The fact that a number of these mites are parasitic on domestic animals suggests that these animals should be kept free from such pests and handled with due regard to their possible presence and transfer to man. Cheyletus eruditus and Acaropsis mericourti are recorded from the external auditory canal of man; Neprophages sanguineus and Carpoglyphus alienus from the urine. Such records call for close scrutiny before regarding them as cases of parasitism. Species of Tarsonemus have been reported from tumors, and Saul has suggested that an etiological relation may exist between these acarids and tumor formation. Borrel has thought that Demodex may transmit leprosy and cancer. Pediculoides ventricosus, normally a predaceous mite, attacks workers in straw or persons using straw mattresses, giving rise to a severe dermatitis; Rhizoglyphus parasiticus occasions cutaneous eruptions in workers on tea plantations; Tyroglyphus siro, $T$. longior and T. farince, the so-called cheese mites or sugar mites, frequently attack persons who handle the products infested with these mites, such as grocers, workers handling grain, vanilla pods, etc., giving rise to the dermatitis and pruriginous eruptions known as "grocer's itch." These mites may also be ingested in infested food products, giving rise to gastric and intestinal catarrh; the mites have been reported from the feces and also from the urine.

\section{SCABIES}

In addition to the common form of scabies in man, and the more severe form known as Norwegian scabies, due to a variety of the usual Sarcoptes, it will be noticed that sarcoptic scabies, due to the numerous varieties of Sarcoptes peculiar to different mammals, including that due to Notodres in the cat, is transmissible in probably all cases from these other animals to man.

Cunningham emphasizes the fact that the only lesion due to the itch mite is the furrow produced by its wandering through the skin. The papules, vesicles and pustules are a secondary dermatitis due to scratching. Since these sequelæ may swamp the original lesions, a differential diagnosis from dermatitis of other origin must be based on the site of the lesions. The itch mite always selects the soft skin of the abdomen, inner aspects of thighs, axillæ, web of fingers and toes, anterior surface of wrist, nipples and penis; the face and head are exempt. Lice occur in the portions of the body covered by the clothing and should be looked for in the underwear, not on the body; vesicular urticaria commonly occurs on the face and scratching brings out new lesions at once. Schamberg and Strickler find that eosinophilia in scabies is less than in the bullous diseases.

Treatment of Acariasis.-For the transient attacks of such temporary parasites as the cheese mites, straw mites, etc., sulphur ointment is probably as serviceable as anything. Such mites cannot reproduce while parasitic on man and the parasitism is consequently 
self-limited. The more vicious 'attacks of ticks may be met with applications of chloroform, turpentine or oils of some sort. In cases of infestation with Olobius megnini in the ears, the ears may be flooded with some bland oil, as olive or linseed oil. The treatment for scabies consists in cleanliness and the persistent application of such classic remedies as sulphur ointment. 


\title{
DISEASES OF THE BLOOD
}

\author{
By Joseph L. Miller, M. D.
} PERNICIOUS ANEMIA

Etiology.-It is generally accepted that pernicious anemia may be associated with bothriocephalus latus infection. The etiology of pernicious anemia exclusive of this form is still unknown. Considering the great prevalence of bothriocephalus latus infection in Finland (2o per cent. of all inhabitants) pernicious anemia occurs only very infrequently. The permanent recovery of the patient in the majority of cases after removal of the worm gives it a definite etiological relation. Schaumann reports, however, many cases where after the removal of the worm and without reinfection the anemia returned. This and the infrequent occurrence of pernicious anemia in the infected indicates that undetermined predisposing factors must play an important rôle. It has been sugggested that pernicious anemia develops only in case the worm dies in the intestinal tract, but this has not yet been proven.

The relation of focal or general infection to pernicious anemia has been suspected but not proven. Herter made a careful bacteriological examination of the feces in fifteen patients. He reports that certain anaërobic bacteria, especially the Bacillus capsulatus aërogenes, largely disappeared from the stool during the remission and for this reason thinks they might bear some relation to the anemia. Syphilis has frequently been associated with pernicious anemia, but when we consider the great prevalence of lues it is probable the combination is only a coincidence. In support of this view no one except Laache has reported a permanent cure after specific therapy. Considering the occasional long remission periods the permanency of a cure is questionable. Cabot in rro cases and Türk in sixty have never found syphilis an etiological factor. Pregnancy has by some been considered an etiological factor. Pernicious anemia occasionally occurs during pregnancy and not infrequently improves after confinement, later to relapse and run the usual course. The pregnancy may be merely a coincidence, or possibly a secondary exciting factor. Türk in fifty cases has seen pernicious anemia develop only once during a pregnancy.

Pernicious anemia has been reported to develop after prolonged secondary anemia, due to loss of blood, especially from hemorrhoids. These cases, however, occur so infrequently that it is difficult to establish any relation between cause and effect. Bad hygienic surroundings and poverty have been considered possible factors, again, however, without any convincing evidence. It does not appear to 
the writer that pernicious anemia is a disease especially of the poor people. This opinion is based upon the relative infrequency of the condition as seen in Cook County Hospital and in outside practice. During the year I9I $3,29,4 \mathrm{I} 3$ patients were treated in this institution, twenty-seven of whom had pernicious anemia.

Although the etiological factors are still undetermined, there is a general agreement that increased blood destruction and not disturbed blood formation is responsible. In the rather infrequent aplastic type disturbed blood formation plays a rôle, only in that pernicious anemia has occurred in an individual with inefficient bone marrow.

That blood hemolysis is responsible for the anemia is shown by the large amounts of iron in the liver, the deposits of blood pigment in the various organs and the great increase of urobilin in the stool-the latter a good index of blood destruction. Eppinger reports I.I4 gm. in the twenty-four hour stools of pernicious anemia, the normal output being about 0.15 gm. The lemon yellow color of the skin is also due to deposits of blood pigment. The changes in the bone marrow are in the nature of a compensatory hyperplasia, an effort to replace the rapidly destroyed blood corpuscles.

There has been considerable speculation on the nature and origin of the hemolytic agent or agents, but up to the present time this has not been determined. Talquist and Faust by macerating the bothriocephalus latus were able to isolate a hemolytic substance from some, but not all worms. This was an oleic acid and probably absorbed in the form of sodium oleate. Hemolytic lipoids have been obtained from various intestinal bacteria, more especially the colon bacilli (Ludke and Fejis). Korschun and Morgenröth, Berger and Tsuchiya and others have demonstrated a hemolytic lipoid in the gastric mucosa of pernicious anemia and normal individuals. At first it was reported that larger amounts were present in the mucosa in pernicious anemia than in the normal, but this was later disproven. None of the investigators have been able to demonstrate increased hemolytic substances in the stool. As might be anticipated, a severe anemia can be produced in animals by injecting these lipoids, but no one by this method has been able to produce experimentally a definite clinical pernicious anemia, and no one has been able to demonstrate hemolytic lipoids in pernicious anemia, that were not also present in the normal. Eppinger and King have reported great increase of the fatty acids in pernicious anemia and consider this as the hemolytic agent. If further reports confirm their findings, it may prove to be a very important observation. Cederberg has advanced the view that as a result of the atrophy of the intestinal mucosa, absorption of incompletely split proteins is permitted, those acting as foreign proteins give rise to pernicious anemia. Iwao has produced severe anemia by the subcutaneous injection of amines derived from bacterial destruction of protein.

There has been a great deal of discussion regarding the relation of the atrophy of gastrointestinal mucosa to pernicious anemia. Some 
have considered it a causal factor-Cederberg as just mentioned; others believe as the result of this condition digestion is disturbed, putrefaction occurs in the bowel contents and the absorption of these products is responsible for the anemia. Others have considered the atrophy a result of the anemia. Schaeffer denies that atrophy of the mucosa occurs in pernicious anemia, that these changes are all postmortem. It seems scarcely probable that this error of observation should have passed detection for so many years. Faber and Block and others have found constantly with the atrophy a round-cell inflammation of the mucosa, apparently the result of chronic irritation. That the atrophy is not necessarily present has been shown by M. Koch, quoted by Türk, who found the mucous membrane normal in two out of five autopsies. Gastric atrophy may precede for many years any clinical manifestations of pernicious anemia, as noted in a case observed by the writer. Gastric atrophy is frequently observed in the bothriocephalus anemia, and after recovery still persists. Summing up all evidence, it would appear that the atrophy of the gastrointestinal mucosa, like the changes in the cord, is a result rather than a cause of the anemia.

Apparently a disease resembling somewhat closely pernicious anemia appears spontaneously in horses. Moffitt refers to the epidemic among horses described by Mack of the University of Nevada. There develops a marked degree of anemia with high color index but without decided changes in the red corpuscle. Paralysis of the posterior extremities is common. The disease runs a chronic course, with a tendency to spontaneous remission. Details regarding the character of the infectious agent are still lacking. Seyderhelm has recently studied the pernicious anemia of horses in Switzerland. This disease has a blood picture closely resembling pernicious anemia in man, but clinically the course of the disease is quite different, resembling an acute or chronic infection, with no history of remission and no report on the presence of spinal cord changes. The disease is bacterial in origin, the blood and urine containing the infectious agent which is ultramicroscopic. The larva of the horse fly, which he found constantly in the stomachs of the affected animal, contained the infectious agent and he expresses the view that these larva may act as a host. As shown from these reports there is considerable doubt whether either of these diseases in horses is of the same character as pernicious anemia in man.

Eppinger has called attention to the possible rôle of the spleen in pernicious anemia, reporting in this condition a thickening of the central artery, with diversion of the blood current to the sinuses in the spleen pulp where the corpuscles are destroyed. The work of Pearce and others has shown that the spleen is concerned in modifying the resistance of the red corpuscle to hemolytic agents. Removal of the spleen increases the resistance of the corpuscle in the normal animal or man, and at the same time stimulates the bone marrow. It has been shown that in pernicious anemia the resistance 
of the red corpuscle is above normal. This phase of the subject will be gone into more in detail when we consider the value of splenectomy as a therapeutic measure.

Course and Prognosis.-The course of pernicious anemia is extremely variable. In rare instances it may' run a rapid course, a steadily progressive anemia with fatal termination within a few weeks. Sandoz reports fatal termination in fifteen days, Eichhorst six weeks and Müller seven weeks. As a rule the course is much more prolonged and rarely goes to a fatal termination without either the anemia coming to a temporary standstill or the patient showing a definite remission with more or less marked improvement. Not infrequently the anemia reaches a certain degree, and may remain practically stationary for a week or even many months, with at most slight remission, but eventually with downward progression and fatal termination. The remissions may be slight in degree and of short duration, or the anemia may entirely disappear and the patient enjoy normal health, and this may continue for many years with finally a recurrence of the anemia, and a fatal termination. In Cabot's large series of cases remissions occurred in 80 per cent., about 2.5 per cent. had five or more remissions, and 20 per cent. did not show any remission within six months. In one patient under my observation, a laboring man, seen at the Cook County Hospital with severe pernicious anemia, recovered apparently, worked steadily for eight years feeling perfectly well, appearing then again in the hospital with a severe and rapidly fatal pernicious anemia. The patient was not seen and the blood not examined in the interval; hence, I am unable to state whether the blood picture in this interval was actually normal. Türk reports a case, however, where in such a period of improvement the microscopical blood picture showed only the evidence of slight secondary anemia. In another patient this period of improvement continued three years, the man working comfortably in this interval, although never with a normal blood picture. McPhedran reports a remission of seventeen years, the patient in the interval in good health. In some of these periods of remission, the regenerative activity of the bone marrow may carry the production of red corpuscles to beyond the normal, as in a case of the writer's, where in November, I906, the patient had $1,400,000$ red corpuscles and 24 per cent. hemoglobin, and the following June $6,600,000$ erythrocytes and IIO per cent. hemoglobin. This improvement continued for three months, then a rapidly progressive return of the trouble with fatal termination.

Remission may occur at any stage of the disease and apparently even when the anemia is so severe that death is impending a remission may appear. Laache reports a remission after a patient had been comatose six days and Renvers in a patient brought to the hospital moribund. The writer had a patient in the Cook County Hospital who was unconscious for three weeks and six weeks later was walking about the ward. Quincke's case with 143,000 red 
corpuscles had a spontaneous remission. These remissions may recur over and over again as in Mathe's patient, a brick mason, who in a period of eleven years had ten remissions and was able to return to his work during each.

It is still a debatable question whether permanent recovery ever occurs in pernicious anemia. Cabot reports two out of 1200 patients who have remained well for four years and where, he thinks, the recovery may be permanent. Nägeli reports two recoveries of eleven years' duration and one of five years; Grawitz five cases well after five years. A considerable number of similar reports may be found in the literature. With our knowledge of the long duration of remissions, it can readily be seen that none of these reported cases are necessarily permanent cures. A number of writers report on the more favorable outlook of those infrequent cases, occurring during pregnancy or due to syphilis. The question arises whether these are true pernicious anemias. The writer has never seen a permanent cure. At best, if such recoveries actually occur, they are so infrequent as to make the prognosis in any individual case exceedingly unfavorable. The only exception is those cases due to bothriocephalus latus. When we discover what is the cause of the remission and why blood hemolysis spontaneously ceases, often for long periods of time, we will be in a position possible to treat the disease more intelligently.

Treatment.-Prophylactic measures cannot be intelligently applied until we have more definite knowledge of etiology.

While bothriocephalus latus infection has been reported in America there are no references to pernicious anemia, developing in any of these cases. It is nevertheless wise in each instance to exclude tapeworm as a possible cause of the trouble, and if present institute the necessary treatment for removal of the worm.

As a cure is improbable the question of importance is whether by treatment we can modify the intensity of the disease or bring about a period of remission. Such remissions are probably due to either a disappearance of the hemolytic agent or to the acquisition of an immunity to it. That such remissions occur spontaneously is generally admitted. The literature contains numerous references to spontaneous remissions occurring in patients who had already reached a comatose state. In a patient of the writer's, where the coma had persisted for several weeks, a remission developed without the aid of any drug, dietary, or special hygienic measures. With such remissions occurring spontaneously and at the most unexpected times, the question must be seriously considered whether anything we do is responsible for this improvement. The great number of remedies for this condition, which has appeared in the literature during the past twenty years, speaks strongly against the value of any one of them. A patient, who has a remission under a certain line of treatment, when a relapse occurs, may not be affected by this same treatment. As further evidence against the specific value of 
any treatment is the relatively few patients benefited by any single line of treatment. With this understanding the various methods of treatment may be considered.

General Care.-On account of the anemia the severe types belong in bed. Milder forms should restrict their exercise to the point where they are free from dyspnea or a sense of exhaustion. Fresh air is essential. As these people are very sensitive to cold, when possible a warm sunshiny climate is desirable where the patient can be kept freely in the open air. Mention will be made later of the special Grawitz diet, but unless there is some special contraindication a simple but general diet should be used. On account of the gastric and intestinal atrophy the foods to assist digestion should be soft and finely divided. This dietary may include all cereals, soft wellcooked root vegetables, puree of green vegetables, fruit especially cooked, or fruit juices, jellies, marmalades, eggs, tender and well-masticated meats, simple desserts, as ice cream, gelatin, tapioca, rice and simple puddings. In case of diarrhea the necessary modification of diet should be made. The bowels should be regulated by diet or a simple laxative. In case the latter is used, care should be exercised on account of the danger of exciting a persistent diarrhea.

Patients with suspected syphilis or a positive Wassermann should be given specific treatment as would be given to any other individual with such an infection. When pernicious anemia develops during pregnancy, early emptying of the uterus is desirable. It is perhaps needless to say that patients with pernicious anemia during periods of remission should avoid pregnancy.

Grawitz Treatment.-Grawitz believed that decomposition of animal protein in the intestinal tract was a marked factor in pernicious anemia. His treatment consists in daily lavage of the stomach and the use of high enemata. Meats and eggs were excluded from the dietary, which consisted largely of finely divided carbohydrates. He gave intestinal antiseptics, as menthol and salol, and to combat the achylia administered dilute hydrochloric acid. This treatment, combined with absolute rest in bed, was continued for three weeks. Grawitz reported very satisfactory results, including several socalled permanent cures, with this line of treatment. In my hands no special benefit has been derived from this treatment. It may, furthermore, be dangerous to attempt gastric lavage in patients with severe anemia.

Focal Infection.-Since Hunter's time the possibility of focal infections being an etiological factor has been considered. Focal infections about the teeth are so common that their possible relation to general conditions is difficult to determine. Infection of this character, however, should be, if possible, corrected, although there is no special evidence to show that they are etiological factors in pernicious anemia.

Blood Transfusion.-Blood transfusion has been used for many years in the treatment of pernicious anemia, interest in the subject 
having been revived by Morawitz in 1907 , largely due to changes in technique, which avoided some of the previous complications. Here, as with other therapeutic measures, favorable results have been reported. Not only has there been temporary improvement as the direct results of the transfusion, but Hanssen reports the appearance of a remission in six of twenty-five cases. An analysis of his report shows very little encouraging. Three of the six patients benefited received more than one transfusion and improvement took place only after a single injection, the others being without effect. Where a single transfusion was beneficial, later during a relapse the results were negative. In only two cases does he report what he considers as striking results, and these are not more striking than may be observed occasionally when no treatment has been given. Some of the patients were given seven transfusions without results. $\mathrm{He}$ used 100 to 300 c.c. of defibrinated and filtered blood. Isolated cases have been reported by other observers but there is no uniformity in the results. Transfusion is not free from danger, whatever method may be employed. Febrile reactions indicating moderate degrees of hemolysis occur in ro to 30 per cent. of cases (Lindermann). Severe hemolysis leading to fatal termination occurred in two out of fifty-four transfusions made by Percy and in five out of 212 reported by Ottenberg and Libman. Careful hemolytic tests will avoid many, but not all of these accidents. Except in extreme emergencies tests should always be made previous to the transfusion.

As some have believed, if beneficial results followed transfusion, this must be due to the stimulating effect on the blood-forming organs. Weber has recommended the repeated intravenous injections of 5 c.c. of blood and Huber the intramuscular injection every five to six days of to to 40 c.c. Robertson in four cases was unable to demonstrate lessened urobilin output after transfusion.

Arsenic has long been used in the treatment of various anemias, and so is one of the best-established remedies in the treatment of pernicious anemia. It is supposed to stimulate the blood-forming organs. The experiments of Beltman and Kuhn and Aldenbowen showed after large doses marked increased blood destruction combined with stimulation of the bone marrow. After its long-continued use in animals a marked anemia developed. The method of action of arsenic in anemia is obscure, but clinical experience would suggest that in moderate doses it is of value in combating simple anemia.

The particular form in which the arsenic is used is rather unimportant. On account of the lack of appetite present in all of these patients it should not be given in sufficient quantities to disturb the stomach. The Fowler's solution on account of the readiness with which the dose can be increased is a deservedly popular form. Beginning with 2 minims in water after meals, this may be slowly increased to 8 or ro minims. This maximum dose may be maintained for two weeks, then slowly reduced to the minimum dose then after 
an intermission of ten days the process may be repeated. For hypodermic use the organic preparation, sodium cacodylate, has been extensively used. Most of the cacodylate is eliminated through the urine. A small portion, just how much has not been determined, is changed in the tissues to the inorganic form, and as such exercises a typical arsenic action; 0.03 to $0.15 \mathrm{gm}$. ( $1 / 2$ to $2 \frac{1}{2} \mathrm{gr}$.) daily is the usual dose.

Salvarsan in moderate doses has been used with varying results. Like any other form of arsenic, when given in large doses, it may increase the anemia. In moderate doses $0.3 \mathrm{gm}$., intravenously or intramuscularly, it produces at most only a moderate temporary fall in the red count. The dose may be repeated in two to four weeks. Leede in I9I I treated five patients with $0.4 \mathrm{gm}$. intramuscularly; four died within thirty-six hours to fourteen days. It is evident all of these were very advanced cases. The other patient after a single injection became much more anemic, the treatment was discontinued, a remission took place and in a later relapse salvarsan again seemed to act unfavorably. Bramwell reports favorable results in four out of seven cases. Boggs reports eleven cases, two of which died shortly after the first treatment; seven after a temporary increase in the anemia went into a period of gradual improvement; two showed very marked improvement. The writer has treated three cases with $0.3 \mathrm{gm}$., intramuscularly, and has not seen any beneficial results. Robertson after the use of salvarsan was unable to demonstrate any reduction of urobilin in the stool, an index of blood hemolysis.

Glycerin.-As oleic acid is hemolytic and this has been considered as the possible hemolytic agent in bothriocephalus latus anemia, Vetlesen recommends glycerin to combine and render inactive the oleic acid. He has used it in two cases and reports favorable results. He recommends a tablespoonful three times a day given in lemonade.

Cholesterin.-Cholesterin inhibits hemolysis, due to the activity of lipoids. Reicher experimentally was able to show that the anemia due to cobra venom could be lessened by feeding cholesterin, and recommended its use in pernicious anemia. He reports two cases in neither of which were the results at all definite. Klemperer, Gerhardt, Turk, Tabora and others have tried it, but have failed to find any evidence of its value. It is difficult to continue its use for a long period of time on account of nausea. It is best given in a 3 per cent. solution in olive oil, two tablespoonfuls three times daily. The addition of oil of peppermint makes it more palatable, or the mixture may be placed in capsules.

Splenectomy.-The latest treatment of pernicious anemia is splenectomy. Eppinger considers that in pernicious anemia the spleen has undergone pathological changes, chiefly in the nature of an obliterative process in the central artery of the follicles. This causes diversion of the blood current into the connective-tissue spaces of the pulp area where hemolysis occurs. During the past few years there has developed a very extensive literature on the function of the 
spleen, R. M. Pearce and his colleagues being leaders in this field. The hemolytic function of the spleen, however, dates back to Kolliker in I 847. Gabbi in I893 first demonstrated that after splenectomy the red corpuscles became more resistant to hemolytic agents, and this function of the spleen is now generally accepted. Splenectomy also apparently stimulates the bone marrow to increased blood formation. Splenectomy is of undoubted benefit in splenic anemia and hemolytic icterus, both of these being associated with great enlargement of the spleen. It was the beneficial results of splenectomy in this latter condition that led Eppinger to try it in pernicious anemia. The results up to the present time have on the whole been rather favorable as indica ting that splenectomy may be a factor in bringing about a remission in the disease. Perhaps as the best index of the value of this treatment is a review of the effect of urobilin output after the operation. Eppinger and Ranzi report five cases where in all after splenectomy the urobilin in the stool dropped to normal. Robertson reports six cases in all but two of which the urobilin reached normal within a short time after the operation. Huber's patient still had a high urobilin output five weeks after, and McCrudden three weeks after splenectomy. Moffitt reports one case where one week after operation the urobilin output was not modified. Moffitt has collected thirty-one cases from the literature where splenectomy has been performed. Eight of these died, the majority of the others showed marked improvement. Muhsam reports eleven cases, three died as a result of operation, two died later, the remaining six were greatly improved. Florchen collected twenty-six cases from the literature, 69 per cent. of which improved, but none cured. Romer reports two cases with sudden great improvement, followed within one to two months by relapse. The writer has personal knowledge of one patient, reported by Harpole and Fox, with severe pernicious anemia, where after splenectomy there was marked improvement in the anemia and now after the lapse of two years the blood examination shows $3,360,000$ reds, 88 per cent. hemoglobin, color index I.24 and microscopically normal. The spinal cord changes which, however, at the time of the operation were slight, progressed steadily for several months after the operation, showing that the agent responsible for these changes was not removed by the operation.

A study of the function of the spleen and a review of the operated cases leaves us in doubt as to the real value of the operation. It would appear, however, that improvement occurs too infrequently after splenectomy to be accounted for by coincident spontaneous remissions. That the results in a majority and probably all of the cases will prove to be transitory is also probably true. Many recurrences have been noted soon after the improvement following the splenectomy. Hirschfeld apparently truly states that this is only a palliative symptomatic therapy. If, however, it proves definitely palliative, provided the mortality is not too high, it may prove a distinct addition to our treatment. A hemoglobin of less 
than 40 per cent. has usually been considered by surgeons as rendering any operation dangerous. However, several patients with pernicious anemia, with 20 per cent. of hemoglobin, have survived this operation; in fact, it is in the most severe types that the most striking results have been obtained (see page 454 ).

Thorium.-By a special process there has been prepared from radiothorium a preparation, which, when placed in a solution of normal salt, would give off into the solution radioactive substances known as thorium $\mathrm{X}$. It was shown that thorium $\mathrm{X}$ when given in large doses caused marked destruction of the blood corpuscles, but when administered in small amounts stimulated the bone marrow. Bickel, Pletsch, and in this country Parks reported favorable results in pernicious anemia by the administration of thorium $\mathrm{X}$, either intravenously or by mouth. As little has been heard of this method of treatment during the past two years it would indicate that it possessed little value. The writer has treated two patients by this method without results.

Other agencies that have been recommended include iron, hydrochloric acid, male fern, intestinal antiseptics and appendicostomy with irrigation of the bowel.

\section{BIBLIOGRAPHY}

I. Austin, J. H., Pepper, O. H. P., The Relation of the Spleen to Blood Destruction and Regeneration, Jour. Exper. Med., I915, xxii, 675.

2. Branwerl, B., Salvarsan in Pernicious Anemia, Brit. Med. Jour., I91 I-1912.

3. Boggs, T. R., Salvarsan in Pernicious Anemia, Johns Hopkins Hosp. Bull., 1913, xxiv, 322 .

4. Cederberg, A., Die Pathogenesis Einiger Anämien mit Besonderer Berüchsichtung der Kryptogenetischen Perniciösen Anämie, Berl. klin. Wochnschr. 1914, li, 585.

5. Eppinger, H., Zur Pathologie der Milzfunction, Berl klin. Wchnschr., 19r3, 1, I509.

6. EPPINGer, H., Charnas, D., Was Lehren uns Quantitative Urobilinbestimungen im Stuhl? Zeitschr. f. klin. Med., r9r 3 , lxxvii, 387.

7. Hansen, O., Bericht Über Bluttransfusion bei Anämie, Verh. d. Deutsch. Kong. f. inner Med., I9I I, xxvii, I4I.

8. Herter, Intestinal Bacteria in Pernicious Anemia, Jour. Biol. Chem., Igo6, ii, I.

9. Klemperer G., Zur Behandlung der Perniciösen Anämie, Berl. klin. WVchnschr., $1908, x l v, 2293$.

ıo. Lindeman E., Reactions Following Blood Transfusion by the Syringe Canula Method, Jour. Am. Med. Assn., Igi6, lxvi, 624.

II. LUtDKE and FEJEs, Untersuchungen Ưber die Genese der Kryptogenetischen Perniziösen Anämie, Deutsch. Arch. f. klin. Med., I913, cix, 433.

12. Leede, C., Zur Frage der Behandlung der Anämie mit Salvarsan, Münch. med. Wchnschr., x9I1, lyiii, II 84 .

I3. Minot, Nitrogen Metabolism Before and After Splenectomy, Johns Hopkins Hosp. Bull., 1914, $\mathrm{xxv}, 338$.

14. Mofritr. H. C., Studies in Pernicious Anemia, Amer. Jour. Med. Sci., I9I4, cxlvii, 8 I 7 .

15. Morawitz, Die Behandlung Schweres Anämien mit Bluttransfusioner, Münch. med. Wchnschr.. 1907, liv, 767 .

r6. Pearce, R. M., Peet, M., Minor, The Relation of Spleen to Blood Destruction and Regeneration and to Hemolytic Icterus, Jour. Exper. Med., 1913, xviii, 499; Ibid., I 914 , xxii, 682.

17. Plesch, J., Falle von Perniciöser Anämie und Leukämie mit Thorium $\mathrm{X} \mathrm{Be-}$ handlet, Berlin, klin. Wchnschr., I912, xlix, 930.

18. Iwao, Intestinal Intoxication (Amines) in Relation to Pernicious Anemia, Biochem. Ztschr., 1914, lix, 436 . 
I9. Robertson, O. H., Urobilin in the Stool in Pernicious Anemia as Influenced by 606, Splenectomy and Transfusion, Arch. Int. Med., I915, xvi, 429.

20. Satterlee, H. S., Hooker, R. S., Transfusion of Blood with Special Reference to the Use of Anticoagulants, Jour. Am. Med. Assn., I9r6, lxvi, 618.

21. Schauman, Verh. des Deutsch. kong. f. innere Med., rgro, xxvii, I36.

22. SchlaePfer, V., Beitrage Zur Histologie des Darmes bei Perniziöser Anämie Deutsch. Arch. f. klin. Med., r9ro, c, 448.

23. Seyderhelm, K. R., Die Ursache der Perniziösen Anämie der Pferde, Arch. $f$ exp. Path. u. Pharm., I9I4, lxxvi, I49.

24. TURK, W., Vorlesungen über Klinische Hæmatologie, I9r2.

25. VOGEL, K. M., MCCURDY, W. F., Blood Transfusion and Regeneration in Pernicious Anemia, Arch. Int. Med., I913, xii, 707.

26. WEBER, A., Über die Behandlung Schwerer Anämien mit Menschenbluttransfusion, Deutsch. Arch. f. klin. Med., 1909, xcvii, r65.

\section{CHIOROSIS}

Etiology.-Chlorosis may be defined as a secondary anemia of unknown origin, occurring chiefly in girls at the time of or soon after puberty. The majority of writers have considered chlorosis a disease sui generis and not merely a symptom. Whether chlorosis ever occurs in man is still a disputed question. In women it occurs most frequently between the ages of fourteen and twenty years. From the definition all secondary anemias of known origin, as hemorrhage or other factors known to produce anemia, cannot be classed as chlorosis.

Unhygienic conditions, as regards fresh air, diet, exercise and deficient elimination through the bowel have been considered important etiological factors; in fact, these combined with puberty have been considered the etiological factors.

Regarding the pathogenesis of the disease little definite can be said. Virchow's theory of hyperplasia of the vascular system and Meinert's of splanchnoptosis have been abandoned. Poor absorption of iron has been considered a cause but there is no definite evidence to support this view. The explanation which has been most widely accepted in recent times is that we are dealing with impaired blood formation, possibly excited by some ductless gland, more especially the ovary. This view is still purely a theory and has not yet been substantiated by experimental or pathological evidence.

Whatever the nature of the trouble it is generally conceded that chlorosis is becoming, at least in the United States, very much less frequent or at least is less frequently diagnosed. In Cook County Hospital with 29,4I3 patients during the year I9I3 the diagnosis of chlorosis was made only once and the same is true of the year 19I5. Although none of the older records are available for comparison, we cannot help but believe that fifteen years ago the diagnosis of chlorosis was made much more frequently.

Cabot expresses the opinion that chlorosis has become less frequent on account of improved conditions of living, especially among the poorer class. It seems hardly probable that these changes have been sufficient to account for the greatly lessened frequency 
of the disease. It must also be borne in mind that chlorosis was not merely confined to the poorly fed and poorly housed, but not infrequently occurred in the homes of the well-to-do. The question really arises, are we now diagnosing many of these cases as secondary anemia, rather than chlorosis? It is the writer's view that this is probably true. With our recent recognition of the great frequency of gastric or duodenal ulcer and recalling that writers on chlorosis referred to their frequently being complicated by gastric ulcer, and gastric symptoms, it would appear reasonable that at present many of these are now diagnosed as secondary anemia due to ulcer. The more careful search for tuberculous or focal pus infection in all anemic individuals may place many of these cases that were formerly called chlorosis into the group of secondary anemias due to infection. There is one argument against the view that many cases of chlorosis are in reality secondary anemia, viz.: the apparent promptness with which the anemia disappeared under the use of iron. This belief is practically universal Morawitz and others have apparently shown that in many chlorotics the symptoms would disappear by iron alone and without modifying hygienic or dietary conditions.

Treatment.-Prophylactic measures should be directed toward proper diet, fresh air, exercise and regulation of bowels in girls at the time of puberty.

General Treatment.-Before undertaking the treatment it should definitely be determined whether we are dealing with a chlorosis or with a secondary anemia due possibly to gastric or duodenal ulcer or some infection. The treatment in general is the same as that described under secondary anemia and will only be briefly mentioned here. In selecting the diet, palatability, digestibility, food value and iron content should all be considered. When constipation is present this should receive careful attention; first, by suitable diet and only in case this is insufficient or not well borne by the patient should laxatives be used, and for this purpose cascara, rhubarb or senna in some form answers well. In the belief that chlorosis might be an intestinal intoxication, antiseptics have been employed but are of little, if any, value. The best method of combating bacterial decomposition in the intestinal tract is by proper regulation of defecation. Fresh air, exercise and proper hours of sleep must all be considered. Iron should be given; the preparation selected should be determined by its iron content and palatability, already considered under secondary anemia. Blaud's 5-grain pill is entirely satisfactory. When thought advisable the modified Blaud's, containing arsenic, may be used. When the stomach will not tolerate iron it may be given hypodermically $0.06-0.12 \mathrm{gm}$. ( $\mathrm{I}-2 \mathrm{gr}$.) of the citrate daily in 5-Io per cent. solution or dependence may be placed entirely on the diet. Regarding the use of organic iron or proprietary preparation this has already been considered. Recurrence of chlorosis may be guarded against by the same method 
used in the treatment. Where the anemia is severe, restriction of exertion should be observed. The chief complication, with the exception, of constipation, is gastric indigestion. This is most frequently the symptomatology of a hyperacidity, although gastric analysis may show a normal or low $\mathrm{HCl}$ content. In all these cases ulcer should be very carefully considered and when in doubt the patient may be given an ulcer cure or placed on a diet of soft foods; cereals, puree of vegetables, eggs, cream soups and soft desserts with a glass of rich milk between meals, following all food with sodium bicarbonate I gm. ( $5 \mathrm{gr}$.), or in case of constipation magnesium oxid $0.6 \mathrm{gm}$. ( $\mathrm{s} \circ \mathrm{gr}$.).

Where the anemia is due to uncinariasis the first effort should be to destroy the parasite by the use of thymol. Ashford recommends that after free purging the patient be given $2 \mathrm{gm}$. (30 gr.) finely powdered in capsules and two hours later this dose is repeated and two hours after this a purge is administered. The patient during the treatment should remain in bed. After taking the thymol all solvents of the drug as alcohol, glycerin and oils should be avoided. This method of treatment was repeated once a week until the stools were free from ova. Betanaphthol is also of value in expelling the hookworm and is administered in the same manner as thymol, except the total amount given in the two doses is $2 \mathrm{gm}$. instead of $4 \mathrm{gm}$. The anemia is treated by the usual methods.

\section{BIBLIOGRAPHY}

There is so little new on this subject that only a few references are given.

I. CAвот, R., Chlorosis, Osler's Modern Medicine, vol. iv, I9I5.

2. Cushney, A., Pharmacology and Therapeutics of the Action of Drugs, Igro.

3. LINDEman, E., Reaction Following Blood Transfusion by the Syringe Canula System, Jour. Am. Med. Assn., rgr6, lxvi, 624.

4. Meyer, H. H., Gottlieb, R., Experimentelle Pharmakologie, Igr I.

5. Sat'rerlee, H. S., Hooker, R. S., Transfusion of Blood with Special Reference to the Use of Anticoagulants, Jour. Am. Med. Assn., r9r6, lxvi, 6r8.

6. TüRK, W., Vorlesungen Über Klinische Hæmatologie, I9I 2.

7. Werl, R., Sodium Citrate in the Transfusion of Blood, Jour. Am. Med. Assn., I9I 5, lxiv, 425 .

\section{ERYTHREMIA OR POLYCYTHEMIIA WITH CYANOSIS AND SPLENOMEGALY}

Etiology.-In this condition with marked increase in the number of red corpuscles there is usually, but not always, associated cyanosis and enlargement of the spleen, but probably always hyperplasia of the erythrocytic bone marrow. Two types are recognized: one, secondary to circulatory disturbance, especially congenital heart lesions; a second idiopathic form. It is known that deficiency of oxygen stimulates the bone marrow and the theory has been advanced that the red corpuscles in the idiopathic type have lessened power to combine with oxygen, but this has been disproven by Pletsch. Knowing the relation of spleen to blood destruction, splenectomy stimulating the bone marrow and increasing the resistance of the red corpuscle, it would appear that disturbed function 
of the spleen might be the primary cause. Türk reports a polycythemia with tuberculosis of the spleen. The fact that the spleen is not enlarged in all cases does not necessarily interfere with this explanation, as patients with typical family hemolytic icterus may occasionally lack a splenic tumor.

Course.-The disease runs a chronic course, death resulting from hemorrhage or broken compensation. Recovery does not occur.

Treatment.-Dietary methods directed toward reducing the iron intake, by removing from the diet foods containing large amounts of iron, have been employed and under this treatment the erythrocytes can be reduced. Türk has accomplished the same end by using large doses of arsenic and Bence and Koranyi by inhalations of oxygen, Koranyi, McLester and others with benzol. These results are interesting from an experimental standpoint, but any line of treatment directed only toward destruction of the erythrocytes would only be palliative. This can probably be accomplished more safely and conveniently for the patient by frequent venesection and this method has been frequently employed. The amount of blood withdrawn should be 500 to 1000 c.c. (I6 to 32 ounces). This will usually give temporary relief and can be repeated as frequently as is necessary. Splenectomy has been tried, usually followed within a few days by fatal termination and is definitely contraindicated. The above methods are merely palliative, as they do not reach the real cause of the disease, namely, the overproduction of erythrocytes, and beyond this is the cause of the hyperplasia of the bone marrow. Roentgen-ray to the spleen has been beneficial in a few cases, and without result in others. Inasmuch as Aldrich and Crummer's patient was much benefited by the roentgen-ray, this should be tried.

\section{BIBLIOGRAPHY}

I. Aldrich, J. F., Crummer, L. R., Polycythemia, Jour. Am. Med. Assn., r907 xlviii, 1 I 63.

2. Lucas, W. S., Erythremia or Polycythemia with Chronic Cyanosis and Splenomegaly (this article has a very complete bibliography), Arch. Int. Med., $1912, x, 597$.

3. McLester, J. S., Benzol in the Treatment of Polycythemia Rubra, Jour. Am. Med. Assn., r9r4, lxii, ${ }_{3} 8 \mathrm{r}$.

4. TÜrK, W., Vorlesungen Über Klinische Hæmatologie, rgr 2.

\section{SPLEINIC ANEMIA}

Etiology.-Splenic enlargement precedes the anemia, and as the anemia disappears after splenectomy, this organ would appear to be the cause of the blood disturbance. Kolliker in 1847 first reported a hemolytic action of the spleen. More recently Paton and Goodall found that splenectomized animals were more resistant to blood toxins. There has been a great deal of additional evidence, all showing that after removal of the spleen the red corpuscle becomes more resistant to hemolytic agents. This resistance continues, as Pearce 
and his colleagues have shown, for at least one year. King has shown that after splenectomy the fatty acids, which are hemolytic, are decreased in the blood and the cholesterin and total fats, which are antihemolytic, are increased.

The enlargement of the spleen is due to a fibrosis, and Banti, Osler and others have considered as the etiological agent a chronic toxemia. Efforts at isolating microörganism have been unsuccessful, although Gibson recently reports the presence of a streptothrix in six cases. Syphilis, rickets and autointoxication have all been considered factors in the splenic enlargement. Warthin and Dock believe thrombophlebitis of the splenic vein plays a rôle.

From a study of the reported cases it is evident that under the head of splenic anemia are included several distinct conditions. Many cases reported in children have apparently been syphilis, as in Caronia's thirty cases in children, where the parents of nineteen gave a positive Wassermann. Septic endocarditis has also been diagnosed as splenic anemia; in fact, any secondary anemia with enlarged spleen could readily be placed in this group. It is possible that a great variety of infections which give rise to chronic splenic tumor may be capable of causing splenic anemia.

The resistance of the red corpuscle in three cases reported by Eppinger was increased. Türk says in some cases it is increased, others diminished.

Course.-The condition is distinctly a chronic one. Osler refers to a patient where the symptom-complex had been present for twentyfive years. Hematemesis is a frequent cause of death.

Treatment.-Splenectomy.-Removal of the spleen may be considered a curative measure. Türk refers to a case in normal health fifteen years after splenectomy. The results are more satisfactory when the operation is performed before evidence of cirrhosis appears, but even where ascites is present most gratifying results may be obtained, as in a case reported by Griffin from Mayo's clinic in good health seven years after splenectomy. Similar cases have been reported by others. The danger of the operation in the later stages is much greater and the chances of a complete cure less. In a series of thirty-six cases reported years ago by Banti the mortality from the operation in early stages of the disease was 25 per cent., in late stages 60 per cent. The mortality in sixty-one cases reported in the literature from I909 to I9I4 was I9 per cent., exactly the same as in fifty-seven cases reported in the nine years previous. In eighteen cases at the Mayo clinic there was a mortality of II per cent. Twelve of the remaining sixteen entirely recovered, and two others showed marked improvement. In this series five patients had reached the stage where definite cirrhosis of the liver had appeared and four of these were restored to excellent health. In some cases several months may elapse after the operation before definite improvement sets in. Early splenectomy is undoubtedly the best method of treatment. 
Salvarsan.-Perussia, Vallardi and others have reported cures after the intravenous use of salvarsan. Although in some of these cases there was a negative Wasserman the question arises were these cases actually splenic anemia or were they merely syphilis? This method of treatment is so simple that it can well be given a trial before turning to splenectomy.

Roentgen-ray.- This method of treatment has been tried repeatedly, applying the roentgen-ray especially to the spleen, but the results have not been encouraging.

\section{BIMLIOGRAPHY}

I. GRIFris, H. L., Clinical Obscrvations Concerning Twenty-seven Cases of Splenectomy, Am. Jour. Med. Sci., 1913, cxlv, $78 \mathrm{I}$.

2. Osler, W., Anemia Splenica, Trans. Assn. Am. Physicians, r9o2, xvii, 429.

3. Perussia, F., Ein Fall von Anemia Splenica der Erwachsenen mit Salvarsan Behandelt, Munch. med. Wchnschr., I912, lix, I482.

4. For a complete bibliography the reader is referred to Krumbhaar's paper -A Classification and Analysis of Clinical Types of Splenomegaly Accompanied by Anemia, Am. Jour. Med. Sci., I915, cl, 227.

\section{HEMOLYTIC ICTERUS}

Etiology.-This condition of chronic icterus with marked anemia, splenic tumor, urobilin, but absence of bile in the urine, freedom from evidence of biliary intoxication and usually showing lessened resistance of the red corpuscle, has been extensively observed during the past five years. Formerly not infrequently considered as a form of splenic anemia or Banti's disease, it is now known to be a clinical entity, closely related to Banti's. Especially those cases with chronic anemia and only recurring icterus in the non-icteric periods could be classed clinically as splenic anemia.

Since it has been shown that these patients are cured by splenectomy the spleen has been considered the probable source of the trouble and, no doubt, must play an important rôle. A hemolytic function of the spleen was first described by Kolliker in 1847 and confirmed the same year independently by Ecker. Gabbi in 1893 demonstrated that the red corpuscles after splenectomy were more resistant to pyradin and toluene, and in I 895 Banti reported that in dogs after splenectomy pyradin did not cause icterus, and since then this has been confirmed by a number of observers, Pel, Paton and Goodall, Pearce, Heinz and others. That after splenectomy the resistance of the red corpuscle to hemolysis is increased has now been abundantly proven. That hyperactivity of the spleen may lower the resistance of the erythrocyte, is suspected. The presence of lowered resistance in hemolytic icterus associated with enlargement of the spleen rather suggests this probability. McNee in his recent study of hemolytic icterus expresses the opinion that in the higher animals the phagocytic endothelial cells in the spleen play the same rôle in blood destruction and formation of bile as the Küpfer cells of the liver in birds. In man and the higher animals there are comparatively few Küpfer 
cells in the liver, while cells of apparently identical character and function are abundant in the spleen. That a marked increase in these cells in the spleen might lead to increased blood destruction is quite possible. On the other hand there is some evidence to support the view that in the familial or acquired hemolytic icterus there may be a disturbance in bone marrow which results in the formation of less-resistant corpuscles. Under these circumstances the splenic tumor might be secondary to increased blood destruction. That the spleen is not the primary factor in lowering the resistance of the erythrocyte in this condition is suggested by the case reported by Dawson, where twenty-seven years after splenectomy, although the patient was apparently cured, the resistance of the corpuscles was still low and a similar finding has been reported by Roth three years after splenectomy for hemolytic icterus. Chauffard's statement that congenital syphilis may be the cause of hemolytic icterus has not been confirmed. Cold may excite increased hemolysis in these patients as in the case reported by Bettman and also by Chauffard. Others have reported that some of these patients are more icteric during the winter months (Aschenheim). Hemolytic icterus may appear in a congenital form, familial type appearing in several generations and as an acquired disease. When not congenital it most frequently appears in the second decade. While there has been.some discussion as to whether the familial and acquired form are identical, although there may be slight differences, these are inconstant, and the evidence points to the two conditions being identical.

Course.-The disease is chronic. The icterus may show marked fluctuation, at times temporarily disappearing, sooner or later however a hemolytic crisis occurs and the jaundice returns or deepens. In many cases the duration of life and efficiency of the individual is little, if any, impaired. For this reason the statement has been made that this is a cosmetic defect rather than a disease, as in the patient referred to by Benjamin and Sluka who lived more than eighty years. This is not true, however, even in the majority of cases, as the recurrent hemolytic crises are often severe, accompanied with fever, pain in the hepatic and splenic region and the sudden increase in the anemia may be sufficient to cause very decided symptoms. The hemolysis may be so marked that hemoglobinemia and even hemoglobinuria may develop. It is probably true that in the majority of cases the efficiency of the individual is definitely impaired.

Treatment.-Until I9II, when Micheli first performed splenectomy on a recognized case of hemolytic icterus, the condition was considered as incurable, as all forms of drug therapy or surgical procedures such as drainage of the gall-bladder had proved ineffectual. Since Micheli's report Elliott and Kanavel in I9I 5 were able to collect forty-eight cases where splenectomy had been performed. The operative mortality in these forty-eight cases was only 4.I per cent., surprisingly low. The forty-six patients who recovered are reported 
as cured. The jaundice rapidly disappeared, beginning within a few days and being complete within two weeks. The recovery from the anemia was somewhat slower but relatively rapid. The hemolytic crises ceased, although Elliott and Kanavel report a crisis in their patient fifteen days after the splenectomy. As only five years have elapsed since Micheli's case it might be said the time was too short to insure permanency. However, before the condition wat recognized as a disease entity splenectomy was performed on patients, where from the symptomatology there can be little doubt that the condition was hemolytic icterus. In this group of permanent cures may be included the case of Sir Spencer Wells, reported by Dawson, operated upon twenty-seven years ago, Banti's case of eleven years, Bland-Sutton's of ten years and Roth's of six years' duration. With this evidence available the value of splenectomy can scarcely be questioned. Operative measures should probably not be urged except in those patients who are inconvenienced by the condition. In those who suffer no inconvenience, the patient may be left to decide whether he wishes to undertake the risk associated with splenectomy.

With hyperfunctioning of the spleen as a factor in the anemia, reduction of the splenic enlargement by means of the roentgen-ray might give results in those patients where operation was refused, or the risk on account of the anemia or splenic enlargement would make an operation questionable. Parisot and Huelly in 1913 report two cases of congenital hemolytic icterus, where after roentgen-ray the anemia and icterus disappeared and the spleen reduced to normal size. The later course of these cases has not been reported.

Where syphilis is suspected specific treatment should be tried. The anemia should be treated by proper foods, hygiene and the use of iron and arsenic.

It should be borne in mind that these patients may have real gall-stone colic, which should not be confused with the hepatic tenderness noted at the time of a hemolytic crisis. In four of the fortyeight cases collected in the literature by Elliott and Kanavel, gallstones had been removed.

\section{BIBLIOGRAPHY}

1. Benjamin, E., Sluka, E., Uber eine Chronische mit Icterus Einhergehende Erkrankung des Blutes, Berl. klin. Wchnschr., I907, xliv, I 065.

2. Bland-Sutron, Observations on the Surgery of the Spleen, Brit. Jour. Surg., I9I3, i, 157 .

3. Dawson, Splenectomy for Congenital Acholuric Jaundice, Proc. Roy Soc. Med., 1914, vii, v, 85 .

4. Elliotr, C. A., Kanavel, A. B., Splenectomy for Hemolytic Icterus, Surg. Gyn. and Obstet., I9I5, p. 21 .

5. Eppinger, H., Charnas, D., Was Lehren uns Quantitative Urobilin Bestimmungen in Stuhl, Zeitschr, f. klin. Med., I9r4, Ixxviii, 387.

6. Griffin, H. L., Clinical Observations Concerning Twenty-seven Cases of Splenectomy, Ám. Jour. Med. Sci., r913, cxlv, $78 \mathrm{r}$.

7. KrumbiaAr, E. B., A Classification and Analysis of Clinical Types of Splenomegaly Accompanied by Anemia, Am. Jour. Med. Sci., r91 5, cl., 227. 
8. PARISOT, J., HUEliy, L., Essai de traitement de l'ictere hemolytique congenital par la radiotherapie splenique, Gaz. d. Hôff., Paris, I913, lxxxvi, 277.

9. Pearce, R., Relation of the Spleen to Hemolytic Jaundice, Jour. Exper. Med., 191 2, xvi, 758.

io. Trleston, W., Griffin, W. A., Chronic Family Iceterus, Am. Jour. Med. Sci., I9I0, cxxxix, 847 .

II. Whipple, G. H., Hopper C. W., A Rapid Change of Hemoglobin to Bile Pigment Outside of the Liver, Jour. Exper. Med., r913, xvii, 612.

\section{HEMORRHAGIC DISEASES, EXCLUSIVE OF HEIMOPHIIIA AND PURPURA} NEONATORUM

Etiology.-In addition to the so-called idiopathic purpura, cutaneous and mucous hemorrhages, apparently identical in character, may occur in any of the acute infectious diseases. They are also noted in leukemia, nephritis, benzol poisoning and other conditions. Such hemorrhages may be either spontaneous or follow slight trauma. Under this section is included a group of hemorrhagic diseases, which at the present time it is impossible to classify clinically. It includes simple purpura, purpura hemorrhagica, Henoch's purpura and purpura rheumatica. Clinically it is often impossible to sharply. differentiate these various forms. It is not improbable that they represent various intensities of the same process, and certainly from a therapeutic standpoint may be considered as identical.

The presence of lessened blood platelets in this condition was first observed by Denys in 1887 and later confirmed by Hayem. Duke, in extensive observations, has found these findings constant. Hess suggests that we include under the purpuras all bleeders with lessened platelets, although in the purpuras complicating acute infections the platelets may be normal (Hess). Morawitz has shown that the platelets contain large amounts of prothrombin and this finding has been confirmed by Jones. This, however, fails to throw much light on the subject as authorities are generally agreed that the clotting time in the purpuras is normal. In spite of this, however, bleeding from a prick in these cases is much more prolonged than in the normal (Duke). Hayem and Denys have suggested that in purpura the platelets become agglutinated, due to pathological changes in the blood, and that these platelet masses may act as emboli with secondary hemorrhage. Howell has shown that there is no disturbance in antithrombin or prothrombin content of the blood in this condition. On account of the evidence of infection in the majority of cases of clinical purpura there is little doubt that bacteria play an important rôle. Rosenow with a strain of streptococcus, with selective tendencies for the blood-vessels, has been able to produce experimental purpura in animals. Hess believes that some cases of purpura show an hereditary tendency.

Treatment.-Prophylactic measures, until we are more familiar with the etiology of the condition, cannot be intelligently applied. The patient with severe purpura should be kept in bed. Unless there are special contraindications a general diet may be given. In 
case the anemia is marked the diet may be modified as recommended in the treatment of chlorosis or anemia. Iron should be given as in other anemic conditions.

Although there is no apparent disturbance of the blood coagulation, the various reports on the results of using fresh blood or serum would indicate that this method of treatment is of value. The most satisfactory method is the subcutaneous or intramuscular injection of either whole blood, rapidly withdrawn from the vein of a donor with a ro to 20 c.c. syringe, and then immediately injected, before coagulation occurs, into the patient. The amount employed is 20 to 40 c.c. and if necessary this may be repeated daily or several times daily until the hemorrhages cease, the further use of fresh blood being determined by the future course of the disease. Where the patient is quite anemic as a result of the purpura, in order to prevent further hemorrhages, this amount of blood should be given daily for four or five days rather than giving one or two treatments, and then waiting for the appearance of fresh hemorrhages.

Citrated blood, according to the method recommended by Weil is also comparatively simple and safe, and offers the additional advantage, that a sufficient supply may be obtained at one sitting to provide for several injections. A sufficient amount of, we will say, a ro per cent. sodium citrate solution is added to a flask, so that when the blood is added, the citrate will be present in about 0.2 per cent. solution. If we are planning to withdraw 100 c.c. $(31 / 3$ ounces) of blood, 2 c.c. or 30 minims of the citrate solution should be placed in the flask. A moderate-sized needle can then be introduced into an arm vein after proper constriction above the elbow and the blood allowed to drop into the flask which should be shaken occasionally, or a syringe may be attached to the needle and the blood aspirated and expelled in the flask. The citrate solution prevents blood coagulation, and blood so collected, if kept cool, will retain its activity for at least a week; 30 to 40 c.c. ( $1-1 \frac{1}{3}$ ounces) of this blood can then be injected intramuscularly or subcutaneously. The frequency with which this should be repeated depends upon the course of the trouble.

Either of these methods offers many advantages and should replace the use of fresh horse serum or diphtheria antitoxins, or the precipitated sera, as with the foreign sera there is always some danger of anaphylactic reaction. Furthermore unless the serum is fresh it has little if any value in this condition. Some have recommended using the serum in ro to 20 c.c. doses intravenously. This is much more apt to give rise to unpleasant symptoms as chill, nausea, etc., and is not especially more efficient than double this amount given subcutaneously or intramuscularly. Intramuscular injection of fresh or citrated blood has the advantage over direct transfusion that there is no danger of blood hemolysis with its serious consequences. Furthermore direct transfusion requires in its execution the presence of a surgeon while the subcutaneous injection 
of whole or citrated blood can be safely carried out by any physician. Where the patient is very anemic blood transfusion might be indicated, but in every case it should be determined whether the blood of the donor is hemolytic to the blood of the donee. When for any reason foreign serum is used, in order to possess value it should not be more than two weeks old. While the precipitated sera on the market are claimed to retain their activity for a year or more, for this purpose they cannot be as satisfactory as the fresh human blood. It was formerly thought that citrates interfered with blood coagulation, but Weil has shown that the addition of citrate to the blood increases its activity in facilitating blood coagulation.

The other remedies that have been used in purpura include the calcium salts and gelatin, as they were thought to hasten blood coagulation. While the evidence at present is somewhat conflicting, it is at least doubtful whether either of these substances modify blood coagulation in either health or disease (Cohen, Rudolph and Cole). If we resort to these agents the calcium can best be given in the form of the lactate I gm. ( 5 gr.) three times a day. The use of gelatin subcutaneously has been discontinued and when given should be taken by mouth in the form of a dessert.

A number of other drugs have been recommended such as arsenic, aromatic, sulphuric acid and turpentine, but no one has furnished any definite evidence that they have any value.

In bleeding from the gastrointestinal or genitourinary tract, the intramuscular or subcutaneous use of fresh or citrated blood should always be thoroughly tried, as more likely to give results than astringents administered by mouth.

After the hemorrhages have ceased, the patient should be treated as any other anemic individual.

\section{BIBLIOGRAPHY}

I. Austin, J. H., Pepper, P., Experimental Observations on the Coagulation of Oxalated Plasma, with a Study of Some Cases of Purpura, Arch. Int. Med., 1913, xi, 305.

2. Duke, W. W., Pathogenesis of Purpura Hemorrhagica with Especial Reference to the Part Played by Blood Platelets, Arch. Int. Med., I 9 I 2, x, 445.

3. Hess, A. F., The Blood and the Blood-vessels in Hemophilia and other Hemorrhagic Diseases, Arch. Int. Med., 1916, xvii, 203.

4. Howels, W. H., The Condition of the Blood in Hemophilia, Thrombosis and Purpura, Arch. Int. Med., I91 4, xiii, 76.

5. Minot, G. R., Denny, G. P., Davis, D., Prothrombin and Antithrombosis Factors in the Coagulation of the Blood, Arch. Int. Med., rgr6, xvii, ror.

6. RUdolf, R. D., Cole, C. E. C., The Coagulation Time of the Blood in Various Diseases, Am. Jour. Med. Sci., I9I I, cxlii, 48I.

7. WeIr, R., Sodium Citrate in the Transfusion of Blood, Jour. Am. Med. Assn., I91 5, lxiv, 425 .

8. WhIPPLE, G. H., Hemorrhagic Diseases, Antithrombin and Prothrombin Factors, Arch. Int. Med., I913, xii, 637 .

\section{HEMORRHAGIC DISEASES OF THE NEWBORN}

Etiology.-This condition usually develops within two to ten days after birth. In some cases infections may be responsible, and syphilis has been considered by some a factor. The majority, 
however, can be designated as idiopathic. Disturbed blood coagulation is present as a rule but not always. Whipple in the case he studied found lessened coagulation associated with lessened prothrombin. It is possible changes in the vessel wall may be a factor, but the evidence points to a biochemical change in the blood. Graham believes disturbances in the liver due to the use of chloroform or from asphyxia of the child is an important etiological factor. He believes a number of toxic agents that interfere with the proper oxygenation of the tissues may excite this condition.

Treatment.-Prophylaxis with our present knowledge of the condition is not applicable. If syphilis, as some believe plays a rôle, the proper treatment during pregnancy would be important.

By far the most satisfactory method of treatment is by the use of fresh human blood. The simplest and most satisfactory method is to withdraw from the arm of one of the parents, or other suitable individual, 30 to 50 c.c. ( $\mathrm{r}$ to $\mathrm{r} 2 / 3$ ounces) and inject it subcutaneously or intramuscularly into the patient. This may readily be accomplished by using a relatively large syringe which holds, we will say, 20 c.c. ( $2 / 3$ ounce). By having two needles, leaving one in the vein, when the syringe is filled, and using the other to inject the infant, it becomes feasible to easily and rapidly administer the treatment. The child should be reinjected every four to eight hours, depending upon the behavior of bleeding. This injection should be continued for one to two days or longer if the tendency to hemorrhage persists.

Another simple procedure is to use an anticoagulant, the best of which is sodium citrate, in about 0.2 per cent. solution. This method has the advantage of permitting more time in the procedure, as there is now danger of the blood clotting. Another point in its favor is that a large amount, say 200 c.c. (7 ounces), may be drawn from the donor into a citrate solution and if kept cool will remain active for a week, and can be injected subcutaneously or intramuscularly in doses of 30 to 50 c.c. (I to $\mathrm{I} 2 / 3$ ounces) every four to eight hours. Assuming we are using a ro per cent. stock solution of sodium citrate and plan to draw from the donor 200 c.c., we could add to the flask in the beginning 4 to 5 c.c. (I dram) of the citrate solution. When diluted with 200 c.c. of blood this would make the mixture of sufficient strength to prevent coagulation. The blood may be drawn from the donor by using a large syringe or simply introducing a rather large needle into the arm vein and allowing the blood to flow into the flask. The use of the fresh or citrated blood, on account of the ease and rapidity of its application, will as a rule take preference over the direct transfusion. Where the infant is exsanguinated, direct transfusion may be desirable.

The use of fresh animal serum or the dried precipitated sera, now on the market, is much less desirable than fresh human blood on account of the danger of anaphylactic reaction and inactivity of the preparation used. 
The use of supposed coagulants, as calcium chlorid or gelatin, which at most are of questionable value since the use of fresh blood, can be dispensed with. Especially should the physician avoid wasting valuable time by trying these remedies first, rather than resorting at once to the use of fresh blood.

\section{BIBLIOGRAPHY}

I. Satterlee, H. S., Hooker, R. S., Transfusion of Blood with Special Reference to the Use of Anticoagulants, Jour. Am. Med. Assn., r916, lxvi, 6r8.

2. Schloss, O. M., Commiskex, J. J., Spontaneous Hemorrhage in the Newborn with Report of Nine Cases, Am. Jour. Dis. Child., rgr r, i, 276.

3. Werr, R., Sodium Citrate in the Transfusion of Blood, Jour. Am. Med. Assn., r9r5, lxiv, 425 .

4. WhipPle, G. H., Hemorrhagic Diseases-Septicemia, Melena, Neonatorum and Hepatic Cirrhosis, Arch. Int. Med., 1912, ix, 365.

5. Graham, E. G., The Pathogenesis of the Hemorrhagic Diseases of the Newborn, Jour. Exper. Med., 1913, xv, 307.

\section{HEMOPHIIIA}

Etiology.-Hemophilia is usually hereditary, affecting chiefly the males, but transmitted through the female. Apparently not all cases are hereditary, as undoubted spontaneous instances have been reported. It may be that in such cases the condition can be transmitted to the offspring. Hemophilia differs from all the other socalled hemorrhagic conditions by the invariable presence of disturbed blood coagulability. Hess has reported a case where for transitory periods the coagulability was normal and it is possible that this may be a common occurrence, but there is a definite inherent disturbance in blood coagulability.

Regarding the underlying cause of the disturbed blood coagulability there are a number of theories. Morawitz and Lossen believe it is due to a deficiency of thrombokinase, which they consider has its origin in the leukocytes and blood platelets. Weil attributes the trouble to excessive antithrombin. Whipple believes there is deficiency of prothrombin and excessive antithrombin. Wright attributed the trouble to deficient calcium. Addis, and still more recently Howell, believe deficient or altered prothrombin rather than excessive antithrombin is responsible. Addis believes an altered prothrombin is responsible, while Howell, accepting prothrombin as at the root of the trouble, believes in a deficient rather than an altered prothrombin. As the prothrombin originates in the blood platelets, abnormalities present in them are responsible for the disturbance in blood coagulation. Wright's view that absence or lessened calcium may be a factor has been disproven, Nolf and others having shown that calcium in this condition is present in normal amounts, and Morawitz and Lossen that the addition of lime salts to hemophilic blood did not hasten coagulation.

Treatment.-Prophylaxis.-The chief prophylactic measures should be directed toward protecting the individual from injury and taking the necessary precautions preceding all surgical measures. 
In families with hereditary hemophilia, the females should be advised in regard to their children, if males, being hemophilic. It is claimed that bleeding is more apt to occur during cold weather and hence the advisability of such patients living in a warm climate.

Active Trealment.-As disturbed blood coagulation is responsible for the condition, therapeutic measures should be directed toward correcting this disturbance. Calcium salts in this condition are of little if any value, and the same is true of gelatin, and therefore, should not be depended upon. Fresh or citrated blood, fresh animal blood, or serum tissue extracts and peptone possess proven value. Nolf has reported that peptone, when given rapidly intravenously, lessen the coagulability of the blood, but when given slowly or when given subcutaneously, increases blood coagulability. Nolf gave ıо to 20 c.c. of a sterilized 5 per cent. Witte's peptone solution intravenously, or 20 to 30 c.c. subcutaneously. When given intravenously there may be a violent reaction, chill, fever and nausea, and when given subcutaneously considerable local reaction. Others have reported good results from rectal injection of peptone solution. The use of peptone at present in this condition is far inferior to the fresh blood or serum. Horse, ox or rabbit serum when fresh, that is not more than two weeks old, is of decided value, the chief objection being the difficulty of obtaining it and the danger of anaphylactic reaction. It may be given intravenously in ro to 15 c.c. doses or much more safely and practically as efficaciously subcutaneously in doses of 20 to 30 c.c. Diphtheria antitoxin if fresh will, of course, answer the same purpose. Fresh rabbit's blood given subcutaneously in 30 c.c. doses will answer the same purpose. The serum should be given once or twice daily until the coagulation time of the blood becomes normal and its further use will depend upon the coagulation time. Usually within four or five weeks the disturbance in coagulation has returned. The duration of action is sufficient, however, to tide the patient over any operative measure.

Fresh or citrated human blood is always available and is far preferable to any other means. Under all circumstances without previous testing it may be given subcutaneously in 30 to 40 c.c. doses, repeated two or three times daily until the desired results are obtained. The method of preparing and administering has already been described under the "Hemorrhagic Diseases of the Newborn." Where the bleeding point is accessible, cotton or gauze soaked in serum or fresh blood may be applied, often with excellent results. The use of ergot, stypticin and remedies of this class have little, if any value.

For bibliographic references the reader is referred to the section on "Hemorrhagic Diseases of the Newborn."

\section{SYMPTOMATIC OR SECONDARY ANEMIA}

Etiology.-Secondary anemia, as seen clinically, may be due to loss of blood, infection, mineral poisons especially lead, intestinal 
parasites especially hookworm, nephritis, malignant growths and certain diseases of unknown etiology, as pseudoleukemia and leukemia. In addition mild degrees of anemia may result from a great variety of causes, such as disturbances of the ductless glands, etc. Whether secondary anemia of high degree exclusive of chlorosis is ever due to hypofunctioning of the bone marrow has not been proven.

When severe secondary anemia results from sudden loss of large amounts of blood, such bleeding usually takes place either from injury to a large vessel through external trauma or from the respiratory, genitourinary or gastrointestinal tract. Occasionally severe anemia may be due to an extensive purpura. The most frequent cause of severe secondary anemia due to loss of blood is either from continuous slow bleeding or repeated hemorrhages of considerable degree; as examples might be mentioned, bleeding hemorrhoids, gastric or duodenal ulcer, renal, vesical, nasal or uterine hemorrhage.

As seen clinically lead more frequently than any other inorganic agent is responsible for anemia. Large amounts of arsenic will also cause anemia and this may be observed in certain industrial occupations, as in the smelting of ore containing arsenic. Mercury and potassium chlorate may also produce anemia.

Of the intestinal parasites causing secondary anemia the hookworm is the most important. Here the anemia is due directly to the blood-sucking proclivities of the parasite. Rather severe anemia has been reported from anguillula infection and mild types from the Ascaris lumbricoides. The anemia of nephritis may be due in part to the associated etiological cause of the nephritis, but chiefly to the loss of albumin and at times of blood. The associated hydremia may also play a rôle in the low blood count and hemoglobin reading.

The anemia of malignant growth is due chiefly to the activity of a hemolytic agent, although hemorrhage and impaired nutrition may also be important factors.

As a result of every acute infection there is probably more or less anemia. Increased blood destruction rather than impaired blood formation is responsible. Sepsis commonly excites a high degree of anemia which becomes especially marked where the infection is prolonged, as in malignant endocarditis. Acute articular rheumatism probably comes next as a factor in anemia. Typhoid, pneumonia and the contagious diseases as a rule cause only mild anemia. Tuberculosis and less commonly syphilis are among the chronic infections responsible for anemia. The rapidly developing anemia seen in malarial infection is due to direct destruction of the corpuscle by the parasite.

Treatment.-General Consideration.-The first step in the treatment of secondary anemia is to determine its cause and correct this, if possible. When this can be accomplished drug therapy becomes a secondary factor. Where the cause of the anemia is obscure the various possible etiological factors must be considered, and here occult bleeding should be carefully considered, especially the possi- 
bility of gastric or duodenal ulcer. Careful examination should be made for intestinal parasites, lead poisoning must be considered and where the patient is febrile careful search for an infection, especially tuberculosis or endocarditis. Malaria should be excluded and where the anemia is progressive and associated with cachexia the possibility of some obscure malignancy. The habits of the patient should be carefully considered as regards character of food, constipation and hygienic surroundings. Where the anemia is due to loss of blood this should first be corrected. Bleeding hemorrhoids, if not permanently relieved by local measures should be removed by operation; excessive uterine bleeding corrected, if necessary, by surgical measures. Where the anemia is due to a bleeding gastric or duodenal ulcer, an ulcer cure or surgical measures should be instituted, rather than relying on preparation of iron. Morowitz and Blumenthal have shown that experimentally repeated moderate bleeding may cause failure of response of the bone marrow, a suggestion that repeated moderate hemorrhages should receive attention. Constipation, which in young people is believed to be an important factor in causing anemia, can usually be corrected by a diet containing large amounts of green vegetables, fruits, coarse breads and fats, and by avoiding cheese and large amounts of lean meats. This should be combined with exercise that brings into play especially the abdominal muscles, as rowing, and by regular habits in going to the toilet.

Enlarged tonsils or adenoids are responsible for many cases of anemia in children and it is often surprising to see the rapid improvement in their health after surgical measures have corrected this condition. In searching for the cause of anemia, focal infectionsespecialiy of the tonsils and teeth-should be considered.

Climate.-There is no doubt that high altitude stimulates the blood-forming organs, resulting in a marked increase both in the corpuscles and hemoglobin, and in mild anemias of obscure etiology where the ordinary lines of treatment have been tried a change to an altitude of 5000 to 6000 feet may be recommended. Especially for children outdoor life in the country usually yields better results than any other measure.

Diet.-The diet prescribed depends in a measure on the digestive organs of the individual. Any diet that is not well borne is unsuited for that particular patient. In arranging the diet we should consider its food value, its iron content, its effect on constipation or diarrhea and its adaptability to the patient's gastric digestion. To a patient with hyperacidity or a gastric ulcer it is inadvisable to give the coarse diet necessary to relieve constipation. While the iron content of the diet should be considered, it should not outweigh other factors. Stockman and Grieg have shown that an average daily diet contains 5 to ro $\mathrm{mg}$. ( $1 / 12$ to $1 / 6 \mathrm{gr}$.) of iron, and this amount serves to preserve the iron equilibrium by supplying an amount equal to that eliminated daily in the stools. The green vegetables are especially rich in iron; 
according to Bunge Ioo gm. of dried spinach contain $33 \mathrm{mg}$. of iron, asparagus 20 , cabbage (green leaves) I 7 , carrots 8.6 , beans 8.3 , rice I to 2 , peas 6.6, potatoes 6.4 , flour I.5, cherries 5 to Io, apples I3, grapes 5.6 , strawberries 8 , huckleberry and raspberries 4 , milk 2.3 , yolk of egg ro to 24 , beef I 7 , blood 226 . This represents the amount of iron in milligrams in $100 \mathrm{gm}$. of the above foods dried. From this list it can be seen that iron is quite generally distributed in our food supply. There is no question that iron, as found in the foods in the organic form, is readily absorbed, and is present in sufficient amounts to make the administration of inorganic iron unnecessary, as far as supplying the actual demands of the body.

Transfusion.-In secondary anemia transfusion is usually employed either in extreme anemia to tide the patient over until the blood-making organs can respond, or for the purpose of checking hemorrhage where there is disturbed blood coagulability. Direct transfusion can now be replaced by the much simpler transfusion of citrated blood, as recommended by 'Weil. The blood may be drawn into a citrate solution, using a sufficient amount, so that the citrate will be present in about 0.2 per cent. Satterlee reports 0.04 per cent. is sufficient to act as an anticoagulant. This prevents blood coagulation and blood so prepared if placed in the refrigerator may be kept a week without deteriorating. By means of a needle introduced into a vein this citrated blood may be easily administrated to the patient in the same manner as an intravenous salt solution. The results of this method of administration are apparently as satisfactory and much simpler than the older method. In all transfusion methods there is some danger of hemolysis and in order to be certain regarding this the blood of the donor and donee should be tested to see if they are homologous. A simple and fairly reliable test is to take a few drops of defibrinated blood from the donor and add to this about an equal amount of blood from the prospective donee, allow the mixture to stand for half an hour-or until the serum separatesand note whether it is blood tinged. For a more accurate hemolysis test the method recommended by Rebling and Weil may be used, as described in the American Journal of Surgery, March, I9o9. Blood transfusion is not free from danger. More or less febrile reactions occur in ro to 30 per cent. of cases, this febrile reaction in all probabilities being due to hemolysis. This hemolysis may be so severe as to result fatally. Ottenberg and Libman in $2 I_{2}$ transfusions report five deaths, Percy in fifty-four cases two deaths. For this reason except in extreme emergencies a preliminary hemolytic test should be made.

When disturbed blood coagulation is a factor in nemorrhage the above method of transfusion may be used, or blood may be drawn from the donor into a syringe containing the citrate solution and then injected intramuscularly or subcutaneously into the patient. For the purpose of controlling hemorrhage 40 to 50 c.c. of blood is sufficient. This method is much better than the use of 
horse serum, either in the fluid or dried form, as there is no danger of anaphylaxis and we are certain of the activity of the preparation.

In cases of severe anemia, when not convenient to transfuse with blood, normal salt solution may be used.

Iron.-Iron has always held first place in the drug therapy of anemia. There was considerable discussion for several years as to whether inorganic iron was absorbed. This has been definitely answered in the affirmative. Abderhalden has also shown that it is utilized in the formation of hemoglobin. In addition to iron supplying any deficit that may exist, it has definitely been shown to stimulate the blood-forming organs (Romberg, Tartakowsky and Fr. Müller). Iron, whether in the organic or inorganic form, is not readily absorbed, a rather large proportion of the iron given appearing in the stool, but by means of foods and iron administered in the inorganic form sufficient can be supplied to meet all demands.

The particular pharmacological preparation of iron given is chiefly of importance as concerns its palatibility and its actual content in metallic iron. In addition on account of their astringent action some preparations are more constipating than others. The ferrous sulphate and ferric chloride are often used in controlling diarrhea. All iron preparations are partially changed in the stomach to the chlorid and then later into the albuminate.

Von Noorden has recommended that the daily dose of metallic iron should be $0.1 \mathrm{gm}$. ( $1 / 1 / 2 \mathrm{gr}$.) This amount is contained in:

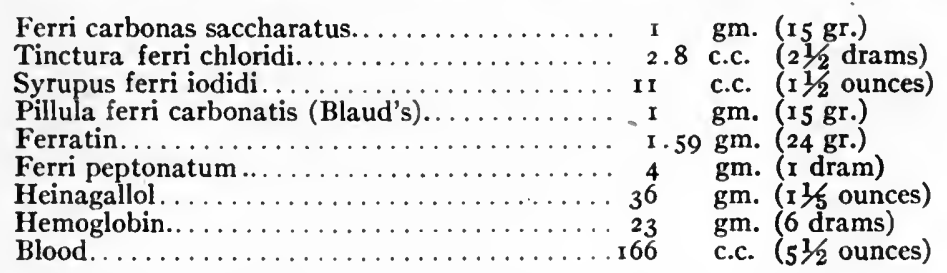

According to Cushny, protein compounds of iron like the albuminate and peptonate possess no advantages over the usual and less expensive preparations. None of the proprietary preparations offer any advantages over the inexpensive Blaud's pills.

Mineral waters containing iron have also been recommended in the treatment of anemia. The amount of iron contained in such waters seldom exceeds $0.1 \mathrm{gm}$. per liter and has no advantages over iron given in any other form. However, the fresh air, change of scene, regular habits established, and psychic effect of suitable mineral springs may make them desirable for certain patients with anemia.

Baths containing iron offer no advantage for the anemic patient, as iron is not absorbed through the unbroken skin.

When the stomach does not tolerate iron well it may be given hypodermatically in the form of the citrate or the ammonium citrate in $0.06-0.12 \mathrm{gm} .(\mathrm{I}-2 \mathrm{gr}$.) in a 5 to ro per cent. solution. 
Arsenic.-Although arsenic holds a position next to iron in the treatment of anemia its action on the blood-forming organs is very obscure. In large doses it is known to cause hemolysis; in smaller doses it is thought to stimulate the bone marrow. In the treatment of anemia the arsenic is administered in moderate doses. The particular pharmacological preparation used is of little importance. The usual practice is to give the arsenic in gradually increasing doses until a certain maximum is reached, remain at this level ten to fourteen days, then gradually reduce the dose to the minimum and then discontinue its use for ten days. After this pause the process is repeated. This method of giving arsenic is based on the readily acquired tolerance for it, and the belief that after continued use the bone marrow becomes tolerant, its susceptibility returning after an intermission in administering the drug. This tolerance, according to Cloetta, is only acquired when the arsenic is administered by mouth and is probably due to decreasing absorption from the intestine, which occurs after prolonged use. When administered hypodermatically an increased tolerance is not acquired.

The arsenic may be given as liquor potassii arsenitis, Fowler's solution $0.05-0.5$ c.c. (I-8 minims) well diluted in water after meals, acidum arseniosum $\mathrm{I}-5 \mathrm{mg}$. $\left(1 / 60^{-1 / 12}\right.$ gr.) three times a day. The sodium cacodylate is a suitable preparation for hypodermic use, the dosage is $0.03-0.12 \mathrm{gm}$. ( $1 / 2-2$ gr.) given once daily.

Some mineral waters contain considerable arsenic. The water of Roncegno, Austria, contains II 5 mg. ( 2 gr.) arsenious acid and Io9 mg. ( $15 / 6$ gr.) of sodium arsenate per liter; Levico, Austria, $8 \mathrm{mg}$. (1/7 gr.) per liter of arsenious acid; La Bourboule, France, $28 \mathrm{mg}$. $(1 / 2 \mathrm{gr}$.) per liter of sodium arsenate. In addition to the above there are a large number of springs containing smaller amounts of arsenic.

\section{LEUKEMIA}

Etiology.-There is a definite growing tendency to consider all types of leukemia as of bacterial origin. In acute leukemia the course of the disease is such as to make an infection highly probable. To this has been added the evidence of Wiczkowski, who injected hens with the pleural exudate from a case of acute leukemia, and was able to produce a lymphatic leukemia which he was in turn able to transmit to other chickens. Ellermann has been able to transmit chicken leukemia. He found the virus filtrable and when injected into a chicken the same virus might produce either lymphatic or myeloid type of the disease, or a pseudoleukemia or a severe anemia. Schmeisser within a few months has reported 105 chickens injected with organ extracts of a chicken with myeloid leukemia, with twentytwo positive results, the blood picture and changes in organs resembling that in man. He reports the usual incubation time as five to six weeks, although once it was prolonged to sixteen weeks.

Trauma was formerly considered a factor. Others have considered the process as related to the neoplasms, and Slye's work on 
heredity in carcinoma in mice, in which she has shown that cancer families are also subject to leukemia, might be considered as evidence in this direction. Several cases of lymphatic leukemia have been reported in roentgen-ray workers.

Course.-Acute leukemia is invariably rapidly fatal, usually terminating within eight weeks. The course of the chronic types is eventually to a fatal termination, although this may be delayed from three to five years or more. Remissions frequently occur during the course of the disease, these appearing either spontaneously, following some acute infection or as a result of treatment. Eventually, however, the patient with rare exceptions succumbs to the disease. Naegeli refers to a case of Klein's still alive at the end of thirteen years, and one of his own who died of pneumonia eight years after the diagnosis of chronic lymphatic leukemia had been made, and mentions a case of Türk's living and well after eleven years. While it is possible and perhaps probable that leukemia may be occasionally actually cured, or recover, this certainly occurs much less frequently than we might suspect. from the reported cures. Several methods of treatment prolong life and the clinical signs and symptoms disappear, as a rule, however, to recur later and eventually to resist all forms of treatment. Warthin has shown that while under roentgen-ray the cells in the leukocytic tissues may be destroyed, causing a great reduction in the leukocytes, this is followed by a reaction in which cells of a more resistant type are formed. This is probably equally applicable to benzol and thorium. While intercurrent acute infection often reduces the leukocytes this is usually an unfavorable omen, and as a rule in case of recovery the leukemia is rapidly progressive.

Treatment.-Until we have acquired further knowledge regarding the etiology of the condition prophylactic measures cannot be considered.

General Consideration.-The patient should have the same general attention as any patient with anemia, as practically all of these patients are more or less anemic. The three methods of treating leukemia most commonly employed are roentgen-ray, benzol and thorium or radium.

Roentgen-ray.-This has now been employed in the treatment of leukemia since 1902 and still holds first place. Although in all probability never curative it is definitely palliative, prolonging life and increasing the patient's working period. This treatment often gives favorable results even in the very late cases and rarely when properly applied do we fail to obtain some degree of favorable results. The danger of doing harm is probably considerably less than with either benzol or thorium. The method of applying the roentgen-ray varies with different operators, and the following rules are taken from Pancoast's reports: It should be applied systematically and with exact dosage to the long bones. At least in the beginning it should be applied daily, and long periods of interruption should be avoided. It should be continued until the myelocytes have disappeared from 
the blood, or the differential count becomes normal. If a return to normal is accomplished, the blood should be carefully studied at least once a month and at the first appearance of trouble the treatment should be resumed. On account of the danger of toxemia exposure of the spleen or enlarged glands should be avoided during the early stages of the treatment. In the beginning of the treatment, the leukocytosis may increase but this should not interfere with continuation of the treatment unless the patient shows evidence of toxemia. The two dangers from roentgen-ray treatment are burns and toxemia. Radiation is contraindicated in acute leukemia as it usually hastens death. Naegeli advises that in all cases where there develops a marked increase in the myelocytes the roentgen-ray should be discontinued.

In refractory cases the roentgen-ray may be combined with arsenic or benzol. Pancoast reports that arsenic under these circumstances may be of considerable value.

The period of marked improvement that follows roentgen-ray treatment may extend over only a few months or several years, as in a case reported by Stengel and Pancoast where the patient remained apparently well for three years and then died of pneumonia.

Benzol.-Since benzol was recommended for the treatment of leukemia by Koranyi in 191 2 it has been extensively used and we are enabled at present to determine its value and limitation. The early enthusiasm regarding its value has somewhat subsided. In certain cases benzol will produce a marked reduction in the leukocytes at times to normal, the splenic tumor subsides, the anemia becomes less marked and the patient gains in weight and strength. These results are, however, only temporary. Although they may continue for one or two years, sooner or later the picture returns and each succeeding treatment with benzol is apt to be less effective until finally the patient fails to react at all. Occasionally a patient who has failed to react to roentgen-rays may be much benefited by benzol, and at times both agents combined give results where either one alone failed. Dr. F. W. Gaarde has furnished me with the results of benzol in thirty patients treated by Dr. Billings during the past three years. In all but two instances the patient was treated with a combination of benzol and roentgen-ray. Twenty-six of the thirty were of the myelogenous type. Twenty-four of the thirty have been followed, and now at the end of three years from the time the first case was treated, twenty of the patients have died. Four are still under observation, none of whom, however, are considered cured.

Benzol may be distinctly toxic. Pappenheim early showed that in rabbits it produced marked parenchymatous changes in the kidney, and areas of peripheral necrosis in the liver. Klemperer and Hirschfeld have reported similar disturbances in their animal research work. That these same changes occur in man after the customary therapeutic dose have been confirmed by autopsy. In 
addition in man there is a disturbance of the gastrointestinal tract characterized by nausea, acidity, loss of appetite and diarrhea. When continued too long it increases the tendency to hemorrhage. The patient receiving benzol should always be under close medical supervision. Frequent blood counts should be made, and when the leukocyte count has fallen to 25,000 or 30,000 the drug should be discontinued, or a leukopenia with serious hemorrhages may follow. Progressive anemia is also an indication for discontinuing the benzol. As a rule in the early stages of benzol treatment the leukocyte count goes up, soon followed in the favorable cases by a fall. But in some patients the only reaction we can obtain with safe doses of benzol is an increase in the number of leukocytes.

The dose recommended is $4-5 \mathrm{gm}$. ( $1 \frac{1}{4}$ drams) daily, using only the chemically pure benzol. Koranyi advised mixing with equal parts of olive oil and placing $0.5 \mathrm{gm}$. ( 8 minims) of the benzol in each capsule, the beginning dose being two capsules three times a day, later increasing two to three capsules after meals. Some patients prefer to take the benzol in the form of emulsion. For the gastric disturbances that follow its use little can be done, except to discontinue for a time the benzol.

Benzol is evidently less satisfactory in the treatment of leukemia than the roentgen-ray. There are many circumstances, however, where it is not feasible to use the roentgen-ray and here under the proper supervision benzol may be given a trial.

Thorium X.- In I9Ix it was shown by Falta and von Noorden that radium emanations had a selective action on the leukocytes. In large doses it produced, at first, a hyperleukocytosis and later a hypoleukocytosis. In I9I 2 there became accessible for clinical use a thorium preparation which gave off its activity into a normal salt solution, which in turn could be administered by mouth, or much better intravenously or intramuscularly. Plesch reports a case, where after a single intravenous injection of 3 million mache units of thorium $\mathrm{X}$ the leukocytes fell rapidly from 109,000 within five days to 8000 , at the end of thirteen days 2155 and after fifty-three days 4 I80 with a disappearance of the myelocytes. Rosenow, on the other hand, treated five cases with intravenous injections of thorium, equivalent to $0.5 \mathrm{mg}$. of radium bromide, with at most very temporary improvement. There is considerable danger associated with the large doses and several fatalities have occurred. The thorium $\mathrm{X}$ is evidently much inferior to the roentgen-ray and probably of less value than the benzol.

Arsenic.-Previous to the introduction of the roentgen-ray, arsenic was the chief reliance in treating leukemia. With quite large doses it was frequently possible to reduce moderately the leukocy tosis. At present it is chiefly used in conjunction with the roentgen-ray or benzol. In order to obtain the best results it should be given to the point of tolerance, which with Fowler's solution may be 10-1 5 minims three times a day. 
Splenectomy.-Splenectomy has been employed in a few cases with disastrous results and this procedure is never warranted.

Bacterial Toxin.-Following the acute infection there may be a temporary reduction in the leukocytes and this has led to the use of Coley's serum. The results, however, do not warrant its use.

\section{BIBLIOGRAPHY}

I. Billings, F., Benzol in the Treatment of Leukemia, Jour. Am. Med. Assn., r9i3, Ix, 495 .

2. Koranyi, A., Die Beinflussung der Leukemie durch Benzol, Berl. klin. Wchnschr., I9I 2, xlix, I357.

3. Nakgeli, O., Leukemie und Pseudoleukemia, r9I3.

4. PaPpenheim, A., Zur Benzolbehandlung der Leukämie und Sonstige Blutkrankheilen, Wien, klin. Wchnschr., r9 13 , xxvi, 4 I.

5. Plesch, J., Falle von Perniciöser Anämie und Leukämie mit Thorium $\mathbf{X}$ Behandelt, Berl. klin. Wchnschr., I912, xlix, 930.

6. Rosenow, G., Klinische Beitrage Zur Therapie der Leukämie mit Thorium $\mathbf{X}$, Munch. med. Wchnschr., I913, lx, 2214.

7. Stengel, A., Pancoast, H. K., The Treatment of Leukemia and Pseudoleukemia with Roentgen-ray, Jour. Am. Med. Assn., I9 1 2, lix, i i66.

8. Warthin, A. S., The Minute Changes Produced in Leukemic Tissues by Exposure to Roentgen-rays, Am. Jour. Med. Sci., I9I4, xlvii, 72. 


\title{
SPLENECTOMY AS A THERAPEUTIC PROCEDURE IN THE ANEMIAS
}

\author{
By E. B. Krumbhaar, M. D., and R. M. Pearce, M. D.
}

Historical.-Removal of the spleen for therapeutic purposes is one of the oldest abdominal operations about which we have definite knowledge(I). Undoubtedly practised by the Greeks and Romans, it was continued at rare intervals through the Middle Ages, as is shown for example by the much quoted operation of Zaccarelli performed in 1549. Although it was early known that the spleen was not necessary for life, splenectomy was at first done only. in the case of rupture or severe injury of the organ. Eventually, however, with the advent of anesthesia and the greater surgical skill of the nineteenth century, it was found practicable to remove the chronically diseased organ and thus widen the field of applicability of the operation beyond that of surgical emergencies. Unfortunately, among the chronic diseases of the organ first attacked were the enlargements incident to cirrhosis of the liver and leukemia. The unfavorable results in these two diseases cast discredit upon the operation; but nevertheless in I900, Bessel-Hagen(2) was able to collect 2 I 6 cases of total extirpation of the spleen with seventy-two deaths. If the cases of leukemia are subtracted, the deaths in the list are reduced to thirty-four, or a mortality of I $_{5} .5$ per cent. Since 1900 our greater knowledge of the physiology and pathology of the spleen has resulted in a better selection of cases, so that now the total mortality has been still further reduced (Laspeyres(3), Michelson(4)).

In the past three years, a more active study of the surgical treatment of certain so-called primary anemias has led to the much wider application of splenectomy, and it is this application of splenectomy that attracts most attention at present. As the technique of splenectomy lies beyond the scope of this chapter, the reader is referred for this phase of the subject to the work of Mayo(5). Considerations of the purely surgical conditions of the spleen such as wounds, torsion of the pedicle, rupture, abscess, cysts, or simple or malignant tumors are also beyond the range of this chapter. Suffice it to say, that in this group, the results of operation have been surprisingly good, and the mortality low.

Contraindications. - It is most important to know when splenectomy should not be done. We now know that in certain diseases, removal of the enlarged spleen is definitely contraindicated. These include the various forms of leukemia, polycythemia, malaria, atrophic cirrhosis of the liver and most cases of tuberculosis and syphilis. In the hypertrophic form of cirrhosis, Eppinger has recently 
advocated splenectomy on account of the evidence of increased blood destruction in this disease, but in the ordinary atrophic forms it certainly should not be considered. Too much emphasis cannot be laid on the necessity of ruling out atypical forms of leukemia, before the splenectomy is undertaken, but on account of the great variety of aleukemic conditions this is often an extremely difficult task. In no case, however, should splenectomy be advised until the blood picture has been carefully studied over an extended period of time, and the presence of leukemia excluded as far as may be possible.

Anything pointing toward a hemorrhagic diathesis should also be given careful consideration. Its presence is, as a rule, sufficient to contraindicate operation, although the repeated hemorrhages from varices or due to other mechanical causes, as in Banti's disease, are more indications for operation than otherwise.

In the severer anemias definite signs of bone-marrow activity should also be forthcoming (nucleated or reticulated cells, Jolly bodies, etc.). If they cannot be provoked by appropriate drugs or by transfusion, it is probable that the marrow is aplastic and splenectomy should not be attempted.

That the removal of the normal spleen is followed by a temporary anemia has been proved both by clinical observation and by animal experimentation; but this should not be considered a contraindication to operation. We have the apparent paradox that while removal of the normal spleen causes a temporary anemia, removal of the spleen in certain blood diseases relieves the existing anemia.

Diseases in which Splenectomy is Indicated.-In certain groups of disease, splenectomy has met with considerable success: these include Banti's disease, Gaucher's disease, the congenital and acquired forms of hemolytic jaundice and, to a lesser extent, pernicious anemia. The cause of the improvement or cure that follows splenectomy in these conditions is but poorly understood. It is probably different in the various diseases mentioned, but a discussion of this problem cannot be undertaken here.

Table I.-Collected Reports of Results of Splenectomy in Blood Diseases

\begin{tabular}{|c|c|c|c|c|c|}
\hline Disease & Author & $\begin{array}{l}\text { No. } \\
\text { cases }\end{array}$ & $\begin{array}{c}\text { Recov- } \\
\text { ered }\end{array}$ & Died & $\begin{array}{l}\text { Per cent. } \\
\text { mortality }\end{array}$ \\
\hline Gaucher's disease.. & $\begin{array}{c}\text { Erdmann and } \\
\text { Moorhead (6) }\end{array}$ & 10 & 8 & 2 & 20.0 \\
\hline Banti's disease. & Isaac $(7) \ldots \ldots$ & 49 & $4 \mathrm{I}$ & 8 & 16.3 \\
\hline Hemolytic icterus, acquired. & Elliott and Kanavel (8) & 16 & 15 & I & 6.2 \\
\hline Hemolytic icterus, congenital. & Elliott and Kanavel(8) & 23 & 15 & I & 4.3 \\
\hline Hemolytic icterus, unclassified. & Elliott and Kanavel(8) & 9 & 0 & $\circ$ & 0.0 \\
\hline v. Jaksch's disease. . . . . . . & Graff(9)...... & 2 & o & o & 0.0 \\
\hline Pernicious anemia... & Krumbhaar(14). . & I53 & 99 & 30 & $\mathrm{xg} .6$ \\
\hline
\end{tabular}

Banti's Disease.-In Banti's disease it is important that the operation should be undertaken before the disease has progressed beyond the first stage of the disease. Splenectomy in the first 
stage is not only accompanied by a low mortality, but in the great majority of cases has caused great and lasting improvement of symptoms, often amounting to a complete cure. When the third stage is reached, with permanent changes in the liver and circulatory system, not only is the operation more dangerous, but the chances of improvement are greatly lessened. As a result of the disease process, the spleen has by this time become largely fibrotic, and its removal could hardly be expected to be attended with marked beneficial effect. Cases have been reported, however, where improvement followed splenectomy even at this late stage.

In 19I2, Isaac(7) collected forty-nine cases with a mortality of I6.3 per cent. With the better understanding of the disease and its treatment that has come about since that time, it is safe to say that the mortality percentage for Banti's disease is now considerably lower.

A patient, then, with the symptoms of Banti's disease, particularly if in the early stages of the disease, should be considered a proper candidate for splenectomy, but the most favorable time for operation should be selected. Before undertaking the operation all other possible causes for such a syndrome (e.g., aleukemic leukemia, tuberculosis, malaria, syphilis, etc.) should be ruled out as far as possible by a complete but not unnecessarily prolonged investigation as to the cause of the disease, with frequently repeated blood examinations.

Gaucher's Disease.-On account of the rarity of this condition and the difficulty of diagnosis without the aid of histological examination, not many cases are available for study. In I9I4, Erdmann and Moorhead(6) collected ten cases of large-celled splenomegaly (Gaucher's disease), in which the spleen had been removed and of these, two died, both within twenty-four hours of operation. While this probably represents too high a mortality, the improvement which followed in the other eight cases cannot always be taken as indicative of eventual cure, for the disease is known to exist independently in the liver, bone marrow and lymph nodes as well as in the spleen. It would, therefore, seem wiser to restrict splenectomy in this disease to those cases that are unusually handicapped by the results of the disease, but are still good surgical risks, and in such cases to limit the prognosis to improvement but not complete cure.

Hemolytic Jaundice. - The field in which splenectomy has been practised with the greatest success is undoubtedly that of hemolytic jaundice. Both in the acquired form (Hayem-Widal) and the congenital or familial type (Chauffard-Minkowski) marked improvement and frequently complete cure have resulted from removal of the spleen. In fact, the success obtained in this type of case, where the chief vitium is that of increased blood destruction; has been a powerful incentive toward extending the operation of splenectomy to the wider range of allied diseases discussed in this chapter. Splenectomy was first recommended in this group by Banti for a condition to which he had given the name, "hemolytic splenomegaly." Success was obtained 
in all the earlier operations (Banti, Micheli, Kahn, Roth) and the procedure would undoubtedly have been in more frequent and intelligent use, in this country as well as Europe, if the several clinical entities, unfortunately grouped together under the cloak of "splenic anemia," had not clouded the worth of the procedure. In I9I 5, Elliott and Kanavel(8) were able to collect forty-eight cases of hemolytic jaundice (sixteen acquired, twenty-three familial and nine unclassified) that had been treated in this way. Of the forty-eight cases, only two died, one shortly after operation, the other from sepsis, six weeks after operation. The other forty-six are reported as "cured;" this result being based upon the disappearance of jaundice and exacerbations, and decrease of the anemia and the urobilin excretion. The effect on the resistance of the red cells was not constant; in some instances the resistance returned almost to normal, but in most cases the red cells remained almost as fragile as before operation.

Pernicious Anemia.-The most important disease to which splenectomy has been applied, from the point of view of its greater frequency and greater severity, is pernicious anemia. The first attempts were made independently by Eppinger and von Decastello in Vienna in r9r3, and were shortly followed by Klemperer in Berlin in the same year, and soon after, on account of theinitial improvement observed, by a large number of surgeons all over the world. Although it is still too early to proclaim any definite decision as to the value of splenectomy in pernicious anemia, it has already become possible to base opinions on substantial evidence. I have recently collected the results in ${ }_{53}$ cases treated in this manner. In thirty instances death occurred either immediately or within six weeks of the operation. Of those surviving the operation, nearly all showed a marked improvement both clinically and in the blood picture. The so-called "blood crisis" (appearance of numerous nucleated red cells and Jolly bodies), indicating a more active bone marrow, usually was observed within a day or two of the operation, and was followed for several weeks or months by a steady rise in hemoglobin and red blood cell count. In a few cases, this increase, coincident with gain in weight and improvement in the patient's strength and general condition, has persisted during the period of observation; in six cases over a period of two years. In the majority, however, the blood picture of pernicious anemia remained, and after several months of improvement, the patient returned to the same condition as previous to operation. Of nineteen individuals that were living at the end of the first year after operation; six died later and five relapsed to the preoperative condition, while eight showed continued improvement at the end of two years. It is noteworthy, though not surprising, that those cases presenting spinal cord changes show no improvement in nervous symptoms, and are otherwise less improved by the operation than are those cases in which spinal cord changes do not exist. 
Although great improvement, therefore, may be said to persist in a certain number of cases, in the majority the effect of the operation is to produce only a remission, such as is characteristic of the natural course of the disease. When, then, should the operation be performed? Should it be withheld, as a trump card, to stave off death for several months when all other measures have failed; or, bearing in mind the minority cases that acquire more lasting improvement, should it be done as soon as a sure diagnosis is made, and before the disease has progressed too far or spinal cord changes set in? Also should every case of pernicious anemia have the spleen removed? Certainly in such cases as those reported by Antonelli(ro) and Mosse(II), verging on the borderline of hemolytic jaundice, with splenomegaly, increased fragility and urobilin output better results should be expected than in cases at the other extreme of the group, with no demonstrable signs of increased hemolysis, and perhaps an aplastic bone marrow. The presence or absence of spinal cord changes may also prove to be a decisive factor. Such questions, however, must remain unanswered until the lapse of time and acquisition of more evidence will allow us to decide conclusively. The operation has already gained sufficient vogue to allow one to predict that such an accumulation of evidence will soon be forthcoming.

Differential Diagnosis.-The differential diagnosis of chronic splenic disease is not only important in the selection of proper cases for splenectomy, but also in order that a more accurate prognosis may be given. For the details of the differential diagnosis, the reader is referred to other text books and medical articles(I 2); but the following points may be alluded to:

The characteristic picture of Banti's disease is that of a disease running a chronic course, usually occurring in otherwise healthy young adults, and divided into three periods. In the first or preascitic period, usually lasting several years, a gradually increasing weakness and pallor is noticed with digestive disturbances and abdominal pain, which may first call attention to the enlarged, hard spleen. A tendency to hemorrhages with a moderate anemia of the chlorotic type is usually present. There is nothing characteristic of this anemia, an increased urobilin excretion being the only sign of increased blood destruction. The resistance of the red cells is unchanged, and signs of a regenerating bone marrow (nucleated and reticulated red cells) are slight or absent. Leukopenia is usually present. The second or intermediate stage, characterized by scanty urine, attacks of diarrhea and dyspepsia and increase in size of liver, lasts but a few months and is usually merged in the general picture. The features of the third stage are cirrhosis, recurrent ascites and jaundice, and increasing emaciation and anemia.

In Gaucher's disease, the onset is more insidious, the symptoms appear in infancy or childhood, and the disease runs a more chronic course. A history of similar trouble in the family is frequently clicited. The huge size of the liver with attending abdominal dis- 


\begin{tabular}{|c|c|c|c|c|c|c|}
\hline 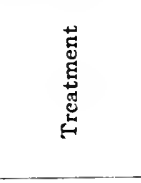 & 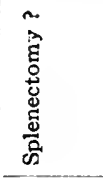 & 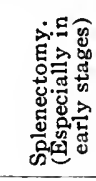 & 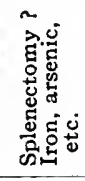 & 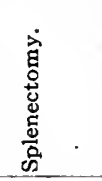 & 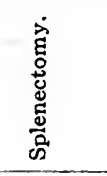 & 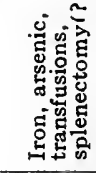 \\
\hline$\stackrel{5}{3}$ & + & 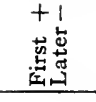 & + & +吅 & + & $\begin{array}{l}\text { हु } \\
\text { है } \\
\end{array}$ \\
\hline $\begin{array}{c}\text { poolq ut } \\
\text { sołкวoyn } 7 \\
\end{array}$ & 1 & 1 & + & 1 & 1 & $15+$ \\
\hline 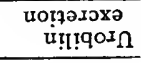 & a & + & $\sim$ & + & + & + \\
\hline 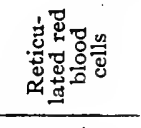 & n. & 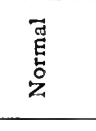 & $n$ & 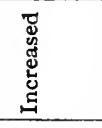 & 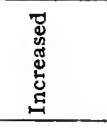 & 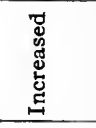 \\
\hline 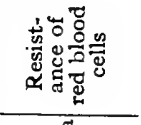 & $n$ & $\begin{array}{l}\text { हु } \\
\text { हू } \\
\text { z }\end{array}$ & $n$ & . & 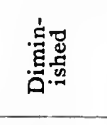 & 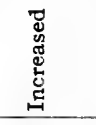 \\
\hline $\begin{array}{l}\stackrel{\pi}{E} \\
\stackrel{g}{\rightleftarrows} \\
\end{array}$ & 葛 & 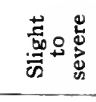 & 壳。岕 & 总 & 壳 & 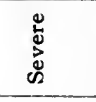 \\
\hline 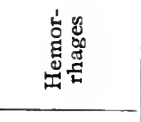 & 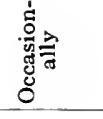 & 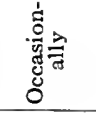 & $\sim$ & 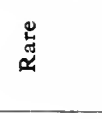 & 0 & ڤّ̆ \\
\hline 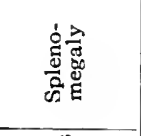 & $\begin{array}{l}+ \\
+ \\
+\end{array}$ & + & $\begin{array}{l}+ \\
+\end{array}$ & $\stackrel{+}{+}$ & + & 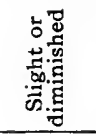 \\
\hline 录 & 总 & ه & 总 & 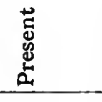 & 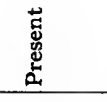 & 窇苞 \\
\hline 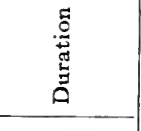 & 窘号 & 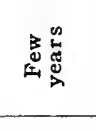 & 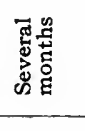 & 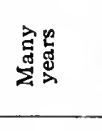 & 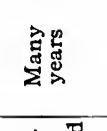 & 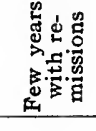 \\
\hline 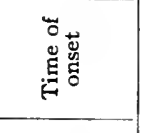 & $\begin{array}{l}\tau \\
0 \\
0 \\
0 \\
0 \\
0\end{array}$ & 泀』 & 总 & 至品 & 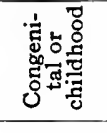 & 苛。 \\
\hline 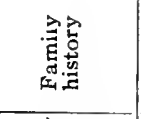 & 总 & 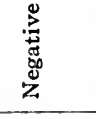 & 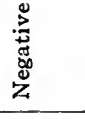 & 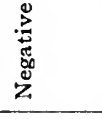 & 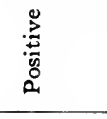 & 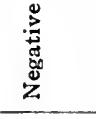 \\
\hline 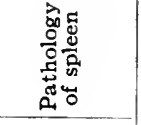 & 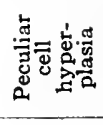 & 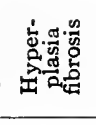 & 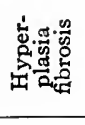 & 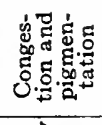 & 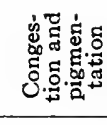 & 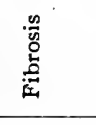 \\
\hline 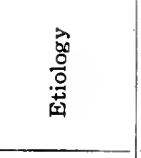 & 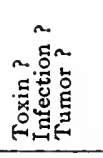 & 蒫 & 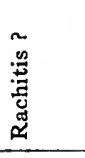 & 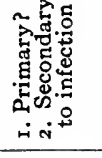 & 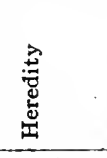 & 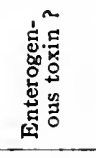 \\
\hline . & 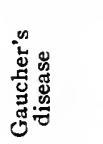 & 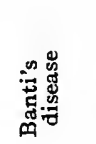 & 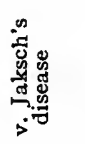 & 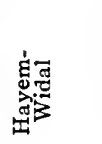 & 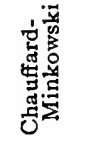 & 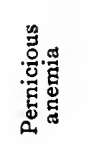 \\
\hline
\end{tabular}


comfort is the most prominent symptom; and histological examination either by splenic puncture, or after splenectomy, reveals the large vesiculated cells with small eccentric nuclei characteristic of this disease. A brownish discoloration of the skin, with "peculiar yellowish wedge-shaped thickening of the conjunctiva" on both sides of the cornex has been noted. The changes in the blood are similar to, but less marked than, those in Banti's disease.

In hemolytic jaundice the characteristic features are the persistent acholuric jaundice, the enlarged spleen and the decreased resistance of the red blood cells to hypotonic salt solution (normally hemolysis begins at 0.45 per cent. $\mathrm{NaCl}$ and is complete at 0.35 per cent). This is usually accompanied by enormous increase in urobilin output and in the percentage of the reticulated red cells. The number of nucleated cells depends on the degree of anemia, which tends to be severe in the acquired form and mild in the congenital or familial type. Crises characterized by increased severity of symptoms and anemia are common to both types.

As von Jaksch's pseudoleukemia is probably not a clinical entity and pernicious anemia is such a well-known condition, they need not be dealt with at this time.

Choice of Time for Operation.-On general principles it may be said that splenectomy should be undertaken as soon as the diagnosis is definitely settled. In certain instances, however, exceptions must be made. A "crisis of deglobulization" in hemolytic jaundice or a severe hemorrhage from a mucous membrane in Banti's disease would indicate postponement of the operation until the usual condition (for the disease) of health is regained. The existence of a possible contributing factor, as indicated for example, by the finding of a positive Wassermann reaction or of malarial organisms, would naturally postpone operation until the influence of such factors had been, as far as possible, eliminated. When the anemia is severe, and this applies particularly to pernicious anemia, a series of blood transfusions (often four or more) should precede operation. If the patient's blood in this way is temporarily enriched, not only is the operative risk lessened, but more lasting benefit ensues. Good results have been obtained in pernicious anemia by the subcutaneous or intraperitoneal injection of splenic extract $\left(\mathrm{r}_{3}\right)$, and in cases where the so-called "blood crisis" fails to materialize after splenectomy, indicating a failure of the bone marrow to respond, this procedure might be tried.

\section{REFERENCES}

I. KrumbiaAR, E. B., The History of the Extirpation of the Spleen, New York Med. Jour., I915, ci, 232.

2. Bessel-Hagen, F., Arch. f. klin. Chir., 1900, lxii, 188.

3. LASPEYRES, R., Central. f. d. Grenzgeb. d. Med. u. Chir., I904, vii, I.

4. Michelsson, F., Ergebn. d. Chir., u. Orth., I913, vi, I.

5. Mayo, W. J., Ann. Surg., I9r 5, lxii, I7r; and Collected Papers by the Staff of St. Mary's Hospital, Mayo Clinic, r912, p. 469.

6. Erdmann, J. F., and Moorlexad, J. J., Am. Jour. Med. Sci., rgr4, cxlvii, 213. 
7. IsAac, S., Schmidt's Jahrb., I912, cccxv, I4.

8. Elliott, C. A., and Kanavel, A. B., Surg., Gyn., and Obstet., I915, xxi, 2 I.

9. GrafF, Verh. d. Deutsch. Ges. f. Chir., r9o8, i, 248; and discussion, p. 252.

10. Antonelli, Semaine méd., igr 2, xlvi, 547.

I I. Mosse, Berlin. klin. Wchnschr., I913, xlv, 2088.

I2. KrumbhaAR, E. B., Am. Jour. Med. Sci., I91 5, cl, 227.

IsAAC, S., Schmidt's Jahrb., I9I 2, cccxv, I4.

Brili, N. E., and Mandlebaum, E. S., Am. Jour. Med. Sci., r9r3, cxlvi, $86_{3}$.

Tileston, W., Am. Jour. Med. Sci., rgro, cxxxix, 847.

ThaYeR, W. S., Ill. Med. Jour., I9I I, xix, I74.

13. Mickhailoff, Russky Vrach, I9I4, xiii, 837. (Abstracted in Jour. Am. Med. Assn., I9I4, 1xiii, 208I).

14. Krumbraar, E. B., Jour. Am. Med. Assn., I9 16, Ixvii, 723. 


\title{
HODGKIN'S DISEASE
}

\author{
By George Dock, M. D.
}

This term within recent years has been limited by careful writers and speakers to cases of lymph-node enlargement having the histologic features associated with the name of Dorothy Reed. It cannot be distinguished positively, without examination of excised glands, from some other diseases of the lymph nodes, especially tuberculosis, and owing to the changes due to degeneration or complicating infections it may be necessary to examine several glands in order to discover the basic disease. In removing tissue for this purpose, which can be done under local anesthesia, but only with complete asepsis, that recently involved should be selected. The clinical features, and in some cases the blood, enable a diagnosis to be made with considerable accuracy, but the anatomic diagnosis should always be made as early as possible, and before such treatment as roentgen-rays or arsenic has been used. Poultices and mercurial ointments, still used to a dangerous extent, share with arsenic and roentgen-rays the risk of exciting changes in the tissue that make more difficult the histologic diagnosis.

No certainty has as yet been attained in the search for the cause of Hodgkin's disease. The work of many investigators from Fraenkel and Much (r9ro), Negri and Mieremet (I9r3), to Bunting and Yates $^{1}$ has revealed the almost constant presence of a pleomorphic diphtheroid organism in the affected tissues, but on the one hand, some observers have failed to find these germs in Hodgkin's disease, others have found them so widely in various diseases as to raise the suspicion of a contamination, or a harmless association.

Increased attention has been paid to the possibility of finding a primary focus of the disease, and in some cases a tonsil or a tooth has been looked upon as the portal of entry, a suspicion strengthened by the frequent beginning of the disease in the cervical glands, and its occasional occurrence in the gastrointestinal tract.

Treatment.-A causal treatment should always be attempted, especially in early cases, and all possible primary foci should be removed. If there seems to be a limited area of involved tissue, early extirpation is proper, but it should always be remembered that other and hidden foci may be present, as in the mediastinal or retroperitoneal glands, or even deep in the neck, that render illusory such an operation.

\footnotetext{
${ }^{1}$ See Yates and Bunting, Jour. Am. Med. Assn., June 1 2, 1915, p. 1953, for the most important references up to that time, as well as a valuable discussion. 
The best chance of healing is given by thorough roentgenization, treating all discoverable foci with adequate dosage. Arsenic, in the form of Fowler's solution or otherwise, can be used at the same time. Under this method the writer, like others, has seen cures lasting up to five to seven years. Cure should not be announced in such cases, but the patients should continue under observation.

Vaccines have been used to a considerable extent, and encouraging results have been reported with the vaccines of $\mathrm{B}$. hodgkini, as well as with other germs, and also with serums such as Coley's erysipelas and prodigiosus preparation. Much more observation, and the publication of the later stages of cases reported as cured under all methods of treatment, are very desirable. 


\title{
DISEASES OF THE ENDOCRINE GLANDS
}

\author{
By George Dock, M. D.
}

\section{EXOPHTHALMIC GOITER}

The most important change in the treatment of exophthalmic goiter, since the appearance of the first edition, is the wide application of surgical treatment, especially the removal of part of the enlarged thyroid. This must be considered in every case and the general practitioner, as well as the internist as a specialist, must be able to form an opinion on the question of operation in each case. Of course the final opinion, and the details of operation, must be left to the surgeon. Only surgeons with special experience in thyroid operations should be consulted. Operations for exophthalmic goiter have been performed on so many patients in the last ten years that a very large mass of testimony is available. One of the most certain of the facts is that the statement made on page 84I, of the first edition "it will be long before even a majority of patients will permit" operative treatment," is no longer true. Dexterous operators have so far removed the fear of the disease that most patients, soon after they learn the name of their complaint, are anxious to undergo operation; many make accurate diagnoses in their own cases, after hearing of operations, and apply directly to the surgeon. It is often difficult to make them understand the conditions governing improvement or recovery even with an operation.

How the operation does good is not yet clearly understood. It is hardly causal. It seems, however, to so distinctly and promptly lessen the cardiac, toxic and nervous symptoms that the organism has a chance to recover more rapidly and to a more marked degree than under non-operative treatment. This promptness of action is often the guide to the decision for an operation. People who can afford to be partial invalids for months or years can take the slower chances of non-operative treatment. Those whose time is important, as a rule, gain much by undergoing operation as soon after a diagnosis is made as possible. Certain symptoms, such as severe dilatation of the heart, with marked functional disturbance, or toxic symptoms such as vomiting, diarrhea, or fever, may require preliminary treatment, but even in serious cases it is usually safer to operate than to wait more than a few days for the symptoms to improve.

The operations of choice are ligation of one or more thyroid arteries and removal of part of the gland. The determination can be based upon the degree of severity of symptoms, but a general rule the author has found valuable is that emphasized by Halsted- 
to remove as much as possible on theoretic grounds, which may leave no more than a fourth of the normal bulk of tissue. After operation all symptoms subside more or less rapidly, though exophthalmos is usually less influenced than the others.

In all cases operated, the patients should be warned of the incompleteness of the method of cure. All possible assisting causes, such as local diseases, functional or organic, worry, loss of sleep, acute infections, should be removed or treated as quickly as possible. Diet and mode of life should be arranged somewhat upon the lines laid down for symptomatic treatment. The possiblity of a second or even third operation should be admitted in the beginning, and its necessity indicated should the rules be neglected.

Since the former edition experience has accumulated regarding other methods then on trial. Preparations of serum from thyroidectomized animals have not maintained their early expectations. Interesting results sometimes follow, but actual curative results seem wanting.

Thyroid preparations such as thyroid extract should not be used, nor should iodine, which is their most certain principle. Temporary improvement will sometimes be seen under these preparations, but the danger of making the disease worse is always great, and in practice many more bad than good results follow both thyroid and iodine treatment.

The same advice holds for thymus, adrenal and other organ preparations. These, as well as substances still to be discovered, are legitimate subjects for experiment, under proper conditions, but no claims can be made for them at present, except that their action is usually nil.

Medicinal treatment for symptoms is often necessary and should be used according to the indications. Specific medicinal "cures," which reappear at short intervals in medical literature, have so far been disappointing.

\section{HYPOTHYROIDISM}

\section{MYXEDEIMA, CRETINISM}

The most important feature in the therapeutics of hypothyroidism concerns its recognition. Not only in the mild forms, in adults and children, but even in the severe cases failure to make an accurate diagnosis too often occurs. In mild cases the therapeutic test may be necessary to confirm the diagnosis.

The treatment, as in so many endocrine anomalies, must be carried on with due regard to the symptoms of the disease in the individual patient, and the expected as well as the untoward symptoms that follow treatment. Beginning with a small dose, say 2 grains (O.I2 gm.) of dried thyroid gland three times a day, the amount is increased until the desired improvement is secured, or until symptoms of overdose occur. The dose is then reduced until, by trial, the requisite 
daily amount is learned. It should be made clear to the patient or the attendant, that at times changes in dosage may be necessary. Experience in treating cases of sporadic cretinism, or typical adult myxedema, seems essential to the successful management of less typical cases.

It is recognized that substitute treatment for hypothyroidism, especially in its typical forms, is at present very crude. Efforts at the isolation of definite components of the secretion have often been attempted since the time of Baumann, but so far without results of value in therapeutics. Future investigations in that field should be followed with interest.

Thyroid Treatment of Various Disorders.-Thyroid extract has been almost abandoned in the treatment of many diseases in which it was once used with enthusiasm. It is still used in obesity, when it often does harm. If used at all, it should only be in cases thoroughly investigated and under constant observation. In Dercum's disease the results are doubtful.

Certain French authors gave rise to the thyroid treatment of chronic arthritis. In the writer's experience, which includes various thyroid diseases, as well as arthritis, such cases are rare, and while a therapeutic test is harmless, it needs only a short time in positive cases to show the relations. Even in these the search for infectious foci, such as the tonsils and teeth, must not be neglected.

In many skin diseases such as urticaria, eczema rosacea, and in the dry and itching skin of old people, thyroid is sometimes almost magical in its effects, but here too, results are prompt or do not occur at all.

\section{PARATHYROID GLANDS}

\section{Tetany, Subtetanic Hypoparathyreosis (Halsted) ${ }^{1}$}

The relation of tetany to parathyroid lesions has been the subject of much investigation since the rediscovery of these small organs. Tetany rarely follows thyroid operations now, owing to improved technique, and in non-operative cases it is impossible to determine the nature and severity of the lesion. A single healthy parathyroid gland will prevent tetany. If accidental removal is discovered at an operation on the thyroid, the parathyroids should be found and implanted. The effects of loss of function can be overcome by injections of parathyroid extract (Halsted). Calcium, introduced by MacCallum, is of temporary benefit. Thyroid extract has seemed to have some value.

The real value of parathyroid preparations in convulsive diseases, such as paralysis agitans, has not been sufficiently investigated.

\section{ADRENAL DISEASE}

But little progress has been made in determining the clinical relations of functional alterations of the adrenal glands. The terms 
"hyperepinephry" and "hypoepinephry," "hypoadrenalism," or "hypoadrenia" are as yet rarely used in a definite sense. Sometimes the symptoms of lowered function are very striking, as described by Lavenson. ${ }^{1}$ In the acute cases the symptoms may be those of acute pancreatitis, with sudden onset and excruciating pain in the epigastrium; or of cholera, or meningitis, going on in some cases to coma, convulsions, and collapse, and in all forms with low arterial pressure. Whether hypoepinephry is the cause of hypotension in such diseases as diphtheria, pneumonia, cholera, septicemia with typhoid state, etc., remains to be proved, and deserves more investigation than it has yet received.

In all cases in which low arterial pressure is a feature, and not explained by other causes, it would be proper to try the effects of adrenal preparations. Ascending doses, best given hypodermatically, should be used and the result tested by the effect on the blood-pressure.

\section{ADDISON'S DISEASE}

The diagnosis of Addison's disease is often made so late that the prospect for successful treatment is very small. Whenever the syndrome is suspected, an effort to exclude other causes than tuberculosis should be made. If there is evidence of syphilis (Wassermann reaction), energetic treatment should be given. If tumor is suspected, an exploratory operation at the hands of a surgeon familiar with the field would be proper.

In the confirmation of a diagnosis of tuberculosis, the various tuberculin tests may be used, but in all, and especially" the subcutaneous test, the dangers and the reasons for risking them should be made known and every precaution taken to prevent dangerous collapse, which may follow even a small dose.

In tuberculous cases general treatment as carried out in special sanatoria is most important, including open air. This may be used even in cold weather, but the patient must be kept warm by clothing and artificial heat in any of the various forms available.

Adrenal Therapy.-Though the dried gland is still official, and may be used in doses of from 2 to ro grains (0.1 2 to $0.6 \mathrm{gm}$.), the active principle epinephrin, under one of several proprietary names, is most frequently used. It is generally used hypodermatically in doses of 5 to 15 minims ( 0.3 to 1.0 c.c.) of the I : 1000 solution. Toxic symptoms such as dizziness, nausea and vomiting, anginoid pain, rapid heart's action and collapse, have followed the hypodermatic, as well as the local use of epinephrin. Secondary hemorrhage often follows the local use of the drug, as in the nose and throat. It is probably this tendency to dangerous or unpleasant symptoms that has prevented a wider use of epinephrin in various conditions in which it seems theoretically useful, especially in collapse in medical or surgical diseases.

${ }^{1}$ Arch. Int. Med., August, I908. 
In asthma the remedy is often of great value in cutting short an attack ( 5 to 15 minims) ( 0.3 to 1.0 c.c.), but it should never lead to a neglect to find some reflex cause, as in the nose, sinuses, or elsewhere.

In osteomalacia epinephrin should be tried thoroughly, giving 0.5 to I c.c. of the I : Io00 solution daily for at least a month, and much longer if untoward symptoms do not occur.

\section{DYSPITUITARISM. HYPERPITUITARISM. HYPOPITUITARISM. ACROMEGALY}

Knowledge of the disease furnished by clinical study, by experiments on animals and by many operations, has advanced very far within the last ten years. The clinical picture has been made clearer, though much fuller in details. The psychical symptoms, the eye symptoms including the ophthalmoscopic changes and fields of vision; the glycosuria or lowered sugar tolerance; all these and many more, with the roentgenoscopy of the sellar region, enable one to make a close estimation of the anatomic and functional changes in the pituitary body in acromegaly, and to recognize the stage of the disease and its rate or direction of progress.

Treatment.-The most pressing question in any case is whether operation is indicated, or rather, just what it may accomplish. Certain indications are imperative, such as those of dangerous cerebral pressure, or of impending loss of sight. The broader question, that of securing recovery cannot be answered as positively. In many patients, after various operations, not only have the pressure or "neighborhood" symptoms been promptly relieved, but many other symptoms, including enlargement of "acra," have markedly improved. Unfortunately, after a few months, the symptoms are likely to return, though usually in a different clinical picture. One practical difficulty consists in the objection of patients to operation in the early stages, the time when the greatest benefit might be hoped for. The details of the operative treatment cannot be entered upon here, but the monograph and other publications of Harvey Cushing are readily available. After any operative treatment, roentgen exposures can be used to increase the effect of the former. Opotherapy can also be carried out, either with whole gland preparations, or those of the separate parts. Owing to the interrelation of the pituitary functions a certain degree of obscurity still surrounds the question in almost every case. The treatment is experimental, in the sense that it must be followed as carefully as any pharmacologic test.

\section{HYPOPITUITARISM}

Synonyms.-Pituitary Dystrophy; Fröhlich's Syndrome; dystrophia adiposo-genitalis (Bartel); syndrome hypophysaire-adiposo-génital (Launois and Cléret). Recognized much later than acromegaly, this important expression of pituitary disease has been made known by 
many clinical examples, and a considerable amount of experimental work in animals (Aschner, Ascoli, Biedl, Cushing, etc.). The commonest example is furnished by the Fröhlich type, with infantile, sometimes large, sometimes dwarfish, body; excessive subcutaneous fat with feminine distribution; arrest of development of genital organs, and the secondary sexual characteristics (face and body hair especially), with alterations of bony growth, and of metabolism. Polyuria and "neighborhood" symptoms may occur. A very striking symptom is the heightened sugar tolerance. Some patients cannot take enough glucose (400 to $500 \mathrm{gm}$. being about the limit of gastric tolerance) to give a reaction in the urine. The body temperature is often low, but reacts to injections of pituitrin solutions (Cushing). Roentgenograms sometimes show alterations of the sella. The condition may be combined with acromegaly. Its relations to adiposity and Dercum's disease are probably close.

Treatment.-As in acromegaly, operation is indicated, and vitally so when there are pressure symptoms.

Experiments with organ preparations should be made. Puncture of the corpus callosum (Anton) and the spinal canal (Herrick) sometimes have striking effects upon symptoms, such as polyuria, but severe symptoms, such as headache, general depression and low blood-pressure may follow.

Pituitary Medication.-Pituitary preparations are available in several forms, such as the powder and tablets of the whole gland, or of the anterior or posterior parts. Solutions are also in use. The dosage is largely problematical, but the powders can be given in doses of $\mathrm{I}$ to 4 grains ( 0.065 to $0.25 \mathrm{gm}$.) three times a day.

Pituitary preparations have been used empirically in many disorders on the basis of their known or suspected physiologic action. As the exact clinical conditions are rarely known, the results are in many cases dubious.

Posterior lobe preparations have been used with marked success in obstetric practice. Pituitrin has been used not only to induce labor at term, but also to bring on premature labor. The dose is 5 to I5 grains ( 0.3 to I.o gm.), repeated as necessary.

Pituitary preparations have also been used in distention of the bowels, postoperative or otherwise. Collapse symptoms have been noted in some cases in which the method was tried.

\section{POLYGLANDULAR AFFECTIONS}

Careful study of ductless gland diseases shows many combinations of symptoms. Among them there are a few that exhibit the more striking symptoms of so many single types that the above term seems appropriate. They combine the bodily, mental and metabolic changes of acromegaly, myxedema, eunuchism, Addison's disease, and others in various types, and on autopsy several of the endocrine glands will be found diseased.

Treatment, at present, is symptomatic only. 


\section{THE PINEAL BODY}

"Hypopinealism," "dyspinealism" and "apinealism" are terms applied by Marburg and Foa to the results of disease of the pineal body. The characteristic features are precocity, both bodily and mental, unusual size and early function of the sexual organs, precocious beard and body hairs in boys, masculine type of hair growth in girls. Adiposity and cachexia later occur, with symptoms of brain tumor.

The treatment is that of brain tumor. 


\title{
SURGICAL TREATMENT OF DISEASES OF THE THYROID GLAND
}

\author{
By Joseph Colt Bloodgood, M. D.
}

\section{EXOPHTHALMIC GOITER}

Our experience since I9II has brought out new facts chiefly in relation to exophthalmic goiter.

Before subjecting these cases to any operation we are searching for some removable cause and attempting by methods of rest to reduce absolutely the mortality of operation.

Since I9I I a large number of my cases has had infected tonsils, purulent accumulations in the sinuses, or infections about the roots of teeth.

All of these cases of exophthalmic goiter were first put to bed in the hospital with ice to the heart and neck, given light diet, and urged to drink large quantities of water. During the first week of rest the investigations go on with the least disturbance to the patient, and everything is done to allay fear and anxiety. It is my rule to tell them that all the things we do are designed to obviate the operation, if possible.

Roentgen-rays are made of the chest, but up to the present time we have been unable to recognize any shadow suggesting thymus hypertrophy. This picture, however, gives one a good idea of the size and position of the heart.

The patients have also been subjected to roentgen-ray treatment, providing they are willing to have it done and are not frightened. I have been unable to find any evidence that roentgen-ray treatment is better than any other form of placebo.

A few of the cases have been treated by injections of boiling water into the glands - too few to allow any conclusions. However, in these cases there was nothing startling by way of improvement.

After seven to ten days, or longer if the patient's condition demands it, one tonsil is removed, later the second tonsil; the sinuses are drained; teeth are extracted. In all-of these cases we have made the operation shorter than we would have in patients with no cardiac handicap.

Rest in bed is continued. The period of time before operation on the thyroid in the severe cases has varied from six weeks to three months.

All of these desperate cases were later subjected to operation. That is, when they were operated upon, they were still exophthalmic goiter cases, but the heart and general condition had improved so much that the operator considered the risk slight. 
There has been no mortality. One patient was taken from the hospital by her parents, because I refused to operate after two months' rest treatment; subjected to operation at another hospital without any further rest, the patient died a few hours after leaving the table.

In all of the cases of extreme exophthalmic goiter seen by me since I9II I have forced the rest over a longer period than before and there has not been a single case in which improvement did not follow this rest.

This rest treatment is a difficult one to manage. The physician must ultimately obtain psychic control.

The improvement after the removal of infected tonsils, draining sinuses, and extracting teeth, has been absolutely distinct. There was no difficulty in recognizing the change. This is in marked contrast to my own observations on hot-water injections. However, my experience is too limited, and I shall continue to employ boiling water during the rest treatment.

The signs of improvement under the rest treatment are gradual, often very gradual; remissions are always observed, because it is impossible to protect these individuals from some kinds of shock. Family, nurse, or intern may do or say something which will upset the patient for one or more days. However, in spite of this they improve.

It is my hope that the mortality of the operation for exophthalmic goiter will be wiped out by not operating on these patients in the extreme state of thyreotoxemia, but give every patient the benefit of prolonged rest until the condition is such that operation offers little or no risk.

The probabilities are that all cases of exophthalmic goiter could get along pretty well if the causes were removed and they were kept in bed. I look upon the operation as a means of restoring their condition to so near normal that they will be able to do their work as well as before they were taken ill.

There is nothing new about rest in exophthalmic goiter, but it will always be found to be a very difficult method of treatment, not only in the hospital, but much more so in the home.

I know of no diet that need be applied to these cases, and I have had no results from the employment of the usual drugs.

These cases should be recognized earlier by the general practitioner. Many cases are overlooked simply because there has been no thorough examination. On the other hand, exophthalmic goiter may develop insidiously. This is especially true when the etiological factor has been some form of a mental trauma. I had the opportunity to observe one case which followed an accident. The girl was thrown from her horse and dragged; one rib was broken, as shown in the roentgenogram. At the end of the year there was nothing in the picture different from the ordinary traumatic neurosis. The first positive sign in this case was an increased lymphocytosis; the second was intermittent attacks of palpitation. 
In a number of recent cases high blood-pressure in young people has been an early sign.

It seems strange that in spite of the publicity given to exophthalmic goiter there has not been a great improvement in the early recognition of the disease. Surgeons are still seeing desperate cases.

Even when the general practitioner is suspicious of exophthalmic goiter, or even when he is certain in some cases, he plans his treatment along the lines of least resistance, advises the family and the patient to do those things easiest for them to do, while, as a matter of fact, he should take the dilemma by the horns, put the patient at absolute rest in bed, change to a hospital if necessary, search for some cause, and remove it if possible.

In one of my recent cases the Wassermann test was positive, and there were suggestive, although very early signs of tabes. This patient was not improved by intravenous and intraneural salvarsan during which time she was allowed to be up and about on so-called modified rest. However, after two weeks of forced rest the first distinct signs of improvement appeared. Antisyphilitic treatment was continued. This is the only case of exophthalmic goiter which I have not subjected to operation at some period. The result in this case is such that I would be glad to have it among my operative group.

To recapitulate, my own experience urges me to emphasize the importance of earlier recognition of exophthalmic goiter, enforced and properly arranged rest treatment, and the postponement of surgery until the patient's condition is such that it makes little difference what form of anesthesia is employed, or whether you "steal" the gland or take it out openly. The object of this operation is to complete the cure, to allow the patient to get out of bed and gradually return to work.

\section{THYROID TUMORS WITH EXOPHTHALMIC SYMPTOMS}

This is a distinct group and should be differentiated from exophthalmic goiter. The recognition is chiefly based on palpation of the tumor in the thyroid rather than upon the general symptoms.

In tumors the swelling of the thyroid gland is asymmetrical, confined to one lobe or the isthmus. The tumor is much more prominent than the gland. In exophthalmic goitre there is a uniform enlargement of the entire thyroid; one lobe, usually the right, may be larger. Among our older cases a number of these were looked upon as exophthalmic goiter. The patients were apparently quite sick, but when subjected to operation, they were much less upset than the patients with Graves' disease, and all of them recovered. In this group the pathological examination showed that we were dealing with an adenoma, or a cyst, or an area of chronic thyroiditis. In all of these cases there was, clinically, a visible and palpable tumor, but the diagnosis was made on account of the general toxic symptoms. As a rule, in this group exophthalmos is not of ten present, but nevertheless all the eye signs have been observed with cysts and adenomas. 
It is of great practical importance to differentiate the group. The danger of the operation in this second group, even when the thyreotoxic symptoms are grave, is slight as compared with the danger of the same operation for Graves' disease.

In this group as a rule one week's rest is sufficient.

During and after operation there may be a very rapid pulse, but the symptoms rapidly subside after the complete removal of the tumor and full convalescence is comparatively short in striking contrast to the convalescence from exophthalmic goiter.

Chronic Thyroiditis-Cancer.-This thyroid group is apparently on the increase. In a certain number of cases the condition has followed grip, and the pathological examination has disclosed a distinct thyroiditis. These patients have the grip; a few remember some discomfort and tenderness in the region of the thyroid; the majority do not. They return to work, experience early fatigue; then follow gradually or rapidly the well-known thyreotoxic symptoms. Sometimes the patient, in other instances the physician on careful examination, finds the thyroid enlargement. To the surgeon these cases present more than one dilemma. On palpation a zone of chronic thyroiditis feels and looks like cancer. In removing the lobe the infiltration is like cancer. But one could feel pretty certain that if it were cancer, it had become a hopeless local condition. For this reason the surgeon does not operate as for cancer, but by blunt dissection, usually with a piece of gauze, strips and tears the indurated lobe of the thyroid from the infiltrated thickened posterior capsule, and thus completes the operation without subjecting the patient to laryngeal paralysis. It is important for every surgeon to know that a cancer of the thyroid which has infiltrated outside of its capsule, is a hopeless disease; that a similar infiltration may be present in chronic thyroiditis which is not a hopeless disease, and in which there is no necessity to do such a radical operation that the recurrent laryngeal nerve is in danger.

Quite a number of cases of chronic thyroiditis have been sent to the Surgical Pathological Laboratory for diagnosis. As a rule both surgeon and pathologist had concluded that the condition was malignant. The chief proof of the correctness of our laboratory diagnosis is that these patients are well in spite of the fact that the local condition was not completely removed.

Cysts and Adenomas. - The tumors are usually single and for reasons not explained there are rarely pressure symptoms. In some the patient has been aware of the swelling before a general breakdown was observed. In others the thyreotoxic symptoms were observed before the appearance of the tumor. To the present time I have the impression that the same etiological factors which lead to Graves' disease may produce the same results in the patient with a cyst or an adenoma. But this theoretical consideration is of no practical importance. I am convinced, however, that we have sufficient evidence which will allow us to urge operation upon every individual with a 
nodule in the thyroid gland. They are fortunate if they feel a nodule first. The removal of this nodule will protect them from cancer, and I am also inclined to believe, from attacks simulating Graves' disease.

Excision of Piece for Diagnosis.-The importance of always investigating the thyroid most carefully is borne out by our larger experience, but there are groups of cases in which we would welcome some exact method of diagnosis. This is especially true in obscure psychic disturbances. Goetsch of the Johns Hopkins, having found a specific stain for exophthalmic hypertrophy, suggests excising a piece of the gland for diagnosis in these doubtful mental cases.

Puberty Hypertrophy.-After puberty and for some years the thyroid may be as prominent as in some cases of exophthalmic goiter. These patients may be as nervous and restless; in fact, they may present many symptoms of Graves' disease. One, of course, is always afraid that the serious lesion may be overlooked, and at puberty and in young individuals exophthalmic goiter is a much more critical disease. To the present time I have had the good fortune to recognize the only two cases of Graves' disease which I have seen at puberty. One I have already mentioned as the one in which the parents refused further rest treatment. The other was a case also observed in St. Agnes Hospital, but before we were so thoroughly convinced of the necessity for prolonged enforced rest. This young girl after two weeks' rest was subjected to operation by a colleague and died of acute dilatation of the heart a few hours after operation.

The other cases of enlargement of the thyroid at puberty have, as far as I know, gradually recovered without the enforced rest treatment or operation. Some occupation seems to be the agent that helps most in puberty hypertrophy. One should, of course, search for a cause, but in the group which I have so far observed, it was rather the exception to find an etiological factor in a pelvic lesion or in an infected tonsil, sinus or tooth.

Thyroid Tumors and Pressure.-It has been very fortunate that our patients with huge goiters or with tumors producing pressure have rarely had any thyreotoxic symptoms. That is, when these toxic symptoms have been present, the goiter on one hand has been relatively small, or the tumor, on the other hand, easily removable.

Huge goiters are not of ten coming to the Baltimore clinic. All other types of thyroid lesions are on the increase. With few exceptions, patients with thyroid tumors and pressure symptoms have known of the tumor before the pressure symptoms set in. Of course, these individuals could have been relieved of the tumor first. In a few pressure symptoms antedated the appearance of the tumor, or at least its discovery. The most common pressure symptoms are loss of voice and cough, associated in some instances with bloody expectoration. I am inclined to think that in most of these cases the tumor could have been palpated at or shortly after the beginning 
of the pressure symptoms. In this group there is always unnecessary delay. One of my patients had waited twenty years with loss of voice and cough.

Roentgen-rays and Goiter.-The roentgen-rays have been very helpful in outlining the position of the trachea in relation to the thyroid and in portraying areas of calcification - a finding sometimes helpful in the differential diagnosis. So far the roentgen-rays have been an absolute failure in aiding us in the recognition of concealed substernal thyroid tumors and in outlining the thymus.

Transplantation of the Thyroid.-In my only observation since IgI I the one case of chronic myxedema in which thyroid tissue had been transplanted into the peritoneal cavity, rectus muscle and marrow of the tibia, there have been no results. 


\title{
DISEASES OF THE CARDIOVASCULAR SYSTEM
}

\author{
By Sir Clifford Allbutt, K. C. B., M. D.
}

The reflection which on revision of this article chiefly occupies my mind is that the cardiac potential, and therefore the purpose and method of treatment of cardiac disorders are concerned more and more definitely with the myocardium. In the experience of the pathological laboratory we are learning that the myocardium is subject not only to fluctuations of tone but is susceptible also of many and various injurious influences; not a few of them, such as the "toxins" of almost all infectious maladies, directly injurious. To these must, of course, be added certain obscure poisons of constitutional origin"katabolites;" and atrophies, such as the fibrotic, or degenerative, results of defective arterial supply or intrinsic decay. On the threshold then of the treatment of any cardiac case our first question is, What is the quality of the myocardium? a condition of which unfortunately we have no very definite means of measurement; and the next, What means, physical or pharmacological, have we of bracing or repairing this muscle? a question which, in the main, seems nowadays to shrink almost to this-When and how are we to use digitalis? At the outset of a consultation on a case of heart disease we ask the family physician-How have you used digitalis, and what response to the drug have you obtained?

For it cannot be said that of late years our medical, our curative, resources have increased in proportion to the great increase of our knowledge of cardiac rhythms and vortices. Indeed as the values and properties of digitalis are better understood, other remedies, at any rate other pharmaceutical remedies, seem to recede into the background. Again, if it would be unreasonable to say that the Oertel boom, or the Nauheim boom, has left nothing behind it, for indeed they have left results of some importance, yet their operations have proved to be far more restricted, and their effects more equivocal, than their prophets had proclaimed: moreover, they are far too costly and troublesome for a large number of cases in which simpler methods may be at least as efficacious.

May we hope then that, as we are learning more of cardiac physiology, normal and abnormal, we are pegging out larger claims for the properties of digitalis? We are, it is true, defining more accurately its special aptitudes, as for instance in dilated chambers and auricular fibrillation; but scarcely are we, as we penetrate into the recesses of cardioarterial function, carrying with us digitalis throughout as our unfailing light. For example, we have not yet decided whether this drug does or does not constrict the muscular 
blood-vessels of the periphery. Dr. W. S. Dixon in the fourth edition of his "Pharmacology" (1915, page 183) says still that it does constrict the vessels; he gives several and various experimental tests of this property, and points out that this action arises both peripherally and centrally. Sir James Mackenzie, on the other hand, almost scornfully denies this effect, but does not relate any experimental disproof of it. If, in the case of the only cardiac drug that has been studied clinically and experimentally with any throughness, this dissent be found, what must be the state of our knowledge in respect of the whole class! Again, in respect of myocardiac potential, the potential that is the foundation of cardiac capacity, we are baffled, for the more directly heart failure is myocardial the less help do we get from our sovereign heart medicine! In what we compendiously call "myocarditis," in all that class and array of cases in which the heart's muscle is impaired by infections or decay, digitalis not only availeth little, but indeed is often mischievous: and the same is true of the myocardial degenerations, and of atrophies due to coronary defect, or senile decay. In injuries to the valves of the heart it helps us only in so far as the myocardium, or much of it, is sound. A patient of mine, aged forty-five, had rheumatic fever eighteen years ago. His mitral valve was affected, but he was very carefully treated and not allowed to exert himself for six months or more. He then returned to his work as a coalheaver, and for some seventeen years in spite of audible mitral regurgitation he pursued this strenuous life without discomfort. Then eight months before admission to Addenbrooke's Hospital he was attacked by that insidious evilinfluenza. On return to work grave symptoms of heart diseasedyspnea and dropsy-appeared in force. At first digitalis, with rest in bed, diet, mercurials, etc., seemed effectual; but their influence seemed to be transitory, and theobromin, salt privation, etc., to do more for his condition. This serious breakdown was not due directly to the mitral lesion, but to poisoning of his myocardium by the influenza; thus our chief hope of amendment, so far as I know, lay essentially in the slow process of muscular repair. This digitalis could not compass for us.

In the protean forms of "functional" cardiac disorder digitalis is, at the heart, of only incidental service, if any. In the so-called soldier's heart it is of no use whatever.

Digitalis then will help the heart especially in mitral regurgitation so far as there is good heart's muscle to respond to it, and no farther; assuredly this is on the whole an invaluable aid; but in respect of progress in cardiac therapeutics it is one which, in spite of our studies, leaves us pretty well where we were fifty years ago.

Nor in our new cardiac physiology have we discovered any more efficient way of using digitalis and its several preparations; no more, at any rate, than may be found in the essay to which this is a postscript. For my part I am less and less ready to plump the patient with large initial doses, such as 0.5 dram doses of the tincture. It 
is better, in my opinion, to test each patient with ordinary doses, and so to feel one's way gradually to bolder exhibitions. But if ordinary doses-such as 45 to 60 minims of the tincture, or an ounce of the infusion in twenty-four hours-fail to benefit the patient big ones rarely prevail, while with them we run the risk of upsetting that precious auxiliary, the stomach. The more one sees of heart disease the more anxiously does one wait upon the favor and the efficiency of the alimentary canal-the via prime. Of the much-advertised new preparations of digitalis, digipuratum and Nativelle's granules seem to maintain their position as stable and efficacious. Digitalin seems to be as stable as any other preparation of the drug, but no more so. Digipuratum, however, containing as I am told, more digitalin, which is less irritating, and less of the acrid digitoxin, may be preferred when the stomach is vexed. And we are assured that digipuratum may be injected subcutaneously or intravenously. Unfortunately we have no ready tests for digitalin and digitoxin, respectively.

Mackenzie, Lewis, and Cowan say that in auricular flutter full doses of digitalis may even set up fibrillation-indeed excessive, doses may do it in any heart--but that if then the drug be intermitted, a normal rhythm may return. However, as Cowan observes,${ }^{1}$ seeing that the administration of digitalis is usually but a part of a rational and many-sided treatment, its own effects cannot always be precisely reckoned.

In partial heart-block digitalis is apt to increase the default, perhaps by irritation of the vagus and certainly by lowering conduction from area to area. Tabora (Ztschr. f. exp. Path. u. Therap., I906) illustrated this by crushing the a-v bridge in animals, and then giving digitalis. Under the drug the beats dropped out much earlier. Atropin on the other hand, by suspending the vagus influence, may often serve as a test of the relative values of the nervous and myocardial coefficients. In hearts affected by auricular fibrillation, paroxysmally or continuously, especially in the later stages of high pressure and its results, the vagus has a strong influence upon the heart (C. Robinson, Rockefeller Rpts. xxi, I9I5). And so it is also in angina pectoris (Wenkebach).

If digitalis be of no service in restoring damaged myocardium it is a means of restoring tone to so much of it as may still be substantially sound; and by its effects may indicate the degree and quantity in which this structure has survived. Nevertheless, even in partial heart-block-prolonged a-c interval- 5 minims of tincture of digitalis may be cautiously tried in the hope of some available cardiac reserve, not forgetting that, if the block be substantial, StokesAdams symptoms might be brought on. But, when extrasystoles are signifying ventricular dilatation or stress, the drug may be specifically needed, perhaps in no inconsiderable dose. For example, in the extrasystoles of aortic disease, if these be due to a laboring

${ }^{1}$ Dis. of Heart, I9r4. 
left ventricle, digitalis may be cautiously tried, with atropin to soothe the vagus, ${ }^{1}$ the patient being kept meanwhile at absolute rest in bed. In this instance a cordial should be given with the digitalis, and perhaps strychnin which is credited with a guardian or auxiliary function (page 483).

Digitalis then is the remedy for $(a)$ dilated chambers and $(b)$ for fibrillation of the auricles; and the typical case for its success is mitral regurgitation of rheumatic or similar origin; the myocardium having virtually recovered itself. During the myocarditis and endocarditis, as indeed, in pyrexia generally, it is of course to be eschewed. In mitral stenosis it is less efficacious. For the "functional" troubles which, partly perhaps from its prevalence in young women, hang more about this mode of cardiac disease than any other, digitalis has not the assuaging influence one might well have expected of it. It has been said, I think by Sir James Mackenzie, that in the accelerated but regular pulse of mitral stenosis digitalis is useless. This is my own impression. In these cases, as Hirschfelder suggests, the vagi may for some reason be out of action. In some cases of mitral stenosis I have thought that strophanthus proved to be a better ally than digitalis; but if auricular fibrillation appear digitalis must surely be wanted. In respect of high degrees of stenosis we have to remember that the harder the right ventricle is driven the more unhappy is the state of the lungs between the opposing forces. We cannot increase the diameter of the contracted mitral orifice; our end is to be gained only by increase of velocity, and it is easy to see that there must be an optimum retromitral pressure, just such as to compass that degree of velocity which may load the left ventricle better, but not exceed what may be consistent with machinery already under strain; a nice point of clinical tact. At critical moments deobstruent methods, and repeated small venesections or leechings, may, by lowering the viscosity of the blood, accelerate its passage. The fretfulness of these hearts must be soothed by remedies proper for more purely functional heart affections; here convallaria-the aqueous extract in $15^{-20}$ gr. doses-or cactus may help us, but in no disease are the heart's action and dimensions so variable and unstable. In some cases it is well to allow the sufferer more freedom of movement, more change of scene. As Sir James Goodhart says, "too much bed should be avoided; if the patient is fit to move gently about he will be the better for it;" if, I would aid, the case be mainly valvular, not myocardial. In reckoning the probable effect of such permissions we may be guided, as it is one of the few cases where we are thus guided, by the murmur itself. A variable "presystolic" murmur, often fading, at times perhaps to disappear or give place to a diastolic murmur, or signs of stress on the lungs, would warn us against more liberty; while a steady presystolic murmur and breezy lungs would favor it.

Some physicians, among them Dr. Windle, advise us, in cases

'See Pflüger Archiv., April, s9ro. 
where digitalis seems definitely indicated, to begin with a few largish doses-say 20 minims of the tincture every four hours; and then to drop to doses of 5 minims. Thus, it is alleged, the full effects of the remedy may be secured while the ills of "accumulation" are avoided.

Another valuable use of digitalis is, so to speak, to stop a leak. In persons in whom a valvular defect is quiescent and the circulation in good balance, some stress, mental or bodily, some transient fatigue or ailment, disorder of the via prime for instance, may from time to time threaten the balance; so that, unless precautions are taken, the heart may fall into trouble. It is at these moments, often to be recognized by the patient rather than by the doctor, that a few small doses of digitalis, perhaps with the addition of a little sodium bromid and a mild mercurial, will steady the heart and its nervous governance, and tide over the peril. Those thus affected must be taught to keep a sharp eye for such episodes, and even to help themselves on occasion in this way:

We have observed that in aortic regurgitation, more than in other kinds of heart disease, nervous and psychical disorders are apt to appear; such as headache, insomnia, excitement, delirium. To describe these symptoms would fall outside our limits, and the specialist's treatment of them will be found in other sections of this work. For the headache, iodid of potassium is useful; for the delirium, hydrobromate of hyoscin, beginning cautiously with $1 / 200$ grain. For the mere insomnia of heart disease, not due to pain or dyspnea, a little port wine, old sherry, or sauterne, undiluted or as negus, is often helpful, or, in cases of depression, champagne. For hospital use, brandy and soda is cheaper. The first effervescence should be allowed to pass off. Beer is to be avoided lest the liver be more burdened, and for this reason in valvular disease alcohol in all its forms should be given with a sparing hand.

While still looking to the myocardium I would call attention to certain advances in our comprehension of that difficult class of cases known variously as the "strained" heart, the "soldier's heart," etc. The late Dr. Michell, and myself in Cambridge, had been much impressed by the caprice of these manifestations in athletes. Many are no doubt, what they seem to be, fatigued, and overstressed or unbalanced circulation; whether this be at the heart, in the vasomotor system, or otherwise. But in many others, perhaps a majority, though this depends much on the conditions at the time, the symptoms appear without obvious proximate causes, and continue beyond all common reckoning. In many such cases, as one or both of us have put on record, we have found, with the athletic stress previously borne with ease, the coöperation of some such intercurrent malady as influenza, or even a "bad cold." For some time past we have warned boating and football men to take rest not only during any such infection but also for some reasonable time after recovery. The severer the effects the longer the rest, or comparative 
rest. Men who fought through such episodes did so sometimes no doubt with impunity, but by no means always; some of them broke down more or less, or even became invalided. It is especially in these cases that the nutrition of the myocardium and the defensive forces of the body should be promoted by open-air life, nutritious diet, and careful attention to digestion and excretion. But I shall return to this subject a few paragraphs later.

Now at the Mt. Vernon Hospital, Hampstead, where Sir W. Osler, Sir James MacKenzie, and myself are consultants, Dr. Lewis, and his assistants, on war service and in coöperation with the National Research Committee, are in charge of a large number of these "functional heart cases" in soldiers. In some of these cases, and in the earlier admissions Dr. Lewis noted a persistent pyrexia, a slow fever of no wide range indeed, but indicative of a mild chronic sepsis and in a few cases streptococci were discovered in the blood. However, from subsequent observations it is clear that chronic sepsis takes no important part in the causation of "soldier's heart." In the case of soldiers returned from foul trenches or other such quarters, some of these cases may be complicated and retarded by a latent sepsis, and while this insidious evil is on foot, treatment founded narrowly on notions of cardiac disorder, must be futile. That distinguished clinician, Dr. DaCosta, who knew nothing of a septic ingredient, treated these very cases on the usual lines, with digitalis and rest and was not unsuccessful in restoring many of them to the fighting line. But there is something more to be said.

The symptoms of these cases are curiously uniform: and consist in dyspnea, giddiness, oppression, frequent pulse, palpitation, pain, and inability for exertion and, in many of them, a nervous instability. This uniformity seems to exclude malingering. Moreover, in not a few cases a history of such symptoms can be carried back before enlistment. With the aid of Mr. Barcroft of Cambridge, these symptoms have been found coincident with a defect in the "buffer" salts of the blood (vid. Brit. Med. Jour., Oct. I4, I9I6), due presumably to some error of metabolism. Hitherto the treatment has been by progressive exercises, which improve metabolism. Administration of the defective salts by the mouth is of no service. So far the results are promising.

In convalescence from the more specific infections, from, let us say, rheumatic or scarlet fever, diphtheria, influenza and the like, a more rigid prescription of rest, which in its proper place is now enforced by competent physicians, is justifying itself by ultimate results. Dr. Cowan lays great stress upon this precaution. $\mathrm{He}$ directs that at least until the rate and rhythm of the heart are normal the rheumatic cardiac patient shall lie absolutely at rest. Then he may sit up a little in bed for short periods, the size of the heart and the pulse rate being watched, so that the periods may be gradually increased. He should, however, be confined to bed for at least two months more, and for another month before he is allowed to stand Vor. IV. -31 
and walk, merely removed to a couch. For three to six months longer exercise should be restricted, and for a year afterward hard exercise forbidden. Massage may be begun very gently as soon as the patient is allowed to sit up. Cowan gives records of cases which under this method showed "surprising results." My own experience is to the same effect, and I would add the warning that in many of these cases, especially perhaps in mild cases of scarlet fever, myocarditis is overlooked; it is not sought for, and the heart suffers accordingly from dilatation and at a later date is regarded as "overstrained." About fifteen years ago a boy, in and after rheumatic fever, presented all the signs of mitral regurgitation and stenosis. He was carefully treated on these lines, but a few months later the signs were still present. Some years later the patient happened to call upon me and I asked leave to examine his heart. To my surprise, I could find nothing wrong with his heart, and later, soon after the war began, he was accepted for military service and is still with the forces. On the other hand, in acute rheumatism we are occasionally baffled, or at any rate I am, by cases of young persons in whom, in spite of careful treatment by salicylates in various doses larger and smaller, a comparatively slight pyrexia will persist week after week. In the absence of ordinary septic and other new infections it would seem that rheumatic endocarditis, and perhaps myocarditis, may thus smoulder on. In these and such cases the long confinement to bed, which seems inevitable, is for young people a grievous penalty. It is, of course, imperative that any other sources of sepsis, such as bad teeth, foul tonsils, discharging ears and the like, should be vigilantly sought for.

With vaccine or serum treatment in cardiac cases of this kind no progress has been made. Normal horse serum has been suggested in rheumatic and malignant endocarditis, but, so far as I know, with no clinical or pathological proofs. Collargol injections likewise have as yet met with no definite success.

From the virtues of strophanthus we have drawn little but disappointment; though the marvellous occasional effect of intravenous injection of strophanthin (or oubain) has been confirmed. Still this operation is one for use only in extremity (see page 8I, Vol. I) and then may frequently be interdicted by an immediately previous course of digitalis to which it is perilous to add the strophanthin. Hirschfelder warns us against the escape of any of the injection into the tissues about the vein, where it is apt to set up an angry reaction. $\mathrm{He}$ adds that the site of the injection should be massaged for a few minutes afterward. Occasionally, he says, the injection is followed by a severe chill and some fever, but a transient duration.

The apparent caprices and failures of strophanthus in clinical medicine, which are in sharp contrast with its behavior in experiment on animals, are said to be due to very wide variations in the solubility of its active principle in the stomach and bowels; if so, its effects on the circulation may be long delayed, and then burst forth incon- 
tinently, and even dangerously. It does not affect the vasomotor system, or but little. I think all users of strophanthus feel that there is something irregular about it, or about our methods of using it. As to that $I$ have little to add to what I said of it on page 8I, Vol. II. The glucosid is not destroyed in the stomach as those of digitalis may be.

In respect of strychnin again we are pretty much where we were. A belief in its virtue as a cardiac tonic or stimulant is widespread, and its use general; but the common belief rests little on proof. For a while indeed experience, both clinical and pharmacological, seemed to contradict the belief, but some more recent experiments ${ }^{1}$ seem to corroborate it. Our knowledge is not yet ripe enough for further discussion of the point; meanwhile we shall no doubt continue in the good rule not to deprive a patient of a remedy of alleged efficacy, whether personally we have full confidence in it or not. For my own part I think Hirschfelder is right in saying that it seems efficacious rather in cardiac dyspnea (falsely called "asthma"), and Cheyne-Stokes respiration; i.e., rather as a respiratory and vasomotor agent than as a myocardial tonic. But for these conditions with diet for a few days, oxygen inhalations and subcutaneous injections of camphor in oil-besides, of course, digitalis or caffein, are far more effectual. For this condition Josué recommends the injection under the skin of I mg. of adrenalin in 250-500 c.c. of serum. There is some painful distention at the swollen spot but the gradual absorption is an advantage. The doses of strychnin, if they are to be effectual, should be stronger than is customary-say in a vigorous adult even up to gr. $1 / 10$ (0.0065 gm.) (see page 54 , Vol. II).

As regards other drugs, alternatives to digitalis, such as caffein, theobromin, theocin, etc., I find little to add to my former article. The chief new point is that, as Dr. Harvey has shown in our laboratories, caffein is, or so far as experiments on animals go seems to be, harmless to the kidneys in congestive or nephritic states, and no less efficacious than when the kidneys are healthy. Presumably this observation, if verified, will apply also to the congested kidney of heart disease. The opinion I have expressed of the considerable remedial value of these drugs, an opinion framed on many years experience, is gaining ground. Caffein, in cases where digitalis is inadmissible, or in any case where a fillip to diuresis is required, as in the dropsy of yielding heart, often has an admirable result. The pure caffein is to be preferred to the citrate, salicylate, or other salts. Unfortunately in some persons its nervous and sleepless effects are a great drawback; if so, theobromin (in cachet gr. 5-15) can be used, and hopefully; though it is not quite so efficacious as caffein. We are told that the salicylic compounds, such as diuretin, have drawbacks which, in agurin or pure theobromin are avoided. Caffein and theobromin

\footnotetext{
${ }^{1}$ See Mann, Guy's Rpts., Jan. 25, 1908, who says a hypodermic injection raises both systolic and diastolic pressure in ten minutes.
} 
can be administered quite well in cachets. I suppose that the use of drugs of this class, not constructive but stimulant or moratory should not be long continued. If, in the individual case, these good effects do not become quickly apparent, say in two or three days, the drug is not likely to prove of service, and should be withdrawn. Of theocin I know little; it is accused of upsetting the stomach, and, as in my occasional trials of it, it seemed at any rate to be of no greater use than theobromin, I have neglected it. In the dropsies of high-pressure cases failing not by apoplexy but by cardiac dilatation, theobromin is often of great service; probably it is on the whole the best of this class of drugs. Atropin is often very useful in vasomotor storms, and as a test for heart-block (page 478). Tincture of aconite, very cautiously used, is sometimes effectual in stilling the throbbings and palpitations in "functional" heart affections. In this place I may remark in respect of operations for anasarca of the legs that some physicians, such as Cowan ${ }^{1}$, have found the safest of them to be as follows: The punctures should be many, and about an inch apart; two or three rows may be made on each side of the legs below the knees. The punctures should not be deep, just through the skin, by means of a sharp broad surgical needle guarded $3 / 8$ inch from the point. This operation may be needed also about the penis or scrotum. The skin should be protected beforehand by some aseptic ointment over which the oozings will run away.

These patients require the most careful nursing. On the heavy insensible heels or ankles, if water pads or cushions are not used to equalize pressure, gangrenous spots may suddenly appear, and the posture of the limbs should be altered from time to time. Also the weight of the sitting body bears heavily upon the coccyx where, if not bedsore, great tenderness and soreness may arise. A water cushion will help to prevent this great discomfort. In bedridden elderly men prostatic difficulties must be anticipated, and the emptying of the bladder carefully obtained. In complete or "absolute" rest is included the use of the bed pan.

In the pyrexial heart diseases, such as pericarditis and endocarditis, when digitalis is contraindicated, I have referred to the use of the ice bag, as urgently recommended by the late David Lees and other authors. I said then that evidence of the effect of the cold upon the tissues, pulses and blood-pressures, was lacking. Now from experiments quoted by Hirschfelder (edition of I9ro) it appears that the ice bag (two bags should be in use, so that no period of reaction should intervene during refilling) does affect these parts and functions very decidedly. The pericardium is cooled from $I^{\circ}$ to $5^{\circ}$; the pulse is retarded by some Io beats, and the bloodpressure may rise to $190 \mathrm{~mm}$. presumably because of large areas of vasoconstriction due to sympathetic chill. The cooling may be good for the heart, and for the pulse, but the great rise of pressure 
is surely a disquieting condition, especially when we know that myocarditis is almost certainly present. Nevertheless Hirschfelder uses this means and continues to recommend it. For my part, although quite disposed to use the ice bags in acute pneumonia, in respect of these cardiac conditions I am awaiting further information.

At first, then, in most perhaps of our severer cases, it is a nice question how far in the individual "myocarditis," or a degenerated myocardium, may we resent digitalis but accept a surrogate such as theobromin, or other "cardiac" drug. On the difficulty of estimating myocardial values, whether in toxic states-such as scarlet fever, rheumatic fever, influenza, diphtheria-or in mechanical or degenerating cases, $I$ have dwelt at length in my book on "Diseases of the Arteries" (I9I5), and have touched upon it in my former essay in this work (page 138). One difficulty of "work tests" lies in the general condition of the patient to be subjected to them, and furthermore their deep implication of psychic and vascular factors. Besides the tests to which I have alluded (on pages 66,67 ), one which I have used but scarcely proved, is as follows: Stopping the wave in the usual way with the cuff, note the maximum pressure; then with a finger gauge (I have used Fleischmann's but Gärtner's would do) try the still higher pressure required to arrest the flow in the finger; i.e., to close the brachial artery completely (not to stop the wave only). If the heart be vigorous 25 to 50 per cent. more pressure may be needed, but the less the weaker the organ. It is said that if at this point digitalis be injected subcutaneously it may restore the arterial flow in the finger, and if so that the presage is relatively better. Again, if in "pulse pressure" (amplitude) the difference between systolic and diastolic is reduced, especially if the mean pressures be weakening, and the diastolic relatively rising the heart (caeteris paribus) may be failing; but these kinds of test have only a temporary and comparative value. Diminished oscillation with raised pressure means of course more peripheral resistance.

What can be done, and must be done, in all cases of any nicety, is to analyze the rhythms, so as to distribute as clearly as possible auricular and ventricular discords, respectively, to distinguish extrasystoles from other arrhythmias, as in cases of accelerated rate, or in "blocking," and thus to make sure of their nature and character as arrhythmias before interfering with our remedies.

In respect of diet, $I$ think $I$ have little to add to what is written in the former article; the main points being economy of slops, flatulent and bulky carbohydrates, and restriction of fluids at or near the meals. Clear soup in a small cup, pounded fish, minced chicken, fresh potted meats-not too highly flavored-on thin toast or biscuit, no coarse vegetables nor pulpy puddings, but plain custards, jellies, junkets and the like, will help us out. Although savories and spices are apt to "rise," some seasonings may be sparingly used, for if the food be not tasty and dainty the appetite may flag. The little earthen porringers, known as "terrines" are very useful for 
invalids, as the food in them comes in small quantities and keeps hot. Acids or acid foods, such as certain fruits, should not follow soon after milk. When the digestive system is disordered, the dyspnea perhaps distressing, and the kidneys inactive, four or five days' restriction to milk diet, or even to butter-milk, is often found very beneficial. We shall remember that for an inactive patient little fuel is required beyond the needs of heat production, and for this purpose milk, sugar or glucose, even per rectum, will keep things going for a short time. In small quantities alcohol is probably a compact and digestible respiratory food; e.g., a little weak brandy and water, or light French wine; but in more liberal quantities it loads the liver which becomes larger and more tender.

The diet in high-pressure cases is considered under "Arteriosclerosis." My experience is still that gouty persons, in whom the disease presents itself mainly as a crippling of the limbs, are not more the subjects of high blood-pressure than other persons of the same age.

Here I may allude to Dr. Goulston's prescription of sugar-the best "Demerara"-in failing heart. He prescribes gradually increasing quantities of it, from I to 6 ounces as tolerated, in the belief that it supplies a ready nutrition to the myocardium. The sugar may be consumed in any way convenient to the patient; as with thin bread and butter, or other food, in tea, or as syrup. Goulston has published more than one series of cases in which the results seemed to be very good. I confess I ought to have made a better trial of it, but cases in consultation are often lost sight of. In a few cases in hospital or otherwise I have made some trial of it and I certainly thought with advantage; the sugar was willingly consumed. It is prescribed especially, of course, in case of failing myocardium.

As regards rest in the chronic heart diseases of adults I have nothing to add to my former essay. This prescription in them cannot be so absolute; habits and temperament must be taken into account, as also the constitutional disorders which a torpid life are apt to entail, and moreover the right of the intelligent patient to balance the advantage of irksome rules against the risks of disobedience.

Concerning baths and heart exercises I have little to add to what I said before. Resisting exercises elicited by a skilled attendant, which may be begun with the arms before the patient leaves his bed, still appear to be the best mode of them; and should invite just so much muscular function as to call forth the tonicity of the myocardium without fatigue of it. Therefore, as Hirschfelder has pointed out, the least sign of stress, such as dilating nostrils, drawing of the corners of the mouth, yawning, sweating, and, still more, any duskiness or pallor, should be noted promptly, the exercise suspended and a more cautious approach made on the next occasion. The exercises should be slow, uniform with intervals of rest and not repeated in the same area; so that the circulation may be promoted over a large system of dilating peripheral vessels. Respiratory 
gymnastics and gentle abdominal massage may be used to empty the mesenteric system and promote discharge of gases. All is well if, after these exercises, and baths in the indifferent zone ( skin shows signs of reaction and warmth. In machine exercises (Zander's and others) these watchful precautions are apt to be neglected or but perfunctorily attended to. Such exercises are only for cases in which the heart is reckoned still to retain some fair capacity, some reserve; in degenerate or myocarditic cases they are inappropriate, at any rate before convalescence is well on the way, and even then these hearts are very tricky.

In discussing exercises we shall remember that systems, such as Oertels or the Swedish, comprehend much more than movements. The amount of liquid to be drunk is carefully measured, as also the amount of flatulent carbohydrates, and of fats. These systems act best in the obese with overladen, but not very degenerate, hearts. Moreover, we shall be careful to regulate muscular exercises by the kind of life the patient has led. They cannot be prescribed in like measure for self-indulgent sedentary persons and for out-door laborers or sportsmen. We shall endeavor to reduce the viscosity of the blood by eliminating carbon dioxide and frictional resistance by opening out the peripheral vessels. Increased tension of carbon dioxide causes dilatation of the heart, both in systole and diastole. We shall not forget also Peter's advice "mittre a laidete des passions emotives."

However, as Hirschfelder added, one great secret of systems, such as the Nauheim, is that by one way and another, the patient is made to feel that a great deal is being done for him, a great effort made for his welfare. Whatever our methods then, we shall take pains to see that he shall not be without this consolation. As regards mineral waters in heart diseases Royat is much to be commended; BourbonLancy also proves useful. To keep the gastro-intestinal system in order is of course of the first importance, and a climate promoting skin action without great vasomotor fluccuations or constrictions or bronchial irritation is to be sought, if possible.

To the section of arteriosclerosis I have nothing to add except that the reader will find these maladies more fully discussed in my book, published subsequently, on "Diseases of the Arteries." I may say, however, that in my farther experience of the high-frequency current in cases of high blood-pressure I have reason to speak with increasing confidence of its beneficial effects; whatever the explanation may be. It seems to act by means of peripheral vasodilatation.

As regards angina pectoris $I$ am more and more convinced that this malady must be treated, whatever our opinion of its nature, as we treat an aneurysm of the aorta (see Diseases of the Arteries, pages I6 3 and I67, Vol. II), if with less severity and for a shorter time. The principle is that the pain must not be repeated; every recurrence makes for its perpetuation. I advise the patient at first to keep his bed altogether, for some weeks or months, as the imminence 
of the pain may dictate. During this quiescence, of course the diet must be proportionately reduced and simplified, even to milk only. For a male adult about 3 pints a day will suffice. Flatulence and constipation must be anxiously avoided. It is better at the outset to explain fully to the patient what lies before him, so that instead of the disheartening rallies and relapses of half measures he may take steps to arrange business and other affairs accordingly, and to provide for the quiet occupations and amusements which, as a man otherwise healthy, he can carry on, not unhappily, in his bed. A nurse is desirable because, at first at any rate, and until the ways of the case are ascertained, the bed pan should be used; often probably with the help of an ordinary or glycerin enema. For further details I may refer again to my book on "Diseases of the Arteries." Then as time elapses, and the pains fall into abeyance, the patient may crawl to the sofa, thence to the easy chair and to the carriage, and so on; in the reasonable hope that the healing effects of time and rest may endure.

Syphilitic diseases of the myocardium can be but guessed at; syphilitic aortitis, although also very often latent, may by both signs and symptoms be more definitely made out. Since my former article was written we have had more experience with salvarsan, and have found it valuable in cardiovascular disease. For the modes of its administration, and its place in the therapeutics of syphiliswhether affecting the heart or other parts the principles are the same-I must refer to the special articles on the disease.

As regards the surgical treatment of heart diseases not much more has been done. In pericardial synechia Max Herz of Vienna has urged the importance of precise diagnosis before operation; as for instance between malformed chest, and mediastinal growth on invasion by tuberculous or other fibrosis, cardiac hypertrophy, and so on. On the other hand, the sort of synechia is more and more clearly seen (see Keith, Lancet, I904, i; Wenkebach, Brit. Med. J., I907). At the German Congress of Inner Med. of I904, Brauer showed three patients relieved by cardiolytic resection of ribs and cartilages. Morison showed a case with good results to the Harveian Society in Nov., r909. The operation seems indicated if there be strong retraction of the ribs and distressful heart, or dropsy and dyspnea not relieved by ordinary means; but it requires much care and experience. Morison's patient, whom I saw, was evidently very much relieved and did not hesitate thankfully to say so. 


\section{DIABETES}

By Theodore C. Janeway, M. D.

Since 1912 the treatment of diabetes has been profoundly modified by studies along three main lines. These three have been: (I) The quantitative estimation of the blood sugar by methods using small quantities of blood; (2) the measurement of acidosis by the $\mathrm{CO}_{2}$ tension of the expired air and other methods; (3) the experimental and clinical work of Allen on the value of undernutrition. Other investigations of fundamental importance have not yet influenced our practical management of diabetics.

The Blood Sugar.- The most satisfactory method for determination of the blood sugar is that of Lewis and Benedict. ${ }^{1}$

The method of Bang, largely used, is more difficult and, except in skilled hands, less accurate. In our experience, puncture of a vein with a small needle is no more painful than the attempt to obtain sufficient blood from the ear or finger by puncture. The LewisBenedict method gives somewhat higher values for blood sugar than the Bertrand method requiring Io to 20 c.c. of blood, the normal fasting individual showing between 0.07 and 0.10 per cent. The determination should always be made before breakfast to avoid the transient hyperglycemia following meals. The studies of Jacobson ${ }^{2}$ have shown that, following the ingestion of carbohydrate food, the blood sugar of the normal individual rises rapidly, reaching its maximum in half an hour, and returning to normal on the average within two hours. The height of the maximum depends upon the amount of carbohydrate ingested. Following $100 \mathrm{gm}$. of glucose he found as high as 0.17 per cent. without the appearance of sugar in the urine. In other supposedly normal persons values up to 0.227 per cent. were reached, with a trace of sugar in the urine. The hyperglycemia never persisted more than three and a half hours. ${ }^{3}$ In the diabetic, on the other hand, the return of the blood sugar to its previous level is very much retarded. In very mild cases there may be only postprandial hyperglycemia, disappearing before the next meal. In such cases the morning urine is sugar-free, and mistakes in diagnosis will be made by physicians who do not examine the mixed twenty-four-hour urine. With progress to a more severe degree of intolerance, the level of blood sugar never falls to the normal between meals, and eventually

1 Jour. Biol. Chem., 19r5, xx, 61.

2 Bio-chem. Zeitschr., I913, lvi, 47 r.

${ }^{3}$ See also Hopkins, A. H., Am. Jour. Med. Sci., I915, cxlix, 254. 
may show an almost complete fusing of the postprandial rises in a constant marked hyperglycemia. The relation between the degree of glycosuria and of hyperglycemia has not yet been completely elucidated. Normal individuals do not usually excrete sugar in the urine until the percentage in the blood exceeds 0.I7 per cent. Longstanding cases of diabetes may develop a rising kidney threshold for glucose and show a smaller concentration of it in the urine with blood sugars of 0.4 and 0.5 per cent. than other patients with only about 0.2 per cent. They may actually remain aglycosuric with a blood sugar of 0.2 per cent. So far as our observations go, young people with severe glycosuria and only moderate hyperglycemia have a less favorable outlook than the chronic cases with extreme hyperglycemia. Occasionally true diabetics may exhibit glycosuria with blood sugar considerably below 0.17 per cent., thus evidencing a lowered renal threshold.

The ideal result of treatment should be the reduction of the blood sugar to its normal level. In the long-standing cases, however, this cannot be obtained. In my opinion, one should at least endeavor to secure a blood sugar below 0.I5 per cent. during the preliminary period of vigorous treatment.

Blood-sugar studies have proved renal diabetes to be a real, though rare, type of glycosuria. Both for prognosis and for treatment it is highly important to recognize it. It is to be suspected whenever an individual shows a persistent slight glycosuria, not strikingly influenced by food, not disappearing on carbohydratefree diet, and not accompanied by polyuria, thirst or other clinical symptoms. If, in such a case, the fasting blood sugar be not over o.I per cent., with glucose in the urine at the same time, a diagnosis of renal diabetes, that is, of a diminished kidney threshold for sugar, may be made. The prognosis, in such cases, is not yet certain, for none have been observed for a sufficient length of time. It is clearly much better than in true diabetes. The treatment should consist merely in limitation of the quickly absorbable sugars, the prevention of gross overeating and the leading of a hygienic life. For the careful study of a case and literature, the reader is referred to Lewis and Mosenthal's recent article. ${ }^{1}$

A second type of glycosuria, dependent upon lowered carbohydrate tolerance, but probably distinct from true diabetes, is that associated with hyperthyroidism. This can be much more clearly recognized by the blood-sugar curve than by the urine, as in the series reported by Geyelin. ${ }^{2}$

I have seen a very few cases of true exophthalmic goiter develop a severe diabetes, but the transition is rare. The treatment should be directed against the disease of the thyroid, and high protein intakes should be especially avoided.

It is probable that further studies will reveal other types of non-

${ }^{1}$ Bull. Johns Hopkins Hos., I916, xxvii, p. I33.

2 Arch. Int. Med., I915, xvi, 975 . 
diabetic carbohydrate intolerance. Hyperglycemia is known to occur in uremic patients and in disease of the pituitary. Sufficient knowledge is not yet at hand to permit of very definite statements about these.

Acidosis.-Until recently the only available measures of acidosis have been the difficult quantitative determinations of the acetone bodies of the urine and the estimation of the $\mathrm{NH}_{3}$ excretion. These have indicated merely the output of acids, but have given no direct information as to the vital question of the amount of retained acids and the impairment of the alkali reserve of the blood and tissues. The work of Haldane and Priestley ${ }^{1}$ on the respiration opened this field, and Straub ${ }^{2}$ showed the great value of their method in the study of diabetic acidosis. Methods ${ }^{3}$ now available for the measurement of the $\mathrm{CO}_{2}$ tension of the expired air give direct evidence of actual acidosis. As the abnormal acids accumulate in the body, they diminish the number of hydroxyl ions in the blood available for combining with $\mathrm{CO}_{2}$, and the amount of the latter in the expired air falls. In the normal man this is about 40, and should never go below 38 . The first and best indication of true acidosis is a fall in this figure. When it goes below 30 , clinical symptoms, such as slight nausea and a little tendency to air hunger, are likely to be present; below 25 , a dangerous degree of acidosis exists; and below 20 , the occurrence of coma is altogether probable unless prompt treatment is instituted. An excellent discussion of the newer work in this field will be found in Peabody's recent article. ${ }^{4}$ Where the apparatus for this determination cannot be had, the test of Sellards ${ }^{5}$ may be applied with advantage. Sellards has also shown ${ }^{6}$ that with simple litmus paper, valuable information as to the alkali tolerance may be obtained from the urine. Palmer and Henderson ${ }^{7}$ demonstrated that the urine of normal individuals usually becomes alkaline on giving $4 \mathrm{gm}$. of sodium bicarbonate by mouth. More than to gm. is never necessary. The amount in excess of this required by a diabetic constitutes a rough, but valuable, index of the severity of his acidosis. Van Slyke, Stillman and Cullen's recently published method ${ }^{8}$ affords exact information as to the alkali reserve of the body.

The Starvation Treatment.-A notable advance in the successful treatment of diabetes, especially in its more severe forms, has been made very recently by Allen. ${ }^{9} \mathrm{He}$ has based this upon the study of the effect of diet on dogs rendered diabetic by the extirpation of a large part of the pancreas.

1 Jour. Physiol., I905, xxxii, 225.

2 Deutsch. Arch. f. klin. Med., I913, cix, 223.

${ }^{3}$ For description of methods see Boothby and Peabody, Arch. Int. Med., 1914, xiii, 497.

Am. Jour. Med. Sci., 1916, cli, 184.

- Bull. Johns Hopkins Hos., I 914 , xxv, ror.

- Bull. Johns Hopkins Hos., I91 2, xxiii, 289.

${ }^{7}$ Arch. Int. Med., I9r 3 , xii, 153 .

8 Proc. Soc. Exper. Biol. and Med., 19r5, xii, 165.

O Jour. Am. Med. Assn., 1914, lxiii, 939. 
Such dogs, if fed all they will eat, show a severe glycosuria and polyuria, emaciate progressively, and die of cachexia within several months. Allen found that he could prevent the glycosuria entirely by fasting, then give a low diet without glycosuria recurring. The dogs remained thin, but lively, and no sign of progressive cachexia ensued. These observations led him to believe that the treatment of diabetic patients should proceed along the same line, and during two years' study of patients in the hospital of the Rockefeller Institute he has developed in detail the technique of what is practically a new plan of treatment for the disease. His methods have been widely applied during the last year, and seem certain to supplant the older methods of treatment in severe diabetes and in the diabetes of young persons, and to become the method of choice in the practical management of most diabetic individuals. ${ }^{1}$

The fundamental difference between Allen's treatment and plans previously in vogue is the logical result of his demonstration that persistent undernutrition is safer for a diabetic than persistent glycosuria and acidosis. Heretofore short periods of semistarvation, such as the green days advocated on page 28 of my former article, or one or two fast days, as advocated by Naunyn, have been used to combat the acidosis of severe cases; but when patients could not be rendered sugar-free, the danger of inanition has been held to be too great, and feeding resumed in spite of an increase in the glycosuria. Allen has proved that the latter, not the former, is therealdanger. It would seem wise not to push this idea too far in every case until our experience extends over a considerable period of years, but I will discuss the limitations of the treatment subsequently.

The technique of the treatment is simple. The patient is first given an initial fast until the urine has been sugar-free for twentyfour to forty-eight hours, preferably the latter. Water may be drunk freely, and a cup of black coffee and a cup of tea may be taken. When the fast must be prolonged, clear meat broth is given. In emaciated patients this is the means of introducing needed salt. Alcohol was formerly used by Allen in the form of whiskey or brandy, well-diluted. With severe acidosis I believe it valuable, but it is apt to be repugnant when no food is taken. Not over 90 c.c. of whiskey should be given.

When the patient has been sugar-free for forty-eight hours, feeding is begun cautiously, the first addition to the broth being $200 \mathrm{gm}$. of green vegetables from the list given in Table I, page 39, Vol. III. which will be repeated here for convenience:

\footnotetext{
Vegetables and salads: Asparagus, beet greens, Brussels sprouts, cabbage, cauliflower, celery, chicory, cresses, cucumbers, egg-plant, endive, kohlrabi, leeks, lettuce, pumpkins, radishes, rhubarb, salsify, sauerkraut, spinach, string-beans, Swiss chard, tomatoes, vegetable marrow.
}

The average carbohydrate content of these is 3 per cent. On the second day the green vegetables may be increased to $300 \mathrm{gm}$. and one

${ }^{1}$ Allen, F. M., Boston Med. and Surg. Jour., I91 5, cl, 480; clxxii, 241. Joslin, E. P., Am. Jour. Med. Sci., I91 5, cl, 485 . 


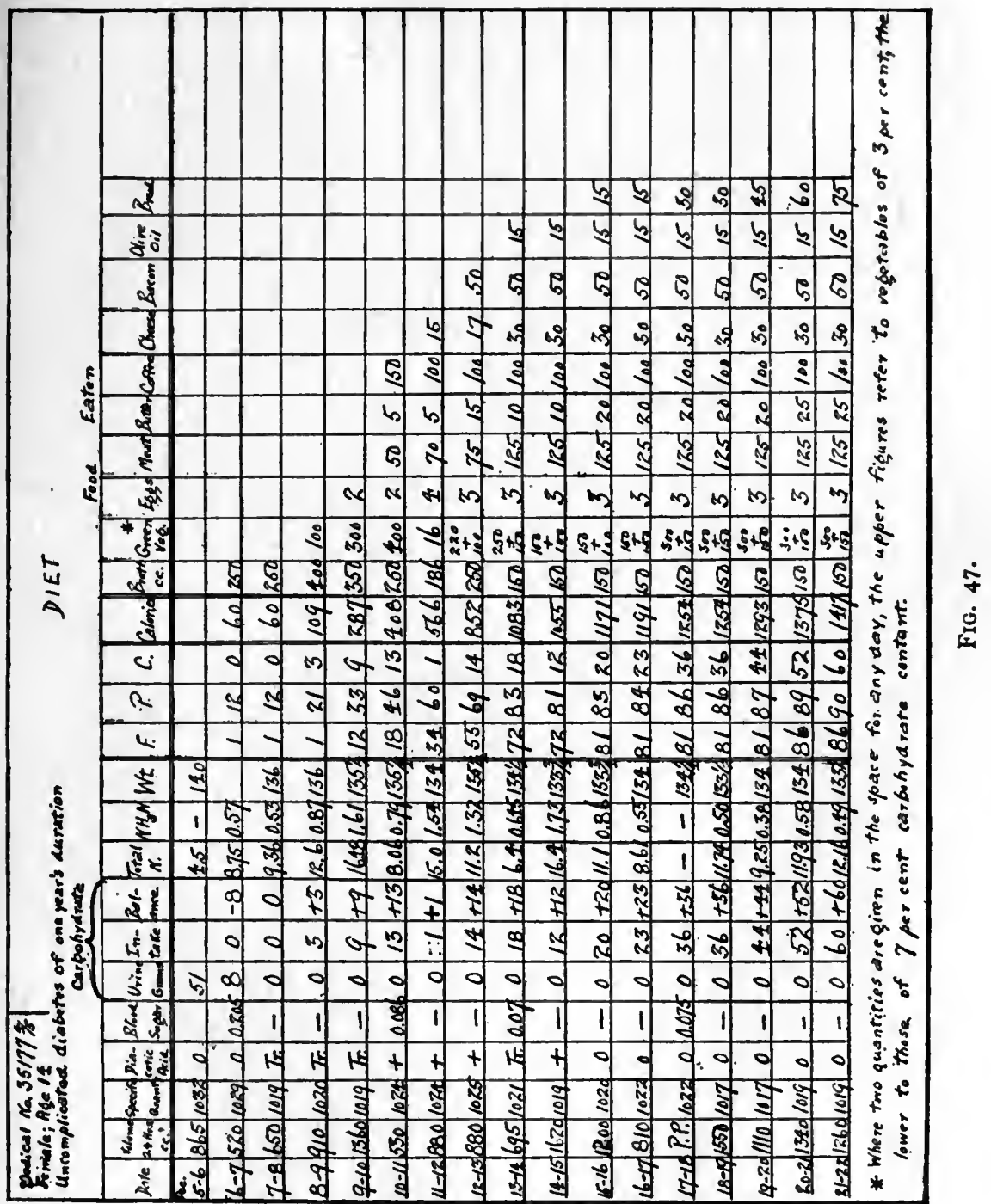




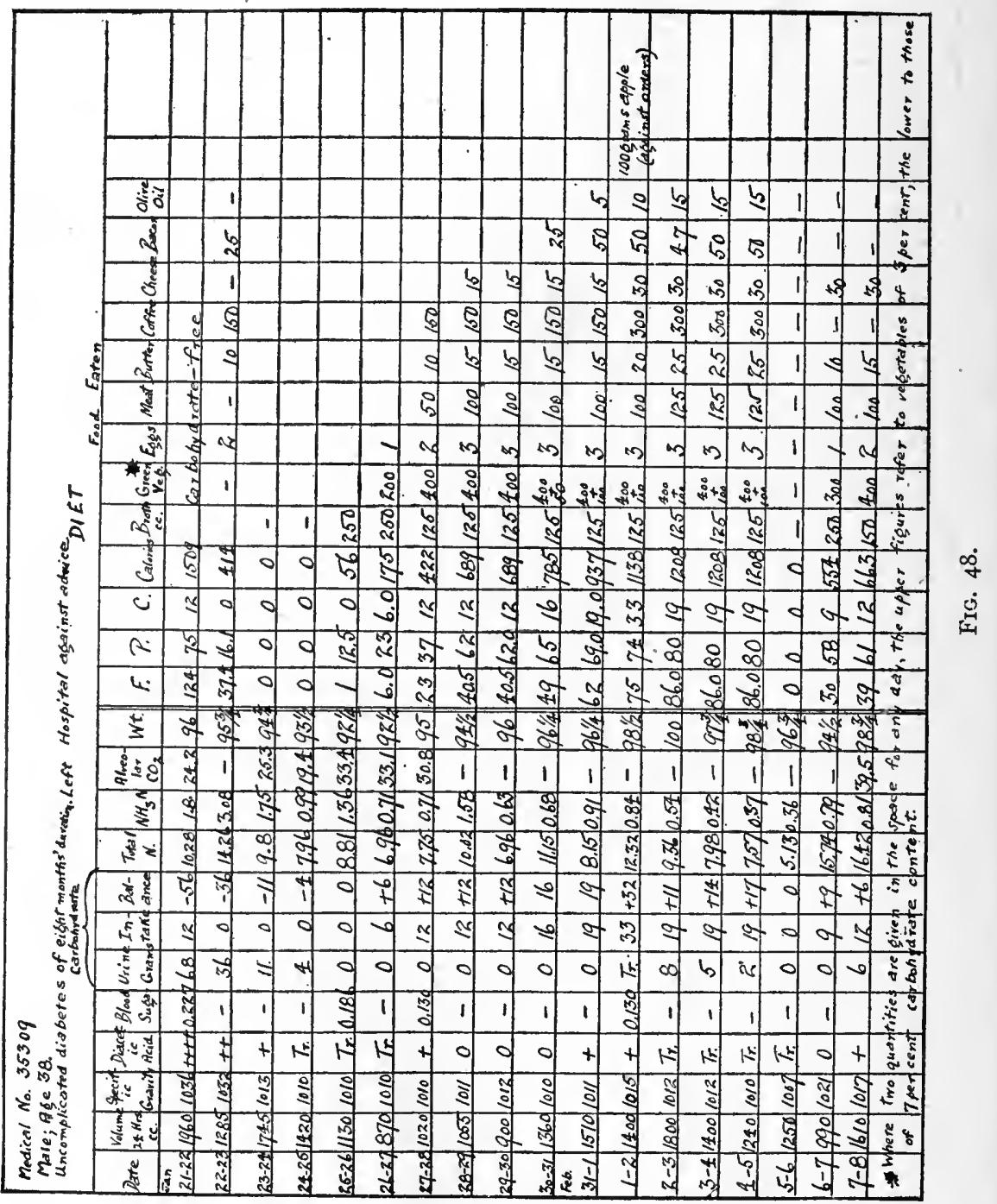


egg given. Gradually more eggs are added, then small amounts of meat, bacon; later, salad oil and butter, when the meat and eggs have been increased to a reasonable amount; and later yet, small weighed amounts of carbohydrate, first as the vegetables of lower carbohydrate content (Table VIII, page 43, Vol. III)-onions, carrots, squash, turnips; and the fruits-grape-fruit, oranges, melons, strawberries and olives. Subsequently, peas and beans, then potatoes and the fruits of higher carbohydrate content, may be added more cautiously, or a weighed amount of bread or biscuit. The carbohydrate intake should not be increased more than $15 \mathrm{gm}$. a day. The urine must be tested daily for sugar, and upon the first reappearance of sugar, a fast day must be given and the diet resumed on a lower level. In the course of weeks, the patient is thus gradually brought up to his maximal tolerance. Allen insists that all patients should be kept below their original weight when well, and that they should never be allowed to return to a full diet, even of carbohydrate-free food. He instructs all his patients to examine their urine daily, and to regulate their own diet by this. In addition, he believes in active exercise, even in severe cases. ${ }^{1}$

The initial fast in all severe cases should be carried out under strict supervision, either in a hospital or with a competent nurse in charge, and the patient kept under observation until his tolerance has been established. For convenience, diets of 500, 1000, 1500, and 2000 calories have been worked out by Dr. Mosentbal and Miss Mann in the Medical Clinic of the Johns Hopkins Hospital, and are appended. The transition between these diets should never be sudden, but should be gradually accomplished by the addition of portions of eggs, meat, carbohydrate-containing food and fats. For convenience in accomplishing this, the tables accompanying the diets, giving I 5 -gm. portions of carbohydrate, and meat portions equivalent to Io $\mathrm{gm}$. of steak, have been prepared. It is clear from the experiences with this plan of treatment that the older diabetic diets contained too much fat, and that in the testing of the tolerance of all but the mildest cases, the total calories of the food in protein and fat, as well as its carbohydrate content, must be considered. The following tables show the method of carrying out the treatment, and its result, in a mild and in a severe case studied in our clinic.

In the majority of cases the initial fast reduces the ketone bodies in the urine as promptly as it does the sugar. In very mild cases, however, ketonuria may appear, just as in carbohydrate starvation in a normal individual. This demands no attention, and will clear up promptly as feeding is resumed. Bicarbonate of soda is no longer given as a routine during starvation. In all severe cases actual acidosis, rather than ketonuria, should be constantly controlled by the observation of the alveolar $\mathrm{CO}_{2}$, or, where this is impossible, by Sellards' test, and the case managed as described below under the treatment of acidosis.

\footnotetext{
${ }^{1}$ Boston Med. and Surg. Jour., I915, clxxiii, 743.
} 
Limitations of the Starvation Treatment.-No method of treatment in any disease can be schematic and applicable to every patient. While our experience with the starvation treatment is as yet incomplete, our observations in the Medical Clinic of the Johns Hopkins Hospital have convinced us of certain limitations. Obese patients during starvation almost always develop ketonuria, which may be difficult to abolish later. I believe that they are more wisely treated by a gradual withdrawal, first of fat, then of carbohydrate, resorting to protracted starvation only if glycosuria and ketonuria persist. Single fast days may be used once or twice a week. Mild diabetics, particularly if elderly, who become sugar-free by the former method of withdrawal of carbohydrate alone, and who have no significant ketonuria, I believe are better treated by the former method described

Medical No. $35257 \frac{1}{2}$. Male, Age 58. Diabetic. One Year. Glucose

\begin{tabular}{|c|c|c|c|c|c|c|c|c|c|}
\hline Date & 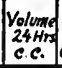 & 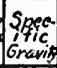 & 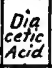 & $\begin{array}{l}\text { Blood } \\
\text { per } \\
\text { cent }\end{array}$ & $\begin{array}{l}\text { Urine } \\
\text { graws }\end{array}$ & & bohylatic & wat. & Diet \\
\hline \begin{tabular}{|l} 
Jan. \\
$8-9$ \\
\end{tabular} & 930 & 1025 & 0 & 0.215 & 11 & 60 & +49 & 1762 & Carbohydrate - free + 90 grams bread \\
\hline $9-10$ & 760 & 1022 & 0 & - & 0 & 10 & +10 & $177 / 2$ & Carbohydrate-free \\
\hline $17-18$ & 1090 & 1026 & 0 & 0.141 & 0 & 10 & +10 & 1754 & \\
\hline $18-19$ & 900 & 027 & 0 & 0.104 & 0 & 0 & 0 & $\mid>44,2$ & Starvation with broth an \\
\hline 19.20 & 1400 & 1028 & $\dot{+}$ & $=$ & 0 & 10 & 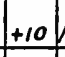 & 1748 & Carbohydrate - free \\
\hline $20-21$ & 1100 & 1028 & + & $=$ & 0 & 25 & +25 & 175 & Carbohydrate-free +30 grams bread \\
\hline $21-22$ & 1060 & 1027 & 0 & 0.117 & 0 & 25 & +25 & 175 & Carbohydrate-free +30 grams bread \\
\hline 25 & 1610 & 1023 & 0 & - & 0 & 40 & +40 & 175 & Carbohydrate-free +60 grams bread \\
\hline $26-27$ & 810 & 1030 & 0 & $=$ & 5 & 48 & +43 & $173 \%$ & Carbohydrate-free +75 grams bread \\
\hline $7-28$ & 1520 & 1020 & 0 & 0.158 & 0 & 35 & +35 & - & Carbohydrate-free +5 \\
\hline
\end{tabular}

FIG. 49.

for mild cases, page 24, Vol. III. A fast day once a week or once in ten days is probably wise for them in addition. I am not convinced that undernutrition is beneficial to them. However, they should clearly avoid overheating. The accompanying chart (Fig. 49) shows this method of management.

The onset of nausea and vomiting during starvation, particularly if the evidence of acidosis persists, calls for the resumption of feeding, using 500 to 1000 calories of a carbohydrate-free diet. Fasting is resumed after the threatening symptoms of acidosis have again subsided. I am also convinced, from personal experience in a very severe case and knowledge of another, that fasting should never be continued more than five days unless complete observations on the urine, the blood sugar, and the alveolar $\mathrm{CO}_{2}$ can be made. If these do not all show steady improvement, and particularly if the $\mathrm{D}$ to 
$\mathrm{N}$ ratio remains 3.65 to $\mathrm{I}$, protein-fat feeding must be resumed, with subsequent short starvation periods. In addition, I believe that the general practitioner outside a hospital should not carry out fasts of over three days' duration. Loss of weight, in itself, does not seem to contraindicate the fasting. Our experience with intercurrent disease is not yet sufficient to warrant conclusions, but certain observations make me believe that great care is necessary in carrying out the starvation treatment in very emaciated patients with tuberculosis. On the other hand, surgical infections call for rigorous starvation treatment except in the severest cases already mentioned.

The Treatment of Acidosis.-The plan of treatment just outlined has proved the most effective means of combating the acidosis of diabetes. Only in severe cases is it now necessary to give alkali. When the ammonia of the urine exceeds $2 \mathrm{gm}$. in the twenty-four hours, or it becomes more than 20 per cent. of the total N, soda should be given. In spite of a growing tendency to discard alkalies altogether, I believe that the indications just mentioned demand the administration of from to to $30 \mathrm{gm}$. of bicarbonate of soda a day. The best measures of the need for alkali are the alveolar $\mathrm{CO}_{2}$ tension or Sellards' test, and it should be given until these tests show a return to normal. In impending coma, with $\mathrm{CO}_{2}$ below 20 , it must be given by intravenous infusion. Magnus-Levy ${ }^{1}$ has shown that sodium bicarbonate solution supplies more sodium ions, with less toxicity, than the sodium carbonate solutions heretofore employed. In addition, sodium bicarbonate is unirritating, and its escape into the tissues will not cause necrosis. It is regularly used in our clinic, and may be very simply prepared from a flask of 2.5 per cent. sodium carbonate solution by passing a stream of $\mathrm{CO}_{2}$ from one of the ordinary carbon dioxid cylinders through the previously sterilized solution. A sterile double perforated rubber stopper, with inlet and outlet tubes, is used, and, if the solution be shaken, all the carbonate is converted into bicarbonate in five minutes. The resulting solution has a concentration of 4 per cent. A drop of phenolphthalein solution is used as an indicator, and shaking should be continued until the solution is completely decolorized.

It seems clear that the administration of large quantities of levulose or other carbohydrate in impending coma should no longer be considered. Instead, fasting is the strict indication, with the administration of as much alkali as may be necessary to overcome the symptoms and neutralize the retained acid. A patient properly treated from the start should never develop these symptoms, though he may have to be kept greatly undernourished to avoid acidosis. It is highly probable, that with the general adoption of Allen's methods, diabetic coma will become a rare event, except in those patients who refuse to persist in the treatment, or who develop grave intercurrent infections. 
Carbohydrate-free Diet, 500 Calories

\begin{tabular}{|c|c|c|c|c|c|c|}
\hline Food & $\begin{array}{l}\mathrm{Gm} \text {. or } \\
\text { c.c. }\end{array}$ & $\begin{array}{c}\text { Protein, } \\
\text { gm. }\end{array}$ & $\begin{array}{l}\text { Fat, } \\
\text { gm. }\end{array}$ & $\begin{array}{l}\text { C-H, } \\
\text { gm. }\end{array}$ & Calories & $\begin{array}{l}\text { Calories } \\
\text { per meal }\end{array}$ \\
\hline \multicolumn{7}{|l|}{ Breakfast: } \\
\hline One egg. & 50 & 6.6 & 6.0 & 0.0 & 83 & \\
\hline Bacon $^{1}$ & 40 & 4.2 & 7.6 & 0.0 & 88 & \\
\hline Black coffee. . . . . . . & $\ldots$ & $\ldots$ & $\ldots$ & $\ldots$ & $\ldots$ & 171 \\
\hline \multicolumn{7}{|l|}{ Dinner: } \\
\hline$\ldots \ldots \ldots$ & I 50 & $3 \cdot 3$ & 0.3 & 0.0 & r6 & \\
\hline Steak $^{2}$. & 40 & 9.4 & $4 . I$ & 0.0 & 77 & \\
\hline Vegetables ${ }^{3} . . . \ldots \ldots$ & 200 & 2.0 & 0.0 & 6.0 & 33 & \\
\hline Butter............ & 5 & 0.1 & $4 \cdot 3$ & 0.0 & 40 & \\
\hline Black coffee. . . . . . & $\ldots$ & $\ldots$ & $\ldots$ & $\ldots$ & $\ldots$ & r66 \\
\hline \multicolumn{7}{|l|}{ Supper: } \\
\hline Broth. & I 50 & $3 \cdot 3$ & 0.3 & 0.0 & r6 & \\
\hline Steak $^{2} \ldots \ldots$ & 40 & $9 \cdot 4$ & $4 \cdot I$ & 0.0 & 77 & \\
\hline Vegetables $^{3}$. & 200 & 2.0 & 0.0 & 6.0 & 33 & \\
\hline Butter........... & 5 & 0.1 & $4 \cdot 3$ & 0.0 & 40 & \\
\hline \multirow[t]{2}{*}{ Plain tea.......... } & $\ldots$ & $\ldots$ & $\ldots$ & $\ldots$ & $\ldots$ & 166 \\
\hline & & 40.4 & 31.0 & 12.0 & & $5 \circ 3$ \\
\hline
\end{tabular}

1 The bacon is weighed uncooked. The fat and protein content is calculated for the cooked product.

2 The caloric equivalent of other carbohydrate-free meat or fish should be frequently substituted from the accompanying list, to furnish variety in the diet.

${ }^{3}$ Two or three different vegetables should be chosen from the accompanying list, which tabulates the vegetables containing 5 per cent. or less of carbohydrates.

Carbohydrate-free Diet, rooo Calories

\begin{tabular}{|c|c|c|c|c|c|c|}
\hline Food & $\begin{array}{l}\mathrm{Gm} \text {. or } \\
\text { c.c. }\end{array}$ & $\begin{array}{l}\text { Protein, } \\
\text { gm. }\end{array}$ & $\begin{array}{l}\text { Fat, } \\
\text { gm. }\end{array}$ & $\begin{array}{l}\text { C-H, } \\
\text { gm. }\end{array}$ & Calories & $\begin{array}{l}\text { Calories } \\
\text { per meal }\end{array}$ \\
\hline \multicolumn{7}{|l|}{ Breakfast: } \\
\hline Eggs (2). & 100 & 13.2 & I 2.0 & 0.0 & I66 & \\
\hline Bacon ${ }^{1} . . . \ldots \ldots$ & 50 & $5 \cdot 3$ & 9.6 & 0.0 & I I I & \\
\hline Butter.......... & 5 & 0.1 & $4 \cdot 3$ & 0.0 & 40 & \\
\hline Black coffee. . . . . . . & $\cdots$ & $\cdots$ & $\ldots$ & $\ldots$ & $\ldots$ & 317 \\
\hline \multicolumn{7}{|l|}{ Dinner: } \\
\hline $\begin{array}{l}\text { Broth.. } \\
\text { Steak }\end{array}$ & 150 & $3 \cdot 3$ & 0.3 & 0.0 & 16 & \\
\hline $\begin{array}{l}\text { Steak } \\
\text { Vegetables } \\
\end{array}$ & 100 & 23.9 & 10.2 & 0.0 & I93 & \\
\hline Vegetables ${ }^{3} \ldots \ldots \ldots$ & 200 & 2.0 & 0.0 & 6.0 & 33 & \\
\hline Olive oil......... & ro & 0.0 & 10.0 & 0.0 & 93 & \\
\hline Butter............ & ro & 0.1 & 8.6 & 0.0 & 80 & \\
\hline Black coffee........ & $\cdots$ & $\cdots$ & $\cdots$ & $\cdots$ & $\cdots$ & $4 I 5$ \\
\hline \multicolumn{7}{|l|}{ Supper: } \\
\hline Steak ${ }^{2}$. & $\begin{array}{r}150 \\
75\end{array}$ & $\begin{array}{r}3.3 \\
17.0\end{array}$ & 0.3 & $\begin{array}{l}0.0 \\
0.0\end{array}$ & $\begin{array}{r}10 \\
145\end{array}$ & \\
\hline Vegetables $^{3} . . . \ldots \ldots$ & 200 & $\begin{array}{r}1.9 \\
2.0\end{array}$ & $\begin{array}{l}7.7 \\
0.0\end{array}$ & $\begin{array}{l}0.0 \\
6.0\end{array}$ & $\begin{array}{r}145 \\
33\end{array}$ & \\
\hline Butter............ & 10 & $0 . \mathbf{I}$ & 8.6 & 0.0 & 80 & \\
\hline \multirow{2}{*}{ Tea (plain)......... } & $\ldots$ & $\ldots$ & $\ldots$ & $\cdots$ & $\ldots$ & 274 \\
\hline & & 71.2 & 71.6 & I 2.0 & $\cdots$ & 1006 \\
\hline
\end{tabular}

${ }^{1}$ The bacon is weighed uncooked. The fat and protein content is calculated for the cooked product.

2 The caloric equivalent of other carbohydrate-free meat or fish should be frequently substituted from the accompanying list, to furnish variety in the diet.

3 Two or three different vegetables should be chosen from the accompanying list, which tabulates the vegetables containing 5 per cent. or less of carbohydrates. 
Carbohydrate-fref. Diet, i 500 Calorifs

\begin{tabular}{|c|c|c|c|c|c|c|}
\hline Food & $\begin{array}{c}\mathrm{Gm} \text {. or } \\
\text { c.c. }\end{array}$ & $\begin{array}{c}\text { Protein, } \\
\text { gm. }\end{array}$ & $\begin{array}{l}\text { Fat, } \\
\mathrm{gm} \text {. }\end{array}$ & $\begin{array}{l}\text { C-H, } \\
\text { gm. }\end{array}$ & Calories & $\begin{array}{l}\text { Calories } \\
\text { per meal }\end{array}$ \\
\hline \multicolumn{7}{|l|}{ Breakfast: } \\
\hline Eggs (2). . & 100 & 13.2 & 12.0 & 0.0 & 166 & \\
\hline Bacon $1 . .$. & 60 & 6.4 & II. 5 & 0.0 & 133 & \\
\hline 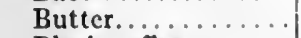 & 10 & $0 . \mathbf{I}$ & 8.6 & 0.0 & 80 & \\
\hline Black coffee........ & $\ldots$ & $\ldots$ & $\ldots$ & $\ldots$ & $\ldots$ & 379 \\
\hline \multicolumn{7}{|l|}{ Dinner: } \\
\hline 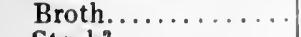 & 150 & $3 \cdot 3$ & 0.3 & 0.0 & 16 & - \\
\hline Stcak $^{2} \ldots \ldots \ldots \ldots$ & 140 & $33 \cdot 5$ & $14 \cdot 3$ & 0.0 & 270 & \\
\hline Vegetables $^{3} \ldots . . .$. & 200 & 2.0 & 0.0 & 6.0 & 33 & \\
\hline Cream cheese...... & 20 & $5 \cdot 2$ & 6.7 & 0.5 & 86 & \\
\hline Olive oil.......... & I 5 & 0.0 & 15.0 & 0.0 & 140 & \\
\hline Butter............ & 15 & 0.2 & 12.9 & 0.0 & I 20 & \\
\hline Black coffce........ & $\ldots$ & $\ldots$ & $\ldots$ & $\ldots$ & $\ldots$ & 665 \\
\hline \multicolumn{7}{|l|}{ Supper: } \\
\hline 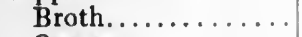 & 150 & $3 \cdot 3$ & 0.3 & 0.0 & 16 & \\
\hline One egg............ & 50 & 6.6 & 6.0 & 0.0 & 83 & \\
\hline Steak $^{2} \ldots \ldots \ldots \ldots$ & 100 & $23 \cdot 9$ & IO. 2 & 0.0 & 193 & \\
\hline Vegetables $^{3} . . . \ldots \ldots$ & 200 & 2.0 & 0.0 & 6.0 & 33 & \\
\hline 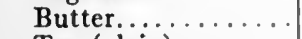 & 15 & 0.2 & 12.9 & 0.0 & 120 & \\
\hline \multirow[t]{2}{*}{ Tea (plain)......... } & $\ldots$ & $\cdots$ & $\cdots$ & $\cdots$ & $\cdots$ & 445 \\
\hline & & $99 \cdot 9$ & 110.7 & 12.5 & $\cdots$ & 1489 \\
\hline
\end{tabular}

1 The bacon is weighed uncooked. The fat and protein content is calculated for the cooked product.

2 The caloric equivalent of other carbohydrate-free meat or fish should be frequently substituted from the accompanying list, to furnish variety in the diet.

${ }^{3}$ Two or three different vegetables should be chosen from the accompanying list, which tabulates the vegetables containing 5 per cent. or less of carbohydrates.

Carbohydrate-free Diet, 2000 Calories

\begin{tabular}{|c|c|c|c|c|c|c|}
\hline Food & $\begin{array}{l}\text { Gm. or } \\
\text { c.c. }\end{array}$ & $\begin{array}{l}\text { Protein, } \\
\text { gm. }\end{array}$ & $\begin{array}{l}\text { Fat, } \\
\text { gm. }\end{array}$ & $\begin{array}{l}\text { C-H, } \\
\text { gm. }\end{array}$ & Calories & $\begin{array}{l}\text { Calories } \\
\text { per meal }\end{array}$ \\
\hline \multicolumn{7}{|l|}{ Breakfast: } \\
\hline Eggs (2).. & 100 & $13.2^{\circ}$ & 12.0 & 0.0 & I 66 & \\
\hline 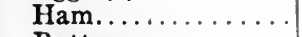 & 75 & 15.2 & I6.8 & 0.0 & 219 & \\
\hline Butter..... & I 5 & 0.2 & 12.9 & 0.0 & 120 & \\
\hline Vegetables ${ }^{2} .$. & 100 & 1.0 & 0.0 & 3.0 & I6 & \\
\hline Black coffee....... & $\ldots$ & $\ldots$ & $\ldots$ & $\ldots$ & $\ldots$ & 521 \\
\hline \multicolumn{7}{|l|}{ Dinner: } \\
\hline Broth. . & 160 & $3 \cdot 5$ & 0.3 & 0.0 & 17 & \\
\hline Steak $^{1} \ldots \ldots \ldots \ldots$ & 160 & 38.2 & 16.3 & 0.0 & 308 & \\
\hline Vegetables $^{2} \ldots \ldots \ldots$ & 300 & 3.0 & 0.0 & 9.0 & 49 & \\
\hline Cream cheese...... & 30 & 7.8 & IO. I & 0.7 & I 29 & \\
\hline Butter............. & 20 & 0.2 & 17.2 & 0.0 & 160 & \\
\hline Olive oil .......... & 15 & 0.0 & 15.0 & 0.0 & 140 & \\
\hline Black coffec........ & $\ldots$ & $\ldots$ & $\cdots$ & $\ldots$ & $\ldots$ & 803 \\
\hline \multicolumn{7}{|l|}{ Supper: } \\
\hline Broth... & 160 & $3 \cdot 5$ & 0.3 & 0.0 & 17 & \\
\hline Eggs (2). & 100 & 13.2 & 12.0 & 0.0 & I 66 & \\
\hline Steak ${ }^{i} \ldots$ & 140 & $33 \cdot 5$ & $14 \cdot 3$ & 0.0 & 270 & \\
\hline Vegetables ${ }^{2} . \ldots \ldots \ldots$ & 300 & 3.0 & 0.0 & 9.0 & 49 & \\
\hline 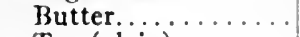 & 20 & 0.2 & 17.2 & 0.0 & 160 & \\
\hline \multirow[t]{2}{*}{ Tea (plain) ........... } & $\therefore$ & $\ldots$ & $\ldots$ & $\ldots$ & $\ldots$ & 662 \\
\hline & & $135 \cdot 7$ & $144 \cdot 4$ & $21 \cdot 7$ & & I9S6 \\
\hline
\end{tabular}

1 The caloric equivalent of other carbohydrate-free meat or fish should be frequently substituted from the accompanying list, to furnish variety in the diet.

${ }^{2} T$ wo or three different vegetables should be chosen from the accompanying list, which tabulates the vegetables containing 5 per cent. or less of carbohydrates. 
Caloric Equivalent of io Gm. of Steak in Carbohydrate-free MEAT OR FISH

\begin{tabular}{|c|c|c|c|c|}
\hline Food & $\mathrm{Gm}$ & Fat, gm. & Protein, gm. & Calories \\
\hline 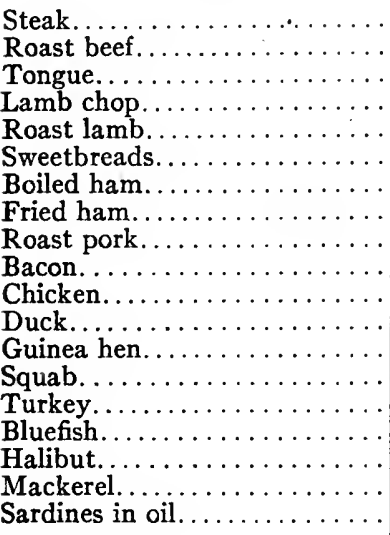 & $\begin{array}{r}\text { IO } \\
5 \\
7 \\
5 \\
8 \\
\text { I I } \\
7 \\
5 \\
9 \\
9 \\
\text { I } \\
9 \\
12 \\
9 \\
7 \\
\text { I3 } \\
\text { I6 } \\
\text { I5 } \\
7\end{array}$ & $\begin{array}{l}\text { I.O } \\
\text { I. } \\
\text { I. } \\
\text { I. } \\
\text { I. } \\
\text { O.I } \\
\text { I. } \\
\text { I. } \\
0.9 \\
\text { I. } \\
\text { I.O } \\
\text { I.3 } \\
0.8 \\
\text { I. I } \\
\text { I. } \\
0.6 \\
0.7 \\
\text { I. } \\
\text { I. } \\
\text {. }\end{array}$ & $\begin{array}{l}2.4 \\
\text { I.I } \\
\text { I. } \\
\text { I.I } \\
\text { I. } \\
4.4 \\
\text { I. } \\
\text { I.I } \\
2.6 \\
0.9 \\
2.4 \\
1.8 \\
2.8 \\
2.1 \\
2.0 \\
3.5 \\
3.3 \\
2.5 \\
\text { I.6 }\end{array}$ & $\begin{array}{l}19 \\
18 \\
20 \\
18 \\
20 \\
19 \\
19 \\
20 \\
19 \\
20 \\
19 \\
19 \\
19 \\
19 \\
20 \\
20 \\
20 \\
20 \\
20\end{array}$ \\
\hline
\end{tabular}




\section{TREATMENT OF ACIDOSIS IN CHIILDREN}

By John Howland, M. D. ANd W. McKim Marriott, M. D.

Definition.-The treatment of acidosis in children requires a clear conception of what acidosis is, an appreciation of the fact that acidosis is present or imminent and a recognition of the type and severity of the acidosis. The term acidosis has been very loosely applied to a variety of conditions. It was originally used by Naunyn to designate the excretion of acetone, aceto-acetic acid and $\beta$-oxybutyric acid in the urine. When these substances are present in large amounts, in diabetes for instance, certain symptoms may arise, the most characteristic of which is hyperpnea. These same symptoms may arise, however, when there is no increased excretion of acetone bodies in the urine and no accumulation of them in the blood. Acidosis indicates a disturbance of the acid-base equilibrium. It is better, therefore, to restrict the term acidosis to those conditions in which a disturbance of this equilibrium arises from any cause and to designate the mere occurrence of acetone bodies in the urine as acetonuria.

Acidosis thus is to be looked upon as a condition in which acid ions are present in the body in relative excess. There is, of course, never any free acid present. But the relative excess of acid ions brings about an actual depletion of the alkali reserve of the body. We may thus define acidosis as a condition characterized by a depletion of the alkali reserve of the body. Acidosis is not present unless there is this depletion. There may be an excess, for instance, of the acetone bodies in the blood, but if they have been completely neutralized without affecting the alkali reserve, acidosis is not present. The acetone bodies are neutralized and eliminated in the urine; acetonuria results but not an acidosis. On the other hand, acidosis may occur of a severity sufficient to cause a fatal termination with no increase of acetone bodies either in the blood or in the urine.

Detection of Acidosis.-Clinical.-The clinical evidence of acidosis is deep breathing, hyperpnea (I). The respirations are usually moderately increased in rapidity, but it is the depth of the individual respirations that is most striking. The ventilation of the lungs is increased. At the beginning, it may be difficult to appreciate that the excursions of the thorax are excessive. Later it becomes unmistakable. The chest heaves, the accessory muscles of respiration are brought into play but there is no evidence of obstruction to the entrance or exit of air: With all the evidences of respiratory difficulty, cyanosis is absent. This hyperpnea is the direct result of stimulation of the respiratory center by blood in which the acid ions 
are in relative excess. In cases in which the acidosis is due to an excess of acetone bodies in the blood, the odor of acetone may be detected in the breath. Care should be taken not to confuse many other odors with the odor of acetone. In our experience it is uncommon to detect the acetone odor.

Laboratory Methods.-Those chiefly used are: I. The determination of the carbon dioxid tension in the alveolar air.

2. The determination of the alkali reserve of the blood.

3. The determination of the oxygen-combining power of the hemoglobin.

4. The determination of the ammonia coefficient in the urine.

The Determination of the Carbon Dioxid Tension in the Alveolar Air (2).-Only with older children who are capable of intelligent coöperation can the sample for analysis be collected according to the method of Haldane or Fredericia. With younger children and with infants, it is necessary to use a modification of the Plesch-Higgins method (3). Analysis of the air thus collected may be made with the Haldane apparatus or more readily by the colorimetric method (4).

If alveolar air has been properly collected, an analysis of it serves to indicate with accuracy the presence or absence of acidosis. Two factors influence the reliability of the results. The determinations are unreliable if there is an hyperirritability of the respiratory center such as occurs with various cerebral lesions and after certain drugs. They are also unreliable if there is a pulmonary lesion interfering with the free exchange of gases between the blood in the capillaries of the lung and the air in the pulmonary alveoli.

The Determination of the Alkali Reserve of the Blood.-Several methods are available. The first one devised for clinical use was that of Sellards (5). The proteins of the blood are precipitated by absolute alcohol. The alcoholic filtrate is evaporated to dryness on a water bath with a few drops of phenolphthalein. A deep purple remains under normal conditions. When acidosis is present the residue is light pink or even colorless. The method is a valuable one. It is very readily carried out and may be used with a few drops of serum. It distinguishes clearly severe grades of acidosis but will not detect the milder grades.

Van Slyke(6) has devised a method for determining the combining power of the plasma for carbon dioxid. In an apparatus especially constructed for the purpose, acid is added to the blood plasma, the carbon dioxid removed by means of a vacuum and measured. By this method not only can the presence of acidosis be appreciated but its severity can be accurately determined. Marriott(7) has made the dialysis indicator method(8) for determining alterations in the hydrogen ion concentration of the blood serum available for the determination of the alkali reserve. According to this method 0.5 c.c. of serum is dialyzed in a collodion capsule against a normal saline solution containing the indicator, phenolsulphonephthalein. Carbon dioxid is blown out of the dialysate and the color of the solution 
compared with solutions of known hydrogen ion concentration. The method is a delicate one for the detection and quantitative estimation of the degree of acidosis.

An indirect measure of the alkali reserve can be obtained by another procedure devised by Sellards (9). Later, Henderson and Palmer(ro) used an almost similar but more accurate method. Samples of urine are collected before and after the adminsitration of bicarbonate of soda by mouth and the reaction of the urine determined by litmus paper or indicator solutions. When the alkali reserve is diminished, excessive quantities of the bicarbonate are required to render the urine alkaline. This method has the advantage that it requires little familiarity with laboratory technique and no apparatus. The method is not an exact one; it frequently cannot be used with infants on account of vomiting.

The Determination of Oxygen-combining Power of the Hemoglobin. - The capacity of the hemoglobin to combine with oxygen is greatly diminished in the blood of patients suffering from acidosis. The method for determining this as described by Barcroft(II) is a difficult one. It gives very valuable information but is unfortunately available for use only in hospitals with well-equipped laboratories.

The Determination of the Ammonia Coefficient in the Urine.-This is one of the oldest methods. A high ammonia coefficient is presumptive evidence of acidosis. The results are more difficult of interpretation in children than in adults for the reason that the coefficient is very dependent upon the character of the diet. It is also a fact that high ammonia coefficients are obtained when there are no other evidences of acidosis and furthermore the ammonia coefficient may not be high in the acidosis of nephritis.

Varieties of Acidosis.-For the diagnosis and intelligent treatment of acidosis it is imperative that we should determine the cause upon which the acidosis depends.

So far as our present knowledge goes, it may depend upon the formation of acetone bodies in excessive amount, or it may result from the failure of the kidney to excrete acid phosphates. It has been suggested that acidosis may result from excessive loss of bases by the bowel (cholera, infantile diarrhea, etc.) or from the formation in excess of organic acids such as lactic acid. At the present time, convincing proof of the truth of these theories is lacking. Evidence of the excessive formation of the acetone bodies may be derived from determinations of these substances in the blood(r 2 ), from determinations of their excretion by the kidney or from determinations of acetone in the breath. The determination of the acetone bodies in the blood is of much more value with infants than their determination in the urine. Less time is required and the difficulty of collecting urine for twenty-four hours is obviated.

A moderate increase ( 15 to $35 \mathrm{mg}$. in $100 \mathrm{gm}$. of blood) of the acetone bodies is found in nearly all febrile conditions and as the result of the ingestion of an insufficient quantity of food. Such amounts do not 
indicate an interference in the acid-base equilibrium. In acidosis clearly dependent upon the formation of acetone bodies much larger amounts (up to $200 \mathrm{mg}$.) are found. The results of qualitative tests for acetone or diacetic acid in the urine have no importance for the diagnosis of acidosis. It is only when many grams of the substances can be determined quantitatively in the twenty-four-hour urine that one is justified in considering the acetone bodies responsible for the disturbance.

Acidosis of a severe grade may be present when there is no increase in the production of the acetone bodies. Such is the acidosis that occurs in nephritis and probably in a number of other conditions. In the acidosis of nephritis it has been proven that the inorganic phosphates of the serum are increased from five to twenty times the normal. There is every reason to believe that this is the result of the selective retention of acid phosphates by a diseased kidney.

Clinical Types of Acidosis. - We may roughly divide acidosis in children into $(a)$ acetone body acidosis and $(b)$ non-acetone body acidosis. Acetone body acidosis almost invariably occurs in cases of diabetes in children unless treatment is carefully and conscientiously directed against its occurrence. The discussion of this form of acidosis will be found under diabetes.

The spontaneous production of acetone bodies in excess is not a very rare occurrence in late infancy and early childhood. The amount may be sufficient to cause a reduction of the alkali reserve with the clinical and laboratory evidences of acidosis. Mild but repeated attacks of this with vomiting pass under the name of "recurrent vomiting." With these repeated attacks the amount of acetone bodies is very rarely sufficient to cause acidosis. It should not fail to be emphasized, however, that with recurrent vomiting the appearance of the acetone bodies is sometimes late and apparently due to starvation.

With ileocolitis, the acetone bodies may be found in amounts sufficient to determine a mild degree of acidosis. Such is not usually the case.

The most frequent cause of acidosis in infancy is severe diarrhea(I3). It is a very fatal condition and marks the terminal stage of many but by no means of all cases. The cause of the acidosis is not yet clear.

In the course of nephritis the evidences of acidosis may develop. It may appear with acute nephritis with marked suppression or near the end of a severe chronic nephritis. We have much reason for believing that it is due to a failure of the diseased kidney to excrete acid phosphate.

Blackfan has demonstrated, in some unpublished observations, the presence of acidosis with severe burns. It has not yet been determined how this arises.

Treatment.-No attempt will be made to discuss the acidosis of diabetes; that more properly belongs in another chapter. 
Something can be done in the way of prophylaxis. With recurrent acetone body acidosis, children should lead a quiet, preferably out-of-door life with no nervous excitement. There should be plenty of opportunity for rest, especially at night and overfatigue should particularly be avoided. The diet should be carefully regulated. There is no good reason for believing that any particular ingredient of the food should be removed entirely from the diet but there should not be a preponderance of any one ingredient. Fats in excess are not well borne, nor is a diet largely of carbohydrates. By observing these rules, especially if the previous régime has been a bad one, some reduction in the number and severity of attacks may be looked for, but an entire prevention of attacks should not be expected.

In the course of severe diarrhea, especially when the stools are large and loose, acidosis may arise. It usually does this when the loss of fluids from the tissues has been great and particularly when there is almost complete anuria. Every effort should be made to prevent the excessive loss by the bowels, the desiccation and the suppression of kidney activity. In addition to the proper dietetic management of the condition, water should be freely given, by mouth if possible. If vomiting renders this out of the question, it may be given in the form of salt solution ( 0.8 per cent. strength) either subcutaneously or intravenously; but however given it must be frequently repeated. One may give from 75 to 200 c.c. of salt solution subcutaneously once or twice a day. When the intravenous route is employed not more than 150 c.c. should be given at one time except to large infants, otherwise there is danger of throwing too great a strain upon the circulatory apparatus. Another method of introducing saline solution is by intraperitoneal injection. The danger of infection is always great and the method is not to be used except when there are all facilities for the employment of aseptic technique. The marked advantage that this method has over the others is that a large quantity may be introduced at one time. We have employed an amount sufficient to distend moderately the whole abdomen. Absorption has been satisfactory in all of the patients with whom we have used this method.

When acidosis is present, treatment should be prompt and energetic. This disturbed acid-base equilibrium should not be allowed to continue. In order to bring about a restoration of normal conditions alkali is necessary. This is best administered in the form of sodium bicarbonate. When the acidosis is of a mild degree (the hyperpnea not excessive, the carbon dioxid tension $30 \mathrm{~mm}$. or more and the alkali reserve not greatly depieted) the bicarbonate may, in the absence of vomiting, be given by mouth. This is especially the case with older children. When the acidosis is severe (the hyperpnea marked, the carbon dioxid tension of the alveolar air 15 to $30 \mathrm{~mm}$. and the alkali reserve greatly depleted) or in any event if there is vomiting, it is far preferable to give the alkali intravenously.

Depending upon the size of the child from I to $5 \mathrm{gm}$. ( 15 to 75 
gr.) may be given by mouth at a dose. It should be repeated every two to four hours. In any event the amount required is large. If to a normal infant of $\mathrm{I} 2$ pounds $3 \mathrm{gm}$. (45 gr.) of bicarbonate is given by mouth, this is usually sufficient to render the urine alkaline and to keep it alkaline for eighteen to twenty-four hours. With infants the subject of acidosis, many times this amount must be given, perhaps 20 to $30 \mathrm{gm}$. ( 300 to $450 \mathrm{gr}$.) will be required. It is, therefore, impossible to lay down precise rules as to the quantity that should be given. It is advisable to continue the medication until the urine is alkaline and then to give only a sufficient quantity to keep it so.

For intravenous use a 2 to 3 per cent. solution of bicarbonate is employed. It should have been thoroughly sterilized. The injection may be made into a superficial vein of the arm or into any vein that may be available. With some infants the external jugular vein may be employed. It is at times impossible, with small infants, to find a vein that can be entered with the needle. The choice lies then between the dissection of a vein or the use of the superior longitudinal sinus. The sinus puncture(I4) requires excellent technique and should be used only by those with skill and experience. The quantity of soda solution should be the same as that of salt solution advised above. More than one injection is usually required. The injections should be repeated with the use of soda by mouth, if this is possible, until the urine is alkaline.

It sometimes happens that vomiting renders medication by mouth impossible and intravenous injection for some reason or other is out of the question. The soda solution may then be injected subcutaneously. The great objection to this is that solutions of soda have a tendency to produce necrosis of the tissues; especially is this true of solutions of the carbonate. Their use is out of the question. When solutions of the bicarbonate of soda are sterilized, the bicarbonate is transformed into the carbonate. It is then necessary to transform the carbonate back into the bicarbonate. This may be done by passing, with all aseptic precautions, a current of carbon dioxid through the cold, sterilized solution to which a few drops of phenolphthalein have been added, until the pink color entirely disappears. The carbon dioxid may be obtained from a cylinder or from a Kipp's generator. A 2 or 3 per cent. solution may be employed and from 50 to 200 c.c. injected at one time. It is best to do it very slowly and to avoid any great overdistention of the tissues. Repetition of the injection will be necessary unless the cessation of vomiting allows the soda to be taken by mouth.

Water is indispensable. The neutralized acids must be removed by the kidneys and their excretion is greatly facilitated if there is a free secretion of urine. Water may be supplied according to the methods described under prophylactic treatment.

With acetone body acidosis, not diabetic in origin, there is some evidence to prove that the sugar content of the blood is low and that 
the excessive formation of the acetone bodies may be in part due to this. Under such circumstances, especially if it is possible to prove by analysis of the blood that the glucose is deficient, the administration of glucose is indicated. Glucose may be given by mouth, by rectum, subcutaneously or intravenously. It is usually impossible to give it by mouth. Occasionally, its administration by the rectum is very successful. It should be administered slowly by the drop method in a solution of 5 to Io per cent. strength. With children beyond the age of infancy, as much as a liter may, at times, be given in the course of six hours. For subcutaneous or intravenous use, a 5 per cent. solution is best.

When food can be taken, cereals and sugars are indicated unless the condition of the digestive tract is such as to contraindicate their use. Fats should be avoided until recovery is complete and they should then be added to the diet with caution.

The methods of treatment of the acidosis occurring in nephritis and following burns are those that have been outlined for the treatment of the acidosis occurring in the course of diarrhea.

The acidosis is in many instances but an incident, though a very important one, in the course of several different diseases. It introduces the necessity for rapid and energetic specialized treatment but when the acidosis is overcome, the underlying condition is still to be recognized and combated.

\section{BIBLIOGRAPHY}

I. The term hyperpnea has been used to designate either deep or rapid breathing. We understand by it, breathing that is essentially deep and not necessarily rapid.

2. For a discussion of the significance of a lowered carbon dioxid tension in the alveolar air see:

Porges, Leimdorfer and Markovici, Zeitschr. f. klin. Med., ig i r,lxxiii, 389.

Higgins and Means, Jour. Pharm. and Exp. Therap., I9I5, vii, I.

Peabody, Am. Jour. Med. Sci., 1916, cli, 184.

Howland and MARriotr, Johns Hopkins Hos. Bull., r9r6, xxvii, 63.

3. Plesce, Zeitschr. f. Exp. Path. u. Ther., 1909, vi, 380.

Higgrns, Carnegie Institute of Washington Pub., 203, I9 15, p. 168.

Boothry and PEABODy, Arch. Int. Med., 1914, xiii, 497

4. Marriott, Jour. Am. Med. Assn., igr6.

5. SellardS, Johns Hopkins Hos. Bull., 1914, $\mathrm{xxv}, \mathrm{I} 47$.

6. VAN Slyke, Proc. Soc. Exp. Biol. and Med., I915, xii, 165.

7. MARRIotT, Arch. Int. Med., igi6.

8. Levy, Rowntree and Marriott, Arch. Int. Med., I915, xvi, 389.

9. Sellards, Loc. cit.

io. Henderson and Palmer, Arch. Int. Med., I913, xil, 153.

Palmer and Henderson, Journ. Biol. Chem., I9I 5 , xxi, 57.

II. BARCroft, Respiratory Function of the Blood, Cambridge, Igr4.

I 2. MARRiotr, Jour. Biol. Chem., I9I4, xviii, 507.

13. Howland and Marriotr, Am. Jour. Dis. Child., I916, xi.

14. TOBLER, Monatsh. f. Kindesh, 1915, xiii, 384 .

Helmholz, Am. Jour. Dis. Child., 1915, x, 194. 


\section{DIABETES INSIPIDUS}

\section{By Thomas B. Futcher, M. B.}

The chief contribution to our knowledge of the etiology of diabetes insipidus since Eric Meyer, in I905, advanced the view that it was due to the inability of the kidneys to secrete a concentrated urine, has been the demonstration that a number of cases have been associated with lesions of the pituitary gland. Schäfer several years ago had demonstrated that the secretion of the posterior lobe possessed a powerful diuretic action.

Frank, in I9 I2, reported a case of diabetes insipidus in a man who had been shot and in whom the roentgen-rays demonstrated that a bullet had lodged in the pituitary fossa. He showed that the patient's kidneys were unable to concentrate urine. From the study of this case and of others reported in the literature, Frank thought that many cases of essential diabetes insipidus could be explained by hyperfunction of the pituitary gland with resultant diuretic action.

In I9I3, Simmonds reported a case in a woman thirty-seven years of age. Two months after an excision of the breast for carcinoma she developed a very marked polyuria. The autopsy showed, in addition to numerous other metastases, one involving and destroying the posterior lobe of the pituitary gland. This metastasis pressed on the pars intermedia of the hypophysis, but did not involve it or the anterior lobe. Simmonds believed that the polyuria was due to the stimulating action of the tumor on the pars intermedia of the posterior lobe.

Later, Eric Meyer himself observed a case of diabetes insipidus in a patient who had bitemporal hemianopsia and dystrophia adiposogenitalis. He showed that this patient was unable to concentrate urine. In the last three years other cases have been reported showing the intimate relationship between essential diabetes insipidus and pituitary disease.

Cushing, in the Shattuck Lecture of $19 \mathrm{I}_{3}$, stated that his animal experiments and surgical experience in hypophyseal disease strongly convinced him that there was a very intimate relationship between the polyuria of diabetes insipidus and pituitary lesions. In roo cases of pituitary disease the polyuria had been so marked that in six a diagnosis of diabetes insipidus had been made before the patients consulted him. He reported a remarkable case of polyuria in a woman, aged forty, and totally blind, who presented symptoms of hypopituitarism. This polyuria, which reached I2 liters daily, followed a sellar decompression operation and lasted a period of three months. He believed that it resulted from an irritative lesion of the posterior lobe resulting from the operation. 
It is a well-known fact, as Oppenheim and Nonne have shown, that many cases of diabetes insipidus with basilar syphilitic meningitis have had bitemporal hemianopsia. So frequently is the latter symptom associated with hypophyseal tumors, that one is naturally inclined to believe that the polyuria of basilar luetic lesions is due to the stimulating action of the luetic process upon the pars intermedia of the posterior lobe.

Sufficient evidence has now been accumulated to warrant us in believing that a very large percentage of the cases of essential diabetes insipidus is due to lesions of the pituitary gland, producing polyuria through their stimulating action on the pars intermedia. The evidence almost justifies us in considering it a manifestation of disease of one of the glands of internal secretion.

Cushing advances the interesting suggestion that the functional nervous polyurias may be due to overstimulation of the hormone of the posterior lobe of the hypophysis through its autonomic nervous connections.

The question naturally arises as to whether pituitary therapy has any effect on the polyuria of diabetes insipidus. If the polyuria be due to an increased production of the diuretic hormone of the posterior lobe, it is hard to see how administration of pituitary preparations could reduce the polyuria; yet Umber, von den Velden, Farini and Roemer, have shown that pituitrin seems to produce a beneficial effect in reducing the polyuria, and increasing the specific gravity, as well as the sodium chlorid and nitrogen concentration. Its effect is transitory and lasts two to three hours and consequently must be regularly repeated. Umber recommends that the pituitrin be administered hypodermically five times daily in 0.5 c.c. doses. The treatment to be repeated from time to time.

Feilchenfeld reports beneficial results from injections of strychnin nitrate. He advises beginning with a total of $0.005 \mathrm{gm}$. (1/12 gr.) daily, increasing the dose until $0.01 \mathrm{gm}$. ( $1 / 6 \mathrm{gr}$.) are given at each dose and that this dose be kept up for a period of ten days. Leick and Stein also report beneficial results with this drug.

Herrick reported a remarkable result following lumbar puncture in which the cerebrospinal fluid was sought for study. A man, forty-three years old, had had diabetes insipidus for four years. The urine varied in amount from 7500 to I I,000 c.c. and had a specific gravity of roor. Following the withdrawal of 5 c.c. of fluid the urine dropped in forty-eight hours to 660 c.c. and never exceeded I800 c.c. during the four weeks he was under observation. The specific gravity averaged ror 5 after the puncture and reached 103 $\mathrm{I}$ in a single specimen. The polyuria later showed evidence of returning. No explanation is advanced as to the cause of the improvement following the puncture. 


\section{ARTHRITIS DEFORMANS}

\section{By 'Thomas B. Futcher, M. B.}

The preponderance of evidence is now in favor of the view that arthritis deformans, whether of the atrophic or hypertrophic type, is the end result of a chronic infectious process. The views, that it is of nervous or metabolic origin, possess few adherents today. Recognizing its infectious origin, it cannot be too strongly insisted upon, that, in any acute or subacute arthritis which tends to leave some periarticular thickening or permanent impairment of joint function, it is most essential that diligent and persistent search for some nidus of infection should be made. It is the failure to search for, find, and eliminate such a focus in the early stages of the disease, when the changes are chiefly in the periarticular tissues, that is largely responsible for these distressing cases of deforming arthritis.

Probably the two commonest sources for infection are the tonsils and teeth. Tonsils not obviously enlarged may, however, have infected crypts or even deep-seated pus pockets. When diseased, tonsillectomy should be performed. Fortunately, the profession is coming to realize the importance of pyorrhea alveolaris, carious teeth, and of abscesses at the roots of teeth as a cause of infectious arthritis. Such infections should be vigorously treated. Film roentgenograms of the alveolar processes often reveal pus pockets at the roots of the teeth, particularly of those that have been crowned. The source of infection, on the other hand, may be in one of the paranasal sinuses-ethmoid, frontal and maxillary, the middle ear, fallopian tubes, broad ligament, prostate or seminal vesicles, appendix, etc. Wherever the infection exists it must be removed. The writer believes that only in rare instances is the intestinal tract a source of infection. Occasionally an arthritis develops in the course of an amebic dysentery. It is often surprising the relief from pain and stiffness that follows such treatment and the patient is spared recurrences of acute exacerbations he otherwise is most sure to have. Billings, particularly, has emphasized the importance of searching for and clearing up any discoverable focus of infection.

In this connection, one must consider the question of the use of vaccines in the treatment of arthritic infections. With many others in this country, the writer wishes to condemn the indiscriminate use of the various stock vaccines on the market. He has yet to see a single case where undoubted benefit has followed their use and has seen many cases where the patient's health has been materially impaired by the violent febrile reactions that frequently follow their use. There is ample justification for trying the effect of autogenous 
vaccines made from the organism isolated from the supposed focus of infection. Even here the results are often disappointing, for it is difficult to be sure that the organism isolated from a focus of suppuration is actually the one causing the arthritis, unless the same organism can also be isolated from the affected joint. The ideal autogenous vaccine would be that made from cultures grown from the fluid in the infected joint. It must be emphasized that vaccine therapy is more likely to be beneficial in the acute stages of an infectious arthritis than after the inflammatory features have subsided and have been followed by the atrophic and hypertrophic bone changes.

Rosenow's very important work on the cultural characteristics of the streptococcus and the selective affinity of various tissues for special strains of the organism offers a prospect for better results from vaccine therapy in the future. By his method of making anaërobic cultures from joints, muscles, glands and various organs he has been able to secure positive growths where others have heretofore failed. In addition to endeavoring to obtain cultures from the affected joints, he finds that he has been able with his technique to frequently obtain positive cultures from the lymphatic glands draining the diseased joint. This affords a much better prospect of securing a true autogenous vaccine.

As was emphasized in the original article discussing the treatment of arthritis deformans, great harm has been done to patients by physicians owing to a too rigid restriction of their diet. Owing to a false conception that disturbed uric acid metabolism bears some causative relationship to the disease, there has been a tendency to markedly restrict the meats. This undoubtedly is detrimental in that there results a marked lowering of the patient's general resistance. From a nervous dyspepsia, the appetites of these patients is very often impaired and they suffer from unconscious starvation. They should be encouraged to drink milk and eat meats and eggs liberally.

In the last few years Ralph Pemberton(I) has published a series of articles giving the results of his metabolic studies and dietetic treatment of this disease. As the result of a study of a series of thirty-seven cases he draws conclusions rather contrary to those expressed above. He advocates a diet of comparatively low caloric value and emphasizes especialy the importance of marked restriction or practical elimination of the carbohydrates and restriction also of the proteins. He even expresses the view that the ingestion of carbohydrates bears a causative relationship to the disease. After clearing up the arthritic features by dieting he claims to have repeatedly precipitated acute joint manifestations by restoring carbohydrates to the diet. He advises working out for each case the carbohydrate and protein tolerance for that particular individual. His results are interesting and are worthy of further investigation.

In the last few years there have been no real advances in our treatment of the disease from a physical or medicinal standpoint. In 
addition to the baking machines used for applying heat to the affected joints it should be mentioned that the heat may be applied by using a battery of electric lights with a reflector.

There has been a belief on the part of some that disturbed function of the thyroid or thymus may be a causative factor in some of the cases of deforming arthritis. Consequently thyroid and thymus preparations have been used but the results have not proven satisfactory.

Atophan has been used in recent years but the results have not been as satisfactory as in the treatment of true gouty arthritis. Where the salicylates are not well tolerated, atophan in $0.5 \mathrm{gm}$. (71/2-gr.) doses every four hours may be tried for the pain. Chase and Fine found some improvement in two out of eleven cases in which it was used.

In recent years radium therapy in its various forms has been strongly recommended in all forms of chronic arthritis. Its use is most vigorously advocated by those who have it to sell. As this agent has been so helpful in other lines and as so much depends upon knowing the best method of administering it as well as the dosage, we must not advise against its use in the future. The results obtained in arthritis deformans up to the present time have been decidedly unsatisfactory. As our knowledge of the best method of administering it increases, it may yet prove to be of some service.

${ }^{1}$ Ralph Pemberton, Am. Jour. 'Med. Sci., rgr2, cxliv, 474; ibid., rgr3, cxlvi, 895; ibid., I914, cxlvii, III; ibid., I914, cxlvii, 265; ibid., cxlvii, 423 . 


\section{GOUT}

\section{By Thomas B. Futcher, M. B.}

The actual cause of gout is still a subject of much speculation, and a detailed discussion of these theories here would not be profitable. Our knowledge of intermediary purin metabolism and the part that the various specific organic ferments play in the ultimate formation of uric acid is gradually being added to. Up to the present time, these researches have not added anything important to our knowledge of the therapy of gout. The disease is most closely related to the formation or elimination of uric acid, but the only point on which all investigators are agreed is, that the blood of true gouty patients always shows an excess of uric acid above the normal. Whether this excess is due to excessive formation or diminished elimination is still a disputed point. That there is a diminished elimination of uric acid during most stages of the disease is believed by most observers. Umber has recently expressed the view that this diminished elimination is due, for some reason, to an increased affinity of the tissues for uric acid, and not to diminished ability of the kidneys to eliminate it, as Garrod and others have held.

The same general principles of treating the disease, as previously described, still hold. Since the previous article a very few additional therapeutic agents have been advocated which may be here described.

A considerable literature has grown up in the last few years concerning the use of radium in the treatment of gout, first advocated by $\mathrm{His}$ and his associates and enthusiastically recommended by them. Although it has been used in various forms of arthritis, yet true gouty arthritis is the only form in which it has really shown any beneficial result. The results have not been striking, but the evidence seems to show that it does good in some cases. It is of some importance to note that the waters of those spas which have obtained the greatest reputation for effectiveness in the treatment of gout have, on investigation, been shown to be most highly radioactive. It should be emphasized that, to be effective, these waters should be drunk when quite fresh, for it is well known that when bottled they lose their radioactivity in two or three days. Water can, however, be made radioactive by charging it with radium emanations. It is conceded that radium is most effective when inhaled in an emanatorium, although improvement has followed the drinking of radioactive waters, by administering radioactive baths, and by injecting radium salts intravenously.

The view generally held as to the form in which uric acid circulates in the blood is that of Gudzent, namely, as the monosodium 
urate. He has shown that this salt exists in two isomeric forms. At first it is in the laktam form, which is unstable but soluble in blood serum to the extent of $18.4 \mathrm{mg}$. per Ioo c.c. of blood serum. In the course of time it passes into the laktim form, which is only soluble to the extent of $8 \mathrm{mg}$. per roo c.c. of blood serum. This difference of solubility is probably of some importance in helping to explain the deposition of uric acid in the tissues. Gudzent believes that the beneficial effects of radium therapy result from the effect the metal has in transforming the relatively insoluble laktim into the relatively soluble laktam salt, thus rendering the uric acid more easily eliminated by the kidneys.

Chase and Fine treated cases in an emanatorium with radium emanations of 0.5 to 150 Mache units per liter of air. The patients were allowed to breathe this for one to two hours daily for two months. Two cases were treated with radioactive water. Five cases were given injections of radium bromid, which were equivalent to 50 to 100 micrograms of the element. In none of their cases was there any change in the uric acid content of the blood. They were, therefore, unable to confirm Gudzent's findings that with concentration of radium emanations with as little as 2 to 4 Mache units per liter of air, the uric acid disappeared from the blood and remained absent for fully a year. In only two cases in their series were they able to observe clinical improvement. These were treated with injections of solutions of radium salts. They felt that the treatment should be continued over longer periods before drawing definite results.

The radium has also been applied locally over affected joints in the form of pastes made with radium salts. Umber states that from a rather extensive personal experience with radium therapy in gout he has yet to see any beneficial effect. The results of the treatment have not, therefore, borne out the early experiences of His, who claimed such remarkable benefit from this line of therapy. It is quite possible that, after the requisite dosage has been more carefully worked out, the results may prove more satisfactory.

Umber has injected intravenously thorium $\mathrm{X}$ in doses up to $\mathrm{I} 1 / 2$ million Mache units without observing any benefit.

The experience with atophan (phenylquinilin-carboxylic acid) since its introduction a few years ago seems to show that in it we have really a very helpful remedy in the treatment of gout. The writer's personal expenrience with it has been very satisfactory. It is put up in $0.5 \mathrm{gm}$. (71/2 gr.) tablets, and Umber advises that it be given in I gm. ( 5 gr.) doses three or four times daily in the acute attacks. It may be continued in these doses for three or four days. If it upsets digestion or does not give relief to the pain at the end of that time it should be discontinued. It frequently has a marked effect in relieving the joint pains. Nicolaier and Dohrn found that it increased the uric acid output more than 200 per cent. on daily doses of $3 \mathrm{gm}$. (45 gr.), and others have reported a marked increase in the output of the uric acid. It not only relieves the pain but several 
have observed decided diminution in the size of the tophi. It is not definitely known how it exerts its effect, but Weintraub claims that it is through increasing the permeability of the kidney to the uric acid salts.

Electricity over the affected joints has been used with beneficial effect in some cases. Remak and Benedikt claim to have obtained good results by the use of the constant current locally applied, both in the acute and chronic cases. Labatat, Jourdanet, and Levinson have reported improvement from the use of electrical endosmosis, or catophoresis, with lithion, in the treatment of the joints and of skin tophi. The galvanic current, with the electrodes moistened with a concentrated solution of lithion chlorid or carbonate, is passed through the affected joint or tophus, or is given in the form of an electric lithion bath.

It is needless to say that all these therapeutic measures are of subsidiary importance to the dietetic and general management of the case previously described. 


\section{LITHEMIA, URIC ACID DIATHESIS}

\section{By Thomas B. Futcher, M. B.}

In addition to what has been previously said on this subject, none of which is here retracted, it may be well to say that the tendency for the formation of uric acid calculi in the pelvis of the kidney or in the bladder is usually included under this nondescript heading.

It is well to point out that the formation of kidney or vesical calculi of uric acid composition has really no connection with gout. There is no disturbance of purin metabolism, nor increase in the uric acid content of the blood in the majority of these cases. The amount of uric acid output here bears a normal relationship to purin intake in the food. There is probably a greater tendency for the uric acid to crystallize out in the urinary tract, if certain conditions which render the urine incapable of properly holding it in solution. Umber claims that some organic nucleus, such as a small mass of epithelial cells, etc., is nearly always a precursor, and about this the uric acid crystals become deposited. The richer the food is in purin-containing ingredients, the greater will be the uric acid output in the urine and consequently the greater the liability for its crystallization about an organic nucleus, should for any reason such exist in the urinary tract.

Certain general principles govern the management of these cases: (I) To reduce the absolute amount of uric acid excreted in the urine as much as possible. This must be brought about essentially by putting the patient on as purin-free a diet as possible, by eliminating meats, liver, kidney, sweetbreads, beef soups, tea and coffee; (2) to reduce the acidity of the urine by administering alkalies freely; (3) to increase the total output of urine as much as possible by drinking plenty of water and thus increasing the ability of the urine to hold the uric acid in solution; (4) to clear up, if possible, any catarrhal or inflammatory trouble in any part of the urinary tract, in order to prevent the possibility of any nucleus of organic material forming, about which uric acid may crystallize.

Unfortunately we have, as has been said in the article on gout, no medicinal agents that are uric acid solvents, notwithstanding the number that have been put on the market and the claims made for them. There is nothing better than plenty of pure water. When positively demonstrated by the use of roentgen-rays or other means, the only way of getting rid of the calculus is by surgical interference, unless it is small enough to spontaneously pass through the ureter or urethra. 


\title{
DISEASES OF THE RESPIRATORY SYSTEM
}

\section{TONSIILITIS}

\author{
By Charles W. Richardson, M. D.
}

\section{FOLLICULAR TONSIILITIS}

This condition is also as frequently designated lacunar or croupous tonsillitis.

Follicular tonsillitis is characterized by a parenchymatous inflammation of the tonsils with the formation of a croupous exudate in the crypts of the tonsils which exudes from the crypts and appears as isolated pearly white spots, or spreading more or less over the surface of the tonsils, forms one large or several white smaller glistening plaques. The exudate rarely extends beyond the confines of the tonsils. The condition is essentially a disease of child life, although no age is exempt from its invasion.

Its predisposing causes are chronic enlarged tonsils, especially those with ragged surface and inflamed crypts, any disturbance of the general physical condition which lowers the power of resistance, and the rheumatic diathesis.

The exciting causes are exposure to cold, draughts, wetting of the feet, or other portions of the surface of the body. At times it is so prevalent that it almost forces the impression that it is contagious.

The microörganisms usually found present are the staphylococci, the streptococci and the pneumococci.

This affection is most prevalent in the wet, moderately cold and changeable temperature of the early spring and fall.

The disturbance is characterized by well-marked constitutional and local symptoms. The onset is almost always sharp and clearly defined. The constitutional symptoms precede the local. The onset is usually in the form of a severe chill, or chilly sensation, with severe headache and marked aching or pain in the muscles of the extremities, neck and back. Frequently chilly sensations persist throughout the attack; and the temperature varies from $102^{\circ}$ to ro $5^{\circ} \mathrm{F}$. Pulse is full, rapid and strong. The other constitutional symptoms are those which attend an infection of similar intensity. Local symptoms are usually manifested within twenty-four hours after the invasion and are at first characterized by a feeling of fullness or soreness in one or both tonsils, quickly followed by sharp lancinating pain which is intensified by speaking, swallowing, or clearing of the throat. Pain is especially intense when the exudate forms 
between the tonsils and pillars of the fauces. There is usually more or less viscid mucous secretion. Both tonsils are usually affected, although one may be only affected at the outset, followed in a few days with exacerbation of the general symptoms and infection of the other tonsil. The pharyngeal tonsil may participate in the process; head pain is usually more pronounced under this circumstance.

Examination of the fauces reveals the inflamed enlarged tonsils with the characteristic pearly white spots disseminated over the surface of the tonsils. Occasionally the exudate spreads more or less over the surface of the tonsil and we have a pearly white plaque instead of points. Should the points have a yellowish or grayish appearance the condition is probably lacunar diphtheria rather than tonsillitis. As complications we have peritonsillar abscess, albuminuria, nephritis and endocarditis.

The invasion terminates in from four days to one week. The prognosis is exceedingly favorable. All patients should have their urine examined during the attack and after recovery.

Treatment.-One affected with tonsillitis should be confined to the bed until recovery is complete. The constitutional treatment should consist in the administration of a calomel purge succeeded by a saline; the administration of 5 grains $(0.3 \mathrm{gm}$.) of salol every three hours until ringing in the ears occurs to combat the infection; and 5 grains $(0.3 \mathrm{gm}$.) of phenacetin every three hours to relieve the head pain and muscular soreness. Alkalies are advantageous and may be given in the form of bicarbonate of soda, and Rochelle salts in half teaspoonful dose in full glass of water three times daily. The diet should be liquid. Locally, I do not believe in gargles as they are not only useless but quite painful to use. Sprays are of no value. A topical application of a ro per cent. solution of nitrate of silver gently applied only to the affected tonsils once daily is very beneficial. The most effective agent for local, as well as of undoubted constitutional value, is the tincture of the chlorid of iron. The iron is best given in a solution of glycerin as,

R. Tinct. ferri chloridi Glycerinæ q. s. ad.

3ii 8.00

范 60.00

Sig.-One-half teaspoonful every three hours, in wine glassful of water to be drunk slowly.

A neck ice bag may be employed if it gives comfort. The urine should be examined during and after the attack, and the heart kept under observation. After recovery the removal of the tonsils should be considered.

\section{PERITONSIILAR ABSCESS, QUINSY}

Quinsy is an inflammation in the connective tissue which surrounds the tonsils, which rapidly takes on a phlegmonous character and terminates in the formation of an abscess.

The inflammation which results in the formation of an abscess 
most frequently occurs in the connective tissue, in the supratonsillar fossa resulting in the formation of an abscess between the tonsil and the upper portion of the anterior pillar. Less frequently the inflammation takes place in the connective tissue posterior to the tonsil, and then the resulting abscess forms in the posterior pillar. Very rarely the pus works its way into the lateral wall of the pharynx.

This affection is most prevalent in young adult life, between the sixteenth and thirtieth year. It occasionally occurs in child life. It shows a tendency to recur once or more a year, usually in the spring and fall. When once established, the tendency to recur seems to abate after the thirtieth year of life has been attained. The spring and fall of the year are the seasons in which it is most prevalent. Males are affected more frequently than the female sex.

The predisposing cause is the so-called rheumatic diathesis. Local predisposing causes are hypertrophied tonsils, especially those with chronic lacunar infection; and tonsils which have been the subject of frequent acute attacks during childhood, causing adhesion between the tonsil and the pillar at the upper pole. As a result of frequent acute attacks of tonsillitis we have adhesions formed between tonsils and pillars and the development of cicatricial tissue in the supratonsillar fossæ in which are formed pockets which are particularly prone to infection under mild parenchymatous inflammation.

The exciting causes are exposure to cold and wet and follicular tonsillitis. The infecting microörganism are the streptococci and staphylococci.

The symptoms are quite characteristic so that one who has ever had an attack can tell when the premonitory symptoms manifest themselves that an attack is about to supervene. The constitutional symptoms are not marked at first. There may be a slight chill or chilly sensation, but this is not usual. The patient has a moderate temperature, about $100^{\circ}$ to $10 \mathrm{I}^{\circ}$. They feel depressed with the usually febrile constitutional disturbances.

The local symptoms commence with a feeling of fulness and soreness in one tonsil. Later the pain becomes most severe, lancinating and throbbing, in character, and frequently radiates to the ear. Speaking and clearing of the throat increase the pain. Deglutition at first painful becomes later not only painful but almost impossible, on account of swelling, infiltration of muscles and edema. The voice is muffled and throaty. Secretion of buccal and faucial mucus and saliva is greatly increased. Edema of the uvula and curtain of the palate becomes marked in some cases. When uvula becomes markedly edematous and hangs in the vestibule of larynx, we have symptoms of suffocation. When the abscess is bilateral, breathing becomes difficult, occasionally impaired. In the maximum of the discomfort, sleep is abolished on account of pain; the ingestion of 
food is impossible; and phonation is so impaired, painful and unintelligent that the patient makes no effort to talk.

Examination.-Early in the case examination is fairly easy. When tumefaction has become marked with infiltration of pharyngeal muscles, the examination becomes more difficult, as the patient finds it impossible or too painful to widely open the mouth. The tonsil rarely participates in the affection. In the early stage and in a few unusual cases, there is not much swelling. Usually the swelling is manifested above one tonsil, involving the anterior curtain, the tonsil being pushed internally toward the center of the fauces. This tumefaction is rounded and the overlying tissues are much congested. Edema of the uvula and the palate is marked in many cases. When the abscess forms in the posterior pillars, it shows out as a fusiform swelling posterior to the tonsil. When the lateral pharyngeal wall is involved, it encroaches inordinately upon the fauces posterior to the tonsil. Digital examination should be carefully made when possible. Digital exploration is imperative when frank evidences of inflammation are not present to differentiate between possible aneurysm and abscess. This abscess is usually unilateral, it may be bilateral, and one tonsil may be affected immediately after the other.

Termination.-Through spontaneous rupture, or artificially through incision. When spontaneous evacuation takes place the pus gains egress between the pillars and tonsils or through the substances of tonsil. The danger of spontaneous rupture is from asphyxiation through entrance of pus into the larynx during sleep.

Complications.--Septicemia; hemorrhage; burrowing of pus along course of posterior pillar or lateral wall of pharynx into deep fascia of the neck causing cervical abscess, mediastinal abscess and purulent pleurisy. Tonsillar fistula and chronic abscess occasionally occur.

Treatment.-Patients with quinsy should be confined to bed until recovery is complete. A calomel purge should be given, followed by a saline. The diet should be soft or liquid. Antirheumatic remedies are of value in the early stage and should be administered. Tincture of aconite in the early stage seems to have some influence on the progress of the case. Bicarbonate of soda both for its constitutional effect and its local action is of undoubted service. Most cases after the infection has set in will not be materially influenced by the constitutional methods adopted, but will progress to the formation of an abscess, which can be relieved only by spontaneous rupture or through surgical measures. Early operative evacuation of pus is the all-important procedure in peritonsillar abscess. Pus may usually be evacuated within forty-eight hours after the initial symptoms have manifested, and before any marked swelling has developed. The incision should be made in the indurated swollen tissue, over the point of greatest protrusion, or on a line drawn between the last upper molar tooth and the root of the uvula, through the anterior pillar, just external to the tonsil. The incision should be made in a direct line vertically. If properly directed, neither deviating in- 
ward or outward, there is no possibility of injuring any vessel, or penetrating anything but the abscess cavity. A bistoury should never be used. A straight narrow-bladed tenotome, with an inch or inch and a half cutting surface forms the ideal knife for this purpose. With the initial penetration and finding of pus, the wound should be enlarged in a downward direction as the instrument is withdrawn. If it is found necessary to enlarge the wound for more ample drainage this may be accomplished by inserting a narrow-bladed hemostat into the wound and withdrawing them opened, or employing an especially adapted instrument for accomplishing the same result. The parts to be incised and only these should have applied to them a ro per cent. solution of cocain, on cotton applicator, some minutes before the incision is made. The patient should be instructed to gargle the throat with warm soda solution every hour or two, after the abscess has been evacuated in order to favor drainage. In the majority of cases there is a rapid convalescence with subsidence of the inflammatory swelling, after the drainage of the abscess. Occasionally there is a reaccumulation of pus due to union of the wound edges or to the cavity being opened at too high a point. Reopening through the initial wound, with its enlargement usually brings these cases to resolution. Coakley suggests the evacuation of the pus through the use of a right-angle probe, the point of which is inserted above the tonsil at the superior commissure. The probe point is pushed outward readily breaking through the thin fold of mucous membrane at this angle and is then pushed outward until the pus cavity is reached. As the probe is withdrawn, by slightly changing the angle of the probe, the wound is enlarged. This procedure is also done under local cocain anesthesia. After the patient has recovered the tonsils should be removed in order to prevent a recurrence of the infection.

\section{SURGICAL REMOVAL OF THE TONSIIS}

There is hardly any division of opinion that when a tonsil is to be removed it should be done in a most thorough manner. The tonsil should be removed in its capsule. Care should be exercised that no, or as little injury should be done to the surrounding tissues as possible, as such injuries are apt to be attended with the development of annoying cicatrical tissue. The tonsillar pillars should not be extensively injured. Many methods are adopted for the removal of the tonsils. It suffices to state that that operation should be cultivated that removes the organs most thoroughly with the least traumatism. The tonsils become surgical when by their size they are obstructive, interfering with respiration, deglutition or phonation; when they present frequent acute disturbances or chronic disease; when they are the portal of entrance of organism causing various types of general infection; and when through their presence they cause disease of neighboring organs.

Obstructive.-Hypertrophied tonsils, which on account of their 
large size narrow so the isthmus of the fauces that they interfere with respiration, especially at night, impede deglutition, and alter the timbre and quality of the voice, should be removed.

Acute Local Disturbances.-Frequent recurrent attacks of acute tonsillitis; under this subdivision we have those acute attacks occurring in children who are attended or have as sequelæ indefinite soreness or actual pain in the muscles of the extremities; and in the same class of cases in the adult where we have fleeting joint pains. In children and adults having frequent acute attacks of follicular tonsillitis. It is in this type of infection that we have occasionally albuminuria and mild infection of the kidneys. The removal here is not only wise to remove the local infection but also to prevent the possible nephritic complication.

In acute recurrent peritonsillar abscess removal is also indicated.

Chronic Local Diseases. - Chronic hyperplasia of the tonsils with broad flat surfaces, with irregular congested marginal circumferences, and wide-open crypts showing over faucial surface. Chronic hypertrophied but not greatly enlarged tonsils wherein the tonsillar folds cover well over the faucial surface of the tonsils-the embedded tonsils.

Chronic Lacunar Tonsillitis.-Characterized by the formation and accumulation of secretion and debris in the tonsillar crypts and pockets.

Fistula of the tonsil, a complication of peritonsillar abscess.

Chronically painful or tender tonsils, the result of adhesion, or deep-seated accumulation of cheesy cryptic secretion or parenchymatous deep-seated abscess.

Remote Infection.-The systemic infection stated to have the tonsils as their portal of entry are legions. Those which seem to have some clinical facts to bear out the possible relation are rheumatism, endocarditis, nephritis and neuritis.

Affection of Neighboring Organs.-In acute and chronic infections of the cervical glands; in acute, subacute and chronic middle-ear catarrh and acute and chronic suppurative middle-ear infammation; in these conditions the tonsils when diseased or enlarged cause infection, and impairment of the ventilation of the middle ear through pressure on the pharyngeal orifice of the Eustachian tube and through impairing the functional activity of the palati muscles, tonsillectomy is indicated.

In considering tonsillectomy for the purpose of removing the source of general infection, one should thoroughly consider the conditions of all the other organs, sinuses, and glands through which the general infection might arise, as well as through the tonsils, as such effort may lead us to the source of infection and spare us the mortification of removing an innocent organ.

I also wish to caution against the too prevalent habit of removing the tonsils simply because they exist, although they manifest no disease either locally or remote. 


\section{DEFECTS OF SPEECH}

\section{By G. Hudson-Makuen, M. D.}

For purposes of study and treatment defects of speech may be classified as: first, stammering, stuttering, or as I have designated it, dyslalia; and second, faulty articulation, baby talk, or pseudolalia.

Stammering appears in two or more less distinct stages, an initial stage and a chronic stage. The initial stage usually begins during childhood and seems to have a purely physical basis, the stammerer himself not having reached a sufficient degree of psychical development to quite understand or even notice his condition, and it is only after his attention has been called to it, either by his own increasing difficulties of speech or, as is more common, by the remark of some well-meaning friend, that the affection begins to take root and grow in his consciousness until it assumes such proportions as to overshadow everything else in life. This is the beginning of the second or chronic stage, in which the stammering may persist and increase in severity even after the original physical basis for it has ceased to exist. In these cases, the secondary manifestations, such as mental confusion, anxiety, fear, and the autosuggestions which always accompany strongly emotional states, appear to assume causal relations to the affection and suffice to aggravate and perpetuate it.

The etiology of stammering has been a subject of discussion for centuries, and yet it is only recently that satisfactory conclusions have been reached. Much confusion has arisen from the fact that no two stammerers are alike, and in seeking the primary cause, the difficulty has been to find one that will explain all the phenomena of the affection.

The causes of stammering are twofold, predisposing and exciting. The predisposing or ultimate cause of stammering is probably congenital and often.inherited, and it consists in an irritable or hyperesthetic condition of the psychophysical mechanisms of speech. In this respect it bears some resemblance to aphasia, and like aphasia it probably has a physical basis, if we were only able to discover it.

Stammering has been called a transient auditory amnesia for the coloring or inflections of speech, and there is much to be said in favor of this theory. That the affection is of psychic origin in the majority of instances has been pretty generally accepted, but there is also a physical element to be reckoned with, especially in considering the treatment of the affection.

Glandular enlargements in the pharynx are frequent in children who stammer, and it has been suggested that the affection may be due to various other glandular disturbances. One of the newer 
theories as to the causation of stammering is that it may be due to an enlargement of the thymus and to a resultant block in this area.

It is too much to expect, however, that the removal of enlarged tonsils and adenoids should cure stammering after it has once become established, but as a preventive measure in those predisposed to the affection the operation undoubtedly has great value, and in like manner a reduction of an enlarged thymus when it causes pressure upon the surrounding parts might remove one of the many exciting causes of stammering.

The speech processes, like all other similar phenomena requiring the action and coördination of muscles, are centrally represented, and the integrity of their performance depends upon certain wellordered if not well-known central stimuli.

Of the four so-called language centers in the cerebral cortex, the auditory and glossokinesthetic centers constitute what Bastian has called the "primary couplet" representing written language. While it is doubtless true, as has been suggested, that individuals vary in respect to the predominance of these various centers in the use of spoken and written language, yet there are good reasons for supposing that the average hearing person depends for his speech cues upon both auditory and glossokinesthetic memories, just as the average seeing person depends upon his visual and chirokinesthetic imagery for his cues in the use of written language. The average stammerer, therefore, is what has been called an auditomoteur, and his chief difficulty must be in the action of one or both of the centers suggested by this expression.

Stammering has long been supposed to be due directly to what Wyllie, of Edinburgh, and others have called a faulty action of the laryngeal mechanism in the production of speech, resulting in delayed vocalization, but the question arises, what causes the faulty laryngeal action and consequent delayed vocalization?

There appears to be in many instances what Dr. Charles K. Mills has called an "aberrant tonic innervation" of the neuromuscular mechanisms employed in speech, which is influenced to some extent at least by the psychic conditions of the patient, and there is a resultant disturbance in the coördination of sensory stimuli and motor discharges.

When we consider that the oral symbols of speech or spoken words have two distinct elements, one of which is largely articulatory while the other is phonatory, and that the stammerer's difficulty is not so much with the articulatory as with the phonatory element, we must conclude that the chief trouble is in the cortical center representing phonation rather than in that representing articulation. Moreover, the articulatory element of a word being represented for the most part kinesthetically and the phonatory element depending for its production largely upon auditory cues, it follows that the stammerer's disability must exist chiefly in the auditory region of the cerebral cortex. This theory may be demonstrated and estab- 
lished by a careful analysis of the stammerer's difficulties of speech. It will be observed that the articulatory movements of speech or those that are kinesthetically represented are as a rule freely negotiated, and the difficulty appears for the most part only when the phonatory element is encountered. For example, we may take the word "fate." The stammerer can easily produce the initial consonant "f," but the vowel " $a$ " which follows it is the element which occasions his difficulty. The consonant sound, being kinesthetically represented, comes easily enough, and owing to delayed or confused auditory imagery, the " $\mathrm{f}$ " sound is either prolonged or repeated by the individual in the effort to arouse into greater clearness the delayed or obscure auditory imagery upon which the complete externalization of the word depends.

The theory, therefore, that the primary cause of stammering in the great majority of instances is a weakness or irritability in the auditory spech center, rendering the patient temporarily unable to arouse adequate speech imagery, is the one that appears to meet the greater number of conditions with reference to the causation of this affection.

Stammering is in some respects very like aphasia, and it is frequently associated with aphasia in its inception as well as in the course of its development. Moreover, stammering like aphasia is more common in the male than in the female, a fact which is explained on the principle of the greater variability of function in the male, and also by the fact that the female appears to possess a greater clearness and intensity of verbal imagery, as is shown by her greater facility in oral expression.

It is worthy of note also that stammering is an affection of childhood and belongs to the developmental speech period. Speech is an acquired faculty, and stammering speech is in a sense an acquired affection, although, as I have said, the underlying physical cause of stammering, namely, a weakness or hyperesthetic condition of the auditory speech center, is often inherited and is probably, therefore, congenital.

The inheritance of the stammerer, like that of the so-called deaf mute, is not the affection itself, but rather the physical anomalies that give rise to the affection, and they may appear only occasionally in the family history, skipping one, two, or even three generations. Given these anomalous conditions, however, the child is extremely liable to the development of the affection. Even slight exciting causes, such as emotional disturbances arising from fright or injury, or one of the infectious diseases, will give rise to the auditory amnesia upon which the affection depends.

The development of normal speech always follows the development of normal mental imagery, and this in turn depends upon the physical development of the psychomotor mechanisms of speech. A delayed or distorted imagery, on the other hand, always results in one or more of the disorders of speech.

Although stammering rarely begins after the age of puberty, iso- 
lated cases have been reported as developing later in connection with the various forms of acquired aphasia. The conditions frequently giving rise to stammering in children are those which have been designated as congenital aphasia. Many children, for no apparent reason, are slow in acquiring speech, and some of them go well into adolescence with very indifferent speech. It is true that stammering seems to develop in only a small proportion of these so-called congenital aphasics, but, nevertheless, the affection must be regarded as an outgrowth of the various other forms of speech disorders.

Pseudolalia is the term that I have used to include all forms of developmental speech defects other than those designated as stammering or dyslalia. It must be borne in mind, however, that pseudolalia is frequently found in conjunction with stammering, or in other words stammering often appears as a complication of pseudolalia.

Pseudolalia is a form of faulty speech in which defective articulation is the important feature, and this defective articulation is merely the result of faulty development of speech. It may be due and often is due in a measure to peripheral obstructions in the respiratory tract, but more frequently, like dyslalia, it is of cerebral origin. Normal speech always depends upon normal psychophysical development, and any deviation from the normal in the psychophysical organs of speech must result in corresponding defects of speech.

Moreover, an accurate articulation of syllables and words can follow only upon an accurate mental imagery of the elements of speech, and this imagery is developed largely through the sense of hearing as it works in conjunction with the psychomotor mechanisms of speech. When there is no hearing there can be no speech development, although the psychomotor mechanisms may be organically perfect.

The treatment of defects of speech, therefore, must be psychophysical in character. The peripheral mechanisms of speech must first be put in good condition and then the central mechanisms must be trained to operate them in much the same way as the violinist is trained to play upon his instrument.

The details of the treatment of defects of speech are not easily described in writing, but they are given more or less fully in my previous article on this subject.

Accepting, however, the somewhat newer theories herein advanced with reference to the causation of dyslalia and also to some extent of pseudolalia, greater emphasis should probably be placed upon the psychotherapy of the defects. Our treatment must be largely of a psychical nature, or better still perhaps, of a psychophysical nature. It is well-known that pure psychotherapy will not of itself correct defects of speech, at least in their chronic stages, because the faulty physical habits of speech have become so thoroughly fixed as to require special-physical or elocutionary training in order to correct them.

If it is true that there is a blurring of the mental imagery in defects 
of speech amounting to a kind of auditory amnesia, then the training must have in view a general clearing up of the imagery by a frequent practice of the words and syllables of speech with their natural inflection and emphasis. It has been shown that the visual imagery may be sharpened and the powers of observation rendered more acute by training, and in the same way it is quite possible to reëstablish and revivify the weakened or blurred auditory imagery employed in speech.

It must be borne in mind, also, that as the development of normal speech depends upon the accurate subjective hearing of speech, so defects of speech are largely the result of a lack of accurate hearing for speech sounds, and the correction of defects of speech must always be accompanied, if not preceded, by a correction of the faulty hearing for speech sounds through educational measures. 


\title{
HAY-FEVER AND HAY-ASTHMA
}

\author{
By J. L. Goodale, M. D.
}

By the term hay-fever is denoted a form of anaphylaxis in which the body is sensitized to plant pollens. The symptoms are seasonal and synchronous with the flowering of the plants in question. If the degree of sensitization is slight or moderate, the clinical manifestations are limited to the mucous membranes of the eyes and nose; if the sensitization is sufficiently high, asthmatic symptoms may supervene.

When the special exciting pollen comes into contact with exposed mucous surfaces in sensitized individuals, its proteins undergo solution and are split up, yielding a product which has for the subject a toxic influence. This toxic material excites edema with profuse transudation of serum together with itching of the terminal nerves. Clinical examination of the nasal mucous membrane shows pallor and swelling with abundant formation of clear, thin fluid.

Recent serobiological studies have thrown light upon the relationships of plants in connection with hay-fever, and have permitted the construction of a phylogenetic tree. If we start with primitive protein of the flowering plants as the main trunk we shall find it to divide into two branches, the monocotyledons and the dicotyledons. Omitting now the various subdivisions which so far have a botanical rather than clinical importance in this connection, we see that the monocotyledons comprise the large family of grasses. The other branch, namely, the dicotyledons, proceeds upward, giving off the various smaller branches of the rose family, the birches, oaks, maples, etc., and terminating in the Compositæ or ragweed family. These families have no serobiological affinity with each other. Consequently, an individual sensitized to one family alone would not necessarily react to the pollen of a distant group. Where two families are closely related, a positive reaction may be expected, as I have, for instance, found in patients with ragweed hay fever, who often react to the pollen of the squash family.

Diagnosis of the causative agent is considerably simplified by keeping in mind the following facts. In the first place our search should be chiefly directed to the monœcious or diœcious plants, since it is those plants which distribute a large amount of pollen in the air which are capable of exciting hay-fever symptoms. Entomophilous plants which depend upon cross-fertilization through the agency of insects are able to be much more economical in the production of pollen, and it is consequently not likely that such pollen can come into contact with exposed mucous surfaces under natural 
conditions. Such pollen, furthermore, is more viscid and sticky, the object evidently being to enable it to cling to the bodies of the insects which visit the plant in question. It is necessary on the other hand, for anemophilous plants which depend for their fertilization upon the agency of air currents, to produce a large amount of pollen, and pollen furthermore which is relatively light, and easily detached from the anthers.

Recognition of these facts enables us to determine with much more certainty the causes of hay fever in a given locality. We may, therefore, pass over the entomophilous plants and confine our attention to the anemophilous representatives. Such are, in the eastern United States, certain trees, especially maples and oaks, the grass family as a whole, and certain species of the wormwood or ragweed family. Although the pines and other gymnosperms produce a very large amount of pollen, yet I have not so far found any instance of hay-fever being caused by them. It is in the next place necessary to consider the season of hay fever symptoms. If they occur previous to the flowering of the grasses, we shall obviously have to look for the exciting cause in the early flowering shrubs and trees, and among these maple and willow are chiefly responsible. About the middle or the last of May the oak begins to flower in New England, and tests should be made for this family. The flowering period of these trees is relatively brief, and consequently the hayfever occasioned by them is less likely to disturb seriously the patient's mode of life.

In the case of grasses we have a flowering period extending from the middle of May in this locality until the first week in July, and the attack of hay-fever which they may excite, is not only more prolonged but more severe. There is apparently a difference in the strength or activity of the pollen of the different species of grass, timothy grass, which is rather a late bloomer being especially potent.

Examination of the Compositæ is very much simplified by recognizing that the protein or active agent of all its members is essentially the same. While minor differences may exist, yet one active pollen will for diagnostic purposes, cover the whole order. Tests, furthermore, of different members of the Compositæ from the earliest flowering ones such as coltsfoot to the late blooming asters show that with sufficiently strong solutions all ragweed cases will develop skin reaction. Under natural conditions, however, throughout the greater part of the eastern United States, the special exciting cause among the Compositx is found in the ragweed, the structure of which will repay examination. It will be seen that the plant is characterized by the possession of long spikes containing the male flowers, and by the localization of the female flowers at the base of the spikes. The amount of pollen which this plant distributes is very large, and it is also extremely light. The flowers open in succession beginning with the central spike, a few discharging daily, and the scattering of the pollen being consequently maintained for a long period. Vor. IV. -34 
Another plant of the Compositæ which resembles the ragweed in its method of flowering is the Baccharis halimifolia, a favorite plant in shrubberies, although its power of causing hay fever has not hitherto been recorded. The plants are diœcious, and the amount of pollen formed is considerable. The more showy members of the Compositæ such as field daisy, golden rod, asters and other garden representatives, possess a relatively small amount of pollen, as well as one of a more generally viscid character. Patients consequently more readily escape. In the western and southern parts of the United States autumnal hay fever is caused by other members of the wormwood family, such as Parthenium hysterophorus, Ambrosia trifida.

Treatment.-Much interest attaches to the question of increasing the tolerance of hay fever patients by the injection of protein extracts derived from the special exciting plants. There seems to be now sufficient evidence that in the majority of hay fever individuals it is possible to accomplish this object.

Preparation of Pollen Protein.-For many plants which furnish an abundance of easily detached pollen, it is sufficient to gather the partly opened flowers, bring them into a room without currents of air, when in the course of a few days, the pollen may be shaken upon smooth paper. This applies particularly to those plants the cross-fertilization of which is effected through the agency of air currents, such as many forest trees, grasses, and ragweed. In the entomophilous plants, where the cross-fertilization is largely effected by the agency of insects, the freshly opened anthers may be clipped and gathered. The pollen is then either placed in the solution for extraction, or it may be preserved dry for an indefinite period.

In the case of ragweed the following method enables one to obtain the pollen easily in a purer form than has hitherto been the case. Plants of ragweed are gathered in the flowering stage; and the heads of the flowers stripped off. The habit of this plant is to open the flower heads in succession, and consequently at a given time some will be found to have discharged their pollen and others to be as yet unopened. After drying in air the heads of flowers are rubbed up in a dish with carbon tetrachlorid and strained through muslin. Most of the pollen comes out in the process and passes through the muslin. The pollen grains are then allowed to dry and are seen to be somewhat paler than those-obtained in the natural way, the difference in color being probably due to the removal of lecithin.

For the extraction of the protein a normal salt solution with alcohol of from I2 to I4 per cent. by volume appears most suitable. The important object to be attained is a solution which will check decomposition, without producing loss of strength of the active principle. Pollen extract in 6 per cent. alcohol or less shows after a few days development of bacteria and protein decomposition. A dilution of 8 to Io per cent. is sufficient to check putrefaction, and if the bottle is completely filled with the solution it remains 
free from alteration. If, however, the bottle is frequently opened or left half full, there is a prompt invasion of the yeast organism with development of vinous odor. This is later followed by the appearance of the Mycoderma vini and general turbidity. Solutions from 12 to 15 per cent. can be left open without danger of infection.

Pollen protein consists of an albumin coagulable by heat and strong alcohol, and of a proteose which endures boiling and remains soluble in alcohol up to 95 per cent. Both are capable of exciting reactions in sensitized individuals. While the proteose is relatively stable the albumin is readily coagulated on exposure to air and is precipitated. The precipitate, however, when injected, excites a delayed anaphylactic reaction, probably from digestion by the cells of the part. Consequently, the protein extract when once filtered clear should not be subsequently filtered if a sediment appears, as we should thus remove the albumin, and have only the proteose for injection.

When the pollen has been gathered and a suitable extract prepared the latter constitutes the stock solution from which the subsequent dilutions are made. In the case of ragweed and plants with abundant easily detached pollen, it is possible to weigh the amount and prepare an extract with a definite protein strength. One gram of pollen soaked in 500 c.c. of I4 per cent. alcohol for twenty-four hours, will give a theoretical strength of $I: 500$, sufficiently strong for diagnostic tests. In the case of plants with adherent pollen as roses, maples and grasses, pure pollen is difficult to obtain and one is obliged to include the anthers and stamens in the preparation of the solution. The point is, however, not essential since it is simple to standardize the extract physiologically and determine in each case the strength to be employed for injection.

The tests are made by making a series of superficial scratches on the skin of the arm, and gently rubbing in a drop of the pollen extract to be tested into a scratch. After five to fifteen minutes the positive reactions are indicated by varying degrees of local disturbance. These disturbances of the skin may be ranged in order of intensity as follows: In some cases the first perceptible alteration consists in a sharply circumscribed white area, not elevated, bordering the scratch for a distance of $1 / 16$ to $1 / 8$ inch. We may find in other individuals the first manifestations to consist of a slightly reddened raised area. In more pronounced disturbances the area of swelling is more extensive, and is more or less white in color, being surrounded by an area of reddening of varying size. When this degree of disturbance appears, it is usually accompanied by itching. It may, in marked cases, attain a considerable size, the edematous area reaching $\mathrm{I}$ inch or more in diameter, and surrounded by $1 / 2$ inch or more of hyperemia.

The intensity of the skin reaction does not always seem to be proportionate to the clinical symptoms of hay fever. I have seen numerous severe cases where the skin reaction was much less than 
in other individuals who apparently suffered from a milder form of the disease. In the case of children the skin disturbances are relatively less pronounced than in the case of adults, and I have observed several under ten years of age, with apparently well-defined hay fever, who showed no reaction to the prevailing pollens borne in the air at the special season.

The pollen extract is injected subcutaneously, and not into the muscles, as pain or stiffness may follow in the latter case.

The dosage is determined in the following way: After the special exciting pollen has been ascertained by the skin test; a second series of scratches is made at a.distance from the first, and different dilutions of the pollen extract in question are applied. It is important not to have this test applied in the vicinity of the skin which has been reddened by the first tests, as an increased excitability of this region is present, and even a simple scratch will cause localized swelling. The dilutions may be most conveniently made by adding a certain amount of the stock solution to alcohol of the same strength, and a 25 per cent., ro per cent., I per cent. and even weaker dilution of the original extract are applied to the second series of scratches. The initial dose is determined by the dilution which fails to excite a definite skin reaction, and for the sake of causing as little smarting from the alcohol as possible, the quantity of material injected should not exceed 5 or Io drops. It is best to postpone the injection of even a small amount until after the reactions from the first skin tests have subsided, since a considerable amount of adsorption from these probably takes place, and the introduction of an additional amount has seemed in a few cases to cause systemic disturbance. The injections may be made at intervals of two days to a week, increasing by a few drops at first, and later by the adoption of stronger percentages of the stock solution. To avoid the risk of anaphylactic disturbance one should advance the strength at first slowly. After five or more injections have been given, the strength may be increased with greater rapidity. The disturbances occasioned by the injection of the pollen extract below the skin consist, when a sufficient strength has been attained, of a more or less well-defined lump, varying from the size of a bean to that of a pigeon's egg, accompanied by a sensation of moderate heat and itching. As a rule these manifestations cause only a trivial degree of discomfort. The total number of injections ranges from Io to 20 or more according to the resistance of the individual.

It is desirable, if possible, to stop the injections before the expected date of attack, since the introduction of protein into the system during the attack may cause a temporary increase in the symptoms.

It seems, however, to be indicated that if the patient presents himself at the beginning of the hay fever season. it may be wise to begin treatment. Under these circumstances, there is apt to be a temporary increase in the severity of the vasomotor symptoms, but 
these tend to subside after reaching a climax, with the result that relief may appear several weeks before the ordinary time.

In reviewing the results thus far obtained in the treatment of hay-fever by hypodermic injection of pollen protein, the general consensus of opinion is to the effect that the majority of patients have been relieved. From those who felt themselves improved, however, a considerable number should be eliminated, as a careful examination of the surroundings, their physical condition, and the season, might permit other explanations of the improvement. $\mathrm{Pa}$ tients are all too ready to attribute a greater freedom from hay-fever symptoms to the treatment employed. It is only where the patients' statements are controlled by clinical tests, that they may be accepted as possessing value. My own impression is that we are not likely with the present methods to do away completely with hay-fever symptoms in the course of a single season, but that nevertheless sufficient relief may be obtained to make it worth while to continue this method.

Where, however, treatment has been carried on during one season with more or less success, it seems evident that a second course of treatment the following season is more distinctly efficacious.

At the close of the first hay fever season one finds that the patient has attained a relatively high degree of immunity which in the course of the winter progressively diminishes. Beginning again next season several weeks before the expected date for hay-fever, one injects a small dose carefully determined in the usual way, and follows this by larger doses. In most cases a rapid, striking diminution in the intensity of the skin reaction is observable, much more speedy than was the case during the first season. Such cases when the hay-fever season arrives are distinctly more benefited than during the previous year, many showing no or exceedingly trifling symptoms.

Anaphylactic Shock.-If an overdose is given, the patient experiences in the course of an hour dizziness with a sensation of fulness in the head. In more severe shock, nausea, vomiting, asthma, exophthalmos and unconsciousness may follow. No fatalities have been reported, but such a result is entirely possible. The symptoms usually pass off in a few hours.

Such general disturbances should not occur if the physiological tests are made in each case with care. Before we undertake the treatment, we should first decide whether the patient's discomfort is sufficient to justify us in starting him upon a course of procedure which may prove time-consuming and not wholly devoid of risk. The treatment is by no means to be compared to typhoid inoculation, but may require numerous visits with careful tests of the degree of progress in order to determine the correct dose for each occasion. I cannot too strongly emphasize the importance of this matter of dosage. We are dealing with agents capable in moderate overdose of causing unpleasant shock, and in sufficient amount, of leading 
promptly to a fatal result. The best advice that can be given one starting in this work is to test continually the patient's resistance, and to increase the doses gradually in conformity with the results of the skin or conjunctival tests.

General Treatment.-Although the immunizing treatment of fever occupies at the present the center of the stage, yet the older aids, both local and general, should not be overlooked. Pathological alterations of the nasal tissues should be corrected if possible, especially deviations of the septum, if obstructive, and diseases of the sinuses. In certain instances a hyperesthetic condition of the nasal mucous membrane exists throughout the year, and caustic applications may be often advantageously made to the points of exit of the anterior ethmoidal nerves.

The general management of hay-fever patients should follow the time-honored lines, namely, restriction in the amount of nitrogenous food, and omission of alcoholic beverages. Water should be drunk freely, and a diuretic may be given to advantage. 


\section{ASTHMA}

By John H. Musser, Jr., M. D.

The treatment of hay-fever and hay-asthma has been discussed by Dr. Goodale (page 528) from the viewpoint of a specific cause, a known plant pollen. There are, however, innumerable cases of bronchial asthma for which a specific cause cannot be found, and in which the injection of a vaccine is of no value. It is this group that will be discussed in this section.

Pathogenesis.-In rgro Meltzer ${ }^{1}$ published a paper in which he called attention to the possibility of bronchial asthma being a phenomenon of anaphylaxis. He wrote that it has been shown that the anaphylactic attack as in asthma is of peripheral, not central origin; that the so-called asthma is due to a stenosis of the bronchioli from spasm, as is also anaphylactic shock; and that the similarity of the two conditions is also shown by other manifestations, as for example, the eosinophilia that occurs both in anaphylaxis and bronchial asthma. This general assumption is based on the work of Biedl and Kraus ${ }^{2}$ who have shown that anaphylactic death in guinea pigs is due to suffocation, the result of spasm of the musculature of the small bronchi, as confirmed by Auer and Lewis, and others.

It seems probable that anaphylactic intoxication may be induced by a large number of plant and animal proteins. Wells and Osborne ${ }^{3}$ have demonstrated definite biological reactions with numerous vegetable proteins such as edestin from hemp seed, gladin from wheat flour, hordein from barley flour, zein from maize and several others. The deduction that may be drawn from these authors' works as applied to anaphylaxis is that there are probably many other vegetable proteins less readily prepared in a pure state in the laboratory, but which in nature are sufficiently pure to sensitize certain individuals in some unknown manner, possibly in the intestinal tract, so that future contact with the specific protein will render them susceptible to an anaphylactic attack, or in its limited sense to an attack of asthma.

For example, an attack of asthma may be induced by various irritants as in the well-known horse or guinea-pig asthma. In these cases the patient usually has come to recognize the cause of his attack from experience.

Although such grossly recognized causes may precipitate an attack, there still remain the patients, excluding also those who have

1 Jour. Am. Med. Assn., I9 ro, Iv, ro2r.

2 Ztschr. f. Immunität., I 9 I0, v. 7 .

3 Jour. Inf. Dis., I9 I I, viii, 66. 
an exciting cause established, as animal emanations, plant pollen and so on for whom the sensitizing factor is not known. So also it is highly probable that points of chronic focal infection in such sites as the nose, the accessory air sinuses, the roots of the teeth, etc., may harbor the offending protein, which, in such a case may be of bacterial origin.

Sewall and Powell ${ }^{1}$ in their work on anaphylaxis induced by the instillation of foreign protein into the nose, say that the mucous membrane of the nose "seems to us to be the organ, par excellence, through which the natural relations between antigens and the living tissues are mediated-every breath of air contains its suspension of organic matter liable to settle on the nasal channel and there set up profound reactions." This work is of particular interest in its relation to asthma because, first, it seems likely that such proteins as might induce asthma are frequently absorbed by inhalation and, secondly, because of the list of cures that have resulted from proper treatment of the nasal membrane. It is to be presumed that local nasal treatment either so effects the mucous membrane that it no longer absorbs the specific protein, or else there are corrected pathological conditions as polyps, which set up chronic ethmoiditis and interfere with free secretion so that absorption of the offending protein takes place more readily than with normal mucous membrane.

In 1907 Besredka $^{2}$ came to the conclusion that calcium chlorid was a supremely effective drug in preventing anaphylaxis, Kastle, Healey and Buckner ${ }^{3}$ confirmed Besredka's work and concluded that calcium in proper doses and at the proper time does protect an animal against anaphylactic shock.

Lewis and Auer ${ }^{4}$ have shown that atropin exerts a favorable influence upon anaphylaxis when given shortly before the intoxicating injection. This work was confirmed in part by Anderson. ${ }^{5}$

Jannschke and Pollak ${ }^{6}$ have shown that intravenous injections of adrenalin into an animal in which a bronchial constriction has been produced results in a dilatation of the bronchioles.

Jackson $^{7}$ verified all the actual observations made by these authors, though he takes exception to their theories as to the mechanism producing its results.

Cannon ${ }^{8}$ again calls attention to the fact that one of the precise actions of the adrenal gland is to assist by means of its internal secretions, in overcoming pathological conditions which may cause an abnormal bronchoconstriction.

1 Arch. Int. Med., I9I 5, xvi, 605.

${ }^{2}$ Compt. rend. Soc. d. Biol., I907, lxii, 1053.

${ }^{3}$ Jour. Inf. Dis., I913, xii, I27.

4 Jour. Am. Med. Assn., I9o9, liii, $45^{8}$.

5 Johns Hopkins Hosp. Bull., 19 ro, xxl, L.

6 Arch. f. exper. Path. u. Pharmakol., I9I I, lxvi, 205.

7 Jour. Pharm. and Exper. Therap., I9I 2, lv, 59 .

8 Bodily Changes in Pain, Hunger, Fear and Rage, New York and London, Appleton \& Co., 1915, p. 204. 
According to the anaphylactic theory, the asthmatic attack then may be considered to be of peripheral origin, and the result of spasm of the circular muscles of the fine and medium-sized bronchi and alveolar ducts. The attack is induced by the absorption, from the respiratory or intestinal tracts or from some site of infection in the body, of a specific foreign animal or vegetable protein to which that person has been previously sensitized. It is upon the assumption that this theory is correct and that the above mentioned drugs have an anti-anaphylactic effect that the rationale of the treatment outlined below is based. It may be added that the treatment in the past three years in a small series of cases has yielded excellent results.

Prophylaxis.-The sufferer from asthma should have a most thorough physical examination. It is necessary for the physician to search out and eradicate such focal infections as may be discoverable. The nose, throat, accessory sinuses of the head, the teeth, the prostate and pelvic organs should be examined most carefully and if there is any question of trouble in the regions accessible to the roentgen-ray, such as the teeth and sinuses, resource should be had to this method of diagnosis. At times it is possible apparently to cure a patient by the surgical removal of some focus of infection, or by the eradication of it through the use of autogenous vaccines. In this connection, I might mention an interesting case which occurred upon my service at the Philadelphia Hospital and which Dr. Schnabel has reported in the Reports of the Philadelphia General Hospital. The patient was a miner with an extremely obstinate case of asthma. He had been treated without avail for some time with the usual asthmatic remedies. When he came upon my service it was proposed to make an autogenous vaccine from his bronchial secretions. Two organisms besides an unidentified streptothrix were used in preparing the vaccine. The improvement was almost immediate and most pronounced. After four injections the vaccine was lost. Additional vaccines were made repeatedly from the bronchial cultures but we were never able to isolate again the streptothrix. The patient's paroxysms of asthma began again shortly after the last injection of the first vaccine and though considerably modified he was never entirely free from them. Even if no site of focal infection is found, any abnormality in the nose should be corrected upon the assumption that this may be the site of absorption of the specfic irritating protein. This known clinical fact of the relation of abnormal nasal conditions to asthma, is elucidated by the experimental work of Sewall cited above.

These preliminary procedures may or may not prevent a recurrence of the asthmatic attack so that further steps should be taken to check the asthmatic seizure.

Upon the basis of the experimental work of Besredka and of Kastle, Healey and Buckner, to which we have already called attention, calcium chlorid should be administered to the asthmatic patients in order to prevent a recurrence of their anaphylactic phenomena. 
It should be given over long periods of time. Probably the best method is to have the patient take it for two weeks and then to stop for two weeks, the process to be repeated indefinitely depending upon the entire freedom from attacks and the general condition of the patient. As a general rule the best procedure is to continue the administration of the drug for at least six months, the possibility being borne in mind that there may be a recurrence of the attacks as long as sensitization persists, which may be for variable lengths of time. The calcium chlorid should be administered in a simple watery vehicle, as it is incompatible with most drugs; the dose should be I gm. four times a day. The drug should not be prescribed in a dry form as it is extremely hydroscopic. The large doses are given because it is impossible to say just how much of the drug is absorbed.

In addition to the measures advised for the overcoming of the anaphylactic tendency, general hygienic measures should be recommended as in all chronic diseases. The patient should eat carefully of a good general diet; the bowels should perform their function daily; a certain amount of exercise should be taken daily in the outside air, and this should be supplemented by light gymnastics upon arising, followed by a cold sponge bath and a brisk rub; lastly, the patient should have regular hours of rest and a sufficiency of sleep.

The patient should be advised as to the nature of his condition and told to try to remember what had instituted attacks. At times the patients come to realize that emanations from certain animals, the odor of certain plants and some type of foodstuffs may promote an attack, and hence they avoid these irritating sensitizing substances.

Treatment of Attack.-As the result of various investigations and work with adrenalin, also mentioned in the forepart of this section, there has been given to us a superbly efficient remedy, to check the actual attack. One-half to $I$ c.c. of a $I:$ Iooo adrenalin chlorid solution injected subcutaneously, frequently checks the attack in a most brilliant manner. If the physician is not at hand to give the adrenalin it may be instilled into the nose. Dr. A. F. Hertz, of London, himself a sufferer with asthma, contends that too large doses of adrenalin are usually given, and that a dose of 0.1 to 0.2 c.c. is usually sufficient to check the attack. ${ }^{1}$

At times resource must be had to morphin, the effect of which is probably in part due to the atropin which is usually given in conjunction with it, substantiating the experimental work of Auer and Lewis, upon the anti-anaphylactic action of atropin.

Alcohol to the point of intoxication is hardly therapy, but occasionally a patient is seen who has, by his own researches, confirmed the work of others, and who will, to get relief, drink himself into a drunken stupor. Other methods to check attacks include such technical procedures as direct intratracheal applications, procedures which belong to the realm of the specialist.

${ }^{1}$ Brit. Med. Jour., I914, l, 965 . 
The present chaotic condition of the treatment of asthma and the failure to recognize what is probably the true genesis of the condition, may be realized by a review of some of the recent literature upon the treatment of the disorder. To enumerate a few examples, Marconowski ${ }^{1}$ reports the curing of severe cases of asthma by psychoanalysis. Freudenthal ${ }^{2}$ recommends direct galvanization and faradization of the bronchi and following local treatment. Crookshank $^{3}$ realizing the transient effect of adrenalin, gives pituitary substance instead of the adrenalin solution. He reports twenty patients as cured of asthma by feeding them 2 grains (0.12 gm.) of pituitary extract twice a day, while Davies ${ }^{4}$ adhering to the theory of a reflex origin of asthma, reports marked improvement or cure by the removal of an encysted testicle in one patient, by the fixation of the uterus in another, by dilatation and curettement in a third, as well as by numerous other procedures in other patients to correct pathological conditions somewhere in the economy.

In England there seems to be tendency to give adrenalin over long periods with apparently favorable results. Waller ${ }^{5}$ writes that he has used adrenalin for five years, giving 5 or 6 minims ( 0.3 to 0.35 c.c.) of a $\mathrm{I}: 1000$ solution. Miller ${ }^{6}$ reports eleven cases treated by injections of adrenalin once a week. Usually eight to ten injections were sufficient to cause marked improvement, but in one case it was necessary to give weekly injections for over a year. The dosage was ro minims (o.6 c.c.) of either a $I: I 5,000$ or 1500 solution.

The conception of the anaphylactic pathogenesis of asthma is becoming more and more widespread, and this idea is reflected in some of the recent procedures reported by various authors, in their effort to discover the sensitizing protein and to build up an immunity against it in order to ward off future asthmatic attacks.

It must be borne in mind that immunity to a sensitizing foreign protein is gained only by giving at short intervals over long periods of time, very small, gradually increasing doses of the specific protein. In line with this procedure is the work of Talbot ${ }^{7}$ dealing with asthma in children due presumably to anaphylaxis of egg poisoning. Children that are anaphylactic to egg albumen are treated by giving small doses of the albumen in capsules, increasing the size of the dose until the patient is immunized.

Babcock $^{8}$ assuming that the anaphylactic phenomena are induced by a foreign protein of bacterial origin rationally combats the attacks by injections of autogenous vaccines, prepared from cultures from some point of focal infection, or failing this, from cultures of the

${ }^{2}$ Jahrb. of Psychoanal. u. Psychopathol.Forsch., I913, v., 529.

2 Amer. Med., 191 5, x, 176.

Lancet, 1914, 1,747 .

- Jour. Am. Med. Assn., rgr4, Ixll, roo6.

Lancet, 1914, No. 2, p. 445 .

- Lancet, 1914, No. 2, p. 158.

$?$ Boston Med. and Surg. Jour., 19r4, clxxi, 708.

8 Jour. Am. Med. Assn., 1915, Lxiv, 2115. 
sputum. Dr. Goodale, in another section of this volume deals with immunity achieved by giving extracts of plant pollens. Morris ${ }^{1}$ also reports upon the benefits derived from active immunization of patients made asthmatic by plant pollens. Laskowit $z^{2}$ supports the anaphylactic nature of asthma and considers that the secretions of the trachea and large bronchi act as the antigen, basing this assumption on Sanford's work from the Mayo clinic. He, therefore, removes the secretion by intratracheal aspiration, treating at the same time the associated chronic bronchitis. The aspirated material is then boiled down to a thick gelatinous mass, to which is added 60 c.c. of normal salt solution and a small quantity of phenol. This is filtered and represents the standard solution. The first injection of this material consists of 0.5 c.c. diluted to one-tenth of its original strength. Injections are given every third day in increasing doses for a period of ten weeks. At the same time he attempts to discover a sensitizing food protein by skin tests, using for this purpose various food proteins.

In following the method of treating asthmatic patients as suggested above, it must be borne in mind that it is not always possible to achieve immediate and permanent cures. The plan of attack is, however, perfectly rational and substantiated by both experimental work and clinical results.

1 Jour. Mich. State Med. Soc., I9I5, xiv, 400.

2 New York Med. Jour., I 91 5, cii, 950. 


\section{HEMOPTYSIS}

\section{By James E. Talley, M. D.}

Common experience would seem to indicate that in the majority of patients with hemoptysis, enforced physical and mental rest and hypodermic injections of morphin, gr. $1 / 16$ ( $0.004 \mathrm{gm}$.) or codein, gr. 1/8 (0.008 gm.) to control excessive cough, pulmonary hemorrhage will cease spontaneously. However, in intractable cases it is necessary to have in mind other drugs and measures which have stood the test of repeated use. For that reason we shall amplify and add to what has already been said in the previous edition.

That there has been advancement in the treatment of intractable hemoptysis one need but refer to the almost universal testimony of the value of artificial pneumothorax in controlling persistent hemorrhage from the lung. By this procedure surgery lends definite aid to what was formerly considered a purely medical condition. Wider experience with common salt, normal blood serum, coagulose and pituitrin make us feel that these comparatively new remedies are very definitely useful and that their use rests upon a good sound basis. The use of all these drugs and artificial pneumothorax merits a fuller discussion.

Sodium Chlorid.-Common salt as a means of arresting hemoptysis still has warm advocates(I). Its ingestion or injection renders the blood strongly salty, disturbs the existing osmotic relations and probably causes a flow of tissue fluids into the blood carrying with them the elements which promote coagulation. It has been found that ro gm. of salt well diluted, taken by the mouth, will in twenty minutes reduce the coagulation time of the blood from three and three-fourth minutes to one minute. This reduction probably reaches its maximum in about a half hour and subsides in the course of an hour. In hemoptysis a teaspoonful or two of salt in a half glass of water can be given at once. Five or ro c.c. of a ro per cent. sterile solution of sodium chlorid given intravenously acts more quickly and decisively and is harmless and practically painless. Its ephemeral action suggests the desirability of repeating the dose to insure its full action and as a safeguard against recurrence of hemorrhage. Its use does not interfere with subsequent use of normal serum as has been shown by actual experience. Sodium or potassium bromid in 30 grain ( 2 gm.) doses by mouth may be given alternately with the sodium chlorid. The bromids not only calm the patient but appear also to reduce the coagulation time of the blood. 
Blood Serum.-Clinical experience has confirmed laboratory experiment in demonstrating the usefulness of normal blood serum in hemorrhage. The injected serum is supposed to furnish the individual with a renewal of those elements which promote coagulation but which have been reduced by the hemorrhage. Since the mechanism and chemistry even of normal coagulation is an unsettled question, it would be futile to discuss here just how normal serum acts as a hemostatic. Those interested are referred to the articles of Howell(2). Specially prepared sera, both human and animal, have been used successfully. In large hemorrhages direct transfusion would be the ideal method were it not for the technical difficulties. Practically the treatment of hemorrhage by serum injections is reduced to the use of normal horse serum and the precipitated horse serum known as coagulose because these preparations are always available.

Normal Horse Serum.-This preparation is supplied by various manufacturers in to c.c. syringes ready for use. Before making a subcutaneous injection of horse serum for hemoptysis it is advisable to determine that the patient is not subject to asthma and has not recently had an injection for other purposes. Other sera, as antidiphtheria serum which has also been used to check hemoptysis when normal horse serum was not available, may produce sensitization and subsequent injection of horse serum may produce anaphylaxis. This contingency may be met by first injecting but I c.c. of the horse serum, and if no toxic effects develop (chill, fever, headache, abdominal pain) then by injecting the full Io c.c. or more as the urgency of the case demands. In case of severe hemorrhage where other methods have failed the full dose may be given at once without regard to the above precautions.

Coagulose.-Coagulose according to Clowes and Busch(3) is prepared from horse serum by precipitation with a suitable mixture of acetone and ether. They claim these advantages: that coagulose is as efficient if not superior to fresh serum, that it is freely soluble in cold water, is sterile, always available, retains indefinitely its capacity to stimulate coagulation of the blood, produces no deleterious effects and can be definitely standardized. Coagulose is supplied in glass bulbs each containing the equivalent of ro c.c. of blood serum, full directions for dissolving the powder in 6 or 8 c.c. of sterile water at $98^{\circ} \mathrm{F}$. are furnished with each package. Since it is apparently non-toxic the same doses may be used for adults and infants and the dose may be repeated according to the necessity of the case.

Pituitary Extract.-Some substance in the posterior lobe (infundibular portion) of the pituitary gland has a marked constricting effect on peripheral vessels. These pituitary preparations are known as pituitary extract, pituitrin, etc. Pituitary extract has been favorably recommended in hemoptysis. Wiggers (4) in his "Experimental Investigations of the Treatment of Hemoptysis" concludes it is the most desirable drug we have in hemoptysis. This is because 
this drug "combines an ability to elevate systemic arterial pressure and simultaneously to lower that in the pulmonary circuit." Pituitrin is supplied in I c.c. ampoules which is the average dose and may be given subcutaneously or intramuscularly and repeated three or four times daily when necessary.

Artificial Pneumothorax (5). - The volume of literature on artificial pneumothorax in the treatment of pulmonary tuberculosis which has appeared in the last decade is large. This method originally suggested by Carson in $182 \mathrm{r}$, practically employed in a number of cases by Forlanini in 1882 , and introduced in America by Murphy in 1898 has gained such universal testimony as to its usefulness in the treatment of pulmonary tuberculosis both without and with hemorrhage as to insure for itself a permanent place among the methods of treatment. Here we are concerned with the treatment of but one symptom of tuberculosis, hemoptysis. The older methods will suffice where there is blood in the sputum only occasionally or where decided hemorrhages do not appear serious and do not occur too frequently. The indications for artificial pneumothorax in hemoptysis are repeated severe hemorrhages uncontrolled by other means. Experience has shown that this method will stop most pulmonary hemorrhages but not all. The earlier the process and the freer the pleural cavity from adhesions the greater the chances of success. If adhesions prevent sufficient collapse of the lung, artificial pneumothorax will not stop the bleeding. This is true because for hemoptysis one needs a slightly greater pressure than for the other uses of artificial pneumothorax. In hemorrhage from a thick-walled cavity, or a fibroid or anthracotic lung, compression may fail because of the consistency of the lung. Hemorrhage may arise from both lungs and the compression of one end in failure. In spite of these exceptions the majority of cases of tuberculous hemoptysis will be checked at once by artificial pneumothorax. A decompensated heart or one on the verge of decompensation is a real contraindication. Otherwise patients in early stages, late stages and even with severe enteritis or nephritis have been successfully treated as far as hemoptysis is concerned. As cough, sweats, fever and excessive expectoration are relieved usually also by the procedure, one does not have to delay the induction of artificial pneumothorax for hemoptysis because of the fear of aggravating other symptoms, nor is there danger of aggravating the progress of the disease in the opposite lung. So though the case is old and adhesions are present, conditions may be urgent and an attempt at compression may be made at several sites.

Technique.-The induction of artificial pneumothorax for hemoptysis is a surgical procedure. The same precautions in strict asepsis should be maintained as in any other surgical operation. When we read of artificial pneumothorax being induced in hemoptysis by means of a hypodermic needle, rubber tubing and air filtered through absorbent cotton, we know the operator is acting in the face of a 
grave emergency and with the only instruments at hand. Even those who have had the greatest experience in the induction of artificial pneumothorax still have accidents but these accidents have lessened with their growing experience. Most operators have had some or all of these complications among their patientsgas embolism, shock, pleural effusion, pyopneumothorax, subcutaneous emphysema, puncture of the lung, dilatation of the heart, pneumothorax of opposite pleura and pain from overstretched adhesions.

Gas embolism was one of the commonest and most dangerous complications of the treatment. Since the introduction of the water manometer and strict vigilance to see that it registers a negative pressure with wide oscillations insuring that the needle is in the free pleural cavity before the gas is introduced, this distressing danger has been reduced to a minimum. If in a left-sided case in seeking sufficient positive pressure to control hemorrhage, the inflation should lead to much cardiac disturbance the gas can be easily evacuated with a needle and vacuum bottle, just as if it were fluid.

The Floyd-Robinson apparatus is used by many operators, other types are offered(6). The patient should lie with the lung to be compressed uppermost; a pillow under the lower side serves to separate the intercostal spaces through which the needle is to be introduced. Nitrogen is commonly used because it is slow of absorption. Oxygen and air are more readily absorbed and a mixture of air and a little nitrogen is suggested by Mace in the treatment of hemoptysis because of its ready absorption, especially with their use more often than with nitrogen a neutral or slightly positive pressure probably will in twenty-four hours be negative again. This may be of advantage in bilateral pulmonary tuberculosis where prolonged compression may prove undesirable. A site where there is good pulmonary resonance, few adventitious sounds, and as far away from the known area of consolidation as possible often proves successful. A common site is in the sixth, seventh, or eighth interspaces somewhere between the anterior axillary and scapular line. Frequently several sites have to be tried before the manometer needle registers a negative pressure with good oscillations showing the operator that he is in a free pleural cavity. Lent finds a free pleural space will give a reading of -4 to - Io $\mathrm{cm}$. Even slight pleural adhesions may reduce this to -2 to $-4 \mathrm{~cm}$. Until the manometer shows negative pressure and free oscillations no gas should ever be injected. In primary inflations and in those with much cough a preliminary injection of morphin, gr. $1 / 8$ ( $0.008 \mathrm{gm}$.), a half hour before operation is desirable. It renders the procedure easier and helps prevent subcutaneous emphysema. The skin may be cleansed in the usual manner taking care not to wet and chill the patient too much. Finally the site of the puncture should be painted with iodine and the excess wiped off with alcohol. Most operators lay stress upon the use of local anesthesia. Montgomery and Speese prefer a r per 
cent. eucain solution; Lent, 2 c.c. of a 0.25 per cent. novocain solution. The skin, intercostal tissues and pleura should be anesthetized which renders the operation practically painless. This seems especially desirable since several punctures may be necessary before free pleural cavity is found. This fact also argues against thoracotomy, a procedure which gives a wider surgical aspect and according to the experience of some seems provocative of subcutaneous emphysema. Many operators puncture the skin with a cataract knife before inserting the needle but thoracotomy in which the incision was made down to the free pleura has almost entirely given way to simple thoracocentesis. There are many factors which will determine the amount of gas to be used. By turning the stopcock and reading the pressure with each introduction of 50 c.c. of gas, warmed by placing the rubber tubing in hot water, 300 to 500 c.c. of gas may be used in an initial inflation without discomfort and especially if we stop with a negative or neutral manometer reading. This slow procedure gives the heart time to adjust itself. If the patient develops dyspnea and pain during the injection it is usually better to stop at once, because displacement and torsion of the heart and great vessels is probable under the circumstances. We should remember that even with a low negative or neutral prèssure collapse of the lung may be effected. Even in severe hemorrhages 4 or 5 c.c. of water pressure should never be exceeded. In cases of general pleural adhesions a small amount of gas may produce unexpected high pressure so it is better to go slowly. Where the pleura is evidently thickened and the oscillations of the needle are small as low as 200 c.c. of gas only may be borne without causing pain by stretching the adhesions. A lung once artificially collapsed for severe pulmonary hemorrhage is more safely kept in that condition for three or four months provided the other symptoms of the disease are bettered by the treatment. This prevents recurrence of hemorrhage, the formation of adhesions which may render difficult subsequent inflations and reduces the danger of the development of bronchopneumonia. Once the latter complication has developed, inflation is useless. The time of reinflation depends upon the time of reabsorption which is determined by the physical examination, by the fluoroscope and occasional roentgenograms. The patient's comfort and the position of the heart are elements to be considered. Subcutaneous emphysema has been known to last for weeks and even months, an element which makes it undesirable to reinflate during its existence. Strapping the chest with adhesive plaster immediately after inflation lessens the occurrence of subcutaneous emphysema.

In addition to artificial pneumothorax and the drugs discussed above, which the writer believes have a definite value in the treatment of intractable pulmonary hemorrhage, he feels that a word should be said about emetin hydrochloride, coagulin-ciba, pantopon, gelatin and salines. 
Emetin Hydrochlorid.-Flandin(7) reports very favorable results in the treatment of hemoptyis by hypodermic injections of emetin hydrochlorid. Guerrero(8) used the same dosage in patients in the second and third stages of pulmonary tuberculosis without benefit. The general use of emetin hydrochlorid in the treatment of pyorrhea in more or less normal individuals by means of hypodermic tablets and ampoules of gr. $1 / 4$ to $1 / 2$ ( 0.015 to $0.03 \mathrm{gm}$.) should not lead us to forget that in hemoptysis we are dealing with a patient probably exhausted by tuberculosis and hemorrhage. Emetin hydrochlorid is a decided respiratory and cardiac depressant; besides it is capable of inducing that undesirable complication vomiting both by its centric influence and local influence when eliminated by the $\operatorname{stomach}(9)$. Depending upon the the condition of the patient from $1 / 4$ to $1 / 2$ grain ( 0.015 to $0.03 \mathrm{gm}$.) may be tried and if necessary it may be repeated once or twice in the twenty-four hours. Even when it is apparently successful the suggestions and restrictions offered by Levy and Rowntree(Io) in the treatment of amebic infections should be followed. One-third to $1 / 2$ grain three times daily subcutaneously for one week at a time is held to be safe. In hemoptysis the injections would hardly have to be kept up so long. As intravenous medication appears to be growing in vogue it seems well to mention the unfortunate results that the above experimenters had with their animals by the use of intravenous injections of emetin. Emetin by the intravenous route is apparently unsafe, it should not be used by that method in hemoptysis.

Coagulen-Ciba (Coagulen, Kocher-Fonio; Coagulin, KocherFonio).-According to the Journal of the American Medical Association, September 25, I 9 I $_{5}$, coagulen, which is more commonly used for staunching local hemorrhage, has also been recommended for pulmonary hemorrhage. It is said to act as a hemostatic by increasing and accelerating the natural process of coagulation. Coagulenciba is a yellow granular powder prepared from blood platelets, I gm. representing $20 \mathrm{gm}$. of dried blood. It is soluble in physiological salt solution and plain water, may be sterilized by boiling for three minutes without decomposition. Fresh 5 per cent. solutions of coagulen-ciba are used locally, intramuscularly and intravenously. The subcutaneous and intravenous injection is contraindicated where there is a tendency to thrombosis or embolism, as for example in arteriosclerosis, aneurysm, marked heart weakness, phlebitis and varicose veins.

Pantopon Hydrochlorid.-Pantopon hydrochlorid is also recommended in pulmonary hemorrhage. Pasch(II) records a case where in two instances the hemorrhage ceased promptly after a hypodermic injection of $0.02 \mathrm{gm}$. of pantopon. In the light of our present knowledge it is difficult to see how this drug should be superior to morphin. As it represents the total alkaloids of opium and is stronger than morphia the doses used would be expected to have all the objectionable features of large doses of morphia in the treatment of pulmonary 
hemorrhage. Until it is proven to have some definite influence in checking hemorrhage it would be better to use it in the simplest doses which would relieve excessive cough and allay mental perturbation.

Gelatin.-With respect to gelatin, Ciuffini(12) found that gelatin promotes coagulation but loses its power if heated to 130 or $135^{\circ} \mathrm{C}$. for half an hour; thus the very necessary sterilization renders this medium inefficient. After heating gelatin to $100^{\circ} \mathrm{C}$. and giving it by the mouth or rectum no effect on coagulation was perceptible.

Salines.-The favorable testimony (1 3$)$ as to the use of magnesium sulphate in cases of hemoptysis witt constipation makes one feel that its possible elimination of toxins which may help to provoke hemoptysis may offer a possible explanation of its action.

In the previous edition the writer speaks of 40 ounces of milk and four to six raw eggs as a possible diet the first twenty-four hours when feeding was resumed, to begin with half of these amounts is probably sufficient. Also among the causes of blood spitting, varices at the base of the tongue and hemophilia might have been included.

\section{BIBLIOGRAPHY}

1. Blomer, Abs. Jour. Am. Med. Assn., Aug. 27, 1910, p. 811.

Müller, Abs. Jour. Am. Med. Assn., Oct. 25, 1913, p. 1578.

2. Howel., Am. Jour. Phys., xxvi, p. 453; xxix, p. 187.

3. Clowes And Busch, New York Med. Jour., Jan. 4, 1913.

4. WigGers, Arch. Int. Med., July I5, igrr.

5. Rothschild, Jour. Am. Med. Assn., Oct. 28, I9I I, p. 1424.

GraY, Jour. Am. Med. Assn., Feb. 14, 1914, p. 564.

SPEese AND Montgomery, Penna. Med. Jour., February, igr 4.

Von Adelung, Bost. Med. and Surg. Jour., July 30, 1914, p. 200. Interstate Med. Jour., St. Louis, August, 1915, p. 846 .

MAcE, Jour. Am. Med. Assn., March 13, 1915, p. 897. Jour. Am. Med. Assn., Dec. 20, I913, p. 224 I.

Lent, Jour. Am. Med. Assn., June 12, 1915, p. 1973.

SACHS, Jour. Am. Med. Assn., Nov. 27, 1915, p. 1861.

LUCAS, Brit. Med. Jour., Aug. 7, I915.

Sloan and Hamman, Bull. John Hopkins Hos., Aug. 28, 1915, p. 827.

6. Robinson AND FlOYD, Arch. Int. Med., April 15, 1912 , p. $45^{2}$.

Vrooman and Wrttich, Jour. Am. Med. Assn., March 21, 1914, p. 929.

7. Flandin, Abs. Jour. Am. Med. Assn., Nov. I, 1913, p. 1667.

8. Guerrero, Abs. Jour. Am. Med. Assn., Feb. 28, 1914, p. 740.

9. Jour. Am. Med. Assn., Nov. 13, I91 5, p. 1730.

10. Levy AND Rowntree, Arch. Int. Med., March 15, 196, p. 420.

11. PASCH, Allg. med. Centr. Ztg.. I912, lxxxi, 614.

12. Crufrini, Polyclinico, Rome, December, Igro.

13. Burns, Jour. Am. Med. Assn., Dec. 20, 1913, p. 2207. 


\section{PULMONARY EDEMA}

\section{By James E. Talley, M. D.}

The acute, recurrent or paroxysmal form needs such immediate and decisive attention that one must have continually a definite plan in mind. For the primary attack morphin sulphate, gr. 1/8 to $1 / 4$ (0.008 to $0.015 \mathrm{gm}$.) with atropin sulphate, gr. 1/50 to $1 / 100$ $(0.0004$ to $0.0006 \mathrm{gm}$.) should be given at once. In fifteen to thirty minutes the atropin may be repeated once if there has been no improvement in the situation. If the patient can swallow, aromatic spirits of ammonia and whisky may also be given. Cardiac stimulation should follow closely. Digitalon preferably in the form of hypodermic tablets or even liquid may be given hypodermatically and repeated every third hour until the pulse is slower and fuller. Digitalon is mentioned because it has been found a uniformly satisfactory preparation and always procurable. Digipuratum and digalen are equally reliable. Many of the cases are associated with high pressure and at least an attempt to relieve this by the repeated injection of nitroglycerin, gr. 1/100 $(0.00065 \mathrm{gm}$.) may be made on the supposition that a part of the pressure may be due to vascular spasm. Oxygen and dry cupping may be helpful and venesection may be necessary. Stengel(I) has also emphasized preventive treatment which is important. The patient should be warned against overeating especially at night, sudden and prolonged physical strain among which rapid stair climbing is one of the worst, emotional excitement, overfatigue and sudden chilling. Even in cases with but little microscopic or chemical evidence of kidney change but with a low phenolsulphonthalein output, electric cabinet or hot vapor baths and carefully considered diet are important. In patients whose attacks tend to recur at short intervals and especially at night, where a nurse or other skilled attendant is at hand, definite directions should be given. On the slightest suspicion of an attack, aromatic spirits of ammonia and whisky should be given at once. Unless there is prompt relief morphin, gr. 1/8 (0.008 gm.), and atropin, gr. $1 / 200(0.0003 \mathrm{gm}$.), should be given. This may be followed later with nitroglycerin and strychnia or digitalis. One practitioner sees too few cases to draw general conclusions but the writer has been impressed with the rapidly fatal termination of patients developing auricular fibrillation concurrently with the attack of paroxysmal pulmonary edema. Those patients with a very rapid but regular pulse have fared better. Ohlmacher(2) has called attention to the association of status lymphaticus and epilepsy and the tendency of these patients to sudden and unusually fatal edema of the larynx, lungs or brain.

\section{BIBLIOGRAPHY}

I. Stenger, Am. Jour. Med. Sci., rgir, cxli, r.

2. Ohlmacher, Am. Jour. Med. Sci., rgio, cxl. 


\title{
PULMONARY COLLAPSE
}

\author{
By James E. Talley, M. D.
}

Several articles have appeared on postoperative collapse of the lung. The condition is also referred to as massive collapse and acute or active lobar collapse. Briscoe points out that massive collapse of the lung is not limited to those who have undergone operation. Postmortem examination reveals more or less complete collapse of the lower lobes of the lungs in patients bedridden for some time and without any symptoms of such lesions being present during life. This condition is the natural result of prolonged quiet breathing in the supine position by people who do not use the abdominal muscles to fix their chests. The condition occurs in complete paraplegia where the diaphragm is the sole muscle of respiration. The collapsed area may become the seat of infection simulating a pneumonia; on the other hand, a bronchitis may precede it and be the causal factor.

Treatment.-In atelectasis of the newborn, from conversation with various obstetricians, one feels convinced that the lungmotor is the best mechanical means of treating this condition. This apparatus sucks out the occluding discharge and gets rid of it through the valve provided for that purpose. The treatment can be kept up longer, perhaps more efficiently and certainly more pleasantly by means of this apparatus than by means of mouth to mouth insufflation.

Elliott(I), Pasteur(2) and Briscoe(3) have written on the treatment of postoperative or massive collapse. The latter considered preventive treatment, treatment when the actual collapse is discovered and also when the lung shows bronchitis and infection of the collapsed area. Prophylaxis seeks a healthy condition of the mouth and respiratory passages before operation, limits the degree of anesthesia when these parts are in an unsatisfactory state and protects the patient from being chilled after operation. After thirty-six hours the patient should be allowed to lie on the side and make spontaneous movements such as raising the head and the taking of food and liquid unaided. He should be supported upon pillows a part of the twentyfour hours and tight bandaging of the abdomen should be avoided. Should collapse without symptoms be detected the above treatment may be persisted in, but in addition the patient should be encouraged to talk, and artificial distention should be sought by means of the blow bottle by adults, and bubble blowing or blowing up of a toy balloon by children. In a severe and sudden attack the patient may be propped up continually, expiratory efforts may be encouraged 
by bimanual pressure at intervals to the lower thorax. Oxygen and quick stimulation should be used. If fever, cough and expectoration appear omit the blow bottles, keep the patient propped up and quiet until the fever subsides. In any stage with marked distention of the large intestines or dilatation of the stomach after operation, these viscera should be evacuated by appropriate means.

\section{BIBLIOGRAPHY}

I. Elitotr, Lancet, 1914, No. 1, p. r308.

2. PASTEUR, Brit. Jour. Surg., I9I4, p. 587.

3. Briscoe, Pract. Encyclop. of Med. Treat., London, rgr 5. Lancet, r9r4, No. I, p. I 247. 


\title{
EMBOLISM, THROMBOSIS AND INFARCTION OF THE LUNG
}

\author{
By James E. Talley, M. D.
}

Prophylaxis is important in those conditions where thrombosis or embolism is prone to occur. Good contraction of the uterus should be sought after labor. In endocarditis undue muscular effort including straining at stool and severe coughing should be avoided. Thane(I) suggests the undesirability of prolonged use of the Trendelenburg position as it may predispose to pulmonary embolism. Ward(2) speaks of the abandonment of the extensive exposure of the levator ani muscle during perineoplasty to prevent bruising of the numerous small veins exposed. This is important because perineal repair is one of the commonest operations done by the general practitioner. In a recognized or suspected phlebitis with a possible thrombosis the patient should remain at rest and the infected part be immobilized where possible. The length of time that an extremity, for instance, should be kept immobilized should be gauged by the disappearance of swelling, tenderness and fever which often takes one or two months. The return to normal activities should be by easy stages. Grober(3) advises where possible the extirpation of the original site of thrombosis. All these precautions, of course, are to prevent possible pulmonary embolism. Where this occurs it is doubly important for the patient to be kept absolutely at rest in bed, as the clot may not yet have occluded a large branch of the pulmonary artery and its displacement may prove fatal. Morphia may be given for distress, and wherever possible cardiac stimulation is avoided because it may provoke the transfer of the clot to a more dangerous area. Where there appears to be actual danger one has to take a chance and then the best cardiac stimulants are intravenous injections of strophanthin followed by digitalon, digalen or digipuratum. In hemorrhagic infarct the first indication usually is to relieve the pain. This may be accomplished by rest in bed, strapping the side, by ice-bag or hot-water bag. This failing morphia or codein may be used, however, where possible as in acute localized pleurisy, wet cupping or leeching usually gives relief. Ordinarily hemoptysis is negligible, only where the amount is unusually large and its appearance prolonged do we need to make use of the measures given in the section on hemoptysis. Pleural effusions arising from pulmonary infarct are treated like similar effusions arising from other causes.

\section{BIBLIOGRAPHY}

I. Thane, Australian Med. Gazette, ror 2, p. 126.

2. Ward, Am. Jour. Obstet., 1913, lxvii, 561 .

3. Grober, Deutsch. med. Wchnschr., Feb. 19, 1914. 


\title{
TREATMENT OF TRACHEAL AND BRONCHIAL OBSTRUCTION
}

\author{
By Chevalter Jackson, M.D.
}

The development of the bronchoscope has recently added greatly to the available means of treatment of tracheal and bronchial obstructions; though, except in the case of foreign bodies, the therapeutic developments have been rather less epoch-making than the diagnostic developments.

Endobronchial therapeutics with the aid of the bronchoscope is in the developmental stage and excellent results are being accomplished. Ingals very wisely advises caution in the development of endobronchial therapy and thinks it should be done only by those who have had special bronchoscopic training. As pointed out by a number of observers ordinary oral inhalations of nebulized fluids are practically worthless for the local treatment of bronchial disease for the reason that the nebula does not penetrate even as far as the trachea. Even where all the air inhaled by the patient is saturated with finely nebulized fluid, it is doubtful whether any appreciable amount reaches the deeper air passages, because of the impinging upon the mucosa at the various turns of the upper air passages. These various surfaces act as baffle plates to remove the minute particles of medication suspended in the inspired air. Intratracheal injections by the aid of indirect laryngoscopy have slightly better results but even here the prompt coughing of the patient will remove the fluid before it can reach the deeper passages. On the other hand, endoscopic applications place the fluid deep down in the bronchi where all bechic efforts to expel it only serve the better to scatter it over the mucosa. Therefore, when local endobronchial medication is desired, the only certain method is the bronchoscopic one. Dr. Emma E. Musson obtained excellent results by the injection of medicated solutions through a long silk-woven tube passed through the larynx with the aid of the direct laryngoscope illustrated at $G$, page 220 , Vol. III.

Malignant disease of the bronchi and lung has been bronchoscopically treated by radium together with deep roentgen-ray applications with apparently beneficial results. The cases have been too few and too recent to give a final estimate of the value; but considering the ease with which the radium can be applied and the fact that if untreated the disease presents Ioo per cent. mortality, the method is certainly worthy of trial in inoperable cases, to which class most cases belong by the time the diagnosis is made. The method is not suitable for diffuse metastatic foci. The radium salt is enclosed in a glass capsule, placed within a gold capsule onto which a casing of hard rubber has been vulcanized. This can be 


\section{PLATE 1}

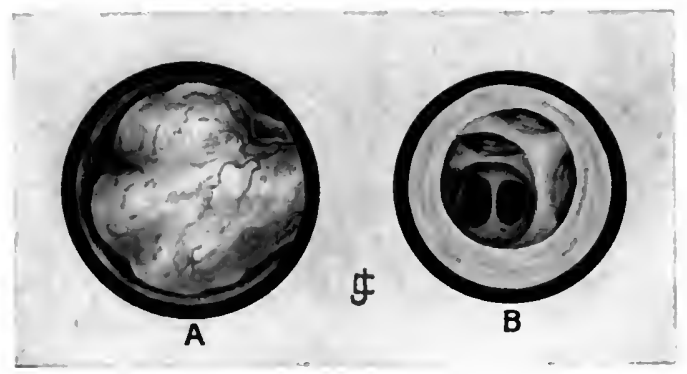

Endobronchial obstructing endothelial tumor in a man aged thirty-five, who complained of coughing, wheezing, and "a sensation as of a ball-valve shutting off his breath," sometimes on inspiration and at other times on expiration. The patient had been treated in a sanitarium for tuberculosis for two years. B, Bronchoscopic view down a normal right main bronchus. $A$, Tumor presenting itself in its self-made bronchial enlargement, when the bronchoscopic tube-mouth reached the location at which the view $(B)$ normally should appear. Tumor removed with forceps through a bronchoscope passed through the mouth. Patient perfectly well in three weeks, and still so nine months later. 

placed with great accuracy with the aid of the bronchoscope. The equivalent of from 50 to roo $\mathrm{mg}$. of radium is left in situ for a period of from a half hour to several hours. Removal of small endobronchial malignant growths through the bronchoscope is quite feasible; but as a rule such removal would seem theoretically unsurgical. That results worth while can be obtained, however, in growths of a low grade of malignancy is shown by the case illustrated in Plate I. This tumor removed by me, was found on histological examination by Dr. Ernest W. Willets to be an endothelioma.

Luetic obstructions of the tracheobronchial tree have been observed bronchoscopically in a number of instances. The treatment of active lesions is general and does not differ from the same lesions in other localities. Cicatricial stenosis following active lesions, if sufficient to require treatment, can be cured by bronchoscopic bouginage with or without prolonged bronchial intubation.

Tuberculosis. - The excellent results yielded in the endoscopic cauterization of laryngeal tuberculosis have led to the effort to obtain the same results from the endobronchial and endotracheal treatment. The application of the cautery here, while perfectly feasible, is not so well adapted because of the essentially different secondary processes in the tracheobronchial tree. The endobronchial injection of solutions of guaiacol and of creosote have been used with apparent benefit, though further observations on such cases are necessary before giving them unqualified endorsement. When bronchi are filled with cheesy débris, granulations, etc., following the erosion through of a tuberculous lymph node, the obstruction in the bronchus may be entirely removed with the mechanical spoon (Vol. III, p. 22I) through the bronchoscope, and air is thus admitted to the wedge of lung supplied by the affected bronchus.

It may not be out of place here to call attention to the fact that quite a number of cases, such as those shown in Figs. 50, 51, 52, and 54, in which patients with a typical clinical picture of tuberculosis failed to improve under ordinary methods of treatment, and were found to be really due to a quickly curable bronchial obstruction secondary to an unsuspected foreign body. Attention may be called also to the case illustrated in Plate $\mathrm{I}$.

Thymic Tracheostenosis.-Since bronchoscopic demonstration of the fact that the thymus gland can and does compress the trachea and that this compression may be increased to a fatal point by engorgement of the hypertrophied gland, treatment of the hypertrophic thymus has received much attention. Hickey, Cole, Grier and others have demonstrated the remarkable efficiency of the roentgenray in these cases. As the dosage must be regulated with great nicety, it is essential that the treatment be undertaken only by those of very large experience with the therapeutic ray.

Gangrene of the lung may follow bronchial obstruction. The best treatment is, as stated by John C. DaCosta,, "Operate as for 
abscess of the lung." It must be borne in mind that gangrene exceedingly rarely follows foreign body in the bronchi; but that all the symptoms of gangrene may be due to the presence of a foreign body followed by saprophytic processes without there being any gangrene whatever. It is, therefore, advisable to do an exploratory bronchoscopy unless the presence of a foreign body can be excluded by the roentgen-ray or other means. Three cases of supposed gangrene have been cured by the removal of foreign bodies.

Pulmonary abscess, properly so-called, has been evacuated bronchoscopically with excellent results in a number of non-tuberculous cases. The best results have, however, been obtained in cases of "drowned lung" (Fig. 56) in which the pus collection has been in more or less normal bronchi rather than in distinct cavities with an abscess wall. In the latter class of cases near the periphery, especially if the pleura is adherent, an external operation is the preferable procedure, unless the abscess is due to foreign body. In foreign-body cases bronchoscopic removal with, if necessary, bronchoscopic dilatation of the fistulous cavitary opening, has been curative in 90 per cent. of the cases in the author's clinic. In one instance a glass collar button (Figs. 55 and ${ }^{6} 6$ ) had been in the lung twentysix years. Pleural empyema had been drained externally. Cure followed peroral bronchoscopic removal of the collar button. The patient at the time of bronchoscopy was expectorating large quantities of foul pus from the pulmonary abscess, and weighed 98 pounds. Two years later she weighed 182 pounds.

Foreign Bodies in the Bronchi.--Hundreds of cases have demonstrated that the primary bronchial obstruction due to a foreign body can be quickly and completely relieved by the removal of the foreign body, if the case is seen before secondary pathologic processes have intervened. A condition of "drowned lung" (Fig. 56) of ten clears completely immediately after the bronchoscopic removal of foreigh body and pus. In cases of prolonged sojourn cicatricial strictures plus the foreign body, plus the granulation tissue and secretions, seem to cause a quite complete obstruction. The strictures, however, do not seem to show the intractable nature of cicatricial strictures in other canals. Of the author's cases, in all but one the preliminary dilatation of the stricture followed by the removal of the foreign body has resulted in a complete cure.

Foreign bodies in the bronchi are now so quickly and safely removed by bronchoscopy that all other methods of treatment have been rendered obsolete. Recent statistics ${ }^{1}$ have shown that of 182 consecutive cases of bronchially lodged foreign bodies in my clinic, I 77 ( 97 per cent.) were removed bronchoscopically through the mouth. In the $\mathrm{I} 82$ cases there were only three deaths from any cause whatever within one month, though not quite all the cases could be followed so long, giving a mortality of 1.7 per cent.

Anesthesia.-It has been found that the coöperation of the patient, ${ }^{1}$ Peroral Endoscopy and Laryngeal Surgery, Chevalier Jackson, I9I5. 
as to holding breath, restraining cough, or coughing, at the request of the operator, is of very great value, contributing largely to a successful outcome in difficult cases and lowering mortality. 'Therefore, it may be said that ideal work is done without any anesthesia, general or local, in children under six years of age. In adolescents and adults, local anesthesia may be used and will suffice for almost all cases. The foregoing statement must not be taken too literally

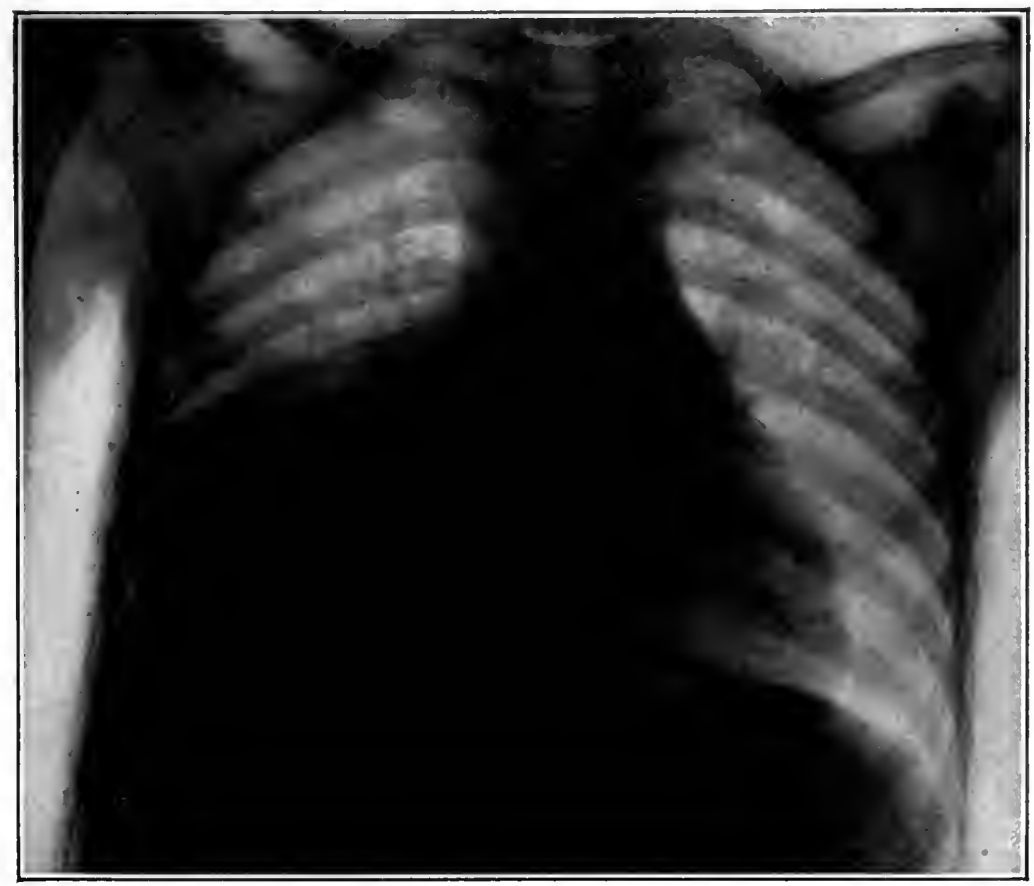

FIG. 50.- Roentgenograph showing tack, in the lung of a man aged 25 years, who had been having intermittent septic attacks with chills and fever followed by purulent expectoration, supposed to be due to tuberculosis. Diagnosis of foreign body made clinically by Dr. George L. Richards. Plates by Dr. M. N. Tennis. Tack removed by the author bloodlessly through the mouth, after bronchoscopic dilatation of the superjacent bronchial stricture. Patient totally relieved of symptoms and has remained well one and one-half years.

because the operator should be free to choose as to anesthesia whatever plan he deems best considering the personal equation of himself and of the particular patient.

The mechanical problems presented in the bronchoscopic disengagement and removal of various foreign bodies are very numerous, because of the great variation in the nature of the foreign bodies. The feasibility of overcoming extremely great difficultics by patient, careful execution of a carefully planned procedure is illustrated in Figs. 59 and 6r. To have attempted to remove the staple by traction with forceps in the position, A (Fig. 59), would have torn 
fatal lacerations in the bronchial walls and would not have succeeded in removing the staple. The patient made a prompt and perfect recovery. The method has been successfully used by the author in four cases. The method of disengaging a large-headed tack is shown in Fig. I2.. Similar tacks have been removed in twelve cases,

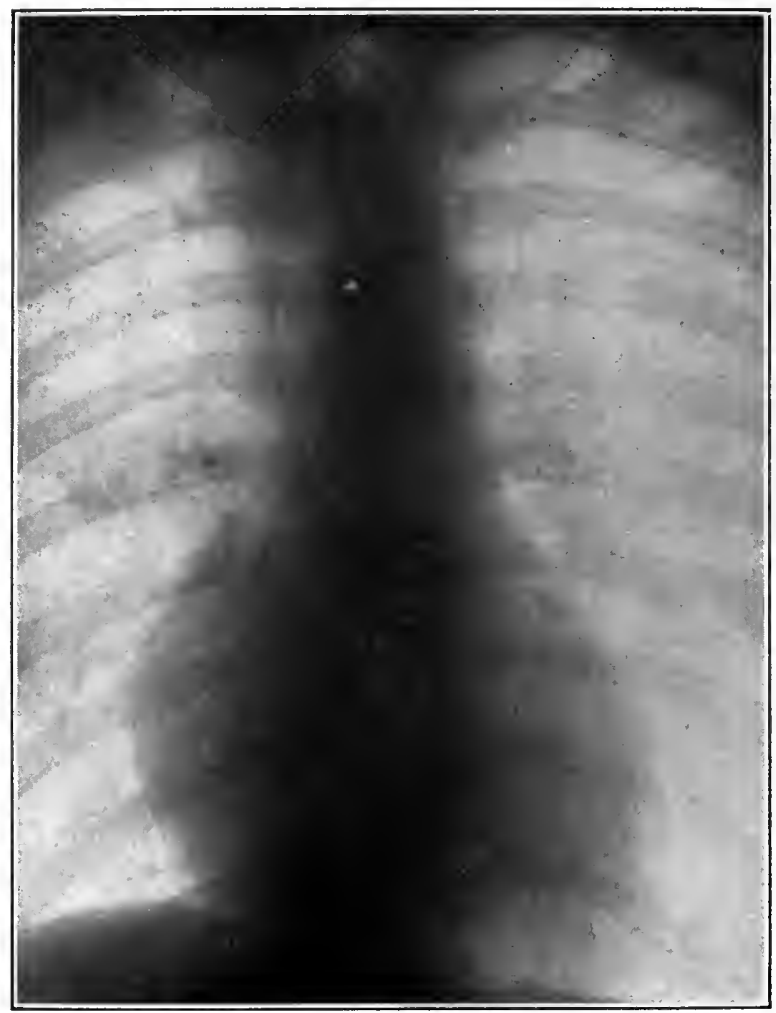

FIG. 51.-Roentgenograph showing a tack in the right bronchus, and the pathology resulting from the obstruction, in the case of a boy, aged $\mathrm{r} 8$ years. Radiograph made by Dr. W. L. Dunn. Diagnosis by P.H. Ringer. Tack removed by the author, bloodless through the mouth, by bronchoscopy. Patient in perfect health one year later. In twelve cases similar tacks have been removed from the bronchi in my clinic.

without mortality. The method of disengaging pointed objects such as pins (Fig. 13) is similar to that illustrated for tacks. The general principles of this method have been successfully used in IO4 cases of pointed objects of various kinds in the author's clinic. 

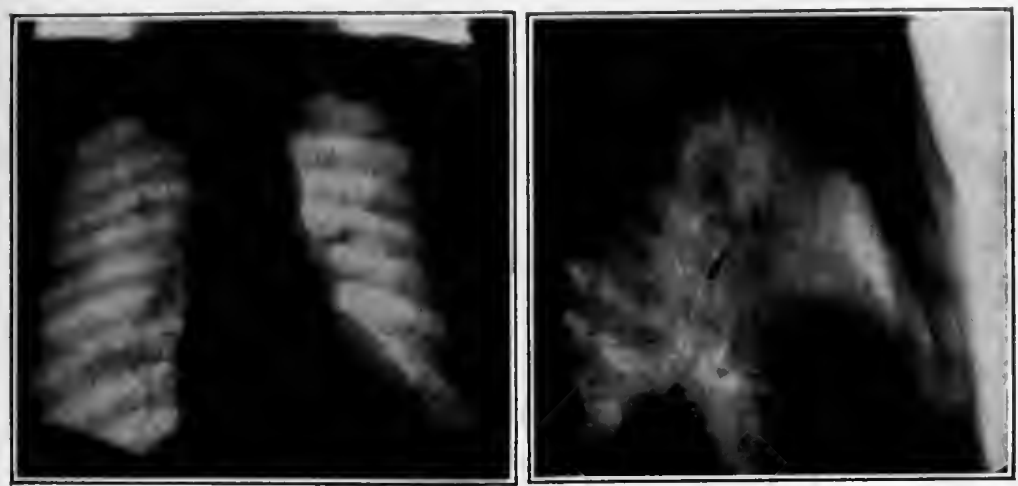

FIG. 52.-Roentgenograph, anteroposterior and lateral, showing a large fence staple in the left bronchus of a man of 45 years. Staple (Fig. 53) was bronchoscopically brought up to the bifurcation, turned and removed through the mouth, under local anesthesia. Perfect recovery. (Plates by Dr. George W. Grier.)

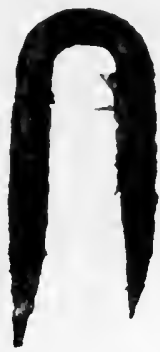

Fig. 53. - Large fence staple removed from lung of a man, aged 45 years. Removed bronchoscopically through the mouth. 


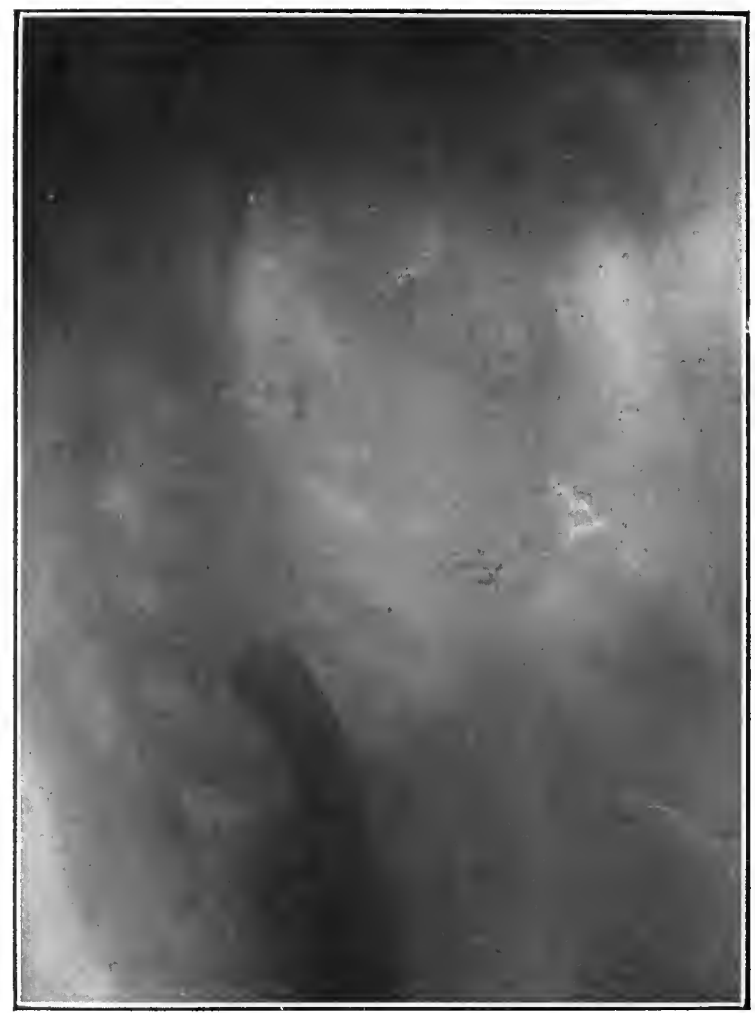

FIG. 54.- Roentgenograph showing collar button that had been for twenty-six years in the lung of a woman, aged 48. Removed by peroral bronchoscopy without anesthesia, general or local. (Plate by Dr. George C. Johnston.) 


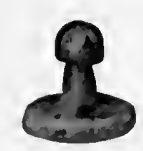

Fig. 55.-Glass collar button removed by peroral bronchoscopy from the lung where it had remained for twenty-six years (see Fig. 54).

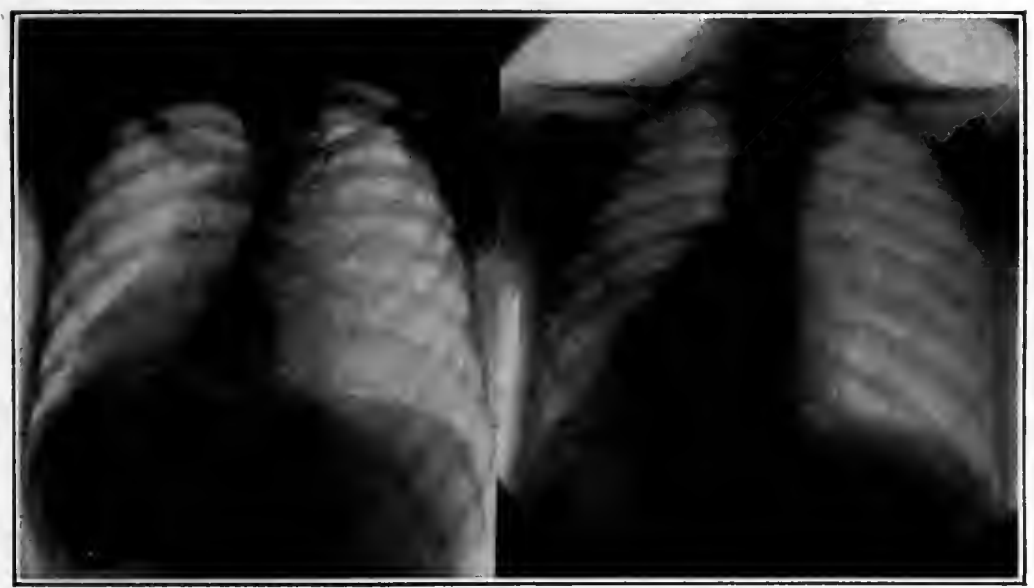

Fig. 56.- Roentgenograph showing the wedge-shaped shadow of "drowned lung" caused by occlusion, by a peanut shell, of a large posterior branch of the left inferior lobe bronchus of a child of eight years. On the right is shown the complete disappearance of the shadow immediately after removal of the peanut shell (Fig. 57), and the pus which it had "corked" in the bronchus. Perfect recovery. (Plates by Dr. George C. Johnston.) Fifty-eight cases of "drowned lung" from bronchial obstruction by foreign bodies have been cured by bronchoscopy in the Pittsburgh clinic.

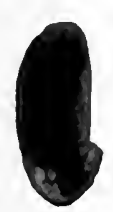

Fig. 57.- Shell of pistachio nut removed from bronchus of child of eight years (see Fig. 56). 

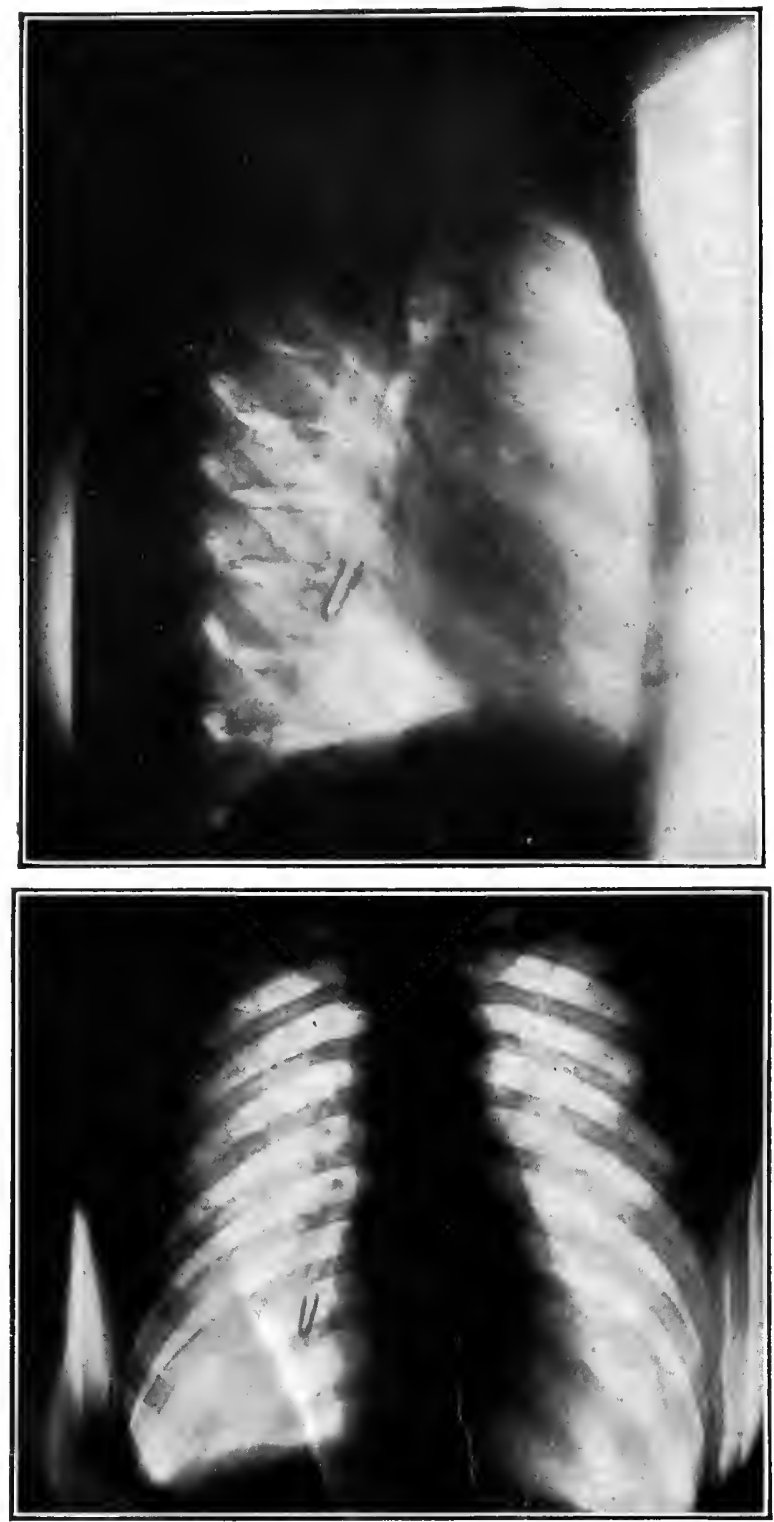

FIG. 58.- Roentgenograph, anteroposterior and lateral showing a staple in a posterior branch of the inferior-lobe bronchus, $10 \mathrm{~cm}$. (4 inches) below the bifurcation of the trachea, in a man aged 44 years. (Plates made by Dr. George W. Grier.) 


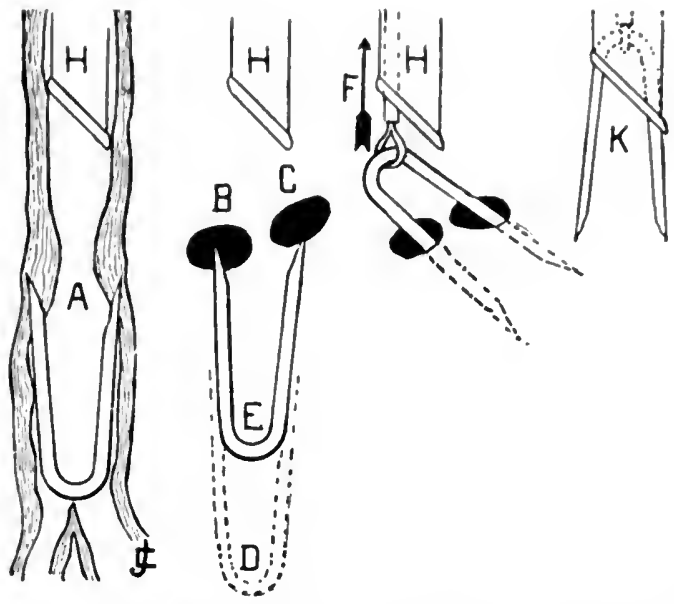

FIG. 59.- Schema illustrating method of removal (by version) of bronchiallylodged staples or double-pointed tacks. $H$, bronchoscope., $A$, swollen mucosa covering points of staple. At $E$ the staple has been manipulated upward with bronchoscopic lip and hooks until the points are opposite the branch bronchial orifices, $B, C$. Traction being made in the direction of the dart $(F)$, by means of the rotation forceps, and counterpressure being made with the bronchoscopic lip on the points of the staple, the points enter the branch bronchi and permit the staple to be turned over and removed with points trailing harmlessly behind $(K)$. The method has been successful in all four of the cases in which it was attemped.

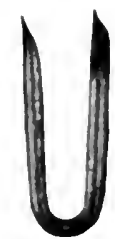

Fig. 60.-Staple (actual size) removed from the right lung (sce Fig. 58), bloodlessly through the mouth, by bronchoscopy, after version as shown in Fig. 59. 


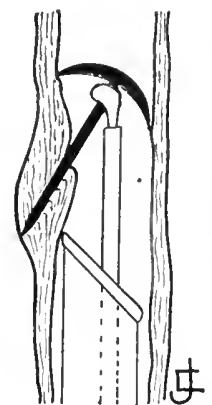

A

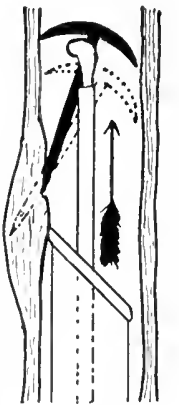

B

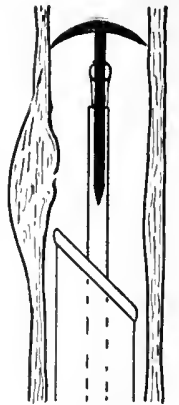

C

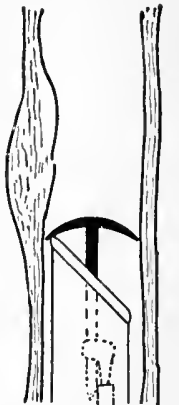

D

FIG. 6r.-Problem of the upholstery tack with buried point. If pulled upon, the imminent perforation of the mediastinum, as shown at $A$, will be completed, the bronchus will be torn and death will follow even if the tack be removed, which is of doubtful possibility. The proper method is gently to close the side-curved forceps on the shank of the tack near the head, push downward as shown by the dart, in $B$, until the point emerges. Then the forceps are rotated to bring the point of the tack away from the bronchial wall. It is usually better at this stage to release the tack and grasp it firmly near the point for withdrawal, $D$. During stages $A, B$ and $C$ the tack is grasped very gently.
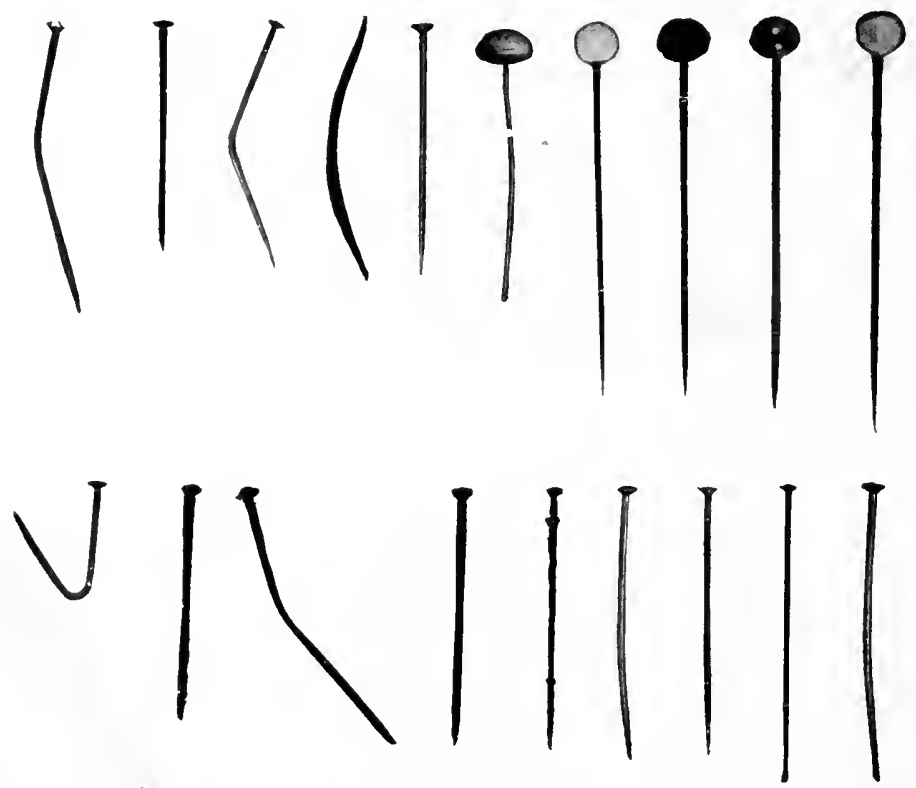

FIG. 62.-Pins removed by peroral endoscopy. (Dr. Jackson's collection.) 


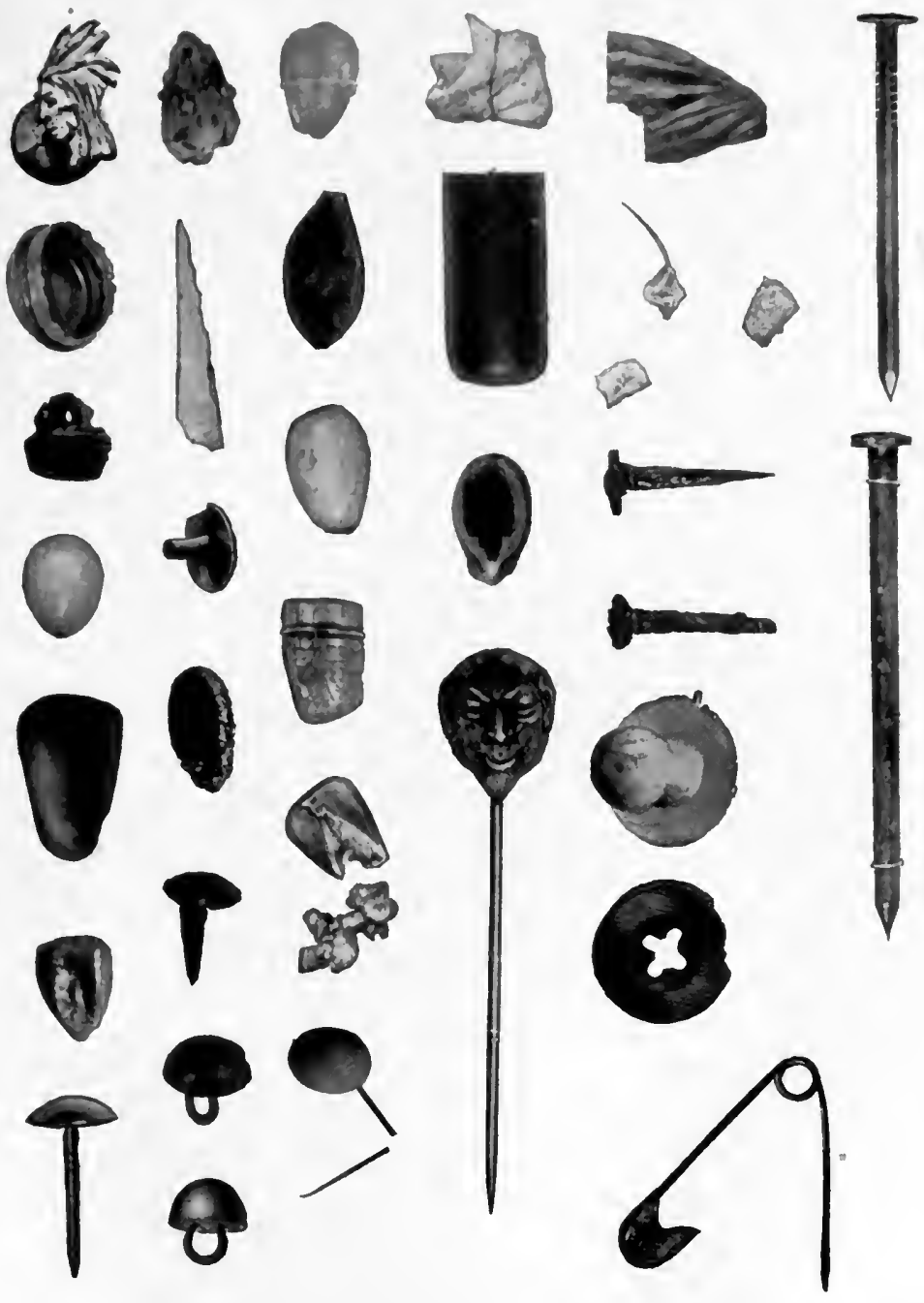

FIG. 63.-A few specimens from the author's collection of foreign bodies removed from the trachea and bronchi by peroral bronchoscopy. 


\title{
PLEURITIS
}

\author{
By Edward H. Goodman, M. D.
}

In discussing treatment of pleuritis, a more consecutive consideration of the subject can be made if some classification be followed. Sharp lines cannot be drawn with certainty between the various stages or form of the disease, but the following classification will be preferred as suitable for an intelligent discussion of treatment:

I. Acute fibrinous pleuritis: (a) primary; $(b)$ secondary.

2. Acute serofibrinous pleuritis.

3. Chronic fibrinous pleuritis.

4. Chronic serofibrinous pleuritis.

5. Purulent pleuritis (empyema).

Although it has not been demonstrated in every case of pleuritis, that a microörganism is the etiologic factor, yet a bacterial agent is certainly responsible in the majority of instances. Any form of pleuritis may be due to one or more organisms in the same or in different cases, and of the organisms commonly considered as etiologic factors, the tubercle bacillus, the pneumococcus and the streptococcus are the most frequently found.

The tubercle bacillus is by far the most common cause of pleuritis, but not the only cause of adhesions and scars so frequently found in the pleura. In serous effusions it is said to be an etiologic agent in from 22 to 85 per cent. of the cases.

The pneumococcus may be the cause of any form of pleuritis, fibrinous or pleuritis with effusion. In the latter class of cases a clear exudate is not the rule, but the pneumococcus variety generally exhibits a turbid fluid or a true purulent effusion.

The streptococcus is not commonly found in the clear serofibrinous exudates but is generally confined to the production of the purulent form (empyema).

Organisms other than those mentioned above have been held to be etiologic factors but apart from the streptococcus aureus these may be left undiscussed.

Although pleuritis is generally secondary to disease of the lung, bronchitis, or some infection elsewhere in the body, there are cases for which an etiologic agent or at all events, a contributory agent must be sought in exposure, alcoholism or rheumatism, but it must be remembered that even in these cases negative findings do not preclude the possibility, even the probability, of it being tuberculous in nature.

Knowledge, then, that the disease is an infection affords a basis of action which is most clear, and treatment should be preceded by 
an attempt to unearth the particular infectious agent in any given case, as the discovery of this agent clcars up materially the path of procedure. Inasmuch as such a large proportion of all cases are known to be tuberculous, it becomes plain that in the greater number of cases of pleuritis the treatment must be that of the treatment of a manifestation of tuberculosis. Moreover, we know that when the infectious agent is the streptococcus, more active interference is demanded than when the pneumococcus is the etiologic offender.

When instituting treatment in pleuritis, it must be borne in mind that a not too limited or narrow view of the individual should be taken; that is, the disease should not be treated entirely symptomatically. Symptomatic treatment, while of course, of utmost importance in giving relief, is yet but a small part of therapeusis. Not only is it essential that one should understand the etiology and the pathology of the disease, but valuable therapeutic indications are derived from the symptoms and clinical course, and from these facts in social and family life, and previous medical history which bear upon the progress of the disease.

As the disease is often secondary, contributing lesions in other parts of the body should be unearthed and reckoned with in the treatment of the infection. Thus, antecedent or associated bronchitis, tuberculosis, pneumonia, streptococcic and staphylococcic infection in any part of the organism, typhoid fever, the acute exanthemata, gonorrhea and syphilis, may each be regarded as a causal factor in producing pleuritis, and the management of all these sources of infection must be carefully considered before treatment can be thoroughly carried out.

The whole process of pleurisy must be regarded as a manifestation of the natural defenses possessed by the organism, and the formation of these defenses should be encouraged and the cause of their production removed or made less influential. The first stage in a pleuritic infection is usually that of fibrinous exudation, called out to protect the necrosis of the endothelium caused by the bacterium, the same as a scab forms to protect a wound. Further irritation by the bacterial agent and by the respiratory movements but serves to increase the output of exudate, and based on this knowledge we apply therapeutic measures to combat both - the first, by supporting the general system to throw off the infecting bacteria; and the second by putting the part at rest.

Further on in the course of the disease, a secondary line of defense is called into action which finds expression in the outpouring of serous fluid as a result of the vascular dilatation. This fluid is serviceable, since it contains antibodies, serves to dilute the irritant, and acts as a fluid splint. On the other hand, it tends to carry the causative factor from the original focus, affords pabulum for bacteria, and by its presence, if too long confined, is conducive to the formation of adhesions.

Resorption of the fluid may take place spontaneously, and may 
be rapid or slow. If there has been primarily but a small area of pleural tissue affected, thickening with localized adherence may be seen, and this varies in degree until enormous adhesions involving the entire parietal and visceral pleural surfaces take place (tuberculosis). Very often in the necropsy room pleural adhesions of even an extensive degree are found quite by accident, their presence having been overlooked by the clinician on account of absence of objective and subjective symptoms.

Remembering that the fluid may be regarded on the one hand as a safeguard against further advance of the infection, and on the other, as a menace against the health of the individual, it is not to be wondered at that physicians have arrayed themselves on either side of the question, the one advocating postponement of aspiration, and the other favoring early removal of the fluid.

Treatment based on symptoms alone, without regard to any other fact of the case, is sometimes, as in severe pain, a sine qua non. From the urgency of a particular symptom the physician is of ttimes driven to its amelioration without conception of its cause. From the relief given by the successful application of a therapeutic measure, the physician is too often influenced by the patient's temporary, perhaps more or less permanent feeling of good health, and ceases to inquire into more intimate causative conditions, and therefore ceases to understand the case on hand. As a starting place, treatment based on symptoms is to be recommended, but as a terminus it has no value. Symptoms which indicate a poor reaction should compel treatment to increase resistance; on the other hand, sedative or depletive measures are not amiss if the reaction is excessive.

The treatment of all forms of pleuritis is influenced by the age and sex of the patient, the habits, the occupation, in as much only as these factors favor or retard the defensive reactions of the organism. While from observation we say the young and the old, the feeble and the robust, the temperate and the intemperate, should have diverse methods of treatment, we know, by our instruments of precision, more precisely the indications for treatment in the special varieties. Our knowledge of the clinical course of the disease under varying circumstances is substantiated and explained by our more precise knowledge from the use of instruments of precision and by experimental studies.

When pleurisy occurs in a patient suffering from chronic heart disease, chronic nephritis, or some chronic infection, again we have to modify the treatment in accordance with the nature and resisting powers of the organism. Finally, the state of the functions of digestion, elimination, and the circulation must be observed. When required, they should receive proper attention. This particularly applies to the eliminative and circulatory powers of the organism, as upon them depend the discharge of toxic products.

To base the treatment of pleurisy on facts of the social, previous medical and family histories, or on etiology, morbid processes or 
on symptoms alone, were the height of folly, but we insist that all of these things should be inquired into, so that the rationale of treatment may be placed upon as sound a basis as possible.

\section{ACUTE FIBRINOUS PLEURITIS}

The first and essential therapeutic indication in this form of pleuritis is pain, and it is this symptom which drives the patient to the physician for relief. Rest is imperative when there is much fever or great pain, but even in the so-called mild cases rest in bed accomplishes a great deal by preventing extensions and even effusion.

Having put the patient to bed, active measures are to be institu ted for the relief of pain, and of them all fixation of the side is probably the best. This is accomplished by the use of adhesive straps, preferably zinc oxid, which, by preventing the rubbing of the inflamed pleural surfaces, gives ofttimes immediate relief. The method of application is as follows: Strips of zinc oxid adhesive plaster, about 4 inches wide and long enough to reach from the paravertebral region around the chest to about 2 inches from the opposite edge of the sternum, are cut. The patient being braced against a firm object, one end of the plaster is applied to the paravertebral region, and as the patient terminates a full expiration and holds his breath, the plaster is wrapped about the chest as tightly as possible. The first strap should be placed over the lower thoracic region, and successive straps are applied, so that each overlaps the one just applied, and this is continued until the affected area is well covered. In women with large breasts this method of procedure may be modified by using narrow strips of plaster and applying them from the paravertebral region downward, and forward from the sternal region downward and backward so that the straps intersect in the axillary region.

Should the chest be hairy, it should be shaved, so that the removal of the straps may be accomplished with as little discomfort as possible. The plaster should not be allowed to remain on longer than a week and it may be readily removed if vaseline is thoroughly rubbed on the straps the night before they are to be removed. The free use of benzin will be found of great assistance, not only in making the removal of the straps much easier of accomplishment, but also in cleaning the chest after the straps have been taken off.

Strapping at times fails to give relief, and in these cases an ice bag or hot-water bottle may be used with advantage. Fomentations or compresses may be found more satisfactory than the hot-water bottle. Flaxseed poultices are sometimes used, and are made by stirring into boiling water flaxseed meal, until it becomes of the consistency desired, that is, of a consistency which permits of the spreading of the paste. After this thickness is obtained, it is taken from the fire, and beaten thoroughly, after which it is spread on gauze or cheese-cloth. The edges of the gauze are turned in to prevent the 
escape of the paste, and the entire poultice is wrapped in gauze and placed on the chest, and covered over with rubber or silk tissue.

Another method of affording relief from the pain is by counterirritation. Of the counterirritants, only mustard should be used, and the writer cautions against the use of iodine and the application of blisters. The commercial mustard leaf or plaster is serviceable and is applied after dipping in warm water. Usually reddening is observed after fifteen or twenty minutes; the plaster is then removed, the part well cleansed and vaseline is applied. In no case should the plaster be left on more than twenty minutes as blistering may readily follow. Should home measures be required, a serviceable mustard paste may be made by mixing I part of mustard with from 2 to to parts of flour according to the strength desired, and then adding enough lukewarm water to make an easily manipulated paste. The white of an egg is often mixed in with the paste, especially when the skin is very tender. This mustard paste is spread on cheesecloth and allowed to remain on the chest from fifteen to twenty minutes. It should be renewed not oftener than every four or five hours. A combination of mustard and flaxseed may be used to good advantage.

Cupping is sometimes employed, though of less value than the foregoing. Cups are manufactured especially for this purpose and are most convenient when required. Should these not be at hand, small wine glasses may be used in the following manner: the rim of the glass should be dry, a small portion of alcohol is placed in the bottom of the glass and ignited, and when the flame is consumed the cups are quickly placed on the chest. The skin will be drawn up in the glass, and the cup should be left on until the congestion is well marked, when it may be easily removed by allowing some air to enter the cup. Wet cups are no longer used. Cups may be reapplied as frequently as every four hours.

Should local measures prove unsuccessful in combating the pain, hypodermic injections of morphin must be resorted to. The presence of kidney disease should prove no contraindication to the judicious use of morphin. In less severe cases phenacetin may be used, gr. 5 ( $0.3 \mathrm{gm}$.) every three hours with or without salicin gr. 5 to Io ( 0.3 to $0.6 \mathrm{gm}$.). Salicylates have earned a well-deserved place in the treatment of pleuritis but to secure the best results large doses must be given. When given with double the amount of sodium bicarbonate, large amounts are borne without any ill effects (gr. I5 to 30-I.0 to $2.0 \mathrm{gm}$.--every three hours). Sodium salicylate is preferable to aspirin, novaspirin, salicin, salophen, and such preparations.

The bowels should be kept open. Calomel in divided doses seems to hold the palm for popularity. It should be followed by an active purge the next morning or if the time of seeing the patient for the first time permits, at the expiration of six hours. The patient's sleep should not be broken during the night by the opera- 
tion of a purge untimely given. A half to $\mathrm{I}$ ounce ( 15 to $30 \mathrm{gm}$.) of Epsom, Glaubers or Rochelle salts may be given, or magnesium citrate ( $\delta$ vi-r8o c.c.) or even the milder acting Seidlitz powder or one of the laxative waters (Hunyadi, Pluto, Apenta). Enemata are not so efficacious as purges in this condition.

The diet should be simple and easily digestible and may comprise, milk, broths, soups, milk toasts, soft-boiled and poached eggs, cereals, custard, junket, ice cream, and fruits, all depending on the desire of the patient for food, and his ability to digest it. Water should be taken freely whenever there is fever and I make it a rule to have 6 ounces administered every hour. The patient should be kept in bed until the temperature is normal.

Remembering that pleurisy is often a manifestation of disease in some other part of the body, the recognition of the source of the infection should be the aim of the physician. If part of a tuberculous condition, treatment should not cease with amelioration of symptoms, but when the first discomfort is over, and even during convalescence, hygienic treatment should be begun (see section on tuberculosis). If part of a pneumococcic infection, treatment should be directed to the primary condition, the pleuritic involvement being treated expectantly, by warmth or cold, or in extreme cases by morphin. Should the pleuritic involvement be but an expression of a rheumatic affection, great benefit is to be expected from the use of salicylates-aspirin, salophen, sodium salicylate, etc., as stated above.

The association of pleurisy with syphilis has led Stintzing, in obstinate cases, to put the patient on antisyphilitic treatment, and he especially recommends the use of iodids, claiming to have seen cures under their use.

After subsidence of the acute symptoms, respiratory exercises are to be undertaken. These will be spoken of, however, when the treatment of chronic fibrinous pleuritis is discussed.

\section{ACUTE SEROFIBRINOUS PLEURITIS}

Aspiration when demanded should be performed slowly; i.e., a large effusion may require from thirty to forty-five minutes for its ultimate withdrawal, but thoracentesis should be discontinued as soon as the patient begins to cough immoderately or commences to get dyspneic, or manifests any symptom of cardiac discomfort.

Injections Following Tapping.-It has been suggested to inject air or oxygen or salt solution into the pleural cavity after aspiration, particularly in those cases where adhesions prevent full expansion of the lung. There are dangers of infection from the use of air and also, there is a possibility that it may of itself prevent reëxpansion of the lung by splinting the lung.

Repeated Tappings.- Usually it is unnecessary to repeat the tapping. The indication for a repetition is reaccumulation of fluid 
but the diagnosis of reaccumulation is difficult, and must be made particularly on signs of cardiac and respiratory embarrassment, although auscultatory signs are not be be neglected.

After tapping, the patient should be kept in bed for about a week, but apart from this, thoracentesis should change in no wise the treatment of the case. As has been said, every case should be treated as tuberculous, and the reader will find this discussed in the chapter devoted to the management of tuberculous patients.

Open Pleural Puncture.-A method of tapping, using only an ordinary trocar and no suction apparatus is described by Schmidt under the name of "open pleural puncture." The patient is placed sidewise on two beds, the head and shoulders on one, and the hips on another, with a space about 2 feet wide between the beds. The diseased side being inferiorly placed, a trocar about $8 \mathrm{~cm}$. by $0.4 \mathrm{~cm}$. is inserted from below at the lowest point of dulness, and the plunger is removed. When there is positive pressure in the pleural cavity fluid runs out, but when there is negative pressure, air first rushes in and then forces the exudate out. After a short space of time following the flow of fluid air is sucked in and forces the fluid from the corners and crevasses, and an increased flow begins. Gradually the stream ceases, air is inspired and expired through the canula. The canula is gradually withdrawn until it is just within the pleural cavity, and at this point the patient is told to breathe out forcibly or to cough. At the height of the expiratory act the canula is removed and the wound closed with broad adhesive plaster.

Schmidt claims that by this method there is no irritation of the pleura by trocar and hence no coughing, fainting, pulmonary edema, and albuminous expectoration, and he asserts there is absolutely no danger from infection through the atmosphere.

\section{HEMOTHORAX}

Hemothorax, of the non-traumatic type, is seen as a result of rupture of intrathoracic vessels, when the seat of aneurysmal dilatation. I have had two cases of this kind, the first of whom recovered completely, and the second of whom recovered from the first rupture, but succumbed to a second break or leakage. ${ }^{1}$ If there are alarming pressure symptoms, thoracentesis if very slowly performed may be advisable, although not too much blood should be withdrawn at one time. In the second case, mentioned above, alarming symptoms of collapse followed the removal of a few hundred cubic centimeters of blood.

\section{CHYLOTHORAX}

Lord advises that in the presence of small amounts of fluid, once the diagnosis is established, strapping should be practised, as by diminishing the respiratory changes in intrapleural tension, an

${ }^{1}$ Jour. Am. Med. Assn., I9I4, Ixii, I242; Jour. Am. Med. Assn., I9I6, 1xvi, 568. 
increase of the fluid may be prevented. 'The repeated withdrawal of large amounts of fluid is inadviasble, inasmuch as the diminution of pleural pressure may favor reaccumulation, and because of the drain on the patient of loss of such fluid.

\section{ECHINOCOCCUS DISEASE}

Primary echinococcus infection of the pleura occurs very rarely (x.73 per cent. of all cases of echinococcus pleura) and echinococcus disease of the pleura whether primary or secondary is a great curiosity. The treatment of this rare condition which seems to have met with favor in some quarters, is the injection into the pleural cavity after removal of the cyst content, 20 c.c. of a $I$ : I000 bichlorid of mercury solution. v. Bokay is especially enthusiastic over the results obtained with this method, suggested by Baccelli, of treating echinococcus pleuræ.

\section{ACTINOMYCOSIS OF THE PLEURE}

This is generally secondary to disease in other parts of the body, notably in the lungs. Treatment is medical and surgical. Vaccines have been tried but have failed. Potassium iodid is the most reliable medical agent, and although it cannot be called a specific in the proper use of the word, yet it does seem to favor resorption of the inflammatory product, and aids materially in melting down the actinomycotic areas. It must be given in large doses, and as much as $\mathrm{I} 8 \mathrm{gm}$. pro die have been administered. When possible, operative treatment should be advised, although when giving this advice, the writer appreciates fully the enormous difficulties to be encountered.

In cases of empyema perforans, surgery promises much, but only if all fistulous openings are thoroughly explored and all diseased areas removed. Karenski has published results of operative interference. ${ }^{1}$

${ }^{1}$ Ergebn. d. Chir. u. Orthop., I914, viii, 424. 


\title{
INFANTILE LA GRIPPE
}

\author{
By IsaAc A. Abt, M. D.
}

La grippe is the most common illness in infants during the cold season of the year. It is the starting point of a great numbee of other diseases, some very serious or even fatal. The infantilr mortality and morbidity from la grippe in the winter, though not so large as the mortality and morbidity from the diarrheal diseases in the summer, are considerable. Pfaundler made the very important observation that many babies sicken with feeding disorders and die as a result of respiratory infections, many of which may be considered grippal in character.

In older children the course of la grippe resembles that in adults, and the details which have special reference to the adult type of the disease will not be taken up here. In infancy the course of an ordinary case is characterized by prostration or malaise, desire to be kept quiet, persistent vomiting (an expression of tnxemia, and not, as is so frequently supposed, of a gastro-intestinal disorder), restlessness, pale or ashen gray skin, and fever. In mild cases, fall of temperature and improvement may follow in a few days; other cases may last weeks or even months.

In another type of the disease the patient has fever for several days more or less complete anorexia (explained in some instances by mechanical interference with breathing when the child tries to take the breast), loss in weight, anemia and prostration, nasopharyngeal inflammation, "fetor ex ore," and in many cases, an irritating cough. It may or may not be possible to palpate enlarged lymph nodes. Auscultation of the chest is frequently negative.

The nasopharyngeal involvement is sometimes difficult to diagnose. Examination of the pharynx may show redness, swelling and accumulation of mucopus on the posterior or lateral wall of the pharynx. In other cases examination of the pharynx is negative and the condition is disclosed chiefly by the nasal voice or by mouth breathing and the infant's failure to apply himself to sucking at the breast or bottle.

Fever.-The fever may be of various, types, continuous or intermittent; terminating in spontaneous cure after one or two days, or lasting three, four or even eight weeks, giving rise to the suspicion of tuberculosis or some obscure infection. In the pyemic type of fever there may be a daily variation of temperature from Ior $^{\circ}$ to I0 $6^{\circ} \mathrm{F}$. This may last four or five days, with complete remission 
for a number of days, followed by a relapse with high temperature and possibly complications. A not uncommon typhoidal form of la grippe gives rise to great difficulty in diagnosis. This is characterized by continuous high fever (10 $5^{\circ}$ to $106^{\circ} \mathrm{F}$.), running for ten days or three weeks, with catarrhal symptoms absent or practically absent, and no other findings of importance beyond restlessness, somnolence, anorexia and some vomiting. Feeble, ill-nourished or premature infants, on the other hand, though suffering from severe infection, may have no fever or may show even a subnormal temperature.

Differential Diagnosis.-The alimentary symptoms of la grippe - constipation or diarrhea, tympany and thin watery stools - sometimes lead to an erroneous diagnosis of gastro-intestinal disturbance. If the withdrawal of food, with substitution, for instance, of tea and toast or tea sweetened with saccharin, for twenty-four hours does not produce improvement, it may be concluded that the condition is due to infection outside of the gastro-intestinal tract. Also, a bad odor from the mouth is almost pathognomonic of pharyngeal or nasopharyngeal disturbance.

Diphtheria bacilli may be demonstrated in cultures from the nose or throat of a "carrier" in a case of la grippe. The diagnosis of clinical diphtheria, of course, is justified only if the clinical symptoms of diphtheria are present.

The onset of la grippe much resembles that of measles or of whooping cough. Often all early signs fail and it is necessary to await confirmation of the diagnosis by the characteristic whoop of true whooping cough, or by the appearance of Koplik's spots in measles.

Complications.-Glandular fever, sometimes known as Pfeiffer's disease, occurs in the winter or the early spring, as a rule, usually after a slight nasopharyngeal infection, very often in epidemic form. It is a true grippal infection. The glands at the angle of the jaw, or lying either anterior or posterior to the mastoid muscles, become swollen. Soon the swelling assumes great proportions, being frequently bilateral. Usually this condition is accompanied by very high fever (perhaps $105^{\circ}$ or $106^{\circ} \mathrm{F}$.), continuing high for several days or weeks. Sometimes, though rarely, there is a middle-ear complication. Occasionally respiratory complications occur, and not infrequently an acute hemorrhagic nephritis complicates the disorder. In cases of true glandular fever suppuration rarely occurs.

Otitis media is perhaps the most frequent complication of la grippe, since the inflammatory secretion readily finds its way into the middle ear and produces a reaction. In older children the diagnosis is rendered comparatively easy by the presence of swelling, redness, fluid in the middle ear and agonizing pain. In young children and infants the examination is sometimes attended with great difficulty, especially in those cases in which the auditory canal is tortuous and in which it is difficult to insert the speculum. Otitis media, as a rule, does not indicate a bad prognosis in young infants. Mas- 
toid infections, meningitis, and cerebral abscess fortunately are rare.

Non-suppurative, "spotted yellow" or "spotted red" encephalitis may occur as a complication, producing hemiplegia or other paralyses, or sudden death according to the localization. These cases of non-suppurative encephalitis are not infrequent in children of any age.

Swollen lymph nodes on the posterior pharyngeal wall may terminate in retropharyngeal abscess, which is diagnosed by the attitude of the child's head (held back and stiffly to one side), by a peculiar muffled cry, indicating fulness in the throat, and by a characteristic snoring breathing.

Pneumonia and bronchitis are complications which, being fully treated under separate headings, do not require discussion here.

Endocarditis with valvular lesions sometimes occurs, though not frequently. The most common cardiac affection in grippe is myocarditis. A disturbed myocardium is a very important factor in la grippe, as in other infectious and contagious diseases.

Peritonitis is an occasional occurrence. While not infrequently a frank pneumonia will produce abdominal symptoms simulating a true peritonitis, on the other hand, a grippal peritonitis may occur without any preceding pleural involvement. These are usually pneumococcus infections. The grippe infections may extend through the lymphatic channels or as the result of a general blood infection.

Osteomyelitis and joint involvement are among the less common, but nevertheless well-authenticated complications.

Renal complications, with albumin and casts, are not infrequent, as in any acute infectious process. Hemorrhagic nephritis may occur, especially where there is extensive lymph node involvement. As a rule, the acute hemorrhagic nephritis in this disease offers. a favorable prognosis, though uremia and convulsions may occur. Probably many cases of hemorrhagic involvement are due to the use of hexamethylenamin or to its administration in too large doses. The formaldehyde which results from the splitting up of the hexamethylenamin in the body is likely to have an injurious effect on an already irritated, congested or partially inflamed kidney. If the drug be continued long enough, hematuria occurs. Another occasional renal complication of la grippe is pyelitis. It is not easy to explain the relation of grippal infection to pyelitis, except on the assumption that the pyelitis was chronic or latent and that the infection produced a recurrence by some unknown irritation of the urinary tract. If pus is present in considerable quantity (it may be necessary to examine several specimens of urine before the pus is found), the existence of pyelitis may be assumed.

A spasmophilic baby that falls ill with la grippe may have a latent spasmophilia transformed into a manifest spasmophilia or tetany. 
Prophylaxis.-Infants and children should be protected as far as possible from contact with persons suffering from "bad colds" or la grippe. The younger the child, the more rigorously should this protection be enforced, since in young infants the antibodies are poorly developed and the child consequently has little resistance against infection. The attempt should be made to render the child's constitution resistant to infection by proper attention to the hygiene of bathing, dressing, outdoor airings and housing.

Treatment.-The child should be loosely clad, kept as quiet as possible, given plenty of fluids, kept in a well-ventilated room of moderate temperature, and given nourishment if he is able to take it. The digestion should not be disturbed by large amounts of physic. The nasal disorder should not be overtreated; the use of forcible syringing and irritant applications to the nostrils should be avoided. A mild, non-irritating solution is the best of all-a little normal salt solution slowly instilled into the nostrils with a medicine dropper, or boric acid solution, argyrol in 5 per cent. solution, or liquid vaseline (the yellow vaseline in preference to the white, which has been bleached by chemicals, and is quite acid to the taste). If the condition does not clear up under this treatment, 3 to 5 drops of epinephrin (adrenalin) solution ( 1 : Iooo diluted with normal saline solution so that the finished product represents an epinephrin solution of $I: 6000$ or $I: 8000$ ) may be instilled into the nose with a medicine dropper two or three times a day. If the baby has fever and is suffering, crying and restless, a tepid bath $\left(95^{\circ}\right.$ or $98^{\circ} \mathrm{F}$.,) may be ordered. Or a tepid, moist compress may be applied, enveloping the baby's entire trunk.

For the fever and restlessness some drugs give symptomatic relief. The use of all forms of depressant drugs should be avoided if pulmonary involvement is present or threatens. Aspirin, $1 / 2$ to $\mathrm{I}$ grain $(0.03$ to $0.065 \mathrm{gm}$.), depending upon the age of the infant, two to four times a day, depending on the degree of temperature and restlessness, or, salophen, phenacetin, antipyrin, in the same dosage, may be employed. In the recent literature one finds glowing reports concerning the efficiency of quinin, especially in the pneumococcus infections. In the German literature optochin or ethyl cuprein hydrochlorid is strongly advised, as suggested by Morgenroth. The preparation is injected hypodermically in $1 / 2$ to $\mathrm{I}$-grain $(0.03$ to $0.065 \mathrm{gm}$.) doses once daily. The dihydrochlorid of quinin or the bisulphate may be used in I-grain $(0.065 \mathrm{gm}$.) doses in solution hypodermically. Aristoquin or euquinin (Merck) may be given in $1 / 2$ to I-grain $(0.03$ to $0.065 \mathrm{gm}$.) doses three or four times a day. These preparations are selected because they are less bitter than the quinin in common use. Expectorant cough mixtures are of doubtful value even when cough and bronchitis are present. Mild sedatives are sometimes required to quiet cough and produce rest. For this purpose minute doses of codein, gr. $1 / 60$ (0.00I gm.) for an infant of one year; Dover's powder, gr. $1 / 4$ to $1 / 2$ ( 0.015 to $0.03 \mathrm{gm}$.) for an 
infant of the same age, or paregoric 5 to ro minims ( 0.3 to o.6 c.c.). Repeat in two or three hours if required.

The acute middle-ear inflammation should be treated conservatively at first, and warm phenol glycerin solution ( 5 or Io per cent.) should .be dropped into the ear at frequent intervals. If pain, inflammation, and bulging of the membrane indicate more radical treatment, incision of the membrana tympani may be indicated. 


\section{DISEASES OF THE DIGESTIVE SYSTEM}

\section{GENERAL HYGIENE OF THE MOUTH}

\section{By George Morris Piersol, M. D.}

During recent years few subjects in medicine have received more attention than has oral sepsis. Whereas formerly the importance of the mouth as a focus of acute and chronic infection was appreciated by comparatively few clinicians, today the significance of septic infection of the oral cavity is being widely recognized by physicians and dentists alike. Widespread recognition of the fundamental importance of this subject, has led to increased coöperation on the part of the medical and dental profession, with the result that much painstaking research and many careful clinical observations have demonstrated the undeniable relationship that exists between the mouth as the primary source of infection and certain diseased conditions of the endocardium, joints, muscles, nerves and other remote structures. One of the most significant evidences that the medical profession as a whole is being aroused to the importance of this subject, is the fact that in nearly all the larger and more progressive hospitals, oral surgeons, visiting dentists and dental residents are being added to the staffs, of which they form a no less important part, so far as the successful management of the patients is concerned, than do the departments of clinical pathology or roentgenology.

The discussion of the general hygiene of the mouth, as presented by Dr. M. H. Cryer in his original contribution, particularly in reference to the care of the teeth from earliest childhood to old age, needs no further amplification, and may be regarded as an authoritative exposition of the subject. The same is to be said of Dr. Cryer's discussion of superficial inflammations of the gums, or marginal gingivitis. Disorders of the deeper structures of the mouth, namely, pyorrhea alveolaris and abscesses of the alveolar process, have excited so much interest of late, that they need further discussion.

\section{PYORRHEA ALVEOLARIS}

Our conception of pyorrhea alveolaris in particular has undergone marked revision since the researches of Barrett and Smith, and later those of Bass, Johns, and others have conclusively shown that an ameba, if not the sole cause, is certainly the chief cause of this affection, and further that this ameba is attacked and destroyed by emetin. Indeed, so great has been the general interest in this

$$
\text { Vox. IV. }-37
$$


disease, that it deserves separate consideration, and therefore is elsewhere discussed in detail (see page ${ }^{8} 8$ ).

\section{ALVEOLAR ABSCESS}

Alveolar abscess next to pyorrhea alveolaris, is one of the most important and common sources of mouth infection frequently associated with grave secondary manifestations elsewhere in the body. It is not surprising, therefore that these abscesses of the alveolæ should have been the subject of much careful investigation. The etiology of alveolar abscesses has always been of much interest. For some time the general impression obtained that the chief exciting cause of these abscesses was Staphylococcus aureus and albus, Recent bacteriological investigation, however, has clearly demonstrated that these organisms, although they do occur in alveolar abscesses, are by no means the predominating ones, and that most of these infections are chiefly the result of streptococcus invasion. The streptococci found, vary from a hemolytic streptococcus in the acute abscesses, to Streptococcus viridans and Streptococcus mucosus in the more chronic forms. Bacillus fusiformis, Staphylococcus aureus and albus, and Micrococcus catarrhalis have also been found in association with the more dominant streptococci. When we consider that alveolar abscesses have not infrequently proved the chief factor in the production of such conditions as acute endocarditis, and that Streptococcus viridans is of not uncommon occurrence in endocardial lesions, these bacterial findings take on an added interest and throw considerable light on the relationship between local infections of the jaw and various more remote foci. Billings is of the belief that chronic alveolar infections may cause chronic infections elsewhere by hematogenous bacterial emboli which produce ischemia of the tissues at the same time that they infect them.

The route by which infecting bacteria reach the jaw structures is of interest. The deep alveolar abscesses are found down in the deeper structures of the alveolar process, and are probably always of hematogenous origin; whereas in the dento-alveolar abscess, that is those situated at the apex of the root, the offending bacteria gain entrance to the root canals through decay of the teeth. Some observers have recorded the epidemic occurrence of alveolar abscesses, and have further suggested that these epidemics of alveolar infection occur in association with or secondary to, streptococcus infection of the nose and throat. In confirmation of this, instances are on record in which the same organism was isolated from the tonsils and nasopharynx that was found in the alveolar abscess. Infectious material from the mouth, nose and throat could easily invade the structures of the jaw, either through the circulation or directly through areas of decay in the teeth.

The diagnosis of alveolar abscesses is a matter of the greatest importance. The deep abscesses associated as they usually are with 
pain, swelling and other definite evidences of inflammation, are rarely overlooked. The so-called "blind" or dento-alveolar abscesses, are more difficult to detect and doubtless frequently escape notice. Careful inspection of the teeth often fails to disclose them. The only sure method of recognizing them is by the roentgen-rays. When roentgenograms are properly made and correctly interpreted by one skilled in such work, we have the only reasonably sure method of detecting blind abscesses. On the other hand, improper technique and inexperience are prolific sources of error in this field of diagnosis. The importance of subjecting all suspected jaws to expert roentgenologic examination, and further the necessity of such examination of the jaws in all cases of chronic infection for which no obvious foci can be found, cannot be emphasized too strongly. Obscure cases of arthritis, myositis, neuritis, persistent anemia and general malnutrition, would often be cleared up more promptly if this procedure were adopted routinely. The author can recall two recent instances of persistent and unexplainable headache which were relieved only after roentgen-ray examinations had disclosed the presence of several blind alveolar abscesses. The headaches cleared up promptly when the infected teeth were extracted and the abscess cavities drained.

In the treatment of these alveolar abscesses, several methods are available. For the deep abscesses associated with definite collections of pus, obviously the only rational treatment is surgical. In the treatment of the more chronic type of superficial abscess, when the infecting organism has been isolated, autogenous vaccines have been used, at times with strikingly good results. Gilmer and Moody have successfully utilized autogenous vaccines made from cultures of both aërobic and anaërobic organisms. The vaccines are administered at five-day intervals in graded doses. This method of treatment, however, is applicable only in certain chronic suppurations. Various methods of local treatment, directed at the contents of the root canal, may prove effective. It should be remembered, however, that apical abscesses are rarely, if ever, found at the roots of vital teeth. Therefore, if local measures are not successful in a reasonably short time, the more radical procedure of removing the teeth should be resorted to before the local infection has had an opportunity to cause too much destruction of the contiguous structures or to produce more serious changes in the remoter organs.

\section{CANCRUM ORIS}

Little new has recently been added to our knowledge of cancrum oris, that severe infection occurring in and about the mouth. Although all observers agree that the nature of the lesion in cancrum oris strongly suggests some specific infection, the causative microörganism has not as yet been found. The fusiform bacillus and spirillum of Vincent, are the organisms most frequently isolated from 
the lesion of cancrum oris. Whether or not these are primary or secondary invaders, is a matter of conjecture. In a few cases the Klebs-Loeffler bacillus appears to have been the sole organism present. Unfortunately no new forms of specific treatment have as yet been devised. for this dread disease, except that in a few cases apparently due to spirilla, salvarsan and neosalvarsan are reported to have done good. Cauterization of the lesion with pure nitric acid is still the preferred method of treatment. What to use after cauterization when dressing the lesion, seems to be a matter of personal preference. In Kerley's opinion, dilute alcohol (equal parts of alcohol and water) has proved more effective in arresting the progress of the disease than either solutions of bichlorid of mercury or boric acid, both of which are frequently employed. The dressings of alcohol should be frequently renewed and in order to prevent evaporation as much as possible, the solution should be applied on double pieces of lint, covered over with rubber tissue.

\section{LUDWIG'S ANGINA}

In considering the etiology and pathology of Ludwig's angina, it should be borne in mind that the acute septic process is primarily . a cellulitis of the tissues of the floor of the mouth and the submaxillary region, affecting chiefly the connective-tissue spaces, and involving the lymph nodes and the submaxillary and sublingual salivary glands secondarily. The lesion is, therefore, not primarily a lymphangitis as was once thought. Additional evidence that the lesion is chiefly a cellulitis is furnished by the fact that the submaxillary glands and lymph nodes usually are found more or less intact lying in the center of the necrotic area. The infection rapidly involves both the floor of the mouth and the cervical tissues. T. Turner Thomas emphasizes the fact that the infection spreads from the floor of the mouth around the posterior border of the mylohyoid muscle. The neck is usually affected bilaterally. As well emphasized by Dr. Cryer in his article on this subject, the most essential feature of the successful treatment of Ludwig's angina, is prompt surgical intervention. The affected part should be incised just as soon as the diagnosis is made, without waiting for the appearance of free pus. The incision and free drainage of the affected structures is done under local anesthesia, general anesthesia being usually out of the question. In addition to making the well-known incision for draining the floor of the mouth, J. W. Price has suggested that the mucous membrane of the floor of the mouth be incised from the midline to the second molar tooth, and that all soft necrotic areas be gently curetted. Price's incision is particularly indicated when there is marked edema of the sublingual tissues. 


\title{
CHRONIC PERIDENTAL SUPPURATIONS, WITH ESPECIAL REFERENCE TO CHRONIC SUPPURATIVE PERICE- MENTITIS OR PYORRHEA ALVEOLARIS
}

\author{
By Allen J. Smith, M. D., and M. T. Barrett, M. Sc., D. D. S.
}

In the general group of oral septic conditions the peridental suppurations occupy a prominent position because of their extreme prevalence, their local effects, and because of the part it is believed they play, along with analogous lesions elsewhere, as primary sources of systemic intoxications and microbic metastases. Aside from their local results it is particularly in these last connections that they are of importance to the internist, who would regard them, along with infections of such other sites as the tonsils, nasal passages and accessory sinuses, middle ear, gall-bladder, appendix, intestine, prostate, endometrium and tubes, as frequent foci of origin of such subinfections and intoxications. The work of the dentist Miller(I) of Berlin, called attention long since to the importance of the mouth as a site of bacterial growth and to the multiplicity of species to be met with there; and, too, impressed the view of oral infections, especially peridental suppurations, underlying serious systemic faults. The papers of Hunter(2) of London, especially served to focus attention upon the chronic suppurative peridental lesions, the likelihood of their persistence under fixed mechanisms applied by dentists, and the dangers to which they subject the host. Appreciation of the need for thorough elimination of these and all analogous foci has within the past few years culminated in a feeling which, as far as the dental phase is concerned, has almost reversed the habit of thought and practice of the dental profession. The anxiety so common among dentists of even a few years ago to "save" affected teeth, and fill their carious cavities, or, after filling, to utilize them as anchorages for bridges is giving way rapidly in the mind of progressive dentists to the belief that no sacrifice is too great to absolutely destroy infections about the teeth, and that the greatest caution is to be observed lest, in filling an exposed canal an unhealed apical abscess be merely concealed, and lest in crowning and in attaching bridges the same fault of uneliminated suppurations be covered in. Both professions have contributed to the development of this present-day attitude, although the impression of the importance of these focal infections to various corporeal complications has especially stimulated medical men to a coöperative interest with the dental profession, the work of Billings and his associates $(3)$ deserving prominent recognition in this country. The list of complications arising either from subinfection or from toxic absorption from the primary foci is an 
extended one, including varying grades of anemia (even of pernicious severity), neuritis, thyroid hyperplasias, endo- and pericarditis, arthritis both acute and of the chronic "deformans" type, lymphadenitis, and renal, myocardial and hepatic degenerations and inflammations, to say nothing of the local peridental changes and of the results of direct extension of the infecting agents and toxic substances to the neighboring tissues and over the surfaces of the mucous membrane of the mouth to nasal and to lower alimentary levels. The evolution of the relative importance of toxic agencies on the one hand and of actual microbic metastasis on the other hand cannot be regarded as established; the older view of the effectiveness of toxic absorption being materially modified by the more recent recognition of the frequent reality of microbic metastasis to the secondary localities(4). That the former cannot be disregarded may be believed from the frequence of negative findings in cultural studies of such complicating affections; and may be asserted by the writers from actual production of lymphadenitis, acute arthritis and acute pleuritis by introduction of extracts of pyorrhea material filtered through porcelain, when contrasted with control injections into the neck, joints and pleura of sterile normal salt solution without comparable effect.

Peridental suppurations include both acute and chronic types; but of these, consideration is here mainly devoted to the latter, since they largely outweigh in a medical sense the former because of their long period of persistence, great prevalence and chance of neglect, with consequent eventual local and general effects of serious grade (5).

From an anatomical standpoint it is essential to recognize lesions limited to the superficial gingival tissues (gingivitis, gingival pyorrhea), lesions which, beginning in the gingivæ, proceed along the roots of the teeth to alveolar levels (alveolar pyorrhea), lesions which begin beneath the intact gums along the dental root (lateral and intermediate alveolar abscesses), and lesions which are extensions from a primarily infected pulp to and about the dental apex (apical $a b$ scesses). From a medical standpoint all of these may serve as sources of metastasis or toxic absorption, the differences of effect arising from the precise nature of the infection, the amount of involvement, and the local conditions afforded for absorption or drainage. In their establishment it is to be presumed that those which develop from the oral surface (true pyorrhea) will from the beginning harbor a multiplicity of microörganisms, some merely saprophytic, others of pathogenic character; while, on the other hand, that both alveolar abscesses and those apical abscesses which do not follow infection of the pulp from carious exposure, being probably due to hemic or lymphatic infection, will primarily (and until penetration to the oral surfaces gives access from the cavity) present an actually or relatively simple infection. 


\section{PYORRHEA ALVEOLARIS}

(Riggs' disease, Fouchard's disease, interstitial gingivitis, alveolitis, alveolodental pyorrhea, alveolar periosteitis, chronic suppurative pericementitis, phagedenic pericementitis, calcic pericementitis, etc.)

Of these chronic peridental suppurations the type to which the above terms have been applied is the most common, and for this reason at least the most important. It is a long-continued and progressive disease beginning as a gingivitis and advancing along the root of the tooth into the alveolus, destroying the tissue of the peridental ligament and producing thereby a suppurating cavity known as a pyorrhea pocket, exposing the cementum, and by chronic inflammatory absorption causing progressive loss of the alveolar bone, with chronic cellular infiltration and sclerosis of the adjacent soft tissues, these changes in their fuller development leading to the loosening and eventually the loss of the tooth or teeth involved.

Undoubtedly the affection has prevailed from the most ancient periods. It is stated that its local effects are appreciable in mummies taken from the earliest Egyptian pyramids and in the jaws of skeletons of prehistoric times. Although unquestionably it must have been referred to by earlier medical and dental writers under such indefinite terms as "spongy gums," "bleeding gums," "ulcerated gums," "loosened teeth," etc., probably to Dr. J. M. Riggs, a dentist of Hartford, Connecticut, should be ascribed the credit of bringing the condition prominently before the dental profession, which he did mainly by a series of lectures and clinics rather than by formal publication, in a period of years from about 1865 onward into the seventies(6), his name becoming associated with the condition (in which he was principally interested in order to demonstrate a mode of cure by establishing surgical drainage) so that even to the present it is frequently spoken of as "Riggs' disease." The commonly applied term, pyorrhea alveolaris, was first employed by the dentist, Rehwinkel(7), of Chillicothe, Ohio, who contributed one of the earliest comprehensive papers upon the subject, with review of American and European references to the condition. The name "chronic suppurative pericementitis" was suggested by the late Dr. G. V. Black(8), of Chicago, who was the first to describe the anatomical features of the pyorrheal pocket, with clear differentiation from alveolar and apical abscesses(9). Confusion of the true pyorrhea lesion with other peridental suppurations existed not only prior to Black's description, but has continued and contributed largely even to the present time to faults in the selection of appropriate modes of dealing with the individual types of these affections.

Etiology.-Among dentists, there has been, moreover, no little confusion from attempts to classify pyorrhea in relation to various general conditions, because of a common belief that it is a local manifestation of general morbid states (anemic or cachectic pyorrhea, diabetic pyorrhea, gouty pyorrhea, nephritic pyorrhea, senile pyorrhea, 
etc.). At present it is more and more coming to be regarded as a local infectious disease; and, while reciprocal relations with various general diseases are not denied, their influence is being increasingly accepted as contributory rather than as primarily causative, and dentists and medical men are coming to look upon some at least of the general affections once thought to be causative as in reality secondary to the local peridental infection.

The disease begins as a gingival lesion, and progresses thence to the alveolar level in the peridental tissues. In its inception there is no reason to refuse the idea that microörganisms growing in the detritus in the gingival groove, especially in a badly kept mouth, may be directly responsible for the ulceration; but, on the other hand, it is generally believed that gingival trauma, as by food impaction, by toothpicks, harsh toothbrushes and the like, may occasior the primary port for microbic penetration into the tissues. Much has been made of the influence of tartar encrustations in producing a similar effect. A mass of salivary tartar, perhaps ledge-like and overhanging the gingival border, is driven down upon the gum margin in forcible occlusion of the jaws with the effect of separating the gum from about the neck of the tooth, infection taking place in such space of separation. Moreover, it is thought that with the development of the suppurative pocket tartar crusts formed upon the exposed root surface may likewise be responsible for further separation of the peridental tissue from the cementum of the root in strong occlusion, by being driven with the tooth deeper into the alveolus and thus pressing upon the peridental tissues. There is no reason why this mechanical influence of tartar should not be recognized in individual cases; but it cannot be an invariable factor, because pericemental suppurative lesions may exist without tartar and vice versa. In the view of the writers these deposits of tartar on the exposed root in the pocket are secondary to the actual lesion, resulting from calcification of necrotic detritus adherent to the exposed surface, and are rarely likely, even if present, to extend so deep in the pocket as to act to mechanically advance the separation.

Whatever the factors thus far alluded to may accomplish in the establishment and promotion of the lesion, the determining importance of local infection cannot be set aside; and since the work of Miller the discovery of a specific agency has been the main object of both dentists and medical investigators. Of the various forms of bacteria met in these lesions may be named the different pyogenic staphylococci and a group of streptococci and diplococci, including prominently Streptococcus viridans (Streptococcus mitis, Streptococcus salivarius, Streptococcus hæmolyticus, Streptococcus fecalis), Streptococcus mucosus, Streptococcus, catarrhalis, pneumococcus, along with Bacillus pyocyaneus, bacillus of influenza organisms of the colon group and of the proteus group, spirochete, and fusiform bacilli, and a large number of less important forms of saprophytic organisms. There are commonly present, too, marked growths of leptothrix threads, and 
often some one or more types of the higher fungi. Of these vegetable organisms, embracing in all dozens of typef, it is believed (mainly upon the basis of work by Rosenow and his associates) that the streptococcus-pneumococcus group are of especial importance. Of these Streptococcus viridans is most uniformly recognized. But in spite of the exceptional work that has been done in the study of this group (especially bearing upon their important metastases and production of arthritic and other distant lesions), their status in the causation of the local peridental lesion cannot be said to be proved. Doubtless they and their associates all take part in the local process; but that they are specific agents in the causation of chronic suppurative pericementitis is not proved. For many years it has been known that there occur in the mouth certain rhizopodial and flagellate protozoan parasites; and in I9I4 the writers, followed by Bass and Johns(ro), urged the pathogenic importance of certain parasitic amebas in establishing and maintaining the pyorrheal types of peridental suppuration. Apparently two species of endamebas(I I) may be encountered, Endamaba gingivalis (Gros) and Endamaba pyogenes (Verdun and Bruyant), although the latter seems to be met in only a small fraction of cases and has not been seen in these lesions by the writers. The basis for the claim of pathogenic importance includes the almost invariable presence of these protozoa in the contents of the suppurative pockets, and the cessation of suppuration when an efficient amebicidal agent, as emetin hydrochlorid, is administered (best by local application to the lesion, but also when given hypodermatically). A wide interest followed this announcement, especially in the dental profession but also in the medical profession; and opinions as to the justice of the claims of the authors became sharply divided between advocacy and refusal. The main grounds for refusal include the assertion that while these endamebas are, it is true, found with great frequency in the contents of pyorrhea pockets, they are found also in normal mouths(I2) and are really no more than saprozoa; and in the second place that whatever value emetin may have as an amebicide in healing amebic lesions elsewhere (as in the bowel in amebic dysentery) it is useless in the treatment of pyorrhea(I3). The writers regret that it has thus far (unless the recently published success of Wherry and Oliver meet acceptance(I4)) been found impossible to artificially cultivate, either in isolation or in known symbiosis, these protozoa so as to make inoculation studies feasible; but maintain the credibility of their views in spite of the criticism referred to. They feel justified in so doing upon the basis of their original claims, strengthened by accumulated experience( I $_{5}$ ); and question in the first place the really normal state of some of the mouths in which these parasites are said to have been found. Further, inasmuch as these parasites also exist in the tonsillar crypts in chronic cryptal tonsillitis(I6), and in the suppurative material in chronic (especially recurrent) inflammations of the nasal passages and accessory $\operatorname{sinuses}(\mathrm{I} 7)$, it is quite possible that from these latter foci the para- 
sites may spread over the oral surfaces (supposing there exist no chronic pericemental suppurative foci); so that in declaring the innocuousness of these parasites in a given individual it is essential not only to exclude as possible sources the most obscure peridental lesions (for which the careful attention of a thoroughly competent dentist is essential), but also ameba-containing lesions in the tonsils, nose and sinuses (demanding again careful investigation at the hands of a trained specialist). The criticism involving the value of emetin in the treatment of the ameba-bearing pyorrhea lesion they believe untenable if there be employed a good preparation of the drug, fresh, and in proper dosage, in the manner to be detailed below; failure depending upon fault in selecting appropriate cases, or in fault in the method of applying the remedy. Used in concentrations recommended by the writers for local employment in the mouth the drug is undoubtedly bactericidal as well as amebicidal; but it possesses a wide margin of amebicidal efficiency in higher dilutions in which no thought of bacterial destruction can be admitted, and these grades are met in the dilution of the emetin in the body fluids when administered hypodermatically. In the latter mode of administration it can be only amebicidal; but clinically is found to cause prompt disappearance of the amebas from the mouth and to be followed by cessation of suppuration about the teeth in a large proportion of cases (less efficiently however than when locally applied, where it may be believed to be lethal both to amebas and to many of the associated bacteria). It is scarcely possible that the amebas are the sole specific agents, alone responsible for the peridental lesions; rather it may be urged that in symbiosis with some or with all of the associated bacteria are their effects produced. These protozoa are actively phagocytic of leukocytes, red blood cells and probably also of tissue elements, and may by their digestion of such substances produce low products of protein dissociation peculiarly suited as pabulum for bacterial growth, and may thus favor a rank flora such as is characteristically found in these lesions. But they also phagocytize bacteria and are of ten found loaded with bacterial fragments. In this destruction of bacteria they may contribute in important degree to the freeing of bacterial toxins (which also are freed by ordinary death and disintegration of non-phagocytized bacteria). In the absence of evidence of any one constant toxic effect of chronic suppurative pericementitis it may be questioned whether the amebas themselves elaborate a toxin (and none is known to be produced by any species of amebas); but as the special toxin of now this, now that bacterium, according to the grade of symbiotic association in the pocket, is thus produced and is absorbed (this varying with the thickness and density of the wall of the pocket, and inversely to the proportion of drainage discharge of the pocket) selective action of these absorbed poisons upon different structures of the body may be reasonably expected. That the filtered extracts from such pockets (bacteria-free) are capable of exciting inflammatory changes in joints, 
serous membranes and lymph nodes has been proved(5); but this does not exclude equal possibility that metastasis of the bacteria to distant positions may also take place, and, alone or in conjunction with the toxins, excite the corporeal complications of the oral sepsis (accepting therefore the importance of the subinfections urged by Billings, Gilmar, Rosenow, Davis and others).

If, however, the symbiotic chain in the pericemental focus be broken by the destruction of the amebas by an efficient amebicide, a particularly favorable feature for bacterial growth and maintenance is eliminated; and the local infection disappears in the great majority of cases with marked promptness.

The writers have found these endamebas in over 95 per cent. of the pyorrhea lesions which they have examined; and for this large group of cases have used the term amebic pyorrhea, in the same sense that the term amebic dysentery is employed for those cases of colonic ulceration, in the stools and lesions of which dysenteric amebas are encountered; and believe that the relations of the parasites in the two conditions are identical (present in both; when destroyed in either by an amebicide the lesions heal; neither species culturally isolated and tested alone for pathogenic ability; in both associated with bacteria which it is possible they may influence symbiotically or which they may merely convey). In addition to these "amebic" cases, however, there are other instances of peridental suppurations which fail to show the presence of endamebas, but which are characterized by special presence of spirochetes or particular types of bacteria. In the first of these the employment of a known efficient spirochetocide, as neosalvarsan, is readily productive of cure; and the authors believe it proper to speak of these as "spirochetal pyorrhea." In the second neither emetin nor salvarsan finds an especial value; but here ordinary bactericidal agents, as iodine, silver preparations, etc., if applied regularly for a few days, usually act efficiently. To these cases the term "bacterial pyorrhea" might well be applied.

In the study of these different microörganisms the usual methods of microscopic examination, including the use of dark field illumination and the methods of bacterial cultivation, are of course all to be employed. For the detection of endamebas the writers prefer immediate search for the living organisms, followed as desired by permanent staining. Others believe they find advantages in making preliminary stained preparations after simple heat fixation, with subsequent examination of the living organisms and preparation of carefully fixed and stained specimens at will. In examining the fresh material the writers place a small drop of normal salt solution upon a clean slide, warm it slightly over the flame, then diffuse in this a bit of the purulent material abstracted from a pocket by means of a convenient carrier, as a scaling tool. This is covered, and examined, with the light-diaphragm well cut down, under a 4-mm. lens and appropriate eyepiece. The diffusion in the saline is practised so as to scatter 
well the mass of leptothrix threads, bacteria and detritus, and thus give sufficient clear field in which to observe the protozoa in their movements - these being scarcely distinguishable otherwise in the mass of obscuring material. (A small drop of saliva will serve equally well as diluent; but water or hypotonic and hypertonic salt solutions are unfit.) The size, activity of motion, shape and number of pseudopods, proportion of clear ectoplasm, degree of granularity and number of coarse englobed bodies in the endoplasm, and the vacuoles are thus well made out. Search for the nucleus in the unstained specimen should be made, although in the common Endamaba gingivalis it cannot be seen until stained (but the larger and more solid nucleus of Endamceba pyogenes should be visible without staining). If one prefer to stain directly for preliminary examination, the material from the pocket should be evenly and quickly spread over a cover or slide, dried in the air until visible moisture is gone from the film, fixed by drawing the preparation several times through the flame (film side away from the flame); after which the film is flooded for a moment with carbol-fuchsin solution, quickly washed in water, flooded without previous drying with Loeffler's methylene blue solution for half a minute to a minute, and washed. The preparation is then dried and mounted in the usual manner, and examined with convenient air objective (or, without mounting, in oil with an oil immersion objective). The protozoa in such preparations are easily distinguished, but the details of structure are not well differentiated. For permanent preparation and careful study films should be wetfixed in Schaudinn's solution (saturated aqueous solution of mercuric chlorid, I ; 90 per cent. or absolute alcohol, 2), being kept in this solution for not less than ten or fifteen minutes (best at body temperature or slightly above). Thereafter the preparation may be kept indefinitely in 70 per cent. alcohol; the mercury to be discharged before staining by immersion for five or more minutes in Gram's iodine-iodid of potash solution and washing in alcohol. To demonstrate the general structure Giemsa's method of staining may be recommended (for details consult laboratory manuals); but for the nuclear structure no method of demonstration is comparable to a good hematoxylin staining (especially Heidenhain's iron-hematoxylin-for which consult laboratory manuals). Endamaba gingivalis (Gros)(II), recently known as Endamoeba buccalis Prowazek, which is, however, only a synonym, ranges in diameter of the round restingparasite up to 30 to 35 micromillimeters; moves with nearly or quite the activity (variable) of Endamoba histolytica Schaudinn, which it closely resembles morphologically. Its pseudopods are few, broad and lobose; ectosarc complete; endosarc granular and often full of englobed bacteria and coarse fragments of chromatin of phagocytized leukocytes and other cells; red cells are quickly hemolyzed by it and are not found in preserved condition within it in numbers comparable to those of the erythrocytes often seen in Endamoeba histolytica. Its nucleus is very like that of the "histolytica stage" of Endamaba 
histolytica, small, vesicular, with a small, of ten dot-like, chromatinized "binnenkörper;" in location it is more often central than is the nucleus of Endameeba histolytica, but may be found in subcentral or eccentric position. Reproductive cysts are not known; but it has been seen to multiply by ordinary binary fission and apparently also by gemmation (denied by many). Endamæba pyogenes Verdun and Bruyant is especially distinguished from the above by its larger nucleus, which contains a large, highly chromatinized binnenkörper.

These endamebas are found widely over the oral mucous surface, and too, in tonsillar crypts and on the mucosa of the nasal passages and accessory sinuses; but are particularly numerous in the depths (Bass and Johns) of pericementitis pockets. They are not readily found in the wall of the pockets; but have been met in material from deep abscesses of the jaw and neighboring soft tissues and are therefore not limited to mere superficial occurrence.

Pathological Anatomy and Symptomatology.-Beginning as an acute or chronic ulcerative gingivitis, with progressive separation of the soft tissue from the tooth, the process advances along the peridental membrane toward the apex of the tooth, with comparatively little tendency to extend laterally into and through the alveolar structures as do apical and other alveolar abscesses. There are met occasional instances of an acute ulcerative gingivitis with especial phagedenic destructive tendencies which differ from the ordinary and less active gingival lesions of the usual type of pyorrhea. In some of these at least, spirochetes and fusiform bacilli of Vincent are especially numerous, this feature and the promptness of relief from the local application of salvarsan, suggesting that they constitute a particular spirochetal form. They are apt to develop rapidly without previous known changes in the gums, to spread extensively, to erode quickly and deeply, to show intense redness and swelling of the gums with hemorrhage and a pasty, whitish discharge from the ulcers, and to be attended by considerable local pain and tenderness and by some systemic reaction. In ordinary pyorrhea alveolaris, however, there is little pain or tenderness at the beginning of the lesion, although the gums become somewhat swollen, reddish and slightly spongy, bleeding when the teeth are brushed or upon even slight food impaction in chewing. Close inspection with good lighting may be required for detection of the small and early lesions. When fairly developed the usual lesion has extended along the root of the tooth into the alveolar level, and involves a variable extent of the circumference of the root. External inspection shows the gums more or less reddened, swollen and spongy; and, exuding from the orifice at the gingival margin, spontaneously or expressed by slight pressure, a purulent material, creamy or yellowish in color, varying in consistence from a milky to a thin paste-like substance. The older the lesion the more likely the gum margin is found shrunken and exposing the root, and the tooth loosened. Only when the process has reached well toward the apex, causing swelling of the deeper 
peridental and apical tissue, tenderness and pain are apt to be complained of. Bleeding from brushing the teeth or from mastication is common throughout the course.

In the more recent and relatively acute examples the pocket is bounded on the one hand by the exposed cementum of the tooth, and on the other by the inflamed soft parts, showing a necrotic lining overlying a granulation tissue base; but in the more commonly observed old, chronically progressing pockets the epithelium of the gum is found grown into the pocket well toward the bottom, the subepithelial tissue being sclerosed from the prolonged inflammation. Only at the end of the pocket as a rule is there left a small extent of exposed granulation tissue surface. The loss of tissue in the formation of this pocket involves primarily the peridental membrane, but extends also to the fibrocellular tissue lining the alveolar bone and the bone itself with result of secondary absorption of the alveolar wall. The loss of the latter materially aids in the opportunity for shrinkage of the gum and exposure of the root, and, too, for the enlargement of the tooth socket. The involved tooth thus becomes more and more loose, and is often eventually lost. Over the exposed root scales of tartar frequently form, but are not invariably present.

In examining microscopically a section of an involved tooth and its surrounding structures one notes primarily the cavity extending from the free gum along the root, in which the mixed content lies as a dense and confused mass of pus, detritus and microörganisms; on the exposed root surface perhaps a thin scale of tartar not reaching to the full depth of the cavity, overlying the cementum, which may show no appreciable change; with the underlying dentine also probably without appreciable change. On the opposite wall of the cavity a thin extension of the gingival epithelium reaches well to the bottom of the cavity, with a small area of ulcerative exposure at the depth. Beneath this epithelium the connective tissue varies in different specimens and at different levels, ranging from recent cellular infiltrations in the deeper to dense cicatricial tissue toward the gingival margin (with sparsely scattered lymphoid and plasma cells in the midst of the fibrous stroma). As may be expected polynuclear leukocytes abound in the cellular infiltrate at the deeper levels, where the ulcerous process is advancing, but are rare in the older and more cicatrized tissue near the gum margins. This infiltrated and sclerosing tissue passes with little differentiation to the surface of the irregularly absorbed bone; about which and into the spaces of which the fibrocellular tissue extends.

It is exceptional that endamebas can be shown within the tissues of the wall of the pocket except in the ulcerating area at the bottom of the cavity. Here they are occasionally seen; but, as is well known, in active suppurating foci in the bone or soft parts (as in osteomyelitic cavities and about exfoliated bits of bone in connection with the process in the soft tissues) they are apt to abound. By appro- 
priate methods bacterial invasion of the tissues of the wall, more frequently than otherwise by streptococci, has been proved.

As complications of such conditions submaxillary and cervical lymphadenitis, arthritis of even the most distant joints, and other lesions already referred to are met; the active phases of which often subside with remarkable promptness when the peridental foci (and other anologous foci) are removed. That the tooth must suffer to no more than the passive degree above suggested is not to be accepted, even though morphologically it shows little alteration. The cementum is a porous structure and both toxic matter and microörganisms doubtless enter its lacunæ and destroy the cementoblasts; thus providing a source for reinfection in case the pocket be mechanically freed from its infectious agents. The dentine is likewise porous, and may permit passage of soluble toxic matter if not the actual microörganisms into the pulp, which in consequence is apt to slowly degenerate and die, even though the tooth shows no exterior carious changes. It is commonly believed, too, that exposure of the denuded root to marked variations of temperature is responsible for the death of the tooth. The chance of hemic or lymphatic infection of this dead pulp is accentuated by lack of vital resistive factors; and in this way (even if not more directly) apical lesions may be secondary results. With the loosening and loss of teeth by faults of dental approximation mastication is disturbed more and more; and the swallowing of improperly masticated food (together with increased swallowing of toxic and infectious agents discharged from the pockets into the mouth) is believed to lead to the very common complications of gastritis and gastroenteritis. These faults associated with other corporeal complications, may in the end seriously affect the general nutrition of the subject.

Treatment.- If the common opinion of the present be correct that this affection is not merely a local manifestation of general diseases, but is rather a primary local infectious lesion, responsible at times for the corporeal conditions accompanying it rather than resulting from them, the rational indications for treatment involve local destruction or elimination of the infecting agents as the primary measure. This does not mean that systemic treatment of complications and measures intended for the improvement of the general health and palliative of the collateral disturbances should be disregarded; these at times may materially influence the local reaction, and attention to them is distinctly indicated.

Long overlooked or disregarded by the medical profession, chronic suppurative pericementitis has with the general dental practitioner been looked upon as a disease of almost hopeless prognosis as far as securing permanent cessation of suppuration is concerned (to say nothing of actual cure with repair of the local changes), but as amenable to palliative measures. And in any case which has advanced to destruction of the peridental membrane, with absorption of the bone of the alveolar ridge and cicatricial shrinkage of 
the gums, question of material repair can scarcely be considered; and the term cure must be accepted as being relative rather than absolute.

Even this as the final result may, however, leave the tooth with sufficient ancharage to make it useful; and in the cicatrization beyond the level of separation some degree of added fixation may be attained. From a medical standpoint, too, the ending of the suppurative process and healing of the inflamed area is the greater point to be attained; and this without reference to incompleteness of local repair should be sought, with the purpose of ending the chance of continued septic absorption and of microörganismal metastasis.

To accomplish these ends local measures of elimination and destruction of the infecting agents occupy first place in the treatment, and measures tending to prevent recurrence must stand in close relation.

Riggs' original treatment, to which his efforts were mainly directed rather than to the demonstration of a special disease entity, included incising the gum and exsecting the tissue to the bottom of the pocket so that a permanent drainage from the pus pocket should exist, preventing retention of microörganisms and permitting the local reactive processes of the tissues to destroy the reduced infection and heal the ulcerating area in the base of the pocket. This measure alone was productive of marked palliative benefit in a large proportion of favorably situated pockets, and continues to be followed in case of deep lesions favorably situated (as on the buccal side of the lower gum) when other measures have failed to give a proper relief, or in association with other measures.

The usual, so-called "prophylactic" dental operative procedure has as its basis the mechanical cleansing of the pocket. Under the belief that tartar encrustations along the root are responsible for deepening the cavity and maintenance of the inflammation, the process has the removal of tartar scales as the major part of its object, this being accomplished by instrumentation according to the skill and persistence of the dental operator, who seeks to remove the scale and the superficial layer of porous cementum (the surface of which is held to be more or less impregnated with microörganisms and toxic matter absorbed from the pocket), leaving the surface as smooth and clean as possible. The pocket is cleansed by repeated flushing with normal saline solution; and, with the same end in view and according to the judgment of the operator, its base and wall in the soft tissue are curetted, and perhaps, in picked cases, drainage established by excision of the gum. Local disinfecting applications are employed in conjunction, but in comparison with their effects elsewhere in sterilization of tissues their results are not so impressive or conducive to reliance upon them. The good effects of such "prophylactic" practice is clearly due rather to the cleansing than to the accessory employment of the ordinary disinfectant agents. Unquestionably the work of those especially skillful and thorough 
has shown the possibility of cure (in the limited sense) of Riggs' disease; and at the hands of most dental practitioners the procedure may be accepted as usually palliative and effective in temporarily staying the active progress of the lesion. In individual cases the added employment of fixation of loosened teeth by some type of appliance and the institution of general medical treatment of associated corporeal faults as anemia, diabetes, rheumatism, etc., have materially aided in obtaining measurable success; although this cannot be said to be true in any very important proportion of cases. In fact in splinting loosened teeth to obtain rest the appliance employed may well add such difficulties to the maintenance of oral cleanliness as to work harm rather than otherwise. In picked cases the indications for collateral medical attention is clear enough and its effects upon the general health valuable; but it is possible to interpret the relations of such treatment to the local oral condition in more than the one sense that such corporeal faults are basically responsible for the development or the maintenance of the local lesion.

However, granting the degree of success just outlined for the method, it cannot but be added that because of the severe pain occasioned by this composite "prophylactic" procedure, the usual necessity for its frequent repetition, the varying skill among operators and the common eventual loss of the affected teeth so treated, much of the unenviable reputation in which chronic suppurative pericementitis has been held popularly and in the minds of the average dental practitioner has arisen; and the plan by no means fulfils all that is to be desired.

In the belief in the existence of a definite and perhaps a specific infectious cause of this widely prevalent affliction the hope of discovery of some efficient disinfecting agent, to be employed either alone or in conjunction with auxilary local cleansing measures, has led to the employment of now this, now that of the long list of known disinfectants, the value of which has been only measurable and the results incomplete. The belief in a bacterial origin of the affection has led also to the employment of vaccines, sometimes of isolated organisms, sometimes mixed, made from cultures obtained from the lesions. Occasional brilliant results have been obtained from vaccines, but more often no influence has been appreciated; and probably true pyorrhea is not the best field for the employment of such agents.

Primarily for the purpose of determining the effect of eliminating the endamebas above discussed, the writers made. use of emetin hydrochlorid solutions by direct application to the pyorrhea pockets. Emetin hydrochlorid had been used successfully in treating amebic dysentery by Sir Leonard Rogers (I8) (its use having been suggested by experimental studies by Vedder of the United States Army Medical Corps intended to determine the constituent of ipecac responsible for the amebicidal effects of the latter drug(Ig)). Voc. IV. -38 
Rogers' published statements as to the efficiency of emetin in dysentery and amebic abscesses of the liver had been amply verified all over the districts of the world in which amebic dysentery prevails; and it was believed by the writers that it would equally serve to destroy the oral endamebas and thus eliminate one fraction of the confused group of vegetable and protozoan microörganisms commonly to be found in pyorrhea cavities. Direct instillation of a 0.5 per cent. solution of emetin hydrochlorid was found not only to destroy these parasites in the course of a few days; but at the same time the flora of the cavities diminished greatly or apparently disappeared and the lesions healed (but without regeneration of lost tissue). It was on the basis of this effect that we have urged the pathogenic importance of these amebic parasites, probably tot as the sole pathogenic factor involved but rather in a symbiotic relation with one or more of the associated bacteria. In spite of a number of adverse reports it is commonly accepted that with proper use this remedy is really curative of the suppurating process, particularly when reasonably aided by accessory attention as will be detailed. A difference in the effect of the drug is shown by different samples, although the reason for such variation is not known. It is most effective when used in freshly made solution, and in a strength of not more than $\mathbf{I}$ part of the drug in 200 of the diluent. (Weaker solutions than indicated are also efficient.) Old solutions are usually found to contain bacterial growths which apparently fix or change the amebicidal moiety of the drug; and higher concentrations used in local application are productive of undesirable local inflammatory reactions. Bass and Johns (20), working and publishing shortly after the writers, proposed the systemic use of emetin hydrochlorid by hypodermic injection; and claimed for the remedy a truly specific effect. This extreme position has not been sustained, however; but there is ample corroboration that in a large proportion of cases the systemic administration is destructive of the amebas in the mouth (just as it is lethal to the intestinal amebas in the dysenteric colon), and that with the destruction of these parasites the suppuration ceases and the lesion heals. It is true that in dilutions of $I: 1250$ and less(2I) emetin in vitro is not only amebicidal but bactericidal as well, and it is inhibitive of the growth of certain spore-forming bacteria even in dilutions of $\mathrm{I}: 5000$ and $\mathrm{I}: 10000$ in vitro. No comparable bactericidal value, however, is known for emetin in the living body. In vitro it is apparently amebicidal in high dilution (changes occurring in the endamebas upon four hours' exposure to solution of $\mathrm{x}$ : over 400,000, which are either indicative of death or of a prelethal condition), proportions which are comparable to the degree of dilution the drug attains when administered in ordinary hypodermatic dosage. While when employed by local instillation into the pockets it probably acts both by its amebicidal and bactericidal influences, it can be safely regarded as acting solely as an amebicide when successfully used by systemic administration; and this the writers believe to be in substantiation 
of their view that these parasitic amebas are important pathogenic factors in this disease. Used locally in appropriate manner and with logical adjuvant treatment the results have been in our hands fully satisfactory almost uniformly; and by hypodermatic administration published statements indicate that over 50 per cent. of cases are either cured or markedly benefited. The local method of employment requires the skilled services of a dentist; and in routine is briefly as follows. Having determined by microscopic examination the prevalence of endamebas in the pocket contents, and having. flushed out all the pockets with normal saline solution by means of a dental syringe, the operator prepares his emetin hydrochlorid solution by dissolving a $0.0 \mathrm{I} \mathrm{gm}$. hypodermic tablet of the drug in 2 c.c. of sterile normal saline solution or water. This amount should not be exceeded for a single treatment, irrespective of the total number of pockets to be filled, since there is invariably some of the drug swallowed, which may in greater quantities occasion unpleasant symptoms of nausea and vomiting. With a small syringe with fine, flexible, blunt-pointed needle, the drug is introduced successively into each of the pockets, the needle made to reach to the bottom of the pocket, and leaving each cavity filled as it is withdrawn. Even in the dilution named ( 0.5 per cent. or $\mathrm{I}: 200)$ the drug is apt to inflame the mucous membrane of some persons; and as a preventive it is appropriate to lightly anoint the gingival and lip surfaces with vaseline before applying the solution. The patient should be advised against swallowing the saliva for a time thereafter. Such applications should be given daily for from five to six days and thereafter every other day for as many times. In conjunction with these applications tartar encrustations should be removed, not all at once and by persistent and energetic scaling, but gradually and gently, so as to leave the mouth clear of such deposit in the course of the full period of treatment. As a rule after the third or fourth application of emetin the suppuration will have ceased to gross inspection; and the swelling and redness of the gums will have disappeared. Continuation of applications thereafter is, however, advisable, to insure complete destruction of any remaining amebas, and thus to prevent an early recurrence. With the cessation of suppuration loosened teeth feel firmer and are firmer to the touch; the increased fixation being probably at first due to condensation of the congested and edematous inflamed peridental tissue, but that which remains permanently after completion of the local applications being surely dependent upon other factors, as peridental cicatrization. Not infrequently, although suppuration is promptly and largely diminished, some slight persistence of discharge remains; and here as a rule the above treatment is properly made to include a terminal application of bactericidal agents, as a solution of iodine in normal saline.

There is no assurance that reinfection may not take place; but with care and cleanliness the pocket may remain healed indefinitely. 
Lesions treated by us in May and June of I9I4 remain without reinfection to the date of writing (nearly two years); but others have recurred. However, the same measures serve again to heal the reinfected foci.

The local use of the drug is unquestionably more efficient than its hypodermic administration, and we believe should always be employed in combating these suppurative foci when confined to the peridental tissues. But when there is reason to believe the infestment by amebas and their associates exists coincidently in localities not easily reached for local applications, as in the tonsils or sinuses, or when the services of an interested dentist cannot be commanded, the drug may be used hypodermatically. For the average adult, doses of from $1 / 3$ grain to a grain, (0.02 to $0.065 \mathrm{gm}$.) or exceptionally even 2 grains, may be administered by deep injection into the tissues (not intravenously). More or less pain persisting after such injections is to be expected for some hours, and tenderness lasting for a day or two. The drug should be thus administered for three or four days, followed by an interval of at least equal duration; after which several similar courses broken by intervals should succeed.

Emetin is not the only remedy of importance as an amebicide. Quinin, thymol and many other drugs also are lethal to these parasites. Quinin applications formed the basis of a method followed and advocated years ago by the late Dr. Truman, of Philadelphia, but abandoned because of the local irritation caused by the drug and because of its unpleasant taste. As early as 1877 , thymol applied in alcoholic solution with a camels-hair pencil was recognized as curative(22). Succinamid of mercury has been urged and is successfully used for the treatment of these affections (believed to act rather as a bactericide). It is given by deep hypodermatic introduction in doses of from $2 / 5$ grain to a grain ( $0.03-0.065 \mathrm{gm}$.), repeated three or more times at intervals of six or seven days.

In the group, above referred to, of spirochetal pyorrheas, the application of arsenic compounds of the type of salvarsan (either by dusting into the lesions or by instillation of solutions of the drug into the pocket) acts usually with marked benefit, often allaying the inflammation within the day of first employment and curing it in the course of two or three days more. In the above-mentioned group of bacterial pyorrheas almost any antiseptic of known value will prove of service if applied in the same way as is the emetin solution in the amebic pyorrhea. Iodine or the various silver preparations are effective; the writers finding a I per cent. solution of iodine in normal saline solution excellent. Here, if at all in true pyorrhea, vaccine medication should find its best application; and doubtless the occasional complete cures reported for vaccines have been of this form

In all advanced cases, finally, with marked loosening of the teeth, where it is doubtful whether, even if the active process is stopped by emetin or other remedy, the teeth can ever recover sufficient 
fixation to permit efficient mastication, the radical treatment of removal of the teeth should be urged. Where an affected tooth has more than one root, and only one of these is seriously involved, amputation of the root close to the crown and its removal by excision of the gum and alveolar bone, may serve the same purpose of thorough eradication without loss of the tooth. Such radical treatment, moreover, is to be advocated when important apical complications add to the seriousness of the septic condition.

In summary the writers feel that, because of its promise of healing the suppuration and because of the ease and painlessness of its application, the use of an amebicide such as emetin should be urged in the usual amebic case of pericementitis; they acknowledge the palliative and occasional curative results of ordinary "prophylactic" instrumentation, but refrain from urging it because of its frequent failure, the usual need for frequent repetition, the extreme pain it occasions to many individuals, and the possibility that it may serve to disseminate the infectious material in the surrounding tissues and lead to extension of the local lesion and the chance of freer toxic absorption and metastasis of bacteria. And they advise in advanced cases, with important local complications or with little prospect of recovery of proper fixation and adaptation of the affected teeth, that radical removal be the only plan of procedure considered.

\section{APICAL AND SUBGINGIVAL ALVEOLAR ABSCESSES}

Suppurations beginning otherwise than by the process described in case of true pyorrhea fall under this heading. They most frequently develop about the apex of a tooth but may develop along the face of the root, above the apex and beneath an intact gum. The former, too, by extension along the root toward the crown may give origin to the lateral and intermediate alveolar suppurations. An apical abscess may originate from primary oral infection of an exposed pulp, or on the other hand may form at the apex of a non-carious tooth by primary hemicinfection of the pulp or by hemic or lymphatic infection of the periapical tissue. The common belief is that such abscesses are necessarily secondary to pulp infection and necrosis; but there is no reason why the original focus may not be outside the pulp at the apex, and give rise secondarily to pulp infection. However, it is the rule to meet the two conditions in association, and undoubtedly the infected pulp by constantly furnishing microörganisms to the periapical tissue serves to prevent the ready correction of the apical lesion by the usual antagonistic agencies of the tissues and body fluids. An apical abscess, therefore, once established is almost sure to pass into a chronic course. Subgingival abscesses are believed, likewise, to depend upon hemic or lymphatic infection of the peridental tissues, although a number of them represent merely an extension of the apical suppuration along the root. In their acute phase both are attended by marked pain. There is tenderness 
on mastication, exaggerated by the elevation of the tooth by the inflammatory swelling at the apex; and the least handling of the tooth, which may be loosened by the inflammation, usually occasions exquisite pain. In neither of these types is there as great a tendency to continuation of the suppuration in and along the peridental membrane as in case of pyorrhea, although there are a number of cases in which the pus finds its way to the oral surface at the gum margin. There is more tendency in the early, active phase of the lesions to extend into the bone of the alveolar process, necrosing it and causing its rapid absorption. Progressing through the bone it tends to advance to and through the periosteum, and finally after causing a marked swelling of the gum, as a "gum boil," to discharge into the mouth, either on the buccal or lingual side of the lower or on the buccal or palatal side of the upper jaw. Further progress may sometimes be seen into and through the cheek (to discharge externally), or downward from the lower jaw into the tissues of the floor of the mouth and even into the neck (giving rise to a cellulitis of the type of Ludwig's angina). In the majority of cases, with or without such burrowing, the original focus becomes limited by the formation of a well-organized fibrous wall, the tooth in extraction having adherent to the side or the apex of the root a pus-containing sac.

The infectious agent involved in these forms of peridental suppuration is for obvious reasons apt to be a simple bacterial one, pure cultures of some one organism being often obtainable. The more common organisms met include the ordinary pyogenic staphylococci, streptococci, pneumococci and occasionally other species. In the acute phases of the abscess systemic symptoms of slight elevation of temperature, malaise, etc., are common; and in any of the chronic examples, because of the difficulty of drainage along established fistulæ or because of the absence of drainage, chronic toxic effects are apt to develop from systemic absorption from the lesions. Toxic and metastatic complications of the same type mentioned in connection with pyorrhea alveolaris are to be expected; and in proportion to the number and size of the lesions are probably even more sure to be encountered than in the latter (from which a better chance for drainage into the mouth exists).

There are in addition to the suppurative infections just noted analogous involvements due to non-pyogenic organisms, leading to varying necrotic and cicatricial changes in the localities involved, and capable in variable degree of giving rise to systemic effects.

In these infections the services of a competent dentist are essential. The method of dealing with them contemplates the opening of the canal of the affected tooth, penetration into the apical pus cavity, disinfection of the periapical focus and of the canal and pulp cavity by suitable antibacterial agents, and finally the proper filling of the canal and pulp chamber and repairing the crown of the tooth. This demands long, delicate instrumentation and disinfectant treat- 
ment. When the abscess is approached through the tooth, opportunity to obtain material for culture and manufacture of autogenous vaccines is afforded. Probably in such cases more than in pyorrhea, vaccines are to be expected to be of really curative value. A surgical procedure available when disinfectant applications through the canal fail of the desired end, but to be advised only when the extent of the lesion is limited, consists in lifting a small flap of the superficial tissue over the alveolar bone, penetrating the latter with trephine or chisel to the affected root and removing the entire sac and amputating the root apex within the sac, with a grinding burr, thus eliminating the focus entirely. Before operation, the canal of the tooth should have been thoroughly cleansed and filled permanently. The operation can readily be performed under local anesthesia. The wound is to be packed with antiseptic dressing for a few days, and allowed to heal from the bottom.

When the suppuration is extensive and any great part of the root involved, with correspondingly marked loosening from necrosis and absorption of the alveolar bone, it is best to proceed at once to extraction. This, too, is advisable as an immediate and radical procedure when there are symptoms of serious systemic complications.

\section{REFERENCES}

I. Dental Cosmos, September, I891; also, The Microörganisms of the Human Mouth, Philadelphia, 1890.

2. Brit. Med. Jour., July 28, 1900, p. 215; Practitioner, December, 1900; London Lancet, Jan. I4, I9I1, p. 79; Brit. Med. Jour., Nov. 19, I904, p. I358.

3. Billings, Arch. Int. Med., I91 2, ix, 484.

GILMER, ibid., p. 499.

Davis, ibid., p. 505 .

Billings, Jour. Am. Med. Assn., Sept. I3, I913, p. 819.

Rosenow, Jour. Inf. Dis., I9I4, xiv, I and 6r.

Rosenow, New York Med. Jour., Feb. 7 , rgr4.

Rosenow, Jour. Am. Med. Assn., April II, I9I4, p. I 146.

Rosenow, Jour. Am. Med. Assn., Sept. 12, 1014, p. 903.

Billings Jour. Am. Med. Assn., Dec. 5, 1914, p. 2024.

Rosenow, Jour. Am. Med. Assn., Nov. 13, I913, p. I687, etc.

4. Vide Billings, sup. cit., articles by Rosenow.

5. Unpublished work by Middleton and Smith, personal communication.

6. Dental Cosmos, 1869, xi, 412.

7. Trans. Am. Dental Assn., 1877, in Dental Cosmos, November, 1877, p. 572.

8. Special Dental Pathology, published by Medico-Dental Publishing Co., Chicago, Ill., I915.

9. Proc. Illinois Dental Soc., 1882 , p. 93.

Io. Suitir and Barretr, Preliminary report by Barrett, Dental Cosmos, August, I9I4.

Smrth, Dental Cosmos, September, r9r4, and January, 19 r5.

BAss and Jorns, New Orleans Med. and Surg. Jour., November, rgr 4 ; see also Chiavaro, abst. in Dental Cosmos, September, rgr4.

II. SMrth and Barretr, Jour. Parasitol, June, I915; September, I91 5.

12. Willians, v. Sholly and RosenberG, Proc. N. Y. Path. Soc., I9I5, n. s. v. I5, p. 34 .

Williams, v. Sholly, Rosenberg and Manx, Jour. Am. Med. Assn., December II, I9I5, p. 2070.

Craig, Jour. Inf. Dis., February, I9i6.

13. Hartzell, Dental Review, September, I915, p. 867.

Talbot, New Orleans Med. and Surg. Jour., March, 1915.

14. WherRy and Oliver, Lancet Clinic, April I, I9IO. 
I5. Smith and Barretr, Oral Health, April, 1915; Dental Review, October, I915; Dental Cosmos, November, I9r 5 .

Kolmer and SMITH, Jour. of Inf. Dis., March, ror 6.

SMITH and BARRETT, Items of Interest, June, I9 I6, p. 45I.

16. SMith, Middleton and BARRetT, Jour. Am. Med. Assn., Nov. 14, I9I4.

Evans, Middleton and SmitH, Am. Jour. Med. Sci., February, I916, 2 Io.

I7. SMITH and BARRETT, Dental Review, October, I9r 5.

Howe, New York Med. Rec., v, 88, p. 604.

18. Rogers, Brit. Med. Jour., June 22 and Aug. 24, I9I2; Therap. Gaz., I9I2, xxxv, 837; London Lancet, Oct. I9, I9 2.

19. Vedper, Bull. Manila Med. Soc., March, Igrr.

20. Bass and Johns, New Orleans Med. and Surg. Jour., November, 1914; Jour. Am. Med. Assn., February I3, I915, p. 553; New Orleans Med. and Surg. Jour., February, r9r 5 .

2I. Kolmer and SMITH, Jour. of Inf. Dis., March, r9i6, p. 247 and p. 266.

22. ReHwinkel, Dental Cosmos, November, 1877, p. 573 .

23. White, U. S. Naval Med. Bull., October, I914; Wright and White, New York Med. Rec., March I3, I9I 5. 


\title{
DISEASES OF THE MOUTH, TONGUE AND SALIVARY GLANDS
}

\author{
By Gegrge Morris Piersol, M. D.
}

In our original paper upon diseases of the mouth, the subject of mouth infection was given considerable importance, and much emphasis was laid upon the relationship which exists between oral sepsis and disturbances of the digestive tract, certain constitutional disorders and infectious processes remote from the mouth. Abundant clinical experience has amply confirmed our belief in the significance of septic conditions about the mouth as primary foci of infection in the production of many disorders. Without again entering into the subject in detail, in view of the ever-increasing importance of oral sepsis in its relation to etiology, diagnosis and prognosis, renewed emphasis must be placed upon our earlier statements in reference to the care that should be exercised in searching for septic foci in the mouth and the promptness and energy with which such sources of infection when once discovered, should be attacked and thoroughly eliminated.

\section{STOMATITIS (CATARRHAL, APHTHOUS, AND ULCERATIVE)}

For the sake of convenience, and possibly as the result of tradition, most writers have classified stomatitis into catarrhal stomatitis, aphthous stomatitis, ulcerative stomatitis, and thrush or parasitic stomatitis. In our original contribution on this subject, this conventional clinical grouping was rigidly adhered to, and under the various divisions may be found sufficiently detailed accounts of the etiology, clinical manifestations and generally accepted methods of treatment of these conditions. Although from the academic viewpoint this division of stomatitis into catarrhal, aphthous and ulcerative is both useful and satisfactory, from a purely clinical standpoint these various types of stomatitis, always excepting thrush, are by no means invariably found so clear cut and sharply defined as might be supposed from the classic descriptions usually accorded them. This is especially true in the case of children, in whom by far the largest number of cases of stomatitis are encountered. It has been pointed out, particularly by pediatrists, that many cases are seen in which the stomatitis begins as a simple catarrhal process, but which may soon become aphthous, a condition which readily passes over into the ulcerative type. Indeed, it is the exception to find an aphthous stomatitis which is not associated with more or less catarrhal inflammation of the mucous membrane of the mouth, and in almost every case of ulcerative stomatitis, there is present a concomitant catarrhal stomatitis. 
The three main types of stomatitis may be looked upon, therefore, with some justice as varying degrees of the same process. There is little doubt but that all these forms of stomatitis must be regarded as of infectious origin. As yet, however, a specific microörganism has not been discovered. A considerable array of mechanical, chemical and thermic factors have been described as the cause of the various types of stomatitis. In reality these are but contributing factors, the true exciting cause being undoubtedly bacterial. It is more than likely that the fusiform bacillus and spirillum of Vincent bear some relationship to the production of ulcerative stomatitis, just as they probably have some etiological significance in gangrenous stomatitis. There are some who hold that gangrenous stomatitis is only a more severe and aggravated manifestation of the same process that is going on in ulcerative stomatitis, and that the difference in the severity of the manifestations of the two conditions is determined by the resistance of the individual and by the degree of virulence of the infecting organism. The fact that from time to time the various forms of stomatitis have proved to be contagious is additional evidence in favor of the probable infectious nature of all the varieties.

\section{THRUSH}

Parasitic stomatitis, mycotic stomatitis, or thrush, has an entirely separate etiology from the above-mentioned forms of stomatitis, and must be considered as a separate type. The exact vegetable parasite which causes this disease is still a matter of discussion. Although formerly the Ö̈dium albicans was generally held to be the specific cause, the opinion of Grawitz, that the infecting parasite is the Saccharomyces albicans, is now the more generally accepted view.

Treatment.-The general prophylactic and curative measures, suitable to the management of the various forms of stomatitis, were given in detail in our original contribution, and the methods of treatment therein described have undergone no noteworthy change in recent years. Minor additions to the treatment of stomatitis may, however, be mentioned. In the case of the catarrhal and aphthous forms, in addition to plain boiled water and boric acid solution, which have proved so useful as mouthwashes, other non-irritating washes may be recommended. Dobell's solution, diluted with equal parts of cold boiled water has been highly recommended by some. In addition liquor antisepticus (I part to 3 or 4 of boiled water), or liquor antisepticus alkalinus in similar dilution, or solutions of sodium borate ( 2 or 3 per cent.) have been advocated. The essential feature in the local treatment of catarrhal stomatitis is to employ washes which are absolutely non-irritating. The use of the more powerful astringent and antiseptic washes urged by some appears to be not only unjustifiable but absolutely injurious. In aphthous stomatitis, in addition to the above-mentioned bland washes, certain astringents may be carefully employed. Holt advocates applying 
to each one of the small ulcers finely powdered burnt alum, applied with a camel's hair brush. Three per cent. boric-acid glycerin has also been recommended as an application to the aphthx. A weak solution of warm sodium bicarbonate has also been found to be a useful remedy in aphthous stomatitis.

In ulcerative stomatitis, potassium chlorate, as both a local and internal remedy, still maintains its prestige. By many it is regarded as a specific. Much has been said in reference to the danger of administering potassium chlorate to children on account of its alleged tendency to produce renal irritation. Kerley is inclined to believe that this danger has been much overestimated and he claims that he has never seen ill effects arise from the administration of potassium chlorate in doses of from ro to 20 grains daily. Hydrogen peroxid, in addition to potassium chlorate, seems to have become one of the most popular and useful mouthwashes in cases of ulcerative stomatitis. It is usually employed diluted with 2 or 3 parts of water. Recently a careful summary of the local treatment of ulcerative stomatitis has been given by Williger. He first proceeds to swab frequently the ulcerated surfaces with undiluted hydrogen peroxid, continuing this until all fetor has disappeared. The tartar is then gently removed from the teeth, and the ulcerations are quickly treated with an 8 per cent. solution of zinc chlorid. For half a day or a day, the patient then rinses the mouth every hour with an exceedingly weak solution of hydrogen peroxid, a teaspoonful of hydrogen peroxid being added to a glass of warm water, and in addition the mouth is cleansed three times a day by the finger wrapped in a thin layer of cotton. The rest of the tartar is then carefully removed. With this treatment Williger, has found that by the end of the third day the ulcers are healing, and at the end of a week the ulcerative stomatitis is cured. Instead of zinc chlorid as a local application to the ulcers, this same author recommends a mixture containing a little iodoform and a few drops of alcohol combined with enough of an 80 per cent. solution of lactic acid to make a thin paste. $\mathrm{He}$ also says that when once the stomatitis is controlled, the toothbrush which has been previously used, must be destroyed and replaced by a new clean brush.

For the treatment of thrush, Holt recommends, in addition to the use of the usual mild antiseptic washes, that twice a day a i per cent. solution of formalin be very gently applied.

\section{HERPETIC STOMATITIS}

In addition to the commoner forms of stomatitis just mentioned, certain rarer types of inflammation of the mouth have been described. Riesman notes a type of herpetic stomatitis characterized by an herpetiform eruption which invades various parts of the mouth. This eruption in the mouth is entirely distinct from any simple herpes that may occur on the lips. The eruption is usually limited to the posterior portion of the mouth, and may be associated with herpes of 
the pharynx. The condition sometimes occurs at the menstrual period. There is no specific treatment for it, but mild alkaline antiseptic washes are recommended.

\section{GONORRHEAL STOMATITIS}

There has been described a form of stomatitis occurring in the newborn, nine to twelve days after birth, due to infection with the gonococcus. The lesion consists of yellowish-white patches, occurring on the anterior two-thirds of the tongue, the hard palate, anteriorly along the free margin of the gums, and sometimes on the posterior border of the alveolar process of the superior maxillary bone. In all instances the affection has been derived from the mother, and the diagnosis is made by discovering the gonococci in the exudate taken from the lesion. In addition to the usual treatment, the application of a I to 2 per cent. solution of silver salt is one of the most effective means of combating the disease. Dilute bichlorid of mercury solution ( $\mathrm{I}: \mathrm{I0}, \infty 00)$, or even a saturated solution of boric acid; have been employed satisfactorily.

\section{TUBERCULOSIS OF THE MOUTH}

Tuberculosis of the mouth may occur. The mouth infection is always secondary to tuberculosis elsewhere, although it is often difficult to establish the primary focus. Tuberculosis of the mouth has sometimes resulted from the extention into the mouth of lupus. A more common method of tuberculous infection of the oral cavity is due to direct contamination of the mouth by the tubercle bacilli from the sputum. These mouth lesions may appear as ulcerations, or as small tuberculous nodules on the gums. The local treatment consists in excision of the tuberculous area or curettment of the ulcers, followed by iodoform dressings, unless the general disease is so far advanced as to make such procedures either unjustifiable or unnecessary.

\section{SYPHIIIS OF THE MOUTH}

Syphilis of the mouth may occur during all stages of the disease, and manifests itself either as a chancre, so-called mucous patches, or as gummatous ulcers. The diagnosis should be readily made by the history, the associated manifestations of lues, and the demonstration of the Treponema pallidum in the smears made from the lesions. In addition to the constitutional treatment of the disease itself, certain local measures should be employed. Scrupulous cleanliness of the mouth and the use of mild antiseptic washes, should be supplemented by local applications to the lesions themselves. For this purpose bichlorid of mercury, in I per cent. solution, or silver nitrate in 20 per cent. solution, or chromic acid in 5 to ro per cent. solution, are recommended. 


\section{ACTINOMYCOSIS OF THE MOUTH}

Actinomycosis of the mouth is rare. The ray fungus may attack the lips, the cheeks, the jaw and the tongue. On the soft parts the lesions produce tumor-like swellings, which tend to break down. In the jaw the infection is usually a periostitis. Ulceration sooner or later occurs, and the ray fungus may be recognized in the discharges. The treatment of this condition is largely surgical, excision being indicated. The administration of potassium iodid internally is also generally employed.

\section{DISEASES OF THE TONGUE}

\section{ACUTE CATARRHAL GLOSSITIS}

In addition to the association of catarrhal inflammation of the tongue with simple stomatitis, glossitis also occurs from a number of causes. Among these should be mentioned trauma, such as irritation of the organ by a carious tooth, the biting of the tongue during convulsions, or irritation of the tongue from contact with hot liquids, acids, alkalines or other irritating chemicals. Tobacco is a frequent cause of simple glossitis. Tobacco has been held responsible for the so-called "smoker's patch," which consists of whitish lines or a triangular patch on the mucosa extending back from the juncture of the lips toward the first molar tooth. Recently Landouzy has maintained that these smoker's patches are found exclusively in syphilitics and that tobacco merely acts as a local irritant. He has also found them in women, but only in those married to syphilitics, or who have acquired lues. Out of I 3 I individuals showing smoker's patches, he found the Wassermann reaction positive in eighty-one. $\mathrm{He}$ also found these patches to retrogress under mercury and iodid, even when the smokers did not alter their tobacco habits. He regards these patches as of the greatest diagnostic importance in the recognition of lues, and routinely examines all patients for their occurrence. Acute glossitis is also found in association with many acute infectious diseases, notably scarlet fever, typhoid fever, erysipelas, smallpox and frequently in pneumonia. The treatment of acute catarrhal glossitis consists in the removal of the cause and the free use of mild antiseptic mouthwashes.

\section{LEUKOPLAKIA}

The uncertainty as to the etiology of leukoplakia still exists. Although leukoplakia cannot be said to be regarded as a definite manifestation of syphilis, there seems to be more than a casual relationship between the two conditions. The most that can be said is that leukoplakia is possibly a parasyphilitic condition. The treatment of leukoplakia is at best unsatisfactory. All observers seem to agree that the cessation of smoking is essential to its successful management. Whereas some authorities maintain that antiseptic treatment and strong applications are positively harmful, there are 
others who recommend applications of a ro per cent. solution of tincture of iodine, or a I:roo solution of salicylic acid. The careful use of the roentgen-rays, has met with some success in the hands of experts. The following procedure is said to have been successful in some cases, provided always the patient first absolutely gave up the use of tobacco. Every day, or every other day, balsam of Peru was thoroughly applied to the diseased area. The mouth was then thoroughly rinsed six to twelve times with a weak salt solution ( 0.5 to 3 per cent.).

\section{DISEASES OF SALIVARY GLANDS}

Salivary Calculi.-In addition to the more common diseased conditions of the salivary glands, such as acute and chronic inflammations, attention should be called to salivary calculi. The most common location for salivary calculi, is in Wharton's duct. Occasionally the calculi may form in the glands themselves. These calculi are nearly always of small size, and consist of calcium phosphate, or calcium carbonate. As is the case with other calculi, the nidus of salivary calculi may consist of minute foreign bodies, or of clumps of bacteria or the products of bacterial infection. Changes in the consistency of the saliva may also be a factor in the production of salivary calculi. The most prominent symptoms of salivary calculi are intermittent swelling of a salivary gland, accompanied by intermittent and often severe pain in the floor of the mouth. The stones may sometimes be palpated, but the easiest and most reliable means of diagnosis at present is the roentgen-rays. Obviously the only treatment is to remove the stone. This may be accomplished sometimes by manipulation and pressure if the stone is small. Larger and more deeply seated calculi must be attacked by surgical measures. 


\title{
ESOPHAGOSTOMY AND INTRATHORACIC RESECTION OF THE ESOPHAGUS
}

\author{
By Charles H. Mayo, A. M., M. D., Ll. D., F. A. C. S.
}

Cancers of the thoracic portion of the esophagus have been regarded as practically inoperable. Operations for excision have, to be sure, been attended with a high mortality; only two successful cases are recorded. Palliative operations to relieve obstruction and to preserve the function of swallowing have been more successful. Investigations, experimental and clinical, have been activated by improved methods of artificially maintaining the oxygenation of the blood despite the embarrassment of respiration attendant upon a wide-open operative pneumothorax. Apparatus for positive and negative pressure though equally efficient has been replaced by the less cumbersome devices for the maintenance of intratracheal insufflation.

Willy Meyer, Sauerbruch, Torek, Zaaijer, Ach, Rehn, Janeway, Greene and Tiegel are essentially responsible for the considerable degree of progress which has occurred. Willy Meyer has conscientiously carried out his original suggestion that all operations upon the esophagus should be recorded regardless of their outcome. Although he has not as yet reported a successful resection, his investigations and detailed reports serve as valuable contributions.

Failure has been the regular sequel to attempts to anastomose the proximal and distal ends of the esophagus, following resections. Efforts to lift the stomach through the diaphragm and to produce an anastomosis between it and the proximal esophageal stump have been likewise unavailing. Tension on anastomosing sutures has been so great that leakage and subsequent pleural infection could not be prevented.

The publication in I9I 2 by Jianu of an operation previously described by Beck and Carrel stimulated enthusiasm for extrathoracic esophagoplasty. The greater curvature of the stomach is converted into a tube, the proximal end of which retains its attachment to the cardiac end of the stomach, the distal end being carried by a route subcutaneous or subpectoral, to the level of the third rib where it is brought through the skin at the left of the sternal border. A new esophagus is thus provided which is almost sufficient in length for the deficit resulting from the resection of the diseased portion; furthermore it is almost long enough to meet the proximal portion of the esophagus which is withdrawn from the thorax through an incision in the neck, and carried thence subcutaneously in the direction of 
the presenting upper end of the new distal portion. The two ends of the new esophagus may be united by a rubber tube (Gluck-Perthes).

It is to be hoped that further development of this technique will render it entirely adequate to restore the ability to swallow masticated food to those afflicted with obstructing cancer of the esophagus, even if the obstructing growth is left in situ.

Ach has contributed definitely to the technique of withdrawing the proximal esophageal stump from the thorax. A wire with looped end is inserted by the narcotizer to the ligated proximal stump, at which point the operator through the thoracic wound stitches the stump to the included wire. Traction on the oral end of the wire will then draw the esophagus out through the mouth until the ligated end lies opposite the neck incision, through which it may be picked up and drawn to the surface. Willy Meyer has successfully repeated this extraordinary method which bids fair to lead to further important developments in surgery of the esophagus.

The intrathoracic resection of the esophagus continues to meet definite obstacles; this is particularly true of that portion situated behind the aortic arch. Serious disturbances of heart action and air exchange are apt to result from retraction or division of the vagus nerves which are generally involved in the growth. Hydrothorax is a usual sequel which complicates the convalescence even if the fluid remains uninfected. Heat loss from prolonged exposure of the intrathoracic viscera contributes to the shock complement of the necessarily long operation.

At the present status of surgery of the esophagus cases of cancer of the intrathoracic portion may be divided into three groups:

r. Patients afflicted with obviously advanced malignant disease and presenting infiltrating inoperable growths, may in case of obstruction be provided with a gastrostomy of the Witzel or Kadert type.

2. Patients with apparent resistance and with circumscribed, perhaps operable, esophageal tumors may be operated upon in two or more stages. A Beck-Jianu operation may first be done. An exploratory thoracotomy is then performed and the nature and extent of the tumor determined. If excision is abandoned (as it doubtless should be in a majority of the cases) the esophagus may be divided above the growth, the ends being invaginated. The proximal end is then withdrawn through the neck incision as described by Ach. The thorax is closed with drainage (Meyer) or without drainage (Sauerbruch).

3. If on the contrary the tumor is circumscribed and favorably situated, excision may be attempted, the proximal stump being treated as above.

Tumors of the esophagus are of the type often amenable to radium therapy. It is conceivable that a tube of radium might be inserted through the esophagoscope to the immediate vicinity of, or even to a point within the limits of a carcinoma of the esophagus. 


\section{REFERENCES}

Ash, Munchen. med. Wchnschr., 19r $3,1 x, 1115$.

BECK, Illinois Med. Jour., 1905, vii, 463.

Surg., Gynec. and Obst., I915, xx, 170.

Jianu, Deutsch. Ztschr. f. Chir., I9r 2, cxviii, $3_{8}{ }_{3}$.

MEYer, Trans. Am. Surg. Assn., I91 2, xxx.

Surg., Gynec. and Obst., I9I 2, xv, 639.

Ann. Surg., I913, lviii, 289-295.

Surg., Gynec. and Obst., I915, 8x, I62.

Ann. Surg., 1915, lxii, 693-709.

Robinson, Surg., Gynec. and Obst., I9i 5, xxi, 774.

ROEPKE, Cent. f. Chir., I9I 2, xxxix, 1569.

Sauerbruch, Technik der Thoraxchirurgie, igir, p. 87.

TIEGEL, Berl. klin. Wchnschr., I91 2, xlix, 867. 


\title{
GASTRIC AND DUODENAL ULCER
}

\author{
By Bertram W. Sippy, M. D.
}

The medical treatment of gastric and duodenal ulcer advocated and described in this article has been gradually evolved and established in practice by the writer. It differs radically in principle from the Leube, Lenhartz and all other forms of medical treatment in common use the world over.

The treatment attacks and effectually destroys the digestive or corrosive action of the gastric juice and thus protects the ulcer from gastric juice corrosion until healing of the ulcer takes place. The greatest known hindrance to the healing of peptic ulcer is thereby removed. Nature thus aided fills in the defect with granulation tissue, and cicatrization of the ulcer occurs approximately as rapidly as it does elsewhere on the surfaces of the body.

The development of the method has been attended by unusually favorable clinical facilities. The length of time the patients have been observed subsequent to treatment has been adequate. The number and variety of cases treated have been sufficient to serve as a basis for conclusions. Approximately 70 per cent. of the cases treated have been of the obstructive type of ulcer. Pyloric obstruction of the highest grade, due to ulcer at the outlet, associated with spasm of the pylorus or acute inflammatory swelling, yields quickly to the treatment. Pyloric obstruction of high grade due to chronically infiltrated and edematous tissue, subsequently to be described as obstruction due to tissue narrowing, may also be made to disappear under the influence of the treatment. The conditions under which such results may be obtained will be described later. A large number of the cases treated gave histories indicating that the disease had been present continuously or as a recurrent affection for a period of several years. - Included among the cases successfully treated are many such as gastroenterostomy and other surgical procedure legitimately applied have failed to relieve.

After an experience of over thirteen years in applying the principle involved in the treatment, the writer is convinced that the vast majority of gastric and more particularly duodenal ulcers now treated surgically, and with indications commonly accepted as justifiable, can be readily cured by the medical treatment advocated. There is reason to believe that the healing of peptic ulcer can be made to progress more rapidly by this method of treatment than by any other devised.

For half a century circumscribed areas of ulceration, such as are 
commonly found in the stomach and duodenum, have been designated by the terms peptic, corrosive and digestive ulcer.

Peptic ulcer of the lower end of the esophagus has been described. "An ulcer may appear wherever gastric juice flows" is an old and time-worn statement. During these many years it has been generally recognized that a peptic ulcer develops in approximately the following manner: A circumscribed area of the mucous membrane or wall of the stomach or adjacent duodenum through malnutrition or necrosis loses its normal resistance to the peptic action of the gastric juice, and becomes digested. The resulting defect is an ulcer. Much discussion and experimental work have been directed toward determining the causation of the malnutrition and necrosis as they occur in these local areas. Various local conditions, such as thrombosis and embolism of the arterial twigs supplying the stomach and upper portion of the duodenum, hemorrhage, passive congestion, corrosive substances, burns, mechanical injuries and pressure have been believed to be causes of the circumscribed areas of malnutrition and necrosis. The recent work of Dr. E. C. Rosenow renders it probable that these local areas of malnutrition and necrosis are commonly produced by hematogenous bacterial (streptococcus) invasion.

From what is known of the behavior of other anatomical structures, there is reason to believe that local defects of malnutrition and necrosis, such as occur in association with peptic ulcer, whether from hematogenous bacterial invasion or other causes, would practically always undergo relatively rapid repair, and without the development of serious symptoms, in the absence of gastric juice corrosion.

The results of clinical and experimental research, together with all present knowledge pertaining to the subject, tend to substantiate the belief that has existed for many years, namely, that an ulcer of the stomach or duodenum would heal approximately as rapidly as an ulcer located elsewhere, if its granulating surfaces were not exposed to the digestive action of the gastric juice. Thus the important relationship existing between the digestive action of the gastric juice and ulcers of the stomach and duodenum has long since been recognized.

If gastric juice corrosion is such an important factor in retarding the healing of peptic ulcer, the rational medical treatment of the disease should be directed toward lessening and, if possible, completely destroying the digestive or corrosive properties of the gastric juice.

For a great many years it has been known that the peptic or digestive action of the gastric juice is due to the solvent action of pepsin on albuminous substances that have been properly permeated by a free acid. It is well known that pepsin is practically inert in alkaline and neutral mediums. It acts slightly in the presence of a combined acid medium, but combined acids are incapable of preparing albumins properly for the solvent action of pepsin. A free acid, such as hydrochloric acid, is required to permeate the albumin and prepare it for the full action of the peptic ferment. Pepsin exerts 
no appreciable solvent or digestive action on the raw and exposed surfaces of a gastric or duodenal ulcer in the absence of free hydrochloric acid.

The treatment advocated consists essentially in protecting the ulcer from gastric juice corrosion until healing of the ulcer takes place. This is accomplished by maintaining approximately an accurate neutralization of all free hydrochloric acid during the time that food and secretion are present in the stomach. The digestive action of the gastric juice is thereby destroyed. Thus the conditions for the healing of both gastric and duodenal ulcer are rendered as ideal as they can be made to be in the light of all present knowledge. Experience with the method has demonstrated beyond doubt that it is entirely possible and remarkably easy to exercise practically absolute control over peptic activity for as long a period as desired.

Diagnosis.-Before instituting treatment in a given case of gastric and duodenal ulcer, one should make a careful study of the conditions that attend the ulcer. It should be understood that the diagnosis of peptic ulcer involves a careful and painstaking consideration of the following points:

I. Is ulcer present?

2. What is its probable location? If at the outlet of the stomach, is it on the duodenal or gastric side of the pylorus?

3. What is the probable duration of the disease? If the clinical history indicates that the disease is of long duration, it should be carefully analyzed to determine whether it is probable that ulcer has been continuously present, or whether it is a recent recurrent affection.

4. What complications and sequels, if any, are present?

An intelligent and systematic search should be made in every case for evidence of the following complications and sequels of ulcer:

I. Secondary malignant disease.

2. Pyloric obstruction: If present, obstruction from indurated tissue narrowing should be differentiated from the more common type of obstruction, due to conditions that may be completely overcome by one or two weeks' application of the accurate medical management to be described. Approximately 90 per cent. of all cases of pyloric obstruction, varying from the lowest to the highest grades of obstruction, belong to the later type. It is probable that pyloric spasm, acute inflammatory swelling and in some instances local peritonitis are the causes of the obstruction in such cases.

3. Continued secretion.

4. Perigastritis.

5. Perforation.

6. Perigastric adhesions.

7. Hour-glass or other deformity of the stomach.

A blood examination and chemical examination of the stool should be made to determine whether hemorrhage is depleting the patient. While it may not be possible to determine with absolute 
exactness every detail relating to diagnosis mentioned, it should be a matter of routine to investigate every case of peptic ulcer with reference to the conditions and complications mentioned. If one knows what to look for and how to prosecute an intelligent study of the case, the time required for such investigation usually does not exceed two or three days, or, at the outside, one week. Often the greater part of what may be learned about the case at that period becomes apparent at the first examination.

Only after the points enumerated and other conditions peculiar to the individual case have been carefully and intelligently studied is one prepared to advise whether that particular case should be treated surgically or medically, and to otherwise direct the management of the case.

Indications for Surgical Treatment.-The following absolute and relative indications for surgical treatment of peptic ulcer should be kept clearly in mind:

I. Secondary carcinoma: If there is a reasonable reason for suspecting that a carcinoma is developing at the seat of an ulcer and no contraindication exists, an exploratory operation should be made at once, and, if accessible, the ulcerated area should be widely removed with due regard to lymphatic distribution.

2. Perforation into the free peritoneal cavity demands immediate operation.

3. Pyloric obstruction of high grade due to indurated tissue narrowing that fails to yield appreciably or completely to an accurate application of the medical treatment to be described should be treated surgically, if no contraindication exists.

In approximately 90 per cent. of all cases of pyloric obstruction due to peptic ulcer the obstruction disappears during the first two or three weeks under the influence of the medical treatment described in this article.

In the remaining ro per cent. of cases the obstruction is due to indurated tissue narrowing. If the medical management described is accurately applied, the obstruction can be made to disappear, the ulcer can be healed, and without serious difficulties in the majority of these cases also.

4. Perigastric abscess should be treated surgically.

5. Hour-glass stomach usually requires surgical relief.

6. Hemorrhage from peptic ulcer is a direct result of corrosion of blood-vessels by the gastric juice. Such corrosion is impossible when the digestive action of the gastric juice is annulled by accurate medical management.

Since serious bleeding practically never occurs after the first week of the medical management, to be outlined, surgical treatment is required only when patients are bleeding when first seen, or within the first week after treatment is begun.

The results of surgical intervention when instituted for serious hemorrhage at the time it is occurring, or immediately thereafter, 
are so discouraging that it is extremely rare that operative procedure is advisable or required for the control of hemorrhage.

7. Foci of infection about the roots of teeth, in the tonsils and elsewhere in the body should be sought and removed. Rosenow's contribution to the etiology of peptic ulcer is of the utmost importance relative to the recurrence of ulcer and the prophylactic treatment of the disease. It is manifestly useless to talk about the endresult of any form of treatment of peptic ulcer when the patient harbors an alveolar abscess. An abscess about the root of a tooth may be responsible for recurrences of ulcer extending over a period of years.

8. Perigastric adhesions resulting from ulcer very seldom, if ever, interfere seriously with the motility of the stomach or produce pain sufficient to require surgical intervention.

If gastric or duodenal ulcer is complicated by any of the conditions, and as described, operative treatment is indicated, provided no contraindication exists. In the vast majority of all other cases the medical treatment to be described should be instituted. When such complications and sequels of ulcer as may be mechanically removed are not present, surgery can do nothing of consequence except when the ulcer is so located that it may be excised, resected or reduced in size by cautery and suture. In a limited number of cases roentgen-ray examination gives evidence of a large or deeply excavated ulcer, such as may justify an attempt to apply at once one of the three last-mentioned surgical procedures.

The former practice of performing gastroenterostomy in practically all cases of peptic ulcer, whether associated with pyloric obstruction or not, has fallen into disrepute since laboratory experiment combined with actual test of the motor power after gastroenterostomy has demonstrated that gravity has little to do with the passage of food and secretion from the stomach. After gastroenterostomy the stomach empties itself of food in approximately normal time. The immediate drainage effect that was formerly supposed to take place after gastroenterostomy does not occur.

While in some cases the roentgen-ray may show that barium solutions leave the stomach after gastroenterostomy more rapidly than normal time, unfortunately, there is no operative procedure that enables the stomach to empty itself of food and secretion appreciably earlier than the physiologic limits of normal time. After gastroenterostomy the stomach continues to empty itself through the pyloric outlet proportionate to the size of the opening through it. Under such conditions, an ulcer at the pylorus or in the duodenum is exposed to the corrosive action of the gastric juice during the several hours each day that the stomach contains food and its accompanying secretion. After gastroenterostomy if an ordinary diet consisting of three meals daily be given, food and corrosive secretion are emptied through the pyloric outlet for a total period of from nine to fifteen or more hours each day. Hence, the relief from chemical corrosion and mechanical irritation after the operation is much less 
than was formerly supposed. A peptic ulcer is protected from gastric juice corrosion by gastroenterostomy to an appreciable extent only, when there is an obstruction at the outlet that is causing retention of food and secretion.

In all cases in which the clear indications for surgical treatment, as outlined, do not exist, the medical treatment to be described should be instituted.

Treatment.-Essential to the treatment of peptic ulcer as conceived by the writer is a careful neutralization of the free hydrochloric acid, thus rendering the digestive action of the gastric juice inert from 7 A. Mr. until about ro.30 P. M., or during the entire time that food and its accompanying secretion are present in the stomach. In addition, it is accurately determined whether an excessive night secretion is present. If so, this is removed each night until the irritability of the gastric glands has subsided. The removal of an excessive night secretion applies almost entirely to cases of duodenal and pyloric ulcer that have been associated with stagnation of food and secretion for one or two months, or longer. Such cases are almost invariably attended by a more or less copious continued secretion during the night, which should be removed by aspiration two or three times each night, if necessary. Usually after three or four days of accurate control of free acidity the excessive night secretion disappears. Subsequently the normal quantity (about ro c.c.) of gastric juice present in the stomach during the night is left undisturbed.

The neutralization of hydrochloric acid is accomplished by frequent feedings and the use of alkalies is carefully regulated but adequate quantities.

According to the conception upon which the treatment advocated is based, the most favorable conditions for the healing of peptic ulcer are maintained when the aspirated stomach contents show absence of free hydrochloric acid during the entire time that food and its accompanying secretion are present in the stomach, and all excessive night secretion is controlled. It should be understood, however, that the presence of free hydrochloric acid now and then for a few minutes each day does not seriously interfere with the healing of the ulcer. Such short periods during which corrosion of the ulcer may be possible amount to relatively little when compared with the many hours of corrosion to which duodenal and pyloric ulcers are subjected after gastroenterostomy. As previously stated, such ulcers are subjected to the corrosive action of the gastric juice during the whole period of normal stomach digestion, which occupies from nine to fifteen hours each day. The majority of pyloric and duodenal ulcers treated by gastroenterostomy show few symptoms after the operation, and probably usually heal in the course of time, the same as the majority of the non-obstructive type of gastric ulcers usually heal without any form of treatment, and without serious clinical symptoms. In either case, however, the conditions for healing are far from ideal. 
Non-obstructive Type of Peptic Ulcer.-In uncomplicated cases of peptic ulcer, with individual exceptions, the writer has found the following management most serviceable: The patient should be put to bed, where it is usually advisable for him to remain for approximately three weeks. At the end of that time he may be allowed to sit up a portion of each day, and finally walk about the room or yard. If such complications as perigastritis, inflammatory swelling, local peritonitis, or hemorrhage exist, a longer period of rest may be required. One can rarely find justification for attempting to treat a case of peptic ulcer without a preliminary period of rest in bed. In the absence of the serious complications mentioned, the patient is usually able to do some or all of his regular work at the end of four or five weeks.

If the patient cannot afford a constant attendant, a well-stoppered bottle, such as an ordinary effervescing citrate of magnesia bottle, may be filled with equal parts of milk and cream and kept in a pitcher of ice water at the bedside, conveniently placed on a stand, together with the powders, drinking water, and a measuring glass. Except when some serious complication is present, the patient can easily manage'his powders and feedings, provided the extras to be described are brought to him when directed.

The uncomfortable preliminary starvation period common to other types of medical management serves no purpose when the acidity is controlled; therefore, nourishment is given from the very beginning of the treatment. A wide variety of soft and palatable foods may be given. The following plan of diet has been found most adaptable: 3 ounces of a mixture of equal parts milk and cream are given every hour from 7 A. M. until 7 P. M. After two or three days soft eggs and well-cooked cereals, such as rice, oatmeal or farina, are gradually added, until at the end of about ten days the patient is receiving approximately the following nourishment: 3 ounces of the milk and cream mixture every hour from 7 A. M. until 7 P. M. In addition, three to four soft eggs, one at a time, and 9 to 22 ounces of a cereal, 3 ounces at one feeding, may be given each day. The cereal is measured after it is prepared. The cereal and egg portions are usually given alternatingly, and are given at the time and in addition to the 3 -ounce mixture of milk and cream.

Cream soups of various kinds, vegetable purées, bread and butter and other soft foods, may be substituted now and then, as desired. The total bulk at any one feeding while food is taken every hour should not exceed 6 ounces. Many of the feedings will not equal that quantity. The patient should be weighed. If dèsired, a sufficient quantity of food may be given to cause a gain of 2 or 3 pounds each week.

A large variety of soft and palatable foods may be used, such as jellies, marmalades, custards, creams, etc. The basis of the diet, however, should be milk, cream, eggs, cereals and vegetable purées, and bread and butter. Lean meats and meat broths are not given 
during the period of accurate observation, since it interferes with the tests for occult blood in the stool and aspirated stomach contents.

The acidity is more easily controlled by feeding every hour and giving the alkalies midway between feedings. The acidity may, however, be controlled by feeding every two, three and four hours. Complete control of free hydrochloric acidity may be maintained by feeding three times daily and giving alkalies in adequate doses and at intervals appropriate to the individual case. With few exceptions, however, the plan of feeding every hour by giving alkalies midway between feedings is best.

Alkalies are given from the beginning of the treatment.

In cases of stomach ulcer unassociated with stagnation of foods and secretion, the free acidity is usually controlled by feeding every hour and giving a powder containing Io grains ( $0.6 \mathrm{gm}$.) each of heavy calcined magnesia and sodium bicarbonate, alternating with a powder containing Io grains $(0.6 \mathrm{gm}$.) of bismuth subcarbonate and 30 grains (2.0 gm.) of sodium bicarbonate, midway between feedings. In addition, the powders should be given every half hour after the last feeding for three or four doses or until the stomach contains no food. The number of powders to be given after the last feeding may be easily determined by aspirating the stomach about one and onehalf to two hours subsequent to the time food was last given. Just before taking a powder it is stirred up in a glass containing from I to 3 ounces of water, as desired.

Heavy calcined magnesia has approximately four times the neutralizing power of sodium bicarbonate. Since its neutralizing effect is prolonged compared with that of sodium bicarbonate, and for other reasons, calcined magnesia should be used between as many feedings as possible. An uncomfortable diarrhea usually prevents its exclusive use as a neutralizer.

It is not difficult to determine the amount of alkali required between feedings to control accurately all free acidity. If an adequate quantity of alkali is being used, it should be possible to aspirate the contents of the stomach at any time during the period that it contains food without finding free hydrochloric acid.

Experience has shown that when the free acidity is found controlled late in the afternoon and just previous to taking the powders or feedings, the acidity is likely to be controlled at all other times during the feeding hours. If not controlled the first few days by giving the usual amount, the dose is gradually increased by adding Io grains ( $0.6 \mathrm{gm}$.) of sodium bicarbonate to each powder until the acidity is regularly found controlled.

The non-obstructive type of peptic ulcer requires relatively little attention to details compared with the obstructive type of duodenal and pyloric ulcer. The dosage of alkali required to control the acidity is smaller, and it is seldom, if ever, necessary to remove the remaining food and secretion at bed time.

The average length of time that a patient with peptic ulcer should 
be under the accurate control and observation of the physician is about four weeks. During this period, if observations have been carefully and intelligently conducted, the finer points essential to a complete diagnosis of peptic ulcer, including such conditions and complications as may attend the ulcer, will have been determined, and the patient will have learned how to manage himself accurately. Thus the gratifying results to be obtained by the management advocated are to be secured. The experience of the writer has been that in the management of no other serious disease is it so easily possible to obtain the intelligent coöperation of all classes of patients. The reasons are probably of this nature: The things required of the patient during the after-management are straightforward and relatively simple; the sufferings of the patient before treatment have often been so severe that he usually required no further stimulus to do his part. Fortunately, the most accurate coöperation is secured from patients who have long suffered from the obstructive type of duodenal or pyloric ulcer.

During the three or four weeks that the patient is under the careful supervision of the physician, the patient learns how to manage his diet and powders so that practically there will be no free hydrochloric acid in the stomach while it contains food and the secretion that accompanies it. Above all, he learns why he is to faithfully continue to do his part. He has become accustomed to the routine and is almost invariably ready and willing to assume the responsibility of managing himself with an accuracy sufficient to insure a complete recovery. Experience with large numbers of all classes of patients has abundantly demonstrated that in the after-management of peptic ulcer it is easily possible to continue with the hourly feedings and with the powders while the patient is attending to his regular work. Thus the ideal conditions for healing may be maintained for as long a period as desired.

If, upon resuming work, conditions are such that it is too inconvenient for the patient to continue with the exact method of feeding, as outlined, practically the same results may be accomplished in other ways. For example, the patient may eat a light breakfast, consisting of such foods as cereal, soft egg, bread and butter, or soft foods of any kind desired, to the extent of ro or I2 ounces. A pint or quart thermos bottle containing equal parts milk and cream is a convenient source of food supply for the hourly feedings. Three or 4 ounces of this mixture should be taken every hour until noontime, when, if desired, a light luncheon of any of the large variety of foods previously mentioned may be taken. Meats of all kinds may now be included in the diet, since, as a rule, the stool is no longer examined for occult blood.

During the afternoon 3 or 4 ounces of milk and cream mixture should be taken each hour until time for the evening meal.

The total bulk of food taken at these meals should not be sufficient to cause a greater gain in weight than is desired. The patient should 
be weighed regularly and the total bulk and character of the food should be influenced thereby.

Now and then, if, for sufficient reason, the patient desires to stop the hourly feedings for a portion or whole of one or more days, and substitute one or three ordinary meals daily, the powders should be allowed to do so. Under such conditions, the powders should be taken every hour for three doses after a light breakfast. After luncheon one powder should be taken, approximately at the end of one hour, two powders at the end of the second and third hours, and one the next or fourth hour after eating. After the evening meal the powders should be taken in the same way.

An hour or more of free hydrochloric acidity will be present each day under such management, but only a fraction of the corrosion that may regularly take place when a duodenal ulcer is treated by gastroenterostomy will be possible.

If desired, at the end of ten or twelve weeks the length of time between feedings may be regularly increased to two hours, and the powders continued midway between feedings, as before. Approximately twice the quantity of food should be taken at each feeding, and two powders midway between feedings. The free acidity is not as accurately controlled under such management as when the food and powders are taken hourly.

At the end of twenty or more weeks the patient may eat three small meals daily and take a glass of equal parts milk and cream about midway between breakfast and the noon meal, and between the noon and evening meals. Two powders should be taken midway between the breakfast and the glass of milk and cream mixture. Two powders should be taken midway between the milk and cream mixture and the noon meal. In like manner, two powders should be taken midway between the afternoon feedings and two powders approximately one and two hours after the evening meal, and again two powders at the end of three hours after the evening meal.

An ulcer that upon roentgen-ray examination shows no evidence of large size or other unfavorable conditions is likely to heal within a few weeks, or four or five months, under the influence of the accurate management advocated. However, because of the seriousness of the disease and the relative ease with which the favorable conditions for continued healing of the ulcer may be maintained, the five feedings each day, with powders midway between, as outlined, beginning twenty weeks after treatment is begun, should be continued for four or five months or longer. No possible harm can come from continuing the management for months after the ulcer has healed. The cost in time and trouble to the patient is insignificant when compared with permanent relief from distress and the dangers that attend an unhealed ulcer. All danger that might come from the continued use of the powders may be obviated by interrupting the powders for a period of one week at the end of each five or six weeks' period after the patient is on his own responsibility. 
In very rare instances individual peculiarities may require various modifications of diet. A patient who claims that he dislikes milk and cream usually finds to his surprise that when given in the quantity outlined he relishes it. The milk and cream may be seasoned with salt or flavored with coffee, tea or cocoa. A small amount of grape juice given after each feeding is often appreciated. The white of an egg may be substituted for milk. Ice-cold butter balls may be substituted for cream. There is no limit to the wide variety of soft and palatable foods that may be taken. The indication is to give small quantities of palatable and nourishing foods often and at regular intervals, with alkalies in adequate quantity to neutralize the free hydrochloric acid. The acidity can be controlled with greater accuracy by so doing. The feedings are as small as outlined because if more is taken, the weight increases too rapidly.

Further details of management applicable to the non-obstructive type of ulcer will be discussed in connection with the treatment of the obstructive type of the disease.

Pyloric Obstructive Type of Peptic Ulcer.-A peptic ulcer located at the pylorus or immediately adjacent to it on its duodenal or stomach side is likely to be attended by a delayed emptying of the stomach. When this occurs, the duration of corrosion is longer than that of a gastric ulcer located in its usual position on the posterior wall or lesser curvature, and sufficiently distant from the pylorus to not interfere seriously with the motor power of the stomach.

An increased duration of corrosion retards the healing of the ulcer, and thereby contributes to such complications as hemorrhage and perforation.

In addition, the distressing symptoms incident to food stagnation and the continued gastric juice corrosion that attends it, is likely to produce a clinical picture that is much more easily recognized than the more common type of uncomplicated peptic ulcer located in the stomach.

It is very probable that the vast majority of all peptic ulcers that develop in the stomach distant from the pylorus, or that for other reasons are unassociated with stagnation of food and secretion, heal relatively rapidly and without more serious clinical manifestations than are generally supposed to accompany an ordinary hyperchlorhydria. Unquestionably, many of the cases that are diagnosed and treated successfully as hyperchlorhydria are in reality cases of uncomplicated peptic ulcer.

Because of the more intense symptomatology, the pyloric obstructive ulcer is the most common type of peptic ulcer that is recognized and treated.

Peptic ulcers located in the pyloric ring, or on the duodenal or stomach side of it, cause pyloric obstruction either through spasm of the pyloric muscle, or more rarely through acute inflammatory swelling or chronic indurated tissue narrowing of the pyloric outlet. The resulting stagnation of food and secretion increases the number 
of hours each day to which the outlet ulcer is subjected to peptic corrosion. The healing of the ulcer is thereby retarded. Usually a normal or hyperacid secretion is present. The nerves of the ulcer thus exposed to the strong hydrochloric acid become irritated, spasm of the pylorus results and pain due to the nerve irritation and the pyloric spasm that attends it usually becomes a prominent feature. The more pronounced the spasm, the greater the degree of stagnation of food and secretion. The retention of food and secretion and the consequent increased irritation of the ulcer are likely to become associated with a hyperexcitability of the gastric glands. This results in the production of an excessive secretion of gastric juice during the time that food is present in the stomach, and a continued secretion is likely to develop. In such cases the patient is usually awakened with pain during the night, at about one or two o'clock. If vomiting occurs at that time, the vomitus is watery and strongly acid. Usually only a small amount of food is contained in the material vomited. Subsequently the nerves in the ulcer subjected to the prolonged irritation of the strong hydrochloric acid may cause a sufficient increase in the spasm of the pyloric muscle to produce an obstruction of the very highest clinical grade.

In about one case in ten, as patients with pyloric obstruction from peptic ulcer present themselves for treatment, the obstruction is due to an anatomic narrowing resulting from tissue infiltration that has attended the ulcer.

The medical treatment of peptic ulcer that is causing obstruction is the same in principle as that described for the treatment of the nonobstructive type of the disease.

The details of management differ chiefly in that usually a somewhat larger quantity of alkali is required to neutralize the day secretion, and that the best results are obtained by aspirating the stomach every night one-half hour after the last powder is taken. The greatest stimulus to an excessive night secretion is thereby removed. Thus the excessive day and night secretion, two conditions antagonistic to the healing of ulcer, are usually easily controlled. Beginning with the powders advised in the treatment of the non-obstructive type of ulcer, if necessary the quantity of alkali may be increased by adding Io or 15 grains ( 0.6 to $1.0 \mathrm{gm}$.) of sodium bicarbonate to each powder, about every second day, until upon aspirating late in the afternoon and just prior to the taking of a feeding or a powder, it is found that the free acid is completely controlled. In rare instances it has required the equivalent of roo grains of sodium bicarbonate every hour midway between feedings, and every half hour after the last feeding, until 9.30 or ro P. M., to completely control the free acidity. As a rule, it is sufficient to add from ro to 30 grains ( 0.6 to $2.0 \mathrm{gm}$.) of sodium bicarbonate to each powder. In any event, the alkali should be gradually increased until the afternoon and evening aspirations show that the free acidity is controlled. In all cases in which a high-grade continued secretion is present, the stomach 
should again be aspirated at $\mathrm{I} 2$ or I o'clock at night, during the first few days of treatment. If 300 or 400 c.c. of gastric juice is found, the aspiration should be repeated at 4 or 5 A. M., when roo or 200 c.c. likely to be present. If so, the stomach should again be aspirated that morning before feeding is begun.

The night secretion usually rapidly disappears under the influence of an accurate control of the free acidity during the day, and a removal of the excessive night secretion. As a rule, after the third or fourth night the stomach is found to contain no more than Io or 15 c.c. of secretion at midnight and thereafter. That quantity is approximately normal. It is not necessary to remove it or control it by alkalies. Subsequently the stomach should be aspirated every night, one-half hour after the last powder is given. If no free acid is present at that aspiration, the free acidity is almost surely controlled during the entire day. All doubt may be removed by aspirating now and then during the afternoon just before the powders or a regular feeding is taken, and testing the acidity.

At the end of one week or ten days from the beginning of the management, an ordinary seven-hour motor meal should be given for the purpose of determining the improvement in the ability of the stomach to empty itself. During the seven hours subsequent to the taking of the motor meal no alkalies are given. At the end of seven hours the stomach is aspirated, after which it is thoroughly washed. The amount of food and secretion aspirated, together with the quantity of food that settles in the washings, denotes approximately the amount of food and secretion retained seven hours after the ingestion of the motor meal. This amount is compared with the amount similarly obtained prior to treatment. Nausea is such an important factor inhibiting the motor power of the stomach that, when present, the motility test should be repeated. In practically all cases of pyloric obstruction due to ulcer the amount of food recovered from the stomach at the end of seven hours will be found very greatly reduced after one or two weeks' treatment. If the treatment advocated has been accurately applied, at the end of two or three weeks no food will be found in the stomach seven hours after the ingestion of a motor meal in approximately 90 per cent. of all cases of pyloric obstruction due to ulcer.

It will be noted that a rather frequent use of the stomach tube is advised in carrying out the treatment advocated. While it is easily possible to exercise an efficient control over the free acidity in practically all cases of the non-obstructive type of ulcer, and in the majority of all cases of the obstructive type of the disease, without the use of the tube, the advantages to be gained by aspirating are sufficient to warrant using the tube when no serious contraindication exists. A very small tube is adequate for all aspirations except when a motor meal is given.

Patients afflicted with peptic ulcer rarely, if ever, object to the use of the tube when they understand the advantages that are to be gained. 
The aspirations serve three very important purposes: First, the aspiration one-half hour after the last powder is taken removes all food and secretion, and thus lessens the stimulus to a night secretion with its attending corrosion. At the end of one or two weeks the evening aspiration usually shows very little, if any, food remaining in the stomach. Under such conditions it is not necessary to continue aspirating the stomach every evening. As previously stated, it is usually unnecessary to aspirate in the evening in treating the nonobstructive type of ulcer. Second, by aspirating now and then to determine whether the acidity is controlled, an efficient neutralization of the corrosive action of the gastric juice may be obtained with minimum use of alkalies. Third, the aspirated stomach contents may be subjected to the chemical tests for occult blood. The absence of occult blood upon repeated examinations enables one to practically exclude a cancer of the stomach developing at the seat of a peptic ulcer. If occult blood was not present in the first aspiration, and if it continues to be absent in the subsequent aspirations, it finally becomes very probable that the ulcer is on the duodenal side of the pylorus. This becomes still more probable if the early examinations of the stool showed occult blood, which, upon subsequent examinations, gradually disappears. As corroborative evidence, the roentgen-ray examination is likely to show a deformed duodenum.

The vast majority of all ulcers causing obstruction are on the duodenal side of the pylorus.

Pyloric obstruction of all grades resulting from peptic ulcer may be readily separated into two clinical types:

r. Pyloric obstruction that completely disappears during the first ten days or two weeks under the influence of the accurate medical management advocated. Approximately 90 per cent. of all cases of pyloric obstruction due to ulcer belong to this type. The anatomical conditions causing the obstruction are of such character as may be explained by pyloric spasm, acute inflammatory swelling and in rare instances, local peritonitis.

A pyloric obstruction of the highest grade, as evidenced by pronounced undulations of the abdominal wall, due to vigorous peristaltic waves of the stomach, the presence of sarcinæ in abundance, and the retention of food from twelve to thirty-six or more hours, may yield so rapidly that after one or two weeks' managment the stomach is found empty six or seven hours after the ingestion of a motor meal. Under such circumstances a tissue narrowing of serious grade may be excluded.

2. Pyloric obstruction that yields to an appreciable degree under the influence of the accurate medical managment advocated, but still shows food retention to the extent of roo c.c. or more, seven hours after the ingestion of a motor meal. Approximately io per cent. of all cases of pyloric obstruction due to ulcer belong to this type. Indurated tissue narrowing is the cause of obstruction in such cases, provided unmistakable signs of obstruction of high grade were pres- 
ent before treatment was begun and the degree of obstruction has been appreciably lessened under the influence of the management. It is necessary to make that statement, since Ioo c.c. and more of food may be found in the stomach of an individual not afflicted with pyloric obstruction or any other serious disorder. When, however, a definite pyloric narrowing has existed for a considerable period of time prior to treatment, the gastric muscles that propel the stomach contents, become hypertrophied. On account of the increased motor power, the stomach will empty itself of food in normal time, provided the opening through which the food passes, the pyloric outlet, is adequate.

It is to be understood that prior to treatment the degree of obstruction, as evidenced by the quantity of food retained seven or twelve hours after the ingestion of a motor meal, the presence of sarcinæ and vigorous peristaltic waves, is no index to the clinical type of obstruction present.

In general, the more severe and pronounced the symptoms, such as pain, tenderness, vomiting and food retention, the more probable it is that the obstruction is due to pyloric spasm of acute inflammatory swelling-conditions attending the ulcer that are readily relievable by the medical management advocated.

When an untreated peptic ulcer is causing pyloric obstruction of high grade, is associated with relatively little pain, an anatomic narrowing from chronically infiltrated tissue is probably present.

Operative experience shows that indurated tissue narrowing of serious grade is found in only approximately ro per cent. of all cases in which the ulcer is located at the pyloric outlet.

After the accurate medical management advocated has been applied for two weeks, the amount of food retained seven hours after an ordinary meal is a fair index to the degree of obstruction provided, nausea and other conditions interfering temporarily with the motor power are not present. In a given case of pyloric obstruction, presumably due to ulcer, the possibility of obstruction from malignant growth should be constantly kept in mind. Since a duodenal ulcer rarely becomes cancerous, in all cases of pyloric obstruction due to tissue narrowing, it is comforting when roentgen-ray examination shows that the bulb or first portion of the duodenum is persistently deformed, that the antrum is without defect, and that it exhibits active peristalsis in all portions quite up to the pyloric ring. Carcinoma may involve the pyloric ring and not invade the antrum sufficiently to be detected by roentgen-ray examination.

Conclusions as to the cause and character of the obstruction may be reached by noting the rapidity with which the obstruction yields under the influence of the management. Pyloric obstruction of high grade, if due to ulcer, is so regularly lessened by the medical treatment advocated that in a given case, if, after two or more weeks' management, the quantity of food retained is appreciably reduced. When an obstruction of high grade resulting from ulcer 
yields so rapidly that within a week or ten days a motor meal is completely emptied in seven hours or less, the cause of the obstruction is practically always pyloric spasm or acute inflammatory swelling. When the obstruction yields much more slowly, so that three or more weeks of treatment are required before the stomach empties itself in normal time, the cause of the obstruction is practically always an infiltrated and indurated tissue narrowing.

All pertinent evidence that arises during the four or five weeks that a patient with pyloric obstruction is advisedly under the accurate observation of the physician should be noted and used in drawing final conclusions as to the exact condition present. Fortified by the knowledge that when pyloric obstruction results from ulcer the lesion is rarely on the stomach side of the pyloric ring, evidence bearing on its exact location should be accumulated. If it can be determined that the ulcer is in its usual position, on the duodenal side of the pylorus, the danger from the development of cancer at the seat of ulcer is reduced to the minimum.

Regular stool examinations for occult blood will show by its rapid disappearance and continued absence that the ulcer is healing. A gastric ulcer that is undergoing malignant degeneration usually continues to bleed. The continued absence of occult blood in the aspirated stomach contents, together with roentgen-ray examination showing a perfectly pliable stomach wall and without defect, renders it improbable that a carcinoma is developing at the seat of an ulcer in the stomach. Evidence of pyloric obstruction combined with absence of occult blood in the stomach contents and the presence of a gradully diminishing quantity of occult blood in the stool during the first week or ten days of management render it probable that the ulcer at the outlet is on the duodenal side of the pylorus. In such cases roentgen-ray examination will usually show that the bulb or first portion of the duodenum is narrowed or deformed.

All pain and discomfort due to peptic ulcer usually disappears in one or two days after the accurate ulcer management advocated is instituted. It cannot be too strongly emphasized that the pain of a peptic ulcer unassociated with such complications as local peritonitis, perforation, perigastric abscess, and secondary carcinoma, is practically always associated with an adequate free hydrochloric acidity. Such pain will almost invariably be temporarily relieved by foodtaking and by the administration of a dram (4.0 gm.) of calcined magnesia, or one or two teaspoonsful of sodium bicarbonate. If, instead of testing the character of the pain by the use of food or alkalies, the stomach tube be used, a free hydrochloric acidity equal to or greater than the normal should be found to account for that particular pain. If no free hydrochloric acid is found at the time the pain is present, one may be sure that something other than an uncomplicated ulcer is causing the distress.

All abdominal distress that arises during the period that the patient is under the accurate supervision of the physician should be Voz. 1V. -40 
reported, and its cause carefully determined. If the free acidity has been accurately controlled from 7 A. M. until ro P. M., and no more than the normal Io or I 5 c.c. of gastric juice is allowed to remain in the stomach during the night, distress appearing at any time four or five days after treatment is begun is not likely to be due to peptic ulcer. When an ulcer is protected from gastric juice corrosion, it quickly begins to heal. Within a very few days thereafter all pain from a peptic ulcer unassociated with such complications as local peritonitis, perforation, perigastric abscess and secondary carcinoma practically always subsides. The newly formed granulation tissue covers the nerves that have been exposed to the irritating action of the gastric juice or the nerves that are exposed because less sensitive to such temporary free acidities as may occur during the management. Distress that appears after the ulcer management has been accurately applied for one week or more is often due to a colon irritated by too much magnesia. Under such circumstances, the stools are usually frequent, and the distress is likely to be temporarily relieved by a bowel movement. An enema of water may increase the distress as it enters the bowel, but upon allowing the water to escape, the distress may be entirely relieved, for a short time at least. Hot applications are likely to give temporary relief. A reduction in the number of magnesia powders during the next two or three days is likely to be followed by great or complete relief.

If it becomes apparent that the colon is not the source of the distress, the possibility of a diseased gall-bladder or some other intraabdominal disorder should be carefully considered. Not infrequently diseased gall-bladder and peptic ulcer coexist. If all other causes are excluded, the persistence of distress directly associated with ulcer suggests the probability of a secondary carcinoma as a complication. Under such circumstances other signs and symptoms suggestive of carcinoma are likely to be present. Roentgen-ray is likely to show a defect. Carcinoma developing at the seat of an ulcer is likely to show visible or occult blood in the aspirated stomach contents. Upon careful investigation one is likely to observe that pain or distress occurs when upon aspirating the stomach the free acidity is found controlled and that the distress is not always relieved by the taking of food and alkalies.

Skill in the differential diagnosis of the various abdominal conditions that may simulate peptic ulcer is attainable through a careful and intelligent analysis of a sufficient number of clinical cases. The writer is convinced that too great emphasis has been given to the supposed difficulties that are to be encountered in diagnosis, because a diseased gall-bladder or appendix may give rise to symptoms closely simulating those of peptic ulcer, and vice versa. In a given case the difficulties are at times insurmountable upon superficial analysis of the case. It is well to understand, however, that in the vast majority of all cases, after careful study, the supposed difficulties disappear and the nature of the disorder may practically always be determined 
with satisfactory accuracy without an exploratory laparotomy. While roentgen-ray examination can be made to have a very decided value in many cases, it usually leads to an enormous confusion in the hands of those not expert in its use. The very great tendency to rely upon the fluoroscopic examinations and the interpretation of roentgenray plates to the exclusion of other clinical methods in the differential diagnosis of peptic ulcer is to be very greatly deplored. The maximum efficiency in the diagnosis of peptic ulcer is to be attained only by utilizing all methods of clinical investigation to the greatest possible extent.

Physicians not expert in the use of the roentgen-ray for diagnostic purposes should be stimulated to do accurate diagnostic work by realizing that in nearly all cases of peptic ulcer the evidence that may be obtained by other methods may be made to be of such value that the most expert roentgenologist will usually be unable to disclose conditions that have not already been even more accurately determined by other methods of investigation. It is to be understood, of course, that now and. then roentgen-ray examination may reveal some coarse defect, a perforative type of ulcer, an hour-glass narrowing, or some other rare condition that cannot be determined by other non-operative clinical means of investigation.

The length of time that is required for a peptic ulcer to heal depends on its size, depth, the amount of induration in its walls, the adequacy of its blood supply, and the accuracy with which the conditions unfavorable to the healing of the ulcer are removed. Nature heals the ulcer. All that medical or surgical treatment can do toward promoting the healing process is to remove as far as possible those conditions that retard or prevent the ulcer from healing.

Assuming that hematogenous bacterial invasion is a common cause of the local malnutrition or necrosis that lessens the normal resistance of the gastric and duodenal wall to the peptic action of the gastric juice, it is probable that the living bacteria that persist in the walls of the ulcer contribute to the chronicity of the disease. No operative treatment except removal of the ulcer or the use of the cautery combined with suture has any influence on the bacteria in the walls of the ulcer. It must be clear that unless a peptic ulcer is removed by excision or resection, it cannot cease to exist except by undergoing a healing process that terminates in cicatrization of the ulcer.

If the commonly accepted conception of peptic uicer is correct, namely, that the digestive action of the gastric juice constitutes the greatest hindrance to healing that is amenable to medical and surgical control, then it must follow that the healing of peptic ulcer is promoted most by that type of management which reduces to the greatest degree the duration and intensity of gastric juice corrosion.

Adequate experience with the method here advocated has demonstrated that it is entirely possible and remarkably easy to exercise 
practically absolute control over peptic activity for many months, and, if necessary, for years. Under the influence of such treatment an uncomplicated gastric or duodenal ulcer of a few weeks' or months' duration is one of the most satisfactory diseases of a serious nature the physician is called upon to treat. Under proper medicinal management the tendency is toward rapid healing. An ulcer of six or eight months' or one and a half years' duration heals more slowly, requiring in some cases, perhaps, a corresponding time to heal. The symptoms, however, may disappear at once and never return, provided proper medicinal management is instituted and maintained. If an ulcer has persisted uninterruptedly for years until the edges have become indurated and anemic from the continued deposit of connective tissue, healing takes place slowly. It is to be understood that an ulcer may become so old, deep and indurated that healing may never take place. Such large ulcers are rare and almost exclusively located in the stomach, where the conditions for extensive surface ulceration are possible. Roentgen-ray examination is likely to give some clew to the extent of the pathological process when unusual difficulties in healing are to be encountered. In such cases the patient may be kept practically free from discomfort by the use of proper diet and alkalies. A real cure may be accomplished only when excision, resection or reduction in size by cautery and suture is possible.

A duodenal ulcer is rarely large enough to present serious diffculties in healing, on account of size and extent. In the surgical treatment of duodenal ulcer, unless the ulcer is very small, it is rarely excised. When small enough to be excised, it should heal quickly under accurate medical management.

In the treatment of all cases of peptic ulcer associated with pyloric obstruction, the best results are to be obtained by teaching the patient to use the stomach tube and to recognize the simple color reaction for free hydrochloric acid. This can easily be done during the three to five weeks that the patient is advisedly under the accurate supervision of the physician. The patient is then in position to practically insure his own recovery. As has been previously stated, in all cases of obstruction at the pylorus the stomach should be aspirated every evening, one-half hour after the last powder is taken, until the obstruction has disappeared.

The gratifying results to be obtained by applying the treatment or the principles involved in the treatment to such cases of gastric and duodenal ulcer as are ordinarily regarded as absolutely intractable to medical management, including also such cases as gastroenterostomy and other surgical procedure legitimately applied have failed to relieve, demonstrate that there can be no lack of incentive for the internist to develop a technique in the medical management of peptic ulcer that is accurate and painstaking. 


\title{
SURGERY OF THE STOMACH AND DUODENUM
}

\author{
By William J. Mayo, A. M., M. D., Sc. D., F. R. C. S., \\ F. A. C. S., LL. D.
}

\section{CANCER OF THE STOMACH}

Cancer of the stomach is a surgical disease. There is no medical side to the question, unless the drug palliation of incurable diseases can be so called. Comprising as it does 30 per cent. of all cancers of the human body, and having been looked upon from a purely medical standpoint, cancer of the stomach has had a death rate of roo per cent. However, the medical profession is now beginning to realize that the medical man should seek surgical consultation upon the suspicions of gastric cancer exactly as he would do if the disease were of the lip, breast or uterus, and it is essential that the physician and the surgeon act together in complete harmony, in order that the patient may have a chance for his existence. For cancer in its inception is a local disease and if removed at that time is curable.

An early diagnosis of gastric cancer by ordinary clinical methods is not yet possible. Since early recognition and operation alone offer a chance of cure, every measure which will increase the number of correct and early diagnoses is of the highest importance. The demonstration of specific ferments in the gastric contents at best is only of correlative value. The roentgen-ray, though yet in a developmental stage, is the most valuable single diagnostic method for it may prove the existence of gastric carcinoma in various stages in as high as 95 per cent. It is of especial value in those cases in which there is an absence of palpable tumor and pyloric obstruction. The great majority of gastric cancers, however, presenting advanced stages of the disease, may readily be diagnosed by the usual clinical methods, the suggestive history, the presence of palpable tumor, obstruction, and the characteristic nature of the gastric contents. The benign ulcer undergoing early malignant degeneration invariably awaits the surgical pathologist for its recognition.

Cancer of the pyloric end of the stomach and of the lesser curvature occurs in about 75 per cent. of the total number, and this is about the percentage which, with an early discovery, might be considered surgically. The diagnosis in this group of cases is aided materially by mechanical symptoms due to obstruction, which call the attention of the patient to his trouble in the early stages.

Cancer of the body and fundus of the stomach occurs in about 25 per cent. of all cases. When located at the cardia, these growths 
may produce early obstruction but surgical removal is as yet in the experimental stage. In the body of the stomach but few are diagnosed sufficiently early to permit operation. Situated as they are behind the margin of the ribs, the tumor cannot be palpated until a late stage and, because of the wide gastric lumen, obstruction is a late development. The nature, situation and approximate extent of such tumors can be ascertained by means of the roentgen-ray, an advantage which now obviates the necessity of many useless exploratory operations and is rapidly increasing the number of successful surgical results.

The indication for surgical intervention is based upon the fact that cancer exists. Should the condition be beyond the possibility of radical cure, the question of palliation remains; but this is of far less importance, as it merely prolongs by a few weary months a hopeless invalidism.

The idea prevalent in the past that the presence of a tumor indicated an inoperable condition has been proved a mistaken assumption. A movable tumor is a favorable condition, especially if accompanied by early obstruction. A fixed growth would be an argument against operation. The same rule applies to tumors situated in the left epigastrium with little or no mobility and without associated obstruction.

A previous history of ulcer with secondary malignant degeneration is often favorable, other things being equal, because, on account of the scar tissue, the growth may be of less rapid progress. In an investigation by Wilson and MacCarty of 2 I 8 cancers of the stomach removed by operation in our clinic, it was found that ulcer or some primary lesion of a similar character existed in more than 50 per cent.

A marked cachexia, free fluid in the peritoneum, the carcinomatous or button umbilicus, carcinomatous glands in the supraclavicular space, and implantation carcinoma in the bottom of Douglas' pouch felt through the rectum prohibit an attempt at radical cure, and render a palliative operation of questionable propriety.

Cancer of the pyloric end of the stomach has two principal methods of extension: First, by the lymphatics; second, by continuity and contiguity of structure. Metastasis by embolism and peritoneal grafting indicate a late and inoperable condition. A moderate amount of adhesion does not add seriously to the difficulties and dangers of extirpation; but adhesions act as avenues of carcinomatous infiltration and if they are close in character, especially to the pancreas, they will add largely to the mortality as well as reduce the percentage of cures.

Haberkant showed a mortality of over 75 per cent. after radical operations with marked adhesions, and Mikulicz showed a mortality of 74 per cent. when there were attachments to the pancreas. In our experience, pancreatic complications in the operable cases have been found to increase the mortality not more than $\mathrm{I}$ per cent. 
Cuneo has made a close study of the lymphatics of the stomach, and has established the fact that there are comparatively few glands along the greater curvature; that these few are set on the pyloric half with the artery and vein, or some little distance from the gastric wall, and that this lymphatic circulation is from left to right. Hartmann has taken advantage of this knowledge and, unless the extent of the growth prevents, he saves the greater curvature as far to the left as these lymphatic nodes, which greatly facilitates the subsequent restoration of the continuity of the gastrointestinal tract. Cuneo further demonstrated that on the lesser curvature the lymphatic structures lie in the wall of the stomach itself, and thereupon Mikulicz was able to establish the dictum that in every case of radical excision of cancer of the stomach the entire lesser curvature to the gastric artery must be removed.

Kocher has called attention to the necessity of removing the group of glands about the pylorus, especially in the groove between the head of the pancreas and the duodenum, where they lie with the distribution of the gastroduodenal artery. The fourth group of glands consists of two or three nodes along the superior pyloric vessel.

The dome of the stomach does not communicate to any great extent with the lymphatic arrangement of the pyloric portion, but drains into a few glands in the splenic area, and especially into those about the cardia. The general receiving glands for the gastric group lie about the celiac axis and the aortic gland, and involvement of these deep glands contraindicates an attempt at radical operation.

In the early stage of the disease the entire pyloric end of the stomach, with its lymphatic apparatus, can be removed en masse. The disease usually cuts off directly at the pylorus and invasion into the duodenum is comparatively rare; but, as pointed out by Robson, wandering carcinoma cells may be found a centimeter or two in the duodenum beyond the growth. It is wise, therefore, in resecting to leave a free margin of apparently normal duodenum on the side of the growth to prevent the possibility of a return at this point.

Pylorectomy and partial gastrectomy was first performed by Péan in 1879, and by Rydygier in I880. Billroth had the first successful case in $\mathrm{I} 88 \mathrm{I}$.

Billroth established two methods of gastric resection. They differ from each other in the manner of effecting the juncture between the intestinal canal and the gastric stump. The first method was to sew the cut end of the duodenum into the gastric stump at its lowest part. In order to do this it was necessary to narrow the gastric end by a series of sutures until it was reduced to the size of the duodenal fragment. At the point where the circular line of sutures met the transverse line of sutures leakage was so frequent that it received the name of the "fatal suture angle." Kocher modified this method in a most noteworthy manner by completely closing the amputated stomach with continuous sutures and inserting the open end of the duodenum at a new point on the posterior surface of the gastric stump. 
This method has given most excellent results in the hands of its illustrious originator. The second method seems to be the one suitable for the average case. It consists in completely closing both the gastric and duodenal ends and making an independent gastrojejunostomy. This is the method we prefer. The third method is the Polya operation, which consists of a direct anastomosis of the end of the stomach to the side of the jejunum after a technique similar to an ordinary gastroenterostomy. This promises to become a popular procedure. The two-stage operation as advocated by Crile and Lilienthal is indicated in certain cases.

The earlier gastric resections were attended by an enormous mortality. Prior to 1887 the death rate ranged from 60 to 70 per cent.; from I 887 to $I 890$ there was considerable improvement, due to the more general acceptance of antiseptic methods of treatment, and the mortality ranged from 30 to 40 per cent. Since the year I 900 , with the development of aseptic methods, together with the use of holding clamps to facilitate the suturing and prevent leakage, the mortality has been still further reduced, and now ranges under to per cent.

Of Mikulicz's resections, I6 per cent. of the patients lived beyond three and one-half years. Kocher had patients alive for fifteen years, and at various periods between that and three years. Kocher calls particular attention to the statement that fourteen months is the average length of life of those who have recurrence, and that practically all of such patients maintain good mechanical gastric function to the last. Krönlein has had a considerable percentage of patients who have lived beyond three years.

During the period between January I, I894, and January I, I9ז6, I 525 patients in our clinic were submitted to operation for cancer of the stomach. There were 574 resections, 386 palliative operations and 565 simple explorations. The operative mortality in these groups averaged 22 per cent., 6 per cent. and 2 per cent., respectively. The mortality depends largely on the patients accepted for operation. Some patients in the very late stages get well and remain well. Therefore, we have considered it advisable to extend the limit of operability, with the result that patients whose conditions are much more advanced are operated on at a later date than was formerly customary.

In some years there has been a mortality under 6 per cent. and in other years, with more perfect technique, as high as I6 per cent. Improved operative methods have made it possible to operate on patients who have hitherto been considered hopeless. There is a prospect of five-year cures in 25 per cent. and of three-year cures in 38 per cent. if the patient recovers from the operation. Comparatively few patients who recover following resection fail to get less than fourteen to eighteen months of comfortable existence.

For pyloric obstruction due to malignant disease which cannot be removed, gastrojejunostomy furnishes a palliation. If the parts 
are fairly movable, a posterior suture operation uniting the jejunum within 3 or 4 inches of its origin to the posterior wall of the stomach, is the method of choice. It rather frequently happens that the fixed condition of the tumor makes anterior gastrojejunostomy the operation to be considered. For obstruction of the cardiac orifice of the stomach, gastrostomy is indicated, but should be done only when the patient elects, after a full explanation has been given him. The Witzel and Stamm-Kader methods of gastrostomy are among the most popular and easy of performance. However, both gastrojejunostomy and gastrostomy for the palliation of inoperable malignant disease have a considerable mortality, depending upon the degree of starvation and cachexia of the individual patient. Jejunostomy by the Witzel method is occasionally a valuable procedure in cases of gastric cancer, or ulcer suspiciously malignant, especially in those high posterior perforations in which a radical operation is not possbile and in which often a gastroenterostomy cannot be done on the proximal side. Jejunostomy in either ulcer or cancer affords adequate feeding and gives complete rest to the involved stomach.

Upon exploring for gastric cancer it frequently happens that a wholly inoperable condition is disclosed, yet one in which there are no obstructive phenomena to lead to the necessity of palliation. Under such circumstances the abdomen should be closed with catgut and buried in silk or linen mattress sutures in the aponeurosis, and the patient allowed to return home to spend the remaining days of his existence with his family and friends.

\section{ULCER OF THE STOMACH}

Without going into the etiology of gastric and duodenal ulcers, two factors are of sufficient importance to demand attention, and are necessary to the proper understanding of the surgical side of the subject.

First.-Duodenal ulcers are more common than gastric, and both duodenal and gastric are more common in the male than in the female. In 2500 patients operated on in our clinic for gastric and duodenal ulcers, which includes all operated on up to July $\mathbf{r}$, 191 5, 27 per cent. were gastric and 73 per cent. were duodenal. The proportion in gastric ulcers was seventy-one males and twenty-nine females; in duodenal ulcers it was eighty-three males to seventeen females.

About 65 per cent. of all gastric ulcers are situated in the pars pylorica, especially near the lesser curvature. Aside from anatomical considerations and theories, the enormous frequency of gastric ulcer in the pyloric end of the stomach may in some way be connected with the traumatism received in the grinding process. Hot fluid carried along the lesser curvature directly to the duodenum may also be a source of chronic irritation. It is a common custom to take fluid in to the stomach hotter than can be held in the mouth. 
Second.-The presence of an excess of acid gastric secretions and, whether cause or effect, the corrosive action that results, are of the utmost etiologic significance. This is shown by the development of duodenal ulcers in the first $21 / 2$ inches of the duodenum below the pylorus, well above the common duct with its alkaline secretions. Further, these changed secretions will occasionally produce a typical jejunal ulcer near the point of a jejunostomy which had been made for the original lesion. The great importance of traumatism and acid gastric secretions in the development of ulcer is shown by the favorable effect of gastroenterostomy made to the left of the muscular pyloric portion, by which the emptying time of the stomach is increased, the period of corrosion by the gastric juice greatly reduced, and the degree of acidity greatly lowered by the reflux of strongly alkaline duodenal contents.

Surgically speaking, the small intestine may be said to begin at the opening of the common duct. The 4 inches of the duodenum lying between this point and the pylorus may be called the "vestibule," and this is so closely related to the stomach that a consideration of gastric ulcer necessarily includes duodenal ulcer.

Ulcers of the stomach may be divided into two groups-acute and chronic calloused.

Acute ulcer does not always show on the outer wall of the stomach, and the diagnosis may be clinical. Well-marked symptoms, including hemorrhages, may exist, and yet a prolonged search of the gastric interior might be necessary in order to detect the lesion. Patients have bled to death from an ulcer so minute as to be discernible only with a lens at postmortem. Ulcers of this description are sometimes merely superficial erosions of small extent, though occasionally they are of enormous size and exhibit fatal characteristics, as pointed out by Dieulafoy. More often a small fissure will be found, or the typical round or oval peptic ulcer. These acute ulcers are usually the result of some gastric toxic substances originating in changed metabolism, i.e., anemia, cirrhosis of the liver, focal infection, etc.

Chronic calloused ulcers invade all of the coats, and are therefore easily diagnosed from the exterior as typical scar-like masses of irregular size. They are frequently found in the pyloric half of the lesser curvature of the stomach. In this situation the induration about the ulcer often takes a saddle shape, riding the lesser curvature and extending flap-like down the anterior and posterior wall. When in the region of the pylorus, it is the most common cause of benign pyloric obstruction. Strange to say, the frequency of calloused ulcer in the first $2 \frac{1}{2}$ inches of the duodenum has only recently been pointed out, and this is the result of surgical observation rather than of postmortem findings.

The calloused condition of the indurated ulcer shows its chronicity, and nearly all ulcers of this group are surgical. The acute ulcer, on the contrary, becomes surgical only in certain emergencies, such as perforation and hemorrhage. 
Hemorrhage may be so copious and repeated at such frequent intervals as to necessitate an emergency operation. Rodman has pointed out that a single large hemorrhage does not necessitate intervention, while a succession of hemorrhages of chronic blecding of small amounts would indicate interference. For acute grave hemorrhages the only safe plan is to open the stomach, find the bleeding point, and either ligate it from the mucous side (Andrews) or excise it. In either case the peritoneal coat should be sutured over its base to prevent perforation. Excision of the ulcer in conjunction with the gastroduodenostomy of Finney is the operation to be preferred in the chronic and less grave forms of hemorrhage; or, more commonly, the vessels in the vicinity of the ulcers are carefully tied, followed by simple gastroenterostomy. It should be borne in mind that a single hemorrhage which is not preceded and followed by the usual signs and symptoms of ulcer is seldom due to ulcer, but has its origin usually in some toxic cause.

There are three varieties of perforations-acute, subacute with abscess formation, and chronic.

In 90 per cent. of cases, as pointed out by Brunner, acute perforations occur through some point in the area of a chronic indurated ulcer, and in the large majority of instances there had been previous symptoms of the lesion which were diagnostic.

The treatment for this condition should be immediate operation. The patient who has suffered from symptoms of gastric or duodenal ulcer and is suddenly seized with violent pain, abdominal rigidity and other symptoms of rupture of a viscus should be explored at once as few patients will be saved after a delay of ten hours. If the pain and local tenderness are on the left side, the perforation is probably gastric; if on the right side, duodenal. The latter group is frequently confused with perforating appendicitis. On opening the abdomen the escape of thin fluid and gas points to the nature of the lesion. The perforation should be closed by sutures placed transversely, if possible, so as not to entail future contraction. A drainage opening into the abdominal cavity immediately above the symphysis pubis should be made and a glass drainage tube introduced into the pelvis. In very early cases the abdomen should be flushed out with quantities of hot saline solution. This is not so necessary in delayed conditions, and if nature has begun to throw out adhesive protections, it is contraindicated. In either case the patient is put in the semisitting posture (exaggerated Fowler position) after the upper wound is closed. This method of treatment (semisitting posture and pelvic drainage) will save a large number of patients. If the condition of the patient warrants it, a gastroenterostomy should be done at the same time.

Subacute perforations with formation of localized collections of pus are not infrequent. When they occur in the stomach, subdiaphragmatic abscesses are the common result. These accumulations may perforate the diaphragm and appear as empyema. 
A very large number of cases have been reported in which operation either just below the diaphragm or, more frequently transpleural, has been followed by recovery. We have had a number of this variety.

Perforation of the duodenum with formation of localized abscess has a tendency to work to the surface of the body, usually in the region of the gall-bladder, and is amenable to incision and drainage. Care should be taken in opening such an abscess not to break down the limiting wall. The perforation will take care of itself and, if necessary later to overcome obstruction, gastrojejunostomy can be done.

Chronic perforations are exceedingly prone to become adherent to the liver, the transverse mesocolon, the gall-bladder, and the abdominal wall. Slight leakage accounts for the recurrent attacks of regional peritonitis which are so characteristic of indurated ulcer. Adhesions and thickened areas can sometimes be mapped out from the surface, or they may be located with great exactness by the patient. Gastrojejunostomy is the operation of choice for this group of cases, and also for the vast majority of pyloric obstructions which are the result of cicatricial contraction.

Hour-glass constriction, the result of chronic gastric ulcer, present in 5 per cent. of the cases, usually has its base on the lesser curvature. More than one such constriction may exist, so that the stomach is bifid or trifid. The hour-glass stomach, if a single loculus, may be treated by gastrogastrostomy after the Watson method, or by the sleeve resection. We have found the results of either of these operations satisfactory. A gastrogastrostomy which is planned in a general way after the Finney gastroduodenostomy will give excellent results. A considerable number of hour-glass stomachs have secondary obstruction in the vicinity of the pylorus, or they are associated with duodenal ulcer. In either case, gastroenterostomy in addition to the treatment of the hour-glass condition is necessary. Where several loculi exist, increasing the lumen of each by a plastic operation of the Heinke-Mikulicz type so that they are converted into one large cavity, and then making a gastroenterostomy, is most efficient.

Chronic gastric and duodenal ulcers are to be considered surgically when medicinal treatment has failed to cure, not only in the group of obstructive cases, but in the much larger group where the patients are anemic from chronic blood loss, and are continuously underfed because of the pain which comes with a proper variety and amount of food. These patients travel from one physician to another, and any temporary improvement gives rise to an erroneous belief on the part of the medical adviser that the patient is cured. It is the old story of appendicitis and gall-stone disease over again; recovery from each attack is considered a medical cure. Munro asks: "Is it any wonder that the victim of chronic peptic ulcer is spontaneously seeking surgical aid?" At least 90 per cent. of patients with gastric ulcer, taking them as they come, will be cured 
or greatly relieved by operation. The mortality at the most is about 3.5 per cent. The results in duodenal ulcer are extraordinarily good, 98 per cent. of the patients being either cured or greatly relieved by the operation. 'The mortality of this group is about 1.5 per cent.

Generally speaking, posterior gastrojejunostomy, uniting the jejunum within 3 or 4 inches of its origin to the posterior wall of the stomach near the greater curvature, has the widest field of usefulness in gastric ulcer, especially if there be interference with motility. In selected cases of narrow strictures, the Finney gastroduodenostomy is the operation of choice. It has the great advantage of leaving the opening in its normal situation.

Excision of gastric ulcer, especially if it is along the lesser curvature and not giving rise to mechanical symptoms or otherwise interfering with drainage, should always be done if possible on account of cancer liability. In addition gastroenterostomy is advisable, since without it patients may not obtain relief. It is probable that complete resection of the ulcer-bearing pyloric end of the stomach and independent gastrojejunostomy, as suggested by Rodman, should be done more frequently. For posterior ulcers of the body of the stomach which have become adherent, especially to the pancreas, transgastric excision of the ulcer has been of service. The method of Balfour, whereby the ulcer and ulcer-bearing area are destroyed by actual cautery, has steadily given good results.

Gastrojejunostomy is the operation to be preferred for duodenal ulcer which has but little liability of undergoing a malignant change. When possible, the ulcer should be enfolded by a few mattress sutures of linen.

\section{DILATATION OF THE STOMACH}

There are two varieties of dilatation of the stomach-acute and chronic. Acute dilatation (in the form which has been recognized) is a rare condition. After some toxic or traumatic cause the stomach suddenly dilates until its cavity may contain a gallon or more of thin dark fluid and a large quantity of gases under great tension. In a few cases gastrojejunostomy has been performed with benefit after lavage had failed. Milder and unrecognized forms of acute dilatation are not uncommon after operations, especially operations on the bile duct, duodenum, and pylorus. If properly treated with gastric lavage, the majority of those so affected will recover.

Chronic dilatation causing the patient to lie in the prone position is a symptom, first, of insufficient power: second, of obstruction. Chronic dilatation due to muscular insufficiency, the so-called "atonic dilatation," is a medical condition. A number of these patients have been subjected to gastrojejunostomy, usually with poor results. The variety of gastric dilatation depending upon benign pyloric obstruction, as has already been pointed out, is usually the result of ulcer. In the more severe forms of this condition gastrojejunostomy is a necessary procedure and gives splendid results. 
Valve formation owing to a sharp bend caused by a short gastrohepatic omentum sometimes takes place at the pylorus. These patients have occasional attacks of vomiting large quantities of ingesta without apparent cause. We have met with a small number of individuals suffering with this intermittent form of obstruction of sufficient severity to demand operation.

\section{PROLAPSE OF THE STOMACH}

Prolapse of the stomach is usually a part of a general enteroptosis. Individuals thus afflicted, as a rule, have general relaxation of all the abdominal visceral supports. The transverse colon will sometimes be found prolapsed, and in a small percentage of cases the liver will be movable. The writer considers prolapse of the stomach as merely one phase of splanchnoptosis rather than as an entity, and he would not often expect operation to give relief. The experience of the medical profession with nephropexy for movable kidney has not been particularly gratifying, and the procedure is decidedly less popular than formerly as a cure for the neurasthenic. It is quite certain that indiscriminate operations for prolapse of the stomach, with or without atonic dilatations and allied neurasthenic conditions, are more apt to be harmful than beneficial. Under such circumstances gastrojejunostomy in particular is meddlesome surgery, because the prolapsed stomach is not accompanied by stagnation of food, and gastric drainage is normal or nearly so. 


\section{DISEASES OF THE INTESTINES}

\section{By Joseph Sailer, A. B., M. D. \\ SCLEROSIS OF BLOOD-VESSELS}

Sclerosis of the blood-vessels of the abdomen is not a rare condition in elderly persons. It usually occurs in all persons subject to a general arteriosclerosis. It may even occur in the young as a result of lead poisoning or syphilis and is said sometimes to occur in young persons with cirrhosis of the liver who have indulged excessively in alcohol, although it may well be suspected in these cases that syphilis is the really important element in the causation. It is rather remarkable in view of the frequency of this condition in old persons that symptoms should so rarely occur, but they do sometimes occur and are as a rule fairly characteristic. Among them are severe pain usually centered in the epigastrium. These attacks of pain commonly occur after some severe exertion and occasionally after some severe mental excitement. They may even follow indiscretion in diet, particularly alcoholic. Usually the onset is sudden, is severe, and the pain bores through to the back. There is a sense of prostration. The skin may become cold and moist and there may even be a manifestation of great fear. The abdomen is extremely tender, and the sense of cramp causes the patient to lean forward in order to relieve pressure. In a longer or shorter time there is nausea which is sometimes followed by vomiting, and vomiting as a rule not only does not relieve the pain but causes it to become more severe. In a short time, however, rest will give relief, but if any physical exertion is undertaken the pain is apt to occur again. Sometimes there is a good deal of accumulation of gas causing distention of the abdomen and eructation and the passage of flatus. In the interval the symptoms are those of sluggish digestion. Sometimes there is epigastric tenderness, nearly always a good deal of flatulence. Occasionally, with eructation, a small amount of gastric contents may be brought into the mouth. The bowels are constipated. There may be mental dulness with a desire to sleep which is probably not due so much to the abdominal as to the cerebral condition. Occasionally an enlarged liver is found but this, probably, also is due not so much to the sclerosis of the abdominal vessels as it is to other conditions which have helped to bring this sclerosis about. In rare cases there is a distinct relation between the pain and eating, the pain coming on in about one hour according to Hunter. This is usually an early symptom and later the paroxysm may occur without reference to food. 
The prognosis is of course hopeless for cure.

Whatever method benefits general arteriosclerosis ought to be employed. The treatment therefore consists of the avoidance of all severe physical effort. The patient should be particularly instructed not to walk in the cold against a high wind. Stair climbing, any violent physical exercise such as tennis, rowing, swimming perhaps golf, should be forbidden. If it is feasible the patient should be advised to retire from business, to avoid active professional or commercial life. Residence in a warm climate is also advantageous. The diet should be of the character described as light, that is to say the heavy pastries, heavy meats, meat salads should be to a certain extent avoided, but it is more important to reduce the quantity of the food than to modify the quality. Nevertheless the nutrition of the patient should be maintained and if the meals are made smaller it is desirable that they be taken more frequently. Constipation in particular should be avoided. This, in many of these cases, can best be controlled by the administration of saline laxatives in small doses at regular times. Five to ro gm. of phosphate of sodium in a small amount of warm water before breakfast, or a small dose of magnesium sulphate or some of the saline laxative waters such as Hunyadi Janos, or Pluto, or a Seidlitz powder each morning before breakfast will serve to evacuate the bowels rather freely and as a rule will diminish the frequency and the severity of the attacks. Occasionally in these cases gastric analysis will show deficiency of hydrochloric acid and of peptic content in the gastric juice. When this is found there should be supplied a small amount of pepsin, about $0.5 \mathrm{gm}$. and sufficient amount of dilute hydrochloric acid as indicated by the result of the analysis of the gastric contents may be given before each meal well diluted.

Of the drugs the nitrites are unquestionably the most efficient. The dose cannot be determined in general but each patient should be given a sufficient amount to relieve the pain or at least to diminish its frequency and severity. Either nitroglycerin or sodium nitrite may be used for this purpose. The patient often experiences comfort if the abdomen is kept warm. One of the ordinary woolen binders is very satisfactory for this purpose. If there is much obesity a binder designed to relieve the strain on the abdomen is often useful and gives a good deal of comfort. As a rule, however, these patients are thin. If syphilis is present specific treatment should be employed.

The differential diagnosis is comparatively difficult. Very frequently in general arteriosclerotics gall-stones are present and may give rise to paroxysms which are falsely considered to be abdominal angina. Chronic disease of the pancreas is also not as rare as has been supposed and may give rise to severe similar attacks. Gastric and duodenal ulcer must also be excluded and renal calculus may under certain conditions be mistaken for this condition. Therefore, when symptoms of paroxysmal and acute abdominal pain are encountered even in an old person with tortuous peripheral arteries and evidences 
of extreme arteriosclerosis in the retinal arteries, a hasty conclusion should not be reached.

\section{DIVERTICULITIS}

Diverticula of the colon are by no means an uncommon finding at the autopsy. In all probability in the majority of cases they give rise to no symptoms during life and are therefore unrecognized. Occasionally, however, they become inflamed and under these circumstances give rise to symptoms which are by no means characteristic but which after a careful study of the case may lead to a suspicion of the condition that exists. The diagnosis is rather important for the reason that treatment either medical or surgical may sometimes relieve the inflammation of the diverticulum and such recognition may also lead to undertaking an operation that, if the condition were known to be malignant, would have been avoided. As a rule the symptoms occur in elderly males, that is, men who have pas ed fifty years. There is usually pain in the left lower quadrant of the abdomen, a palpable mass that is tender, constipation, or pseudodiarrhea, slight fever particularly in the evening, and a moderate leukocytosis. These symptoms may subside only to recur at longer or shorter intervals and finally to become chronic. The patient usually feels a lack of physical energy, tires easily, worries considerably about his condition, the stools commonly show a trace of occult blood, not infrequently a good deal of mucus, and occasionally some pus. As a rule, examination with the sigmoidoscope is negative, the diverticula either being situated above the point to which the sigmoidoscope can penetrate or else showing very little that can be recognized in the mucous membrane of the bowel on inspection. It may be of value to describe a typical case. The patient, after having for some time experienced severe pain in the left lower quadrant of the abdomen, rather acutely developed a mucous diarrhea. There were from six to twelve movements every day, each consisting almost exclusively of mucus and each occurring with suddenness so that it was necessary immediately to rush to the toilet. There was almost complete anorexia, slight headache, an evening temperature between 99 and roo, leukocytes were persistently in excess of 1 2,000, no fecal matter was seen for a period of about four days. There was a distinct mass in the left lower quadrant situated near the median line just above the left spine of the pubis. This mass seemed to be about as large as a hen's egg. It was movable, and distinctly tender and palpation caused a desire for stool. The nutrition of this patient was excellent. The evacuations from the bowel contained a small amount of pus, much mucus, and occasionally a trace of occult blood. The diagnosis was confirmed by the roentgen-ray and showed small masses of bismuth remaining to one side of the sigmoid flexure after the bowel had been thoroughly emptied. The patient, a physician, was convinced in the beginning that he suffered fron a carcinoma of the lower bowel. A somewhat 
similar case, also seen in a physician was characterized by a constipation, considerable pain in the left lower quadrant, a palpable mass which was tender and movable, slight evening fever, anorexia, and general malaise. In this case also careful roentgen-ray studies showed a single diverticulum extending from the side of the sigmoid toward the median line.

Treatment of this condition is either medical or surgical. If the condition is seen early and the attack is mild, medical treatment is distinctly indicated. It consists first of emptying the colon as thoroughly as possible by enemata. For this purpose a preliminary enema of oil seems to be most efficacious, about 8 ounces of warm cottonseed oil being injected and retained as long as possible. On the following day an enema preferably consisting of magnesium sulphate, glycerin and water should be given and if a large amount of fecal material is dislodged the attack usually rapidly subsides. Sometimes it is necessary to give these enemas several times and at the same time to use general laxatives by the mouth. For this purpose the liquid paraffin oils combined occasionally with cascara or senna and sometimes laxative mineral waters seem to be most efficient. The patient should be advised to remain in bed excepting when it is necessary to rise to go the the toilet. The diet should be very light consisting chiefly of milk, egg, broth and a small amount of toast, pulled bread or zwiebach. When the acute stage has subsided the bowels should be kept rather freely moved and for this purpose agar-agar combined with some vegetable laxative, or a saline before breakfast, seems to be most efficient. It is probable that the large mass of the agar-agar scours the wall of the intestines and prevents a reinfection of the diverticulum. If constipation should develop at any time, it should be vigorously combated either by giving calomel in fractional doses or by the use of an oil enema followed by either a soda or magnesium sulphate enema. The patient should take moderate exercise, and while the condition is latent there is no need to forbid habitual employment. The diet does not seem to have any particular influence and during the latent period the ordinary diet may be freely taken. It is sometimes of advantage to add certain laxative substances, particularly fruit. The fruit in my experience is best taken either before breakfast or at bedtime. Particularly at bedtime figs and apples seem to be advantageous. Stewed fruit is also useful in these cases. Should the condition fail to yield to medical treatment, and should the tumor, tenderness, fever, leukocytosis persist and there be constantly a trace of occult blood, mucus and pus in the stools, operation should be undertaken without hesitation. According to Griffin diverticula not infrequently become carcinomatous. Indeed, he thinks that carcinomas of the colon may frequently arise from this cause and this is an added reason for early operation in order to remove the possibility of the development of subsequent malignancy. The importance of this condition may be illustrated by the brief recital 
of a case seen about a year ago. The patient, a man of sixty-five, developed a large mass in the right lower quadrant which was distinctly tender. His leukocytes ranged from i2,000 to 20,000 . The stools contained blood, mucus and pus. The temperature ranged between 99 and 102. His general condition was poor as the symptoms of infection had, when first seen, persisted for some weeks. A diagnosis had been made by several previous consultants of malignancy of the lower bowel and the patient declined operation. An alternative diagnosis of diverticulitis was made and operation strongly urged. A subsequent consultant, however, insisted that the condition was carcinomatous. Several months later the patient developed a large abscess that pointed under Poupart's ligament and which was opened and discharged a considerable amount of pus. He refused an operation and died. At the autopsy a large diverticulum that had ruptured was found and the pus had extended upward in the left flank as far as the kidney. The wall of the diverticulum was sectioned and failed to show any sign of carcinomatous change.

In addition to the differential diagnosis of carcinoma of the colon the possibility of perisigmoiditis ought to be considered. This is a condition to which Simmonds has called attention as a result of his autopsy examinations. It is characterized clinically by longstanding constipation, occult blood in the feces, and a palpable tumor in the left hypochondrium. It consists actually of an inflammatory condition of the serous wall of the sigmoid which results in adhesions to the surrounding tissues and some changes in the mucous membrane. The treatment, of course, is purely surgical.

\section{INTESTINAL STASIS}

Intestinal autointoxication and intestinal stasis are two conditions that seem to amount to about the same thing. Not, however, in every case of alleged intestinal autointoxication is constipation present. Whether intestinal autointoxication actually exists or not is still undecided. No toxin has ever been discovered. We assume the existence of a toxin from some of the general symptoms manifested by the patient and we consider this assumption is justified if, after treatment supposed to relieve the stasis of the intestines, all these symptoms disappear. As these symptoms are by no means constant or definite the problem is still more complicated and opinion varies from that of men who deny such a thing as intestinal autointoxication to enthusiasts like Lane and Reid who believe that practically all chronic symptoms and even definite pathological lesions in other organs such as tumors of the breast may be due to this cause. It may be assumed that in this, as in other conditions, the true solution is in the middle ground; that is to say all symptoms of all chronic diseases need not be ascribed to intestinal autointoxication, neither need it be denied that as a result of stasis in the 
colon general symptoms may be produced. It is not, however, necessary to assume that they are the result of the existence of a toxin. They may, indeed, be the result of defective absorption by the colon, for it is well known that various substances are absorbed in the colon, possibly the result of the products of the ultimate digestion of certain carbohydrate material, and if this absorption is defective it may well be that symptoms will be produced. Moreover, it is quite possible that the intestinal stasis is merely one manifestation of some other process, perhaps defective small intestinal digestion which is actually the cause of the other symptoms. Of course, if any proof could be found for the latter assumption, then the various forms of treatment designed to relieve the colonic stasis would be unscientific and probably would result in no benefit to the patient. It is furthermore possible that we may have disturbances of the general metabolism as a result of any one of the three suggested causes and if it were possible therefore to differentiate between the different types, various forms of treatment could be employed which would be more rational and probably would lead to better results.

Great stress has been laid upon the presence of indican in the urine as an evidence of faulty colonic absorption. Indican, however, has been shown to occur in a variety of conditions and it would be at least irrational to diagnose intestinal autointoxication in every case of indicanuria. There are unquestionably cases of chronic colonic stasis in which indicanuria does not occur, so that it can in no sense be regarded, as some have insisted, as a pathognomonic sign. Again, I am inclined to believe that the truth lies somewhere between these two extremes; that the presence of indican in the absence of any definite evidence of other causation may lead to a suspicion that the gastrointestinal tract is disturbed, possibly in the form of stasis, and as an indication for further investigation, indicanuria may therefore be valuable, but as a definite sign I think it would be well to deny it any preëminent value whatever. It is, of course, well known that in cases of intestinal obstruction with fermentation of the contents, the indican in the urine becomes very excessive. The symptoms of chronic colonic stasis are exceedingly various, headache, anorexia, nausea, malaise, loss of weight, early fatigue are common enough in a great variety of conditions. More definite symptoms can possibly be related to this condition. Among these are headache, asthma, urticaria, glycosuria, melancholia, degenerative changes in the eyes, etc. Of great value are careful roentgen-ray studies of the motility, position and size of the colon. If the bismuth column advances with extreme slowness and there is distinct evidence that the colon retains its contents too long; at least there is a definite mechanical condition present which is an indication for treatment and if, as a result of this treatment, the symptoms disappear, the patient may be satisfied, but the physician feels that the connection between the two has not been as definitely established as he might wish. 
There can, of course, be no general prognosis devised for these conditions. Each case is to a certain extent unique and depends upon the degree of stasis and the amount of general disturbance caused thereby.

Treatment.-The treatment of chronic intestinal stasis is by no means fixed and constant. It varies with the manifestations in each case and as there are a variety of indications to be met it would be well to consider these in detail. The first indication is, of course, to relieve the mechanical condition present, that is the slow advance of the column of the intestinal contents. There is every reason to believe that absorption in the colon is fairly active as the fecal mass is usually dry and well hardened. To that extent, therefore, the functions of the large bowel are not disturbed. On the other hand, the motor function of the bowel seems to be distinctly defective. This defect may be due to several causes, either defective action of the muscles of the bowel itself or to obstruction due to kinks which may be produced by abnormal elongation of certain portions of the colon such as for example the sigmoid, to ptosis of the whole colon, possibly to cecum mobile, although this is doubtful, to dilatation and atony of the cecum, or to excessive innervation of the bowel so that spasm occurs, to excessive absorption so that the feces become too dry. If it were possible to differentiate clearly between these defects, treatment more or less rational might be devised that would overcome the particular fault present in the case but this is rarely possible. Palpation of the abdomen is sometimes of advantage. The atonic dilated cecum can often readily be detected, particularly by the presence of a succussion splash in the right lower quadrant. If spastic contraction occurs, it may be suspected by the hard cord-like feel of the descending colon. Excessive absorption may be suspected, if the feces are excessively dry. If there is much gas, faulty starch digestion may be present. The roentgen-ray will often show the presence of kinks which may be indicated by the stoppage of the column of bismuth at some point beyond which the column becomes much more slender or does not appear at all, and it will often show twists and redundancy of the large bowel that cannot be detected by palpation. Examination of the feces may indicate that there is faulty digestion of the meats, the starches or the fats, which would suggest that the trouble is higher up, either in the secretions of the stomach, pancreas, the liver or the intestines. Occasionally a functional test of the pancreas or of the liver may yield some information. Indican, as has already been stated, may or may not indicate putrefactive changes taking place somewhere in the intestinal tract preferably in the large intestines. In any event the evacuation of the bowels is the first consideration. This may be facilitated in a variety of ways. First, by hygienic treatment. This involves life in the open air; as much exercise as is compatible with the patient's strength; freedom as far as possible from mental anxiety; and certain special exercises which 
have for their object what may be called an automassage of the large intestine. Fernet has devised the following system: Before arising, the patient should lie on the back in bed and take five or six deep inspirations with the mouth closed, protruding and retracting the abdomen with each inspiration. He then relaxes for a brief period and repeats this exercise until he has done it about five or six times. He then arises, bathes, breakfasts very slowly and immediately thereafter makes an effort to evacuate the bowel. If this is unsuccessful he performs a sort of rectal gymnastic by pressing and retracting the anus a number of times. In the course of a few days normal movements should be established without the use of laxatives or enemas and the patient should be regarded as cured with, however, the necessity of continuing the exercises regularly. Bastedo has modified these slightly but apparently to no advantage. He suggests that food should be of a type that leaves a considerable residue. The second element in the treatment is dietetic. No fixed diet can be given. If the examination of the feces does not indicate that there is a special form of indigestion the character and amount of the diet should be determined by the nutrition of the patient. If underweight then the patient should be overfed, carefully avoiding any tendency to produce indigestion. In general, it may be said that those foods which have a fair amount of residue are of advantage. Breakfast should consist of fruit, cereal with cream, eggs, if desired some vegetable such as potatoes, rice, and if desired tea and coffee, oatmeal muffins, cornmeal bread, Graham bread, whole wheat bread or bran bread. The luncheon should consist of meat, some coarse vegetable such as spinach, cauliflower, cabbage, beans, particularly string beans, some of the coarser breads and a light dessert. The dinner should consist of a coarse vegetable soup, meat, vegetables as at luncheon, green salad and a light dessert, the coarser breads and if the patient is accustomed to it a demitasse. Very often either at luncheon or dinner stewed fruit may be substituted for other forms of dessert. If there is marked emaciation, light luncheons between the principal meals consisting of a small amount of cream, coarse bread and butter with, if desired, honey, dried fruits such as dates or figs may be taken or, if the patient prefers, buttermilk or some other form of fermented milk and crackers. If the patient is overnourished but shows no particular defect in the digestion of various foods, a reduction of the diet is probably indicated. This should be sufficient to reduce the weight slowly but not too rapidly and at the same time not to weaken the patient too much. The following is the type of diet $I$ have used for some time in these conditions with beneficial effects. It represents to my mind the lowest diet that is safe for an individual who is not bedfast to take. Obviously in some cases additions must be made to it in order to prevent too rapid reduction and to supply enough food to maintain the nutrition during work, etc. It is, therefore, not to be taken as a fixed type of diet but merely as a minimum to 
which additions may be made. Breakfast: Orange or grapefruit (no sugar), one egg, one slice of toast, one cup of black coffee, no sugar or cream. Luncheon: One cup of bouillon, I or 2 ounces of meat, two tablespoonfuls of green vegetables, salad (lettuce, Romaine, celery) with French dressing, jelly or fruit dessert. Dinner: The same as luncheon. Drink water freely. Use saccharin or crystallose in the place of sugar. If hungry between meals, eat a few grapes.

If as a result of examination of the gastric contents there is any reason to suppose that there is defective digestion, two measures may be employed; either to supply the lacking ferment or to reduce in the diet those articles of food which are poorly digested. It is obvious, of course, that both measures may be used at the same time in the same case, sometimes with advantage. If there is subacidity and apepsia, pepsin and hydrochloric acid is indicated, depending upon the degree of acidity. From 0.5 to $1.0 \mathrm{gm}$. of pepsin may be administered dissolved in a sufficient amount of hydrochloric acid. Cinnamon water makes an excellent vehicle for this preparation. From 0.5 to 2.0 c.c. of dilute hydrochloric acid may be safely given. If there is faulty starch digestion, various diastatic ferments may be employed. Of these the most effective are pancreatic extract or takadiastase. The latter may be administered directly in the tablets which are put upon the market. The former should be given only when protected against destruction by the acid in the gastric juice. This may be accomplished by using glutoid capsules, by enclosing it in capsules coated with salol or by mixing the pancreatic extract with some protective substance such as tannic acid or derivative from it, such as the pankreon now prepared for sale.

It must be remembered that there are certain cases in which the secretion of acid is adequate for a light meal but appears to be limited and therefore is inadequate for a full meal. A single test is therefore not sufficient but in these cases not only should an Ewald meal be used but also a protein meal such as that suggested by Fisher which consists of ground tenderloin steak, bread and water. For the faulty digestion of fats the pancreatic extract may also be employed and in addition the patient may be given some preparation of bile such as fel bovis. Of the pancreatic extract the usual dose is from 0.3 to $0.5 \mathrm{gm}$. Of the fel bovis from 0.2 to $0.3 \mathrm{gm}$. each to be taken immediately after meals. The regulation of the diet in these cases is fairly obvious. If the proteins arc poorly digested their amount in the diet should be distinctly restricted. All heavy meats should be avoided and a sufficient amount of animal protein obtained, preferably from milk and fresh eggs. If the starches are defective the starchy vegetables should be excluded. These include particularly potatoes, macaroni, cereals and breads. Rice seems less subject to fermentation than the other starchy foods and may be permitted in moderation. If the fats are defective, butter, cream, bacon, oil salad dressings should be excluded from the diet or at least reduced to a 
minimum. It must be remembered that the indications obtained from a single specimen of feces may not be entirely correct and repeated examinations should be made to obtain as great a degree of certainty as possible. Moreover, after the diet has been instituted, these examinations should be made in order to determine whether it is reasonably effective or not.

Mechanical treatment has been greatly lauded by various individuals, not only physicians but also masseurs, osteopaths and fadists. My experience has been somewhat disappointing. Masseurs and masseuses have frequently assured my patients that they would be able to cure them of chronic constipation and I have been willing that they should undertake the task. Occasionally there is temporary improvement but I have hitherto not seen a case that has been permanently relieved or indeed whose relief persisted during the period of massage. The iron ball rolled over the abdomen following the course of the colon gives considerable satisfaction to some patients but apparently has not been followed by any very permanent results. As an adjunct massage is often useful, but I think the massage should be general for the purpose of improving the tone of the patient. With osteopathy I have had no personal experience. I do not recommend it to my patients. Those who have taken it upon their own volition and whom I have seen subsequently have not been satisfied with the results. The direct treatment may be regarded under two heads; drugs administered by the mouth and enemas. Frequently considerable relief can be obtained by the use of various laxatives. Of these cascara occupies the first place. It should be given in a dose sufficient to cause a movement every day but not to produce purgation. This must be regulated for each patient and can usually readily be determined. Next is senna which also can be given over long periods of time. Rhubarb, particularly if combined with magnesia, is also very valuable and may in some cases be administered in capsules three times daily after meals. The dose of the extract varies from 0.05 to $0.2 \mathrm{gm}$. as required by the patient. Salines are also very efficient. Of these the best are probably magnesium sulphate or sodium phosphate taken in hot water on arising in the morning. Purgation should be avoided and as nearly as possible an effort should be made to obtain a natural movement. Milk of magnesia is also useful and from 4 to 16 c.c. may be taken in the morning well diluted upon arising or in the evening just before going to bed. If there is no fat indigestion olive oil is often sufficient and a tablespoonful may be taken after meals two or three times a day. Castor oil and calomel should of course be used only occasionally for the purpose of producing free evacuation when the stasis has been somewhat prolonged. A few patients have done well on small doses of podophyllin from 0.005 to $0.008 \mathrm{mg}$. taken once daily at bedtime. With any of these laxatives a very useful aid is the mineral oil extensively exploited for commercial purposes. Different preparations seem to 
me to be about equal in value. There is no particular advantage in the preparations prepared from Russian petrolcum. The dose is not fixed. As much as the patient can comfortably take one to three times a day may be given and sometimes it alone suffices to soften the intestinal mass and to promote the evacuation. If there is much fermentation various intestinal antiseptics may be employed. Salol is rather dangerous on account of the tendency to irritate the kidneys. Betanaphthol, guaiacol, thymol are all fairly useful and preparations of lactic acid bacilli and particularly soured milk are also useful.

Treatment by enemata is perhaps the most important in these conditions. A thorough lavage of the colon daily is habitually employed with asserted benefit by many persons. From I to 3 quarts of water should be allowed to run into the colon either upon arising or shortly after breakfast. This apparently can be used for long periods of time without any injurious effect to the general nutrition. Salt solution, either the physiological salt solution or one slightly stronger may be substituted for the plain water. Soda may also be employed with advantage. About 3 per cent. solution seems to be the most generally useful. It consists of I ounce of soda bicarbonate to a quart of water. Various substances may also be introduced with the enema, either for the purpose of stimulating the bowel more vigorously or for checking fermentation. Of the former glycerin is particularly advocated. Hertz, indeed, starts with 50 per cent. solution of glycerin and gradually increases the glycerin and diminishes the water until pure glycerin is employed. I have not obtained by this method the results that he describes and it often causes considerable discomfort. Small amounts of magnesium sulphate dissolved in the water are powerful stimulants to the contraction of the bowels and in very sluggish cases seem to be necessary. An enema consisting of magnesium sulphate, glycerin and water in varying proportions according to the needs of the case is often of great value particularly in the early stages of the treatment. As a rule it can be discontinued in a short time and a less stimulating enema employed that is equally efficient. Enemas of oil have been recommended by a variety of authorities. They are of most value in my opinion when used occasionally as adjuncts to other forms of treatment. The following method I have found to work well in a few cases. On the first day of the treatment an enema of oil is given that is retained as long as conveniently possible by the patient. Often it can be retained over night. The next day an enema of 3 per cent. soda solution is given and this commonly results in a very free evacuation of the colon. The third day no enema is given and on the fourth day the cycle recommences. As the condition of the patient improves the rest days are increased so that the cycle consists of oil, soda and two, three, or more days of rest. In several cases of asthma of long standing which appeared to have some relation to constipation and fermentation in the colon the asthmatic attacks were almost whoily relieved by this treatment. In one case there was a 
distinct reaction to ragweed pollen extract but immunization to ragweed although it reduced, did not wholly cure the attacks. In cases of severe headache it has been similarly successful, when there was good reason to believe that the headache was dependent, at least partly, upon the condition of the colon. In other cases where a similar relation seemed to exist it has, however, failed, but in these cases the failure was probably due to a too ready acceptance of the causal relation of the intestines to the headaches. A variety of antiseptics may be employed in the enema. I believe formalin is probably the most efficient; 0.05 to 0.2 per cent. may be used preferably in a soda solution, the amount to be reduced if any burning or discomfort is caused. Thymol in o.I per cent. solution usually in physiological salt or in plain water is also helpful. Various silver salts have been employed, nitrate of silver, protoargol, collorargol, etc. The latter, however, are more useful in ulcerative colitis. Attempts, however, have been made to secure a laxative that could be administered hypodermatically. Hormonol, to which reference has been made elsewhere, has been highly recommended but the evidence of its danger has steadily accumulated and it has never come into general use, probably deservedly. Sennatin has also been recommended but I have no personal experience with it and feel that the general repugnance of the profession against its employment is well founded.

The treatment of associated conditions such as hyperchlorhydria, gastric catarrh, chronic pancreatitis, hepatic insufficiency belongs properly in other sections of this book and I shall not refer to them. When their existence is discovered it is obviously important that they should be corrected, if possible. An interesting phase of chronic intestinal stasis is its possible relation to the autonomic system. This has been particularly mentioned by Spitzen and von Noorden. Reference has already been made to it. It is conceivable that intestinal stasis may be due either to an under- or overaction of the autonomic nervous system. If there is any reason to suspect that it is due to overaction of the vagus and pelvic nerves, the administration of belladonna or atropin is indicated and in sufficient doses to produce slight physiological effects. If there is evidence that the vagus and pelvic nerves are depressed it is conceivable that pilocarpin or adrenalin would be of value. Pituitrin which is a stimulant to the nonstriated muscular system might also be considered but it has not, however, fulfilled the claims made for it in intestinal conditions.

Although it will be considered elsewhere, perhaps reference may be made to some of the medical aspects of surgical intervention. If there is mechanical obstruction, it should obviously be relieved. The results of such operative measures are sometimes brilliant in the extreme. One of the most remarkable cases occurred in a small boy of four years who had an almost insuperable constipation. Roentgen-rays showed an excessively long sigmoid flexure which was resected by John G. Clark with prompt disappearance of the constipa- 
tion and great improvement in the patient's nourishment. The operation devised by Lane, of short-circuiting the colon and thereby eliminating the possibility of chronic stasis, the retention of feces in the colon and possible absorption of toxins has been advocated and decried. I do not feel competent to discuss the ultimate results. Personally I believe that the reports of various men who use these operations extensively are too optimistic. In the few cases that I have seen there was no obvious benefit and indeed some of the cases complained of feeling worse than they were before the operation. On the other hand the reports of Lane, Reid and others claim cure not only of the ordinary symptoms of intestinal autointoxication, but also for such conditions as diabetes, breast tumors, tuberculosis of the hip, etc. This seems almost irrational and should require ample confirmation on the part of others and this confirmation is at present lacking. The mere fact that the colon is not an organ having definite and well-established functions in the economy is by no means proof that it is indispensable and the fact that patients survive this operation for many years is not sufficient proof of this assertion. On the other hand, there is no reason to believe that the entire destruction of its functional activity is a trivial matter for the organism and there should be very ample evidence that such a destruction is not only indicated for the manifestations of the disease from which the patient suffers, but also will not result in harm in some other direction before the operation is undertaken. Personally I do not believe that this evidence has as yet been presented in incontrovertible form and that therefore Lane's operation should not be undertaken recklessly in any form of chronic disease.

\section{CHRONIC DIARRHEA}

The distinction between the various forms of chronic diarrhea is not nearly as sharp as one would judge from the elaborate classification in the text-books on medicine and works devoted to the gastrointestinal tract. Mucous colitis, chronic colitis, ulcerative colitis shade into each other by imperceptible degrees. In a few cases the etiological factor can be discovered and there is an increasing tendency especially in the South to regard some of these chronic diarrheas as possibly associated with certain forms of tropical disease, particularly pellagra and sprue. It cannot be said that this hypothesis has added much from the standpoint of therapeutics. For pellagra no satisfactory treatment has been devised and for sprue the treatment with milk and fresh fruit seems distinctly less efficient in our southern States than it does in tropical countries where the disease is common. Of some interest is the possible relation of chronic intestinal disease to the disturbance of the autonomic or sympathetic nervous system. Spitz has called attention to this. The autonomic nervous system is the motor innervator of the bowel. As a result of stimulation of the vagus peristalsis is increased and 
therefore the intestinal contents are pushed onward. If this increased peristalsis is sufficiently prolonged then diarrhea will ensue. There is some reason now to believe that not only is the peristalsis of the walls of the hollow organs actively increased but at the same time relaxation of the sphincters occurs so that the onward movement is very considerably facilitated. These conditions are conceivably of three types. First, the persistent excessive secretion of the hormone which stimulates the vagus. This is probably a part of the internal secretion of the pancreas. Second, constant stimulation from the central nervous system, at least that part of it not directly a portion of the autonomic system. This is not entirely hypothetical. It is known, of course, that severe emotional disturbance may cause excessive activity for a time in the autonomic system. Third, the depression of the physiological antagonist of the autonomic system, that is the sympathetic system. A case of this kind may be worth noting. The patient, sixty-five years of age, was brought to my service at the Presbyterian Hospital suffering from persistent uncontrollable diarrhea. The nature of the case was not determined during life. All forms of treatment were futile even including appendicostomy and lavage of the colon through the appendix. The patient died. Hypernephroma and metastasis to lungs was found and both suprarenals were markedly diseased. The patient had not shown any of the conventional signs of Addison's disease. The mucous membrane of the large and small bowel was normal macroscopically and microscopically. It was, therefore, surmised that the destruction of the adrenals and the consequent loss of the hormone stimulating the sympathetic system had permitted an overaction of the autonomic system. Assuming that such a condition may arise and could be recognized during life there would be two lines of treatment available. First, the administration of sufficient doses of atropin or belladonna to depress the autonomic system. Second, the administration of adrenalin to stimulate the sympathetic system. Of the two, the former would appear to be more rational. It should be remembered that cases suffering from vagotonia can stand large doses of belladonna preparations without apparently manifesting physiological effects.

Very little that is new has been suggested in the way of treatment for the various forms of colitis. The treatment suggested by von Noorden consisting of oil enemata, hypernutrition and certain laxative measures has been on the whole so successful that nothing has been devised to replace it: There is still, however, a great desire to introduce antiseptics into the bowel and thereby sterilize the intestinal contents in the hope that when this sterilization occurs there will be no further irritation and the inflammation will rapidly subside. The nature of the infectious process, however, has not yet been determined. I mean by this whether, when it is an actual bacterial infection, the bacteria grow and proliferate in the cavity of the bowel or whether they grow and proliferate beneath the mucous 
membrane. In the latter event intestinal antiseptics would probably not accomplish much. Theoretically it would seem quite possible to administer enough of certain antiseptics to the patient if these antiseptics should be distributed throughout. the entire content of the intestines to produce a solution sufficiently strong to inhibit bacterial growth. Practically this does not seem to occur. Apparently no new intestinal antiseptics have been devised. 'Those which secure the most approbation are the salicylate of bismuth, thymol, the silver salts, betanaphthol, and guaiacol. The use of sterile food, of frequent small meals has also been suggested. For enemata, which are still very popular, Wegele recommends the following: If there is much pus, a solution of I per cent. ichthyol or 0.25 per cent. collargol. If there is much blood an enema of gelatin or calcium chlorid is useful. The gelatin should be about io per cent., made as the ordinary gelatin medium is made without the addition of Witte's peptone. The calcium chlorid solution should be about 0.5 to I per cent. In addition, in severe cases, hydrogen peroxid may be used with advantage but should be allowed to flow out through the rectal tube and no effort made to retain it. If there is much ulceration an emulsion of iodoform may be of advantage. A new method is the transduodenal lavage. This has been devised by Jutte. The duodenal tube is introduced in the usual way and its location in the duodenum determined by withdrawing some bile-stained contents, or by the roentgen-ray. The outer end is then attached to a reservoir containing about 2000 c.c. of fluid. This is allowed to trickle directly into the bowel through the duodenal tube for about ten minutes by which time at least Iooo c.c. has been injected. The solution used should vary with the nature of the case. For ordinary lavage of the intestines normal salt solution is most satisfactory. When it is desirable to stimulate the kidneys distilled water may be used. Castile soap is also employed. In catarrhal enteritis some astringent may be added. In amebic dysentery quinin is added to the solution. Apparently it has not of ten been used as yet. An interesting suggestion is that in cases of edema a solution of salt stronger than normal would serve to cause the osmosis of considerable water into the gastrointestinal tract. This appears to me to be a theoretical suggestion that might not be borne out in practice. The effort to get solutions directly into the bowel, without permitting them to pass through the stomach, that might effect their direct action upon the mucous membrane of the interior has lead in the first place to enemas, in the second place to appendicostomy, an operation that is now rarely performed, and in the third place to the use of the duodenal tube as recently described. There has always been a feeling that the introduction of gas into the intestines might be of some value. Even in Gulliver's travels the idea has been suggested. Later sulphureted hydrogen enjoyed considerable vogue as a specific remedy for tuberculosis. Recently Schmidt has suggested the injection of oxygen 
into the intestines through the rectal tube and Gross has used the duodenal tube for the same purpose. As there have apparently been no subsequent reports upon this procedure there is little likelihood that it is of any benefit.

\section{DYSCHEZIA}

Dyschezia, a term that has been used by Singer and Hertz, is a condition in which the lower portion of the rectum loses its expulsive power. The symptoms are a feeling of heaviness in the rectum and a desire to have a movement of the bowel which can be gratified only slightly or not at all. The patients usually complain of constipation. Upon examination of the rectum it is usually filled with a fecal mass which is usually in the form of hard lumps but may be distinctly pasty. The condition is usually chronic. It may occur in women after childbirth or in patients who have had an anal or rectal operation. In this condition laxatives by the mouth are unavailing. The rectum must be relieved by enemas and occasionally in cases where the condition is longstanding by a preliminary mechanical evacuation of the contents. The most efficient enemas are oil, glycerin and water and glycerin, water and magnesium sulphate. Occasionally lavage of the rectum is sufficient. Treatment must be carried out daily and at the same time it is of advantage to the patient to give moderate doses of strychnia with the hope of increasing the tone of the rectal muscles. As a rule this tone can be restored and in the course of time the enema may be used less frequently and finally discarded altogether. 


\section{CONSTIPATION}

\section{By George Morris Piersol, M. D.}

Physiology.-The physiology of the large intestines is by no means an absolutely settled question. In the main, the statements made in our original article on constipation, may be regarded as embodying the generally accepted views on the physiology of the large bowel. However, attention should be called to some more recent opinions.

Several years ago Meyer Betz studied normal peristalsis, and came to the conclusion that the large bowel is practically never at rest, but is constantly and periodically in motion. This is contrary to the opinion expressed by Holzknecht, who was among the first to observe peristalsis by means of the roentgen-rays, and who believed that the large bowel is quiescent for the greater part of each day, during which time there occur three or four great waves of contraction lasting a few seconds each, which are capable of propelling the contents about a third the leng th of the large bowel. Holzknecht's views, however, have been opposed by other observers. Meyer Betz has further shown that the chief seats of reflex stimulation for the large intestine are the stomach and the rectum. When food is taken regularly in to the stomach, this organ becomes the most important physiological stimulus of intestinal peristalsis. A substance such as glycerin introduced into the rectum, closely simulates the normal impulse of defecation, and sets up throughout the intestines reflex contractions which progressively involve the large bowel from the rectum to the hepatic flexure. In studying the effects of laxatives on intestinal peristalsis, he found that those laxatives produce the greatest intestinal movements which liquefy the contents of the bowel, such as the various salines, jalap and castor oil.

From studying a normal man placed upon a diet of uniform consistency, Schwartz found that ingested food was propelled as far as the sigmoid and rectum by the end of the first day, but in spite of this, food taken on a given day was not completely discharged from the rectum until the third day. In connection with these observations, it is interesting to note that the character of the food, whether it is stimulating or bland, produces decided variations in the peristaltic waves. The greatest variation is observed during the first six hours in the activity of the small bowel and the first part of the large intestine. However, after twenty or thirty hours the difference in the degree of progress noted between irritating and non-irritating food is negligible.

Cannon, whose contributions to intestinal physiology have been 
of such signal service to both clinicians and physiologists, describes in great detail the mechanics of the act of defecation in man. The intestinal contents, after coming through the descending colon, normally are blocked at the acute angle made by the junction of the pelvic colon and the rectum. The greater the distention of the pelvic colon, the more this angulation is obliterated, until finally the waste material can advance into the rectum. When the fecal mass once enters the rectum, there is a desire to defecate. This desire is made more urgent by the taking of food, exercise; etc. Defecation itself is actually brought about by the contraction of the diaphragm, which forces downward the entire transverse colon, thus compelling the ascending colon and cecum to become a globular mass and the intraabdominal pressure to be markedly raised. The rise in intraabdominal pressure increases the rectal pressure four to eight times that of normal. The rise in rectal pressure also causes more material to enter the rectum, which becomes overdistended, and this distention induces reflexly contractions of the colon, produces contractions of the abdominal wall and relaxation of the anal sphincters. Normally there follows the complete emptying of the rectum and pelvic colon. Indeed, there are some who hold that under entirely normal conditions at a single stool the large intestine is completely evacuated from the splenic flexure on.

Causes.-So far as the causes of constipation are concerned, chief interest has centered about the various local conditions which give rise to retardation in the onward progress of the intestinal contents. As far back as 1902 Cannon observed antiperistalsis in the intestine of a cat. Whether or not similar antiperistalsis occurs in the human intestines, has been a matter of controversy. More recently several observers, notably Roith, Bloch, Stierlin and Albrecht, claim to have demonstrated the occurrence of antiperistalsis in the intestinal tract of man, and further, some of these observers are of the opinion that this antiperistalsis, or anastalsis, which prevents the contents of the bowel from leaving certain portions of the intestines with normal rapidity, is responsible for various degrees of constipation.

Among the local causes of constipation may be mentioned lesions of the ascending colon. These have been studied with care by Duval and Roux, who have found that the lesions of this part of the intestines which are most often responsible for constipation, consist of, first, abnormal mobility of the cecum associated with ptosis and duplication of the ascending colon; second, inflammatory pericolitis, and third, ectasia of the cecum. Clinically cases of this kind exhibit right-sided abdominal pain, periods of constipation, varying with shorter periods of diarrhea, and general impairment of health. The importance of the cecum and ascending colon in the production of constipation, is also emphasized by the careful work of Hopmann, who from a detailed study of twenty cases of constipation found that in fourteen cases large fecal accumulations were present in the cecum 
and ascending colon at eighteen to twenty-four hours, and that these fecal accumulations did not entirely come away for two or three days. In each of these instances the fecal mass contained an admixture of mucus. In four other cases, four to seven days were necessary for the fecal accumulations to come away. In these latter cases, mucus was not present in the stool until a laxative diet and mild purgatives were given, when definite microscopic mucus was found. It is of considerable significance that mucus occurred in the stools in all these cases. He believes that it indicates a catarrhal inflammation of the cecum, ascending colon and possibly the small intestines, which is the underlying cause of the constipation. Hopmann concludes that the presence of mucus hinders the normal absorption of fluids from the intestinal contents, which ordinarily takes place in eighteen hours. Hence, the fecal mass is forced to lie longer than normally in the ascending colon. He calls this form of constipation, ascending constipation. He has also observed that the symptoms which accompany it are identical with the symptoms of so-called spastic constipation. He, therefore, concludes that this latter condition is the result of catarrhal changes in the cecum, ascending colon or the small intestines.

Torpor recti, or dyschezia, is another local cause of constipation to which attention has been called. Dyschezia means a condition in which the rectum is not thoroughly emptied at each defecation, so that instead of the rectum being empty as it normally should be, except just before a bowel movement is about to take place, the rectum is found more or less constantly full of fecal material. This condition has been observed to be present in about 60 per cent. of cases of chronic constipation. Dyschezia is divided into two kinds: (I) Insufficient defecation; and (2) obstruction to a complete defecation. The cause of this condition is supposed to be long-continued inhibition of the normal act of defecation. This may be brought about by irregularity of the stool, or by failure to respond promptly to the desire to defecate. Anything that persistently diminishes the volume of the fecal mass, such as a diet too rich in meat, but too poor in cellulose, or the excessive and too rapid absorption of water from the fecal mass in the colon, is also held to be responsible for this condition.

In recent years some enthusiasts have attributed an enormous number of symptoms including ileal stasis and chronic constipation, to certain folds or bands of the peritoneum, probably congenital in origin, which are alleged to cause undue traction on certain portions of the bowel, and give rise to kinks which produce varying degrees of chronic obstruction. In this connection Lane's ileal band, which produces the now famous Lane kink, is held by some to be of particular importance. It will be recalled that Lane's kink is the result of traction on the left or inferior side of the ileum at its junction with the cecum, produced by a fold of the peritoneum, probably congenital in origin, which runs from the under side of the ileum in this locaVoL. IV. -42 
tion down to the parietal peritoneum which covers the psoas muscle near the pelvic brim. Among surgeons and internists alike, much controversy and discussion has arisen over Lane's kink and the various symptoms some attribute to it. It is doubtful whether it is as important a factor in the production of intestinal stasis as its discoverer would have us believe.

Treatment.-General Hygiene.-It has already been pointed out that the amount and kind of food present in the stomach is an important stimulus to the normal evacuation of the bowels. Agéron basing his observations upon this physiological fact, describes a form of constipation which he terms "gastrogenous." He has found that the uniform and rapid passage of food from the stomach into the the small intestines, and from thence into the ascending colon is of extreme importance in producing normal bowel movements, and that therefore if there is any diseased condition of the stomach which produces gastric retention, food enters the small bowel at irregular times and too slowly. It consequently reaches the colon in small masses which do not produce the proper stimulus to normal colonic contraction. He has also observed that the proper filling of the stomach with food plays an important part in emptying the bowels. In abnormal gastric conditions this stimulus is of ten lacking and inadequate, because of the inability of the patient to take sufficient quantities of food or fluid. His observations lead him to certain conclusions about the treatment of constipation. First of all he is of the opinion that it is of great importance to ascertain the condition of gastric digestion and the state of gastric motility. The condition of the stomach having been studied, the next step is to correct, if possible, the gastric abnormality. In cases where there is a tendency to gastric retention, Agéron suggests that solid food be given during the day, and that upon retiring liquids should be administered. $\mathrm{He}$ believes that bowel movements may be greatly facilitated by the administration of fluids at the proper time, and he recommends that 500 c.c. of milk be taken on a thoroughly empty stomach before going to bed. In place of milk, thin oatmeal gruel containing two teaspoonfuls of milk sugar may be employed. He is opposed to Carlsbad salts in cases of impaired gastric motility and dilatation of the stomach, as they induce the pouring out of an undue amount of fluid into the stomach. He also decries the use of honey, fruit, marmalade, compotes, etc., on the ground that they are retained too long in cases of gastric retention; that by their fermentation they produce gaseous distention of the bowel; and finally that they do not produce sufficient waste to aid materially the fecal bulk. He thinks laxatives should be given in the early morning or late in the evening, that is, at the time they will leave the stomach most promptly. In all conditions in which laxatives would be retained unduly in the stomach, he advocates enemas. He believes that little benefit has been derived from mineral waters in chronic constipation, since the relief is on the whole only a temporary one. Agéron's views on the importance of 
regulating the gastric digestion in the treatment of constipation, bears out the opinion expressed at some length in our original contribution on constipation in which we discussed the dramatic results often obtained in certain cases of chronic constipation when the state of gastric digestion was carefully investigated and corrected.

In the discussion of the physical and dietetic measures necessary to combat chronic constipation, Bastedo lays great emphasis upon the well-known fact that every effort must be made to establish a regular habit of going to stool. The effort to have a bowel movement must be persisted in daily, even if there is no desire to defecate, and while this attempt is being made, the mind should be concentrated on the act; hence he disapproves of converting the bath room into a reading room, as one soon falls into the all too popular but pernicious habit of perusing an interesting book or the daily paper while at stool.

Exercise--Exercise is a well-known adjunct to the successful treatment of chronic constipation. Yet it has been observed that athletes in spite of the excessive exercise in which they indulge are prone to be constipated. The explanation of this fact is that certain exercises are more successful in stimulating peristaltic movement than others. Among the most useful exercises for the purpose of overcoming constipation are the simple procedures of raising the legs when lying on the back, or bending the trunk when standing. Goodman reports good results from having his patients adopt the following exercises: On awakening in the morning, the patient is instructed to remove the pillow from under the head, and while lying flat in bed, deep inspirations are taken. While the breath is held, the abdomen is protruded rapidly five times. A deep expiration is then made, and the abdomen is forcibly retracted five times. This sequence of movements should be repeated five times. In addition, the patient is instructed to try to have a bowel movement at a definite time each day. While waiting for defecation to occur, the same muscular exercises are performed. Between each cycle of inspiration and expiration, the rectal sphincter is alternately relaxed and contracted several times. While these rectal gymnastics are being performed, under no condition should there be any straining. Both the abdominal and rectal exercises are similar to those originally described by Fernet. Fernet had his patients lie flat on the back and take five to ten deep breaths with the mouth closed, protruding and contracting the abdomen with each respiration. After a few natural breaths the procedure was repeated until this cycle had been performed five or six times. He claims that by means of this deep breathing the abdominal organs are subjected to a form of massage. In the periods of normal breathing, Fernet had his patients manipulate the abdomen manually. If no bowel movement occurred when the patient went to stool after breakfast, the rectal exercises above described were practised. Fernet maintains that if a patient will persist conscientiously in this form of exercise, the 
constipation may be overcome without the use of laxatives, enemas, or suppositories. As useful forms of exercise to overcome constipation may be mentioned: rising from the horizontal to the sitting positions without the use of the arms; bending the trunk forward, backward and sideways while deep breaths are taken, and bending the hips and knees on one side, while standing on the other foot. Such exercises should be done twice a day. They should be done slowly and should never be carried to the point of fatigue, the number of exercises being gradually increased as the patient's muscles become accustomed to them.

Water Drinking.-Rather excessive water drinking has at times been advocated in the treatment of constipation. As has been pointed out by some authors recently, this procedure is by no means as useful as has been thought, since the water is so rapidly extracted from the intestines that it adds nothing to the bulk of the stool. An adequate amount of water should, of course, be taken between meals, but its chief utility is in maintaining the soft consistency of the fecal mass.

Diet.-Diet unquestionably is, and always has been regarded, as one of the most important factors in successfully combating constipation. Some observers report remarkably good results in curing long-standing constipation by dieting alone. Among these is Rosenfeld, who has had great success by placing patients first upon oatmeal, rice, cocoa and chocolate, excluding all meat, butter, milk, fruit and vegetables. This diet is kept up for three or four days. He then adds a little coffee and boiled ham for two days. For two more days roast fowl is added. The next addition to the diet consists of tablespoonful doses of boiled cream. In a few days the amount of boiled cream is added by the cupful. This period of dieting is kept up for two or three weeks, and finally asparagus, spinach, potatoes, green peas, and lastly, fruits are added. It will be observed that this diet by which Rosenfeld has so successfully cured chronic constipation, is typically a diet suited to the treatment of diarrhea. In spite of this fact, it seems to act well, possibly because the treatment is initiated by having the patient remain absolutely alone in a room for several days, during which time all visitors are excluded and the patient is urged to frequently go to the water closet in an attempt to have a bowel movement, even when there is no desire for defecation. This is done in order to regain the lost control of the bowel.

Another rather rigorous form of dietetic treatment, which is alleged to be exceedingly successful in combating constipating that has been refractory for years, is laid down by Kohnstann. His method is to have all meat, fish and poultry absolutely dropped from the diet, and the patient placed upon a diet consisting of milk, and dishes made with milk, cocoa, milk soups, etc. As an aid to the digestion of vegetables and milk dishes, he allows at times, some meat soups and broths. After one or two weeks, depending upon the 
condition of the patient, meat can be resumed once a day, at first only white meats being given. $\mathrm{He}$ bases this dietetic procedure upon his conception that constipation is due largely to the meat, and the object of this form of treatment is to replace the meat putrefaction bacteria by a milk fermentation flora, which when once established, is apt to continue and to predominate even when meat is resumed in the diet.

Bastedo recommends a diet which is more in keeping with the conventional idea of a proper diet for constipation. He urges the free use of vegetables, salads, lettuce, spinach, carrots, parsnips, oyster plant, turnips, cabbage, cauliflower and squash, all of which contain much cellulose and fibrous tissue, and which leave a large residue in the intestines. Inasmuch as meats, eggs and milk leave little residue, and therefore do not favor increased peristalsis, they are not recommended and should not comprise the bulk of the diet. He also advises farinaceous food, such as cereals and starchy vegetables. Bran and bran bread are recommended as superior to ordinary white bread. Fats should be given freely, preferably in the form of cream, butter and olive oil. Bastedo also believes that fruits are valuable adjuncts to such a diet, but calls attention to the fact that they are apt to induce flatulence and are contraindicated in cases of gastric hyperacidity.

A practical diet list which is here given in detail, has been recommended by Newburgh.

\footnotetext{
Breakfast: Fruit: apples, grapes or berries.

Cereal: large helping of oatmeal, cracked wheat or cornmeal.

Eggs in any form.

Bread: Graham or whole wheat, toasted or not, as desired.

Coffee or tea.
}

Luncheon: Small helping of fish or meat, with a large helping of spinach, cauliflower, cabbage, tomatoes, green peas or beans.

Two or more slices of whole wheat or Graham bread, or oatmeal crackers. Dessert as desired.

Dinner: Unstrained vegetable soup; small helping of meat, fish or poultry.

A baked potato, baked in its jacket which may be eaten to advantage, and peas, beans, cauliflower, and spinach.

Salad of lettuce, celery or asparagus.

Bread as at luncheon.

Dessert as desired, and coffee.

Drug Treatment.--It is safe to say that for the last few years, no drug has been more widely employed in the treatment of intestinal stasis and chronic constipation, than liquid petrolatum, or Russian mineral oil. Although various forms of petrolatum have been used from time to time to overcome chronic constipation, it required the ardent enthusiasm of Sir Abuthnot Lane to popularize this form of therapy, and its present widespread use is the direct result of his energy. Since the drug has become popular, it is known by a variety of names, no less than fifty being mentioned by the Council of Pharmacy and Chemistry of the American Medical Association. Petrolatum, or Russian mineral oil, is divided into two grades: the lighter 
which is used as a vehicle, especially for oil sprays and is not suited to internal administration, and the heavier and more viscid oil, which was originally used chiefly as the base for ointment, but which is now so extensively employed in intestinal conditions. Paraffin oil suitable for internal administration should be absolutely colorless, have no taste or odor, and should also be free of acids and alkalies. Most authorities agree that for intestinal stasis, a heavy oil is absolutely essential, that is one having a specific gravity of 0.885 to 0.889 , at $15^{\circ} \mathrm{C}$. Observations tend to indicate that paraffin oil is in no sense absorbed by the intestinal tract, nor is it dissolved. It acts in an entirely mechanical way. No untoward results have ever been reported from its use; it is said to have a soothing action upon the bowel, particularly when spasmodic contraction is present. It is also alleged to retard intestinal absorption. Some enthusiasts have even gone so far as to claim that his drug inhibits the action of the putrefactive bacteria in the bowel. The question has been raised as to whether or not the long-continued use of paraffin oil may not produce cancer. It seems, however, that such an occurrence is most unlikely by reason of the fact that liquid paraffin is one of the later products in the distillation of crude petroleum, and paraffin cancer seems to be due only to the intermediate oil fractions. Moreover, liquid paraffin does not contain any auxetics, and it has been shown that it is the auxetics that are the predisposing factor in the production of pitch cancer. Furthermore, paraffin oil has now been used extensively for a number of years by a large number of people of cancer age, but in spite of this fact, no increase in intestinal cancer has been reported that could be attributed to the use of petrolatum. The drug may, therefore, be considered safe so far as the production of carcinoma of the intestinal tract is concerned. Thus far it has not been made exactly clear just which forms of chronic constipation are most successfully treated by paraffin oil. Experience indicates that all forms do not yield to this drug with equal ease. It is probably most useful in those forms of constipation associated with irritation or spasm of the intestine and those types in which the fecal mass is extremely hard and dry. The oil is usually given in two ways, in small doses, one to three teaspoonfuls half an hour before meals, or in one large dose, 0.5 to I ounce ( 5 to 30 c.c.) on retiring. The oil should not be given with or after meals, inasmuch as it is said to exercise some inhibition on the proper digestion of food. The oil is not soluble, it has to be taken raw, and although it is tasteless, it undoubtedly leaves behind. a rather disagreeable, oily sensation in the mouth. This taste may be removed by following the oil by a swallow of warm water, or even better, warm milk or a bite of dry bread.

Other medicinal agents have been employed with the idea of increasing the bulk of the feces, and in this way relieving constipation. Agar-agar was first introduced for this purpose by Adolph Schmidt, who when he found that agar alone was not satisfactory, added to 
it cascara sagrada. This combination of agar-agar and cascara has been known by the name of regulin. Agar is useful in conditions in which the feces are dry, hard and reduced in quantity. As it passes through the intestinal canal, agar takes up fluid, and thereby increases considerably its original bulk. Following the lead of Schmidt, Einhorn has succeeded in impregnating agar with a number of preparations, thus greatly increasing the efficiency of the drug in chronic intestinal stasis. Medicated agar is prepared by Einhorn as follows: The medicinal agent is dissolved in boiled agar water solution, and this is then evaporated to its original agar volume. The impregnated agar is then ground into flakes. For use in chronic constipation, Einhorn has devised a phenolphthalein agar of which each level teaspoonful represents gr. $1 / 2(0.03 \mathrm{gm}$.) of phenolphthalein, and a rhubarb agar, of which each teaspoonful represents I c.c. or 16 minims of the official fluidextract of rhubarb. The advantage of this form of medication is that the agar-containing remedy first absorbs fluid, and later through osmosis liberates the medical substance it contains. This process is slow, and at the same time covers a large area of the intestinal tract, thus greatly increasing the efficiency of the medicinal agents.

As substitutes for agar-agar in the treatment of chronic constipation, linseed and fleaseed have been highly recommended. As these mucilage-containing seeds are tasteless, they may be added to any soup or other dish, the intestines do not seem to become habituated to their use, and they never cause irritation. By reason of the mucilage which they contain, they lubricate as they pass along the bowel, aiding the action of the normal mucus present. Both linseed and fleaseed take up fluids, thus increasing in volume and adding to the bulk of the feces, at the same time that they keep the fecal mass moist.

Within a few months it has been announced that exhausted orange peel has been used with great success in the French army as a substitute for agar-agar in the mechanical treatment of chronic constipation. The peel is prepared as follows: The orange peel is boiled for half an hour in water until it becomes soft. The decoction is then decanted. The marc is again boiled for thirty minutes in a fresh quantity of slightly sweetened water. It is then drained and dried on a plate. It is held by Rosenthal, that his exhausted orange peel not only acts mechanically by increasing the bulk of the feces, but that it also stimulates the flow of bile.

There has always existed an insistent demand for a cathartic which, when administered hypodermically, would prove a successful stimulant to the intestines. This dream seemed to be realized when in I9I I Zülzer brought forth a substance which he termed "hormonal," and which he regarded as a specific peristaltic stimulant. Zülzer believed that the normal intestinal peristalsis was excited chemically by a hormone, and he discovered that the spleen contained considerable quantities of this hormone, which was absent in other organs. 
Therefore, he put upon the market an extract of the spleen under the name "hormonal." After the introduction of the drug, Zülzer along with a number of clinicians, were loud in its praise, claiming that the drug when given intravenously, stimulated normal peristalsis and was almost a specific in various forms of constipation. Zülzer declared that the use of the drug produced no bad symptoms other than at times a headache and a little rise in temperature. It was not long, however, before it began to appear that hormonal was by no means a harmless drug. Repeated instances were reported of serious collapse following its administration. It is doubtless true that the drug has some value in certain cases, but the overwhelming weight of clinical evidence indicates clearly that hormonal is a dangerous drug, capable of producing serious cardiovascular failure and even death. Aside from any effect which it may have upon the intestinal tract, it is proved that it is a decided depressor of bloodpressure, and that this depression may be accompanied by marked changes in cardiac and respiratory functions. Lately the wave of enthusiasm for hormonal seems to have largely subsided. The preparation is mentioned here only in order to warn against its employment.

Several years ago, Credé prepared a new remedy to be used subcutaneously known as sennatin. This drug was prepared from senna leaves, and was said to stimulate the peristaltic activity to a marked degree. It is claimed that no serious or untoward symptoms accompany its use. The average adult dose is 2 grains (0.I2 gm.), given preferably deeply into the gluteal muscles. It is supposed to be particularly effective in paralysis of the intestines, such as occurs in acute infections or in the course of peritonitis. Good results have been reported in eighty-three cases out of two hundred and one. It is even claimed that its proper use prevents the occurrence of intestinal adhesions. On the whole it seems that sennatin may have some advantages, and by the few who have used it, is regarded as a reliable means of combating constipation.

A now well-recognized and thoroughly satisfactory drug, which in addition to other useful properties has the power of stimulating the non-striated muscles of the intestine, is pituitrin, a preparation derived from the pituitary gland. The drug is dispensed in ampoules of I c.c. each, ready for hypodermic administration. In postoperative distention, and in the distention which accompanies acute infections, notably in pneumonia, the hypodermic use of pituitrin is indicated and has often done good, relieving the intestinal paresis and at the same time elevating the blood-pressure. 


\title{
THE SURGICAL TREATMENT OF INTESTINAL STASIS
}

\author{
By John G. Clark, M. D.
}

A careful analysis of the results of my operations performed for the correction of anomalies productive of intestinal stasis has in no way caused me to essentially modify the opinions stated in the first edition of this work. I cannot accept the extravagant assertions of Lane and his followers that the colon is the source of all evil and with its removal, marvelous changes ensue: nor can I agree with the opposite view that the subject contains no element of truth. I am convinced that in certain cases, surgery is the only means at our disposal which offers any prospect of cure. The great majority of cases included as types of intestinal stasis can be benefited or cured by appropriate medical treatment; the lesion becomes surgical only when these measures have failed and the clinical history, substantiated by thorough roentgenographic studies, demonstrates a definite mechanical obstruction in the terminal ileum or along the course of the colon.

The type of operation must, of necessity, depend upon the nature of the anomaly. The various forms of colonic suspension have, on the whole, been followed by unsatisfactory results; for this reason I have largely discarded them in favor of excision of the offending segment. An exception to this general principle is the cecum mobile. Thus far, I have limited my operative procedure to a suspension to the lateral parietal peritoneum, but I am gradually coming to the belief that the better operation would be excision and anastomosis of the ileum with the ascending colon.

Routine examination of the terminal ileum, ascending colon and sigmoid not infrequently reveals the presence of peritoneal bands which are of anatomic interest but in no way interfere with the normal function of the bowel. The occasional case presents symptoms which are directly referable to an angulation or obstruction produced by these bands, when their division is indicated.

My experience with complete excision of the colon is limited to twelve cases representing the extremes of colonic ptosis. This operation carries with it too high a mortality and the ultimate postoperative results are too unsatisfactory to justify so hazardous a procedure except in the rarest instances. The dangers of peritoneal adhesions, which have caused one death in our series four months later and have necessitated a second operation in two others, constitute a postoperative risk too real to be ignored. I am of the opinion that a less radical procedure must be employed and incline to the limitation of the colectomy to the ascending and middle of the trans- 
verse colon, with a lateral anastomosis of the ileum into the transverse colon. In this way, the omentum is preserved and there is far less traumatism to the mesentery. Any form of anastomosis between different segments of the colon or between the cecum and sigmoid flexure with the expectation of diverting the fecal current into this new channel, will almost invariably be doomed to failure.

My cases have shown the ileum will not uniformly assume the function of the colon; the backward pressure from the colon through the anastomotic opening, when it is low down, will in a definite proportion of cases cause dilatation and permanent impairment of the ileum.

In severe, obstructive constipation, I believe our cases demonstrate that colectomy may be a very efficacious operation if carefully restricted, and may be added to our surgical therapeutic list. The immediate and remote dangers make it, however, too hazardous to extend it into the wider fields so ardently advocated by Lane. It may be efficacious, but it possesses no miraculous power. 


\title{
DIGESTIVE DISORDERS OF INFANTS
}

\author{
By Maynard Ladd, M. D.
}

The general principles of feeding and of the treatment of intestinal disturbances in infants are to be found in the article on the "Dietetics of Infants" (Vol. i) . and "Gastroenteric Diseases of Infants and Children" (Vol. iii) of the first edition. Since then a large amount of work has been done on the bacteriology of the intestinal tract and the metabolism of infants. It is impossible to correlate into rules for practical feeding all of the conclusions of recent investigation. Much of the data is incomplete and so subject to change that their application to practical feeding can be made only to a limited extent. The mineral salts of the food, for instance, have been shown to be closely connected with disturbances of digestion and nutrition and investigation of their metabolism is much to the fore in modern scientific research, yet little has been learned that one can apply in a useful way. On the other hand, the part played by the ingredients of milk modifications, their relation to each other in the processes of digestion, the effects of bacterial fermentation and of infection in the production of diarrhea are much better understood and have added definiteness to the indications for feeding.

The necessary ingredients of an infant's food are fats, carbohydrates, proteins, mineral salts and water. They are present in milk in an unstable mixture easily broken down by digestive fluids and bacterial action. Deficiency or excess of digestive secretions, too much or too little of one or more of the ingredients of the food, and abnormal forms of bacterial activity disturb the processes of digestion and nutrition and result in states in which it is difficult to distinguish between cause and effect.

This interrelation of the food elements to each other and the possibility of confusing primary with secondary conditions must be keenly appreciated by the careful feeder, or erroneous conclusions may be drawn and serious errors of treatment made.

When digestion is seriously impaired as a result of bacterial activity or by overfeeding of one ingredient, there are set in motion a series of consequent changes which eventually produce a clinical picture extremely confusing to differentiate as to their relation to each other. So many factors enter into the physiology of digestion and nutrition, that it is not difficult to understand the lack of una nimity of views which prevail in regard to principles of feeding. A consideration of some of the factors concerned may contribute to a more intelligent understanding of a difficult but most important branch of therapeutics. 
The calculation of a food formula in percentages of its ingredients represents the easiest and most rational way of expressing the relation which the different ingredients bear to each other. The quantity of food taken provides the other factor necessary to determine the energy value of the food as a whole. An individual child has its own equation of heat unit requirements, (i.e., the number of calories per kilogram or pound of weight); and in his food requires a certain amount of nitrogen for the building up of tissues. The human body cannot advantageously fulfil its requirements from one ingredient alone, such as from fat, carbohydrate or protein. For the successful exercise of its functions it must have all three elements (and in addition mineral matter and water). Percentage feeding, therefore, merely reduces these factors to terms capable of being expressed intelligently and represent, so to speak, the alphabet on which is built the language we use in treating and studying the problems of infant feeding. The alphabet and language must be learned, but that is the responsibility of the man who wishes to enlarge his point of view and better fit himself to direct the feeding of those who are unfortunate enough not to have their natural food supply. Looked at from this point of view it is hard to see why the percentage idea of feeding, checked by the estimation of the caloric value of the food, should antagonize any one not too old or too lazy to learn new methods.

Cow's milk can never be modified in such a way as to make it the same chemically as human milk. The milk of each mammal is adapted to its young, and therefore when used as a substitute food for another species requires modification in several respects. The fat of cow's milk, for example, is of coarser emulsion and contains much larger proportions of volatile fatty acids than human milk, and therefore is less readily digested and more likely to produce irritation of the intestine than the fat of human milk.

The sugar is lactose in both milks, but some difference of opinion exists as to whether the lactose is chemically the same. Clinically speaking the difference if it exists is relatively unimportant.

The protein in cow's milk is largely casein, the soluble proteins being small in amount, while the reverse holds true of human milk. The casein molecules are probably different chemically and their properties of coagulation vary greatly.

The various milk ferments, nitrogenous extractives and mineral matter are radically different, but for practical feeding we cannot take advantage of what is known about them.

Many of these differences can be changed or the effects neutralized by methods of milk modification so that in a large majority of cases of feeding an efficient substitute can be provided. In rare cases there does exist in infants a true milk idiosyncrasy; but most of the cases so reported are not as represented. When a milk idiosyncrasy does exist, it is probably due to anaphylaxis to the protein, probably from too early administration of milk, or from too large a proportion of 
casein, by which sensitization to a cow's milk protein is produced. True cases of milk anaphylaxis are rarely seen by experienced feeders. The supposed intolerance generally disappears when the previous feeding is carefully analyzed and the milk properly adapted to the individual peculiarities of the child.

The caloric value of an infant's food is generally said to range from roo to $\mathrm{r} 20$ calories to the kilogram of body-weight in the first year and is about 90 in the second year. This statement based on averages varies widely according to weight development and individual peculiarities. Emaciated babies require a higher energy quotient, even as much as 160 or 180 , while the wellnourished infant sometimes thrives on 90 or less. Very active babies require food of higher energy than those who are phlegmatic. The principal value of the caloric estimation of the food is to determine whether the infant is being overfed or underfed. Fats and carbohydrates are not equally efficient or interchangeable in the processes of digestion and nutrition, and a certain amount of protein must be present to supply the nitrogen requirements of tissue growth. Hence the importance of determining the relation of the different elements of the food to each other, a relationship which can be accurately expressed only by the percentage composition of the food.

In the early days of percentage feeding too much emphasis was laid upon special types of indigestion caused by fat or sugar or protein. There was not sufficient appreciation of the fact that indigestion is of ten the result of improper proportions of the ingredients to each other. Still one cannot feed a baby scientifically without realizing the part each ingredient plays in digestion and nutrition and it may be profitable to review the essential facts which one may reasonably accept.

Fat.-The quality of cow's fat cannot be altered in milk modification, but owing to its greater difficulty of digestion, lower percentages are given in the early months. A newborn baby who takes readily 4 per cent. of breast milk fat may find 2 per cent. of cow's fat its maximum percentage. This limitation of tolerance can be overcome in normal babies by very gradual increases so that many healthy infants of six months can digest the full 4 per cent. of cow's fat. This amount should never be exceeded. If, however, the fat digestive function has been overtaxed in the early months, the infants' intolerance to cow's fat is greatly increased and may reach such proportions that even minute amounts in the food will precipitate the most serious digestive disturbances. It is often a matter of months to restore the fat digestion to its normal. A large proportion of cases of so-called difficult feeding are infants of this class.

The difference in the emulsion of the fats in cow's milk can be overcome by a process of homogenization which will be spoken of later, but this while undoubtedly efficient is a matter of special laboratory apparatus. 
The quantity of fat which may be given depends to some extent upon the proportion of sugar and proteins in the mixture. If the latter are given in high percentages, so much energy is used up in digestion and assimilation that only relatively low amounts of fats can be tolerated. If, in a case of severe fat indigestion, very high sugar and protein have from necessity been given to supply the caloric needs of the infants, their percentages should be gradually reduced as the fat is reintroduced into the mixture. This is a fundamental principle in building up an overtaxed fat digestion.

The substitution of a vegetable fat, such as olive oil, which can readily be homogenized into an emulsion finer than that of human milk, and which is relatively free from the irritating volatile fatty acids, is the most efficient means of dealing with these difficult cases of fat indigestion. By this method extremes in administration of sugar and proteins can be avoided and the proper relations of fats to the sugar and proteins be reëstablished.

There are two principal sources of danger in producing fat intolerance. The first is an overzealous effort to make a baby gain rapidly in weight in what is generally an unhealthy competition with other infants of the neighborhood. The second error is generally unconscious and arises from the lack of exact knowledge of the percentage of fat in the cream or top milk which is used in making up a mixture. Cream does not always rise by gravity according to fixed rules. An average gravity cream, that is cream down to the apparent line of separation, is generally 16 per cent. fat, but owing to the difference of temperature at which milk sets, to difference in the fat composition of the whole milk and to what is called the viscidity of milk, such a cream may vary from ro to 20 per cent. fat. If some check is not made in reference to this point it is easy to understand how the fat percentage of a mixture may vary greatly from that which has been calculated.

Symptoms of Fat Indigestion.-The earliest symptoms of fat indigestion are loss of appetite, and either diminished rate of gain, or loss of weight. This is followed by sour regurgitation and later by severe vomiting, the vomitus being strongly acid in odor and reaction from the presence of butyric and other fatty acids. The stools are increased in frequency, containing many small soft curds, accompanied by mucus and presenting an appearance similar to curdled milk. In long-continued cases the stools become grayish in color, hard and dry. The fatty acids may, in the absence of alkaline bases, such as calcium and magnesium, appear in the stools free and from their irritating action produce watery, strongly acid movements which cause marked scalding of the buttocks. When combined with alkaline bases these fatty acids form the typical soap stools. If the fat is in great excess of the infant's tolerance, the fatty acids draw from the tissues alkaline salts, and this brings about a relative acidosis with increased elimination of ammonia in the urine. If allowed to continue, acid intoxication results with the characteristic 
rapid respiration, restlessness, cherry red lips and eventual coma and death.

In less severe cases, becoming chronic, the drain on the tissues of its magnesium, sodium and calcium salts results in disturbances of metabolism which eventually tends to severe malnutrition, rachitis, and atrophy. Many of the worst cases of infantile atrophy have their origin in a primary fat indigestion which so disturbs the general processes of metabolism that the whole mechanism is upset and intolerance not only to fats but to carbohydrates and protein is developed in the later stages.

The appearance of soaps in the stool alone is not necessarily an unfavorable symptom, if associated with a keen appetite and normal rate of gain. After the first weeks of life from 14 to 25 per cent. of the normal dried stool is composed of soaps. Too much attention to the stool without regard to the general condition of the patient may lead to too radical reduction of the fat contents of the food. This is a serious mistake, for the continuous use of a fat free or low fat mixture in itself lowers the tolerance for fat, which is an essential element for normal growth and nutrition.

The ability of infants of this class to digest the high percentages of fat in breast milk suggests that the intolerance is not due so much to fat as to certain of the volatile fatty acids such as butyrin which is present in large quantities in cow's milk and to a slight extent in human milk. Theoretically one might expect similar favorable response when cow's fat is changed to a properly emulsified olive oil. My recent work on homogenized olive mixtures, showed that the symptoms of indigestion are rapidly controlled by this method and the normal rate of gain reëstablished almost as effectively as when breast milk is used.

This subject of fat intolerance cannot be properly considered alone, however, but must be studied in connection with the digestion of carbohydrates.

In a well-established case of fat indigestion radical measures should be employed at once and all fat removed from the food for a period. Slight reduction of the fat percentage generally proves a temporizing and unsuccessful policy. After a period of rest, when the symptoms of hunger become urgent and the stools are again well digested, a small amount, not exceeding 0.5 per cent. may be added to the food. If this does not cause vomiting, and loss of appetite, the fat should gradually be raised at intervals of several days until the baby begins to show a satisfactory gain in weight. The reappearance of soaps in the stools does not contraindicate this procedure, as long as the child is eager for its food and does not vomit nor have a marked increase in the frequency of its movements. It is a question of the amounts of soaps; if they are constantly increasing with the readministration of the fats there will not be a continuance of these favorable symptoms, of keen appetite and gain in weight. The reappearance of fatty acids in the stools, if in any considerable amount, 
almost always means that the intolerance has not been overcome, and it is necessary to go back to the fat-free diet and provide a longer period of rest. One or even several setbacks should not lead to the conclusion that the fat intolerance is permanent. Repeated trials, controlled by good judgment, bring almost always their reward in a successful restoration of normal fat digestion.

Carbohydrates.-Carbohydrates are never absorbed as such from the intestines but must first undergo changes by amylolytic ferments. Lactose, maltose, invertin, and diastase which are the ferments required for the reduction of milk sugar, maltose, cane sugar and starch respectively, are all present in the small intestines of infants, but the diastase is weak and inactive in the early months of life.

So far as ferments are concerned, any of the sugars should be suitable for infant feeding. As all milks contain lactose to the exclusion of the other carbohydrates, this would appear to be the sugar best suited to the infant. The best evidence is against the theory that sugar has a specific intoxicating effect. It is, however, a favorable culture medium for bacteria which by their activity can develop products which are harmful to the body. This is a most important point to remember in connection with disturbances of digestion in infants.

Lactose and cane sugar are absorbed slowly, maltose more rapidly, and dextrose still more so. Lactose and maltose increase the retention of nitrogen because the intestinal bacteria will utilize sugar in preference to protein, and thus form less nitrogen to be excreted in the stools. Neither of these sugars influence the retention of mineral matter nor the absorption of fats.

In abnormal conditions the bacteria play an important part in breaking down the carbohydrates, forming acids (lactic, acetic, butyric) which like the fatty acids formed in fat indigestion are prolific sources of intestinal disturbances. Their irritant action causes increased peristalsis, hastening the excretion of the food and consequently causing diminished absorption, and disturbances of metabolism. The same is true of starches, but as their molecule is more complex their conversion is a longer process.

Lactose.-The arguments in favor of the use of milk sugar in infant feeding may be briefly stated: First, it is the form of sugar contained in breast milk. Second, being more slowly absorbed than other sugars, it is more likely to favor the development of the normal fermentative bacteria in the intestines and is least susceptible to butyric and alcoholic fermentation. On the other hand, its energy value is less than that of malt sugar, and the gain in weight is less than when malt sugar is given. When given in excess it is more likely to produce diarrhea owing to its slow absorbability and greater fermentability. Also many brands of commercial milk sugar are adulterated. Milk sugar has no specific pyrogenic action, but when given with sodium salts in diseased conditions of the intestines may produce fever. In acute disturbances of digestion, therefore, it is usually less well borne than dextrin-maltose. 
Milk sugar should never be given in greater percentages than seven, and even this amount may cause trouble to susceptible infants.

The causes of digestive disturbances arising from milk sugar are due partly to faulty digestion and partly to bacterial fermentation of the lactose. It is not profitable or possible to differentiate between these two factors. Whichever the casc, the characteristic symptoms are watery, greenish, acid stools which cause marked irritation of the buttocks and genitals. The odor of the stool is of acetic, lactic or succinic acid. The same odor may be detected in the vomitus, but vomiting is a much less frequent symptom in sugar than in fat indigestion.

In severe acute cases there is sharp loss in weight, high fever of temporary duration and severe symptoms of nervous disturbance, with extreme prostration and sighing and irregular respiration. Rarely, the symptoms are fulminating and death occurs.

There are chronic cases without the fever and severe toxic symptoms. Those make up a fair number of the cases of chronic intestinal indigestion with malnutrition which may be seen in any feeding clinic.

The treatment of diarrheas due to excess of, or to abnormal fermentation of milk sugar depends principally upon the elimination or radical reduction of the milk sugar, after preliminary catharsis and water diet for twelve or twenty-four hours. It is necessary to remember that in all cases of sugar indigestion fats are not well tolerated so that the fats also must be temporarily reduced to about one-half of the amount previously taken. Skim milk and whey contain even more sugar than whole milk so that if used considerable dilution is required to bring the milk sugar down to the necessary amount. It is in these acid fermentative diarrheas that Eiweiss milch or precipitated casein finds its greatest usefulness, owing in part to the lower sugar content and in part to the fact that much higher casein percentages may be given because of its subdivided condition. A food rich in protein tends toward the production of an alkaline stool and is therefore antagonistic to acid fermentation of the sugars.

When the acute symptoms have subsided, sugars should be added to the food in gradually increasing amounts, but it is advisable to utilize another kind such as maltose or dextrin-maltose, limiting the amount of milk sugar to that contributed by the cream and milk in the formula prescribed.

In rare cases of the chronic type, prolonged reduction of the lactose being indicated, the food must consist mostly of fat and proteins in higher percentages than usual in order to supply the caloric needs. The best method of dealing with such cases is to utilize a higher percentage cream to get whatever percentage of fat is required and to use a precipitated casein for the high protein percentages. In this way the lactose can be kept down to the minimum. Homogenized olive oil mixtures with precipitated casein are also very useful in VoL. IV. -43 
such cases, as the irritating volatile fatty acids of the cream are in this way also eliminated. As these mixtures are not conveniently obtained outside of a milk laboratory it may be more practicable to provide a wet nurse, for breast milk in spite of the high lactose percentage is generally well borne, a fact difficult to explain except on the ground that there is some subtle difference between commercial lactose and that excreted in human milk, a point which lacks positive chemical proof.

In the great majority of cases, fortunately, the temporary withdrawal of the lactose and the subsequent use of dextrin-maltose preparations, with properly adjusted fat percentages results in the reëstablishment of normal digestion and weight development.

Maltose.-Malt sugar, as used in infant feeding is never a pure maltose, but is a mixture of dextrin and maltose, the proportions varying according to the preparation. The larger the proportion of dextrin, the less rapid its absorption. Both maltose and dextrin are absorbed as dextrose, the same as lactose, but being lower sugars they are more quickly reduced and absorbed and are of greater energy value as weight producers. The assimilation limit of maltose is twice as great as that of lactose and the sugar is more directly utilized. The dextrins are reduced to maltose before being converted to dextrose. The larger percentage of maltose in the preparation the greater the laxative action, while the dextrins produce the opposite effect, a fact which may profitably be taken advantage of in determining the best preparation to use in babies with marked tendency to constipation or diarrhea. It is possible that the laxative action of dextrin-maltose depends upon the different salt contents and upon the presence of a substance called phytin. If made from potato starch there is a small amount of mineral salts and phytin; if from barley the percentages of both salts and phytin are much higher. One of the commerical preparations which is distinctly costive in action is said to be derived from potatoes. It also has a high dextrin and low maltose content as compared to other malt preparations, the majority of which are distinctly laxative in their effect. The efficiency of a malt sugar mixture is increased usually by the addition of cereal decoctions, like barley water, probably because they increase the colloidal action of the mixture on the proteins.

Dextrin-maltose does not undergo lactic acid fermentation but is more susceptible to butyric acid fermentation than lactose. It is absorbed wholly as dextrose; whereas milk sugar is converted into dextrose and galactose and the galactose requires further energy to be converted into glycogen before it is utilizable. There is, therefore, definite physiological reason for preferring dextrin-maltose preparations in difficult cases of feeding with malnutrition, and in my experience they have proved of greater practical usefulness. In normal healthy infants, however, lactose serves its purpose sufficiently well. 
Excessive proportions of malt sugar will cause a diarrhea, in appearance similar to that caused by excessive amounts of lactose, except for the color of the stool, but the acute disturbance is less severe and more amenable to treatment.

Cane Sugar.-Cane sugar, like lactose is split into half dextrose and half lactose, the latter not being utilized until further changed. It has therefore no advantage over lactose, and is less useful than the dextrin-maltose preparations which are reduced wholly to dextrose. It has the disadvantage of undergoing alcoholic fermentation instead of lactic acid fermentation and is, therefore, more likely to produce gas and symptoms of colic. A further disadvantage of cane sugar is its excessive sweetness, so that when given in as large amounts as lactose or dextrin-maltose, it is likely to impair the appetite.

Starch.-The amylolytic ferments are present in the saliva and pancreatic secretions at birth, but not in sufficient amounts to be utilized for the digestion of starch until the second half year, and generally not until the ninth month. This function varies with different infants, however, and as starch decoctions, such as barley water, have a distinctly colloidal action on proteins, their use at any age may clinically appear to be favorable by making the casein curds flocculent and, therefore, more easily digested. Cereal diluents may therefore be useful at all periods; in the early months because of their mechanical action, in the later months both for their colloidal action and food value, and in preparation for the digestion of starchy foods of the second-year diets. Clinical experience had led to the custom of fixing the maximum percentage of starch in an infant food in the first year at 0.75 per cent. Lower percentages than these are inefficient for effective colloidal action on the protein, and higher percentages are rarely utilizable. The fat and protein in cereal diluents are practically without influence upon nutrition owing to the extent to which they are reduced in the dilution. Barley flour is the starch used by preference in the first year. It is more constipating than the sugars owing to its complex molecule and slow disintegration into sugars, as a result of which fermentation goes on more slowly and there is less fermentation of irritating end products.

The best results in feeding are often to be obtained by combining the advantages of several carbohydrates. Sufficient lactose is generally present in the cream and milk of a formula to supply food for the development of the normal fermentation bacteria, additional sugars being obtained by dextrin and maltose while cereal decoctions are added chiefly for their colloidal action on the protein.

Protein.-An infant requires protein to replace nitrogenous waste and furnish material for new cell formation, a requirement which fats and carbohydrates cannot meet. Anything above this nitrogen requirement is disadvantageously utilized for heat production which is essentially the function of the fats and carbohydrates. The protein need of infants varies from r.5 to $2 \mathrm{gm}$. per kilogram ( 0.7 to $0.9 \mathrm{gm}$. per pound) of body-weight. Expressed in other terms 
Rubner has calculated that an infant should have 7 per cent. of the calories in the form of protein. The average modified milk mixture greatly exceeds this amount. The increase of protein intake at first results in increased nitrogen retention, which is followed by increased excretion.

The amount of fat in the food influences nitrogen retention little if at all, but carbohydrates increase nitrogen retention, unless given in amounts sufficient to produce diarrhea in which case there is considerable loss of nitrogen. Increase of the protein in the food generally increases nitrogen retention, but it does not result. in gain in weight. There is, therefore, no logical reason for very high proteins in an infant's food, except in cases in which fats and sugars cannot be given in sufficient amounts to supply the caloric needs, or in order to make a stool temporarily alkaline to counteract acid fermentation. The continued use of very high proteins makes more difficult the giving of fats and carbohydrates in proper proportions. While it is essential that the minimum protein requirements should be provided, it is an error in feeding to continue very high proteins for too long a time and at the expense of the other ingredients of the milk. When, therefore, a disturbance of digestion has been corrected by the use of a high protein milk, such as Eiweiss milch, it is good policy to reduce the protein to $\mathrm{I} .5$ to $2 \mathrm{gm}$. per kilogram of body-weight when the time comes to reintroduce fats and to increase carbohydrates in the diet.

There is a tendency at present to minimize the power of protein to cause disturbances of digestion, for the effects of such overfeeding are less easy to recognize than in cases of fat and carbohydrate indigestion. It has been shown that very high protein long continued may cause fever and irritation of the kidneys and there is reason to believe that the equilibrium of metabolism may be disturbed in other ways and result in loss of fats and carbohydrates from the body. While it is probable that the baby artificially fed on cow's milk wastes more nitrogen or can utilize a smaller proportion of the protein constituent of the food, and so requires a higher minimum for'normal nitrogen retention, it is safe to follow the indication set down by nature in the composition of breast milk, and not to exceed in the first half year a protein percentage of $\mathbf{I . 5 0}$, or in the last six months of 2.00 or at the most 2.50 per cent. In many cases of disturbed digestion, much lower percentages can be given with advantage for a limited time.

The digestibility of protein is greatly influenced by the form in which it is administered. The casein of cow's milk being greatly in excess of the soluble protein, is naturally coagulated in large indigestible masses in the stomach, with the result that the contents are not readily expelled and digestion and assimilation are slowed. This may favor fermentation and putrefaction and cause various types of indigestion. On the other hand, if the casein is protected by the colloidal action of cereal diluents, or if it is rendered incapable of coagulation 
by previous application of heat (boiling) or if it has been precipitated and pulverized and reincorporated in the milk as in protein or Eiweiss milch, or if by the action of lactic acid cultures it has been reduced to the lactate of casein, the digestibility of the protein will be greatly enhanced and correspondingly favorable results obtained in finding.

Another method of rendering the casein curd more flocculent and at the same time retarding its precipitation in the stomach, thus allowing more rapid emptying of the contents into the duodenum, is by the use of alkalies. Lime water, bicarbonate of soda and citrate of soda and milk of magnesia are generally used for this purpose. Lime water causes the mucoid protein to swell, making the curd more flocculent. Ten to I5 per cent. of the total mixture is generally required to be effective. Bicarbonate of soda acts by carbonic acid gas formation, rendering the casein curd more porous. Sodium citrate acts by forming a sodium, instead of the usual calcium caseinate, which is split into the soluble sodium paracaseinate instead of the usual insoluble calcium paracaseinate.

Another possible effect of alkalies is to supply in pancreatic digestion bases to unite with fatty acids formed by the splitting of fats and the fermentation of carbohydrates. The calcium and magnesium bases unite with the fatty acids to form insoluble soaps, while the sodium salts form soluble soaps. Alkalies also serve to neutralize the acidity of milk. With such possibilities of action it is difficult to say how much, if any, may be added to milk mixtures with advantage. Clinically it is customary to add $\mathrm{I}$ or 2 grains of sodium bicarbonate or sodium citrate to each ounce of milk and cream.

The character of the casein may also be altered by partial peptonization or pancreatization of the milk previous to digestion, but this method is less used than formerly.

Protein Milk.-This is a mixture first suggested by Finkelstein and Meyer under the name of Eiweiss milch, it is also described as "casein" and "albumin" milk. Its method of preparation is as follows:

Four teaspoonfuls of essence of pepsin are added to a quart of milk heated to $100^{\circ} \mathrm{F}$. The whey from the resulting curd is strained off through a linen cloth and the curd washed, and then forced through a fine sieve to divide it minutely. To the pulverized curd is added a pint of water and a pint of buttermilk. The composition of the food so prepared is

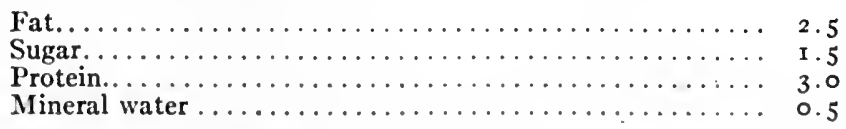

The analysis will vary considerably, however, according to its method of preparation. If a small amount is made considerable fat is caught in the linen mesh, and the percentage runs lower than that given. If large amounts are made at one time, using the same strainer, higher percentages of fat are obtained. There is also con- 
siderable variation, depending upon the fat percentage in the milk, the temperature at which the milk is heated, the length of time the curd is forming, and the extent to which it is washed.

There are several points in such a mixture to which favorable results may be attributed, the low fat, the very greatly reduced sugar, and fine division of the casein curd and the presence of living lactic acid organisms. The salts are somewhat higher than are obtained in the usual modified milk mixture. A quart of this mixture has a caloric value of $37^{\circ}$ calories. Its efficiency is unquestioned in fermentative diarrheas due to sugar indigestion, owing to the low. sugar, the presence of lactic acid bacilli and the finely divided casein. Its relatively high fat content, however, militates against its usefulness in case of fat indigestion and its high protein in cases with putrefaction. As a food for continued use, or in normal cases of feeding, its low carbohydrate content must be made up by addition of some sugar, preferably maltose. The preparation is difficult to make outside of hospitals or milk laboratories and it is highly doubtful if it offers any advantage over methods previously in use in American milk laboratories. Kendall has shown that fermentation always precedes putrefaction. Proteins are absorbed as amino-acids, which are chemically but one step removed from the toxins which result from putrefactive processes. In the presence, therefore, of putrefactive bacteria either in the milk or in the intestines, excessive amounts of amino-acids form favorable conditions for the development of such organisms and may result in serious conditions of toxemia. In diarrheas with stools of foul odor and strongly alkaline there may be considerable danger in the continued use of high protein mixtures.

By the use of fat-free lactic acid milk, very concentrated creams and precipitated casein we may in American milk laboratories prescribe formulæ which combine all the advantages claimed for Eiweiss milch, which are much more susceptible of exact variation and adaptation to individual needs. The fats, for instance, may be eliminated or increased at will, the protein (in the form of lactate of casein or precipitated casein) may be raised or lowered. The strain of lactic acid bacilli may be selected. Carbohydrates in any amount and variety may be prescribed according to the indications. In other words instead of prescribing a food of a fixed composition to all needs and conditions of digestion, we may utilize the favorable features of Eiweiss milch and still follow the principles of percentage feeding.

Protein Indigestion.-Protein disturbance in babies who are artificially fed, arise with few exceptions, from indigestion of the casein. The indigestion which is sometimes met with in babies fed upon high whey-protein percentages is to be referred to the sugar or possibly the salt content of the whey and not to the whey-protein itself. Casein indigestion is characterized by vomiting of large curds with much flatulence and colic. The stools are increased in frequency 
and partly loose and watery, of foul odor, alkaline and contain mucus and large, tough curds. Coincidently, if the diarrhea is allowed to continue, there are set up fermentation processes of the other elements of the milk, and fever of temporary duration results. If the amount of protein at the outset is radically reduced, or if the casein is made more digestible by one of the methods previously described, the disturbance is easily regulated. In the more serious cases, the additional use of lactic acid cultures tends to inhibit the growth of the putrefactive bacteria and is probably the most effective way of controlling a dangerous solution.

In breast-fed infants protein indigestion is the most common of all forms of indigestion and is less easily controlled, because of the diffculty of altering the composition of the breast milk. Very high protein elimination occasionally results from fatigue or nervous conditions affecting the mother and the reaction on the child is often serious. Temporary withdrawal of the breast milk is then the safest treatment, while exercise and methods désigned to remove or alleviate the cause of worry or excitement of the mother, are carried out. The symptoms are much the same as in protein indigestion from cow's milk, but the nervous disturbances in the child are more severe. Convulsions and fever may occur. The analysis of the milk in such cases, while usually showing a high protein, sometimes as much as 3 or 3.50 per cent., does not always follow the rule. The disturbances may possibly be due to elimination of other toxic material about which little is known.

The use of whey-protein mixtures is especially valuable in cases of protein indigestion, either as a temporary substitute food, while the breast milk is being regulated or, when the infant is artifically fed, as a means of supplying low casein percentages without reducing the total protein to a point below the minimum protein requirement. If, however, the protein indigestion is associated with fermentation, the high sugar content of whey may render it unsuitable and better results are obtained from precipitated casein mixtures. The high amino-acid content of the lactalbumin of whey (lysin, cystin) compared with casein, substances which Mendell and Osborne have shown to be essential for normal growth, probably explains the efficiency of the whey-protein in mixtures in the early months when high casein percentages are not well tolerated.

Indigestion with Fermentation.-In ordinary indigestion of the food elements which have been described, it is always a question as to whether the symptoms are due wholly to an absolute or relative insufficiency of the digestive fluids, or to this factor combined with abnormal fermentations. Theoretically, simple indigestion should apply to conditions in which the fermentation processes play an unimportant part in the disturbance, but as a simple indigestion may be the first step leading to abnormal fermentation, and as in severe cases, fermentation is always the predominating factor in producing symptoms it is not practical to differentiate simple in- 
digestion from indigestion with fermentation except in cases that are very mild and of short duration.

It is, however, of very practical importance to differentiate indigestion with fermentation from infectious diarrhea. All the severe forms of diarrhea can be put in one of these two divisions, bearing in mind that an infectious diarrhea, meaning a definite infection of the intestinal mucosa with various pathological lesions, may coexist with a state of indigestion with fermentation of the intestinal contents.

Indigestion with fermentation, therefore, deserves consideration in more detail than has been given it in connection with simple indigestion of the food elements. Its etiology is directly connected with abnormal bacterial activity in the intestinal tract, either directly upon the food constituents or, secondarily, upon the products of their incomplete digestion. The bacteria may be introduced into the tract along with the food, developing increased activity under the favorable conditions existing therein, or they may be normal constituents of the intestinal tract which under the influence of an unbalanced food find the conditions favorable for development from saprophytic to pathological agents. It is easily understood, therefore, how the two factors, an unbalanced or unsuitable food, together with an infected milk produce the etiological factors first of indigestion, then of fermentation and finally if not checked of actual invasion of the mucosa and submucosa with the production of pathological lesions and the final clinical factors of infection.

The causes concerned with the production of simple indigestion have already been considered, as well as some of those which have to do with fermentation. In the normal digestion of fats, the neutral fat is split into glycerol and fatty acids by the pancreatic lipase (steapsin). A portion of the fatty acids so formed are dissolved by the bile and absorbed as fatty acids, the rest unite with alkalies of the pancreatic fluid and of the food to form soluble soaps which are emulsified by the bile and so absorbed. If the acid formation is excessive the bile may be decomposed and fail to dissolve the fatty acids preparatory to absorption and lose its properties of emulsifying the soapsThe excessive fatty acid formation may arise from excess of fat in the food or from the bacterial fermentation of the carbohydrates. The interrelation of these two food constituents is thus apparent. The presence of excessive volatile fatty acids from whatever source causes increased peristalsis from their irritant action which lessens the rate and degree of absorption and causes a marked increase in bulk of excreted stools. The waste in neutral fats, fatty acids, soaps and ash is markedly increased and with the lessened power of absorption there is a greatly diminished power to split fats.

Nature tends to check this riotous process by an outpouring of alkalies from the tissues and by the utilization of the alkaline bases in the food to neutralize the fatty acids by soap formation. The drain of calcium and magnesia from the tissues and the breaking down of the protein results in excessive liberation of nitrogen in the 
urine and a relative acidosis develops which often determines the clinical manifestations of the condition.

Another chemical process antagonistic to acid production is the breaking down of the protein constituents of the food with the formation of alkaline end products. If these are utilized to neutralize excessive acid production there are less protein products available for absorption and consequent disturbance of metabolism and nutrition. Normal digestion and absorption, therefore, can proceed only when all these digestive and fermentative processes are in a state of equilibrium. A disturbance in any one of them, whether originating in indigestion from an unbalanced food, or from bacteria introduced from without or from hyperactivity of the normal intestinal flora, results in a condition of digestive chaos, with the clinical picture of diarrhea.

The bacteria chiefly concerned in the production of fermentation are the $\mathrm{B}$. perfringens, the butyric acid bacilli, the B. acidophilus, and the B. putreficus. It is also probable that the normal intestinal flora, such as the colon bacillus and the normal lactic acid bacilli may as a result of disturbance set in motion by indigestion act as fermentative bacteria. In any event the etiological factors are mixed and probably numerous and the bacteriological examination of the stools yields little conclusive evidence as the cause.

The pathological changes in the intestines in fermentative diarrhea as opposed to infectious diarrhea are slight, showing little beyond hyperemia of the mucosa and mild catarrhal inflammation.

Symptoms of Fermentative Diarrhea.-The onset is acute with high fever ( $103^{\circ} \mathrm{F}$. to $105^{\circ} \mathrm{F}$.) with rapid pulse and respirations. Vomiting may or may not occur. The abdomen is moderately distended and the toxic symptoms usually severe. Diarrhea rapidly sets in, the stools being frequent, watery, fatty, often green in color, and sour and acid or foul and alkaline according to whether the fermentation is of the carbohydrates and fats, or proteolytic. There is much mucus and irritation of the buttocks when acid fermentation is present. Blood is not present and the fever, while apt to be high, is not maintained, but drops as the intestines clear themselves of the fermentated contents. Continued use of food favorable to the continuance of the fermentative processes results in the renewal of the fever, and in such cases the toxemia may assume such proportions as to cause death. Rapid loss in weight is the rule. The urine is diminished and moderate, leukocy tosis ( $I_{5}, 000$ to 20,000 ) is present. If not promptly and intelligently handled such diarrhea overwhelm the system with the toxic products of fermentation or may lower the resistance of the tissues and pave the way for direct infection of the intestinal mucosa.

The differential diagnosis from infectious diarrhea, while not always to be definitely determined, is made by the relatively rapid subsidence of the toxic symptom after free catharsis, the temporary elevation of the fever, which lasts rarely for more than two or three 
days, the absence of blood in the discharges and the characteristic fermental stools. The prognosis is never to be regarded lightly while the toxemia is severe and the temperature remains high. Cases in which the stools are watery, dark brown and of musty odor are usually more serious than those with definitely sour or foul odor. The symptoms may become fulminating with excessive vomiting, hyperpyrexia or meningismus and death rapidly ensue; if the child survives the first three or four days, recovery usually takes place, but if the feeding is not properly directed the diarrhea becomes chronic and malnutrition with intolerance for all the food ingredients results.

Treatment of Fermental Diarrhea.-At the very onset of the diarrhea free catharsis with castor oil or calomel, followed by irrigation of the bowel with saline solutions two or three times the first day and then once or rarely twice a day subsequently should be instituted. While this clearing out process is being carried out food should be withheld for a period of twelve or twenty-four hours or until the temperature breaks, and water given freely, in amounts equal to that of the previous feeding. Prolonged starvation is not desirable, especially if the fermentation is proteolytic in type, for the causative bacteria will turn to the protein constituent of the tissues for food, and cause a secondary and more serious disturbance. The object to be attained is the introduction of food which will inhibit the growth of the causative bacteria by organisms of antagonistic nature. In the majority of cases this can be accomplished by the use of pure cultures of lactic acid bacilli, of which the B. bulgaricus is the most efficient. These may be given in broth cultures or in a ripened fatfree milk sufficiently diluted not to overtax the digestion with high proteins. Eweiss milch serves the same purpose. After the acute symptoms have subsided a gradual return to the previous percentage formula is attempted, bearing in mind that the fats and sugars are not well tolerated. The modified milk should continue to be ripened with the B. bulgaricus cultures for some time, and these naturally should never be killed by pasteurization or boiling. The dried tablets of the B. bulgaricus are less reliable than the broth or milk cultures.

If a fermentative diarrhea does not yield to such treatment the inference is that the cause of the fermentation is a lactic acid producing organism, such as in the B. acidophilus. In such cases lactic acid milks of any variety are contraindicated, and the most rational treatment is to attempt to alter the reaction of the intestinal contents by the use of precipitated casein. When the stools have become alkaline, less fermentable carbohydrates than milk sugar should be used, such as dextrin-maltose, and preferably a preparation that is relatively high in dextrin. The rapidity with which fats may be added depends largely upon the amount of butyric acid fermentation which is present. Olive oil homogenized in the milk mixture are much better tolerated than cow's fat, owing to the almost complete absence of the volatile fatty acids.

When the fermentation is proteolytic and the stools alkaline, 
the theory of treatment is exactly opposite from that described. The lactic acid mixtures are still indicated, but the protein should be reduced to the minimum and high carbohydrates given. In either case the fats are best entirely excluded at first and then given in small and gradually increasing percentages only when the child is convalescent. Beef juice, broths and albumin are best never given in any of the fermentative diarrheas, as they are foreign proteins and in the impaired condition of the intestinal walls, may pass through and sensitize the infant. It is perfectly practicable to obtain an appropriate type of food to meet the type of fermentation by manipulation of the natural ingredients of milk, following the general principles of milk modifications.

Infectious Diarrhea.-Infectious diarrhea, in contradistinction to diarrhea with fermentation, assumes an invasion of the intestinal mucosa and submucosa by bacteria with the production of pathological lesions. These vary according to the infectious agents and their virulence. There may be only catarrhal inflammation with superficial ulcerations or hyperplasia of the solitary follicles and Peyers patches, often with deep ulcerations, and with pseudomembranous exudates involving large areas of the intestines. The severity of the symptoms are generally but not always in proportion to the severity of the ileocolitis. Hyperplasia of the mesenteric lymph nodes, rarely going on to suppuration with degenerative changes in the parenchyma of the liver, spleen and kidneys occur as secondary lesions.

Infectious diarrhea should be looked upon as an acute infectious disease, not unlike typhoid fever in its origin, but differing in its onset and symptomatology according to the agent of the infection. The bacteria are present within the tissues, producing their toxins from their growth on the tissue cells themselves. The toxemia resulting is primarily caused by such action and only secondarily by the products of the fermentation of the intestinal contents. The onset of infectious diarrhea is usually acute, with high fever and considerable severe toxemia. The stools at once become frequent, loose, with much mucus and blood and usually pus after a few days. They may or may not show signs of fermentation and are usually alkaline. The characteristic of the fever is its persistence; it is high or low depending on the virulence of the infecting bacteria. When ulceration is present, blood is also persistent. Pain and tenseness is prominent, and the abdomen, often distended at first, is soon sunken and only slightly tender. Vomiting may or may not be present.

The bacteria which are the cause of the infectious diarrhea may be divided into three classes.

I. The various types of the dysentery bacillus (chiefly the Shiga and Flexner bacilli).

2. The B. Welchii (or gas bacillus).

3. Other organisms such as the streptococcus, colon bacillus and $\mathrm{B}$. pyocyaneus. 
The type of infection can only be determined by the examination of the stools and not by the symptom or cause of the disease. The exact bacteriological differentiation is not practical outside of a well-equipped laboratory, but it is comparatively easy to determine whether the gas bacillus is present in considerable numbers, a point of considerable importance in the treatment. The dysentery bacillus thrives best on proteins, the gas bacillus on carbohydrates. It is, therefore, important in the dysentery types of diarrhea to cut down protein food to the minimum and to give relatively high carbohydrates, while in gas bacillus infections carbohydrates should be excluded so far as possible and moderately high proteins given. Fats are best entirely excluded during the active stages of the disease.

The clinical test for the gas bacillus is as follows: A portion of the stool is added to a test-tube of milk or sugar solution and gently boiled for a few minutes to kill all bacteria except those in a spore state. The contents of the tube are then added to a sterile fermentation tube and incubated for twenty-four hours. The displacement of a considerable amount of the fluid ( $\mathrm{I}$ inch or more) indicates the presence of the gas bacillus in sufficient numbers to warrant the reduction of carbohydrates to the minimum in the subsequent feeding. The test naturally gives no evidence as to the presence or absence of the dysentery bacilli or the other possible organisms. If the gas test is negative, carbohydrates may be given with reasonable safety. Whatever the result of the bacteriological test, there is little reason to give more than the minimum protein requirement. If the stools are alkaline, the $\mathrm{B}$. bulgaricus cultures, or diluted lactic acid milk with low proteins and moderate carbohydrates, preferably dextrin-maltose, make a reasonably safe diet after the initial period of starvation, catharsis and irrigation.

While these indications for feeding have proved useful, it is not meant that there is any specific food to control an infectious diarrhea. Fermentation of the intestinal contents can be discouraged, food of a kind least adapted to the growth of the infecting agent can be given, but as the infection is within the walls of the intestines, it must run its course very much as in typhoid fever. While the disease is active, the bacteria cannot be starved out or killed by antiseptics, nor will the stools become normal and free from mucus, pus and blood, until natural reparative processes have been established. As this is a matter of time, it is necessary to provide food of some sort and the question is limited to what kind, in view of the nature of the infections, is most likely to keep down fermentation and provide the least favorable media for growth of the active agents of the infection. Having determined these factors, the management of the case is that of any acute infectious disease, with toxemia and deficient elimination. These are matters of general treatment into which it is not necessary to go (see Vol. I). 


\title{
DISEASES OF LIVER AND GALL-BLADDER
}

\author{
By Charles G. Stockton, M.D.
}

Cholecystitis. - The method most advisable for dealing with an infected gall-bladder is a matter not yet sufficiently developed to admit of a general answer. It remains a question of "many men of many minds." Therefore, a judicial attitude is advised; it depends upon the case. During the past few years it has been the conclusion of a large number who consider themselves as advanced that, given an infected gall-bladder or choleduct, it should be immediately operated. An increasing number hold that the operation for infective cholecystitis should be exsection. This is due to the recognition that many cases subsequent to drainage operations continue to suffer.

We have not as many cases of cholecystectomy as cholecystostomy on which to base conclusions. Undoubtedly there are now more unfortunate results following simple drainage than following resection. How this will be ten years hence remains to be seen.

Unfortunately, surgical results like medical results do not admit of numerical averages. Results depend upon the surgical judgment and technique on the one hand and upon the character of the medical treatment on the other. Bad surgical results may follow unskilled handling of tissues, continued infection or scar contraction that fixes in unnatural position the liver, the stomach, the duodenum or other parts of the intestine. Thus is caused disturbance in the function of the ducts of the liver and of the stomach and intestine. There is more rather than less pain, and more disturbance with digestion. On the other hand, with an attempt to treat an unsuitable case by medical means only, the patient's condition of ten becomes deplorable. He suffers from adhesions and deformities similar to those of unsatisfactory surgery. In addition, through chronic infection and intoxication, he becomes a victim of myocarditis, endarteritis, nephritis, and other lesions too numerous to mention.

As it stands, too many, and at the same time too few cases have surgical treatment. Cases for operation should be thoroughly selected. Early diagnosis, followed by a thoroughgoing plan of treatment, may make medical treatment successful. Too commonly, the patient is treated only until the painful symptoms subside, when he goes his own course, with but casual assistance. until a recrudescence occurs. It should not be assumed that every case of cholecystitis is at once surgical. It is absurd to state, as some do, that cholecystitis does not recover without operation. Clinical and autopsy experience constantly prove to the contrary. It must be admitted that these recoveries are, for the most part, spontaneous. 
That is, the treatment received has been too temporary and sporadic to have been the probable cause of recovery. It is the character of the infection and the resistance of the individual that are the decisive factors.

While the nature of the infection may be beyond medical control, the same is not true of the resistance. Resistance to infection may be raised by the exercise of proper control in an individual. It is important to make a diagnosis before the immunity is seriously lowered. It is not enough merely to relieve symptoms, not enough merely to use measures supposed to act directly upon the liver and biliary tract and digestive apparatus. All these measures are well enough when advisedly carried out, but if one hopes to avoid recurrences and surgical intervention, he should study how best to raise the resisting power of the patient against the infection at work.

Often this is a question of guarding against fatigue, especially against fatigue which involves the back and abdomen, although it may be fatigue in a general sense, even mental overtaxing. At times it is a question of correcting the metabolism. A dietary including proteins in excess, or, for that matter, excess in other proximate principles may lead to disturbance in metabolism by means of which the special infection is favored and resistance depressed. For instance, infection by the colon bacillus is favored by excessive fatigue and by acidosis, by the state described by the term "biliousness," "goutiness," "lithemia." As cholecystitis frequently depends on infection from the colon bacillus, it will be seen how we possess a means of preventing recurrences of exacerbations and how we may assist nature in the case of the disease. It may be safely assumed that we shall never possess a specific for curing such infection of the biliary apparatus. The wisest course is to prevent infection by looking to secure natural drainage and immunity. When, however, infection takes place we should never omit to give chief attention to the application of these same principles in helping the patient to overcome the infection.

Reference has been made to "natural drainage." By this is meant, the results that follow the right posture of the individual, reasonable use of skeletal muscles, the relief of adiposis and emaciation, and finally, abandoning the type of garment that hinders the proper position and motion of the abdominal viscera.

It is by the exercise of principles of treatment such as are here discussed that many patients may be helped to recover from infection, whether of the gall-bladder or of other parts, without entering the as yet debated field of surgical relief.

\section{LOWERED FUNCTIONAL POWER OF THE LIVER}

The functional power of the liver is known to suffer impairment as the result of various structural diseases of that organ.

Likewise, in cardiovascular and renal disease hepatic function is 
lowered, partly because of the depressing effect of the accompanying intoxication and partly as the result of retardation of the blood mass. In these cases the liver structure is usually more or less faulty, so that it is impossible to distinguish to what extent the weakened function of the liver is due to primary hepatic degeneration.

Practically considered, the evidence of an incompetent liver calls for a reduction of the excessive physiological strain from which it suffers. We may judge somewhat the extent to which the liver is overtaxed by studying the excretions, by determining the retention in the blood of waste matter, and by observing the results of retarded movement of the blood mass upon the volume and the tension of the liver. Naturally, the failure of hepatic function, in cardiovascular and renal disease, will be somewhat in ratio with the structural changes of the liver. The view is not based upon general impression. Autopsy findings considered in connection with the course of the disease confirm the view.

This problem of interrelated structural and functional involvement to the liver confronts us not only in cardiovascular and renal disease, but also in the severe infections and intoxications, and in grave systemic exhaustion or shock. Today attention is keenly directed toward the study of metabolism and its modifications by disease.

In this regard must be considered a class of cases which shows high blood-pressure, or which have done so in the earlier stages, and in which the liver is found large and, perhaps, tense and tender. The urinary findings may show little of the true nature of the disturbance. It is exactly in these cases that treatment may be greatly confused and mistaken if complete and frequent analyses are not made. It is in these cases that such questions as those of the amount of carbohydrate, protein and fat intake must be considered. The intake of fluid and its excretion must be studied, and the excretion of sodium chlorid must be considered in its relation to edema or ascites. Particularly must be considered the question of the administration of alkalis or of antirheumatic or antilithemic drugs.

The blood should be examined as to hydrogen ion concentration, and as to the degree of retention of glucose, urea, total nitrogen, of uric acid and other acids. The carbon dioxid tension of the alveolar air is easily determined and its measurement may give indirect conclusions concerning the presence of acids in the blood. The earliest indication of approaching acidosis is perhaps given by the increase of "alkali tolerance," as shown by the administration of sodium bicarbonate with the view to determine the quantity necessary to make the urine alkaline. Peabody ${ }^{1}$ states that his test apparently tells when even a small inroad has been made on the reserve supply of bases in the tissues. He believes that it may be considered as a guide to acidosis in the tissues themselves.

${ }^{1}$ Peabody, F. W., Clinical Studies on the Respiration, Arch. Int. Med., 1915, xlvi, 954. 
In some cases, with marked retention in the blood of urea and total nitrogen, with high hydrogen ion concentration and low carbon dioxid tension in the alveolar air, improvement will follow treatment directed to lessen the physiological strain, the character of which is evidenced in the nature of metabolic defect.

In some of these cases treatment may be followed by a decline of acidosis, the nitrogen retention becoming less, but at the same time the uric acid and sometimes the carbohydrate load in the blood will be found to have increased. Such a finding would indicate that while the heart and kidneys are showing more efficiency, the liver remained incompetent. The following case is illustrative:

In the wards of the Buffalo General Hospital is a patient who entered four months ago, having arthralgia, dyspnea, edema, occasional delirium and insomnia. At times he was semicomatose. There were nephritis and arteriosclerosis with the usual cardiac accompaniments. The liver was large. With the phenolsulphonphthalein test the kidneys showed an extremely low kidney function. The blood study was very enlightening. It showed the following:

Uric acid.................. $2.1 \mathrm{mg}$. in 100 c.c. blood.

Urea................... $52.0 \mathrm{mg}$. in 100 c.c. blood.

Total nitrogen................. I04.0 mg. in 100 c.c. blood.

In other words, while the uric acid was high, considering that the patient was having a limited diet, the urea and total nitrogen were relatively much higher, about four times the normal amount.

Treatment consisted of a preliminary fast of forty-eight hours, allowing the drinking of water. Then for four days, the "Karell diet" was employed, and the patient was given 2 drops of the fluidextract of apocynum every two hours. Immediately there was a gain in blood-pressure and an increase in urinary output; the anasarca and the dyspnea decreased.

Diaphoretic baths were now added to the treatment and the diet increased, yet salt was excluded and animal proteins also, with the exception of milk in limited amount. Following this plan there was continued improvement, not only in the symptoms but in the blood retention.

A second examination, one month later, showed:

Uric acid. .....................

Urea.

$2.01 \mathrm{mg}$. in roo c.c. of blood.

$38.0 \mathrm{mg}$. in roo c.c. of blood.

The patient was now able to sit up, the mind was clear, the complexion more natural, and there was no more dyspnea.

However, after a few weeks, there was a recurrence of arthralgia. At this time a blood examination showed:

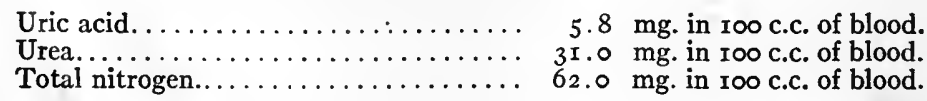

As will be noticed, the urea and total nitrogen had greatly 
decreased, although the uric acid had risen to nearly $6 \mathrm{mg}$. in 1oo c.c. of blood. The liver was smaller than it was formerly, owing to improvement in the circulation, although it was still larger than normal; ascites was no longer evident. The liver was believed to be in a state of fatty degeneration with a moderate fibrous growth.

The diet was kept low in proteins and the total intake of food daily was limited to I 700 calories. Alkalies, sodium et potassium tartras, were given in doses of $2 \mathrm{gm}$. (gr. $\mathrm{xxx}$ ) three times daily and occasionally small doses of magnesium salicylate, gm. $\quad 0.3$ (gr. v) three times daily, for two or three days. Following this the arthralgia ceased and the uric acid dropped.

This case illustrates the effect of hepatic incompetency in a syndrome often encountered; it also shows the beneficial effect of treatment of an incompetent and diseased liver by dietary and medication based upon metabolic study.

In cardiovascular disease the rôle of the liver is too often neglected. Undoubtedly good may follow the use of hepatic stimulants, such as glycocholate of soda, aspirin and at times mercury, and sodium sulphate, but these remedies accomplish little unless at the same time the work demanded of the liver is moderate and controlled by knowledge of the metabolic state of the patient. The careful regulation of the diet, in cases having a failing hepatic function, is nearly as important as it is in diabetes. In fact, glycosuria is at times a part of the symptom complex of defective function of the liver, either with or without corresponding disease of the pancreas. This is illustrated in the following case:

A man, aged seventy-two, retired since the age of forty-five. At this age he had what he called nervous breakdown for which he took extensive treatment, both in this country and abroad. During the years from the age of forty-five to the present time he has given most careful attention to his absorptive and eliminative processes, for the reason that he had marked indicanuria and transient glycosuria. On a most carefully selected diet, with alkaline waters, well-directed exercises and baths, this man at the age of seventytwo has systolic blood-pressure of 120 , diastolic 90 , with a remarkably good urine for a man of his age. His blood had a uric acid content of $1.8 \mathrm{mg}$. and a urea of $7.8 \mathrm{mg}$., total non-protein nitrogen of I9.2, chlorids $68 \mathrm{mg}$., blood sugar 0.10 , hydrogen ion concentration of his blood 7.8. This man when he presented himself was taking about $40 \mathrm{gm}$. of proteins, I 60 of fat, I00 of carbohydrate, amounting to about 2000 calories. For study he was placed on a considerably higher dietary, consisting of protein 90 , fat I90, carbohydrate 250 , or about 3500 calories. On this diet the blood showed the following: Uric acid, 2.4, urea I4.2, total non-protein nitrogen 29.6 His blood sugar remained the same: his hydrogen ion concentration rose slightly. His urinary acidity, which was 25 per cent. at the beginning of his study, quickly rose to 85 acidity per cent., after being on the increased diet for three days. His urinary indican, which was VoL. IV. -44 
I3 mg. for twenty-four hours at the beginning, quickly rose to 42 $\mathrm{mg}$. for twenty-four hours. This study shows, as we have noticed in numerous other cases, that the nitrogenous waste is considerably modified by diet. Not only were the nitrogenous waste matters piled up in the blood serum, but many other metabolic disturbances were noticed. His urinary ammonia rose, as did the hydrogen ion concentration. Before the increased diet was given, his nitrogen balance was only slightly positive. On the increased diet he showed an increased nitrogen retention. This case shows an increasing retention of the waste nitrogen with increased feeding, but we can reverse the conditions and show records of cases which came to the hospital with markedly increased retention which, upon proper dietary, fell promptly to within normal limits. In others it required from four to six weeks to reduce the nitrogen percentage in the blood. A few cases failed to respond to treatment at all. It is especially those who have neglected themselves too long, so long that the urea retention is above $60 \mathrm{mg}$. When the urea nitrogen is much above 60 mg., the prognosis begins to be grave. It is as necessary for these patients to remain on a low protein diet after they have been relieved of their retention, as it is for a diabetic to remain on a low carbohydrate diet after he has been relieved of his hyperglycemia.

In the case just detailed, the hepatic function was particularly low, although all the metabolic and eliminative processes were below the normal.

In addition to the dietetic treatment, the patient should be safeguarded from overfatigue, mental or physical. Symptoms of intoxication may be relieved by small doses, 0.3 to $0.6 \mathrm{gm}$. ( $\mathrm{gr} \mathrm{v}$ to $\mathrm{x}$ ) of magnesium salicylate, two or three times a day. This may be more effectual if given with sodium glycocholate $0.12 \mathrm{gm}$. (gr. ii), and an occasional dose of mercury.

\section{CANCER OF THE LIVER}

Besides the usual hygienic treatment and the allaying of symptoms such as must be carried out for the comfort of the patient, attention must be given to the possible prevention of certain grave conditions which are in the nature of sudden accidents accompanying the course of cancer of the liver. Sudden death may be due, in certain cases, not to hepatic insufficiency, but to a more general intoxication similar to that of diabetes. The increased destruction of albuminoids gives rise to the formation of acetone, diacetic acid and oxybutyric acid. Coma or convulsions may occur. Study of the urine and blood may give indications for therapy which, though there is no question of curing the disease, may help to avoid these accidents and to prolong life. Carbohydrates and fats should be increased in the diet. Proteins should be decreased. Large doses of alkalies should be administered. Diuretics are useful in ridding the organism of toxic substances. In these cases apparent improvement may be suddenly interrupted with fatal termination. 
Likewise hemorrhagic and infectious complications are of serious import. Inflammation of the liver may be avoided to some extent by frequent disinfection of the intestinal tract. The patient should be protected from alimentary intoxications, and exposure to infection of any sort should be avoided.

However, granting due importance to these measures, the most important therapy for cancer of the liver is that which takes into account the ever-present possibility of a mistaken diagnosis of what actually is syphilis of the liver. Treat cancer of the liver as syphilis of the liver. Negative history, absence of signs of the disease, negative Wassermann, even supposed evidence of cancer on exploratory operation, may all be disproved by recovery of the patient under vigorous antisyphilitic treatment.

\section{SYPHILIS OF THE LIVER}

The frequency with which the liver is involved in syphilis is coming to be generally recognized. The author drew attention to the frequency of this complication many years ago, but since the advent of the Wassermann test the number of cases recognized has been greatly augmented. Once more it is insisted upon that in a fair proportion of cases syphilis progresses in the liver without other striking evidence of the disease and even in the absence of a Wassermann reaction. Such a case is reported in another work. ${ }^{1}$ As is well known the complement fixation will be found positive, in a proportion of cases, only after a provocative injection of salvarsan or mercury.

Often it is necessary to continue the treatment of the case for a long time before improvement is manifest. Not only should salvarsan be used intravenously, but mercury should be given by the mouth. It is believed that when absorbed through the alimentary canal mercury traverses the portal system of veins, thus reaching the liver directly.

\section{TROPICAL ABSCESS OF LIVER}

The good results obtained by the use of emetin hydrochlorid, in the destruction of the Amaba histolytica of dysentery, led soon to its employment in amebic abscess of the liver. The use of emetin, as a preventive of abscess, was easily appreciated, for it was seen that by its early application and the clearing of the walls of the intestine from the living protozoa that there would be slight possibility of hepatic abscess. The work in India of Rogers, followed by the results obtained in France and French possessions, such as those reported by Dopter, Chauffard and others, showed that by subcutaneous injections of emetin, with or without the local use of the drug, a large proportion of cases were cured. Experience, both abroad and in America, while confirming the importance of the treat-

${ }^{2}$ Stockton, Diseases of the Stomach, New York, 1915, p. 624 . 
ment has shown that in well-developed abscess, it is not advisable to depend upon unaided injections of emetin. Although a "living" abscess may thus be converted into a "dead" abscess, although the protozoa may be killed, the abscess remains and needs to be evacuated. Spontaneous opening and discharge of necrotic tissue may occur, but whenever possible such a process should be anticipated by surgical opening and drainage.

Preceding and following the evacuation of the abscess emetin should be injected hypodermically. The effect is death of the amebas and a fall in fever and a relief of symptoms. In a small minority of cases the treatment is ineffective; occasionally there are recurrences. In most cases the amebas quickly disappear, the extension of the abscess to the lung, stomach or other parts readily heal, and the course of the cases is in every way strikingly satisfactory.

The subcutaneous dose of emetin must be sufficiently large, from 0.03 to $0.08 \mathrm{gm}$. (gr. $1 / 2$ to $\mathrm{x} / 3$ ) daily for one or two weeks is usually sufficient.

Certain preparations of emetin are unduly toxic and their use in an efficient dose is harmful or dangerous. The toxic symptoms are deceiving, since they simulate the symptoms of dysentery itself, and since they do not occur directly following the use of the drug. At present, even the name of a well-known supply house does not necessarily guarantee the purity of the drug. No evil results need be apprehended in the employment of a good specimen. ${ }^{1}$

Emetin appears to be useful, as would be expected, only in the amebic type of abscess. However, its curious property of overcoming hemorrhage is not limited to lesions depending on the ameba. In cirrhosis of the liver it is useful for the gastrointestinal hemorrhages secondary to the cirrhosis.

'Johns Hopkins Bulletin, Feb., I9r6. 


\title{
SURGERY OF THE LIVER AND GALL-BLADDER
}

\author{
By John B. Deaver, M. D., LL. D., Sc. D .
}

\section{INFECTIONS}

Surgery of the biliary tract is called for most often as a result of infection of the liver or bile passages. The portion of the tract which is most vulnerable to infection is the gall-bladder. The recent work of Rosenow indicates that the infection frequently arrives by way of the blood stream, having previously gained entrance to the circulation through any one of the numerous small atria of infection which exist in the upper respiratory tract or along the course of the alimentary tract from the mouth to the anus. Appendicular disease, tonsillar and sinus infections and peridental suppuration are presumably the chief portals of entry for this type of infection, the organism found being most frequently the streptococcus. Doubtless, however, these are not the sole sources of infection. The work of Adami has shown that microörganisms frequently find their way to the liver through the portal vein and are in time excreted into the bile. Here, under conditions of stagnation and especially in the gall-bladder, they may multiply and effect a lodgment in the tissues. Again, though probably exceptionally, organisms may ascend the common duct directly from the duodenum. Finally, in some cases the liver, the gall-bladder or extrahepatic ducts may become infected through extension from a neighboring viscus, such as a duodenal or gastric ulcer which has contracted adhesions to their exterior. The important point which has not received the attention it deserves in diagnosis and practice is the extraordinary frequency of such infective processes. Certainly not less than one in ten adults are at some time the victims of infective hepatitis or cholangitis which for the most part passes unrecognized as one of the forms of "indigestion."

Infection once established in any portion of the bile passages does not exist as a simple catarrhal irritation of the mucosa but always is to some degree an interstitial process. Here it follows the usual course of interstitial infections in spreading by way of the lymphatics. At times it may ascend against the lymphatic current and involve the interlobular tissues of the portal spaces. More commonly it takes the usual direction of lymphangitis and descends with the current to involve to a greater or less degree the structures next in order. In this manner the common duct and the periductal tissues become infected and show the lesions of acute or chronic lymphangitis. Often enlarged hyperplastic lymph nodes are formed along the course of the cystic and common ducts. The peripancreatic lymphatics are also involved, causing swelling of the head of 
that organ where it is in close relation to the common duct. At times the inflammation may be communicated to the pancreatic substance itself. The pancreas, therefore, is concerned in no small degree in the treatment of lesions of the bile passages.

Infections of the bile passages may resolve spontaneously or with the aid of treatment and leave little or no disability. In other cases the infectious process may advance and per se give rise to conditions which require surgical treatment. Again, certain complications or sequelæ develop which of themselves, even after the subsidence of the infective process, are sufficient to cause symptoms that can be reached only by the surgeon. Most often the condition is a combination of alternating periods of exacerbation of the infection together with the establishment of various complications, each condition serving to aggravate the symptoms and excite the activity of the other.

Much has been said of the tendency of biliary infection to die out and it is undoubtedly true that infection here, as elsewhere in the body, if not too severe or resistant is gradually overpowered by the forces of immunity and the tract becomes again sterile. Too little attention, however, has been drawn to the remarkable persistence of many biliary infections. Thus the typhoid bacillus has been recovered from the gall-bladder forty years after an attack of typhoid fever and countless instances are at hand of infections of the gallbladder which persisted for many years. In such cases the infection frequently becomes attenuated and only at intervals finds the conditions present for resumption of virulence and exacerbation of symptoms. There is abundant reason to believe that recurrent symptoms of biliary infection do not point to reinfections but to a natural recrudescence and activity on the part of an infection which is never entirely eradicated. The local lesions of persistent infection are progressive and give rise to a variety of conditions. The chief of these are increasing thickening and interstitial fibrosis of the gall-bladder and biliary passages, ulceration and desquamative processes in the mucosa, pericholecystic and periductal adhesions, interlobular hepatitis and cirrhosis, gall-stones, and, in a percentage of cases, as a result of continued chronic irritation, carcinoma.

The systemic effects of persistent infection even in the absence or comparative mildness of local signs and symptoms are certain toxic degenerations of essential organs and tissues. Babcock has forcibly called attention to the myocarditis due to latent gall-bladder disease. The kidneys may suffer in a similar fashion and a host of other toxic disorders may on occasion be traced to an infected biliary tract which is giving off a slow poison to be carried to the tissues by the blood. The pancreas, as noted above, is most likely to suffer, and a certain percentage of cases of diabetes take their origin from primary infection of the bile passages which is later communicated to the pancreas. The close interdependence of function of the organs of the upper gastrointestinal tract is the explanation of 
various digestive abnormalities affecting the stomach, duodenum, pancreas and liver in cases of diseased gall-bladder and bile ducts. Particularly is this true of the secretory, motor and nervous phenomena of the stomach and in many cases gastric symptoms and demonstrated disturbances of stomach function are but secondary to cholecystic disease.

It is apparent that the prime object of all treatment directed toward infective disease of the biliary tract must be the eradication of the infection. Medical measures are to be given a fair trial, but should be abandoned in a favor of surgery when they are seen to be inadequate. Hyperacute infections resulting in perforation, suppuration or gangrene demand immediate intervention. Persistent infections including the recurrent type which are not curable by medical means indicate surgical procedures. Gall-stones and mechanical embarrassments of function, such as may be due to adhesions, call for surgery in proportion to the disability which results therefrom. Operations of choice in the earlier stages of gall-bladder disease often forestall operations of necessity due to late complications and in many instances increase longevity by rescuing the body from chronic digestive disturbances and toxic degenerations.

Treatment.-The surgical measures which are employed in the treatment of infections of the duct tract and its consequences fall under the heads of (I) drainage, (2) extirpation.

Drainage operations are either external or internal. External drainage is accomplished by placing the gall-bladder, the cystic or common duct in communication with the exterior. This is done by bringing one of these structures, usually the gall-bladder, into direct relation with an incision or stab through the parietes. More often the communication is affected by means of a rubber tube which is placed in the gall-bladder or duct and brought out through the abdominal wall. This creates a sinus by the formation of adhesions about the tube. Such a sinus shows a strong tendency to close spontaneously after withdrawal of the tube. Unless an obstruction of the duct exists distal to the drainage opening, closure usually takes place in two to six weeks. An obstruction to the cystic duct in case of gall-bladder drainage will result in a persistent mucous fistula, owing to the continuous accumulation and discharge of the mucous secretion of the glands of the gall-bladder mucosa. In such cases the only cure is removal of the gall-bladder.

A persistent fistula which discharges bile is indicative of an obstruction between the artificial opening and the normal point of discharge of the bile into the intestine, namely, the papilla of Vater. This also can be relieved only by operation either removing the obstruction, reconstructing the duct or performing a sidetracking operation between a portion of the duct tract above the opening and the intestine, whereby the bile may flow unimpeded into the intestine. Persistent biliary and mucous fistulas may be avoided as a rule by appropriate procedures at the primary operation, 
forestalling the occurrence of obstructions distal to the point of drainage.

The tendency toward spontaneous closure of the drainage sinus is so marked as to necessitate some thought on the part of the surgeon as to the length of time which it is desirable that drainage should persist. It is well, therefore, to consider what may be accomplished by simple drainage.

It has been shown in many thousands of cases in simple infectious conditions, and indeed in many of the graver infections with marked anatomical changes that drainage alone for a number of weeks will suffice to resterilize the biliary tract. This takes place as a result of favoring the secretion of bile, eliminating back pressure upon the gall-bladder and ducts with resulting stagnation and especially because in this way the bile channels can be placed at rest, being no longer subject to alternating distention and contraction, factors which in every hollow muscular viscus are strongly hostile to the eradication of infection.

Simple drainage, therefore, to be effective must be proportionate to the degree and resistance of the infection present. These conditions are, naturally, not capable of exact valuation at the time of operation, but require for their estimation a combination of appreciation of the extent of pathological changes together with special knowledge derived from previous experience in this line of work. In general it may be stated that it is safer to use a maximum-sized drainage tube in the gall-bladder or ducts and the tube should be allowed to remain until it comes away practically without force. As such tubes are fastened in place, as a rule, by chromicized catgut, they will be found to be loose in from one to two weeks when they may be lifted out. By this time a fairly firm fibrous sinus tract has been established which will require several weeks longer to effect spontaneous closure. During all this time the duct tract is at rest, the bile is mechanically cleansing the sinus and in all but the more resistant infections by the time the drainage ceases the bile tract has become sterile.

When more trouble in effecting sterilization is to be apprehended owing to the extensive alteration in the ducts and especially when the pancreas is involved, it may be advisable to establish drainage for a longer period than can be done in the manner described above. This can be done through the medium of the gall-bladder which may be brought up to the wound and stitched to it in such a way that as healing progresses the mucosa of the gall-bladder may join with the ingrowing epidermis. Such a sinus will persist indefinitely and can be maintained until the lapse of months and the absence of symptoms are convincing evidence of subsidence of the infective process. The margins of the opening can then be freshened and brought together by suture which will often suffice to bring about closure in the event that no obstruction exists to the normal discharge of the bile into the intestine. It is equally safe and much 
surer to dissect out the sinus and repair the opening in the gallbladder.

Another method of maintaining drainage for any period at the pleasure of the operator is to place a T-tube in the common duct. This tube, introduced by $\mathrm{Kehr}$, is, as its name implies a rubber tube with a long arm and a short cross bar at one end. The short ends are introduced into the duct, one end running downward toward the duodenum, the other passing upward toward the liver. The long arm is brought out through the abdominal wound. The bile can now pass through the short arm lying in the lumen of the common duct and if no obstruction is present, it will find its way into the duodenum. If there is obstruction to this normal channel the excess will find its way out of the long arm to the surface. The effect is that of an artificial gall-bladder which protects the liver and ducts against pressure and distention, but instead of acting as a tension and storage bulb in the manner of the gall-bladder, the tube promptly discharges its bile upon the surface, there being no possibility of exceeding its limit of capacity or giving an opportunity for stagnation in its cavity or recesses. This is especially applicable in chronic pancreatitis which constitutes a vicious circle with infective cholangitis. The infection from the biliary tract involves the pancreas which in turn becomes swollen and indurated and creates more or less obstruction to the passage of bile down the common bile duct, which in two-thirds of all cases runs for a short distance through the head of the pancreas before piercing the posterior wall of the duodenum. As a result of this obstruction the infective phenomena in the biliary tract are furthered and each condition becomes mutually disadvantageous to the other. The best guarantee against early recurrence of symptoms in this type of case we have found to be long-continued drainage by the T-tube.

This operation is particularly indicated in chronic cholangitis of long or short standing, with more or less jaundice. I will illustrate my remarks by stating a case occurring in the practice of my friend and colleague, Dr. David Riesman. The patient was a woman in middle life, with chronic gall-stone disease, with a history of hepatic colic, jaundice and so forth. At operation-a cholecystectomy at the hands of the writer-calculous cholecystitis was found with no evidence of common duct obstruction or pancreatic involvement. The patient recovered and remained well for six months, when she again became sick, complaining of general malaise, indisposition, loss of appetite, epigastric distress after eating, gradual loss of flesh, slight jaundice and so forth.

Second operation: Drainage of the common duct; small, single rubber tube, the end of which was engaged in the hepatic duct. Prompt recovery from operation. The liver at the time of operation was enlarged, not markedly so, however, but tougher than normal to the touch. The patient remained well for about nine months, when recurrence of the same set of symptoms as complained of before 
the last operation appeared in an aggravated form, severe rigors, high temperature, profuse sweats, mild jaundice, no pain. This continued until Dr. Riesman again asked me to see the patient with him, at which time he agreed with me that another operation was imperative. The common duct was again opened and a rubber T-tube introduced. The liver at the time was enlarged, presenting a streaked appearance, its consistency being tougher to the touch than at the second operation. No obstruction of the common duct or disease of the pancreas was found. A singular remark that the patient made to me when she came to the hospital for the third operation was that the sweating at night was so profuse that she would wear six nightgowns in one night. Great improvement followed the operation, there were no further chills, sweats, practically no temperature. It is now over three years since the last operation and the patient remains well and continues to wear the tube.

Internal drainage of the biliary tract can be accomplished by an anastomosis between the gall-bladder or one of the ducts and some portion of the gastrointestinal tube, preferably the duodenum or stomach. This is a less simple procedure than external drainage and is, therefore, not to be used when drainage is the sole indication. Its field, par excellence, is in obstruction of the common duct by stricture or by enlargement of the head of the pancreas. It has been advocated as a drainage operation in the treatment of chronic pancreatitis and possibly in cases presenting a marked obstructive element it may be the best procedure. In cases that do not cause obstruction of the common duct it is fallacious to attempt to establish permanent drainage by a side-tracking operation with the intestine, as the new opening will be shunned by the bile in favor of the normal course and in time the anastomotic opening will become obliterated. In short, in all others but obstructive cases when it becomes necessary to save the secretion of bile for the intestines, the obstruction insuring the permanence of the new opening, internal short-circuiting drainage operations do not justify the increased difficulties and dangers inherent to their performance.

Extirpation.-The most satisfactory method of dealing with infection of the gall-bladder is removal, cholecystectomy. Like the infected appendix and Fallopian tubes, the gall-bladder, which is the seat of infection, harbors within its glandular recesses and throughout its walls numerous bacteria in a more or less quiescent stage. The researches of Rosenow emphasize the importance of the interstitial infection, having shown in practically every instance of macroscopic disease of the gall-bladder that organisms, in the majority of cases streptococci, can be recovered by culture of the gall-bladder wall even though the contained bile be sterile. Clinical evidences of renewed activity of gall-bladder infection after drainage operations also show that such operations are no guarantee against reinfection or recrudescence. There is, therefore, a very decided trend toward removal of the gall-bladder as the most satisfactory 
operation from the standpoint of permanent cure. The mortality of cholecystectomy has hitherto been slightly in excess of that of cholecystostomy but this is chiefly because of the fact that removal of the gall-bladder has been reserved mainly for the more severe forms of the disease in which naturally a heavier mortality is to be expected whatever the form of treatment. In the same class of cases the dangers of cholecystectomy in skilled hands are but slightly if at all greater than in cholecystostomy. Drainage of the biliary tract may be and in the majority of cases should be combined with cholecystectomy by placing a tube in the stump of the cystic duct or directly in the hepatic or common duct.

The final step in the operation of cholecystectomy as we have practised it in the German and University Hospitals is the placing of a small rubber tube down to and just beyond the free border of the gastrohepatic omentum, which is retained for four or five days so that in the event of a ligature on the cystic duct giving way the bile will have an exit. Occasionally a glass tube is used which is replaced the following day by a rubber tube, the latter being small enough to be carried down the glass tube and the glass tube withdrawn. When the gall-bladder is so large that access to the cystic duct is difficult, it may be aspirated or opened and emptied, care being taken to guard against infecting the field of operation, when the removal, as above described can be made. In some cases of gangrenous and phlegmonous gall-bladders the operation is made a little more difficult than in ordinary cases, yet this technique can be carried out.

Drainage of the common duct by way of the stump of the cystic duct is only a temporary procedure. When, as is frequently the case, it is necessary to have prolonged drainage of the common duct, I open the common duct and introduce a T-shaped rubber drainage tube. I have a number of patients wearing these tubes. This form of drainage is introduced in certain cases of pancreatic lymphangtis, chronic interstitial and interacinar pancreatitis.

The discharging sinuses created by tube drainage in the gallbladder or one of the ducts usually require no treatment except for the purpose of ordinary cleanliness.

The tubes themselves are allowed to remain until the catgut sutures by which they are held in place soften and permit of easy removal. We are accustomed to say that they should stay in until they fall out of themselves, in order to emphasize the fact that there should be no tugging on them while the sutures are still intact. If these directions are followed out there is no damage done to the deeper structures and the tube remains in place long enough to create a moderately firm sinus wall that will drain for several weeks. Ordinarily the tubes may be removed in from ten days to two weeks and the resulting sinus will close in from four to eight weeks. The sinus needs no irrigation, cauterization or cleansing. Closure is certain unless there be obstruction distal to the opening. If the cystic duct be obstructed in the case of drainage of the gall- 
bladder the result will be a persistent mucous fistula which can be cured in no other way than by removal of the gall-bladder. If the common duct be obstructed the bile will continue indefinitely to be poured out of the artificial opening until the obstruction is relieved or a new communication made between the gastrointestinal tract and the biliary tract above the point of obstruction. The rule, therefore, is that simple sinuses close spontaneously, while persistent sinuses, meaning those that exist without marked diminution of discharge for more than three months require operation. The latter are rare and depend usually upon an obstruction unrecognized at operation. Occasionally inflammatory processes about the ducts cause destruction or blockage of the channels, requiring reoperation.

When a T-tube has been placed in the common or hepatic duct a small amount of force must be used in its removal since this is accomplished by "buckling" the short arms of the tube in order that they can be pulled out. When the gall-bladder has been marsupialized by stitching it to the anterior abdominal wall, the resulting fistula may require operation to close it, since the sinus may become completely covered by epithelium which will not obliterate by cohesion of its walls as in the case of a granulating sinus. It is important in every case that requires opening of the bile passages, whether this be by cholecystostomy, cholecystectomy or choledochostomy, to place a drainage tube in the abdomen in proximity to the opening even if it be ligated or sutured, since the vis a tergo of the bile within the passage may be sufficient to force the ligature or suture and allow escape of the bile, an accident which, in the absence of a passageway to the surface inevitably results in peritonitis and death. Such tubes should be left in situ for eight to ten days when they may be gradually removed by shortening from day to day. If a biliary discharge makes its appearance the sinus may be handled in the same manner as if created by design.

During convalescence while bile is being discharged externally the patient may have the ordinary varied diet suitable to that period. Rather frequent feedings are of some advantage in that the orifice of the common duct is thereby relaxed and the bile encouraged to flow into the duodenum for use in digestion. Fluids should be given in abundance to reduce the consistency and increase the amount of the bile secretion. There is no objection to the use of the so-called bile antiseptics such as hexamethylenamin, ox gall and the salicylates, but they are unnecessary and of doubtful utility. The bowels should be carefully regulated by enema, or an occasional saline draught.

The local and general consequences of infective disease of the gall-bladder and bile ducts, with or without stones, justify the surgeon in urging operation more frequently and at earlier periods than is now commoniy the case. The mortality in simple cases ranges from 0.5 per cent. to 2 per cent. This would be lowered if operation were advised and accepted in that large class of cases that suffer 
from persistent or recurring indigestion due to derangement of the bile apparatus but without the more serious symptoms or sequelæ. By this means a vast amount of semi-invalidism could be remedied and a large proportion of degenerative disease, as well as acute illnesses not commonly recognized as dependent upon or associated with the toxemia and functional disturbances of biliary disease, would be prevented. The later and more serious complications also would be anticipated and the higher mortality of those common conditions be converted to the lower mortality of the earlier stages.

As the chief indication for operative interference, therefore, we would place:

r. Chronic, persistent or recurring indigestion due to infective disease of the biliary tract or its consequences in the shape of gall-stones, adhesions, etc.

There can be no question of the advisability of operation in:

2. Perforation of the gall-bladder.

3. Gangrene of the gall-bladder.

4. Acute purulent cholecystitis.

bladder.

5. Obstruction of the cystic duct with $(a)$ hydrops or (b) empyema of the gall-

6. Obstruction of the common duct.

7. Suppurative cholangitis.

The field of medical treatment is in:

I. The early less serious manifestations of the disease.

2. Cases showing mild attacks at very infrequent intervals.

3. Cases in which operation is contraindicated by definite acute or chronic organic disease not dependent upon the disease of the biliary tract.

It is worthy of special remark that obstructive jaundice due to gall-stones demands early operation. An impacted stone in the common duct is best removed at once or as soon as the primary shock, if shock be present, has subsided. If this cannot be done the surgeon should be governed by the general and local condition except in the case of suppuration, perforation or gangrene which call for immediate interference. Often it is best to delay operation until the inflammatory condition has subsided. This will usually take place within a few days under treatment designed to give physiologic and anatomic rest to the parts. Practically nothing should be given by mouth. A few chips of ice or teaspoonful doses of hot water or tea may be given at intervals of one to three hours.

Fluid should be supplied for bodily needs by rectal injections of normal saline, or better by continuous enteroclysis by Murphy's method. The patient should be in the recumbent position and an ice bag applied to the epigastrium and right hypochondrium. Small doses of morphia should be given if necessary. When tenderness and rigidity have subsided, the curative operation should be performed. The counsel so often given to wait two or three months is very unwise. Patients who have been jaundiced for several weeks are subject to much increased risk from operation. Shock, hemorrhage and infection all take an increased toll in such a group. 
Concerning the long-noted hemorrhagic tendency due to chronic jaundice much remains to be known. Not all patients show it, while others seemingly no more jaundiced exhibit it in uncontrollable form. By way of prophylaxis jaundiced patients should receive large doses of calcium for three to five days before operation. The best preparation is the lactate of calcium which is soluble in water $\left(\mathrm{r}: \mathrm{I}_{5}\right)$ and may be given in doses of $\mathrm{r}$ or $2 \mathrm{gm}$. (gr. $\mathrm{I}_{5}$ to 30 ) to a total of 100 grains a day. It is best given on an empty stomach as it is likely to be precipitated by the phosphates of the food. Saline aperients should be avoided for the same reason. The solution of calcium lactate should be clear or nearly clear. If a white precipitate is present it should be discarded.

If the hemorrhagic tendency is observed during operation coagulen, Kocher-Fonio in 5 to ro per cent. solution may be applied locally to the bleeding surfaces. Packing with gauze may be advisable. If bleeding continues after operation, horse serum in doses of 15 to 30 c.c. intravenously or subcutaneously may be used freely. Diphtheria antitoxin will answer the same purpose. If the amount of hemorrhage becomes dangerous to life direct transfusion of blood from a suitable donor is strongly indicated both for the purpose of restoring blood to the circulation and to check the hemorrhage. It is wise to arrange for this possibility before operation. It is well to understand that the cause of the hemorrhagic tendency in chronic jaundice is not known nor is there any method of determining to a certainty in advance which cases will prove to be bleeders. Coagulation time and "bleeding time" are indicators and should always be estimated and if unduly prolonged operation should be postponed until conditions improve unless the condition is of the nature of an emergency. The difficulties and uncertainties of the therapy of this condition are a strong argument against undue delay in the care of operable cases of chronic jaundice.

\section{ABSCESS OF THE LIVER}

Abscess of the liver occurs in two chief forms: (a) the large solitary (tropical) abscess; and (b) small multiple abscesses. Occasionally to use a Hibernianism the solitary abscess is multiple also, that is, there may be two or more large independent abscesses.

The small multiple abscesses are usually the consequence of portal vein infection (pylephlebitis). Less commonly they result from biliary duct infection or from infective processes involving the hepatic artery or hepatic vein. They are difficult of recognition and not amenable to treatment though in the cases of duct-borne infection, drainage of the gall-bladder and common duct is not out of place.

The solitary abscess which is usually secondary to bacillary or amebic dysentery, though not invariably so, is likewise difficult of recognition and usually escapes detection until in its enlargement it 
impinges upon the parietes, giving local evidence of its presence through the signs of tumor, tenderness, or other signs of inflammation. When it is located in the dome of the liver and expands toward the diaphragm it is difficult or impossible to distinguish from a subdiaphragmatic abscess to which in fact it may be converted. Again it may perforate the diaphragm and first having contracted adhesions with the lung eventually empty itself spontaneously through the bronchi. Such an outcome is exceptional.

The ideal treatment is that of abscesses in general, namely, incision and drainage. When the abscess has contracted adhesions with the anterior abdominal wall it may be opened directly with little danger. Similarly abscesses which point upward may compress the diaphragm against the chest wall and by adhesive inflammation shut off an area from the thorax through which an incision may be carried directly into the abscess. Diagnostic puncture is as a rule too dangerous since puncture into an abscess unprotected by adhesions will be likely to result in peritonitis or empyema, as the case may be. A diagnostic incision under conditions properly arranged for whatever surgical procedure may be found necessary is justifiable in the case of strong suspicion that abscess of the liver is present. The special difficulties arise in this type of case through the dangers of contamination of the pleural or peritoneal cavities during and after evacuation of the abscess.

The first step, therefore, after opening the peritoneum in search of abscess is the careful walling off of the suspected focus from the general abdominal cavity. Puncture may then be employed to locate the collection, or if it be evident it may be opened directly or aspirated by trocar and cannula before free incision. Tube and gauze drainage should then be inserted into the abscess cavity and a surrounding tampon of gauze so arranged as to protect the abdominal cavity. In some cases it is wiser to bring the surface of the abscess beneath the incision which is left open in part and packed with gauze down to the liver surface. In a few days when the adhesions are sufficiently firm the incision may be made into the abscess without danger.

When the pleura is opened in search for abscess of the dome of the liver the dangers of immediate evacuation are much increased. Nevertheless, it may be better to proceed at once to open it, protecting the pleura as well as possible with gauze pads. The case may than be treated as an empyema. If mechanical conditions are such as to render it possible it is preferable here also to create a closed tract before incising. This may at times be done by suturing the parietal pleura to the diaphragmatic pleura. Often this is impossible. As a general rule the operator should avoid entering the chest and adhere scrupulously to the area which he has reason to believe is closed off from the pleura, making his exploration of the liver by puncture rather than by examination of the diaphragmatic surface. A portion of rib should be resected as in the operation for 
empyema, as liver abscesses if at all large are slow in closing and free drainage is necessary.

The after-treatment of drained abscesses consists in maintaining free outlet for the pus and secretions by means of tubes or gauze lightly packed. As the liver shrinks the relative position of the liver and incision may change so that an ample incision is wise. Irrigation should never be practised until the tract is safely closed off from the peritoneum or pleura and even then only infrequently if at all. Postural drainage is best if the location of the abscess renders it practicable.

\section{ANOMALIES OF FORM AND POSITION OF THE IIVER}

Very exceptionally in cases of visceroptosis the liver may be so abnormally movable as to occasion symptoms and cause difficulties of diagnosis and treatment. Usually the condition is but a part of a general ptosis and requires no surgical procedure. Hepatopexy or fixation of the liver to the parietes has been performed, first by Billroth, and following him by a number of surgeons. Its performance should be reserved for outspoken cases of wandering liver with definite symptoms.

The linguiform (Riedel's) lobe of the liver is an elongated lappet of liver substance which extends downward from its anterior and lower border in the region of the fundus of the gall-bladder. It is most often accompanied by and has been thought to be a consequence of disease of the gall-bladder itself. This is not invariably the case. At times a constriction exists at the junction of the process with the liver. It may be felt as a mass in the abdomen which is exceedingly difficult of interpretation. At times it is congested and tender. Under such circumstances it may be advisable to remove it, as has been done on a few occasions. Usually the treatment is that of the associated cholecystitis or cholelithiasis.

In situs inversus the greater lobe of the liver and the gall-bladder is on the left side and in at least one recorded instance this condition was found at operation.

\section{TUMORS OF THE LIVER AND BILE DUCTS}

Benign neoplasms of the liver and ducts are rare and are more often discovered accidentally than because of symptoms demanding operation. The fibroma, adenoma, and hemangioma are occasionally met with. Hemangioma at times gives rise to symptoms which are presumably the result of congestion. This form of growth also shows a tendency to invade the adjacent liver substance, though the process is slow and never accompanied by metastasis unless sarcomatous transformation takes place. Excision of such growths may be done, providing that operative difficulties and dangers are not prohibitive.

Malignant growths may be either primary or secondary, of which the latter are more common. Such metastatic growths are commonly 
secondary to carcinoma of the stomach, breast, pancreas or lower intestinal tract. As a rule they are multiple and represent such an advanced and widespread process that nothing is to be hoped for by surgical intervention.

Primary carcinoma of the liver parenchyma is rare, but primary carcinoma of the gall-bladder or bile ducts is of more frequent occurrence. It affects the gall-bladder usually, the common duct less commonly and the smaller ducts within the substance of the liver still less frequently. Statistics indicate that it is definitely associated in the majority of instances with antecedent infective or calculous disease of the gall-bladder or ducts. When it is so situated that it causes early obstruction of the cystic or common duct, sufficient symptoms may arise to indicate operative intervention before any great spread of the disease has occurred. In such cases excision of the affected parts should be made. When the growth has invaded tissues beyond the reach of excision, it is as a rule useless or worse to remove any portion. When the growth obstructs the common duct, the cystic duct and gall-bladder being unaffected, relief from jaundice and the obstruction symptoms and prolongation of life may be effected by anastomosis of the gall-bladder with the stomach, duodenum or intestine.

In all cases of tumor of the liver it is imperative to bear in mind that gumma of this organ is not uncommon and may give rise to remarkable masses and all the symptoms of obstruction and cachexia characteristic of neoplasm. A history of syphilis should be sought for and a Wasserman reaction made and if doubt exists the therapeutic test is advisable. Syphilomas of the liver yield promptly to specific treatment so that no great delay is necessary in coming to a decision.

In general it may be said that the most practical conclusion for treatment to be drawn from what we know of malignant disease of the bile passages is in the way of prevention of at least a proportion of such conditions by timely treatment of cholelithiasis and biliary infections.

\section{CYSTS OF THE IIVER}

Cysts are usually of hydatid origin and in this country are seen most frequently in those of alien birth. Small cysts in accessible situation may admit of excision or enucleation but as a rule they are to be treated by incision and drainage. Occasionally bacterial invasion of such a cyst occurs, transforming it into an abscess and demanding appropriate treatment. In dealing with hydatid cysts whether infected or not it is necessary to exercise great care to avoid soiling the abdominal cavity with the contents; and in favorable cases the surface of the cyst may be connected with the exterior of the body through an incision directly over it, allowing adhesions to form which exclude it from the peritoneal cavity before it is opened. Drainage should be free and the casting off of the cyst wall may be aided by gentle curetting of its surface.

vos. IV. -45 


\title{
DISEASES OF THE PANCREAS
}

\author{
By James M. Anders, M. D., LL. D.
}

While our knowledge of the pancreas and its diseases during the past five years has been distinctly enriched by the labors of Korte, Robson, von Noorden and others, the advances gained have had but slight influence upon the therapeusis of pancreatic affections. Thanks to recent progress, however, we are in a position today to make an assured diagnosis in not a few cases which formerly proved to be inscrutable; hence we can avail ourselves of a more rational mode of treatment at present writing than formerly.

The labors of Wohlgemuth and later those of Bickel have shown that certain drugs can influence the amount of pancreatic secretion. For example, sodium chlorid, alcohol, pilocarpin and hydrochloric acid stimulate, while atropin, opium, adrenalin and the alkalies diminish the functional activity of this gland.

The diet also influences pancreatic secretion. Bouillon, and more especially carbohydrates (based on the experimental findings of Pawlow, in the fistulous dog), tend to increase it; per contra a fat diet tends to decrease pancreatic function. Chiari claims that the physical character of the food, whether coarsely or finely divided has a marked influence on pancreas secretion. For example, the more finely divided the articles of food taken, the greater the stimulating effect upon the secretion of this organ. Probably, no form of rectal alimentation causes the pancreas to secrete.

The results of the experiments of Tileston indicate that the administration of either raw pancreas or pancreatic extract tends to increase materially both fat and protein absorption where the pancreatic juice is deficient. It is to be recollected, however, that such feedings cannot supplant the internal secretion of the pancreas, e.g., in diabetes. In pancreatic feeding, it has been found that the raw gland is preferable to the extracts, which, however, are more easily procured. Pankreon enjoys a wide popularity for the purpose; "it is best given after meals with an equal amount of calcium carbonate, in rather large doses, I to $3 \mathrm{gm}$., I 5 to 45 grains" (Tileston). Lombroso and Manetta ${ }^{1}$ have called attention to the fact that following splenectomy the pancreatic secretion increases.

\section{ACUTE PANCREATITIS}

During recent years surgical has largely replaced medical treatment. At present, non-operative management is confined largely to

\footnotetext{
${ }^{1}$ Policlinico, Röme, March, 1915, Medical Section, No. 3.
} 
milder forms, in which actual collapse is absent. Some of the cases, which pursue a mild course, however, terminate in abscess, when they should be operated.

During the active stages of the disease it is recommended to employ rectal feeding, allowing the stomach to remain at perfect rest. Following acute pancreatitis and recovery from operations in this disease, certain indications for medical treatment of ten present themselves, more particularly diabetes mellitus. In pancreatic diabetes we must allow carbohydrates, and "oatmeal days should be instituted at regular intervals, and large doses of bicarbonate of soda given internally" (Held).

\section{CHRONIC PANCREATITIS}

Recent observers have emphasized the importance of a correct dietary. For example, A. Schmidt ${ }^{1}$ contends that the diet should be based on the findings in the stools after the test-diet, restricting the fat (no butter, cream, very little milk), and the most minute division of all the meats and eggs eaten. Schmidt also finds gelatin dishes extremely useful, as gelatin makes but slight demands on pancreas function and gives but little occasion for putrefactive processes. In his experience pancreas preparations help in some cases, but fail in others.

A. Albu states that in his experience a large, if not the largest, contingent of chronic pancreatitis is caused by lues. In cases in which the diagnosis of pancreatic syphilis is fully established by the history and a positive Wassermann reaction, salvarsan should be brought into requisition. It is a question, however, as pointed out by Albu, to what extent resorption of newly formed fibrous connective tissue in the pancreas can be effected, although prospect of its accomplishment is brighter in syphilitic than in non-luetic varieties of chronic pancreatitis. Diabetes mellitus dependent upon syphilitic pancreatitis also calls for antisyphilitic measures, and cures have recently been reported as the result of this method of treatment.

Certain recent observers have emphasized the importance in the treatment of chronic pancreatitis of balneotherapy for its influence upon the functions of the intestines, as the result of depletion of the portal circulation, the saline alkaline mineral waters and also the purely saline waters, being especially indicated.

Porter ${ }^{2}$ holds that any pathogenic bacteria, or even the colon bacillus, under abnormal intestinal conditions may become the inciter of a pancreatitis with ensuing suppression or deficiency in one or other of the pancreatic ferments. He raises the question of the value of vaccines in these circumstances and states that in one case the results following their use were so striking that it seemed impossible to explain them on the ground of mere coincidence.

${ }^{1}$ Mittheil. a. d. Grenzgeb. d. Med. u. Chir., Jena, r913, xxiv, No. r.

2 Am. Jour. Dis. Child., August, rgr 3. 


\section{PANCREATIC CALCULI}

Albu holds that pancreatic calculi are oftener a complication of cholelithiasis than occurring alone. The condition is rare and seldom recognized in vivo. The alkaline and saline alkaline waters such as are to be found at certain spas, are rapidly winning, if they do not already hold, first place in the treatment of the earlier stages. The taking of large quantities of certain foods, especially the carbohydrates would tend to stimulate free pancreatic secretion.

\section{PANCREATIC ACHYLIA}

The condition rarely obtains as a primary affection, in contrast with achylia gastrica. It is much more often a complication of the latter. If in the course of chronic atrophic gastritis, nutritive disturbances arise, pancreatic achylia may be inferred after exclusion of latent carcinoma. It is also possible to make a diagnosis from a careful, systematic examination of the feces after an appropriate test meal, consisting of scraped beef, Ioo gm., milk, I liter, and butter, Ioo gm. If there ensue macro- or microscopic creatorrhea and steatorrhea, soon the one, soon the other predominating, then disturbance of the pancreatic function may be safely inferred.

Einhorn ${ }^{1}$ has, by the use of the agar-tube method, determined the amount of the various ferments that are present in the duodenal contents-a point of importance from the standpoint of pancreatic therapy. Crohn states that the quantitative estimation of the duodenal ferments is the most rational and accurate method of studying the external secretion of the pancreas. The diminution of enzyme activity of the pancreas is a reliable sign of organic disease of the gland. Occasionally a diminution of the ferments occurs as a symptom of organic disease elsewhere in the body.

It is to be recollected that fat and nitrogen may be absorbed from the intestine independently of the external secretion. Absorption may be poor with an intact gland, or good with a gland, of which only a fragment survives the disease. The functional activity of the gland, not its organic condition, determines the degree of absorption, and the absorption tests give the index of the functional activity of the pancreas.

.As in the case of achylia gastrica, so in achylia pancreatica, balneotherapy deserves a careful trial. Owing to the disposition to diarrhea in this condition, an appropriate diet, or one composed largely of carbohydrates, is indicated. The mineral acids, on account of their influence upon pancreatic secretion, are useful.

\section{PANCREATIC INFANTILISM}

Pancreatic infantilism, which was first described by Bramwell, is believed to be due to defective or arrested pancreatic secretion. The characters of the infantilism are: arrested bodily and arrested

'Jour. Am. Med. Assn., May 29, I915, p. 1872. 
sexual development; intelligence good, no mental defect; no deformity or structural defect of the bones; no visceral disease or derangement except chronic diarrhea and flatulent distention of the abdomen. Bramwell ${ }^{1}$ believes that the arrested pancreatic secretion is due to chronic pancreatitis. The condition is completely cured, in some cases at least, by the persistent administration of pancreatic extracts. In one of the author's cases, sexual development, which when the patient came under observation was quite infantile, gradually became complete, the patient losing his child-like appearance and high-pitched childish voice.

1 Edinburgh Med. Jour., May, 19r5. 


\section{VISCEROPTOSIS}

\section{By Joseph Sailer, M.D.}

Less and less attention has been paid to this condition as a definite morbid entity. It appears to be a contributing factor to various disturbances of nutrition but not in itself, unless there is evidence of obstruction, a definite pathological state. As a result, operative procedures for the relief of this condition are perhaps less used than formerly. On the other hand mechanical support is used more than ever before and it is a very common thing in going around the hospital wards to observe patients on whom adhesive plasters supports have been applied in the hope of relieving some of their discomfort. A considerable number of methods have been devised for applying the straps, none of which apparently are any great improvement on the methods of the past. Probably the most efficient consists of one or two broad bands placed around the body in two portions, the ends overlapping in the front and back. This binder was fully described in the previous article. It is best applied with the patient standing and using a fair degree of force in order to have it as tight as possible. The lower strap should be put on first. The Lane abdominal support has also been exploited considerably. It consists of a thick pad in front, large enough to cover the lower portion of the abdomen but to lie well within the anterior superior spines of the pubis. The pad is in two parts with a median vertical hinge. Two ordinary truss springs extend from the middle of either half around the back and terminate in pads which rest against the spine. This support is particularly available in patients who are thin, because it presses upon the lower part of the abdomen irrespective of the amount of protuberance and does not rest at all upon the bones of the pelvis excepting in the back. It should be placed in position with the patient lying on the back and preferably with the hips elevated on a pillow. It can be worn indefinitely without discomfort and indeed often gives a great degree of relief so that the patient feels exceedingly uncomfortable if he attempts to stand without it in position. As in all other efficient binders it tends to improve nutrition by preventing kinks and by facilitating the onward movement of the intestinal contents. All the objections to binders of any kind of course apply to this and it should not be used where there is any suspicion of an inflammatory condition or a tumor of the abdomen.

More and more attention is being paid to the general malformation of the body in these cases and less to the actual condition of the intestinal organs. There seems to be no doubt that the most general cause of visceroptosis is a diminution of the infradiaphragmatic space 
that is particularly brought about by the bending inward of the edges of the lower ribs and perhaps by lordosis of the lumbar spine and some kyphosis of the dorsal spine. Measures, therefore, that will in any way tend to increase the infraabdominal space are distinctly useful in this condition. Obesity, if it can be brought about, will nearly always cause the ribs to project somewhat, and if it can be maintained will probably in time improve the shape of the abdominal cavity. It is in this way in all likelihood that the permanent cures reported by Einhorn and others may be obtained. In addition there is every reason to believe that special exercises and proper spinal support is of advantage. The former consists of all those exercises which are designed to improve nutrition. The setting-up exercises in the United States army, used also in other military organizations, are of great advantage. These are best undertaken under the direction of a competent gymnasium director who will devise exercises for each individual case according to his or her needs. Naturally the exercises should be carried out systematically and regularly, gradually increasing in complexity, vigor and duration. It is preferable, I think, to have them taken at least twice each day. It is not necessary, however, that the director should be present at every exercise but he should see the patient two or three times every week in order to note if they are carried out efficiently. If there is much feebleness of the body and emaciation they must be very light in the beginning. As strength increases they can also be increased until finally in the majority of cases, thoroughly vigorous exercise can be taken night and morning without unreasonable fatigue. The proper station is important. The abdomen should be held in, lumbar lordosis overcome as much as possible by muscular contraction, the shoulders thrown back, the head thrown up and back, and an effort made to expand the upper portion of the chest. At first this will be done only when the patient is thinking of it, but if the exercises are continued it may become more or less of a habit. In addition to this exercises on the rings or horizontal bar are useful, the rings particularly are available for women and in a short time they can learn to support themselves and even carry out simple gymnastic procedures. They have a distinct tendency to spread the lower part of the chest. Orthopedic apparatus in addition to the various forms of support may also be useful. They are designed to straighten the spinal column as much as possible. A single rod attached to a firm pelvic binder curved to conform to the spine but at the same time to improve its position is often of advantage. This has been suggested by Goldthwait. It is not to be regarded so much as a support as a corrective, tending to improve the general station of the patient and from time to time, as the station improves, the curve should be corrected so that the maximum improvement will ultimately be obtained.

The prophylactic treatment is receiving more attention than it did formerly. The pot belly of the rachitic child is really nothing more than one of the manifestations of general splanchnoptosis due 
to the curving in of the lower edges of the thoracic cage and the diminution of the infraabdominal space. Such children are greatly benefited by a vigorous gymnasium course. This should be adapted to their particular physical needs and carried out under the direction of a competent gymnasium director. Fortunately, more and more of these competent directors are being supplied as the result of the work of various schools in the country. No definite group of exercises can be described. Military marching, however, is particularly useful. The swinging exercises on the rings, the trapeze, the horizontal bar, boxing, wrestling, are all of advantage. The exercises should be made interesting to the child. Otherwise they will probably fail in their purpose. If possible they should be competitive, that is, class work as a rule is better than individual instruction. The time should be arranged with proper reference to meal time and with an opportunity of at least a brief period of rest afterward and there should, of course, be such a regulation of the diet that the child will eat at regular intervals, and have an ample supply of nutritious food that throws the least stress on the digestive organs. Practically it is merely a general physical improvement, but it is sometimes amazing how the physical condition of a child will improve under such a régime although previously there had apparently been an ample opportunity for open-air exercise. The improvement in the station and the shape of the thorax, the disappearance of the protuberant abdomen, the increase in the musculature of the limbs is often exceedingly gratifying and seems to be associated with a general improvement in energy, in the disposition and often in the mentality of the child.

\section{CECUM MOBILE}

Reference was made in the preceding article to cecum mobile as one of the elements in splanchnoptosis. Practically speaking, this is not correct. Cecum mobile is due to a defect in the mesenteric attachment of the cecum and ascending colon whereby the lower portion of the ascending colon may become more movable. This condition is by no means uncommon. Dr. H. K. Pancoast has been studying it by the roentgen-ray for several years and finds that it exists in a considerable number of cases and can be readily demonstrated. To a certain extent it is one of the associated conditions of splanchnoptosis, because almost invariably in these conditions the hepatic flexure is much lower than it normally should be. Cecum mobile is a potential but not an actual disease. It becomes a morbid condition affecting the health of the patient only if the motility and the relaxation of the surrounding tissues is sufficient to permit the cecum to move enough to produce a kink. Under these circumstances signs of obstruction arise, the cecum becomes distended, ultimately atonic, that is, the state known as typhlotonia, and the patient will suffer from pain and attacks of colic and chronic constipation. Unless the displacement of the cecum is considerable and kinks 
occur, it is not a serious condition and docs not often endanger life and may only, to a moderate extent, affect the general health. The symptoms of the disease are those of chronic constipation, that is flatulence, loss of appetite, sense of pressure in the abdomen, headache, and some malaise. The symptoms referable to the condition itself are the attacks of colicky pain, a sense of fulness in the right side of the abdomen, and often soreness in this region. They resemble on the whole those of chronic appendicitis with which, I believe, the condition has often been confused, and the existence of this condition is, I believe, one of the reasons why not infrequently patients who have had a so-called chronic appendix removed are but little benefited by the operation. The physical signs are first a palpable cecum. This is usually irregular, has according to Klose a cushion-like sensation and if the mass is rolled under the fingers there is often distinct gurgling. An audible splash is by no means uncommon and can be best heard by placing the stethoscope over McBurney's point and then practising succussion in the right lower quadrant. The motility of this mass is often easily demonstrated. It can be moved sometimes 5 or $6 \mathrm{~cm}$. toward the median line and back again. There is usually distinct tenderness over McBurney's point and often tenderness can be elicited in the surrounding region, being not as sharply localized as it is in chronic appendicitis, but as this is a quantitative rather than a qualitative variation it is of subordinate value in making the diagnosis. The most important element in the diagnosis is the roentgen-ray examination. About eighteen hours after a bismuth meal, when the cecum is still quite full of bismuth, if the picture is taken with the patient lying on the back and then lying upon the left side the change in the position of the cecum is often quite remarkable. It falls toward the median line and often distinctly upward. Mere retention in the cecum, which was the feature originally described by Wilms, is not nearly as valuable as the actual demonstration of the change in the position of the cecum itself. It must be admitted that Craemer and Bevan have denied the importance of a movable cecum and have particularly declared that it was not a disease entity. In this, I believe, they are wholly correct. As I stated at the beginning of this paragraph it is not actually a disease in itself but simply contributes to the occurrence of certain changes in the cecum, that is, kinking with colic sensation and gradual atony and dilatation, leading to chronic constipation. As in all other conditions the anatomical defect, unless it leads to functional disturbance, is of no significance to the patient and is only of importance in so far as the application of preventive measures may subsequently lead to the manifestation of functional disturbance.

The treatment may be either medical or surgical. The former is probably the more important, particularly as surgical intervention will probably not be undertaken until the condition has been severe for some time and has lod to a dilatation and atony of the cecum 
that medical interference could not correct. The most important feature of the medical treatment is first to overcome the chronic constipation. Second, to relieve any putrefactive changes that may occur in the retained fecal contents. Third, to increase nutrition and improve the condition of the abdominal musculature so that the cecum will have less opportunity to swing into an abnormal position and produce kinks. Of the measures used to combat the constipation the most efficient, at least in the beginning of the treatment, are probably enemas. These may consist of oil injected daily or every other day as the case may require. From 250 to 500 c.c. of neutral cottonseed oil appears to be best for this purpose. It may be efficacious to alternate the oil enemas with alkaline enemas, usually about 3 per cent. bicarbonate of soda. This seems to loosen any mucous accumulation on the wall of the intestine more efficiently and apparently stimulates the muscles to more vigorous contraction. In addition paraffin oil may be taken by the mouth and mild laxatives. Vigorous purgation is distinctly contraindicated. Probably among the best of the milder laxatives are cascara, senna, and if there is an associated hyperchlorhydria, as there often is, milk of magnesia. The diet should be regulated by a careful study of the feces. If there is much vegetable detritus, vegetables should be decreased. If there is hyperchlorhydria, fruit should be reduced or absolutely removed from the diet. If, on the other hand, there are meat fibers in the feces, the amount of heavy meat should be diminished. The presence of a large number of starch grains in the feces is rather an indication for aiding starch digestion by some of the diastatic ferments than for restricting the intake of starch, unless there is excessive fermentation and meteorism. Disinfection of the intestines is theoretically desired. Practically it is very difficult to obtain. The various disinfectants that may be administered by the mouth (betanaphthol, salicylates, thymol, guaiacol, creosote) are all useful but should be used in moderate doses. Their actual efficiency is doubtful but at least they diminish the offensive odor of the bowel movements and the flatus in many cases. Disinfectants may also be added to the enemas sometimes with distinct advantage. Among these in my own experience the most valuable are thymol and formalin. Thymol should be given in O.I per cent. solution and may advantageously be added to the soda enemas. Formalin may also be added to the enemas in amount of 0.5 to I c.c. in 500 c.c., that is, a proportion of 0.1 to 0.2 per cent. It occasionally causes some burning but as a rule is well borne and a rather immediate effect is noted in the reduction of the amount of flatus and the diminution in the odor of the feces. In addition the use of soured milk or lactic acid bacilli has been highly praised. My own experience has been somewhat disappointing. Actual evidence of improvement in the condition of the bowels, the diminution of gas and of the malaise of the patient is difficult to perceive in the majority of cases, but very often buttermilk carefully soured with pure cultures and 
made from a good quality of whole milk with a fair fat percentage, is of great advantage in improving nutrition and in this way helps the patient considerably. The administration of liquid cultures or tablets of lactic acid organisms is not as a rule very successful. I have, however, seen apparent results if the administration has been continued over a long period of time, but, as in all these cases other methods of disinfection were employed, it was difficult to determine to which to ascribe the improvement. Mechanical treatment is also of advantage. The ordinary abdominal binder giving support and preventing movement of the intestinal viscera usually gives a considerable degree of relief. If the cecum is large and can be shifted easily toward the median line the introduction of a pad in the binder, which should lie just to the right of the median line, seems to prevent this abnormal motility and perhaps, by directly compressing the cecum, improves its tone and the condition of the patient. It often, in a short time, appears to abolish the pain entirely. Surgical treatment seems to be less popular than it was at the time of the discovery of this condition. Unquestionably it is rarely indicated. It may, indeed, be a disadvantage, as Craemer has pointed out, to have the cecum fixed during pregnancy; therefore it should not lightly be undertaken in married women or in young women who are likely to marry. As I have already said the mere fixation of the cecum will not improve very materially an existing atony and dilatation. In one case of mine the operation was carried out by Dr. Carnett on a young woman who had suffered for years from pain in the right lower quadrant and who had had her appendix removed without any benefit whatever. The results were rather startling. The pain almost entirely disappeared, the fermentation was distinctly improved, the very obstinate constipation was easily controlled, there was improvement in nutrition and strength and also in what had been supposed to be a severe hysterical or neurasthenic state. 


\title{
THE POSTURAL TREATMENT OF THE ABDOMINAL AND THORACIC VISCERAL DISTURBANCES
}

\author{
By Joel E. Goldthwait, M. D., F. A. C. S.
}

To appreciate all that is involved in a subject of this kind it must be remembered that the human family is not all of one type, in spite of the fact that the text books of anatomy and most of the anthropometric reports would give one to believe this to be the case. That there are three general anatomic types there can be no question, if individuals and not groups are studied, and that each type has its own more or less definite potential of disease there is also but little question.

The type which is described in the text books of anatomy, the intermediate type (Fig. 64), naturally exists, and the general anatomic characteristics are similar to the description found in these works.

Besides this there are two other types which are common, with characteristics so widely different that their structure must be appreciated if proper interpretation of the symptoms is made. Of these the most common is the slender type (Fig. 65), the so-called "Congenital Visceroptotic," the "Carnivorous," the "Narrow-backed," etc., in which practically all of the tissues of the body are different in shape or structure from that which is the text book normal. The skeleton is smaller and lighter, the individual vertebræ are narrower and consequently the spine is more flexible than is normal. The ribs are more delicate and much more round instead of the distinctly flat shape of the so-called normal or the heavier type. The neck is long, the face is narrow, the ears prominent, the skin much softer than normal, with the growth of hair more excessive than in the normal type. The extremities are slender, the muscles are of slender fibers, the hands and feet are slender with tapering fingers and toes. Viscerally the stomach is generally of the tubular or J shape, as it is seen when the patient is standing, having a much looser attachment than is normal. The small intestine is much shorter, having from io to 15 feet of length instead of the 20 feet which is considered normal. The colon practically always has a free mesentery on the ascending portion, with frequently a free mesentery on the descending portion, and the whole colon is usually shorter than is normal.

The other type, the heavy (Fig. 66), or the so-called "Herbivorous" or "Broad-backed" type, is also different from the text book normal, all of the characteristics being heavier and designed apparently for strength and endurance rather than agility. The skeleton is heavier, the vertebræ are not only broader laterally, but are heavier in their general structure. The torso is relatively shorter than in the slender type and the lumbar vertebræ have broader and longer 
transverse processes than the text book normal. The ribs are heavier, broad and flat instead of the more or less rounded shape of the slender type. 'The neck is short and thick, the shoulders heavy, the chest is high and barrel-shaped, the muscles are of the coarse-fibered type, so that the extremities are heavy in appearance, the hands and feet are broad, the fingers short. The visceral condition shows the

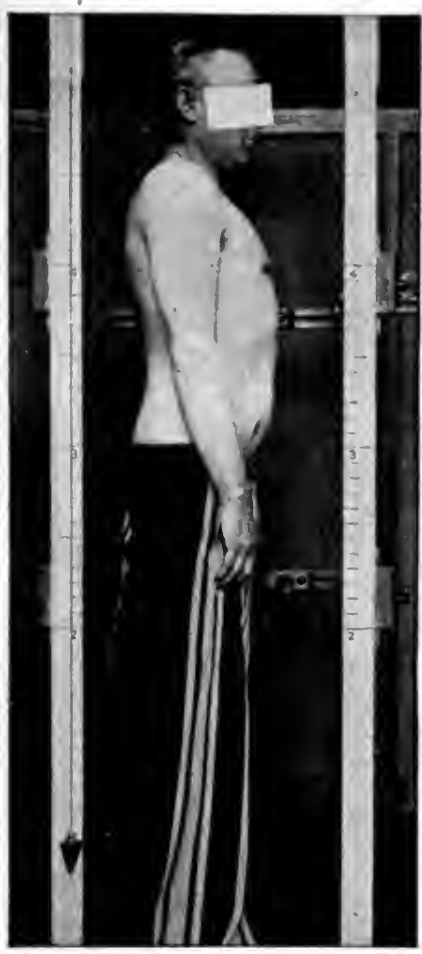

Fic. 64. The normal human type as described in the text-books of anatomy. This is intermediate in all its characteristics.

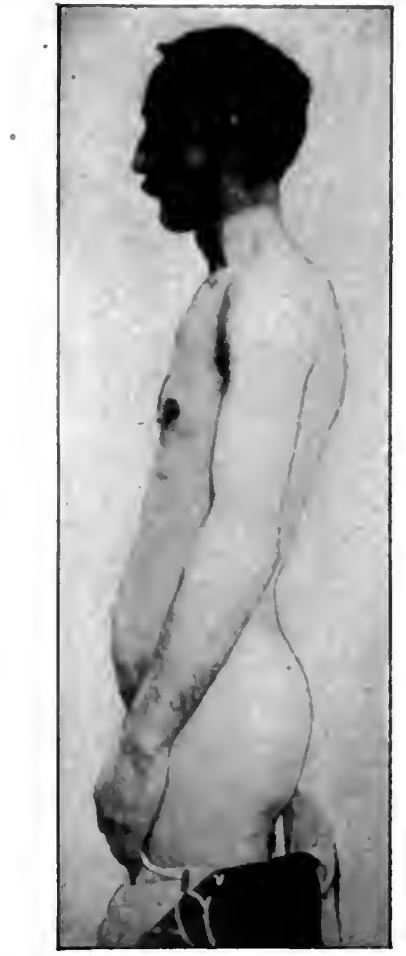

FIG. 65. The slender congenital visceroptotic or carnivorous type, showing the proportionately greater length of body, the increased low lumbar curve, forward inclination of pelvis, forward position of the shoulder, flat chest, prominent lower abdomen, narrow upper abdomen, forward position of head, etc.

stomach pear-shaped and placed high in the abdomen, the small intestine having from 25 to 39 feet in length instead of the normal 20 , the colon being attached retroperitoneally, both the ascending and descending portions, with usually a much longer transverse colon than is normal. The sigmoid flexure is also excessive in length.

With each of these types the postures assumed must vary, and can only be understood by appreciating the anatomic form of the given case. With the slender type the individual practically always 
develops, unless carefully trained, with the flattened chest, so that the chest is commonly used with the neutral point at full expiration. All the breathing is done from the low point, which must, of course, mean that the lungs are used much below their normal capabilities. In standing the.pelvis is usually tipped forward, so that the vertical axis is inclined downward in front much more than is normal. This

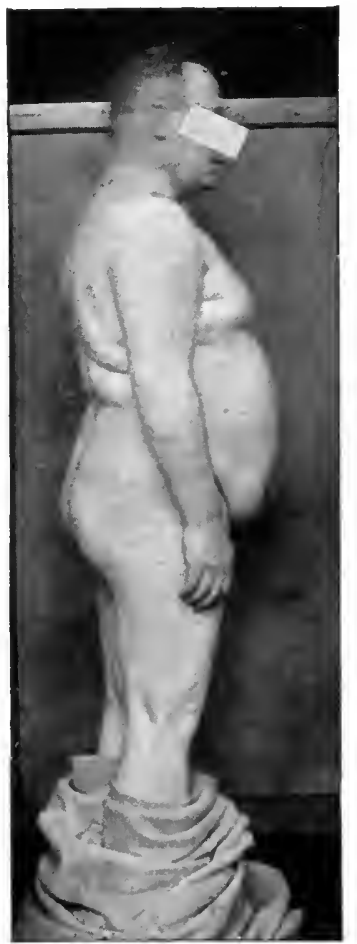

FIG. 66. The heavy or herbivorous type, showing the large, heavy build of the body throughout, the relatively straight position of the pelvis, the backward bend occurring in the upper lumbar region rather than in the low lumbar, showing the round-shaped head, flat ear, broad neck, with the shoulder well back on the thorax.

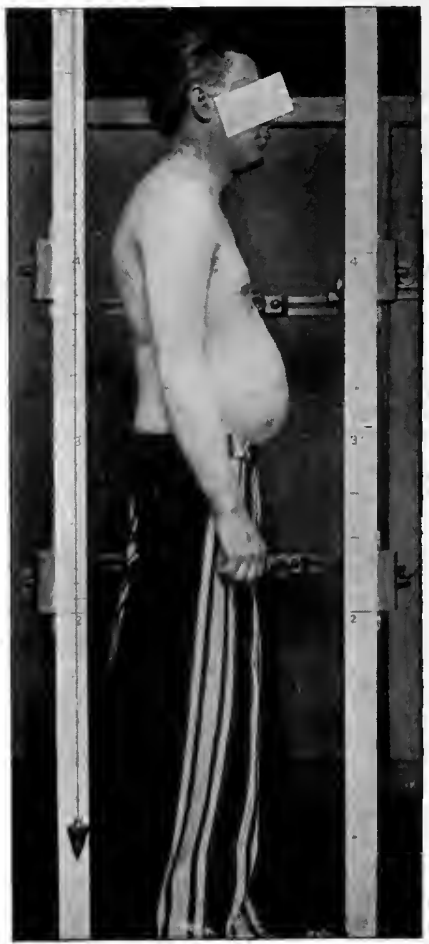

FIG. 67. The same individual as is pictured in Fig. 64, and taken on the same day. The drooped posture naturally leads to a true visceroptosis, with the crowding of the organs downward and forward. The erect position (Fig. 64) draws the organs at once back into place.

makes the hips more prominent and is associated with a sharp lordosis at the lumbosacral juncture, the spine being quite straight but inclined upward and backward from this point to the upper third of the dorsal spine, when it bends forward fairly abruptly, with the sharp backward bend in the cervical region. This results in the head being carried forward much more than is normal. In this position because of the lowering of the ribs there will naturally be the forward or dropped position of the shoulders. In this position with the lowering of the ribs, the diaphragm is naturally pushed downward, the 
anterior abdominal wall is relaxed, and the abdominal viscera are not only pushed downward, because of the lowering of the diaphragm and the ribs, but sag downward because of the absence of the normal support which the anterior abdominal wall should give. In this type of anatomy the viscera are always much below their normal position, partly because of the loose attachments and partly because of the mechanical conditions just described. With the downward displacement of the organs, the fat pads in the upper part of the abdomen will gradually be absorbed until ultimately there is comparatively little of the ordinary posterior fat. This, of course, means that

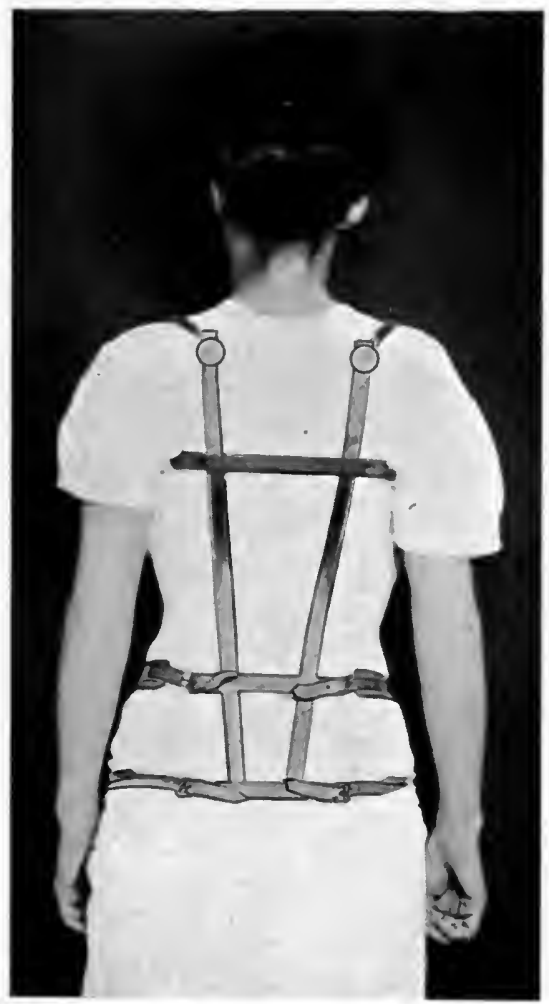

Fig. 68.-Back brace used to maintain the body in the fully erect position.

the kidney and adrenal bodies must be much less protected or supported than is normal, and also the protection which the abdominal fat should give to the abdominal ganglia is lost.

In the normal type, because of the structure of the body, the positions assumed may be with the body fully erect and with the organs in place, or if the body is relaxed the degree of sag and the effect upon the thorax and the abdomen will naturally be much less than in the more flexible and slender type just described. In the so-called normal in standing, when relaxed, the ribs are never lowered 
to the position of full expiration, but they may be lowered enough so that the neutral position is much nearer to full expiration than is normal. In this position also the abdominal viscera are never forced downward as much as in the slender type, but they may be lowered enough to result in quite marked interference with the function of the special organ. In this type in the relaxed position (Fig. 67 ) the bulk of the abdomen is crowded forward and gives the impression of a much larger abdomen than is justified. This is usually entirely corrected when the body is raised to the fully erect position (Fig. 64). Since in this type the colon is attached in the ascending and descending portions, with the downward displacement of the stomach and liver which is a natural part of the sag of the diaphragm, it is not uncommon to have a sharp angle in the colon formed at the hepatic and splenic flexures, with the natural inter-

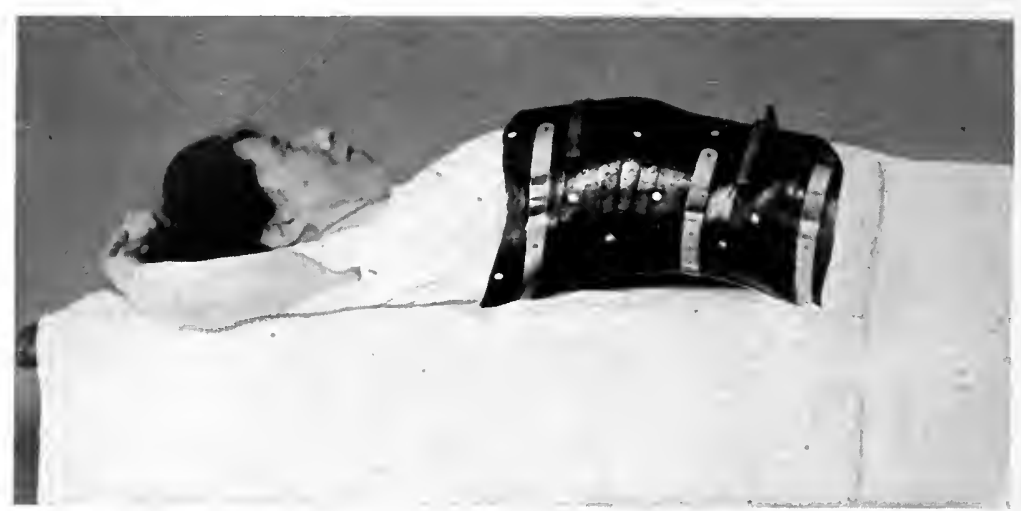

FIG. 69.-The jacket for use at times of recumbency, which has been fitted with the body hyperextended, or with the ribs raised, ensuring the greatest amount of space in the upper abdomen.

ference with the drainage of the organ. In this type, in the relaxed position, the pelvis is usually tipped forward somewhat more than is normal, but never to the extent that is seen in the slender type.

In the heavy type, since the spine is so inflexible, the individuals usually develop so that the body is carried in very good poise all through the growing period. After this, because of the usual increase in weight with the especial increase in the weight of the abdomen, there is commonly a backward inclination of the body, the bend taking place practically wholly at the dorsolumbar juncture instead of at the lumbosacral juncture, as is the case in the slender type. The heavy, non-flexible type of spine explains this peculiarity of the curve in this heavy type. In this type the pelvis is always more nearly vertical than is considered quite normal, and one never sees the marked forward inclination which is present in the slender type. In the backward inclination the lower ribs are always crowded together, 
and while there is some relaxation of the chest, the neutral standing position is but little below the midway position between full inspiration and full expiration. The shoulders are dragged forward moderately, but never to the extent that is seen in the slender type, because the ribs cannot be lowered enough to allow the shoulder to sag to this extent. The abdominal viscera may be moderately displaced, as is true in the so-called normal, and because of the usual increase in the length of the transverse colon, the effect of the downward displacement of the liver and stomach upon the colon will be more marked than in the so-called normal, with greater probability of interference with the passage of the waste of the body by the hepatic flexure and by the splenic flexure. In this type, with the marked increase in the abdominal fat, the weight of the organs results in the gradual stretching of the anterior abdominal wall so that there is

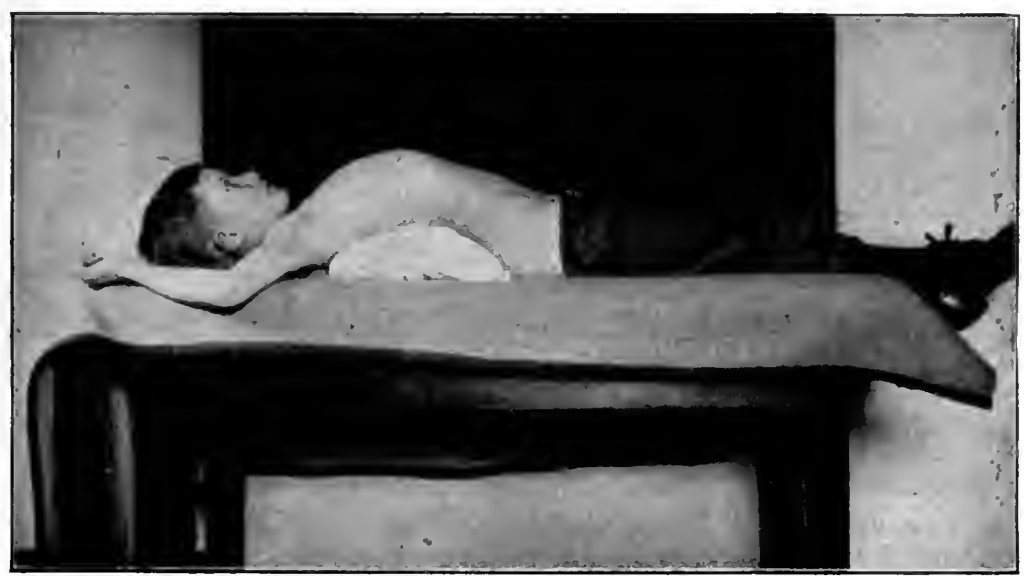

FIG. 70.-The hyperextended position. Note the position of the pillow at the dorsolumbar juncture, with the marked prominence of the anterior portion of the lower ribs.

much less of the normal support, with the result that the organs drag forward and downward as the patient stands, and probably some of the obscure abdominal symptoms referred to the kidney, pancreas, stomach, liver, or bowel, are due to this drag.

The practical significance of this is that in the first place patients should not be treated in routine manner, but that the special form of each should be appreciated in the interpretation of the symptoms, and secondly that the best function of the body as a whole or of any individual part is impossible unless the body is used with all the parts in proper adjustment.

The lungs cannot work rightly if the chest is flattened and the diaphragm is lowered. The same is true of the action of the heart. The abdominal organs cannot work rightiy if they are forced out of place or crowded against each other. If the ribs are lowered in front Vou, IV, -46 
or are narrowed in at the side, there cannot exist the space in the upper part of the abdomen for the organs of that region. The stomach must be low and will very likely have difficulty in emptying itself because of the usual high attachment of the duodenum and the drag upon this which the displaced stomach must cause. The liver must be low and its function may be interfered with, as well as the gall-bladder and its duct. The transverse colon, because it is attached to the stomach and liver, will also be displaced, with many possible resulting disturbances. The kidneys under such conditions are less well protected because of the absorption of the abdominal fat, and are frequently displaced. The other organs may also be displaced and since the displacement will always be partly downward, it is not

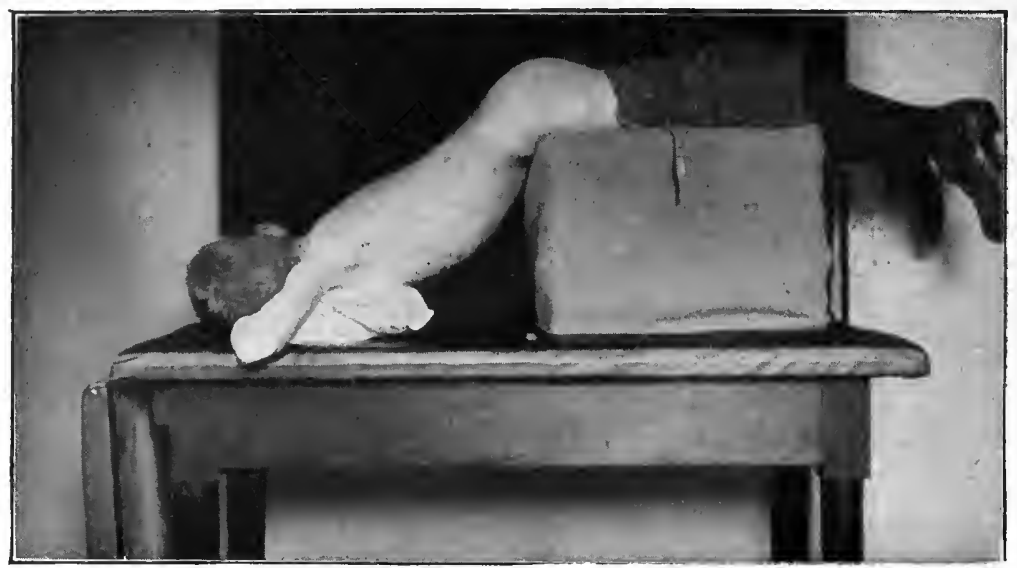

Frg. 71.-The prone hanging position. In this position, if maintained for a few minutes so that the muscles relax, the organs must sag in to the upper abdomen, with the freeing of intestinal kinks, the relieving of an over-loaded stomach, etc., etc.

hard to understand some of the interference with the pelvic organs which is so often seen.

The appreciation of any one feature or symptom without regard to its relationship to the other parts will naturally be unsatisfactory. To try to replace organs without first remodelling the body so that the proper space exists for the organ is obviously irrational. To try to assist an organ by medicine, without trying to relieve the mechanical conditions which may be causing the symptoms, is also irrational. To get the body in good form seems reasonable whether surgery or medicine may also be needed.

The ideal which the orthopedist has in the treatment of such conditions is to restore the body as nearly as possible to the normal poise, in which the proper relationship may exist in all its parts. In this he fully appreciates that it is impossible to change the general shape of the special organ or the general character of the ligamentous attachments. In other words, the special type that the individual 
represents will always exist. He appreciates, however, that the best function of an imperfect organ, as well as the best function of a perfect organ, cannot take place unless all acquired or unnecessary interference is removed. It is well known to those who have given special study to this subject that even when the slender type exists, these patients rarely have symptoms in early life indicative of visceral disturbance. The symptoms which have developed later are undoubtedly due to the increase of the downward displacement of

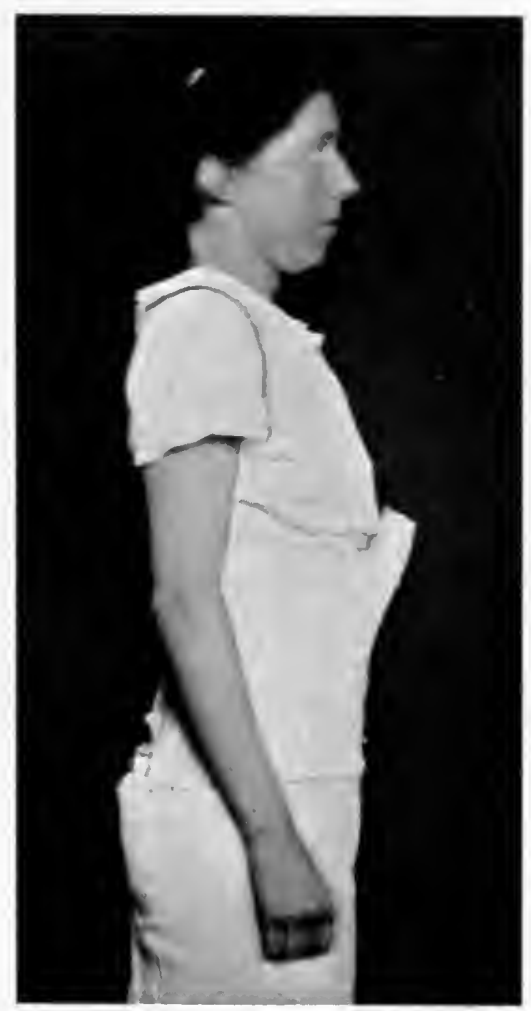

FIG. 72.-A marked congenital visceroptotic individual, held in the fully erect position by means of a back brace (Fig. 68) and well fitted corset which makes support over the low abdomen and hips, with no pressure over the upper abdomen.

the organ or the peculiarity in its formation which must come from the long-continued use of the body in the upright position. Since nature's plan is to repair damage, it is reasonable to expect that the relief of the strain, which resulted in such symptoms, will be followed by improvement, with the contraction of the visceral ligaments, exactly the same as takes place in a relaxed joint with the removal of the strain.

It is felt by the writer, both from general reasoning as well as from extensive experience, that a good prognosis is usually possible 
in such conditions, if the acquired features of disturbance of poise and visceral sag can be overcome.

The correction of the poise or the remodeling of the body is at times comparatively simple and can be accomplished by the use of simple apparatus and special exercises without interfering with the active life of the individual, but at other times the condition is so severe that more radical measures are necessary.

Whenever the disturbance of poise in such cases has been longexistent not only is the relationship between the bones changed, but there is a marked change in the muscle tone of the body. The anterior muscles of the trunk weaken as the result of disuse, since in this position the strain is thrown almost wholly upon the muscles of the back, with the result that there is a gradual weakening of these back muscles. To attempt to improve the position of the body by exercises alone with such a muscular condition present means increasing the muscle weakness, with a distinct interference with the general recovery. The first step, therefore, is to rest the parts which have been overstrained and to stimulate the parts which have been weakened from disuse. This at times can be accomplished by the use of a brace (Fig. 68), which not only has for its purpose the relief of the strain to the spinal muscles, but also the correction of the poise, the elevation of the chest, the forcing of the lower ribs forward and upward, and furnishing support for the pelvis and the lower part of the abdomen. While this apparatus is being worn, massage, stimulating bathing, etc., are all of advantage in hastening the repair. As soon as the evidences of muscle fatigue have passed, active exercises should be started with the idea of gradually strengthening all the muscles of the trunk so that ultimately the poise of the body can be maintained without artificial aid. The brace at such times is usually omitted, the pelvic belt and lighter supports being substituted for varying periods.

In the severer cases recumbency is absolutely necessary, and since in such cases considerable visceral disturbance may take place as the result of the recumbent posture, care must be taken that the body is so held that this tendency is made as little as possible. The common experience that the nights are times of much distress, with disturbed sleep, much gas in the bowel, abdominal pain, the dragged feeling in the morning with the weak pulse and the subnormal temperature, finds explanation if the anatomic formation is considered.

To prevent the sag of the organs which must occur as the muscle reflexes disappear in sleep, a leather or plaster-of-Paris jacket may be used, which is molded from a cast of the body taken when the body is fully extended and with the ribs fully raised. Such a jacket (Fig. 69) is worn during the night, and in such a jacket the patient is allowed to move about or change from back to side or face at will, knowing that whatever position is assumed, harmful interference with the viscera is reduced to the minimum. The quality of the sleep resulting from such support is at times in striking contrast to the quality existing before the apparatus was applied. 
In the severe cases recumbency is insisted upon both day and night at times for periods of many weeks, this being necessary partly because of the faulty position of the ribs, with the retraction of the ligaments at both the costosternal and vertebral articulations. With the body horizontal, the downward sag of the organs is naturally relieved, but it must be remembered that sag in other directions may be quite as harmful in the function of the organs as occurs when the patient is erect. The attacks of vomiting when the patient is kept for long upon the back, with the many and irregular painful abdominal conditions, usually find an anatomic explanation and are usually relieved by change of posture. The position insisted upon in the daytime is with the patient upon a flat bed, with not more than one pillow under the head, preferably none at all, with a small pillow under the back to prevent the backward sag of the lumbar spine, or with the jacket above described with frequent changes of position to the side or face. Occasionally the foot of the bed is raised to still further assist gravity in the replacement of the organs. The hyperextended position (Fig. 70), in which the diaphragm is raised to the extreme, should be taken for at least a half hour after each of the meals, when the stomachic digestion is most active. Besides this, either the knee-chest position or the prone hanging position (Fig. $7 \mathrm{I}$ ) should be taken at intervals, not only to raise the diaphragm and elevate the ribs but also to allow the abdominal organs to sag into the upper part of the abdomen. If the stomach has had difficulty in discharging its contents into the duodenum, because of its low position, not infrequently the mere assuming of the knee-chest position or the prone hanging position is sufficient to relieve the difficulty. Not only is this true, but usually such positions are followed by improvement in the function of the intestine, so that not only is that part of the digestion carried on better, but the interference with the movement of the bowel is markedly lessened.

In such cases, as part of the bad postures, the lower ribs are drawn in, producing an acute angle at the border of the costal cartilages. The recumbent position and the special positions with the apparatus should be continued until the lower ribs have not only been raised, but until they have flared sufficiently to change the costal angle from an acute angle to at least a right angle. This at once assures the space in the upper abdomen under the ribs in which normally the stomach and liver lie.

The recumbent treatment should be maintained also until the general nutrition of the body has improved sufficiently for the retroperitoneal fat, which is almost entirely lacking in the extreme cases of the congenital ptosis, to be replaced. This can easily be determined by palpation through from front to back in the loin and over the lateral spinal spaces.

When it is considered wise for the patient to go about, the brace (Fig. 68) is worn at first, and if the patient is a woman, over this a carefully fitted corset (Fig. 72) is also worn. Special exercises are 
given for the purpose of increasing the muscle tone, and as the strength increases the brace is omitted, the pelvic belt with low abdominal support being substituted. With women this is needed for only a few weeks, after which a corset fitted to give firm support over the pelvis and low abdomen, with no constriction to the upper abdomen or low ribs, is all that is required. With men, the pelvic belt should be worn until the muscles are fully strong and the understanding of the correct poise is so perfect that there is no tendency to acquire the bad postures.

Supervision of such a case should naturally be continued at intervals for a long time for the purpose of preventing relapses. The patients should be made to understand the harm of badly fitted costumes and the great care that should be taken at all times to see that, whatever the occupation, the body is not allowed to flex "in the middle," the bending which may be necessary being at the hips, the trunk as a whole being kept straight.

If such an understanding of the subject is held, the writer feels that not only is the prognosis good in many of these cases now considered hopeless, but that such understanding offers opportunities of team work between the physician, the surgeon, and the orthopedist that shows our profession at its best. 


\title{
SURGICAL TREATMENT OF VISCEROPTOSIS
}

\author{
By John H. Gibbon, M. D.
}

It may be said to the credit of surgery that in the past few years articles on the operative treatment of visceroptosis have been few in number. In our original discussion of the subject a very conservative attitude was taken in regard to operations for this condition and the only object in saying anything further on the subject is to confirm and accentuate this conservatism. Since what is said here is limited to the surgical treatment, it must necessarily be brief.

For general visceroptosis, especially the congenital type, surgery offers very little, and in the acquired type but very little more. It is, therefore, wise to confine operative procedures to the displacement of single organs, such as the uterus and kidney. Operations for ptosis of the liver, the colon, and the stomach are becoming more and more restricted. Extensive operations for general visceroptosis of the congenital type offer about as much hope of success as an endeavor by trimming, bending and binding to straighten out and make symmetrical the shape of an old tree. It is by training and exercise during the period of growth and by support during later life that we should treat this condition.

There is nothing new in the operative fixation of displaced kidneys. The operation of nephropexy should be absolutely confined to the cases in which the displacement gives rise to definite obstruction and other localizing symptoms. And the same statements apply to ptosis of the liver.

No change in surgical opinion which has taken place in recent years has offered the writer greater satisfaction than that regarding fixation and the so-called short-circuiting of the colon. In a recent clinic, one of the most distinguished and progressive surgeons of the country remarked that these operations when done for constipation or for ptosis were absolutely unwarranted. This has been my own opinion for years, notwithstanding the dramatic results claimed by Mr. Lane and others. Many American surgeons who entered enthusiastically into this field of surgery have now lost their enthusiasm and confine their operations only to cases in which there is definite obstruction. The time had certainly come for such a change and we may expect to see fewer futile, mutilating and extensive operations on the colon. Of course, there will always be a certain number of cases of torsion of the sigmoid that can be relieved only by surgical means.

Ileosigmoidostomy is not only a useless operation but, unless accompanied by resection of the colon, leaves the patient in a far 
more distressing condition than before the operation; because in the large majority of instances the colon, through reversed peristalsis, becomes hopelessly distended with fecal matter and gas. No one can thoroughly appreciate this condition who has not operated for its correction. Resection of the colon is a very serious operation and to undertake it for the correction of intestinal stasis is unjustifiable. Acute and chronic obstructions of the small intestine are not unusual sequels of the operation.

Gastropexy is an operation which has a distinct place in surgery, but the indications for it are even more limited than when this subject was discussed in the original article. Many surgeons believe, myself among the number, that in many of the cases of gastroptosis drainage of the stomach in its displaced position by means of a posterior gastroenterostomy or a Finney operation is preferable to gastropexy. 


\title{
PERITONITIS
}

\author{
By John H. Jopson, M. D.
}

\section{INTRODUCTION}

The treatment of peritonitis of all varieties has undergone no revolutionary changes in the time which has elapsed since the publication of the article on this subject in a previous volume of this work. No such strides as marked the progress of surgery in this field in the previous decade have been witnessed. There has rather been a slow but steady progress in the direction of reduction of mortality in peritonitis, and a recognition of what may be termed physiological methods of treatment, with a tendency to simplification of operative measures for its relief. Large series of cases have become available from time to time which throw light on the bacteriology and pathology of appendicitis and peritonitis, and the symptomatology of the less common lesions which give rise to a diffuse or local type of infection has been further elucidated, and a corresponding improvement in the results has been obtained. Additional series of cases showing the end results of treatment in acute diffuse peritonitis in its early and late stages, and of tuberculous peritonitis have appeared from time to time, and attempts have been made to contrast the results of differences of technique on the mortality in both acute and chronic lesions. The abandonment of the older and more radical methods of treatment by multiple incisions, evisceration, mechanical cleansing, and free lavage has been abundantly justified. There has been a more general appreciation of the value of the natural defenses, both local and constitutional, against intraabdominal infections, as well as the necessity of a conservation of the normal resistance to peritoneal infection, and the avoidance of measures which weaken or destroy it. The importance of acid intoxication in peritonitis has influenced the operative treatment and the after-treatment. The further application of the so-called kinetic theory of Crile has influenced to a certain extent the mind and hand of the profession in combating this common surgical disorder. Differences of opinion are still present as to the value of drainage in peritonitis, of immediate operation in the later stages of diffuse peritonitis, and in the operative treatment of tuberculous peritonitis, as well as to minor points in technique. These points will be discussed in detail later.

\section{SEPTIC PERITONITIS}

The classification of cases of septic peritonitis according to their origin, their bacterial flora, the character of the inflammation, and the extent to which the peritoneal cavity is involved, is of great impor- 
tance in discussing the question of treatment. The classification of peritonitis by Flexner into primary, exogenous and endogenous is still of value. Primary cases are those in which the infecting organism reaches the peritoneum by way of the blood stream or lymph channels, and are held by some to be of considerable importance especially in relation to pneumococcic and streptococcic peritonitis. While usually considered rather rare, primary peritonitis according to Fishbein is more common than is usually appreciated. In a series of ro4 cases which he studied, there were thirty-nine which he classified as primary from a pathological standpoint. He found the infection more often of the pure than of the mixed type, and rising from a suppurating focus at a distance, especially in combination with diabetes, cirrhosis, etc. Its importance to the surgeon is lessened by the fact that it is usually a terminal infection. The exogenous cases in which the infection arises from without as from gunshot wounds, operations and abortions are comparatively infrequent, and are more often due to a single organism than to a mixed infection. The endogenous variety is the most common, rising as it does from foci in relation to the abdominal cavity, some of which, as the lower bowel, are loaded with many varieties of microörganisms. This type is more often due to double or multiple infection. The colon bacillus is most frequently recovered by culture in cases of septic peritonitis, partly due to the fact that it inhibits the growth of other organisms in culture. It is usually considered to be associated with a type of peritonitis of lower mortality than when streptococcic infection is present, either alone or in combination. The following table compiled by A. V. Moschowitz in an analysis of 2000 consecutive cases of appendicitis is of interest in showing the mortality of peritonitis in relationship to different varieties of bacterial infection.

\begin{tabular}{|c|c|c|c|}
\hline & Cases & Recovered & Died \\
\hline Bacterium coli... & II 6 & 89 & 27 \\
\hline Bacterium coli and streptococcus. & $I_{4}$ & Iо & 4 \\
\hline Streptococcus . . . . . . . . . . & 13 & 9 & 4 \\
\hline Friedlander's bacillus. & 4 & 4 & 0 \\
\hline Staphylococcus alb.... & 3 & 2 & r \\
\hline Streptococcus and Staphylococcus alb ........... & 4 & 2 & 2 \\
\hline 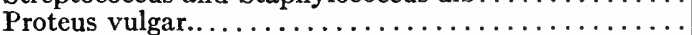 & 4 & 2 & 2 \\
\hline Proteus and Bacillus coli..... & 2 & I & I \\
\hline Bacillus coli, pyocyaneus, staphylococcus and strepto- & & & \\
\hline coccus.. . ..................... & 2 & $\mathbf{I}$ & r \\
\hline Bacillus coli and Staphylococcus aureus. & I & $\mathrm{I}$ & o \\
\hline Streptococcus and Friedlander......... & I & I & $\circ$ \\
\hline
\end{tabular}

Moschowitz has since stated, however, that in the cases studied at the Mt. Sinai Hospital, 500 to 600 in number, no difference was present in the mortality in cases of infection by the colon bacillus, streptococcus or staphylococcus alone or in combination. Rutherford Morrison and Noetzel hold similar views. Streptococcic peri- 
tonitis is prone to be attended by marked inflammation of the peritoneum with but little fluid exudate and spreads rapidly, due to the absence of fibrinous exudate and consequent localization. The staphylococcus, small intestine diplococcus, and the gonococcus are less dangerous. The gonococcus is especially difficult to demonstrate in culture and is therefore often overlooked. In fatal cases of peritonitis bacteremia is often and perhaps usually present.

When we come to consider the pathological lesions of the peritoneum in their relation to treatment, which is usually combined with the treatment of the causative lesion, it must be borne in mind that many of the manifestations of peritonitis are in the nature of defensive processes on the part of the organism to limit and overcome the infection. The exudate, which is usually poured out in large quantities, is itself possessed of bactericidal properties. The lymph is rich in bacteriolysins, while the leukocytes which are present in large numbers once the process of inflammation is established, are vigorous in their phagocytic action. The omentum as well as the parietal peritoneum plays a large and important part in the defensive process, not only contributing heavily to the formation of the exudate, but frequently surrounding and sealing up a perforation, or walling off an infecting inflammatory focus to which it is attracted by chemotaxis. The peritoneum having the two-fold function of absorption and secretion, the bacterial products are carried into the lymph and blood channels for destruction and neutralization, although these natural defenses may be overwhelmed, and septicemia and bacteremia or pyemia follow. The fibrinous exudate which forms in the later stages when bacteria are present in overwhelming numbers, by blocking the channels of absorption and by surrounding and walling off purulent collections, is often life-saving in itself, and makes surgical treatment possible in the presence of suppuration. The paralysis of the bowel at the height of the inflammatory stage aids in localizing a septic process, the spread of which would be favored by normal or excessive peristalsis. The rigidity of the overlying muscles of the abdominal wall, as Crile points out, is a phylogenetic protecting agency against trauma of the inflamed peritoneum and viscera. The methods of treatment which we adopt today aim at the conservation of these natural physiological methods of defense against peritoneal infection. The action of the peritoneum in the direction of secretion and consequent neutralization of infection can be effectively increased while the other normal defensive agents are carefully protected.

The pathological changes which are most striking are, first, the presence of the exudate, at first clear or slightly cloudy, and later becoming opaque and finally purulent. It is often present in considerable quantities within a few hours of the development of an acute appendicitis, when it is usually sterile on culture, while in the late stages it is loaded with bacteria and toxins. Peritoneal infection frequently follows acute inflammation of the appendix without visible 
macroscopic perforation as the diffuse inflammatory process readily permits bacterial migration, an important point recently emphasized by Stanton. Pus may be thick, creamy and foul smelling, especially when yielding colon bacillus to culture, but less virulent than the streptococcic infection which is accompanied by a thin, scanty and sanious pus. The appearance of the peritoneum itself is at first unchanged or slightly injected, retaining its smooth and glistening surface, but later it loses its luster and becomes decidedly injected, roughened, coated with fibrin, or presenting areas of necrosis, or is the site of localized abscesses of which it forms part of the wall. In streptococcic peritonitis where exudate is scanty, it is dry, sticky and inflamed. Its appearance, as well as the character and amount of the exudate, is of great importance to the surgeon in prognosis and treatment, especially as regards the latter question in relation to the problem of drainage, the value of which is still a matter of dispute.

The location of the point of infection has much to do with determining the course and probable outcome of the resulting peritonitis. Exogenous infections, as shown by abdominal wounds and operativs infections, are exceedingly rapid and fatal. In upper abdominal lesions such as perforating gastric and duodenal ulcer, the contente of the upper gastrointestinal tract being comparatively free from bacteria, the prognosis with early operation is fairly good and drainage can sometimes be dispensed with. The large quantities of fluid poured into the peritoneum in these cases, often with the presence of much foreign material, activates the peritoneal infection and produces at the same time such excessive exudation that the prognosis is made progressively worse by every hour of delay in operative intervention. In gall-bladder disease of ordinary severity localization of the inflammatory process to the subhepatic region is the rule unless perforation occurs, when, partly due to the activating influence of the escaping bile, the peritonitis which ensues is attended by a very high mortality. In the lower gastrointestinal tract and the appendix the richness and virulence of the bacterial flora favor a more rapid and dangerous form of peritonitis, which is unfortunately the commonest of all. In pelvic infections, the result of tubal disease and gonorrheal in origin, localization of the process under rational measures is the rule, the gonococcus being of comparatively low virulence. In the streptococcic puerperal infections the mortality is high and little influenced by operative treatment. General septicemia and bacteremia are the rule and the process takes largely the nature of a subperitoneal cellulitis and lymphangitis, which in this region is virulent and largely beyond surgical control. The perforation of any hollow viscus is attended by the continuous outpouring of a secretion which may be sterile or mildly infectious of itself, but which results in an irritative inflammation of the peritoneum and the quick migration of bacteria through the inflamed intestine. Perforations from any cause affecting the stomach, duodenum, gall-bladder and 
urinary bladder are examples in point, and when infection is already present in the viscus the action of the escaping contents is of course enormously increased.

Treatment.-We have reviewed the above-mentioned points in pathology in the attempt to show their influence on the modern conception of the treatment of septic peritonitis. No matter what its origin may be, we aim to preserve always and to increase when possible the processes by which nature overcomes infections, and usually after a prompt removal of the source of infection; and acting in our operative and non-operative measures with due regard to the local conditions, as well as to the general state of the patient. The best treatment of septic peritonitis lies in its prevention and in all types but the primary, which is rare and surgically of minor importance, there is a stage in the history of causative lesions when surgical intervention of an ideal character will prevent its onset. In the cases of exogenous origin as in intestinal wounds, this stage is measured in hours, as it is in the cases of acute appendicitis. In the chronic lesions of the stomach, duodenum, appendix and gall-bladder, the danger signals may be present for months and even years before perforative lesions develop.

The question of treatment naturally divides itself into operative and non-operative, including in the latter class all therapeutic measures not associated with the actual surgical technique. The vast majority of cases will call for both forms of treatment, and while the occurrence of operation, whether in the early or late stages of peritonitis, may have a modifying influence on the purely medical treatment, the general principles of the latter are the same in the preoperative as well as in the postoperative stage.

In the treatment of acute septic peritonitis there are certain cardinal principles to be borne in mind by the medical and surgical attendant. They are often neglected in the preoperative stages, both before and after the correct diagnosis has been arrived at, and on this neglect the mortality is in part dependent. It would seem unnecessary in this day to dwell on the importance of immediate surgical consultation were it not for the fact that many physicians still neglect to share the responsibility and persist in attempts to avoid operative intervention. This is the first and most important point. In all acute intraabdominal lesions which may be attended by the development of peritonitis, we must bear in mind the methods of natural defense already frequently referred to and institute treatment accordingly. The first step is to institute measures to allay peristalsis, the influence of which on the diffusion of infection is well recognized. The ingestion of food and liquids is attended by increased peristalsis, and in the presence of threatened peritonitis, as well as when it is already present, they should be withheld. Purgation, once a favorite treatment for peritonitis, is now recognized as being a potent factor in increasing mortality, and it does this by increasing peristalsis, favoring perforation, spreading local infection 
until it becomes diffuse or general, and causing reversed peristalsis, gastric dilatation and vomiting, with their attendant dangers. The statistics of Pfeiffer are striking in this connection. He studied sixty-three cases of appendicitis complicated by peritonitis and all operated upon. . Of these fifty-six had been purged before admission, and of these seven died. Only seven had not been purged and all recovered. My own experience is in accord with that of surgeons generally, that the most dangerous cases are those that have been purged before operation. It is unfortunate that many physicians have clung to the idea that the so-called unloading of the bowel will relieve appendicitis in its early stages, an idea which is not founded on appreciation of the pathology of appendiceal inflammation.

The prompt institution of postural treatment in peritonitis is also obligatory. By this we mean the adoption of the so-called Fowler treatment by which the patient is placed in the sitting or semisitting posture to favor the drainage of the peritoneal exudate into the pelvis, and aid in its localization in this region. Absorption of fluids in this region is less active than in the neighborhood of the upper abdomen, especially the diaphragm, and drainage by operation is comparatively easy. The risk of pulmonary complications is materially diminished, the patient soon becomes accustomed to the position and usually prefers it after a time to the supine position. Coffey has pointed out that the patient's body should be elevated to an angle of not less than $5 \mathrm{r}$ degrees to insure drainage of the renal pouches into the pelvis. The use of a special bed frame as devised by Gatch makes the maintenance of this position easy.

Hypodermoclysis and intravenous injections of saline and alkaline solutions have their place in the treatment of peritonitis, especially the former. They are used to combat shock, relieve thirst, stimulate excretion, counteract toxemia and restore the body fluids, however they may have been depleted. To Murphy is due the credit for the introduction of proctoclysis in the treatment of peritonitis, a method which has since been popularized in other infections. While many surgeons continue to use normal saline solution exclusively, others use plain boiled water or tap water for rectal instillation, while in cases where acid intoxication is to be feared and especially where food is withheld for long periods, the glucose and soda bicarbonate solution of Crile in 2 per cent. to 5 per cent. strength is an excellent anti-acid and possesses some stimulating and nourishing qualities. More complicated formulæ are recommended by Schiassi and others but are not generally employed. The technique of administration has been fully described in my previous article.

In persistent vomiting, gastric lavage is the most valuable method of treatment. Where the stomach continues to refill at frequent intervals, continuous siphonage by a small tube introduced through the nares, has been recommended and seems reasonable.

Treatment by drugs is symptomatic. Crile and Yates have recommended a return to the opium treatment introduced by Alonzo 
Clark and Flint, and popular with internists and surgeons twentyfive years ago. It is credited with controlling peristalsis, and thereby limiting infection, relieving pain and thus protecting the organs of the kinetic system especially the brain from the nerve exhausting sensory impulses (kinetic drive), and inhibiting metabolism and diminishing the necessity for food. These are strong arguments, but I do not regard them as sufficient in the majority of cases to make us deviate from the usual plan of using morphin sparingly and under definite indications, to relieve pain after a specific course of treatment has been instituted.

Eserin and pituitrin are reliable drugs for the treatment of tympanites and paralytic ileus. In spasmodic ileus a full dose of atropin sometimes relieves in striking fashion. Mechanical ileus of course demands prompt operation.

Adrenalin, pituitrin and camphorated oil are the quickest and most reliable agents to employ in postoperative shock, the former in combination with saline solution administered intravenously or beneath the skin, while strychnin and digitalis are slower in action but can be used where prolonged stimulation is necessary.

Vaccine Treatment.-The serum and vaccine treatments have little value in septic peritonitis. The nature of the infection is beyond the control of this form of therapy with its present limitations. Only in the chronic forms could benefit be expected from vaccines. Van de Velde has reported what seems to be a rational treatment of pelvic abscess, however. After drainage of the abscess through the vagina, he prepares an autogenous vaccine from the pus and administers this before practising laparotomy for the radical treatment of the diseased appendages.

Operative Treatment.-The lesson of the value of prompt operation in the earliest stages of peritonitis has been well learned by surgeons, and is constantly brought home by all statistics which show the percentage of recovery as regards the time of operation. In the treatment of advanced diffuse peritonitis, or what is commonly but incorrectly called general peritonitis, I still believe the method of Ochsner is sometimes preferable to immediate operation. While its value in pelvic surgery is generally recognized, it appears to have lost ground in the favor of surgeons in the last few years. Having seen many examples of its value as a preparatory treatment and as an aid to the localization of infection, I practice it in those cases of general peritonitis which, with a history of long-standing or fulminating infection, exhibit generalized distention, paralysis of the bowel, and frequent vomiting, oftentimes combined with a flagging circulation. The Ochsner treatment is not a substitute for operation, but should be persisted in until the return of peristalsis, cessation of vomiting, subsidence of distention, and localization of rigidity and tenderness over one point marked usually by an intraabdominal mass, give the signal for drainage of the abscess which has resulted.

The general principles of operative technique have not been ma- 
terially modified or changed in the last five years. Rapidity of operation and removal of the cause of the inflammation, and in some cases provision for drainage, continue to be the essential features of operative treatment. In the choice of anesthetics nitrous oxide and oxygen in combination have won wide and increasing favor, being attended with much less shock and depression than is ether in those cases already toxic by reason of absorption, and where resistance is greatly lessened. Infiltration anesthesia, preferably with novocain, is especially valuable in such conditions as typhoid perforation where collapse is often present, and operation must be performed without delay.

The intra-abdominal technique should be as simple and brief as is consistent with the needs of the patient. It must be pointed out that it is influenced by the local or general nature of the peritoneal infection, as well as by the stage of the disease, the condition of the patient and the nature and location of the infecting focus. Here again minor differences of opinion are present among surgeons, which in some cases concern the results as regards the recovery from the peritonitis, by far the most pressing and important consideration, while others affect the prospects of cure of the cause whatever it may be. The first should always be borne steadfastly in mind. As an example may be mentioned the procedures to be adopted in acute perforation of gastric ulcer. In the majority of cases the immediate needs of the patient are served by suture of the perforation and the institution of drainage. The performance of gastroenterostomy at the same time would seem at first thought to be a hazardous and unnecessary complication, prolonging the time of operation and exposing the patient to the dangers of spreading infection to the lesser peritoneal cavity and elsewhere. While Ellsworth Eliot favors simple suture of the ulcer, I think that the papers of Deaver have shown that the immediate mortality is actually lowered by the performance of gastroenterostomy, the danger of secondary perforation being materially lessened, while the end results are also proportionately much improved. Inow practise this procedure in gastric and duodenal perforation. The same considerations apply for and against the search for and removal of the appendix, in localized peritonitis with abscess formation. It is still argued by many surgeons that the operative procedure in these cases should be confined to the drainage of the abscess, and that the appendix should not be searched for, and should be removed only when it readily presents itself. In my previous article I favored its removal in the great majority of cases, as during its removal other collections were frequently uncovered and drained. It might be added as Van Buren Knott points out, that by removal of the appendix the convalescence is materially hastened, fecal fistulæ are much less frequently encountered, secondary abscesses are rare. and the danger of recurrent attacks of perforative appendicitis, which are common when it has not been removed, is obviated. The 
proportion of cases of recurrence of appendicitis when the abscess has been treated by simple drainage has been estimated as high as 25 to 30 per cent. (Knott). Knott has reported 50 r cases of periappendicular abscess without general peritoneal infection, all treated by opening of the abscess, separation of adhesions, removal of the appendix and the institution of pelvic drainage by a large split rubber tube and gauze wicks. He emphasizes all the above-mentioned arguments for primary appendectomy, as well as the fact that the MurphyFowler treatment has robbed the operation of the dangers of spreading a peritonitis by these radical measures. Knott's statistics of six deaths in 5 or cases treated by this method are the best that have been published. G. G. Ross and Eastman have also spoken strongly in favor of removal of the appendix. There are a few cases of large appendiceal abscess, especially of the postcecal type, which are best treated by extraperitoneal incision and drainage without appendectomy at the time. If these are so treated the mortality from septicemia, which is present in any method of treatment, will be materially reduced.

Irrigation has been practically abandoned in the treatment of peritonitis, and discussion of its merits and disadvantages is no longer necessary. It is still of value in perforations attended by the escape of gastrointestinal contents throughout the abdomęn, as a means of rapid removal of the same. Even mopping and sponging of the exudate is usually unnecessary, in view of what has been said as to the protective nature of the exudate especially in the early stages. The suction apparatus of Kenyon and Pool offers an efficient means of rapid removal of an abdominal effusion when desirable. It consists of a large bottle in which negative pressure is produced by continuous water or steam pressure, and which is connected by tubing with special suction tips which can be carried to dependent portions of the abdomen and pelvis, and the fluids rapidly exhausted. While irrigation per se has been largely abandoned, at least in this country, the practice of using antiseptics to combat peritoneal infection is still introduced from time to time. The use of weak tincture of iodine on the inflamed peritoneum, and of ether lavage, as advocated and practised by Morestin and Souligoux, and in this country by DeTarnousky, may be mentioned. The latter method has found some favor in France in other hands than its originators. The introduction into the abdomen of camphorated oil, strength I per cent., at the conclusion of the operation, in quantities of 200 to 300 c.c. is another example, its object being not only to inhibit toxic absorption, and to act as a cardiac stimulant, but also to prevent adhesions. The continuous injection of oxygen after operation, as recommended by Goodwin and Amand may be mentioned as another attempt to increase the local resistance of the peritoneum.

- The value of drainage after operations for septic peritonitis is a matter of much dispute. The tendency among surgeons is to limit the use of drainage as far as possible. Experimental evidence VoL. 1V. -47 
tends to disprove its value as a means of drainage of the abdominal cavity for more than a few hours. At the expiration of this time the drainage tract is shut off by adhesions, and the secretions which then escape are from the granulation tissue forming its walls. In clinical practice, however, where infection is already present, its more prolonged action is a matter of daily observation, and I believe with Murphy that in the free peritonal cavity tubular drainage, when used in the presence of advanced infection, continues to act for a longer time. In the early stages of appendiceal inflammation the clear or turbid exudate, as has been pointed out, is protective and usually sterile and drainage is generally unnecessary. At a later period it is not possible to rely on its sterility or its immunizing qualities by mere inspection. The extensive and unprotected gauze packing formerly employed has given place to tubular drainage, usually in the form of a large rubber tube split throughout its length and containing a wick of gauze which is introduced into the cul-de-sac of Douglas, or carried to the point of infection. Cigarette drains are used as adjuvants to tube drainage, or as precautionary measures where slight localized infection is present or suspected. Unprotected gauze is harmful as it quickly excites adhesions, becomes clogged and ceases to act as a drain, and its removal is difficult and painful, and it also increases the risk of obstruction of the bowel. It is occasionally useful to control oozing or to wall off an actively leaking point of infection. Cigarette drains are removed early, and tubes are shortened as the drainage lessens and removed when no longer needed.

Postoperative treatment is conducted along the lines already laid down under the head of medical treatment. In the case of localized parietal abscesses, in upper abdominal infections requiring drainage and even in diffuse peritonitis, some surgeons recommend the horizontal or lateral prone position after operation, in preference to the Fowler method. This is a rational method if drainage of the pelvis is not necessary, or the lateral position may be used in combination with elevation of the head of the bed to favor pelvic drainage.

Results.-It is difficult to estimate the mortality of septic peritonitis in reference to different methods of treatment owing to the differences of opinion as to what constitutes local, diffuse and general peritonitis. Every feature of the pathology of peritonitis as regards the source and nature of the inflammation, the time of operation, the virulence of the infecting organism, the extent of involvement of the peritoneum, as well as the preoperative treatment, has a bearing on the result, and of tentimes these features are of much more moment than the nature of the operative treatment.

A study of all statistics shows such a wide variation in results that it is at once apparent that different conditions are being dealt with under the same name, and what is described in one series as general peritonitis, will be classified by another as local peritonitis with peritoneal irritation, or the early stage of diffuse peritonitis. 
It is this feature which gives the widest variation to the results, and renders it impossible to compare a series showing a general mortality of 15 per cent. in general peritonitis, so-called, with one which runs as high as 50 to 60 per cent. We must bear in mind that those series which contain a large number of cases of such character as acute gastric and duodenal perforations from ulcer, in which the mortality may rise as high as 35 or 45 per cent., or of typhoid perforation with its attendant mortality of 65 to 75 per cent., or of hemorrhagic pancreatitis or puerperal sepsis, cannot be justly compared with another series of cases of peritonitis of appendicular origin which may show only 15 per cent. of deaths in cases in all stages, and a much lower mortality in early cases alone. The duration of the disease from the time of onset of perforation is perhaps the best guide in judging the results of treatment, and then only when cases due to the same cause are grouped together for purpose of study. In all instances this clearly demonstrates the necessity of early operation. In cases of acute perforation aside from appendicitis the mortality rises with every hour that passes before operation is performed. In appendiceal peritonitis it may be somewhat slower, but each twelve hours that elapses is marked by a steady rise in the death rate. Murphy reported forty-nine cases of peritonitis, most of them due to appendicitis, with two deaths, all operated under forty hours, and Deaver reports I 8 cases with I.5 mortality, all operated within fifty hours. Within twenty-four hours the mortality in acute perforative appendicitis is so low as to be almost negligible, while in perforative cholecystitis, as in rupture of any other hollow viscus into the free peritoneum, it quickly rises to 50 per cent. Gibbon reports I 2 cases of operation for perforated typhoid ulcer with a mortality of 75.9 per cent. and quotes Hay's series of thirty-eight cases with 36 per cent. of recoveries as the best he has found. Deaver reports a remarkable number of recoveries from perforation of gastric and duodenal ulcers. In twenty-five cases there was but one death, and of these twenty-three were subjected to primary gastroenterostomy. The nature of the exudate is of some value in prognosis, as indicating the stage of the disease. The mortality, as shown by the statistics from the Obouchoff Hospital, was 18.3 per cent. in seropurulent cases, and 78 per cent. in tue fibrinopurulent types. The table on page 740, compiled by Herbert A. Bruce, shows the importance of early operation and is derived mainly from European sources (p. 740).

A strong argument in favor of the Ochsner treatment of advanced diffuse peritonitis is found in a comparison of the results of American and European surgeons. As Fay points out, this treatment originated in and appears to be still largely confined to this country. While the mortality of peritonitis from perforation and other lesions which demand immediate operation in any stage is about the same in Europe as in this country, it is difficult to explain the mortality of appendiceal perforation with advanced peritonitis in some foreign statistics without consideration of the operative technique. I believe the gen- 


\begin{tabular}{|c|c|c|c|c|c|c|}
\hline Surgeon & Variety of peritonitis & $\begin{array}{l}\text { No. of } \\
\text { cases }\end{array}$ & Time since onset & Cures & Deaths & $\begin{array}{c}\text { Mor- } \\
\text { tality. } \\
\text { per } \\
\text { cent. }\end{array}$ \\
\hline Hartmann.. & Appendicular. & I5 & Within 36 hours... & I5 & $\circ$ & 0.0 \\
\hline Hartmann.. & Appendicular. & Io & Within 48 hours. & 9 & I & 10.0 \\
\hline Hartmann.. & Appendicular. & I3 & From 2 to 4 days. & 8 & 5 & $3^{8} \cdot 5$ \\
\hline Hartmann.. & Appendicular. & 8 & After $4^{\text {th }}$ day.. & $\circ$ & 8 & 100.0 \\
\hline Hartmann.. & $\begin{array}{l}\text { Perforating gastric and } \\
\text { duodenal ulcer....... }\end{array}$ & 53 & Within I 2 hours. & 37 & I6 & 30.0 \\
\hline Hartmann.. & $\begin{array}{r}\text { Perforating gastric and } \\
\text { duodenal ulcer...... }\end{array}$ & 38 & In I 2 to 24 hours & I6 & 22 & 58.0 \\
\hline Hartmann.. & $\begin{array}{c}\text { Perforating gastric and } \\
\text {.duodenal ulcer..... }\end{array}$ & 29 & In 24 to 48 hou & & 22 & 76.0 \\
\hline $\begin{array}{l}\text { Murphy.. } \\
\text { Siegel... }\end{array}$ & $\begin{array}{l}\text { Chiefly appendicular. } \\
\text { Wounds involving di- } \\
\text { gestive tract....... }\end{array}$ & 50 & $\begin{array}{l}\text { In } 3 \text { to } 40 \text { hours.. } \\
\text { Within } 4 \text { hours... }\end{array}$ & $4^{8}$ & 2 & $\begin{array}{r}4.0 \\
15.0\end{array}$ \\
\hline Siegel. & $\begin{array}{l}\text { Wounds involving di- } \\
\text { gestive tract......... }\end{array}$ & & & & & n \\
\hline Siegel & $\begin{array}{l}\text { Wounds involving di- } \\
\text { gestive tract......... }\end{array}$ & & & & & 63.6 \\
\hline Krylof & Typhoid perforation. & 8 & Within 4 hours. & 4 & 4 & 50.0 \\
\hline Kryloff & Typhoid perforation. & 3 & 4 to I 2 hours. & & 3 & 100.0 \\
\hline Kryl & Typhoid perforation. & 8 & I 2 to 24 hours. & $\circ$ & 8 & 100.0 \\
\hline Kryloff. & Typhoid perforation. & 5 & In 24 hours... & I & 4 & 80.0 \\
\hline Krylof & Typhoid perforation. . & 2 & In 2 days. & I & I & 50.0 \\
\hline Kryloff & Typhoid perforation. . & I & $4^{\text {th }}$ day. & ० & I & 100.0 \\
\hline Kryloff. & Typhoid perforation. & I3 & No definite history & 2 & II & 84.6 \\
\hline
\end{tabular}

eral use of the Murphy-Fowler treatment constitutes one important feature contributory to the excellent statistics of American surgeons, and the combination with a preparatory course of treatment under the Ochsner plan is a second. The reduction of mortality from acute appendicitis in Ochsner's own clinic to 2.I per cent., and this includes many late cases, is another convincing argument against immediate and indiscriminate operation in cases of advanced peritonitis. The influence of technique in the treatment of appendiceal abscess has already been discussed, as has the general question of drainage. Rotter and Bauer are strong advocates of the elimination of drainage where possible, and whereas most surgeons agree with them in principle, they furnish strong statistical evidence. Rotter's mortality in drainage cases was 40 per cent., while in cases of acute appendicitis with peritonitis, not drained, it was $\mathrm{r} 6.5$ per cent. and fell finally to 5.9 per cent. in the latest years. Bauer reports a mortality of but 7.5 per cent. in undrained cases. The fact that they employ drainage under unfavorable conditions modifies to a certain extent the value of their conclusions, as Murphy pointed out.

\section{PNEUMOCOCCUS PERITONITIS}

The proportion of pneumococcic to other infections in suppurative peritonitis is very small but the condition is of sufficient importance to merit special attention, as the treatment of the resulting peritonitis is materially modified where the diagnosis can be made before operation. In Moschowitz's series of I7 I cases there were 
three cases of pneumococcus infection. Netter found it twice in 140 cases, while Salzer found 9 cases in 300 of purulent peritonitis in children. It is most common in children, especially girls, under thirteen years of age, and is claimed by some observers to be primary in the peritoneum in 50 per cent. of cases. It is frequently secondary to or associated with pneumonia, which may escape detection, is sometimes thought to effect the peritoneum bydirect extension through the diaphragm, and rarely arises from the gastrointestinal tract or the Fallopian tubes. When primary it is associated with pneumococcic septicemia, the organisms reaching the abdomen by way of the blood or lymph channels. The general symptoms are high fever, cyanosis and grave constitutional depression or typhoid state along with high leukocytic count, and are early associated with the usual abdominal symptoms of pain and vomiting common to other types of peritonitis. Diarrhea is present instead of constipation as a conspicuous symptom, and the constitutional symptoms in cases of pneumococcus peritonitis are out of proportion to the local objective symptoms, which include a moderate rigidity only, or what is described by Salzer as a doughy resistance of the abdominal wall, and slight distention. In children it must always be remembered, however, that the rapid progress of any type of septic peritonitis is often marked by the absence of the marked rigidity and tenderness of adults, and advanced lesions may surprise the operator at the time of operation in these cases.

Where the diagnosis of pneumococcus peritonitis is clear, especially where pneumonia is present, operation in the acute stages is unwise and it should be deferred until a localized abscess has developed. Salzer and Kahn emphasize this and Deaver and Pfeiffer are in accord with their conclusions. This is in the presence of a well-developed generalized peritonitis in which, if operated upon, one finds the abdomen filled with greenish-yellow, flaky and odorless pus, without local foci in the way of an inflamed appendix, tube or gall-bladder, to explain its presence. Bacteriological examination of the pus clears the diagnosis in such cases. When the diagnosis can be made at this stage before operation, the preparatory treatment described under the general treatment of acute diffuse peritonitis (Ochsner's treatment) should be adopted and persisted in until a localized collection of pus has formed, when the abscess should be carefully opened and drained; a procedure attended by recovery in a high percentage of cases, estimated by Rohr at 87 per cent. The resulting abscess may be central or pelvic, and if not operated upon sometimes points at the umbilicus. In the meantime special measures of a supporting nature will be needed to combat the depression and the general pneumococcic infection. If the peritoneum be opened during the acute stage, Kahn recommends immediate closure without drainage, and a continuation of the waiting plan until abscess develops, rather than to run the risk of introducing a secondary infection by drainage. While the mortality of operation is high in this stage, we 
would prefer to treat it by drainage once the abdomen has been opened, but the results of future observations may confirm Kahn's views.

\section{TUBERCULOUS PERITONITIS}

The observations already made in regard to the treatment of peritonitis in general, and its development in the last five years, apply equally well to tuberculous peritonitis. The review of the literature will disclose no striking innovations or revolutionary changes in our method of treatment or operative technique. Rather has it impressed upon us the limitations in the way of the possibility of a cure of what is a secondary manifestation of a tuberculous infection, the primary focus of which is to be sought for and eradicated when possible, but is sometimes beyond the reach of the surgeon. A consideration of the end results has a sobering influence, when we realize that the percentage of permanent cures is materially diminished over what was formerly thought to be obtained. It has been found that we must follow our operative cases for at least four or five years before we can determine what percentage of them is permanently relieved. The discussion as to the relative merits of operative and non-operative treatment still continues, and we find Borchgrevink, Rose, Krönig and Murphy advocating and practising the latter, as showing a higher percentage of cures. In every case of peritoneal tuberculosis which presents itself a careful preliminary study is necessary before any form of treatment is decided upon. This preliminary study should be made with a view of determining the type of peritoneal tuberculosis which is present in the particular case, the previous duration of the disease, the probable location and accessibility of the primary focus, the presence of imperative indications for immediate operation, the presence or absence of positive contraindications to operation, and the social status of the patient as it bears upon the possibility of prolonged hygienic or sanitarium treatment as an alternative to operation. The data derived from such a study must be carefully analyzed before an opinion is arrived at as to the form of treatment which promises the best results. The decision as to the institution of or persistence in non-operative measures of treatment, as hereafter described, or the performance of operation, and the time at which the surgical treatment is to be undertaken, is based upon the results of the study of the data obtained.

In briefly reviewing the above-mentioned points we must lay emphasis upon the usual differentiation of peritoneal tuberculosis into the ascitic or "wet" cases, or those presenting more or less marked abdominal effusion; the adhesive or "dry" forms; and the ulcerative or suppurative type. The first form, that accompanied by effusion, has long been recognized as the most favorable for operative treatment and as furnishing by far the largest percentage of cures. It was the form in which operation first demonstrated its efficacy, a fact stumbled upon by Sir Spencer Wells. The adhesive form, on 
the contrary, has usually been considered unsuitable for operative treatment by reason of the difficulties and dangers of the operation, the large percentage of postoperative complications, and the unsatisfactory end results in cases surviving or escaping these perils. It is this type which furnishes a large percentage of the small intestine fistulæ which are a dreaded complication of operation, and often the forerunners of a fatal termination. In recent years, however, a surprising percentage of cures in this type of peritoneal tuberculosis has been reported by Caird, Bagozzi and Coenen. The ulcerative type is also considered unsuitable for operation unless abscess forms. This is usually the result of mixed infection or perforation, and in such a case furnishes one of the positive indications for operation in the shape of incision and drainage.

The duration of the disease is important in determining the choice of treatment. In cases in the early stage, or in those in which no systematic treatment has been instituted, a trial of medical and hygienic measures is usually indicated. In a certain proportion of cases, including those with good resistance, and who have been laboring under the disadvantages of bad environment or poor nourishment, this will result in cure. It is always indicated during the period of high fever. Bagozzi urges operation as soon as this stage has passed, while others would persist in medical treatment for from three to six months.

The detection of an intraabdominal lesion which acts as a continuously infecting primary focus of tuberculosis, is of the greatest importance in deciding on the steps which should be taken. When there is a history of recurrent appendicitis preceding this disease, or where tubal infection is present, and tuberculosis of the Fallopian tube is suspected or diagnosed, prompt operation may result in a brilliant cure. A hyperplastic tuberculosis of the cecum may lend itself to excision of that portion of the intestine, while an ulcerative lesion of the same region would furnish an unfavorable prognosis. The accessibility of the primary focus from a surgical standpoint is of prime importance, but the type and extent of the peritoneal involvement must also be taken into consideration.

Among the positive indications for operation are those complications which threaten life, including the formation of an abscess as the result of perforation in ulcerative lesions, perforative peritonitis from intestinal ulceration into the free peritoneal cavity, and mechanical obstruction of the bowel resulting from adhesions. All of these complications demand prompt operative intervention.

Contraindications to operation are found in those types of peritoneal tuberculosis which experience has shown are not benefited, or in which operation is dangerous. The adhesive type has already been referred to. It has been my experience in children, among whom this type is especially common, that the results are almost uniformly bad. The peritoneal cavity is frequently obliterated by adhesions. Attempts to separate the coils of the bowel results in profuse hem- 
orrhage and laceration or necrosis of the bowel, postoperative fistulæ are frequent, and there is no opportunity for reactive inflammation which is one means of bringing about cure. Extensive ulceration of the bowel precludes hope of benefit from operation, as does very advanced pelvic.disease with preëxisting adhesions and fistulæ. Advanced pulmonary tuberculosis always precludes operation, except for the positive indications already enumerated.

The social status of the patient is especially emphasized by Härtel in determining treatment. In some cases this precludes the possibility of prolonged treatment, especially sanitarium treatment, which is so valuable in these cases and it influences one in the direction of early operation to hasten cure.

Medical Treatment.-All observers will acknowledge the benefit and necessity of medical treatment as an adjuvant to operation, while some claim a higher percentage of cures by its persistent use than is obtained by operation. In some types of disease it is the only treatment to be recommended. Some statistics showing as high as 8I per cent. of cures having been published, while the absence of such complications as fistulæ, hernia and the spreading of infection to fresh areas are enumerated among its advantages. Medical treatment includes primarily the placing of the patient in good environment, preferably a sanitarium, and the Germans lay especial stress on the desirability of high altitudes. Rest is indicated as in other forms of acute tuberculosis, and a diet rich in nourishment and adapted to the special needs of the patient is necessary. In cases with ascites Borchgrevink and his associates emphasize the value of a diet poor in salt to increase absorption and favor diuresis. Heliotherapy and the local use of hot air to the abdomen are more or less standard methods of treatment in sanitoria, while roentgen therapy is also used and has some support in the experimental observations on animals by Falk. Tuberculin in the old form should be given a trial, and in combination with roentgen therapy is highly spoken of by Murphy. The local measures described probably act by exciting a local reaction with outpouring of a bactericidal effusion, or by the production of hyperemia and inflammatory reaction with encapsulation of the tubercles, or a combination of both these results. The value of medical treatment is not confined to non-operative cases or to the preoperative stage. As a postoperative treatment it is invaluable and should always be directed in at least a modified form for a long period until the patient regains his health, or until the case is seen to be hopeless.

Surgical Treatment.-The weight of authority is still in favor of surgical treatment of tuberculosis of the peritoneum in selected cases, subject to the limitations which have been described. It embraces such minor procedures as aspiration of fluid, simple laparotomy and the evacuation of localized or general collections of fluid, the removal of primary foci in the shape of a tuberculous appendix or Fallopian tube, and in some instances a supravaginal hysterectomy. It also 
includes the excision of, or short-circuiting of a loop of ulcerated or hyperplastic tuberculous gut, and in some cases the introduction into the peritoneum of some chemical reagent to excite increased peritoneal reaction. We are still ignorant of the exact method by which cure is brought about, but two theories are commonly advanced. Gatti ascribed marked bactericidal properties to the fluid poured out after operation. Postoperative hyperemia and fibrinous exudation with subsequent encapsulation of the tubercles is urged by Hildebrand. In women the possibility of continued reinfection of the peritoneum through the Fallopian tubes is a possible factor, and the ends of the tubes in these cases are often seen to be stiff, open and outstanding. Removal of the exudate permits sealing of the tube ends by the parietal peritoneum (Mayo) and may explain a temporary or permanent betterment.

Where aspiration alone is performed Brückner follows it by the injection of nitrogen in amounts of from 500 to 600 c.c., the injection being repeated in a few days and again in two or three months if the fluid reaccumulates, a method somewhat similar in technique to that used in cases of pulmonary tuberculosis. Laparotomy in ascitic cases or those without marked adhesions should be accompanied by a search for the focus of infection. Where one is found in the appendix it should be removed. It would seem advisable to attempt to get beyond the limits of tuberculous infiltration in the cecum lest a fistula follow, a complication which I have observed. If the Fallopian tube is primarily diseased and not merely studded with secondary tubercles, it should be excised along with the horn of the uterus from which it springs, or a supravaginal hysterectomy may sometimes be justified in extreme pelvic involvement. A badly diseased portion of the bowel may be resected or short-circuited if removal is impossible. Small groups of glands in the ileocecal angle are sometimes removed with safety. Evler has proposed a means of utilizing the immunizing properties which some believe to be possessed by the ascitic fluid, by the formation of a permanent fistula from the peritoneal cavity into the subcutaneous tissues to provide for its continuous absorption, somewhat after the manner employed in cases of ascites due to cirrhosis of the liver. Others would refrain entirely from drainage of the fluid, pleading its local bactericidal properties. The immediate injection into the subcutaneous tissues of some of the fluid removed from the abdomen as an auto-serotherapeutic measure has been recommended, and I have used it myself in this manner. The application of chemical reagents to the tuberculous peritoneum, to increase the reaction and the therapeutic benefits of operation per se, continues to find advocates. Thus Hoffman and Falkner, before closing the abdomen, paint a ro per cent. iodine solution over the peritoneum, while camphorated oil, nitrogen, oxygen gas, and formalin gelatin are among the reagents which have been used at the time of operation. The prevention of postoperative adhesions by the introduction of olive oil or vaseline, in a method 
similar to that which is used in adhesive peritonitis in general, is recommended by Moynihan.

Drainage should always be dispensed with unless a mixed infection is present. Its use favors fecal fistula, secondary infection, and the development of subsequent hernia, while the prolonged drainage of the fluid externally is not desirable or even possible. Reaccumulation of the fluid may call for a repetition of laparotomy. In any event postoperative treatment must be prolonged, and carried out as far as possible along the lines already mentioned. A return to bad environment may result in a relapse or the development of pulmonary tuberculosis.

In estimating the curability of tuberculous peritonitis by comparison of the results of the various methods of treatment, it must be emphasized that recurrence may take place years after apparent cure, and observations must be continued for four or five years before reliable end results are obtained. Borchgrevink and others claim permanent cures by the non-operative method in 35 to $8 \mathrm{I}$ per cent. This wide variation in the number of cures is significant. We must reckon in our figures the mortality from the other forms of tuberculosis, remembering that the peritoneal involvement is secondary. The estimates as to cures after operative treatment vary as widely as after non-operative treatment. Härtel emphasizes, that while the immediate advantages of laparotomy are striking, the longer the observations are continued the greater the tendency for the advantage to be lost. This is especially true in patients returning to bad environment. Moynihan and others still claim that cures can be obtained in from 60 to 70 per cent. of cases operated upon where ascites is present. In a small group of cases reported by Caird, thirty-one in number, the ages varying from thirteen to sixty years, the average being twenty-two years, there were twentyeight operative recoveries and three deaths, all of the dry forms recovering from operation. Of this series, ten cases could not be traced, and of the remainder two were not materially benefited, nine died, and eight were alive and well from two to nine years after operation. A conservative estimate of the permanent cures following operation would be from 25 to 40 per cent., and this in the types most favorable for operation. 


\title{
THE URINARY SYSTEM
}

\section{THE TREATMENT OF NEPHRITIS}

\author{
By Leonard G. Rowntree, M. D.
}

\section{INTRODUCTION, ETIOLOGY AND CLASSIFICATIONS}

To the thinking physician the treatment of nephritis does not consist merely of applying measures of relief. The essence of treatment consists in recognizing the condition and its cause, in understanding the mechanism involved in its production and in the development of its clinical manifestations, in knowing the type, extent and probable outcome of the resulting morphological and functional injury, in valuing correctly the significance of clinical and laboratory findings, and in knowing the indications for and the best methods of applying the measures for its prevention, abortion, amelioration or cure.

In nephritis, as in most diseases, the indications for treatment are derived from three sources.

(A) Etiology.--Removal of the cause constitutes radical treatment and brings a cure in conditions in which irreparable damage has not already resulted. Unfortunately, this can be employed but rarely in nephritis, owing to ignorance concerning etiology in some instances and in others to the fact that the cause is beyond attack.

(B) Pathology.-This is used in its widest sense and refers more to pathological physiology than to anatomical changes. In nephritis it is extremely important, since the treatment of the disease rests upon our conception regarding the functional changes underlying its various manifestations. Indeed the underlying principle of therapeutics is the utilization of our knowledge of pharmacology, physiology and hygiene for the correction of a perverted or pathological physiology. It is, therefore, of the utmost importance in a rational treatment of nephritis to have a definite grasp of the functional changes associated with or resulting in the more important clinical manifestations, such as uremia, edema, and hypertension.

(C) Symptoms and Clinical Course.-Ordinarily symptomatic treatment is unjustifiable and to be avoided. In nephritis as elsewhere it is capable of much harm. However, where other indications fail and when the symptoms are urgent we are sometimes forced to adopt purely symptomatic measures, even when the underlying pathological physiology is obscure.

In the following pages it is the intention of the author to outline, in as simple a way as possible, the method of approaching the 
indications for treatment as weil as to describe the methods of applying remedial measures. A general view will be first taken of the subject of nephritis, which will include its etiology and its various classifications. The various clinical types will be presented along with a consideration of etiology, functional changes, prophylaxis and treatment.

Etiology of Nephritis.-The recognition of the etiology of a disease and its reproduction in the laboratory constitute the most promising channels to its rational and successful treatment. In nephritis these conditions have not been attained. However, a brief consideration of the etiology will not be out of place from the standpoint of prophylaxis and treatment. Considerable is known concerning the association of nephritis with other diseases and with intoxications, but the etiology of a large proportion of the cases still remains obscure. Perhaps no feature of nephritis has been less successfully explored than its etiology. It offers at present a wide field for research.

A working classification of the factors concerned in the etiology of nephritis is proposed by Herrick(I) in his excellent and comprehensive review of the literature of the subject:

I. Infectious diseases, acute and chronic.

2. Chemical, i.e., toxic substances, acute or chronic, in their action.

(a) Exogenous.

(b) Endogenous: (I) Gastro-intestinal; (2) metabolic.

3. Cold.

4. Pregnancy.

5. Heredity.

1. Infectious Diseases.-Acute and Chronic.-Acute nephritis is not an infrequent complication or sequela in some of the acute infectious diseases. Whether it is due to the specific organism, to their toxins or to other causes is not yet known. Scarlet fever undoubtedly heads the list among infectious diseases. Jt is an extremely common disease in childhood. Nephritis develops in from to to 20 per cent. of the cases. It appears most frequently in the second or third week, but sometimes not until the fourth. A severe nephritis may develop in a very mild attack of scarlet fever. The earlier its appearance the severer the course as a rule. The glomeruli are particularly affected. The nephritis may be severe or mild, ending in complete recovery or going over into a chronic form.

Diphtheria is a somewhat less frequent cause. Nephritis may occur quite early in the disease, setting in occasionally with complete suppression of urine. General dropsy is not so common in this as in the scarlet fever variety. The tubules are particularly involved. A point of special interest in connection with this form may be mentioned. The injection of the toxins of the diphtheria bacillus is followed by the appearance of nephritis, thus affording direct proof 
that toxins are capable of setting up a nephritis. The other common diseases of childhood-i.e., measles, German measles, whooping cough, mumps and cerebrospinal meningitis-are only rarely complicated by Bright's disease.

True nephritis is rare in typhoid, pneumonia and acute rheumatic fever, although febrile albuminuria is quite common in these diseases. In typhus, smallpox, cholera and yellow fever it is not an uncommon complication, but these diseases are rare in this country.

An attack of acute tonsillitis, of influenza, or an every-day sore throat may be the starting point, affording a channel of entrance for infection or for the products of infection which result in the development of acute or chronic nephritis. A focus of infection in any part of the body may be responsible for its origin. Consequently in all cases of nephritis of obscure origin it is necessary to search carefully for such foci. Chronic inflammation of the paranasal sinuses (including the antrum of Highmore), chronic tonsillitis, chronic otitis media, pyorrhea alveolaris, alveolar abscess, chronic bronchitis, chronic ulcerative enteritis, chronic appendicitis, cholecystitis, chronic pyelitis, chronic cystitis, chronic urethritis, chronic prostatitis, chronic salpingitis and chronic endometritis, may be mentioned as possible foci. When present, any of these conditions call for radical treatment.

Acute and subacute parenchymatous nephritis is of ten associated with tuberculosis. Many of the supposed cases of nephritis are in reality amyloid. Secondary infection with pyogenic organisms, tuberculous toxins, exposure to cold, drugs and overfeeding probably play some rôle in its etiology.

With septicemia and pyemia, depending on the streptococcus, staphylococcus, pneumococcus, etc., nephritis is not uncommon. In many instances focal metastatic infections result from mycotic emboli from an infected heart valve or from a thrombosed vein. A pyelonephritis, a suppurative nephritis, or a surgical kidney may thus develop. In other cases a true acute nephritis is encountered. A primary epidemic infectious nephritis has been described in Italy, and epidemic nephritis has been observed among British soldiers in the trenches.

While a causal relationship of the infectious diseases to nonsuppurative nephritis is accepted, our knowledge of the exact mechanism whereby it is produced is not satisfactory. In acute nephritis there is a strong tendency to consider bacterial action as the cause. Bacterial toxins are known to be capable of producing nephritis, and they have been held responsible at least in part for its occurrence. Attempts at cultivation of organisms in the urine of patients suffering from nephritis have as a rule failed to reveal organisms other than saprophytes, which occur in the normal urethra. Quite recently G. F. and G. R. Dick(2) have made a successful attempt using newer methods to cultivate organisms in the urine of nephritics. Streptococci or small anaerobic bacilli were found in 
practically all of the cases of nephritis studied, whereas they were always absent on applying the same method to normal individuals and patients suffering from diseases other than Bright's.

The bacteria isolated from the urine of nephritic patients are capable of producing both the urinary and anatomical findings of nephritis. Such findings naturally have a direct bearing on the treatment of nephritis. Urinary antiseptics, however, have not played a rôle in its treatment. These findings suggest the advisability of giving them a trial.

Some of the protozoal diseases are also complicated by nephritis. Much discussion has centered around syphilis. By some the mercury employed in its treatment is ascribed as the cause of nephritis rather than the syphilis itself. Certainly mercury has a striking affinity for the cells of the renal tubules, particularly those of the fourth part of its proximal convoluted tubules, and is capable of setting up a nephrosis and consequently it must be considered a possible cause of injury. It is, however, essential to the treatment of syphilis. Special attention must, therefore, be paid to the kidney throughout its administration. The rôle of syphilis in producing chronic sclerotic changes, generally and also in the kidney, is almost beyond question. Arsenic, on the other hand, is capable of setting up a type of nephritis in which the glomeruli are injured preponderantly. In all cases of nephritis in which lues is considered as a cause the evidence should be very clear before intensive antiluetic treatment is instituted. ${ }^{1}$

Malaria must also be considered as a cause. Thayer, who analyzed the cases occurring in Baltimore, found nephritis in 4 per cent. of the patients with æstivoautumnal infection. Febrile albuminuria is very common in malaria. Nephritis has also been described in relapsing fever and trypanosomiasis.

It is extremely important to recognize the association of nephritis with infectious diseases or with a foci of infection somewhere in the body, since the proper treatment of the infection is the best prophylactic measure we have for the prevention of nephritis.

2. Toxic Chemical Substances.-The nature of the toxic substances responsible for nephritis in infectious diseases is not known. Undoubtedly in the majority of cases of nephritis the toxic agent, exogenous or endogenous, is unknown. There are, however, many substances that are capable of its production and which are responsible unquestionably in some cases.

(a) Exogenous Substances.-Drugs; arsenic, cantharides, mercury, salts of chromium and uranium, potassium chlorate, salicylic acid and its compounds, oxalic acid, the mineral acids, alcohol,

${ }^{1}$ In a case of nephritis occurring in the course of secondary lues $0.45 \mathrm{gm}$. salvarsan was followed by an immediate and marked change for the worse. The phthalein dropped from $5 \mathrm{I}$ to 22 per cent. excretion, the albumin increased from 20 to $96 \mathrm{gm}$. per liter, while the condition was considered hopeless. Within three weeks tremendous improvement had occurred, and the patient left the hospital after six weeks with a normal phthalein and only a trace of albumin. 
chloroform, phosphorus, tartrates, lead, vinyl amin, phenol, turpentine, balsam of Pine, diphtheria toxin and cobra venom, when given in the proper dosage result in renal changes, in nephritis, or in conditions closely resembling it. Many of these drugs are not used medicinally, and hence their presence in the body is the result of accident or of intent to injure.

Peculiar interest attaches to some of these bodies because of the type of injury produced. ${ }^{1}$ Thus arsenic and cantharides produce lesions which are chiefly glomerular, while those of chromium, mercury and tartrates are chiefly tubular. It must be remembered, however, that the injuries are never purely tubular or purely glomerular. The lesions are degenerative in character, not inflammatory. Functional studies have been carried out in these experimental nephroses and have been made the basis in attempts at classification of the nephritides. Uranium produces lesions at first chiefly tubular, later diffuse. It is of peculiar interest because of the possibility of producing edema in this form. Chronic nephritis closely resembling the clinical type (interstitial nephritis) has been produced by its prolonged use in small doses. Vinyl amin results in papillary changes only. Lead is perhaps the most important exogenous intoxication leading to hypertension. It is responsible for hypertension in plumbism. Arteriosclerotic and chronic interstitial renal changes are frequent clinical accompaniments in chronic lead poisoning. Phosphorus and chloroform injure the kidney to some extent, but as a rule the liver injury predominates.

The question of adulterated foods is also important. Ptomain poisoning may result in severe renal injury. In mushroom poisoning (3) (amanita phalloides) tubular degeneration analogous to that of chromium posioning may be found. The too free use of highly seasoned food is considered a possible source of injury in some cases.

Alcohol in itself is probably not capable of producing nephritis. However, it is a great contributing cause. Its abuse frequently leads to exposure to cold which is followed by nephritis. Bacchus as well as Venus must be held to account for some of the cases of luetic origin. Poverty and decreased resistance, the result of alcoholism, are responsible for still other cases. Lastly, alcohol probably plays a considerable rôle in some instances of the arteriosclerotic type.

(b) Endogenous Substances. - Nephritis occurs at times in acute gastrointestinal disorders, especially in children. Whether this results from organisms, from abnormal products produced from food or from both is not known. O'Hare (4) has produced, through the administration of colon bacilli and uranium nitrate, a renal lesion in rabbits similar to that of chronic nephritis in man.

Gout is regarded as a frequent cause of nephritis, particularly of the chronic form exhibiting associated cardiac changes. This is not always the case, since hypertension and nephritis are sometimes

${ }^{1}$ In this connection see Suzuki Zur Morphologie der Nierensekretion, Jena, 1912; also Schlayer u. Takayasu Deutsch. Arch. f. klin Med., 1910, xcviii, 7 . 
absent in gout which has persisted throughout a long life. Occasionally nephritis appears in the course of diabetes. In jaundice, albumin and casts are commonly present, but these disappear with the jaundice; in some instances a renal lesion persists.

Martin Fischer(5) describes an acid condition of the parenchymal cells of the kidney as the fundamental pathological change in nephritis. According to this author the renal cells constitute a colloidal membrane interposed between the blood and the urine. When these cells become acid the colloidal albumin becomes soluble and appears in the urine. Fischer's experimental studies which he brings as proof for this theory do not reproduce conditions as they exist in the body, most of the work being carried out with mineral acid and in a concentration infinitely greater than is possible in the organisms. He has not proved his contention, but the view is new and deserving of considerable investigation. Clinically the use of alkalies advocated on the basis of this theory has been disappointing on the whole; but anuria is occasionally temporarily or permanently relieved by their employment, particularly in cases of acute and chronic parenchymatous nephritis.

3. Cold.-Exposure to cold and wet is one of the most frequent causes of acute nephritis and of acute exacerbations in the chronic form. This is particularly apt to follow exposure after a drinking bout. How cold acts is not clear. It may be that exposure favors the action of bacteria and that it lowers the resistance of the kidney to infection or to the action of the toxins. It is ascribed as the cause in probably 50 per cent. of the cases of acute and subacute nephritis in adults.

4. Pregnancy.-The development of pathological findings referable to the kidney is common in pregnancy. True nephritis is not rare. Two possibilities as to its etiology have been advocated-that suggested by Rayer ascribing it to increased intraabdominal pressure with pressure on the renal vessels and ureter, and secondarily one by Virchow, who considered it the result of a toxemia depending upon altered conditions of life, lack of exercise, neglect of hygiene, particularly of the skin and bowels, constipation, etc. Perverted metabolic processes, particularly in nitrogenous metabolism, may result in the development of toxins.

5. Predisposing and Contributing Factors.-Age.-Parenchymatous forms of nephritis are more frequent in the young, when they are often dependent on infectious diseases. In later life arteriosclerotic and hypertensive cardiovascular disease (included under interstitial forms) are more common.

Heredity. - A tendency toward nephritis is unquestionably transmitted from one generation to another. This holds true more for the chronic form with vascular changes. Arteriosclerosis may be a family disease, the kidney sharing in the general process. Dickinson(6) records a remarkable family history including four generations. Three out of five in the first generation, four out of the six in the 
second, five out of a family of six in the third, and one, a child, of the fourth, were affected with albuminuria. Herrick also has described a family with many members showing evidence of arteriosclerosis and nephritis.

Sex.- The disease is more common in the male than in the female, because of greater exposure and greater wear and tear in life. Pregnancy and pelvic infection are prolific sources of nephritis and tend to make the distribution more even.

Occupation.-Those coming constantly into contact with alcohol, such as brewery workers, saloonkeepers and bartenders are particularly susceptible to the disease. Workers in mercury, lead, and those exposed to extremes of heat and cold are also prone to it. Arteriosclerosis and hypertension develop in individuals the victims of a strenuous business life. Nephritis may develop from extensive lesions of the skin, such as burns or chronic disease. Myocardial insufficiency is a frequent accompaniment of nephritis. It is the result rather than the cause in the majority of instances. However, congestion of the kidney as a result of a cardiac lesion may presumably be an etiological factor at times.

The epidemic of acute nephritis ${ }^{1}$ occurring in the ranks of the British army in the present war is of peculiar interest in many respects. Throughout the exposure of a hard winter practically no cases of acute nephritis were observed. In February I915, it made its first appearance. By months the incidence of acute nephritis were as follows: February, 72: March, 138: April, 220: May, 211: and June, 326 cases. Cold and exposure apparently had no causal relationship to this epidemic of acute nephritis. Attempts to explain it in relation to tonsillitis, ascending infection, blood infection were futile. The type was a diffuse nephritis and the cause remains unknown.

But in many instances, no cause may be found. Nephritis is often very insidious in onset, careful study of the patient and the anamnesis failing to reveal a cause. In a rather large proportion of chronic cases we are loath to ascribe any cause. Excessive eating, drinking, or use of tobacco, or a too strenuous life, is often made the etiological scapegoat.

Classification of Nephritis.-Definition.-The term nephritis means inflammation of the kidney and, as usually employed, signifies a non-suppurative type of inflammation. It is commonly considered as synonymous with Bright's disease or morbus Brightii. While this is not absolutely correct, since Bright's disease includes only types with edema and albuminuria, custom has sanctioned this use of the term. Strictly speaking, inflammation of the kidney is not present in all the conditions classified under nephritis. Kidneys showing degenerative lesions, granular atrophy and cloudy swelling are commonly included. Pure congestion and amyloid changes in the kidney are usually considered under, separate heads.

${ }_{1}^{1}$ Paris Letter, Jour. Am. Med. Assn., Dec. 18, 1915 , p. 2181.

Vol. IV. -48 
Since the appearance of Bright's(7) first paper many attempts have been made to classify the nephritides. These have usually proceeded along the clinical and anatomical and occasionally along etiological lines. Of late years similar attempts have been made utilizing physiological variations as revealed through renal functional studies as the basis for classification. All efforts in this direction, however, have been attended with great difficulty and have met with little success. Although definite types are distinguishable the gradations and variations are such that following the acceptance of any classification a large proportion of cases are atypical and become difficult to pigeonhole.

There is general agreement as to the division of all cases of nephritis into two classes-acute and chronic. Clinically and anatomically even this is not always easy. Greater difficulty, however, is encountered in subclassifying chronic nephritis. While there is a tendency on the part of some clinicians and pathologists to attempt many subdivisions and exact diagnoses in nephritis, there is also a tendency in the opposite direction on the part of others. There are excellent physicians who prefer to make a diagnosis simply of chronic nephritis, and good pathologists who report chronic diffuse nephritis.

The classifications which appear most helpful to the author are given below.

On clinical grounds nephritis can be divided into acute and chronic forms. In acute nephritis the etiology may be clear, as in scarlet fever and diphtheria, malaria and pregnancy, etc. The cause, however, is so frequently obscure that an etiological basis does not answer well for a general classification. Two forms of chronic nephritis are commonly recognized. To one form, characterized by edema, marked albuminuria and cylindruria, the name chronic parenchymatous nephritis is applied; while to the other, with marked cardiovascular changes, slight albuminuria and cylindruria, the name chronic interstitial nephritis is given. As synonymous for the former, the large white kidney, chronic desquamation and chronic tubal nephritis, chronic diffuse nephritis with exudation, may be mentioned; and for the latter, secondary contracted or small white kidney, primary contracted or small red kidney, cirrhotic, sclerotic granular atrophy, gouty kidney, arteriosclerotic kidney and senile kidney. Since the cardiovascular phenomena play a very important rôle, the name hypertensive cardiovascular disease has also been proposed.

On Anatomical Grounds.-Pathology has afforded no more successful field for discussion than that of nephritis. Indeed so little agreement exists that Aschoff(8) writes: "Hier jeder Pathologe sein eigenes Glaubensbekentniss hat." Where discussion is still rife among pathologists a detailed consideration along pathological lines would be entirely out of place in an article dealing with the treatment of nephritis. However, a word or two concerning the trend of the classifications would not be amiss. 
Aschofi divided nephritis into acute inflammations:

Nephropathia inflammatoria chronica.

Nephropathia degenerativa chronica.

Nephropathia circulatoria chronica.

Volhard and Fahr(9) make the following divisions:

A. Nephrosis (degenerative nephritis). This is unaccompanied by increase in blood-pressure and may or may not show amyloid changes.

I. Acute stage.

2. Chronic stage.

3. Terminal stage (contracted kidney-very rare).

B. Nephritis (inflammatory).

I. Diffuse glomerular nephritis (acute, chronic, and terminal stage). This is accompanied by elevation of bloodpressure. In terminal stage renal insufficiency may develop.

2. Focal nephritis (no elevation of blood-pressure).

(a) Focal glomerular nephritis (acute and chronic).

(b) Acute interstitial non-suppurative nephritis.

(c) Embolic nephritis.

C. Arteriosclerotic kidney (associated with high blood-pressure).

I. Pure arteriosclerotic kidney. (Little or no glomerular change. No evidence of renal insufficiency. "Benign Hypertension.")

2. Combination form. (Scleroses associated with secondary glomerular changes. Renal insufficiency common. "Malignant Hypertension.")

The classification by Senator is simple and practical and is the one most familiar to the profession generally.

A. Acute nephritis.

B. Chronic nephritis.

I. Chronic parenchymatous nephritis, or chronic diffuse nephritis without induration.

2. Chronic interstitial nephritis, or chronic diffuse nephritis with induration.

(a) Primary chronic interstitial nephritis.

(b) Secondary chronic interstitial nephritis.

(c) Arteriosclerotic kidney.

3. A diffuse nephritis, a combination of forms one and two.

The correlation of clinical and autopsy findings has been the basis for a great amount of work. It has met with only a measure of success, the autopsy findings being very different from those expected.

On Functional Grounds. - Recently methods for studying the function of the kidney in health and disease have been introduced. This 
subject, though still in its infancy, promises to shed much light on pathological physiology of the kidney in its various diseases, and to be of great importance from the standpoint of diagnosis, prognosis and treatment.

Through changes in general blood-pressure and responses of the renal vessels resulting from various stimuli and from the ability of the kidney to excrete certain test substances Schlayer(ro) and his coworkers have attempted a functional classification of nephritis. On the assumption of selective excretion by: different parts of the kidney-e.g., that lactose and water are excreted specifically by the glomeruli while chlorids and iodids are excreted only by the tubules, an attempt has been made by them to divide nephritis into the following types-glomerular, glomerular tubular, tubular glomerular and tubular. However, proof that these substances are handled specifically by different parts of the kidney has not been established. Granting that they are so handled we(II) have met with insurmountable difficulties in attempting this classification in clinical nephritis, and with other investigators have encountered great difficulty in making such a classification in experimental forms of nephritis, such as the nephroses resulting from chromium and mercury on the one hand and cantharides and arsenic on the other. Some idea as to the relative degree in some instances in both experimental and clinical conditions, but a classification at this time on this basis is impracticable. The work has resulted, however, in a definite advance in our knowledge of the pathological physiology involved and has had an important bearing on our attitude toward certain clinical phenomena, such as polyuria, oliguria, hyposthenuria, etc., and on our treatment, particularly in relation to the administration of water, salt and diuretics.

Widal has recently attempted to divide nephritis into two types -chloremic and azotemic-which are primarily unable to excrete sodium chlorid and non-protein nitrogen. While it is undoubtedly true that there is a type of nephritis which cannot excrete sodium chlorid but can excrete nitrogen, it is exceptional to find a case in which there is normal chlorid excretion associated with retention of nitrogen.

From my personal experiences with functional studies I feel that in the present state of our knowledge it is inadvisable to attempt classifications solely on functional grounds, except in the effort to learn, from an academic point of view, what correlation is possible between clinical, functional and anatomical findings. On the basis of functional and clinical studies it is possible to divide nephritis, with regard to the degree of involvement, into mild or severe forms with or without cardiac involvement.

For the better understanding of nephritis and for improvement in its management, functional studies are essential. Attempts at correlation of the clinical, anatomical and functional findings are also of the greatest importance. A complete diagnosis of a given 
case should include the clinical, anatomical and functional, and, when possible, the etiological considerations.

The functional studies are not of so great value for the classification of nephritis but serve as guides in prognosis and treatment. The ability of the kidney to excrete water, salt and nitrogen can be readily determined, thus affording criteria in relation to dietary control.

\section{ACUTE NEPHRITIS}

Etiology.- Since the general subject of etiology of nephritis has been already considered in some detail, only a few words need be said as to the more important causes of the acute type.

I. Cold.-Exposure to wet and cold is one of the most common causes in adults, particularly when the exposure occurs during or immediately following a drinking bout.

2. Infectious Diseases.-Scarlet fever is responsible for the majority of cases of acute Bright's disease in children. Measles, diphtheria, typhoid fever, chickenpox, smallpox, malaria, cholera, yellow fever and meningitis are occasionally accompanied or followed by it. Syphilis is also a prolific source, cold often being ascribed as the exciting cause. It is not uncommon in tonsillitis. In tuberculosis, septicemia or rheumatism it may also appear.

3. Toxic Agents.-The use of turpentine, cantharidin, potassium chlorate and carbolic acid in too large doses or over too long a period occasionally results in renal injury and sometimes in acute nephritis. Nephritis may follow mercury and arsenic administered therapeutically or taken with suicidal intent.

4. Acute nephritis not uncommonly develops in pregnancy. Sometimes it is only an acute exacerbation of a chronic form, while in other patients it appears where previously the kidneys have been perfectly normal.

5.'Extensive Skin Lesions.-Burns or chronic skin diseases, trauma and operative procedures on or about the kidney are occasionally responsible.

Morbid Anatomy.-This has been briefly summarized by Osler(r 2). For a brief description we probably cannot do better than to quote him: "The kidneys may present to the naked eye in mild cases no evident alterations. When seen early in more severe forms, the organs are congested, swollen, dark, and on section may drip blood. Bright's original description was as follows:

"The kidneys $* * *$ stripped easily of their investing membrane, were large and less firm than they often are, of the darkest chocolate color, interspersed with a few white points and a great number nearly black, and this, with a little tinge of red in parts, gave the appearance of a polished, fine-grained pophyry or greenstone. $* * *$ On section these colors were found to pervade the whole cortical part, but the natural striated appearance was not lost, and the external part of each mass of tubuli was particularly dark. *** 
A very considerable quantity of blood oozed from the kidney, showing a most unusual accumulation in the organ.

"In other instances, the surface is pale and mottled, the capsule strips off readily and the cortex is swollen, turbid and of a grayish red color, while the pyramids have an intense beefy red tint. The glomeruli in some instances stand out plainly, being deeply swollen and congested; in other instances they are pale.

"The histology may be thus summarized:

" (a) Glomerular Changes.--The tufts suffer first and there is either an acute intracapillary glomerulitis, in which the capillaries become filled with cells and thrombi, or involvement of the epithelium, of the tuft and of Bowman's capsule, the cavity of which contains leukocytes and red blood corpuscles.

" (b) The alterations in the tubular epithelium consist in cloudy swelling, fatty change and hyaline degeneration. In the convoluted tubules, the accumulation of altered cells with leukocytes and blood corpuscles causes the enlargement and swelling of the organ.

"(c) Interstitial Changes. - In the milder forms, a simple inflammatory exudate-serum mixed with leukocytes and red blood corpuscles-exists between the tubules. In severer cases, areas of smallcelled infiltration occur about the capsules and between the convoluted tubes."

With the use of all the methods at our command, it is possible during life to determine at best only approximately what part of the kidney is chiefly or primarily involved. On the other hand, for purpose of treatment, extremely important information concerning the degree of renal functional injury can be obtained.

Prognosis.-Two features enter into consideration-death and the development of chronic nephritis. The prognosis as to death during the attack is largely dependent upon the etiology. Scarlatinal nephritis in young children has a very high mortality, death resulting in about one-third of the cases, while in acute Bright's dependent on cold, recovery is more frequent. Certain symptoms are always of serious import, i.e., low arterial tension, uremia, and effusion into the serous sacs. The persistence of dropsy for more than a month, with large amounts of albuminuria and extreme pallor points to the disease becoming chronic.

When associated with specific fever, it becomes almost impossible at times to ascertain whether the patient is suffering from a toxemia due to non-excretion or one due to a specific toxin of the fever. The capacity of the kidney to excrete can be readily determined, but this means but little prognostically on account of the rapidity with which marked changes in this respect occur. Clinically, I have seen cases of scarlatinal nephritis with Io per cent. phthalein output for two hours excrete 28 per cent. four days later and the normal amount within two weeks. Experimentally, chromium animals with a zero output for two hours have returned to a normal excretion within ten days, while twenty-four hours later, more chromium having been 
given, the phthalein was again not excreted. It is evident, therefore, that frequent repetitions of tests are essential to prognosis in acute nephritis. But when a patient exhibits no phthalein, no lactose, together with a high blood urea concentration, the case must be considered a grave one, though not hopeless. The immediate danger from the renal inadequacy factor is at least determined.

Cases of acute nephritis, especially the scarlatinal type, may clear up rapidly, edema disappearing within a weck and the urine being normal at the end of a month. Dropsy may recur and yet complete recovery ensue even after the lapse of a year. When the albuminuria and anemia persist over several months, the disease is very apt to go over into the chronic form. Death may occur from uremia, especially in the young, from anuria, from edema, and rarely from cerebral hemorrhage or acute anemia. Cardiac weakness may develop suddenly.

The Treatment of Acute Nephritis.-This consists of prophylactic measures for its prevention, the treatment of the nephritis itself and the management during convalescence.

Prophylaxis.- Just how much can be accomplished in a given case by methods designed to prevent the development of nephritis, it is difficult to say. However, all wisely chosen measures of prevention in conditions in which its development is a possibility are worthy of trial. Chief among these conditions are the infectious diseases. The judicious use of specific remedies, of quinin in malaria and of antitoxin in diphtheria, undoubtedly wards off many cases of Bright's disease. In arsenic and mercury, the specifics for syphilis, we have a two-edged sword. However, through properly controlling their administration and in eradicating syphilis, a prolific source of nephritis is removed.

In the other infectious diseases, careful nursing and proper treatment probably have some effect in decreasing the incidence of nephritis. Generally speaking, their proper treatment ${ }^{1}$ is a prophylaxis against nephritis. The free use of water combined with catharsis may be the means of preventing nephritis. However, the determining factors in the development of the nephritis are the susceptibility of the individual and the intensity of the infection. The latter plays the minor rôle since severe nephritis may appear in a mild attack of scarlet fever.

Mild infections, such as simple sore throat, should receive proper attention. Tonsils, the seat of repeated tonsillitis, should be removed. All foci of infection anywhere in the body, so far as possible, should be treated radically.

Extreme care should always be practised in the use of drugs that are irritant to the kidney, but this is especially true in all diseases

${ }^{1}$ In scarlet fever, for instance, Jaccoud insists upon the value of a milk diet in the prevention of nephritis. When the arterial tension becomes markedly increased and large amounts of coloring matter appear in the urine, a brisk saline purge may avert nephritis. Decrease in the amount of urine and the appearance in it of albumin and blood call for unusual care. 
in which nephritis is a frequent complication. The writer has seen the medicinal use of turpentine immediately followed by marked albuminuria, decrease in the amount of urine and the appearance of red cells, and has also seen these symptoms immediately disappear following its wițhdrawal. The administration of following drugs should be attended with careful observation of renal functionurotropin, turpentine, potassium chlorate, mercury, carbolic acid, cubebs, copaiba and salicylates. Decreased urinary output, cylindruria, albuminuria and red cells appearing during their administration call for an investigation to determine the cause of their appearance.

The question arises at times as to whether cold baths in acute fevers do not result in injury to the kidney. In typhoid, where the baths are most used, there is no evidence in favor of this idea. The good results outweigh many times the hypothetical injury to the kidney. If indicated, they should be given, unless actual evidence of nephritis appears. In scarlet fever there is commonly more hesitation in regard to their use. Senator(I3) was convinced that they did no harm. To me, it seems inadvisable to use very cold baths (under $80^{\circ} \mathrm{F}$.) in scarlet fever, since great good can be accomplished by sponging, cold packs and baths at this and at higher temperatures.

The value of moderate exercise and hygienic care of the skin and bowels in pregnancy cannot be too much emphasized. Symptoms of nephritis rapidly developing in the last month of pregnancy may call for induction of premature labor. It is possible to induce this at times by the administration of quinin sulphate, gr. $x$ ( $0.6 \mathrm{gm}$.), followed in half an hour by an ounce (30 c.c.) of castor oil. This failing, it may be necessary to resort to the use of the bougie.

Treatment.-There is no specific treatment for acute nephritis. There are underlying cardinal principles that must ever be kept in -mind: (I) Remove the cause when possible; (2) obtain rest for the kidney by minimizing its work through restriction of water and restriction and selection of diet; (3) increase the activity of those organs capable of assisting.in part at least, the function of the kidney, i.e., skin and bowels; (4) meet symptoms and complications as necessity arises; (5) carefully consider all contemplated measures of relief and avoid those capable of further injury to the kidneys unless the indications are paramount.

Removal of thel cause is sometimes possible as in infectious diseases, in syphilis, malaria and diphtheria. These diseases should receive appropriate treatment, remembering that care must attend the use of arsenic and mercury. Removal of tonsils or other foci of infection may beimmediately followed by a clearing up of nephritis. Drugs irritating to the kidney should be discontinued if in use: When the nephritis occurs in pregnancy, emptying the uterus may be necessary. If in the last month of pregnancy, quinin and castor oil, or the bougie may be utilized as has been already indicated.

Rest.-The patient should be put to bed and kept at rest 
until the acute manifestations have completely disappeared. Rest is of paramount importance. It is sometimes extremely difficult, however, to decide how long rest in bed should continue. Many patients are kept in bed unnecessarily long, harm undoubtedly resulting, particularly in children during the developmental stage. Albuminuria may persist for months or even years. The physician attempts to protect himself from criticism in the event of the development of chronic nephritis by keeping the patient in bed often for very protracted periods. Von Noorden (I4) has laid down the following guide, which is of great value in this connection:

"The most important criterion for the time when it is permitted to leave the bed is the disappearance of red cells and kidney epithelium from the urine. As long as these elements are present, the acute stage of irritation is not over. After their disappearance, one should wait two weeks, and then despite continuance of albuminuria, the patient should be allowed to get up, provided, of course, that all the other symptoms of nephritis have disappeared. It will then frequently be seen that in the first days after giving up complete rest the albuminuria increases somewhat. This lasts only a short time, and should not deter us from holding firmly to our conclusions. On the other hand, the recurrence of red cells and renal epithelium should be regarded as proof that we have been too hasty, and then it is necessary to order rest in bed once again."

As sweating plays an important part in the treatment, the patient should be accustomed to blankets, if possible. He should be clad in canton flannel, and kept as warm as is consistent with comfort. Fresh air is absolutely essential, chilling, however, must be avoided. The patient must be protected from draughts. The temperature of the room should be warm but not uncomfortably so. Dependence should be placed in coverings, not in a high atmospheric temperature for keeping the patient warm.

Diet.-Much discussion centers around the questions of diet and of fluid intake in acute nephritis. The underlying principles are to feed such foods as demand the least work on the part of the kidneys, while avoiding substances irritating to the kidneys. The kidneys excrete: (I) end products of nitrogenous metabolism; (2) salts, especially chlorids; phosphates and sulphates; and (3) water. Substances particularly irritating to the kidneys are condiments, and certain vegetables such as onions, celery and radishes, which naturally must be avoided.

The onset of nephritis, particularly the scarlatinal form, may be very acute and characterized by marked nausea and vomiting. Under such conditions, no food at all may be retained. The administration of water even may set up violent retching. For a day or so thereafter it may be necessary to withhold all food. Heretofore, milk has been considered the best available diet during the acute symptoms. No hard and fast lines as to the diet can be laid down since patients differ in their response even to foods. 
It would seem rational, however, to follow the lead of von Noorden and the Vienna school limiting proteins and excluding them altogether from the dietary. Thereby, the amount of nitrogen and of salts, particularly the phosphates and sulphates, is markedly reduced. The.end products of carbohydrate metabolism are water and carbon dioxid. The carbon dioxid is discharged by way of the lungs, and the water in part by the lungs and also by the skin and bowels. Therefore, their excretion entails little or no work on the kidneys. The carbohydrate diet is better suited for the protection of the kidneys than is starvation, since it reduces to a minimum the breaking down of body proteins. Therefore, in a moderate carbohydrate diet, we have the best method of securing rest for the kidney, of supplying the caloric need of the body and of aiding the return of the kidney to normal.

The von Noorden diet consists of considerable amounts of sugar (fruits rich in sugar), fruit juices, gruels of finely ground rice, corn, wheat, or potato starch. Washed butter may be added, since pure fat is also metabolized to carbon dioxid and water. Von Noorden uses this diet throughout the first week or two while the symptoms are most severe and considers the results better than with the generally used milk diet.

Milk Diet.-If taken alone, from $x$ to $1 / 2$ quarts are taken daily. This, naturally, soon becomes monotonous. It is not sufficient to supply the daily caloric need. To the milk, therefore, may be added, as it can be taken and according to the dictates of appetite, cream, which is rich in calories, lactose or cereals, such as oatmeal, sago, rice, etc. Gruels in the form of arrowroot or oatmeal are given. Junkets or custards may be tried. Since fruits are not injurious, apple sauce, baked apple, cooked fruits or jellies, oranges, grapefruit or lemonade may also be allowed. Lactose may be added to the milk, dr. i to ii ( 4 to $8 \mathrm{gm}$.) to the glass as it is in the treatment of typhoid. In the very acute stages, the protein in the milk is considered sufficient ( 30 to $40 \mathrm{gm}$. to the liter). Some allow $50 \mathrm{gm}$. and still others up to $80 \mathrm{gm}$. of protein. During the acute stage, it is almost universally agreed that meat and meat broths must be interdicted.

The objections to the milk diet are these: the amount of water is large, consequently entailing work for the kidney. Though poor in chlorids and extractives, milk contains 30 to $40 \mathrm{gm}$. of protein to the liter and is rich in phosphates and sulphates. It burdens the kidney with considerable work and its caloric value is low.

Von Noorden withholds the milk until the acute symptoms have passed. When the quantity of urine increases, with an increased salt concentration and decreasing amounts of albumin, milk is added to his diet outlined above. As the acute symptoms disappear, the menu is gradually enlarged to include bread and butter, saltfree crackers, puree of vegetables and lettuce, watercress, etc. Small amounts of tea, coffee, or cocoa are allowed also. Salt must be 
restricted until the edema has disappeared and only in the later stages are eggs, fish and meat allowed. Where the acute stage is very protracted and craving for meat is great, a little fresh fish, chicken or even dark meat may have to be allowed occasionally. Phipps(15) has recently claimed marked improvement in subacute or protracted acute cases following a more liberal protein allowance.

Fluid Intake.-As to whether it is possible to or not to flush out the kidneys in acute nephritis through the administration of large quantities of water is another much discussed question. In the majority of cases it is not possible. However, in certain cases without marked edema and with a good urinary output, the kidney is fairly permeable to water, so that rather large quantities can be excreted. On the other hand, there are cases with oliguria or anuria with marked edema where water is excreted not at all or only with the greatest difficulty.

The answer to the water question is found in the permeability of the kidney. Each patient should be treated individually. In the majority of cases, water is handled with great difficulty and the total fluids should be limited to 1000 or 1500 c.c. a day. This rule should always be followed where edema, marked oliguria or anuria are present, since these indicate renal impermeability to water. The more severe the edema, the greater should be the restriction. Water administered does not flush out the kidney, it only increases the edema. The skin, lungs and bowels are capable of excreting considerable water, so that patients suffering markedly from thirst can be given up to $1 \frac{1}{2}$ liters daily.

Later, when the urine quantity is augmented and salt and water are being excreted and the edema is diminishing, more water can be allowed. In many of the febrile cases, dropsy is not a prominent feature. Also in some instances of acute nephritis following exposure to wet or due to the action of drugs, the edema is not marked and the permeability of the kidney is good. Here restriction of water is not so strongly indicated.

What has been said of water naturally applies to other drinks, such as alkaline mineral waters, lemonade, or lemonade and cream of tartar, etc. When water is allowed, these drinks may be substituted if desired. During the height of the disease, if acidosis exists, alkaline water should be substituted for plain water.

Fischer has used a formula containing $\mathrm{I} 4 \mathrm{gm}$. of sodium chlorid and ro to $20 \mathrm{gm}$. of sodium carbonate (crystalline) to the liter of water. For intravenous work naturally this must be sterile. I have occasionally resorted to this method, substituting sodium bicarbonate up to 4 per cent. for the carbonate. This was sterilized in tightly stoppered bottles in the autoclave at 15 pounds pressure for twenty minutes, care being taken to scatter a little bicarbonate free in the autoclave.

Diuretics.- Since water is one of the best diuretics, it is advisable here to consider the question of the use of diuretics in acute nephritis. 
Osler considers water the best diuretic in this connection. If acidosis is present, undoubtedly moderate doses of alkalies, sodium bicarbonate, potassium citrate or potassium acetate are indicated up to the point of bringing the hydrogen ion concentration of the blood to normal. The alkali (combined with sodium chlorid) may be given in solution by mouth or by rectum by the drip method, and in desperate conditions even intravenously, by the method advised by Fischer. The indications and contraindications for most of the other diuretics are much the same as for water. They are contraindicated in the acute stage. Christian(I6) and his co-workers have demonstrated that diuretics have little or no good effect in acute experimental nephritis in animals and that they often do harm. In rabbits suffering from acute uranium poisoning, the duration of life was considerably shortened by the administration in therapeutic doses of caffein, sodium theobromin salicylate, theocin, potassium acetate, spartein and water. Diuresis, as a rule, does not result from their use, except where circulatory disturbances exist. It is, therefore, advisable in acute nephritis to avoid the use of diuretics. If used at all, they should be given in small doses and for one day only, keeping in mind that the kidney ( $I 7$ ) is easily overtaxed; the good effect, if elicited, being watched until it disappears before the drug is again administered.

Where the arterial tension is low and where circulatory disturbance (passive congestion) exists, diuretics such as digitalis or strophanthin often bring prompt results. The purin diuretics also answer well at times under these conditions.

The diuretics should always be used in moderate doses only: theocin, gr. iii ( $0.2 \mathrm{gm}$.), theobromin, gr. $v$ to viii ( 0.3 to $0.5 \mathrm{gm}$.), diuretin (theobromin sodium salicylate), gr. $\mathrm{x}$ to $\mathrm{xv} \cdot(0.6$ to $\mathrm{r.0}$ gm.), caffein, gr. ii to iv (0.1 5 to $0.25 \mathrm{gm}$.), and caffein citrate, gr. $\mathrm{v}$ to $\mathrm{x}(0.3$ to $0.6 \mathrm{gm}$.) and preferably for one day only. Of the digitalis preparations, the freshly prepared infusion, dram ii to iv (8 to I6 c.c.) the tincture, minims $\mathrm{xv}$ to $\mathrm{xx}$ ( 1.0 to I.3 c.c.), q. four hours for twenty-four or forty-eight hours may be used. Digipuratum ${ }^{1}$ is a reliable standardized preparation of digitalis. It is marketed in tablet form, each tablet equivalent to $\mathrm{I} 1 / 2$ grains (o.I $\mathrm{gm}$.) of the dried leaf, of which four are given the first day and three the second day. It may also be had in ampoules for intramuscular or intravenous use. Strophanthin is unquestionably the best preparation of strophanthus, and is administered intravenously or preferably intramuscularly ( $1 / 4$ to I mg.) followed by twenty minutes' local massage. In acute exacerbations of chronic nephritis, with high blood-pressure and myocardial insufficiency; digitalis or strophanthus are indicated. In the presence of high blood-pressure no fear of using these preparations need be entertained since arterial hypertension not infrequently decreases markedly during their administration.

Sodium Chlorid. - Salt should be withheld. It is excreted with

${ }^{1}$ When a standardized potent leaf is available it is not necessary to use digipuratum. 
difficulty by the diseased kidney. If retained, it increases the edema. It is in the acute form of nephritis particularly that salt is injurious. The salt intake should, therefore, be as low as possible (see table of food values). Fruits, fruit preparations and other carbohydrates mentioned above have only a small salt content. Milk has approximately $1.6 \mathrm{gm}$. per liter; bread, 0.5 to $0.7 \mathrm{I}$; and ordinary butter I per cent. Unsalted bread, crackers and washed butter should be used when these articles of diet are first allowed. It is practically impossible to get an absolutely salt-free diet. A diet containing I to $2 \mathrm{gm}$. of salt per diem should be used for a few days or for the first week in severe cases. Later 2 to $5 \mathrm{gm}$. may be allowed, and this may be gradually increased.

Catharsis.-The bowels should be kept free. The extent of purging is guided by the patient's clinical condition. Marked edema and impending uremia call for intensive purgation. Ordinarily, the saline purgatives, if retained, meet all the indications. Daily doses of Epsom salts, 7 to $\mathrm{r} .5$ ( 30 to $45 \mathrm{gm}$.): Rochelle salts, $\mathrm{F}$ ss ( 5 gm.): sodium sulphate, 5 ss ( 5 gm.) or sodium phosphate, 5 ss ( $5 \mathrm{gm}$.) in a half glass of hot water, given in the morning before breakfast, answers admirably if well borne by the stomach. Effervescent preparations, such as Seidlitz powder, liquor magnesii citra tis,

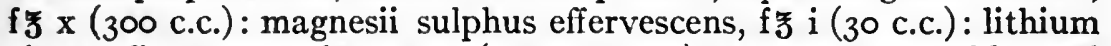
citras effervescens, $f \xi$ I to 2 ( 30 to 60 c.c.) are more palatable, and may be preferred by the patient, but the large quantities of water involved may make them undesirable. More brisk purgation may be necessary, when the compound cathartic pill, compound jalap powder, $3 \mathrm{ss}(2 \mathrm{gm}$.) or elaterin, gr. $1 / 20$ to $1 / 10$ ( 0.003 to $0.006 \mathrm{gm}$.), will be found useful. Calomel may be used occasionally, but is not suited for continuous use in acute nephritis. Croton oil should be used in the presence of coma.

If the dropsy is only moderate, the urine not markedly concentrated and uremia neither present nor impending as indicated by clinical and functional studies, the bowels should be kept loose without violent purgation. Vegetable laxatives, such as cascara, senna or a pill of aloes, belladonna, cascara and strychnina, then suffice.

Diaphoresis.-Sweating is indicated particularly where the edema is marked or where uremia is impending and renal function markedly depressed. It should be procured with as little depression to the patient as possible and without subsequent exposure to cold. Dropsy is best combated with hydrotherapy - either the hot bath, the hot pack or the hot air bath. For children, the hot pack is usually best. The bed is covered with a rubber sheet; a large, heavy blanket is dipped in hot water, wrung out, and rapidly wrapped about the naked patient, care being taken that it is not hot enough to burn. A dry blanket is wrapped about the wet one and several hot water bags placed about the patient and an ice cap placed on the head. The bath should last for one hour. Care should be taken to have the room warm - no draught should be permitted. If sweating does not 
appear early, a drink of cold water, alone or with spiritus etheris nitrosi, i to ii ( 4 to 8 c.c.), is given. Where sweating still fails, resort to a small dose of pilocarpin, gr. $1 / 20$ ( 0.003 gr.), may be necessary. The pack should be given daily, if great need exists, twice daily.

For adults, the hot air bath is usually employed. The method as employed in the wards at the Johns Hopkins Hospital is described in the section dealing with therapeutic technique.

If sweating does not readily appear, it can be often facilitated by the administration of cold drinks, water or a diaphoretic mixture, such as sweet spirits of niter. Pilocarpin in small doses, gr. 1/20 ( $0.003 \mathrm{gm}$.) hypodermically, occasionally has to be combined with the above procedure.

Hot baths of fifteen to twenty minutes' duration, immediately followed by wrapping the patient in hot blankets, answer very well in some cases. The patient can be wrapped in hot blankets, surrounded by hot bricks or by hot water bottles according to the technique described later.

Recourse can be had naturally to electric cabinets in which the patients can remain in the recumbent position, if such are at hand.

The sweats are given daily. Where the edema is extreme or uremia is threatening or present, they may be used twice a day. Pilocarpin is not advisable except where the other methods fail. It can be used as an adjunct to them in small doses. If this fails, and uremia supervenes, it can be given hypodermically, gr. $1 / 6$ to $1 / 8$ ( 0.01 to $0.008 \mathrm{gm}$.) to adults and gr. $1 / 20$ to $1 / 12(0.003$ to $0.005 \mathrm{gm}$.) to children of from two to ten years.

Treatment of Symptoms and Complications.-Uremia.-This calls for intensive treatment along the lines which will be discussed later.

Edema is combated by the general measures just described, particularly by restriction of fluid and salt intake, by free purgation and by sweating. If these measures fail to reduce the dropsy, or if the dropsy is extreme, the fluid may be drained from the subcutaneous tissues by one of the methods of drainage incision punctures or Southey's tubes which are described under therapeutic technique. But these measures are only indicated where the edema is extreme and sloughing is threatened. They are efficacious-resulting in the removal of as much as 4 liters of fluid a day at times. Unless extreme care is exercised, erysipelas may develop.

In rare instances ascites is extreme and demands an abdominal paracentesis, or a Southey's tube can be inserted and the fluid generally drained off. Occasionally a thoracic paracentesis is necessary.

Anuria.-This is usually associated with edema. The Paquelin cautery, or dry or wet cups, warm poultices, or the hot water bag should be applied locally over the kidney. Sometimes the hot bath relieves the anuria. Excretion by other channels must be encouraged and the fluid intake kept as small as possible. Wet packs are given 
once or twice a day and free purgation elicited. The administration of water does no good. The fluid intake should be reduced to a minimum. To assuage the thirst, small pieces of ice may be sucked or water be given in teaspoonful doses. The patient should be kept warm, especially the feet. Absolute anuria is usually of short duration, the patient dying or the urinary output increasing within a few days. Enteroclysis with water $102^{\circ}-120^{\circ} \mathrm{F}$. for fifteen minutes is claimed by some to relieve renal congestion. Where all other methods have failed, and especially where acidosis exists, the administration of alkalies intravenously as advised by Fischer may start the kidneys excreting again.

Backache.-This is common in acute nephritis, particularly in the early stages. Much relief follows the local application of hot poultice, a hot water bag, or an electric pad. Local massage also proves of value.

Headache.-These are usually the result of toxemia and indicate the necessity of intensive eliminative treatment. Locally heat or cold may be used. Where the circulation is normal and the headache intense, small doses of one of the coal-tar products, pulv. acetanilid comp., gr. iii to $\mathrm{v}$ ( 0.2 to $0.3 \mathrm{gm}$.), acetphenetidin (phenacetin), gr. v to $x(0.3$ to $0.6 \mathrm{gm}$.), antipyrin, gr. iii to $\mathrm{v}(0.2$ to $0.3 \mathrm{gm}$.), or potassii bromidum, gr. $\mathrm{x}(0.6 \mathrm{gm}$.) may be given.

Nausea and vomiting, uremic in origin, may be relieved by sucking small pieces of ice, by restriction of food and by active elimination. They are usually manifestations of uremia and are central in origin. Chloral, gr. v ( $0.3 \mathrm{gm}$.) every four hours is effective at times. At times, however, they can be relieved by drop doses of creosote, iodine or carbolic acid or by dilute hydrocyanic acid (drops ii) with bismuth subnitrate, gr. $\mathbf{x x}$ (r.3 gm.) three times a day. Small doses of morphia given as a last resort are usually most effective. Persistent lavage sometimes yields considerable relief.

Itching is best treated with hot alkaline baths. Substances irritating to the kidneys must be avoided when sedatives are considered.

Dyspnea.-The cause must be sought. Large accumulations of fluid in the abdominal or thoracic cavities should be removed through tapping. Bilateral hydrothorax, even if small, often causes extreme dyspnea and calls for tapping. The dyspnea is often of cardiac origin when digitalis or strophantin may give relief. At times a good sweat in the evening brings relief and permits a good night's sleep. Where sleep is impossible on account of the dyspnea, morphia is undoubtedly the drug par excellence. Small or moderate doses do not interfere with renal excretion, although it has a tendency to decrease elimination by the skin and bowels. These effects, however, can be overcome readily by purgatives and by the methods for eliciting sweating described above.

Edema of the glottis may require scarification or even tracheotomy. Local cold to the neck should be tried first. 
Pulmonary Edema.-Early bleeding is indicated. Where the blood-pressure is low, strophanthin or digitalis should be administered. The subcutaneous administration of camphorated oil, min. $x x$ (r.3 c.c.) or the administration of aromatic spirits of ammonia, dr. $i$ (4 c.c.) may, ward off the attack. Atropin is of value at times. Miller(I8) however, is convinced that morphin constitutes the best remedy for pulmonary edema. It is unquestionably of great value.

For the anemia associated with acute Bright's disease, iron should be used, but not until the acute symptoms have subsided. In adults, Blaud's pills, tr. ferri perchloridi, min. $\mathrm{x}$ to $\mathrm{xx}$ ( 0.6 to I. 3 c.c.) or Basham's mixture (liquor ferri et ammonii acetatis), dr. i to iv (4 to 16 c.c.) should be employed, while for children, the syrup of iodid of iron, min. $\mathrm{v}$ to $\mathrm{xx}$ ( 0.3 to I.3 c.c.) or the syrup of phosphate of iron, dr. ss (2 gm.) are preferable.

Urinary antiseptics have played but a minor rôle in the part. Where renal infection is proved, their judicious use may be considered.

Measures Injurious to the Kidney to be Avoided.--Undoubtedly, much harm has been wrought in the past and is still produced by illadvised treatment. The injudicious use of diuretics and the administration of large quantities of water must be particularly avoided.

Treatment in Convalescence.-Patients, particularly children, must not be kept in bed unnecessarily long,. as has been already indicated. Extra care is necessary in avoiding cold and in the control of diet. No article should be added to the menu without due consideration and careful trying out. Attention to anemia, particularly when convalescence is delayed, is frequently indicated. Some of the iron preparations already mentioned may be used. Much good may also result from the use of a bitter tonic. A change of air is frequently of benefit, particularly a residence in a more equable climate.

\section{CHRONIC PARENCHYMATOUS NEPHRITIS}

Acute nephritis following exposure to cold or due to scarlet fever or pregnancy may pass over into the chronic parenchymatous form. Cold and dampness may account for its frequent appearance in icemen, bakers, and butchers. It frequently develops insidiously and often without any assignable cause. One or another of the infectious diseases, tonsillitis or a focus of infection somewhere in the body may be responsible. Syphilis, tuberculosis and chronic infection of ten result in its development, under which conditions amyloid disease is associated. Malaria is responsible in some instances. It occurs more often in young adults and is not infrequent in children. It is more frequent in males than in females. Alcohol is believed to play a rôle in a certain proportion of cases. Factors which, acting suddenly, cause acute nephritis may, if acting less violently and over a considerable length of time, produce this chronic form.

Although a chronic process, its course is frequently interrupted by acute exacerbations which closely resemble acute nephritis. These 
exacerbations may result from exposure to cold, acute infection, errors in diet, or overexertion. In other instances no cause can be assigned.

Morbid Anatomy. - The process is always diffuse-never limited strictly to the parenchyma. The large white kidney which Wilkes has described is usually regarded as the type. The kidney is light gray, often nearly white in color, the capsule is thin and strips readily, leaving a white, non-granular surface. The organ is soft. On section the cortex is smooth, white, pale gray or pale yellow, with here and there opaque spots, while the pyramids are dark and congested, contrasting strikingly with the pale cortex. Microscopically the epithelium is found to be desquamated, fatty and granular in character. The tubules of the cortex are most affected; they are distended, the epithelial cells encroaching on the lumina. Casts of different kinds, degenerated epithelial cells, fat globules, red and white cells and débris may fill the lumina of the tubules.

The glomeruli are large with thickened capsules. Swollen desquamated and degenerated cells may fill the capsular space. The epithelium of the tuft is extensively altered. The tuft itself may be pushed to one side, the space being filled with inflammatory exudate, degenerated cells, red or white blood cells. Hyaline changes may be present in the walls of the capillaries, and the capillaries themselves may be thrombosed.

The interstitial tissue is increased throughout, though not to an extreme degree.

Another form that is found at times is the small white, or secondarily contracted, kidney, which is of ten the end result of the large white kidney and which at other times probably develops independently of it. In this form the interstitial changes are more advanced, the kidney is small and firm with a decreased cortex. The capsule is thick and adheres in places, presenting a rough granular surface. Bowman's capsule is thickened, and the glomeruli are of ten destroyed and replaced with fibrous tissue. The tubules are frequently distended with a fatty, granular material, appearing in the cortex as white or opaque foci. The epithelium of the convoluted tubes is degenerated. The interstitial tissue is markedly increased, and the arteries are noticeably thickened.

The Functional Picture.-It is this form of nephritis which earlier brought functional studies into disrepute. In some cases the excretory power of the kidney is very little disturbed except in regard to water and salt. Apparently the functional injury to the kidney is not always proportional to the severity of the disease. Marked edema characterizes the condition, and it may be that edema of the brain or of other structures is responsible in part for the clinical picture, and possibly also in part for the mortality. Or the factors at work in setting up the nephritis, acting elsewhere, may be responsible. As a rule the functional studies underestimate rather than overestimate the injury.

Vou. IV. -49 
Different pictures may be seen in different cases. Some of these are fairly distinctive and worth recording. In severe cases the excretory capacity of the kidney may be tremendously reduced: the phthalein may be zero, lactose, iodid and salt and water excreted with great difficulty, and cumulative phenomena be present in the blood. A case of this type, of luetic origin, with marked anasarca, great dyspnea, with $30 \mathrm{gm}$. albumin to a liter of urine, and with a phthalein output practically zero, came to autopsy at the end of two months.

On the other hand, in cases showing equally marked edema and albuminuria the phthalein, lactose, iodid and diastase excretion has been found to be normal, the blood nitrogen, blood urea and freezing point also being normal. Salt, however, was excreted with extreme difficulty and the responses to water were abnormal. Here then we have a clinically severe nephritis with a normal function except in regard to water and salt. One of these patients was under observation for a period of two years, without any appreciable change in the functional picture. Death occurred in this instance as the result of an acute intercurrent infection. Anemia is commonly associated with the picture.

Hyperpermeability (I9-2O) of the kidney for substances other than salt may occasionally occur in this form, as much as 80 per cent. phthalein for one hour being recorded.

Prognosis.- The ultimate prognosis is extremely grave. Only occasionally does recovery take place. Some difficulty is experienced at times in distinguishing this form from acute nephritis. The edema may disappear and the secondary contracted kidney develop. Recovery is extremely rare is cases persisting for more than a year, except in children, when it has taken place even after two years. Death occurs from exhaustion, uremia, anemia, or by edema and secondary implication of the serous membranes. Patients rarely live longer than two years.

The functional tests are of definite value prognostically. In cases showing marked decrease in renal function uremia commonly develops and frequently ends in death. A zero phthalein in chronic nephritis indicates a very grave prognosis while a blood creatinin above $5 \mathrm{mg}$. per roo c.c. points to an early exitus. In other types, in which renal function is good except in regard to salt and water, it can be predicted that death from renal insufficiency is not imminent. The degree of functional injury in acute exacerbations can also be determined. Care must be exercised in making a good prognosis in the presence of adequate renal activity because of the frequency of acute exacerbations and because of the fact that death occurs from causes other than renal insufficiency.

Treatment.-What has been said in regard to the prophylaxis of acute nephritis applies also to this form. Little can be accomplished in an individiual case outside of advice to the alcoholic and to workers exposed to damp and cold. 


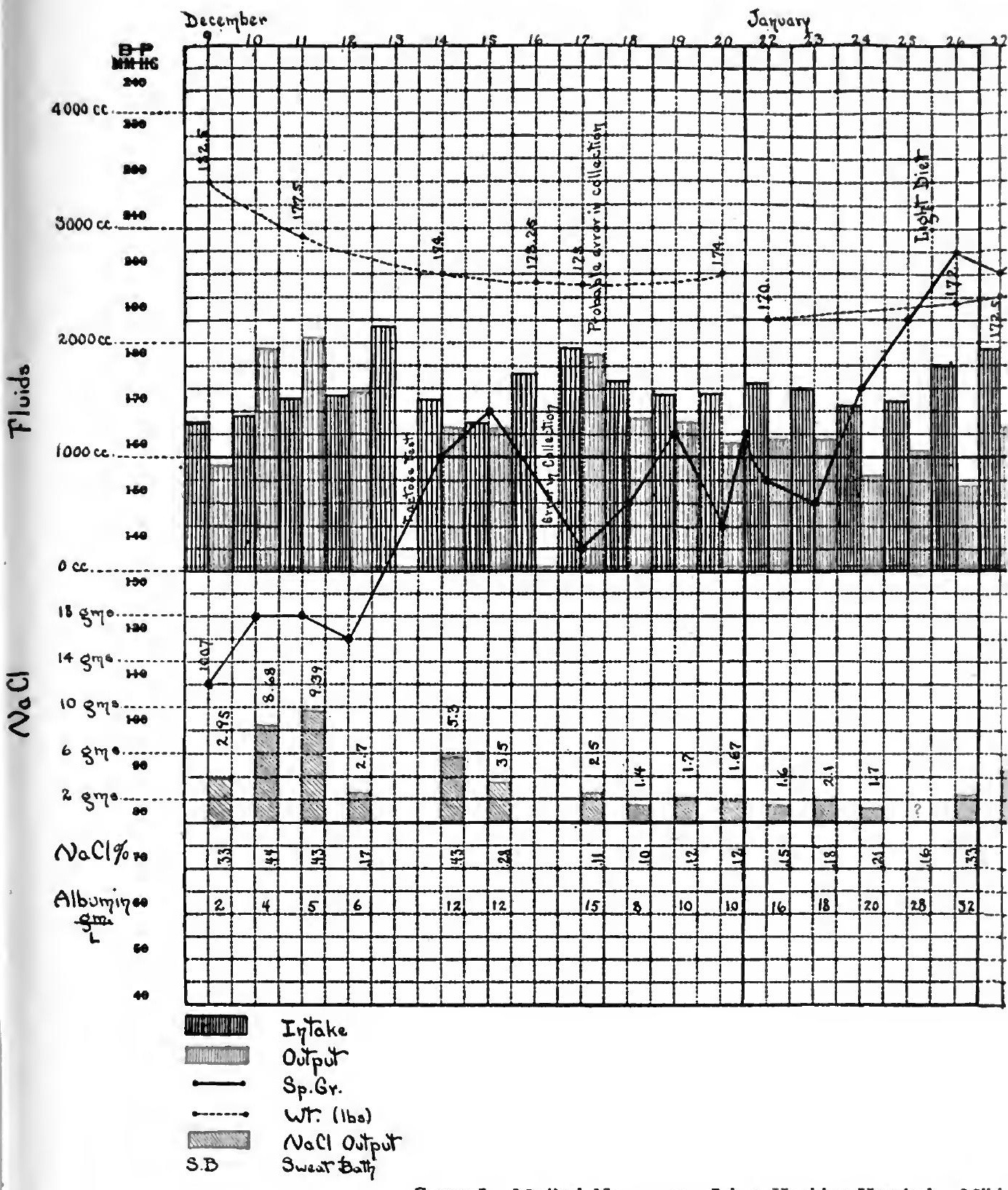

Charr I.-Medical No. 33424, Johns Hopkins Hospital. Mild 


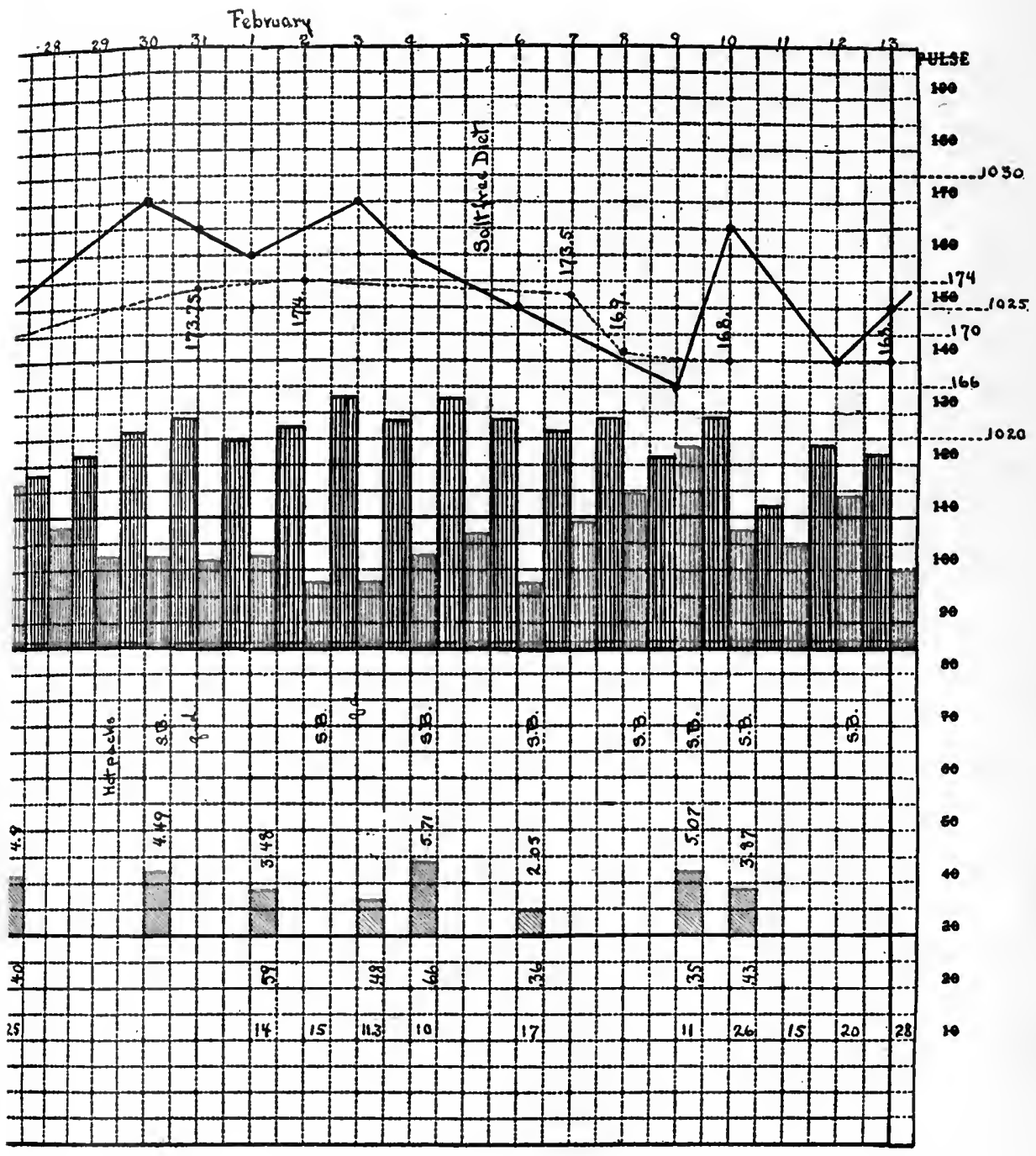

chronic diffuse nephritis with edema, no hypertension. 
Dute Decils

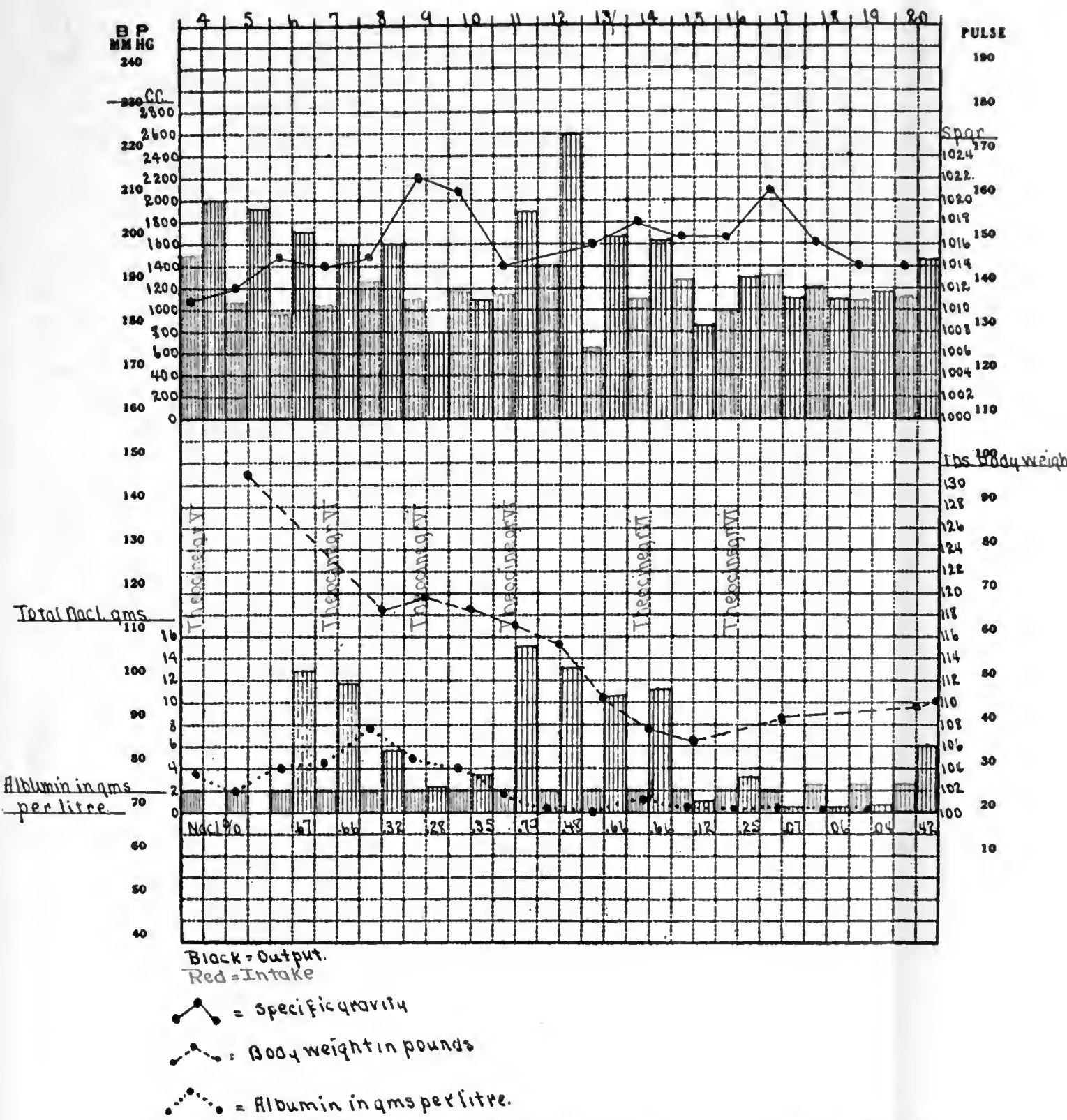

Chart III.-Medical No. 35138, Johns Hopkins Hospital. Chronic parenchymatous nephritis; sinusitis, right antrum. 

Generally speaking, the treatment is the same as that of the acute form. Certain modifications are necessitated by the chronic course of the disease. These concern mainly the question of exercise, diet, climate, and the condition of the heart. But the underlying principles are the same: (I) removal of the cause when possible; (2) rest for the kidney by minimizing its work through restriction of water and restriction and selection of diet; (3) increasing the activity of those organs capable of carrying on renal function vicariously; (4) meeting the symptoms and complications; and (5) avoidance of remedial measures capable of injury to the kidney.

I. Removal of the Cause.-This is of ten possible when the nephritis is of luetic origin or due to a focus of infection or to pregnancy. This has already been discussed.

2. Rest for the Kidney.-Complete rest in bed early in the disease and in acute exacerbations is important; also if edema is severe or if acute uremia develops; otherwise, it is not advisable or justifiable to confine the patient to bed. A certain amount of muscular exercise should be encouraged, and under suitable climatic conditions the patient should be out of doors, care being taken to see that he is warmly clad, preferably with woolen garments next to the skin. Even if the albuminuria increases when the patient is up, it is advisable, unless red cells appear in the urine, to keep him up, since the increase is as a rule only temporary. In mild protracted cases the patient may occasionally be allowed to attend to indoor work, provided great bodily and mental fatigue is not entailed, in which case work must be scrupulously avoided. Exercise must be supervised by the physician with regard to the heart as well as to the kidney.

Diet.-Here again the principles laid down under acute nephritis apply. But the diet obviously cannot be the same. In a disease lasting months or possibly years, such a marked restriction is unjustifiable and provocative of great harm. The caloric needs of the patient must be met. The arrangement of a proper diet is one of the chief functions of the physician as well as one of his greatest difficulties. The diet must be individualized according to the needs and appetite of the patient. It should be under constant supervision and revision. In controlling it the feeling of the patient, his weight and appetite, the extent of the edema, the level of the non-protein nitrogen in the blood and the character of the urine should all be considered. Judgment and resourcefulness are required to keep the diet suitable and palatable.

Carbohydrate and fat entail no great amount of work on the kidney. These with milk, therefore, form the basis of the diet. Since the disease is chronic, proteins cannot be indefinitely withheld. An average daily allowance of 80 to. $100 \mathrm{gm}$. of protein, in the form of milk, meat, fish, eggs or vegetable protein, usually best meets the requirements.

Milk alone is unsatisfactory. It is monotonous, involves too large a bulk, results in loss of appetite, and often nausea and diarrhea 
and loss of strength. It should form a part of the diet, about I liter a day being given-this contains about 30 to $40 \mathrm{gm}$. of protein. Some protein should be given in the form of fresh meat. Of meat weighed raw I 20 to I $50 \mathrm{gm}$. corresponds to 30 to $40 \mathrm{gm}$. of protein. The kind of meat is immaterial so long as it is fresh. Fish up to 200 gm. (weighed raw) may be substituted. Protein in the form of eggs or of vegetable albumin is also allowable. This amount of protein together with carbohydrate and fat assures a minimum breaking down of body proteins and, generally speaking, is well adapted to the patient's needs.

The amount of carbohydrate and fat will depend upon the condition of nutrition. Sugar, cereals such as ordinary breakfast foods, rice, tapioca, and sago, or the various gruels, may be freely given. Lactose may be added to the milk. Fruits and fruit juices are admirably suited. Bread, toast, crackers, and some of the simple vegetables such as potatoes, peas, beans and corn may also be allowed. Fat in the form of cream or butter may be added. All highly seasoned food, rich meat broths, fried and greasy foods, pastries, pies, and fancy desserts should be avoided. With the protein sufficient carbohydrate and fat should be taken to avoid weakness and loss of weight but not enough to make the patient fat. Since the appetite is commonly poor, more difficulty is experienced with undereating than with overeating. When feasible the diet should be controlled by actual metabolic studies.

A diet of 75 to $100 \mathrm{gm}$. of protein corresponds approximately to a nitrogen intake of $\mathrm{I} 2$ to $\mathrm{I} 6 \mathrm{gm}$. of $\mathrm{N}$ daily. When acute exacerbations occur, the diet advised for acute nephritis should be temporarily adopted, the protein intake being decreased to 50 to $60 \mathrm{gm}$. or even lower. The same holds true when metabolic studies show that the kidney is unable to excrete the I 2 to $16 \mathrm{gm}$. nitrogen and when its total non-protein nitrogen and urea are piling up in the blood. A diet containing $5 \mathrm{gm}$. of $\mathrm{N}$ continued for one week results in some cases in a decided decrease in the level in the blood of non-protein nitrogen and urea, together with subjective improvement in the patient's condition.

Water Intake.-Marked edema calls for marked restriction of fluids to $\mathrm{I}$ to $\mathrm{I} 1 / 2$ pints a day. When the edema is disappearing and polyuria developing a somewhat larger fluid intake may be allowed if desired. The ease with which the kidney excretes water is a guide in determining the amount allowed. Small amounts of tea or coffee once a day will do no harm where the patient is accustomed to it, unless insomnia or palpitation results. Lemonade or drinks containing fruit juices can be substituted for water. Alkaline drinks are desirable when acidosis is present. Beer, wines, and alcoholic drinks of all kinds should be interdicted.

Sodium Chlorid.--Here, as in the acute form, salt should be restricted, because of the inability of the kidney to excrete it and because of its influence in increasing edema. Salt, however, cannot 


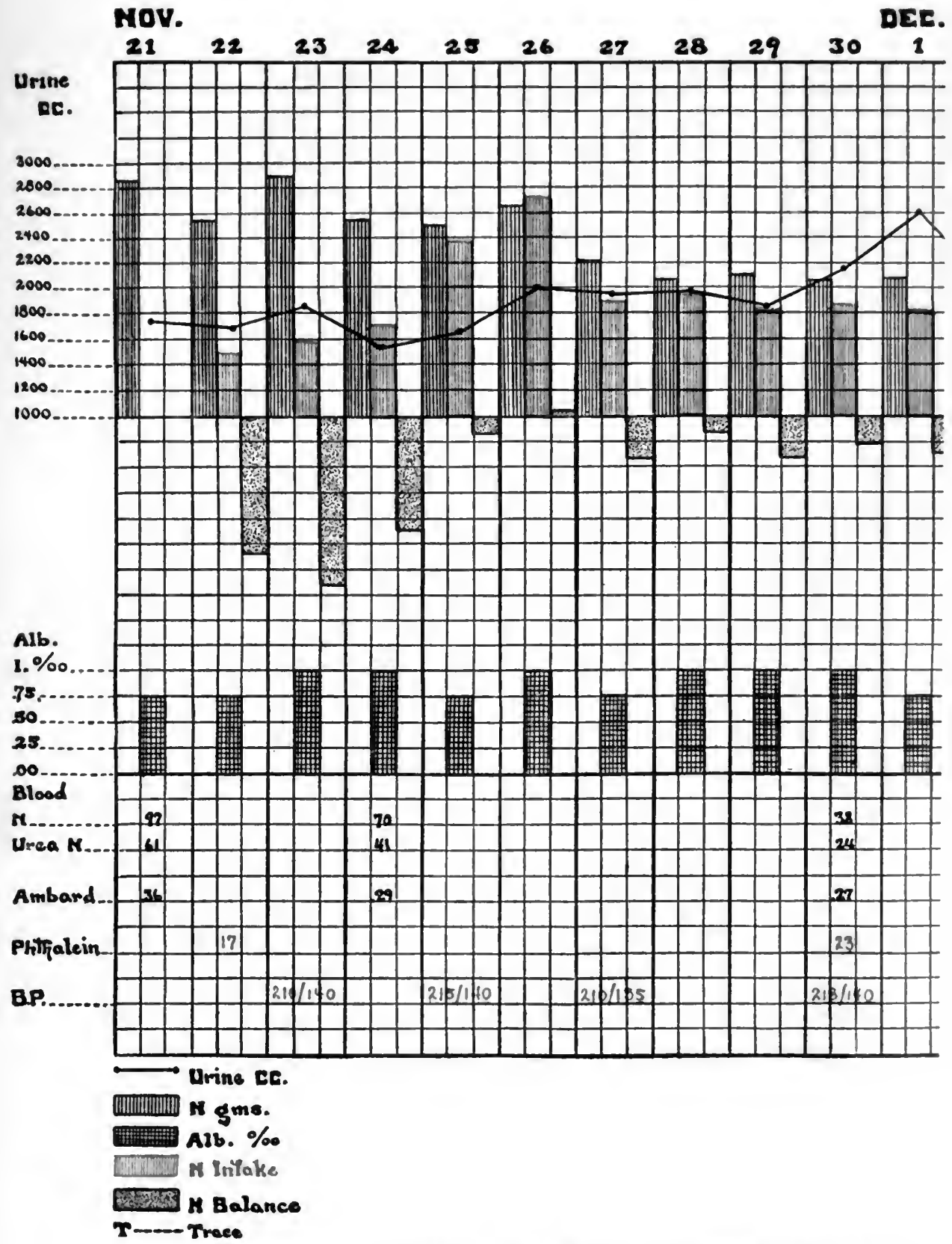

Chart II.-Medical No. 33588, Johns Hopkins Hospital. arteriosclerosis; albuminuric retinitis; syp 
Low protein diet throughout. Nitrogen bolayce from urige only. (Feces Nitroger not deterringed).

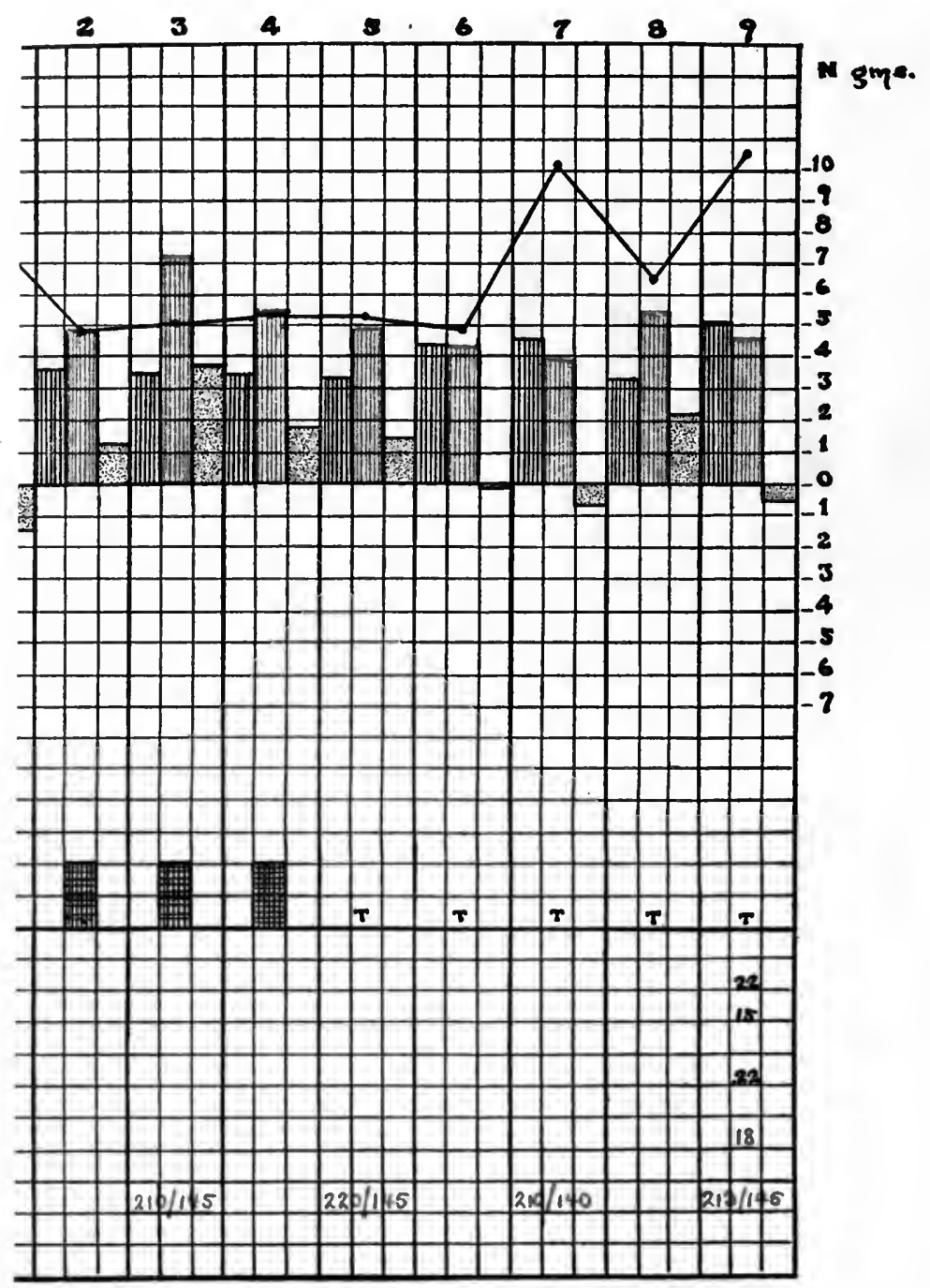

Chronic diffuse nephritis; hypertension; hilis; chronic tonsillitis. 
be altogether withheld for long periods of time. If marked edema exists a diet such as that outlined above can be employed. If due care is given and salt-free bread and washed butter are employed, the sodium chlorid content can be readily held as low as $\mathrm{I} 1 / 2$ to $3 \mathrm{gm}$.

By controlling salt and water intake it is possible in a large proportion of instances to control the edema and the weight of the patient to a considerable extent. The increase in weight following increase in salt is readily demonstrated in this class of cases. I have sometimes been questioned if it is not possible perhaps to curtail the salt to too great an extent. The question arose as the result of two observations.

I. Following the above regimen the edema may be very considerably reduced, but not entirely removed while the albuminuria may be very marked. Occasionally it has been observed that following somewhat larger amounts of salt the albumin decreases considerably, but the weight increases, i.e., the edema increases. By instituting sweats the edema can be fairly well controlled while the albuminuria is decreased temporarily at least.

2. On very rare occasions an additional $5 \mathrm{gm}$. of salt given as a renal test when the patient was on extreme salt restriction resulted in no increased elimination, whereas this amount given after a somewhat higher daily salt intake resulted in a fair increase in salt elimination. This might be interpreted as indicating too great deprivation of salt and as tissue need for salt. Fischer cites several cases in which the alkali-salt mixture referred to under acute nephritis resulted in prompt increase in urinary output, decrease in albuminuria and edema. He also claims to have seen cases of chronic parenchymatous nephritis in which ordinary measures produced no results clear up remarkably under large amounts of salt, e.g., $25 \mathrm{gm}$. a day. On the other hand, the writer has convinced himself that the administration of the salt alkali mixtures often results in increase in the edema.

As the edema disappears and polyuria develops, salt may be allowed in gradually increasing amounts.

Elimination by Other Organs.-Catharsis needs no special consideration. Constipation should never be allowed. When edema or uremia develops one of the cathartics is employed by the method already outlined under acute nephritis, but otherwise it is only necessary to see that the bowels move regularly. If mild laxatives are indicated small doses of the salines, cascara or phenolphthalein may suffice.

Diaphoresis.-Even in mild cases daily warm baths are advisable. Here again acute exacerbations, edema and uremia may call for measures insuring free elimination by the skin. These have been already described. In chronic nephritis we have an additional measure in climate. A warm, dry climate is to be preferred. $\mathrm{Pa}$ tients with weak hearts should not be sent to hot climates, but in cases of chronic parenchymatous nephritis edema which has resisted all 
other measures may disappear in a continuously warm climate, though the albuminuria is unaffected. Exacerbations are also less common when cold is avoided. In Europe patients convalescing from acute nephritis are often sent to "winter in Egypt." Southern California in this country offers an excellent climate throughout the entire year.

\section{CHRONIC INTERSTITIAL NEPHRITIS}

Three types of kidney are commonly found in this disease: (I) the primary contracted or red granular kidney; (2) the secondary contracted or pale granular kidney, which is considered by some to be the end stage of the chronic parenchymatous nephritis and by others to be an independent disease; and (3) the arteriosclerotic kidney. In many cases evidence of nephritis is wanting, the lesion being an atrophy with secondary fibrosis-which has led to objections to the term nephritis. The greatest difficulty is experienced in attempting to diagnose these types clinically, and even sometimes pathologically. It may be absolutely impossible clinically and by laboratory studies to differentiate between the primary and secondary contracted kidney, although the history of chronic parenchymatous nephritis, of course, suggests the latter. Marked cardiovascular changes are encountered in all forms and during life hypertension is apt to be a prominent feature, although these manifestations are lacking in some of the cases of secondary contracted kidney.

The secondary contracted kidney may result from the chronic parenchymatous form. Bradford, however, believes that the pale granular kidney is a distinct entity, related neither to the large white nor to the small red kidney. The primary contracted kidney is so named because no history of previous renal disease is available.

Following the lead of Gull and Sutton(2I) the genuine contracted kidney is often looked upon, as has already been shown, not as a nephritis and not even as a primary nephropathy but as renal atrophy of circulatory origin due to a sclerosis of the small renal vessels which is but a part of general "arteriocapillary fibrosis." Accepting this conception, the disease should not be looked upon simply as a renal disease, but as a part of a general condition, for which the name of "hypertensive cardiovascular disease" has been proposed. In the early stages anatomical evidence is lacking. The hypertension is thus supposed to be dependent upon vasoconstriction, functional in nature, resulting from increased sensitiveness of the vasomotor mechanism.

As has been stated, the underlying pathological lesion in the genuine contracted kidney is an arteriocapillary fibrosis, or a disease of the small arterioles. When large arteries are involved in the sclerosis, particularly when arteries going to the kidney are involved, a patchy atrophy and contraction results, the kidneys showing a coarsely granular or warty surface, or one whole pole shrunken. This is referred to as the arteriosclerotic form. 
Etiology.-Since the secondary contracted kidney may result from the chronic parenchymatous or large white kidney, obviously they have a common etiology. This has already been discussed. The cause of arteriosclerosis is closely bound up with the etiology of the two other types. Such factors as heredity, syphilis, alcohol, gout, overwork (physical or mental), overeating and worry are thought to play a rôle. As a matter of fact little or nothing is acutally known concerning the underlying cause of either the arteriosclerosis or the nephritis. It is advisable, however, to comment on some features.

Age and Sex.-It is a disease, generally speaking, of middle and later life and is more common in the male. The arteriosclerotic form rarely appears before the forty-fifth year. Chronic interstitial nephritis does occur in childhood and even in infancy. A lad of twelve years, recently under the observation of the writer, suddenly developed uremia and died. At autopsy extensively contracted kidneys were found.

Heredity.-A hereditary tendency is unquestionable at times, several members of one or of successive generations showing evidences of nephritis or of hypertensive cardiovascular disease.

Gout.-This is considered a frequent cause in countries where it is common. On the other hand, no evidence of hypertension, arteriosclerosis, or of nephritis may be found in undoubted gout of years' duration.

Lead.-Although practically all attempts at the experimental production of chronic interstitial nephritis by lead have been futile, clinical studies upon workers in lead show an unquestionable connection. Furthermore, lead can produce vasomotor crises and also hypertension. Gout also results from it, and in many instances the nephritis is ascribed to the gout.

Alcohol.-Similarly, experimental proof is lacking in regard to the etiological relationship of alcohol, though clinically it appears to be established beyond question.

Acute Infectious Diseases and Infections.-The question of infection has already been sufficiently discussed. It has been proved that infection plays a rôle in at least some cases of acute and of parenchymatous nephritis. Occasionally a patient is followed through these stages, and sometimes later to autopsy, when a contracted kidney is found. It is quite conceivable that many cases may result from renal injury which though insignificant at the time leads to secondary processes which slowly progress until fibrous tissue replaces and destroys much of the renal tissue, the contracted kidney resulting.

It would lead too far afield to attempt to discuss the relationship between nephritis on the one hand and gastrointestinal disturbance, faulty metabolism, dietetic errors, overeating, and the abnormal function of thyroids, suprarenals, liver and other organs, all of which have been the subject of considerable research and speculation. 
A strenuous life and heredity are held largely responsible, particularly in the arteriosclerotic form. Poor vascular material and excessive wear and tear account for many of the cases, men of thirty revealing vessels sixty years of age. There are many middle-aged men in whom syphilis, alcoholism, excess in food and drink and chronic infection can be excluded who are the victims of arteriosclerosis, the result of a strenous life and of long-continued high-pressure work. Osler states that the victims are in reality fortunate when the high pressure and urinary changes are recognized early, since much can be done to prolong life, health and enjoyment by the correction of work, habits, etc.

Prognosis.-Chronic interstitial nephritis is an incurable disease. Recovery rarely if ever occurs. The disease, however, is compatible with years of comfort, enjoyment and of some activity. As it is encountered in the hospital wards the outlook is as a rule very dark, for the majority of the patients come to the hospital in the end stages. It must be borne in mind that most cases are latent at first. The patient in the ward may have been the subject of the disease for years without having suspected any illness. The first symptoms, however, may be the last. Many patients enjoy fair health for as long as ten or fifteen years. The onset of uremia, perisistent nausea and vomiting, sudden convulsions or coma, dilatation of the heart, development of serous effusion or of Cheyne-Stokes respiration, are all of serious import. Functional studies at times throw much light on the question of prognosis.

The majority of the patients do not die of renal insufficiency. As has been indicated, the heart, the vessels and the kidney are all involved. Many cases die a cardiac death, others by apoplexy and some in uremia. Janeway (22) has tabulated the cause of death in 2I2 carefully studied private cases:

\begin{tabular}{|c|c|c|c|c|c|}
\hline \multirow{2}{*}{ Causes of death } & \multicolumn{2}{|c|}{$\begin{array}{l}\text { Male, } \\
\text { per cent. of total } \\
\text { known- } 137\end{array}$} & \multicolumn{2}{|c|}{$\begin{array}{c}\text { Female, } \\
\text { per cent. of total } \\
\text { known- } 47\end{array}$} & \multirow{2}{*}{ Total } \\
\hline & No. & Per cent. & No. & Per cent. & \\
\hline Gradual cardiac insufficiency....... & 48 & 35.0 & I 2 & $25 \cdot 5$ & 60 \\
\hline $\begin{array}{l}\text { Uremic convulsions, coma, or gradual } \\
\text { uremia. }\end{array}$ & T & 206 & 2 & & \\
\hline 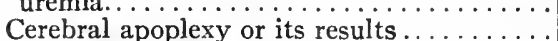 & $\begin{array}{l}31 \\
20\end{array}$ & $\begin{array}{l}22.0 \\
14.6\end{array}$ & $\begin{array}{r}15 \\
9\end{array}$ & $\begin{array}{l}31.9 \\
10.1\end{array}$ & $\begin{array}{l}40 \\
29\end{array}$ \\
\hline Angina pectoris............... & 10 & $7 \cdot 3$ & ? & & Iо \\
\hline Edema of lungs. . & 6 & 4.4 & I & $2 . I$ & 7 \\
\hline Progressive anemia.. & I & 0.7 & 2 & $4 \cdot 3$ & 3 \\
\hline Pericarditis......... & I & 0.7 & ○ & & \\
\hline Complicating acute infectious disease. & 9 & 6.6 & 4 & 8.5 & 13 \\
\hline Other accidental causes................ & 7 & 5.1 & 2 & $4 \cdot 3$ & 9 \\
\hline Unknown $\ldots \ldots \ldots \ldots \ldots \ldots \ldots \ldots \ldots$ & 25 & .... & 3 & & \\
\hline \multirow[t]{2}{*}{ 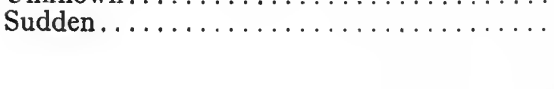 } & 4 & 2.9 & 2 & $4 \cdot 3$ & 6 \\
\hline & 162 & & 50 & & 212 \\
\hline
\end{tabular}


"While the largest total number, sixty (32.6 per cent.), died a gradual cardiac death-if one groups together deaths from uremia and from apoplexy, a 'cerebral group,' as did Bright-seventy-five (40.8 per cent.) are thus included." His results closely agree with those of Bright.

That the early symptoms associated with hypertension are significant prognostically and therapeutically, Janeway has also indicated. Early myocardial weakness and angina pectoris point to a probable death from myocardial insufficiency and call for protection and treatment of the heart. Polyuria and headache suggests the probability of an uremic death: many of the headache cases die of apoplexy. The heart does not call for special treatment unless cardiacsymptoms appear. A high systolic pressure (well above 200) points to uremia or apoplexy, "but the exact height of the blood-pressure in millimeters of mercury does not have much bearing on the expectancy of life." In his series four years for men and five years for women was the average duration of life after the onset of symptoms with high blood-pressure, 25 per cent. of the cases living for more than ten years.

The Functional Condition of the Kidney.-In the early stages, according to the findings of the various functional tests, renal function is approximately normal, except in regard to salt, water, and lactose. Polyuria, nycturia, and a somewhat low and fixed specific gravity are also frequently encountered. Polyuria is readily induced. On applying the salt test, a vascular hyposthenuria is found; the sodium chlorid being smoothly excreted within twenty-four hours, with great increase in the amount of urine, low specific gravity, and a low salt concentration. Lactose excretion is lightly delayed and the amount somewhat low.

On the other hand, in the end stages with a markedly contracted kidney, renal function is tremendously reduced and uremia common. The functional picture is apt to be that of uremia, i.e., low phthalein and lactose secretion with cumulative phenomena in the blood, a high constant of Ambard, together with a tubular hyposthenuria and inability of the kidney to concentrate.

Between these two extremes, all gradations are found, depending upon the degree of renal involvement. Functional findings are of extreme value, particularly when marked decreased function and impending uremia are indicated. On the other hand, these findings must be used with extreme caution. A good renal function does not mean a good prognosis, as death from extrarenal factors is extremely common, myocardial insufficiency, as already indicated being the most frequent mode of exitus. Clinically, it is often difficult to determine whether, in a given case, the clinical picture is the result of myocardial or renal insufficiency. By functional studies, the relative rôle played by each is readily determined. Renal insufficiency may be indicated by functional studies long before uremia or clinical manifestations suggest it. On their evidence uremia may be 
successfully predicted in many instances. On the contrary, death does not invariably follow closely on a zero phthalein output. One such case lived for approximately a year but always in or on the verge of chronic uremia. An ever-increasing total non-protein-nitrogen in the blood, together with a constantly increasing constant of Ambard, and particularly a blood creatinin above $5 \mathrm{mg}$. to Ioo c.c. would indicate approaching death. In myocardial insufficiency cumulative phenomena are extremely uncommon and the phthalein excretion is usually good. In extreme myocardial insufficiency, however, the phthalein may be markedly depressed, but with the first signs of cardiac improvement it is increased markedly. Simultaneously, the edema is apt to become less marked, the urinary output increased with increase in total and relative amount of salt, while, on the other hand, when the renal factor is concerned chiefly, the phthalein is low and remains low, no marked sudden increase resulting from treatment. Cumulative phenomena are extremely common.

Treatment.-Prophylactiçally, little can be accomplished except in so far as checking the progress of the disease is concerned. Occasionally, but not often, timely warning given to the victim of the strenous life and to the subject of hereditary taint bears fruit. Advice to the gouty, alcoholic, to the painter and worker in lead, if followed, may also prove of value. The proper treatment of infectious diseases and of the renal foci of infection undoubtedly prevents the development of some cases. But prophylactic measures are of value chiefly for the arrest of progress.

It is extremely important that all sources of infection be sought and removed. Particular care should be given to the teeth and mouth. Pyorrhea alveolaris should always receive appropriate treatment. The treatment can be divided into general hygienic measures and the treatment of symptoms and complications. The patients fall into two grups-those in whom renal and vascular manifestations predominate and those in whom myocardial insufficiency is the prominent feature. The general treatment here prescribed applies to group I. Myocardial insufficiency calls for special treatment and also for great caution in the application of some of the measures advocated for group I.

The principles underlying the treatment of acute and of chronic parenchymatous nephritis apply here also, i.e., (I) remove the cause where possible; (2) obtain rest for the kidney by minimizing its work through restriction and selection of diet; $(3)$ increase the activity of organs capable of assisting in part the function of the kidney; (4) meet the symptoms and complications as they arise; (5) guard against measures capable of further injury to the kidney. But this does not suffice. The kidney is only one source of danger, the heart and vessels also demand attention. The treatment can be briefly summarized as follows: Regulate the life. Remove strain from vessels, kidney and heart: Spare and protect the kidney. Protect and control the heart. 
General Hygienic Measures.-In the early stages, regulation of the patient's life is the most important feature. 'The high-pressure life must be given up. The incessant strain, work, and worry of the overactive business or professional life must be removed. Moderation becomes the patient's watchword. The picture of the insidious course of the disease and of its tragic ending should be portrayed to the patient. The influence of the moderate life in delaying further development and in possibly warding off severe symptoms should be discussed with him. The comfort and length of life to be had through moderation should be contrasted with the downward course and fatal ending which is so apt to result from persistence in the overactive life. The patient must see the danger and make his own decision. The physician must see that relaxation is permanent.

A fair amount of activity is compatible with a fair degree of health. Business and professional duties should be greatly curtailed but not always abandoned. Business responsiblilities should be shifted to other shoulders. Many patients do better when allowed sufficient work to sustain their interests in life. At first, absolute absence from work, with a complete change, such as can be obtained from a trip abroad, etc., may be necessary in order to break the regular routine of life. By such a measure, the subsequent curtailment of work, which must be insisted upon is frequently more readily obtained. A fair amount of physical exercise is desirable, but overexertion must be avoided on account of the heart. The cardiac condition is of extreme importance. Anything that is apt to throw strain on the heart, which is already overtaxed, must be avoided. Signs of bodily or mental fatigue call for immediate interference on the part of the physician.

Rest, Sleep and Exercise.-Regulation of all of these is essential: The amount of rest necessary naturally depends upon the condition. In the milder cases of hypertension, slight curtailment of business or professional duties may suffice, with one or two hours' entire rest each day. With the severe grades, and particularly where the myocardium is weak, business may have to be given up entirely, the patient resting almost constantly.

Rest in bed for a week with a milk diet is often the best method of reducing blood-pressure. Mental and physical relaxation are necessitated. Psychical treatment and suggestion are often necessary for the former.

Normally, sleep is associated with a considerable drop in bloodpressure - of ten 30 to $50 \mathrm{~mm}$. Although this amount is, perhaps, not obtained in chronic nephritis, there is a tendency to lower pressure during sleep. Frequent awakenings may interfere with this. In insomnia, the night pressure may exceed that of the day; consequently, measures to insure sleep are necessary at times. Warm baths, mustard baths or general massage may suffice. When these fail, resort can be had to bromids, paraldehyde, veronal, sulphonal, or chloral. 
Outdoor exercises are preferable when weather conditions are suitable. Walking is an excellent form of exercise. Milder sports, such as motoring, sailing, canoeing, fishing, golf, croquet, and bowling on the green, may be indulged in to a moderate extent. All exercise should be deliberate. Sudden effort and hurry must be sedulously avoided. Marked general improvement is often manifested immediately upon a regimen which includes outdoor exercise. The appetite and digestion improve, the patient feels better, sleeps better and takes a deeper interest in life. Mental as well as physical fatigue must be avoided.

The wealthy patient deprived of business responsibilities is not usually content to stay at home. If business is resumed at all the working hours are fewer and the vacations longer. Naturally, he turns to the physician as to where he should go. The following locations are admirably suited to his needs: in the winter, the dry, warm climate of southern California, Florida, New Mexico, Jamaica, Bermuda, Southern France, Southern Tyrol or Egypt, while for the summer months, the cooler bracing climate of Canada, Scotland, Scandinavia, or the mountains of Colorado, New York, New England, West Virginia, or North Carolina. Mountain climbing, however, must be carefully suppressed or interdicted. Altitudes greater than 3000 feet should be avoided. Considerable stress is often laid upon the value of visits to mineral springs. Mineral waters are not necessary, and, unless wisely chosen, of ten result in harm. Radioactivity is possibly responsible in part for some of the benefit afforded by the watering places, since hypertension is undoubtedly often decreased by radium. But the chief benefit of watering places is derived from the change in the life of the patient, from the relaxation from business and the attention given to diet and exercise.

Other hygienic measures, such as clothing, bathing, etc., are also important. No matter in what climate, the patient should be guarded against cold. Light woollen underwear is preferable. When the nights are chilly extra wraps should be always available. Bathing is a matter for care. Daily tepid sponge or tub baths should be taken. Under ideal climatic and water conditions, outdoor bathing in smooth water may occasionally be allowed in very mild types, but as a rule it is inadvisable. Swimming is rather too active a form of exercise and should be allowed only in the greatest moderation and this only in the early stages of the disease.

Hydrotherapy. - The influence of hot and cold baths must be kept in mind in hypertension. Hot baths produce a primary rise in blood-pressure and an increase in pulse rate, followed by a secondary fall in pressure, and finally, a permanent increase in pressure which is accompanied by increase in pulse rate and in systolic output. Hot baths are indicated in uremia when the myocardium will stand the extra strain. They are also valuable from the standpoint of bloodpressure, a drop of to to $20 \mathrm{~mm}$. of ten persisting some time after a hot bath that results in a good sweat. The condition of the myocardium 
must always be considered before prescribing a hot bath. Cold baths also increase blood-pressure. The colder and more suddenly they are given, the greater the rise in blood-pressure. They are dangerous, vertigo, apoplexy, and attacks of precordial distress often being precipitated. On the other hand, warm or tepid baths $-37^{\circ}$ to $38^{\circ} \mathrm{C}$. generally lower blood-pressure through peripheral vasodilatation.

Carbon dioxid and oxygen baths at a temperature of $34^{\circ}$ to $36^{\circ} \mathrm{C}$. are sometimes employed. They exert essentially a cardiovascular tonic effect. With the $\mathrm{CO}_{2}$ baths, either a rise or fall in pressure may occur-which, cannot be predicted. The oxygen baths more often result in decreased pressure. At a temperature lower than $34^{\circ} \mathrm{C}$., hypertension is apt to be increased by their use.

Diet.--Spare the kidney but maintain nutrition. Enthusiasm in regard to the former often results in disregard of the latter. The disease is protracted, dragging on from ten to fifteen years in many instances. Nutrition and strength must be maintained, else the patient falls a victim to other diseases. Enervating types of treatment must be discarded and yet physiological economy in regard to protein must be observed.

The important factors to consider are:(r) maintenance of strength and weight; (2) as low a nitrogen intake and output as is compatible with maintenance of strength and weight; (3) maintenance of blood nitrogen at normal level or at as low a level as possible; (4) maintenance of appetite and relish for food; (5) restriction of all substances stimulating and irritating to the kidney; (6) changes in diet to meet special conditions.

In regard to the first, we are guided by the work of Chittenden. Proteins are absolutely essential to health and strength and cannot be excluded from the diet. But they can be reduced to a considerable degree for long periods of time without endangering bodily health or strength. But the tendency at present is to over-rather than to under-restriction of diet. The rules of diet suitable to acute nephritis are not infrequently blindly applied to chronic nephritis. Overzealousness on the part of both physician and patient may lead to serious injury.

The patient requires 80 to roo gm. of protein daily. ${ }^{1}$ The addition of carbohydrates and fat assures a minimum tissue protein breakdown. In what form is this protein best administered? A mixture of various proteins of animal and vegetable origin is best. Milk to the extent of I liter a day ( 30 to $40 \mathrm{gm}$. protein) is advisable. Milk, obviously, cannot be the sole or main article of diet. Fresh meat, white or red, can be taken once a day to the extent of roo to $150 \mathrm{gm}$. (weighed raw), or fish $(200 \mathrm{gm}$.) can be substituted. Eggs are allowable and vegetable proteins such as are present in peas, beans, corn, etc. The caloric and protein values of many of the commoner foods can be ascertained from the tables which follow. A mixed

\footnotetext{
${ }^{1}$ Short periods of greater restriction, e.g., a week or two on a $4 \mathrm{gm}$. $\mathrm{N}$ intake, are frequently indicated, but the physician should attempt to meet the caloric needs.
} 
and varying diet containing the prescribed amount of protein can be chosen each day, special attention being paid to the likes and dislikes of the patient.

The bulk of the diet should be in the form of carbohydrates and fat. The amount of these will depend upon the condition of nutrition and the caloric requirements of the patient. Cereals and breakfast foods with sugar and cream are desirable forms of food. Ripe fruits may be added. Fruit juices may be used as such or in the form of drinks. Simple, well-cooked vegetables, such as potatoes, corn, peas, beans, etc., may be taken, due attention being paid to their protein content. Bread, toast, and biscuits of various kinds are also allowable in considerable quantities. Fats are most suitable in the form of butter, cream, ice cream, etc., although a certain amount may be allowed as fat meat. Simple salads, if not highly seasoned may also form part of the menu. Simple desserts in the form of gelatin, fruit jellies, jams and compotes, or as puddings, or rice, sago, or tapioca are often pleasing to the patient and do no harm.

It is apparent that considerable liberty is allowed the patient in the selection of his food. Simple, well-cooked foods can be taken in moderate amounts at regular intervals. Care must be exercised, however, to see that the amount ingested is not greatly in excess of the caloric needs. The physician should see that the patient is sufficiently nourished but that the kidney is not made to work uselessly for the mere gratification of the patient's palate.

When possible, the level of the non-protein nitrogen of the blood should be ascertained. When this is high, a proper change of diet may be instituted, i.e., cutting down the protein intake, while increasing, perhaps, the carbohydrate and fats. A patient on a daily I2 to $5 \mathrm{gm}$. nitrogen intake may fail to excrete this much. The patient exhibits a positive nitrogen balance and the blood nitrogen may attain double its normal level. A diet of $4 \mathrm{gm}$. of nitrogen continued for a week will, in many cases, cause the non-protein nitrogen of the blood to return to normal; and coincidentally, the patient may feel considerably better, although the blood-pressure may be unaffected and objective evidence of improvement be lacking. Foster and Davis have recently(23) insisted upon the necessity of forcing water as well as limiting protein intake for the successful excretion of nitrogenous waste products particularly in cases of chronic nephritis exhibiting a low and fixed specific gravity urine.

Sufficient is not yet known concerning the necessity or desirability of maintaining blood nitrogen at the normal level, but unquestionably at times subjective improvement results from a low nitrogen intake. That in many cases the blood nitrogen can be lowered by suitable diet has been indicated. On the other hand, when the nephritis is far advanced and when uremia is present, the blood non-protein-nitrogen level is often beyond dietary or medicinal control. Even when all food is withdrawn, the nitrogen of the blood may continue to mount until a level five to ten times the normal is 
reached. This is probably dependent in some instances upon an increased protein catabolism. An antemortem increase is not uncommonly encountered.

Highly seasoned meat broths and meat extracts, pickles, relishes and sauces of various kinds, and indigestible, fried and greasy foods, pastries, pies, and fancy desserts are undesirable and should be avoided.

The development of uremia or of acute exacerbations calls for changes in diet along the lines already discussed. Myocardial insufficiency develops with great frequency and also necessitates dietary changes. When edema is extreme, a Karell diet given for a few days is best, the patient being allowed milk only-600 c.c. to I liter a day. Later, a diet poor in salt and protein is prescribed, such as is used in parenchymatous nephritis.

Water.-The water intake also demands careful supervision. The ingestion of abnormally large quantities of water is harmful, on the following grounds: (I) After absorption it circulates in the blood until eliminated by the kidney - thus tending to make extra demands on the heart and to increase ${ }^{1}$ or maintain a high blood-pressure(24). (2) Increased renal activity is necessary for its elimination. On the contrary, one must remember that the concentrating power of the kidney is lost in the advanced stages of the disease and that considerable quantities of urine are necessary to insure excretion of waste products of metabolism. In the early stages the kidney is hypersensitive and responds readily to increased water intake; but it tires easily, and undoubtedly water should be somewhat restricted in these cases. The thirst is not always a safe guide. A total fluid intake of $11 / 2$ liters a day usually satisfies the patient's thirst and yields a urinary output large enough to carry off waste products even when the excreting power is somewhat poor. But in tubular hyposthenuria when the specific gravity is low and fixed at least 2 or 3 liters a day is necessary. Von Noorden emphasized the necessity during restriction of a "drink day" once a week, the patient then being allowed $21 / 2$ liters

It is not necessary to resort to mineral waters. Ordinary pure water, and water combined with fruit juices, are best. Mild alkaline water may be used at times, but care should be exercised in its choice, chlorids being avoided. Patients often derive benefit from an annual visit to one of the springs. The Poland, Bedford or Saratoga springs in this country, and the Bruekenau, Wildungen, Neuenahr and Vichy springs in Europe are suitable.

Beverages.-Tea, coffee or cocoa in moderate quantities may be allowed once a day. Alcohol should be prohibited altogether as a rule. Occasionally, however, its use in small amounts is justifiable

1 In a recent study of blood volume the writer and his co-workers were forced to conclude that the blood volume is not increased but that it is rather decreased in the majority of instances of hypertension. The vascular system is too small for the volume of blood rather than the blood volume being too great for the patient. 
for patients who have been accustomed to it and in whom loss of appetite and digestive disturbances follow its withdrawal. In this case only a small quantity of a light wine should be permitted before or with meals once a day. Similarly the patient is better off without tobacco, and its use should be interdicted. A daily pipe or cigar may be permitted to those to whom its withdrawal is a great hardship, provided the physician is certain that this allowance will not be exceeded.

Sodium Chlorid.-The necessity for marked restriction of the salt intake is not so great here as in the chronic parenchymatous form. Salt is necessary for proper nutrition and the disease is a protracted one. The excretion of salt necessitates work on the part of the kidney. In pursuing the general plan of protecting the kidney the salt intake should be restricted. This can be accomplished by the patient's giving up his salt-shaker. He must not add salt to his food and must avoid salty foods, such as ham and salt fish. Such a regimen has the additional advantage of decreasing the thirst for water. This amount of restriction suffices except in the event of acute exacerbations or of myocardial breakdown.

Care of the Skin and Bowels. - It is desirable to keep both active. Obviously, however, it is impossible for these organs to assume much renal work over a protracted period, and they are called on for special work only in the event of uremia, exacerbations, etc. Daily tepid baths and moderate exercise will keep the skin active and are the only measures necessary except under the special conditions mentioned. Laxatives are unnecessary if the bowels move regularly and efficiently. Constipation must be avoided. The patient should be taught to go to stool regularly at certain hours. Coarse foods with considerable residue, and fruits, are important dietary considerations. Mild laxatives, such as agar-agar, Russian oil, phenolphthalein gr. i ( $0.065 \mathrm{gm}$.), syrupus cascaræ aromaticus $3 \mathrm{ss}$ to ii. ( 2 to 8 c.c.), pulvis glycyrrhizæ compositus $3 \mathrm{i}$ to ii ( 4 to $8 \mathrm{gm}$.), a Seidlitz powder or a mild pill of aloes belladonna, cascara, and strychnia, may be indicated from time to time. An occasional saline purge is desirable. Purgatives are necessary at times, then any of the drugs mentioned under uremia may be employed.

Ordinarily diuretics are not called for. In the event of oliguria of cardiac origin they assume great importance. Cardiac drugs such as digitalis and strophanthin are indicated and often suffice. But in addition, the xanthin diuretics, caffein, theobromin, diuretin or theocin may be necessary. Theocin is usually most effective (gr. iii ( $0.2 \mathrm{gm}$.) t.i.d. for one day). Saline diuretics, such as potassium citrate or acetate, are also of value. The question of their use is more fully considered under myocardial insufficiency which is not infrequent in this form of nephritis.

Treatment of Symptoms and Complications.-Hypertension.This constitutes one of the most important therapeutic problems of the disease. With it must be identified most of the clinical manifes- 
tations of the disease (cardiac, renal and cercbral). Obviously, therefore, its treatment is worthy of grave consideration.

The height of systolic pressure is not the determining factor prognostically or therapeutically. Many patients tolerate high pressures above $200 \mathrm{~mm}$. Hg. better than others do lower grades of hypertension. Attention should be paid to a high diastolic pressure.

The most important questions are: (I) Is the hypertension a compensatory affair necessary for effective renal excretion? (2) Can it be reduced without injury to the patient? (3) When is it necessary to reduce the pressure? (4) How can this be most successfully accomplished?

A certain degree of hypertension is necessary and unavoidable in chronic interstitial nephritis. Therefore, it is not always to be combated. Nature may be overcompensating or faulty modes of life may result in an arterial tension higher than is necessary or desirable. The excess only is to be combated. Great reduction of pressure is undesirable and harmful, resulting in anuria and effusion into the serous cavities, while too high a pressure may lead to myocardial insufficiency or to apoplexy. As already indicated, it is not the degree but the tolerance of the hypertension in the individual case which determines our therapeutic attitude toward it. A happy medium must be sought in order to avoid dangers lying on either side.

Nevertheless the physician and patient both feel somewhat comforted by maintaining the blood-pressure at as low a level as is compatible with effective renal activity. The general hygienic measures (moderation in physical and mental work, freedom from worry, control of diet and of water intake and proper attention to sufficient activity on the part of the bowels and skin) tend to achieve this end without undue risk.

These measures may not suffice, however. Dyspnea and myocardial insufficiency, uremia, extreme headache and insomnia, may develop, and apoplexy may threaten or occur. Under such conditions the patient is put to bed. Further nitrogen restriction is instituted and the patient is thoroughly purged. A week in bed on a milk diet may greatly reduce the hypertension. In urgent cases in plethoric individuals venesection is advisable. When the heart is in a good condition sweating may be employed with beneficial results. The medicinal treatment of increased blood-pressure is considered in the chapter dealing with hypertension.

Dyspnea usually indicates myocardial insufficiency, present or impending, and calls for cardiac tonics such as digitalis and strophanthin. Outspoken myocardial insufficiency demands its own treatment-rest in bed, limitation of food, water and salt, depletion through venesection, purgatives or puncture, together with support of the heart by strophanthin or digitalis. Uremia calls for treatment along the lines already discussed. The details in regard to the treatment of these various conditions are given below. 


\title{
RENAL, FUNCTION IN HEALTH AND DISEASE
}

\author{
By Leonard G. Rowntree, M. D.
}

The Function of the Kidney in Health.-To separate from the blood substances carried to it for elimination and to pass these substances on into the urine constitutes the chief function of the kidney. The exact nature of the processes by which this is accomplished has been and is at present more a question of theory than a matter of actual conclusive demonstration. According to Ludwig it is brought about by the physical forces of filtration and diffusion. Adherents of this theory, however, have been forced to admit that selective absorption and selective diffusion, depending upon unknown peculiarities of structure of the epithelial cell, must play a rôle, and they thereby abandon the purely mechanical theory. Bowman and Heidenhain(25) assume that in the glomeruli water and inorganic salts are excreted, while urea and related bodies are eliminated through the activity of the epithelial cells in the convoluted tubes. They further assume active participation of the cells through which substances are excreted, and a selective excretory capacity for various parts of the kidney. A large literature has arisen as the result of the controversy waging over the two theories. The facts accumulated are not such as to demonstrate clearly one view or the other, but they have led the majority of workers to lean toward the theory of Bowman and Heidenhain to the extent at least of recognizing that the physical laws of filtration and diffusion (as now known) do not afford a satisfactory explanation of the facts.

The question of selective excretion is of great importance, and has actually been made the basis clinically in attempts at classification of nephritis. This subject, however, is still more within the province of the physiologist $(26)$ than of the internist. It must be admitted that at present the ultimate physics and chemistry of the excretion of any substance by any part of the kidney is not known.

The kidney is not only an organ of excretion: It is capable of synthesis, hippuric acid being synthesized by it from glycocoll and benzoic acid. It has been credited with an internal secretion by means of which it exercises control over nitrogenous metabolism. The existence of this internal secretion has not been proved, but it is here mentioned since it forms a basis for one theory of uremia. The kidney is also an important link in the mechanism maintaining correct equilibrium between acids and bases of the organism-e.g., in maintaining the reaction of the blood and tissues.

The sum total of renal activity in health is, therefore, the removal of waste and unnecessary material from the blood, their excretion 
in the urine, and the maintenance of various substances in the blood and tissues at the most propitious levels.

The Urine of Heallhy Man.-The urine is a yellow liquid secreted or excreted by the kidney. It has a variable specific gravity -usually, however, between IOI 5 and 1025. It is acid in reaction, the acidity being largely due to acid sodium phosphate $\left(\mathrm{NaH}_{2} \mathrm{PO}_{4}\right)$ and in part to organic acids. The degree of acidity varies with the diet and water intake. Its average daily amount in adults approximates I 500 c.c. but varies with the water and food intake and with the seasons. Normal individuals do not arise to empty the bladder during the night, therefore, the normal night amount does not exceed the capacity of the bladder which is 300 to 400 c.c.

Its composition is very complex. In addition to water and inorganic salts the following constituents are of importance-urea, ammonia, the purin bodies (uric acid, xanthin, hypoxanthin), hippuric acid, amino-acids, creatinin, calcium oxalate, several conjugated sulphates and conjugated glycuronates, several aromatic oxyacids and nitrogenous acids, fatty acids, dissolved gases ( $\mathrm{N}$ and $\mathrm{CO}_{2}$ ), its urinary pigments, urochrome and urobilin, and certain enzymes. Of the inorganic salts sodium chlorid is the most important, from Io to $15 \mathrm{gm}$. being excreted daily. The nitrogen output varies with the diet, an average man or an average diet excreting $\mathrm{I} 4$ to $\mathrm{I} 8 \mathrm{gm}$. per diem. Urea $\mathrm{N}$ constitutes ordinarily 80 to 90 per cent. of the total nitrogen.

A striking phenomenon is the variability of the quantitative composition of the urine in response to increase or decrease in substances brought to the kidney by the blood. In health the kidney is not working at its maximum and can readily respond to increased demands made upon it. These responses occur in more or less sharply defined manner in health, but vary widely from this in disease. The features deserving particular study along these lines are the amount of urine, day and night excretion; the specific gravity; chlorid, nitrogen and urea content; and the changes in reaction.

\section{METHODS OF DETERMINING THE FUNCTIONAL CAPACITY OF THE KIDNEYS}

The development and introduction of numerous renal functional tests ${ }^{1}$ are in accord with the general trend of medicine of today, the importance of knowing the ability of any organ to carry on its work, rather than the appearance of the cells engaged in the work, being emphasized.

Renal functional capacity is usually ascertained in one of three ways: (I) By tests of excretory capacity through the quantitative

'A fairly complete consideration of this subject was presented in the symposium on "The Study of Renal Function" before the Congress of American Physicians and Surgeons, Trans., I9I3, ix, I, and before the Am. Med. Assn., Jour. Am. Med. Assn., Sept. 23,1916, p. 927. 
determination of the excretion of various substances in the urine, dyes-methylene blue, indigo, carmine, rosaniline, phenolsulphonephthalein, other chemicals-potassium iodid, lactose, salicylates, sodium chlorid, urea, sugar following phloridzin, and the enzyme diastase. (2) By tests of retention through quantitative determination of the concentration of certain substances in the blood, ions through electrical conductivity, molecules through cryoscopy, incoagulable nitrogen urea, creatinine uric acid and cholesterin. (3) By the quantitative response of the kidney to a definite stimulation, Ambard's constant.

For practical purposes many of these methods are unnecessary. Through recognizing the value and peculiar significance attaching to the findings of the various tests, a profitable selection is possible. In the majority of instances the phthalein and the blood urea give all the necessary information. When edema exists the salt and water balance should be determined.

The Contrast in the Response to Physiological Stimulation of the Normal and Diseased Kidney.-I. Response to Water Tests of the Concentrating and Diluting Capacity of the Kidney.In health, water is usually excreted in amounts of about two-thirds of the fluid intake, and the response to large amounts of water is prompt. The bulk of it is ordinarily excreted by day. Nycturia is a frequent phenomenon in cases exhibiting hypertension and chronic nephritis. The response of the kidney is sluggish throughout the day but on assuming the recumbent position at night the output is markedly augmented, the night urine often exceeding that of the day.

It is generally admitted that the excretion of water is accomplished chiefly by the glomeruli. The kidney has the ability of diluting the urine in response to unusual stimulation, as for instance, a large fluid intake, and also the power of concentrating the urine, that is, of increasing its specific gravity, particularly on a dry diet.

In disease different types of response are observed. Polyuria may develop, and the response to water or any other diuretic is then more pronounced than normal. The polyuria may be continuous, and is supposedly dependent upon small amounts of diuretic substances in the food. According to Schlayer(27) the glomerular vessels are hypersensitive. This condition is often encountered (along with other evidences of vascular disturbance) in cases with hypertension, in mild types of nephritis, and in the type with primary contracted kidney. On the other hand oliguria may persist despite the administration of large quantities of water. This Schlayer ascribed to fatigue of the vessels or to a torpid condition of the vessels, as a result of which they are unable to excrete the usual amount of fluid. The oliguria is frequent in acute nephritis, in chronic parenchymatous nephritis, and in congestion, and is usually associated with marked renal injury. It denotes greater injury to the glomerular vessels than does polyuria. Finally the 
kidney may shut down entirely, absolute anuria supervening temporarily or until death.

It must be remembered, however, that water excretion is subject to many extrarenal factors. The need of the tissues for water, their salt content and their reaction, the permeability of the vessels, passive congestion, and many other factors affect materially the excretion of water and may overshadow the local conditions in the kidney.

Where unilateral disease is present, the diseased kidney almost invariably responds less readily and less promptly than the normal. Its reserve capacity is diminished and it tends to work constantly at its maximum and, therefore, cannot respond to additional stimulation as readily as does the normal. This was the finding of Albarran on whose work these later studies are based.

The Specific Gravity.-Normally this fluctuates, varying inversely with the amount of urine, the urine becoming promptly dilute in polyuria and concentrated in oliguria. It may vary markedly from day to day. In disease the specific gravity still depends to a large extent upon the quantity of urine, in oliguria being high, in polyuria low as a rule. It changes somewhat with the amount of urine, but there is a greater tendency for it to remain fixed.

The ability of the kidney to concentrate is of great importance. On a dry diet the urine of a normal kidney gives a specific gravity of 1025 or 1030. In disease this ability may be entirely lost and a specific gravity of greater than IOI 2 is impossible. Naturally a urine normal in amount with a specific gravity of ror 2 may be incapable of carrying off the waste products of metabolism. It is desirable under such conditions to accomplish this by a polyuria through increased water intake.

The concentrating capacity is often lost while the ability to excrete water is retained. Indeed the diluting capacity of the kidney is often one of the last functions to disappear. While it is often lost in a passive congestion, in acute glomerular nephritis and in nephrosis, it may remain unimpaired until the end stages of chronic glomerulonephritis or of the arteriosclerotic type of nephritis at which period marked renal insufficiency is indicated by practically all the functional tests. Eventually it may disappear and the kidney excrete a urine of constant specific gravity and of relatively fixed composition.

In tubular hyposthenuria the concentrating power of the kidney is almost entirely lost, the specific gravity remaining constantly low and fixed. In acute nephritis oliguria and a fairly high specific gravity may persist despite the administration of large quantities of water and despite the use of diuretics.

The water test as used by $\operatorname{Cottet}(28)$ is as follows:

$6.30-7.00 \mathrm{~A} . \mathrm{MI} \ldots \ldots \ldots .600-800$ c.c. water.

$9.00 \mathrm{~A} . \mathrm{M}$........... a light breakfast +250 c.c. milk products.

Noon................ light meal +400 c.c. liquid.

7.00 P. . . . . . . . . . . light meal +400 c.c. water. 
The urine is collected at 7 A.M. to 9 P.M. and from 9 P. M. to 7 A.M. Normally a marked diuresis occurs promptly, the day urine being three times as great as the night urine. With decreased function this does not occur, in some instances the night urine being greatly in excess of that of the day. Other features such as the influence of posture, the specific gravity, and the chlorid content may also be considered. Cottet feels that by such a study the type of renal injury is revealed.

Volhard and $\operatorname{Fahr}(29)$ administer I 500 to 2000 c.c. of water in the course of an hour. Normally the kidney can eliminate 500 c.c. in the next half hour and the full amount in addition to the normal urinary excretion in the course of twenty-four hours.

In interpreting the results of water tests extrarenal factors must . always be kept in mind or excluded. These are salt intake, sweating, diarrhea, atmospheric conditions, etc.

These $\mathrm{H}_{2} \mathrm{O}$ tests are all modifications of the test of Albarran(30) which was used to show the difference in response to increased water intake by the normal and diseased kidney in unilateral disease of the kidney.

II. Response to SAlt.-On an ordinary diet, Io to I2 $\mathrm{gm}$. of sodium chlorid are excreted daily, in a concentration of from 0.4 to 0.8 per cent. Additional salt, up to at least ro gm., is usually excreted promptly within twenty-four hours. Large amounts of salt are excreted in one of two ways. If the salt is given without extra water it is almost entirely excreted within twenty-four hours, without diuresis, by increased concentration in the urine (according to Volhard and Fahr this may reach 1.8 per cent.); if given with an excess of water it is excreted partially through increased concentration in the urine and partially through diuresis.

In disease of the kidney the excretion of salt is very different. Naturally with a substance so uniformly distributed throughout the organism extrarenal factors influence its secretion markedly. Some forms of nephritis, congestion of the kidney, edema, etc., and diseases unassociated with the kidney-e.g., pneumonia-all play a rôle. The ability to concentrate salt is unquestionably lost in some forms of nephritis, a concentration greater than 0.3 or 0.4 not being attained even with a large salt and small water intake. Certain definite types of response to chlorids are recognized and have been described by Schlayer.

Where a mild vascular injury to the kidneys exists, the administration of salt may be followed by a marked diuresis, all of the salt being smoothly excreted in twenty-four hours without its percentage content in the urine being at all increased. This is usually associated with a somewhat low and fixed specific gravity, and the syndrome is spoken of as vascular hyposthenuria. Here the inability to concentrate is not due to any incapacity of the tubules to excrete salt, but to the hypersensitiveness of the vessels which respond to the salt administration with diuresis. In more severe vascular injury the vessels do not react in the same way, oliguria characterizing the urinary picture. The added salt may be slowly excreted over several days or may be retained in the tissues. Following its administration the total chlorid output may not be at all increased; in fact, it may be definitely diminished. In severe tubular de- 
struction a urine of fixed low specific gravity is obtained, the quantity of which is not materially affected by the administration of salt, and the salt content of which is not augmented by the administration of an extra amount of chlorids because of the inability of the tubules to excrete it. Such a condition is spoken of as tubular hyposthemuria. The specific gravity of the urine is low and fixed, the concentrating ability of the kidney being lost.

In passive congestion the kidney's ability to excrete salt is very variable. As a rule it is markedly decreased. Clinically this is true and also experimentally. In artificially induced passive congestion (constricting bands being placed on the renal veins) the concentration and total salt output was usually decreased although the extent varied markedly. With the clearing up of passive congestion in clinical cases large quantities of salt were swept out of the tissues, as a rule with polyuria, but the concentrating capacity of the kidney for salt is often high.

The Salt Test. ${ }^{2}-$ For this test it is advisable to have the patient on a constant diet and constant water intake. Without change in regard to these factors 5 to $10 \mathrm{gm}$. of sodium chlorid are given, preferably in the early part of the day. The amount of salt excreted is determined by the Lütke Martius method. The percentage concentration of salt, the total output and the effect upon the amount of urine are all-important features for consideration.

III. Response to Nitrogen Intake.-The output of nitrogen in the urine depends primarily upon the total amount of protein metabolism of the body. This is further dependent on the nitrogenous intake. Nitrogen usually constitutes one-sixth of the protein molecule. The nitrogen intake can therefore be roughly calculated by dividing the number of grams of protein of the food by 6.25. Thus $100 \mathrm{gm}$. of protein equals approximately $16 \mathrm{gm}$. of nitrogen. In feeding an attempt is made to meet the requirements of the body. Ordinarily I 2 to $20 \mathrm{gm}$. (the Voit diet, I4 to I8 $\mathrm{gm}$.) of nitrogen are consumed and excreted daily. It is possible to obtain nitrogen equilibrium where the intake and output of nitrogen perfectly balance. Increased nitrogen intake is followed in health by prompt response on the part of the kidneys, i.e., prompt increase in output. The addition to the diet of even $20 \mathrm{gm}$. urea is followed by its prompt excretion.

Extensive investigation has been made on the partition of the urinary nitrogen between the urea and ammonia under normal conditions and with different amounts of nitrogen in the diet. A large part of the earlier work is vitiated by the use of inaccurate methods of analysis. Only the more recent studies or those in which reliable methods of analysis were employed are quoted here. Schultz(3I) found, with a daily excretion of 26 to $36 \mathrm{gm}$. nitrogen, the urea to be 86.3 to 91.6 per cent. and with 17 to $22 \mathrm{gm}$. nitrogen,

${ }^{1}$ It is necessary to sound a warning note against the indiscriminate administration of salt, particularly in cases of acute nephritis. 
83.7 to 88.4 per cent. Gumlich(32) gives the following averages from his investigations:

Urea $\mathrm{N}$

$\mathrm{NH}_{3} \mathrm{~N}$

On mixed diet...........85.6 per cent........4.95 per cent.

On meat diet.............87.0 per cent.........4.77 per cent.

On vegetable diet.........79.2 per cent.........4.47 per cent.

Camerer(33) found urea $\mathrm{N} 84$ to 87 per cent. and $\mathrm{NH}_{3} \mathrm{~N}_{4.6}$ to 5.4 per cent. with an excretion of $\mathrm{I} 2$ to $\mathrm{I} 7 \mathrm{gm}$. nitrogen, while with excretion of 20 to $22 \mathrm{gm}$. the urea $\mathrm{N}$ was increased to 88 to 9I per cent.

Probably the most careful investigation of the partition of urinary nitrogen in health has been made by Folin. On a diet according to Voit's standard, a man of average weight excretes daily between $\mathrm{I} 4$ to $\mathrm{r} 8 \mathrm{gm}$. of nitrogen in the urine. The partition of the nitrogen between the urea and ammonia is in this case, according to Folin(34) urea N 86 per cent. and ammonia N 3.3 to 5 per cent. However, the distribution of the total nitrogen depends on the absolute amount present. When protein metabolism is reduced the ammonia percentage of the total nitrogen rises, unless the food is such as to yield an alkaline ash and the urea suffers a relative as well as absolute decrease. With a reduction of the total nitrogen to 2 to $3 \mathrm{gm}$., only about 60 per cent. of it is excreted in the form of urea. Haskins (35) confirms Folin's conclusions in regard to the effect of low protein diet on urea excretion, but finds that the ammonia $\mathrm{N}$ is a constant per cent. of the total $\mathrm{N}$. With low protein diet he found the urea $\mathrm{N}$ per cent. 71.4 to 82.2 His figures for ammonia $\mathrm{N}$, however, are much higher than those given by other investigators even on a high protein diet. Gammeltoft $(36)$ on a high protein diet with excretion of ro to $\mathrm{I} 2 \mathrm{gm}$. of urinary nitrogen finds the urea $\mathrm{N}$ to be 83.9 to $86 . \mathrm{x}$ per cent. and the ammonia N 3.9 to 4.4 per cent. of the total, while on a low protein diet with an excretion of 5 to $7 \mathrm{gm}$. of urinary nitrogen, the urea $\mathrm{N}$ amounts to 76.9 to $8 \mathrm{r}$ per cent. and the ammonia $\mathrm{N}$ to 5.4 to 6 per cent. of the total.

The limits of normal with varying levels of protein metabolism are, therefore, rather hard to define sharply. However, one can probably safely conclude that with a urinary nitrogen of Io gm. or more the lower limit for urea $\mathrm{N}$ is 80 per cent. and the upper limit for $\mathrm{NH}_{3} \mathrm{~N} 5$ per cent., while with a nitrogen excretion of 5 to ro gm. the lower limit for urea is 70 per cent., and the upper limit for ammonia $\mathrm{N}$ is 8 per cent., while on 2 to $3 \mathrm{gm}$. the urea may fall as low as 60 per cent.

Furthermore, excessive acid production in the organism is accompanied by a lowering of the urea percentage and increase in the ammonia percentage of the total nitrogen.

For the interpretation of findings in disease these wide variations in the nitrogen partition occurring in health solely as the result of dietary changes must be kept in mind. Thus in a series 
of sixty-five cases of renal and cardiorenal disease investigated by Lewis(37) in regard to Ambard's constant, he found urea $\mathrm{N}$ less than 60 per cent. of the total nitrogen in only two instances. The urea per cent. of the total nitrogen must always be considered in connection with the protein intake.

Indeed, the excretion of nitrogen by the kidney proceeds along such regular lines that Ambard has been led to construct certain laws in regard to its rate of excretion in relation to its concentration in the blood. This resulted in a constant for health and the variations from this in disease gives an index to the renal functional capacity which is of decided value clinically. The recent introduction by Marshall of accurate and simple methods for the determination of the urea in urine and of blood tremendously enhances the value of this constant.

Ambard's Coefficient of Urea Excretion.-Ambard and Hallion $(38-39)$ have shown that renal function is governed by the following mathematical laws:

I. If the concentration of urea in the urine remain constant, then the rate of output in the urine will vary directly with the square of the concentration of urea in the blood.

2. If the concentration of urea in the blood remain constant, the rate of output will vary inversely as the square root of the concentration of urea in the urine.

3. If the concentration of urea in blood and urine both vary, then the rate of output will vary directly with the square of the concentration of urea in the blood and inversely as the square root of the concentration in the urine.

The coefficient of urea excretion is a constant relation which exists between the concentration of urea in the blood and the "rate of output" of urea in the urine. It is determined by means of the following formula, based on the first and second laws:

$$
K=\frac{U r}{\sqrt{D \times \frac{70}{p} \times \frac{\sqrt{C}}{\sqrt{25}}}}
$$

where $K=$ coefficient of urea excretion.

$U r=$ concentration of urea in the blood in grams per liter.

$D=$ output of urea in grams per twenty-four hours.

$C=$ concentration of urea in the urine in grams per liter.

$P=$ weight of individual in kilograms.

Twenty-five grams per liter is the standard concentration of urea in the urine, and $70 \mathrm{~kg}$. the standard weight, to which all concentrations and weights are compared.

Procedure.- The patient is given 200 c.c. of water to ensure a proper flow of urine during the test period. Thirty minutes later,

1 Rate of output is the output of any substance in grams per unit of time. Ambard has chosen twenty-four hours as the unit of time 
the bladder is emptied, and this urine is discarded. A second specimen is obtained exactly two hours after the first. This is measured, analyzed, and from these values the twenty-four hour output and the concentration of urea in the urine are calculated. Midway between the voidings, ${ }_{5} 5$ c.c. of blood are withdrawn into a vessel containing a small amount of potassium oxalate. The urea in the blood and urine is determined by Marshall's urease method.

Normal values ${ }^{1}$ of the coefficient(40) lie between 0.07 and 0.09 . Impaired function is evidenced by a rise in the constant, proportional to the degree of renal insufficiency.

In nephritis, values of 0.09 to 0.12 mean very slight impairment of renal function; 0.13 to 0.2 , moderate impairment; and 0.2 to 0.35 , very marked interference with the excretory power of the kidney. Coefficients above 0.4 are met with only in the severest cases, and mean death within a few months.

In chronic passive congestion there is a slight rise in the coefficient-rarely above 0.15 . This rapidly falls to normal with the return of compensation.

In cardiorenal disease, the variations depend on the relative importance of the cardiac or renal features in the symptom complex. Higher values are obtained when renal disease predominates; lower when cardiac insufficiency is the main factor in the functional disturbance.

In normal cases with fever there is a lowering of the coefficient which is not thoroughly understood as yet.

The Excretion of Additional Urea.-Since urea is the chief nitrogenous constituent, it has been utilized in determining the response of the kidney to increased nitrogen intake. Monakow(4I) has observed that in health the addition of $20 \mathrm{gm}$. of urea to the diet results in prompt excretion by the kidney within twenty-four hours, whereas in disease the response is delayed, often days being required. The curve of excretion indicates the functional condition of the kidney.

The total nitrogen is determined by an ordinary Kjeldahl-Gunning Method.

The urea content of the twenty-four hour specimens of urine is best determined by the Marshall method(42). The principle of this method consists in the conversion of the urea into ammonium carbonate by means of an enzyme, urease, present in an extract of the soy bean, and the titration with standard hydrochloric acid and methyl orange. Two 5 c.c. portions of urine are measured into flasks of 200 to 300 c.c. capacity and diluted with distilled water to about roo to I25 c.c. Two c.c. of the enzyme solution are added to one flask, a few drops of toluol to each and the solutions allowed to remain, well stoppered, at room temperature over night. The fluid in each flask is titrated with $0.1 \mathrm{HCl}$ and methyl orange. The amount of hydrochloric acid required for neutralization of the contents of the flask containing the urine and enzyme solution, ${ }^{2}$ less the amount required for neutralization of 5 c.c. urine alone and 2 c.c. enzyme solution alone corresponds to the urea originally present in the urine. This value multiplied by 0.6 gives the number of grams of urea per liter of urine.

The ammonia of the urine is determined by Folin's $(43)$ method.

Test Meals or Test Days.-An attempt has been made to combine several studies in one, i.e., to determine the response of the kid-

1 McClean has modified the form of expression of Ambard's coefficient.

2 One or two tablets of Dunning's urease may be substituted. 
neys to diuretic substances contained in the ordinary diet. For this purpose Monokow(44), Hedinger and Schlayer(45) introduced a test day. The former requires fifteen days of observation and the latter three days. Mosenthal (46) has attempted to cover the observations in a single day. The urine is collected at two-hour intervals during the test day. The urinary output, specific gravity, its total and percentage content of sodium chlorid and nitrogen, the amount excreted by day and by night are determined. A more or less typical response to the meal occurs in health with interesting variations in disease. Thus responses greater than normal occur in some cases of mild nephritis while in severer grades slight or no immediate effect was obtained, the patients exhibiting nocturia. Test meals or test days are now being employed by Mosenthal in the wards of the Johns Hopkins Hospital and by Christian and his collaborators in Boston. Christian(47) states that for the Hedinger-Schlayer test there is no normal curve. Normality consists in the ability to vary from period to period the amounts and concentration of the substances quantitated-abnormality consists in a more or less marked degree of fixation from period to period in all these values.

Sufficient work has already been done to indicate that such studies are of considerable value in determining the type of renal injury and also, what is more important, as a guide to the intelligent treatment of nephritis.

Mosenthal's directions for the test meals are contained in the following memorandum, given in mimeographed form to the nurse in charge of the case:

\section{Test Meals for Renal Function}

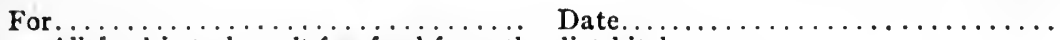

All food is to be salt-free food from the diet kitchen.

Salt for each meal will be furnished in weighed amounts. ${ }^{1}$

All food or fluid not taken must be weighed or measured after meals and charted in spaces below.

Allow no food or fluid of any kind except at meal times.

Note any mishaps or irregularities that occur in giving the diet or collecting the specimens.

Breakfast, 8 A. M.

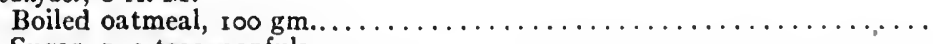

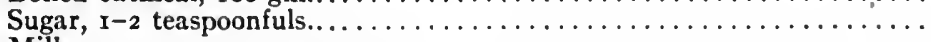

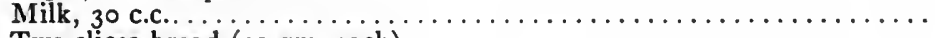

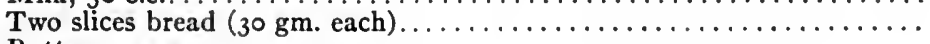

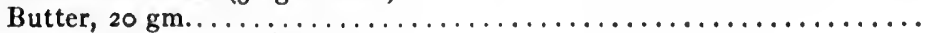

Coffee, 160 c.c.

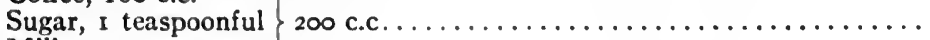

Milk, 40 c.c.

Milk, 200 c.c.......................................

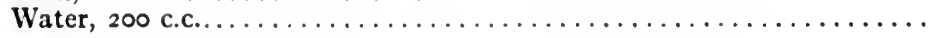

Dinner, 12 Noon.

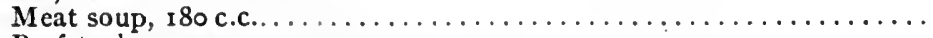

Beefsteak, $100 \mathrm{gm}$.

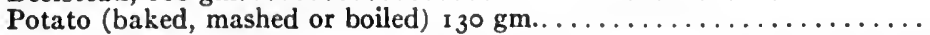

${ }^{1}$ One capsule of salt, containing $2.3 \mathrm{gm}$. of sodium chlorid, is furnished for each meal. The salt which is not consumed is returned to the laboratory, where it is weighed, and the actual amount of salt taken is calculated. 


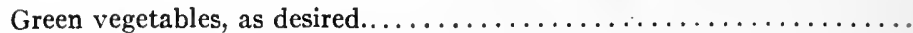

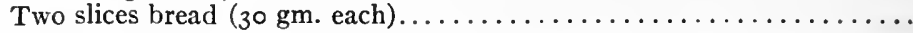

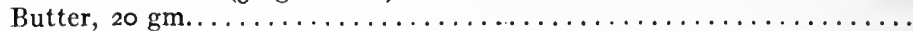

Tea, I 80 c.c.

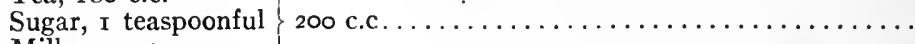

Milk, 20 c.c.

Water, 250 c.c......

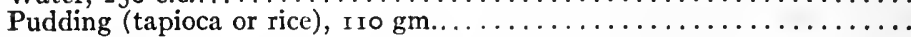

Supper, 5 P. M.

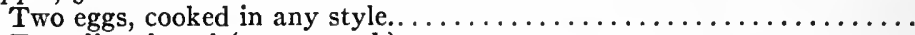

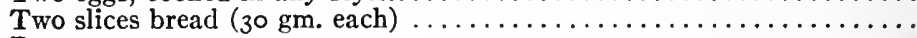

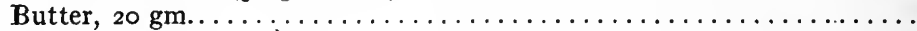

Tea, 180 c.c.

Sugar, I teaspoonful

Milk, 20 c.c.

Fruit (stewed or fresh) I portion

$8 \mathrm{~A}$. M. No food or fluid is to be given during the night or until 8 ; clock the next morning (after voiding), when the regular diet is resumed.

Patient is to empty bladder at $8 \mathrm{~A}$. M. and at the end of each period, as indicated below. The specimens are to be collected for the following periods in properly labeled bottles.

8 A. M. -10 A. M.: ro A. M. -12 N.: I 2 N. -2 P. M.: 2 P. M. 4 P. M.: 4

P. M. -6 P. M.: 6 P. M. -8 P. M.: 8 P. M. -8 A. M.

Specimens are to be left in ward until called for at $8.30 \mathrm{~A}$. M. by attendant from the chemical laboratory.

The above dietary contains approximately $13.4 \mathrm{gm}$. of nitrogen, $8.5 \mathrm{gm}$. of salt, I 760 c.c. of fluid, and a considerable quantity of purin material in the meat, soup, tea and coffee. All these substances act as diuretics, and it is on the mode of excretory response to such stimuli that the present study of renal function depends. Spaces are provided to chart the amounts of food not eaten by the patient, and corresponding allowances can be made in calculating the food intake.

Creatinin $(48,49)$ has recently been utilized for the determination of renal function. The curve of its excretion in the urine for six-hour periods is determined. One or $1 \frac{1}{2} \mathrm{gm}$. of creatinin are administered by mouth or hypodermically and the effect upon the curve determined. Normally the creatinin is promptly excreted while in disease it is delayed.

Similarly the level of creatinin in the blood is important $(50,51)$. Ordinarily this does not exceed $2 \mathrm{mg}$. per roo c.c. In renal insufficiency, however, it is decidedly increased of ten reaching in fatal cases $5 \mathrm{mg}$. or more per 100 c.c. of blood.

IV. Response ro AlKalies.-Although the urine is usually acid, in health it can readily be rendered alkaline by the administration of sodium bicarbonate ( $10 \mathrm{gm}$. intravenously). In acidosis this is not the case. The severity of the acidosis is indicated by the amount of alkali required to render the urine alkaline, 50 and even $100 \mathrm{gr}$. failing to do this in some instances. Quantitative studies along these lines have been carried out by Sellards(52), Palmer and Hender$\operatorname{son}(53)$, and others.

Other Methods of Determining the Functional Capacity of the Kidneys.-In addition to the abnormal response on the part of the kidney to physiological stimuli, numerous other methods of studying renal function have been devised and are in common use. Of these, the tests of greatest value are the excretion of phthalein and 
of lactose and the determination of the level of the total non-protein nitrogen and urea nitrogen in the blood.

The value of any of the excretory tests is purely empirical, because of lack of sound physiological information dealing with the ultimate physics and chemistry of the excretion of any substance by any part of the kidney tubules or glomeruli. Experience has taught us, however, that the failure of phthalein to appear in urine or its excretion in mere traces in the course of chronic nephritis indicates impending uremia and grave prognosis, even in the absence of any definite knowledge concerning the excretion of any other substance. In other words, failure to excrete phthalein empirically signifies incapacity on the part of the kidney to carry on its work, and the degree of reduction indicates to a great extent the reduction in the renal function. The phthalein test is the most valuable single test for renal function. On account of its simplicity, ease of application, and reliability it should always be used. If the phthalein excretion is at all decreased the blood urea or blood nitrogen should be estimated or the constant of Ambard determined.

The Phthalein Test(54).-Twenty minutes to half an hour before administering the test the patient is given 200 to 400 c.c. of water, to insure free urinary secretion, and before giving the test is asked to void, in order to have the bladder empty. One c.c. of solution of phenolsulphonephthalein $(6 \mathrm{mg}$.) is injected intramuscularly, preferably into a lumbar muscle by means of an accurately graduated syringe. ${ }^{1}$ At the end of an hour and ten minutes the patient is instructed to void into a receptacle, and at the end of two hours and ten minutes to void into a second receptacle. Only in cases with obstruction, or when the patient is unable to void, is it necessary to use a catheter. Sufficient sodium hydroxid (25 per cent.) is added to each specimen to make it decidedly alkaline, in order to elicit the maximum color. Each specimen is placed in a I liter measuring flask, and distilled water is added to bring it accurately up to I liter. Each flask is then inverted, to insure a uniform mixture, and a small sample is taken from each and compared against a standard made up of $I$ c.c. of the dye to a liter of water, containing sufficient alkali to bring out the maximum color. The determination can be made in an R. and G. modification of the Autenrieth-Königsberger colorimeter, or in a Dunning or a Duboscq colorimeter. The phthalein output is an index of the total excretory capacity of the kidney.

Normally 50 to 60 per cent. of the dye is excreted in the first hour and 60 to 80 per cent. in the two hours. An excretion of less than 50 per cent. for two hours indicates decreased renal function. The extent of reduction of renal function is indicated by the amount of reduction in phthalein output.

The Lactose Test $(55,56,57,58)$. - This is not necessary for general use. It is of importance in detecting very slight grades of renal injury as occur early in hypertension and arteriosclerosis. The excretion of lactose is always delayed when congestion is present. When its excretion is suppressed a serious interference with renal function is indicated.

Two and one-half grams of pure lactose are dissolved in 25 c.c. of freshly distilled water, placed in small cotton stoppered Erlenmeyer flasks and pasteurized for four hours daily on four successive days, at a temperature between 75 and $80^{\circ} \mathrm{C}$. By this method the dose used is slightly over $2 \mathrm{gm}$. of the sugar in 20 c.c. of water. A fresh solution is used for each injection. Unless the lactose solution is so carefully prepared and sterilized, severe chills and other constitutional symptoms may follow its adninistration. After injection all the urine is collected at the end of four hours and every hour or two thereafter up to twelve hours. Each specimen is tested for the presence of sugar with

${ }^{1}$ The 2 c.c. Record syringe graduated in fifths of a centimeter answers the purpose admirably. 
Nylander's reagent, using constant amounts of urine, solution, and a constant length of time for boiling. In this way excretion time is determined. Polarimetric readings must also be made to quantitate the total amount of sugar excreted. Normally 60 per cent. or more of the dose injected is regained in from four to six hours. Abnormality in excretion, both as to time and amount, affords an index to the degree of glomerular functional disturbance.

The Non-protein Nitrogen of the Blood in Health and in Renal Insufficiency.-As the result of failure on the part of the kidney to excrete certain substances they accumulate in the blood and tissues. Their presence in quantities larger than normal can readily be demonstrated. The extent to which they accumulate has an important bearing on prognosis and treatment.

In health the blood or serum contains-according to Folin-22 to $26 \mathrm{mg}$. of non-protein nitrogen to 100 c.c. However, diet markedly affects this level and on a high protein diet anything under $40 \mathrm{mg}$. to roo c.c. can be considered normal. Approximately 50 per cent. of this is represented by urea nitrogen. In many conditions in which no evidence of serious renal involvement is recognizable the total non-protein nitrogen and urea increase considerably. It is unusual, however, to find the total non-protein nitrogen higher than $50 \mathrm{mg}$. per 100 c.c. of blood, or the urea higher than $55 \mathrm{mg}$. per 100 c.c. (urea in $26 . \mathrm{mg}$.), except in the presence of renal insufficiency. When such figures are attained they point to renal inadequacy and attain prognostic significance. In cases of chronic nephritis, with the piling up of these substances(59 to 68) to 100, I 50 and $200 \mathrm{mg}$. per roo c.c. death is imminent.

The partition of the nitrogen is also changed. Whereas urea $\mathrm{N}$ ordinarily forms 40 to 60 per cent. of the total non-protein nitrogen it may increase so as to constitute 80 to 90 per cent. of the total. The accumulation of nitrogenous products is not confined to the blood alone. It has been shown by Marshall(69) and Davis that the urea of the tissues generally closely approximates that of the blood.

Decrease in the excretory capacity of the kidney is undoubtedly the chief factor in the high level of non-protein nitrogen. That it is the only factor is unlikely, as has been demonstrated in experimental uranium nephritis(70) and in phosphorus $(7 \mathrm{I})$ and mushroom(72) poisoning. Increased protein catabolism undoubtedly plays a part at times. It may play a considerable rôle in pneumonia and in other conditions where a great rise is encountered immediately antemortem.

The Freezing Point of Blood (73 to 76).--Increased molecular concentration of the blood and serum, as evidenced by a depression of the freezing points, is usually associated with the increase of nitrogen. A freezing point below $-0.60^{\circ} \mathrm{C}$. is rarely found except in renal insufficiency where it may be -0.75 or even lower.

The term cumulative phenomena has been introduced to designate these changes in the blood. By this a total non-protein nitrogen content greater than $50 \mathrm{mg}$. to roo c.c., a urea content greater than $55 \mathrm{mg}$. to roo c.c., and a blood freezing point below $-0.60^{\circ} \mathrm{C}$. are indicated. 
The Micro-Kjeldahl for the Determination of Total Non-protein NitroGEN (77). - Five c.c. of blood or serum are mixed with nine volumes of 95 per cent. ethyl alcohol and allowed to stand for twenty-four hours or longer and are then filtered through a dry paper. Ten c.c. of this filtration (corresponding to I c.c. of serum or blood) are used for the determination and are transferred to a large Jena test-tube. One drop of sulphuric acid, I drop of kerosene and a pebble are added, and thealcohol is evaporated off by immersing the test-tube in a beaker of boiling water for five to ten minutes. When the alcohol is removed 1 c.c. of concentrated $\mathrm{H}_{2} \mathrm{SO}_{4}, 1 \mathrm{gm}$. of potassium sulphate and a drop of copper sulphate solution are added, and the mixture is boiled over a fla me of a microburner for ten to thirty minutes until the solution is entirely colorless. From this digested mixture the ammonia is removed in the usual manner-by an air current or by distillation -and collected in a second large test-tube, previously charged with I c.c. of $\frac{\mathrm{N}}{10}$ acid and 2 to 3 c.c. of water. An appropriate amount of Nessler's reagent (diluted $1: 5$ ) is added ${ }^{1}$ The resulting colored solution is washed into a 25-c.c. measuring flask and water added to this. When larger amounts of Nessler's reagent are required a 50- or roo-c.c. flask is used.

The color is immediately read against a freshly prepared standardized Nessler's reagent.

The calculation of analytical results to milligrams of $\mathrm{N}$ for 100 c.c. of blood is simple. The standard solution contains I mg. $\mathrm{N}$ (as ammonium sulphate) Nesslerized in a 5o-c.c. flask, and the colorimeter (Duboscq) prism of the standard is set at $20 \mathrm{~mm}$. The formula is $\frac{20}{R} X D$, in which $R$ stands for the reading of the unknown and $D$ represents the volume to which its ammonia has been diluted.

Blood Urea Determined According to Marshall's Method(78).-Three to 5 c.c. of blood are measured into a small test-tube. One c.c. of the enzyme solution or one tablet of the urease preparation is added, and the tube allowed to stand to complete decomposition. Half an hour at room temperature usually suffices. The contents of the tube is washed into tall cylinders, Caprylic alcohol and sodium carbonate are added, and the ammonia removed by means of a strong air current. The ammonia is collected in flasks containing 25 c.c. $\frac{N}{50}$ hydrochloric acid. When all the ammonia has been driven over, the excess of acid in the flasks is titrated with $\frac{\mathrm{N}}{50}$ sodium hydroxid and sodium alizarin sulphonate. The amount of hydrochloric acid required to neutralize the ammonia represents the urea present in the amount of blood taken. The ammonia can also be determined calorimetrically by means of Nessler's solution.

1 Usually 7 to 8 c.c. suffices for normal blood, but where the non-protein nitrogen is high, more is needed. 


\title{
THE CAUSE, PATHOLOGICAL PHYSIOLOGY, AND TREAT- MENT OF THE MORE IMPORTANT CLINICAL MANIFES- TATIONS OF NEPHRITIS
}

\author{
By Leonard G. Rowntree, M. D.
}

Certain clinical features may dominate the clinical picture of nephritis. Thus in one case edema may be the outstanding feature, while in another it may be hypertension or myocardial insufficiency. Again any combination of these manifestations may be present in one case. Hence we speak of the edematous type, hypertensive type, etc. Some of the manifestations of nephritis are so constant, so complex in origin, and so intimately associated with its rational treatment that a somewhat detailed consideration of their origin, their physiological pathology and their treatment is necessary.

In the vast majority of cases nephritic albuminuria and cylindruria attain. In febrile states these are also extremely common, being associated with cloudy swelling of the kidneys. Their presence may or may not signify nephritis. The casts may disappear or persist with no increase in blood-pressure and no other manifestations of nephritis. The diagnosis of nephritis seems scarcely justified and one is content at times to class such cases simply as instances of albuminuria.

Albuminuria is the simplest type and from this the more complex pictures of nephritis can be built up. Thus hypertension or edema or both may be added. From the standpoint of therapy this idea is important and nephritic patients may be classified as follows:

I. Albuminuria with or without cylindruria.

2. Albuminuria and general edema without myocardial insufficiency.

3. Nephritis with hypertension.

4. Nephritis with hypertension and myocardial insufficiency.

5. Nephritis with advanced renal insufficiency or uremia. Uremia may be superimposed on any of the above groups or may occasionally appear suddenly when no suspicion of nephritis has existed.

\section{ALBUMINURIA}

By this is meant the constant presence of more or less albumin in the urine over considerable periods of time, or its appearance at frequent intervals. The physiological albuminuria which appears after excessive exercise such as long walks, football games, a race, etc., are naturally not included.

Following the observation of $\operatorname{Bright}(79)$ in 1836 that albuminuria was directly associated with pathological changes in the kidney, the 
profession exhibited a natural tendency to regard the presence of albumin in the urine as a positive sign of nephritis. However, cases rapidly accumulated showing albuminuria without other evidence of ill health and with favorable outcome. Their accumulation soon established the belief that albuminuria is not of necessity an indication of severe pathological change in the kidney. This belief has led to the adoption of numerous terms such as "albuminuria in the apparently healthy," "functional albuminuria," "intermittent albuminuria," "cyclic albuminuria," "physiological albuminuria," "albuminuria of adolescence," "orthostatic albuminuria" and "postural albuminuria."

Albuminuria denotes a change in the permeability of the renal cells to albumin. It indicates some change in the tubule or glomerular epithelium, a change which is transient, slight and unimportant, perhaps functional, on the one hand; and on the other, one that is organic, permanent and perhaps progressive in character.

Albuminuria may be divided as follows:

\section{ALBUMINURIA WITHOUT COARSE ORGANIC RENAL CHANGES}

A. Orthostatic Albuminuria. ${ }^{1-A l t h o u g h}$ albuminuria has been previously noted in adolescents by Ultzman and Gull, Moxon in 1878 was the first to call attention to the fact that albumin was frequently present in the day urine of young men of delicate complexion. Following this, numerous communications appeared, the most important of which was published by $\operatorname{Pavy}\left(8_{3}\right)$ in $188_{5}$ in which he insisted upon the importance of the upright position and excluded the influence of diet which was being championed by Rendall(84). He applied to it the name "cyclic albuminuria." Stirling(85) called it "postural albuminuria" and Tessier(86) "orthostatic albuminuria," the terms which are not most frequently employed.

This is a most interesting condition. It occurs most frequently at the period of adolescence, usually between the ages of ten and fifteen years, and is more common in boys than in girls. It is usually discovered accidentally.

Neuroses are frequent among the parents of these patients. Well-marked vasomotor disturbances are often present. It is a common condition particularly in adolescence and puberty and is most frequently encountered in boys in training.

Etiology.-Sex and adolescence are important. The sexual development along with masturbation was held responsible by some of the earlier observers. Disturbance of digestion and general nutritional disturbances have also been held accountable.

Posture is the most important factor. With the patient lying down the albuminuria disappears to reappear with the assumption of the upright position. This is not due to exercise since exercises

1 Excellent articles on the subject are published, see references 80,81 and 82 .

Vor. 1V. -51 
taken in the prone position fail to produce the albuminuria. Moderate exercise in the upright position tends to decrease the albuminuria. Merely standing upright, particularly in the position of lordosis, is the surest method of bringing out the albuminuria in this type. Jehle ascribes the albuminuria to lordosis, postulating pressure on the renal vessels as the cause.

Vascular and vasomotor disturbances are also thought to play a rôle. Craig(87) considered low arterial tension, Edel(88) vasomotor disturbances as the essential feature. A careful study by Erlanger and Hooker(89) showed that a low pulse pressure was the only constant circulatory phenomenon associated with the albuminuria. Hooker $(90)$ succeeded in producing albuminuria in the perfusion of the isolated kidney through decreasing the pulse pressure. He advances the theory of temporary interference with the oxygen supply of the renal tubules occasioned by the decrease in pulse pressure as an explanation of the altered permeability to protein.

Teissier(9I), another careful student of the subject, believes that the condition is dependent upon a developmental defect in the glomeruli.

Langstein, who gives an excellent consideration of the subject, states that Huebner's opinion as to the character of orthostatic albuminuria remains valid today, supported as it is by pathological and anatomical research: "It is a special and long-continued excretion of albumin by the kidneys, not the result of a tissue decrease of the renal substance and not confined to periods of organic development. It is the expression of a general state of organic weakness which cannot be satisfactorily and sufficiently explained."

The urine as a rule contains only a small quantity of albumin but this is extremely variable. Large quantities of albumin of ten appear within a few minutes after assuming the upright position, particularly a lordotic position. The night urine is absolutely free from albumin. A large proportion of the cases can indulge in considerable exercise in the upright position without its occurrence, whereas standing in a lordotic position for fifteen minutes may immediately call it forth. The urine as a rule is normal in quantity and color and in specific gravity. On the other hand, it may be somewhat increased and of a high color. Casts, glycosuria, and a protein precipitable by acetic acid without heat, are present at times. The pulse and blood-pressure are usually normal and no abnormalities are found in the heart.

Prognosis.- The outlook in true orthostatic albuminuria is good. Most students of the subject feel that it has no influence on the expectancy of life. Goodhart (92) who studied the after-history in 250 cases, holds "that the albuminuria of the adolescent has no sinister effect on health or upon duration of life and that with due circumspection such cases ought not to be excluded from the advantages of life insurance or from clerkship in the bank and private office."

In most cases the albuminuria disappears by the twentieth year. In a few cases the albuminuria persists and indications of organic 
renal changes appear. Senator (93) believed that albuminuria always indicated organic disease. He wrote in 1904 as follows: "With the lapse of time I am more strongly confirmed in the view which I expressed years ago, that a slight irritation or inflammatory condition which may progress toward recovery or toward a diffuse chronic nephritis is responsible for most if not all of the cases of 'cyclic' or 'orthostatic' albuminuria."

Clinical and life insurance studies show, however, that the majority of cases disappear with advance in years. Insurance companies generally reject all cases of albuminuria except this type.

B. Febrile Albuminuria (Extremely Common).-The presence of the albumin is usually ascribed to slight glomerular changes, such as cloudy swelling which is induced by the fever. It occurs in the majority of cases of pneumonia, diphtheria, typhoid and scarlet fever. In malaria and in fever accompanying tonsillitis it is apt to appear. As a rule the amount of albumin is small and clears up as the fever disappears. In the vast majority of cases no permanent injury results. Occasionally hyaline and epithelial casts are also present. Nephritis, acute or subacute, develops in a few instances particularly in scarlet fever, diphtheria, and tonsillitis.

C. The Kidney of Pregnancy.-Many cases of slight albuminuria occur in pregnancy without pathological lesions other than cloudy swelling to account for it. In the marked albuminuria of pregnancy organic changes in the kidney may appear. The condition may progress into a true acute diffuse nephritis.

D. Hemic Changes.-Purpura, leukemia, and profound anemia may all be associated with albuminuria. Abnormal ingredients such as bile may also cause albumin to appear in the urine.

E. Diabetes.-Albuminuria is tolerably frequent in diabetes. The amount of albumin varies markedly. In many instances it is slight and unaccompanied by casts and apparently is of little consequence. In other instances it is associated with edema or casts or increase in blood-pressure. Naturally organic renal and vascular lesions are often found associated with diabetes.

\section{ALBUMINURIA WITH DEFINITE LESIONS OF THE URINARY ORGANS}

A. Passive Congestion.-Congestion(94) in itself is capable of causing the appearance of albuminuria, granular and hyaline casts and red blood cells. Details concerning this type of renal lesion will be found in the section dealing with myocardial insufficiency.

B. The various forms of acute and chronic nephritis which have already been considered.

C. Various affections of the pelvis, bladder, ureters, and of the kidney such as pyelitis, hydro- and pyonephrosis, pyelonephritis, tuberculosis of the kidney, etc.

The Treatment of Albuminuria.-Naturally this depends upon the severity and cause of the albuminuria. In a mild albuminuria 
no special treatment is necessary other than that for the underlying disease. It must be frankly admitted that we have no known means of combating albuminuria per se. It will often persist for very long periods despite our every effort. This must be recognized since attempts at treatment frequently result in harm rather than good.

Three good rules can be laid down, however: (I) Every case showing considerable albumin and casts in the urine should be placed in bed, put upon a milk diet and for one week given the treatment already described under acute nephritis. If this proves helpful it can be persisted in judiciously. If it fails to produce considerable evidence of improvement, it should not be persisted in. Many cases, particularly young and growing children, are subjected to prolonged periods of inactivity which do not in any way improve the albuminuria but which do interfere with proper development. (2) Every case of this type, unless the cause is apparent, should be subjected to a search for a focus of infection. The removal of such a focus may result in the immediate clearing up of the condition. (3) Every patient who fails to respond to either of the previous lines of treatment should be put upon an invigorating regimen. Tonics, fresh air, and an abundant supply of food should be insisted upon. With general improvement of nutrition the albuminuria may entirely disappear.

Special comment is advisable in regard to the treatment of orthostatic albuminuria. The albuminuria disappears on putting the patient to bed. This, however, must not be taken to indicate that the patient is to be kept there. The albuminuria appears immediately on assuming the upright position in many cases. Such patients should be up and around under an invigorating regimen and not confined to bed or to the house.

Randall and Dukes(95) reported cases in which diet played a great rôle in controlling the albuminuria. Many of these patients are frequently put upon a milk diet or a very limited diet. This is also a mistake. Diet has very little influence on the excretion of albumin. The diet and also the exercise should be subject to much the same regulation as those of a healthy child.

An open air and active life should be advised. Gymnastics are of great importance provided they are taken short of fatigue. The circulation and the skin should be toned up through massage and friction. Tonics should be administered-bitter stomachics when the appetite is wanting and iron for anemia. In short, the child should be treated much as a normal child, but tonics should be given at times and an outdoor life with sufficient exercise should be assured: In albuminuria associated with oganic lesions of the genitourinary tract, naturally the treatment of the underlying condition is the important consideration. This will be considered, in part at least, under nephritis, myocardial insufficiency, etc. 


\section{EDEMA}

Edema is a prominent feature in nephritis. Here, however, it is not always of renal origin. In acute and in the so-called chronic parenchymatous nephritis its origin is referred to as renal. In chronic interstitial nephritis with hypertension it is of ten encountered in association with myocardial insufficiency which must be ascribed as its cause. Occasionally it is anemic in origin. This is particularly true in hemorrhagic types of nephritis.

The edema which is considered renal in origin is found in the following conditions: in acute diffuse nephritis, in the so-called parenchymatous nephritis, in amyloid disease, and in certain types showing tubular degeneration, the so-called nephroses. ${ }^{1}$

Edema means an excess of fluid in the tissue. The great problem is its cause. In this connection many theories and hypotheses have been advanced. One is forced to admit that in finding the cause of edema one must take into consideration a larger problem, i.e., the factors controlling the water content of protoplasm generally, since all tissues contain fluid.

The development of edema is invariably associated with a disturbance in the excretion of water and almost invariably with retention of salt. When administered, these are not excreted in the normal way but are retained. Edema is therefore associated with water and salt retention. These are factors that are easily demonstrable in most cases of edema. V. Monakow (96) confirms this claim, but states that while edema and chlorid retention are usually associated either may occur without the other. In chlorid retention with edema he finds that water is retained in amounts to yield prac. tically a physiological saline solution. He suggests that this is an intercellular retention. In chlorid retention without edema, water is not retained. He considers this possibly an intracellular salt retention.

Theories as to Cause.-Many theories have been advanced to explain the cause of edema. Hydremia, vascular injury, increased permeability of the peripheral vessels, renal injury with decreased renal permeability, changes in osmotic pressure, circulatory changes, etc., have all been considered. Many of these factors undoubtedly play a rôle. Unquestionably circulatory changes, vascular injury, etc., do play a rôle in the production of edema but the magnitude of their influence is still in doubt. Recently Fischer(97) has advanced the theory that the cause of edema is to be found in the tissues themselves. According to him, edema is due to an increased affinity of the colloids of the tissues for water, i.e., the tissues become more hydrophilic. This he ascribes to the accumulation in the tissues of acids due either to their increased production or to their diminished elimination.

1 Volhard and Fahr state that edema is the most striking clinical manifestation in the majority of cases of nephrosis. It must be admitted, however, that it is not seen clinically in cases of bichlorid of mercury poisoning nor is it encountered in any of the experimentally induced types of tubular degeneration. 
Irrespective of its ultimate cause certain factors play a rôle in its development which are sufficiently interesting to warrant comment. Its production experimentally throws some light on some of these factors.

An excess of either water or salt alone does not result in the appearance of edema. Tremendous quantities of a physiological saline solution can be injected without causing its appearance. Cohnheim and Lichthein $(98)$ injected saline up to 92 per cent. of the body-weight without causing a subcutaneous edema. Injury to the skin of sick animals such as is produced by iodine or exposure to the sun resulted in its appearance. According to Cohnheim this is the result of injury to the vessel walls in the skin and subcutaneous tissues.

The work of Magnus(99) seems to confirm this. He showed that infusion of salt which did not produce edema resulted in its appearance if vascular toxins such as arsenic, chloroform, or chloral were previously administered. In tubular types of nephrosis the injection of salt solution does not give edema. Thus in chromate and bichlorid poisoning edema does not result. Edema does not develop in clinical cases of bichlorid poisoning. Schlayer(roo) has studied these experimentally induced types, determining their vascular response by means of the oncometer. In tubular type, the vascular response is normal or exaggerated to epinephrin, caffein, salt and to tobacco smoke blown into the nostrils of the rabbit. This we have confirmed. Such kidneys are permeable to water. Indeed polyuria or even diuresis follows the use of salt or caffein in these conditions. Pearce(ror) also found that edema failed to appear on administering water or salt to these types. However, when he added the effect of vasomotor irritants such as arsenic, ricin or snake venom to the existing tubular injury, edema could be readily obtained. Most investigators who have worked in this field feel that three factors are necessary for the production of edema, these being renal injury, excess of fluid and vascular injury.

Uranium nephritis is the only experimental type in which edema appears. Richter(IO2) demonstrated that an excess of water administered by stomach tube resulted in edema. Schlayer and Hedinger investigated the vascular response in this type also. Caffein and salt were found to resul ' in vascular dilatation just as in the normal or chromate kidney. But whereas the increase in renal volume is ordinarily accompanied by diuresis, this failed to appear in one stage of the uranium poisoning - the stage in which edema results from the administration of water. These investigators, therefore, established the fact that the renal cells are impermeable at this time. They postulate an increase in the permeability of the peripheral vessels as an etiological factor.

Heineke (IO3) has published an extremely interesting obśervation in connection with uranium nephritis. The serum of the uranium poisoned animal injected into another animal with chronic nephritis results in the development of edema. 
Widal(IO4) has laid great emphasis upon the retention of salt and the part it plays in edema and has demonstrated beyond question the great value of a salt restriction in the clearing up of edema. Experimental work seems to indicate that salt retention is not an absolute requisite to its production and that water plays a more important rôle. The cause of salt retention is interesting. Unquestionably there are types of nephritis in which there is a relative impermeability of the kidney to salt. On the other hand, it is not always due to the inability of the kidney to excrete but due to the retention of salt by the tissues of the body. Castaigne(105) demonstrated the ability of the dog's kidney to excrete salt in certain forms of experimental nephritis accompanied by its retention. The chlorids when injected directly into the renal artery were readily excreted, whereas if administered in the ordinary way they were retained. The importance of the part played by the tissues is increasing in the light of Fischer's studies. (Meltzer (ro6) has given an excellent presentation of the subject of edema.)

Fischer discards circulatory influence, renal and vascular injury. He claims that the cause of the edema is to be found in the tissues themselves. Colloids vary in their ability to take up and hold water according to their acid base and salt content. The majority of acids and alkalies increase the hydrophylic property of protoplasm. Salts, including sodium chlorid, decrease it. Tissues increase in volume through inhibition of water in the presence in the tissues of an excess of acid or alkali. Accumulation of acid from increased production or decreased elimination occurs in certain forms of nephritis. The tissues become hydrophilic, water is inhibited, swelling and edema result. The treatment recommended by him is the neutralization of the acid present with alkali and the administration of salt which results in a decrease of the ability of the tissue to hold water. Fischer injects intravenously a solution of sodium chlorid, gm. 0.14, sodium carbonate, gm. 0.20 , to I liter of water. In less urgent cases the alkali and salt can be administered by mouth or rectum. His theory and treatment are still matters of lively discussion. His ideas are revolutionary. His treatment is now being "weighed in the balance."

Although the fundamental considerations appeal to the reason and although the swelling of colloids is subject to experimental proof, the treatment is far from meeting with universal success. The writer has seen edema unquestionably increased by the application of this treatment.

The Management of Edema.-It is essential to determine the type of edema. Although the same general principles apply to the treatment of the anemic, cardiac, and renal types, yet the etiological distinction is of great importance.

The type due to anemia is met by the administration of preparations of iron or arsenic or both. Where the anemia is severe, transfusions may prove of great value. In the cardiac cases the treatment of the heart-rest and digitalis-play a tremendous rôle 
in its treatment. In all forms of edema the following measures are of importance.

Rest.-The patient should be put to bed and kept at rest until the acute manifestations have completely disappeared. Rest is of paramount importance. It is sometimes extremely difficult to decide how long rest in bed should continue. Many patients are kept in bed unnecessarily long, harm undoubtedly resulting. This is particularly true in children during the developmental stage. Albuminuria following acute nephritis or in the chronic parenchymatous form may persist for months, sometimes in association with slight grades of edema. Von Noorden has laid down the following guide in acute nephritis which is of great value in this connection. So long as red cells and renal epithelium persist in the urine the condition is acute and the patient should be confined to bed. Two weeks following their disappearance the patient may be allowed to get up provided all other symptoms of nephritis are absent. The author believes that mere traces of edema at this stage would suggest the advisability of prolonging the rest somewhat. On the other hand, when persistence in treatment and rest fail to remove the last traces of edema, general improvement follows when the patient is allowed to sit up or take very restricted exercise. An acute nephritis may pass over into a secondary contracted kidney over a period of years. Slight grades of edema may persist despite treatment over prolonged periods, during which too great restriction is inadvisable. Acute exacerbations indicate the treatment advised for acute nephritis.

Clothing.-As sweating may play an important part in the treatment, the patient should be accustomed to blankets, if possible. He should be clad in canton flannel and kept as warm as is consistent with comfort. Fresh air is absolutely essential but chilling must be avoided. Screens should be employed to protect from drafts. The temperature of the room should be kept warm but not uncomfortably so. Dependence for warmth should be placed in coverings, not in a high atmospheric temperature.

Diet.- This must be adapted to the individual case, its choice depending on the cause underlying the edema. Two requisites must be kept in mind, i.e., chlorid and water restriction. The diets suitable to different types of nephritis are discussed in connection with the various types. Thus, in acute nephritis the von Noorden or milk diet; in chronic parenchymatous nephritis, a somewhat more liberal diet; and in myocardial insufficiency, a Karrell diet may be indicated.

Fluid Intake.-The answer to the question as to the amount of water to be allowed is found in the permeability of the kidney. Each patient should be treated individually. In the majority of cases with edema water is excreted with difficulty indicating that the amount of fluid should be restricted to from $1 / 2$ to $\mathrm{I} 1 / 2$ liters a day. This is always indicated in marked edema, marked oliguria, and in anuria associated with edema, since these phenomena point to renal 
impermeability. The more severe the edema the greater should be the restriction. Water administered in large volume in marked edema does not flush out the kidney as a rule. It contributes to the extent of the edema. Since the skin, lungs and bowels are capable of excreting considerable water the thirst can be met with $I$ to $I \frac{1}{2}$ liters of fluid daily without injury.

As the urine quantity increases with the clearing up of the edema more water can be allowed. There are types of acute nephritis following exposure to wet and cold in which the renal permeability is fair. Here so great restriction is not called for.

Salt.--Sodium chlorid should also be withheld. It is excreted with difficulty by the diseased kidney. If retained, as it usually is in edema, it increases the edema. It is in the acute and chronic parenchymatous nephritis particularly that salt is injurious. The salt intake should, therefore, be as restricted as possible. Fruits, fruit juices, and cereals have a very small salt content. Milk has approximately $\mathrm{I} .6 \mathrm{gm}$. per liter, bread 0.5 to $0.7 \mathrm{I}$, and ordinary butter I per cent. Unsalted bread and crackers and washed butter should be used when edema is extreme. A salt-free diet is unnecessary and practically impossible. In marked edema a salt content of $\mathrm{I}$ to $2 \mathrm{gm}$. daily should not be exceeded. Later 2 to $5 \mathrm{gm}$. can be allowed as in the cardiac dict here described. Later more salt can be allowed as the edema clears up.

Diuretics in Edema.- The advisability of administering diuretics, their choice and dosage varies with the cause of the edema. Water is one of the best diuretics but has the disadvantage of contributing to the edema if not excreted. Its use in connection with the various types is discussed elsewhere. If acidosis is present, undoubtedly moderate doses of alkalies, sodium bicarbonate, potassium citrate or potassium acetate are indicated up to the point of bringing the hydrogen ion concentration of the blood to normal. The alkali (combined with sodium chlorid) may be given in solution by mouth or by rectum by the drip method, and in desperate conditions even intravenously, by the method advised by Fischer. The indications and contraindications for most of the other diuretics are much the same as for water. They are contraindicated in the acute stage. Christian( $(107)$ and his co-workers have demonstrated that diuretics have little or no good effect in acute experimental nephritis in animals and that they of ten do harm. In rabbits suffering from acute uremic poisoning, the duration of life was considerably shortened by the administration in therapeutic doses of caffein, sodium, theobromin salicylate, theocin, potassium acetate, spartein and water. Diuresis, as a rule, does not result from their use, except where circulatory disturbances exist. It is, therefore, advisable, in acute nephritis to avoid the use of diuretics. If used at all, they should be given in small doses and for one day only, keeping in mind that the kidney is easily overtaxed, the good effect, if elicited, being watched until it disappears before the drug is again administered. 
Where the arterial tension is low and where circulatory disturbance (passive congestion) exists, diuretics such as digitalis and strophanthin often bring prompt results. The purin diuretics also answer well at times under these conditions.

The diuretics should always be used in moderate doses only: Theocin, gr. iii ( $0.2 \mathrm{I} \mathrm{gm.);} \mathrm{theobromin,} \mathrm{gr.} \mathrm{v} \mathrm{to} \mathrm{viii} \mathrm{(} 0.3$ to $0.5 \mathrm{gm}$.); diuretin (theobromin sodium salicylate), gr. $\mathrm{x}$ to $\mathrm{xv}$ ( 0.6 to $\mathrm{I} .0 \mathrm{gm}$.); caffein, gr. ii to iv (0.12 to $0.25 \mathrm{gm}$.), and caffein citrate, gr. $\mathrm{v}$ to $\mathrm{x}$ ( 0.3 to $0.6 \mathrm{gm}$.), and preferably for one day only. Of the digitalis preparations, the freshly prepared infusion, 3 ii to iv ( 8 to $x 6$ c.c.), the tincture, $m x v$ to $x x$ (I to $\mathrm{r} .3$ c.c.), q. four hours for twenty-four or forty-eight hours may be used. Digipuratum is a reliable standardized preparation of digitalis. It is marketed in tablet form, each tablet equivalent to $\mathrm{x} / 2$ grains (0.1 gm.) of the dried leaf, of which four are given the first day and three the second day. It may also be had in ampoules for intramuscular or intravenous use. Strophanthin is unquestionably the best preparation of strophanthus, and is administered intravenously or preferably intramuscularly ( $1 / 4$ to I mg.) followed by twenty minutes' local massage. In acute exacerbations of chronic nephritis, with high blood-pressure and myocardial insufficiency, digitalis or strophanthus are indicated. In the presence of high blood-pressure no fear of using these preparations need be entertained since arterial hypertension not infrequently decreases markedly during their administration.

Catharsis.-The bowels should be kept free. The extent of purging is guided by the patient's clinical condition. Marked edema and impending uremia call for intensive purgation. Ordinarily, the saline purgatives, if retained, meet all the indications. Daily

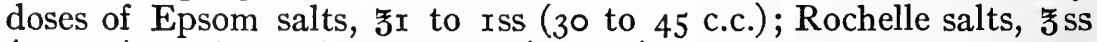
( $15 \mathrm{gm}$.); sodium sulphate, 5 ss. (I $5 \mathrm{gm}$.); or sodium phosphate, 5 ss ( $5 \mathrm{gm}$.) in a half glass of hot water, given in the morning before breakfast, answers admirably if well borne by the stomach. Effervescent preparations, such as Seidlitz powder, liquor magnesii citratis, $5 \times$ (300 c.c.); magnesium sulphas effervescens, 5 i (30 c.c.); lithium citras effervescens, 5 I to 2 ( 30 to 60 c.c.), are most palatable, and may be preferred by the patient, but the large quantities of water involved may make them undesirable. More brisk purgation may be necessary, when the compound cathartic pill, compound jalap powder, 3 ss $(2.0 \mathrm{gm}$.), or elaterium, gr. $1 / 20$ to $1 / 10$ ( 0.003 to 0.0065 gm.), will be found useful. Calomel may be used occasionally, but is not suited for continuous use in acute nephritis. Croton oil should be used in the presence of coma.

If the dropsy is only moderate, the urine not markedly concentrated and uremia neither present nor impending as indicated by clinical and functional studies, the bowels should be kept loose without violent purgation. Vegetable laxatives, such as cascara, senna or a pill of aloes, belladonna, cascara and strychnina, then suffice. 
Diaphoresis.-Sweating is indicated particularly where the edema is marked or where uremia is impending and renal function markedly depressed. It should be procured with as little depression to the patient as possible and without subsequent exposure to cold. Dropsy is best combated with hydrotherapy - either the hot bath, the hot pack or the hot air bath. For children, the hot pack is usually best. The bed is covered with a rubber sheet, a large, heavy blanket is dipped in hot water, wrung out, and rapidly wrapped about the naked patient, care being taken that it is not hot enough to burn. A dry blanket is wrapped about the wet one and several hot-water bags placed about the patient and an ice cap placed on the head. The bath should last for one hour. If sweating does not appear early, a drink of cold water, alone or with spiritus etheris nitrosi, $3 \mathrm{i}$ to ii (4 to 8 c.c.), is given. Where sweating still fails, resort to a small dose of pilocarpin, gr. 1/20 (0.003 gm.), may be necessary. The pack should be given daily, if great need exists, twice daily.

For adults, the hot air bath is usually employed. The method employed in the wards at the Johns Hopkins Hospital is described under the section dealing with therapeutic technique.

If sweating does not readily appear, under this treatment it can be often facilitated by the administration of cold drinks, water or a diaphoretic mixture, such as sweet spirits of niter. Pilocarpin in small doses, gr. $1 / 20$ ( $0.003 \mathrm{gm}$.) hypodermically, occasionally has to be combined with the above procedure.

Hot baths of fifteen to twenty minutes' duration, immediately followed by wrapping the patient in hot blankets, answer very well in some cases. The patient can be wrapped in hot blankets, surrounded by hot bricks or by hot water bottles according to the technique described under uremia.

Recourse can be had naturally to electric cabinets in which the patients can remain in the recumbent position, if such are at hand.

The sweats are given daily. Where the edema is extreme or uremia is threatening or present, they may be used twice a day. Pilocarpin is not advisable except where the other methods fail. It can be used as an adjunct to them in small doses. If this fails, and uremia supervenes, it can be given hypodermically, gr. $1 / 6$ to $1 / 8$ (0.0I to $0.008 \mathrm{gm}$.), to adults and gr. $1 / 20$ to $1 / 12$ ( 0.003 to $0.005 \mathrm{gm}$.), to children from two to ten years.

Edema is combated by the general measures just described, particularly by restriction of fluid and salt intake, by free purgation and by sweating. If these measures fail to reduce the dropsy, or if the dropsy is extreme, the fluid may be drained from the subcutaneous tissues by one of the following procedures: $(a)$ incisions $1 / 2$ to I inch long over each malleolus; $(b)$ Io or 15 punctures are made into the subcutaneous tissue with a bistoury needle or trocar; $(c)$ three or four Southey's tubes, small perforated silver trocars, or three or four ordinary trocars are inserted into the subcutaneous tissue.

These measures are only indicated where the edema is extreme 
and sloughing is threatened. They are efficacious-resulting in the removal of as much as 4 liters of fluid a day at times. Unless extreme care is exercised, erysipelas may develop. The technique and dangers of these procedures is considered later.

Ascites may be extreme, which demands an abdominal paracentesis, or a Southey's tube can be inserted and the fluid gradually drained off. A thoracic paracentesis is necessary at times when much fluid collects in the pleural cavities.

\section{HYPERTENSION}

The term hypertension is used to designate a blood-pressure which is greater than normal, and in adults this is usually a systolic pressure of $160 \mathrm{~mm}$. Hg. or more. The condition ${ }^{1}$ is a clinical manifestation commonly encountered in $(a)$ chronic nephritis, particularly in forms associated with contracted kidney and with arteriosclerosis; (b) in uremia; (c) apart from any demonstrable renal lesion, when it may be considered as essential hypertension; and $(d)$ in association with myocardial insufficiency.

Since the time of Bright(I08) the cause of hypertension and the relationship between it and chronic nephritis, arteriosclerosis and cardiac hypertrophy have greatly interested physicians and pathologists. Bright himself recognized the existence of an intimate relationship between the heart and kidneys in chronic nephritis. He speculated as to the cause of the cardiac changes and set in motion problems that even today have not been satisfactorily solved. $\mathrm{He}$ compared the clinical and autopsy findings in a hundred cases of nephritis.

He writes: "The deviations from health in the heart are well worthy of observation: they have been so frequent as to show a most important and intimate connection with the disease of which we are treating. . . ., Out of fifty-two cases of hypertrophy, no valvular disease whatsoever could be detected in thirty-four. . . . This naturally leads us to look for some less local cause, for the unusual effort to which the heart is impelled: and the two most ready solutions appear to be, either that the altered quality of the blood affords irregular and unwonted stimulus to the organ immediately; or, that it so affects minute and capillary circulation as to render greater action necessary to force the blood through the distant subdivisions of the vascular system."

What is the relationship between hypertension and chronic nephritis and cardiac hypertrophy? As the result of much thought and investigation a definite view is now entertained by many of the foremost workers in this field. Following the lead of Gull and Sutton(rog), the genuine contracted kidney is looked upon not as a true nephritis or as a primary nephropathy, but rather as renal

1 The recent work of Sir Clifford Allbutt on "Diseases of the Arteries" will be found interesting. 
atrophy of circulatory origin due to a sclerosis of the small renal vessels which is but a part of a general "arteriocapillary fibrosis." Arteries, kidney and heart each play a rôle. The term "hypertensive cardiovascular disease" (I I 0 ) has been proposed. Unfortunately this name does not include the renal factor. Bright recognized all three, but chose to emphasize the renal factor, as evidenced in the name. The term hypertensive cardiovascular disease is very significant, as indicating the importance attached to the other factors by one of the foremost students of the subject. The term admirably fits one type of hypertension as will be seen later. The primary lesion is considered by most authors to be arteriolar, the renal and cardiac manifestations being secondary.

The Causes of Hypertension.-In the majority of cases the cause is unknown. Some of the factors, however, are known. It can be produced experimentally:

(A) By a sufficient reduction of renal substance (III-II2-II3). The reduction must be extreme (about two-thirds) whereby the factor of safety is abolished and the remaining part is working at its maximum. In other words, hypertension develops when the excretory function is diminished to the minimum compatible with life. The explanation nearest at hand is that products are retained which set up hypertension which is ascribed to vascular tonicity.

$(B)$ By partial bilateral obstruction or partial obstruction to the ureter after removal of one kidney. Hydronephrosis results under these conditions together with increase in the blood nitrogen and decrease in the excretory capacity of the kidney(II4). The same explanation seems plausible here also.

(C) By lead. Lead poisoning is the most important exogenous intoxication leading to hypertension. Indeed hypertension is produced directly by lead in plumbism and indirectly by gout, arteriosclerosis, and nephritis, all of which are common in lead poisoning. Lead colic and lead encephalopathy in their onset are associated with marked increase in blood-pressure. According to Norris(I I 5) " 80 per cent. of lead workers exhibit high arterial tension over years of time even when free from symptoms of poisoning." Pal(I I6) looks upon lead colic as a spasm of the vessels of the splanchnic area. It is, therefore, analogous in origin to angina pectoris and calls for treatment by nitrites.

Clinically hypertension is frequently associated with uremia, which is regarded as a toxemia and in which nitrogen retention is common. It is clear, therefore, that retention due to inadequate renal function often goes hand in hand with hypertension.

On the other hand, hypertension is found clinically apart from nitrogen retention, when it is frequently associated with hypersensitiveness or overirritability of the renal vasomotor mechanism. The hypersensitiveness of the renal vessels can be demonstrated, but the excretory capacity is excellent. Vascular irritability may be -and is usually supposed to be-general. This, however, has not 
yet been proved. The renal factor in these cases is secondary, years often elapsing before renal function is disturbed. Eventually a primary contracted kidney and a hypertrophied, dilated heart may be found. Hypertension can, therefore, exist without any demonstrable decrease in renal excretion( 1 I 7-1 18 ).

This is as far as the facts at hand take us. What is the character of the arteriolar changes underlying the hypertension? What is its mechanism? Is it functional or organic?

Two views have been entertained:

(A) Mechanical Origin.-Traube(II8) believed that the obliteration of great numbers of capillaries in the kidney raised the arterial pressure, which resulted in hypertrophy of the heart. Cohnheim(120) held that the circulation through the kidney at any one moment is not dependent upon the need of the kidney for blood but rather upon the amount of material in the blood which is to be excreted in the urine. When the circulation on the part of the renal tissue is interfered with through atrophy of the renal substance the blood flow through the remaining kidney substance must be as great as it would have been for the whole organ, had it remained intact. In order to secure such a blood flow through the restricted capillaries an increased pressure is necessary. The extra pressure is supplied by the left ventricle, which hypertrophies, a corresponding resistance on the part of the other arterial territories being necessarily maintained. This explanation covers both the hypertension and the cardiovascular changes.

However, ligation of both renal arteries fails to result in hypertension. Extensive cutting off of renal arterioles by emboli(I I I) (paraffin) is not accompanied by increased tension. The purely mechanical theory, therefore, does not suffice.

Loeb(122) has claimed that the degree of hypertension and the extent of glomerular changes run somewhat parallel. He considers hypertension as a regulatory function which is evoked reflexly when, owing to capillary obliteration, and decreased glomeruli, vasodilation in the kidney is no longer capable of producing an adequate flow of blood through that organ. It has been demonstrated, however, that hypertension may be most pronounced in cases with but slight glomerular lesions. The parallelism is by no means absolute.

Recently Volhard and Fahr(r23) have admirably summarized our knowledge concerning the relation of hypertension to renal disease. They particularly emphasize its association with diffuse glomerulonephritis. Indeed, to them, hypertension constitutes the most reliable criterion for distinguishing clinically between simple degenerative nephritis and diffuse glomerulonephritis. They have also divided hypertension into two forms, the "benign," in which they claim that there is intimal thickening in the smallest renal vessels unassociated with changes in the vast majority of glomeruli and secondly the "combination or malignant" form in which primary arterial sclerosis is associated with secondary glomerular changes 
which results in a contracted kidney. The different types depend upon the presence or absence of histological changes in the glomeruli, these glomeruli being normal in the former type while the majority of them show atrophy, hyalinization or fibrosis in the latter. Intimal thickening in the smallest renal vessels is capable of setting up hypertension, but unless a large proportion of the glomeruli are involved renal function is little, if any, disturbed. The cause of the glomerular changes they were unable to determine.

Benign Hypertension.-This is characterized by very high bloodpressure with practically a normal renal function as regards nitrogen, chlorid, water, phthalein, etc., as long as the myocardium remains in the stage of compensation. Hypersensitiveness of the vessels, nycturia and a slight delay in lactose excretion may be the only abnormal functional findings.

The concentrating capacity of the kidney is retained, a specific gravity of 1025 or 1030 being reached on a dry diet. Albumin and casts-hyaline and granular-are commonly associated. The eye grounds reveal sclerosis, but not albuminuric retinitis.

The incidence of the disease increases with each succeeding decade. Its course may be exceedingly prolonged. Fifteen, twenty, and even thirty years may pass without either extreme discomfort or serious accident.

Cardiac and asthmatic symptoms are prominent. Myocardial insufficiency is extremely common bringing with it its usual train of, clinical and functional renal changes. Vascular and cerebral symptoms are also frequent. Convulsive seizures referred to as eclamptic types of uremia may develop. ${ }^{1}$ Apoplexy or cerebral thrombosis usher in the fatal termination. At section the kidney may be normal in size or constricted and in appearance may present a smooth lobulated or granular surface. The heart is always hypertrophied, often dilated. This type is in reality primarily and chiefly a vascular disease. The choice of Volhard and Fahr is a fitting name for the condition considering the frequency with which it runs a prolonged benign course. The name suggested by Janeway"hypertensive cardiovascular disease"-also answers admirably for this type.

Malignant Hypertension.-In a small proportion of cases of the former type signs of renal insufficiency develop while the heart is perfectly compensated. True uremia may develop. One of the earliest evidences of transition from the "benign" to the "malignant" form is the development of albuminuric retinitis. Polyuria, low fixed specific gravity, interference with nitrogen elimination, or the piling up of nitrogen and urea in the blood, and inability to concentrate chlorids ensue. The phthalein output may be reduced

\footnotetext{
${ }^{1}$ One such case recently seen in our wards presented practically normal renal function. Convulsions developed repeatedly at one- to four-week intervals. The patient eventually developed what appeared to be a cerebral apoplexy. At section hemorrhage in the medulla and pons was found, the kidney being approximately normal.
} 
to a trace or zero and cumulative phenomena and high Ambard constant appear as renal insufficiency or uremic onset. Death from uremia is the rule, though about half the cases show cardiac edema prior to death.

The histological study of the kidney reveals intimal thickening of the smallest renal vessels, extensive glomerular atrophy, hyalinization, or fibrosis of the glomeruli, and often marked tubular changes.

(B) Chemical Origin.- Johnson's(124) view is the one most frequently accepted-that excretory failure on the part of the kidney results in the accumulation of waste products which cause vasomotor spasm. This view has already been discussed, together with some of its shortcomings. Simple retention of urea and other waste products, so far as we know them, fails to explain the facts.

Various substances have been held responsible. Chief among them is adrenin (epinephrin). Hypertension has been explained on the grounds of adreninemia(125-126). ${ }^{1}$ Adrenin cannot be absolutely excluded, but the recent work makes it seem improbable as the cause of hypertension.

Renin.-Tigerstedt and Bergmann in 1898 (127) obtained a substance from the kidney of the rabbit which on injection caused a rise of blood-pressure. This they called renin. Subsequent work has failed to show the constant presence of such a substance when care is exercised to test the extract on the species from which the extract is obtained.

The two views generally held at present are that hypertension arises as the result of an accumulation of toxins due to the failure of the kidney to excrete them, or secondly as a primary irritability of the vasoconstricting mechanism of unknown origin.

In the latter type we are dealing with an arteriolar anomaly. Although often spoken of as an arteriocapillary fibrosis, the early obstruction may be functional in character rather than organic. Eventually widespread narrowing of arterioles and capillaries, organic in character, i.e., arteriosclerosis, does develop.

Although the ultimate factors concerned are as yet unknown, certain factors are recognized as having some bearing on the incidence of hypertension.

Etiology of Arteriosclerosis.-Osler(128) says: "There are four great factors in the causation of arteriosclerosis-the normal wear and tear of life, the acute infections, the intoxications, and those combinations of circumstances which keep the blood tension high." Unquestionably the three former play a great rôle in the production of hypertension.

1 Space does not permit a full discussion in regard to this. Janeway has discussed the present status of the problem. Suffice it to say that the individual tests employed in the identification of adrenalin are not specific. Consequently, utilizing non-coagulated blood, a group of three tests must be employed for its identification-i.e., constriction of the carotid artery, dilatation or relaxation of the coronary artery and of intestinal strips. The result of the application of the three tests points to the presence of a substance with properties differing from those of adrenalin and which more closely resembles barium. 
The wear and tear of life is greatest when the candle is kept burning at both ends. The victim of the strenuous life at thirty-five or forty years of age often awakes with a start to the fact that hypertension and arteriosclerosis have insidiously laid hold upon him while he has been attending assiduously to business. There are all grades of arteries. Some wear better than others but the best grades are apt to fail when the strain is constant and great. Individuals inheriting poor vascular material escape only when the greatest moderation is continuously observed in regard to work, eating and drinking.

Overeating.-According to Allbutt, "One main cause of rising arterial pressure in middle life is excess of feeding, that is to say, of food in excess of work and excretion." Osler assents to this, stating that his "attention has been repeatedly called to the frequency of arteriosclerosis in persons who have been temperate in every respect except at the table."

The Acute Infections.- In this connection syphilis is of the greatest importance. Its predilection for the arteries is universally recognized. It induces acute productive lesions and also degenerative lesions as seen in chronic arteritis. Mesaortitis affecting the thoracic aorta particularly, may develop slowly after infections and arteriosclerosis with hypertension are both extremely frequent following luetic infection. Scarlet fever, diphtheria, measles, smallpox, influenza, typhoid fever, and tuberculosis may all play a rôle.

Age.-Hypertension is infrequent in the young, rarely appearing before thirty. The vast majority of cases, 80 to 90 per cent. according to Janeway's studies, occur in the fifth, sixth and seventh decades. His series showed a greater proportion of cases over eighty years of age than below thirty. Normal vessels are the exception after forty years of age.

Sex.-Males are affected more frequently than females. Men, as a rule, have a more strenuous life and are subjected almost constantly to greater strain. The preponderance in males is decided.

Heredity is unquestionably concerned. The quality of, as well as the stress put upon, the vessels is a determining factor.

Intoxications.-Alcohol, tobacco, and lead are exogenous poisons commonly believed to have important influences. In regard to lead there is no question. Crucial proof, so far as alcohol and tobacco are concerned, has not been brought. Longevity is not at all uncommon in individuals who have been constantly addicted to their moderate use throughout life. Unquestionably they play a rôle in vascular crises such as angina pectoris, but that they initiate the primary changes is still a matter to be proven.

Hypertension and Renal Function.-According to the findings of functional tests, in the early stages renal function is usually normal, except in regard to salt, water and lactose Polyuria, nycturia and somewhat low specific gravity are apt to be encountered. Polyuria is readily induced. On applying the salt test a vascular Vou. IV. -52 
hyposthenuria is found, the sodium chlorid is smoothly excreted within twenty-four hours with great increase in the amount of urine, low specific gravity indicating a low salt concentration. Lactose excretion is slightly delayed and the amount of urine is somewhat low.

On the other hand, in the end stages with markedly contracted kidney, renal function is tremendously reduced. Uremia frequently develops. The functional picture is apt to be that of uremia-i.e., low phthalein and lactose secretion, and cumulative phenomena in the blood together with a tubular hyposthenuria and inability of the kidney to concentrate. The extremes are well illustrated by the "benign" and "malignant" types of Volhard and Fahr. Between these two extremes all gradations are found depending upon the degree of renal involvement.

Functional findings are of extreme value particularly when marked decreased function and impending uremia are indicated. On the other hand these findings must be used with extreme caution. A good renal function does not mean a good prognosis as death from extrarenal factors is extremely common. Myocardial insufficiency, as already indicated, is the most frequent cause. In this connection, however, the functional studies are of extreme importance. Clinically it is often difficult to determine whether in a given case the clinical picture is the result of myocardial or renal insufficiency. By functional studies the relative rôle played by each is readily determined as a rule. In myocardial insufficiency alone, cumulative phenomena are extremely uncommon and the phthalein excretion is relatively good. In extreme myocardial insufficiency, however, the phthalein may be markedly depressed but with the first signs of cardiac improvement the phthalein output increases markedly. Simultaneously the edema is apt to become less marked, the urine increased with increase in total and relative amount of salt. When the renal factor is chiefly concerned the phthalein is low and remains low, showing no marked sudden increase as the result of treatment and at the same time cumulative phenomena are extremely common.

Great prognostic significance also attaches to eye-ground changes.

Prognosis.-The majority of these patients with hypertension do not die of renal insufficiency. As has been indicated in the discussion of chronic interstitial nephritis, the heart, the vessels and the kidney are all involved. Many cases die a cardiac death, others by apoplexy and some in uremia. Janeway has tabulated the cause of death in $2 \mathrm{I} 2$ carefully studied private cases:

"While the largest total number, sixty (32.6 per cent.) died a gradual cardiac death-if one groups together deaths from uremia and from apoplexy a "cerebral group" as did Bright-seventy-five (40.8 per cent.) are thus included." His results closely agree with those of Bright.

That the early symptoms associated with hypertension are significant prognostically and therapeutically, Janeway has also indicated. Early myocardial weakness and angina pectoris point to a 
CAUSES OF DEATII JN JANEWAY'S SERIES

\begin{tabular}{|c|c|c|c|c|c|}
\hline \multirow[t]{2}{*}{ Causes of death } & \multicolumn{2}{|c|}{$\begin{array}{l}\text { Male, } \\
\text { per cent. of total } \\
\text { known-13i }\end{array}$} & \multicolumn{2}{|c|}{$\begin{array}{l}\text { Female. } \\
\text { per cent. of total } \\
\text { known-47 }\end{array}$} & \multirow[t]{2}{*}{ Total } \\
\hline & No. & Per cent. & No. & Per cent. & \\
\hline Gradual cardiac insufficiency...$\ldots \ldots \ldots$ & 48 & $35 \cdot 0$ & I 2 & $25 \cdot 5$ & 60 \\
\hline Uremic convulsions, coma, or gradual uremia & 31 & 22.6 & 15 & 31.0 & 46 \\
\hline Cerebral apoplexy or its results.......... & 20 & 14.6 & 9 & I9. I & 29 \\
\hline 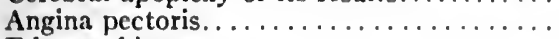 & 10 & $7 \cdot 3$ & $\circ$ & $\ldots$ & 10 \\
\hline 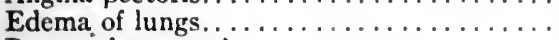 & 6 & $4 \cdot 4$ & 1 & 2.1 & 7 \\
\hline 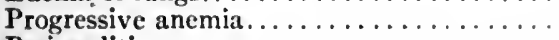 & I & 0.7 & 2 & $4 \cdot 3$ & 3 \\
\hline 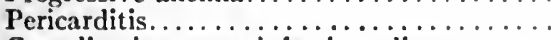 & I & 0.7 & $\circ$ & & \\
\hline Complicating acute infectious diseases... . & 9 & 6.6 & 4 & 8.5 & 13 \\
\hline Other accidental causes.............. & 7 & $5 \cdot I$ & 2 & $4 \cdot 3$ & 9 \\
\hline Unknown $\ldots \ldots \ldots \ldots \ldots \ldots \ldots$ & 25 & $\ldots$ & 3 & & \\
\hline \multirow[t]{2}{*}{ 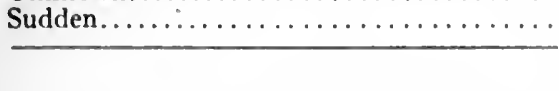 } & 4 & 2.9 & 2 & $4 \cdot 3$ & 6 \\
\hline & 162 & $\ldots$ & 50 & $\ldots$ & 212 \\
\hline
\end{tabular}

probable death from myocardial insufficiency and call for protection and treatment of the heart. Polyuria and headache suggest the probability of an uremic death; many of the headache cases die of apoplexy. The heart does not call for special treatment unless cardiac symptoms appear. A high systolic pressure (well above 200) points to uremia or apoplexy, "but the exact height of the blood-pressure does not have much bearing on the expectancy of life." Four years for men and five years for women was the average duration of life after the onset of symptoms with high blood-pressure, 25 per cent. of the cases living for more than ten years.

Volhard and Fahr classified 268 cases of their series as benign hypertension and only thirty-six as malignant. In the former, death by cardiac decompensation and edema, or by vascular accident is the rule, while in the latter uremia preceded death.

Prophylactically little can be accomplished except in so far as checking the progress of the disease is concerned. Occasionally, but not often, timely warning given to the victim of the strenuous life and to the subject of hereditary taint bears fruit. Advice to the gouty, alcoholic and to the painters and workers in lead, if followed, may also prove of value. The proper treatment of infectious diseases and the removal of foci of infection undoubtedly prevent the development of some cases, but these measures are of value chiefly for the arrest of progress. It is extremely important that all sources of infection be sought and removed. Particular care should be directed to the teeth and mouth. Pyorrhea alveolaris and alveolar abscesses should always receive appropriate treatment.

Active Treatment.-The principles underlying the treatment of nephritis apply here also-i.e., (I) remove the cause when possible; (2) obtain rest for the kidney by minimizing its work through restriction and selection of diet; (3) increase the activity of organs capable in part of assuming the function of the kidney; (4) meet 
the symptoms and complications as they arise; (5) guard against measures capable of further injury to the kidney. But this does not suffice. The kidney is only one source of danger; the heart and vessels also demand attention. The treatment can be briefly summarized as follows: regulate the life; remove strain from vessels, kidney and heart; spare and protect the kidney; protect and control the heart.

The treatment can be divided into general hygienic measures and the treatment of symptoms and complications. The patients fall in to three groups: ( $\mathrm{x}$ ) those in which renal and vascular manifestations predominate; (2) those in whom myocardial insufficiency is superimposed, and (3) cases exhibiting no symptoms whatever. Myocardial insufficiency calls for the special treatment later described and also for great caution in the application of some of the measures advocated for group III. Evidences of renal insufficiency or of apoplexy naturally demand special attention.

Hypertension per se does not constitute an indication for medicinal treatment. In chronic nephritis a certain degree of hypertension is necessary for the proper carrying out of renal function. It is a provision of nature, a mechanism for compensating an existing functional or organic visceral defect. At times the renal factor is almost negligible. On the other hand, hypertension is not devoid of danger. It throws extra work on the heart, results in accidents to the vascular system and often leads to local organic or functional changes which may be accompanied with discomfort, pain and danger. Only hypertension which exceeds physiological necessity or endangers the heart or vascular system calls for treatment.

The rational view assumes that a certain degree of hypertension is necessary, that nature sometimes oversteps the bounds of necessity and overcompensates deficiencies, and that only when danger threatens the heart or vascular system, or when hypertension occasions great discomfort as in angina pectoris, and headache, should it be combated by measures other than those of general hygiene. Otherwise, the use of nitrites and other blood-pressure lowering drugs áre not justifiable.

General hygienic considerations dealing with rest, with moderation in all phases of life, with questions relating to exercise, climate, diet, water and salt intake, care of the skin and bowels, etc., are of the utmost importance. Proper attention to these considerations is always advisable, and in many instances results in the patient leading a life of considerable activity and enjoyment. Details in regard to these matters and in the use of nitrites are worthy of consideration.

General Hygienic Measures.-In the early stages the treatment consists of advice. Regulation of the patient's life is the most important factor. The methods of doing so have been considered fully in the section on treatment of chronic interstitial nephritis.

Rest and Sleep.-These are both essential. The amount of rest 
which is necessary naturally depends upon the condition and the same general plan should be followed as outlined in the treatment of chronic interstitial nephritis.

Care of the Bowels.-Active purgation is not indicated, yet constipation should be avoided. Regular habits should be established, the patient going to toilet night and morning and remaining ten minutes on each occasion if necessary. Exercise and diet are controlled from the point of view of hypertension. Some selection as to diet is possible. Fruits such as oranges and grape fruit, stewed prunes, oils such as olive oil and foods with a large residue such as coarse breads, etc., are of value. Bulk can be furnished the intestinal content by the use of agar-agar or Russian oil. Mild laxatives such as phenolphthalein, gr. i, aromatic cascara, 3 ss to ii ( 2 to 8 c.c.), or compound licorice powder, $3 \mathrm{i}$ to ii (gr. I2), are necessary at times. Enemata and suppositories are useful at times. Purging must be resorted to occasionally. The water and sodium chlorid intake and the use of beverages are subject to the same consideration as in chronic interstitial nephritis. They have been already discussed in this connection.

Diet.-Chittenden(I29) has shown that proteins are absolutely essential to health and strength and cannot be excluded from the diet. With a good phthalein output the diet can be somewhat more liberal in nitrogen than that discussed in the treatment of chronic interstitial nephritis. The blood nitrogen does not increase as a rule until after the phthalein is appreciably decreased. Folin, Dennis and Seymour(I3O), and Seymour(I3I) state that the nitrogen of the blood does not accumulate until the phthalein drops to as low as 40 per cent. for two hours.

Sufficient is not yet known concerning the necessity or desirability of maintaining blood nitrogen at the normal level, but unquestionably at times subjective improvement results from a low nitrogen intake. That this can be accomplished in many instances has been indicated by the work of Seymour( $\left.\mathrm{r}_{3} \mathrm{I}\right)$, Folin, Dennis and Seymour(r 30 ), Frothingham and Smillie(132) and Mosenthal(133).

Highly seasoned and indigestible foods must be shunned.

Lowering of Blood-pressure Medicinally.-The nitrites are the most important drugs in this class. They have, however, been -greatly abused in the past. As already stated, a certain degree of hypertension is necessary. This hypertension cannot be permanently abolished. Nature reasserts her claims and the tension rises again as the affect of the drug disappears. Too great reduction in tension, even if temporary, may result in harm. Hygiene and dietetic measures usually suffice to keep the tension at a level as low as is compatible with best renal activity. Beyond this it is not advisable to go. Consequently vasodilators and blood-pressure lowering drugs have a very limited field of usefulness. It is true that they are often employed, but it is also often to the detriment of the patient, resulting sometimes in oliguria, anuria, and serous effusions. 
In the wards of the Johns Hopkins Hospital the use of nitrites is restricted at present to cases in which there is localized arteriosclerosis or arterial spasm in a vital part, i.e., to angina pectoris and to cases in which as the result of high blood-pressure a vascular accident such as apoplexy is threatening or is feared. Occasionally in severe headaches with marked hypertension, when other measures have failed the nitrites are given, but often it is without relief. The colic of lead poisoning is often associated with hypertension and in these cases nitrites have sometimes been of benefit. Formerly they were extensively used when dyspnea developed during the course of hypertension, particularly in the absence of edema. The digitalis bodies, however, yield better results under these conditions and have supplanted the nitrites, the dyspnea being interpreted as indicating myocardial weakness, hence calling for cardiac tonics rather than for vasodilators. Under the influence of digitalis the dyspnea disappears and the arterial tension often falls considerably.

Certain features common to all the vasodilators must be kept in mind. Their action is rapid in onset and is usually of short duration. Tolerance is rapidly established. Therefore, in order to obtain results increasingly large doses are necessary; and they should be given frequently and in increasing doses. The onset and the duration of their effect tend to vary inversely. Wallace and Ringer(I34) have studied the effect of the various members of the group on renal subjects and in arteriosclerosis, with the following results:

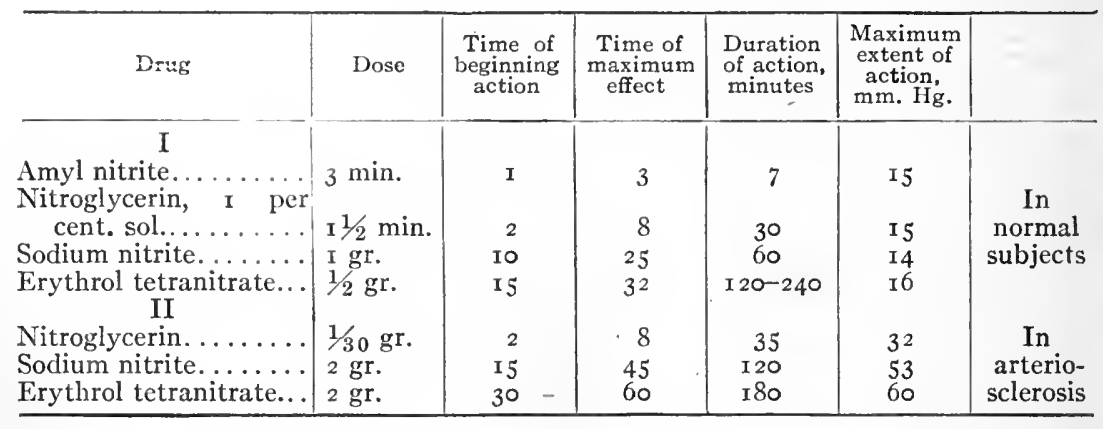

The physiological action of nitrites is, generally speaking, 'the. same, the variation appearing in promptness, duration and intensity of action. The blood-pressure is lowered through vasodilatation. Simultaneously the pulse rate is accelerated, the systolic output is increased and the blood flow also increased. The splanchnic vessels, those of the head and the superficial vesisels of the upper part of the body are chiefly affected.

Mode of Administration and Dosage of Nitrites.-Amyl nitrite is best given in the form of perles, $\mathrm{mii}$ to $\mathrm{v}$ (0.15 to $0.3 \mathrm{gm}$.), which are crushed in a handkerchief and inhaled. These ampoules must be kept in the dark until used. The fall in blood-pressure is 
usually immediate, but is of ten extremely transient. Nitroglycerin is used in I per cent. solution as spiritus glycerylis nitratis; or in the form of tablets containing gr. $1 / 100(0.00065 \mathrm{gm}$.). This dissolved on the tongue is rapidly absorbed and gives more rapid results than when taken by the stomach. The dose may be increased up to gr. $1 / 10(0.0065 \mathrm{gm}$.). Sodium nitrite is also commonly used in the tablet form, gr. ss to ii ( 0.03 to $0.3 \mathrm{gm}$ ), or even v. These may be dissolved on the tongue or swallowed. Erythrol tetranitrate gives the most lasting effect. It is dispensed in tablet form, gr. $1 / 4$ to $1 / 2$ (o.01 5 to $0.03 \mathrm{gm}$.). Mannitol nitrate is sometimes used in gr. $\mathrm{i}(0.065$ gm.) doses.

Nitroglycerin is the preparation most frequently employed, and it is used in increasing doses. The nitrites are best given after meals and should always be well diluted. Sudden vasodilatation is undesirable.

Idiosyncrasies in relation to nitrites are not uncommon, and variation in tolerance is very marked. Untoward effects are flushing, headache, syncope and oliguria. Occasionally anuria and serous effusion results from the too free or long-continued use in chronic interstitial nephritis.

Iodids.-These are frequently given over long periods in conditions associated with hypertension, but particularly in those cases exhibiting painful crises, such as angina pectoris and the colic of lead poisoning. The mechanism of their action has been much discussed but is little understood. In certain instances the relief apparently suggests that the hypertension is of luetic origin. Macht(I35) considers that an ionic action of potassium is exhibited since iodin itself tends to constrict vessels. The excretion of iodids involves as much work for the kidney as does the excretion of chlorids. Unless specifically indicated, or unless the therapeutic value in the individual case is beyond question, they should not be administered.

Radium and radium emanations $\left({ }_{1} 3^{6}\right)$ also tend to decrease bloodpressure. The writer has seen a decrease in tension of from 20 to $30 \mathrm{~mm}$. $\mathrm{Hg}$ persist for some weeks during the administration of emanation of radium. Large single doses of radium salts may be followed by several weeks of decreased tension. It is questionable, however, whether the use of radium in this connection is justifiable. Certainly the criticisms advanced against nitrites hold for radium.

\section{MYOCARDIAL INSUFFICIENCY}

Hypertrophy of the heart is almost invariably encountered in chronic interstitial nephritis. Hypertension and arteriosclerosis also usually play a prominent rôle. Bright recognized these cardiovascular manifestations and was inclined to ascribe the cardiac hypertrophy to increased difficulty experienced by the heart in forcing the blood through the capillary system. The problem of cardiac hypertrophy and hypertension are intimately connected. The 
theories advanced to explain hypertension have already been discussed; they apply also to cardiac hypertrophy.

It is usually assumed that the hypertrophy results from the hypertension, i.e., that it is secondary. Schlayer and others(I37I $38-139$ ), assert that they have seen the hypertrophy precede the development of hypertension. The left ventricle hypertrophies first but later the enlargement becomes general. The two processeshypertension and cardiac hypertrophy-develop hand in hand. The origin of both is still under discussion.

The hypertensive type of nephritis on physical examination usually reveals the following findings. The apex of the heart is displaced to the left and downward. The aortic second is accentuated and is often ringing in character, a very characteristic sign of hypertension. The first sound at the apex may be reduplicated. A systolic murmur usually develops at the apex, the result, as a rule, of relative insufficiency. It is loud and blowing in character and frequently transmitted to the axilla. These are the usual findings during the stage of hypertrophy with compensation.

Later, however, hypertrophy fails-the heart dilating. The symptoms and signs of myocardial insufficiency appear. Dyspnea, orthopnea, cyanosis, edema of legs or anasarca, palpitation and cardiac distress, nausea and vomiting, or anginal attacks may make their appearance.

On physical examination the apex beat is usually found outside of the nipple line. It may be feeble and diffuse, the point of maximum intensity being difficult to locate. The area of pulsation may be wide and marked venous or arterial pulsations may be seen in the neck. The cardiac area on percussion may be found greatly increased, to the left or right or both. On auscultation the heart sounds may be feeble and distinct, or murmurs, soft as a rule, may be heard at both apex and base. Gallop rhythm may be present. The pulse may be slow or rapid, irregular and intermittent, less frequently it is full and bounding and regular. There is no constant sign or combination of signs in cardiac failure, sometimes feeble sounds and a low-tension pulse being the only evidence.

The importance of cardiac involvement was apparent to Bright. In correlating the clinical and autopsy findings in a hundred cases of chronic nephritis he found cardiac hypertrophy in fifty-two cases and in thirty-four of these no valvular lesion could be found.

According to Janeway(140), it is the most frequent cause of death in hypertensive nephritis-sixty cases in 212 studied. Protection of the heart, therefore, is one of the most important concerns in the treatment of this type of nephritis. This has already been discussed under hypertension.

Myocardial Insufficiency and Renal Function-Myocardial insufficiency may occur independently but in a large proportion of cases it develops in association with nephritis. It is often impossible, on purely clinical lines, in an individual case to decide 
whether the kidney or heart is primarily responsible for the clinical picture encountered. In this connection renal functional studies are of the greatest assistance.

Marked renal insufficiency may result from pure chronic passive congestion. Very exceptionally, clinically and experimentally, the functional studies reveal a decrease in function equaling that seen in the most severe grades of nephritis. Since the congestion for this must be of a most extreme grade, cardiac death is imminent. As a rule in myocardial insufficiency, with a symptomatic and urinary picture identical with that seen in a moderately advanced nephritis alone, or in nephritis associated with a cardiac break, renal function as indicated by both excretory and retention tests is surprisingly good. When low renal function is followed by an increased phthalein output, the amount of increase gives a fair approximation of the extent of cardiac improvement.

In this connection also urea and total non-protein nitrogen studies are of great value. In pure passive congestion an increase in total non-protein nitrogen above $50 \mathrm{mg}$. to IOo c.c. of blood is extremely rare. In only three instances among several hundred cases studied has the author encountered it. ${ }^{1}$ Foster has lately reported three more instances. The finding of normal nitrogen figures, therefore, is of considerable diagnostic significance. A phthalein rapidly returning to normal associated with a low level of blood urea and total non-protein nitrogen speaks strongly for passive congestion as the underlying process.

Treatment.-Myocardial insufficiency constitutes a pathological indication for treatment and calls for treatment notwithstanding the other conditions are present. The principles underlying this treatment are rest, limited diet, limited salt and fluid intake, depletion through bleeding, purgatives, diuresis or tapping, and support of the heart. In addition certain symptoms may call for special attention.

Rest.-This is essential and must be complete at first-absolute rest of short duration. The patient should be confined to bed and should use a urinal and bed pan. No other treatment may be necessary. Rest alone suffices at times but one is not justified in using it to the exclusion of other methods except in milder attacks or for purposes of demonstrating its efficacy. It should be continued until the serious manifestations disappear.

A back rest often affords great comfort. Many patients cannot lie down and must be propped up with pillows or a back rest. Those with side rests are preferable. The extent to which they are used depends entirely on the patient whose comfort must be considered. Although most patients with a serious cardiac decomposition are most comfortably propped up in bed, others are not. In the most severe condition the most comfortable position is assumed by the patient and not prescribed by the physician. Thus, the patient may

${ }^{1}$ Rowntree, Marshall and Baetjer, loc. cit. 
sit straight up in bed, may assume the knee-chest position, may kneel at the side of the bed or hang forward over the back of a chair. In severe dyspnea he may arise from the bed, lean over the back of a chair and gasp for breath. Under such conditions bleeding and strophanthin and other methods of relief about to be discussed are urgently indicated. As soon as possible the patient should be gotten back to bed and rest insisted on.

An additional reason for absolute rest is found in the serious consequences which may attend exercise while the patient is under the influence of digitalis. Not infrequently, in order to escape military service in countries where it is compulsory, digitalis is taken to the point of producing cardiac irregularities. . Exercise under such conditions has been known to result in sudden death.

A state of more or less chronic or partial decompensation which lasts over months is sometimes seen. Here absolute rest and confinement to bed over so long periods is impossible. As much rest as possible must be obtained but such patients often do better when allowed to sit up in an ordinary chair or wheel chair in which they may be taken out of doors.

Diet.- This must be restricted in three ways as to (I) the quantity taken at one feeding, (2) salt content, and (3) water intake. Rest for the stomach as well as for the heart must be insisted on. Do not overfeed. This is a good rule often broken. At first only minimal quantities of food should be given and the patient should be thoroughly purged. Later more food can be allowed.

The most severe breaks require the greatest limitation. Marked nausea and vomiting are frequently met by withdrawal of all food for twelve hours. Water-logged cases in which the anasarca is extreme especially need marked limitation. For such cases the Karell diet is best. This consists of milk and milk only, 600 c.c. to I liter a day being given, 3 or 4 ounces at a time. Sometimes where nausea is very marked, the addition of lime water, $3 \mathrm{i}$ to the ounce is advisable. The Karell diet may be continued for three to six days. During its employment tremendous diuresis with great loss of weight and clearing up of edema may be seen.

At other times milk up to Iooo c.c. together with albumin water (whites of four to six eggs) may be used a day. Occasionally a small amount of whisky or brandy is desirable particularly where the patient is accustomed to their use and the craving is great.

So great restriction is not always necessary. But the additional meals should be small. It is preferable to allow several (five or six) small feedings rather than to overload the stomach at one feeding. The following diet is restricted in both salt and water content and meets the requirement of most patients. It has been used by the author in many cases. Its caloric values are about 2700 calories it contains approximately $2 \mathrm{gm}$. of sodium chlorid and meets with the approval of the patient in the majority of cases.

When the patient desires, water can be substituted for milk. 


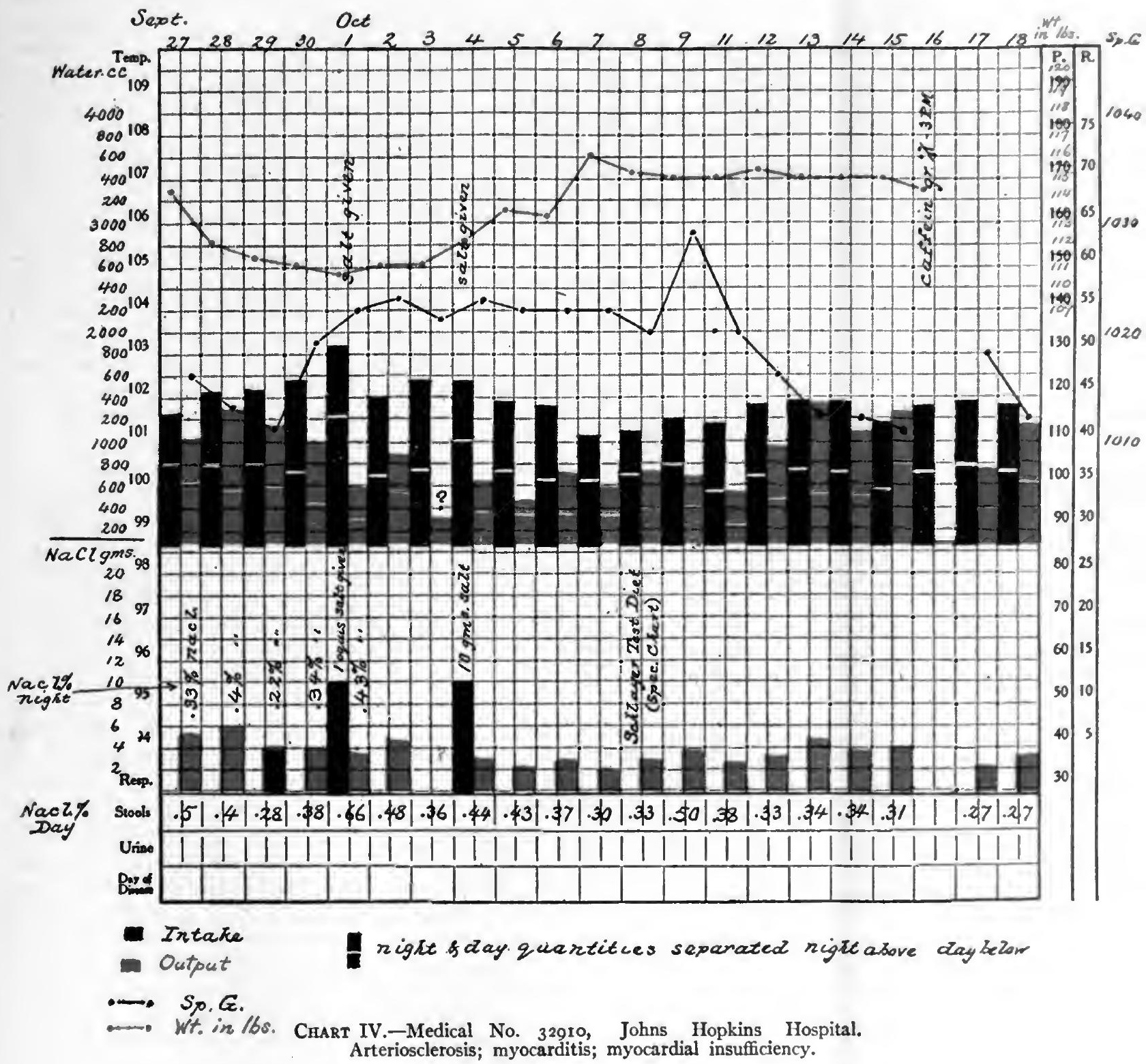




\begin{tabular}{|c|c|c|c|c|c|}
\hline A. M. & 5 A. M. & o A. M. & I P. M. & S P. M. & 9 P.M \\
\hline$\frac{\text { Milk } 200 \text { c.c. }}{1 \mathrm{egg}}$ & $\begin{array}{l}\text { Milk } 200 \\
\text { c.c. }\end{array}$ & $\begin{array}{l}\text { Milk } 200 \text { c.c. } \\
\text { I s h r ed ded } \\
\text { wheat biscuit. } \\
\text { I egg, soft-boiled } \\
\text { or poached. } \\
\text { I slice of toast } \\
40 \text { gm., salt- } \\
\text { free. } \\
\text { I butter ball } 15 \\
\text { gm., salt-free. }\end{array}$ & $\begin{array}{l}\text { Soup (chicken } \\
\text { or tomato) } 200 \\
\text { c.c., no salt. } \\
\text { Lamb chop, 100 } \\
\text { gm. or chicken, } \\
\text { r50 gm. } \\
\text { Mashed pota- } \\
\text { tocs, roo gm. } \\
\text { r soda cracker, } \\
\text { salt-free. }\end{array}$ & $\begin{array}{l}\text { Milk } 200 \text { c.c. } \\
\text { I egg, soft-boiled } \\
\text { or poached. } \\
\text { I slice bread } 4^{\circ} \\
\text { gm., salt-free } \\
\text { Rice or tapioca } \\
\text { pudding, roo } \\
\text { gm. }\end{array}$ & $\begin{array}{l}\text { Milk } 200 \\
\text { c.c. }\end{array}$ \\
\hline
\end{tabular}

As the edema clears up the same diet may be used without making the articles of diet salt-free.

Fluids. - The intake of water and fluids should be restricted, the more the edema the greater the restriction. A special fluid chart indicating the intake and urinary output should be kept in all cases with marked edema. The total fluid intake should be limited to I to $1 \frac{1}{2}$ liters a day at first, more fluid being allowed as diuresis is established and edema disappears. Enormous quantities of water may be lost in the course of a few days - a decrease of 20 to 30 pounds in the course of five to six days not being infrequent. In one of the author's cases 70 pounds was lost in one week, anasarca disappeared, and the phthalein output increased from 16 per cent. to normal.

Sodium Chlorid.-Widal has shown how important is the restriction of sodium chlorid. In the diets described above the salt content is very small. An absolutely salt-free diet is practically impossible and is not necessary. It is next to impossible to obtain a salt content less than I gm. a day. Milk contains o.16 per cent. sodium chlorid, so that any diet containing milk of necessity contains some salt.

So great restriction of salt is often not advisable over prolonged periods. The guide to the amount allowed is found in the ability of the kidney to excrete it. Where fair amounts are excreted and particularly where its concentration in the urine is good, more can be allowed. Many patients are kept on a salt-free diet long after its necessity has passed. Great quantities of salt are excreted as a rule with the clearing up of edema and anasarca. Prolonged use of saltfree diet may lead to deprivation of the tissues in sodium chlorid.

It is advisable where possible to chart fluid and salt intake and output. The following form of chart used extensively in the wards of the Johns Hopkins Hospital is particularly valuable for indicating at a glance the ability of the patient to handle water and salt. The weight of the patient and specific gravity of the urine is also indicated.

In arranging the diet, the table of food values on page 850 will be found of the greatest assistance. Small amounts of whisky or brandy$3 \mathrm{i}$ to ii ( 4 to 8 c.c.) - or of wine may be advisable for the psychical effect 
to those who are accustomed to them. Similarly in many cases a small cup of tea or coffee may be allowed once a day if much desired, provided sleeplessness and palpitation do not accompany their use. The diuretic effect is of distinct disadvantage at times.

Methods of Depletion.-Bleeding, tapping of the pleural, pericardial or peritoneal cavities, diuresis and purgation may all be indicated at times. Sweating is contraindicated owing to the strain put upon the heart.

Bleeding.-Venesection is indicated in acute dilatation of the heart - particularly of the right heart. Dyspnea, cyanosis and marked edema are usually present. The orthopnea may be extreme. In such conditions venesection offers the quickest and most lasting relief. The amount of bleeding varies naturally with the patient but in the majority of cases the withdrawal of 400 to 600 c.c. is advisable. The method employed in venesection is unimportant, the amount of blood removed being the chief factor. In the majority of cases it is necessary to make only a small incision through the skin, exposing the vein. This is cut transversely half through its lumen either with scissors, scalpel or bistoury. A local anesthetic is advisable at times. A large needle or a Lindeman transfusion needle attached to a suction apparatus answers admirably in cases with large prominent veins.

With a hemoglobin below 70 per cent. the question of the advisability of letting blood arises. Smaller bleedings may here suffice but with acute dilatation the phlebotomy may be absolutely necessary for averting death.

Following the phlebotomy, strophanthin, gr. 1/2 ( 0.03 gm.), intravenously or preferably intramuscularly is very frequently employed. Local massage for fifteen to twenty minutes should follow its intramuscular use. Unquestionably bleeding and strophanthin are lifesaving in many instances.

Paracentesis.-Large accumulations of fluid in the pleural or peritoneal cavities may call for tapping. This should be resorted to whenever the accumulation is sufficiently large to seriously embarrass respiration or the heart. Occasionally small bilateral pleural effusions lead to a most pronounced dyspnea which promptly yields to tapping. The necessity for pericardial paracentesis is much less frequent but it should be resorted to when large pericardial effusions occur.

Draining of the subcutaneous tissue is usually unnecessary. However, when the edema is extreme and gangrene or bursting of the skin threatens it should be resorted to. Erysipelas is a frequent complication when drainage of the subcutaneous tissues s employed. Sloughing may occur, and healing is usually markedly delayed. Numerous small punctures over the dependent parts is occasionally employed. The best method, however, is the insertion into the subcutaneous tissues of Southey's tubes or ordinary needles or trochars and cannulæ to which are attached long rubber tubes leading to a vessel beneath 
the bed. The needles are held in position by adhesive plaster. The strictest asepsis is necessary in this, the extremities being surrounded by cotton wrung out of boric acid solution. The drainage may be continued for one or two days, as much as 4 liters a day occasionally being removed in this way.

Purgation.-This is employed in practically all cases of outspoken myocardial insufficiency. It is useful for the removal of water, for the removal of putrefactive material from the intestine and also for the relief of intestinal distention. It may be employed without fear even where asthenia is marked and the pulse feeble. Hydragogues should be used. Magnesium sulphate, $5 \mathrm{ss}$ ( 15 to $45 \mathrm{gm}$.) to I ss, given in concentrated form each morning on an empty stomach is the most satisfactory method of inducing purgation in the majority of cases. This usually results in two or three large fluid stools each day. When this is not well borne by the stomach, that is, when it occasions nausea and vomiting, one or two compound cathartic pills each night or compound jalap powder, gr. $\mathrm{xv}$ to $\mathrm{xlv}$ ( $\mathrm{I}$ to $3 \mathrm{gm}$.), or compound ela terium powder, gr. i to v ( 0.06 to $0.3 \mathrm{gm}$.), may be tried. Elaterin, gr. $1 / 20$ to $1 / 10(0.003$ to $0.006 \mathrm{gm}$.), in alcohol is sometimes efficacious when other methods have failed. Enemata may occasionally be necessary.

After the anasarca disappears milder purgatives or laxatives such as cascara and liquorice powder may be required for regulating the intestines.

Diuresis. - This is usually obtained through the use of digitalis. Where this is not effectual recourse is had to one of the caffein diuretics. Theocin is most satisfactory, gr. iii $(0.2 \mathrm{gm}$.) t.i.d. for one day. The effect is noted and the drug repeated on alternate days if necessary.

Support of the Heart.-Digitalis is the drug par excellence in this connection. Introduced by Withering in 1785 for the relief of dropsy, it has become our mainstay in the treatment of myocardial insufficiency. Myocardial insufficiency constitutes the indication for its use regardless of valvular lesions. Auricular fibrillation demands intensive digitalis therapy and in such cases we see its most striking effect. Its best diuretic effect is seen in dropsy dependent upon circulatory changes in the kidneys.

Digitalis is manifold in its action. It acts on the heart ${ }^{1}$ itself increasing its irritability and strength of contraction, on the vagus slowing the rate and on the vascular system through the vasomotor center and directly on the vessel walls bringing out vasoconstriction and increase in blood-pressure. It slows the heart, increasing its

${ }^{1}$ In this connection the work of Schliomensun(14I) is extremely interesting. An alcoholic phosphatid was extracted from the hearts of animals receiving digitalis therapy, which when injected into a second animal produced all of the biological reactions of digitalis. This he claims indicates a direct combination of digitalis with the heart muscle. Similar extracts from all other tissues of these animals failed to yield such a product, indicating that it was specific to heart muscle. 
force and the output per beat and tends, in passive congestion resulting from myocardial insufficiency, to shift the blood from the venous side where it has collected to the arterial side of the vascular system. In therapeutic doses its use results in improved circulation through the kidney (a relative vasodilating effect upon the renal vessels being claimed) diuresis resulting. It finds its greatest value in cases of mitral disease with marked edema and small, rapid and irregular pulse, although it is of value in all cases of myocardial insufficiency irrespective of the nature of valvular lesion.

Digitalis should be administered in courses and its use should be intensive from the beginning irrespective of the preparation used. A single course may suffice. Repeated courses are usually indicated. In auricular fibrillation intensive treatment is indicated and also more or less continuous treatment after compensation is reëstablished. In this condition small doses can be taken almost continuously or at frequent intervals for months or years. Patients may feel perfectly well on this regimen who do badly without it.

The preparations of digitalis are numerous but four stand out preëminently: These are the powdered leaf, the tincture, the infusion, and digipuratum. Each preparation has its advocates. Results can be obtained with any of them provided the preparation is an active one, that it is properly administered and the case is suitable.

The preparation most commonly used in the wards of the Johns Hopkins Hospital is the infusion. This is given, 5 ss. ( 5 c.c.) q., three or four hours for forty-eight hours. The infusion is an aqueous extract and contains relatively more digitonin than the tincture. As a diuretic it is particularly valuable. It should be prepared fresh-a new supply being obtained once a week.

The tincture is also an excellent preparation. It is an alcoholic preparation containing relatively more digitoxin, digitalin and digitophyllin than the infusion. It is administered $m x v$, to Xx (I to I.3 gm.) q. three or four hours for forty-eight hours. It is usually combined with tincture aromatic amarin or some other bitter and is well diluted with water.

The powdered leaf is given in gr. I to $\mathrm{I} 1 / 2$ ( 0.065 to $0 . \mathrm{gm}$.) doses q. three or four hours for forty-eight hours. Digipuratum is a standardized preparation of digitalis put up in tablets each corresponding to gr. $\mathrm{I}^{1} / 2(\mathrm{O}$ ( $\mathrm{I} \mathrm{gm}$.) of the digitalis leaf. Four tablets are given during the first twenty-four hours, three the second, two the third, and one the fourth. It is an active preparation and well standardized but much more expensive than any of the foregoing preparations and no better, provided these are properly standardized. It is also marketed in liquid form in ampoules. This preparation can be given intravenously or intramuscularly without marked irritation. The ampoule contains I c.c. which corresponds to I gm. of the leaf.

The doses given above represent the routine of the hospital. It 
cannot be too strongly emphasized, however, that digitalis should not be measured in grains or hours but by results. 'The chief requisite is an active preparation and knowledge as to the potency of any preparation is readily obtained. Unquestionably this is of great importance.

Standardization of Digitalis. - This is a matter of extreme simplicity and should be undertaken in every good institution.

Two methods are in common use: the frog and the cat method. The frog method consists of injecting the digitalis preparation into the anterior lymph sac of the frog and determining the amount necessary to bring about systolic standstill in one hour in the frog (Rana pipiens). The results are expressed in heart tonic units. This conveys no impression as to the activity of the preparation unless one knows what constitutes a heart tonic unit. According to Houghton(I42) a heart tonic unit is ten times the normal fatal dose per gram of frog, to Edmunds(I43) it is the amount per $20 \mathrm{gm}$. weight of frog necessary to bring about systolic standstill in one hour, while according to Hale (I44) it is the amount necessary per gram of body-weight. A heart tonic unit may therefore vary 400 per cent. according to what constitutes the standard. Nevertheless the standardization is of value if one accepts any standardized preparation and learns. to use it intelligently. This method is not satisfactory for the standardization of a dilute preparation such as the official infusion since such large quantities must be introduced into the lymph sac that absorption is often not complete at the end of an hour.

The cat method (Hatcher and Brodie)(I45) is simple and satisfactory. The preparation to be tested is slowly run into the femoral vein of a cat until death results. The number of cubic centimeters per kilogram of cat constitutes a cat unit. The technique as employed by the author(146) in standardizing ${ }^{1}$ the infusion is as follows: the cat is given just sufficient ether to permit a cannula being placed in the femoral vein. By means of a burette or a syringe ro c.c. of the filtered infusion is injected in the course of five minutes and I c.c. every two minutes thereafter until death. The total amount is noted and the amount per kilogram, or the cat unit is calculated.

The following emphasizes the importance of standardization. Leaves from various sources (German, English and American) obtained for the hospital pharmacy infusions were prepared according to the U. S. Pharmacopeia. Some of these infusions required only 6 to 7 c.c. per kilogram to kill, whereas others required Io and 12 , and one (the German leaf) required 23 c.c. per kilogram to kill the cat. A variation of 400 per cent. was therefore found in leaves in the hospital pharmacy. An infusion from an American (Wisconsin) leaf -7 to 8 c.c. constituting a cat unit-is the one now in use in the hospital. An equally potent Minnesota leaf is in use in the University of Minnesota Hospital.

Eggleston(147) has recently claimed that 0.143 cat unit per pound of body-weight is necessary for therapeutic effects. The additional claim is also made that this full amount can be given in twenty-four rather than in forty-eight hours-one-half being given in the first dose, one-third four to six hours later and small doses at four-hour intervals until the calculated amount is reached. Doses up to 50 c.c. of the infusion now in use in the Johns Hopkins Hospital have been given as the initial dose without untoward effect.

Since there are so great variations in the potency of digitalis, it becomes imperative in order that it be used intelligently that the physician be familiar with the potency of the preparation which he is administering. The only other alternative is to push the drug until the therapeutic effect is obtained provided the case is one suitable to digitalis therapy.

In order to do this one must have well in mind what constitutes the therapeutic stage of digitalis treatment and what criteria are to be accepted as indicating the desired digitalis effect. Slowing of

1 The method here described is based on the one devised by Hatcher. 
the pulse is sometimes erroneously accepted as the criterion. It should not be accepted since slowing of the pulse does not always occur in the therapeutic stage and since it is not attained by digitalis in certain types of myocardial insufficiency. Thus Edens(I48) states that slowing does not occur when hyperthyroidism is present, in acute myocarditis, idiopathic hypertrophy or in the small heart of a tuberculous diathesis. In such conditions the toxic manifestations appear before slowing.

When the case is closely followed, the therapeutic stage is the first toxic manifestation and is usually readily recognized. The following should be closely observed: (I) the urinary output in relation to the intake, since diuresis usually characterizes the therapeutic stage and oliguria the toxic stage of digitalis; (2) the outline of the heart; (3) the character of the heart beat and heart sounds; (4) the symptomatic condition of the patient in regard to dyspnea, cyanosis and edema; (5) the effect on blood-pressure and character of the pulse and (6) the pulse deficit if it is present; (7) conduction time and inversion of the $T$ wave in the electrocardiogram.

Digitalis is slow in action, usually twenty-four to thirty-six hours being needed, and also cumulative in its effects and consequently should be given in courses. When the desired amount is prescribed the digitalis should be stopped and the effect noted. The toxic effects are nausea and vomiting, vertigo, syncope, and diminished urinary secretion. The pulse may become either slow (vagus effect) or fast (increased muscle instability). Irregularities may develop. Bigeminal and trigeminal pulse is often encountered in this stage or irregular extrasystoles or auricular fibrillation may develop in some cases.

Sudden cumulative effects are said to occur but the more closely a patient is watched the less sudden as a rule are the toxic effects of digitalis. Nausea and vomiting, which herald toxic action, often go unheeded.

The effect on blood-pressure is deserving of comment since digitalis in animal experiments leads to increase in blood-pressure until, and often after, toxic stage is reached. Clinically, little or no influence on blood-pressure may bé seen. In some cases a rise of pressure is encountered while in others a considerable fall may be seen which is synchronous with unquestionable clinical improvement. Naturally rest, diet, and purgation probably also play a rôle in determining the effect on blood-pressure.

The time element in digitalis therapy is important. It is unusual to get digitalis effects from any preparation given by mouth in the ordinary dosage in less than thirty-six hours. Digitalis given today does not manifest its action until tomorrow. Where immediate effect is necessary recourse is had to strophanthin.

Substitutes for Digitalis.-For routine use no drug can replace digitalis. Strophanthus, however, is unquestionably the best substitute and in certain conditions it is preferable. Strophanthin is 
much the most valuable preparation - the tincture of strophanthus comparing in no way with that of digitalis.

Strophanthin is given in $1 / 4$ to $1 / 2 \mathrm{mg}$. doses intravenously and in $1 / 4$ to $\mathrm{r} \mathrm{mg}$. doses intramuscularly. Local massage for fifteen minutes at the point of injection obviates the local irritant effect otherwise encountered. When used in $\mathbf{I ~} \mathbf{m g}$. dose it cannot be repeated within twenty-four hours; doses of $1 / 2 \mathrm{mg}$. may be repeated in twelve hours if necessary although in the majority of cases it is sufficient; doses of $1 / 4 \mathrm{mg}$. should be repeated in eight to twelve hours. Strophanthin is given as is digitalis in courses of two to three days' duration.

Warning is necessary in its use where digitalis has been in use. As already stated, digitalis requires twenty-four to thirty-six hours to demonstrate its effect. The addition of $1 / 2 \mathrm{mg}$. strophanthin at the end of a course of digitalis may bring out alarming toxic manifestations. On the other hand, strophanthin is admirable when used in the beginning of a course of digitalis, as follows: A patient suffering from acute dilatation of the right heart is immediately bled, $1 / 2 \mathrm{mg}$. strophanthin is given intramuscularly and then a course of digitalis is started in the ordinary way. From this procedure an almost immediate digitalis effect is secured and maintained.

Strophanthin acts pharmacologically and therapeutically much as digitalis but it has somewhat less effect as a vasoconstrictor. It constricts the splanchnic terminals as does digitalis but the vessels of the extremities and cerebellum are not constricted but may even undergo slight dilatation at times. Considerable controversy has been waged over its effect upon the coronaries. Digitalis constricts the coronaries and, therefore, decreases the blood supply to the heart muscle. Loeb(I49) claims that strophanthin here exerts a dilating influence. Voegtlin and Macht( $\left.{ }_{15} \mathrm{O}\right)$ using arterial rings find a constricting influence for digitalis and a dilating one with strophanthin. The chief effect of both drugs is identical, however, i.e., shifting of the blood from the venous to the arterial side of the vascular system.

The time necessary for manifestation of their physiological effect is the chief point of difference. The strophanthin effect is almost immediate, whereas digitalis requires twenty-four to thirty-six hours. Strophanthin is, therefore, used in preference to digitalis where the need is urgent.

Caffein and members of the caffein group are sometimes substituted for digitalis where the latter fails. When slowing results from its use the pharmacological effect resembles that of digitalis. However, the pulse rate is more frequently accelerated rather than retarded. Unfortunately, its use is also frequently attended with the development of palpitation, insomnia, and sometimes nausea and vomiting, and delirium. These untoward effects often appear just as early as the effect on the heart, consequently caffein is seldom employed in this country as a substitute for digitalis. 
But these purin derivatives are excellent diuretics according to

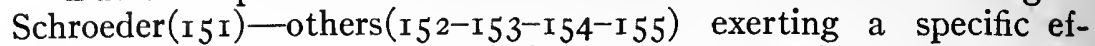
fect on the cells of the renal tubules; consequently they are often. employed in myocardial insufficiency not as a substitute for digitalis but as a synergist from the point of view of renal secretion. Theobromin has a more constant renal effect than caffein and is frequently used in doses of gr. 5 to 8 (gm. 0.3 to 0.5 ) t.i.d: or in the form of sodiosalicylate of theobromin, gr. I5 (gm. r) t.i.d. The most valuable preparation of the caffein group, however, is theocin which is administered in 3-grain (0.2 gm.) doses three times a day for one day. This can be repeated on alternate days if its effect disappears rapidly. Where the diuretic effect of digitalis is lacking, our practice is to turn to theocin which is given in addition to digitalis in the manner just indicated.

Certain other remedies sometimes substituted are perhaps worthy of mention. Squill is of value at times. It has a very mild digitalis effect and is an excellent diuretic. It is given as the tincture, 5 to I 5 min. ( 0.3 to I c.c.) or as the syrup, $30-60$ min. ( 2 to 4 c.c). Niemeyer's pill which contains I grain each of calomel, digitalis and squills is a valuable preparation. Apocynum as the fluid extract, 5 min. ( 0.3 c.c.), convallaria as the tincture ( 0.3 to I c.c.) and adonidin, gr. $1 / 6$ to $1 / 3$ (o.or to $0.02 \mathrm{gm}$.), are occasionally used but of very doubtful therapeutic value. Spartein sulphate, gr. I to 2 ( 0.06 to $0.12 \mathrm{gm}$.) is rarely employed now. Cactus grandiflorus is absolutely inert and should be deleted from the pharmacopeia.

Adjuncts to Digitalis Therapy.-Anemia is not at all infrequent in myocardial insufficiency. Iron and arsenic are here of the greatest value. Strychnia is occasionally of value. Alcohol in small doses for its psychical effect is employed at times when craving is great in those accustomed to its use.

The value of charts, such as those suggested above, in quickly conveying the effect or need of treatment from the standpoint of the kidney is worthy of emphasis. At a glance, one accustomed to their use grasps the condition of renal activity.

The Symptomatic Treatment of Myocardial Insufficiency.At times certain symptoms become so pronounced as to call for special treatment in addition to the general treatment described above.

Edema is usually controlled by rest, diet, restriction of fluids and salts and by digitalis alone or together with one of the caffein bodies. Where these fail, or where the anasarca is extreme so that pressure interferes with the action of the heart or lungs, tapping of the cavities concerned is necessary. Where edema of the extremities is so severe as to threaten gangrene, drainage may be employed with success.

Dyspnea.-The cause of the dyspnea should be determined. If mechanical, tapping of the large cavities may be indicated or special attention to diet when due to pressure from intestines which are filled with gas. The back rest may bring great relief. For orthopnea, 
morphin, gr. 1/6 (0.01 gm.), and atropin, 1/150 (0.00045 gm.), often brings immediate relief. When these fail and when marked dilatation of the right heart is found, venesection and strophanthin answer best.

Formerly nitrites were much used in dyspnea associated with hypertension. The dyspnea is now considered as evidence of beginning myocardial weakness and interpreted as indicating digitalis, not vasodilators.

Palpitation and Cardiac Disiress.-Occasionally the gastrointestinal tract is at fault and its repsonsibility should be investigated. Local treatment may suffice. The ice bag is frequently the most prolific source of comfort. Small blisters and belladonna plasters are of value at times. Internally, potassium iodid is frequently employed with success. Tr. aconite, $m$ I to 5 ( 0.06 to 0.3 c.c.) may help at times,

Gastric Symploms.--Nausea and vomiting are commonly encountered. They frequently yield rapidly to the general treatment already described. Their time of origin must always be determined since their appearance after instituting digitalis therapy should suggest the possibility of digitalis as their cause. Their treatment may then be the withdrawal of digitalis. Similarly Epsom salts may be responsible.

All food should be stopped for twelve hours if nausea and vomiting become extreme. Nothing should be allowed by mouth but crushed ice. Later milk and lime water are allowed in small amounts. Gastric sedatives such as sips of cold effervescing drinks: champagne, apollinaris water or an ordinary syphon are often of value. Bismuth, gr. $x x$ ( $1.3 \mathrm{gm}$.), creosote, $\mathrm{mi}$ to ii ( 0.06 to o.I 2 c.c.), dilute hydrocyanic acid, $\mathrm{m}$ i (o.06 c.c.), cocain hydrochlorid, gr. $1 / 10$ to $1 / 4$ (o.06 to $0.15 \mathrm{gm}$.), or a mixture of tr. nux vomica, $\mathrm{mv}$ ( 0.3 c.c.), and soda bicarbonate, gr. x ( 0.6 gm.), may be tried. Counterirritation as by a mustard plaster on the abdominal wall is occasionally helpful.

Naturally in myocardial insufficiency in chronic nephritis, nausea and vomiting may be the evidences of uremia. All of the above methods may be tried without success while some general sedative such as chloral, gr. $v(0.3 \mathrm{gm}$.) q. four hours or morphin, gr. 1/6 (0.0I gm.), may bring relief.

Persistent nausea and vomiting, particularly if associated with a markedly enlarged and pulsating liver, is an extremely serious complication often ending fatally.

Cough.-The cough is usually due to changes in the lungs and responds to the cardiac treatment. Expectorants are as a rule contraindicated.

Hemoplysis.-Though most alarming to the patient, hemoptysis in myocardial insufficiency is seldom serious. The patient should be assured that the hemorrhage is salutary and does away with the necessity of doing a venesection. Assurance and absolute quiet 
and an ice bag over the chest usually' suffice. When the patient is markedly upset a small dose of morphia is often desirable.

Edema of the Lungs. - This is an extremely serious complication and calls for quick action. The patient should be bled 400 to 600 c.c. Morphia, gr. $1 / 4$ (0.0I $5 \mathrm{gm}$.), should be given hypodermically. Atropin, $1 / 150$ (0.00045 gm.), is used at times but morphin is of greater value.

Insomnia.-This is frequently a very troublesome symptom. A good back rest with side supports often allows a comfortable sleep in a sitting posture. Any of the following hypnotics or sedatives can be tried-paraldehyde, $31 / 2$ to 2 ( 2 to 8 c.c.) in capsules, sulphonal gr. ro to 30 ( 0.6 to $2 \mathrm{gm}$.), Hoffman's anodyne, 3 ss to $\mathrm{i}$ ( 2 to 4 c.c.), spirits of chloroform, $m_{5}$ to Io ( 0.3 to 0.6 c.c.), spirits of camphor, 3 ss to $\mathrm{i}(2$ to 4 c.c.) alone or $3 \mathrm{ss}(2$ c.c.) in combination with ether $3 \mathrm{ss}$ (2 c.c.), veronal, gr. v (o.3 gm.), or urethane, gr. Io to 3 o ( $\mathrm{I}$ to $2 \mathrm{gm}$.). Where these do not give relief it is better not to waste valuable time. Morphia, gr. $1 / 6$ to $1 / 4$ (0.010 to $0.015 \mathrm{gm}$.), alone or in combination with atropin, $1 / 150$ (0.00045 gm.) should be given hypodermically.

\section{UREMIA}

Uremia $^{1}$ is a term used to designate a group of symptoms, the manifestation of a toxemia resulting from renal insufficiency from any cause. It develops in the course of nephritis, in conditions associated with anuria, in bilateral surgical diseases of the kidney, and ushers in the fatal termination in a large proportion of renal diseases. The nature and source of the toxin or toxins are unknown, but they show a predilection for the nervous system.

Uremia may be latent. Its appearance is often sudden and unexpected and its course acute and severe, rapidly ending in death, or chronic, lasting through months. Its signs and symptoms are protean and not exhibited in a determinate order. The symptom complex embraces a large number of morbid manifestations of widely varying character and source, only a few of which appear in an individual case. The grouping of symptoms is so bizarre that two cases of uremia may appear to have very little in common. Hence different clinical types are recognized.

Almost invariably the renal insufficiency underlying uremia is evidenced by functional changes which are easy of demonstration. The findings of certain functional tests are much more constant than are the symptoms. Such findings when associated with any of its clinical manifestations make the diagnosis of uremia a certainty. In the absence of symptoms functional studies often reveal an impending uremia.

Theories of Uremia.-Of the many suggested theories four stand out prominently:

1 Any one interested in uremia should read the classic of Ascoli-Vorlesungen über Uremie, Jena, 1903. 
I. That it is due to the accumulation in the blood of the products of a normal or abnormal metabolism which are ordinarily secreted by the kidney. A marked increase in the non-protein nitrogen of the blood does occur in the majority of cases, the urea fraction being particularly increased. Urea, however, is relatively non-toxic and is not considered to be responsible for the symptoms. On the other hand, a small proportion of the cases of uremia, particularly those of the eclamptic type; show no increase in the nitrogenous bodies of the blood. Ammonia carbamate and carbonate, trimethylamin( $\left.{ }_{5} 5^{6}\right)$ creatinin and the salts of potassium( I $_{57}$ )have been suspected, but proof of their responsibility has not been established.

Recently two interesting researches bearing on uremia have been reported. Foster $\left(\mathrm{r}_{5} 8\right)$ has isolated a toxin from the blood of cases with uremic convulsions which is rapidly fatal in guinea pigs. Its chemical structure is not yet determined. Possibly it is one of the poisons concerned. Hartman( $\mathrm{I}_{59}$ ) has isolated from the urine a bright yellow oil which gives the reactions of a cyclic ketone and to which he has ascribed the empirical formula $\mathrm{C}_{6} \mathrm{H}_{8} \mathrm{O}$. This oil has the odor of urine and to it he has given the name "urinod." Normally it is conjugated in the urine. In its free state it is extremely toxic. In the mouse $1 / 300$ part of the body-weight administered proved fatal. Injected into the frog, twitching, dyspnea, increased irritability, convulsions with respiratory death results. Hartman experimented on himself with small injections which resulted in intense nausea, extreme headache, loss of appetite and great weariness, irritability of temper, restlessness, insomnia, and increased frequency of urination.

2. That it is due to changes in normal kidney metabolism, particularly to a disturbance of the internal secretions of the kidney. In favor of this theory are the findings that removal of more than three-quarters of the renal substance leads to marked increase in the nitrogen bodies of the blood associated with emaciation. But the excretory capacity is simultaneously decreased. The presence of the internal secretion is certainly not proven.

3. That it is due to nephrolysin. Broken up renal substance when injected into an animal causes injury to the renal cells, and the serum when injected into a second animal causes nephritis with albuminuria. As pointed out by $\mathrm{F}$. Müller uremia is most common in conditions associated with great destruction of renal substance.

4. That it is due to localized edema of the brain. This is supposed to hold true particularly for the forms of uremia associated with coma and convulsions. The theory is an old one and has had few adherents of late years. Fischer seems inclined to accept this theory. Widal and Strauss are inclined to think that it holds for the eclamptic type. Foster( $(160)$ has reported three cases with practically normal renal function who at autopsy exhibited edema of the brain in addition to renal changes.

Since the nervous symptoms play so prominent a part, the rôle 
of the central nervous system must be considered in determining the mechanism involved in the production of uremic manifestations.

5. Acidosis ${ }^{1}$ has been ascribed as the cause. Studies by numerous investigators indicate that acidosis is associated with nephritis and with uremia at times but that it is in causal relationship as extremely doubtful.

The Functional Picture in Uremia.-Uremia is characterized by definite functional changes. The sulphonephthalein excretion is markedly reduced, usually to a trace, and of ten to zero for the two hour period. The total non-protein nitrogen of the blood and serum is markedly increased, usually to more than $50 \mathrm{mg}$. to roo c.c. The urea nitrogen of the blood is particularly increased and constitutes as a rule from 75 to 86 per cent. of the total non-protein nitrogen. The freezing point of the serum and blood is considerably depressed; indicating an increase in its molecular concentration. The characteristic picture, therefore, is a low phthalein output associated with cumulative phenomena-i.e., a total blood non-protein nitrogen greater than $50 \mathrm{mg}$. to Ioo c.c., a blood urea greater than $55 \mathrm{mg}$. per roo c.c. (urea $\mathrm{N} 26 \mathrm{mg}$.) and a freezing point less than -0.60 . The persistence of this functional syndrome, even in the absence of symptoms, indicates that uremia is impending.

The decreased phthalein output is the most constant functional finding in uremia. In a small proportion of cases an output of from 20 to 35 per cent. is encountered. This is most frequent in cases associated with marked cardiac and circulatory changes. In very rare instances uremia has been observed with a normal phthalein excretion. These cases have usually been of the so-called eclamptic type and cerebral arteriosclerosis as their cause must yet be excluded. The cumulative phenomena are absent in a somewhat larger number of cases.

Riess(I6I) has classified uremia into the following groups: (I) asthenic, (2) psychical, (3) convulsive and (4) mixed types. He states that markedly increased urea content and increased molecular concentration of blood characterizes groups $\mathrm{I}$ and 4 but that these phenomena are apt to be lacking in the psychotic and convulsive forms. Foster(I62) on the other hand, believes that the convulsive form of uremia is likely to evidence higher blood nonprotein nitrogen values than is the asthenic type.

Acidosis, as evidenced by a decrease in the $\mathrm{CO}_{2}$ tension of the alveolar air, by increased alkali tolerance and by increase in the $\mathrm{H}$ ion concentration of the blood, is present in a small proportion of cases. It is usually accompanied by respiratory changes, especially by hyperpnea. In many cases, however, no evidence of acidosis can be elicited.

Symptoms.- Since much of the treatment of uremia is symptomatic, an outline of the symptoms is presented. We recognize acute and chronic forms of uremia. Acute uremia may develop in

1 Straub and Sehlayer, Münch. med. Wchnschr., I gr 2, No. 2. 
any form of nephritis. It may apparently come as a bolt from a clear sky and end in death. The chronic form is most frequent in the arteriosclerotic and granular kidney and in the kidney resulting from back pressure from obstruction in the lower urinary tract. For convenience the symptoms may be grouped as cerebral, respiratory, gastrointestinal, and miscellaneous.

(A) Cercbral Symptoms. - Psychical Manifestations.-The patient commonly exhibits more or less delirium or confusion, is sleepless, talkative, noisy and restless, or on the other hand is drowsy and apathetic. Mania may develop, sometimes quite suddenly. Delusional insanity or melancholia may appear.

(B) Headache.-This is a frequent and often an early and persistent manifestation. It is usually occipital and intense, and often extends to the neck.

(C) Convulsions.- These are common and may appear suddenly or be preceded by restlessness, twitching, headache, and gastrointestinal symptoms. They vary in degree from mere muscular twitching to fully developed convulsions. When general convulsions appear suddenly from time to time with normal periods intervening, the condition is spoken of as the eclamptic type of uremia. Cerebral arteriosclerosis, however, is usually responsible for the symptoms. Absence of cumulative phenomena in the blood, together with decreased renal capacity is apt to characterize this type.

(D) Coma.-This is apt to develop gradually where drowsiness and apathy have been conspicuous. It may follow and persist after a sudden convulsive seizure. Coma may or may not be associated with other cerebral manifestations such as delirium and twitching. It is commonly accompanied by respiratory symptoms. Its duration may be short or prolonged for weeks or months.

(E) Other Nervous Manifestations.-Paralyses-as hemiplegia or monoplegia-are not uncommon, an organic basis other than edema being absent at autopsy. Other nervous manifestations such as intense itching, numbness and tingling and cramps in the extremities are sometimes seen.

Respiratory Symptoms.-Dyspnea is common. It may be paroxysmal or continuous, or of the Cheyne-Stokes type. It is often cardiac in origin. Uremic asthma may develop and is usually nocturnal. The breathing, especially in coma, may be noisy and stridulous. It is sometimes associated with acidosis. Recovery may occur after Cheyne-Stokes respiration has persisted for months.

Gastrointestinal Symptoms.-Nausea and vomiting may develop gradually and be associated with a furred tongue and fetid breath and loss of appetite, or they may develop abruptly and be uncontrollable. Diarrhea, alone or with vomiting, is common. An uremic stomatitis is sometimes seen.

Miscellaneous Symptoms.-Fever occasionally occurs. In some instances there is a marked secondary anemia. Uremic amaurosis 
may follow the convulsions, in some cases persisting and in others clearing up with the general improvement of the patient.

Prognosis.-Uremia always indicates a serious condition, always calls for immediate therapeutic action, always suggests a grave prognosis, but does not always indicate a hopeless condition. Two features must be considered: (I) recovery from the actual attack; and (2) the likelihood of recurrence at an early, date. The uremia of acute nephritis may end in death or in complete recovery, whereas recovery from uremia in chronic nephritis is apt to be temporary.

Many factors must, therefore, be considered-the symptoms, the functional findings, the underlying cause and whether or not this is amenable to treatment. A decreasing phthalein excretion, a constantly increasing blood urea and especially a high blood creatinin, increase the gravity of the prognosis. Death does not always occur from renal insufficiency. Terminal infection-such as acute peritonitis, pericarditis, pleurisy, meningitis and endocarditis-apoplexy and myocardial insufficiency often usher in the exitus.

Prophylaxis.- It is not necessary to wait for the appearance of the actual symptoms before beginning the treatment. By the aid of functional studies we are in a better position to ward off uremia. Functional findings indicating its proximity call for the institution of antiuremic measures. In chronic nephritis uremia may be long postponed, while in acute cases it may be altogether prevented.

In any form of nephritis in which uremia is threatened, antiuremic measures should be immediately employed. The diet should receive attention. It may be well to limit the food temporarily to milk, carbohydrate, and to fruit juices. Purgation and sweating should be instituted, and in suitable cases venesection is indicated.

Young's preoperative treatment-i.e., constant catheter, forced water and dietary control-not only prevents uremia in some cases of enlargement of the prostate but converts a bad or hopeless case into a good operative risk. Following operation renal function may become practically normal.

Treatment.-The patient should usually be put to bed except in cases of very mild uremia of the chronic type or where insomnia or dyspnea preclude it. The underlying disease must receive its appropriate treatment. The treatment of the various forms of nephritis has been considered earlier. Uremia dependent upon renal changes consequent upon obstruction in the lower tracte.g., renal insufficiency following hypertrophy of the prostate-is best met by the treatment introduced by Young. A retention catheter is introduced, or the patient is catheterized at frequent intervals. Water is forced, up to from 3 to 6 liters per diem. The diet is low in nitrogen. Such measures will frequently cause a disappearance within a few days of all the uremic manifestations. This is usually accompanied by marked functional improvement, which, when sufficiently advanced, permits the removal of the 
prostate, and this is usually followed by marked improvement in the renal function.

The most successful line of treatment is based on the assumption that toxins, nitrogenous in character, accumulating in the blood and tissues, are responsible for uremia. The attempt is, therefore, made to keep the concentration of these toxins as low as possible, by partial removal through venesection, by increasing their vicarious elimination by various means, and by limiting their production through dietary control.

Diet.-Uremia is itself so variable and is associated with such varying underlying conditions that no especial diet can be advised. In acute uremia the symptoms may appear unexpectedly and the patient may die suddenly. In chronic uremia the diet is important. It should be low in nitrogen content, since patients usually feel more comfortable on such a diet. It has also been demonstrated that the level of the non-protein nitrogen of the blood can frequently be lowered by a diet poor in nitrogen-e.g., containing 4 to $5 \mathrm{gm}$. of nitrogen. The amount allowed depends in part on the ability of the organism to handle nitrogenous fluids. This is determined in each case by the ability of the kidney to excrete nitrogen on an ordinary diet and on one rich in nitrogen, and by the level of non-protein nitrogen and the effect on it of high and low nitrogen intake, or by the response to renal test meals. In cases with fixed low specific gravity, increase in water as well as low nitrogen intake is necessary for the sweeping out from the blood and tissues of accumulated waste nitrogen products. However, in some instances, despite low nitrogen intake, the total non-protein nitrogen continues to increase until death.

The caloric need of the patient must be considered in all chronic uremic conditions. Where a low $\mathrm{N}$ diet is persisted in for any length of time calories must be supplied. This is best done with a carbohydrate diet. In cases associated with edema low chlorid and water intake is necessary, the fluids being limited to rooo to I 500 c.c. and the amount of salt decreased according to the amount of edema and the ability of the kidney to excrete chlorids.

Bleeding.-This is often regarded as the most important measure for combating uremia. The amount of blood removed depends upon the urgency of the case and upon the condition of the patient's blood. Robust, full-blooded individuals should be bled 500 to 600 c.c. In weak and debilitated patients, particularly if anemia is present, venesection has often proved harmful. These conditions are apt to exist in chronic forms of uremia. Under such circumstances the patient may be bled, the venesection being immediately followed by transfusion of an equal or a large quantity of blood from a suitable donor. The procedure is repeated as needed. In our hands excellent immediate results have attended this line of treatment. In such cases if a transfusion is not contemplated the bleeding should be light and followed by an infusion of salt 
solution of equal or even double the quantity of blood removed. In uremia associated with evidences of myocardial insufficiency the effects of venesection may be striking, since in these cases the circulatory disturbance is undoubtedly a contributing factor.

Increased Elimination.- $(A)$ By the Bowels.-Purgation is important and should be carried out in all cases. Hydragogue cathartics are of undoubted value. The saline purgatives, if retained by the stomach, meet all the indications. Daily doses of Epsom or Rochelle salts or of the effervescing solutions of magnesium citrate are most frequently used. Where edema is present magnesium sulphate should be employed by the method of Mathew Hay, as follows: Half an hour to an hour before breakfast 0.5 ounce to an ounce ( $\mathrm{r}_{5}$ to $30 \mathrm{gm}$.) of the salts is given in concentrated form; this usually produces from three to five liquid evacuations. Compound jalap powder, $3 \mathrm{ss}$ to $3 \mathrm{i}$ ( 2 to $4 \mathrm{gm}$.), is also excellent and may be given over considerable periods without injury to the intestines. Elaterium, gr. $1 / 10$ to $1 / 16$ ( 0.006 to $0.004 \mathrm{gm}$.), or elaterin, gr. $1 / 20$ ( $0.003 \mathrm{gm}$.$) , may be given, and repeated every four hours until$ full purgation is obtained. The addition to either of extract of hyoscyamus, gr. $1 / 8$ ( $0.007 \mathrm{gm}$.), lessens the tendency to griping. Calomel in divided doses, gr. $1 / 10(0.006 \mathrm{gm}$.), with sodium bicarbonate, or as a single 2-grain (0.12 gm.) dose, may be used. The compound cathartic pills, one or two each night, is the purgative probably most frequently employed in our wards. With an unconscious patient $x$ or 2 drops of croton oil is placed on the back of the tongue. Even where the pulse is very feeble these hydrag ogue cathartics are well borne.

(B) By the Skin.-Sweating is one of the most effective measures used in combating uremia. It should be tried in all severe cases unless contraindicated. It is of benefit both in the presence and absence of edema. However, when there is marked myocardial insufficiency it should not be utilized, since the additional strain on the heart is dangerous. The secretion of sweat normally participates in the regulation of the temperature and the water exchange of the body. From the standpoint of excretion the water is the most important constituent, the solids excreted being of minor importance in comparison with the amounts eliminated by the kidneys. The sweat is a secretion of low specific gravity (roo4 to ror $x$ ). The constituents of sweat are in general the same as those of the urine-urea, ammonia, uric acid, amino-acids, creatinin, chlorids, phosphates, sulphates and certain enzymes. The quantity of these substances eliminated by the skin is far less, normally, than that eliminated through the kidneys. According to Schwenkenbecher and Spitta( $\left(\mathrm{I}_{3}\right)$ about $1 / 3 \mathrm{gm}$. each of sodium chlorid and nitrogen are eliminated daily through the skin, while Benedict(I64) found during rest about $0.07 \mathrm{x} \mathrm{gm}$. of nitrogen, and during hard work I to $2 \mathrm{gm}$. are excreted in twenty-four hours. Favre and Kast(I65) have shown that no relationship exists between the 
relative amounts of chlorids, phosphates and sulphates excreted in the urine and sweat. Mironowitsch(166) found mercury more readily eliminated by the skin than the kidneys following its subcutaneous injection. Riggs( $\left(\mathrm{I}_{7}\right)$ showed a fair constancy in the relation of urea and ammonia to the total nitrogen in sweat, which, however, differs from that in the urine. Plaggemeyer and Marshall(r68) in a comparison of the concentration of nitrogenous substances eliminated in the sweat and urine with those of the blood, pointed out that the sweat glands concentrate urea and ammonia, but that diastatic activity is less in sweat than in either blood or urine and that uric acid is present in less concentration than in the bloud, that the concentrating power of the skin and kidneys for urea and ammonia are very different, and that the commoner dyestuffs which have been used for estimating renal function are not excreted at all through the skin. Furthermore, Austin and Miller(I69) later showed that the non-protein and urea nitrogen of the blood in nephritics was not lowered by sweating. This is not to be wondered at when we know that it is unusual to find more than $0.5 \mathrm{gm}$. urea in 500 to 600 c.c. of sweat.

Taking into consideration, the above facts, it is evident that the rationale of sweating is not clear. In any short period of time (one-half hour) the amount of waste products which can be eliminated is too small to account for the improvement in the patient's condition. The facts that the skin cannot vicariously assume any great part of the renal function under normal conditions and yet that sweating is of definite therapeutic value may indicate that the toxic substances of uremia (as yet unknown) are more readily eliminated by the skin, such as is the case with mercury. Considering all the available facts, the removal of known toxic substances or waste products of normal metabolism by the sweat bath would not seem at all sufficient to account for the therapeutic effect. The problem is similar to that of the beneficial effects of venesection in uremia. The amount of active toxic substance or substances removed is so small in comparison to the total content of the body that it does not seem adequately to account for the therapeutic effects which are beyond question. Sweating, therefore, although recognized as distinctly valuable is an empirical measure. Various methods of inducing sweating are employed, most of them being modifications of the Turkish bath in which dry heat is employed. The methods most commonly employed are the hot air bath, the brick method, electric light baths, hot packs and hot baths. Details in relation to these methods will be found on page $85 \mathrm{I}$.

In the mild chronic uremia where it is impossible to give the treatment by any of the above methods, an ordinary hot bath proves of benefit. It should last from fifteen to twenty minutes, and after it the patient is wrapped in blankets. The duration and number of sweat baths depends upon the severity of the symptoms and the degree of functional involvement. At most they should 
not be employed more than twice daily. Very careful observation is necessary when the heart is weak. The bath should be immediately discontinued when the temperature reaches $I 0{ }^{\circ} \mathrm{F}$. if the patient complains of feeling weak and faint and the pulse becomes weak and rapid. Cardiac stimulants should be kept at hand.

Pilocarpin is, wisely, regarded with less favor than formerly and is now rarely used except as an adjuvant to one of the methods described above, when it is given in $1 / 10$ to $1 / 12$-grain ( 0.006 to $0.007 \mathrm{gm}$.) doses. Following its hypodermic injection perspiration usually appears in from fifteen to twenty minutes. It must be borne in mind that pilocarpin is a marked cardiac depressant, that it exerts a peripheral action upon the nerve terminals, ${ }^{1}$ that it induces marked salivation and often profuse secretion from the bronchial mucous membrane, that edema of the lungs is apt to accompany its use, and that the sweating following its employment is prolonged and cannot be checked if necessity arises except perhaps through the use of atropin. On the other hand, pilocarpin often causes sweating when all other methods have failed. Consequently it may occasionally have to be resorted to.

(C) By the Kidney.-It is frequently impossible to increase the excretory capacity of the kidney, and in the attempt to do so, harm is often done. In the uremia of acute nephritis all diuretics are apt to fail-indeed they are apt to do more harm than good. It is impossible to flush out the kidneys by increased water intake. In chronic nephritis diuretics accomplish most in the cases associated with myocardial insufficiency and circulatory disturbance of the kidney. It must always be remembered that in some instances the kidney is hypersensitive and responds more readily than normally to diuretics, to water and to salt, but that such kidneys also tire easily, and consequently diuretics must be used sparingly in small doses for a day or so only, the effect being observed to have worn off before they are again given. Water is the only diuretic necessary. Digitalis is indicated when circulatory changes are present. Theocin, gr. iii. (0.2 gm.) t.i.d. for one day is often of the greatest value. The other purin diuretics or alkaline salts may be used. In the kidney resulting from back pressure in the lower urinary tract water may be pushed to several liters a day. Where acidosis exists sodium bicarbonate should be given by mouth or in 4 per cent. solution intravenously, in doses sufficient to bring the hydrogen ion concentration of the blood back to normal. The administration of alkali frequently results in marked polyuria.

Use of Thyroid Extract.-Percy(I70) has advocated the use of large doses of thyroid extract in the treatment of nephritis. Phipps(I7I) has been somewhat impressed with results obtained by

${ }^{1}$ Sweat glands are supposedly innervated only by the sympathetic but they respond to drugs which affect only the autonomic system, do not respond to epinephrin which affects only the sympathetic. They, therefore, behave as organs of autonomic innervation. 
this treatment. The writer has used desiccated thyroid, gr. 2 (0.12 gm.) t.i.d. in a case of uremia without secing any effect whatever from its use. However, a further trial of this treatment seems justifiable.

Symptomatic Treatment.-In all severe cases the general treatment described above should be pushed vigorously and persistently. Other measures should be adopted as needed.

(A) Treatment of the Nervous Manifestations.-Such symptoms as delirium, restlessness, talkativeness are best met with quiet, a darkened room and sedatives. Protection of the patient from himself may be necessary. If violent, canvas sides to the bed, and perhaps a restraining sheet, may be called for. When restlessness and insomnia are persistent and do not yield to diaphoretics and other general measures, sedatives such as veronal, $\mathrm{gm}$. O.I at 2 P. M., 5 P. M., and 8 P. M. daily, potassium sodium bromid, gr. Io to 15 ( 0.6 to $1.0 \mathrm{gm}$.), every four to six hours, or better, morphin, gr. $1 / 6$ to $1 / 4$ (0.OI to $0.015 \mathrm{gm}$.), should be given. Care must be exercised in the use of morphin, but a few hours of good sleep usually will overbalance the harm resulting from its use. The patient will often sleep better sitting in a chair. Vasodilators before lying down are sometimes efficacious. For drowsiness, apathy and coma dependence should be placed chiefly on the general measures already mentioned, particularly bleeding, sweating, and purging. Where acidosis exists the administration of sodium bicarbonate intravenously; by mouth, or by rectum, occasionally results in a clearing up of the patient's mental condition, but such a result is rare if coma is established.

For the headache an ice cap may be tried. This sometimes brings relief. The analgesics ordinarily employed for headache, such as the bromids, coal-tar products and acetyl salicylic acid may be tried, but they usually fail in effect, as does also nitroglycerin in the majority of cases. Relief may follow venesection. These measures failing, spinal puncture may be resorted to. This is of particular value in types associated with increased in tracranial pressure or with outspoken cerebral vascular disturbances. Resort may finally be had to morphin. This should be used sparingly except in cases of uremia with excruciating headache in advanced chronic nephritis, where its free employment is justifiable, just as it is in the end stages of carcinoma.

Convulsions are met by the administration of chloroform inhalations. Morphia, gr. 1/4 (0.015 gm.), hypodermically, or chloral, gr. $\mathrm{xx}$ to $\mathrm{xxx}$ ( $\mathrm{I} .3$ to $2.0 \mathrm{gm}$.), and sodium brumid, gr. 30 (2.0 gm.), by rectum should be given immediately and a venesection done. The patient should be freely sweated and if the convulsions persist the chloral or morphia may be repeated. Spiritus ætheris compositus, 32 to 4 in 4 to 6 ounces physiological saline by rectum has been advised by Le Fevre when chloroform is unavailable or contraindicated. 
(B) Treatment of Respiratory Distress.-Respiratory changes may be dependent upon many sources. Among these must be recognized mechanical interference, as by hydrothorax or ascites and occasionally edema of the lungs, myocardial insufficiency and acidosis. Dyspnea of myocardial origin, usually recognized readily after a physical examination, associated with uremia, may be quickly relieved by venesection or by thoracic or abdominal paracentesis when they are indicated. Digitalis therapy is indicated. Strophanthin, gr. $1 / 60$ (0.000I gm.), is given intravenously or intramuscularly when rapidity of action is needed, or digitalis, in the infusion 3 ii to iv ( 8 to 16 c.c.), or as the tincture, $m \times x$ (I.3 c.c.), three to four hours for forty-eight hours, where the need is less urgent. Strophanthin can be injected intramuscularly without fear of local injury, provided the part is massaged, thoroughly for twenty minutes. Camphor, as the camphorated oil, $m \times v$ (I.O to r.3 c.c.) subcutaneously, occasionally benefits temporarily.

When acidosis is present alkalies should be given intravenously or by mouth until the reaction of the blood is corrected.

Edema of the lungs is best treated by venesection and the hypodermic administration of morphia, gr. $1 / 4$ (0.015 gm.), or atropin sulphate, gr. $1 / 100$ (0.00065 gm.).

Morphia is undoubtedly the best drug for uremic asthma. For the nocturnal attacks, morphia, gr. $1 / 4$ to $1 / 6$ (0.015 to 0.01 gm.), alone or combined with atropin, frequently results in a quiet sleep with the disappearance of the dyspnea within a few minutes. Morphia is not entirely devoid of danger but is of the greatest value. Chloral and bromid are helpful and are worthy of trial when fear of using morphia necessitates a substitute.

Treatment of Gastrointestinal Symptoms.-Nausea and vomiting frequently offer great difficulty therapeutically. It must be remembered that they result from the toxemia and are usually central in origin. They are manifestations of the action of a toxin which resembles apomorphin in its effect on the vomiting center. Consequently gastric sedatives, which are the measures usually employed, have frequently little or no effect. The most good results from the general sedatives such as chloral, gr. v. (0.3 gm.) q. four hours, or morphia, or from the general measures described above.

In the mild cases attention to diet and to the bowels will prove of benefit. It may be advisable to discontinue all food by mouth for from twelve to twenty-four hours, allowing only crushed ice, or sips of iced water, or of one of the carbonated waters (an ordinary siphon of Apollinaris, or ginger ale), champagne or one of the other sparkling wines. On resuming feeding, milk and lime water in small amounts should be tried first. Gastric sedatives such as bismuth subnitrate, gr. xx (r.3 gm.); cocain, gr. $1 / 4$ (o.or 5 gm.); phenol, $\mathrm{mi}$ to ii (0.06 to 0.12 c.c.); creasote, $\mathrm{mi}(0.06) ; \mathrm{HCl}, \mathrm{mi}$ to ii (0.06 to 0.12 c.c.); tincture iodine, $\mathrm{mi}$ ( 0.06 c.c.); or diluted hydrocyanic acid, $\mathrm{mi}$ ( 0.065 c.c.); may be tried. Alkalies, such as sodium 
bicarbonate, gr. $\mathrm{x}$ to $\mathrm{xxx}$ ( 0.6 to $2.0 \mathrm{gm}$.), or the heavy magnesium carbonate, gr. xv (r.o gm.), sometimes help.

Diarrhea, unless very severe and prolonged, is not to be combated since it is salutory in its effects. It is one of nature's ways of controlling uremia by vicarious elimination, and interference in it may result in the appearance of other uremic symptoms and in death. Purgatives, alone or with high colon irrigation, should be first administered. After thorough evacuation the diarrhea usually become less marked.

Attention should be directed to keeping the mouth clean, by frequent use of a toothbrush and of an antiseptic mouthwash such as Dobell's solution.

Anemia.-Anemia developing in the course of chronic nephritis may be marked and call for treatment with iron or asenic or both. Transfusion of 500 to 600 c.c. has proved of value in our hands.

Itching.-This is not infrequent in uremia and is best met by hot alkali baths or application. Care must be taken to avoid the use of substances which are irritating to the kidney.

Hypertension in the past has usually been combated by means of nitrites in the form of nitroglycerin, amyl nitrate, erythrol tetranitrate or sodium nitrite. Nitrites are never used in this connection in the wards of the Johns Hopkins Hospital except in cases associated with coronary sclerosis or in cases in which a vascular accident is threatening. Dependence is placed upon rest in bed, diet, purgation and diaphoresis. Venesection sometimes results in a considerable decrease in pressure; again it may have no effect, or a very temporary one. When digitalis is indicated for an associated myocardial insufficiency, it should be given without fear of increasing hypertension, as marked decrease in pressure is often encountered despite its use. Digitalis or strophanthin is indicated in low blood-pressure associated with myocardial insufficiency. This has been considered in greater detail in relation to the treatment of hypertension.

In the presence of anuria or marked oliguria the general treatment should be forced. Diuretics avail little as a rule except in the presence of circulatory disturbance. Heat or cupping locally over the kidney region will sometimes prove of great benefit, particularly in the anuria of acute nephritis. The administration of alkalies intravenously may result in reëstablishing urinary secretion. Glucose in ro per cent. solution may be given up to 500 c.c. and often results in immediate diuresis.

\section{GENERAL THERAPEUTIC MEASURES}

Diet in Nephritis.-In the selection of food for nephritic patients three factors enter particularly into consideration, namely: the salt content, the protein content and the caloric value. Information concerning the salt content may be had from the tables published by Levy $\left(\mathrm{I}_{72}\right)$, while the protein content and caloric values are given in the tables constructed by Atwater and Bryant(I 73). Locke( I74) 
has furnished us with a valuable table which facilitates the choice of a menu for special diseases such as nephritis. The little volume by Gephart and Lusk(I 75) on "The Analysis and Cost of Ready-toServe Foods' furnished a useful guide for protein values for both practitioner and patient.

I have constructed a short table giving the chlorid, protein and caloric values of some of the more commonly used foods, raw and cooked. The figures for the greater part are obtained from the abovenamed sources (tables of Levy and Atwater and Bryant). A few of the chlorid values were determined in the chemical laboratory of the Johns Hopkins Hospital.

TABLE I

\begin{tabular}{|c|c|c|c|}
\hline Food prepared for table use & $\begin{array}{l}\mathrm{NaCl} \text { content, } \\
\text { per cent. }\end{array}$ & $\begin{array}{l}\text { Protein, } \\
\text { per cent. }\end{array}$ & $\begin{array}{l}\text { Calories, } \\
\text { per I00 gm. }\end{array}$ \\
\hline \multicolumn{4}{|l|}{ Meats: } \\
\hline Roast beef. & 0.98 & $22 \cdot 3$ & 357 \\
\hline$\ldots \ldots \ldots \ldots \ldots \ldots \ldots \ldots \ldots$ & I. $04 / 1.29$ & $23 \cdot 5$ & 286 \\
\hline 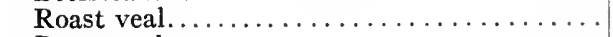 & I. II & 28.4 & 132 \\
\hline 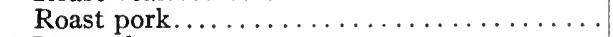 & 1. 54 & 28.4 & 210 \\
\hline 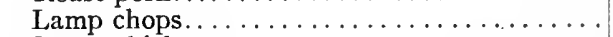 & 0.97 & 29.9 & 367 \\
\hline 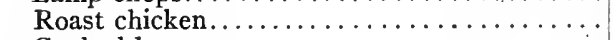 & 0.39 & 32.1 & $18 \mathrm{r}$ \\
\hline 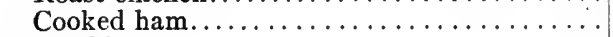 & I. $85 / 5 \cdot 35$ & 22.0 & 282 \\
\hline \multicolumn{4}{|c|}{ Vegetables: } \\
\hline 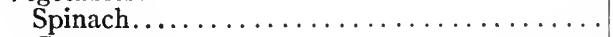 & 0.91 & 2. I & 57 \\
\hline 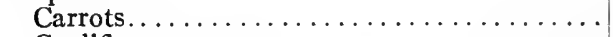 & 0.46 & 0.53 & 18 \\
\hline 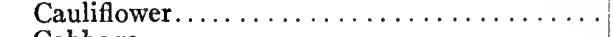 & 0.49 & 0.9 & 7 \\
\hline 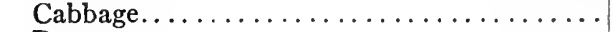 & $\ldots \ldots \ldots$ & 0.6 & 5 \\
\hline \multicolumn{4}{|c|}{ Potatoes: } \\
\hline Boiled .. & $\ldots \ldots \ldots$ & $2 \cdot 5$ & 65 \\
\hline Baked...$\ldots \ldots \ldots \ldots$ & $\ldots \ldots \ldots$ & 2.9 & 90 \\
\hline Mashed and creamed... & $\ldots \ldots \ldots$ & 2.6 & $\operatorname{Ir} 2$ \\
\hline \multicolumn{4}{|c|}{ Desserts: } \\
\hline Apple sauce. & $0.03 \mathrm{I}$ & 0.2 & 120 \\
\hline Pear compote & 0.019 & & \\
\hline Tapioca pudding.. & 0.27 & $5 \cdot 3$ & 140 \\
\hline \multicolumn{4}{|l|}{ Bread and starchy food: } \\
\hline White bread.......... & $0.018 / 0.70$ & $9 \cdot \mathrm{I}$ & 270 \\
\hline 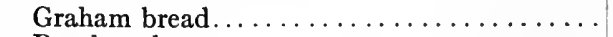 & $0.6 \mathrm{I}$ & 8.9 & 267 \\
\hline 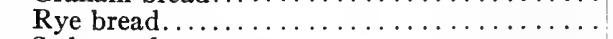 & $0.18 / 0.59$ & 9.0 & 260 \\
\hline Soda crackers.... & $\ldots \ldots \ldots$ & 9.8 & 424 \\
\hline Graham crackers...... & $\ldots \ldots \ldots$ & 10.0 & 429 \\
\hline
\end{tabular}

TABLE 2

\begin{tabular}{|c|c|c|c|}
\hline Food & $\begin{array}{l}\mathrm{NaCl} \text { content, } \\
\text { per cent. }\end{array}$ & $\begin{array}{l}\text { Protein, } \\
\text { per cent. }\end{array}$ & $\begin{array}{l}\text { Calories, } \\
\text { per roo gm. }\end{array}$ \\
\hline Cow's milk. & 0.16 & $3 \cdot 3$ & 72 \\
\hline$\ldots \ldots \ldots \ldots \ldots \ldots$ & 0.16 & 3.0 & 36 \\
\hline 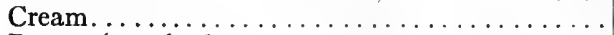 & 0.13 & 2.5 & 201 \\
\hline 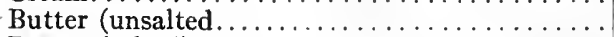 & $0.02 / 0.21$ & & \\
\hline 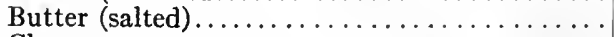 & $1.02 / 2.0 / 3.0$ & I.O & 795 \\
\hline Cheese: & & & \\
\hline American... & $28.8 / 29.0$ & $\cdots$ & $453 / 477$ \\
\hline Cottage... & $0.20 / 1.15$ & 20.9 & 112 \\
\hline 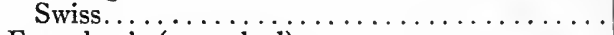 & 2.0 & 27.6 & 443 \\
\hline Eggs, hen's (uncooked) $\ldots \ldots \ldots \ldots \ldots \ldots \ldots$ & $0.2 \mathrm{I}$ & I 3.4 & I 59 \\
\hline
\end{tabular}


Table 2.-(Continued)

\begin{tabular}{|c|c|c|c|}
\hline Foord & $\begin{array}{l}\mathrm{NaCl} \text { content, } \\
\text { per cent. }\end{array}$ & $\begin{array}{l}\text { Protein, } \\
\text { per cent. }\end{array}$ & $\begin{array}{c}\text { Calories, } \\
\text { per too gm. }\end{array}$ \\
\hline 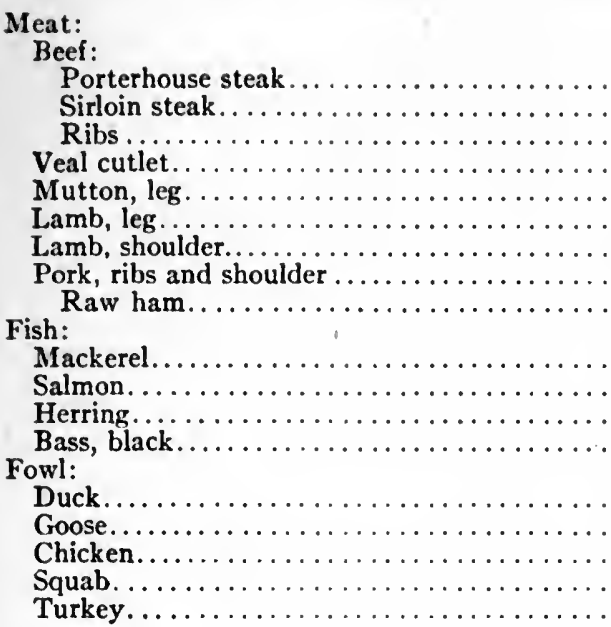 & $\begin{array}{l}0.11 \\
0 . \cdots \\
0.13 \\
\ldots \ldots \\
\ldots \ldots \cdots \\
0.17 \\
0.10 \\
4.15 / 5.86 \\
0.28 \\
0.61 \\
0.27 \\
\ldots \ldots \ldots \\
0.14 \\
0.20 \\
0.14 \\
0.15\end{array}$ & $\begin{array}{l}21.9 \\
18.9 \\
17.8 \\
19.9 \\
18.7 \\
18.6 \\
18.1 \\
17.3 \\
15.7 \\
18.7 \\
22.0 \\
19.5 \\
18.6 / 20.6 \\
18.3 \\
16.6 \\
20.7 \\
18.6 / 22.8 \\
22.2\end{array}$ & $\begin{array}{l}280 \\
249 \\
302 \\
1 ; 4 \\
239 \\
287 \\
351 \\
360 \\
375 \\
142 \\
149 \\
209 \\
146 \\
886 / 103 \\
284 \\
366 \\
196 \\
315 \\
305\end{array}$ \\
\hline
\end{tabular}

TABLE $2 A$

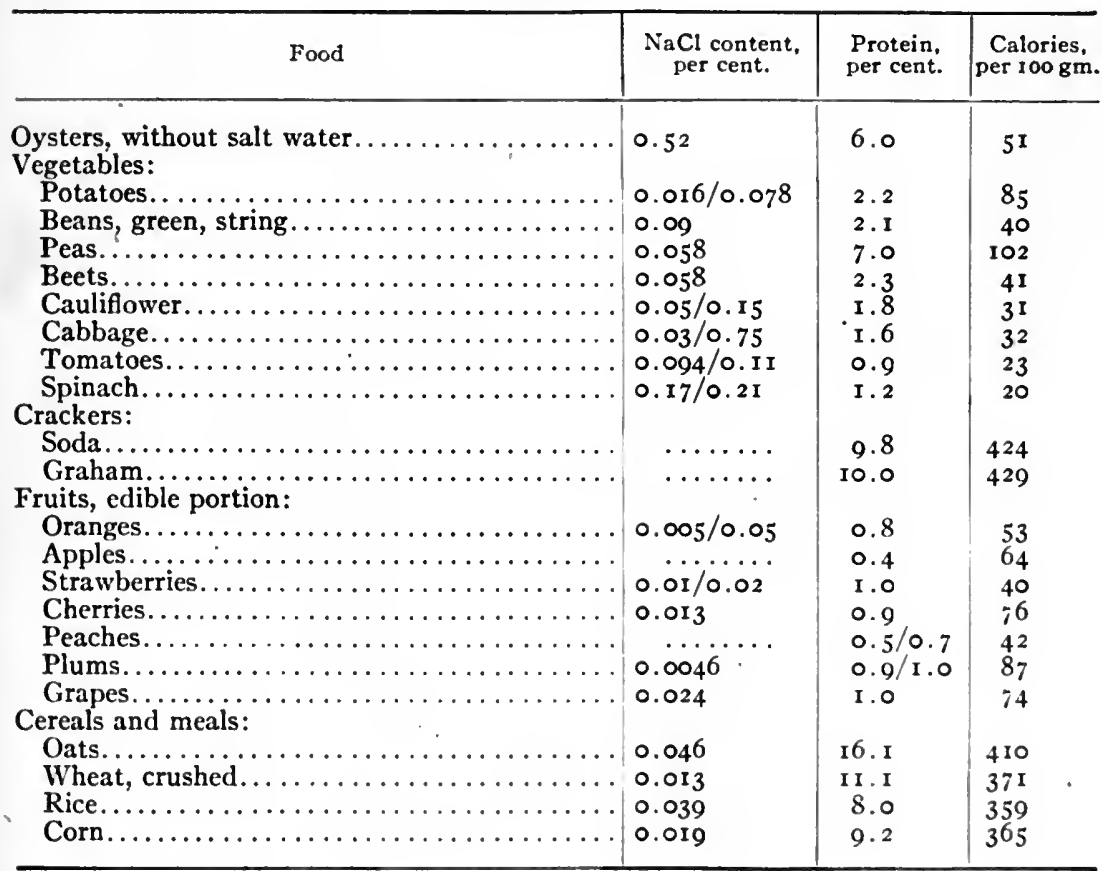


Table ${ }^{1}$ in Use in the Chemical Laboratory of Johns Hopkins Hospitai Approximate Percentages of $\mathrm{N}$ and $\mathrm{NaCl}$ of Food in the "Salt-free Diet"

\begin{tabular}{|c|c|c|c|c|c|}
\hline Food & $\begin{array}{c}\mathrm{N} \\
\text { per cent. }\end{array}$ & $\begin{array}{l}\mathrm{NaCl} \text {, } \\
\text { per cent. }\end{array}$ & Food & per cent. & $\begin{array}{l}\mathrm{NaCl} \text {, } \\
\text { per cent. }\end{array}$ \\
\hline Apple sauce......... & 0.3 & 0.0 & Milk............... & $0.5^{2}$ & 0.17 \\
\hline Apples............. & 0.06 & 0.0 & ( & & \\
\hline Beefsteak... & 2.50 & 0.01 & $\begin{array}{l}\text { Oatmeal } \ldots \ldots \ldots \ldots \ldots \ldots \\
\text { Oil (olive) } \ldots \ldots \ldots \ldots \ldots \ldots\end{array}$ & $\begin{array}{l}0.3^{0} \\
0.0\end{array}$ & $\begin{array}{l}0.03 \\
0.0\end{array}$ \\
\hline Beef (scraped)........ & 2.59 & $0 . I$ & 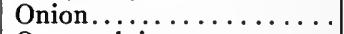 & 0.19 & $0 . I$ \\
\hline Beets............... & 0.26 & $0 . I$ & Orange juice........... & 0.0 & 0.06 \\
\hline Bread............... & I. 5 & 0.0 & & & \\
\hline$\ldots \ldots \ldots \ldots$ & 0.3 & 0.07 & Parsnips............. & 0.25 & O. I \\
\hline 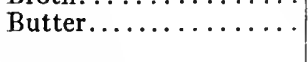 & 0.16 & 0.0 & 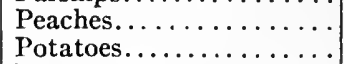 & $\begin{array}{l}0.1 \\
0.4\end{array}$ & $\begin{array}{l}0.1 \\
0.03\end{array}$ \\
\hline Cabbage............. & 0.25 & $0 . I$ & 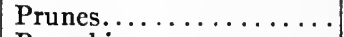 & 0.1 & $0.0 \mathrm{I}$ \\
\hline Carrots.... & 0.18 & $0 . I$ & Pumpkin............ & 0.18 & $0 . I$ \\
\hline Celery............... & 0.18 & $0 . I$ & & & ( \\
\hline Cheese............. & 0.83 & I. 5 & Rice.............. & 0.45 & 0.0 \\
\hline Chicken............ & 3.1 & 0.04 & Rice pudding.......... & 0.64 & 0.17 \\
\hline 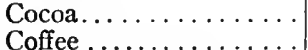 & 0.64 & 0.17 & & & \\
\hline Coffee ............ & 0.18 & 0.06 & Salsify......... & 0.2 & $0 . \mathbf{I}$ \\
\hline Crackers (S.F.)....... & $5 \cdot 2$ & 0.005 & Shredded wheat... & I. 7 & 0.0 \\
\hline Custard.............. & 0.9 & 0.17 & $\begin{array}{l}\text { Spinach } \ldots \ldots \ldots \ldots \ldots \ldots \\
\text { Squash } \ldots \ldots \ldots \ldots \ldots \ldots\end{array}$ & $\begin{array}{l}0.34 \\
0.23\end{array}$ & $\begin{array}{l}0.21 \\
0.1\end{array}$ \\
\hline Eggs.. & $2 . I$ & 0.14 & 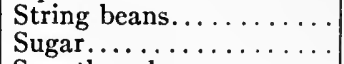 & $\begin{array}{l}0.13 \\
0.0\end{array}$ & $\begin{array}{l}0.1 \\
0.0\end{array}$ \\
\hline Figs....... & 0.69 & 0.01 & Sweetbreads............ & 2.7 & 0.25 \\
\hline Fruit...... & 0.03 & 0.01 & Tapioca pudding........ & 0.53 & 0.17 \\
\hline Ice cream. & 0.8 & 0.17 & 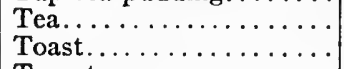 & $\begin{array}{l}0.18 \\
\text { I. } 8\end{array}$ & $\begin{array}{l}0.06 \\
0.0\end{array}$ \\
\hline Junket. & 0.5 & 0.17 & 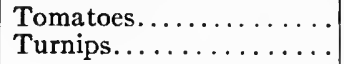 & $\begin{array}{l}0.14 \\
0.2\end{array}$ & $\begin{array}{l}0.1 \\
0.1\end{array}$ \\
\hline & 2.6 & 0.07 & & & \\
\hline Lettuce.. & 0.19 & 0.13 & Wine jelly. & 0.7 & 0.1 \\
\hline
\end{tabular}

${ }^{1}$ This table and the following diet lists were furnished the writer by Dr. H. O. Mosenthal, who is in charge of metabolic studies in the Johns Hopkins Hospital.

Breakfast: Sherry, 30 c.c.

\section{Low Protein Diet}

Baked apple, stewed prunes, orange.

"Hominy cornstarch cereal."

Cream, is 5 c.c.

Dinner: Sherry, zo c.c.

Potato, baked or mashed.

String beans, cabbage, carrots, lettuce, onions, tomatoes, cucumber pickles. Fruit cornstarch pudding, fruit tapioca pudding.

\section{Supper: Same as dinner.}

Weigh or measure all food eaten except salt, sugar, and butter, which may be used as desired, and need not be weighed.

\section{Nitrogen Content of Food Used in Low Protein Diet}

\section{Article of food}

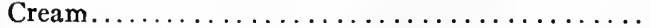

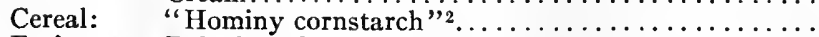

Fruit:

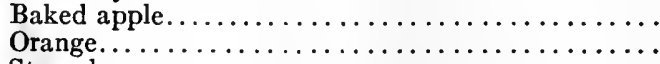

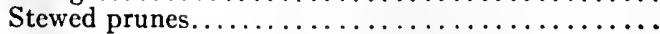

$22 / 3$ hominy, $1 / 3$ cornstarch.
Percentage of nitrogen

$0.4 \mathrm{I}$

0.13

0.04

0.16

0.14 
Nitrogen Content of Food Used in Low Protein Diet

Article of food

Percentage
of nitrogen

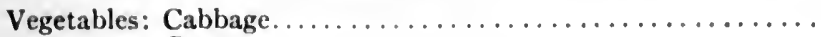

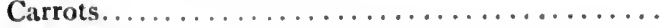

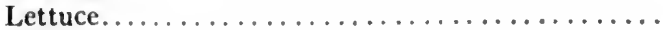

Onions. . . . . . . . . . . . . . . . . . . .

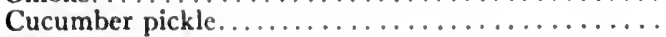

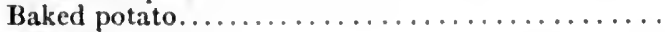

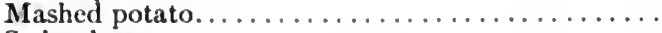

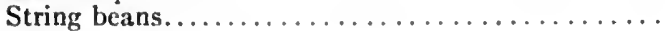

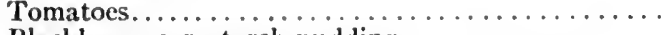

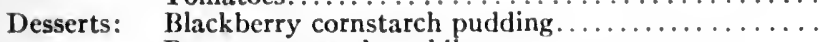

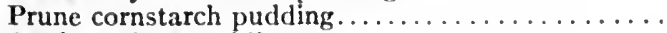

Apple tapioca pudding.

Peach tapioca pudding.

o. 16

0.10

0.24

0.17

o. 10

0.48

0.40

0.23

0.23

0.05

0.07

0.02

0.06

Methods of Inducing Sweating.-The Hot-air Bath.-The patient is placed in bed and a low tent is constructed over his naked body. He lies upon blankets which are placed over a large mackintosh or rubber sheet. Cradles are placed on the bed and over these the blankets and mackintosh are drawn. The blankets are closely adjusted about the patient's neck. Over the tent another blanket is thrown. Into the tent is brought a small stovepipe, which by means of an elbow reaches close to the floor. Heat, generated by an alcohol lamp or by a Bunsen burner fixed below the lower end of the pipe, is carried into the tent in such a way that it does not come directly in contact with the patient. An ice cap is placed on the patient's head. The temperature under the covering quickly reaches from $45^{\circ}$ to $50^{\circ} \mathrm{C}$. A temperature higher than $55^{\circ} \mathrm{C}$. is not advisable. Sweating usually appears withing fifteen to twenty-five minutes, the patient's condition being carefully watched, and the pulse being taken repeatedly. At the end of the sweat the flame is extinguished, the blankets removed-one every five minutes - the patient is rubbed dry and is placed between dry sheets which have previously been arranged beneath the lower rubber blanker.

Sweating is increased by the administration of cold drinks, such as water, lemonade or a mixture containing spiritus ætheris compositus or liquor ammonii acetitis. If these measures fail, a small dose of pilocarpin, gr. $1 / 20$ ( $0.003 \mathrm{gm}$.), may be given hypodermically as an adjuvant to the heat.

For patients not confined to bed the same principle may be $\mathrm{cm}-$ ployed. The patient is stripped and seated on a cane-seated chair. A large blanket is pinned about his neck, making a tent covering his body below that point, reaching the floor. An alcohol lamp is placed beneath the chair. With this method great care must be exercised in order to avoid blistering the patient or setting fire to the blanket.

The method used in the Presbyterian Hospital in Chicago is described by Herrick ( $\mathrm{I} 76$ ) as follows: "The patient lies between blankets, and outside each blanket - of the one under as well as the one over the patient - is a heavy rubber sheet. Hot bricks, in bags for 
convenience in handling, are placed about the patient, the blankets being so rolled that the body is protected from the bricks by the cloth of the bags and by the thickness of the blanket. Alcohol is poured over the bricks and the blankets are tucked in around the patient. A cold cloth is kept on the forehead during the sweat."

Electric Light Baths. - These have lately been introduced and answer the purpose admirably. All danger of fire is avoided. They can be improvised utilizing the tent described above and attaching a series of bulbs (six to ten) to the cradles used in constructing the tent. Regular electric cabinets can be obtained on the market which will accommodate patients, sitting or lying, the head projecting from the.top or from the end. The box is lined with 20 or 30 bulbs, so that a great amount of heat is thrown off in a few minutes, the temperature quickly reaching 45 to $50^{\circ} \mathrm{C}$. Sweating is allowed to continue for from fifteen to twenty minutes, and the patient is then dried and returned to bed.

Hot Pack.- When moist heat is used it is generally in the form of the hot pack. The bed is covered by a rubber sheet. A large heavy blanket is dipped in hot water, wrung out and rapidly wrapped about the naked patient, care being taken that it is not too hot. A dry blanket is folded over the patient and several hot water bags placed about him. Finally, the rubber sheet is wrapped about him and an ice cap placed on the head. The bath should last for an hour. Cold water is administered if sweating does not result. The pack is stopped if the patient's temperature goes higher than Ior $^{\circ} \mathrm{F}$.

\section{BIBLIOGRAPHY ON NEPHRITIS}

I. Herrick, Nephritis, Osler's System, r9i4, Vol. iii, p. 845 .

2. Dick, G. F., and G. R., Jour. Am. Med. Assn., 1915, Ixv, 6.

3. Clark, Marshall and Rowntree, Jour. Am. Med. Assn., 1915, lxiv, 230.

4. O'HaRe, Arch. Int. Med., I9I3, xii, 6r.

5. Fischer, Edema, N. Y., I9I0; Nephritis, N. Y., I9I 2; Edema and Nephritis, N. Y., Wiley and Sons, I915.

6. Drckinson, A Treatise on Albuminuria, Wood, N. Y., I88I, Chap. viii, p. 107.

7. BRIGHT, Guys Hosp. Reports, I836, I, p. 338 .

8. Aschoff, quoted by VolHard and FaHR, Brightische Nierenkrankheit, Berlin, rgi 4 , p. I.

9. Volhard and FAHR, Brightische Nierenkrankheit, Berlin, 1914, p. I.

io. Schlayer and Takayasu, Deutsch. Arch. f. klin. Med., igro, cl, 333.

II. RownTree and Collaborators:

Rowntree and Fitz, Arch. Int. Med., I9I3, xi, 258.

Rowntree, Marshall and BaetJer, Arch. Int. Med., 1915, xv, 543.

I2. OsLeR'S Practice of Medicine, 8th Edition, I9I4, p. 697.

13. Senator, Nothnagels Encycloped. of Practical Med., I905, p. I7.

I4. Von Noorden, Postgraduate, I913, xxviii, 3 .

15. PhIPPS, Boston Med. and Surg. Jour., I9I6, clxxiv, 73.

I6. Christian and Co-workers.

Christian and O'Hare, Arch. Int. Med., I913, x, 517.

I7. Schlayer, igth Congress f. Inn. Med., I9I 2, p. 501 .

I8. Miller, J. L., Forchheimer's, Therapeutics, I9I4, vol. iv, p. Io.

19. Pepper and Austin, Am. Jour. Med. Sci., I9I3, cxlv, 254.

20. Baetjer, Arch. Int. Med., I9I3, xi, 593.

21. Gull and Sutron, Med. Chir. Trans., I872, lv; 273.

22. Janeway, Arch. Int. Med., I9I3, xii, 755 .

23. Foster and Davis, Am. Jour. Med. Sci., igr6 cli, 49. 
24. Keitr, Rowntree and Geragity, Arch. Int. Med., 1915, xvi, 547.

25. Heidenhain, Hermann's Handbuch de Physiol., v, 348.

26. Howell's Text Book of Physiology.

For discussion of the function of glomeruli and tubules see:

Nussbaum, Arch. f. Physiol., r878, xvi, 139, 1878, xvii, 580.

AdAMr, Jour. Physiol., 1886, vi, 382.

BEDDARD, Jour. Physiol., 1902, xxviii, 20.

Cullis, Jour. Physiol., 1906, xxxiv, 250.

Barcroft and Straub, Jour. Physiol., I9II, xli, 145.

Rowntree and GeragirTy, Arch. Int. Med., I91 2, ix, 284.

27. Schlayer and 'Takayasu, Deutsch. Arch. f. klin. Med., rgro, ci, 333.

28. Cotret, Ann. de. méd., Paris, 1914, 1, 283.

29. VolmaRd and FAHR, Loc. cit.

30. Albarran, Ann. d. mal. d. org. génito-urin., Paris, 1904, 81.

31. Schultze, Arch. f. Physiol., 1889, xlv, 401 .

32. Gusurch, Ztschr. f. phys. Chem., I893, xvii, ro.

33. CAIIERer, Ztschr. f. Biol., I902, xliii, 13.

34. Folin, Am. Jour. Physiol., I905, xiii, 45.

35. HASkins, Jour. Biol. Chem., 1906-07, ii, 217.

36. Gammeltort, Skand. Arch. Physiol., 1913, No. 28, p. 325.

37. Lewis, D. Sclater, Personal communication.

38. Ambard and Hallion, Compt. rend. Soc. d. biol., igr 2, lxxii, ibid., Igr 2, lxxviii.

39. McLean, Jour. Am. Med. Assn., 1916, lxvi, 4I5.

40. Lewis, D. Sclater, Personal communication to be published later.

41. Monakow, Deutsch. Arch. f. klin: Med., April i2, I9Ir.

42. Marshall, Jour. Biol. Chem., I913, xiv, 283; ibid., xv, 495.

43. Folin, Ztschr. f. Physiol. Chem., r9r2, xxxvii, i6r.

44. Monokow, Loc. cit.

45. Hedinger and Schlayer, Deutsch. Arch. f. klin. Med., I9I4, cxiv, 120.

46. Mosenthal, Arch, Int. Med., 1915, xvi, 733.

47. Christian, Jour. Am. Med. Ássn., I 915 , lxv, 1947.

48. V. Neubauer, Munch. med. Wchnschr., April, 1914, p. 47.

49. Van Hoogenhuyze, Ztschr. f. Chir., I9r4, p. 377.

50. Detwiler and Guffiet, New York Med. Jour., I915, cii, 798.

51. Meyers and Lough, Arch. Int. Med., 1915, xvi, 536 .

52. Sellards, Johns Hopkins Hos. Bull., I912, xxiii, 289; ibid., 1914, xxv, 101; ibid., 1914, $\mathrm{xxv}, \mathrm{I} 4 \mathrm{I}$.

53. Palmer and Henderson, Arch. Int. Med., I913, xii, 153.

54. Phthalein 'Test:

Rowntree and Geraghty, Arch. Int. Med. I912, ix, 284.

Rowntree and Fitz, Arch. Int. Med., 1913, xi, 258.

Rowntree, Marshall and Geraghty, Surg., Gynec. and Obst., I914, xv, I96.

Rowntree, Marshall and BaetJer, Arch. Int. Med., 1915, xv, 543.

55. Lactose Test:

Schlayer and Takayasu, Deutsch. Arch. f. klin. Med., 1910, xcviii, 17.

Scirlayer, ibid., I911, cii, 31 I and I9I I, ci, 333 .

56. Vort, Deutsch. Arch. f. klin Med., 1897, lviii, 545.

57. De Bonss, Giorn, Intern. d. science med., I907, xxix, 446.

58. RownTREe and Fitz, Loc. cit.

59. Prevost and Dumas, Quoted by Schondorff, Arch. f. d. ges. Physiol I899, lxxiv, 307.

6o. Bright, Guys Hos. Rept., I 836 , i, 358 .

61. Ascoli, Arch. f. d. ges, Physiol., 1901, lxxxvii, 103.

62. Strauss, Die chronische Nierentzündungen in ihrer Einwirkung auf die Blutflussigkeit und deren Behandlung, Berlin, r9o2, Hirschwald.

63. MulLER, Verhandl. der deutsch. path. Gesellsch., 1904-05, vii-ix, Ergänzungsheft, 80.

64. Von Noorden, Metabolism and Practical Medicine, 1907, 1i, 486.

65. Obermayer and Popper, Ztschr. f. klin. Med., r909, lxvii, 332.

66. Holweg, Deutsch. Arch. f. klin. Med., 1912, civ, 2 I 6.

67. Widal, Bull. et mém. d. hôp., Paris, series 3, I9I1, xxxii, 627.

68. RowNTREe, Loc. cit.

69. Marsilall and Davis, Jour. Biol. Chem., I9I4, xviii, 53.

70. Mosenthal, Proc. Soc. Exper. Biol. and Med., New York, I913-14, xi, 133.

71. Marshall and Rowntree, Jour. Exper. Med., 1915, xxii, 333.

72. Clark, Marshall and Rowntree, Jour. Am. Med. Assn., 1915, lxiv, 1230. 
73. Dreser, Arch. f. exper. Pathol. u. Pharm., I892, xxix, 303.

74. KoranyI, Ztschr. f. klin. Med., I897, xxxiii, r.; ibid., I898, xxxiv, 1 .

75. HabMurger, Centralbl. f. inn. Med., 1900, xxviii, 297.

76. LeON BERnard, La presse méd., Feb. I7, 1900, I 59.

77. Folin and DenIS, Jour. Biol. Chem., I9I 2, xi, 527.

78. Marshall, Jour. Biol. Chem., I9I3-I4, Xv, 487.

79. BRIGHT, Loc. cit.

80. Hooker, Arch. Int. Med. I910, v, $49 \mathrm{r}$.

8I. JEHLE, Die Albuminurie, Ergeb. f. int. Med. u. Kinderheilk., Leipsic, 1913, 8089I2.

82. Langstein, Pfaundler and Schlossman, Handbuch, I908, iv, 29.

83. Pavy, London Lancet, I885, No. 2, p. 706.

84. ReNDALI, A Thesis to Paris, I 884 . Quoted by Hooker.

85. Sterling, Brit. Med. Jour., I887, No. 2, p. II57. Quoted by Hooker; ibid., I 889 , No. I, p. 807 . Quoted by HookeR.

86. Teissier, Semaine méd., I 899, xix, p. 425. Quoted by Hooker.

87. Craig, Brit. Med. Jour., I886, No. i, p. 333 .

88. EDEL, Münch. med. Wchnschr., I90I, xlviii, I833; Deutsch. med. Wchnschr. I $903, \mathrm{xxix}, 639$.

89. ErLanger and Hooker, Johns Hopkins Hos. Rept., I904, xii, 145.

90. Hooker, Arch. Int. Med., igro, v, 49r.

9I. Teisser, Rev. de méd., I905, xxv, 233.

92. GooDhart, Brit. Med. Jour., I89o, No. i, p. I I 2 r.

93. Senator, Deutsch. med. Wchnschr. I904, xxx, part 2, p. 1833.

94. RownTrEe and FITz, Arch. Int. Med., I9I3; xii, 24.

95. Dukes, Brit. Med. Jour., 1878, No. 2, p. 794; ibid., 1889, No. I, p. 625.

96. Monakow, Deutch. Arch. f. klin. Med., 1914, cxv, 224; ibid.; 1914, cxvi, 127.

97. FISCHER, Loc. cit.

98. Cohnheim and Lichrhein, Arch. f. Path. Anat. 1877, lxix, 106.

99. Magnus, Arch. f. Exp. Path. u. Pharm., I899, xlii, 250.

roo. Schlayer and Schmidt, Deutsch. Arch. f. klin. Med., I909, civ, 60.

ior. Pearce, Arch. Int. Med., I909, iii, 422.

I02. RichteR, Berlin, klin. Wchnschr., I905, xlii, 384 .

ro3. Heineke, Deutsch. Arch. f. klin. Med., r907, xc, ror.

I04. Widal, La Cure de Dechloruration. Book.

105. Castaigne, Semaine méd., I903, xxiii, 309.

Io6. Meltzer, Am. Med., I904, viii, 59.

107. Christian and Co-workers, Loc. cit.

I08. BRIGHT, Loc. cit.

109. Gull and SUtToN, Loc. cit.

I 10. Janeway, Arch. Int. Med., r9r 3, xii, 755.

I II. BRADFORD, Jour. Physiol., I899, xxiii, 4I 5 .

I 2. PASSLER and HeINEKe, Verhandl. d. deutsch. path. Gesellsch., 1905, ix, 99.

II3. Janeway, Am. Jour. Med. Sci., I913, cxlv, 625 .

II4. KeITH and SNowDEN, Arch. Int. Med., I915, xv, 239.

I I5. NoRris, Blood Pressure, Philadelphia, I9I4.

I16. PAL, Gefasskressęr, Leipzig, I905.

II7. Gull and Sutton, Loc. cit.

118. JoREs, Verhandl. deutsch. path. Gesellsch., I908, xii, I87.

I I9. Traube, Gesammelte Abhandl., Berlin, I856, ii, 290 ; iii, 440.

1 20. Connheim, Vorlesungen ueber allgem. Pathol., Berlin, I880, ii, 355.

I2I. MÜller and MaAs, Ztschr. f. klin. Med., I9I I, lxii, I89.

122. Loes, Deutsch. Arch. f. klin. Med., I905, lxxxv, 348.

123. VOLHARD and FAHR, Loc. cit.

124. Jornson, Pathology of Contracted Granular Kidney, London, 1896.

125. Janeway, Am. Jour. Med. Sci., I9 13 , cxlv, 625 .

126. Other interesting articles dealing with this subject are found in:

Vaquez, Hypertension, Proc. Congrés. Francais de méd., r. 1904, p. 338.

SChUR and WeISEL, Deutsch. med. Wchnschr., r 1907 , xxxiii, 2136.

STEWART, G. N., Jour. Exper. Med., I9I1, xiv, 377; ibid, I913, xvii, I 52.

O'Connor, Arch. f. exp. Path. u. Pharmakol, I9I I-I 2, levii, I95.

JANEWAY and PARK, Jour. Exper. Med., I9 I 2 , xvi, p. 54I.

I27. Tigerstadt and Berganan, Arch f. Physiol., I898, viii, 223.

128. OsLer, Arteriosclerosis, System of Medicine, iv, 452.

I29. ChitTEnden, Physiological Economy in Nutrition, New York, I907.

I3o. Folin, Dennis and Seymour, Arch. Int. Med., I914, xiii, 224. 
131. Seymour, Boston, Med. and Surg. Jour., 1913, clxix, 795.

132. Frothingham and Sirllie, Arch. Int. Med., 1915, xv, 204.

133. Mosential, Loc. cit.

134. Wallace and Ringer, Jour. Am. Med. Assn., 1909, liii, 1629.

I35. Macirt, Jour. Pharm. and Exper. Therap., 1913-I4, v, 514.

136. Rowntree and BaEtjer, Jour. Am. Med. Assn., 1913, xli, 1438.

137. SCHLAYER, Loc. cit.

138. JOREs, Verhandl. deutsch. path. Gesellsch., 1908, xii, 187.

139. Marchand, Verhandl. d. Cong. f. inn. Med., I904, xxi, 60.

140. JANEWAY, Loc. cit., 21.

141. B. Schltemensun, Arch. f. exper. Path. u. Pharmkol., Ixiii, 294.

142. Hovghton, Am. Jour. Pharm., October, 1909, p. 461.

I43. EDsunds, Jour. Am. Med. Assn., 1907, xlviii, 1744.

EDsunds and Halle, Bull, 48, Hyg. Lab. U.S. P. and M. H.S., Wash.

144. Hale, Jour. Am., Med. Assn., I9 Io, liv, 35.

145. Hatcher and Brodie; Am. Jour. Pharm., 1910, lxxxii, 360.

146. Rowntree and Macht, Jour. Am. Med. Assn., igi6, lxvi, 870.

147. Eggleston, Arch. Int. Med., 1915, xvi, I.

148. EDENs, E., Deutsch. Arch. f. klin. Med., I9I1, civ, 512.

149. LoeB, Quoted by Hirschfelder, Diseases of Heart and Aorta, Phila., 1913, p. 237.

150. Voegtin and Machi, Jour. Pharm. and Exper. Therap., 1913-14, v, 77.

51. V. Schroeder, Arch. f. exper. path. u. Pharmakol, 1886, xxii, 39, and 1887, xxiv, 85 .

152. AUTEN, Arch. internat. de pharmacodyn., I90 r, viii, 453.

153. AcH, Arch. f. exper. path. u. Pharmakol, Igoo, xliv, 3 I9.

154. Cullis, Jour. Physiol., 1906, xxxiv, 250.

I55. Barcroft and Straub, Jour. Physiol., igir, xli, 145.

I56. Golla, Quart. Jour. Med., I908, ii, 23r.

157. SMILliE and Wilson, Arch. Int. Med., 1915, xvi, 330.

158. Foster, Arch. Int. Med., igr 2, x, 4 r4.

159. Hartman, Arch. Int. Med., I915, xvi, 98.

I60. Foster, Arch. Int. Med., I913, xii, $45^{2}$.

16r. REISs, EMIL, Ztschr. f. klin. Med., IgI4, lxxx, 97, and I9I4, lxxx, 424.

162. Foster, Arch. Int. Med., 1915, xv, 356.

163. Schwenkenbecker and SPITta, Arch. f. exper. Path. u. Pharmakol., 1906-o7, lvi, 284.

164. BenEDICT, Jour. Biol. Chem., 1905-06, i, 263.

165. Kast, Ztschr., f. phys. Chemie, I887, xi, 501 .

166. Mironowitsch, Med. ko. Obostenz., St. Petersburg Med. Biol., I895, No. I2, p. 33 .

167. RigGS, Jour. Med. Research, 1911, xxiv, 285.

168. Plaggemeyer and Marshali, Arch. Int. Med., 1914, xiii, 150.

169. Austin and Miller, Jour. Am. Med. Assn., i9i4, lxiii, 944.

r7o. Percy, Jour. Am. Med. Assn., r9 12, lix, r 708; ibid., r9 13, lxi, 380.

171. Phipps, Boston Med. and Surg. Jour., 1916, lcxxiv, 73.

172. LeVY, Arch. f. Verd. Krank., xvi.

i73. Atwater and Bryant, U. S. Dept. of Agr. Bull. No. 28, 1906.

174. LoCke, Food Values, r9i1.

175. Lusk, Analysis and Cost of Ready to Serve Foods, Jour. Am. Med. Assn., 1915, lxiv, 1717.

176. Herrick, Loc. cit. 


\title{
SURGICAL INDICATIONS IN URINARY TRACT DISEASES
}

\author{
By Guy L. Huńner, M. D.
}

On reviewing the present chapter in the light of progress in the past five years it is difficult to determine what additions to our knowledge of treating these diseases should receive special discussion in this brief summary.

The author craves the reader's indulgence for injecting some of his own recent observations as of prime importance in the diagnosis and treatment of these diseases.

\section{URETER STRICTURE}

In our former discussion of suppurative diseases of the kidney, ureter stricture was merely mentioned as one of the conditions of partial obstruction determining a pyelonephritis. Recent studies have shown that ureter stricture plays a far more important rôle than we have formerly realized in the origin of hydronephrosis, pyelitis, and pyonephrosis, and that it probably determines some cases of pyelonephritis, as well as furnishing fertile soil for the formation of stone in the ureter.

Ureter stricture with its sequelæ leads to many useless operations for appendicitis, and to other exploratory laparotomies in an effort to determine the cause of obscure abdominal pain; and an early discovery of this lesion would save many operations of a mutilating and destructive character on the upper urinary tract.

Incidence.-Excluding tuberculous stricture and those strictures immediately surrounding stone, the author's cases of what may be called simple stricture or infiltration of the ureter wall observed up to November I, I9I5, numbered fifty as contrasted with forty-nine nephrectomies for tuberculosis and thirty-nine cases of stone in the ureter. Since becoming particularly interested in the subject, the cases have been discovered much more frequently and in the six months since November I, twenty-one new cases have been treated. In the first fifty cases, twelve were bilateral and in the last twentyone cases, seven were bilateral. This latter proportion is probably nearer the true state of affairs as some of these were accidentally discovered in catheterizing both sides for roentgenograms, the patient coming because of symptoms on one side only. Roentgenography has been avoided until the recent introduction of thorium.

Etiology.-Far too much of the literature on this subject is devoted to the so-called congenital stricture of the ureter. There can be no doubt that some cases have a congenital origin but by 
far the greater number are acquired, and many at an early period in life. Other causes that have been given a prominent place in recent literature are gonorrheal infection, descending infection from the kidney, and ascending infection from the bladder. Trauma, especially that due to childbirth and pelvic operations, is a recognized cause. The trauma of serious falls and violent accidents occasionally leads to stricture, and one of the author's late cases developed hydronephrosis and serious symptoms eight years after a gunshot injury to the ureter.

By far the greater number of ureter strictures are due to systemic infection by way of the blood stream or lymphatics, and this infection arises in the tonsils, sinuses, teeth, gastrointestinal tract, or some such distant focus that we have learned to look for in most cases of infectious arthritis. (See Hunner, Chronic Urethritis and Chronic Ureteritis Caused by Tonsillitis. ${ }^{1}$ ) According to this theory, which seems too well supported in an experience with seventy-one cases to be only a theory, the infection settles in the ureter wall, and in most cases it is only later when contraction and urinary stasis has taken place that the urine becomes infected. It is probable that in some cases the acute edema of the early infection causes a temporary stasis and an infection of the urine practically synchronous with the ureter wall infection.

Morbid Anatomy.-The lesion in the ureter wall as observed grossly in twelve operations and by microscopic study in three cases, is one of typical chronic infiltration. On palpation at operation, it is sometimes difficult to differentiate between the nodular thickening in the ureter wall and the thickening and density characteristic of an encapsulated stone.

The ureter lesion is most often located in the broad ligament region-in fifty-three out of sixty-two ureters (twelve out of the first fifty cases being bilateral). The next most frequent site is near the glands of the iliac vessels, eight of the strictures being in this region, and only one being located next to the kidney pelvis.

The secondary lesions affect the ureter above the stricture, the kidney pelvis, and finally the kidney substance. These are usually first dilated as a simple hydroureter and hydropelvis, with clear noninfected urine. Later the pelvic enlargement encroaches on the kidney substance even to the latter's practical destruction. In exceptional cases the dilatation is confined to the pelvis almost exclusively. In one case the stricture was located $3 \mathrm{~cm}$. from the bladder, the ureter above this point was only slightly dilated and the kidney pelvis held 360 c.c. The urine was absolutely clear and contained only the usual number of epithelial cells, a phenolsulphonephthalein test showing the same percentage of work being performed by each kidney. On resection of the large saccular pelvis, it was noted that the calices were only slightly dilated and the kidney substance seemed of normal thickness. 'Two months after resection the

1 Jour. Am. Med. Assn., 1911, lvi, 937. 
kidney pelvis held 7.5 c.c., the normal amount, thus showing normalsized calices.

As a rule the stasis caused by the stricture is followed by infection and we then have to deal with a pyelitis, an infected hydronephrosis, or a pyonephrosis. Some recent cases seen within a few months of the onset of symptoms have shown infection with only a slight degree of hydronephrosis, and some have had infection before an appreciable hydronephrosis could be detected.

In one group of cases the measured content of the pelvis is less than normal. One might expect this condition in old infections, as due to the contraction of infiltration overcoming the resistance of the stricture, but the narrowing is found in some non-infected cases as well. Some of these may be cases with former infection which has cleared up, and it is possible that some have an unusually sensitive area at the stricture site and that the irritation of the urine keeps up a state of reflex spasm resulting in a narrowing of the upper tract. In some I have seen a lessened capacity at the time of an acute attack with a normal or slightly increased capacity soon after. This is probably due to the acute swelling of the kidney encroaching on the pelvis.

Symptoms and Diagnosis. - Local pain is a symptom in some cases but perhaps the majority complain of pain in the kidney region or of vague abdominal symptoms. Many have vesical symptoms particularly when the pain in the lumbar region is increased. A few have the symptom-complex characteristic of stone in the ureter, viz., pain in the flank radiating to the back, to the bladder, and down the thigh, vesical and rectal tenesmus, and frequency of voiding with blood, or blood and leukocytes in the urine. Meteorism, nausea and vomiting, elevation of temperature and a chill help to complete the picture of a pyelitis attack due to stone.

It is only by getting a negative roentgenograph and by passing a wax tip catheter, which meets an obstruction but comes back free of scratch marks, that one changes the diagnosis from that of stone to that of probable stricture. The finding of hydronephrosis makes the diagnosis more certain; and, if at a later examination one adds to his catheter a spindle of wax a few centimeters back of the tip and gets a definite "hang" of the wax bulb and feels it grate over the scar tissue area on its withdrawal, the diagnosis is practically certain.

This may be definitely and visually verified by a pyelo-ureterogram with a thorium injection, which demonstrates the area of stricture narrowing and usually a dilated ureter above this point and a dilated and more or less deformed kidney pelvis.

One should suspect a ureter stricture in any case, which, after an ordinary catheter examination, has an unusual amount of kidney colic or a pyelitis attack, these often being due to the swelling shut of a traumatized stricture area. In any case of pyelitis which resists repeated lavage treatments, one should suspect stricture even though the catheter has not been appreciably obstructed. The wax bulb 
test will then demonstrate the infiltration area if such be present, and the pyelitis will generally quickly yield to treatment after thorough dilatation of the previously unsuspected stricture area.

Many of my first fifty cases were dispensary patients, and occurring before special interest was aroused in this subject, the statistics on the pelvis capacity and conditions of infection are not as complete as we could wish. Of sixteen non-infected cases the average age was thirty-eight years, the average duration of symptoms two and one-half years, and the average size of the kidney pelvis in ten of the cases was I9 c.c. Three of these held 8, I I, and I 2 c.c. respectively and the other seven held from 15 to 30 c.c. In one exceptional case with symptoms of four years' duration, the pelvis held 360 c.c. without becoming infected. In five of the non-infected cases there was no note on the pelvic capacity.

Of eighteen infected cases the average age was thirty-five years, the average duration of symptoms was four years, and the average size of the kidney pelvis in fifteen cases in which a record was made was 98 c.c. In four of these fifteen cases the pelvis was of normal capacity, 7 to 8 c.c., showing for the eleven dilated cases an average capacity of 130 c.c. In the cases with both pelves dilated the capacity is figured on the larger pelvis only.

It is probable that the infection of the urine in most cases is secondary and due to the mechanical obstruction and stasis, and therefore throws no light on the nature of the original ureter wall infection. Of the eighteen cases in which cultural studies were positive, colon bacillus was grown in thirteen, in five of these from both kidneys. In four cases staphylococcus only was grown-in one case a pure typhoid culture was obtained. In one of the above colon bacillus cases colon was grown from one kidney and staphylococcus from the other, making five cases in which staphylococcus was grown.

The urine in the non-infected cases is not a reliable guide in making a diagnosis. It usually contains at least microscopic blood or leukocytes or both, particularly after an exacerbation of pain, but in the quiescent stage, particularly after the patient has been in bed a few days, it may be impossible even with the most careful centrifuging to demonstrate abnormal elements in the urine.

Treatment.-The ideal treatment consists in dilating the stricture area by the vesical approach. This is accomplished most easily in the female subject by using the knee-breast posture and the Kelly speculum through which can be passed many different instruments, ranging from the finest whalebone filiform up to the French elastic bougie of size 16 with a diameter of $51 / 3 \mathrm{~mm}$. This is the largest size I have used by the vesical route but larger ones could be used if necessary. The flexible metal dilator of Bransford Lewis is the most useful instrument devised for use through the Nitze type of cystoscope, although there are many male cystoscopes now in use through which large-sized renal catheters may be used for dilating a stricture area. 
Too frequent trauma is detrimental. The stricture should not be treated more frequently than once a week, and a ten-day or twoweek interval is preferable.

In eight of my non-infected cases symptoms were completely relieved by dilatation through the vesical approach and the pelves which averaged 25 c.c. for the eight cases before treatment, averaged ro c.c. in five cases measured after treatment.

In five cases of infection, two of them bilateral, the symptoms and the infection were cleared up by dilatation of the stricture and pelvic lavage. One of these cases with a pelvis of 30 c.c. capacity and a staphylococcus infection had sterile urine and a pelvis of 8 c.c. six months after treatment; one with only one kidney and a hydronephrosis of 40 c.c. probably of long standing, and a colon bacillus infection probably of ten months' duration, had sterile urine and a pelvis of 30 c.c. two months after treatment. Three cases with infection are free of symptoms and probably have a cure, but this has not been verified by cystoscopy.

Three cases with bilateral strictures and infection ceased treatments as soon as their more severe pyelitis symptoms were relieved and we have not been able to learn of their ultimate condition. One case with bilateral pyonephrosis, the left pelvis holding I20 c.c. of thick purulent urine and the right too c.c., was promptly relieved by dilating the strictures and lavage treatment. Within three weeks the urine became macroscopically clear but it never ceased to harbor a mild colon bacillus infection and contain pus in microscopic amount. The patient has had four years of apparently good health, doing an unusual amount of work, and having only occasional slight pain in either kidney region. She has had dilatation of her strictures once or twice a year and in November, I9I 5 , the kidneys took 50 and 40 c.c. to the point of discomfort, each side returning 45 c.c.

Treatment by Operation.-If all methods of vesical approach fail, we must consider the operative relief. No form of operation will be undertaken until as complete investigation as possible has been made of both sides. Stricture of the ureter is so often bilateral that we cannot afford to take anything for granted in dealing with these cases.

If investigation shows stricture of but one ureter, associated with a kidney of little or no functional value, conservatism usually calls for extirpation of the morbid kidney. This was done in six of my cases with entirely satisfactory results.

If the stricture is high at the junction of the kidney pelvis with the ureter, we may follow Fenger in doing some form of pyelo-ureteroplasty. Actual stricture at this point is extremely rare and the valve-like obstruction formed by floating kidney can usually be overcome by mere high fixation of the kidney as I have done in a number of cases with excellent results.

If careful examination at the time of operation leads one to suspect an organic narrowing at the pyelo-ureter junction, a pyelotomy 
and careful dilatation may be done in addition to the kidney fixation, or if the pelvis is very large, a partial pyelectomy may be done, being careful to dilate if the orifice into the ureter is at all narrowed.

If the stricture is lower and about the lumbar or pelvic brim region, it has been recommended to sever above the stricture and implant into the colon or to bring the ureter out on the loin region.

If the stricture is low and near the bladder, as a vast majority of these strictures are, it has been the custom to implant the severed healthy end into the colon or bladder. I have never made a colon transplantation as I have but little faith in our ability to make the transplant in a manner to prevent future infection of the kidney. I am familiar with Coffey's brilliant results on the dog and with the apparent satisfactory results of his and Mayo's colon transplants in the human, but I fear time will not bear out the perfection of these results.

In the type of cases under discussion the problem is particularly difficult, for in a large proportion of them the ureter is much enlarged above the stricture, making intestinal implantation difficult, and the kidney is already infected or has its resistance so lowered that intestinal infection is invited.

I have done bladder implantation in two of these fifty stricture cases with indifferent or questionable results in both. I say "questionable results" because in neither case could I later enter the ureter with a catheter from below. Both cases have been in good health since the implantation, but I suspect in both of these cases there may have formed stricture at the site of the implantation with gradual destruction of the kidney.

Retrograde Dilatation.-I wish to emphasize a method for handling these cases by operation which I have not seen mentioned in the literature, but which I am sure must have been done by some surgeons and which has probably been described before, viz., the treatment by retrograde dilatation. Certainly every surgeon must follow his ureter stone extractions by dilatation of the usual area of infiltration about the stone.

The ureter is exposed by an extraperitoneal incision, incision is made into its dilated portion above the site of stricture, and increasing sizes of the French gum elastic bougies or metal sounds are passed until the stricture is dilated to a diameter of from 5 to $7 \mathrm{~mm}$. The ureter incision is then closed with catgut, reinforced, if thought necessary, with silk or linen. A wick drain is usually left in the extraperitoneal incision for forty-eight hours to take care of possible contamination by the escaped urine at the time of operation, or of postoperative leakage and the excessive serum secretion following the extraperitoneal operation.

If the dilatation has not been entirely satisfactory, or if there has been much trauma to the ureter, I leave it open or close it carelessly with catgut to favor urine drainage in case of temporary swelling and to shut off the traumatized stricture area. In such cases two or three 
small wicks are dropped to the area of ureter incision and left some days or until there is certainly no urine leakage.

A McBurney incision is suitable for most of these cases but a semilunar line incision is more useful, for it can be enlarged up or down to suit the exigencies of the case and through a moderately long semilunar line incision, one can easily palpate from kidney pelvis to bladder. With care one can preserve the intercostal vessels and nerves crossing this incision to the rectus muscle simply by deflecting and working between them.

I have treated eight cases by this retrograde dilatation, five cases in which it was impossible to dilate from below, two cases in which stricture of the ureter was found when stone was being looked for, and in one case in which ureter stricture had been successfully treated from the vesical approach one year previously, but in which the stricture again swelled shut sufficiently to cause kidney symptoms in the course of an attack of acute gonorrheal salpingitis.

The results in these eight cases treated by retrograde dilatation have been perfect in six, so far as measured by relief of symptoms and ability to easily catheterize later from below.

The cases least suited for retrograde dilatation are those in which previous testing of the capacity of the kidney pelvis and ureter, and pyelography have shown an absence of marked enlargement of the lumen above the site of stricture. In these cases the ureter is found too small above the stricture to admit large dilators, and if it is at all possible to get by from the vesical approach, one should be satisfied to do as well as possible by this route although it may require a long tedious course of treatment.

\section{THORTUM}

One of the most valuable recent technical advances in urinary work has been the introduction of thorium as a roentgenographic material. ${ }^{1}$ Burns, after much experimenting, recommends a neutral solution of thorium nitrate dissolved with sodium citrate as an ideal solution for pyelography. Being a heavy metal, a ro to I 5 per cent. solution casts a good shadow and it is non-toxic, non-irritating, and of perfect fluidity. It leaves the urinary tract rapidly and is perfectly clean, not staining the skin or clothing.

In any case in which the total phthalein secretion is low or in which from former examinations one can be reasonably certain that the kidney function is near the borderline of failure, pyelography of both sides should not be attempted at one sitting.

In the case above referred to as having double pyonephrosis fours years ago and pelves holding from 40 to 50 c.c. in November, I915, I got a double thorium picture in May, I9I6, and the patient died eight days later from uremia. I do not charge thorium with

1 Thorium-A New Agent for Pyelography, J. Edward Burns, Jour. Am. Med. Assn., lxiv, 2126. 
this death and believe the complete shutdown would have occurred had both kidneys been simply irrigated with silver nitrate at the same sitting.

Tuberculosis of the Urinary Tract.-Two suggestions of interest have recently been made concerning the care of cases of tuberculosis of the urinary tract. Time and further experience will determine whether there is real value in these departures from accepted customs. Mayo ${ }^{1}$ advises the closure of the wound after nephrectomy in the hope of getting primary union and doing away with the too frequent ugly, slowly healing, drained tuberculous nephrectomy wound. So far as commented upon by those who have tried this method, the consensus of opinion scems to be in favor of drainage, because of the likelihood of later abscess formation and increased time of ultimate healing in those cases which heal per primam.

Crabtree and Cabot in reporting from the Massachusetts General Hospital, "The End Results of Seventy Cases of Renal Tuberculosis Treated by Nephrectomy,"2 find disappointing end results in the early cases, and suggest that this may be due to the lack of time for the establishment of an immunity. They suggest that we must either find some means of securing the vessels early in the operation before the kidney is handled, and thus prevent squeezing infectious material into the general circulation; or that possibly we may defer operation and take measures to hasten the development of an immunity.

\section{CHRONIC CYSTITIS}

In our previous chapter emphasis was laid on the fact that many so-called cystitis cases are devoid of cystitis and that the symptoms are due to a tuberculous or kidney stone or to some lesion in the trigonum or urethra.

Mention was made of certain chronic urethritis cases in which gonorrhea could be excluded as a cause, and in which the removal of diseased tonsils cured the "bladder neurosis" by doing away with the original focus supplying the periurethral tissues with infection or toxins (see Hunner, ibid). Later observations have shown that some of these cases get their supply of irritating material from infected sinuses and teeth and that the chronic urethral lesion clears up spontaneously as soon as the original focus is drained or removed.

Since writing that chapter we have been interested in the study of a new type of bladder ulcer or inflammation, the recognition and treatment of which will do away with another section of that group of cases, which has been going about with the encouraging diagnosis of "cystalgia," "neurosis of the bladder," "irritable urethra," etc., but with the discouraging experience of finding no relief.

Preliminary reports on the first eight cases have been made in the Boston Medical and Surgical Journal (I9I5, clxxii, 660) and in the

1 Nephrectomy without Drainage for Tuberculous Kidney, Surg., Gyn. and Obstet., $1912, \mathrm{xv}, 523$.

2 Surg., Gyn. and Obstet., 1915, xxi, 669. 
Southern Surgical and Gynecological Transactions for I9I4. Since those preliminary reports I have studied six more cases, eleven of the fourteen cases having been operated for removal of the inflammatory area.

I classified this lesion as a simple chronic ulcer because of the urinary findings and because of its apparent origin within the bladder wall rather than from an infected urine.

It was contrasted with the simple ulcer of E. Hurry Fenwick, the latter occurring on the base or fixed portion of the bladder, and resembling round ulcer of the stomach in its deep necrosing character, and in having hemorrhage as one of its marked symptoms. The ulcer under consideration has always been found in the vertex or free portion of the bladder. It is of such small size as to have been universally overlooked, even by the best cystoscopists, and in none of the cases have we found more than a microscopic quantity of blood in the urine.

The urine is macroscopically clear and in some cases it is only by careful centrifuging that a few leukocytes and a few red blood corpuscles can be found. At certain stages of temporary healing of the ulcer area, the urine may be quite free of pathologic elements, only to show them again at the next examination. From none of the cases has a culture been grown.

Through the cystoscope one's attention is as likely to be arrested by an extra white scar area as by the small ulcer. In some cases there is only the scar area with small groups of congested vessels near the scar. Touching these slight areas of congestion with an instrument causes immediate bleeding showing their exposed ulcerated character.

At the first few operations on these cases I was surprised to find a large area of bladder wall surrounding the ulcer involved in congestion of the mucosa and thickening of all the coats of the bladder wall. At times this area shows edema after the manipulation necessary to free the bladder from its surrounding tissues, and in some cases this edema seems to arise during the excision of the area after the bladder has been opened. In one case a sector of this edema area, which extended well into the base of the bladder, was left at operation and this is the only patient who has had a return of her symptoms after operation.

Since becoming familiar with this large congested area at operation I have been able to detect through the cystoscope a wide area around the ulcer, having either a deeper red hue than has the normal bladder mucosa or a paler scarred appearance. In two cases I have seen edema areas through the cystoscope which were present at certain examinations and absent at others.

One has no trouble in outlining this wide inflammatory area after opening the bladder, and during the operation one can quickly detect with the scissors when passing from the thin normal bladder tissue to this thickened inflammatory area. 
Treatment.-So far as we have been able to determine after eight years of experience with these ulcers, there is no curative form of treatment other than excision. This has to be carried through all coats of the bladder including the thickened peritoneum if the inflammatory process happens to subtend the peritoneal walls. When possible the peritoneum is stripped from the bladder and the operation is entirely extraperitoneal. If the peritoneum must be entered, it is carefully protected until the close of the bladder excision when it is closed separately and the bladder edges are then brought together, usually leaving a suprapubic retention catheter in the bladder and one or two finger drains beside the catheter to the space of Retzius.

Irrigations with silver nitrate solution are begun about the third day through the retention catheter and are kept up daily by this route until the withdrawal of the catheter on the tenth to the fourteenth day after which the irrigations are given through the urethra, until the infection, which always follows operation, is cleared.

Most of these cases have been treated over a period of months with various forms of cystitis and ulcer treatments before subjecting them to operation. This delay in the operative relief has had two purposes: first, the desire to make sure of the diagnosis; and second, the desire to study the effects of different forms of treatment, although we were quite convinced after.observing the first few cases and finding the markedly extensive lesion at operation that no ordinary form of cystitis treatment would suffice.

Most of these cases had been suffering for many years (an average of seventeen years in our first eight cases) and they had been subjected to various forms of treatment before we saw them.

In brief, the areas excised from the bladder present a picture of chronic inflammatory tissue. The reader is referred to the above articles for a more detailed description of the microscopic picture.

\section{TUMORS OF THE BLADDER}

In no department of urinary surgery has such progress been made in the past five years as in that dealing with bladder tumors.

New methods of treatment have been largely responsible for the enthusiasm that has resulted in earlier diagnosis, more accurate methods of classification, and more logical application of our methods of treatment.

The introduction by Beer ${ }^{1}$ of the Oudin monopolar current, and by Keyes ${ }^{2}$ of the D'Arsonval bipolar current as practical methods of attacking bladder tumors through the cystoscope has awakened a new interest in a field that previously was clouded by uncertainties in diagnosis and disappointments in treatment. The more recent

1 Removal of Neoplasms of the Urinary Bladder. A New Method, Employing High Frequency (Oudin) Currents through a Catheterizing Cystoscope, Jour. Am. Med. Assn., r 1910 , liv, 1768 .

${ }^{2}$ Am. Jour. Surg., July, rgro.

Vor. IV. -5.5 
introduction of radium promises to add another valuable means of treatment.

Summarizing our knowledge to date and recognizing that with all our advances we are still in the empirical stage of treating bladder tumors, we may state that we cannot determine by cystoscopic vision whether a given papilloma is non-malignant or malignant except when one of the latter type has grossly invaded the surrounding tissues. A papilloma may have its major portion malignant or non-malignant. Non-malignant and malignant papillomata may exist in the same bladder.

According to Buerger ${ }^{1}$ the malignant changes occurring in a primary papilloma begin in the periphery rather than in the base.

The reaction of a given papilloma to the high-frequency current is of diagnostic value, the benign papilloma disappearing rapidly, while the carcinomatous papilloma is more resistant to treatment.

All papillomata whether benign or malignant should be treated by fulguration unless there be infiltration of the bladder walls. Very recent observations by Young and Geraghty and by Kelly and Burnam seem to indicate that some malignant papillomata which are extremely resistant to fulguration become amenable to this form of treatment after treatment by radium.

Every surgeon will make exceptions to the foregoing rule according to his judgment in the individual case. In case of large mutiple papillomata filling the bladder there is probably less danger from hemorrhage and from the development of intractable cystitis, and the treatment may be shortened, by doing the open suprapubic operation first and then following the case carefully with fulguration in the event of recurrences.

In case of open operation for non-infiltrating growths the Paquelin or electric cautery should be used to excise the pedicle and desiccate the base.

In operating for malignant bladder tumor of whatever type the excision should be done to include all coats of the bladder, leaving as wide a margin about the tumor as practicable and the excision should be done by actual cautery in order to destroy possible extensions, and especially to sear the fresh edges against implantation by broken-off bits of tumor. It is probable that in the future radium will prove a valuable adjunct in such operations, a large protected charge being left in the bladder wound for a few hours after operation.

Every case of bladder tumor should be followed, after operation, by repeated cystoscopy. In the cases with single tumor which the microscope shows free of cell transformations of suspicious character, two or three examinations in the first year should suffice.

The occurrence of multiple tumors is in itself a promise of future trouble even though the rapid disappearance on desiccation treatment

${ }^{1}$ The Pathological Diagnosis of Tumors of the Bladder with Particular Reference to Papilloma and Carcinoma: A Study of $\mathrm{II}_{3}$ Neoplasms, Surg., Gyn. and Obstet., I9I5, Xxi, I79. 
and the microscopic study show an absence of malignancy. Such cases should have more frequent examinations during the first year and should be watched at longer intervals after a year of freedom from recurrence.

Cases with mutiple tumors in any of which the microscope demonstrates malignant changes, and cases of papillomata which show an unusual resistance to fulguration, and particularly cases which have been operated for frankly malignant tumors, should be watched frequently and over a period of several years although the chances for recurrence after one year are probably slight. 



\title{
THE NERVOUS SYSTEM
}

\section{SURGICAL ASPECTS OF CERTAIN LESIONS OF THE CENTRAL NERVOUS SYSTEM}

\author{
By Charles H. Frazier, M. D.
}

Pituitary Lesions.-Lesions of the pituitary body require consideration independently of other surgical lesions of the brain. The indications for surgical treatment may be visual disturbance, headache, accession of fat, sexual impotence, but of these the most constant is visual disturbance. For this reason the neurological surgeon and the ophthalmologist should work hand in hand in the management and direction of the treatment. Apart from the characteristic bilateral temporal hemianopsia there is an insidious atrophy of the optic nerve, not preceded by the papillo-edema of brain tumors, which if not recognized in the early stages will reach a stage when restoration of vision is out of the question.

In all cases of pituitary disorder, where vision is not threatened and the patient continues under the observation of the ophthalmologist, glandular feeding should be given a trial. From 3 to 5 grains of pituitary extract, with as much thyroid extract as the patient will tolerate, should be given three times daily. In the exceptional case the symptoms may subside under this régime, but this is not usually the case. As a rule pressure must be relieved by surgical intervention and the operation best adapted to this purpose is a sella decompression by the transphenoidal route. In many instances in addition to the removal of the floor of the sella turcica the contents of the sella may be in part removed.

The results of this operation, performed in my clinic under local anesthesia, are excellent providing intervention has not been too long delayed.

When the roentgenologic studies determine the lesion to be extrasellar and a sella decompression fails, as it sometimes will, to give relief, the tumor must be approached by the transfrontal route.

Tumors of the pituitary body differ from those of the brain in one important particular; a very large percentage are benign, and even when the tumor is malignant it seems to run in this region a comparatively benign course. This pathological characteristic gives to pituitary lesions a more favorable aspect than would at first seem probable, and for this reason operative intervention should be undertaken before the damage is irreparable. The diagnosis with the aid of the roentgenogram and studies of the eye ground, is devoid of 
difficulty, so that delay in the recognition of the disease, and in recourse to appropriate treatment is unpardonable.

Hydrocephalus.-Before the treatment of any case of hydrocephalus is undertaken, the phenolsulphonephthalein test should be applied to differentiate the obstructive from the non-obstructive type (Frazier and Peet). Generally speaking, there may be said to be three types of hydrocephalus classified on the basis of their pathogenesis: (x) the obstructive type; (2) the type due to delayed absorption, and (3) that due to hypersecretion.

Of these, the first or obstructive is the only one in which any of the various methods designed to drain the ventricles should be applied. The simplest method of draining the ventricles is callosal puncture. If the fontanelles be closed, a small opening is made in the skull, $2 \mathrm{~cm}$. in front of the midpoint between glabella and inion, and an equal distance from the median line. A canula designed for the purpose is introduced toward the median line and directed along the surface of the falx cerebri until it penetrates the corpus callosum. It is then moved from side to side until an opening $\mathrm{I} \mathrm{cm}$. is made. The ventricles may be drained by a platinum tube, bent at right angles; one limb of which penetrates the ventricle, the other lying between the dura and brain. When hydrocephalus is accompanied by headache, vomiting and choked disc, relief may be afforded by temporal decompression.

The non-obstructive type of hydrocephalus, due to delayed absorption offers a difficult, if not insurmountable obstacle. At the present time there is no accepted method of dealing with this problem, other than repeated lumbar puncture, which in a few instances has been of permanent benefit. The author believes it may be possible to drain the subarachnoid space into the pleural cavity.

To the hypersecretory form, the same methods of treatment may be applied, and as experimentally it has been shown that thyroid extract retards the activity of the choroid plexus, the author recommends a trial of thyroid feeding in doses appropriate to the age of the patient.

Trigeminal Neuralgia.-It is important to differentiate between trigeminal neuralgia and those expressions of pain in the distribution of the trigeminal nerve, which are due to some local focus of infection, as sinus disease, or to some general toxic influence. The clinical pictures are quite distinct and readily differentiated.

There are but two methods of dealing with trigeminal neuralgia, alcoholic injections and avulsion of the sensory root.

Alcoholic injections have entirely supplanted the so-called peripheral operation, and are recommended as the only means of affording relief in which the major operation, avulsion of the sensory root is not clearly indicated. The period of relief is extremely variable, but in the majority of cases recurrence may be expected in a year. In the author's experience, alcoholic injections not infrequently become less potent as time goes on, that is the interval of relief is 
shorter and the degree of relief is less with each successive injection. The fourth injection in as many years, often fails to give any relief whatsoever.

Technique of Alcoholic Injection.-Alcohol may be injected peripherally into the supraorbital, the infraorbital, the inferior dental, the mental and the lingual branches. It may be injected into the second or third divisions at their exit from the skull, or into the ganglion itself.

Peripheral Injections. - The infraorbital and supraorbital nerves are readily reached by introducing the needle into the infraorbital and supraorbital foramina respectively. To reach the infraorbital foramen introduce the needle through the cheek $0.5 \mathrm{~cm}$. to the outer side of the upper border of the ala nasi. The needle should be pushed upward and outward. To inject the superior maxillary division at its exit from the foramen rotundum the needle is introduced on the inferior margin of the zygoma, $0.5 \mathrm{~cm}$. behind a vertical line corresponding to the posterior border of the orbital process of the malar bone. The needle is directed into the pterygomaxillary fossa, slightly upward and forward, a distance of 4.5 to $5 \mathrm{~cm}$. The pterygoid plate lies about $\mathrm{I} \mathrm{cm}$. external to the foramen rotundum and serves as a useful guide.

The third or inferior maxillary division is more readily injected. Introducing the needle at a point on the inferior border of the zygoma $2.5 \mathrm{~cm}$. in front of the external auditory meatus the needle is directed slightly upward and backward a distance of 4 to $4.5 \mathrm{~cm}$. when it penetrates the nerve. Whether or not the point of the needle is within the substance of the nerve can be readily determined by the resistance offered as the fluid is injected. Care should be taken not to direct the needle too far backward in order to avoid injuring the Eustachian tube. As guides to the foramen ovale the spine of the sphenoid lies just below and behind, while the external pterygoid plate lies just external and anterior. The needle may impinge against either of these structures in its passage.

Injection of the Gasserian Ganglion.-There are two accepted methods of injecting the ganglion, the indirect method of Harris and the direct method of Härtel. The technique of Harris is similar to that of Levy and Baudouin for injection of the third division, although in his early cases he introduced the needle at a point either on, or slightly below, the line joining the incisura notch to the ala nasi instead of below the lower border of the zygoma, and directed it slightly more upward. He now inserts the needle below the zygoma, however, and finds that by injecting I.5 c.c. alcohol, a few drops at a time, into the nerve at the lips of the foramen the whole territory of the trigeminal nerve gradually becomes anesthetic, the anesthesia appearing first in the third division and then in the second and first. While in many subjects it is possible to push the needle through the lips of the foramen ovale into the ganglion itself, Harris believes that this is rarely necessary. One should be extremely careful to discon- 
tinue the injection at once if pain is felt deep in the ear, as deafness might ensue; this pain, however, must be distinguished from pain which may occasionally be referred to the outer ear as a result of irritation of the auriculotemporal branch of the third division of the foramen ovale. ' Care should also be taken to avoid penetrating the pharynx by directing the needle too low. This will rarely happen, however, if one takes the under surface of the sphenoid as a guide.

In rgr 2 Härtel reported a new technique for injecting the Gasserian ganglion which was based on most elaborate anatomical studies. This procedure is briefly as follows: The needle is introduced into the cheek at the level of the alveolar margin of the second upper molar tooth, then directed between the ascending ramus of the lower jaw and the maxillary tuberosity around the buccinator muscle to the infratemporal fossa, thus avoiding the mucous membrane. By hugging the hard surface of the planum infratemporal, the foramen ovale is reached, and the needle advanced in the same direction as before until pain is experienced in the distribution of the second division.

Complications of Alcoholic Injections.-Experience seems to indicate that injection of the ganglion may be followed by the same complications and necessitates the same precautions as does the radical operation. That a subsequent keratitis may develop cannot be denied, but it is also true that it may usually be prevented here as after the radical operation by careful after-treatment. This should consist, according to Härtel, in keeping the patient in the hospital, from one to several weeks after the injections, in wearing a closefitting goggle, in the daily use of boracic lotions and a weekly installation of atropin. In Härtel's series of twenty-four cases, there was permanent anesthesia of the cornea in fourteen, and keratitis developed in six, but in five of the six instances of keratitis, the patient was treated as an ambulant patient. Only once did keratitis develop when the above prophylactic measures were observed, and in this instance the patient insisted upon leaving the hospital too soon. Harris states that it should be impossible to inject 0.5 c.c. alcohol after total anesthesia appears without risk of the anesthesia being permanent, providing the entire amount injected does not exceed $\mathrm{r} .5$ c.c. If more than r.5 c.c. is injected, the risk of the development of a keratitis is great, and Harris's practice is to close the eyelids if total anesthesia of the eyeball persists more than half an hour after the injection, and, if it still persists the following day, the lids should be sewed, leaving just a chink at the canthus. He has been obliged to resort to this procedure in seven cases.

Until clinical experience gives us more positive evidence as to the permanency of the results following injections of the ganglion, I feel that its field should be much more limited than Härtel's conclusion would seem to indicate. In view of the fact that the effects of ganglionic injections thus far are variable, and because of the possibility of subsequent complications, I believe that it should be used as a substitute for the radical operation only in those cases in which such an 
operation is contraindicated, either because of the advanced age of the patient or other deterring factors. In all other cases which have failed to respond to peripheral injections, I believe, for the time being at least, we should resort to the radical operation. In those cases in which a radical operation is out of the question, one should be sure to give the peripheral injections a thorough trial, particularly when the neuralgia is confined to the first and second division, deep injections being given for the second division, and a superficial injection at the supraorbital foramen for the first. There have been no deaths after avulsion of the sensory root in the last fifty cases so that the mortality in my hands has been reduced almost to nil and as compared with other major operations may be said to be a negligible quantity. This is rather extraordinary when one takes into account the fact that the majority of patients are over fifty and many over sixty years of age. 


\title{
DISEASES OF THE SPINAL CORD
}

\section{TABES DORSALIS}

\author{
By E. W. TAylor, M. D.
}

Since the publication of the previous article on the treatment of tabes, the final, definite establishment of the disease as a late, degenerative stage of syphilis has naturally strongly influenced its treatment. It has become increasingly evident that the main hope of checking or ameliorating the disease lies in the use of anti-syphilitic remedies in intensive form, a feeling which did not prevail to the same degree when tabes was regarded as a parasyphilitic affection without ascertainable pathological relations to the original infection. Under the present conditions it is desirable to determine as far as may be where we stand in the treatment of the disease by antisyphilitic remedies, particularly salvarsan and its allied remedies, diarsenol and similar products. In general it may be said that rapidly widening experience leaves small reason for doubt that in the early stages the disease may be checked by the use of salvarsan and that, at any stage, a favorable influence may be exerted on many of its symptoms. In the present state of our knowledge, salvarsan should unquestionably be administered in all cases of tabes unless there is some definite counterindication in the patient's general condition, especially in relation to the cardiorenal system. In general it may be said, that when carefully given under surgical precautions, the danger of intravenous salvarsan is so slight as to be negligible. This holds true in practically the same degree with intraspinous medication.

Whatever the final classification may prove to be, tabes must be regarded as a degenerative type of the disease syphilis. In this type symptoms appear insidiously, often many years after the primary lesion are primarily paralytic in type and to a much less degree irritative. The pathological process is essentially a degeneration of nerve elements and spirochetes are found, especially in tabes, with extreme difficulty. Of the degenerative types, we are at present concerned especially with that which is confined primarily to the spinal cord and its nerves. The treatment of these degenerative cord lesions of which tabes is the most frequent example, offers hope, at least, of improvement in selected cases. Before beginning the treatment of a frank case of tabes, one should reach as clear a conception as possible of the activity of the pathological process and for this purpose it is necessary to differentiate between symptoms and signs. For example, pains are usually significant of an advancing process, though not always; paresthesiæ, constriction sensations and 
crises also signify a progressive process; ataxia, on the other hand, is a sign of lost function, as is loss of bladder control and libido. More important than these symptoms is the condition of the spinal fluid which, in active degeneration shows increase of cells and proteins, with positive Wassermann and Gold reactions. The extreme desirability of determining by tests the degree of activity of the process lies in the fact that to treat with strong antisyphilitic measures a "dead" process is quite unnecessary and may prove harmful, whereas a process having been diagnosticated as active, a vigorous course of treatment is indicated with the expectation that some improvement at least in symptoms and laboratory findings will result. In general, if progress is not made by other means, it is well to place the patient early on serum therapy in one or another of its forms (Swift-Ellis). Such a course is presumably safer and more efficient than to increase overmuch the dosage of other medication. When to stop treatment is a difficult problem, since it is seldom possible to render a tabetic sound, as regards symptoms or signs. In some cases we must be satisfied with moderate improvement since overradical treatment adds merely a further burden of discomfort; on the other hand, certain patients of considerable physical vigor may properly be treated until all active symptoms have disappeared and until the tests are finally returned negative.

In our enthusiasm for newer methods of treatment it should not be forgotten that in every case of tabes a considerable amount of accessory treatment is required. The ataxia, for example, may be greatly benefited by the Fraenkel exercises as detailed in the earlier article. Pain is naturally one of the most frequent reasons for consultation with a physician. While the systematic, antisyphilitic medication will often ultimately help pain, its frequency may increase at the outset and for some time thereafter. To meet this difficulty aspirin in full doses is at times sufficient, but usually some stronger drug is required. Pyramidon in 5-grain doses repeated every three hours will dull moderately severe attacks of pain, but morphin should naturally in such a condition be dispensed with until the possibilities of all the less dangerous drugs be determined. Occasionally tincture of iodine painted over a localized pain area gives relief, or rubbing the affected area with ice, or the employment of dry heat may at times be effective. Hydrotherapy, the electric light bath and the leucodescent lamp are also means which may be employed. Indigestion and gastric crises are symptoms of great persistence. Roentgen-ray examination with bismuth often shows irregularities of peristalsis, at times hypermotility and at times atony and dilatation. This condition seems to be less amenable to antisyphilitic treatment than almost any condition met with in the disease. The gastric crises must be treated symptomatically, with attention to diet and bowel function. Atropin at times is beneficial. Posterior root resection has properly had no consistent following. Trophic disturbances require physical treatment, and especially the 
Charcot joints. For the latter orthopedic apparatus may be indispensable, especially when the joints involved happen to affect hip, knee, ankle or spine. Cutting operations should be undertaken only with great caution.

Various forms of the newer antisyphilitic medication, including mercurial injections, intravenous salvarsan and intraspinal injections have been employed within the last four years in the Neurological Department of the Massachusetts General Hospital, under the direction of Dr. James B. Ayer, in the treatment of tabes, with the following general methods and results:

Mercurial injections in tabes have been found relatively inert. They should certainly be continued over a long period of time and have a certain disadvantage over mercury given in other ways, that once injected, should unpleasant symptoms arise, there is no means of removing the drug from the tissues. Mercury inunctions should still be given a place of usefulness although naturally not so effective in the treatment of tabes as in the earlier stages of syphilis. That mercury and possibly iodid of potash either preceding or along with salvarsan or diarsenol should prove more efficacious than when any of these drugs is given alone, is a somewhat natural expectation. The usefulness of this combined method has, however, not been proved as efficient as in the primary or secondary stages. Salvarsan intravenously should be given under ordinary circumstances to adults in 0.3 to $0.4 \mathrm{gm}$. doses. It is seldom desirable to give larger doses as at first recommended. The injection should be repeated in from ten days to two weeks, depending upon the condition of the patient, until from four to six doses have been given. At the same time, as suggested above, mercury is given for a period of six weeks by injection, either the oxycyanate or salicylate (Burroughs and Wellcome) in doses of I grain twice weekly or I.5 grains once weekly. If possible, the effect of the treatment should be determined by lumbar puncture every two months. If relatively small doses of salvarsan, diarsenol or neosalvarsan are given the not infrequent unpleasant results of the injection are very largely avoided. At the end of approximately two months' treatment, as outlined above; it is desirable to treat the patient with various tonic drugs such as strychnia with iron, or the elixir of the phosphates of iron, quinina and strychnia, for a period of about three weeks. The beneficial effect of the original treatment is often noticed during this period, rather than while the treatment is in progress. If the patient shows a distinct tendency toward improvement and continues to gain after cessation of the treatment, it is well not to force it further. If, on the contrary, symptoms begin to return or a previous gain has ceased, another series of injections should be undertaken at an interval of from three to five months. If no progress has been made after a second series of intravenous injections, and if the various tests, serologic and spinal, have not been markedly influenced, a combined intravenous and intraspinal treatment after the technique of Swift-Ellis should be undertaken. 
Four or five intraspinal injections should be given at intervals of from ten days to two weeks or until the tests have been brought to normal.

Inthe great majority of cases, if this method of treatment is faithfully carried out, leaving the Swift-Ellis intraspinal method as a final resort, distinct improvement in the symptoms of the disease results. The gastric crises are apparently particularly stubborn and no definite improvement as a result of treatment has been observed. Various gastrointestinal symptoms have also not been helped. Optic atrophy has not been influenced nor has the Argyll-Robertson pupil or the knee-jerk been restored to normal. On the other hand it may definitely be said that in most cases pain has been decidedly helped although at times rendered temporarily worse. In about 50 per cent. of the cases bladder conditions have improved, and in occasional instances also sexual potency has returned after two or three years absence. There has also been improvement in general condition, strength, appetite, weight, etc. If treated within six months ocular motor palsies have recovered under, treatment. Trigeminal neuralgia, apparently associated with the tabetic process has been helped in a limited number of cases. Both subjective and objective numbness have improved. Ataxia has ultimately been improved although in certain cases it has been rendered temporarily distinctly worse and in one or two cases acute ataxia was apparently induced by the treatment.

Certain investigators, notably Bernard Sachs of New York, have steadily maintained that the intraspinal method of treatment has no advantages over the intravenous, basing their conclusions in part upon the exceedingly small arsenic content of the injected serum. The weight of opinion, however, is still in favor of the intraspinal method in cases which prove refractory to intravenous injection or to the mercurials Swif $\mathrm{t}^{1}$ has suggested that a combination of intravenous and intraspinal therapy may at times be advantageous where the intravenous alone is ineffectual. This he thinks is particularly true of those cases in which the symptoms and abnormal spinal fluids are not influenced by other therapeutic measures.

\section{ACUIE ANTERIOR POLIOMYEIITIS}

Treatment.-The problem of treatment in poliomyelitis has developed considerably since the publication of the first edition of this book, but still lies rather with the end results of the disease than with its onset. Although much knowledge has been gained through recent years of its etiology through the investigations of Flexner and his colleagues at the Rockefeller Institute for Medical Research, the exact nature of the causative agent is still elusive. Enough, however, has been learned through the study of epidemics as well as through laboratory investigation, to prove that prophy-

${ }^{1}$ Swift, Jour. Am. Med. Assn., 1915, 1xv, 209. 
laxis is in a measure possible. Recently enacted laws in many States including poliomyelitis among reportable diseases has forced upon public attention the fact that it may be regarded as in some sense transmissible. This has led to precautions of an extreme and no doubt often of an unnecessary sort to avoid the spread of the disease through disinfection, isolation, and various quarantine regulations. The fact, however, still stands out prominently that exceedingly few cases of direct transmission from patient to patient have been demonstrated. In epidemics an explanation of the appearance of the disease in several members of the same family may often be explained by a common cause rather than by direct transmission. It is, however, undoubtedly the part of wisdom to take undue precautions until we are surer of our facts than is at present the case.

Owing to the known effects of hexamethylenamin as a prophylactic in certain forms of meningitis, as pointed out a number of years ago by Crowe, this drug has been widely used both when the disease has developed and as a possible preventive in unaffected persons. The evidence seems to show that it has slight prophylactic capacity and presumably is inefficacious in persons already attacked by the disease. It may be given, depending upon the age of the patient, in doses of from 5 to 20 or even more grains a day, care being taken to observe its effect upon the kidney.

Except at times of epidemic, the onset of the affection is often regarded lightly by parents until the paralysis has revealed the nature of the infection. Should a physician be called at the early stage, the treatment should be that of other infectious processes, namely; absolute rest in bed, free opening of the bowels, and a low diet. A counterirritant and diaphoretic treatment may also in certain cases be advisable. When the paralysis has developed and as long after as pain and discomfort persist, no active treatment should be undertaken. This period naturally varies with the violence of the infection from a few days to a number of weeks, so that any hard and fast rule cannot be laid down as to the exact time of beginning active mechanical treatment. This acute phase demands no measures beyond those which would be prescribed in any condition of infection.

A disease identical with acute poliomyelitis in infants occurs also in adults, though far less frequently. The pathologic alterations are the same as in the infantile form, though, perhaps, somewhat more extensive in distribution. The clinical course is naturally similar, modified only by the greater age of the patient. The onset is often less sudden than in the young child.

Meddlesome therapeutics is distinctly harmful. The patients are often exceedingly uncomfortable, both on account of the paralysis and especially because of the pain and tenderness which may reach an extreme degree, and, therefore, much care must be taken in moving them and in attending to their daily needs. To combat the pain, heat, aspirin, phenacetin, codein or morphin should be used as necessary. 
When the tenderness has disappeared, more active treatment may be begun, and it then resolves itself into the restoration so far as possible of damaged muscles and the avoidance of deformity. For the former purpose, massage and muscle training ${ }^{1}$ are undoubtedly the most important means at our disposal, electricity occupying an entirely subordinate place. The massage should be gentle, designed to improve the general nutrition at first, gradually going over into active movements and resistive exercises as the muscles increase in strength. From the first, the utmost care must be taken not to tire the already weakened muscles, to prevent undue stretching, and to avoid the development of contractures through unopposed muscular pull. Particularly important is the almost inevitable foot-drop in paralysis of the legs, from weakness of the anterior group of muscles and overaction of the stronger posterior group. The foot should, therefore, be maintained in a position of dorsal flexion, which may easily be secured by placing the foot in a padded box at right angles to the leg, or by some other simple device. The value of electricity has been much in dispute, and on the whole much overrated. In this connection, however, the experimental work of Langley and $\mathrm{Kato}^{2}$ should be mentioned. These observers found experimentally that in animals in which motor nerves were cut, improvement took place relatively more quickly when the affected muscles were treated by electricity rather than by massage or passive movement. Such experimental evidence should naturally be given much weight in the determination of the efficiency of these forms of treatment. In young children the use of electricity is often alarming, the fat layer may be thick, rendering a satisfactory stimulation of the muscles practically impossible by currents which can be borne. In older patients, electricity may be used in skilled hands to advantage, stimulating the muscles affected, both directly and indirectly by the form of current which induces the best contraction. In muscles showing reaction of degeneration, the galvanic anode as the stimulating electrode is presumably more efficient than the cathode. As time goes on, the various forms of mechanical treatment must be modified or elaborated to meet special indications. In strong children, without extreme paralysis, certain gymnastic exercises may be desirable. It should always be borne in mind that, although certain nerve cells may be wholly incapable of regeneration, improvement goes on indefinitely through the development of unaffected muscles and through instinctive adaptation to the changed conditions. It should also be remembered that what the patient does for himself is very much more important than what is done for him, and he should, therefore, be encouraged to use his muscles, and not become too dependent upon mechanical exercises.

In spite of all efforts, deformities inevitably result in many cases,

${ }^{1}$ Wright, Muscle Training in the Treatment of Infantile Paralysis, Boston Med. and Surg. Jour., I9 1 2, clxvii, 567 .

2 Jour. Physiol., I915, xlix, 432; editorial in Jour. Am. Med. Assn., May 13, 1916. 
which demand the use of apparatus and braces. At this stage, the treatment becomes definitely orthopedic. Finally, operative interference may be necessary through the transplantation of muscles and the correction of high degrees of deformity, to which no detailed allusion need be made in this place. In general, destructive as the disease is, the results of treatment are often unexpectedly satisfactory, but this can only be brought about by unremitting care and patience, by a quick recognition of impending deformity, and by complete coöperation between the physician and the orthopedic surgeon. ${ }^{1}$

During the recent (I9I6) epidemic in New York, and certain of the eastern states, a trial has been made of blood serum taken from patients recovered from the disease and injected intraspinally in amounts not exceeding ro c.c. into the subdural space of patients acutely ill. It appears probable that this treatment should be instituted in the preparalytic stage if definite results are to be obtained, but the evidence is, as yet, too conflicting and the time of observation too short to make any definite statement as to the efficiency of this form of treatment.

${ }^{1}$ Lovett, Principles of the Treatment of Infantile Paralysis, Jour. Am. Med. Assn., I914, lxii, 25 . 


\title{
TREATMENT OF CEREBROSPINAL SYPHILIS
}

\author{
By Homer F. Swift, M. D.
}

In understanding the treatment of syphilis of the central nervous system, it is essential to consider the various manifestations of the disease, and as far as possible, the reasons for these manifestations. It is well established that spirochetes circulate in the blood of practically all patients in the late primary and early secondary stages, and during this period the central nervous system is frequently involved. In fact, with such an universal dissemination of the virus it is difficult to conceive how any organ escapes infection. Even though different observers report wide variation in the relative frequency with which the cerebrospinal fluid of patients in the secondary stage shows alteration, we know that only a small proportion of syphilitic patients have nervous involvement in later years. It is evident, therefore, that in many cases early infection must undergo spontaneous resolution. This phenomenon corresponds with the course of syphilis elsewhere in the body and is probably due to a tissue immunity which develops as a result of prolonged contact between parasite and host. As a result of this tissue immunity the secondary eruption disappears, and cutaneous relapses may never recur even in untreated patients. If, however, cutaneous manifestations do recur they become more and more localized, tend to group and to involve deeper structures with finally gummatous changes.

A similar process appears to occur in the central nervous system. Following the initial inflammation, the organisms deposited in the meninges during the period of spirochetemia are usually destroyed, but in some cases they remain latent only to become active after a period of months or years. This is the usual course of syphilitic infection in untreated or poorly treated cases. The character of the picture may, however, be altered by the institution of intensive treatment. It has long been observed that cases of primary syphilis, relapsing after unsuccessful attempts at abortive treatment with mercury, are liable to show severe skin lesions. Similar deep nodular or ulcerative lesions are frequently seen in the relapses following inadequate salvarsan treatment. This phenomenon of increased severity noted in relapsing lesions of the skin, occurs also in the central nervous system, and explains the occurrence of meningitis with cranial nerve paralysis in many of the inadequately treated cases of secondary syphilis. In these patients the greater part of the infecting organisms have been destroyed by the remarkable spirocheticidal action of salvarsan. This has, moreover, been accomplished so rapidly that the usual tissue immunity which develops as a result of prolonged contact between parasite and host, is lacking. As a result a small focus of spirochetes, tucked away safely in the tissues of the central nervous system, and thus escaping the spirocheticidal action of the salvarsan, can develop in the susceptible host with great 
rapidity and severity. The severity of the meningitis is evidenced by the cerebrospinal fluid which shows a marked pleocytosis, considerable globulin excess, and usually a strongly positive. Wassermann reaction. The Wassermann reaction in the blood is frequently negative, showing how well the active syphilis is limited to the central nervous system. The failure to recognize this possibility of active meningitis in the early stages of syphilis with a negative Wassermann reaction in the blood, may result in the failure to resume treatment at a most critical period. It should be emphasized that the development of this condition is due to inadequate intensive treatment and that a few doses of salvarsan, without continued treatment, may result in more severe nervous manifestations than if milder therapy had been applied.

The recognition of the early involvement of the central nervous system is important not only because of the possibility of the development of an early meningitis, but also from the standpoint of prophylaxis of the latter and more destructive conditions. The best treatment of brain gummata, tabes and paresis will always be their prevention.

Before starting treatment in any case, it is well to try to recognize the character of the involvement, for the subsequent therapy is to a certain extent determined by the type of manifestation. These manifestations depend upon the tissues involved, and upon the reaction of those tissues to the irritative effect of the spirochetes. The disease may implicate the meninges, blood-vessels or parenchyma. While it is conceivable that any one of these structures may be affected alone, still the intimate relation of one structure to the other makes it almost impossible for one to be diseased without the others sharing to a certain extent in the pathological process. There is a type of nervous disease due to syphilis in which the infection seems to involve the vessels in the form of an endarteritis. In this condition the cerebrospinal fluid shows little if any abnormality, and the symptoms are referable to the arterial sclerosis of the cerebral vessels rather than to actual interstitial or parenchymatous disease. The response of this type of case to treatment depends upon the extent to which the endarteritis can be favorably affected, and not upon the resolution of a syphilitic exudate in the meninges.

Following the demonstration of the Treponema pallidum in paretic brains a new classification of lues of the brain and cord has arisen. This comprises (I) interstitial types, which include the lesions previously classified under cerebrospinal syphilis; and (2) parenchymatous types, which include paresis and tabes dorsalis.

In both the interstitial and parenchymatous forms, the one common lesion is the perivascularitis which may vary to a wide degree, from a simple mantling of the vessel, to a collection of cells in the form of miliary gumma, and when combined with a similar process in the meninges may result in large gumma formation. In the interstitial forms, which include meningitis, menigoarteritis and 
gumma, the disturbance in function is due either to an actual destruction of the nervous elements or to a cutting off of nutrition to the nerve tracts and centers. This latter condition may be brought about by the narrowing of vessels and compromising of the lymphatic spaces, to the edema surrounding active inflammation, and to pressure from the tumors. In fact, the remarkable improvement of many of these cases under treatment can be explained only on the basis that the pathological process has been largely limited to the vessels and meninges. In the parenchymatous forms the outstanding picture is degeneration of nervous elements, combined with symptoms of irritation of these structures. Unfortunately it is impossible to examine microscopically the tissues in these parenchymatous forms until late in the disease when the degeneration is far advanced. But even at this time perivascularitis and focal meningitis are usually seen, and it does not seem unreasonable to consider that much of the degeneration is secondary to a disturbance of the nutrition of the cell or axis cylinder, which has resulted from a blocking of the perivascular, perineural or perineuronal lymph spaces by the cellular exudate.

In paresis the presence of the spirochete in the brain substance may, by the liberation of toxins, result directly in the death of the cell. In tabes dorsalis, however, the active inflammatory process is not usually found in the most obviously degenerated areas, viz., the columns of Goll and Burdach, but rather in the dorsal roots and in the meninges surrounding them.

The evidence brought forward by Stargardt(I) and by Spiller(2) seems to prove that optic atrophy and oculomotor palsy in tabes and paralytica dementia is due to a round-cell infiltration of the cranial nerves. This infiltration is usually secondary to a low-grade leptomeningitis. Thus it appears that the classification of the various tabetic optic atrophies as primary is only an attempt to explain them in terms of the older opinion that these diseases were parasyphilitic in nature. As a matter of fact they all appear to be secondary to a chronic syphilitic meningitis.

Chronicity is probably an important element in the development of parenchymatous syphilis. The outstanding symptoms of most cases of tabes or paresis develop from the tenth to twelf th year, after the primary infection. Upon careful questioning of many of these patients, it will be found that some single symptom, such as pain or isolated nerve paralysis, often transitory in character, has occurred years previously, and their nature is often recognized only when the disease is well developed. These facts point to the probability that a low-grade inflammation with subsequent atrophy has been going on for years, and only after a sufficient number of tracts or centers are involved to interfere with the patient's usual activities does he seek the aid of the physician. On the other hand the majority of cases of the interstitial type develop in the first three years after infection. The course is more rapid and the symptoms 
appear with greater violence, no doubt due to the fact that there is a more extensive and rapid development of the meningeal and perivascular exudate.

In both tabes and cerebrospinal syphilis, then, we are dealing with diseases of a similar nature. The variation in the manifestations seems to depend upon the difference in the rapidity and degree of development as well as upon the localization of the inflammatory process. In tabes the infiltration being less intensive and slower, the picture of degeneration is brought more vividly into the foreground. The spinal fluid in the two diseases is practically the same, even to the type of curve in the gold reaction. Both also respond to specific treatment in much the same manner, although at a different rate, and while the symptoms due to irritation are improved, those due to degeneration are little if any affected.

In paresis, on the other hand, we appear to be dealing with a disease which is not strictly comparable with the two just discussed. There are certain points of similarity, viz., in the meningitis and perivascularitis. The direct action of large masses of spirochete in the substance of the cerebral cortex may possibly result directly in the destruction of cortical cells and fibers. The peculiar type of curve in the gold reaction, moreover, points to some essential difference between this disease and tabes. The difference in response to treatment is also most striking. While most cases of tabes will show the favorable influence of treatment both. in the spinal fluid and in marked and persistent clinical improvement, the response of most paretics to a similar, or more intensive treatment is much less noticeable, and the ultimate results are usually discouraging. Whether this difference in response is due to difference in accessibility of the active lesion, or to the fact that in paresis the disease attacks portions of the central nervous system, which are more essential to the orderly carrying on of vital functions, are questions which are as yet unanswered.

It goes without saying that the best preventive of syphilis of the central nervous system is the prophylaxis of syphilis, but this problem is far from solved. Next in importance is the proper treatment of syphilis in the early stages. It should be emphasized at this point that no case should be released from treatment until the cerebrospinal fluid has been shown to be normal, in so far as pleocytosis and Wassermann reaction are concerned. Whether a slight excess of globulin is an indication for continuation of treatment, if all other abnormal elements are absent, is still an open question.

While the incidence of cerebrospinal involvement in syphilis varies in different races and in the different sexes, the experiences of Mattauschek and Pilcz(3) are representative. These authors followed 4I 34 cases of syphilis in officers in the Austro-Hungarian army who were infected between 1880 and 1900 . These cases were followed until r9r2. The results of these investigations are shown in Table I. A striking feature of the table is the difference in the per cent. of 
cases that developed tabes and paresis who were untreated or poorly treated compared with those who had been energetically treated. Practically a quarter of the poorly treated cases developed paresis contrasted with a little over 3 per cent. of those who were well treated. Similarly from $\mathrm{I} I$ to $\mathrm{I} 2$ per cent. of the poorly treated ones developed tabes, while only a fifth of this proportion of well-treated patients developed this disease. A single course of treatment seemed to increase the liability to the cerebrospinal form of lues. This observation is of added interest in view of our present knowledge of the results of insufficient salvarsan treatment on the development of cerebrospinal syphilis already discussed in an early part of this paper. A similar parallelism between early efficient treatment and diminished incidence of tabes and paresis is seen in the last part of the table. In the period between 1880 and 1884 , the treatment was relatively insufficient, while in the period between 1895 and 1899 there was an improvement in the general treatment of the disease with a corresponding decrease in the incidence of parasyphilis. It is striking that the incidence of cerebrospinal syphilis in the two groups is the same.

If the difference between poor application of mercury and fairly efficient application is so marked, it is reasonable to expect that under the modern intensive treatment with both mercury and salvarsan, the incidence of parasyphyilis should be even more markedly reduced.

From the standpoint of prophylaxis of tabes or paresis it is also important to realize that they frequently develop slowly and that signs or symptoms are often present for years before the disease is fully manifest. Lately more attention has been directed to the monosymptomatic forms of syphilis of the central nervous system. Some authors claim that these forms are more frequent today than formerly and that tabes is assuming a milder character; but it is more probable that their detection is due to refinement in diagnosis.

Mattauschek and Pilcz.

TABLE I

Developed paralytica dementia............ I98 $=4.8$ per cent.

Developed tabes dorsalis................ II $3=2.7$ per cent.

Developed cerebrospinal lues...............

Total.................... $443=10.5$ per cent.

\begin{tabular}{|c|c|c|c|}
\hline & EFfect & TREatMent & \\
\hline & None & One course & Repeated energetic \\
\hline aresis.. & $\begin{aligned} & 100 \\
= & 25 \mathrm{per} \text { cent }\end{aligned}$ & $3 I=23$ I 34 per cent & $30=3.2$ per cent. \\
\hline oped tabes....... II & $=$ II per cent. & $16=$ II. 9 per cent. & $25=2.7$ per cent. \\
\hline ped cer sp. lue & 3 per cent & $21=15.6$ per cent. & $7 \mathrm{I}=7.6$ per cent. \\
\hline
\end{tabular}

\begin{tabular}{|c|c|c|}
\hline & $\begin{array}{l}\text { Poorly treated } \\
1880-84\end{array}$ & $\begin{array}{l}\text { Better treated } \\
\quad 1895-99\end{array}$ \\
\hline No. of cases & 617 & II 39 \\
\hline $\begin{array}{l}\text { Developed paresis } \ldots \ldots \ldots \ldots \ldots 60 \\
\text { Developed tabes............ }\end{array}$ & $\begin{array}{l}0=9.7 \text { per cent. } \\
2=3.5 \text { per cent. }\end{array}$ & $\begin{array}{l}37=3.2 \text { per cent. } \\
16=1.4 \text { per cent. }\end{array}$ \\
\hline veloped cer. sp. lues........ I 5 & $5=2.4$ per cent. & $28=2.4$ per cent. \\
\hline
\end{tabular}


While fairly numerous cases are reported in which the monosymptomatic forms remained free from further symptoms for many years in spite of lack of treatment, it is wiser to regard all such cases as being early forms of late serious disease. Years ago Erb called attention to the importance of ophthalmoplegia interna as a forerunner of tabes or paresis. Many patients with tabes give a history of transitory squint which was present years before any other symptom. Often the condition has improved after the administration of a small amount of iodids or mercury and the patient has been allowed to escape from further observation. Auditory nerve lesions are also described early in tabes as well as in cerebrospinal syphilis. These patients usually consult the otologist. It is important for the specialist to realize that these isolated nerve lesions are a part of the general disease, syphilis of the central nervous sytem, and that they are usually manifestations of a basilar meningitis. Surgeons also see early manifestations in abdominal disturbances. Nuzum(4) recently reported that among Iooo cases of tabes admitted to Cook County Hospital, eighty-seven had previously undergone laparotomy because of the mistaken diagnosis of their abdominal pain. It is important to remember that gastric crises, either mild or severe, may be present as the first manifestation of tabes for years before full development of the disease. Other single symptoms which are often present for a longer or shorter period are bladder disturbances, lightning pains in the leg and "rheumatism." The general practitioner or the specialist should recognize the nature of the symptoms and place the patient under proper treatment, for it cannot be emphasized too strongly that prevention of degeneration is worth more than the treatment of symptoms once they have fully developed.

The examination of the blood and cerebrospinal fluid is of the greatest help in clearing up the diagnosis in doubtful cases, and to a certain extent in determining the type of syphilitic involvement of the central nervous system. While these examinations are of great value, they do not replace but only complement a careful clinical examination. The alterations in the cerebrospinal fluid which have practical bearing in cerebrospinal lues are indicated by: (r) pleocytosis; (2) increase in globulin and albumin; (3) Wassermann reaction; (4) type of curve obtained with the Lange gold reaction. It is also well to note the pressure of the fluid which is often increased in acute syphilitic meningitis or when large gummatous tumors exist. We have not found the estimation of the sugar content by means of Fehling's solution to give information of importance.

Pleocytosis.-The degree of pleocytosis is a fairly accurate guide to the intensity of an existing meningitis. The highest counts occur in secondary syphilitic meningitis. On the other hand, in pure endarteritis syphilitica the number of cells is usually normal and in syphilitic spinal spastic paralysis where the lateral tracts are supposed to degenerate secondarily to syphilitic disease of the blood-vessels supplying them the counts are usually between 5 and 30 . In 
tertiary cerebrospinal lues the count varies between normal and several hundred according to the amount of meningitis. Likewise, in tabes pleocytosis depends upon the character of the case. If irritative symptoms are prominent the counts are often high, if the disease has been of long duration and the degenerative picture most prominent, or if there has been an arrest in the process the counts are usually low. In paresis there is usually a moderate increase in the number of cells, although counts of several hundred per cubic millimeter are occasionally found. A number of authors claim that the percentage of plasma cells is much higher in paretic fluids than in other conditions. Under treatment the cell count shows a more rapid return toward the normal than is seen with any other of the abnormal elements. This is true in all stages of the disease, and following practically any efficient form of treatment.

Globulin.-The increase in soluble protein is usually measured by an excess of globulin. This is probably an evidence of vascular disturbance in the central nervous system, and indicates an abnormal transudation from the vessels. It occurs in a great variety of conditions, such as tumors, in arteriosclerosis, and in acute or chronic inflammatory processes involving any portion of the cerebrospinal tract. In the majority of cases in any stage in which there is actual syphilis of the brain, cord or meninges there is some globulin excess. In the slight involvement in the early secondary period this may be the only abnormal element; the same is often true in endarteritis syphilitica. In secondary syphilitic meningitis the increase is often marked, but in paresis a marked increase is most often found. In tertiary cerebrospinal lues and tabes there is a wide variation in the degree of increase. In tabes of long standing where the disease has been quiescent for years there may be no increase.

Wassermann Reaction.-While pleocytosis and globulin excess are only signs of inflammation in some part of the cerebrospinal axis a positive Wassermann reaction in the fluid indicates the presence of active syphilis in some part of the cerebrospinal axis. The point should be emphasized, viz., that without injury to the central nervous system a positive Wassermann reaction is not found in the fluid. The converse, however, is not true, and there may be definite pathological processes due to syphilis without the presence of a positive reaction in the fluid.

Of extreme importance is the titration of the strength of the Wassermann reaction in the spinal fluid. The designation of 4 plus or negative Wassermann reaction in the fluids means but little unless we know the amount of fluid that was used in obtaining the readings. This is specially true where the effect of treatment is being estimated. For example if 0.2 c.c. or 0.4 c.c. has been the only amount used to test the fluid a relatively small amount of treatment may change the reaction to negative with the same amount. On the other hand, if only I c.c. has been used a large amount of treatment may not affect the reading, although as a matter of fact the reaction may have been 
much weaker at the end of the treatment. The only way to judge accurately of the effect of treatment in the reaction is to test the fluid with diminishing amounts of fluid between, corresponding to amounts between 2 c.c. and 0.2 c.c. in the original Wassermann test (smaller amounts if necessary), and determine the point at which the reaction is completely positive. If then with a given amount of treatment the positive point has moved from 0.2 c.c. to 0.6 c.c. or higher, we know that the treatment is having some effect. This of course presupposes that constant methods are used in testing the fluids at different times, for if a very sensitive method is used at one time, and a comparatively unsensitive method at another, the relative value of the two tests is lost. At present we feel that all fluids should be tested with quantities up to 2 c.c., for occasionally reactions are negative with I c.c. quantities but positive with double this amount.

In the early secondary period when a slight increase in cells and globulin is often found, the Wassermann reaction is usually negative. A positive reaction in the fluid in this stage indicates a severe infection of the central nervous system, and in patients with positive findings in this stage there are usually symptoms and clinical signs of a severe meningitis. Positive reactions in this stage usually disappear rapidly under vigorous general antisyphilitic treatment. In tertiary cerebrospinal syphilis we have found positive reactions in about 95 per cent. of fluids where I c.c. was used, but in only 33 per cent. with 0.2 c.c. quantities. Our findings in untreated tabetics corresponded closely to the percentages in cerebrospinal lues. Only rarely is the fluid negative in tabes, and in most instances in which a negative reaction is found the disease is quiescent. In paresis the fluid is positive in nearly every case and in the majority it shows a strong reaction with small quantities, i.e., o.2 c.c. or less. As will be pointed out later the initial strength of the reaction in tabes is of some value in determining the amount of treatment which will be required.

Lange Gold Reaction.-This test requires for its performance a proper solution of colloidal gold which is red in color. The spinal fluid is diluted with 0.4 per cent. NaCL as follows: I-IO, I-20, $I-40, I-80, I-I 60, I-320, I-640, I-I 280, I-2560, I-5120$. Then the colloidal gold solution is added, well shaken and allowed to stand over night after which the readings are made. With abnormal fluids the red color in certain of the tubes is changed. These changes are designated by numbers, i.e., I red blue, 2 lilac or purple, 3 blue, 4 pale blue, 5 colorless. Thus the reading 5555421000 indicates that the first four dilutions were completely decolorized, the fifth dilution blue, the sixth red blue and the next three unaffected. This is the typical paretic curve in which the first four to six or more tabes. show discolorization. In the luetic type of curve there is not a complete decolorization of the first dilution and the maximum change occurs in the second to the fourth or fifth tabes, i.e., 3334310000 , or I 221000000 . 
The luetic type of curve given by the fluid in cerebrospinal lues and tabes is of confirmatory value. The test's great usefulness, however, seems to be in differentiating paralytica dementia from these two conditions. The fact that the fluid from a patient with a clinical diagnosis of tabes, or cerebrospinal syphilis gives a paretic curve does not militate against it, for it is well known that between Io and 15 per cent. of tabetics develop paresis and at times it is with the greatest difficulty that the differential diagnosis between cerebral syphilis and paresis can be made. If future observations confirm what now seems most probable, viz., that these paretic curve findings in the spinal fluids of patients clinically not paretic point to a possible development of the disease, it will be of importance in prognosis and of value in determining why symptoms of mental disorder appear in spite of vigorous and prolonged treatment.

The problem of treatment of syphilis of the central nervous system is complicated by peculiarities of the subarachnoid space which in addition to the larger spaces and cisterna, is directly continuous with the perivascular, perineural and perineuronal lymph spaces. It does not seem reasonable to suppose that the infection is carried by the cerebrospinal fluid along these channels, and the various meningeal and perivascular lesions can be most easily explained in this way.

The reaction to the spirochete is meningitis and perivascularitis. It is probable that the nerve cells and fibers obtain their nutrition to a considerable degree through these spaces, hence plugging of them with exudate not only compromises the nutrition of these structures, but also probably increases the difficulties with which therapeutic agents are brought in to contact with the exudate. At presentitseems almost superfluous to recite the numerous experiments which prove the separateness of the subarachnoid space from the general lymphatic and blood vascular systems. Antibacterial immune bodies, vital stains and most drugs, when administered by mouth or parenterally, do not reach the subarachnoid space in demonstrable quantities. It is true, however, that many drugs, given either by mouth or subcutaneously, do act on the central nervous system, but these drugs administered subdurally act in much smaller quantities.

In many cases, the general administration of mercury, iodids and salvarsan affects favorably the course of syphilis of the cerebrospinal axis. This favorable influence is especially marked in the early forms of the disease. The tendency, however, of cerebrospinal syphilis to relapse in spite of energetic mercurial and iodid treatment has long been noted by syphilographers and neurologists, and the failure of most cases of tabes to respond to the antiluetic treatment of the presalvarsan era made therapeutic nihilists of most neurologists. Even the brilliant clinical improvement of most cases of cerebrospinal syphilis and tabes after salvarsan intravenously, has not always been followed by a parallel improvement in the cerebrospinal fluid. Dreyfus(5) who gives courses of salvarsan of 4 to $6 \mathrm{gm}$. combined with to to 
I 2 injections of calomel or gray oil, notes that many such courses are necessary to eliminate all signs of the disease. In only three out of I 25 cases of cerebrospinal syphilis did the fluid become normal after one or two courses. Gennerich(6), who is famous for the intensity of his antisyphilitic treatment says that he feels that all cases with meningeal involvement in any stage should have intraspinal treatment. Many observers report cases of cerebrospinal syphilis or tabes which having come to a standstill both clinically and pathologically under general treatment. have shown renewed improvement under intraspinal therapy.

The desirability of subarachnoid therapy has been well established. The problem was to find some beneficial therapeutic substance which could be repeatedly introduced without injury to the delicate nervous tissues, for only by repeated and prolonged treatment can we hope to arrest any form of cerebrospinal lues permanently. Last year, the writer $(7)$ reviewed the use of these various agents and the methods of their administration. Suffice it to say that the preparations which have stood the test of time are: (I) the serum obtained from patients shortly after intravenous injections of salvarsan; (2) serum to which small quantities of salvarsan have been added; (3) neosalvarsan in small quantities and weak concentration; and (4) mercuralized serum.

The direct application of mercury in the form of albuminate as devised by Byrnes (8) should theoretically be of value, and the reports seem to indicate that if the amount of mercury is kept under the irritating dose beneficial results may be expected from its injection. It is well to call attention to the danger of repeated injections of mercurialized horse serum, which may lead not only to a general anaphylactic state to horse serum, but may cause the meninges to become hypersensitive to the foreign protein and repeated injections over a number of months may lead to a chronic meningitis similar to the condition we are trying to combat.

The injection of neosalvarsan in concentrated solutions as recommended by Ravaut or even in one pro mille solutions has been proven by numerous observers to be a dangerous procedure, since it is apt to be followed by various degrees of urinary retention and incontinence, rectal paralysis, paresthesia of the legs, ataxia and girdle sensations and, in severe cases, by paralysis of the lower extremities and death. A given dose may be well tolerated several times, but later be followed by severe symptoms. Because of the uncertainty of unfavorable sequelæ, intraspinal injections of neosalvarsan should be given with extreme caution. Gennerich lately reported that by using very dilute solutions he has been able to avoid these unpleasant symptoms. $\mathrm{He}$, however, warns against the use of more than 0.5 $\mathrm{mg}$. in spinal cord disease, and not over I or $2 \mathrm{mg}$. in patients with paresis or syphilitic meningitis. Personally, I feel that repeated doses of this size are dangerous.

The additions of small quantities of salvarsan to serum as recom- 
mended by Ogilvie was devised to give a known amount of salvarsan instead of the uncertain amounts in the serum salvarsanized in vivo. Marinesco and Minea have noted that neosalvarsan is less irritating when diluted with serum than when dissolved in water or saline. Both Ogilivie and Fordyce, however, have noted bladder disturbance and paresthesia in the legs when more than I mg. of salvarsan was added to the serum and now recommend that the dose should never be over $0.5 \mathrm{mg}$., usually less. With these quantities intraspinal treatment has been followed by distinctly beneficial results.

The use of serum of patients withdrawn after intravenous salvarsan injections has been criticized chiefly because of the small amounts of salvarsan it contained. We have found that the majority of the sera one hour after an intravenous injection contained 0.01 mg. or more per cubic centimeter. When $I 2$ to 20 c.c. of serum are used each treatment represents the injection of from 0.12 to 0.2 mg. of salvarsan. As above noted, it is unsafe to inject intraspinally more than a fraction of a milligram of either salvarsan or neosalvarsan. The uncertainty of the dosage is an objection which cannot be overcome unless definite quantities of salvarsan are added.

The "autosalvarsanized serum" is, however, definitely spirocheticidal and the salvarsan is present in a colloidal combination with the serum which cannot be dialyzed through a celloidin membrane, as can salvarsan alone. Salvarsan in this colloidal state is probably more slowly diffused out of the cerebrospinal fluid, hence is longer in contact with the syphilitic exudates. Moreover, it seems to us that some of the beneficial effect from serum injection may be due to the introduction of the serum per se, for we have been able to bring the cell count to normal and cause the positive Wassermann reaction to disappear from the cerebrospinal fluids of a number of patients with tabes, by the injection of non-salvarsanized serum.

The explanation of these effects are theoretical, but the experimental facts stand, and it is safe to say that the injection of autosalvarsanized serum is followed by fewer permanent, unpleasant effects than result from the intraspinal injection of any other antisyphilitic remedy.

In some patients the pain which follows the injection is so severe that the treatments must be discontinued, but such instances are the exception. The willingness of most patients to return for treatments over long periods is sufficient proof that the method is not too unpleasant for practical application.

In order to save time both the intravenous and intraspinal treatment may be given the same day, and the patient can ordinarily return to his home the following noon. If it is necessary to keep the patient in bed a longer time, it is well to give the treatments on Saturday so the patient may return to his work Monday morning with the loss of only a single working day.

It has seemed to us that the treatments are better borne if not repeated oftener than once in two weeks. This is especially true 
in tabetics or patients with spinal syphilis. In paretics or patients with cerebral syphilis the intervals may be shorter. At times it seems wiser to lengthen the intervals. It is well to bear in mind that a certain amount of irritation always follows the introduction of any foreign substance into the subarachnoid space, and the effect of this should be allowed to disappear before the treatment is repeated. A good rule to follow in any form of treatment of cerebrospinal syphilis is not to push the treatment so hard that the patient's general health is depressed. With each treatment there should be some improvement in the general condition, at least there should be no regression, for if a depressing treatment is repeatedly added to a more and more depressed condition, the ultimate effect will be injurious rather than beneficial.

The treatment of the several forms of syphilis of the central nervous system presents different problems according to the stage of the disease and the individual patient. One general principle which should always be considered is that in any patient who shows evidence of involvement of the cerebral meninges or brain, salvarsan treatment should be preceded by a short course of mercury to prevent the possible occurrence of a Herxheimer reaction in the region of vital nervous centers. Fortunately such distressing reactions are rare, and can be obviated by proper preliminary treatment. If the presence of gummata is suspected, a vigorous course of potassium iodid is often followed by marked improvement. It has seemed to us that the response of cases in the tertiary stage to salvarsan has been more marked and lasting, if the courses of salvarsan have followed treatment with mercury and iodids. Gummatous exudates resolve under this preliminary treatment and the spirocheticidal effect of the salvarsan is more readily brought into play.

In the meningitis of the secondary period the response to alternate courses of salvarsan and mercury has been prompt and permanent in all of our cases. Gennerich is of the opinion that all these cases respond more rapidly to combined intravenous and intraspinal therapy and advises giving two intraspinal treatments after the cerebrospinal fluid has become normal. Following any form of treatment in this condition the cerebrospinal fluid should be examined in two or three months after treatment has been stopped, and every six months for two or more years thereafter to forestall relapses.

In the tertiary forms of the disease, the so-called interstitial forms, alternate course of mercury and iodid, and of salvarsan are usually followed by decided improvement, both clinical and in the condition of the cerebrospinal fluid. If possible, the fluid should be examined after each course or each pair of courses. Not infrequently it will be found that the fluid has become normal after a few months' treatment. If so, the prognosis is good and treatment should be conducted along general lines. On the other hand, if the strength of the Wassermann reaction is the same or only slightly altered after four or six months of intensive general treatment, it may be well to resort to intraspinal therapy. 
In tabes dorsalis, because of the apparent sensitiveness of many cases to mercury, it is better to start the treatment with small intravenous doses of salvarsan, gradually increasing and giving a treatment every week for a course of six or eight injections. If at the end of this course the Wassermann reaction in the fluid is considerably weaker, a course of mercury may be tried, followed by another course of salvarsan, alternate courses being given with periods of rest until the fluid is brought to normal. Many cases will be found, however, in which the strength of the reaction is only slightly altered by such a plan of treatment. The substitution of combined intraspinal and intravenous therapy in these patients will usually result in an improvement in the condition of the fluid. I am convinced that our aim should be to bring the fluids to normal and keep them there, if we intend to speak of the cure of syphilis. This can be done in a majority of cases of tabes if the treatment is carried out in a systematic way. In our patients in whom the fluid has become normal and remained so, there have been very few relapses, and no progression of the tabetic symptoms, while in those in whom the fluid has not become normal, the tendency to relapse has been more marked.

In the presence of a rapidly advancing case of tabes or of optic atrophy where it is desirable to arrest the progress of the disease, in an important organ, the institution of combined intravenous and intraspinal therapy at the beginning should be seriously considered, as rapidity of cure is the end to be accomplished.

Compared with the results in other forms of lues of the central nervous system, the treatment of paralytic dementia has been disappointing. With the proof of the active syphilitic nature of this disease, it was thought that a cure might be expected from intensive treatment, but there is something in the nature of the disease which apparently prevents the remedies from reaching the spirochete in sufficient quantities to eradicate them completely. It is true that most of the cases improve clinically and in some of them there is a marked improvement in the condition of the spinal fluid. Still, with corresponding amounts of treatment this response is much less marked than in tabes. In addition, there is a decided tendency for pleocytosis and Wassermann reaction to increase in intensity when treatment is discontinued. In two of our patients there was at first almost as marked clinical improvement and diminution in abnormal elements in the cerebrospinal fluids as is seen in tabes. Both have had two relapses and with each relapse the response to treatment has been less marked. This indicates that as the paretic process grows older it is less amenable to treatment and probably explains the difference in results from state hospitals for the care of the insane and those from private physicians. In state institutions the cases are usually much more advanced than those which come under the observation of private institutions or practitioners. Thus Runge reports 37.4 per cent. remissions, Raecke 55.4 per cent. improved, on salvarsan intravenously, Cotton with combined intravenous and 
intraspinal therapy $5^{8}$ per cent. arrested or much improved, and Ogilvie 34 per cent. with complete remissions, and 40 per cent. incomplete remissions, a total of 74 per cent. improved. In four of Ogilvie's cases both the blood and spinal fluids were brought to normal. When we compare these results with the number of remissions in the presalvarsan era, 5 and 8 per cent., the treatment of paretics seems justified. The return of a paretic to his business, even though it be for only six months or a year is of ten of vital importance to his associates and family. Compared with our therapeutic efforts in chronic cardiac or renal disease, the results obtained thus far in paresis are decidedly encouraging, although in none of these diseases can we speak of a cure.

In the treatment of syphilis of the central nervous system we should never forget the patient, and that he may be suffering from disease of other vital organs. If there is marked ataxia, Fraenkel's reëducation movements are useful; if he is emaciated, attention should be directed toward diet and general nutrition. It is unwise to keep a patient with tabes in bed for long periods for the ataxia is often markedly increased after prolonged quiet. Most patients with any form of cerebral syphilis show a certain amount of mental abnormality. Here psychotherapy is of value. In other words, treatment must be individualized and while a general plan should be followed, it is well to remember that different response is to be expected at different stages of the disease, and also that each case may demand a certain deviation from the regular course to meet his peculiar condition.

Technique.-The successful injection of serum demands two main conditions: first, rigid attention to bacteriological asepsis; second, the ability to do easily a successful lumbar puncture. The technique may be placed under four headings: first, obtaining of blood; second, preparation of serum; third, lumbar puncture and injection of serum; fourth, examination of the spinal fluid.

The Obtaining of Blood.-From one-half to one hour after an intravenous injection of salvarsan, about 50 c.c. of blood is obtained by venous puncture. This is most easily done by using a MacRae venous puncture needle, which is inserted in a rubber stopper which fits closely into a 50 c.c. bottle-shaped centrifuge tube. The MacRae needle is a double needle provided with a shoulder which fits firmly against the upper surface of the rubber stopper. Immediately above this shoulder the shorter arm of the needle turns abruptly outward and ends in a round bulbous enlargement, over which a piece of rubber tubing of fine bore can be passed. A sterile glass mouthpiece plugged with two cotton pledgets is inserted in the other end of the rubber tubing. By creating suction with the mouth through this tubing, a partial vacuum is formed in the tube and the flow of blood hastened. A supply of centrifuge tubes and glass mouthpieces, both plugged with cotton, can be sterilized by dry heat and kept on hand for use as required. The MacRae needle with its rubber stopper and piece 
of rubber tubing may be sterilized by boiling immediately before use and the rubber stopper carefully dried in a flame before inserting it in a centrifuge tube. A more convenient way is to keep the MacRae needle, rubber tubing sterilized and on hand. In using this form of apparatus the patient should be sitting up, in order that the blood does not touch the neck of the tube.

Preparation of the Serum.-After the blood has been obtained, it is allowed to clot, and then centrifugalized at high speed for about thirty minutes. The clear supernatant serum so obtained is then pipetted off into another sterile test-tube. If it is desired to use dilute serum, normal sodium chlorid solution is now added. The usual dose of diluted serum is 30 to 40 c.c. of a 40 or 50 per cent. serum. If the serum is injected pure, as is now recommended, no further preparation is necessary, except that it is heated for one-half hour at $56^{\circ} \mathrm{C}$. The diluted serum is also heated. It is important that the serum after centrifugalization should be perfectly clear and no red cells shoula be injected, as a small quantity of red blood cells in the serum causes the reaction following injection to be much more severe.

Ogilvie Method.-Serum is obtained from either an untreated or treated patient in the same manner as above described. Ten c.c. is pipetted into a sterile tube and to it the requisite amount of a weakly alkaline salvarsan solution is added and well mixed. This serum-salvarsan mixture is then heated to $56^{\circ} \mathrm{C}$. for one-half hour, when it is ready for injection.

Mercurialized Serum (Byrnes Method).-To I 2 c.c. of serum is added I c.c. of a solution of mercuric chlorid in freshly distilled water, so made that each cubic centimeter contains $0.0013 \mathrm{gm}$. (1/50 gr.) of mercuric chlorid. To the serum thus prepared is added sufficient quantity of normal salt solution to make a total volume of 30 c.c. If concentrated solution is used, this step is omitted. It is then heated at $56^{\circ}$ for half an hour when it is ready for injection.

Lumbar Puncture and Injection of Serum.-Lumbar puncture is performed with the patient lying on his right side in bed. The most essential procedure in the technique is the preliminary arrangement of the patient, who should be on the extreme edge of the bed, lying on his right side, and an attempt should be made to have the lower back as nearly straight in both planes as possible. The knees should be drawn up so that the thighs are at right angles to the trunk. Care should be taken to have the patient's shoulders straight, that is, both shoulders should be at right angles to the surface of the bed. This prevents a sagging of the body and consequent delignment of the spine in the lumbar region. In stout women a small pillow placed in the curve between the crest of the ilium and the costal margin is often of service. Attention to details in this stage of the procedure increases greatly the ease of lumbar puncture.

The intervertebral space at the level of the iliac crest is the one usually selected for puncture, but the space immediately above or 
below below this may be used as desired. After sterilization of the surrounding parts the skin and underlying tissue in the area selected are anesthetized with a little 2 per cent. novocain. This makes a great difference in comfort to the patient, and is most essential where repeated punctures are to be carried out. The lumbar puncture needle is then inserted in the midline toward the lower border of the intervertebral space selected. The needle should be kept at right angles to the body in both planes when, if the patient has been properly placed, it should pass into the canal without touching bone in its course. If the space below the iliac crest is selected a slight cephalic direction of the needle must be used. The needle should be inserted until its point is felt just to touch the anterior wall of the vertebral canal. It is to be remembered that nearly all unsuccessful attempts with such a technique are due to a misdirection of the point of the needle downward, that is to the right of the canal. If, therefore, fluid is not obtained, the needle should be partially withdrawn and its butt strongly depressed; this raises the point of the needle, which then, on reinsertion, frequently reaches its desired destination. As soon as fluid is obtained, if it is desired to estimate the pressure, the manometer is attached to the lumbar puncture needle. After the pressure has been ascertained, the manometer is detached and the spinal fluid allowed to flow into several test-tubes. If pure serum is injected, from to to $\mathbf{2} 2$ c.c. of fluid are removed. If 30 to 40 c.c. of diluted serum are to be injected practically the same amount of cerebrospinal fluid is removed. The serum is then injected by gravity. A simple apparatus for this injection consists of the barrel of a 20 c.c. Luer syringe, to which is attached a piece of rubber tubing about $40 \mathrm{~cm}$. in length. At the other end of the rubber tubing is a small metal applicator which fits accurately into the lumbar puncture needle. The empty syringe is attached to the needle and depressed so that the tubing fills with cerebrospinal fluid, thus dispelling all air. The mouth of the test-tube containing the serum which has been kept at a temperature of $100^{\circ} \mathrm{F}$. should be thoroughly flamed and the serum poured into the barrel of the syringe, the top of which is covered with a sterile sponge. On elevating the syringe the serum will flow into the subarachnoid space.

- Examination of the Fluid.-The routine measures usually employed are estimation of pressure, determination of clearness or turbidity of fluid, cell count, globulin reaction, Wassermann reaction, gold curve.

Cell Count.-The preparations should be made as soon as the fluid is removed, as the cells settle rapidly and adhere to the sides and bottom of the tube if the fluid is left standing; they may be counted at leisure as long as sufficient time does not elapse for evaporation to occur. The counts are made with the pipette ordinarily used for leukocyte counts and the Thoma-Zeiss counting chamber, Neuberg ruling. Two pipettes and two chambers are used in each estimation. For diluting fluid the following formula is best: methyl violet o.I 
gm., glacial acetic acid 2 c.c., aq. dist. 50 c.c. This is drawn to the "I" mark on the pipette, which is then filled to the "I I" mark with spinal fluid. This gives a dilution of spinal fluid of nine in ten within the chamber. The fluid in the capillary portion of the tube is then blown out, and a drop of fluid from the chamber run on to the counting stage. The cover-slip is then placed on the slide and, while firm pressure is being applied, pushed across the stage. With this method Newton rings are easily obtained on all four sides, thus assuring the constant depth of the preparation. The cells on the whole ruled surface are then counted. This ruled surface in the ThomaZeiss Neuberg-ruling chamber has an area of $9 \mathrm{sq} . \mathrm{mm}$. As the chamber is only $0.1 \mathrm{~mm}$. in depth, the total count gives the number of cells in $0.9 \mathrm{cu} . \mathrm{mm}$. of the diluted fluid. The figure so obtained must, therefore, be multiplied by $1 \%$ to give the number of cells per cubic millimeter, and a further multiplication by $1 \% \frac{1}{\text { is required to }}$ correct the dilution; or, combining the two calculations, we can multiply by $10 \% / 81$, that is, approximately $1 \% / 8$. The result gives the number of cells in I cu. mm. of undiluted fluid. The two chambers should correspond closely. Dreyfus has suggested the following standard in considering the results of cell estimations:

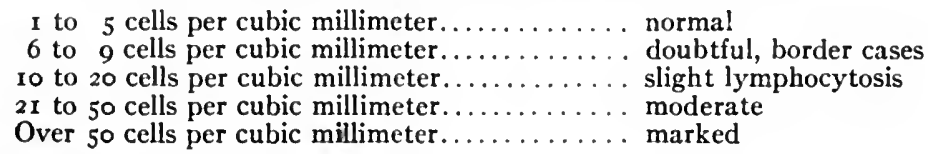

Globulin.- Numerous methods are in use for the determination of abnormal amounts of globulin in the spinal fluid. A simple and satisfactory method is that of Noguchi. The reagents used in the Noguchi reaction are butyric acid (Merck) diluted to ro per cent. in 0.85 per cent. sodium chlorid and a 4 per cent. solution of sodium hydrate. To 0.2 c.c. of spinal fluid 0.5 c.c. of butyric acid is added, and the mixture brought to the boiling point over a small flame; o.I c.c. of 4 per cent. sodium hydrate is then added, and the whole again heated to boiling. If carefully observed, the reaction can be delicately shaded and relatively quantitative readings made. The tubes should be watched carefully for the first ten minutes, and read in half an hour, and again in two hours. When possible, several fluids should be done at the same time to obtain a comparison. In reading the Noguchi reaction we have adopted the following arbitrary standard:

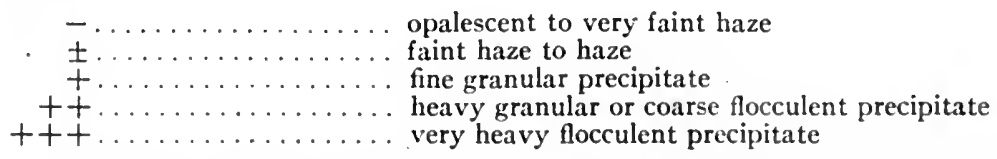

The Nonne-Apelt ammonium sulphate method is even simpler than that of Noguchi, but is less delicate and, therefore, less satisfactory. This reaction is performed by mixing equal parts of a saturated solution of ammonium sulphate and cerebrospinal nuid;

Vol. IV. -57 
I cc. of each is quite sufficient. It is advisable to layer the fluid on the sulphate solution; if the globulins are increased, there occurs a more or less distinct ring at the plane of contact. After this preliminary observation the mixture is well shaken and the result may be read within three minutes. If it is distinctly opalescent or cloudy it is designated as a positive "Phase I" reaction.

Pandy's Test.-This is the simplest of all tests for increased protein content. The reagent consists of a saturated aqueous solution of carbolic acid. To 0.5 or I c.c. of the reagent is added I drop of the spinal fluid. The immediate formation of a bluish white ring or cloud is the evidence of an increased protein content.

Wassermann Reaction.- This test must be carried out in a wellequipped laboratory by a competent worker. It is well for the clinician to select one laboratory on which he can rely and to insist that the results be comparable from time to time.

Lange Gold Reaction.-This test must also be carried out in a wellequipped laboratory. One c.c. of fluid for this test should be collected in a separate clean tube. It is very essential that no blood or serum be in the fluid to be tested with this method(Io).

After-treatment of patients following spinal injection consists of rest in bed for at least twenty-four hours. The pains of back and legs which frequently follow the injection, are best controlled by the use of phenacetin, aspirin, and codein. Only exceptionally should morphin be used.

It should be urged that the treatment of the late chronic forms of cerebrospinal syphilis is a prolonged one, requiring at least three, months and often one or two years to reverse the abnormal elements in the cerebrospinal fluid to normal. The slow disappearance of these abnormal elements(II), therefore, should not be a cause for discouragement, for it is important for both the physician and patient to realize that the disease has been years in developing, hence treatment should not be expected to be effective in a short time. The prolonged treatment, however, is worth while if we can affect the arrest of a chronic disease which is progressive in character and in the vast majority of cases leads to incapacity or death.

\section{BIBLIOGRAPHY}

1. Stargardt, Allg. Ztschr. f. Psych., t9iz, lxix, 735.

2. SpILler, Jour. Nerv, and Mental Dis., I9I5, xlii, I5.

3. MattauscheK and Pilcz, Ztschr. f. ges. Neur. u. Psych., Bd. 8, S. 133; ibid., I9I3, Bd. I5, I908.

4. Nuzum, Jour. Am. Med. Assn., I9I6, 1xvi, 482.

5. DREYFus, Münch. med. Wchnschr., I914, lxi, 525 .

6. GenNerich, Münch. med. Wchnschr., I915, lxii, I696.

7. Swift, Jour. Am. Med. Assn., I9I 5, lxv, 209.

8. Byrnes, Jour. Am. Med. Assn., I914, lxiii, 2182.

9. Ogilvie, Jour. Am. Med. Assn., I914, lxiii, I 936.

10. For a full discussion of Lange's gold reaction, see Milier, Brush, Hammers and FeLtoN, Johns Hopkins Hos. Bull., r 1915, xxvi, 39r.

II. For typical responses to intraspinal and intravenous therapy, see case reports by SWIFT and Elis, Arch. Int. Med., I913, xii, 33 I. 


\title{
THE SURGICAL TREATMENT OF DISEASES OF THE SPINAL CORD
}

\author{
By Charles A. Elsberg, M. D.
}

The operator who does much spinal surgery must be a good neurologist. He must have a thorough knowledge of the gross and minute anatomy of the spinal cord and a sympathetic understanding for the functions of the fiber tracts. He must be prepared not only to expose and to treat disease on the outside of the spinal cord, but also to invade the tissue of this organ in order to remove tumors from its substance, to drain collections of fluid or blood or to divide certain of its fiber tracts. It has become evident, therefore, that special training and experience are necessary for successful work in this field, and that for the special worker, a spinal operation is seldom a difficult or a dangerous one.

The Exposure of the Spinal Cord by Laminectomy and the Recognition of Lesions in and Around the Cord.-Technically, there is little difference of opinion as to the manner in which a laminectomy should be done. The osteoplastic methods described by Marion(I), Cavicchia(2), Bickham(3) have been well-nigh forgotten for they are too time-consuming and too complicated and are often followed by wound complications. Complete removal of the spines and laminæ is preferable to the so-called unilateral operation. The best technique for a complete laminectomy is briefly the following: An incision is made over the spines that are to be removed and is rapidly deepened. The fascia and muscles of one and then of the other side are cut off close to the spines and laminæ and are held back by an automatic retractor. Bleeding is seldom excessive and is well controlled by the retractor. The spines are bitten off at their bases with large rongeur forceps and the laminæ removed with small rongeurs. In the cervical and dorsal regions, the removal of part of the laminæ will widely expose the spinal canal; in the lower dorsal and lumbar regions, the laminæ must be removed up to or into the articular processes.

On account of the relative small size of the vertebræ, laminectomy is easiest in the cervical and upper dorsal regions. For most operations two, three or more vertebral arches have to be removed. Most operators, Horsley(4), Krause(5), von Eiselsberg(6), Frazier(7), Cushing(8), Kuettner(9), Kocher(ro), and Elsberg(II), perform complete laminectomy, but A. S. Taylor(I2) still prefers hemilaminectomy. In the majority of instances the normal mobility of the vertebral column is completely regained after the operation.

In the lower dorsal and lumbar regions, the ligamenta subflava are very thick and they must of ten be separately cut off before the 
dura is exposed. The outer surface of the dura is covered by a layer of fat which must frequently be stripped off before the dura is exposed. The incision of the dura is easily accomplished but the surgeon must be careful not to injure the cord underneath. A small incision is first made between traction sutures and the opening is then enlarged with a grooved director, the dura tearing easily in a longitudinal direction.

Excepting in the special cases that will be mentioned, the dura should always be closed by suture and the muscles, fascia and skin edges united without drainage.

The recognition of pathological conditions in and around the cord requires considerable experience. Normally, the outer surface of the dura is smooth and glistening. A discoloration or redness or granulation tissue on its outer surface signifies that there is an inflammatory process of the surrounding bone or of the dura itself. The arachnoid is a thin transparent membrane, in contact with but not adherent to the inner surface of the dura. Calcareous placques are often met with on its outer surface, but they have no known pathological significance.

The cord is normally of a creamy white color and the vessels on it are fairly prominent. Marked pallor of the cord is met with in old age and in atrophic cords below the level of an old transverse lesion. The cord is also of a dead white color in advanced sclerosis. In meningitis or meningomyelitis, the cord is often pink in color due to a marked hyperemia of the pial blood-vessels. This appearance must be differentiated from the venous congestion which is often observed below the level of a spinal compression. In the latter condition, the return flow in the posterior spinal veins is obstructed and the vessels stand out as abnormally large blue strands on the normal creamy white cord.

If there is no escape of cerebrospinal fluid when the arachnoid is divided, the surgeon can be certain that there is some obstruction above the level at which he is operating and he must explore the spinal canal upward with a probe bent at the appropriate angle. An obstruction to the passage of the probe may be due to adhesions between the membranes, or the end of the probe may be in contact with a nerve root. The obstruction due to an extramedullary tumor causes an elastic resistance which should be recognized by the surgeon. Frequently, slight pressure with the probe is followed by the sudden escape of cerebrospinal fluid.

The spinal cord has normally a firm consistency; it should be palpated as little as possible. Sometimes it feels abnormally soft (injuries, hydromyelia, etc.) and one can often recognize that it contains a collection of fluid or that there is a tumor within its substance.

Fractures of the Spine with Injury to the Spinal Cord and Nerve Roots. - The importance of a spinal fracture or dislocation depends upon the harm that has been done to the contents of the spinal 
canal-the spinal cord and its nerve roots. The cord has considerable range of motion and is elaborately protected against injury.

In the cervical region, the spinal canal is large and the vertebra are relatively small and are more freely movable upon each other than in the dorsal and lumbar regions. In the lower dorsal and lumbar regions, on the other hand, the vertebra are relatively large, the spinal canal is small and the vertebre are firmly bound together by strong ligaments. As a result of these anatomical peculiarities, dislocations are more frequent in the cervical region where some crushing injuries of the cord may occur without fracture, while in the lower portions of the spine, injury of the cord is usually associated with extensive fractures of the vertebra.

The cord symptoms are not always due to a direct injury by fractured or dislocated bone. The cord may become edematous from the concussion, a hemorrhage (hematomyelia) may occur into it, or it may not be actually injured but may be compressed by blood, or by fractured and dislocated bone. The patients may at first present all of the symptoms and signs of a transverse lesion, and after some days or weeks improve very much.

Because it is often difficult or impossible to determine beforehand whether an irremediable injury to the cord has occurred, surgeons are usually in one of two groups - those who believe that every patient should be given the benefit of an exploration, and those who would operate only on strict indications. Many hopeless cases have been operated upon and, therefore, very poor results obtained from surgical interference. At the present time, the majority of neurologists and surgeons are very conservative in their recommendations of operative interference in fresh fractures of the spine. A number of writers (Sherman, Coste(I3), Kocher(I4), etc.) have endeavored to formulate exact indications and contraindications, but all make tacit acknowledgment that the diagnosis of the extent of the injury to the cord is often uncertain, and that operative interference may be indicated if the symptoms are not those of a transverse lesion. If there is complete motor and sensory loss up to a level, with complete or almost complete abolition of all of the reflexes, operative interference should not be attempted. If some power and sensation, and some of the reflexes are left, an operation may do much good (Thompson(I 5), Schloffer(I6), Bottomley(17), Coriat and Crandon(I8), etc.).

Old fractures of the spine, with residual cord symptoms, can often be much benefited by operative interference. A much narrowed spinal canal can be widened by the laminectomy; pressure of bone upon the cord and upon nerve roots can be relieved; a marked angulation of the cord can be straightened out. A careful study of the symptoms and good roentgen-ray pictures will help the surgeon to make up his mind as to the prospect of improvement after an operation.

A thorough after-treatment is important. A few weeks after the operation, massage, electricity and active and passive exercises may be begun and should be continued for several years. In some patients 
tenotomies or posterior root sections to relieve spasticities may be necessary. Others can be put upon their feet again by means of appropriate splints or shoes that will compensate for the drop-foot. Whether persistent bladder symptoms can be benefited by intradural nerve anastomoses (Frazier(I9), Cadwallader and Sweet(20)) is still an open question.

Bullet and Stab Wounds of the Cord.-The general symptoms of bullet wound of the spinal cord are similar to those of spinal fracture - with the difference that the wound is always a compound one and, therefore, the danger of infection is added. Many bullet wounds heal without suppuration, but shrapnel injuries are almost regularly infected.

The following general rules of treatment may be laid down:

I. When the symptoms are those of a complete transverse lesion, it is inadvisable to operate unless the roentgen-ray shows that the canal is narrowed or deformed by splintered fragments of bone or by the bullet. In these cases, operation is justifiable as soon as some signs of returning power in the limbs appear.

2. In incomplete lesions of the cord, operative interference is indicated within the first few days or weeks of the injury, unless there is suppuration along the track of the bullet, or unless the symptoms are very slight.

The laminectomy may reveal easily remediable lesions. The dura may be found much thickened and the incision or excision may relieve pressure upon the cord, or there may be a localized serous meningitis, or splinters of bone or bullet fragments may have to be removed.

Wounds of the spinal cord by cutting instruments do not differ either in their symptoms or the indications for their treatment from other injuries of the cord.

Tumors of the Spinal Cord and Membranes.-All writers agree that the differential diagnosis between intramedullary and extramedullary growths is often very difficult; a painless beginning and dissociation of sensations may occur with extramedullary tumors, and root pains are not so rare in intramedullary disease. This has been pointed out by $\operatorname{Schultze(2I),~Ropke(22),~Rothmann(23)~and~}$ others. The roentgen-ray is of negative value for the diagnosis of spinal tumor, although absorption of bone may occur with extradural grow ths. The fluid obtained by lumbar puncture is often of a yellow color (xanthochromia) with an increased amount of globulin and a normal number of cells (Nonne(24), Kaplan(25), Raven(26), etc.).

Greater refinements in the technical removal of extramedullary spinal tumors have been followed by increasingly good results. The greatest care should be taken of the cord. If a growth is adherent to the cord or under the pia, it should be removed in two steps. If a tumor grows under a nerve root or a slip of the dentate ligament, it is advisable in most instances to cut the root or the ligament, rather than to pull out the tumor. As extramedullary growths are usually 
well encapsulated, of slow growth and clinically benign, it is preferable to leave part of the capsule behind if this is closely adherent to the cord. Tumors in the upper cervical region should not be at once removed, as the sudden change of pressure may be followed by medullary symptoms.

Great care must be taken in the removal of the large tumors which are met with around the conus and roots of the cauda equina. These tumors (giant endotheliomata) are so closely connected with the nerve roots that paralysis of the bladder and rectum is apt to follow their removal. At the first operation, the growth should be exposed and the dura left wide open. At a second operation, the growth can be removed if it is not too closely connected with the nerve roots. Otherwise as much of the tumor as can be excised without injury to the caudal roots, should be excised, and the dura then left wide open for decompressive purposes.

The results that can be obtained by the operative removal of spinal cord tumors vary with the location of the growth, its connection with the cord and the duration and severity of the symptoms. Complete recovery is frequent and often rapid (Rothman(27), Van Gehuchten and Lambotte(28), Hecht(29), Frazier(30), Babinski(3I), Pussep(32), Martins(33), Kennedy(34), Elsberg(35), Clarke(36), Hunt and Woolsey $(37)$, etc.). If a paraplegia has existed for a longer time, six months to two years, the outlook for recovery of function is not so good. Considerable power may return in the paralyzed limbs, but more or less spasticity, weakness and sensory disturbance will remain (Rothmann(38), Pussep(39), Rotstadt(40)Schultze, Nonne(4I), Redlich(42), Sato(43), Elsberg(44), etc.). If the paralysis has existed for a number of years, there is rarely much, if any, improvement after the operation.

Unless the cord has been gravely injured by the much prolonged pressure of a tumor, all of the symptoms of cord compression will disappear within a few months. A long-continued after-treatment -massage, active and passive exercises, electricity-may be necessary before complete restoration of function occurs. The spasticity which may persist after the successful removal of a spinal tumor can often be much relieved by a well-selected posterior root section.

Intramedullary Surgery.-Up to within the last few years, intramedullary growths were considered hopeless, but it has been shown that localized growths occur within the cord substance, and that, with a proper technique these can be removed. Successful operations have been reported by Ropke, von Eiselsberg, Schultze, Elsberg and Beer, Elsberg, Foerster and others. Some good results were obtained by incision of the cord and peeling out of the tumor, but usually such manipulations have caused a transverse softening of the cord. In the case of Ropke, a tumor was found which was partly intra- and partly extramedullary. The cord was incised longitudinally, the entire growth easily removed, and the patient was much improved after the operation. 
Based on anatomical and physiological studies, the writer has shown that an incision can be made in the posterior columns near the posterior median septum without causing any marked loss of function. The incision must be very carefully made, with a fine "Graefe" knife with avoidance of any bleeding.

In order to do away with all manipulation of the cord, Elsberg devised the method called "delivery of intramedullary growths by extrusion." In this method the cord is incised near the posterior median septum, down to the tumor, and the muscles, fascia and skin closed for the time being. The tumor is gradually pushed out of its bed in the cord, so that at the second operation, about one week later, it is found to lie outside of the cord, so that it can be removed with a minimum amount of handling of the cord tissue. This "method of extrusion" allows the processes of nature to push out the tumor in the attempt to equalize pressure conditions. The growth should never be removed, unless it is complete, extruded and well encapsulated; if this is not the case, as much as possible is excised.

Not only have intramedullary growths and foreign bodies been removed from the substance of the spinal cord, but the cord has been incised as a decompressive method in infiltrating tumors (Cushing, Elsberg, etc.), an incision has been made in the posterior columns near the posterior median septum as a decompressive method in edema secondary to trauma (Allen), in gliosis, for the drainage of hydromyelia and intramedullary cysts.

Spiller and Martin have suggested that the anterolateral tracts can be divided in order to relieve persistent pain due to malignant disease. The cord is exposed in the usual manner, and the anterolateral tracts, which convey sensations of pain and temperature from the opposite side of the body, are divided by an incision of about $2 \mathrm{~mm}$. in depth. If the operation is carefully done and not too much of the cord is divided, no symptoms are caused by the incisions excepting a loss of sensation for pain and temperature below the level of the lesion. If the pain is bilateral, the anterolateral tracts of both sides must be divided; if unilateral, the contralateral tract is to be cut.

It is needless to add that these operations in which the cord is invaded, require a delicacy of manipulation and an exactness of technique that are only possessed by an operator experienced in this field, and that an incision in the spinal cord should be made only in carefully selected cases.

An incision into the cord may sometimes be made in hematomyelia, to evacuate blood or in syringomyelia, to drain an intramedullary collection of fluid. In spinal gliosis, a few cases of benefit from a decompressive incision of the cord have been reported, but this method of treatment is still in its infancy.

\section{BIBLIOGRAPHY}

I. Marion, Chir. du Système Nerveux, Paris, rgo4.

2. Cavicchia, in Marion.

3. Bickham, Ann. Surg., Igro, li, 529. 
4. Horsley, Brit. Med. Jour., August, 1906.

5. KraUSE, Chir. des Gehirns u. Rueckenmarks, 1911.

6. voN Eiselsberg, Arch. f. klin. Chir., 1913, cii, 465.

7. Frazier, Surg., Gyn. and Obstet., September, 1910.

8. Cusilng, Bull. Johns Hopkins Hosp., November, 19 ro.

9. Kuettner, Verhandl. d. deutsch. Gesellsch. f. Chir., Igo8.

10. Kocuer, Chir. Operationslehre.

II. ElSberG, Surg., Gyn. and Obst., I913, xvi, I 17.

12. TAYLOR, A. S., Ann. Surg., 1910, li, 529.

13. Coste, Deutsch. mil. ärztl. Ztschr., Berlin, 1912, xii, 55.

I4. Kocher, Cor.-Bl. f. Schweiz. Aerzte, 1912, xiii, 415.

15. Thospson, Texas State Jour. Med., 1911, vii, 296.

16. SCHLOFfer, Muench. med. Wchnschr., 1912, lix, 2927.

17. Botromley, Boston Med. and Surg. Jour., I9I 2, clxvii, 69r.

18. Coriat and Crandon, Med. and Surg. Rep. City Hos., Boston, 1913, 235.

19. Frazier and Mills, Jour. Am. Med. Assn., 1912, lix, 2202.

20. Cadwallader and Sweet, Med. Record, 1913, lxxxiv, 800.

21. Schultze, Deutsch. med. Wchnschr., 1912, xxxviii, 1676.

22. Ropke, Arch. f. klin. Chir., I9I I, xcvi, 963.

23. Rothman,, Berl. klin. Wchnschr., I913, $5^{28}$.

24. Nowne, Deutsch. Ztschr. f. Nervenheilk, Leipzig, I913, xlvii.

25. KaplaN, New York Med. Jour., August, 19 3 .

26. Raven, Deutsch. Ztschr. f. Nervenheilk., I9I3, xlix, No. 2.

27. RothManN, loc. cit.

28. Van Gehuchten and Lambotte, Bull. Acad. royal de mèd. de Belg., Brux., 1912, N. S., xxvi, 697.

29. HechT, Med. Record, I9I 2.

30. Frazier, Surg., Gyn. and Obst., September, igro.

31. BABINSKI, Rev, neurol., I912, xxiii, r.

32. Pussep, Mitt. a. d. Grenzgeb. d. Med. u. Chir., I9II-I9I 2, xxiv, 339.

33. Martins, Jahrb. d. Hamburg. Staats krankenaustaltungun, I9II, XV, 213.

34. KeNNedy, Jour. Nerv. and Ment. Dis., I9I I, xxviii, 689.

35. ElsBerg, loc. cit.

36. Clarke, Brit. Med. Jour., I912, i, I 75.

37. Hunt and Woolsey, Ann. Surg., rgro, lii, 289.

38. RothmanN, Berl, klin. Wchnsch r., I913, 528.

39. Pussep, loc. cit.

40. Rotstadt, Nouv. iconog. de la Salpêtrière, I913, xxvi, 36.

41. NONNE, loc. cit.

42. REDuich, Wien. klin. Wchnschr., I912, xxv, 2026.

43. SAto, Zur Extirpation maligner Geschwülste des Rückenmarks, Gottingen, I9I I.

44. Elsberg, Surg., Gyn. and Obst., February, 1914. 


\title{
THE SO-CALLED FUNCTIONAL NERVOUS DISEASES
}

\author{
By F. X. Dercum, M. D.
}

Little has been added of recent years to our knowledge either of the symptoms or of the treatment of functional nervous disorders. However, a very decided change has taken place in our conceptions regarding their pathology and this in a measure influences our therapeutics. Into many of the functional nervous disorders there enters, as has been long recognized, an element of exhaustion. Of this fact neurasthenia and the neurasthenoid states afford comprehensive examples. In others again, as in hysteria, psychic factors play a dominant rôle; but in almost all of them there are, in addition, elements indicative of toxicity. We are gradually beginning to recognize that in affections which present no gross organic pathology, we have-save in the limited number that are psychic in origin-to deal with toxins.

The various functions of the body, including the elimination of nervous and muscular energy, are convertible into terms of tissue chemistry, and it is the derangement of this chemistry which constitutes disease-a derangement which may have its origin spontaneously in some fault of the organism or which may be secondary to some cause introduced from without, such as a poison or infection. "Disturbances of the metabolism of the body necessarily entail states of intoxication and in these states of intoxication must be sought the explanation of many nervous and mental disorders. The earlier observations of Mendel, Bouchard and others are today only of historical interest. Chemistry was not yet sufficiently advanced in their day to render their observations of value nor to prevent incorrect interpretations from gaining a foothold. This is likewise true of the extensive rôle at one time assigned to the uric acid group. Only in a measure do the leukomaines still demand consideration. The failure of treatment directed toward their elimination conclusively proved that their importance had been greatly overestimated.

When we turn our attention to the subject of autointoxication as revealed at the present, we learn that much has been gained from our rapidly accumulated knowledge as to the rôle played in the organism by poisons of exogenous origin, notably alcohol and the infections. Poisons, among which the bacterial toxins are of course to be included, may act directly upon the nervous system or they may bring about disturbances in the functions of other organs, disturbances leading to changes in the general metabolism of the body, to the formation of endogenous toxins and to the consequent autointoxication of the entire organism. Notable examples of such autointoxications are 
the long-continued confusional insanity persisting in alcoholic subjects long after the alcohol has ceased to be ingested or persisting in an infection long after the infectious process has run its course. Obviously we have in both instances to do with the secondary toxemias of a deranged metabolism.

With these considerations before us, let us turn our attention to autointoxication proper, that is, to primary or endogenous poisoning. A moment's reflection reveals that primary autointoxication is of two kinds: first, one that is gastrointestinal in origin, and another that arises in the tissues of the body itself. This distinction may in practice be a very difficult one. An intoxication by way of the intestinal tract may give rise to secondary disturbances of metabolism within the body, e.g., derangements of the liver or of the thyroid gland, or, intoxications having their origin within the body proper may be followed by a secondary intestinal intoxication; of this neurasthenia, melancholia, and other affections in which the innervation of the intestinal tract is diminished, afford abundant examples. In considering gastrointestinal intoxication as a possible primary factor, we must bear in mind that in the normal or healthy individual, intestinal poisons are destroyed by the liver, the thyroid and by the other glands and tissues generally; or, they are eliminated by the various excretory organs. Poisoning of the nervous system is thus effectively guarded against. However, in diseased and defective states, poisoning may occur and may in turn produce, as above pointed out, secondary disturbances of metabolism. The presence of phenol, indol and skatol in the urine in large amount bespeak, of course, intestinal intoxication; but, on the whole, it may be safely said that when there are present marked or persistent nervous symptoms, we have to deal with a coexistent and probably primary interstitial or endogenous poisoning.

Intoxications of short duration probably play a relatively unimportant rôle; in brief intoxications we have to deal with poisonings which the organism successfully resists and, so to speak, disposes of speedily and promptly. For such resistance the organism is admirably equipped. We have in the very beginning the defensive action of the gastric and intestinal juices. If injurious substances, notwithstanding, gain access to the organism they are submitted to the action of the liver and later to that of other glands and tissues. Substances are oxidized, deoxidized, hydrated, dehydrated or variously combined or disintegrated and finally eliminated. Such is probably the course of intoxications of short duration, but in many of those of long duration such as are commonly observed in nervous and mental disorders, the organism doubtless has recourse to the formation of immune bodies, though many times with an indifferent success. The greater number of functional nervous disorders are essentially chronic and persistent and do not present the wave-like course so frequently observed in the acute infections and cannot be paralleled with the latter. However, their course does not differ essentially 
from that of the chronic infections, e.g., syphilis and tuberculosis or from that of the chronic intoxications, e.g., alcohol or lead. Further, the rôle played by the toxins may be very complicated. A striking instance of a chronic intoxication of the nervous system and one that in addition is purely an autointoxication is that furnished by dementia præcox. Here as has been shown by Fauser and a large number of subsequent investigators we have to deal with a toxic hormone which gains entrance into the circulation from the sex glands. This toxic hormone evokes the formation of a defensive ferment, but it so happens that the latter, instead of protecting the organism, has the unfortunate property of digesting cortical tissue. The fact of a defensive reaction on the part of the organism does not therefore imply its successful protection. This reaction, as we know, commonly fails also in some of the infections, e.g., tuberculosis and syphilis.

The problem of autotoxicity presents itself in still another aspect. Thus, in manic-depressive insanity, neurasthenia, psychasthenia and neurasthenic states generally, defensive ferments are never found. In these states it is safe to infer that foreign substances do not gain access to the circulation, but that the symptoms are due to a quantita tive and not to a qualitative change in substances normally present in the organism. Such substances, if excessive or abnormally deficient, have all the values of poisons. Of this fact the thyroid secretion is a familiar instance. Both hypo- and hyperthyroidism are attended by symptom groups expressive of toxicity.

With these considerations before us, let us turn our attention to neurasthenia. Here there are many facts that indicate that toxic factors also are at work. First, the symptoms of themselves point unmistakably to a toxicity, and for a consideration of this point the reader is referred to the writer's article in Vol. III. In addition, certain general clinical facts also indicate the same truth. For instance, it is known that in neurasthenia there is a very high resistance-a relative immunity - to tuberculosis, just as there is in gouty states. The first thought that arises is that this immunity is due to the presence of an excess of the purin bodies, but that this cannot be the entire story of the toxicity of neurasthenia is shown by the failure of treatment directed to the elimination of these substances to relieve the symptoms. Finally, that metabolism in neurasthenia is profoundly disturbed in some way not as yet explained is proven by the remarkable fact that in neurasthenia the lymphocytes in the blood are increased-not the leukocytes generally, but only the lymphocytes. Bearing in mind the conclusions indicated by the negative findings as regards defensive ferments, does the truth lie in quantitive disturbances-an imbalance of theinternal secretions? This I regard as very probable. It is further suggested by the phenomena clearly referable to disturbances of the autonomic and sympathetic nervous systems. The physiologist Langley renamed the sympathetic nervous system the autonomic nervous system, largely on the basis that the structures supplied by the sympathetic 
nerves are independent of the will. The cerebrospinal nerves which execute the mandates of the will, that is, carry out voluntary movements, terminate only in striated muscular fiber. The sympathetic nerves, on the other hand, terminate in smooth muscular fiber and in glandular tissue; in other words, the sympathetic fibers include all efferent nerve fibers except those which go to the voluntary muscles. In accepting the term autonomic as a substitute for the term sympathetic nervous system, we should bear in mind, however, that the first or proximal neurone of this apparatus is always situated in the gray matter of the cerebrospinal axis, i.e., in the cord or in the brain stem. Further, this proximal neurone gives off an axone which passing out by way of the rami communicantes terminates in an arborization about the distal neurone situated in the sympathetic ganglion. Thence fibers pass to smooth muscle or gland. The sympathetic nervous system thus has an origin which is primarily cerebrospinal and it is really an integral portion of the cerebrospinal apparatus. It is certainly not an independent system and is only in a measure autonomic.

In recent years this sympathetic or autonomic nervous system has come to be divided into the so-called autonomic nervous system proper and the so-called sympathetic nervous system proper. The word autonomic is applied to that portion above the second dorsal segment of the cord and to that portion below the second lumbar segment, while that portion included between these levels is spoken of as the sympathetic proper. This division which has no biological basis, is made rather arbitrarily upon the differences in function which obtain in some measure between the two. For instance, fibers of the sympathetic proper pass from the upper dorsal cord through the superior cervical sympathetic ganglion to the dilator muscle of the iris, to the constrictor fibers of the vessels supplying the ear, the oral and nasal cavities, the eyeball and the salivary glands. On the other hand, autonomic fibers so-called, originate in proximal neurones in the midbrain, accompany the oculomotor nerve and terminate in arborizations about cells in the ophthalmic ganglion, from which fibers in turn originate which supply the ciliary muscle and the constrictor fibers of the iris; at the same time other autonomic fibers supply the dilator fibers of the vessels of the mucous membrane of the mouth, throat, nose and nasal sinuses and also the salivary glands. A similar duplex arrangement exists in the innervation of the heart; for example, a sympathetic innervation through the accelerator nerves and an autonomic innervation through the vagus. Likewise, duplex innervations can be traced in the glands of the mucous membrane of the trachea and bronchi, the muscles of the trachea and bronchi and the glands and muscles of the intestinal tract from the esophagus to the anus and also in the case of the urinary bladder, genital organs and the vessels of these structures. To a degree these two systems are physiologically antagonistic to each other, and are each capable of separate stimulation. For instance, adrenalin induces 
contraction of the blood-vessels, acceleration of the heart's action, dilatation of the pupils, drying of the mucous membranes, inhibition of the salivary glands, inhibition of the motility and secretion of the stomach and of the intestines. Adrenalin, therefore, stimulates the sympathetic fibers proper and may, therefore, be said to be sympathicotropic and sympathicotonic.

Just as adrenalin stimulates the sympathetic system so does pilocarpin stimulate the autonomic system. Pilocarpin is a vasodilator and in full doses slows the heart's action. At the same time it stimulates glandular action, the perspiration and saliva being greatly increased. The pupil also is contracted and gastric and intestinal movements, as has been shown in experiments on animals, are increased. Pilocarpin can, therefore, be spoken of as autonomicotropic or to employ the term actually in use vagotropic and vagotonic.

Again, atropin acts as a paralyzer of the autonomic apparatus. There is here because of the paralysis of the autonomic apparatus dilatation of the pupils and arrest of the secretions. In full doses the heart beats become exceedingly rapid, the pulse ranging from I 20 to 160 . Atropin is therefore vagotropic but is paralyzing in its action instead of being vagotonic.

Less is known about substances or poisons which, being sympathicotropic, also paralyze the sympathetic. In paralysis of the sympathetic there would be vascular dilatation, contraction of the pupils, slowing of the heart's action, increased glandular activity; but when we come to look for poisons having such an action we cannot find one which will fill all of the requirements. Morphin which contracts the pupils, and slows the heart's action, is an indifferent vasodilator and instead of increasing the secretions inhibits them, unless it be that of the skin. Similarly, when we turn back to stimulating . drugs of the autonomic apparatus, among which may be mentioned in addition to pilocarpin, physostigmin, cholin, digitalis, we find that there are many variations in the details of their action which do not admit of an explanation limited to an exclusive and stimulant action upon the autonomic apparatus. This it may be added is also true of the paralyzing drugs.

Now, when we turn our attention to the action of the internal secretions, e.g., that of the thyroid or pituitary glands, we meet with similar difficulties. Space will not permit of a detailed discussion, but it is clear that poisons do not necessarily limit themselves in their action to one or the other subdivisions of the sympathetic system. A complete antagonism, such as outlined, does not always obtain. Sometimes portions grouped on the one hand among the autonomic or on the other hand among the sympathetic, are variously acted upon by the same poison. However, for us, the fact of the direct action of special substances upon various parts of the sympathetic nervous sustem is one of the very greatest significance, and in the recognition of this fact must lie the explanation of many of the 
symptoms met with in functional nervous disorders. Among these symptoms are constipation, atony and dryness of the digestive tract, the phenomena presented by the circulation, the pallor, or lividity, dampness or dryness, coldness, and other appearances of the body surface, the dilatation or the contraction of the pupil, and other symptoms as well. The point which should be emphasized is that these phenomena must be referred to a toxic action, an action which expresses itself through the sympathetic nervous system. In neurasthenia, the symptoms presented by the digestive tract, the circulatory apparatus, the secretions and certain very special forms of apparatus such as the iris permit of no other explanation. Indeed, they point to a direct toxic action. There can be no doubt that an explanation is here afforded of many symptoms observed in neurasthenia and the neurasthenoid states generally. In many other functional nervous disorders, special toxic factors appear to be at work, as in migraine, epilepsy and paralysis agitans, while in others, such as chorea and tetany, the morbid agency is probably an infection.

In a large number of disorders, e.g., the psychasthenias, tics and hysteria, there appear to be present in addition to exhaustion and toxicity certain definite peculiarities of nervous action which determine the character of the clinical picture presented. These peculiarities have their basis in an inherent pathological structure of the nervous system, a neuropathy, which manifests itself both in qualitative and quantitative changes in function. These changes are observed especially in the neurasthenoid states, the psychasthenias, and in hysteria. The qualitative changes consist in a tendency to the formation of pathological associations such as lead to the special fears, phobias and obsessions. This has already been sufficiently considered in the original article, Vol. III, page 897. The quantitative changes manifest themselves by deficiencies of will, deficiencies of inhibition and by a feebleness of decision which leads in its full development to folie du doute (loc. cit.).

These phenomena depend upon a quantitative subnormality of function which Janet has characterized as "un abaissement du niveau mental"-a depression of the mental level. The writer many years ago used the expression "reduction of the field of consciousness" to convey the same idea; and it was the recognition of this condition which led Janet to devise the term "psychasthenia." This state is essentially an asthenic one, and the full appreciation of this fact is, of course, of prime importance from the standpoint of therapeutics.

A consideration of the pathology of the so-called functional nervous disorders brings to light three groups of facts: first, those dealing with exhaustion; secondly, those dealing with toxicity; and thirdly, those dealing with an inherent pathological structure of the nervous system. These facts lead from the standpoint of therapeutics, first, to a consideration of rest as an element of treatment. This has already been abundantly discussed in the article in Vol. III. The 
second problem that presents itself is that of the toxicity. Inasmuch as this toxicity is commonly a protein toxicity it is obvious that little is accomplished by efforts at elimination, a conclusion which is confirmed by experience. Again our knowledge of the toxins at work is so limited that pecific medication is as yet out of the question. Chief reliance must be placed upon full feeding and at times upon massive feeding. Among other and more direct results of massive feeding is the entrance of a certain amount of unchanged protein into the blood and the consequent formation of ferments in the plasma. That there is here a factor which may play a powerful rôle in the battle with toxicity goes without saying. Among other things overfeeding leads to the production of an increased amount of lipoid substance in the blood. Such substances are both antitoxic and antihemolytic and there can be no question that by this means the resistive power of the organism is increased while in a general way its biochemical processes are raised to a higher and more active level. The other aspects presented by full feeding have already been considered in the article in Vol. III.

In some instances, more especially in the neurasthenoid (the psychasthenic) states symptoms are present indicating the employment of glandular therapy. Thus, every now and then symptoms of thyroid deficiency-usually slight-or of thyroid excess, are present suggesting the administration of thyroid extract or of antithyroidin. Usually it is wisest to begin-especially with thyroid extract-with very small and tentative doses, later employing the remedy in larger doses according to circumstances. Not infrequently pituitrin proves of decided value in cases with cardiac and general circulatory deficiency. In given cases also in which menstrual abnormalities are related to functional nervous states distinct gains are made by the administration of lutein or it may be of a general ovarian extract.

The method of dealing with inherently neuropathic states, has already been sufficiently considered in the article in Vol. III. Suffice it to say here that emphasis must be laid upon the simple physiological life. Among other things the proper portion between physical and mental activities on the one hand and rest and feeding on the other must be determined for a given case and rigidly adhered to.

Of the long list of functional nervous disorders considered by the writer in Vol. III, little can be said in detail that is new. They all demand the application of general therapeutic principles, principles which the recent advances in our views as to their pathology outlined above, profoundly influence. 


\title{
TREATMENT OF INSANITY
}

\author{
By James Hendrie Lloyd, M. D.
}

A selective method is required in the preparation of the present chapter, for since the appearance of the original chapter, there have been more changes and more apparent progress in some ways than in others. The whole subject of mental therapy will not be reviewed, but merely parts of it.

\section{PARESIS}

Since Schaudinn, a zoölogist, discovered the Spirochata pallida, which is now recognized to be the living organism of syphilis, our conception of paresis and tabes has undergone a change. ${ }^{1}$ For many years it had been taught that these two diseases were parasyphilitic (or metasyphilitic). The idea seemed to be that, although general paresis and locomotor ataxia were due to syphilis, yet it was in a remote and roundabout way - a way, in fact, which nobody was able to describe plainly. This notion was countenanced by the fact that the pathology of paresis and tabes presented a different picture from that of ordinary cerebrospinal syphilis. In the parasyphilitic diseases the essential process was in the neurones, or nerve cells themselves; in other words, it was a parenchymatous disease, whereas in ordinary cerebrospinal syphilis the disease was located in the bloodvessels and meninges, presenting the well-known endarteritis of Heubner, with gummatous exudation and lymphocyte infiltration. Two such distinct processes, it was thought, could not be due to the same cause acting in the same way.

Noguchi, in I9r 3 , showed the falsity of this orthodox view, when he announced that he had found the spirochetes in the brain in forty-eight among 200 cases of general paresis, and in one case among twelve of tabes dorsalis. ${ }^{2}$ The protozoa were deeply embedded in the nervous tissue-in fact in the very neurones themselves, which would seem to show that they were quite competent to account directly for a parenchymatous, or neuronic, degeneration. Noguchi also infected rabbits directly with the tissue from general paretics, and produced a typical syphilitic lesion in the testicle of the animal, from which he recovered the spirochetes $;^{3}$ and Foster and Thomasczewski, by employing the method of brain puncture, were able to

${ }^{1}$ See a paper by the author, Syphilis of the Pons, Medulla, and Upper Spinal Cord, in the International Clinics, vol. 4 , I913, from which the statement in the text is taken.

2 Jour. Exper. Med., Feb. I, I913, p. 232; also a second paper in Jour. Cut. Med., August, 1913, p. 543 .

Jour. Am. Med. Assn., July 12, 1913, p. 85.

Vol. 1V. -58 
demonstrate living spirochetes in the aspirated cortical substance from patients suffering with general paresis. ${ }^{1}$ Others have confirmed these observations, ${ }^{2}$ and the conclusion is plain that both general paresis and tabes are simon-pure syphilitic diseases, caused by the actual presence of the organisms in the tissues. It thus appears that we may as well abolish the term "parasyphilitic" for good.

The effect of these discoveries, coming so soon after the introduction of salvarsan by Ehrlich, was for the time to revolutionize our outlook upon both paresis and tabes. In common with all forms of cerebrospinal syphilis, these affections were at once swept together into a group of diseases in which there was supposed to be a fighting chance.

Since the introduction of salvarsan and neosalvarsan, a great impetus has thus been given to the treatment of all forms of syphilis of the nervous system, and general paresis has received its due share. The literature of the subject has already grown to large proportions, but no attempt will be made to review all this literature in these pages, for space would not admit, nor would the task be altogether profitable. Great hopes were raised that a remedy had at last been found for paresis, but these hopes have not as yet been realized in anything like the measure that had been anticipated by some enthusiasts. There is, in fact, a general sense of disappointment; and a more conservative mental attitude is already apparent on this subject. It is perhaps too soon to reach a final judgment. This will require time and the criticism of unbiased observers all over the world. The feeling of the present writer is that a good case has not yet been made out for salvarsan as a cure for paresis.

It must never be forgotten that general paresis is a disease of most variable course, of marked remissions and of fatal relapses, even after long intervals. The hope of cure, or even of permanent improvement and arrest, has so often been dashed to the ground, that the wise therapeutist will not lose sight of the fact that nothing is easier in the whole range of psychiatry than to draw false conclusions in the treatment of this dread disease.

Salvarsan may be given under the skin, or into the muscles, or into a vein, or directly into the subarachnoid space.

In the earlier days the drug was given, especially in Germany, in large intramuscular or subcutaneous injections. ${ }^{3}$ These injections were painful, and often caused abscesses and sloughs. The dosage was also rendered uncertain; for it could not be determined how much of the drug remained in the indurated tissue, i.e., unabsorbed. The intramuscular method is still practised, but it is not recommended in

${ }^{1}$ Wile, Jour. Am. Med. Assn., Sept. I3, I913, p. 866.

2 McIntosh and Filder (Brain, vol. xxxvii) found the spirochetes in six out of seven cases of general paresis examined. The organisms were almost always confined to the gray matter.

${ }^{3}$ Nonne, Syphilis and the Nervous System, translated by Ball, p. 376 . 
these pages. It has no advantages over the intravenous injections. The same is to be said of the subcutaneous method.

The intravenous method has several advantages. It admits of an exact dosage; it is not painful, and as a rule, if done properly, leaves no severe local reaction; and the remedy is more quickly distributed and, therefore, gives a more certain result. Precautions are necessary. Thus the solution should be neutral or alkaline; rigid asepsis should be observed; air bubbles should not be allowed to enter the vein; and it should not be given in cases of advanced arterial sclerosis, diabetes, Bright's disease, cachexias, or advanced cases of tabes or paresis. Care must be observed that the needle is properly inserted into the vein, otherwise the solution will flow into the subcutancous tissue, and may cause abscesses and sloughing.

The following covers briefly the points concerning salvarsan and neosalvarsan, from memoranda given by Dr. M. C. Albright, Chief Resident Physician of the Methodist Hospital.

Salvarsan.-Introduced in I909, commonly known as "606." Chemical formula; $\mathrm{HClNH}_{2} \mathrm{OC}_{6} \mathrm{H}_{3} \mathrm{As}=\mathrm{As} \mathrm{C}_{6} \mathrm{H}_{3} \mathrm{OHNH}_{2} \mathrm{HCl}+2 \mathrm{H}_{2} \mathrm{O}$. Contains 3 I.5 per cent. of metallic arsenic. Readily oxidizes when exposed to the air, hence kept in vacuum tubes, and should be used immediately after the tube is opened. Soluble in water with acid reaction. The drug is marketed in various-sized ampules containing from 0.3 to $0.6 \mathrm{gm}$. The maximum dose is most frequently used. As a general rule, no symptoms follow the injection; nevertheless, very severe reactions and even fatalities have occasionally resulted. The following symptoms are more commonly met with, headache, giddiness, nausea, vomiting, diarrhea, dyspnea and tremor. In fatal casea edema, cyanosis, coma, and collapse are the precursors of dissolution.

Neo-salvarsan.--Introduced in I9 I 2, commonly known as "9 I4." Chemical formula: $\mathrm{NH}_{2} \mathrm{OHC}_{6} \mathrm{H}_{3} \mathrm{As}=\mathrm{AsC}_{6} \mathrm{H}_{3} \mathrm{OHNHCH} \mathrm{OHSONA}_{2} \mathrm{OASO}$ Readily oxidizes when exposed to the atmosphere. Three parts of neosalvarsan are equivalent to 2 parts of salvarsan. Dose, 0.3 to $0.9 \mathrm{gm}$. Administered almost exclusively by the intravenous method, since it is even more irritating and more prone to cause sloughing than salvarsan when injected subcutaneously or intramuscularly. Untoward effects are rarely met with after the injection of neosalvarsan. Moderate fever, nausea, vomiting and chills may follow. Neosalvarsan has superseded the mother product, salvarsan, in popularity chiefly because of the facility of its administration and its lesser degree of toxicity.

The contraindications, which apply to both preparations, are advanced cardiac and renal disease, marked arteriosclerosis, optic atrophy, diabetes and advanced pulmonary tuberculosis.

Soon after the introduction of salvarsan doubt began to be felt whether this drug, when given by any of the above-named methods, reached the central nervous system, for observation seemed to show that it could not be recovered in the cerebrospinal fluid withdrawn 
by lumbar puncture. The idea must have occurred to many minds that a more direct way would be to inject it directly into the spinal subarachnoid space. Marinesco was one of the first to do this. $\mathrm{He}$ injected neosalvarsan in doses of $5 \mathrm{mg}$. intraspinously in thirteen patients, and had "unpleasant" results. Swift and Ellis injected high dilution of salvarsan in monkey serum intraspinously into monkeys, and found it too irritating to warrant its use in human beings. They then proposed their method of injecting salvarsanized serum. ${ }^{1}$

Subarachnoid Therapy.-The methods of subarachnoid therapy may be briefly stated as follows, after Swift: ${ }^{2}$

I. Substances used:

(a) Neosalvarsan dissolved in water, normal saline solution, or cerebrospinal fluid.

(b) Serum salvaranized in rivo.

I. Human serum.

2. Serum of animals.

(c) Serum salvaranized in vitro.

I. With salvarsan.

2. With neosalvarsan.

(d) Mercury:

I. Mercuric chlorid lavage of the subarachnoid space.

2. Mercuric cyanid dissolved in saline.

3. Mercuralized serum.

2. The mode of application:

(a) Into the spinal subarachnoid space by lumbar puncture.

(b) Into the cranial subarachnoid space through a trephine opening.

I. Subdurally.

2. Into lateral ventricles.

(c) Into the large basal subarachnoid cistern through the sphenoidal fissure.

(a) In Germany, according to Hassin, ${ }^{3}$ the methods of Gennerich and of Schubert are preferred. In Gennerich's method O.I $5 \mathrm{gm}$. of neosalvarsan are dissolved in 300 c.c. of normal sterile salt solution, of which 60 c.c. are introduced into the spinal canal by means of a syringe, after an equal amount has been withdrawn and mixed with the normal salt solution. In Schubert's method nothing but the spinal fluid of the patient is used to dilute the drug. The reactions to the injections are shooting pains in the back and legs; sometimes also in the arms; headache, nausea, vomiting, retention of urine, and slight fever. Hassin reports some very bad results in advanced cases, one of tabes and one of cerebrospinal syphilis. He seems to prefer the internal use of "plain" arsenic or arsenic teroxid.

${ }^{1}$ New York Med. Jour., July, rgr 2.

${ }^{2}$ Swift, Jour. Am. Med. Assn., July I7, 1915, p. 2 Io. in detail.

Journ. Am. Med. Assn., Dec. II, I9I 5, p. 2078, in which the methods are described 
Swift, ${ }^{1}$ in discussing the various modes of giving neosalvarsan intraspinously, such as advocated by Ravaut, Wile and others, says that because of the sequelæ (some of which have caused death), intraspinal injections of neosalvarsan should be given with extreme caution, if at all, and the dose should never exceed $0.5 \mathrm{mg}$. Deaths have been reported by Gordon, Sachs and others; the latter with Strauss and Kaliski, had two deaths in paretics, and they hesitate now to urge any form of intraspinous treatment.

(b) Serum Salvarsanized in Vivo.-This method, as proposed by Swift and Ellis, ${ }^{2}$ consists in the intraspinous injections of serum obtained from a patient who had previously had an intravenous injection of salvarsan and will be specifically discussed by Dr. Swift (page 88I). Marie and Levaditi ${ }^{3}$ suggested the use of the serum of animals which had received large doses of neosalvarsan; but there are objections to the introduction of a "foreign" protein.

(c) Serum Salvarsanized in Vitro.-Ogilvie's ${ }^{4}$ method is to add a small amount of faintly alkalinized salvarsan to human serum. By this method a known dose of the drug is given instead of the uncertain dose of "autosalvarsanized serum." He reported encouraging results, but emphasizes the necessity of not giving more than $\mathrm{Img}$. Fordyce ${ }^{5}$ warns against the addition of more than $0.5 \mathrm{mg}$. and has used even smaller doses with advantage. Swift found the spirocheticidal effect of this serum superior to that of serum obtained from patients after salvarsan intraspinously, and thinks that Ogilvie's method is an advance. Marinesco and Minea ${ }^{6}$ used subarachnoid injections of inactivated serum to which from 6 to $\mathrm{I} 2 \mathrm{mg}$. of neosalvarsan were added. In some cases severe bladder disturbances resulted, but these observers believe that neosalvarsan can be given more safely in serum than in saline solution. However, the dose employed by them seems very large, and such doses are not recommended here.

(d) Mercury.-I. Horsley ${ }^{7}$ recommended subarachnoid lavage with a solution of mercuric chloride, but the suggestion has not met with favor.

2. The same may be said of Sicard's plan of subarachnoid injections of mercuric cyanide.

3. Byrnes ${ }^{8}$ recommends a solution of from $\mathrm{I} \cdot 3$ to $2.6 \mathrm{mg}$. of mercuric chloride in $\mathrm{I} 2$ c.c. of human serum, diluted with I 8 c.c. of normal saline solution, for spinal subarachnoid injection. This method has met with favor; some reports are very good; and these injections may be used alternately with the Swift-Ellis injections. It seems highly desirable that the local application of mercury should be tried.

I Jour. Am. Med. Assn., July 17, 1915, p. 2 I I.

2 Arch. Int. Med., 1913, xii, 331.

3 Compt. rend. soc. d. Biol., I913, lxxvi, 567.

${ }^{4}$ Jour. Am. Med. Assn., Nov. 28, 1914, p. 1936.

5 Jour. Am. Med. Assn., Aug. 15, 1914, p. 552.

- Rev. Neurolog., 1914, No. 5.

${ }^{7}$ Neurolog. Centralbl., I910, xxix, ir 70.

8 Jour. Am. Med. Assn., Dec. I9, 1914, p. 2182. 
Intracranial injections, either with salvarsan or mercury, have been used, especially in paretics, but their superior utility is doubtful, and the difficulty of the operation is objectionable. This applies to the yarious procedures recommended by Marinesco, Sicard, Ballance, Hammond and Sharpe, and others. With our present knowledge, these methods cannot be recommended in these pages. There is evidence, moreover, that the spinal injections are rapidly diffused throughout the whole cerebrospinal subarachnoid space, and therefore that the intracranial route is not called for. Experiments with phenolsulphonephthalein and with India ink prove that these substances when introduced into the spinal subarachnoid space can be recovered in fluid drawn from the ventricles in from a few minutes to several hours, as shown by Dandy and Blackfan. ${ }^{1}$

Wardner ${ }^{2}$ has developed a technique for intracranial puncture; as suggested by Levaditi, Marie and Martel. The salvarsan is given intravenously in the usual manner, and the serum collected as in the Swift-Ellis method. The next morning an incision is made over the precentral gyre, and a trephine opening made with a Hudson drill. As soon as the dura is exposed a lumbar puncture is done, and sufficient fluid is withdrawn to reduce the intracranial pressure. The serum, about 30 c.c. in amount, is then injected beneath the dura. But Wardner was unable by this method to reduce the globulin content or to influence very materially the Wassermann reaction in the cerebrospinal fluid. The blood Wassermann, however, was much reduced. Cotton thinks that this method, used in combination with the Swift-Ellis and the Ogilvie methods, may prove efficacious. Hammond and Sharpe's method consists in introducing salvarsanized serum into the lateral ventricles. ${ }^{3}$ Cotton proposes a method of introducing mercurialized serum into the ventricles. ${ }^{4}$ There is not space here for full details of these various methods; reference must be made to the original articles. ${ }^{5}$.

Swift has answered the objections to his form of intraspinal therapy, objections based on the claims that intravenous injections yield equally good results; that the difficulties of the operation, the pain and the occasional bad results are contraindications to it; that the cerebrospinal fluid sometimes contains arsenic after intravenous injections of salvarsan; and that blood serum, withdrawn one hour after the intravenous injection, does not contain sufficient arsenic to render it of value for intravenous use. In spite of these objections the Swift-Ellis treatment is now largely in favor, although Swift himself acknowledges that not all patients require intraspinal injections. Many of them respond well to intravenous injections com-

1 Am. Jour. Dis. Child., December, I9I4, p. 406.

2 Am. Jour. Insanity, January, I9I5.

${ }^{3}$ Jour., Am. Med. Assn., Dec. I8, I9I 5, p. 2I 47.

${ }^{4} \mathrm{Op}$. cit.

${ }^{5}$ Passek (abstract in Rev. Neurolog., Mar., I9I 5, p. I97) made trephine openings over both frontal lobes and scarified the pia; once a week colloidal mercury was applied to this surface, and the patients also received intravenous injections of salvarsan. Two patients, it is claimed, showed improvement. 
bined with the use of mercury and iodids. Both the clinical and biological results must be the criteria. Whatever treatment is used, it should be controlled by repeated examinations of the blood and the cerebrospinal fluid.

Results.-As already said, it is difficult, if not too early, to estimate results. We are concerned in these pages with paresis especially, and although not a few favorable reports have been published, it is noteworthy that these have to do more with "improvement" than with "cure." To judge by the biological or laboratory tests, paresis remains a most rebellious disease to any form of salvarsan or neosalvarsan treatment, for it is generally acknowledged that in this disease it is next to impossible to obtain a cerebrospinal fluid that is negative to all the tests. This is probably due to the fact that the spirochetes are deeply embedded in inaccessible regions of the brain, and are widely distributed, and have been long entrenched. We are not yet able to claim that we can dislodge and destroy them. Then, too, even if they could be destroyed, they would doubtless leave irreparable damage behind them. This is also suggested by the fact that the Argyll-Robertson pupil, once established, is not "cured" by salvarsan.

Dr. Henry A. Cotton has made extensive trial of intraspinous therapy in paretics in his service at the New Jersey State Hospital at Trenton. ${ }^{2} \mathrm{He}$ has treated more than roo patients. In old demented cases the results were not encouraging. In 33 per cent. of all his other cases he reports that "improvement has been marked." These patients have been able to do a considerable amount of hard work, and they have been saved thus far from the final bed-ridden stage of the disease. Relapses, he finds, are difficult to account for; they are most apt to occur in tabetic types, and may be due to the fact that treatment was not intensive enough. He thinks the Ogilvie method overcomes this better than the Swift-Ellis method. Early cases, of course, give better results. His method is to combine these two methods, using them on alternate weeks. But owing to the difficulty of obtaining salvarsan, he has lately used the Byrnes method with mercurialized serum, and thinks he is getting better results with it than with salvarsan. The old mercury treatment he regards as of no value in paresis. Dr. Cotton believes that a good start has been made in the treatment of paresis, but that present methods are not efficient enough. He is very conservative about claiming cures.

$\mathrm{McDonagh}^{3}$ claims that he has demonstrated a life cycle in the organism of syphilis, which organism he names Leukocytozoōn syphi-

${ }^{1}$ Head and Fearnsides (Brain, 1914, vol. 37, p. I) hold that in so-called parasyphilitic diseases, when the disease process has come to an end, leaving behind it irreparable degeneration, the Wassermann reaction may diminish in strength, or even become negative.

$2 \mathrm{Am}$. Jour. Insanity, July, rgi5. The reference in the text above is to a personal communication from Dr. Cotton to the author.

${ }^{3}$ Biology and Treatment of Venereal Diseases, London, rors. 
lidis, and that the spirochete is only one phase of that cycle. Paresis, or degenerative encephalitis, according to him, is caused by the spores escaping from the vessel walls and undergoing further development in the nerve tissue. Hence, they are difficult to reach, if not indeed inaccessible to drugs. He believes that the blood-vessels of the nervous system and the meninges are invaded in the early or generalized stage of infection, and he holds that treatment should be begun early in order to avoid serious later involvement of the cerebrospinal parenchyma. When the organisms have once developed, as stated, in the nervous tissue proper, that is, when paresis has developed, he seems to have faint hope of controlling them by treatment, and he says (page 24I) that he has decided not to prescribe antisyphilitic treatment in cases of degenerative encephalitis. He even believes that treatment usually results in hastening the end (page 240). In one case, in the very early stages of paresis, he gave eight intraspinal injections of salvarsanized serum, the number required to render the cerebrospinal fluid absolutely normal; but the only apparent result was to precipitate the patient into an advanced and hopeless stage of the disease. He, however, describes a "meningeal" form of paresis, in which the prognosis, so far as remissions are concerned, is rather better.

Charles F. Read ${ }^{1}$ reports a series of twenty cases of paresis in which doses ranging from 3 to $6 \mathrm{mg}$. of neosalvarsan were given intraspinously. The Ravaut method was used, the drug being diluted in at least ro c.c. of the patient's own spinal fluid. The results were not brilliant. Read concludes that intralumbar treatment with neosalvarsan in small quantities is without effect, and that when pushed with the object of obtaining results, it is extremely apt to prove injurious. He thinks its use in this manner is to be discouraged.

Gilpin and Early have proposed drainage of the cerebrospinal fluid by lumbar puncture, the idea being that by decreasing the pressure within the subarachnoid space it might be possible to cause "an osmosis," or in some other way, because of difference of pressure, to cause substances such as mercury and arsenic to pass from the blood into the cerebrospinal fluid. For some months they have tried this method. Clinically their patients have improved. They claim that when they thoroughly mercurialize a patient by inunction and drain the cerebrospinal fluid weekly, they secure a negative Wassermann test in both the blood and fluid, and reduce the number of lymphocytes and the globulin. Although the method shows these changes in paresis as well as in tabes, they think it is too soon to speak distinctly of results; but the method appears to be of benefit. However, they have not demonstrated either mercury or arsenic in the cerebrospinal fluid.

Space does not admit here of a review of the results claimed by all those who have used these various methods in paresis. The subject.

${ }^{1}$ New York Jour., Sept. II, r915, p. 56r. This paper contains a good review of the 
writer is confident that the time has not yet come for a final judgment. The whole subject is still in the experimental stage. It is a safe prediction, however, that no remedy will be found to restore tissue which has once been destroyed; hence comparatively early cases are the only ones likely to derive benefit. This conclusion seems to be indicated in most of the reported cases. How prolonged or how durable the improvement will remain, is still an open question. Nonne says that the consensus of opinion is that salvarsan is no more a cure for paresis than mercury and iodid. ${ }^{1}$

In his latest paper Swift ${ }^{2}$ says that the treatment of paresis by the various subarachnoid methods is disappointing. Something prevents the remedy from reaching the spirochetes, which are lodged in the substance of the cerebral cortex. Many cases improve clinically, and in some the cerebrospinal fluid improves, but the response is much less marked than in tabes. Also there is a tendency to relapse when the treatment is discontinued. With each relapse the response to treatment is less marked. Ogilvie brought the blood and cerebro-spinal fluid to normal in 4 cases. Swift, however, holds that the treatment of paresis is justified by the results, although in no case can we speak of cure. He believes that a very early intensive treatment of generalized syphilis tends to diminish the number of cases of late tabes and paresis; but it seems to the present writer that not sufficient time has yet elapsed to support even this claim. The preparations which, according to Swift, have stood the test of time, are (I) autosalvarsanized serum; (2) serum to which small quantities of salvarsan have been added; (3) neosalvarsan in small quantities in weak solution; (4) mercurialized serum. The Lange colloidal gold reaction has its great field of usefulness in differentiating paralytic dementia from cerebrospinal syphilis; and this distinction has significance in therapeutics because in cerebrospinal syphilis, and probably also in tabes, the lesions are secondary to a chronic syphilitic meningitis; whereas in paresis the disease depends upon masses of spirochetes lodged in the cerebral cortex.

Mercury.- The use of mercury by inunction in paresis is disappointing. Given by the mouth in this disease the drug is probably useless. Various preparations of mercury are used by hypodermic and intramuscular injections, but even in these more thorough methods the advantages in paresis are still subjudice. The hypodermic use of mercury salicylate in the treatment of syphilis is very general at present, but Nelson and Anderson, ${ }^{3}$ using $100 \mathrm{mg}$., or $\mathbf{I} 1 / 2$ grains, found its value so slight as to make it not worth the time spent in its administration. It does not influence the serum reaction. This being so, its use in paresis may be ignored.

Most of the reports on these various hypodermic uses of mercury are based on the treatment of general syphilis, and conclusions drawn

I Op. cit., p. 385 .

${ }^{2}$ Am. Jour. Mled. Sci., Oct., I916, p. 490.

'Jour. Am. Med. Assn., Nov. 27, I915, p. 1905. 
from them as to any possible value in paresis are not warranted. If the test in this latter disease is to consist of a perfectly normal cerebrospinal fluid, then the value of this mode of treatment is still far from being made out. It would seem, as already said, that the best method of using mercury in paresis is the Byrnes method of giving mercurialized serum intraspinously. Swift holds (op. cit.) that mercury and the iodids, "properly administered," may be useful adjuncts to the intravenous injections of salvarsan in some cases of nerve syphilis and tabes, but he does not mention paresis in this connection.

The following are some of the formulæ used for injection:

R. Hydrarg. salicylav .................. r.o

$$
\text { Paraffin liquid ................................ }
$$

Sig.-To be well shaken before using. To be injected every four or five days into the nates.

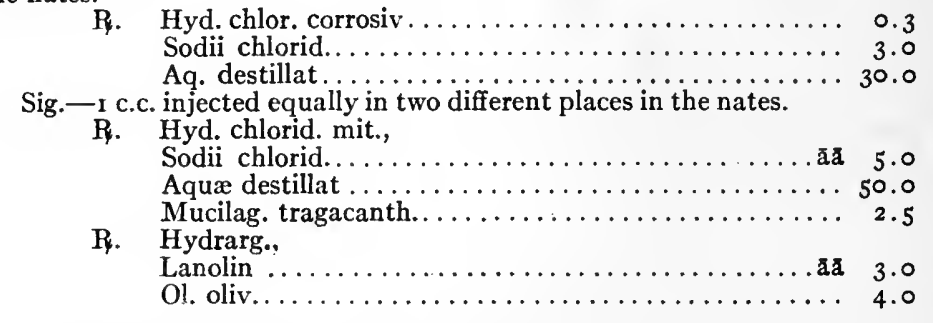

The dose of each of these latter preparations is I cc.. injected once every fourth or fifth day into the nates. But these injections of calomel and oleum cinereum are liable to cause abscesses. Nonne, ${ }^{1}$ from whom the above formulæ are taken, says that very often in the initial stages of paresis he has administered large doses of mercury by inunction and injections, and only in very rare instances has "improvement" occurred. He is entirely sceptical about their curative value.

The Iodids.- Since the introduction of salvarsan the iodids have fallen into considerable disrepute, although some practitioners still hold that they are of value in late or tertiary syphilis of the nervous system, as tending to promote the absorption of exudates. ${ }^{2}$ That they have any spirocheticidal action is more than doubtful. Experience has not proved that they are of value in paresis.

\section{AUTOINTOXICATION}

Bouchard, in 1887 , gave an impetus to the study of autointoxication and its relation to disorders of the gastrointestinal canal as a cause of mental disorder. Buckley ${ }^{3}$ has written on this subject. He cautions agains the theory that indicanuria is the same thing as autointoxication; nevertheless he believes that there are poisonous products which accumulate as a result of defective functions of the gastrointestinal apparatus. Arbuthnot Lane holds that in conse-

\footnotetext{
1 Op. cit., p. I 90 and pp. 358,359 .

2 Nonne, op. cit., p. 363 .

3 Penna. Med. Jour., April, rg 5.
} 
quence materials exist in the circulation which produce degenerative changes. The theory may have been overworked; but Buckley holds that in mental cases constipation is an evil to be carefully guarded against; in cases of melancholia especially there is a sluggish state of the body as well as of the mind, and gastrointestinal stasis is a part of the symptom complex. Buckley believes that laxatives are indicated as an important part of the routine treatment, in order to secure a complete intestinal cleansing. The salines are probably the best, with an occasional dose of calomel.

\section{DELIRIUM TREMENS}

Dr. E. A. Leonard, at the Philadelphia Hospital, has treated patients suffering with delirium tremens, with intraspinous injections of magnesium sulphate. The treatment was suggested by the good results obtained in a case of tetanus. Lumbar puncture was done, and varying amounts of cerebrospinal fluid withdrawn according to the degree of pressure found, i.e., from ro to $30 \mathrm{c.c}$. Then a 25 per cent. solution of magnesium sulphate was injected in doses of $\mathrm{I}$ c.c. for every 25 pounds of body-weight, at a temperature of about $95^{\circ}$ or $100^{\circ}$. No case received a second treatment. Of twelve cases there were ten recoveries and two deaths. In most of the cases a transient paraplegia developed with some loss of pain and touch sensibility, weakness of the bladder and sphincter ani, and abolition of the deep reflexes. This paraplegia lasted for only a day or two. The temperature rose $I^{\circ}$ or $2^{\circ}$ and there was increased rate of respiration and pulse Under this treatment the delirium subsided rapidly without the use of sedative drugs, and sleep followed. The two deaths occurred in patients whose condition was bad when they were admitted.

\section{INTERNAL SECRETIONS}

Our knowledge of the relationship of disorders of the internal secretions to mental diseases is still in such an unsatisfactory state that a profitable review of the subject is not possible. In certain cases of cretinism there is a high grade of idiocy, but the physical state is the predominant one. In exophthalmic goiter certain of the psychoses may appear. In acromegaly and other pituitary disorders there are also in some cases very marked mental disorders. The treatment of all these affections, however, is determined by the physical rather than the mental state. ${ }^{1}$

There has been a tendency to look for disorders of the internal secretions in cases of dementia precox, but the whole subject is still in a speculative stage. The writer has known thyroid extract to be given by some practitioners in cases of adolescent insanity, but this treatment is entirely empirical, and such practitioners themselves would be unable to give any rational excuse for it. If anything is

1 Vincent, Internal Secretion and the Ductless Glands. In this very complete work very little account is made of mental disorders. Dementia pracox is not even mentioned. 
to be discovered in this field, it will not be by a hit-or-miss polyglandular dosing. We must wait for some scientific knowledge of the subject.

Dercum, in a recent paper, ${ }^{1}$ has reviewed the subject of metabolism in insanity and the rôle of the internal secretions. In dementia precox Fauser found defensive ferments against the sex glands and aginst the cortex. He assumes that a primary dysfunction of the sex glands leads to the entrance into the blood of unchanged sex gland protein, and that in the subsequent breaking up of this protein, substances are formed which are injurious to the cortex. The substance which enters the circulation is, of course, the internal secretion, the hormone, of the sex gland, not the germinal product. The indications for treatment, based upon this occult subject, are still uncertain.

Berkley and Folis ${ }^{2}$ and Kanaval ${ }^{3}$ have reported good results from partial thyroidectomy, on the theory that the thyroid secretion was in excess. Berkely also tried lecithin, testicular, ovarian and thymus juices, nucleins, parathyroid and thyroid extracts, iodothyrin, epinephrin-in fact, the whole gamut of organotherapy. ${ }^{4}$ Ludlum and Corson White, ${ }^{5}$ using the Abderhalden tests, found patients with dementia præcox showing a type similar to that seen in animals from which the thymus had been removed; and they used thymus extract with excellent results in three out of six cases. ${ }^{6}$

\section{PSYCHOANALYSIS}

Much has been written about psychoanalysis, and the subject unfortunately lends itself to endless controversy. There has grown up a school, or a cult, founded largely on the teachings of Freud. ${ }^{7}$ The central doctrine is that of repressed emotions. The patient has at some time suffered a painful emotion, such as fear or mortification, especially on some sexual subject, and by a process of repression has succeeded in banishing the memory of this sexual insult into the background, or rather in crowding it down into the subconscious. Here it continues an independent activity, unknown to the patient, and gradually forces into consciousness a series of reactions, which become the symptoms of the various hysterical and neurasthenic psychoses. Much importance is attached to dreams, in which these repressed

1 Jour. Am. Med. Assn., lxvi, p. I 183.

2 Am. Jour. Insanity, January, Igog.

3 Illinois Med. Jour., September, I 909.

${ }^{4}$ Sajous, New York Med. Jour., Nov. 23 , 1915, for a review of the whole subject.

5 Am. Jour. Insanity, April, I9I 5.

${ }^{6}$ See also a paper by B. Holmes on Dementia Præcox and the Nucleates in the Chicago Med. Recorder, I915, xxxvii, 88. Also a paper on Adrenalin Mydriasis in Dementia Præcox in the Lancet Clinic, 1915, July 31, p. 94. This is a discussion of the physiological fact that adrenalin causes mydriais in dementia præcox, and the possibility of disturbance of the glands of internal secretion acting as a cause of "the infection on which dementia præcox depends."

${ }^{7}$ Hitschmann, Freud's Theories of the Neuroses; Jung, Theory of Psychoanalysis; also articles in the Psychoanalytic Review, New York. 
subconscious emotions are supposed to hold high revel, and in the proper interpretation of which much light is thrown on the underlying mechanism of the psychoses. The interpretation of this subliminal state is also helped by a process of association of ideas, by which the patient is gradually helped to recover, or to resurrect into consciousness, the offending repressed emotions. Thus cure is promoted by a process of "mental catharsis," by which these emotions are cast out.

It is needless to say that in such an occult science there is a wide field for much that is fanciful and extravagant; and even a rapid survey of the literature reveals that it is often the mind of the doctor rather than the mind of the patient that is furnishing a good part of the psychoanalytic output.

The present writer believes in a certain kind of psychoanalysis, and has long practised it, as has probably every physician who has had much to do with nervous patients. Sexual emotional "insults," or memories, associated especially with a sense of shame or personal unworthiness, have long been known to be operative in the psychoses; but the criticism of Freud's doctrine may be based on several objections.

First, it is a pure assumption to say that all hysterical and psychasthenic symptoms are due to such repressed emotions, and it is not clearly made out how such emotions are translated into symptoms. Moreover, the disturbing emotions in these patients are not always repressed, but are sometimes very vividly retained in memory.

Second, dreams have no such substantial foundation in the subconscious as is assumed, but not proved, by this school. The so-called "interpretation" of dreams is often a mere process of auto-suggestion, in which both doctor and patient may be deluded. There is an unlimited field here for pseudoscience.

Third, the process of resurrecting these repressed emotions by using association of ideas is often futile, and the time consumed is not only wasted but the process may be offensive and injurious to the patient.

Fourth, even assuming that the repressed emotions can be recovered, or restored to consciousness, and the patient be tutored to see in them the cause of his or her mental malady, it does not follow that cure will result. Sometimes it is rather the reverse, for the patient may be made distinctly worse.

The present writer has no intention of ignoring what may be of real value in psychoanalysis, but he believes harm may come from trying to construct an elaborate but artificial system upon the basis of a few simple and elementary laws of psychology. Emotions and dreams and the association of ideas are all real factors in the mental life of every individual, and require special study in psychiatry; but it is another thing to erect around them a sacred cult, with all its mystic rites and sacraments. 


\section{HYDROTHERAPY}

This subject was discussed at length in the first edition of this work. ${ }^{1}$ The value of the prolonged neutral bath as a hydrotherapeutic agent for producing quiet and sleep in certain nervous conditions, hysteria, mild excitements, maniacal and anxiety states, has long been recognized. Brierre de Boismont in France and Scholz in Germany used a modified continuous bath for excited patients many years before Kraepelin's observations brought this method into more or less general use. In fact, the use of the bath is as old as Hippocrates. Among the moderns, Currie, in $\mathrm{r} 796$, and Guislain, somewhat later, were advocates of a most roborant "water cure."

About twenty years ago Kraepelin noticed that confused, restless and debilitated patients were greatly benefited by several hours spent in the warm baths. He gradually increased the time to an entire day; and finally the unsatisfactory condition of his excited patients during the untreated or night period led him to attempt the experiment of a really prolonged or "Dauer-bad." Patients are now frequently kept in the baths for weeks or even months without any untoward results. The "Dauer-bad". rapidly came into general favor, until at this time practically every modern mental hospital is equipped with this useful therapeutic adjunct.

The writer has had an opportunity of observing this treatment at the Pennsylvania Hospital for the Insane under the management of Dr. Copp. The following statement has been prepared by Dr. Edward A. Strecker, assistant physician.

The continuous baths have been in use at this hospital for about two years, during which time probably 150 patients have, at some time or another in the course of their psychoses, received this method of treatment. There are two large sunny bath rooms, each containing three tubs, fitted with heavy plate glass windows and doors and equipped with ventilating devices. With the doors and windows closed the rooms are practically soundproof. The temperature is controlled from a temperature table, and the nurse who, is in constant attendance, makes frequent readings from an ordinary bath thermometer. The patients are clothed in a loose-fitting, one-piece garment, permitting great freedom of movement. There is an observation window facing the hall through which every part of the bath room can be easily seen.

Observations of the bodily temperature, pulse rate, respirations and general condition, which were recorded over a period of about six months, show practically no variation from the normal. There are at times fleeting vasomotor disturbances, usually manifesting themselves as deep flushing of the face and chest. They disappear in a short time and do not seem to have any especial significance. Occasionally ephemeral rashes and exfoliations of the skin have been noted. Anointing the body with lanolin has been advised. There have been several cases of discharging ear, usually a furunculosis of

${ }^{1}$ Vol. iii, p. 1028. 
the canal, following the baths, and the plan of plugging the ears with cotton moistened with liquid albolene is being tried. Probably the thorough drying of the ears is the most important prophylactic measure. Kraepelin regards any existing ear disease as a contraindication to the use of the tubs. Most clinicians interdict the use of the tubs during the menstrual period. After the menses have been well established, however, there seems to be no good reason why a greatly excited patient should not be given moderate tub treatment with close observation. The few patients for whom it was deemed advisable, showed no deleterious effects. Advanced cardiac and pulmonary diseases contraindicate the prolonged baths, but even markedly arteriosclerotic patients do not seem to show any ill effects.

The estimation of just how much any isolated therapeutic measure contributes toward a recovery, must, of course, be largely speculative. It would be incorrect to say that the continuous baths cure or even definitely shorten the course of any psychosis; that they constitute an extremely valuable and practical method of controlling excited states, however, cannot be denied. Probably the cases in which the best results are obtained are the confused and restless states, frequently associated with considerable physical debility. Many of the infective-exhaustive group are a good illustration of this type. After five or six hours in the tubs the larger percentage of such patients become quiet and drowsy, and often fall into a sound slumber shortly after being placed in bed. Some clinicians advocate sleeping in the tubs; but when a quiet sleepy state is reached, transferring the patient to bed is desirable. A few of these patients, after they reach a convalescent condition, have expressed the opinion that their recovery was largely due to tub treatment. The excited periods of manic-depressive insanity respond to the prolonged bath in a fair percentage of cases. Here again the treatment is purely symptomatic and not specific. States of excitement of dementia præcox are not infrequently controlled. There is a patient in fairly well advanced dementia pracox in the hospital who begs to be put in the tub at the beginning of an excited period. The restless, excited states, principally of involutional depression, appear to be less favorably affected by tub treatment. A few seem to be benefited, but the larger number show little change. Epileptics are apparently not improved. Patients with general paralysis and delirium tremens have been treated in the baths with a fair degree of success.

The "Dauer-bad" should never be regarded as a specific remedy for any condition. It may possibly have some effect in shortening the course of restless, confusional states, but here again too many factors are operative, and an accurate estimate of the individual value of each remedial measure is difficult. In a fairly large number of excited episodes, varying somewhat with the nature of the psychosis under consideration, the prolonged baths have distinct advantages over other methods of treatment. They require less preparation, and may be continued for much longer periods of time than other 
measures; for instance, than wet packs. The temperature of the water may be easily varied and can at any time be ascertained to the exact degree. No restraint is necessary. If mechanical means are required to keep a patient in the tub, some other form of treatment had better be resorted to. These baths effectively cleanse the body, an important consideration in untidy patients. They permit a wide range of motor activity under good conditions, which may possibly contribute to the production of normal fatigue. They frequently induce sleep. In a certain group of cases they seem to stimulate the appetite and general bodily vigor.

Perhaps the principal disadvantage of the baths is their cost, both as regards installation and operation. Furthermore, the intensive nursing required is a matter of considerable expense. Intelligent care, before, during and after the bath, is imperative, and is the only sure method of avoiding accidents and of realizing the greatest possible value of the treatment. . Probably if the amount saved, under the conditions of the bath in caring for an untidy and destructive patient, could be computed, the importance of these factors would be materially lessened.

It seems a conservative conclusion that the prolonged bath is the most satisfactory method we have of treating a fairly large group of the excited states, which are met with in many of the psychoses. One advantage is that it obviates to a great extent the necessity of giving depressive drugs, although it does not do this in all cases. 


\title{
VASOMOTOR AND TROPHIC DISEASES
}

\author{
By H. C. Moffett, M. D.
}

We are still unable satisfactorily to explain the varied vasomotor and trophic phenomena. Clinical and experimental work of recent years has emphasized the chemical or physical basis of many reactions formerly regarded as primarily of nervous origin. In the varied manifestations of anaphylaxis vasomotor phenomena play a major rôle. The protein poisons of chronic infections and of intestinal disease explain many skin, arthritic and visceral symptoms that formerly were referred to primary vasomotor disturbance. The clinic has abundantly demonstrated the profound trophic changes of soft parts and bones that may accompany aberration of ductless gland function. The mobilization of thyroid, adrenal and other gland secretions at the command of fear and anger has been emphasized of late by Crile and by Cannon and may explain in part the vasomotor and trophic manifestations of emotional disturbances. The studies of Jacques Loeb and MacCallum with calcium and those of Meltzer with magnesium salts have shown the great importance of chemical action in the regulation of nervous phenomena. Based upon the great work of Langley on the sympathetic and autonomic systems pharmacological and clinical investigation has demonstrated more and more frequently the control of many vasomotor and trophic functions by chemical action of hormones or drugs (Meyer and Gottleib, Eppinger, Herz, Barker and Sladen and many others).

No matter just how it may be regulated we nevertheless must still assume that the nervous system exercises great influence over nutrition of the various tissues. Certain facts may be reviewed in regard to trophic mechanisms and trophic disease. From operations on the Gasserian ganglion, it has been shown conclusively that sensory loss alone does not necessarily lead to trophic disturbance (Kraus, Cushing). The influence of disordered rather than diminished function, first suggested by Charcot and Mitchell, has been emphasized by the clinical reports of recent years and by the neurological experiences in the present great war. Possibly special trophic centers in brain cord and sympathetic may exist and may be excited pathologically by partial destruction, by direct irritation or by abnormal impulses from a diseased periphery or conducting apparatus. Wier Mitchell held that irritation of sensory nerves might be transmitted centrifugally and produce peripheral trophic lesions and it is of interest to note from recent descriptions of injuries to nerves by shrapnel and shell that trophic phenomena may develop in distant parts of the body not innervated by the injured nerve. Marinesco

Vol. IV. -59 
and Goldscheider have shown that sensory impressions from the periphery as well as impulses from cortical centers were necessary for the preservation of the integrity of the spinal trophic center for the muscles. Raymond and Hoffa have demonstrated that reflex muscle atrophy in joint affections may be inhibited if the posterior roots be divided and sensory irritations be prevented from acting on the anterior horn cells. Lervaschew showed that irritation of the sciatic may lead to changes in arteries and veins of the extremity. Lapinsky observed vascular changes as the result of neuritis and following section of vasomotor nerves. Recently Lowenthal has studied changes in skin, blood and lymph glands in rabbits following section of the sciatic nerve and has made the interesting observation that eosinophiles increase and collect in distant parts of the body. Not infrequently vasomotor and trophic disturbances are the result of organic lesions of the brain, cord, sympathetic system or peripheral nerves; they are most marked in lesions of the central gray matter of the cord. In the great group of cases for which no sufficient organic nervous lesion can be found there can be no question that personal idiosyncrasy plays a most important part in vasomotor instability. We are wholly unable to define the chemical or physical basis upon which this instability depends. We know that it is frequently inherited. We know that it tremendously increases the individual reaction (probably through the nervous system) to chemical or physical irritants, to poisons like alcohol, or tobacco, to drugs, to protein intoxications, to infections like influenza, to disturbances of internal secretion as of the thyroid or gonads. The various articles of Solis-Cohen, and the monograph of Herz deal fully with this personal or family instability of "vasomotor ataxia.".

In spite of some of the newer conceptions of these disorders there have been no essential changes in their treatment and but few minor changes since the appearance of the original articles in Vol. III. In the treatment of angioneurotic edema calcium lactate sometimes seems of decided benefit in gram doses several times daily. The bromides, valerian preparations or asafetida should be given a trial. Quinin, arsenic, strychnin, nitroglycerin, atropin, epinephrin and pituitrin have all been recommended. Epinephrin and calcium solutions are of value locally in mucous membrane swelling. Highfrequency currents influence subjective symptoms favorably and seem to have some effect in preventing return of the swelling. Treatment by autoserum injections or by intramuscular injection of foreign blood may be mentioned.

Additional cases of scleroderma have shown the worthlessness of thiosinamin. An acute case improved while taking thyroid extract. As noted before thyroid extract has apparently been of value in the hands of Osler, Paulesco and others. Epinephrin had no effect in two cases with pronounced pigmentation. The high-frequency current has continued to be of service in relieving paresthesiæ and seems to influence indurative patches to a slight degree. Persistent 
baking has relieved one of my patients and Cassirer speaks highly of this method of treatment. Until more is known of the etiology and pathogenesis little can be done for patients aside from protecting them from exposure, keeping their nutrition at the best, and avoiding drastic and irritating modes of treatment.

Three cases of the remarkable affection, progressive facial hemiatrophy, have been seen by the author since the first report was written. In two there were remarkable combinations of ductless gland symptoms, suggesting pituitary and adrenal involvement. In another the process attacked both sides of the face and the pectorals and skin covering them were indurated and atrophic. Possibly dystrophy limited for long periods to fat tissue alone may later involve muscle and bone.

The etiology of the disease is still wholly uncertain. It has close relations with scleroderma. There has been no further evidence to support the infectious theory of Moebius. There have been no further encouraging reports in regard to treatment. Resection of the sympathetic has given no favorable results. Temporary cosmetic results may be achieved from the injection of paraffin of high melting point but the method is associated with the danger of embolism and of later lumpy distortion and is not to be advised.

From an excellent review of hypertrophic pulmonary osteoarthropathy recently by Locke of Boston there can be no question that ordinary clubbed fingers seen so frequently clinically and the outspoken phenomena of the long-standing condition described by Marie as osteoarthropathie hypertrophiante pneumonique are simply different stages of the same process. Treatment of the condition is limited to the control of the underlying disease or intoxication. 



\section{INDEX}

ABdomen, sclerosis of blood-vessels of, 639 Abdominal rigidity in septic peritonitis, 731

support in cecum mobile, 715

in visceroptosis, 710

visceral disturbances, postural treatment, 7 I 6

Abscess, alveolar, 578. See also Alveolar abscess.

apical dental, 582

cavities, bismuth paste treatment, 144

dento-alveolar, alveolar abscess and, differentiation, 578

from administration of salvarsan, 284

of liver, 702. See also Liver abscess.

of lung, artificial pneumothorax in, 250 in lobar pneumonia, 215

pelvic, autogenous vaccines in, 735

perigastric, in peptic ulcer, treatment, 6r3

peritonsillar, 5 r8. See also Quinsy.

pulmonary, bronchoscopy in, 554

retropharyngeal, in infantile la grippe, 574

subcutaneous, in erysipelas, 275

tooth, iritis from, 386

Acapnia, 95

Acariasis, 4I 7

chloroform in, 417

linseed oil in, 419

olive oil in, 419

sulphur ointment in, 418

treatment, $4 \mathrm{I} 8$

turpentine in, 419

Acetanilid in acute nephritis, 767

Acetone as antiseptic, 27

bodies, excessive, in acidosis in children, 503,504

Acetonuria in mumps, 336

Acetphenetidin in acute nephritis, 767

Achylia gastrica, pancreatic achylia associated with, 708

pancreatic, 708. See also Pancreatic achylia.

Acidosis as cause of uremia, $8_{3} 8$

control of, in Allen treatment of diabetes, 495

definition, $50 \mathrm{r}$

in children, 5 or

bibliography, 507

causes, 503

clinical types, 504

detection, 501

by determination of carbon dioxid tension in alveolar air, 502

by laboratory methods, 502
Acidosis in children, determination of atkali reserve of blood in, 502

of ammonia coefficient of urine in, 503

of oxygen-combining power of hemoglobin in, 503

diagnosis, $5 \circ 3$

diet in, 505,507

from diarrhea, 504

treatment, 505

from excessive formation of acetone

bodies, 503, 504

glucose in, 507

hyperpnea in, 501

in burns, 504

in ileocolitis, 504

in nephritis, 504

intravenous saline solution in, 505

prophylaxis, 505

recurrent vomiting in, 504

sodium bicarbonate in, 505,506

treatment, $50 \mathrm{O}, 504$

Van Slyke's test for, 502

varieties, 503

water in, 506

in diabetes, $49 \mathrm{I}$

effect of Allen treatment on, 497

estimation of, $49 \mathrm{r}$

hyperpnea in, 501

sodium bicarbonate solution in, 497

in edema, treatment, 809

in lowered functional power of liver, 687

in uremia, treatment, 845

Acids, fatty, in feces in pernicious anemia, $42 \mathrm{I}$

Acne, staphylococcic vaccines in, 42 vulgaris, roentgen-ray therapy in, $\mathbf{x} 3$

Aconite in functional heart diseases, 484

in quinsy, 520

Acquired anaphylaxis, 157

Acromegaly, 467

treatment, 467

Acroparesthesia, high-frequency effluve in, $1 \times 5$

vacuum electrode in, 115

Actinomycosis of mouth, 605 treatment, 605

of pleura, treatment, 571

Addison's disease, 466

adrenal therapy in, 466

diagnosis, 466

epinephrin in, 466

tuberculosis in, treatment, 466

Adenitis, tuberculous, deep roentgen-ray therapy in, 136

Adenoids, removal of, in dyslalia, 524 
Adenoma of bile-ducts, 704

of liver, 704

of thyroid gland, treatment, 47.3

Adhesive strapping after artificial pneumothorax in hemoptysis, 545

in acute fibrinous pleuritis, 567

in chylothorax, $57^{\circ}$

Adrenal disease, 465 symptoms, 466

preparations in low arterial pressure, 466

therapy in Addison's disease, 466

Adrenalin chlorid in aneurysm of aorta, I 55

effect of, on bronchial constriction, 536

on pancreatic secretion, 706

on sympathetic nervous system, 9 10

in anaphylactic shock of food poisoning, I 59

in asthma, 538,539

in cardiac dyspnea, 483

in Cheyne-Stokes respiration, 483

in chronic diarrhea, 652

in intestinal stasis, $65^{\circ}$

in pertussis, 333

in septic peritonitis, 735

in septicopyemia, 270

in yellow fever, 356

Adrenin as cause of hypertension, 816

African trypanosomiasis, 371. See also Trypanosomiasis, African.

Agar-agar in constipation, $66_{2}$

in diverticulitis, 642

in hypertension, $82 \mathrm{I}$

Age in etiology of arteriosclerosis, 8I 7 of chronic interstitial nephritis, 775 of nephritis, 752

Agglutination test in diagnosis of undulant fever, 353,354

Agglutinins in blood after typhoid inoculation, I95

Ague cake with cachexia in malaria, treatment, $35^{2}$

Air-passages, upper, acute catarrhal diseases of, autogenous vaccines in, 28

Air-space required for factory workman, I 72

Albumen as antidote, 163

in corrosive sublimate poisoning, 164

Albuminuria, 800

anemia associated with, 803

cyclic, $80 \mathrm{I}$

exercises in, 804

febrile, 803

from passive congestion, 803

hemic changes in, 803

hygienic treatment, 804

in diabetes, 803

in infectious diseases, $8 \mathrm{O}_{3}$

in nephritis, 800

in pregnancy, 803

in yellow fever, 308

leukemia associated with, $8 \circ 3$

orthostatic, 801

adolescence in, etiology, 8or
Albuminuria, orthostatic, diet in, 804

effect of exercise on, 802

etiology, 801

Huebner's theory of, 802

prognosis, 802

sex in, etiology, 801

treatment, 804

urine in, 802

vascular disturbances in, etiology, 802

physiologic, 800

postural, 8or

etiology, 8or

purpura associated with, 803

treatment, 803

with definite lesions of urinary organs, 803

without coarse organic renal changes, $80 \mathrm{I}$

Albuminuric retinitis in malignant hypertension, 8I 5

Alcohol and lead-water in erysipelas, 277 chronic interstitial nephritis from, 775 dressings in cancrum oris, 580 effect of, on pancreatic secretion, 706 in Allen treatment of diabetes, 492

in asthma, 538

in delirium tremens, 167

in heart diseases, 486

in myocardial insufficiency, 828

in septicopyemia, 270

in yellow fever, 314

injection in trigeminal neuralgia, 870

in trigeminal neuralgia, complicacations, 872

duration of relief in, 870

keratitis from, prevention of, 872 nephritis from, $25 \mathrm{I}$

Alcoholic poisoning, I65. See also Delirium tremens.

stimulants in aortic regurgitation, 480 Aleppo boil, $35^{8}$

Algiers, recurrent fever of, 362

Alkali excretion in diseased kidney, 796 in healthy kidney, 796

Alkalies, administration of, in non-obstructive peptic ulcer, 617

effect of, on gastric secretion, 29

Allen treatment of diabetes, $49 \mathrm{I}$

alcohol in, 492

charts in, 493, 494

control of acidosis in, 495

diet tables for, 498-500

effect on acidosis, 497

exercise in, 495

fasting in, 492

first feeding in, 492

green vegetables in, 492

increasing carbohydrates in, 495

initial fast in, 495

in surgical infections, 497

in tuberculosis, 497

ketonuria in, 495

limitations, in general practice,496, 497

meats in, 495 
Allen treatment of diabetes, prepared diet lists in, 495

principles of, 492

technique, 492

testing urine in, 495

Altitudes, effect of, on hemopoiesis, 94

Alum in aphthous stomatitis, 603

Aluminum filters for roentgen rays, 132

Alveolar abscess, 578

apical, 597

autogenous vaccines in, 599

bibliography, 599

cellulitis in, 598

course, 598

dental treatment, 598

metastatic complications in, 598

micrö̈rganisms in, 598

origin, 597

pain in, 598

surgical treatment, 599

systemic symptoms, 598

as cause of chronic infections, 579

autogenous vaccines in, 579

dento-alveolar abscess and, differentiation, 578

diagnosis, 578

epidemic occurrence, 578

etiology, 578

gingival, course, 598

hematogenous origin, 578

intermediate, $5^{82}$

lateral, 582

microörganisms in, 578

nephritis from, 749

roentgen rays in diagnosis, 579

subgingival, 597

autogenous vaccines in, 599

bibliography, 599

cellulitis in, 598

dental treatment, 598

metastatic complications in, 598

microörganisms in, 598

origin, 597

pain in, 598

surgical treatment, 599

systemic symptoms, 598

treatment, 579

periosteitis, 583

pyorrhea, 582

Alveolitis, 583

Alveolodental pyorrhea, 583

Ambard's coefficient of urea excretion, 793

Amblyopia from optochin in lobar pneumonia, 236, 238

Ameba dysenterix, carriers of, $34^{\circ}$

effects of emetin on, 339

encystment, after attack, $34^{\circ}$

Amebic dysentery, 337

carriers of, 340

castor oil in, $337^{\circ}$

chronic diarrhea in, treatment, 653

emetin in, 30, 337, 338

administration per os, 338

clinical cure, 339

dose, 339
Amebic dysentery, emetin in, duration of treatment, 339

effect on liver abscess, 339

effects and results of, 330

intravenous injection, 339

limitations of, 339

methods of administration, 338

subcutaneous method, $33^{8}$

untoward symptoms from, 338

encystment of ameba after, 340

ipecac in, 337

method of transmission, 340

tincture opii in, 337

treatment, 337

Amebic pyorrhea, 587

America, relapsing fever of, $36 \mathrm{I}$

American forest leishmaniasis, 359

spas, 89

trypanosomiasis, 377

Ammonia, aromatic spirits, in pulmonary edema, 548

coefficient in urine, determination of, in acidosis in children, 503

in erysipelas, 276

Ammonium chlorid in intestinal teniasis, 393

Amorphous quinin in malaria, advantages of, 350

Analgesia in leprosy, 341

Anaphoresis, 98, II 3

Anaphoric influence of constant electric current, 98

Anaphylactic shock, $x_{58}$

from pollen extract in hay fever, 533

treatment, 159

Inaphylaxis, acquired, 157

alien proteins in, 157

cows' milk, 668

definition, $\times 57$

food poisoning from, animal inoculation in, diagnosis, 159

clinical manifestations, $15^{8}$

cure of, 159

prevention, 159

skin diseases in, 158

treatment, 158,159

Von Pirquet test in, 158

from foreign proteins instilled into nose, 536

in asthma, 535

calcium chlorid in, $53^{6}$

inherited, 157

in cerebrospinal meningitis, 79

method of entrance of alien protein in, 158

reacting dose ir, $x_{57}$

relation of immunity to, 157

sensitizing dose in, 1.57

to food proteins, 157

to plant pollens in hay fever, 528

Anasarca. See Dropsy.

Anelectrotonus, 98

Anemia, arsenic in, 807

associated with albuminuria, 803

edema in, treatment, 807 
Anemia in acute nephritis, treatment, 768

in hemolytic icterus, treatment, 43 ?

in myocardial insufficiency, treatment, 834

in uremia, treatment, 847

pernicious, 420

appendicostomy with irrigation of bowel in, 429

arsenic in, 426

atrophy of gastrointestinal mucosa in, $42 \mathrm{I}$

bibliography, 429

blood transfusion in, 425

bone marrow changes in, $42 \mathrm{I}$

Bothriocephalus latus in, 420

cholesterin in, 427

color of skin in, $42 \mathrm{I}$

course, 423

diarrhea in, treatment, 425

dietetic treatment, 425

disappearance of Bacillus capsulatus aërogenes in, 420

etiology, 420

fatty acids in feces in, $42 \mathrm{I}$

focal infection in, treatment, 425

from blood hemolysis, $42 \mathrm{I}$

from secondary anemia, 420

general care, 425

glycerin in, 427

Grawitz treatment, 425

hydrochloric acid in, 429

in pregnancy, 420

treatment, 425

iron in, 429

male fern in, 429

of horses, 422

prognosis, 423

recovery in, 424

relation of focal infection to, 420

remission in, 423

salvarsan in, 427

sodium cacodylate in, 427

spleen in etiology, 422

splenectomy in, 427,456

blood crisis after, 456

effect of, on urobilin, 428

splenectomy in, results, 457

syphilis in, 420

treatment, 425

thorium $X$ in, 429

treatment, 424

open-air, 425

urobilin in feces in, $42 \mathrm{I}$

secondary, 443. See also Anemia, symptomatic.

splenic, 433

autointoxication as cause, 434

bibliography, 435

course, 434

etiology, 433

from chronic toxemia, 434

hematemesis in, 434

rickets as cause, 434

roentgen rays in, 435

salvarsan in, 435
Anemia, splenic, splenectomy in, 428, 434 syphilis as cause, 434

thrombophlebitis of splenic vein as cause, 434

treatment, 434

symptomatic, 443

arsenic in, 448

blood transfusion in, 446

climatic treatment, 445

diet in, 445

due to infection, chlorosis and, differentiation, 431

etiology, 443

from constipation, treatment, 445

from extensive purpura, 444

from infection, 443

from intestinal parasites, 444

from loss of blood, 444

treatment, 445

from malignant growth, 444

from mineral poisons, 443

from tuberculosis, 444

from typhoid fever, 444

in acute articular rheumatism, 444

in uremia, 839

intravenous injection of citrated blood in, 446 of saline solution in, 447

iron in, 447

mineral waters in, 447

pernicious anemia after, 420

treatment, 444

temporary, after splenectomy, 454

Anesthesia in bronchoscopy, $\mathbf{5 5 4}$

in septic peritonitis, 736

local, in artificial pneumothorax, $25 \mathrm{I}$ in hemoptysis, 545

Aneurysm of aorta, 155

spontaneous cure in, ${ }_{55}$

treatment, 155

quinsy and, differentiation, 520

saccular, constant electric current in, 118

wiring with electrolysis in, 155 technic, 156

Angina, Ludwig's, 580

pectoris, treatment, 487

Angioneurotic edema, treatment, $93^{\circ}$

Angiostomoidea superfamily, 400

Animal inoculation in diagnosis of anaphylactic food poisoning, 159

irritants, asthma from, 535

parasites, 389

methods of examination of feces for, 389.

technic of microscopic examinations for, 389

Anions, 98

Ankylostoma duodenale, 406

Ankylostomiasis, 406

betanaphthol in, 407

castor oil in, 407

chlorosis from, treatment, $\mathbf{4 3 2}$

eucalyptol in, 407

oleum chenopodii in, 407

thymol in, 407

treatment, 407 
Anode effects of constant electric current, 99

Anthelmintics, 393

Anthomyia pluvialis, 410

Anthrax, 325

carbolic acid injections in, 325

excision of focus in, 325

septicemia, 325

serum treatment, 325

surgical treatment, 325

Antianthrax serum, 325 preparation of, 325

Antibacterial serum, 217 titration of potency of, 220

Antibodies in blood after lobar pneumonia, 222

specific, human convalescent blood with, therapeutic use of, $8 \mathbf{I}$

Anticoagulins, use of, in blood transfusion, 155

Antidiphtheritic serum, 242 average dose for immunization, 242

characteristics, 242

dosage, 58,62

duration of immunity from, 242

effects of, 245

factors regulating dosage, 243

importance of early use, 60

in hemophilia, 443

in hemorrhage in chronic jaundice, 702

intramuscular injection, 61, 243

intravenous injection, 61,243

method of administration, 59

modes of injection, 243

nephritis from, 749

subcutaneous injection, $6 r$

table of dosage, 60

therapeutic use, 58

time for injection, 243

transmission to child in utero, 66

United States standard, 242

use of, in doubtful cases, 243

Antidotes, chemical, to poisons, 163

Antimeningitis serum, 77

addition of preservatives to, 78

effect of, on mortality, 78

Flexner's, 296

gravity method of injection, 78

intraspinal injection, 77

method of administration, 78

repetition of administration, 79

size of dose, 79

standardization of, 77

Antimony in African trypanosomiasis, 375

in kala-azar, 357

Antiperistalsis in constipation, 656

Antipneumococcus serum, 216 antigenic relations of strains in, 220

history of development, 218

in lobar pneumonia, 218

Neufeld and Händel's, 220

Pane's, 218

Rockefeller Institute, 223

effects of, un progress of disease, 226
Antipneumococcus serum, Rockefeller Institute, effects of, on temperature, 225

method of administration, 225

preparation, 224

results from, 225

Römer's, 2 ig

sterilization of blood by, 226

Antipyrin in acute nephritis, 767

in pertussis, 333

Antiseptic, Dakin's, 268. See also Dakin's antiseptic.

Antiseptic treatment, 27

Antiseptics, cutaneous, 27

in chronic diarrhea, 653

in septic peritonitis, 737

in septicopyemia, 272

intestinal, 29

local, in tetanus, 326

urinary, in acute nephritis, 768

Antistaphylococcus serum, 8r

Antistreptococcus serum, 79

action, 80

deterioration, 80

dosage, 80

effects, 80

for prevention of infection, $8 \mathrm{I}$

hemolyzing type, 80

in erysipelas, 277

in scarlet fever, 80, 246

local application, 8r

method of injection, 80

mixed, 80

selection of cases for, 8 r

Antitetanic serum, 7 1, 326

exacerbation of symptoms after use, 73

intraneural injection, 328

intraspinal injection, 72

disappearance from spinal canal after, 73

results, 74,75

intrathecal injection, 329

intravenous injection, $7 \mathrm{I}$

method of applying to wounds, 326

Park and Krumwiede's method of injection, 74

prophylactic use, 71,326

Antithrombin in hemorrhagic diseases, 438

Antithyroidin in functional nervous dis-

eases, 9 I 2

Antitoxin, diphtheria. See Antidiphtheritic serum.

tetanus, 7 r, 326. See also Antitetanic serum.

Antityphoid vaccines, 192

methods of administration, 192

Anuria in acute nephritis, treatment, 766

in uremia, treatment, 847

Aorta, aneurysm of, 155 spontaneous cure in, 155

treatment, I 55

Aortic regurgitation, 480 alcoholic stimulants in, 480 delirium in, treatment, 480 
Aortic regurgitation, headache in, treatment, 480

hyoscin hydrobromate in, 480

insomnia in, treatment, 480

potassium iodid in, 480

Aphasia, congenital; dyslalia from, 524, 526

Aphthous stomatitis, 601

treatment, 602

Apical alveolar abscess, 597. See also Alveolar abscess, apical.

dental abscess, $5^{82}$

Apinealism, treatment, 469

Apocynum in lowered functional power of liver, 688

Apomorphin hydrochlorate as emetic, 29 in delirium tremens, 165

Appendicitis, chronic, cecum mobile and, differentiation, 7 I3

nephritis from, 749

from oxyuriasis, 406

septic peritonitis in, surgical treatment, 736

septicopyemia from, treatment, 267

Appendicostomy with irrigation of bowel in pernicious anemia, 429

Appetite, stimulation of, in septicopyemia, 270

Arachnida, 4I7

Armed tapeworm, 393

Arrhythmia in septicopyemia, 266

Arsacetin in African trypanosomiasis, 374.

Arsenic in African trypanosomiasis, 374

in anemia, 807

in chlorosis, 431

in erythremia, 433

in hemolytic icterus, 437

in Hodgkin's disease, 462

in leprosy, 342

in leukemia, 137,451

in pernicious anemia, 426

in symptomatic anemia, 448

in syphilis, 282

nephritis from, $750,75^{\mathrm{I}}$

Arsenophenylglycin in African trypanosomiasis, 374

Arterial circulation, deficient local, baths in, 85

pressure, low, adrenal preparations in, 466

tension, low, in edema, treatment, 810

Arteriosclerosis, 8 I 6

acute infections in etiology, 817

chronic interstitial nephritis from, 775 etiology, 816

heredity in etiology, $8 \mathrm{I} 7$

high-frequency current in, I 20, 487

contraindications to, I 21

hypertension in etiology, $8 \mathrm{I} 6$

in nephritis, $8 \mathrm{r} 6$

intoxications in etiology, 817

overeating in etiology, $8 \mathrm{I} 7$

sclerosis of blood-vessels of abdomen in, 639
Arteriosclerosis, sex in etiology, 817 syphilis in, etiology, 8I7

Arteriosclerotic kidney in chronic interstitial nephritis, 774

Arthigon, 45

Arthritis deformans, 510

atophan in, 5 I 2

autogenous vaccines in, 5 ro

chronic infections as cause, 5 ro

degenerative, baths in, 86

thyroid treatment, 465

dangers from restricted diet in, 5 I I

diseased teeth as cause of, 5 ro etiology, 5 Io

infected tonsils as cause of, 5 to

nasal sinus infections as cause of, 510

radium in, 512

treatment, 510

in erysipelas, 275

purulent, pneumococcus in, 2 I 5

rheumatoid, electrotherapy in, I 6

secondary septic, in septicopyemia, 266

Articulation, faulty, 523. See also Pseudolalia.

Articulatory element of speech in dyslalia, 524

Artificial pneumothorax, 249. See also Pneumothorax, artificial.

Ascariasis, 404

castor oil in, 405

chloroform in, 405

oleum chenopodii in, 405

prevalence in children, 405

prophylaxis in, 405

symptoms, 405

treatment, 405

wormseed in, 405

Ascaris lumbricoides, 404

Aschoff's classification of nephritis, 755

Ascites in acute nephritis, treatment, 766 in edema, surgical treatment, 8II in uremia, treatment, 846

Ashford's modification of James' method of staining malarial parasite, 346

Aspiration in acute serofibrinous pleuritis, 569

injections following, 569

repeated, 569

in tuberculous peritonitis, 744

of chest, novocain injection in, 142

Robinson's method, 142

Aspirin in tabes dorsalis, 875

in yellow fever, 315

Asthma, 533

adrenalin in, 538,539

alcohol in, 538

anaphylaxis in, 535

calcium chlorid in, 536

atropin in, 538

autogenous vaccines in, 537

baths in, 87

bronchial, in anaphylactic food-poisoning, 158

calcium chlorid in, 538

epinephrin in, 467 
Asthma, eradication of focal infections in, 537

faradization in, 539

from animal irritants, 535

from plant irritants, 536

galvanization in, 539

hay, 528. See also Hay fever.

hygienic treatment, 538

immunization in, 539,540

in intestinal stasis, treatment, 649

in uremia, treatment, $\mathbf{8 4 6}$

morphin in, 538

of bacterial origin, 536

pathogenesis, 533

peripheral origin, 537

physical examination in, 537

pituitary extract in, 539

prophylaxis, 537

psychoanalysis in, 539

treatment of attack, 538

Atelectasis of newborn, pulmotor in, 549

Atmospheric air, sterile, in artificial pneumothorax, 253.

Atophan in arthritis deformans, $5 \times 2$ in gout, 514

Atoxic vaccines, 37

Atoxyl in African trypanosomiasis, 374

Atoxyl-emetic in African trypanosomiasis, 375

Atrophy from fat indigestion in infants, $6 ; \mathrm{r}$

of gastrointestinal mucosa in pernicious anemia, $42 \mathrm{I}$

of kidney in chronic interstitial nephritis, 774

of optic nerve in pituitary lesions, 869 of testicles in mumps, prevention, 336

Atropin, effect of, on autonomic nervous system, 9 IO

on pancreatic secretion, 706

in asthma, 538

in chronic diarrhea, 652

in heart diseases, 479

in intestinal stasis, $65^{\circ}$

in opium habit, 169

in tabes dorsalis, 875

sulphate in anaphylactic shock of food poisoning, 159

in pulmonary edema, $54 \delta$

Auchmeromyia luteola, 4 ro

Auditomoteur, 524

Auricular fibrillation, digitalis in, 32, 479

Autochthonous vaccines in undulant fever, 354

Autocondensation couch, I 13

Autogenous vaccines in acute catarrhal diseases of upper air passages, 28

in alveolar abscess, 579

in apical alveolar abscess, 599

in arthritis deformans, 510

in asthma, 537

in lobar pneumonia, 231

in pelvic abscess, 735

in pyorrhea alveolaris, 28

in septicopyemia, $\mathbf{2 7 2}$

in subgingival alveolar abscess, 599
Autointoxication in etiology of dementia precox, 908

of manic-depressive insanity, 908

of melancholia, 907

of neurasthenia, 907

of psychasthenia, 908

in insanity, treatment, 922

intestinal, 643. See also Intestinal stasis.

splenic anemia from, 434

Autolysates, 36

Automassage of large intestine in intes-

tinal stasis, 646

Automatonism, mental, in African trypanosomiasis, 373

Autonomic nervous system, effect of atropin on, 910 pilocarpin on, 9 ro

relation of intestinal stasis to, 650

Autosalvarsanized serum in cerebrospinal syphilis, $89 \mathrm{I}$

Autoserotherapy in hypopyon keratitis, 388

in typhoid fever, 200

Autotoxemic iritis, treatment, 386

Avitaminoses, 157,160

Azodolen in corneal ulcers, 385

Azotemic nephritis, 756

B ABy talk, 523. See also Pseudolalia.

Bacillary dysentery, serum treatment, 76

vaccines in, 50

Bacillus acidophulus in intestines in typhoid, 205

aërogenes capsulatus, septicopyemia from, 265

Bordet-Gengou, in whooping-cough, 330 bronchisepticus, 330

capsulatus aërogenes, disappearance of, in pernicious anemia, 420

carriers, chronic, 56

coli in scptic peritonitis, 730

septicopyemia from, 265

vaccines from, $5 \mathrm{I}$

enteritidis vaccines, 35

Friedländer infections, vaccines in, 52 septicopyemia from, 265

lepræ, $34 \mathrm{I}$

lymphangiticus in filariasis, 400

mucosus capsulatus vaccines, 235

pertussis, 330

pneumonix in lobar pneumonia, 215

proteus, septicopyemia from, 265

vaccines from, 5 I

pyocyaneus, septicopyemia from, 265 vaccines from, $5 \mathrm{I}$

Sanarelli's, in yellow fever, $30 \mathrm{I}$

tubercle, in pleuritis, $5^{64}$

typhoid. See Typhoid bacilliw.

$X$ in yellow fever, 302

Back brace in visceroptosis, 719,723 , 724

Backache in acute nephritis, treatment, 767 
Bacteremia, disappearance of, after passive immunization in typhoid, 198

in septic peritonitis, $73 \mathrm{I}$

in typhoid fever, 183

Bacteria, digested, vaccines from, 36 fermentative indigestion in infants, 680 in infectious diarrhea in infants, 683

in urine in nephritis, 749

killed, injection of, in scarlet fever, $24^{6}$, 247

vaccines from, 36

live, vaccines from, 37

sensitized, vaccines from, 37

Bacteria-free vaccine virus, 294 advantages of, 295

Bacterial extracts, 36

flora of intestines in typhoid, changes in, 205

pyorrhea, iodine in, 587,596

vaccines in, 596

vaccines, 33

Bahr's classification of sprue, 367

Baking treatment in scleroderma, 93I

Balboni's needle for artificial pneumothorax, 262

Baldwin's adjustable guard for Floyd's artificial pneumothorax needle, 262

Balneotherapy. See Baths.

Bananas in sprue, 370

Banti's disease, differential diagnosis, 457 therapeutic splenectomy in, 454

Barium chlorid in anaphylactic shock of food poisoning, I 59

platinocyanide pastilles of SabouraudNoire in estimating dosage of roentgen rays, I 30

Bartonella bacilliformis, 355

Bassler's modification of Finhorn's stomach electrode, I 7

Bastedo's diet in constipation, 66I

Bastian's primary couplet, 524

Bath spa, principal characteristics, 87 static, effect of, ror technic for application, i ro

train for troops in the field, 92

Baths, cold, effect of, in acute nephritis, 760 in typhoid fever, 92

electric cabinet, in pulmonary edema, $54^{8}$

for workmen, I77

hot, for contusions and bruises, $8_{3}$ in opium habit, 170 vapor, in pulmonary edema, 548

hyperthermal, in acute cases in military practice, 83

in acute cases in military practice, 83 fibrositis, 85 nephritis, 765

in asthma, 87

in cardiac dilatation, 86

in cases after operation in military practice, 85

in chronic degenerative arthritis, 86 eczema, 87 interstitial nephritis, 780
Baths in chronic pancreatitis, 707 parenchymatous nephritis, $\mathbf{7 7 3}$ rheumatism, 85

synovitis, 86

in circulatory disorders, 86

in colon irritation 87 ,

in constipation, 87

in convalescence from rheumatic fever, 86

in cutaneous disorders, 87

in defective peripheral circulation, 86

in deficient local arterial circulation, 85

in digestive disorders, 87

in dyspepsia, 87

in edema, $81 \mathrm{I}$

in fractures with osteitis and necrosis, 84

in frost-bite, 85

in Graves' disease, 86

in hepatic disorders, 87

in imperfectly healed fractures, 84

in incipient tuberculosis, 87

in insanity, 926

contraindications to, 927

results, 927

in insomnia, 86

in joint strains and sprains, 84

in leprosy, 342

in lumbago, 86

in medical cases in military practice, 85

in melancholia, 87

in military practice, contraindications to, 84

in muscular rheumatism from fatigue and exposure, 85

in nephritis, 851 , 852

in nervous disorders, 86

excitement, 86

in neuralgia, 86

in opium habit, 169

in palsies, 87

in pancreatic achylia, 708

in respiratory catarrhs, 87 disorders, 87

in rheumatic disorders, 85

in sciatica, 86

in septicopyemia, 270

in surgical cases in military practice, 83

in tabes dorsalis, 875

in tachycardia, 86

in treatment of pancreatic calculi, 708

in unresolved effusions, 84

in uremia, 843

in visceroptosis, 724

in yellow fever, 3 I 5

prolonged in painful scars, 84

in unhealed wounds, 84

subthermal in acute cases in military practice, 83

sweat, in corrosive sublimate poisoning, 165

thermal, in acute cases in military practice, 83

in fractures near joints with immobility, 84

Bed bugs, extermination of, 4 I 4 
Beef tapeworm, $39 I^{\prime}$

Behring's vaccine in diphtheria, duration of immunity from, 242

Bell electrode, I I I

Belladonna, in chronic diarrhea, 652 in intestinal stasis, 650 in pertussis, 333

Benzidin colors in African trypanosomiasis, 375

Benzine in pediculosis, 415

Benzol in erythremia, 433

in leukemia, $137,45^{\circ}$

dosage, 451

effects of, 450

precautions in administration, $45 \mathrm{I}$

Beri-beri, I60, 364

bibliography, 367

cardiac crises in, 366

insufficiency in, 364

constipation in, treatment, 366

dietetic treatment, 365

digitalis in, 366

dry, $3^{6} 5$

edema in, 364

electrical stimulation in, 366

etiology, 364

deficiency of vitamines in, 364

exercise in, 366

Funk's base in, 365

geographic distribution of, 160,364

infantile, $36_{5}$

treatment, 366

massage in, 366

muscle cramps in, treatment, 366

neuritis in, treatment, 160

peripheral neuritis in, 364

polished rice in etiology, 364

prophylaxis, 160,367

rice in etiology, 160

ship, 365,366

strychnin in, 366

symptoms, 160, 364

treatment, 161,366

dietetic, 366

wet, 365

Besredka's sensitized typhoid vaccine, 47

Betanaphthol in ankylostomiasis, 407

in chlorosis, 432

in chronic diarrhea, 653

in intestinal stasis, 649

Bichlorid of mercury poisoning, 164. See also Corrosive sublimate poisoning.

Bile-ducts, adenoma of, 704

carcinoma of, treatment, 705

common, drainage of, 699

fibroma of, 704

hemangioma of, 704

infections, indications for operation in, 701

infections, medical treatment in, indications for, $70 \mathrm{I}$

tumors of, 704

Bile-passages, infections, drainagc opera-

tions in, 695

carcinoma from, 694

pancreas in, 694
Bile-passages, infections, surgical treatment, 695

systemic effects, 694

treatment, 695

Bilharzia hematobia, 395

Bilharziasis. See Distomiasis, venal.

Biliary fistula, surgical treatment, 695

tract, internal drainage of, 698

Billroth's method of partial gastrectomy in cancer of stomach, 631

Biological products, distribution of, by Pennsylvania Department of Health, 22

Bismuth in scpticopycmia, 270 paste, preparation, 145

technic of injection, 145

treatment of empyema, 145

of suppurating sinuses and abscess cavities, I 44

of tuberculous coxitis, 145

salicylate in chronic diarrhea, 653

salts in trichinosis, 402

subcarbonate in corrosive sublimate poisoning, $16_{5}$

in non-obstructive peptic ulcer, 617

Biting flies, adult, varieties, $4 \mathrm{I} 2$

Black vomit in yellow fever, 308 treatment, 315

Blackwater fever, yellow fever and, differentiation, 310

Bladder, inflammation of, chronic, 863 nephritis from, 749

papillomata of, fulguration in, 866

surgical treatment, 866

simple chronic ulcer of, 864 treatment, 865

tumors, 865

D'Arsonval bipolar current in, 863

Oudin monopolar current in, $86_{5}$

Paquelin cautery in, 866

radium in, 866

treatment, 865

Blaud's pills in chlorosis, 431

Bleeding. See Venesection. gums, $5^{83}$

Blepharitis, ionic medication in, $38 \mathrm{x}$ sulphate of zinc in, 381

treatment, 381

Blindness with choked disk in tetanus, 331

Block-tin electrodes for diathermy; I I 3

Blood, alkali reserve of, determination, in acidosis in children, 502

antibodies in, after lobar pneumonia, 222

biochemical change in, in hemorrhagic diseases of newborn, $44^{\mathrm{I}}$

citrated, injection of, in hemorrhagic diseases, 439

in symptomatic ancmia, 446

coagulability in hemophilia, 442

coagulation in hemorrhagic discases of newborn, 44I

crisis after splenectomy in pernicious anemia, $45^{6}$

cultures in diagnosis of typhoid fever, I 86

technique, 187

of undulant fever, 354 
Blood diseases, 42

treatment, 32

examination for prognosis in typhoid fever, 187

in diagnosis of cerebrospinal syphilis, 886

in typhoid in prognosis, 187

formation, impaired, chlorosis from, 430

freezing-point of, in renal disease, 798

hemolysis, pernicious anemia from, 42I

human convalescent, with specific anti-

bodies, therapeutic use, $8 \mathrm{I}$

immune bodies in, in lobar pneumonia, 226

in African trypanosomiasis, 373

in chronic diarrhea, treatment, 653

loss of, symptomatic anemia from, 444

treatment, 445

non-protein nitrogen in, in health, 798 in renal disease, 798

micro-Kjeldahl method of determining, 799

occult, in obstructive peptic ulcer, 625

organisms in, in septicopyemia, 266

platelets, lessened, in hemorrhagic diseases, 438

pneumococcus in, in lobar pneumonia, 226

rabbit's, subcutaneous injection, in hemophilia, 443

serum, convalescent, in scarlet fever, $8 \mathrm{I}$ human, in poliomyelitis, 82

in acute anterior poliomyelitis, 880

in hemoptysis, 542

sterilization of, by antipneumococcus serum, 226

transfusion, 146. See also Transfusion, blood.

urea in, determination, by Marshall's method, 799

whole, transfusion of, in hemorrhagic diseases, 439

Blood-examination in lowered functional power of liver, 687

in peptic ulcer, 61 2

Blood-letting, 142. See also Venesection.

Blood-pressure, falling, in septicopyemia, treatment, 270

high, in acute nephritis, 764

in exophthalmic goiter, 472

in lowered functional power of liver, 687

in septicopyemia, 266

Blood-sugar, estimation of, in diabetes, 489

normal, 489

Blood-vessels, diseases of, electrotherapy in, 118

of abdomen, sclerosis of, 639

Boards of Health, city, establishment of,

in Pennsylvania, I7

Body louse, 4I4

Boehm apparatus for administration of salvarsan, 284

Boil, Aleppo, 358

Bombay, recurrent fever of, $36 \mathrm{I}$
Bone black, as antidote, ${ }^{6} 6_{3}$

lesions from typhoid bacillus, 185

marrow changes in pernicious anemia, 421

effect of splenectomy on, 428

Bordet-Gengou's bacillus in whooping cough, 330

Boric-acid glycerin in aphthous stomatitis, 603

Bothriocephalus latus in pernicious anemia, 420

Bournemouth Spa, principal characteristics, 88

Bowman and Heidenhain's theory of kidney function, 786

Bradycardia in mumps, 335

in septicopyemia, 266

Brain complications in pertussis, 332 edema of, as cause of uremia, 837 softening of. See Paresis.

Brass-point electrode, single, I I I

Brazilian dermal leishmaniasis, 358 methylene-blue in, 359

Breeze, static, effects of, IOI in headache of neurasthenia, 1 I 5 technique for application, Ios

Bridge of Allan Spa, principal characteristics, 88

Brighton Spa, principal characteristics, 88

Bright's disease. See Nephritis.

Brill's disease. See Typhus fever.

Broad-backed human type, 716,718

Bromids in delirium tremens, 167

in septicopyemia, $27 \mathrm{I}$

in trichiniasis, 402

Bronchi, foreign body in, bronchoscopy for removal, 554 methods, 561,562

malignant disease of, radium in, 552 roentgen rays in, $\mathbf{5 5 2}$

Bronchial constriction, effect of adrenalin in, 536

treatment, 552

Bronchiectasis, artificial pneumothorax in, 250

Bronchitis, acute, pneumococcus in, $2 I_{5}$

chronic, nephritis from, 749

vaccines in, 55

Bronchopneumonia from pneumococcus, 215

in diphtheria, mortality in, 62

in septicopyemia, 266

Bronchoscope, endobronchial therapeutics with, 552

Bronchoscopy, anesthesia in, 555

in drowned lung, 554

in foreign body in bronchi, 554

in lung, 554

in gangrene of lung, 553

in luetic obstructions of tracheobronchial tree, 552

in pulmonary abscess, 554

in removal of foreign body from bronchi, methods, 561,562

in tuberculosis, 553

therapeutic, $\mathbf{5 5 2}$ 
Bruises, baths for, 83

Brush discharges, static effects of, 101 technique for application, 108

Byrne's method of preparing mercurialized serum in treatment of cerebrospinal syphilis, 895

Bubonic plague, 298

fleas as carriers, $4 \mathrm{I} 6$

from rat fleas, 298

prophylaxis in, 298

rats in, etiology of, 298

vaccines in, 51, 298

Bugs, varieties, 414

Bullet wounds of spinal cord, treatment, 902

Burns, acidosis in children in, 504

Butyrin, fat indigestion in infants from, 671

Buxton Spa, principal characteristics, 88

Cachexia, ague cake with, in malaria, treatment, 352

Cactus in functional heart disease, 479

Caffein in acute nephritis, 764

in heart diseases, 483

in myocardial insufficiency, 833

in septicopyemia, 270

Calcic pericementitis, 583

Calcium chlorid enemata in chronic diarrhea, 653

in anaphylaxis in asthma, 536

in aneurysm of aorta, 155

in asthma, 537

in hemorrhage in chronic jaundice, 702

in hemorrhagic diseases, $44^{\circ}$

in tetany, 465

Calculi, pancreatic, in cholelithiasis, 708 treatment, 708

salivary, 606

causes, 606

location, 606

symptoms, 606

treatment, 606

Callosal puncture in hydrocephalus, 870

Calomel in acute fibrinous pleuritis, 568

in follicular tonsillitis, $5^{18}$

in malaria, 349

in oxyuriasis, 406

in quinsy, 520

in trichiniasis, 402

in yellow fever, 312,313

Calymatobacterium granulomatis, 354

Camphor for flea-bites, 416

in erysipelas, 276

in lobar pneumonia, 233

Camphorated oil in cardiac dyspnea, 483

in Cheyne-Stokes respiration, 483

in septic peritonitis, 735,737

in septicopyemia, 270

in tuberculous peritonitis, 745

Cancer from infections of bile-passages, 694

of bile-ducts, treatment, 705

of esophagus, bibliography, 6 Io

intrathoracic, grouping of, 608
Cancer of esophagus, radium treatment, 608

of gall-bladder, 705

of liver, 690,705 .

complications in, treatment, $69 \mathrm{I}$

syphilis of liver and, treatment, $69 \mathrm{r}$

treatment, 69 I

of stomach, 629

contraindications to operation, 630

diagnosis, 629

excision of pyloric end in, $63 \mathrm{I}$

gastrojejunostomy in, 633

gastrostomy in, 633

indications for operation, 629

inoperable, management, 633

jejunostomy in, 633

operative mortality in, 632

partial gastrectomy in, 631

fatal suture angle in, 631

Polya operation in, 632

pylorectomy in, $63 \mathrm{I}$

pyloric, methods of extension, 630

roentgen ray diagnosis, 629

surgical treatment, 629

of thoracic portion of esophagus, surgical treatment, 607

of thyroid, chronic thyroiditis and, differentiation, 473

pitch, predisposing factors in, $66_{2}$

secondary, in peptic ulcer, treatment, 613

Cancrum oris, 579

alcohol dressings in, 580

microörganisms in, 580

neosalvarsan in, 580

salvarsan in, 580

treatment, 580

Cane sugar in infant feeding, 675

Cantharides, nephritis from, $75 \mathrm{I}$

Canula method of blood transfusion, 146

Carbohydrate diet in acute nephritis, 762 in chronic interstitial nephritis, 781

Carbohydrates in infant feeding, 672

increasing, in Allen treatment of diabetes, 495

Carbolic acid injections in anthrax, 325

Carbon-point electrode, III

Carcinoma. See Cancer.

Cardiac crises in beriberi, treatment, 366

dilatation, baths in, 86

dyspnea, adrenalin in, 483

camphor in oil in, 483

strychnin in, 483

edema, treatment, 807

insufficiency in beriberi, 364

Cardiorenal disease, coefficient of urea excretion in, 794

Cardiovascular disease, hypertensive, 815 lowered functional power of liver in, 687

system, diseases of, treatment, 476

Camivorous human type, 716,717

Carrel-Dakin antiseptic solution, 327

Carriers, cerebrospinal meningitis, 296

cholera asiatica, 297

chronic, of bacilli, $5^{6}$ 
Carriers, malaria, 346

of ameba dysenteriæ, $34^{\circ}$

of bubonic plague, fleas as, 416

of disease, diptera as, 4 I3

hemiptera as, 4I4

of undulant fever, 354

typhoid fever, I 85

detection and elimination of, 190

Carter's incubation theory of yellow fever, 302

Cascara in intestinal stasis, 648

Casein mixtures, precipitated in protein indigestion, 679

Castile soap in chronic diarrhea, 653

Castor oil in amebic dysentery, 337

in ankylostomiasis, 407

in ascariasis, 405

in sprue, 370

Cat tapeworm, 391

Cataphor esis, 98, I 13

lithion, in gout, $5^{15}$

Cataphoric influence of constant electric current, 98

Catarrhal conjunctivitis, etiologic factors in, 381

diseases, acute, of upper air passages, autogenous vaccines in, 28

stomatitis, 601

treatment, 602

Catarrhs, respiratory, baths in, 87

Catelectrotonus, 98

Catharsis in acute nephritis, 765

in chronic parenchymatous nephritis, 773

in edema, 810

Catheterization in yellow fever, 314

Cathode effects of constant electric current, 98 of Coolidge tube, I 25

Cations, 98

Cavernous nevis, radium therapy in, I 35

Cecum, abnormal mobility of, constipation from, 656

ectasia of, constipation from, 656

mobile, 7 I 2

abdominal support in, 7 I 5

causes, 7 I 2

chronic appendicitis and, differentiation, 713

constipation in, $7 \mathrm{I} 3$

treatment, $7 \mathrm{I} 4$

cottonseed oil in, 714

diagnosis, 7 I 3

diet in, $7 \mathbf{I} 4$

enemata in, $71_{4}$

etiology, 7 I 2

formalin in, 714

hyperchlorhydria in, treatment, 714

lactic acid bacilli in, 7 I 5

laxative treatment, 7 I 4

mechanical treatment, 715

paraffin oil in, 7 I4

physical signs, 7I3

relation of, to splanchnoptosis, $7 \times 2$

roentgen rays in diagnosis, 7 I 3

surgical treatment, 7 I 5
Cecum mobile, symptoms, 71 2, 713

thymol in, 7I 4

treatment, 713

typhlotonia in, 712

Celli's method of preventing relapse in malaria, 346

Cells of organism, relation of drugs to, 25

Cellulitis in apical alveolar abscess, 598

in subgingival alveolar abscess, 598

Cephalic myiasis, 4IO, 4II

chloroform in, 4II

cocain in, 4II

mortality, 4 I I

symptoms, 4I I

treatment, 4 II

Ceratophyllus fasciatus, 298

Cerebral syphilis, salvarsan dosage in, 283

Cerebrospinal fluid, drainage of, in paresis, 020

meningitis, 296

anaphylaxis in, 79

carriers of, 296

early diagnosis, 296

Flexner's serum in, 296

serum treatment of, 77. See Antimeningitis serum.

syphilis, 88r. See also Syphilis, cerebrospinal.

Cerium oxalate in septicopyemia, 270

Cestodes, varieties, 390

Chancre, syphilitic, treatment of, 279

trypanosome, 372

Charcoal as antidote, $\mathrm{I}_{3}$

Charcot joints in tabes dorsalis, treatment, 876

Chaulmoogra oil in leprosy, $34^{2}$

Cheltenham Spa, principal characteristics, 88

Chemotherapy, history, I5

in lobar pneumonia, 233

Chest, aspiration of, novocain injection in, 142

Robinson's method, I42

Cheyne-Stokes respiration, adrenalin in, 483

camphor in oil in, 483

strychnin in, 483

Children, acidosis in, 50r. See also Acidosis in children.

prevalence of ascariasis in, 405

typhoid fever in, 197

yellow fever in, 309

Chill after vaccination, 40

Chloral hydrate in tetanus, 329

in trichiniasis, 402

Chloremic nephritis, 756

Chloroform in ascariasis, 405, 4ro

in cephalic myiasis, 4I I

in subcutaneous myiasis, $4 \mathrm{II}$

in trichuriasis, 404

nephritis from, $75 \mathrm{I}$

Chlorosis, 430

arsenic in, 431

betanaphthol in, 432

Blaud's pills in, $43 \mathrm{I}$

constipation in, treatment, 431 
Chlorosis, diet in, 431,432

etiology, 430

from impaired blood formation, 430

from uncinariasis, treatment, 432

gastric indigestion in, treatment, 432

general treatment, 431

hygienic treatment, 431

incidence of, 430

iron in, 43I

laxatives in, 43 I

magnesium oxid in, 432

prophylactic treatment, $43 \mathrm{I}$

secondary anemia due to infection and, differentiation, 43I

sodium bicarbonate in, 432

theories, 430

thymol in, 432

treatment, 431

Choked disk with blindness in tetanus, 331

Cholecystectomy in gall-bladder infections, 698

mortality, 699

versus cholecystostomy, 685

Cholecystitis, 685

diet in, 686

guarding against fatigue in, 686

natural drainage in, 686

nephritis from, 749

prophylaxis, 686

resistance to infection in, 686

septicopyemia from, treatment, 267

surgical treatment, 685

treatment, 686

Cholecystostomy versus cholecystectomy, 685

Cholelithiasis, pancreatic calculi in, 708

Cholera asiatica, 297

carriers of, 297

epinephrin in, 298

examination of feces of immigrants for, 297

injection of hypertonic saline solution in, 298

pituitrin in, 298

prophylaxis, 297

rectal injection of saline solution in, 298

symptoms of collapse in, 298

treatment, 297

vaccines in, 51

Cholesterin in pernicious anemia, 427

Chromic acid in syphilis of mouth, 604

Chromium salts, nephritis from, 751

Chrysomyia macellaria, 4 I0, 4 I I

Chylothorax, adhesive strapping in, 570

Cimex lectularius, 4 I 4

rotundatus in kala-azar, $35^{6}$

Cinchonin in malaria, 351

Circulation, defective peripheral, baths in, 86

Circulatory disorders, baths in, 86 system, disorders of, treatment, 32

Cirrhosis of liver, hypertrophic, therapeutic splenectomy in, 454

Citrate method of indirect blood trans-

$$
\text { fusion, } 154
$$

Vor. IV. -60
Citrate method of indirect blood transfusion, advantages, 154

fatalities in, 155

in hemorrhagic diseases, 439

in symptomatic anemia, 466

Claypole and Gay's sensitized typhoid vaccine, preparation of, 193

Climate, medical concept of, 93

Climatic treatment of symptomatic anemia, 445

Climatotherapy, 93

Clonorchis sinensis, 397

Clothing for workman, 177

in treatment of edema, 808

Coagulation, blood, in hemorrhagic diseases of newborn, 44 I

Coagulen in hemorrhage in chronic pancreatitis, 702

Coagulen-Ciba in hemoptysis, $54^{6}$

Coagulose in hemoptysis, 542

Cocain habit, treatment, I 7 I

in cephalic myiasis, $4 \mathrm{II}$

in septicopyemia, 270

Codein, hypodermic injection of, in hemoptysis, 54I

in infantile la grippe, 575

in infarction of lung, 551

in septicopyemia, 270, 271

Cold, effects of, on workmen, treatment, I $8 \mathrm{I}$

in treatment of septicopyemia, 268

pack in typhoid fever, 92

Colds, avoidance of, in workmen, $18 \mathrm{I}$ common, vaccines in, 54

Colectomy in constipation, 666

Coleman's diet in typhoid fever, 205, 206 results from, 208

Coley's toxins in Hodgkin's disease, 462 in leukemia, 452

Colitis, chronic. See Diarrhea, chronic.

Collapse in yellow fever, treatment, 3 I 6

pulmonary, 549

artificial distention in, 549

bimanual pressure in, $55^{\circ}$

in complete paraplegia, 549

preventive treatment, 549

treatment, 549

Collargol enemata in chronic diarrhea, 653

in filariasis, 409

in septicopyemia, 272

Colloidal gold reaction in cerebrospinal syphilis, 888,898

Colon, ascending, lesions of, constipation from, $65^{6}$

bacillus in septic peritonitis, 730 vaccines, 35

excision of, in intestinal stasis , 665

fixation of, indications for, 727

irritation, baths in, 87

resection of, sequels, 728

with ileosigmoidostomy, 727

roentgen-ray study of, in intestinal stasis, 644

Colonic absorption, faulty, indicanuria in, 644

Coma in uremia, 839 
Communicable diseases, physician's precautions in, 18

Community immunity to malaria, 347

Complement fixation in diagnosis of undulant fever, 354

in sprue, $3^{60}$

in syphilis of liver, $69 \mathrm{r}$

test in pertussis, $33 \mathrm{I}$

in plastic iritis, 387

Congenital visceroptotic human type, 7 I6, 7 I 7

Congo floor maggot, 4 IO

Conjunctiva, diseases of, treatment, $38 \mathrm{I}$ tubercle of, treatment, 384

Conjunctivitis, catarrhal, 381

etiologic factors in, $38 \mathrm{I}$

gonorrheal, iced physiologic salt solution in, 383

spohol in, 382

neonatorum, iced physiologic salt solution in, 383

spohol in, $3^{82}$

pneumococcus in, 2 I 5

simple, 38I

etiologic factors in, $38 \mathrm{r}$

special forms, 382

swimming-bath, 382

inclusion bodies in, 382

Conorrhinus rubrofasciatus, in kala-azar, $35^{6}$

Constant electric current, 97

anaphoric influence, 98

anode effects, 99

cataphoric influence, 98

cathode effects, 98

electrotonic effects, 98

in exophthalmic goiter, 116

in neuralgias, I I 5

in occupation neuroses, I 6

in saccular aneurysm, $1 \mathbf{r} 8$

interpolar effects, 98, 99

methods of application, ro3

muscular contractions from, 99

negative effects, 98

polar actions, 98

positive effects, 99

therapeutic uses, $\mathrm{IO}_{3}$

Constipation, 655

administration of liquids in, $65^{8}$

agar-agar in, 662

antiperistalsis in, 656

ascending, causes, 657

Bastedo's diet in, $66 \mathrm{I}$

baths in, 87

causes, 656

chronic, in cecum mobile, $7 \mathrm{I}_{3}$

colectomy in, 666

diet in, 66o, 66I

drug treatment, $66 \mathrm{I}$

electrotherapy in, 118

enemas in, 658

establishing regular habits in, 659

etiology, 656

exercise in, 659

exhausted orange peel in, 663

Fernet's exercises in, 659
Constipation, fleaseed in, 663

from abnormal mobility of cecum, 656

from dyschezia, 657

from ectasia of cecum, 656

from inflammatory pericolitis, 656

from lesions of ascending colon, $65^{6}$

from torpor recti, 657

gastric retention in, treatment, $6_{5} 8$

gastrogenous, 658

general hygiene in, 658

hormonal in, $66_{3}$

in beriberi, treatment, 366

in cecum mobile, treatment, 7 I4

in chlorosis, treatment, $43 \mathrm{I}$

in chronic interstitial nephritis, treatment, 784

parenchymatous nephritis, treatment, 773

in diverticulitis, $64 \mathrm{I}$

treatment, 642

in sclerosis of blood-vessels of abdomen, treatment, 640

Kohnstann's diet in, 660

Lane's kink in, 657

linseed in, $66_{3}$

liquid petrolatum in, $66 \mathrm{I}$

local causes, $6_{5} 6$

location of fecal accumulations in, 656

mucus in feces in, 657

Newburgh's diet in, $66 \mathrm{I}$

paraffin oil in, 662

phenolphthalein agar in, $66_{3}$

pituitrin in, 664

regulation of gastric digestion in, 659

regulin in, 663

relations of amount and variety of food to, $65^{8}$

of gastric digestion in, 658

rhubarb agar in, $66_{3}$

Rosenfeld's diet in, 660

Russian mineral oil in, $66 \mathrm{r}$

sennatin in, 664

spastic, causes, 657

state of gastric motility in, 658

surgical treatment, 666

symptomatic anemia from, treatment, 445

time for laxatives in, $65^{8}$

treatment, 658

water drinking in, 660

Contagious hospital staffs, value of Schick test for, 70

Continuous bath in delirium tremens, 927 in insanity, 926

Contusions, baths for, 83

Convalescence after acute nephritis, treatment in, 768

Convalescent serum in scarlet fever, 245

Convallaria in functional heart disease, 479

Convulsions in uremia, 839

treatment, 845

Coolidge tube, I 23,124 advantages of, I 26

for deep roentgen ray therapy, I 27 anticathode, of 125

cathode of, I25 
Coolidge tube, character of output, 132 diagram of operation, 125 homogeneous penetration of, 132 invention of, 122 method of operating, I 25, I 26 protecting tube box for, 127 standardization of roentgen-ray dosage with, 129

target of, I 25

watercooling device for, $\mathrm{I} 28$

Cooling applications in acute cases in military practice, 83

Copper sulphate in chronic trachoma, 384

Cordylobia anthropophaga, 410

Corn in etiology of pellagra, I6I

Cornea, diseases of, treatment, $3^{84}$

ulcers of, $\mathbf{5 8 4}$

azodolen in, 385

pellidol in, 385

pneumococcus, 215

optochin in, 388

radium in, 305

scarlet red in, 384

treatment, 384

zinc in, 384

Corpus callosum, puncture of, in hypopituitarism, 468

Corradi-Moore treatment of aneurysm, II 8

Corrosive poisoning, emetics for, 29

sublimate in gonorrheal st omatitis, 604 in syphilis of mouth, 604

poisoning, 164

after-effects of, 164

albumen as antidote, 164

bismuth subcarbonate in, 165

effects, 164

hot packs in, 165

hypodermoclysis in, 165

Murphy's drip method of enteroclysis in, 165

stomach lavage after, $16_{4}$

stripping kidney in, 165

suppression of urine in, 165

sweat baths in, 165

treatment, 164

water drinking in, $16_{4}$

solution in oxyuriasis, 406

in echinococcus disease of pleura, 571

ulcer, 6r 1. See also Peptic ulcer.

Corset in visceroptosis, 723,725

Cottet's water test of renal function, 789

Cottonseed oil in cecum mobile, 714

Couch, autocondensation, II3

Cough in myocardial insufficiency, treatment, 835

increased, in artificial pneumothorax, 253

whooping. See Pertussis.

Counterirritation in acute fibrinous pleuritis, 567

County medical inspectors of Pennsylvania, 19

Cows' milk anaphylaxis, 668

fat of, 668
Cows' milk for infant feeding, 668 protein of, 668 sugar of, 668

Coxitis, tuberculous, bismuth paste treatment, 145

Crab louse, 414

Creatinin test of renal function, 796

Creeping eruption, 44

Creole foot bath in yellow fever, $3 I_{3}$

Creosol for fleas, 416

Creosote, endobronchial injection of, in tuberculosis, 553

Cretinism, desiccated thyroid in, 464 treatment, 464

Cross-fire method of roentgen-ray therapy, 135

Croupous tonsillitis, 517

Cuba, extermination of yellow fever in, 304.

Cupping in acute fibrinous pleuritis, 568

Cuspidors, factory, I 75

Cutaneous antiseptics, 27 disorders, baths in, 87 myiasis, 410

Cyclic albuminuria, 801

Cylinder method of blood transfusion, 146

Cysticercosis, 393

Cysticercus bovis, 391

cellulosx, 391, 393

Cystitis, chronic, $86_{3}$

nephritis from, 749

Cysts of liver, hydatid, surgical treatment, 705

of thyroid gland, treatment, 473

Dakin-Carrel antiseptic, 327

in tetanus, 327

preparation of, 327

Dakin's antiseptic, 268 administration by injection, 269

by Murphy drip apparatus, 268

for infected wounds, preparation of, 268

in gas-forming wounds, 268

in military practice, 269

in necrotic wounds, 268

injection, 269

preparation, 268

properties, 269

sterilization of wounds by, 269

uses, 268

D'Arsonval bipolar current in bladder tumors, 865

oscillator with resonator, 102

with two condensers, ror

Deafness from optnchin in lobar pneumonia, 238

Deek's method of preventing relapse in malaria, 346

Deep roentgen-ray therapy, I35

Defecation, mechanics of, 656

Delirium in aortic regurgitation, treatment, 480

tremens, 165 alcohol in, 167

apomorphin hydrochlorid in, 165 
Delirium tremens, bromids in, 167

continuous bath in, 927

diet in, 165

digitalis in, 167

ergot in, 167

hypnotics in, $16 \dot{6}$

induction of sleep in, I 66

intraspinal injection of magnesium sulphate in, $x 67$

ipecacuanha in, I 65

magnesium sulphate injections in, 923

morphin in, 166

paraldehyde in, I 66

rest in, 166

restraint in, 166

scopolamin in, 166

stimulation of circulation in, 167

strychnin in, 167

treatment, 165,923

of exhaustion in, 165

Dementia præcox, autointoxication in, etiology, 908

Deneke's apparatus for artificial pneumothorax, 257, 259

Dengue fever, yellow fever and, differentiation, 3 Io

Dento-alveolar abscess, alveolar abscess and, differentiation, 578

Dermacentor andersoni in Rocky Mountain spotted fever, 417

Dermal leishmaniasis, $35^{8}$ Brazilian, $35^{8}$

Dermatitis from tick, 417

straw mattress, $4 \mathrm{I} 8$

Dermatobia cyaniventris, 410

Desiccation treatment of leukoplakia buccalis, I 33 of senile keratosis, I 34

Dextrin-maltose in infant feeding, 674

Diabetes, 489

acidosis in, estimation of, $49 \mathrm{I}$

hyperpnea in, 501

sodium bicarbonate solution in, 497

albuminuria in, 803

Allen treatment, 49I. See also Allen treatment of diabetes.

estimation of blood sugar in, 489

hyperglycemia in, 480

in exophthalmic goiter, 490

insipidus, 508

etiology, 508

in lesions of pituitary gland, 508

lumbar puncture in, 509

pituitrin in, 509

strychnin nitrate in, 509

treatment, 500

mellitus in acute pancreatitis, 707

mild, diet chart for, 496

pancreatic, pankreon in, 707

sodium bicarbonate in, 707

renal, diagnosis, $49^{\circ}$

Sellard's test in, 49 I

starvation treatment, 49r. See also Allen treatment of diabetes.

Diaphoresis in acute nephritis, 765
Diaphoresis in chronic parenchymatous nephritis, 773

in edema, 811

Diaphoretic baths in lowered functional power of liver, 688

Diarrhea, acidosis from, in children, 504 chronic, $65 \mathrm{I}$

adrenalin in, 652

antiseptic treatment, 653

atropin in, 652

belladonna in, 652

betanaphthol in, 653

bismuth salicylate in, 653

blood in, treatment, 653

castile soap in, 653

causes, ${ }_{55} \mathrm{I}, 65_{2}$

collargol enemata in, 653

edema in, treatment, 653

gelatin enemata in, 653

guaiacol in, 653

hydrogen peroxide enemata in, 653

hypernutrition in, $65^{2}$

in amebic dysentery, treatment, 653

in catarrhal enteritis, treatment, 653

in pellagra, $65 \mathrm{I}$

in sprue, $65^{1}$

ichthyol enemata in, 653

injection of sulphureted hydrogen in, 653

iodoform enemata in, 653

oil enemata in, 652

oxygen injection in, 654

pus in, treatment, 653

quinin in, 653

thymol in, 653

transduodenal lavage in, 653

treatment, 652

ulceration in, treatment, 653

fermentative, of infants, 68I

infectious diarrhea and, differentiation, 68I

symptoms, 68I

treatment, 682

from acidosis in children, treatment, 505

from maltose in infant feeding, 675

in diverticulitis, $64 \mathrm{I}$

in infants from milk sugar, treatment, 673

in opium habit, treatment, 170

in pernicious anemia, treatment, 425

in pneumococcus peritonitis, $74 \mathrm{I}$

in septicopyemia, treatment, 270

in uremia, 847

infectious, fermentative diarrhea of in-

fants and, differentiation, 680

in infants, 683

bacteria in, 683

course, 683

etiology, 683

fermentative diarrhea and, differentiation, 68r

management, 683

Diarsenol in tabes dorsalis, 874,876

Diathermy, 102

electrodes for, $\mathrm{I}_{3}$

in neuritis, II5 
Diathermy in sciatica, 115

in thromboangitis obliterans, 121 uses, 114

Diathesis, rheumatic, as cause of quinsy, 519 quinsy and, relation, 5 19

uric acid, 516

diet in, 516

treatment, 516

Dibothriocephalus latus, $39 \mathrm{~s}$

Dicotyledons in etiology of hay fever, 528

Diet, carbohydrate, in acute nephritis, 762

effect of, on pancreatic secretion, 706

fruit and vegetable, in sprue, 370

in acidosis in children, 505, 507

in acute fibrinous pleuritis, 569 nephritis, 761

in arthritis deformans, $5 \mathrm{II}$

in cecum mobile, 714

in chlorosis, $43 \mathrm{r}, 432$

in cholecystitis, 686

in chronic interstitial nephritis, $78 \mathrm{I}$ pancreatitis, 707 parenchymatous nephritis, 771

in constipation, 660,661

in delirium tremens, 165

in diverticulitis, 642

in edema, 808

in follicular tonsillitis, 510

in heart diseases, 485

in hemorrhagic diseases, 439

in hypertension, 821

in intestinal stasis, 646

in leprosy, 342

in lithemia, 516

in lowered functional power of liver, 689,690

in myocardial insufficiency, 826

in nephritis, 847

in non-obstructive peptic ulcer, 616 modifications, 620

in orthostatic albuminuria, 804

in pancreatic achylia, 708

in pernicious anemia, 425

in quinsy, 520

in sclerosis of blood-vessels of abdomen, 640

in septicopyemia, 270

in symptomatic anemia, 445

in typhoid fever, 203

in uremia, 841

in uric acid diathesis, 516

in yellow fever, mild solid, 314

liquid, in yellow fever, $3 \mathrm{I} 3$

milk, in acute nephritis, 762

strict meat, in sprue, 370 milk, in sprue. 360

von Noorden in acute nephritis, 762

Digalen in pulmonary edema, 548

embolism, 55 I

thrombosis, $55 \mathrm{I}$

Digestion, defective, in intestinal stasis, treatment, 646

Digestive disorders, baths in, 87 of infants, 667
Dig estive system, diseases of, 577

treatment of, 28

ulcer, 6ri. See also Peptic ulcer.

Digipuratum in acute nephritis, 764

in heart diseases, 478

in pulmonary edema, 548

embolism, 55I

thrombosis, $55 \mathrm{I}$

Digitalin in heart diseases, 478

in yellow fever, 315

Digitalis, dosage, in heart disease, 477

effect of, on heart block, 478

in acute nephritis, 764

in auricular fibrillation, 32, 479

in beriberi, 366

in delirium tremens, 167

in dilatation of heart, 479

in edema, 807

in erysipelas, 276

in functional heart disease, 477

in heart disease, 476,477

in mitral regurgitation, 479

stenosis, 480

in myocardial insufficiency, $829-832$

adjuncts to, 834

effects, 830

method of administration, 830

preparations, 830

substitutes for, 832

toxic effects, 832

in septic peritonitis, 735

in septicopyemia, $270,27 \mathrm{r}$

in soldier's heart, $48 \mathrm{I}$

in uremia, 844

in valvular heart disease, 477,480

standardization, methods of, $83 \mathrm{I}$

Digitalon in pulmonary edema, 548

embolism, 551

thrombosis, 551

Dilatation of stomach, 637 acute, gastric lavage in, 637 surgical treatment, $6_{37}$

chronic, surgical treatment, 637

Dionin in opium habit, 169

Dioxydiamido-arseno-benzene dihydrochlorid, 282. See also Salvarsan.

Diphtheria, 24I

antitoxin. See also Antidiphtheritic serum.

bronchopneumonia in, mortality in, 62

clinical symptoms, 241

immunity to, active, $6_{3}$

natural, $6_{3}$

passive, 63

Schick's test'for, 63

infantile la grippe and, differentiation, 573

local treatment of nose and throat in, 244

membranes affected by, 24 I

nephritis in, 748

physiologic saline solution in, 244

prophylaxis in, 242

Schick test in, 63, 241. See also Schick test for immunity to diphtheria.

systemic symptoms, $24 \mathrm{I}$ 
Diphtheria, toxin-antitoxin immunity from, resistance of horses and guinea-pigs to, 69

mixtures, immunity from, 67

treatment, 24 I

Diphtheroid bacilli, vaccines, 35

Diphyllobothrium latum, 39 I

Diplococcus pneumonix. See Pneumococcus.

Diptera, 409

as carriers of disease, 413

Dipylidium caninum, $39 \mathrm{r}$

Disease germs, relation of drugs to, 25 modern treatment, I I

Diseases, infectious, 183

Disinfectants, intestinal, in cecum mobile, 714

oral, in pyorrhea alveolaris, 593

Disinfection of houses, 18 in kala-azar, $35^{8}$

Dispensaries, tuberculosis, in Pennsylvania, 2 I

Distoma hepatica, 398

Distomiasis, 395

hepatic, 397

kamala in, 398

male fern in, 398

treatment, 398

intestinal, 399

male fern in, 399

thymol in, 399

treatment, 399

pulmonary, 397

treatment, 397

venal, 395

male fern in, 396

treatment, 396

Diuretics in acute nephritis, 763

in edema, 809

in myocardial insufficiency, 829

in uremia, 844

Diverticulitis, 64 I

agar-agar in, 642

carcinomatous change in, 642

constipation in, 642

treatment, 642

diagnosis, 64 I

diarrhea in, 64I

diet in, 642

enemata in, 642

fruit in, 642

laxative treatment, 642

operation in, 642

perisigmoiditis and, differentiation, 642

regulin in, 642

symptoms, $64 \mathrm{I}$

treatment, 642

Dobell's solution in aphthous stomatitis, 602

in catarrhal stomatitis, 602

Dog tapeworm, 391, 394.

Donors in blood transfusion, 147 grouping of, 147 methods of testing, 148

Dosage of roentgen-rays, i 29

Dover's powder in infantile la grippe, 575
Dracunculus medinensis, 409

Drainage in tuberculous peritonitis, 745

natural, in cholecystitis, 686

postoperative, in septic peritonitis, 737

Dreams, in etiology of insanity, 925

Drinking water for factories, 175

Droitwich Spa, principal characteristics, 88

Dropsy, epidemic, 366

in acute nephritis, treatment, 765

in edema, surgical treatment, $8 \mathrm{II}$

of heart disease, nursing in, 484

operation for, 484

theobromin in, 484

Drowned lung, bronchoscopy in, 554

Drug habits, $16_{3}$

poisoning, 163

treatment, general principles of, 25

Drugs, Ehrlich's theory of the action of, on cells and microörganisms, 27 relation of, to cells of organism, 25 and disease germs, 25

Duodenal ulcer, 6ro. See also Peptic ulcer.

Duodenum, surgery of, 629

Dust control in factory ventilation, I73

protection from, in factories, 175

Dyschezia, 654

causes, 657

constipation from, 657

enemata in, 654

lavage in, 654

strychnin in, 654

symptoms, 654

treatment, 654

varieties, 657

Dysentery, amebic, 337

carriers of, 340

castor oil in, 337

chronic diarrhea in, treatment, $\sigma_{53}$

emetin in, 337,338

administration per os, 338

clinical cure, 339

dose, 339

duration of treatment, 339

effect on liver abscess, 339

intravenous injection, 339

limitations, 339

subcutaneous method, 338

untoward symptoms from, 338

encystment of ameba after, 340

method of transmission, $34^{\circ}$

tincture opii in, 337

treatment, 337

bacillary, vaccines in, 50

serum treatment, 76

Dyslalia, 523

aberrant tonic innervation in, 524

acquired, 525

articulatory element of speech in, 524

chronic stage, 523

congenital, 525

enlargement of thymus gland as cause

of, 524

etiology, 523

from congenital aphasia, 526 
Dyslalia from emotional disturbances, 525 glandular enlargements in pharynx as cause, 523

hereditary predisposition to, 525

in childhood, 525

initial stage, 523

phonatory element of speech in, 524

psychic origin, 523

psychotherapy in, 526

relation of, to aphasia, 525

to pseudolalia, 526

removal of adenoids in, 524

secondary manifestations, 523

tonsillectomy in, 524

treatment, 526

weakness of auditory speech center as cause, 525

Dyspepsia, baths in, 87

Dyspinealism, treatment, 469

Dyspituitarism, 467

treatment, 467

Dyspnea, cardiac, adrenalin in, 483 camphor in oil in, 483

strychnin in, 483

in acute nephritis, treatment, 767

in artificial pneumothorax, 253

in myocardial insufficiency, treatment, 834

in uremia, treatment, 846

Dystrophia adiposogenitalis, 467

Dystrophy, pituitary, 467

Ear, diseases of, pneumococcus in, 215

Earth, fuller's, as antidote, 164

East Africa, recurrent fever of, $36 \mathrm{I}$

Eastbourne Spa, principal characteristics, 88

Ecchymoses in septicopyemia, 266

Echinococcus discase of pleura, treatment, 571

granulosus, 394

Echinostoma ilocanum, 399

maylayanum, 399

Ectasia of cecum, constipation from, 656

of stomach, 637. See also Dilatation of stomach.

Eczema, chronic, baths in, 87

dosage of roentgen-rays in, 132

in food-poisoning from anaphylaxis, 158 rosacea, thyroid treatment, 465

Edebohls' operation of stripping kidney in corrosive sublimate poisoning, 165

Edema, 805

acidosis in, treatment, 809

angioneurotic, treatment, 930

ascites in, surgical treatment, $8 \mathrm{r}$ I

baths in, 811

cardiac, treatment, 807

cathartics in, 810

causes, 805

clothing in, treatment, 808

diaphoretics in, 811

diet in, 808

digitalis in, 807

diuretics in, 800

dropsy in, surgical treatment, 81 I
Edema, etiology, 805

Fischer's treatment, 807

fluid intake in, 808

from excess of fluid, 806

from renal injury, 806

from vascular injury, 806

hot packs in, $81 \mathrm{I}$

in acute nephritis, treatment, 766,808

in anemia, treatment, 807

in beriberi, 364

in chronic diarrhea, treatment, 653

parenchymatous nephritis, treatment, 774

in myocardial insufficiency, treatment, 834

in nephritis, 805

in nephrosis, 805

iron in, 807

laxatives in, 810

low arterial tension in, treatment, 8 ro

management, 807

of brain as cause of uremia, 837

of glottis in acute nephritis, treatment, 767

in erysipelas, 275

of lungs, 548. See Edema, pulmonary.

of toxic origin, 805

paracentesis in, $8 \mathrm{I} 2$

passive congestion in, treatment, 810

pilocarpin in, 8 I I

potassium acetate in, 809

citrate in, 800

pulmonary, 548

aromatic spirits of ammonia in, 548

atropin sulphate in, $54^{8}$

bibliography, $548,55^{\circ}$

digalen in, 548

digipuratum in, 548

digitalon in, 548

electric cabinet baths in, 548

hot vapor baths in, 548

in acute nephritis, treatment, 768

in myocardial insufficiency, treatment, 836

in uremia, treatment, 846

morphin sulphate in, 548

nitroglycerin in, $54^{8}$

oxygen in, $55^{\circ}$

prophylaxis, 548

strychnin in, 548

treatment, $54^{8}$

whisky in, 548

purgatives in, 810

rest treatment, 808

sodium bicarbonate in, 800

chlorid restriction in, 809 retention in, 805

surgical treatment, 8I I

theories as to cause, 805

water retention in, 805

Effluve electrodes, I 1 I

high-frequency, in acroparesthesia, I I 5 in headache of neurasthenia, 115

Effusion, serous pleural, in artificial pneumothorax, 255

unresolved, baths in, 84 
Ehrlich's discovery of salvarsan, 282 theory of the action of drugs on cells and microörganisms, 27

Einhorn's stomach electrode, Bassler's modification, II 7

Eiweiss milk. See Protein milk.

Electric cabinet baths in pulmonary edema, 548

current, constant, 97. See also Constant electric current.

faradic, 99

galvanic, 97. See also Constant electric current.

high frequency, IOI

induced, 99

sinusoidal, 100

static, IOI

Tesla high-frequency, I02

fan ventilation for factories, 173

light baths in nephritis, 852

in tabes dorsalis, 875

osmosis, 98

stimulation in beriberi, 366

Electricity in acute anterior poliomyelitis, 879

in gout, 515

Electrode, block-tin, for diathermy, II 3

effluve, III

glass, for obtaining heat and local hyperemia, II 2

vacuum, II 2

method of holding, in electrotherapy, IO4

proper positions of, in electrotherapy, I05

stomach, Einhorn's, Bassler's modification of, II 7

universal handle for, with interrupter, ro5

Electrolysis, wiring with, in sacculated aneurysm, 155

technique, 156

Electron roentgen-ray tube, 124

Electronegative drugs, II 4

Electrophysiology, 97

Electropositive drugs, II 4

Electrotherapy, 97

for exercising paralyzed muscles, I I4

in constipation, 118

in diseases of blood-vessels, 118 of intestines, I 18

of muscles and joint, II 6

of nervous system, II4

of stomach, I 6

in lumbago, $\mathrm{I} x 6$

in muscular rheumatism, I 16

in myositis, 116

in Raynaud's disease, 121

in rheumatoid arthritis, 116

in spasm of esophagus, 118

in stiff neck, I 16

method of holding electrodes in, I04

methods of application, ro3

precautions in, ro4

proper positions of electrodes in, 105 psychic influences of, IO3
Electrotherapy, special, I 14

Electrotonic effects of constant electric currents, 98

Elsberg's extrusion method in intramedullary spinal cord tumors, 904

Embolism, gas, from artificial pneumothorax, 254

of lung, prophylaxis, 55I

pulmonary, 55 I

bibliography, 55I

digalen in, $55 \mathrm{I}$

digipuratum in, $55 \mathrm{I}$

digitalon in, $55 \mathrm{I}$

from Trendelenburg position, $55 \mathrm{I}$

morphin in, $55 \mathrm{I}$

strophanthin in, $55 \mathrm{I}$

treatment, $55 \mathrm{I}$

Emetin, effects of, on Ameba dysenteriæ, 339

hydrochlorate in amebic dysentery, 30 in hepatitis, 30

hydrochlorid in hemoptysis, 546

in tropical abscess of liver, $69 \mathrm{I}$

in amebic dysentery, 337,338 . See also Amebic dysentery, emetin in.

in pyorrhea alveolaris, 586,593

dosage, 593, 596

methods of administration, 593, 596

results, 595

technique, 595

Emotions, repressed, in etiology of insanity, 924

Emphysema in artificial pneumothorax, 254

Empyema, bismuth paste treatment, 145 perforans, treatment, 571

pneumococcus in, 215

Encephalitis in infantile la grippe, 574 in pertussis, 332

Encystment of Ameba dysenteriæ after attack, $34^{\circ}$

Endameba buccalis, microscopic appearance, 588

gingivalis, Endameba buccalis and, differentiation, 587

in pyorrhea alveolaris, 585

method of staining, 588

microscopic examination for, $5^{87}$

pyogenes, Endameba gingivalis and, differentiation, 589

in pyorrhea alveolaris, 585

Endarteritis in cerebrospinal syphilis, $88_{2}$

Endemic goiter, American trypanosomiasis and, differentiation, 379

Endobronchial therapeutics with bronchoscope, $55^{2}$

Endocarditis, acute, Streptococcus viridans in, 578

ice bag in, 484

in infantile la grippe, 574

in septicopyemia, 266

pneumococcus in, 215

rheumatic, strophanthin in, 482

Endocrine glands, diseases of, 463

Endogenous septic peritonitis, 730 substances, nephritis from, 751 
Fndometritis, chronic, nephritis from, 749 Enemata, calcium chlorid, in chronic diarrhea, 653

collargol, in chronic diarrhea, 653

formalin, in intestinal stasis, 650

gelatin, in chronic diarrhea, 653

glycerin, in intestinal stasis, 649

hydrogen peroxid, in chronic diarrhea, 653

ichthyol, in chronic diarrhea, 653

in cecum mobile, 714

in constipation, 658

in dyschezia, 654

in intestinal stasis, 649

in oxyuriasis, 406

in trichuriasis, 404 .

in yellow fveer, 315

iodoform, in chronic diarrhea, $6_{53}$

oil, in chronic diarrhea, 652

in intestinal stasis, 649

England, spas of, $87-89$

English Medical Research Committee's antiseptic powder in tetanus, 327

Enteritis, catarrhal, chronic diarrhea in, treatment, 653

chronic ulcerative, nephritis from, 749

Enteroclysis in septicopyemia, 270 Murphy drop, in typhoid fever, 92

Eosinophilia in trichiniasis, 402

Epidemic dropsy, 366

Epidemics, malarial fulminant, 347

Epididymitis, gonococcus vaccines in, 44

Epilation of scalp with massive dosage of roentgen-rays, 133

Epinephrin in Addison's disease, 466

in asthma, 467

in cholera asiatica, 298

in infantile la grippe, 575

in osteomalacia, 467

Ergot in aneurysm of aorta, ${ }_{55}$

in delirium tremens, 167

in yellow fever, 316

Eruption, creeping, 4I I

Erysipelas, 273

acute glossitis in, 605

administration of water in, 276

age of occurrence, 274

ammonia in, 276

antistreptococcus serums in, 277

arthritis in, 275

average duration, 276

camphor in, 276

characteristics, 273

complications, 275

degenerative changes in, 274

diagnosis, 275

digitalis in, 276

disinfection in, 276

drug treatment, 276

edema of glottis in, 275

etiology, 273

facial, appearance, 274

desquamation in, 275

extension, 275

metastatic areas in, 275

site of, 274
Erysipelas, gangrene in, 275

ichthyol in, 277

immunity in, 275

in wound infection, 273

incubation period in, 274

individual susceptibility to, 274

inflammation in, 274

of lymph spaces in, 274

isolation of cases, 274

lead-water and alcohol wash in, 277

liquor ferri chloridi in, 276

local treatment, 277

magnesium sulphate in, 277

medical, 274

site of, 274

meningitis in, 275

method of spread, 274

migrans, 275

mortality in, 276

nephritis in, 275

of mouth, 275

treatment, 277

of navel, 275

of pharynx, 275

treatment, 277

of vagina, 275

ointments in, 277

pathology, 274

pericarditis in, 275

phlebitis in, 275

pleuritis in, 275

pneumonia in, 275

predisposing conditions, 274

prevention of spread, 277

prognosis, 276

prophylaxis, 274,276

protection of attendants in, 276

puerperal, 274

purgative treatment, 276

pyemia in, 273, 275

quinin in, 276

relapse in, 275

seasons in etiology of, 274

secondary acute lymphadenitis in, 274

septicemia in, 273

Streptococcus erysipelatis in, 273

pyogenes in, 273

vaccines in, 42

strychnin in, 276

subcutaneous abscesses in, 275

suppuration in, 274

symptoms, 275

surgical, 274

symptoms, 274

temperature in, 275

theories of, 273

tonsillitis in, 275

treatment, 276

usual site of, 274

Erythema in septicopyemia, 266

Erythremia, 432

arsenic in, 433

benzol in, 433

bibliography, 433

course, 433

dietetic treatment, 433 
Erythremia, etiology, 432

forms, 432

idiopathic, 432

oxygen in, 433

prognosis, 433

relations of spleen in, 433

roentgen rays in, 433

secondary to circulatory disturbance, 432

treatment, 433

venesection in, 433

Eserin in septic peritonitis, 735

Esophagoplasty, extrathoracic, 607

Esophagostrostomy, 607

Esophagus, cancer of, bibliography, 610

intrathoracic, grouping of, 608 radium treatment, 608

thoracic portion, surgical treatment, 607

intrathoracic resection, 607

hydrothorax from, 608

obstacles, 608

spasm of, electrotherapy in, II 8

Ether as antiseptic, 27

lavage in septic peritonitis, 737

Ethyl-chlorid anesthesia in artificial pneumothorax, 25 I

Ethylhydrocuprein. See Optochin.

Eucain-adrenalin anesthesia in artificial pneumothorax, 25I

Eucalyptol in ankylostomiasis, 407

Europe, relapsing fever of, 361

Eusol-eupad for gas gangrene, 27

Examination, physical, of workmen, 182

Exercise, effect of, on orthostatic albuminuria, 802

for workmen, I80

general, in mitral stenosis, 479

graduated, in tuberculosis, 248

in Allen treatment of diabetes, 495

in beriberi, 366

in chronic interstitial nephritis, 780

in constipation, 659

Exercises, Fraenkel, in tabes dorsalis, 875 heart, in heart diseases, 486

in albuminuria, 804

in visceroptosis, 7 I I , 7 I 2,724

Exhaust system of factory ventilation, I 73

Exhaustion element in functional nervous diseases, 906

in delirium tremens, treatment, 165

Exogenous septic peritonitis, 730

Exophthalmic goiter, 470

baths in, 86

constant electric current in, I I6

deep roentgen-ray therapy in, 136 , I 38

diabetes in, treatment, $49 \circ$

high blood-pressure in, 472

importance of early diagnosis, $47 \mathrm{I}$

injections of boiling water in, 470

operation in, results of, 464

operations of choice in, 463

operative treatment, 463

preoperative treatment, 470

rest treatment in, $470,47 \mathrm{I}$
Exophthalmic goiter, roentgen rays in, 470 diagnosis, 475

surgical treatment, $47^{\circ}$

symptomatic treatment, 464

syphilis with, treatment, 472

trauma as cause, $47 \mathrm{r}$

treatment, 463

with infected tonsils, treatment, 470

with peridental infections, treatment, 470

Exposure, nephritis from, 752, 757

Extrathoracic esophagoplasty, 607

Exudate in septic peritonitis, $73 \mathrm{I}$

Eye, gonococcic infections of, treatment, 387

pneumococcic infections of, treatment, 388

streptococcic infections of, treatment, 388

tuberculosis of, treatment, 387

tuberculin in, 387

Eyelid, diseases of, treatment, $38 \mathrm{I}$

Eyes of workmen, care of, I 79

FACTORIES, work hours in, 176

air-space required for workmen, 172

cleaning of, I 75

cuspidors, 175

drinking water for, $\mathbf{I} 75$

dust removal, I75

first-aid kits in, I 76

flooring, damp, prevention of, 174

keeping clean, 174

prevention of slipperiness, 174

of vibration of, 174

hygiene, 172

lighting, I 74

proper location of, 174

lockers, 174

lunch hour, I 75

proper flooring for, 174

protection from dust in, 175

of receptacles in, 176

special hygiene of, I 75

toilets, 175

ventilation, I 73

artificial moisture in, 173

dust control in, 173

exhaust system, 173

obstructions to, 173

open window, 173

personal comfort in, 173

with electric fans, I 73

Faget's law in yellow fever, 307

Fahr and Volhard's classification of nephritis, 755

Failing heart, sugar in treatment, 486

Fannia canicularis, 4I 2

Faradic dry brush in numbness, II 5

electric current, 99

in pertussis, 333

methods of application, ro3

therapeutic uses, to3

Faradization in asthma, 539

Fasciola hepatica, 398 
Fasciolopsis buski, 399 goddardi, 399

Fat in infant feeding, 669

indigestion in infants, 669

atrophy from, 67 I

emulsified olive oil in, $67 \mathrm{I}$

feces in, 670

from butyrin, 67I

malnutrition from, 671

rachitis from, $67 \mathbf{I}$

soap stools in, 670

symptoms, 670

trea tment, 671

of cows' milk, 668

Fatigue fever, baths in, 85

Fats in diet in chronic interstitial nephritis, $78 \mathrm{r}$

in typhoid fever, 205

Fatty acids in feces in pernicious anemia, $42 \mathrm{I}$

Favus, massive dosage of roentgen rays in, 133

transmission of, by pediculi, 415

Febrile albuminuria, 803

Feces, chemical examination of, in peptic ulcer, 6 I $2^{2}$

examination of, in immigrants, for cholera asiatica, $297^{\circ}$

fatty acids in, in pernicious anemia, $42 \mathrm{I}$

in fat indigestion in infants, 670

methods of examination of, for animal parasites, 389

mucus in, in constipation, 657

urobilin in, in pernicious anemia, $42 \mathrm{I}$

Feeding in yellow fever, 314

infant. See Infant feeding.

Feet, care of, in workmen, 178

Fel bovis in intestinal stasis, 647

Fermentative diarrhea of infants, infectious diarrhea and, differentiation, 68I

symptoms, $68 \mathrm{I}$

treatment, 682

indigestion in infants, 680

bacteria in, $68 \mathrm{r}$

diarrhea in, 680

etiology, 680

infectious diarrhea and, differentiation, 680

Fernet's automassage in intestinal stasis, 646

exercises in constipation, 659

Fever, hay, 528 . See also Hay fever.

in infantile la grippe, 572

in trichiniasis, 402

in uremia, 839

in yellow fever, treatment, $3 \times 5$

typhoid, I 83

Fibrillation, auricular, digitalis in, 32

Fibrinous pleuritis, acute, 567. See also Pleuritis, acute fibrinous.

Fibroids, uterine, roentgen-ray therapy in, contraindications to, $14 \mathrm{I}$

Fibroma of bile ducts, 704 of liver, 704

Fibrositis, acute, baths in, 85
Ficus laurifolia in trichuriasis, 404

Filaria bancrofti, 408

loa, 409

perstans, 409

varieties, 408,409

Filariasis, 408

Bacillus lymphangiticus in, 409 collargol in, 409

Filarioidea superfamily, 408

Filtration of roentgen-rays, 132

Finlay's mosquito theory of yellow fever. 301

Finney operation in gastroptosis, 728

First-aid kits in factories, 176

Fischer's solution in yellow fever, 316 treatment of edema, 807

Fish tapeworm, 391

Fistula, biliary, surgical treatment, 695

Flaxseed poultice in acute fibrinous pleu. ritis, 567

Fleas, 416

as carriers of bubonic plague, 4 I 6

bite of, treatment, 416

methods of extermination, 4 I 6

oil of pennyroyal for, 416

prophylaxis, 416

rat, bubonic plague from, 298

varieties, 416

Fleaseed in constipation, $66_{3}$

Flies, 409

adult biting, varieties, 4 I 2

Floor maggot, Congo, 410

Flooring, proper, for factory, I 74. See also Factory flooring.

Flower pollen in etiology of hay fever, 529

Floyd's apparatus for artificial pneumothorax, 258,260

needle for artificial pneumothorax, 262 Baldwin's adjustable guard for, 262

Fluid intake in myocardial insufficiency, 827

Flukes, 395

liver, 398

lung, 397

Fluoroscopic examination in pulmonary tuberculosis, 250

Fly-bite, $35^{8}$

Fly-speck vomit in yellow fever, 308,312

Focal infection in relation to systemic disease, 54

Folkestone Spa, principal characteristics, 88

Follicular tonsillitis, 517

characteristics, 5 I 7

complications in, 518

duration, 5 I 8

etiology, 517

exciting causes, 517

microörganisms in, $5 \times 7$

pearly white spots in, 518

predisposing causes, 517

prognosis, 5 I 8

salol in, 518

symptoms, 517

treatment, 518 
Food absorption in typhoid fever, 204 poisoning by reptiles and insects, I 57

from anaphylaxis, 157. See also Anaphylaxis, food poisoning from.

from hypersusceptibility or anaphylaxis, 157

proper, for workmen, I8I

proteins, anaphylaxis to, 157

supply, protection of, in prevention of

typhoid, I88

Foods, caloric value of, $84^{8-85^{\circ}}$

chlorid values of, $848-850$

infant. See Infant feeding.

protein values of, $848-850$

Foot-and-mouth disease in man, 325

Foreign body in bronchi, bronchoscopy in, 554

methods, $56 \mathrm{I}, 562$

lung, bronchoscopy in, 554

Forlanini's apparatus for artificial pneumothorax, 256, 258

Formalin enemata in intestinal stasis, $65^{\circ}$

in cecum mobile, 714

in thrush, 603

Formalin-gelatin in tuberculous peritonitis, 745

Fouchard's disease, 583 . See also Pyorrhea alveolaris.

Fowler postural treatment in septic peritonitis, 734

solution in Hodgkin's disease, 462

Fractures, imperfectly healed, baths in, 84 near joints with immobility, thermal baths in, 84

with osteitis and necrosis, 84

Fraenkel exercises in tabes dorsalis, 875

France, spas of, 89

Friedländer's bacillus, septicopyemia from, 265

Fröhlich's syndrome, 467

Frost-bite, baths in, 85

Fruit and vegetable diet in sprue, 370

Fulguration in papilloma of bladder, 866

Full feeding in functional nervous diseases, 912

Fuller's earth as antidote, I 64

Fulminant epidemics of malaria, 347

Fumigation in pediculosis, $4 \mathrm{I} 5$

in scarlet fever, 2.45

in yellow fever, 319

Functional heart diseases, aconite in, 484 nervous diseases, 906 . See also Nervous diseases, functional.

Fungus, thrush, in sprue, 368

Funk's base in beriberi, 365

Furunculosis, staphylococcus vaccines in, 42

GALL-BLADDER, carcinoma of, 705 diseases, 685

in peptic ulcer, 626

extirpation of, in gall-bladder infections, 698

infections, carcinoma from, 694

cholecystectomy in, 698

drainage operations in, 695
Gall-bladder infections, external drainage in, 695

extirpation of gall-bladder in, 698

indications for operation in, 7or

medical treatment, indications for, 701

myocarditis from, 694

surgical treatment, 693, 695

treatment, 695

surgery of, 693

Gall-stones, obstructive jaundice from, treatment, 701

Galvanic electric current, 97. See also Constant electric current.

Galvanization in asthma, 539

Galyl in recurrent fevers, $36_{3}$

in relapsing fevers, $36_{3}$

Ganglion, Gasserian, injection of, in trigeminal neuralgia, $87 \mathrm{I}$

Gangrene, gas, antiseptic solution for, 27 in erysipelas, 275

of lung, bronchoscopy in, 553

Gas embolism from artificial pneumothorax in hemoptysis, 544

in artificial pneumothorax, 254 gangrene, antiseptic solution for, 27 roentgen-ray tube, 124

Gas-forming wounds, Dakin's antiseptic in, 268

Gasoline for fleas, 4I 6

in pediculosis, $4 \mathrm{I} 5$

Gasserian ganglion, injection of, in trigeminal neuralgia, $87 \mathrm{I}$

Gastrectomy, partial, in gastric cancer, $63 \mathrm{I}$ in gastric cancer, fatal suture angle in, 631

Gastric cancer, 629. See also Cancer of stomach.

crises in tabes dorsalis, treatment, 875 digestion, relations of, in constipation, 658,659

hemorrhage in yellow fever, treatment, 315

indigestion in chlorosis, treatment, 431

juice, destruction of corrosive action in treatment of peptic ulcer, 6ro, 612

function of pepsin in, $6 \mathrm{II}$

in etiology of peptic ulcer, $6 \mathrm{I} I$

lavage in acute dilatation of stomach, 637

motility, state of, in constipation, ${ }_{55} 8$

retention in constipation, treatment, 658

secretion, effect of alkalis on, 29

ulcer, 6ro, 633. See also Peptic ulcer.

Gastrodiscus hominis, 399

Gastroduodenostomy for hemorrhage in peptic ulcer, 635

Gastroenteritis in pyorrhea alveolaris, 59I

Gastroenterostomy for acute perforation in peptic ulcer, 635

for hemorrhage in peptic ulcer, 635

for hour-glass constriction in peptic ulcer, 636

in peptic ulcer, 614

posterior, in gastroptosis, 728 
Gastrogastrostomy for hour-glass constriction in peptic ulcer, 636

Gastrogenous constipation, 658

Gastrointestinal autointoxications, iridocyclitis from, 387

diseases, nephritis in, $75 \mathrm{I}$

infantile la grippe and, differentiation, 573

mucosa, atrophy of, in pernicious anemia, 422

myiasis, $4 \mathrm{I} 2$

treatment, 412

Gastrojejunostomy for acute dilatation of stomach, 637

in cancer of stomach, 633

in chronic dilatation of stomach, 637 peptic ulcer, 636

in subacute peptic ulcer with localized abscess, 636

Gastropexy, indications for, 728

Gastroptosis, 638

Finney operation in, 728

posterior gastroenterostomy in, 728

surgical treatment, 728

Gastrostomy in cancer of stomach, 633

Gaucher's disease, differential diagnosis, 457

splenectomy in, 455

Gay and Claypole's sensitized typhoid vaccine, preparation of, 47,193

Gelatin enemata in chronic diarrhea, 653 in aneurysm of aorta, 155

in hemoptysis, 547

in hemorrhagic disenses, 440

Giemsa's prophylactic solution in malaria, 348

Gingival pyorrhea, $5^{82}$

Gingivitis, $5^{82}$

interstitial, 583

Glanders, vaccines in, $35,5 \mathrm{I}$

Glandular fever from infantile la grippe, 573

therapy, history, 14

Glass vacuum electrodes, I I 2

Gliosis, spinal, surgical treatment, 904

Globulin estimation in serum treatment of cerebrospinal syphilis, 897

excess of, in cerebrospinal syphilis, 887

Glossina morsitans in African trypanosomiasis, $37 \mathrm{I}$

palpalis in African trypanosomiasis, 37 I

Glossitis, acute catarrhal, 605

causes, 605

in acute infectious diseases, $60_{5}$

treatment, 605

Glottis, edema of, in acute nephritis, treatment, 767

in erysipelas, 275

Glucose in acidosis in children, 507

Glycerin, boric-acid, in aphthous stomatitis, 603

effect of, on vaccine virus, 294

enemata in intestinal stasis, 649

in pernicious anemia, 427

Glycosuria in lowered functional power of liver, 689
Glycosuria with hyperthyroidism, 490

Goiter, endemic, American trypanosomiasis and, differentiation, 379

exophthalmic. See Exophthalmic goiter.

Gold reaction in cerebrospinal syphilis, 888

Gonococcemia, gonococcus vaccines in, 44

Gonococcus in plastic iritis, 387

infections of eye, treatment, 387

vaccines, 34,43

administration, 44

dosage, 44

in conditions due to dissemination, 44

in epididymitis, 44

in gonococcemia, 44

in para-arthritis, 44

in pelvic infections, 44

in prostatitis, 44

in urethritis, 43

in vulvovaginitis, 44

intravenous administration, 45

Gonorrheal conjunctivitis, iced physiologic salt solution in, 383 spohol in, 382

iritis, gonococcus vaccines in, 44

stomatitis, 604

treatment, 604

Gout, 513

atophan in, 514

chronic interstitial nephritis from, 775

electricity in, 5 I 5

etiology, $5 \mathrm{I}_{3}$

lithion cataphoresis in, 515

monosodium urate in, forms of, $5^{1} 3$

nephritis from, $75 \mathrm{I}$

radium in, 513

thorium X in, 514

treatment, 513

by injection of solution of radium salts, 514

in radium emanatorium, 514

Governmental prophylaxis of disease, I 7

Graduated exercise in tuberculosis, 248

Granular lids, 383

Grass pollen in etiology of hay fever, 520

Graves' disease. See Exophthalmic goiter.

Grawitz treatment of pernicious anemia, 425

Grip. Sce La grippe.

Grocer's itch, 4 I 8

Guaiacol, endobronchial injection of, in tuberculosis, 553

in chronic diarrhea, 653

in intestinal stasis, 649

Guinea worm, 409

Gumma of liver, treatment, 705

Gummata in cerebrospinal syphilis, $\mathbf{8 8 2}$

HABIT, cocain, treatment, I 7 I opium, 167

Habits, drug, 163

Hair of workmen, care of, 179

Halzoun, 398

Hampson radiometer for estimating Roentgen-ray dosage, I 30 
Händel and Neufeld's antipneumococcus serum, 220

Hands of workmen, care of, $1 \% 8$

Harrogate Spa, principal characteristics, 88

Hay fever, 528 anaphylaxis to plant pollens in, 528 asthmatic symptoms in, 528 clinical manifestations in, 528 diagnosis of causative agent, 528 dicotyledons in etiology of, 528 flower pollen in, 529

general treatment, 534

grass pollen in, 529

immunity to, from pollen extract, 533 injection of pollen extract in, 530, 532 monocotyledons in etiology, 528

pollen extract in, anaphylactic shock from, 533

determination of initial dose, 532 dosage, 5.32

preparation of, 530

results from, 533

skin reaction from, $53 \mathrm{I}$

relation of plants to, 528

treatment, 530

tree pollen in, 529

Head louse, 4 I 4

Headache in acute nephritis, treatment, 767

in aortic regurgitation, treatment, 480

in intestinal stasis, treatment, $65^{\circ}$

in uremia, 839

treatment, 845

of neurasthenia, static breeze in, $\mathbf{\text { r } 5}$

Health Department, Pennsylvania, I 7. See also Pennsylvania Department of Health.

kidney function in, 786

urine in, 787

Heart block, effect of digitalis on, 478

dilatation of, digitalis in, 479

disease, alcohol in, 486

atropin in, 479

caffein in, 483

chronic, rest treatment, 486

diet in, 485

digipuratum in, 478

digitalin in, 478

digitalis in, 476

dosage, 477

dropsy of, nursing in, 484

operations for, 484

theobromin in, 484

functional, cactus in, 479

convallaria in, 479

digitalis in, 477

heart exercises in, 486

milk diet in, 486

mineral waters in, 487

Nativelle's granules in, 478

strychnin in, 479,483

surgical treatment, 488

syphilis in, treatment, 488

theobromin in, 483

treatment, 476
Heart disease, valvular, digitalis in, 477 , 480

venous section in, 142

work tests in, 485

displacement of, in artificial pneumothorax, 255

exercises in heart diseases, 486

failing, sugar treatment, 486

hypertrophy of, 823 . See also Myocardial insufficiency.

muscle, effect of disease toxins on, 476 soldier's, 480. See also Soldier's heart. stimulation of, in septicopyemia, 270 strained, 480. See also Soldier's heart.

Heat, glass electrodes for obtaining, I I 2

in treatment of septicopyemia, 268

Heavy human type, 716,718

Hectine in malaria, $35 \mathrm{I}$

Heidenhain and Bowman's theory of kidney function, 786

Heliotherapyin tuberculous peritonitis, 744

Helminthiasis, intestinal symptoms, 392

Hemangioma of bile-ducts, 704 of liver, 704

Hematemesis in splenic anemia, 434

Hematomyelia, surgical treatment, 904

Hemiatrophy, progressive facial, treatment, 931

Hemiplegia in artificial pneumothorax, 254

Hemiptera as carriers of disease, 4 I 4 varieties, 4 I 4

Hemoglobin, determination of oxygen combining power of, in acidosis in children, $5 \circ 3$

Hemoglobinemia in hemolytic icterus, $43^{6}$

Hemoglobinophilic vaccines, 35

Hemoglobinuria in hemolytic icterus, 436 in malaria, treatment, $35^{2}$

Hemolysis, blood, pernicious anemia from, $42 I$

Hemolytic icterus, 435. See also Icterus, hemolytic.

splenomegaly, splenectomy in, 455

vaccines, 34

Hemophilia, 442

active treatment, 443

blood coagulability in, 442

transfusion in, 443

diphtheria antitoxin in, 443

etiology, 442

pepton solution in, methods of admin. istration, 443

prophylaxis, 442

sera in, 443

subcutaneous injection of rabbit's

blood in, 443

theories of, 442

treatment, 442

Hemopoiesis, effect of altitudes on, 94

Hemopty sis, 54I

artificial pneumothorax in, 543

accidents in, 574

adhesive strapping after, 545

gas embolism in, 544

local anesthesia in, 545 
Hemoptysis, artificial pneumothorax in, site of, 544

technique, 543

thoracentesis in, 545

bibliography, 547

blood serum in, 542

Coagulen-Ciba in, 546

coagulose in, 542

emetin hydrochlorid in, 546

gelatin in, 547

hypodermic injection of codein in, $54 \mathrm{I}$ of morphin in, 54 I

in infarction of lung, treatment, $55 \mathrm{I}$

myocardial insufficiency, treatment, 835 normal horse serum in, 532

pantopon hydrochlorid in, 546

pituitary extract in, $\mathbf{5 4 2}$

potassium bromid in, 54I

rest treatment, $54 \mathrm{I}$

salines in, 547

sodium bromid in, $54 \mathrm{I}$

chlorid in, 541

treatment, $54 \mathrm{I}$

Hemorrhage, artificial pneumothorax in, 250

from lung puncture in artificial pneumothorax, 255

from lungs. See Hemoplysis.

gastric, in yellow fever, treatment, 315

in chronic jaundice, treatment, 702

in peptic ulcer, 635

treatment, $6 \mathrm{I}_{3}$ surgical, 635

in quinsy, 520

in yellow fever, 308

Hemorrhagic diseases, 438

antithrombin in, 438

bacteria in, etiology, 438

bibliography, 440

calcium in, 440

diet in, 439

etiology, 438

gelatin in, 440

injection of citrated blood in, 439

iron in, 439

lessened blood-platelets in, $43^{8}$

of newborn, $44^{\circ}$

blood coagulation in, 44 $\mathrm{I}$ transfusion in, 44I

etiology, $44^{\circ}$

sodium citrate in, $44 \mathrm{r}$

treatment, $44 \mathrm{I}$

of whole blocd in, 439

prothrombin in, 438

transfusion of blood in, 440

treatment, 438

Hemothorax, thoracentesis in, 570

treatment, 570

Henoch's purpura, 438. See also Hemorrhagic diseases.

Hepatic. See Liver.

distomiasis, 397,398

kamala in, 398

male fern in, 398

treatment, 398

Hepatitis, emetin hydrochlorate in, 30
Hepatoptosis, indications for operation in, 727

Herbivorous human type, 716,718

Hereditary syphilis, treatment, 282

Heredity in etiology of arteriosclesosis, 817

- of chronic interstitial nephritis, 775 of nephritis, 752

Herpes zoster ophthalmicus, $38 \mathrm{r}$

Herpetic stomatitis, 603

treatment, 604

Heterophyes heterophyes, 399

Hexamethylenamin as prophylactic in acute anterior poliomyelitis, 878

in septicopyemia, 272

Hiccoughs in yellow fever, 308

High-calorie diet in typhoid fever, 205, 206 in typhoid fever, results from, 208

High-frequency currents, roI contraindications to use of, I I I

in arteriosclerosis, 120,487

contraindications to, 121

in scleroderma, $93^{\circ}$

methods of application, I IO, III

Tesla, Io2

therapeutic uses, 103

effluve, 112

in acroparesthesia, II 5

in headache of neurasthenia, II 5

spray discharge, $\mathrm{Ir} 2$

Higueron in trichuriasis, 404

Hodgkin's disease, $46 \mathrm{I}$

arsenic in, 462

Coley's toxins in, 462

diagnosis, 46I

Fowler's solution in, $4^{62}$

possible causes, $46 \mathrm{I}$

roentgen rays in, 137,462

treatment, $46 \mathrm{I}$

vaccines in, 55,462

Hog tapeworm, 393

Hook worm, 406

disease. See Ankylostomiasis.

Hormonal in constipation, $66_{3}$

Horse serum for typhoid immunization, I 98

in hemorrhage in chronic jaundice, 702

normal, in hemoptysis, 542

Horses, pernicious anemia of, 422

resistance of, to immunity from diphtheria toxin-antitoxin, 69

Hot-air baths in acute nephritis, 766 in nephritis, 851

treatment of tuberculous peritonitis, 744

Hot baths in acute rephritis, 766

packs in acute nephritis, 765

in corrosive sublimate poisoning, 765 in edema, $8_{I}$ I

in nephritis, 852

weather, precautions for workmen in, $18 \mathrm{I}$

Hotels, inspection of, in Pennsylvania, 23

Hour-glass stomach, peptic ulcer in, surgical treatment, 613,636 
House disinfection, 18

in kala-azar, $35^{8}$

Housing division of Pennsylvania Department of Health, 23

Huebner's theory of orthostatic albuminuria, 802

Human type, broad-backed, 7I6, 7 I8

carnivorous, 7 16, 7 I 7 congenital visceroptotic, 7 I6, 7 I 7

heavy, 716,718

posture of, 7 I 9

visceroptosis in, 7 I 9

herbivorous, 716,718

intermediate, 716,717

narrow-backed, 7 I6, 7I 7

normal, posture of, 7 I 9

visceroptosis in, 720

slender, posture of, 7 I 7

visceroptosis in, 719

Hydatid cysts of liver, surgical treatment, 705

Hydrocephalus, callosal puncture in, 870 from delayed absorption, 870

lumbar puncture in, 870

from hypersecretion, 870

thyroid extract in, 870

obstructive, 870

phenol-sulphonephthalein test in, 870

temporal decompression in, 870

treatment, 870

types of, 870

Hydrochloric acid, action of, in peptic ulcer, 6r 2

effect of, on pancreatic secretion, 706 in intestinal stasis, 647

in pernicious anemia, 429

neutralization of, in treatment of peptic ulcer, 6r 5

Hydrocyanic acid in septicopyemia, 270

Hydrogen dioxid for flea-bite, 4 I 6

peroxid enemata in chronic diarrhea, 653

in ulcerative stomatitis, 603

Hydrology in military practice, 83

Hydronephrosis, ureter structure in, 856

Hydroquinin in malaria, $35 \mathrm{I}$

Hydrotherapy. See Baths.

Hydrothorax from intrathoracic resection of esophagus, 608

in uremia, treatment, 846

Hygiene, occupational, for workman, 177 of factory and workroom, I 72

Hymenolepis diminuta, 39I nana, 391

Hyoscin hydrobromate in aortic regurgitation, 480

in opium habit, 169

in septicopyemia, $27 \mathrm{I}$

Hyperchlorhydria in cecum mobile, treatment, 7 I 4

Hyperemia, local, glass electrodes for obtaining, I I 2

Hyperepinephry, 466

Hyperesthesia in leprosy, 34I

Hyperextension in visceroptosis, 721,725

Hyperglycemia in diabetes, 489
Hyperleukocytosis after vaccination in typhoid fever, 193

Hypernutrition in chronic diarrhea, $6_{52}$

Hyperpituitarism, 467

treatment, 467

Hyperpnea in acidosis in children, sor in diabetes, 501

Hyperpyrexia in septicopyemia, treatment, 270

in yellow fever, treatment, 313

Hypersecretion, hydrocephalus from, 870

Hypersusceptibility, I57. See also Anaphylaxis.

Hypertension, 8 22

adrenin as cause, 816

agar-agar in, $82 \mathrm{I}$.

benign, 8 I 5

care of bowels in, 821

cause, 8 I3 $_{3}$

chronic nephritis with, venous section, 142

constipation in, treatment, $82 \mathrm{I}$

diet in, 821

etiology, 8r3

from lead-poisoning, 8 r $3_{3}$

from reduction of renal substance, $8 \mathrm{r}_{3}$

from ureteral obstruction after nephrectomy, $8 \mathrm{r} 3$

general hygienic measures in, 820

in nephritis, chronic, $8 \mathrm{I}_{2}$

interstitial, treatment, 779,784

in etiology of arteriosclerosis, 816 . See also Arteriosclerosis.

in myocardial insufficiency, $8 \mathrm{r}_{2}$

in nephritis, heart findings in, 824

in uremia, $8 \mathrm{I}_{2}$

treatment, 847

iodids in, 823

laxatives in, 821

malignant, 8r 5

albuminuric retinitis in, 815

mechanical origin, theory of, $8 \mathbf{r}_{4}$

medicinal treatment, $82 \mathrm{I}$

nitrites in, 821

dosage, 822

mode of administration, $\mathbf{8 2 2}$

prognosis, 8I 8

prophylactic treatment, 8I 9

radium in, 823

relation of myocardial insufficiency to, 824

renal function in, 817

renin as cause, $8 \mathrm{I} 6$

rest in, 821

Russian oil in, $82 \mathrm{I}$

sleep in, 821

treatment, active, 8 ro

uremia in, 818

Hypertensive cardiovascular disease, 8 I 5

Hyperthyroidism, deep roentgen-ray therapy in, 139

glycosuria with, 490

Hypertonic saline solution, injection of, in cholera asiatica, 298

Hypertrophy of heart, 823. See also Myocardial insufficiency. 
Hypertrophy of thyroid gland at puberty, 474

Hypnotics in delirium tremens, 166

Hypoadrenalism, 466

Hypoadrenia, 466

Hypoderma bovis, 4 I I

diana, 4 I I

lineatum, $4 \mathrm{II}$

Hypodermic injection of magnesium glycerophosphate in tetanus, 327

of magnesium sulphate in tetanus, 327

Hypodermoclysis in corrosive sublimate poisoning, 165

in septic peritonitis, 734

in septicopyemia, 270

Hypoepinephry, 466

Hypoparathyreosis, subtetanic, 465

treatment, 465

Hypopinealism, treatment, 469

Hypopituitarism, 467

appearance in, 468

pituitary medication in, 468

puncture of corpus callosum in, 468

spinal puncture in, 468

symptoms, 468

synonyms, 467

treatment, 468

Hypopyon keratitis, autoserotherapy in, $3^{88}$

Hyposthenuria, tubular, concentrating power of kidney in, 789

vascular, 790

Hypothyroidism, desiccated thyroid in, 464 treatment, 464

Hysterectomy, supravaginal, in tuberculous peritonitis, 744

ICE CREAM in yellow fever, 3 I 3

Ice-bag in endocarditis, 484 in follicular tonsillitis, 518

in pericarditis, 484

Ichthyol enemata in chronic diarrhea, 653 in erysipelas, 277

in septicopyemia, 268

Icterus, chronic, hemorrhage in, treatment, 702

hematogenous, in septicopyemia, 266

hemolytic, 435

acquired, $43^{6}$

anemia in, treatment, 437

arsenic in, 437

bibliography, 437

congenital, 436

syphilis as cause, 436

course, 436

differential diagnosis, 459

duration, 436

etiology, 435

hemoglobinemia in, 436

hemoglobinuria in, 436

iron in, 437

recurrent crises in, 436

roentgen rays in, 437

spleen in, etiology, 435

splenectomy in, $428,436,455$

Vol. IV. -6I
Icterus, hemolytic, symptoms, 435 syphilis in, treatment, 437

treatment, 436

in yellow fever, 308,312

obstructive, from gall-stones, treatment, 70 r

Idiopathic erythremia, 432

Ileocolitis, acidosis in, in children, 504

Ileosigmoidostomy; resection of colon with, 727

Ileus, paralytic, in septic peritonitis, treatment, 735

Immune bodies in blood in lobar pneumonia, 226

Immunity, duration of, after typhoid vaccination, 192,196

from Behring's vaccine in diphtheria, 242

from diphtheria antitoxin, 242

in erysipelas, 275

in syphilis, $28 \mathrm{I}$

relation of, to anaphylaxis, 157

to African trypanosomiasis, 373

to diphtheria. See Diphtheria, immunity to.

to hay fever from pollen extract, 533

to malaria, community, 347

typhoid, duration, 48

Immunization, active, to typhoid fever, 200

in asthma, 539,540

in mumps, 336

in yellow fever, 317

with antidiphtheritic serum, average dose for, 242

Impetigo, transmission of, by pediculi, 4 I 5

Inclusion bodies in swimming-bath conjunctivitis, 382

Incubation theory of yellow fever, 302

Indian kala-azar, 356

Indicanuria in intestinal stasis, 644

Indigestion, 'fat, in infants. See Fat indigestion in infants.

gastric, in chlorosis, treatment, $43^{2}$

Indirect blood-transfusion, I 49

Induced electric current, 99

Industrial diseases. See Occupational diseases.

Infant feeding, calculation of formula for, 668

caloric values in, 669

cane sugar in, 675

carbohydrates in, 672

cow's milk for, 668

dextrin-maltose in, 674

fat in, 660

indigestion of fat in, 670 . See also Fat indigestion in infants.

ingredients necessary, 667

lactose in, 672

malt sugar in, 672

maltose in, 674

diarrhea from, 675

milk sugar in, 672

diarrhea from, Eiweiss milk in, 673 
Infant feeding, milk sugar in, diarrhea from treatment, 673

digestive disturbances from, 675

olive oil in, 670

percentage, 668

phytin in, 674

protein in, 675

effect of alkalies on digestion of, 677

indigestion in, 676,678

precipitated casein mixtures in, 679

whey-protein mixtures in, 679

milk in, 677

quantity of fat in, 670

starch in, 675

sugars in, 672

vegetable fat in, 670

Infantile beriberi, 365

treatment, 366

la grippe. See La grippe, infantile.

paralysis. See Poliomyelitis, acute anterior.

Infantilism, pancreatic, treatment, 708

Infants, digestive disorders of, 667

fat indigestion in. See Fat indigestion in infants.

fermentative diarrhea of, 68I

infectious diarrhea and, differentiation, 68I

symptoms, 68I

treatment, 682

indigestion in, 679

etiology, 680

infectious diarrhea and, differentiation, 680

foods for, ingredients necessary, 667

infectious diarrhea of, 683

diarrhea in, bacteria in, 683

course, 683

etiology, 683

fermentative diarrhea and, differentiation, $68 \mathrm{I}$

management, 683

Infarction of lung, $55 \mathrm{r}$

bibliography, 55I

codein in, $55 \mathrm{I}$

hemoptysis in, treatment, $55 \mathrm{I}$

morphin in, 551

treatment, $55 \mathrm{I}$

Infected wounds, Dakin's antiseptic in, 268

Wright's treatment, 28

Infectious diseases, .183

acute nephritis from, 757

albuminuria in, 803

chronic interstitial nephritis from, 775

etfology of nephritis, 748

ocular complications of, $38 \mathrm{I}$

treatment, special lines of, 387

symptoms of, septicopyemia and, differentiation, 267

parotitis, 335 . See also $M u m p s$.

Infiltration anesthesia in septic peritonitis, 736

Influenza. See La grippe.

bacillus infections, vaccines in, 53
Influe nza, nephritis from, 740 vaccines, 35

Infusions, saline, I42

Inhalation treatment of tuberculosis, 28

Inherited anaphylaxis, 157

Insanity, autointoxication in, treatment, 922

baths in, 926

contraindications to, 927

results, 927

continuous baths in, 926

dreams in, etiology, 925

glandular therapy in, 923

manic-depressive, autointoxication in, etiology, 908

partial thyroidectomy in, 924

prolonged neutral bath in, 926

psychoanalysis in, 924

relation of internal secretions to, 923

repressed emotions in, etiology, 924

thymus extract in, 924

treatment, 913

warm baths in, 926

Insects, food poisoning by, I 57

Insomnia, baths in, 86

in aortic regurgitation, treatment, 480

in myocardial insufficiency, treatment, 836

in uremia, treatment, 845

Intermediate human type, 7 16, 7 I 7

Internal secretion of kidney, 786 relation of, to insanity, 923

Interpolar effects of constant electric current, 98,99

Interstitial gingivitis, $5^{8} 3$

keratitis, 385

nephritis, chronic, 774. See also Nephritis, chronic interstitial.

Intertracheal insufflation in tetanus, 328

Intestine, large, automassage of, in intestinal stasis, 646

Intestinal antiseptics, 29

autointoxication, 643. See also Intestinal stasis.

disinfectants in cecum mobile, 7I4

distomiasis, 399

male fern in, 399

thymol in, 399

treatment, 399

helminthiasis, symptoms, 392

parasites, symptomatic anemia from, 444

sprue, 367

stasis, 643

adrenalin in, $65^{\circ}$

asthma in, treatment, 649

atropin in, $65^{\circ}$

automassage of large intestine in, 646

belladonna in, $65^{\circ}$

betanaphthol in, 649

cascara in, 648

constitutional symptoms from, 642

defective digestion in, treatment, 646

diet in, 646

enemata in, 649

excision of colon in, 665

fel bovis in, 647 
Intestinal stasis, formalin enemata in, 650 glycerin cnemata in, 649 guaiacol in, 649

headache in, treatment, 650

hydrochloric acid in, 646

indicanuria in, 644

iron ball massage in, 648

Lane's operation in, $65^{\circ}$

magnesium sulphate in, 648

massage in, 648

mechanical treatment, 645

milk of magnesia in, 648

mineral oil in, 648

oil enemata in, 649

olive oil in, 648

pancreatic extract in, 647

pepsin, 646

pilocarpin in, 650

podophyllin in, 648

prognosis, 645

relation to autonomic system, 650

rhubarb in, 648

roentgen-ray study of colon in, 644

senna in, 648

sodium phosphate in, 648

surgical treatment, 665

symptoms, 644

taka diastase in, 647

test mcals in, 647

thymol in, 649

treatment, 645

teniasis, 390. See also Teniasis, intestinal.

Intestines, diseases of, 639

electrotherapy in, 118

treatment, 29

in typhoid, changes in bacterial flora of, 205

large, physiology, 655

seats of reflex stimulation for, 655

Intoxication, food, by reptiles and insects, I57

gastrointestinal, iridocyclitis from, 387

in etiology of functional nervous diseases, 906

Intracranial injection treatment of paresis, 918

Intramuscular administration of salvarsan, $28 \mathrm{r}$

Intraneural injection of antitetanic serum, 328

Intraspinal injection of antimeningitis serum, 77

of magnesium sulphate in delirium tremens, 167

in tetanus, 327

of tetanus antitoxin, 72

disappearance from spinal canal after, 73

Intrathecal injection of antitetanic serum. 329

Intrathoracic resection of esophagus, 607 hydrothorax from, 608 obstacles to, 608

Intravenous administration of salvarsan, 285
Intravenous injection of diphtheria antitoxin, 71

of emetin in amchic dysentery. 339

saline solution in septic peritonitis, 734 in malaria, 349

Intussusception in pertussis, 332

Iodids in aneurysm of aorta, 155

in hypertension, 823

in paresis, 922

Iodine as antidote, $\mathrm{x}_{6}$

in bacterial pyorrhea, 587,596

in leukoplakia, 606

in normal saline solution in pyorrhea alveolaris, 595

in pertussis, 333

in septic peritonitis, 737

in septicopyemia, 272

in tabes dorsalis, 875

in tuberculous peritonitis, 745

Iodine-mercury treatment of tertiary syphilis, 282

Iodoform enemata in chronic diarrhea, 653

Ionic medication in blepharitis, $38 \mathrm{r}$

in pyorrhea alveolaris, 28

Iontophoresis, zinc, in purulent keratitis, 384

Ipecac in dysentery, 337

Ipecacuanha as emetic for children, 29 in delirium tremens, 165

Iridocyclitis, 385

from gastrointestinal autointoxications, 387

in African trypanosomiasis, 372

Iritis, 385

autotoxemic treatment, 386

from pyorrhea alveolaris, 386

from syphilis, 386

from tooth abscess, 386

gonorrheal, gonococcus vaccines in, 44

mercury in, 386

neosalvarsan in, 386

plastic, complement fixation test in, $3^{87}$.

gonococcus in, 387

local treatment, 387

pilocarpin in, 387

potassium iodid in, 387

potassium iodid in, 386

rheumatic, treatment, 386

secondary to mucous membrane infection, 386

sodium cacodylate in, 386

toxemic, treatment, 386

Wassermann test in, 386

Iron ball massage in intestinal stasis, 648

chlorid in yellow fever, 314

content of vegetables, 446

elixir in yellow fever, 314

in acute nephritis, 768

in chlorosis, 431

in edema, 807

in hemolytic icterus, 437

in hemorrhagic diseases, 439

in pernicious ancmia, 429

in pertussis, 333

in symptomatic anemia, 447

in tabes dorsalis, 876 
Iron, tincture of chlorid, in follicular tonsillitis, 518

Irrigations in septic peritonitis, 737 saline, $\mathrm{I}_{4} 2$

contraindications to, 142

Iso-agglutination, testing of, in blood transfusion, 148

Isolation in prophylaxis of typhoid, r 89

Itch, grocer's, 418

Itching in acute nephritis, treatment, 767

in uremia, treatment, 847

JAMEs' method of staining malarial parasite, 346

Ashford's modification, 346

Jaundice. See Icterus.

Jejunostomy in gastric cancer, 633

Jianu's operation of esophagoplasty, 607

Joints, diseases of, electrotherapy in, I 6

fractures near, with immobility, thermal baths in, 84

sprains and strains of, baths in, 84

KALA-AZAR, 356

antimony in, 357

arsenical preparations in, 357

causal organism, 356

cause of death in, $35^{8}$

characteristics of, 356

diagnosis, methods, 357

extermination of vermin in, 358

house disinfection in, $35^{8}$

Indian, 356

Mediterranean, 356

mortality, 356

prognosis, 358

prophylaxis, 358

quinin in, 357

segregation in, 358

treatment, 357

vectors of, 356

Kamala in hepatic distomiasis, 398

Kaolin as antidote, I 64

Karell diet in chronic interstitial nephritis, 783

in lowered functional power of liver, 688

in myocardial insufficiency, 826

Katayama nasophora, 396

Kehr's tubes for surgical drainage of biliary tract, 697

Keloids, roentgen therapy in, I 34

Kenyon and Pool's suction apparatus in septic peritonitis, 737

Keratitis from alcohol injections in neural. gia, prevention of, 872

hypopyon, autoserotherapy in, 388

interstitial, 385

parenchymatous, 385

mercurial inunctions in, 385

neosalvarsan in, 385

potassium iodid in, 385

salvarsan in, 385
Keratitis, pare nchymatous, treatment, 385 tuberculin test in, 385

Wassermann test in, 385

phlyctenular, treatment, 384

purulent, zinc iontophoresis in, 384

Keratosis, senile, treatment, 134

Kerosene for fleas, 416

in pediculosis, $4^{\mathrm{I}} 5$

Ketonuria in Allen treatment of diabetes, 495

Kidney, arteriosclerotic, in chronic interstitial nephritis, 774

chronic passive congestion, coefficient of urea excretion in, 794

concentrating power of, in tubular hyposthenuria, 789

function, Bowman and Heidenhain's theory of, 786

Cottet's water test of, 789

creatinin test for, 796

in disease, 786

in health, 786

in hypertension, 817

lactose test, 797

Ludwig's theory, 786

in myocardial insufficiency, 824

phthalein test of, 797

salt test of, 79 I

test days for determining, 795

test-meals for, 794

tests of, 787

functional condition, in chronic interstitial nephritis, 777

in disease, alkali excretion in, 796

contrast in response to physiologic stimulation in healthy kidney, 788 nitrogen excretion in, 793

non-protein nitrogen in blood in, 798 oliguria in, 788

polyuria in, 788

sodium chlorid excretion in, 790

specific gravity of urine in, 789

in health, alkali excretion in, 796

contrast in response to physiologic stimulation in diseased kidney, 788

factors governing water excretion in, 789

nitrogen excretion in, 791

response to water tests, 788

sodium chlorid excretion of, 790

specific gravity of urine in, 789

internal secretion of, 786

large white, in chronic parenchymatous nephritis, 769

lavage of pelvis, in ureter stricture, 860

of pregnancy, 803

primary contracted, in chronic interstitial nephritis, 774

rest for, in chronic parenchymatous nephritis, 771

secondarily contracted, in chronic parenchymatous nephritis, 769

secondary contracted, in chronic interstitial nephritis, 774

selective excretion of, 786 
Kidney, stripping of, in corrosive sublimate poisoning, 165 synthesis in, 786

Kidneys in yellow fever, 306

Kienböck quantimeter for measuring roentgen-ray dosage, 129 normal scale, 129 test strips, 129

$\mathrm{X}$ unit of roentgen-ray dosage, $\mathrm{x} 29$

Killed bacteria, injection of, in scarlet fever, 246,247 vaccines from, 36 cultures of typhoid bacillus, as vaccines, 192

Kimpton-Brown glass cylinders for bloodtransfusion, 149

Kink, Lane's, in constipation, 657

Knee-chest position in visceroptosis, 725

Kohnstann's dictetic treatment of constipation, 660

Kornmann's apparatus for artificial pneumothorax, 260

Krumwiede and Park's method of injection of tetanus antitoxin, 74

LABORATORY work of Pennsylvania Department of Health, 23

Lactic acid bacilli in cecum mobile, 715

in tubercle of conjunctiva, 384

Lactose in infant feeding, 672

in typhoid fever, 205

test of renal function, 797

Lacunar tonsillitis, 517

La grippe, infantile, 572

codein in, 575

course, 572

differential diagnosis, 573

diphtheria and, differentiation, 573

Dover's powder in, 575

encephalitis in, 574

endocarditis in, 574

epinephrin in, 575

fever in, 572

gastro-intestinal disease and, dif-

ferentiation, 573

glandular fever in, 573

measles and, differentiation, 573

mortality, 572

myocarditis in, 574

nasopharyngeal involvement in, $57^{2}$

otitis media in, 573 treatment, 576

paregoric in, 576

peritonitis, 574

Pfeiffer's disease in, 573

prophylaxis, 575

quinin in, 575

renal complications in, 574

retropharyngeal abscess in, 574

salicylates in, 575

treatment, 575

typhoidal form, 573

whooping cough and, differentiation, 573
Laktam monosodium urate in gout, 513

Laktim monosodium urate in gout, $5^{13}$

Laminectomy, 899

in bullet wounds of spinal cord, 902

in stab wounds of spinal cord, 902

Lane abdominal support in visceroptosis, 710

kink in constipation, 657

operation in intestinal stasis, 651

Lange colloidal gold reaction in cerebrospinal syphilis, 888

Lanolin in leishmaniasis, 359 after serum treatment, 898

Lavage, ether, in septic peritonitis, 7.37 gastric, in septic peritonitis, 734 in corrosive sublimate poisoning, $\mathrm{I}_{4}$ in dyschezia, 654

transduodenal, in chronic diarrhea, 653

Laxatives in edema, 810

in hypertension, 821

time for, in constipation, 658

Lead-poisoning, chronic interstitial nephritis from, 775

hypertension from, 813

nephritis from, $75 \mathrm{I}$

sclerosis of blood-vessels of abdomen from, 639

Lead-water and alcohol in crysipelas, 277

Leamington Spa, principal characteristics, 88

Leishmania donovani, 356

infantum in kala-azar, 357

tropica, $35^{8}$

Leishmaniasis, American forest, 359

bibliography of, 360

Brazilian dermal, $35^{8}$

lanolin in, 359

mutilation in, 359

nasopharyngeal lesions in, 359

tartar emetic in, 359

treatment, 359

ulcers in, 359

vaseline in, 359

dermal, $35^{8}$

tartar emetic in, 355

Leprosy, 34I

analgesia in, $34 \mathrm{I}$

arsenic in, $34^{2}$

bacteriologic diagnosis, $34 \mathrm{I}$

baths in, $34^{2}$

cause, 341

chaulmoogra oil in, 342

clinical symptoms, $34 \mathrm{I}$

diagnosis, $34 \mathrm{I}$

diet in, 342

experimental inoculation, $34 \mathrm{r}$

hyperesthesie in, 34I

macules in, $34 \mathrm{I}$

mercury in, 342

mode of transmission, 34 1

segregation in, $34 \mathrm{I}$

sodium salicylate in, $34^{2}$

strychnin in, 342

treatment, $34 \mathrm{I}$

tubercle, $34 \mathrm{I}$

Leptomeningitis in pertussis, 332 


\section{Leukemia, 448}

arsenic in, $137,45 \mathrm{I}$ associated with albuminuria, $8 \circ 3$

benzol in, $137,45^{\circ}$

dosage, $45 \mathrm{I}$

effects of, 450

precautions in administration, $45 \mathrm{I}$

bibliography, $45^{2}$

Coley's toxins in, $45^{2}$

course, 449

deep roentgen-ray therapy in, 136

etiology, 448

mortality in, 449

radium therapy to spleen in, $\mathrm{I} 37$

remission in, 449

roentgen rays in, 449

dangers of, 449, $45^{\circ}$

results of, 450

rules for application, 449

splenectomy in, $45^{2}$

thorium $\mathrm{X}$ in, $45^{\mathrm{I}}$

treatment, 449

Leukocytosis in pertussis, $33 \mathrm{I}$

Leukopenia, periodic mononucleosis with, in malaria, 348

Leukoplakia, 605

buccalis, treatment of, I33

parasyphilitic origin, 605

roentgen rays in, 606

treatment, 605

Lewis-Benedict method of estimating blood sugar in diabetes, 489

Lewisohn's technique for citrate method of blood transfusion, I 54

Lice, 4I4. See also Pediculosis.

Lids, granular, 383

Lighting, factory, I 74

Linseed in constipation, $66_{3}$ oil in acariasis, $4 \mathrm{I} 9$

Liquor antisepticus in aphthous stomatitis, 602

in catarrhal stomatitis, 602

ferri chloridi in erysipelas, 276

Lithemia, 5 I 6

diet in, 516

treatment, $5 \mathrm{I} 6$

Lithion cataphoresis in gout, 515

Live bacteria, vaccines from, 37

Liver, abscess, 702

after-treatment, 704

etiology, 702

from pylephlebitis, 702

in amebic dysentery, emetin in, 339

large solitary, 702

small multiple, 702

surgical treatment, 703

treatment, 703

tropical, 691, 702

varieties, 702

adenoma of, $\mathrm{TO}_{4}$

anomalies of form and position, 704

cancer of, 690, 705

complications in, treatment, $69 \mathrm{I}$

syphilis of liver and, treatment, 69I

treatment, 69I

diseases of, 685
Liver, fibroma of, 704

fluke, 398

gumma of, treatment, 705

hemangioma of, 704

hydatid cysts of, surgical treatment, 705

hypertrophic, cirrhosis of, therapeutic splenectomy in, 454

in yellow fever, 305

infections of, surgical treatment, 693 systemic effects, 694

lowered functional power of, 686 acidosis in, 687

apocynum in, 688

blood-examination in, 687

determination of extent, 687

diaphoretic baths in, 688

diet in, 689,690

glycosuria in, 689

high blood-pressure in, 687

in cardiovascular disease, 687

Karell diet in, 688

lessening physiologic strain in, 688

magnesium salicylate in, 689,690

mercury in, 690

potassium tartrate in, 689

prognosis, 690

sodium glycocholate in, 690 tartrate in, 689

treatment, 688

Riedel's lobe of, treatment, 704

situs inversus, 704

surgery of, 693

syphilis of, 691. See also Syphilis of liver.

syphiloma of, treatment, 70

tumors of, 704

Living cultures of typhoid bacillus as vaccines, 193

Llandrindod Spa, principal characteristics, 88

Lloyd's reagent as antidote, $\mathrm{I} 64$

Loa loa, $4 \circ 9$

Lobar pneumonia, 21 2

abscess of lung in, 215

antibodies in blood after, 222

antipneumococcus serum in, 218

autogenous vaccines in, 231

Bacillus pneumoniæ in, 215

bacteria associated with pneumococcus in, 215

camphor in, 233

care of patient in, 21 2

chemotherapy in, 233

clothing for patient in out-door treatment, 212

course of, 216

effect of pneumococcus vaccines on, 230

duration of attack, $2 \mathrm{I} 6$

ethylhydrocuprein in, 234

geographic distribution, 215

immune bodies in blood in, 226

initial characteristics, 215

modified pneumococcus vaccines in, 
Lobar pneumonia, Neufeld and Händel's antipneumococcus serum in, 221

Northrup's treatment, 212

open-air treatment, 212

opsonic index in, 230

optochin in, 235

amblyopia from, 236

deafness from, 238

results in treatment with, 237

pneumococcus in blood in, 226

in etiology of, 215

vaccines in, 228

quinin derivatives, 233

results from pneumococcus vaccines in, 220

Rockefeller Institute research on, 222 roentgen-rays in, $2 \mathrm{I} 3$

sensitized vaccines in, 232

serum therapy in, 216

sex in, 215

specific serum therapy in, 222

sphygmomanometry in, 213

strophanthin in, 213

strophanthone in, 213

vaccine therapy in, 228

Lockers, factory, 174

Locomotor ataxia. See Tabes dorsalis.

Loosened teeth, 583

Lotion douche in typhoid fever, 92

Louse, crab, 4I 4

Leukocytozoön syphilidis in paresis, 9 r 9

Lucilia macelaria, 4 Io

Ludwig's angina, 580

etiology, 580

pathology, 580

surgical treatment, 580

theory of kidney function, 786

Ludyl in recurrent fevers, 363

in relapsing fevers, 363

Luetic obstructions of tracheobronchial tree, bronchoscopy in, $55^{2}$

Luetin in functional nervou; diseases, 912

Lugol's solution as antidote, $\mathrm{I}_{3}$

Lumbago, baths in, 86

electrotherapy in, I 6

Lumbar puncture in cerebrospinal meningitis, 296

in diabetes insipidus, 509

in hydrocephalus from delayed absorption, 870

in serum treatment of cerebrospinal syphilis, $895-898$

Lunch hour in factories, 175

rooms, factory, I 75

Lung abscess, artificial pneumothorax in, $25^{\circ}$

bronchoscopy in, 554

in lobar pneumonia, 215

collapse of, 549 . See also Collapse, pulmonary.

drowned, bronchoscopy in, 554

edema of, 548 . See also Edema, pulmonary.

flukes, 397

foreign body in, bronchoscopy in, 554 lung, gangrene of, bronchoscopy in, 553

hemorrhage from. See Ilemoplysis.

infarction of, $55 \mathrm{r}$. Sec also Infarction of lung.

puncture, hemorrhage from, in artificial pneumothorax, 255

Lupus vulgaris, roentgen-ray therapy in, 134

Lymphadenitis in erysipelas, 274

in pyorrhea alveolaris, 591

Lymphangitis, pancreatic, drainage of common bile-duct in, 699

Lymphatics of stomach, surgical rela. tions, 631

Macules, leprous, $34 \mathrm{t}$

Maggot, Congo floor, 410

Magnesia, calcined, in non-obstructive peptic ulcer, $6 \mathrm{r} 7$

Magnesiumglycerophosphate, hypodermic injection of, in tetanus, 327

oxid in chlorosis, 432

salicylate in lowered functional power of liver, 689,690

sulphace, hypodermic injection of, in tetanus, 327

in erysipelas, 277

in intestinal stasis, 648

in malaria, 349

in oxyuriasis, 406

in trichiniasis, 402

injections in delirium tremens, 923

intraspinal injection of, in delirium tremens, 167

in tetanus, 327

Malaria, 345

ague cake with cachexia in, treatment, $35^{2}$

bibliography, $35^{2}$

calomel in, 349

carriers, 346

cinchonin in, $35 \mathrm{I}$

community immunity to, 347

diagnosis, suggestions for, 348

ethylhydrocuprein in, $35 \mathrm{I}$

fulminant epidemics, 347

hectine in, 351

hemoglobinuria is, treatment, $35^{2}$

hydroquinin in, $35 \mathrm{I}$

intravenous salt solution in, 349

latent forms, 345

magnesium sulphate in, 349

mosquito proofing houses in, 348

neosalvarsan in, $35 \mathrm{I}$

nephritis from, $75^{\circ}$

optochin hydrochlorid in, $35 \mathrm{I}$

periodic mononucleosis with leukopenia in, 348

pernicious, dosage of quinin in, 349

prophylaxis, 348

clearing and draining land in, 348

Giemsa's solution in, 348

mosquito net for, $34^{8}$

repellant in, $34^{8}$ 
Malaria, quinidin in, $35 \mathrm{I}$

quinin bichlorid in, 349

byproducts in, $35 \mathrm{I}$

hydrochlorid in, 349

quinin in, $349,35 \mathrm{I}$

acid salts, dangers of, $35 \mathrm{I}$

amorphous, advantages, $35^{\circ}$

daily prophylactic doses, 348

elimination of, $35^{\circ}$

indications for increased dosage of, $35^{\circ}$

intramuscular injections, $35 \mathrm{I}$

injections in, 349

treatment, 348

quinoidin in, $35 \mathrm{I}$

relapse in, 345

Celli's method of preventing, 346

Deek's method of prevention, 346

prevention of, 350

theories of, 347

treatment, 349

Ross' endemic index in, 347

salvarsan in, $35 \mathrm{I}$

Schlesinger's urobilin reaction in, 348

splenectomy in, indications for, 352

treatment, in Panama, 349

vomiting in, treatment, 349

Malarial parasite, cultivation of, in vitro, 347

James' method of staining, 346

Ashford's modification, 346

remittent fever, yellow fever and, differentiation, 310

Male fern in hepatic distomiasis, 398

in intestinal distomiasis, 399

teniasis, 393

in pernicious anemia, 429

in somatic teniasis, 395

in strongyloidosis, 401

in trichuriasis, 404 ,

in venal distomiasis, 396

Malt sugar in infant feeding, 672

Malta fever, 353. See also Undulant fever.

Maltose in infant feeding, 674 diarrhea from, 675

Mania à potu, I65. See also Delirium tremens.

Manic-depressive insanity, autointoxication in, etiology, 908

Manson's definition of yellow fever, 299

Marasmic toxemia in sprue, 367

Margate Spa, principal characteristics, 88

Marshall method for testing urea in urine, 794 of determining urea in blood, 799

Massage in acute anterior poliomyelitis, 879

in beriberi, 366

in intestinal stasis, 648

in soldier's heart with specific infections, $48 r$

in visceroptosis, 724

Massive dosage of roentgen rays, 129 contraindications to, $\mathrm{I}_{32}$

feeding in functional nervous diseases, 9I 2
Mattock Spa, principal characteristics, 89

Measles, infantile la grippe and, differentiation, 573

Meat diet, strict, in sprue, 370

Mediastinum, rupture of, in artificial pneumothorax, 255

Medical cases in military practice, baths in, 85

Medicine, modern, contributions of synthetic chemistry to, I 6

Mediterranean kala-azar, $35^{6}$

Melancholia, autointoxication in etiology, 907

baths in, 87

Meningitis, cerebrospinal, 296 anaphylaxis in, 79

carriers of, 296

early diagnosis, 296

Flexner's serum in, 296

lumbar puncture in, 296

serum treatment of, 77. See also Antimeningitis serum.

complicating typhoid fever, 199

epidemic cerebrospinal, vaccines in, 45

in artificial pneumothorax, 255

in cerebrospinal syphilis, 882

in erysipelas, 275

in septicopyemia, 266

in syphilis, salvarsan dosage in, 283

pneumococcus, ethylhydrocuprein in, 239

pneumococcus in, 215

Meningoarteritis in cerebrospinal syphilis, 882

Meningococcus vaccines, 34

Meningoencephalitis, chronic, in African trypanosomiasis, 372

Meningomyelitis, chronic, in African trypanosomiasis, 372

Menopause, premature, production by deep roentgen-ray therapy, 136

Menthol for flea-bite, 416

Mercurial ointment by inunction in syphilis, 293

Mercury, albuminate of, in cerebrospinal syphilis, 890

bichlorid, See Corrosive sublimate. poisoning, 164. See also Corrosive sublimate poisoning.

by inunction in tabes dorsalis, 876

in iritis, 386

in leprosy, 342

in lowered functional power of liver, 690

in parenchymatous keratitis, 385

in paresis, 921

in syphilis, 280, 292

by mouth, 293

combined with neosalvarsan, 288

hypodermic injection, novocain solution in, 293

of liver, $69 \mathrm{I}$

injection of, in paresis, 917

in tabes dorsalis, 876

nephritis from, $75 \mathrm{I}$

succinamid in pyorrhea alveolaris, 596 
Mercury iodine treatment of tertiary syphilis, $2 \mathrm{~S}_{2}$

Mercury-salvarsan combined treatment of syphilis, 280

Metabolism in typhoid fever, 204

Metaschizotrypanosis of American trypanosomiasis, 378

Methylene blue in leishmaniasis, 359

Micrococcus gonorrhœa, septicopyemia from, 265

melitensis in undulant fever, 353 vaccines, 45

tetragenus in mumps, 335 septicopyemia from, 265

Micro-Kjeldahl method of determining non-protein nitrogen in blood, 799

Middle-ear disease, tonsillectomy in, 522 infections, vaccines in, 55

Military practice, contraindications to baths in, 84

Dakin's antiseptic in, 269

hydrology in, 83

Milk, cows', anaphylaxis, 668 for infant feeding, 668

diet in acute nephritis, 762

in heart diseases, 485

strict, in sprue, 369

in typhoid fever, 205

of magnesia in intestinal stasis, 648 sugar in infant feeding, 672

Mineral oil in intestinal stasis, 648 poisons, secondary anemia from, 443 waters in heart diseases, 487

Minot's modification of Rous and Turner's iso-agglutination test in blood-transfusion, I 48

Mites, varieties infecting man, 4 I 7

Mitral regurgitation, digitalis in, 479

stenosis, digitalis in, 480

gentle exercise in, 479

strophanthus in, 479

venesection in, 479

Mixed vaccines, 36

in treatment of paratyphoid fever, 30

Moisture, artificial, in factory ventilation, I 73

Molded jacket in visceroptosis, 720,724

Molestia de Chagas, 377

Monilia albicans in sprue, 368

Monocotyledons in etiology of hay fever, 528

Mononucleosis, periodic, with leukopenia, in malaria, 348

Monosodium urate, laktam form, in gout, 513

Moore-Corradi electric treatment of aneurysm, i i 8

dangers of, 120

results of, 120

technique, I 19

Morphin, hypodermic injection, in hemoptysis, 54 I

in acute fibrinous pleuritis, 568

nephritis, 767

in asthma, 538

in delirium tremens, 166
Morphin in infarction of lung, 551

in pulmonary embolism, $55^{1}$

thrombosis, 551

in septicopyemia, 271

in tetanus, 328

sulphate in pulmonary edema, 548

Mosenthal's test meals for determining renal function, 795

Mosquito experiments in yellow fever, 302 net in prevention of malaria, 348

proofing houses in malaria, 348

repellant in prophylaxis of malaria, 348

theory of yellow fever, 301

transmission of yellow fever, 299

Mosquitoes in yellow fever, breeding habits, 317

destroying adult, 317,319

destruction of breeding places, 316 , 317

habits, 318

protecting well from, 317

reducing number of, 320

screening sick from, 317

transportation of, 318

Mountain sickness, explanation of, 95

Mouth, actinomycosis of, 605

treatment, 605

diseases of, 601

erysipelas of, 275

treatment, 277

general hygiene of, 577

infections, treatment of, 28

vaccines in, 55

syphilis of, treatment, 604

tuberculosis of, 604

treatment, 604

workman's, care of, I 79

Movable cecum, 712. See also Cecum mobile.

Mucus in feces in constipation, 657

Multiple-point electrode, I I I

Mumps, 335

acetonuria in, 336

atrophy of testicles in, prevention, 336

bradycardia in, 335

complications, 335

immunization in, 336

involvement of ovaries in, 335

of pancreas in, 335

of testicles in, 335

Micrococcus tetragenus in, 335

orchitis in, treatment, 336

pharyngitis in, 335

temporary sterility after, 335

treatment, 336

Muralt's apparatus for artificial pneumothorax, 259

Murphy drip apparatus in administration of Dakin's antiseptic, 268

method of enteroclysis in corrosive sublimate poisoning, 165

in septic peritonitis, 734

in typhoid fever, 92

Muscle contractions from constant elec-

tric current, 90

cramps in beriberi, treatment, 366 
Muscle, diseases of, electrotherapy in, I 16

paralysis, electrotherapy for, I I 4

training in acute anterior poliomyelitis, 879

Mushroom poisoning, nephritis from, $75^{\mathrm{I}}$

Mustard foot bath in yellow fever, 313

plaster in acute fibrinous pleuritis, 567

Mycosis cutanea chronica, $35^{8}$

Mycotic stomatitis, 602. See alsoThrush.

Myiasis, 409

cephalic, 4 IO, 4 I I

chloroform in, 4 II

cocain in, $4 \mathrm{I} I$

mortality, 4I I

symptoms, 4II

treatment, $4 \mathrm{II}$

cutaneous, 4 IO

gastrointestinal, 4I 2

treatment, 412

subcutaneous, 410

chloroform in, 4 I I

treatment, 4 I I

varieties, $4 \mathrm{IO}$

vesical, 4I 2

Myocardial insufficiency, 823

alcohol in, 828

anemia in, 834

caffein in, 833

cardiac distress in, treatment, 835

cough in, treatment, 835

diet in, 826

digitalis in, $829-832$. See Digitalis in myocardial insufficiency.

edema of lungs in, treatment, 836

fluid intake in, 827

gastric symptoms in, treatment, 835

hemoptysis in, treatment, 835

hypertension in, 8I 2

in chronic interstitial nephritis, treatment, 785

insomnia in, treatment, 836 .

Karell diet in, 826

methods of depletion in, 828

orthopnea in, treatment, 835

palpitation in, treatment, 835

paracentesis in, 828

physical examination in, 824

relation of, to hypertension, 824

renal function in, 824

rest treatment in, 825

sodium chlorid restriction in, 827

strophanthus in, 832

surgical treatment, 828

symptomatic treatment, 834

theobromin in, 834

theocin in, 834

treatment, 825

venesection in, 828

vomiting in, treatment, 835

Myocarditis, digitalis in, 477

from infection of gall-bladder, 694

in infantile la grippe, 574

Myocardium, effect of disease toxins on, 476

Myositis, electrotherapy in, I 16
Myxedema, chronic, transplantation of thyroid gland in, 475

desiccated thyroid in, 464

treatment, 464

NABARRo's method of diagnosis in African trypanosomiasis, 374

Nails of workman, care of, 178

Naphthalin for fleas, 4I6

Narrow-backed human type, 7 16, 7 1 7

Nasal electrode, i 22

sinus infections as cause of arthritis deformans, 5 ro

Nasopharyngeal lesions in dermal leishmaniasis, 359

Nativelle's granules in heart diseases, 478

Nausea in acute nephritis, treatment, 767

Navel, erysipelas of, 275

N. C. I. powder in pediculosis, 416

Necator americanus, 406

Neck, stiff, electrotherapy in, I I 6

Necrotic wounds, Dakin's antiseptic in, 268

Nematodes, 399,400

Neosalvarsan, 287

in African trypanosomiasis, 375

in cancrum oris, 580

in cerebrospinal syphilis, 800

in iritis, 386

in malaria, $35 \mathrm{I}$

in parenchymatous keratitis, 385

in paresis, 915

in recurrent fevers, 362

in relapsing fevers, 362

in spirochetal pyorrhea, 587

in syphilis, $31,280,287$

administration, 289

apparatus for, 289

combined with mercury, 288

in concentrated solutions, 292

intramuscular, 292

intravenous, 289

after-care, 289

apparatus for, 289

introduction into vein, 290

position of patient, 290

precautions before, 289

preparation of patient, 289

of solution, 289

technique, $290,29 \mathrm{I}$

temperature after, 292

methods, 289

preparatory mercurial treatment, 288

subcutaneous, 292

after-effects, 292

advantages over salvarsan, 288

appearance, 288

contraindications to, 289

dosage, 288

how obtained, 288

indications for, 289

intervals between doses, 288

precautions in use of, 288

salvarsan and, comparison, 287

in tabes dorsalis, 876 
Nephrectomy in tuberculosis of urinary tract, 863

Nephritis, acidosis in, in children, 504

acute, 654,757

acetanilid in, 767

acetphenetidin in, 767

administration of water in, $7^{-\sigma_{3}}$

alterations in tubular epithelium in, $75^{8}$

anemia in, treatment, 768

antipyrin in, 767

anuria in, treatment, 766

ascites in, treatment, 766

backache in, treatment, 767

baths in, 766

caffein in, 764

carbohydrate diet in, 762

catharsis in, 765

complications of, treatment, 766

diaphoresis in, 765

diet in, $76 \mathrm{I}$

digipuratum in, 764

digitalis in, 764

diuretics in, 763

dropsy in, treatment, 765

dyspnea in, treatment, 767

edema in, treatment, 766,808

of glottis in, treatment, 767

effect of cold baths on, 760

etiology, 757

exudative, in septicopyemia, 266

fluid intake in, 763

from exposure to cold, 757

from extensive skin lesions, 757

from pregnancy, 757

from toxic agents, 757

fruits in, 762

glomerular changes in, $75^{8}$

gruels in, 762

headache in, treatment, 767

high blood-pressure in, 764

hot baths in, 766

packs in, 765

interstitial changes in, $75^{8}$

iron in, 768

itching in, treatment, 767

measures to be avoided in treatment, 768

milk diet in, 762

morbid anatomy, 757

morphin in, 767

nausea in, treatment, 767

pilocarpin in, 766

potassium acetate in, $76_{3}$

bromid in, 767

citrate in, 763

prognosis, $75^{8}$

prophylaxis, 750

in pregnancy, 760

pulmonary edema in, treatment, 768

removal of cause, 760

rest treatment, 760

saline purgatives in, $76_{5}$

sodium bicarbonate in, 763

carbonate in, 763

chlorid in, 763,764
Nephritis, acute, strophanthin in, 764

sweating in, treatment, 701

symptoms of, treatment, 766

theobromin in, 764

theocin in, 764

treatment, 759,760

in convalescence, 768

uremia in, treatment, 766

urinary antiseptics in, 768

vegetable laxatives in, 765

vomiting in, treatment, 767

von Noorden diet in, 762

age in etiology, 752

albuminuria in, 800 . See also $A: b u-$ minuria.

arteriosclerosis in, 816. See also Arteriosclerosis.

azotemic, 756

bacteria in urine in, 749

baths in, 851,852

bibliography, $852-855$

chloremic, 756

chronic, 654

hypertension in, 812

interstitial, 654

age in etiology, 775

arteriosclerosis from, 775

arteriosclerotic kidney in, 774

baths in, 780

beverages in, 783

carbohydrate diet in, $78 \mathrm{I}$

care of skin in, 784

causes of death in, 777

climatic treatment, 780

constipation in, treatment, 784

diet in, $78 \mathrm{r}$

diuretics in, 784

etiology, 775

exercises in, 789

fats in diet in, $78 \mathrm{r}$

from gout, 775

from infectious diseases, 775

functional condition of kidney in, 777

heredity in etiology, 775

hygienic treatment, 778

hypertension in, treatment, $779,78_{4}$

Karell diet in, 783

laxatives in, 784

myocardial insufficiency in, 778

treatment, 778,785

nitrogenous diet in, 782

nycturia in, 777

polyuria in, 777

primary contracted kidney in, $7 i+$

prognosis, 776

prophylaxis in, $77 \mathrm{~S}$

protein diet in, $78 \mathrm{I}$

regulation of patient's life in, 779

renal atrophy in, 774

rest treatment in, 779

secondary contracted kidney in, 774

sex in etiology, 775

sleep in, 779

solium chlorid restriction in, $78_{4}$ 
Nephritis, chronic interstitial, treatment, 778 of symptoms, 784

water intake in, 783

morbid anatomy, 769

nycturia in, 788

parenchymatous, 654,768

acute exacerbations in, 768

baths in, 773 .

carbohydrates in, 772

catharsis in, 773

causes, 768

climatic treatment, 773

constipation in, treatment, 773

diet in, $77 \mathrm{I}$

edema in, treatment, 774

etiology, 768

excretory capacity in, 770

fluid intake in, 772

functional picture in, 769

large white kidney in, 769

prognosis, 770

proteins in, 772

removal of cause in, 770

rest for kidney in, $77 \mathrm{I}$

secondarily contracted kidney in, 769

sodium chlorid restriction in, 773

treatment, 770

with hypertension, venous section in, 142

classification, 753

Aschoff's, 755

on anatomic grounds, 654

on clinical grounds, 654

on functional grounds, 755

Senator's, 755

Volhard and Fahr's, 755

Widal's, 756

clinical course in indications for treatment, 747

manifestations, cause, 800

pathological physiology, 800

treatment, 800

coefficient of urea excretion in, 794

contributing factors in, $75^{2}$

definition, 753

diet in, 847

diffuse acute epidemic, 753

edema in, 805 . See also Edema.

electric light baths in, 852

etiology, 748

in indications for treatment, 747

from acute infectious diseases, 757

from alcohol, $75 \mathrm{I}$

from alveolar abscess, 749

from arsenic, $750,75 \mathrm{I}$

from cantharides, $75 \mathrm{I}$

from chloroform, $75 \mathrm{I}$

from cholecystitis, 749

from chromium salts, $75 \mathrm{I}$

from chronic appendicitis, 749

bronchitis, 749

cystitis, 749

endometritis, 749

prostatitis, 749

pyelitis, 749
Nephritis from chronic salpingitis, 749 ulcerative enteritis, 749 urethritis, 749

from diphtheria antitoxin, 749

from drugs, $75^{\circ}$

from endogenous substances, 751

from exposure to cold and wet, $75^{2}$

from gout, 75 I

from inflammation of paranasal sinuses, 749.

from influenza, 749

from lead, 75I

from malaria, $75^{\circ}$

from mercury, $75 \mathrm{I}$

from mushroom poisoning, $75 \mathrm{I}$

from otitis media, 749

from phosphorus, $75 \mathrm{I}$

from pregnancy, $75^{2}$

from ptomain poisoning, $75 \mathrm{I}$

from pyorrhea alveolaris, 749

from relapsing fever, $75^{\circ}$

from sore throat, 749

from tonsillitis, 749

from toxic chemical substances, 750

from trypanosomiasis, $75^{\circ}$

from vinyl amin, $75 \mathrm{I}$

general therapeutic measures, 847

heredity in etiology, $75^{2}$

hot-air baths in, $85 \mathrm{I}$

hot packs in, $85^{2}$

hypertension in, 8I 2. See also Hypertension.

in diphtheria, 748

in erysipelas, 275

in gastrointestinal disorders, $75 \mathrm{I}$

in pyemia, 749

in scarlet fever, 748

in septicemia, 749

indications for treatment, 747

infectious diseases in etiology, 748

low protein diet in, $850,85 \mathrm{I}$

methods of inducing sweating in, $85 \mathrm{I}$

myocardial insufficiency in, 823. See also Myocardial insufficiency.

occupation in, etiology, 753

parenchymatous, tuberculosis associ-

ated with, 749

pathologic changes in, 752

pathology in indications for treatment, 747

predisposing factors in, $75^{2}$

primary epidemic infectious, 749

salt-free diet in, $85^{\circ}$

Schlayer's classification, $75^{6}$

sex in etiology, 753

symptoms in, indications for treatment, 747.

syphilis associated with, 750

treatment, 747

uremia in, 836. See also Uremia.

Nephrolysin as cause of uremia, 837

Nephropexy, indications for, 727

Nephrosis, edema in, 805

Nerve complications in pertussis, 332

Nervous diseases, functional, 906

antithyroidin in, 9 I 2 
Nervous diseases, functional, exhaustion element in, 906

full feeding in, 912

internal secretions in, 9 ro

intoxication in, 906

luetin in, 912

massive feeding in, 912

neuropathy in, $9 \mathrm{II}$

ovarian extract in, $9 \mathrm{r}^{2}$

pituitrin in, 912

psychasthenia in, $9 \mathrm{rr}$

reduction of field of consciousness in, gir

thyroid extract in, 912

treatment, 906,912

baths in, 86

excitement, baths in, 86

system, autonomic, effect of atropin on, 910

effect of pilocarpin on, 910

central, invasion of, by typhoid bacillus, $x 85$

lesions of, surgical aspects, 869

syphilis of, 881. See also Syphilis, cerebrospinal.

diseases of, electrotherapy in, II4

involvement of, in African trypanosomiasis, 372

sympathetic, effect of adrenalin on, 910

Neufeld and Händel's antipneumococcus serum, 220

Neuralgia, baths in, 87

trigeminal, 870

alcohol injection in, 870 complications, 872

duration of relief from, 870

keratitis from, 872

avulsion of sensory root in, 870

injection of Gasserian ganglion in, $87 \mathrm{I}$ peripheral injection in, 870

constant electric current in, II 5

Neurasthenia, autointoxication in etiology, 907

etiology, 908

headache of, static breeze in, I 5

Neuritis, diathermy in, I I 5

endemic multiple. See Beriberi.

peripheral, baths in, 87

in beriberi, 364

Neuropathy in functional nervous diseases, 9 I I

Neuroses, occupation, constant electric current in, I 6

Nevus, cavernous, radium therapy in, 135

flat superficial, radium therapy in, I 34

Newborn, a telectasis of, pulmotor in, 549

hemorrhagic diseases of, 440. See also Hemorrhagic diseases of newborn.

Newburgh's diet in constipation, $66 \mathrm{I}$

Nitrites in hypertension, dosage, 822 effect of, 822

mode of administration, 822

Nitrogen excretion in diseased kidney, 792 in healthy kidney, 791

gas in artificial pneumothorax, 253
Nitrogen injection in tuberculous peritonitis, 744

non-protein, determination of, in blood, by micro-Kjeldahl method, 799

in blood in health, 798

in renal disease, 798

Nitroglycerin in pulmonary edema, 548

in sclerosis of blood-vessels of abdomen, 640

Nitrous oxid-oxygen anesthesia in septic peritonitis, 736

Non-hemolytic vaccines, 34

Non-obstructive peptic ulcer, treatment 616

North Berwick Spa, principal characteristics, 89 .

Northrup's treatment of pneumonia, 212

Norwegian scabies, 4 I 8

Nose, anaphylaxis from foreign bodies instilled into, 536

diseases of, pneumococcus in, 215

local treatment of, in diphtheria, 244

workman's, care of, I 79

Novocain anesthesia in septic peritonitis, 736

injection in aspiration of chest, 142

solution in hypodermic injection of mercury in syphilis, 293

Novocain-adrenalin anesthesia for artificial pneumothorax, $25 \mathrm{I}$

Numbness, faradic dry brush in, I 15

Nycturia in chronic nephritis, 788 interstitial, 777

Obesity, effect of, in visceroptosis, 7 I I thyroid treatment, 465

Obstructive peptic ulcer, 620

Occult blood in obstructive peptic ulcer, 625

Occupation in etiology of nephritis, 753

neuroses, constant electric current in, I I 6

Occupational diseases, prophylaxis, 172 treatment, 172

hygiene for workman, 177

Ochsner treatment in advanced diffuse peritonitis, 735

Ocular complications in infectious diseases, treatment, 387

Oestrus ovis, 4 I I

Oidium albicans in thrush, 602

Oil enemata in chronic diarrhea, 652 in intestinal stasis, 649 of pennyroyal for fleas, 416 paraffin, in constipation, $66_{2}$

Oleoresin of male fern in intestinal amebiasis, 393

Oleum chenopodii in ankylostomiasis, 407 in ascariasis, 405

in trichuriasis, 403

Oliguria, 788

in uremia, treatment, 847

Olive oil, emulsified in fat indigestion in infants, 671

in acariasis, 419

in infant feeding, 670 
Olive oil in intestinal stasis, 648 injections in yellow fever, 314

Open-air treatment of tuberculosis, 248

Operation, cases after, in military practice, baths in, 85

Ophthalmia neonatorum, iced physiologic salt solution in, $\hat{\dot{j}} 83$

spohol in, 382

Ophthalmoplegia interna in cerebrospinal syphilis, 886

Ophthalmo-reaction in typhoid fever, I 86

Opium, effect of, on pancreatic secretion, 706

habit, I67

atropin in, 169

convalescence in, treatment, I 7 I

diarrhea in, treatment, I 70

dionin in, 160

hospital treatment, 170

hot baths in, I 70

hydrotherapy, I69

hyoscin in, 169

mental diversion in, treatment, I 70

purgation in, 168

removal of cause, 167

scopolamin in, I69

sedatives in, 169

so-called cures, 167

treatment of abstinence symptoms, I 69

withdrawal stage, 168

in septic peritonitis, 735

in septicopyemia, 271

in yellow fever, $3 \mathrm{I} 5$

Opsonic index in lobar pneumonia, 230

in septicopyemia, 272

Optic nerve atrophy in pituitary lesions, 869

Optochin hydrochlorid in malaria, $35 \mathrm{I}$

in lobar pneumonia, 234,235

amblyopia from, 236,238

deafness from, 238

results in treatment with, 237

in malaria, $35 \mathrm{I}$

in pneumococcic ulcers of cornea, 388

in pneumococcus corneal ulcer, 239 meningitis, 239

Oral hygiene, 577

Orange peel, exhausted, in constipation, 663

Orchitis in mumps, treatment, 336

Organism, cells of, relation of drugs to, 25

Oriental sore, $35^{8}$

causal organism, $35^{8}$

incubation period, $35^{8}$

mode of infection, 358

Ornithodorus moubata, 36 I

in relapsing fever, 417

Oroya fever, 355

Orpiment in African trypanosomiasis, 375

Orthopedic apparatus in visceroptosis, 7 I I

Orthopnea in myocardial insufficiency, treatment, 834

Orthostatic albuminuria, 801. See also Albuminuria, orthostatic.

Oscillator, D'Arsonval, with resonator, IO2
Oscillator, D'Arsonval, with two condensers, IOI

Osmosis, electric, 98

Osteitis, fractures with, necrosis and, baths in, 84

Osteoarthropathy, pulmonary hypertrophic, treatment, 93I

Osteomalacia, epinephrin in, 467

Otitis media in infantile la grippe, 573 treatment, 576

nephritis from, 749

Otobius megnini, 4I 7

Oudin monopolar current in bladder tumors, 865

Ovarian extract in functional nervous disorders, 912

Ovary, involvement of, in mumps, 335

Overeating in etiology of arteriosclerosis, 8 I 7

Oxygen in artificial pneumothorax, 253 in pulmonary edema, $55^{\circ}$

inhalations in erythremia, 433

injection in chronic diarrhea, 654

in septic peritonitis, 737

Oxygen-combining power of hemoglobin, determination of, in acidosis in children, 503

Oxyuriasis, 406

anal pruritus in, 406

appendicitis from, 406

calomel in, 406 .

corrosive sublimate solution in, 406

enemata in, 406

magnesium sulphate in, 406

prophylaxis, 406

treatment, 406

Oxyuris vermicularis, 406

Pachymeningitis in pertussis, 332

Pain in acute anterior poliomyelitis, treatment, 878

fibrinous pleuritis, treatment, 567

in apical alveolar abscess, 598

in peptic ulcer, treatment, 625

in pyorrhea alveolaris, 589

in sclerosis of blood-vessels of abdomen, 639

in septicopyemia, treatment, $27 \mathrm{I}$

in subgingival alveolar abscess, 598

in tabes dorsalis, treatment, 875

in yellow fever, treatment, $3 \times 3,315$

severe, baths in, 85

Palsies, baths in, 87

Panama routine treatment of malaria, 349

Pancreas, diseases of, 705

in infections of bile passages, 694

involvement of, in mumps, 335 .

secretion, effect of diet on, 706

of drugs on, 706

of splenectomy on, 706

Pancreatic achylia, 708

associated with achylia gastrica, 708

baths in, 708

diagnosis, 708

diet in, 708 
Pancreatic achylia, treatment, 708

calculi in cholelithiasis, 708 treatment, 708

diabetes, pankreon in, jo7

sodium bicarbonate in, 707

extract in intestinal stasis, 647 in pancreatic infantilism, 709

infantilism, treatment, 708

Pancreatitis, acute, 706 diabetes mellitus in, 707 treatment, 707

chronic, 707

baths in, 707

diet in, 707

drainage of common bile-duct in, 697 in syphilis, treatment, 707

treatment, 707

vaccines in, 707

Pandy's test after serum treatment of cerebrospinal syphilis, 898

Pane's antipneumococcus serum, 218

Pankreon in pancreatic diabetes, 707

Pantopon hydrochlorid in hemoptysis, 546

Papaverin hydrochlorid in pertussis, 333

Papillomata of bladder, fulguration in, 866

surgical treatment, 866

Paquelin cautery in bladder tumors, 866

Para-arthritis, gonococcus vaccines in, 44

Paracentesis in edema, $8 \mathrm{r} 2$

in myocardial insufticiency, 828

Paraffin oil in cecum mobile, 714 in constipation, 662

Paraffin-coated bulbs in blood transfusion, 146

tubes in blood transfusion, 146

Paragonimus ringeri, 397

westermanii, 397

Paraldehyde in delirium tremens, 166 in septicopyemia, $27 \mathrm{I}$

Paralyses in uremia, 839

Paralysis, infantile. See Poliomyclitis, acute anterior.

tick, 4I 7

Paralytic dementia, antisyphilitic treatment, 893

Paranasal sinuses, inflammation of, nephritis from, 749

Paraplasma flavigenum in yellow fever, 305

Paraplegia, complete, pulmonary collapse in, 549

Parasites, animal, 389

methods of examination of feces for, 389

technique of microscopic examinations for, 389

Parasitic stomatitis, 602. Sec also Thrush.

Parathyroid extract in tetany, 465 glands, diseases of, treatment, 465

Paratyphoid bacillus, infection with, $r 8_{5}$ vaccines, 35

fever, mixed vaccine treatment, $30,5 \circ$ prophylactic vaccination in, 50

Paregoric in infantile la grippe, 576
Parenchymatous keratitis, mercurial inunctions in, 385

neosalvarsan in, 385

potassium iodid in, 385

salvarsan in, 385

treatment, 385

tuberculin test in, 385

Wassermann test in, 385

nephritis, chronic, 768. See also Nephrilis, chronic parenchymalous.

Paresis, 913

antisyphilitic treatment, 914

contraindications to salvarsan in, 915

course, $9 \mathrm{I} 4$

drainage of cerebrospinal fluid in, 920

etiology, 9r 3

injection of mercury in, 917

intracranial injection treatment, 918

iodids in, 922

Leukocytozoön syphilidis in, 9 r9

mercury in, $92 \mathrm{r}$

neosalvarsan in, 915

relation of syphilis to, 913

results of treatment in, 919

salvarsan in, $9 I_{4}, 915$

salvarsanized serum in, 9 I 6

Spirochata pallida in etiology, 913

subarachnoid therapy in, 916

Paresthesia, faradic dry brush in, 115

Park and Krumwiede's method of injection of tetanus antitoxin, 74

Parotitis, infectious, 335. See also Mumps.

Pascha-churdj, 358

Passive immunization in typhoid fever, I98

Paste, bismuth, preparation, I 45 technique of injection, 145

Pasteur's vaccine in rabies, 324 preparation of, 324

Pediculi, extermination of, $4{ }^{1} 5$

in etiology of typhus fever, $343,4{ }^{2} 5$ of spirilloses, 360

Pediculicides, 4I 5, 4 I 6

Pediculoides ventricosus, dermatitis from, 418

Pediculosis, 4I 4

benzine in, $4 \mathrm{I} 5$

fumigation in, 415

gasoline in, 415

kerosene in, 415

N. C. I. powder in, 416

treatment, 4I5

vaseline in, $4^{15}$

Pediculus capitis, 4I4

vestimenti, 4I 4

Pellagra, 16I

chronic diarrhea in, $65 \mathrm{I}$

symptoms, I6I

corn in etiology of, $16 \mathrm{r}$

dietetic treatment, 162

ctiology, $\mathbf{1} 6 \mathrm{I}$

geographic distribution, $16 \mathrm{r}$

predisposing conditions, $16 \mathrm{r}$

prevention, I6r

symptoms, $16 \mathrm{I}$ 
Pellagra, treatment, I6I

Pelletierin in intestinal teniasis, 393

Pellidol in corneal ulcers, 385

Pelvic abscess, autogenous vaccines in, 735

belt in visceroptosis, 724

infections, gonococcus vaccines in, 44

Pennsylvania Department of Health, bureau of vital statistics, 2 I county medical inspectors of, 19 disinfection of homes by, 18 distribution of biological products by, 22

division of housing, 23 of public service, 23 of sanitary engineering, 20 of tuberculosis sanatoria and dispensaries, $2 \mathrm{I}$

duties of, I7

laboratory division, 22

medical division, I 8

inspection of schools by, Ig

precautions of physicians required

by, in communicable diseases, I 8 quarantine regulations of, $\mathrm{x} 8$

sanitary inspection of schools by, I9

Pepsin, function of, in gastric juice, $6 \mathrm{II}$ in intestinal stasis, 646

rôle of, in peptic ulcer, $6 \mathrm{r} \mathrm{r}$

Peptic ulcer, 6ro

action of hydrochloric acid in, $6 \mathrm{r}_{2}$ acute, 634

perforation in, surgical treatment, 635

blood examination in, 6 I 2

causes, 6II

chemical examination of feces in, $6 \mathrm{I}_{2}$

chronic calloused, 634

course of, 628

surgical treatment, $6_{3} 6$

complications in, symptoms, 626

diagnosis, 61 2

differential, 626

etiology, 6I I

excessive gastric acidity in etiology, 634

excision of, 637

from hematogenous bacterial invasion, 6II

gall-bladder disease in, 626

gastric juice in etiology of, 6 I I

gastroenterostomy in, 614

grades of pyloric obstruction in, 623

groups of, 634

hemorrhage in, treatment, $6 \mathrm{I}_{3}$ surgical, 635

hot fluids in etiology, 633

in hour-glass stomach, treatment, $61_{3}, 636$

incidence of, 633

locations of, 633

medical treatment, 6ro, 6r 5

mode of development, $6 \mathrm{II}$

non-obstructive, administration of alkalies in, $6 \mathrm{I}_{7}$
Peptic ulcer, non-obstructive, bismuth subcarbonate in, 617 .

calcined magnesia in, 617

diet in, 616

modifications, 620

period of observation in, 618

rest treatment, $6 \mathrm{r} 6$

sodium bicarbonate in, $6 \mathrm{r} 7$

treatment, 616

obstructive, 620

alkalies in, $62 \mathrm{r}$

cause of obstruction in, 62 I

details of management, $62 \mathrm{I}$

diagnosis, 620

medical treatment, $62 \mathrm{I}$

motor meal in, $62 \mathrm{x}$

occult blood in, 625

pyloric spasm in, $62 x$

sodium bicarbonate in, 621

stomach tube in, 622

symptoms, 620

pain in, treatment, 625

perforation in, varieties, 635

into free peritoneal cavity in, treatment, $6 \mathrm{I}_{3}$

perigastric abscess in, treatment, $6 \mathrm{r}_{3}$ adhesions from, treatment, $6 \mathrm{I}_{4}$

points in diagnosis, 612

pyloric obstruction in, cause of, 624 high grade, treatment, $6 \mathrm{I} 3$

results of surgical treatment, 636

rôle of pepsin in, 6rI

secondary carcinoma in, treatment, 6 I 3

subacute, with localized abscess, surgical treatment, 636

surgical treatment, 633

teeth infections in, treatment, $6 \mathrm{I}_{4}$

time required for healing, 627

tonsil infections in, treatment, $6 \mathrm{r}_{4}$

treatment, destruction of corrosive action of gastric juice in, $6 \mathrm{ro}, 6 \mathrm{r} 2$ neutralization of hydrochloric acid in, $6 \mathrm{I}_{5}$

surgical, indications for, $6 \mathrm{r}_{3}$

Peptone solution in hemophilia, methods of administration, 443

Percentage infant feeding, 668

Percy tubes for indirect transfusion of blood, I 53

Perforation into free peritoneal cavity in peptic ulcer, treatment, 6 I 3

Pericardical synechia, surgical treatment, 488

Pericarditis, ice-bag in, 484

in erysipelas, 275

pneumococcus in, 215

septicopyemia in, 266

Pericementitis, calcic, 583

chronic suppurative, 581,583 . See also

Pyorrhea alveolaris. phagedenic, 583

Pericolitis, inflammatory constipation from, 656

Peridental infections, exophthalmic goiter with, treatment, $47^{\circ}$ 
Peridental suppurations, chronic, $58 \mathrm{I}$ systemic infections arising from, 581 types, $5^{82}$

Perigastric abscess in peptic ulcer, treatment, 613

adhesions from peptic ulcer, treatment, 614

Periosteitis, alveolar, $5^{83}$

Peripheral injection in trigeminal neuralgia, 870

Perisigmoiditis, diverticulitis and, differentiation, 642

Peristalsis, allaying of, in septic peritonitis, 733

Peritoneum, appearance of, in septic peritonitis, 732

Peritonitis, 729

advanced diffuse, Ochsner treatment in, 735

in infantile la grippe, 574

in septicopyemia, 266

pneumococcus, $2 \times 5$

diarrhea in, $74 \mathrm{I}$

indications for operation in, $74 \mathrm{I}$

pneumonia associated with, 741

preoperative treatment, $74 \mathrm{I}$

septicemia in, $74 \mathrm{I}$

symptoms, 74I

septic, 729

abdominal rigidity in, $73 \mathrm{r}$

adrenalin in, 735

allaying peristalsis in, 733

anesthesia in, 736

antiseptic treatment, 737

appearance of peritoneum in, 732

Bacillus coli in, 730

bactcremia in, 731

bactericidal powers of exudate in, 731

camphorated oil in, 735, 737

classification, 729

dangers of purgation in, 734

digitalis in, 735

endogenous, 730

eserin in, 735

ether lavage in, 737

exogenous, 730

exudate in, 731

Fowler postural treatment in, 734

gastric lavage in, 734

hypodermoclysis in, 734

in appendicitis, surgical treatment, 736

infiltration anesthesia in, 736

intravenous saline solution in, 734

iodine in, 737

irrigation in, 737

Kenyon and Pool's suction apparatus in, 737

microörganisms in, 730

mortality in, $73^{8}$

Murphy's proctoclysis in, 734

nitrous oxid-oxygen anesthesia in, 736

novocain anesthesia in, 736

operative treatment, 735

intra-abdominal technique, 736 opium in, 735

VoL. IV. -62
Peritonitis, septic, oxygen injection in, 737 paralysis of bowel in, 731

paralytic ileus in, treatment, 735

pathological changes in, 731

pituitrin in, 735

postoperative drainage in, 737

shock in, treatment, 735

primary, 730

prognosis, 732

pus in, 731

results of treatment, 738

strychnin in, 735

treatment, 733

postoperative, 737

tympanitis in, treatment, 735

vaccines in, 735

vomiting in, treatment, 734

streptococcic, 730

tuberculous, 742

adhesive, 742

application of chemical reagents in, 745

ascitic, 742

treatment, 744

aspiration in, 744

camphorated oil in, 745

contraindications to operation in, 743

drainage in, 745

formalin-gelatin in, 745

heliotherapy in, 744

high altitudes in, 744

hot-air treatment, 744

indications for operation in, 743

injection of ascitic fluid in, 745

iodine in, 745

laparotomy in, 744

medical treatment, 744

nitrogen injection in, 744

prevention of postoperative adhesions in, 745

prognosis, 746

recurrence in, 746

results of treatment in, 746

roentgen-rays in, 744

sanitarium treatment, 744

supravaginal hysterectomy in, 744

treatment, 743

surgical, 744

tuberculin in, 744

ulcerative, 742

Peritonsillar abscess, 5 18. See also Quinsy.

Perivascularitis in cerebrospinal syphilis, 882

Pernicious anemia, 420. See also $A$ nemia, pernicious.

Pertussis, 330

antipyrin in, 333

belladonna in, 333

Bordet-Gengou's bacillus in, $33^{\circ}$

brain complications in, $33^{2}$

choked disk with blindness in, $33 \mathrm{I}$

complement fixation test in, 33 I

complications, $33 \mathrm{I}$

diagnosis, 331

encephalitis in, $33^{2}$

faradic currents in, 333 
Pertussis in adrenalin chlorid, 333 infantile la grippe and, differentiation, 573 intussusception in, 332

iodine in, 333

iron in, 333

leptomeningitis in, 332

leukocytosis in, 331

mortality, 332

nerve complications in, 332

pachymeningitis in, 332

papaverin hydrochlorid in, 333

potassium iodid in, 333

prophylaxis, 332

quinin in, 333

silver nitrate in, 332

specific treatment, 333

strychnin in, 333

treatment, 332, 333

vaccines in, 52, 333

dosage, 53

Petechiæ in septicopyemia, 266

Petrolatum, liquid, in constipation, 66r

Pfeiffer's disease in infantile la grippe, 573

Phagedenic pericementitis, 583

Pharyngitis in mumps, 335

Pharynx, erysipelas of, 275 treatment, 277

glandular enlargements in, as cause of dyslalia, 523

Phenacetin in acute fibrinous pleuritis, 568 in follicular tonsillitis, 518 in yellow fever, 315

Phenolphthalein agar in constipation, $66_{3}$

Phenolsulphonephthalein test in hydrocephalus, 870

Phlebitis in erysipelas, 275

Phlyctenular keratitis, treatment, 384

Phonatory element of speech in dyslalia, 524

Phoresis, 98, I I 3

Phosphorus, nephritis from, 75 I

Phrenic nerves, section of, in tetanus, 328

Phthalein test of kidney function, 797

Phthirius pubis, 414

Phthisis. See Tuberculosis.

Physical examination of workmen, I 82

Physicians' precautions in communicable diseases, 18

Phytin in infant feeding, 674

Pilocarpin, effect of, on autonomic nervous system, 9ro

on pancreatic secretion, 706

in acute nephritis, 766

in edema, 8I I

in intestinal stasis, $65^{\circ}$

in plastic iritis, 387

in uremia, 844

Pineal body, diseases of, treatment, 469

Pitch cancer, predisposing factors in, 662

Pituitary body, lesions of, surgical treatment, 869

tumors of, 869

dystrophy, 467

extract in asthma, 539

in hemoptysis, 542
Pituitary extract, in pituitary lesions, 869

gland, diseases of, treatment, 467

lesions, diabetes insipidus in, 508

optic nerve atrophy, 869

pituitary extract in, 869

thyroid extract in, 869

medication in hypopituitarism, 468

Pituitrin in cholera asiatica, 298

in constipation, 664

in diabetes insipidus, 509

in functional nervous diseases, $9 \mathrm{r} 2$

in obstetric practise, 468

in septic peritonitis, 735

Plague, 298

from rat fleas, 298

prophylaxis in, 298

rats in etiology of, 298

vaccine therapy in, 298

Plant irritants, asthma from, 536

pollens, anaphylaxis to, in hay fever, 528

Plastic iritis, complement fixation test in, 387

gonococcus in, 387

local treatment, 387

pilocarpin in, 387

potassium iodid in, 387

Pleocytosis in cerebrospinal syphilis, 886

Plerocercoides prolifer, 394

Plerocercoids, 39r

Pleura, actinomycosis of, treatment, 57 I echinococcus disease of, treatment, 57 I infection of, in artificial pneumothorax,

Pleural effusion, serous, in artificial pneumothorax, 255

puncture, open, in acute serofibrinous pleuritis, $57 \circ$

reflex or shock in artificial preumothorax, 254

Pleurisy. See also Pleuritis.

Pleuritis, 564

acute fibrinous, 567 adhesive strapping in, 567

calomel in, 568

counterirritation in, 567

cupping in, 568

diet in, 569

flaxseed poultice in, 567

in syphilis, treatment, 569

local applications in, 567

morphin in, 568

mustard plaster in, 567

pain in, treatment, 567

phenacetin in, 568

pneumococcic infection with, treat-

ment, 569

purging in, 568

rest treatment, 567

rheumatism in, treatment, 569

salicylates in, 568

sodium bicarbonate in, 568

salicylate in, 568

treatment, 567

serofibrinous, 569

aspiration in, 569 
Pleuritis, acute serofibrinous, aspiration in, injections following, 569 repeated, 569 open pleural puncture in, 570 associated with chronic conditions, treatment, 567 classification, 564 contributory agents in etiology, 564 determining infectious agent in, 565 etiology, 564

factors influencing treatment, 566

fibrinous exudative stage, 565

formation of adhesions in, 566

in erysipclas, 275

microörganisms in, 564

pneumococcus in, 564

purulent, streptococcus in, 564

secondary, 565

septicopyemia in, 266

serous fluid in, functions of, 565

symptomatic treatment, 565,566

tubercle bacillus in, 564

Pneumococcus, bacteria associated with, in lobar pneumonia, 215

biologic differences in strains of, 220 varieties, 222

bronchitis, acute, 215

classification of disease-producing types, 223

conjunctivitis, 215

corneal ulcer, 215 optochin in, 239

cultivation of, 214

differentiation from streptococcus, 214

diseases, 215

of ear, 215

of nose, 215

dominant groups of, 223

empyema from, 215

endocarditis from, 215

in blood in lobar pneumonia, 226

in etiology of lobar pneumonia, 215

in pleuritis, 564

infections, bibliography of, 239

of eye, treatment, 388

specific therapy of, 214

isolation of, 214

meningitis, 215

ethylhydrocuprein in, 239

mucosus, 223

pericarditis, 215

peritonitis, 215,740 . See also Peritonitis, pneumococcus.

purulent arthritis from, $2 \times 5$

saprophytic existence 2 I 4

septicopyemia, 265

ulcers of cornea, optochin in, 388

vaccines, 34

dosage of, 43

effect of, on course of lobar pneumonia, 230

in lobar pneumonia, 228

in pneumonia, 28

results from usc of, 227

modified in lobar pneumonia, $23 \mathrm{I}$

variation in virulence of strains of, 224
Pneumonia, various groups of, 223

acute glossitis in, 605

associated with pneumococcus peritonitis, 741

in erysipelas, 275

lobar, 212. See also Lobar pneumonia.

pneumococcus vaccine in, 28

Pneumothorax, artificial, 249

accidents in, 255

after-treatment, 252

amount of gas injected in serious

hemorrhage, 253

apparatus for, 26 I

Balboni's necdle for, 262

beneficial effects of, 253

cautions to observe in, 250, 255

complete collapse of lung in, 253

complications, 254

contraindications to, 257

to reinflation in, 257

Deneke's apparatus for, 257, 259

displacement of heart in, 255

of organs in, 253

dyspnea in, 253

emphysema in, 254

ethyl-chlorid anesthesia in, $25 \mathrm{I}$

eucain-adrenalin anesthesia in, 25 I

fatalities in, 255

Floyd's needle for, 262

Baldwin's adjustable guard for, 262

Forlanini's apparatus for, $256,25^{8}$

gas embolism in, 254

gastric disturbances from, 255

hemiplegia in, 254

hemorrhage from lung puncture in, 255

in abscess of lung, 250

in advanced cases, 250

in bilateral moderately advanced cases, 250

in bronchiectasis, 250

in early cases, 249,250

in hemoptysis, 250,543

accidents in, 544

adhesive strapping after, 545

gas embolism in, 544

local anesthesia in, 545

site of, 544

technique, 543

thoracentesis in, 545

in moderately advanced cases, 250

in persistent harassing cough, 250

in rapidly progressive cases, 250

in slowly progressive cases, 250

in unilateral advanced cases, 249

increased cough and expectoration in, 253

indications for, $25^{\circ}$

infection of pleura in, 255

intervals between gas injections in, 252

Kornmann's apparatus for, 260

length of time of compression in, 252

local anesthesia in, 251

loss of weight from, 253

meningitis in, 255 
Pneumothorax, artificial, Muralt's apparatus for, 259

needles for, 260,262

nitrogen gas in, 253

novocain-adrenalin anesthesia in, $25^{I}$

origin of, 249

oxygen in, 253

partial collapse of lung in, 253

preparation of patient for, $25 \mathrm{I}$

quantity of gas injected in, 252

relief of symptoms in, 253

results from, 252

Robinson and Floyd's apparatus for, 258

rupture of mediastinum in, 255

selection of cases for, 249

serous pleural effusion in, 255

site of puncture in, $25 \mathrm{I}$

sterile atmospheric air in, 253

symptoms in, 253

technique, $25 \mathrm{I}$

of operation in, 251

Podophyllin in intestinal stasis, 648

Poisoning, alcoholic, 165. See also $D e$ lirium tremens.

chemical antidotes, ${ }^{6} 6_{3}$

corrosive sublimate, 164 . See also Corrosive sublimate poisoning.

drug, ${ }^{6} 63$

food, by reptiles and insects, I 57 from anaphylaxis, I57. See also Anaphylaxis, food poisoning from.

opium, r67. See also Opium habit.

Polya operation in cancer of stomach, 632

Polar effects of c onstant electric current, 98

Poliomyelitis, acute anterior, 877

after treatment, $8 \div 9$

avoidance of deformity in, 879

blood serum in, 880

hexamethylenamin as prophylactic in, 878

human blood serum in, 82

massage in, 879

muscle training in, 879

orthopedic treatment, 880

pain in, treatment, 878

prophylaxis, 878

treatment, 877

Pollen extract, dosage of, in hay fever, 532

in hay fever, anaphylactic shock from, 533

determination of initial dose, 532 immunity to, 533

injection, $53^{2}$

preparation, $53^{\circ}$

results from, 533

skin reaction from, 531

flower, in etiology of hay fever, 529

grass, in etiology of hay fever, 529

tree, in etiology of hay fever, 529

Polycythemia, deep roentgen-ray therapy in, 138

radium therapy in, 138

with cyanosis and splenomegaly, 432.

See also Erythremia.

Polyglandular diseases, treatment, 468
Polyuria, 788

in chronic interstitial nephritis, 777

Polyvalent vaccines, 36

Ponos, 357

Pool and Kenyon's suction apparatus in septic peritonitis, 737

Pork tapeworm, 39I

Position of workmen, while working, 180

Postural albuminuria, 801

Potassium acetate in acute nephritis, 763 in edema, 809

bromid in acute nephritis, 767

in hemoptysis, 54I

chlorate in ulcerative stomatitis, 603

citrate in acute nephritis, $76_{3}$

in edema, 809

iodid in actinomycosis of mouth, 605 of pleura, 57 I

in aortic regurgitation, 480

in cerebrospinal syphilis, 892

in iritis, 386

in parenchymatous keratitis, 385

in pertussis, 333

in plastic iritis, 387

in tabes dorsalis, 876

tartrate in lowered functional power of liver, 689

Precipitin tests in diagnosis of undulant fever, 354

Pregnancy, acute nephritis from, 757 albuminuria in, 803

in pernicious anemia, treatment, 425

nephritis from, $75^{2}$

pernicious anemia in, 420

prophylaxis against acute nephritis in, 760

Price's incision in Vincent's angina, 580

Primary septic peritonitis, 730

Prolapse of stomach, 638 . See also Gastroptosis.

Prolonged neutral bath in insanity, 926

Prone hanging position in visceroptosis, 725

Prophylactic vaccination in typhoid fever, I9I

in typhoid fever in military practice, I9I

Prophylaxis, governmental, of disease, I7

Prostatitis, chronic, nephritis from, 749

gonococcus vaccines in, 44

Protecting tube box for Coolidge tube, I 27

Protein, alien, method of entrance in anaphylaxis, 158

diet in chronic interstitial nephritis, $78 \mathrm{I}$ in infant feeding, 675

effect of alkalies on digestion of, 677

indigestion in infant feeding, 676,678

precipitated casein mixtures in, 679

whey-protein mixtures in, 679

milk, advantages, 678

caloric value of, 678

in infant feeding, 677

preparation of, 677

in infantile diarrheas, 673

of cows' milk, 668 
Proteins, alien, in anaphylaxis, 157 food, anaphylaxis to, 157

foreign, instilled into nose, anaphylaxis to, 536

in diet in typhoid fever, 205

Prothrombin in hemorrhagic diseases, 438

Prowazek bodies in trachoma, 383

Pruritus, anal, in oxyuriasis, 406

from tick, 4 I 7

roentgen therapy in, 135

Pseudolalia, 523, 526

defective articulation in, 526

psychotherapy in, 526

relation of, to dyslalia, 526

treatment, 526

Pseudoleukemia, 46r. See also Hodgkin's disease.

Psilosis. See Sprue.

Psoriasis, dosage of roentgen-rays in, 133

Psychasthenia, 9I I

autointoxication in etiology, 908

Psychoanalysis in asthma, 539

in insanity, 924

Psychotherapy in cerebrospinal syphilis, 894

in dyslalia, 526

in pseudolalia, 526

Ptomain poisoning, nephritis from, $75 \mathrm{I}$

Puberty hypertrophy of thyroid gland, 474

Public eating places, inspection of, in Pennsylvania, 23

Health Service work in scarlet fever, 304 service division of Pennsylvania Department of Health, 23

Pudendum, ulcer of, 354. See also Ulcera pudendum.

Pulmonary abscess, bronchoscopy in, 554 collapse, 549. See also Collapse, pulmonary.

distomiasis, 397

treatment, 397

edema, 548. See also Edema, pulmonary. embolism, 551. See also Embolism, pulmonary.

infarct, 551. See also Infarction of lung.

osteoarthropathy, hypertrophic, treatment, 931

thrombosis, 55r. See also Thrombosis, pulmonary.

Pulmotor in atelectasis of newborn, $\mathbf{5 4 9}$

Pulse-rate, decreasing, in yellow fever, 306

Pumpkin seed in intestinal teniasis, 393

Puncture, open pleural, in acute serofibrinous pleuritis, $57^{\circ}$

Purgation, dangers of, in septic peritonitis, 734

in opium habit, 168

Purgatives, bland and mechanical, 30

in edema, 810

in myocardial insufficiency, 829

in uremia, 842

Purity of Waters Act in Pennsylvania, 20

Purpura associated with albuminuria, 803 extensive, secondary anemia from, 444 hemorrhagica, 438 . See also Hemorrhagic diseases.
Purpura, Henoch's, 438. See also Hemorrhagic diseases.

neonatorum, 440. Sec also IIemorrhagic diseases of newborn.

rheumatica, 438 . See also Hemorrhagic diseases.

simple, 438. See also Hemorrhagic diseases.

Pus, evacuation of, in quinsy, 520

in chronic diarrhea, treatment, 653

septic peritonitis, $73 \mathrm{I}$

Pyelitis, chronic, nephritis from, 749

ureter stricture in, 856

Pyelography, thorium in, 862

Pyelonephritis, ureter stricture in, 856

Pyelotomy in ureter stricture, $86 \mathrm{~s}$

Pyelo-ureteroplasty in ureter stricture, 860

Pyemia, 264. See also Septicopyemia.

Pylephlebitis, liver abscess from, 702

Pylorectomy in gastric cancer, $63 \mathrm{I}$

Pyloric obstruction, cause, in peptic ulcer, 624

grades of, in peptic ulcer, 623

of high grade in peptic ulcer, treatment, 613

spasm in obstructive peptic ulcer, $62 \mathrm{I}$

Pyogenic infections, 264. See also Septicopyemia.

Pyonephrosis, ureter stricture in, 856

Pyorrhea alveolaris, $577,582,5^{83}$

anemic, $5^{83}$

as primary source of systemic intoxications, $58 \mathrm{I}$

autogenous vaccines in, 28

bleeding in, 590

cachectic, 583

complications, $59 \mathrm{I}$

course, 584,589

diabetic, $58_{3}$

effects of, on cementum, 59I.

on pulp, 59 I

emetin in, 586, 593

dosage, 593,596

methods of administration, 593, 596

results, 595

technique, 595

endameba gingivalis in, 585

pyogenes in, 585

etiology, $577,5^{83}$

extraction of teeth in, 597

fixation of loosencd teeth in, 593

gastroenteritis in, $59 \mathrm{I}$

gouty, 583

history, $5^{83}$

iodine. in normal saline solution in, 595

ionic medication in, 28

iritis from, 386

loosening of teeth in, 590

lymphadenitis in, $59 \mathrm{r}$

mercury succinamid in, 596

microörganisms, $5^{84}$

nephritis from, $58_{3}, 749$

oral disinfectants in, 593 
Pyorrhea alveolaris, origin, 584 pain in, 589 pathological anatomy, 589 prognosis, 59I prophylactic dental treatment, 592 quinin in, 596 radical treatment, 597 recurrence of, .595 Riggs' treatment, 592 senile, $5^{83}$

streptococcus viridans in, 585 synonyms, $5^{83}$

tartar in etiology, 584

thymol in, 596

treatment, $59 \mathrm{r}$

vaccines in, 593

amebic, 587

bacterial, iodine in, 587,596 vaccines in, 596

gingival, $5^{82}$ symptomatology, $5^{89}$

spirochetal, neosalvarsan in, $5^{87}$ salvarsan in, 596

Pyramidon in tabes dorsalis, 875

Pyrethrum powder for fleas, 4I 6

QUANTIMETER, Kienböck, for measuring roentgen-ray dosage, I 27

Quarantine in scarlet fever, 245

in yellow fever, $32 \mathrm{I}$

at foreign infected ports, 322 suspected ports, $32 \mathrm{I}$

at southern ports of United States, 322

period of detention in, $32 \mathrm{I}$

regulations of Pennsylvania Department of Health, I8

Quinidin in malaria, $35 \mathrm{I}$

Quinin bichlorid in malaria, 349

byproducts in malaria, $35 \mathrm{I}$

daily prophylactic doses, in malaria, 348

derivatives in lobar pneumonia, 233

dosage of, in pernicious malaria, 349

hydrochlorid in malaria, 349

in erysipelas, 276

in infantile la grippe, 575

in kala-azar, 357

in malaria, $347,348,35 \mathrm{I}$

acid salts, dangers of, $35 \mathrm{I}$

amorphous, advantages of, $35^{\circ}$

elimination of, 350

indications for increased dosage of, 350

intramuscular injections, 35 I

in pertussis, 333

in pyorrhea alveolaris, 596

in septicopyemia, 271,272

in tabes dorsalis, 876

in yellow fever, 3 I 4

injections in malaria, 349

Quinoidin in malaria, $35 \mathrm{I}$

Quinsy, 518

abscess formation in, 519

aconite in, 520

age of incidence, 5 I9
Quinsy, aneurysm and, differentiation, 520

calomel in, 520

characteristics, 5 I 8

diet in, 520

evacuation of pus in, 520

examination in, 520

exciting causes, 5 I9

hemorrhage in, 520

microörganisms in, 519

predisposing causes, 5 I9

rheumatic diathesis as cause, 519

septicemia in, 520

sodium bicarbonate in, 520

surgical treatment, $52 \mathrm{I}$

symptoms, 5 I9

temperature in, 5 I9

termination, 520

treatment, 520

RABBIT's blood, subcutaneous injection, in hemophilia, 443

Rabies, 324

Pasteur's vaccine in, 324

preparation of, 324

prophylaxis, 324

Rachitis from fat indigestion in infants, 671

Radioactive water in gout, $5 \mathrm{I} 4$

Radiotherapy, advances in, 16

Radium, advantages of, over roentgenrays, I4I

application to spleen in leukemia, 137

emanatorium treatment of gout, $5 \mathrm{r} 4$

in arthritis deformans, 512

in bladder tumors, 866

in cancer of esophagus, 608

in cavernous nevus, 135

in corneal ulcers, 385

in flat superficial nevus, 134

in gout, 513

in gynecology, I4I

in hypertension, 823

in leukoplakia buccalis, 133

in lupus vulgaris, I 34

in malignant disease of bronchi, 552

in polycythemia, 138

in senile keratosis, 134

in synovial lesions of skin, 134

in warts, I34

Rat tapeworm, 39I

Rat-bite fever, 343

causal organism, 344

cauterization in, 344

incubation period, 344

prevention, 344

salvarsan in, 344

symptoms, 344

treatment, 344

Rats in etiology of plague, 298

Raynaud's disease, electrotherapy in, I 2 I

Reaction, Schick. See Schick test.

to vaccines, $4 \mathrm{I}$

Wassermann. See Wassermann reaction.

Widal. See Widal reaction.

Reagent, Lloyd's, as antidote, I64

Rectal electrode, II 2

feeding in yellow fever, 3 I4 
Rectal injection of saline solution in cholera asiatica, 298

Recurrent fever, 361

bibliography, $3^{6} 3$

galyl in, 363

ludyl in, 363

neosalvarsan in, 362

of Algiers, 362

of Bombay, $36 x$

of East Africa, 36r

of Tonkin, 362

of West Africa, $36 \mathrm{I}$

prophylaxis, 363

salvarsan in, 362

treatment, 362

results, 363

Reed's mosquito experiments in yellow fever, 302

Yellow Fever Board, 302 conclusions of, 303

Reflex, pleural, in artificial pneumothorax, 254

Regulin in constipation, $66_{3}$

in diverticulitis, 642

Reinflation in artificial pneumothorax, contraindications to, 257

Relapsing fever, 360

bibliography, 363

galyl in, 363

ludyl in, 363

neosalvarsan in, 362

nephritis from, 750

of America, 361

of Europe, 36r

Ornithodoros moubata in, 417

prophylaxis, 363

salvarsan in, 363

treaiment, 362

results, 363

yellow fever and, differentiation, 3 10

Renal. See Kidney.

Renin as cause of hypertension, 816

Reptiles, food poisoning by, 157

Resonator electrode, III

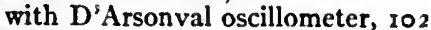

Respiratory disorders, baths in, 87

spasm, control of, in tetanus, 328

system, diseases of, 517

treatment of, 28

Rest, complete, in yellow fever, 314

in delirium tremens, 166

treatment in acute nephritis, 760

in chronic heart diseases, 486

interstitial nephritis, 779

in edema, 808

in myocardial insufficiency, 825

in soldier's heart, $48 \mathrm{I}$

with specific infections, 48 I

Restraint in delirium tremens, 166

Retinitis, albuminuric, in malignant hypertension, 815

Retropharyngeal abscess in infantile la grippe, 574

Rheumatic disorders, baths in, 85

Rheumatic endocarditis, strophanthin in, 482
Rheumatic fever, convalescence from, baths in, 86

iritis, treatment, 386

Rheumatism, acute articular, symptomatic anemia in, 444

articular chronic, baths in, 86

chronic, baths in, 85

muscular, electrotherapy in, 116

from fatigue and exposure, baths in, 85

pleuritis in, treatment, 569

Rheumatoid arthritis, electrotherapy in 116

Rhinitis, atrophic, vaccines in, 52

Rhinoscleroma, vaccines in, 52

Rhubarb agar in constipation, 663 in intestinal stasis, 648

Rice in etiology of beri-beri, 160,364

Rickets, splenic anemia from, 434

Riedel's lobe of liver, treatment, 704

Riess' classification of uremia, 838

Riggs' disease, 583. See also Pyorrhea alveolaris.

treatment of pyorrhea alveolaris, 592

Rigor mortis in yellow fever, 305

Robinson's apparatus for artificial pneumothorax, 258

method of aspiration of chest, 142

Rochelle salts in follicular tonsillitis, 518

Rockefeller Institute antipneumococcus serums, 223

research on lobar pneumonia, 222

Rocky Mountain spotted fever, Dermacentor andersoni in, 417

Roentgen-ray therapy, 122 advances in, 122

advantages of radium therapy over, I 41

corrections in technique, 131,133

cross-fire method, 135

deep, I 29, I 35

advantage of Coolidge tube for, 127

in exophthalmic goiter, 1 36, 138

in hyperthyroidism, 139

in leukemia, 136

in polycythemia, 138

in thymic enlargement, 140

in tuberculous adenitis, 136

in uterine hemorrhage in, 140

production of premature menopause with, 136

special applications for, 136

fractional dose method, 131

tube, Coolidge, I 23, I24. See also Coolidge tube.

electron, 124

gas, I 24

Wehnelt, 124

Roentgen rays, aluminum filters for, 132 dosage, 129

fractional, I3 1

Hampson radiometer for estimating, 130

in eczema, 132

in psoriasis, 133

Kienböck X unit of, 129

massive, contraindications to, 132 
Roentgen rays, measuring by Kienböck quantimeter, I 29

Sabouraud-Noire pastille method of reckoning, I 30

standardization, with Coolidge tube, I 29

filtration of, I 32

in diagnosis of alveolar abscess, 579

of cecum mobile, 713

of exophthalmic goiter, 475

of gastric cancer, 629

of pulmonary tuberculosis, 250

of salivary calculi, 606

of spinal tumors, 902

of ureter stricture, 858

of urinary tract disease, thorium in, 862

in acne vulgaris, 134

in erythremia, 433

in exophthalmic goiter, 470

in hemolytic icterus, 437

in Hodgkin's disease, 462

in keloids, I34

in leukemia, 449

dangers, $449,45^{\circ}$

results, 449

rules for application, 449

leukoplakia, 606

in lobar pneumonia, 213

in location of primary focus in septicopyemia, 267

in lupus vulgaris, I34

in malignant disease of bronchi, 552

in pruritus, 135

in senile keratosis, I 34

in splenic anemia, 435

in thymic tracheostenosis, 553

in tuberculous peritonitis, 744

in uterine fibroids, contraindications to, I4I

massive dosage, I 29, I3 I

epilation of scalp with, I33

in favus, 133

in sycosis, 133

in tinea tonsurans, 133

new nomenclature, I 22

reflection, I 23

relation to ultraviolet light waves, I 23

short wave-length of, I 23

sole leather filters for, 132

sterility from, I 40

study of colon in intestinal stasis with, 644

Rogers' method of treating cholera asiatica, 298

Romberg's sign in African trypanosomiasis, 373

Römer's antipneumococcus serum, 2 I 9

Rosenfeld's dietetic treatment of constipation, 660

Ross' endemic index in malaria, 347

Roundworms, 399, 400

Rous and Turner's iso-agglutination test

in blood-transfusion, 148

Minot's modification, I 48
Russian mineral oil in constipation, $66 \mathrm{r}$ in hypertension, 82 I

SABOURAUD-NOIRE pastille method of estimating roentgen-ray dosage, I 30

Saccharomyces albicans in thrush, 602

Saccular aneurysm, constant electric current in, I I8

wiring with electrolysis in, 155 technic, I 56

Salicylic acid in leukoplakia, 606

Salicylates in acute fibrinous pleuritis, 568

in infantile la grippe, 575

Saline infusions, 142

irrigations, 142

contraindications to, $\mathrm{I}_{43}$

Salivary calculi, 606

etiology, 606

location, 606

roentgen-ray diagnosis, 606

symptoms, 606

treatment, 606

glands, diseases of, 606

Salol in follicular tonsillitis, $5 \times 8$

Salpingitis, chronic, nephritis from, 749

Salt solution in acidosis in children, 505 in diphtheria, 244

in cholera asiatica, 298

in gonorrheal conjunctivitis, $38_{3}$

in malaria, 349

in symptomatic anemia, 447

in yellow fever, 316

iodine in, in pyorrhea alveolaris, 595 preparation of, $\mathrm{I} 43$

test of kidney function, 79 r

Salvarsan, 282

description, 283

discovery of, by Ehrlich, 282

formula for, 283

in African trypanosomiasis, 375

in cancrum oris, 580

in cerebral syphilis, dosage, 283

in malaria, 351

in meningitis, dosage, 283

in pancreatic syphilis, 707

in parenchymatous keratitis, 385

in paresis, 914, 915

in pernicious anemia, 427

in rat-bite fever, 344

in recurrent fevers, 363

in relapsing fevers, 363

in secondary stage of syphilis, dosage, 280

in spirochetal pyorrhea, 596

in splenic anemia, 435

in syphilis, $30,280,282$

administration, abscess from, 284 apparatus for, 284

intramuscular, 287

dosage, 287

preparation of solution, 287

site of injection, 287

intravenous, 285

after-effects, 286

dosage, 280,283 
Salvarsan in syphilis, administration, intramuscular, fatalities in, $\mathbf{2 8 4}, 286$ introduction into vein, 286 preparing solution for, 285 sodium hydroxid solution for, 285 temperature after, 286

methods of, 284

preparation of patient, 285

subcutaneous, 286

after-care, 287

apparatus for, 286

preparation of patient, 287 of solution, 287

site of injection, 287

advantages of neosalvarsan over, 288 combined with mercury, 293

contraindications to, 283

indications for, 283

of liver, $69 \mathrm{I}$

primary stage dosage, 280

provocative dose, 286

tertiary stage, dosage, 281

in tabes dorsalis, 874,876

neosalvarsan and, comparison, 287

Salvarsanized serum in paresis, 916

Salvarsan-mercury combined treatment of syphilis, 280

Sanarelli's bacillus in yellow fever, zor

Sanatorium treatment of tuberculosis, 248 in Pennsylvania, 21 of tuberculous peritonitis, 744

Sanitary engineering division of Pennsylvania Department of Health, 20

inspection, state, in typhoid prophylaxis, 180

Santonin in trichuriasis, 404

Sapremia, 264. See also Seplicopyemia.

Sarcophaga carnaria, 4II

Sarcoptic scabies, 418

Scabies, 418

Norwegian, 418

sarcoptic, 418

sulphur ointment, 4 ro

Scalp, epilation of, with massive roentgenray dosage, 133

Scarborough Spa, principal characteristics, 89

Scarlet fever, 245

acute glossitis in, 605

antistreptococcus serum in, 246

convalescent serum in, $8 \mathrm{x}, 245$

methods of administration, 246

preparation, 246

results from, 246

drug treatment, 245

fumigation in, 245

injection of killed bacteria in, 246, 247

Marmorek's serum in, 80

nephritis in, 748

quarantine in, 245

Schick test in, 65

specific therapy in, 245

red in corneal ulcers, 384

Scars, painful, prolonged baths in, 84

Schick's test for immunity to diphtheria, 63,241
Schick's test for immunity to diphtheria, clinical value, 69

combined, 64

control in, 64

diagnostic value, 69

diluted toxin for, 64

equipment for, 64

for staff in contagious disease

hospitals, 70

in scarlet fever, 65

injection of toxin in, 64

pseudoreaction to, 63

reactions in, 65

reliability of, 69

summary of, in normal children, 66

Schistosoma hematobium, 395

japonicum, 396

mansoni, 396

Schistosomiasis. See Dislomiasis, venal.

Schizotrypanum cruzi, 377

Schlayer's classification of nephritis, 756

Schlesinger's urobilin reaction in malaria, 348

Schools, medical inspection of, in Pennsylvania, 19

Sciatica, baths in, 86

diathermy in, 115

Scleroderma, baking treatment, $93 \mathrm{I}$

high-frequency currents in, 930

thyroid extract in, $93^{\circ}$

Sclerosis of blood-vessels of abdomen, 639 constipation in, treatment, 640

diagnosis, 639

diet in, 640

differential diagnosis, 640

etiology, 639

from lead-poisoning, 639

in arteriosclerosis, 639

in s:philis, 639

nitroglycerin in, 640

pain in, 639

prognosis, 639

sodium nitrite in, 640

symptoms, 639

treatment, 640

vomiting in, 639

Scopolamin in delirium tremens, 166

in opium habit, 169

Scotch douche for contusions and bruises, 83

Screwworm, 4ro

Secondary anemia, 443. See also A nemia, symplomatic.

Sedatives in septicopyemia, $27 \mathrm{I}$

Segregation in leprosy, $34 \mathrm{I}$

Sellards' method of determining alkali reserve of blood, 502

test in diabetes, 491

Senator's classification of nephritis, 755

Senna in intestinal stasis, 648

Sennatin in constipation, 664

Sensitized bacteria, vaccines from, 37

cultures of typhoid bacillus as vaccines, 193

vaccine sediment, intravenous injection, in typhoid fever, 201 
Sensitized vaccines in lobar pneumonia, 232

Sensory root, avulsion of, in trigeminal neuralgia, 870

Septic peritonitis, .729. See also Peritonitis, septic.

toxemia, 264. See also Septicopyemia. Septicemia, 264. See also Septicopyemia.

Septicopyemia, 264

acute exudative nephritis in, 266

adrenalin in, 270

alcohol in, 270

anthrax, 325

antiseptics in, 272

arrhythmia in, 266

autogenous vaccines in, 272

baths in, 270

bismuth in, 270

blood-pressure in, 266

bradycardia in, 266

bromids in, 27 I

bronchopneumonia in, 266

caffein in, 270

camphorated oil in, 270

cardiac stimulation in, 270

cerium oxalate in, 270

cocain in, 270

codein in, $270,27 \mathrm{I}$

cold in treatment of, 268

collargol in, 272

conditions predisposing to, 264

conserving strength and resistance of patient in, 270

constitutional symptoms, 264

course of, 266

dental origin of metastatic focus in, 267

diarrhea in, treatment, 270

diet in, 270

differential diagnosis, 266

digitalis in, 270, 27I

ecchymoses in, 266

endocarditis in, 266

enteroclysis in, 270

erythema in, 266

falling blood-pressure in, treatment, 270

from appendicitis, treatment, 267

from Bacillus aërogenes capsulatus, 265 coli, 265

proteus, 265

pyocyaneus, 265

typhosus, 265

from cholecystitis, treatment, 267

from focal infections, 265

from Friedländer's bacillus, 265

from Micrococcus gonorrhea, 265

tetragenus, 265

from pneumococcus, 265

from Staphylococcus pyogenes aureus, 265

from Streptococcus mucosus, 265 viridans, 265

fulminant forms, 266

heat in, treatment, 268

hematogenous jaundice in, 266

hexamethylenamin in, 272

hydrocyanic acid in, 270
Septicopyemia, hyoscin in, $27 \mathrm{I}$

hyperpyrexia in, treatment, 270

hypodermoclysis in, 270

ichthyol in, 268

in erysipelas, 273,275

in nephritis, 749

in pleuritis, 266

in pneumococcus peritonitis, 741

in quinsy, 520

in septic arthritis, 266

infecting organisms in, 265

intravenous injection of normal saline in, $27 \mathrm{I}$

iodine in, 272

isolation of exciting organism in, 265

local treatment, 268

locating metastatic focus in, 266,267

meningitis in, 266

mild forms, 266

morphin in, 27 I

nephritis in, 749

opium in, 271

opsonic index in, 272

organisms in blood in, 266

pain in, treatment, $27 \mathrm{I}$

paraldehyde in, 271

pericarditis in, 266

peritonitis in, 266

petechiæ in, 266

physical examination in, 267

prognosis, 267

puerperal origin of, 267

quinin in, 271,272

regulation of bowels in, 271

sedatives in, $27 \mathrm{I}$

serum treatment, 273

sleeplessness in, treatment, 271

specific treatment, $27 \mathrm{I}$

stimulating appetite in, 270

subcutaneous injection of albumoses and nucleins, $27 \mathrm{I}$

surgical origin, 267

symptomatology, 266

symptoms of infectious diseases and, differentiation, 267

temperature in, 266

treatment, 267

trional in, $27 \mathrm{I}$

veronal in, $27 \mathrm{r}$

vomiting in, control of, 270

Sera, bacterial, 33

general observation on therapeutic and immunizing uses, 57

in hemophilia, 443

Sero-culture in diagnosis of undulant fever, 354

Serofibrinous pleuritis, acute, 569. See also Pleuritis, acute serofibrinous.

Serous pleural effusion in artificial pneumothorax, 255

Serum, antianthrax, 325 . preparation of, 325

antibacterial, 217

titration of potency, 220

antidiphtheritic, 242. See also Antidiphtheritic serum. 
Serum, antimeningitis, 77. See also $A$ nlimeningilis serum.

antipneumococcus, 216 . See also Ansipreumococcus serum.

antistaphylococcus, 8I

antistreptococcus, 79. See also Antistreptococcus serum.

antitetanic, 71, 326. See also Antitetanic serum.

autosalvarsanized, in cerebrospinal syphilis, 89 r

blood, in hemoptysis, 542

convalescent blood, in scarlet fever, 81

human blood in poliomyelitis, 82

normal horse, in hemoptysis, 542

salvarsanized, in paresis, 916

treatment, history, $\mathrm{r}_{4}$

of anthrax, 325

of cerebrospinal syphilis, 800

Bryne's mercurialized serum in, 895

globulin estimation in, 897

Lange colloidal gold reaction after, 898

lumbar puncture in, $895-898$

obtaining blood for, 894

Ogilvie method, 895

Pandy's test after, 898

preparation of serum, 895

technique, $894-898$

Wassermann reaction in, 898

of dysentery, 76

of lobar pneumonia, 216

of pertussis, 333

of septicopyemia, 273

of tabes dorsalis, 875

Shaffer and Coleman's diet in typhoid fever, 205, 206

results, 208

Ship beriberi, 365,366

Shock, anaphylactic, 158

treatment, 159

pleural, in artificial pneumothorax, 254

Shoes for workmen, best, 178

Sickness, mountain, explanation of, 95

Silver nitrate in follicular tonsillitis, 518

in pertussis, 332

in syphilis of mouth, 604

salt in gonorrheal stomatitis, 604

Sinus infections, vaccines in, 55

inflammations, exophthalmic goiter with, treatment, 470

Sinuses, suppurating, bismuth paste treat-

ment, 144

Sinusoidal electric current, 100

methods of application, 103

therapeutic uses, 103

Siphonaptera, 416

Sippy's treatment of gastric and duodenal ulcer, 6ro

Skin, care of, in chronic interstitial nephritis, 784

color of, in pernicious anemia, $42 \mathrm{I}$

diseases in food-poisoning from anaphylaxis, $15^{8}$

thyroid treatment, 465
Skin lesions, staphylococcus vaccines in, 42 reaction from pollen extract in hay fever, 531

synovial lesions of, treatment, 134

Sleep for workmen, 180

in chronic interstitial nephritis, 779

induction of, in delirium tremens, 166

Sleeping sickness. See Trypanosomiasis.

Sleeplessness in septicopyemia, treatment, 271

Smallpox, acute glossitis in, 605

Smoker's patches, syphilitic origin, 605

Snow's technique for application of static spark, 107

Soamin in African trypanosomiasis, 374

Sodium bicarbonate in acidosis in children, 497, 505, 506

in acute fibrinous pleuritis, 568 nephritis, 763

in chlorosis, 432

in edema, 800

in follicular tonsillitis, $5 \mathrm{I} 8$

in non-obstructive peptic ulcer, 617

in obstructive peptic ulcer, $62 \mathrm{I}$

in pancreatic diabetes, 707

in quinsy, 520

in uremia, 844

borate in aphthous stomatitis, 602

in catarrhal stomatitis, 602

bromid in hemoptysis, 541

cacodylate in iritis, 386

in pernicious anemia, 427

carbonate in acute nephritis, $76_{3}$

chlorid, effect of, on pancreatic secretion, 706

excretion in diseased kidney, 790

in acute nephritis, $76_{3}, 76_{4}$

in healthy kidney, 790

in hemoptysis, 54I

restriction in chronic interstitial nephritis, 784

parenchymatous nephritis, 773

in edema, 800

in myocardial insufficiency, 827

retention in edema, 805

citrate in hemorrhagic diseases of newborn, 441

solution in blood transfusion, 147

glycocholate in lowered functional power of liver, 690

hydroxid solution for intravenous administration of salvarsan, 285

nitrite in sclerosis of blood-vessels of abdomen, 640

phosphate in intestinal stasis, 648

salicylate in acute fibrinous pleuritis, 568

in leprosy, 342

sulphate in yellow fever, 313

tartrate in lowered functional power of liver, 689

Softening of brain. See Paresis.

Soldier's heart, 480

causes, 48 I

digitalis in, $48 \mathrm{r}$

massage in, 48I 
Soldier's heart, rest treatment, $48 \mathrm{I}$ specific infections with, treatment, $48 \mathrm{I}$

symptoms, $48 \mathrm{r}$

treatment, $48 \mathrm{I}$

Sole leather for filtering roentgen rays, I 32

Somatic teniasis, 393

male fern in, 394

treatment, 394

Sore, oriental, 358

throat, nephritis from, 749

South America, yellow fever in, 300

Spanish-American war, yellow fever in, 300

Sparganum mansoni, 394

prolifer, 394

Spark, static, effects of, Ior

technique for application, 107

Spas of America, 89

of England, 87-89

of France, 89

principal characteristics, 87

Spasm of esophagus, electrotherapy in, II 8

Specific diseases, vaccines in, $4 \mathrm{I}$

therapy, 33. See also Vaccines.

Speech, articulatory element of, in dyslalia, 524

Bastian's primary couplet, 524

center, auditory, weakness of, as cause of dyslalia, 525

defects of, 523

elements or oral symbols of, 524

normal, development, 525

phonatory element of, in dyslalia, 524

Sphygmomanometry in lobar pneumonia, 213

Spiders, $4 \mathrm{r} 7$

Spinal canal, disappearance of tetanus antitoxin from, after intraspinal injection, 73

cord, bullet wounds of, treatment, 902 diseases of, 874 surgical treatment, 899

injury of, in spinal fracture, surgical treatment, 900

lesions of, surgical diagnosis, 900 operative exposure of, 899

stab wounds of, treatment, 902

tumors of, after-treatment, 903

intramedullary, surgical treatment, 903

prognosis, 903

roentgen rays in diagnosis, 902

surgical treatment, 902

xanthochromia in, 902

gliosis, surgical treatment, 904

puncture in hypopituitarism, 468

surgery, bibliography, 904

Spine, fracture of, injury of spinal cord

in, surgical treatment, 900

Spirilloses transmitted by lice, 360 by ticks, 360

Spirochæta pallida, discovery of, 278

Spirochetal pyorrhea, neosalvarsan in, 587 salvarsan in, 596
Splanchnoptosis, relation of cecum mobile to, $7 \times 2$

Spleen, deep roentgen-ray therapy to, in leukemia, ${ }^{3} 6$

hemolytic function of, 428,433

in etiology of pernicious anemia, 422

relations of, in erythremia, 433

Splenectomy, 453

bibliography, 459

choice of time for, 459

contraindications to, 453

effect of, on bone marrow, 428

on pancreatic secretion, 706

history, 453

in Banti's disease, 454

in Gaucher's disease, 455

in hemolytic icterus, $428,436,455$ splenomegaly, 455

in hypertrophic cirrhosis of liver, 454

in leukemia, 452

in malaria, indications for, 352

in pernicious anemia, 427, 456

blood crisis after, 456

effect of, on urobilin, 428

in splenic anemia, 428,434

indications for, 454

temporary anemia after, 454

Splenic anemia, 433. See also Anemia, splenic.

Splenomegaly, hemolytic, splenectomy in, 455

polycythemia with cyanosis and, 432 . See also Erythremia.

types of, differential diagnosis, 458

Spohol in gonorrheal conjunctivitis, 382

Spongy gums, 583

Sprains, joint, baths in, 84

Spray, static, effects of, ror

technique for application, 107

Sprue, 367

Bahr's classification, 367

bananas in, 370

bibliography, $37 \mathrm{r}$

biologic conclusions, 369

castor oil in, 370

characteristics, 367

chronic diarrhea in, $65 \mathrm{I}$

clinical conclusions, 368

complement fixation in, 369

complete, 367

dietetic treatment, 369 results, 370

epidemiologic conclusions, 368

fruit in, 370 .

incomplete, 367

intestinal, 367

marasmic toxemia in, 367

Monilia albicans in, 368

mortality in, $37 \mathrm{I}$

strawberries in, 370

strict meat diet in, 370

milk diet in, 369

thrush fungus in, 368

tongue, 367

treatment, 369

dietetic, 369 
Sprue, yeasts in stools in, 367

Stab wounds of spinal cord, treatment, 902

Stammering, 523. See also Dyslalia.

Staphylococcus pyogenes aureus, septicopyemia from, 265

vaccines, 34

administration, 42

in acne, 42

in furunculosis, 42

in skin lesions, 42

in sycosis vulgaris, 42

Starch in infant feeding, 675

Starvation treatment of diabetes, 491.

See also Allen treatment of diabetes.

Stasis, intestinal, 643. See also Intestinal stasis.

Static bath, technique for application of, I 10

breeze in headache of neurasthenia, I I 5 technique of application, 108

brush discharge, technique for application, 108

electric current, ror

methods of application, ro6

precautions in using, 106

therapeutic uses, ro3

spark, technique for application, ro7

spray, technique for application, ro?

wave current, technique for application, rog

Stegomyia calopus in transmission of yel-

low fever, 299

Sterility from roentgen rays, 140

temporary, after mumps, 335

Sterilization of wounds with Dakin's antiseptic, 260

Sternberg's alkaline mixture in yellow fever, $3{ }_{3}$

Bacillus $X$ in yellow fever, 302

Stomach, cancer of, 629. See also Cancer of stomach.

dilatation of, 637. See also Dilatation of stomach.

diseases of, electrotherapy in, I 16

ectasy of, 637 . See also Dilatation of stomach.

electrode, Einhorn's Bassler's modification of, I I 7

hour-glass, peptic ulcer in, treatment, 613

irritability of, in yellow fever, treatment, 3 I 5

lymphatics of, surgical relations, 631 prolapse of, 638 . See also Gastroptosis. surgery of, 629

tube in obstructive peptic ulcer, 622

ulcer of, 610,633. See also Peptic ulcer.

Stomatitis, aphthous, 601. See also Sprue.

catarrhal, 60 r

treatment, 602

gonorrheal, 604

herpetic, 603

treatment, 604

mycotic, 602. See also Thrush.

parasitic, 602. See also Thrush.
Stomatitis, relation of types of, 602 ulcerative, $60 \mathrm{r}$

treatment, 603

Stools, yeasts in, in sprue, 367

Strained heart, 480 . See also Soldier's heart.

Strains, joint, batbs in, 84

Strathpeffer Spa, principal characteristics, 89

Straw mattress dermatitis, 4 I 8

Strawberries in sprue, $37^{\circ}$

Streptococcus, differentiation from pneumococcus, 2 I 4

erysipelatis in erysipelas, 273

in purulent pleuritis, 564

infections of eye, treatment, 388

mucosus, septicopyemia from, $265^{\circ}$

peritonitis, 730

pyogenes in erysipelas, 273

vaccines, 34

dosage, 43

in erysipelas, 42

in general infections, 43

in local infections, 42

viridans in acute endocarditis, 578

in pyorrhea alveolaris, 585

septicopyemia from, 265

Streptothrix muris ratii in rat-bite fever, 344

Stricture of ureter, 856 . See also Ureter stricture.

Strongyloidea superfamily, 406

Strongyloides intestinalis, 400 stercoralis, 400

Strongyloidosis, 400

male fern in, 401

sulphur in, 401

thymol in, 401

treatment, 4 or

Strophanthin in acute nephritis, 764

in lobar pneumonia, 213

in myocardial insufficiency, 828

in pulmonary embolism, $55 \mathrm{I}$

thrombosis, 551

in rheumatic endocarditis, 482

Strophanthone in lobar pneumonia, 213

Strophanthus in myocardial insufficiency, 832

in mitral stenosis, 479

Strychnin in beriberi, 366

in cardiac dyspnea, 483

in Cheyne-Stokes respiration, 483

in delirium tremens, 167

in dyschezia, 654

in erysipelas, 276

in heart diseases, 479,483

in leprosy, $34^{2}$

in pertussis, 323

in pulmonary edema, 548

in septic peritonitis, 735

in tabes dorsalis, 876

in yellow fever, 314

nitrate in diabetes insipidus, 500

Stuttering, 523. See also Dyslalia.

Subarachnoid therapy in paresis, 916

Subcutaneous administration of salvarsan, 286 
Subcutaneous injection of emetin in amebic dysentery, $33^{8}$

myiasis, 410 chloroform in, $4 \mathrm{II}$ treatment, $4 \mathrm{II}$

Subgingival alveolar abscess, 597. See also Alveolar abscess, subgingival.

Subtetanic hypoparathyreosis, treatment, 465

Subthermal baths in acute cases in military practice, 83

Sugar in infant feeding, 672

of cows' milk, 668

treatment of failing heart, 486

Sulphate of zinc in blepharitis, 38r

Sulphonephthalein excretion in uremia, 838

Sulphur in strongyloidosis, $40 \mathrm{I}$ ointment in acariasis, 418

in scabies, 419

Sulphureted hydrogen injection in chronic diarrhea, 653

Superfamily angiostomoidea, 400 filarioidea, 408 strongyloidea, 406 trichinelloidea, $40 \mathrm{I}$

Suppurating sinuses, bismuth paste treatment, I 44

Suppuration in erysipelas, 274

Suppurative cementitis, chronic, $58 \mathrm{I}$. See also Pyorrhea alveolaris.

Suprarenal involvement in American trypanosomiasis, 378

Surface electrode, I 2

Surgical cases in military practice, baths in, 83

Sweat baths in corrosive sublimate poisoning, 165

Sweating in treatment of acute nephritis, 761

Sweatshop ventilation, 173

Swimming-bath conjunctivitis, 382 inclusion bodies in, 382

Sycosis, massive dosage of roentgen rays in, 133

staphylococcus vaccines in, 42

Sympathetic nervous system, effect of adrenalin on, 9 10

Symptomatic anemia, 443. See also Anemia, symptomatic.

Syndrone, Fröhlich's, 467

hypophysaire-adiposo-génital, 467

Synechia, pericardial, surgical treatment, 488

Synovial lesions of skin, treatment, 134

Synovitis, chronic, baths in, 86

Synthesis in kidney, 786

Synthetic chemistry, contributions of, to modern medicine, 16

Syphilis, 278

arsenic in, 282

as cause of arteriosclerosis, $8 \mathrm{I} 7$

associated with pernicious anemia, 420

cerebral, salvarsan dosage in, 283

cerebrospinal, 88r

abdominal pain in, 886
Syphilis, cerebrospinal, after-treatment, 898

auditory nerve lesions in, 886

autosalvarsanized serum in, $89 \mathrm{I}$

bibliography, 898

blood-examination in, 886

classification, 882

course, 884

diagnosis, 882

endarteritis in, 882

globulin excess in, 887

gumma formation in, 882

incidence, 884

interstitial, 882

intraspinal treatment, 890

Lange gold reaction in, 888

meningitis in, 882

meningoarteritis in, 882

neosalvarsan in, 890

ophthalmoplegia interna in, 886

parenchymatous, 882

perivascularitis in, 882

pleocytosis in, 886

potassium iodid in, 892

prophylaxis, 884

psychotherapy in, 894

relapse after treatment, 889

serum treatment, 890 . See also Serum treatment of cerebrospinal syphilis.

symptoms, 886

syphilitic treatment as preventative, 885

treatment, 890

Wassermann reaction in, 882,887

chancre in, excision of, 279

chronic pancreatitis in, treatment, 707

combined mercury and salvarsan treat-

ment, 280

congenital, as cause of hemolytic icterus, 436

constitutional treatment, 280

diagnosis, 278

early, importance, 279

microscopic, 278

from secondary symptoms, 278

positive, 280

dosage of salvarsan in, 280

elimination of poison from patient's system in, 279

excision of chancre in, 279

hereditary treatment, 282

immunity in, 28I

in heart diseases, treatment, 488

in hemolytic icterus, treatment, 437

in pernicious anemia, treatment, 425

initial lesion in, treatment, 279

iritis from, 386

meningitis in, salvarsan dosage in, 283

mercurial ointment by inunction in, 293

mercury in, 280, 292

by mouth, 293

combined with salvarsan, 293

hypodermatic use of, 293

hypodermic injection, novocain solution in, 293 
Syphilis, neosalvarsan in, $31,280,287$. See also Neosalvarsan in syphilis.

nephritis associated with, $75^{\circ}$

of liver, 691

cancer of liver and, treatment, 691 complement fixation in, 691

mercury in, 691

salvarsan in, 691

treatment, $69 \mathrm{r}$

Wassermann test in, $69 \mathrm{I}$

of mouth, 604

treatment, 604

pleurisy in, treatment, 569

primary, 280

recent discoveries in therapy of, 278

recurrence after supposed cure, 279

relation of, to paresis, 913

to tabes dorsalis, 874

resemblance of African trypanosomiasis to, 373

salvarsan in, 30, 280, 282. See also Salvarsan in syphilis.

sclerosis of blood-vessels of abdomen in, 639

secondary, 280

smoker's patches in, 605

Spirochæta pallida as specific cause, 278

splenic anemia from, 434

tertiary, treatment, 28I, 282

treatment, 279

combined mercury and salvarsan, 280 constitutional, 280

in primary stage, 280

in secondary stage, 280

neosalvarsan-mercury, 288

of initial lesion, 279

of tertiary stage, $28 \mathrm{I}$

Wassermann reaction in, discovery of, 278

with exophthalmic goiter, treatment, 472

Syphiloma of liver, treatment, 70

Syringe method, modified, of blood transfusion, 146

Syringe-canula method of blood transfusion, 153

Syringomyelia, surgical treatment, 904

Systemic disease, focal infection in relation to, 54

TABES dorsalis, 874

antisyphilitic treatment in, 874

aspirin in, 875

atropin in, 875

baths in, 875

Charcot joints in, treatment, 876

diarsenol in, 874,876

electric light bath in, 875

Fraenkel exercises in, 875

gastric crises in, treatment, 875

iodine in, 875

iron in, 876

mercurial injections in, 876

inunctions in, 876

neosalvarsan in, 876

pain in, treatment, 875
Tabes dorsalis, potassium iodid in, 876 pyramidon in, 875

quinin in, 876

relation of, to syphilis, 874

salvarsan in, 874,876

serum therapy in, 875

strychnin in, 876

symptoms, 874

Tachycardia, baths in, 86

Tænia saginata, 39I

solium, 391, 393

Tæniarhynchus saginatus, $39 \mathrm{I}$

Takadiastase in intestinal stasis, 647

Tapeworm, armed, 393

beef, 391

cat, $39 \mathrm{I}$

dog, 39I, 394

fish, $39 \mathrm{r}$

hog, 394

pork, 39I

rat, $39 \mathrm{r}$

varieties, 390,393

Tapping in acute serofibrinous pleuritis, 569

Tartar emetic in African trypanosomiasis, 375

in leishmaniasis, 355, 359

in ulcera pudendum, 354

salivary, in etiology of pyorrhea alveolaris, 584

Teeth, diseased, as cause of arthritis deformans, 510

extraction of, in pyorrhea alveolaris, 597

infections, in peptic ulcer, treatment, 614

loosened, in pyorrhea alveolaris, .590 fixation of, 593

of workmen, care of, 180

Temperature in prognosis of yellow fever, 3 II

in quinsy, 519

in septicopyemia, 266

in yellow fever, 306

rise after vaccination, 40

Temporal decompression in hydrocephalus, 870

Teniafuges, 393

Teniasis, 390

intestinal, ammonium chlorid in, 393

diagnosis, 390

oleoresin of male fern in, 393

pelletierin in, 393

pumpkin seed in, 393

symptoms, 392

thymol in, 393

treatment, 392

somatic, 393

male fern in, 394

treatment, 394

Terra silicea purificata as antidote, 164

Tesla high-frequency current, 102

transformer, connections of, $\mathrm{IO}_{2}$

Test days for determining kidney function, 795

Test, Schick. See Schick test.

Test, Wassermann. See Wassermann reaction. 
Testicles, atrophy of, in mumps, prevention, 336

involvement of, in mumps, 335

Test-meals for determining kidney function, 794

in intestinal stasis, 647

Tetanus, 326

antitoxin, 71, 326. See also Antitetanic serum.

Carrel-Dakin antiseptic solution in, 327 chloral hydrate in, 329

control of respiratory spasm in, 328

early symptoms, 329

English Medical Research Committee's antiseptic powder in, 327

hypodermic injection of magnesium glycerophosphates in, 327

sulphate in, 327

intraspinal injection of magnesium sulphate in, 327

local antiseptics in, 326

management of wound in, 328

morphin in, 328

nutrition of patient in, 328

prophylaxis, 326

section of phrenic nerves in, 328

segregation in, 326

serum, 326. See also Antitetanic serum. treatment, 326,328

Tetany, calcium in, 465

parathyroid extract in, 465

thyroid extract in, 465

Theobromin in acute nephritis, 764

in dropsy of heart disease, 484

in heart diseases, 483

in myocardial insufficiency, 834

Theocin in acute nephritis, 764

in myocardial inefficiency, 834

in uremia, 844

Therapeutic procedures, miscellaneous, 142

Thermal baths in acute cases in military practice, 83

Thermopenetration. See Diathermy.

Thim'ni, 4I I

Thoracentesis in acute serofibrinous pleuritis, 569

in artificial pneumothorax in hemoptysis, 545

in hemothorax, 570

Thoracic visceral disturbances, postural treatment, 716

Thorium in roentgen-ray diagnosis of urinary tract disease, 862

$X$ in gout, 5 r 4

in leukemia, 451

in pernicious anemia, 429

Threadworms, 399,400

Throat electrode, I 12

local treatment of, in diphtheria, 244

Thrombo-angiitis obliterans, diathermy in, 121

Thrombophlebitis of splenic vein, splenic anemia from, 434

Thrombosis, pulmonary, $55^{\mathrm{I}}$ bibliography, $55^{\mathrm{I}}$
Thrombosis, pulmonary, digalen in, 551 digipuratum in, $55 \mathrm{x}$

digitalon in, 551

morphin in, $55 \mathrm{I}$

prophylaxis, 551

strophanthin in, 55I

treatment, 551

Thrush, 602

etiology, 602

fungus in sprue, 368

Oidium albicans in, 602

Saccharomyces albicans, 602

treatment, 602, 603

Thymic enlargement, deep roentgen-ray therapy in, I39

tracheostenosis, roentgen-rays in, 553

Thymol in ankylostomiasis, 407

in cecum mobile, 714

in chlorosis, 432

in chronic diarrhea, 653

in intestinal distomiasis, 399

stasis, 649

teniasis, 393

in pyorrhea alveolaris, 596

in strongyloidosis, 401

in trichiniasis, 402

in trichuriasis, 404

Thymus extract in insanity, 924

gland, enlargement of, as cause of dyslalia, 524

Thyroid cancer, chronic thyroiditis and, differentiation, 473

desiccated, in cretinism, 464

in hypothyroidism, 464

in mysedema, 464

extract in functional nervous diseases, 912

in hydrocephalus from hypersecretion, 870

in pituitary lesions, 869

in scleroderma, 930

in tetany, 465

in uremia, 844

gland, adenomas of, treatment, 473

cysts of, treatment, 473

diseases of, surgical treatment, 470

excision of piece for diagnosis, 474

hypertrophy of, at puberty, 474

involvement in American trypanosomiasis, 378

transplantation of, in chronic myxedema, 475

treatment of chronic arthritis, 465

of eczema rosacea, 465

of obesity, 465

of skin diseases, 465

of urticaria, 465

tumors, asymmetric, treatment, 472 pressure symptoms in, 474

Thyroidectomy, partial, in insanity, 924

Thyroiditis, chronic, cancer of thyroid and, differentiation, 473

treatment, 473

Tick, dermatitis from, 4 I 7

fevers, 360

Ornithodoros moubata in, 4 I 7 
Tick, paralysis, 4 I 7

pruritus from, 417

spirilloses transmitted by, 360

varieties, $4 \mathrm{I} 7$

Tincture opii in amebic dysentery, 337

Tinea tonsurans, massive dosage of roentgen rays in, 133

Toilets, factory, 175

Tongue, diseases of, 605 sprue, 367

Tonkin, recurrent fever of, 362

Tonsil infections in peptic ulcer, treatment, 6r 4

Tonsillectomy for acute local disturbances, 522

for removal of source of infection, 522

in affection of neighboring organs, 522

in chronic lacunar tonsillitis, 522

local diseases of tonsils, 522

in dyslalia, 524

in hypertrophy of tonsils, $52 \mathrm{I}$

in middle-ear disease, 522

in obstructive tonsillitis, $52 \mathrm{I}$

in remote infections, 522

indications for, 521,522

Tonsillitis, 5 I 7

chronic lacunar, tonsillectomy in, 522

croupous, 5 I 7

follicular, 5r7. See also Follicular tonsillitis.

calomel in, 518

diet in, 518

ice bag in, 518

phenacetin in, 518

Rochelle salts in, 518

silver nitrate in, 518

sodium bicarbonate in, 518

tincture of chlorid of iron in, 518

in erysipelas, 275

lacunar, 517

nephrit is from, 749

Tonsils, acute local disturbances, surgical removal, 522

chronic local diseases, surgical removal, 522

hypertrophic, surgical removal, $52 \mathrm{I}$

infected, as cause of arthritis deformans, 510

exophthalmic goiter with, treatment, 470

Tooth abscess, iritis from, 386

Torcel, 4 I0

Torpor recti, constipation from, 657

Torquay Spa, principal characteristics, 89

Toxemia, chronic, splenic anemia from, 434

marasmic, in sprue, 367

septic, 264. See also Septicopyemia.

Toxemic iritis, treatment, 386

Toxins, Coley's. See Coley's toxins.

Tracheal obstruction, treatment, $55^{2}$

Tracheobronchial tree, luetic obstructions of, bronchoscopy in, $\mathbf{5 5 2}$

Tracheostenosis, thymic, roentgen rays in, 553

Trachoma, 383

VoL. IV. -63
Trachoma, acute varicties, 383

chronic, copper sulphate in, 384

varieties, treatment, 384

geographic distribution, 383

Prowazek bodies in, 383

racial distribution, $3^{8} 3$

Transduodenal lavage in chronic diarrhea, 653

Transformer, Tesla, connections of, 102

Transfusion, blood, 146 canula method of, 146

cylinder method, 146

direct, in hemorrhage in chronic jaundice, 702

donors in, 147

grouping, 147

methods of testing, ${ }_{4} 8$

in hemophilia, 443

in hemorrhagic diseases, 440

of newborn, $44 \mathrm{I}$

in pernicious anemia, 425

in symptomatic anemia, 446

in uremia, 841

indirect, citrate method, advantages, I 54

fatalities in, I 55

Lewisohn's technique, 154

methods, I49

Percy tubes for, I 53

syringe-canula method, I 53

Unger syringe-canula method, I 53

Vincent tubes for, I 53

Kimpton-Brown glass cylinders for, I 49

modified syringe method, 146

testing iso-agglutination in, 148

use of anticoagulins in, 155

of whole blood in hemorrhagic diseases, 439

Transplantation of ureter in stricture, $86 \mathrm{I}$

Tree pollen in etiology of hay fever, 529

Trematodes, 395

Trendelenburg position, pulmonary embolism from, 551

Triatoma megistus, 4I4

as carrier of Trypanosoma cruzi, 414

Trichina spiralis, $40 \mathrm{I}$

Trichinella spiralis, $40 \mathrm{I}$

Trichinelloidea superfamily, 4 or

Trichiniasis, 401

bismuth salts in, 402

bromids in, 402

calomel in, 402

chloral in, 402

eosinophilia in, 402

fever in, 402

in hogs, 4 or

magnesium sulphate in, 402

prognosis, 402

prophylaxis in, 402

stages, 401,402

symptoms, 401

thymol in, 402

treatment, 402

Trichuriasis, $4 \circ 3$

chloroform in, 404 
Trichuriasis, enemata in, 404

Ficus laurifolia in, 404

higueron in, 404

male fern in, 404

oleum chenopodii in, 403

santonin in, $4 \circ 3$

symptoms, 403

thymol in, 404

treatment, $40^{\circ} 3$

Trichuris trichiura, 403

Trigeminal neuralgia, 870 . See also $\mathrm{Neu-}$ ralgia, trigeminal.

Trikresol as preservative for antimeningitis serum, 78

Trional in septicopyemia, $27 \mathrm{I}$

Trophic diseases, treatment, 929

Tropical abscess of liver, $69 \mathrm{I}$ emetin hydrochlorid in, 69 1 treatment, $69 \mathrm{I}$ diseases, 345

Trypaflavin in African trypanosomiasis, 375

Trypanides, 372

Trypanosoma cruzi, Triatoma megistus as carrier of, $4 \mathrm{I} 4$

in American trypanosomiasis, 377

gambiense in African trypanosomiasis, $37 \mathrm{I}$

rhodesiense in African trypanosomiasis in, $37 \mathrm{I}$

Trypanosome chancre, 372

Trypanosomiasis, 37 I

African, $37 \mathrm{I}$

antimony in, 375

arsacetin in, 374

arsenic in, 374

arsenophenylglycin in, 374

atoxyl in, 374

atoxyl-emetic in, 375

benzidin colors in, 375

bibliography, 380

blood in, 373

characteristics, $37 \mathrm{I}$

chronic meningomyelitis in, 372

diagnosis, 374

emaciation in, 373

evolution of, 373

fly-extermination in prophylaxis, 377

forms, 373

Glossina morsitans in, $37 \mathrm{I}$

palpalis in, $37 \mathrm{I}$

immunity to, 373

involvement of nervous system in, 372

iridocyclitis in, 372

mental automatonism in, 373

Nabano's method of diagnosis, 374

neosalvarsan in, 375

orpiment in, 375

pathology, 372

prognosis, 373

prophylaxis in, 376

resemblance to syphilis, 373

Romberg's sign in, 373

salvarsan in, 375

second period in, 372
Trypanosomiasis, African, soamin in, 374 symptomatology, 372

tartar emetic in, 375

third period in, 373

treatment, 374

conclusions from, 376

results, 375

trypaflavin in, 375

trypanides in, 372

Trypanosoma gambiense in, $37 \mathrm{I}$

rhodesiense in, 371

tryparosan in, 375

American, 377

acute form, 378

bibliography, 380

cardiac form, 378

causal organism, 377

characteristics, 378

chronic form, 378

diagnosis, 378

endemic goiter and, differentiation, 379

forms, 378

metaschizotrypanosis of, 378

nervous form, 378

prognosis, 379

pseudomyxedematous form, 378

sequelæ, 378

suprarenal involvement in, 378

symptomatology, 378

thyroid involvement in, 378

treatment, 379

vectors, 377

nephritis from, $75^{\circ}$

period of debut in, 372

sleeping syndrome in, 373

treatment, $3 I$

tsetse fly in, 376

Tryparosan in African trypanosomiasis, 375

Tsetse fly in African trypanosomiasis, $37 \mathrm{I}$

Tubercle bacillus in pleuritis, 564

of conjunctiva, treatment, 384

of leprosy, 34I

Tuberculin, 248

in tuberculosis of eye, 387

in tuberculous peritonitis, 744

test in parenchymatous keratitis, 385

Tuberculosis, 248

after-treatment, 249

Allen treatment of diabetes in, 497

artificial pneumothorax in, 249. See also Pneumothorax, artificial.

associated with parenchymatous nephritis, 749

bronchoscopy in, 553

endobronchial injection of creosote in; 553

of guaiacol in, 553

fluoroscopic examination in, 250

graduated exercise in, 248

in Addison's disease, treatment, 466

incipient, baths in, 87

inhalation treatment of, 28

of eye, treatment, 387

tuberculin in, 387 
Tuberculosis of mouth, 604

treatment, 604

of urinary tract, surgical treatment, $86_{3}$ open-ait treatment, 248

roentgen-ray examination in, 250

sanatoria and dispensaries in Pennsylvania, 2 I

sanatorium treatment, 248

symptomatic anemia from, 444

treatment, 248

tuberculin in, 248

vaccines in, 35,54

Tuberculous adenitis, deep roentgen-ray therapy in, 136

coxitis, bismuth paste treatment, 145

peritonitis, 742. See Peritonitis, tuberculous.

Tubular hyposthenuria, 790

Tumors of bile ducts, 704

of bladder, 865 . See also Bladder, tumors.

of liver, 704

of pituitary body, 869

of spinal cord, 902

after-treatment, 903

intramedullary, surgical treatment, 903

prognosis, 903

roentgen-rays in diagnosis, 902

surgical treatment, 902

xanthochromia in, 902

thyroid, pressure symptoms in, 474

Tunbridge Wells Spa, principal characteristics, 89

Turner and Rous' iso-agglutination test in blood transfusion, 148

Minot's modification, 148

Turpentine in acariasis, 419

Tympanitis in septic peritonitis, treatment, 735

Typhlotonia in cecum mobile, 7 I 2

Typhoid bacillus, bone lesions from, i 85 detection and elimination of, I9o extracts of, for vaccination, I 92 invasion of central nervous system by, 185

invasive power, in man, $\mathrm{I}_{4}$

killed cultures of, as vaccines, 192

proliferation, in body, 184

vaccines, 35

fever, ${ }^{8} 83$

active immunization to, 200

vaccine dosage in, 200

acute glossitis in, 605

autoserums in, 200

Bacillus acidophilus in intestines in, 205

bacteremia in, 183

bibliography, 208-2 I I

culture in, technique, 187 examination in prognosis in, $I \delta_{7}$ carriers, I 85

detection and elimination of, 100 changes in intestinal bacterial flora in, 205

cold baths in, 92
Typhoid fever, cold packs in, 92

combined serum and vaccine treatment, 202

diagnosis by blood cultures, I 86

diet, fats in, 205

precautions in, 208

protcins in, 205

carly diagnosis, I 86

estimating severity of attack, 187

food absorption in, 204

general prophylaxis, 188

high calorie diet in, 205, 206 results from, 208

immunity to, duration, 48

immunization in, passive, 198

importance of early diagnosis in, 186 in children, I 97

intravenous injection of sensitized vaccine sediment, $20 \mathrm{r}$

lactose in, 205

liberal feeding in, 204

lotion douche in, 92

meningitis in, 199

metabolism in, 204

method of spread, 188

milk in, 205

mode of entrance of infection in, I 84

Murphy drop method of enteroclysis in, 92

ophthalmo-reaction in, 186

passive immunization in, anaphylactic reactions after, 199

disappearance of bacteremia after, 198

dosage of serum in, 198

horse serum for, 198

intravenous injection of serum in, 199

precautions before, 199

types of serum for, 198

pathogenesis, 183

prognosis, blood examination in, 187

prophylaxis in, 188

duties of attending physician in, 180

isolation of individual cases in, 189

protection of food supply in, 188

of water supply in, 188

rules for patient's family in, I 89

state sanitary inspection in, I 89

vaccination in, I9I

degree of immunity from, 192

in military practice, $19 \mathrm{I}$

relative incidence in vaccinated and

non-vaccinated, 197

revaccination in, 196

severity of, in vaccinated and non-

vaccinated, 107

Shaffer and Coleman's diet in, 205, 206

results from, 208

symptomatic anemia from, 444

treatment, 197

vaccination in, 192,198

agglutinins in blood after, 195

application of, 48 
Typhoid fever, vaccination in, best time for, 195

character of reaction in, 201

comparative value of vaccines in, I96

duration of immunity after, 196

extracts of bacteria in, 192

hyperleukocytosis after, I93

immune substances in blood after, 195

infection after, r95

intravenous, abuse of, 203 relapse after, 202

killed cultures of bacillus in, 192

living cultures of bacteria in, 193

local reaction after, 194

methods of administration, $\mathrm{r}_{92}$

mixed vaccines for, 196

negative phase in, 48 , 195

number of injections in, I96

reaction after, 193

results in exposed persons, 195

résumé of cases treated by, 203

sensitized cultures of bacteria in, I93

specificity of reaction in, 203

summary of intensity of reaction after, 194

vaccine, 46 , 192

action, 39

administration, 47

intravenous injection, 49

prophylactic, 46

reaction from, 47

selection, 47

sensitized, 47

subcutaneous injection, 49

therapeutic use, 49

Widal reaction in, 186

Typhus fever, 343

mode of transmission, 343

pediculi in etiology, 4 5

vaccines in, 46

ULCERA pudendum, 354

causative organism, 354

duration of disease, 354

recurrence, 355

tartar emetic in, 354

treatment, 354

Ulcerated gums, 583

Ulcerative stomatitis, 6or

Ulcer, corrosive. See Peptic ulcer.

duodenal. See Peptic ulcer.

gastric. See Peptic ulcer.

of bladder, simple chronic, 864 treatment, $86_{5}$

of cornea. See Cornea, ulcers of. of stomach. See Peptic ulcer.

peptic. See Peptic ulcer.

Ultraviolet light waves, relation of roentgen rays to, 123

Uncinariasis. See Ankylostomiasis.

Undulant fever, 353 autochthonous vaccines in, 354
Undulant fever, bibliography, 354

carriers, 354

diagnosis, 353

agglutination test in, 353,354

blood cultures in, 354

complement fixation in, 354

precipitin tests in, 354

sero-culture in, 354

urine cultures in, 354

diagnostic tests in, relative value, 354

duration of disease, 354

Micrococcus melitensis in, 353

modes of infection in, 354

treatment, 354

yeast therapy in, 354

yellow fever and, differentiation, 310

Unger's syringe-canula method of indirect blood transfusion, 153

apparatus for, 154

United States standard antidiphtheritic serum, 242

Urea excretion, additional, 794

Ambard's coefficient' of, 793

Marshall method of determining, 794

in blood, Marshall's method of determining, 799

Uremia, 836

acidosis in, 838

treatment, 845

anemia in, treatment, 847

anuria in, treatment, 847

ascites in, treatment, 846

asthma in, treatment, 846

baths in, 843

blood theory of, 837

transfusion in, $84 \mathrm{I}$

cerebral symptoms, 838

changes in kidney metabolism as cause, 837.

chronic, 836

coma in, 839

convulsions in, 839

treatment, 845

delirium in, treatment, 845

diaphoresis in, 842

diarrhea in, 847

diet in, $84 \mathrm{r}$

digitalis in, 844

diuresis in, 844

dyspnea in, treatment, 846

edema of brain as cause, 837

of lungs in, treatment, 846

fever in, 839

functional picture in, 838

gastrointestinal symptoms, 839 treatment, 846

headache in, 839

treatment, 845

hydrothorax in, treatment, 84.6

hypertension in, 812,818

treatment, 847

in acute nephritis, treatment, 766

increased elimination in, 842

itching in, treatment, 847

latent, 836

nephrolysin as cause, 837 
Uremia, nervous symptoms, 839 treatment, 845

oliguria in, treatment, 847

paralyses in, 839

pilocarpin in, 844

prognosis, 840

prophylaxis, 840

psychical manifestations, 839

purgatives in, $\mathbf{8 4 2}$

respiratory symptoms, 839 treatment, 845

Riess' classification, 838

secondary anemia in, 839

sedatives in, 845

sodium bicarbonate in, 844

sulphonephthalein excretion in, 838

sweating in, 842

symptomatic treatment, 845

symptoms, 838

theocin in, 844

theories, 836

thyroid extract in, 844

treatment, $84^{\circ}$

vomiting in, treatment, 846

Young's treatment, 840

Ureter stricture, 856

acquired, 857

age of, 859

catheter diagnosis, $85^{8}$

congenital, 857

dilation in, 859

etiology, 856

from systemic infection, 857

from trauma, 857

in hydronephrosis, 856

in pyelitis, 856

in pyelonephritis, 856

in pyonephrosis, 856

incidence of, 856

lavage of kidney pelvis in, 860

morbid anatomy, 857

opcrative treatment, 860

pyelo-ureteroplasty in, 860

retrograde dilatation in, $86 \mathrm{r}$

roentgen rays in diagnosis, $85^{8}$

transplantation in, 86I

treatment, 859

urine in diagnosis, 859

Urethral electrode, I 12

Urethritis, chronic, nephritis from, 749

gonococcus vaccines in, 43

Uric acid diathesis, 516

diet in, 516

treatment, 5 I 6

Urinary antiseptics in acute nephritis, 768 tract diseases, surgical indications in, 856

tuberculosis, surgical treatment, 863

Urine, bacteria in, in nephritis, 749

cultures in diagnosis of undulant fever, 354

determination of ammonia-coeflicient of, in acidosis in children, 503

in diagnosis of ureteral stricture, 859

in health, 787

in orthostatic albuminuria, 802
Urine in yellow fever, 308

microscopic appearance, in yellow

fever, 310

normal, composition of, 787

quantity of, in yellow fever, 311

specific gravity of, in diseased kidney, 789

in healthy kidney, 789

suppression of, in corrosive sublimate poisoning, $16_{5}$

in yellow fever, treatment, 314,315

testing of, for sugar, in Allen treatment of diabetes, 495

variability of quantitative composition, 787

Urobilin, effect of splenectomy on, in pernicious anemia, 428

in feces in pernicious anemia, 421

reaction in malaria, 348

Urticaria, thyroid treatment, 465

Uterine electrode, I I 2

fibroids, roentgen-ray therapy in, contraindications to, $14 \mathrm{I}$

hemorrhage, deep roentgen-ray therapy in, 140

Uveal tract, diseases of, treatment, 385

Uveitis, $3^{85}$

\section{VACCINATION, 294}

chill after, $4^{\circ}$

prophylactic in typhoid fever, 191

typhoid fever, in military practice, I9I

temperature rise after, $4 \circ$

Vaccine, Behring's, in diphtheria, duration of immunity from, 242

Pasteur's, in rabies, 324 preparation of, 324

sediment, sensitized, intravenous injection, in typhoid fever, 201

typhoid, $39,46,192$. Sec also $T y$ phoid fever vaccine.

virus, bacteria-free, 294

advantages of, 295

effect of glycerin in, 294

Vaccines, 33

atoxic, 37

autochthonous, in undulant fever, 354

autogenous. See Aulogenous vaccines.

Bacillus enteritidis, 35

bacterial, 33

colon-bacillus, 35

combined prophylactic, $5 \mathbf{I}$

deterioration, 37

diphtheroid bacilli, 35

dosage, 4 I

from Bacillus coli, 5 I

proteus, 5 I

pyocyaneus, 5 I

from digested bacteria, 36

from killed bacteria, 36

from live bacteria, 37

from sensitized bacteria, 3?

general considerations, 33

glanders, 35 
Vaccines, gonococcus, 34, 43. See also Gonococcus vaccines.

hemoglobinophilic, 35

hemolytic, 34

history, $\mathbf{r} 4$

in atrophic rhinitis, 52

in bacillary dysentery, 50

in B. Friedländer infections, 52

in bacterial pyorrhea, 596

in bronchitis, 55

in bubonic plague, $5 \mathrm{I}$

in cholera, $5^{I}$

in chronic pancreatitis, 707

in common colds, 54

in epidemic cerebrospinal meningitis, 45

in glanders, $5 \mathrm{I}$

in Hodgkin's disease, 55, 462

in influenza bacillus infections, 53

in lobar pneumonia, 228

in middle-ear infections, 55

in mouth infections, 55

in paratyphoid fever, $5^{\circ}$

in pertussis, 52,333

dosage, 53

in plague, 298

in pyorrhea alveolaris, 593

in rhinoscleroma, 52

in scarlet fever, 245

in septic peritonitis, 735

in sinus infections, 55

in specific diseases, 4 I

in tuberculosis, 54

in typhus fever, 46

influenza, 35

leukocytic response to, 39

meningococcus, 34

Micrococcus melitensis, 45

mixed, 36

in typhoid fever, 196

mode of injection, 4 I

negative phase in use of, 4 I

non-hemolytic, 34

non-specific, non-specific response to, 38

paratyphoid bacillus, 35

pneumococcus, 34

dosage, 43

polyvalent, 36

reaction to, $4 \mathrm{I}$

selection of dosage, 55

specific, non-specific response to, 38

staphylococcus, 34, 42. See also Staphylococcus vaccines.

streptococcus, 34, 42. See also Streptococcus vaccines.

theoretic considerations, 37

tuberculosis, 35

types, 36

type of case for, 37

typhoid bacillus, 35

Vaccinia, 294

Vacuum electrode, glass, I 2

in acroparesthesia, II 5

Vagina, erysipelas of, 275

Valvular heart disease, venous section in I42

Van Slyke's test for acidosis, 502
Vapor baths, hot, in pulmonary edema, 548

Vascular hyposthenuria, 790

Vaseline, carbolated, for flea-bite, 4I6 in leishmaniasis, 359

in pediculosis, 4I 5

Vasomotor diseases, treatment, 929

Vegetable and fruit diet in sprue, 370

Vegetables, iron content of, 446

Venal distomiasis, 395 male fern in, 396 treatment, 396

Venesection, I4 2

in chronic nephritis with hypertension, 142

in erythremia, 433

in mitral stenosis, 479

in myocardial insufficiency, 828

in uremia, $84 \mathrm{I}$

in valvular heart disease, 142

Ventilation, factory, I73. See also Factory ventilation.

recent experiments on, 95,96

Ventnor Spa, principal characteristics, 89

Ver de Cayor, 410

Macaque, 4 ro

Vermicides, 30

Vermifuges, 30, 393

Vermin, extermination of, in kala-azar, 358

Veronal in septicopyemia, $27 \mathrm{I}$

Verruga Peruviana, 355

bibliography, 355

Vesical myiasis, 4I 2

Vianna's antimony treatment of kala-azar, 357

Vibration of factory flooring, prevention, I74

Vichy in yellow fever, $3 I_{5}$

Vincent's angina, Price's incision in, 580 mixture for preparation of transfusion tubes, 149

tubes for indirect blood transfusion, $\mathbf{I}_{53}$

Vinyl amin, nephritis from, $75 \mathrm{I}$

Virus, vaccine, bacteria-free, 294 advantages of, 295

effect of glycerin on, 294

Visceral disturbances, thoracic and abdominal, postural treatment, 7 I 6

Visceroptosis, 7 ro

back-brace for, $719,723,724$

baths in, 724

binder in, 7 Io

causes, 7 ro

effect of obesity in, $7 \mathrm{II}$

etiology, 7 IO

exercises in, $7 \mathrm{I} I, 7 \mathrm{I} 2,724$

from malformation of body, 7 ro

hyperextended position in, 721,725

in heavy human type, 719

in normal human type, 720

in slender human type, 719

knee-chest position in, 725

Lane abdominal support in, 7 to

massage in, 724

mechanical supports in, 7 ro 
Visceroptosis, molded jacket in, 720, 724 orthopedic apparatus in, $7 I I$

pelvic belt in, 724

postural treatment, 725

prognosis, 726

prone hanging position in, 722, 725

proper standing position in, $7 \mathrm{II}$

prophylactic treatment, $7 \mathrm{II}$

recumbent treatment, 724,725

surgical treatment, 727

treatment, 710,724

vomiting in, treatment, 725

Visceroptotic human type, congenital, 716 , 717

Vital statistics, bureau of, in Pennsylvania Department of Health, 2 I

Vitamins, 157,160 deficiency of, in etiology of beri-beri, 364

in etiology of pellagra, 161

Volhard and Fahr's classification of nephritis, 755

Vomit, black, in yellow fever, 308 treatment, 315

Vomiting in acute nephritis, treatment, 767

in malaria, treatment, 349

in myocardial insufficiency in, treatment, 835

in sclerosis of blood-vessels of abdomen, 639

in septic peritonitis, treatment, 734

in septicopyemia, control of, 270

in uremia, treatment, 846

in visceroptosis, treatment, 725

recurrent, in acidosis in children, 504

Vomito, 299

Von Noorden diet in acute nephritis, 762 treatment of chronic diarrhea, $65^{2}$

Von Pirquet test in food poisoning, 158

Vulvovaginitis, gonococcus vaccines in, 44

WARM baths in insanity, 926

Warts, radium therapy in, 134

Wassermann reaction, 278

in acute catarrhal glossitis, 605

in cerebrospinal syphilis, 882,887 aiter serum treatment, 898

in iritis, 386

in parenchymatous keratitis, 385

in syphilis, discovery, 278 of liver, $69 \mathrm{I}$

negative, after treatment, $28 \mathrm{I}$

positive, after treatment, $28 \mathrm{I}$

Water for factories, 175

in acidosis in children, 506

in acute nephritis, 763

in constipation, 660

in erysipelas, 276

in yellow fever, 315

intake in chronic interstitial nephritis, 783

retention in edema, 805

supply, protection of, in prevention of typhoid, 188
Water-cooling device for Coolidge tube, 128

Watsonius watsoni, 399

Wave current, static, ror

technique for application, rog

Wehnelt roentgen-ray tube, 124

West Africa, recurrent fever of, $36 \mathrm{I}$

Whey-protein mixtures in protein indigestion, 679

Whisky in pulmonary edema, 548

Whooping cough, 330. See also Pcrtussis.

Widal reaction in typhoid fever, 186 technique, 188

classification of nephritis, $75^{\circ}$

Williger's treatment of ulcerative stomatitis, 603

Wire brush electrode, II I

Wiring with electrolysis in sacculated aneurysm, 155 technique, 156

Wohlfahrtia magnifica, 410

Wood Hall Spa, principal characteristics, 89

Work hours in factories, 176

room. See Factory.

tests in heart diseases, 485

Workman, avoidance of colds by, $18 \mathrm{r}$

bathing for, $x 77$

best shoes for, 178

care of eyes, 179

of feet, 178

of hair, 179

of hands and nails, 178

of mouth, I 79

of nose, 179

of teeth, 180

clothing for, 177

effects of cold, treatment, $18 \mathrm{r}$

exercise for, 180

factory air space required for, 172

occupational hygiene for, 177

physical examination, 182

position of, while working, I80

precautions in hot weather for, 181

proper food for, I 8I

Worm, guinea, $4 \circ 9$

Wormseed in ascariasis, 405

Wounds, infected, Dakin's antiseptic in, 268

erysipelas in, 274

Wright's treatment of, 28

method of applying antitetanic serum to, 326

sterilization of, by Dakin's antiseptic, 269

unhealed, prolunged baths in, 84

Wright's treatment of infected wounds, 28

XaNthochromia in tumors of spinal

cord, 902

Xenapsylla cheopsis, 298

YAws, bibliography, 353 
Yeasts in stools in sprue, 367

Yeast therapy in undulant fever, 354

Yellow fever, 299

adrenalin in, 316

albuminuria in, 308

alcohol in, $3 \mathrm{r} 4$

aspirin in, 315

Bacillus $\mathrm{X}$ in, 302

baths in, 315

black vomit in, 308

treatment, 3I 5

calomel in, 3 r 2,313

Carter's incubation theory of, 302

catheterization in, $3 \mathrm{r} 4$

cause of endemics, 320

collapse in, treatment, 316

continued type, 307

Creole foot bath in, $3 \mathbf{r}_{3}$

decreasing pulse-rate in, 306

definition, 299

dengue fever and, differentiation, 3 ro

descending type, 307

diagnosis, 300

digitalin in, 315

drug treatment, 312

duration of fatal cases, 309

elixir of iron in, 314

enemata in, 315

ergot in, 316

extermination of, in Cuba, 304

Faget's law in, 307

feeding in, $3 \mathrm{r} 4$

fever in, treatment, $3 I_{5}$

Finlay's mosquito theory of, 301

Fischer's solution in, $3 \mathrm{r} 6$

fly-speck vomit in, 308, 31 2

fresh air in, 324

fumigation in, 3 ro

gastric hemorrhage in, treatment, 3 I 5

geographic distribution, 299

hemorrhage in, 308

hiccoughs in, 308

historic epidemics, 299

history, 299

hydrotherapy in, 3r 5

hyperpyrexia in, treatment, $3 \mathrm{r} 3$

ice cream in, $3{ }^{1} 3$

icterus in, 308, 3 I 2

immunization in, 317

importance of complete rest in, 314

in children, 309

in South America, 300

in Spanish-American War, 300

intravenous saline solution in, 3 r 6

iron chlorid in, 314

irritability of stomach in, 3I 5

jaundice in, 308, 312

kidneys in, 306

liquid diet in, $3 \mathrm{r}_{3}$

liver in, 305

Malta fever and, differentiation, 3 ro method of onset, 306

microscopic appearance of urine in, 310
Yellow fever, mild solid diet in, 3 I $4_{4}$

mortality, 3 I I

mosquito transmission, 299

mustard foot bath in, 3 I 3

non-communicability of fomites in, 303

old quarantine methods in, 300

olive oil injections in, 3 r 4

opium in, 3 I 5

pain in, treatment, $3 \mathrm{r}_{3}, 3 \mathrm{r} 5$

Paraplasma flavigenum in, 305

pathology, 305

period of congestion, 307

infection in, 307

phenacetin in, $3 \times 5$

physical appearance in, 306

prognosis in, 3 Ir

prophylaxis, 3 I 6

financial cost of, 320

mosquitoes in, breeding habits, 3 r 7

sanitary measures, 3 r6, 3 r 8

Public Health Service work in, 304

purgatives in, 312

quantity of urine in, $3 \mathrm{rr}$

quarantine in, $32 \mathrm{I}$

at foreign infected ports, 322

suspected ports, $32 \mathrm{r}$

period of detention in, $32 \mathrm{I}$

regulations in, $32 \mathrm{I}$

quinin in, $3 \times 4$

ravages of, in former years, 300

in old times, 300

rectal feeding in, 3 I 5

Reed Board, 302

conclusions of, 303

relapses in, 309

relapsing fever and, 3 ro

remitting type, 307

rigor mortis in, 305

Sanarelli's bacillus in, 301

stages, 307

Sternberg's alkaline mixture in, $3 r_{3}$

suppression of urine in, treatment, $3 \mathrm{r}_{4}, 3 \mathrm{r}_{5}$

susceptibility in, $3 \mathbf{r}$

symptoms, 306

temperature in, 306,307

in prognosis, $3 \mathrm{Ir}$

transmission of, by Stegomyia calopus, 299

treatment, 3 I 2

urine in, 308

Vichy in, 3 I 5

water in, 3 I 5

jack, 299

Yokogawa yokogawa, 399

Young's treatment of uremia, 840

ZINC chlorid in ulcerative stomatitis, 603

in corneal ulcers, 384

iontophoresis in purulent keratitis, ${ }^{\prime} 84$ 




\section{SAUNDERS' BOOKS}

\section{.

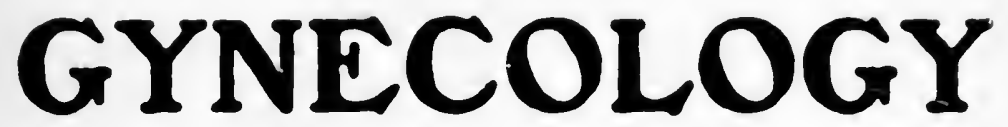 \\ and

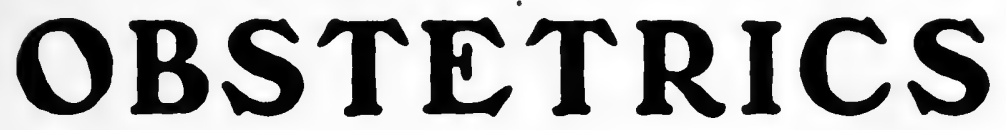

W. B. SAUNDERS COMPANY WEST WASHINGTON SQUARE PHILADELPHIA

\section{HENRIETTA STREET COVENT GARDEN, LONDON}

\section{Graves' Gynecology}

Text-Book of Gynecology. By William P. Graves, M. D., Professor of Gynecology at Harvard Medical School. Large octavo of 770 pages, with 425 original illustrations, many in colors. Cloth, $\$ 7.00$ net; Half Morocco, $\$ 8.50$ net.

\section{TWO PRINTINGS IN FIVE MONTHS}

This new work presents gynecology along new lines. An entire section is devoted exclusively to the physiology of the pelvic organs and to correlated gynecology - the relationship of gynecology to organs of internal secretion, breast, skin, organs of sense, digestion and respiration, blood, circulatory apparatus, abdominal organs, nervous system, bones, and joints. A special section is devoted to enteroptosis, intestinal bands, and movable kidney.

The second portion of the book is devoted to special gynecologic disease and is arranged particularly for the convenience of medical students. The first two parts (covering 500 pages) are entirely non-surgical, giving only drug and mechanical therapy and material invaluable to the general practitioner. The third part is exclusively a treatise on surgical gynecology, and includes profusely illustrated descriptions of those gynecologic operations that to the author seem most feasible. A number of new operations are given and illustrated. 


\section{De Lee's Obstetrics}

Principles and Practice of Obstetrics. By Joseph B. DE LEE, M. D., Professor of Obstetrics in the Northwestern University Medical School, Chicago. Large octavo of 1087 pages, with 938 illustrations, I 75 in colors. Cloth, $\$ 8.00$ net; Half Morocco, $\$ 9.50$ net.

\section{TWO EDITIONS-FOUR REPRINTS}

\section{The Most Superb Book on Obstetrics Ever Published}

You will pronounce this new book by Dr. De Lee the most elaborate, the most superbly illustrated work on Obstetrics you have ever seen. Especially will you value the 938 illustrations, practically all original, and the best work of leading medical artists. Some 175 of these illustrations are in colors. Such a magnificent collection of obstetric pictures-and with really practical value-has never before appeared in one book.

You will find the text extremely practical throughout, Dr. De Lee's aim being to produce a book that would meet the needs of the general practitioner in every particular. For this reason diagnosis is featured, and the relations of obstetric conditions and accidents to general medicine, surgery, and the specialties brought into prominence.

Regarding treatment: You get here the very latest advances in this field, and you can rest assured every method of treatment, every step in operative technic, is just right. Dr. De Lee's twenty-one years' experience as a teacher and obstetrician guarantees this.

Worthy of your particular attention are the descriptive legends under the illustrations. These are unusually full, and by studying the pictures serially with their detailed legends, you are better able to follow the operations than by referring to the pictures from a distant text-the usual method.

Dr. M. A. Hanna, University Mediral College, Kansas City

"I am trank in stating that I prize it more highly than any other volume in my obstetric library, which consists of practically alt the recent books on that subject."

\section{Prof. W. Stoeckel, Kiel, Germany}

“ Dr. DeLee's Obstetrics deserves the greatest recognition. The text and the grz very beautiful illustrations prove that it is written by an obstetrician of ripe experience and of exceptional teaching ability. It must be ranked with the best works of our literature."

Dr. George L. Brodhead, New York Post-Graduate Medical School

"The name of the author is in itself a sufficient guarantee of the merit of the book, and I congratulate him, as well as you, on the superb work just published." 


\section{Band ler's \\ Medical Gynecology}

Medical Gynecology. By S. Wyllis Bandler, M. D., Adjunct Professor of Diseases of Women, New York Post-Graduate Medical School and Hospital. Octavo of 790 pages, with I 50 original illus. t*ations. Cloth, \$5.00 net; Half Morocco, $\$ 6.50$ net.

\section{THIRD EDITION-60 PAGES ON INTERNAL SECRETIONS}

This new work by Dr. Bandler is just the book that the physician engaged in general practice has long needed. It is truly the practitioner's gynecology-planned for him, written for him, and illustrated for him. There are many gynecologic conditions that do not call for operative treatment; yet, because of lack of that special knowledge required for their diagnosis and treatment, the general practitioner has been unable to treat them intelligently. This work not only deals with those conditions amenable to non-operative treatment, but it also tells how to recognize those diseases demanding operative treatment.

\section{American Journal of Obstetrics}

"He has shown good judgment in the selection of his data. He has placed most emphasis on diagnostic and therapeutic aspects. He has presented his facts in a manner to be readily grasped by the general practitioner."

\section{Bandler's Vaginal Celiotomy}

Vaginal Celiotomy. By S. WVllis Bandler, M. D., New York Post-Graduate Medical School and Hospital. Octavo of 450 pages, with 148 original illustrations. Cloth, $\$ 5.00$ net; Half Morocco, $\$ 6.50$ net.

\section{SUPERB ILLUSTRATIONS}

The vaginal route, because of its simplicity, ease of execution, absence of shock, more certain results, and the opportunity for conservative measures, con. stitutes a field which should appeal to all surgeons, gynecologists, and obstetricians. Posterior vaginal celiotomy is of great importance in the removal of small tubal and ovarian tumors and cysts, and is an important step in the performance of vaginal myomectomy, hysterectomy, and hysteromyomectumy. Anterior vaginal celiotomy with thorough separation of the bladder is the only certain method of correcting cystocele.

\section{The Lancet, London}

"Dr. Bandler has done good service in writing this book, which gives a very clear description of all the operations which may be undertaken through the vagina. He makes out o strong case for these operations." 


\section{Ashton's \\ Practice of Gynecology}

The Practice of Gynecology. By W. Easterly Ashton, 'M. D.; LL.D, Professor of Ginecology in the Medico-Chirurgical College. Philadelphia. Handsome octavo volume of 1092 pages, containing I097 original line drawings. Cloth, $\$ 6.50$ net; Half Morocco, $\$ 8.00$ net.

\section{NEW (6th) EDITION}

The continued success of Dr. Ashton's work is not surprising to any one knowing the book. The author takes up each procedure necessary to gynecologic step by step, the student being led from one step to another, just as in studying any non-medical subject, the minutest detail being explained in language that cannot fail to be understood even at first reading. Nuthing is left to be taken for granted, the author not only telling his readers in every instance what should be done but also precisely how to do it. A distinctly original feature of the book is the illustrations, numbering 1058 line drawings made especially under the author's personal supervision from actual apparatus, living models, and dissections on the cadaver.

From its first appearance Dr. Ashton's book set a standard in practical medical books; that he has produced a work of unusual value to the medical practitioner is shown by the demand for new editions. Indeed, the book is a rich store-house of practical information, presented in such a way that the work cannot fail to be of daily service to the practitioner.

Howard A. Kelly, M. D.

Professor of Gynecologic Surgery, Johns Hopkins University.

"It is different from anything that has as yet appeared. The illustrations are particuiarly clear and satisfactory. One specially good feature is the pains with which you describe so many details so often left to the imagination."

\section{Charles B. Penrose, M. D.}

Formerly Professor of Gynecology in the University of Pennsylvania

"I know of no book that goes su thoroughly and satisfactorily into all the details of every. thing connected with the subject. In this respect your book differs from the others."

\section{George.M. Edebohls, M. D.}

Professor of Diseases of Women, New York Post-Graduate Medical Schoo?

"A text-book most admirably adapted to teach gynecology to those who must get their knowledge, even to the minutest and most elementary details, from books." 


\section{Kelly and Cullen's}

\section{Myomata of the Uterus}

Myomata of the Uterus. By Howard A. Kelly, M. D., Professor of Gynecologic Surgery at Jolnns Hopkins University; and THomas S. Cullex, M. B., Associate in Gynecology at Johns Hopkins University. Large octavo of about 700 pages, with 388 original illustrations, by August Horn and Hermann Becker. Cloth, \$7.50 net; Half Morocco, $\$ 9.00$ net.

\section{ILLUSTRATED BY AUGUST HORN AND HERMANN BECKER}

This monumental work, the fruit of over ten years of untiring labors, will remain for many years the last word upon the subject. Written by those men who have brought, step by step, the operative treatment of uterine myoma to such perfection that the mortality is now less than one per cent., it stands out as the record of greatest achievement of recent times.

\section{Surgery, Gynecology, and Obstetrics}

"It must be considered as the most comprehensive work of the kind yet published. It will always be a mine of wealth to future students."

\section{Cullen's Adenomyoma of the Uterus}

Adenomyoma of the Uterl's. By Thomas S. Cullen, M. B. Octavo of 275 pages, with original illustrations by Hermann Becker and August Horn. Cloth, $\$ 5.00$ net; Half Morocco, $\$ 6.50$ net.

"A good example of how such a nonograph should be written. It is an excellent work, worthy of the high reputation of the author and of the school from which it emanates."-The Lancet, London.

\section{Cullen's Cancer of the Uterus}

Cancer of the Uterus. By Thomas S. Cullex, M. B. Large octavo of 693 pages, with over 300 colored and half-tone text-cuts and eleven lithographs. Cloth, $\$ 7.50$ net; Half Morocco, $\$ 8.50$ net.

"Dr. Cullen's book is the standard work on the greatest problem which faces the surgical world to-day. Any one who desires to attack this great problem must have this book."-Howa RD A. K F.L.Y, M. D., Johns Hopkins Liniversity. 


\section{Kelly $\mathcal{E}$ Noble's Gynecology and Abdominal Surgery}

Gynecology and Abdominal Surgery. Edited by HowARD A. Kelly, M. D., Professor of Gynecology in Johns Hopkins University; and Charles P. Noble, M. D., formerly Clinical Professor of Gynecology in the Woman's Medical College, Philadelphia. Two imperial octavo volumes of 950 pages each, containing 880 illustrations, some in colors. Per volume: Cloth, $\$ 8.00$ net; Half Morocco, \$9.50 net.

TRANSLATED INTO SPANISH

\section{WITH 880 ILLUSTRATIONS BY HERMANN BECKER AND MAX BRÖDEL}

In view of the intimate association of gynecology with abdominal surgery the editors have combined these two important subjects in one work. For this reason the work will be doubly valuable, for not only the gynecologist and general practitioner will find it an exhaustive treatise, but the surgeon also will find here the latest technic of the various abdominal operations. It possesses a number of valuable features not to be found in any other publication covering the same fields. It contains a chapte- upon the bacteriology and one upon the pathology of gynecology, dealing fully with the scientific basis of gynecology. In no other work can this information, prepared by specialists, be found as separate chapters. There is a large chapter devoted entirely to medical gynecology written especially for the physician engaged in general practice. Abdominal surgery proper, as distinct from gynecology, is fully treated, embracing operations : ipon the stomach, intestines, liver, bile-ducts, pancreas, spleen, kidneys, ureter, bladder, and peritoneum.

\section{Davis' Manual of Obstetrics}

Dr. Davis' Manual is complete in every particular and fully illustrated with original line-drawings. You get chapters on anatomy of the normal and abnormal bony pelvis, physiology of impregnation, anatomy of the birth canal in pregnancy, growth and development of the embryo; pregnancy, its diagnosis, physiology, hygiene, pathology (complications) : labor, its causes, physiology, pathology (complications), management; the puerperal period, care of the mother and infant; obstetric asepsis and antisepsis ; obstetric operations-use of forceps, version, embryotomy, prevention and repair of lacerations, injury to the bony pelvis, induction of labor, cesarean section (abdominal and extraperitoneal), symphysiotomy, pubiotomy, lessening size of sacral promontory, rupture of uterus ; fetal pathology ; injuries to fetus in labor; mixed feeding; medicolegal aspects.

ramo of $4^{6} 3$ pages, with I7 I original line-drawings. By EDWARD P. DAvis, M. D., Professor of Obstetrics, Jefferson Medical College, Philadelphia.

Cloth, \$2.25 net. 


\section{Webster's}

\section{Text-Book of Obstetrics}

A Text-Book of Obstetrics. By J. Clarence Webster, M. D. (Edin.), F. R. C. P. E., Professor of Obstetrics and Gynecology in Rush Medical College, in affiliation with the University of Chicago. Octavo volume of 767 pages, illustrated. Cloth, \$5.00 net; Half Morocco, $\$ 6.50$ net.

\section{BEAUTIFULLY ILLUSTRATED}

In this work the anatomic changes accompanying pregnancy, labor, and the puerperium are described more fully and lucidly than in any other text-book on the subject. The exposition of these sections is based mainly upon studies of frozen specimens. Unusual consideration is given to embryologic and physiologic data of importance in their relation to obstetrics.

\section{Buffalo Medical Journal}

"As a practical text-book on obstetrics for both student and practitioner, there is left very little to be desired, it being as near perfection as any compact work that has been published."

\section{Webster's}

\section{Diseases of Women}

A Text-Book of Diseases of Women. By J. Clarexce Webster, M. D. (Emrn.), F. R. C. P. E., Professor of Gynecology and Obstetrics in Rush Medical College. Octavo of 7 I 2 pages, with 372 text-illustrations and Io colored plates. Cloth, \$7.00 net: Half Morocco, \$\$.50 net.

Dr. Webster has written this work especially for the general fractitioner, discussing the clinical features of the subject in their widest relations to general practice rather than from the standpoint of specialism. The magnificent illustrations, three hundred and seventy-two in number, are nearly all original.

\section{Howard A. Kelly. M, D.}

Professor of Gynecologic Surgery, Johns Hopkins Unizersity.

"It is undoubtedly one of the bes! works which has been put on the market wathin recent years, showing from start to finish Dr. Webster's well-known thoroughness. The illustrations are also of the highest order." 


\section{Hirst's \\ Text-Book of Obstetrics}

\section{Seventh Edition}

A Text-Book of Obstetrics. By Barton Cooke Hirst, M.D., Professor of Obstetrics in the University of Pennsylvania. Handsome octavo of Ior 3 pages, with 895 illustrations, 53 of them in colors. Cloth, $\$ 5.00$ net; Half Morocco, $\$ 6.50$ net.

\section{INCLUDING RELATED GYNECOLOGIC OPERATIONS}

Immediately on its publication this work took its place as the leading text-book on the subject. Both in this country and in England it is recognized as the most satisfactorily written and clearly illustrated work on obstetrics in the language. The illustrations form one of the features of the book. They are numerous and the most of them are original. In this edition the book has been thoroughly revised. Recognizing the inseparable relation between obstetrics and certain gynecologic conditions, the author has included all the gynecologic operations for complications and consequences of childbirth, together with a brief account of the diagnosis and treatment of all the pathologic phenomena peculiar to women.

\section{OPINIONS OF THE MEDICAL PRESS}

\section{British Medical Journal}

"The popularity of American text-books in this country is one of the features of recent years. The popularity is probably chiefly due to the great superiority of their illustrations over those of the English text-books. The illustrations in Dr. Hirst's volume are far more numerous and far better executed, and therefore more instructive, than those commonly found in the works of writers on obstetrics in our own country."

\section{Bulletin of Johns Hopkins Hospital}

"The work is an admirable one in every sense of the word, concisely but comprehensively written."

\section{The Medical Record, New York}

"The illustrations are numerous and are works of art, many of them appearing for the first time. The author's style, though condensed, is singularly clear, so that it is never necessary to re-read a sentence in order to grasp the meaning. As a true model of what a modern textbook on obstetrics should be, we feel justified in affirming that Dr. Hirst's book 's without a rival." 


\section{Hirst's \\ Diseases of Women}

A Text-Book of Diseases of Women. By Bakton CoOKE Hirst, M. D., Professor of Obstetrics, University of Pennsylvania ; Gynecologist to the Howard, the Orthopedic, and the Philadelphia Hospitals. Octavo of 745 pages, with 701 original illustrations, many in colors. Cloth, \$5.00 net; Half Morocco, \$6.50 net.

\section{SECOND EDITION-WITH 701 ORIGINAL ILLUSTRATIONS}

The new edition of this work has just been issued after a careful revision. As diagnosis and treatment are of the greatest importance in considering diseases of women, particular attention has been devoted to these divisions. To this end, also, the work has been magnificently illuminated with yor illustrations, for the most part original photographs and water-colors of actual clinical cases accumulated during the past fifteen years. The palliative treatment, as well as the radical operative, is fully described, enabling the general practitioner to treat many of his own patients without referring them to a specialist. An entire section is devoted to $\approx$ full description of all modern gynecologic operations, illuminated and elucidaied by numerous photographs. The author's extensive experience renders this work of unusual value.

\section{OPINIJNS OF THE MEDICAL PRESS}

\section{Medical Record, New York}

"Its merits can be appreciated only by a careful perusal. . . . Nearly one hundred pages are devoted to technic, this chapter being in some respects superior to the descriptions in many other text- boks."

\section{Boston Medical and Surgical Journal}

"The author has given special attention to diagnosis and treatment throughout the book, and has produced a practical treatise which should be of the greatest value to the student, the general practitioner, and the specialist."

\section{Medical News, New York}

"Office treatment is given a due amount of consideration, so that the work will be as useful to the non-operator as to the specialist." 


\title{
GET \\ THE BEST \\ American \\ THE NEW \\ STANDARD \\ Illustrated Dictionary
}

\author{
New (8th) Edition-1500 New Words
}

The American Illustrated Medical Dictionary. A new and complete dictionary of the terms used in Medicine, Surgery, Dentistry, Pharmacy, Chemistry, Veterinary Science, Nursing, and kindred branches; with over 100 new and elaborate tables and many handsome illustrations. By W. A. Newman Dorland, M.D., Editor of "The American Pocket Medical Dictionary." Large octavo, II 37 pages, bound in full flexible leather. I'rice, $\$ 4.50$ net; with thumb index, $\$ 5.00$ net.

\section{IT DEFINES ALL THE NEW WORDS-MANY NEW FEATURES}

The American Illustrated Medical Dictionary defines hundreds of the newest terms not defined in any other dictionary-bar none. These new terms are live, active words, taken right from modern medical literature.

It gives the capitalization and pronunciation of all words. It makes a feature of the derivation or etymology of the words. In some dictionaries the etymology occupies only a secondary place, in many cases no derivation being given at all. In the "American Illustrated" practically every word is given its derivation.

Every word has a separate paragraph, thus making it easy to find a word quickly.

The tables of arteries, muscles, nerves, veins, etc., are of the greatest help in assembling anatomic facts. In them are classified for quick study all the necessary information about the various structures.

Every word is given its definition-a definition that defines in the fewest possible words. In some dictionaries hundreds of words are not defined at all, referring the reader to some other source for the information he wants at once.

\section{Howard A. Kelly, M. D., Johns Hopkins University, Baltimore}

"The American Illustrated Dictionary is admirable. It is so well gotten up and of such convenient size. No errors have been found in my use of it."

\section{J. Collins Warren, M. D., LL.D., F.R.C.S. (Hon.), Harvard Medical School}

"I regard it as a valuable aid to my medical literary work. It is very complete and of sonvenient size to handle comfortably. I use it in preference to any other." 


\section{Penrose's \\ Diseases of Women}

\section{Sixth Revised Edition}

A Text-Book of Diseases of Women. By Charles B. Penrose, M. D., PH. D., formerly Professor of Gynecology in the University of Pennsylvania; Surgeon to the Gynecean Hospital, Philadelphia. Octavo volume of 550 pages, with 225 fine original illustrations. Cloth. $\$ 3.75$ net.

\section{ILLUSTRATED}

Regularly every year a new edition of this excellent text-book is called for, and it appears to be in as great favor with physicians as with students. Indeed, this book has taken its place as the ideal work for the general practitioner. The author presents the best teaching of modern gynecology, untrammeled by antiquated ideas and methods. In every case the most modern and progressive technique is adopted and made clear by excellent illustrations.

Howard A. Kelly, M.D.,

Professor of Gynecologic Surgery, Johns Hopkins Universty. Baitimore.

"I shall value very highly the copy of Penrose's 'Diseases of Women' received. I have already recommended it to my class as THE BEST book."

\section{Davis' Operative Obstetrics}

Operative Obstetrics. By Edward P. Davis, M.D., Professor of Obstetrics at Jefferson Medical College, Philadelphia. Octavo of $48_{3}$ pages, with 264 illustrations. Cloth, $\$ 5.50$ net; Half Morocco, $\$ 7.00$ net.

\section{INCLUDING SURGERY OF NEWBORN}

Dr. Davis' new work is a most practical one, and no expense has been spared to make it the handsomest work on the subject as well. Every step in every operation is described minutely, and the technic shown by beautiful new illustrabons. Dr. Davis' name is sufficient guarantee for something above the mediocre. 


\section{Norris' \\ Gonorrhea in Women}

Gonorrhea in Women. By Charles C. Norris, M. D., Instructor in Gynecology, University of Pennsylvania. With an Introduction by John G. Clark, M. D., Professor of Gynecology, University of Pennsylvania. Large octavo of 520 pages, illustrated. Cloth, $\$ 6.50$ net.

\section{A Classic}

Dr. Norris here presents a work that is destined to take high place among publications on this subject. He has done his work thoroughly. He has searched the important literature very carefully, over 2300 references being utilized. This, coupled with Dr. Norris' large experience, gives his book the stamp of authority. The chapter on serum and vaccine therapy and organotherapy is particularly valuable because it expresses the newest advances. Every phase of the subject is considered : History, bacteriology, pathology, sociology, prophylaxis, treatment, gonorrhea during pregnancy, parturition and puerperium, and all other phases.

\section{Ashton's Obstetrics}

New (8th) Edition

Essentials of Obstetrics. By W. Easterly Ashton, M. D. Revised by John A. McGlins, M.D. 12 mo of 287 pages, Iog illustrations. Cloth, \$I.25 net. In Saunders' Question-Compend Series.

\section{Schäffer $\mathcal{E}$ Webster's Operative Gynecology}

Atlas and Epitome of Operative Gynecology. By Dr. O. Schaffer, of Heidelberg. Edited, with additions, by J. Clarence Webster, M. D. (Edin.), F. R. C. P. E. I 38 pages, illustrated. Cloth, \$3.00 net. In Saunders' Hand-Atlas Series.

\section{Cragin's Gynecology}

New (8th) Edition

Essentials of Gynecology. By Edwin B. CRAGIN, M.D. Revised by Frank S. Mathews, M. D. Crown octavo, 232 pages, 59 illustrations. Cloth, \$1.25 net. In Saunders' Question-Compend Series.

\section{American Pocket Dictionary}

New (9th) Edition

The American Pocket Medical Dictionary. Edited by W. A. Newman Dorland, A. M., M. D. 693 pages. \$I.25 net; with patent thumb index, \$I.5O net. 


\section{Montgomery's Care of Surgical Patients}

Care of Patients Undergoing (jynecologic and Abdominal Procedures

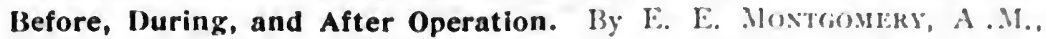
M. D., LL. D., F. A. C. S., Professor of Cynecology in Jefferson Medical College, Philadelphia. $12 \mathrm{mo}$ of 149 pages, illustrated. Cloth, $\$ 1.25$ net.

Every abdominal operation is definitely covered, and each step in it set down in detail, giving the reasons for every procedure. The duties of the nurse and the assistants are explained clearly, giving you cletailed instruction and specific fnformation on every operation in the field of gynecologic and abdominal surgery. It is a book to lessen your labor and increase your efticiency. It is pre-and post-operative care complete.

\section{Schäffer and Edgar's Obstetric Diagnosis and Treatment}

Atlas and Epitome of Obstetric Diagnosis and Treatment. By Dr. O. SchäfFER, of Heidelberg. Edited, with additions, by J. Cliftox Edgar, M. D., Professor of Obstetrics and Clinical Midwifery, Cornell University Medical School, New York. With 122 colored figures on 56 plates, 38 textcuts, and 315 pages of text. Cloth, $\$ 3.00$ net. Saunders Hand-Atlases.

\section{Schäffer and Norris' Gynecology}

Atlas and Epitome of Gynecology. By DK. O. SCHÄFFER, of Heidelberg. Edited, with additions, by RICHaRD C. Norris, A. M., M. D., Gynecologist to Methodist Episcopal and Philadelphia Hospitals. With 207 colored figures on 90 plates, $6 ;$ text-cuts, and 308 pages of text. Cloth, $\$ 3.50$ net. In Saunders' Hand-Atlas Series.

\section{Galbraith's Four Epochs of Woman's Life}

New (3d) Edition

The Four Epochs of Woman's Life: A Studr ix Hrgiexe. By Axis M. Galbraith, M. D., Fellow of the New York Academy of Medicine, etc. With an Introductory Note by JoHx H. Musser, M. D., University of Pennsylvania. I 2 mo of 296 pages. Cloth, s1. 50 net.

\section{Birmingham Medical Review, England}

"We do not, as a rule, care for medical books written for the instruction of the public. But we must admit that the advice in Dr. Galbraith's work is, in the main, wise and wholesome." 




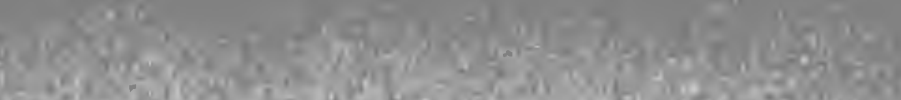

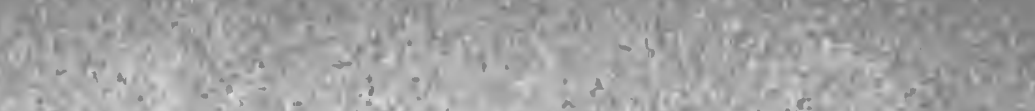
a W.

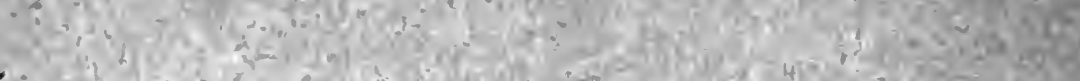
. It

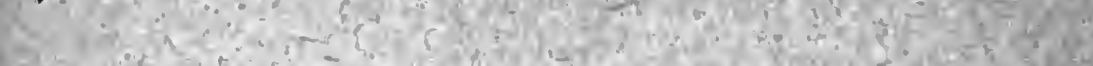
mif

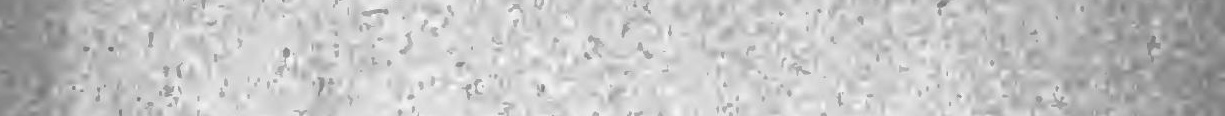

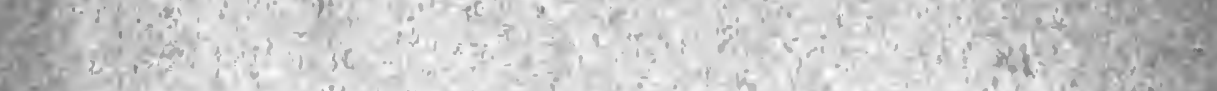

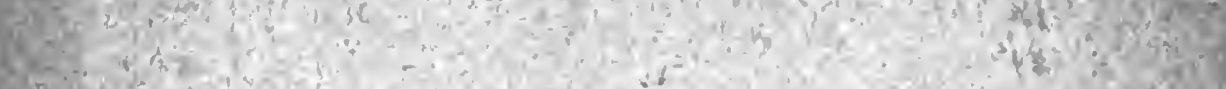

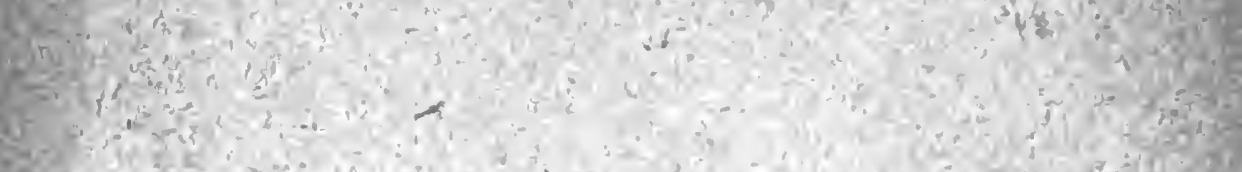

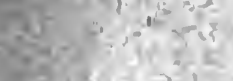
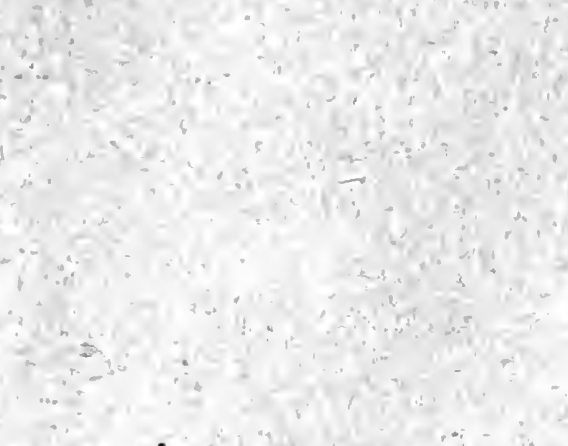



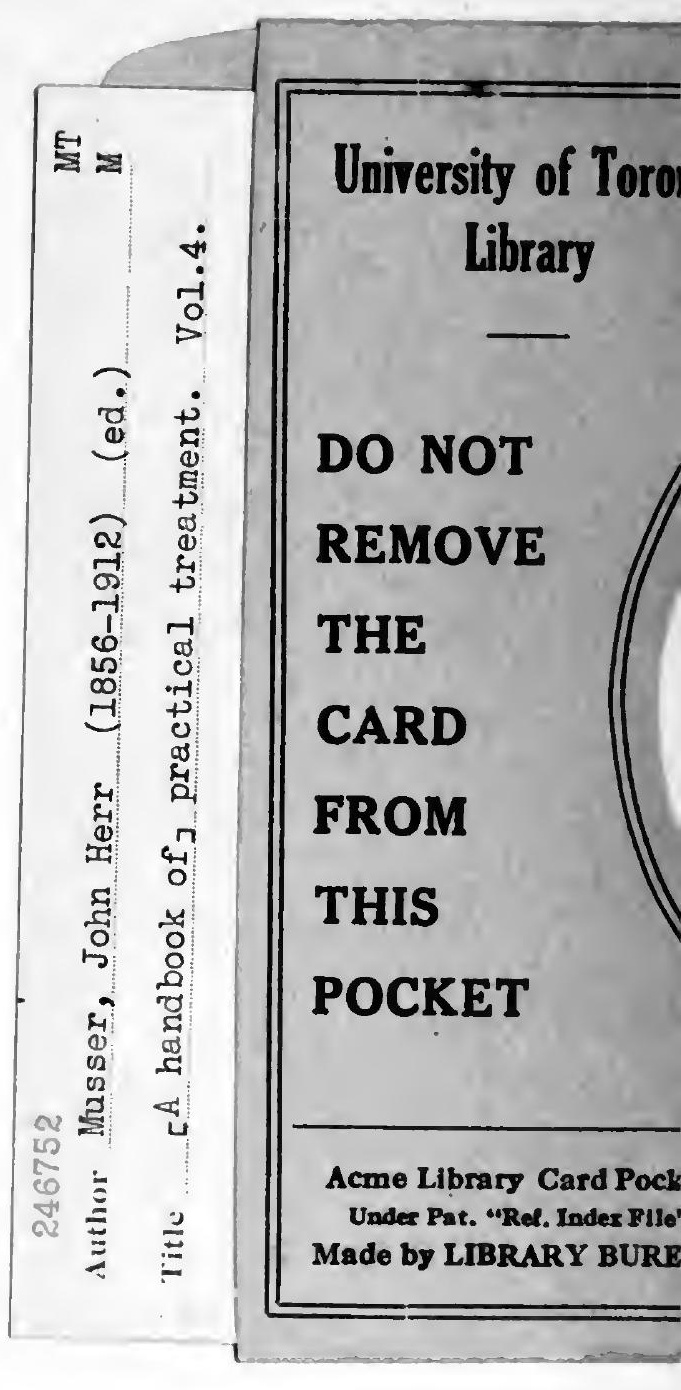


9 